\title{
Prehabilitation and Rehabilitation for Major Joint Replacement
}




\section{Prehabilitation and Rehabilitation for Major Joint Replacement}

Prepared for:

Agency for Healthcare Research and Quality

U.S. Department of Health and Human Services

5600 Fishers Lane

Rockville, MD 20857

www.ahrq.gov

Contract No. 75Q80120D00001

Prepared by:

Brown Evidence-based Practice Center

Providence, RI

Investigators:

Kristin J. Konnyu, Ph.D.

Louise M. Thoma, P.T., D.P.T, Ph.D.

Monika Reddy Bhuma, B.D.S, M.P.H.

Wangnan Cao, Ph.D.

Gaelen P. Adam, M.L.I.S., M.P.H.

Shivani Mehta, M.P.H.

Roy K. Aaron, M.D.

Jennifer Racine-Avila, M.B.A.

Orestis A. Panagiotou, M.D., Ph.D.

Dan Pinto, P.T., D.P.T., Ph.D.

Ethan M. Balk, M.D., M.P.H.

AHRQ Publication No. 21(22)-EHC033

November 2021 
This report is based on research conducted by the Brown Evidence-based Practice Center (EPC) under contract to the Agency for Healthcare Research and Quality (AHRQ), Rockville, MD (Contract No. 75Q80120D00001). The findings and conclusions in this document are those of the authors, who are responsible for its contents; the findings and conclusions do not necessarily represent the views of AHRQ. Therefore, no statement in this report should be construed as an official position of AHRQ or of the U.S. Department of Health and Human Services.

\section{None of the investigators have any affiliations or financial involvement that conflicts with the material presented in this report.}

The information in this report is intended to help healthcare decision makers - patients and clinicians, health system leaders, and policymakers, among others-make well-informed decisions and thereby improve the quality of healthcare services. This report is not intended to be a substitute for the application of clinical judgment. Anyone who makes decisions concerning the provision of clinical care should consider this report in the same way as any medical reference and in conjunction with all other pertinent information, i.e., in the context of available resources and circumstances presented by individual patients.

This report is made available to the public under the terms of a licensing agreement between the author and the Agency for Healthcare Research and Quality. This report may be used and reprinted without permission except those copyrighted materials that are clearly noted in the report. Further reproduction of those copyrighted materials is prohibited without the express permission of copyright holders.

AHRQ or U.S. Department of Health and Human Services endorsement of any derivative products that may be developed from this report, such as clinical practice guidelines, other quality enhancement tools, or reimbursement or coverage policies, may not be stated or implied.

AHRQ appreciates appropriate acknowledgment and citation of its work. Suggested language for acknowledgment: This work was based on an evidence report, Prehabilitation and Rehabilitation for Major Joint Replacement, by the Evidence-based Practice Center Program at the Agency for Healthcare Research and Quality (AHRQ).

Suggested citation: Konnyu KJ, Thoma LM, Bhuma MR, Cao W, Adam GP, Mehta S, Aaron RK, Racine-Avila J, Panagiotou OA, Pinto D, Balk EM. Prehabilitation and Rehabilitation for Major Joint Replacement. Comparative Effectiveness Review No. 248. (Prepared by the Brown Evidence-based Practice Center under Contract No. 75Q80120D00001.) AHRQ Publication No. 21(22)-EHC033. Rockville, MD: Agency for Healthcare Research and Quality; November 2021. DOI: 10.23970/AHRQEPCCER248. Posted final reports are located on the Effective Health Care Program search page. 


\section{Preface}

The Agency for Healthcare Research and Quality (AHRQ), through its Evidence-based Practice Centers (EPCs), sponsors the development of systematic reviews to assist public- and private-sector organizations in their efforts to improve the quality of healthcare in the United States. These reviews provide comprehensive, science-based information on common, costly medical conditions, and new healthcare technologies and strategies.

Systematic reviews are the building blocks underlying evidence-based practice; they focus attention on the strength and limits of evidence from research studies about the effectiveness and safety of a clinical intervention. In the context of developing recommendations for practice, systematic reviews can help clarify whether assertions about the value of the intervention are based on strong evidence from clinical studies. For more information about AHRQ EPC systematic reviews, see https://effectivehealthcare.ahrq.gov/about/epc/evidence-synthesis.

AHRQ expects that these systematic reviews will be helpful to health plans, providers, purchasers, government programs, and the healthcare system as a whole. Transparency and stakeholder input are essential to the Effective Health Care Program. Please visit the website (www.effectivehealthcare.ahrq.gov) to see draft research questions and reports or to join an email list to learn about new program products and opportunities for input.

If you have comments on this systematic review, they may be sent by mail to the Task Order Officer named below at: Agency for Healthcare Research and Quality, 5600 Fishers Lane, Rockville, MD 20857, or by email to epc@ahrq.hhs.gov.

David Meyers, M.D.

Acting Director

Agency for Healthcare Research and Quality

Craig Umscheid, M.D., M.S.

Director

Evidence-based Practice Center Program

Center for Evidence and Practice

Improvement

Agency for Healthcare Research and Quality
Arlene S. Bierman, M.D., M.S.

Director

Center for Evidence and Practice

Improvement

Agency for Healthcare Research and Quality

David W. Niebuhr, M.D., M.P.H., M.Sc.

Task Order Officer

Center for Evidence and Practice Improvement

Agency for Healthcare Research and Quality 


\section{Acknowledgments}

The authors gratefully acknowledge the following individuals for their contributions to this project: Professor Emerita Carol A. Oatis, P.T., Ph.D., from Arcadia University, Glenside, PA; Associate Editor Margaret A. Maglione, B.A., M.P.P., from the RAND Evidence-based Practice Center, Los Angeles, CA; Task Order Officer David W. Niebuhr, M.D., M.P.H., M.Sc., from AHRQ; and Christine Chang, M.D., M.P.H., Associate Director, Evidence-based Practice Center Program, from AHRQ.

\section{Key Informants}

In designing the study questions, the EPC consulted several Key Informants who represent the end-users of research. The EPC sought the Key Informant input on the priority areas for research and synthesis. Key Informants are not involved in the analysis of the evidence or the writing of the report. Therefore, in the end, study questions, design, methodological approaches, and/or conclusions do not necessarily represent the views of individual Key Informants.

Key Informants must disclose any financial conflicts of interest greater than $\$ 5,000$ and any other relevant business or professional conflicts of interest. Because of their role as end-users, individuals with potential conflicts may be retained. The TOO and the EPC work to balance, manage, or mitigate any conflicts of interest.

The list of Key Informants who provided input to this report follows:

Matt Collins, M.D., M.B.A.

Blue Cross and Blue Shield

Sherborn, MA

Patricia Franklin, M.D., M.P.H., M.B.A. ${ }^{\dagger}$

Northwestern University

Chicago, IL

Stephen Hunter, P.T., D.P.T, O.C.S., FAPTA*广

Intermountain Healthcare

Salt Lake City, UT

Amye L. Leong, M.B.A.

Healthy Motivation

Santa Barbara, CA

Jay Lieberman, M.D.

University of Southern California

Los Angeles, CA

*Provided input on Draft Report.

${ }^{\dagger}$ Also a Technical Expert Panel member.
Carol A. Oatis, P.T., Ph.D.

Arcadia University

Glenside, PA

Ajay Kumar Srivastava, M.D.* ${ }^{\dagger}$

American Academy of Orthopedic Surgeons

OrthoMichigan

Flint, MI

Brian Stello, M.D. ${ }^{\dagger}$

Lehigh Valley Health Network,

Lehigh Valley, PA

Chuck Washabaugh, Ph.D. ${ }^{\dagger}$

National Institute of Arthritis and

Musculoskeletal and Skin Diseases

Bethesda, MD

Elizabeth Wonsetler, P.T., D.P.T., Ph.D.

American Physical Therapy Association

Nashville, TN 


\section{Technical Expert Panel}

In designing the study questions and methodology at the outset of this report, the EPC consulted several technical and content experts. Broad expertise and perspectives were sought. Divergent and conflicted opinions are common and perceived as healthy scientific discourse that results in a thoughtful, relevant systematic review. Therefore, in the end, study questions, design, methodologic approaches, and/or conclusions do not necessarily represent the views of individual technical and content experts.

Technical Experts must disclose any financial conflicts of interest greater than $\$ 5,000$ and any other relevant business or professional conflicts of interest. Because of their unique clinical or content expertise, individuals with potential conflicts may be retained. The TOO and the EPC work to balance, manage, or mitigate any potential conflicts of interest identified.

The list of Technical Experts who provided input to this report follows:

John Philip Andrawis, M.D.*

Torrance

Memorial Physician Network

Torrance, CA

Karen Lohmann Siegel, P.T., M.A.*

Department of Veterans Affairs

Washington, DC

Alisa L. Curry, P.T., D.P.T., G.T.C., G.C.S.*

Elena Losina, Ph.D.

American Physical Therapy Association

Hayward, CA

Brigham and Women's Hospital

Boston, MA

Patricia Franklin, M.D., M.P.H., M.B.A.

Northwestern University

Chicago, IL

David Mino, M.D., M.B.A.

CIGNA Corporation

Washington Crossing, PA

Stephen Hunter, P.T., D.P.T, O.C.S., FAPTA*

Nicolaas P. Pronk, Ph.D., M.A.*

Intermountain Healthcare

Salt Lake City, UT

HealthPartners Institute

Minneapolis, $\mathrm{MN}$

Daver C. Kahvecioglu, Ph.D.

Brian Stello, M.D.

Center for Medicare and Medicaid Innovation

(CMMI), Centers for Medicare \& Medicaid

Services (CMS)

Bethesda MD

Lehigh Valley Health Network,

Lehigh Valley, PA

Chuck Washabaugh, Ph.D.

National Institute of Arthritis and

Ajay Kumar Srivastava, M.D.*

Musculoskeletal and Skin Diseases

American Academy of Orthopedic Surgeons

Bethesda, MD

OrthoMichigan

Flint, MI

*Provided input on Draft Report. 


\section{Peer Reviewers}

Prior to publication of the final evidence report, EPCs sought input from independent Peer Reviewers without financial conflicts of interest. However, the conclusions and synthesis of the scientific literature presented in this report do not necessarily represent the views of individual reviewers.

Peer Reviewers must disclose any financial conflicts of interest greater than $\$ 5,000$ and any other relevant business or professional conflicts of interest. Because of their unique clinical or content expertise, individuals with potential nonfinancial conflicts may be retained. The TOO and the EPC work to balance, manage, or mitigate any potential nonfinancial conflicts of interest identified.

The list of Peer Reviewers follows:

David C. Ayers, M.D.

Chair, Department of Orthopedics \&

Physical Rehabilitation Medicine

UMass Memorial Health Center

Worcester, MA

Michael Bade, P.T., D.P.T, Ph.D.

Associate Professor, Physical Therapy

(SOM)

University of Colorado Anschutz Medical

Campus

Aurora, CO

Robert G. Marx, M.D.

Professor of Orthopedic Surgery

Hospital for Special Surgery

Weill Cornell Medical College

New York, NY
Kenneth L. Miller, P.T., D.P.T.

Assistant Professor, Physical Therapy

University of North Texas Health Science

Center

Fort Worth, TX

Leif I. Solberg, M.D.

Senior Investigator, Behavioral Health

HealthPartners Institute

Minneapolis, MN 


\section{Prehabilitation and Rehabilitation for Major Joint Replacement}

\section{Structured Abstract}

Objectives. This systematic review evaluates the rehabilitation interventions for patients who have undergone (or will undergo) total knee arthroplasty (TKA) or total hip arthroplasty (THA) for the treatment of osteoarthritis. We addressed four Key Questions (KQs): comparisons of (1) rehabilitation prior ("prehabilitation") to TKA versus no prehabilitation, (2) comparative effectiveness of different rehabilitation programs after TKA, (3) prehabilitation prior to THA versus no prehabilitation, (4) comparative effectiveness of different rehabilitation programs after THA.

Data sources and review methods. We searched Medline ${ }^{\circledR}$, PsycINFO $^{\circledR}$, Embase $^{\circledR}$, the Cochrane Register of Clinical Trials, CINAHL ${ }^{\circledR}$, Scopus ${ }^{\circledR}$, and ClinicalTrials.gov from Jan 1, 2005, to May 3, 2021, to identify randomized controlled trials (RCTs) and adequately adjusted nonrandomized comparative studies (NRCSs). We evaluated clinical outcomes selected with input from a range of stakeholders. We assessed the risk of bias and evaluated the strength of evidence (SoE) using standard methods. Meta-analysis was not feasible, and evidence was synthesized and reported descriptively. The PROSPERO protocol registration number is CRD42020199102.

Results. We found 78 RCTs and 5 adjusted NRCSs. Risk of bias was moderate to high for most studies.

- $\quad$ KQ 1: Compared with no prehabilitation, prehabilitation prior to TKA may increase strength and reduce length of hospital stay (low SoE) but may lead to comparable results in pain, range of motion (ROM), and activities of daily living (ADL) (low SoE). There was no evidence of an increased risk of harms due to prehabilitation (low SoE).

- KQ 2: Various rehabilitation interventions after TKA may lead to comparable improvements in pain, ROM, and ADL (low SoE). Rehabilitation in the acute phase (initiated within 2 weeks of surgery) may lead to increased strength (low SoE) but result in similar strength when delivered in the post-acute phase (low SoE). No studies reported evidence of risk of harms due to rehabilitation delivered in the acute period following TKA. Compared with various controls, post-acute rehabilitation may not increase the risk of harms (low SoE).

- KQ 3: For all assessed outcomes, there is insufficient (or no) evidence addressing the comparison between prehabilitation and no prehabilitation prior to THA.

- KQ 4: Various rehabilitation interventions after THA may lead to comparable improvements in pain, strength, ADL, and quality of life. There is some evidence of no increased risk of harms due to the intervention (low SoE).

- There is insufficient evidence regarding which patients may benefit from (p)rehabilitation for all KQs and insufficient evidence regarding comparisons of different providers and different settings of (p)rehabilitation for all KQs. There is insufficient evidence on costs of (p)rehabilitation and no evidence on cost effectiveness for all KQs. 
Conclusion. Despite the large number of studies found, the evidence regarding various prehabilitation programs and comparisons of rehabilitation programs for TKA and THA is ultimately sparse. This is a result of the diversity of interventions studied and outcomes reported across studies. As a result, the evidence is largely insufficient or of low SoE. New high-quality research is needed, using standardized intervention terminology and core outcome sets, especially to allow network meta-analyses to explore the impact of intervention attributes on patient-reported, performance-based, and healthcare-utilization outcomes. 


\section{Contents}

Evidence Summary ............................................................................................................................. ES-1

Introduction ................................................................................................................................ 1

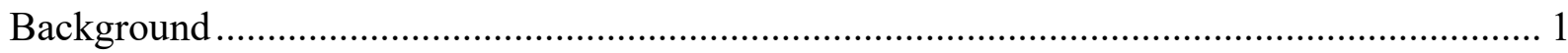

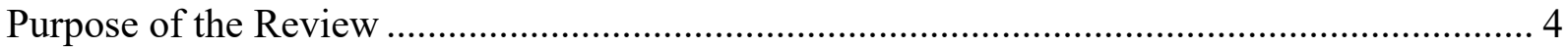

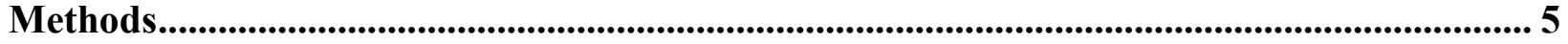

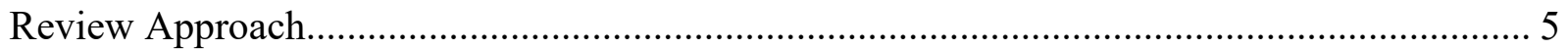

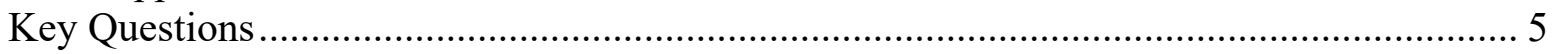

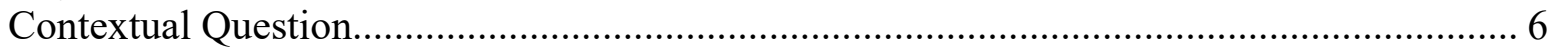

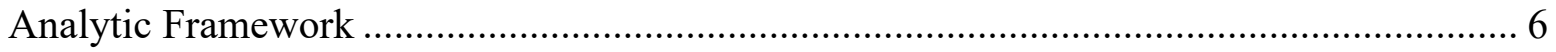

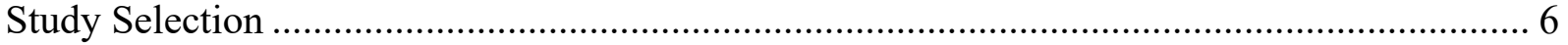

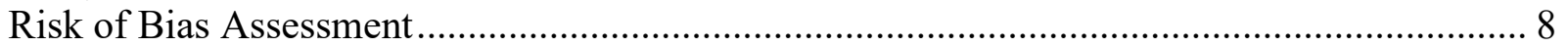

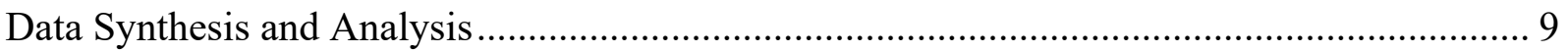

Grading the Strength of the Body of Evidence ................................................................... 9

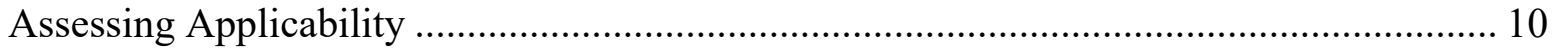

Addressing the Contextual Question ............................................................................ 10

Contextual Question ........................................................................................................................ 11

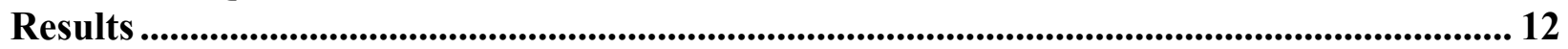

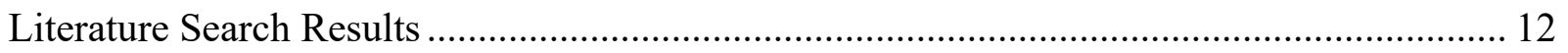

Description of Included Evidence..................................................................................... 12

Key Question 1: Prehabilitation for Total Knee Arthroplasty ………………………….......... 13

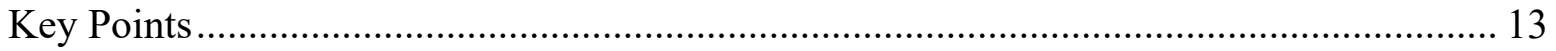

Findings Pertaining to Prehabilitation for Total Knee Arthroplasty.................................... 13

Heterogeneity of Treatment Effects (Subgroup Differences) ................................................ 39

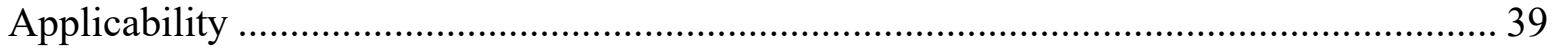

Summary of Comparison of Prehabilitation Versus No Prehabilitation for Patients

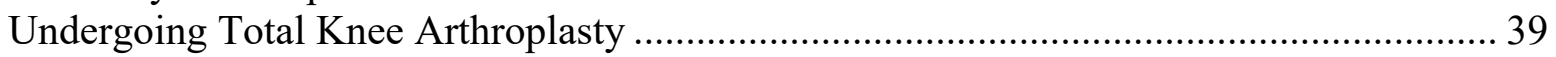

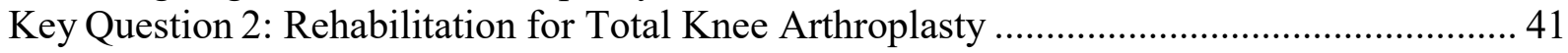

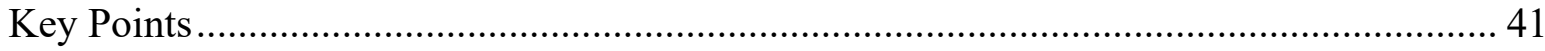

Findings Pertaining to Rehabilitation for Total Knee Arthroplasty.................................... 41

Summary of Comparison of Acute Rehabilitation Versus Various Controls for Total Knee

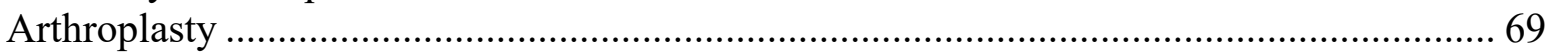

Heterogeneity of Treatment Effects (Subgroup Differences) ........................................... 119

Applicability .......................................................................................................... 119

Summary of Comparison of Rehabilitation Versus Various Controls for Total Knee

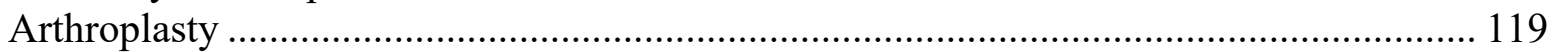

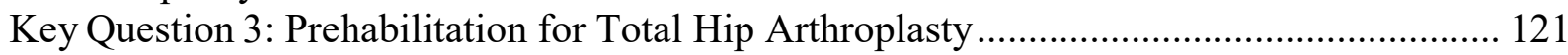

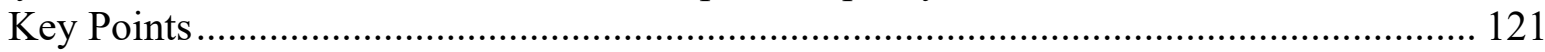

Findings Pertaining to Prehabilitation for Total Hip Arthroplasty ..................................... 121

Heterogeneity of Treatment Effects (Subgroup Differences) ............................................ 139

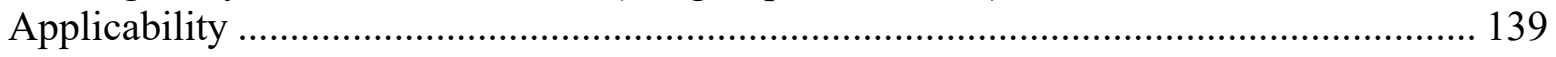

Summary of Comparison of Prehabilitation Versus No Prehabilitation for Patients

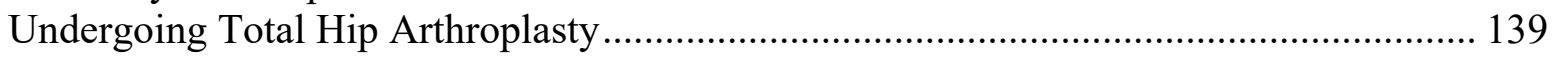

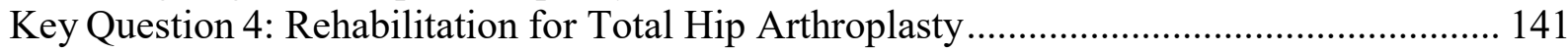

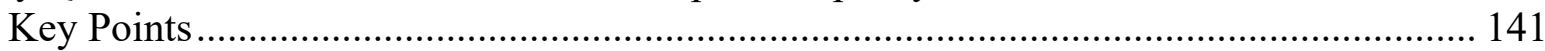

Findings Pertaining to Rehabilitation for Total Hip Arthroplasty ..................................... 141 
Heterogeneity of Treatment Effects (Subgroup Differences) ………….............................. 170

Applicability …….................................................................................................. 170

Summary of Comparison of Rehabilitation Versus Various Controls for Total Hip

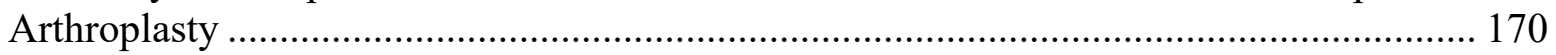

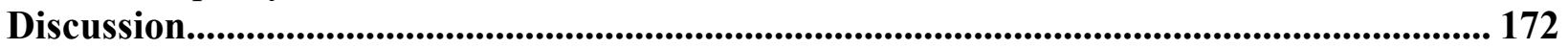

Findings in Relation to the Decisional Dilemmas ......................................................... 172

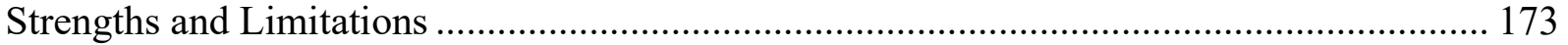

Strengths and Limitations of the Evidence Base ............................................................. 173

Strengths and Limitations of the Systematic Review Process ........................................... 174

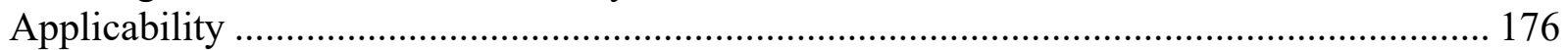

Implications for Clinical Practice ………………….................................................... 176

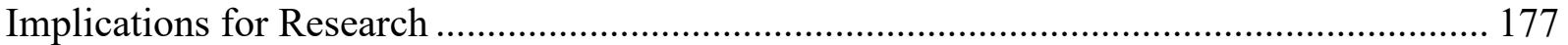

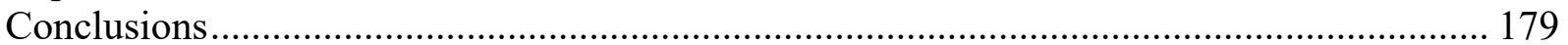

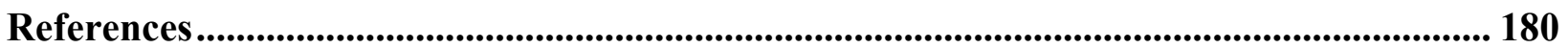

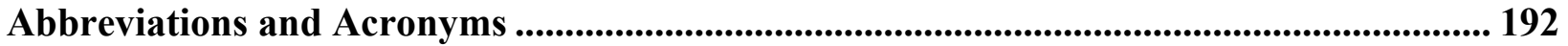

Tables

Table 1. Study eligibility criteria for Key Questions .................................................................. 7

Table 2. Number of studies addressing each Key Question, by study design .............................. 12

Table 3.1. Goal components strength, aerobic, and flexibility and their specific exercise components for prehabilitation interventions versus no prehabilitation for total knee arthroplasty

Table 3.2. Goal components balance-motor-learning-agility, task specific training, patient education, and adjunctive modalities and their specific exercise components for prehabilitation interventions versus no prehabilitation for total knee arthroplasty ................ 17

Table 4. Prehabilitation versus no prehabilitation for total knee arthroplasty - continuous

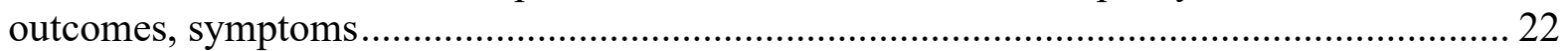

Table 5. Prehabilitation versus no prehabilitation for total knee arthroplasty - continuous

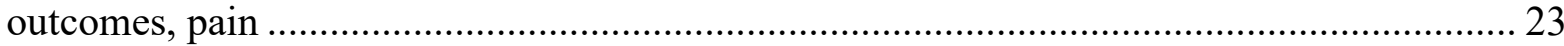

Table 6. Prehabilitation versus no prehabilitation for total knee arthroplasty - continuous

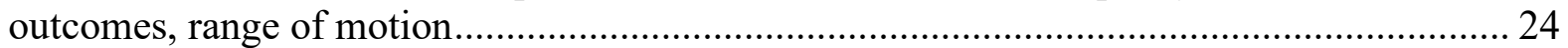

Table 7. Prehabilitation versus no prehabilitation for total knee arthroplasty - continuous

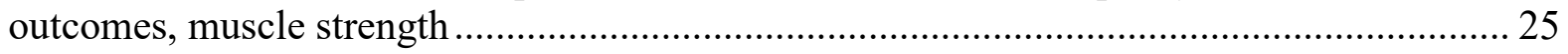

Table 8. Prehabilitation versus no prehabilitation for total knee arthroplasty - continuous

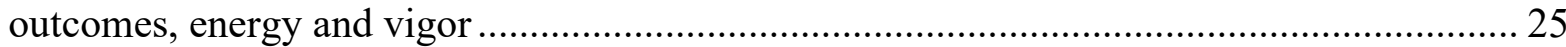

Table 9. Prehabilitation versus no prehabilitation for total knee arthroplasty - continuous outcomes, emotional functioning (stress/coping) ................................................................ 26

Table 10. Prehabilitation versus no prehabilitation for total knee arthroplasty - continuous outcomes, physical function and activities of daily living................................................... 29

Table 11. Prehabilitation versus no prehabilitation for total knee arthroplasty - continuous outcomes, repeated stand test (sit-to-stand) ......................................................................... 30

Table 12. Prehabilitation versus no prehabilitation for total knee arthroplasty - continuous

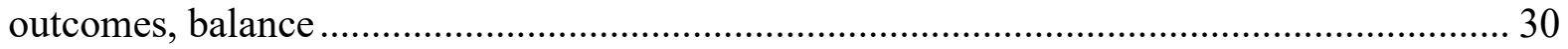

Table 13. Prehabilitation versus no prehabilitation for total knee arthroplasty - continuous

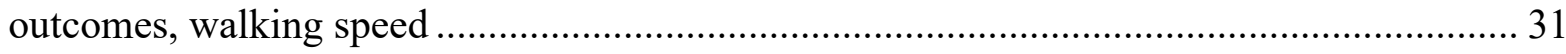


Table 14. Prehabilitation versus no prehabilitation for total knee arthroplasty - continuous

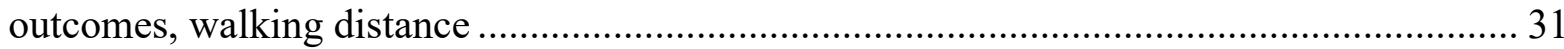

Table 15. Prehabilitation versus no prehabilitation for total knee arthroplasty - continuous

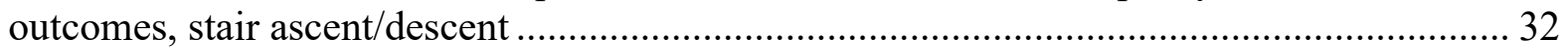

Table 16. Prehabilitation versus no prehabilitation for total knee arthroplasty - continuous

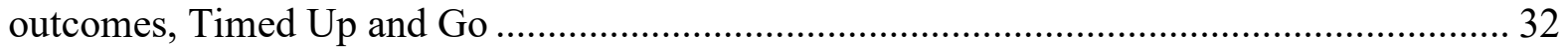

Table 17. Prehabilitation versus no prehabilitation for total knee arthroplasty - continuous

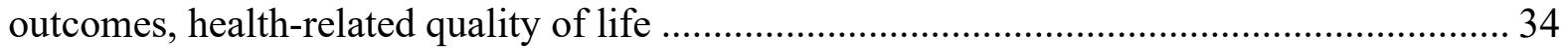

Table 18. Prehabilitation versus no prehabilitation for total knee arthroplasty - continuous outcomes, patient global assessment........................................................................... 35

Table 19. Prehabilitation versus no prehabilitation for total knee arthroplasty - continuous

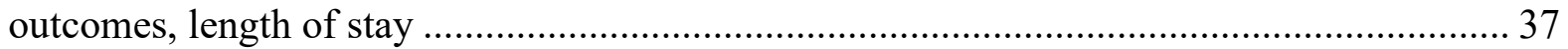

Table 20. Prehabilitation versus no prehabilitation for total knee arthroplasty - categorical outcomes, need for postoperative procedures .......................................................... 37

Table 21. Prehabilitation versus no prehabilitation for total knee arthroplasty - continuous outcomes, other healthcare utilization outcomes ......................................................... 38

Table 22. Evidence profile: Prehabilitation versus no prehabilitation for total knee arthroplasty 40

Table 23.1. Goal components strength, aerobic, and flexibility and their specific exercise components for acute-rehabilitation intervention versus various controls for total knee

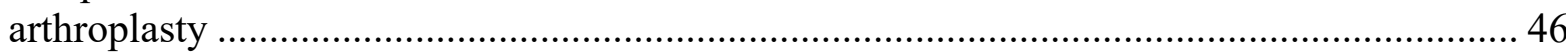

Table 23.2. Goal components balance-motor-learning-agility, task specific training, patient education, and adjunctive modalities and their specific exercise components for acuterehabilitation intervention versus various controls for total knee arthroplasty......

Table 24. Acute rehabilitation versus various controls for total knee arthroplasty - continuous

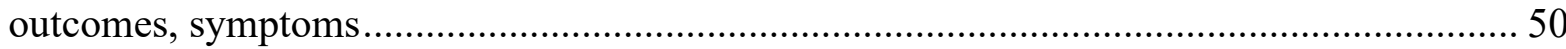

Table 25. Acute rehabilitation versus various controls for total knee arthroplasty - continuous outcomes, pain 6

able 26. Acute rehabilitation versus various controls for total knee arthroplasty - continuous

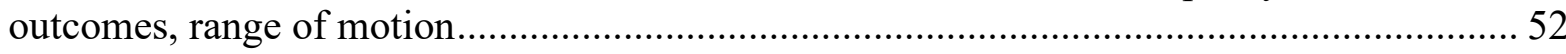

Table 27. Acute rehabilitation versus various controls for total knee arthroplasty - continuous

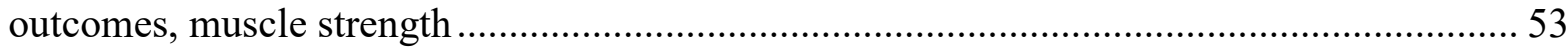

Table 28. Acute rehabilitation versus various controls for total knee arthroplasty - continuous outcomes, emotional functioning (stress/coping) .................................................... 55

Table 29. Acute rehabilitation versus various controls for total knee arthroplasty - continuous outcomes, physical function and activities of daily living ............................................ 58

Table 30. Acute rehabilitation versus various controls for total knee arthroplasty - continuous outcomes, transfers

Table 31. Acute rehabilitation versus various controls for total knee arthroplasty - continuous outcomes, balance

Table 32. Acute rehabilitation versus various controls for total knee arthroplasty - continuous outcomes, mobility.....

Table 33. Acute rehabilitation versus various controls for total knee arthroplasty - continuous outcomes, Timed Up and Go

Table 34. Acute rehabilitation versus various controls for total knee arthroplasty - continuous outcomes, quality of life 
Table 35. Acute rehabilitation versus various controls for total knee arthroplasty - continuous

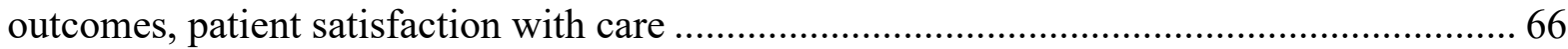

Table 36. Acute rehabilitation versus various controls for total knee arthroplasty - continuous outcomes, patient global assessment.

Table 37. Acute rehabilitation versus various controls for total knee arthroplasty - categorical

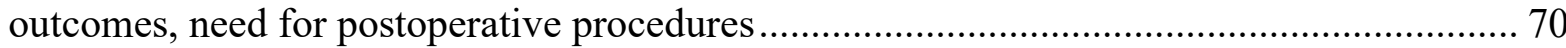

Table 38. Acute rehabilitation versus various controls for total knee arthroplasty - continuous

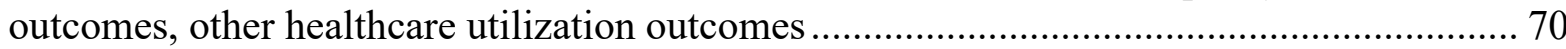

Table 39. Evidence profile: Acute rehabilitation versus various controls for total knee arthroplasty

Table 40.1. Goal components strength, aerobic, and flexibility and their specific exercise components for post-acute-rehabilitation interventions (part 1) versus various controls for total knee arthroplasty.

Table 40.2. Goal components balance-motor-learning-agility, task specific training, patient education, and adjunctive modalities and their specific exercise components for post-acuterehabilitation interventions (part 1) versus various controls for total knee arthroplasty ....... 76

Table 41.1. Goal components strength, aerobic, and flexibility and their specific exercise components for post-acute-rehabilitation interventions (part 2) versus various controls for total knee arthroplasty....

Table 41.2. Goal components balance-motor-learning-agility, task specific training, patient education, and adjunctive modalities and their specific exercise components for post-acuterehabilitation interventions (part 2) versus various controls for total knee arthroplasty ........ 78

Table 42. Post-acute rehabilitation versus various controls for total knee arthroplasty continuous outcomes, symptoms ..................................................................... 83

Table 43. Post-acute rehabilitation versus various controls for total knee arthroplasty continuous outcomes, pain

Table 44. Post-acute rehabilitation versus various controls for total knee arthroplasty continuous outcomes, range of motion

Table 45. Post-acute rehabilitation versus various controls for total knee arthroplasty continuous outcomes, muscle strength and function

Table 46. Post-acute rehabilitation versus various controls for total knee arthroplasty continuous outcomes, energy and vigor.

Table 47. Post-acute rehabilitation versus various controls for total knee arthroplasty continuous outcomes, emotional functioning .....

Table 48. Post-acute rehabilitation versus various controls for total knee arthroplasty continuous outcomes, physical function and activities of daily living...

Table 49. Post-acute rehabilitation versus various controls for total knee arthroplasty continuous outcomes, transfers

Table 50. Post-acute rehabilitation versus various controls for total knee arthroplasty continuous outcomes, balance

Table 51. Post-acute rehabilitation versus various controls for total knee arthroplasty continuous outcomes, mobility

Table 52. Post-acute rehabilitation versus various controls for total knee arthroplasty continuous outcomes, Timed Up and Go.

Table 53. Post-acute rehabilitation versus various controls for total knee arthroplasty continuous outcomes, quality of life. 
Table 54. Post-acute rehabilitation versus various controls for total knee arthroplasty continuous outcomes, satisfaction with care.

Table 55. Post-acute rehabilitation versus various controls for total knee arthroplasty continuous outcomes, patient global assessment.

Table 56. Post-acute rehabilitation versus various controls for total knee arthroplasty - need for postoperative procedures

Table 57. Post-acute rehabilitation versus various controls for total knee arthroplasty - harms 117

Table 58. Evidence profile: Post-acute rehabilitation versus various controls for total knee arthroplasty

Table 59. Goal components and their specific exercise components for prehabilitation interventions versus no prehabilitation for total hip arthroplasty....

Table 60. Prehabilitation versus no prehabilitation for total hip arthroplasty - continuous outcomes, symptoms....

Table 61. Prehabilitation versus no prehabilitation for total hip arthroplasty - continuous outcomes, pain

Table 62. Prehabilitation versus various controls for total hip arthroplasty - continuous

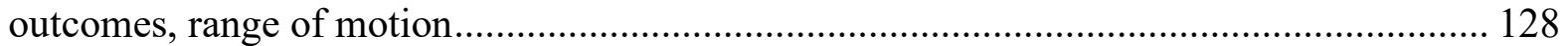

Table 63. Prehabilitation versus various controls for total hip arthroplasty - continuous outcomes, muscle strength ................................................................................. 128

Table 64. Prehabilitation versus no prehabilitation for total hip arthroplasty - continuous

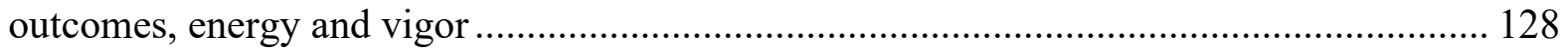

Table 65. Prehabilitation versus no prehabilitation for total hip arthroplasty - continuous outcomes, emotional functioning (stress/coping).....

Table 66. Prehabilitation versus no prehabilitation for total hip arthroplasty - continuous outcomes, patient-reported physical function and activities of daily living

Table 67. Prehabilitation versus no prehabilitation for total hip arthroplasty - continuous outcomes, transfers

Table 68. Prehabilitation versus no prehabilitation for total hip arthroplasty - continuous outcomes, balance

Table 69. Prehabilitation versus no prehabilitation for total hip arthroplasty - continuous outcomes, mobility...

Table 70. Prehabilitation versus no prehabilitation for total hip arthroplasty - continuous outcomes, Timed Up and Go .....

Table 71. Prehabilitation versus no prehabilitation for total hip arthroplasty - continuous outcomes, quality of life

Table 72. Prehabilitation versus no prehabilitation for total hip arthroplasty - continuous outcomes, length of stay

Table 73. Prehabilitation versus no prehabilitation for total hip arthroplasty - continuous outcomes, other healthcare utilization outcomes....

Table 74. Evidence profile: Prehabilitation versus no prehabilitation for total hip arthroplasty 140

Table 75.1. Goal components strength, aerobic, and flexibility and their specific exercise components for rehabilitation interventions versus various controls for total hip arthroplasty

Table 75.2. Goal components balance-motor-learning-agility, task specific training, patient education, and adjunctive modalities and their specific exercise components for rehabilitation interventions versus various controls for total hip arthroplasty.... 146 
Table 76. Rehabilitation versus various controls for total hip arthroplasty - continuous outcomes, symptoms

Table 77. Rehabilitation versus various controls for total hip arthroplasty - continuous outcomes, pain.

Table 78. Rehabilitation versus various controls for total hip arthroplasty - continuous outcomes, range of motion

Table 79. Rehabilitation versus various controls for total hip arthroplasty - continuous outcomes, muscle strength

Table 80. Rehabilitation versus various controls for total hip arthroplasty - continuous outcomes, energy and vigor 156

Table 81. Rehabilitation versus various controls for total hip arthroplasty - continuous outcomes, emotional functioning (stress/coping)...................................................................... 156

Table 82. Rehabilitation versus various controls for total hip arthroplasty - continuous outcomes, physical function and activities of daily living

Table 83. Rehabilitation versus various controls for total hip arthroplasty - continuous outcomes, transfers 162

Table 84. Rehabilitation versus various controls for total hip arthroplasty - continuous outcomes, mobility 162

Table 85. Rehabilitation versus various controls for total hip arthroplasty - continuous outcomes, Timed Up and Go..... 163

Table 86. Rehabilitation versus various controls for total hip arthroplasty - continuous outcomes, quality of life 165

Table 87. Rehabilitation versus various controls for total hip arthroplasty - categorical outcomes, satisfaction with care

Table 88. Rehabilitation versus various controls for total hip arthroplasty - continuous outcomes, patient global assessment 166

Table 89. Rehabilitation versus various controls for total hip arthroplasty - categorical outcomes, harms from rehabilitation. 169

Table 90. Evidence profile: Rehabilitation versus various controls for total hip arthroplasty ... 171

\section{Figures}

Figure 1. Overview of studies of prehabilitation and no prehabilitation interventions for total knee arthroplasty....

Figure 2. Overview of studies of acute-phase rehabilitation interventions and various controls for total knee arthroplasty....

Figure 3. Overview of studies of post-acute phase rehabilitation interventions for total knee arthroplasty 74

Figure 4. Overview of studies of prehabilitation interventions for total hip arthroplasty ......... 123

Figure 5. Overview of studies of acute and post-acute rehabilitation interventions versus various controls for total hip arthroplasty

\section{Appendixes}

Appendix A. Methods

Appendix B. Excluded Studies

Appendix C. Search Results, Study Design, Arm Details, Baseline Characteristics, and Risk of Bias Assessments 


\section{Evidence Summary}

\section{Main Points}

- Prehabilitation for Total Knee Arthroplasty

- Compared with no prehabilitation, prehabilitation prior to total knee arthroplasty (TKA), may reduce length of hospital stays and increase in strength but may lead to comparable outcomes of pain, range of motion, and activities of daily living (ADL) after TKA (low strength of evidence [SoE] for all).

- Prehabilitation prior to TKA may not increase the risk of harms (low SoE).

- There is insufficient evidence regarding the impact of prehabilitation on quality of life (QoL) or need for postoperative procedures.

- There is no evidence on patient's satisfaction with care after prehabilitation or the impact of prehabilitation on posthospital disposition.

- Rehabilitation for Total Knee Arthroplasty

- Compared with various controls (usually less intensive active rehabilitation), rehabilitation in the acute phase after TKA (initiated within 2 weeks of surgery) may result in increased strength (low SoE) and similar satisfaction with care (low SoE), whereas rehabilitation delivered in the post-acute phase may result in comparable strength (low SoE). Rehabilitation in the acute and post-acute phase after TKA may result in comparable pain, range of motion (ROM), and ADL (low SoE). Additionally, rehabilitation in the post-acute phase after TKA may result in comparable QoL.

- There is insufficient evidence on the impact on QoL (for acute rehabilitation), satisfaction with care (for post-acute rehabilitation), and the need for postoperative procedures (both acute and post-acute rehabilitation).

- No studies addressed the risk of harms due to rehabilitation delivered in the acute phase after TKA. Compared with various controls, there was no evidence of an increased risk of harms due to more active rehabilitation delivered in the post-acute phase (low SoE).

- Prehabilitation for Total Hip Arthroplasty

○ There is insufficient evidence on the impact of prehabilitation prior to total hip arthroplasty (THA) on pain, strength, ADL, QoL, length of stay, or posthospital disposition.

- No studies compared prehabilitation to no rehabilitation on satisfaction with care or risk of harms due to prehabilitation.

- Rehabilitation for Total Hip Arthroplasty

- Compared with various controls, rehabilitation in the acute and post-acute phase after THA may result in comparable pain, strength, QoL, and ADL (low SoE).

○ There is insufficient evidence regarding the impact of rehabilitation on satisfaction with care or ROM.

- Compared with various less active rehabilitation controls or no rehabilitation, rehabilitation following THA may not lead to increased risk of harms (low SoE).

- All Evidence

- There is insufficient evidence regarding which patients may most benefit from (p)rehabilitation for TKA or THA. 
- There is insufficient evidence on the effectiveness of specific (p)rehabilitation intervention components at the level of goals (e.g., strength, flexibility) or the presence of specific exercise components to address these goals for TKA or THA.

$\circ$ There is insufficient evidence regarding comparisons of different providers of (p)rehabilitation for TKA or THA.

○ There is insufficient evidence regarding comparisons of different settings of (p)rehabilitation for TKA THA.

\section{Background and Purpose}

Total joint replacement, which includes total knee arthroplasty and total hip arthroplasty, is one of the most successful therapies to manage pain and dysfunction of the hip and knee joints for end-stage osteoarthritis. As the prevalence of osteoarthritis has increased, the numbers of TKAs and THAs have increased and are now the most common inpatient surgical procedures covered by Medicare. Patients may be offered rehabilitation prior to surgery (i.e., "prehabilitation") or after surgery, with the goal of optimizing postoperative function, reducing pain, and returning to normal ADL. The topic of prehabilitation and rehabilitation (hereafter "(p)rehabilitation") is of interest to health systems to enable evidence-based decision making regarding which interventions should be offered to adults undergoing TKA or THA for osteoarthritis to achieve best clinical outcomes, reduce avoidable complications or joint failures, and be cost- and resource-effective for the health system, patients, and their caregivers.

This systematic review (SR) aims to inform healthcare systems, guideline developers, orthopedic surgeons, physical therapists and other rehabilitation professionals and providers of care for patients who have undergone (or will undergo) TKA or THA for osteoarthritis about (p)rehabilitation options. The SR addresses four Key Questions (KQs): (1) prehabilitation for TKA, (2) rehabilitation for TKA (3) prehabilitation for THA, (4) and rehabilitation for THA.

\section{Methods}

We used methods consistent with Agency for Healthcare Research and Quality Evidencebased Practice Center Program Methods Guidance (https://effectivehealthcare.ahrq.gov/topics/cer-methods-guide/overview). The protocol was developed with input from stakeholders on a Key Informants and a Technical Expert Panel, including Learning Health Systems sponsors. Our searches targeted randomized controlled trials (RCTs) and adequately adjusted nonrandomized comparative studies (NRCSs) from January 1, 2005, to May 3, 2021. We extracted intervention details into Excel and all other study data into the Systematic Review Data Repository (SRDR). The evidence base was too heterogenous to allow for meta-analysis. We assessed the risk of bias and evaluated the SoE using standard methods. The PROSPERO protocol registration number is CRD42020199102.

\section{Results}

We found 83 primary studies comprising 14,533 patients in total. These included 78 RCTs $(n=8,397$ patients) and 5 adjusted NRCSs $(n=6,156$ patients). Studies were of mostly moderate risk of bias, primarily related to a lack of blinding. The studies were highly heterogeneous. With only two exceptions, studies reported a unique (p)rehabilitation intervention and a wide range of disparate outcomes. The majority of both prehabilitation and rehabilitation interventions included components to increase strength ( $86 \%$ of studies) and flexibility (75\%) and, to a lesser 
extent, components to increase task-specific training (67\%) and balance (41\%). Studies varied widely in terms of the timing and intensity of the evaluated (p)rehabilitation interventions.

- Prehabilitation for TKA: Thirteen RCTs evaluated prehabilitation for TKA. Compared with no prehabilitation, prehabilitation may lead to increased strength and reduced lengths of acute hospital stays following TKA surgery (low SoE). Prehabilitation may result in comparable pain, range of motion, and activities of daily living (low SoE). There is insufficient evidence regarding the impact of prehabilitation on QoL or need for postoperative procedures and no evidence addressing satisfaction with care or posthospital disposition outcomes associated with prehabilitation prior to TKA. Prehabilitation prior to TKA may not increase the risk of harms (low SoE).

- Comparison of Rehabilitation Interventions for TKA: Forty-nine RCTs and 4 NRCSs evaluated various rehabilitation interventions and comparators following TKA. Various rehabilitation programs in the acute and post-acute phase following TKA may result in comparable improvements in outcomes of pain, ROM, and ADL (low SoE). Acute-phase rehabilitation programs resulted in similar satisfaction with care (low SoE for all). More intensive rehabilitation (e.g., via virtual rehabilitation or with neuromuscular stimulation) may result in increased strength when delivered in the acute phase. More intensive rehabilitation led to similar outcomes of strength among rehabilitation programs delivered in the post-acute phase. There is insufficient evidence on the impact on QoL (for acute rehabilitation), satisfaction with care (for post-acute rehabilitation), and the need for postoperative procedures (both acute and post-acute rehabilitation). We found no evidence regarding harms from acute-phase rehabilitation. Post-acute rehabilitation may have comparable risks of harms among various rehabilitation interventions compared (low SoE).

- Prehabilitation for THA: Six RCTs evaluated prehabilitation for THA. There is insufficient evidence for various patient-reported, performance-based, and healthcareutilization outcomes when comparing prehabilitation to no prehabilitation prior to THA.

- Comparison of Rehabilitation Interventions for THA: Fourteen RCTs and one NRCS evaluated rehabilitation for THA. Rehabilitation in the acute and post-acute phase following THA may result in comparable improvements in patients experience of pain and QoL and performance of strength and ADLs (low SoE). There is insufficient evidence for ROM and satisfaction with care. There is no evidence of increased risk of harm from rehabilitation interventions compared with less active rehabilitation or no rehabilitation controls (low SoE).

\section{Limitations}

Although we found a large body of mostly RCT evidence, the evidence was ultimately sparse since relatively few studies reported the same outcomes pertaining to similar comparisons. With the exception of two interventions evaluated in two studies each, all studies reported unique (p)rehabilitation interventions. Reporting of intervention content was also highly variable, ranging from a few words (e.g., "inpatient rehabilitation") to comprehensive (p)rehabilitation protocols. This variability made coding of intervention content challenging. Thus, evidence regarding prehabilitation (compared with no prehabilitation) and rehabilitation interventions (compared with other rehabilitation interventions) is largely insufficient or of low SoE. Very limited subgroup data was reported, precluding most evaluation of heterogeneity of treatment effects (differences in effect across subgroups). The included studies were mostly at moderate to 
high risk of bias. Several prioritized outcomes, including strength, ROM, satisfaction with care, and QoL, were infrequently reported.

\section{Implications and Conclusions}

Our analysis of all prehabilitation and rehabilitation interventions for TKA and THA found no clear evidence of the effectiveness of prehabilitation versus no prehabilitation, or the comparative effectiveness of diverse rehabilitation programs compared with each other. However, there was some evidence of improved outcomes in specific (p)rehabilitation programs, and a strength of this review is its thorough standardized extraction and synthesis of all (p)rehabilitation interventions. In the absence of definitive evidence on which programs to implement, stakeholders may need to rely on other decision-making factors to decide which (p)rehabilitation program to implement or evaluate. Our detailed categorization of the components of (p)rehabilitation interventions and how they were delivered could be used to guide the efforts to better standardize and improve the evidence base. A strategic and coordinated program of research is needed to address the questions related to (p)rehabilitation, specifically to identify which components of interventions work best and under what circumstances (e.g., setting, personnel, or modes of delivery). To improve interpretation and allow for future meta-analyses, researchers (and funders of research) should consider the use of standardized terminology of intervention content and core outcome sets to measure intervention effects, combined with a universal expectation of robust and transparent reporting of both. Future studies should also consider collecting data on the direct and indirect costs of (p)rehabilitation programs and conduct cost-effectiveness analyses alongside effectiveness analyses to contribute a more complete evidentiary picture to inform evidence-based decision-making regarding which interventions should be offered to adults undergoing TKA or THA for osteoarthritis. 


\section{Introduction}

\section{Background}

Osteoarthritis is a leading cause of joint disability in the United States. Approximately 54 million people ( $23 \%$ of adults) have osteoarthritis, and, of these, 24 million are limited in their daily activities due to osteoarthritis. ${ }^{1}$ Total joint replacement — total knee arthroplasty (TKA) and total hip arthroplasty (THA) - have been some of the most successful therapies in managing pain and dysfunction of hip and knee joints for end-stage arthritis. ${ }^{2-5}$ Patients who have undergone a TKA or THA experience reduced pain and improved function and quality of life. ${ }^{2,6,7}$ As the prevalence of osteoarthritis has increased, the numbers of TKAs and THAs have correspondingly increased, ${ }^{8}$ and they are now the most common inpatient surgical procedures covered by Medicare. ${ }^{9}$ In 2014, an estimated 680,150 patients in the United States underwent a TKA and 370,770 underwent a THA. It is expected that by 2030, 1.26 million patients will undergo a TKA annually (an 85\% increase from 2014) and 635,000 will undergo a THA (71\% increase). ${ }^{10}$

THA involves the removal of the femoral head and part of the femoral neck, the reshaping of the acetabulum and the replacement of the joint with prosthetics made of titanium or cobalt chrome alloy (femoral stem) and either cobalt chrome or ceramic (femoral head). To minimize wear, a variety of materials have been used for the acetabular component of the prosthesis, including metal, ceramic, and cross-linked high-density polyethylene. ${ }^{11-16}$ Newer highly crosslinked polyethylenes have been most successful of these materials and are most commonly used. 13, 17-19 TKA, on the other hand, does not involve replacement of the entire joint but rather is a resurfacing of the diseased bone and cartilage to provide a new bearing surface for the joint. The muscles, ligaments, and capsule are left in place, although they may be adjusted. The arthritic cartilage and bone at the distal head of the femur are replaced by cobalt chrome and the bearing surfaces of the patella and tibia are covered in polyethylene. Due to the more delicate alignment of the knee joint and the greater soft tissue manipulation necessary in TKA compared with THA, there is greater potential for scarring after TKA and the rehabilitation burden is typically greater. Surgical approaches are fairly standardized for TKA. The joint is entered anteromedial to the extensor mechanism and patella. In contrast, for THA, there are three approaches: anterior, anterolateral, and posterior. ${ }^{20-22}$ Although each has its unique peculiarities, advantages, and disadvantages, overall, the outcomes from all three are similar.

While most patients experience improvements in pain after TKA or THA, deficits in functional performance and strength commonly persist a year after surgery for many patients. ${ }^{23-26}$ Full recovery of muscle strength and physical function to a normal level is rare. ${ }^{27,28}$ Approximately $20 \%$ of patients who undergo TKA report dissatisfaction a year after TKA. ${ }^{29}$ Physical rehabilitation is commonly offered to patients undergoing either TKA or TKA with the goal of optimizing postoperative outcomes, including strength, physical function, pain reduction, and return to normal activities of daily living. Increasingly, "prehabilitation" (rehabilitation services provided prior to surgery) is also considered to maximize patients' functional status prior to surgery to improve postoperative outcomes.

The topic of prehabilitation and rehabilitation (hereafter "(p)rehabilitation") is of interest to health systems to enable evidence-based decision making regarding which interventions should be offered to adults undergoing TKA or THA for osteoarthritis to achieve best clinical outcomes, reduce avoidable complications or joint failures, and be cost- and resource-effective for the health system, patients, and their caregivers. The Agency for Healthcare Research and Quality's Learning Health System Panel nominated this topic as being of particular interest. 
Rehabilitation programs are complex interventions that incorporate multiple specific interventions (components) and multiple actors, including various rehabilitation specialists and personnel, orthopedic surgeons and other clinicians, other caregivers, and the patient. The provided rehabilitation services can occur at different times (i.e., before or after surgery) and in different settings. Furthermore, rehabilitation is commonly personalized for individuals, depending on their specific circumstances and their response to surgery and the rehabilitation. These factors are likely to be important determinants of the effectiveness of the rehabilitation programs to improve strength and mobility, reduce pain, and return the patient to their bestpossible overall function. Nevertheless, they all have cost and resource use implications related to the features of the rehabilitation program, the personnel involved, and the location of services. Thus, there are four interrelated considerations at play in deciding on a rehabilitation program:

1. Timing: Rehabilitation can occur prior to surgery ("prehabilitation") or after

2. Type: Rehabilitation may comprise various types or components (e.g., strength training, education, balance training) delivered alone or in combination

3. Setting: Rehabilitation may occur in various settings (e.g., acute inpatient, skilled nursing facility, outpatient rehabilitation facility, home-based)

4. Cost and resource use: Each aspect of a rehabilitation program has its associated costs (e.g., due to specialized personnel, equipment, facility overhead)

Regarding timing, rehabilitation after TKA or THA is the most common practice in the United States. Previous reviews have reported that successful rehabilitation improves pain control, walking and gait, balance, and strength, and reduces length of stay. ${ }^{30,31}$ Though less commonly used, prehabilitation has also been recommended. ${ }^{32}$ Preoperative health status is a strong predictor of favorable postoperative outcomes ${ }^{2,33}$ including reduced pain and improved functioning. ${ }^{34}$ Thus, prehabilitation is hypothesized to accelerate improvement of function and strength postoperatively. ${ }^{35}$ However, the effectiveness of prehabilitation prior to TKA or THA is unclear. A 2017 systematic review on prehabilitation for TKA or THA found conflicting evidence, with some studies finding no added benefit and others finding improvements in postoperative function, quadriceps strength, and length of stay. ${ }^{36} \mathrm{~A}$ subsequent review of prehabilitation for patients about to undergo TKA found prehabilitation was associated with decreased length of stay while limited data suggested prehabilitation made no difference on patient-reported outcomes of pain or function, stiffness, and physical role. ${ }^{37}$

Prehabilitation and rehabilitation are complex interventions, with many facets that are typically individualized for given patients' needs, goals, capabilities, and even personalities. The type of (p)rehabilitation includes various concepts, including what the intervention is, how it is implemented, and who delivers it. The overall intervention program may include various components (typically in combination) that are aimed at improving a range of body function and structure, as well as activity and participation domains, defined by the International

Classification of Functioning, Disability and Health (ICF) ${ }^{38}$ to improve the mobility and stability of joint function, movement control, power and tone of muscles, gait, endurance; along with the related goal of reducing pain. Examples of components may include stretching exercises to improve muscle movement and range of motion; use of weights to improve muscle tone, power and endurance; and stair-climbing to improve mobility, muscle power, and movement control. In addition to the components that directly impact the ICF domains, numerous adjunctive modalities are employed to facilitate the performance of components to help the patient better achieve their rehabilitation goals. Examples of these include cryotherapy to reduce swelling and 
acute pain; mindfulness programs to reduce stress, anxiety, and pain; neuromuscular electrical stimulation to increase muscle control and coordination and biofeedback devices to provide positive feedback and incentives. Each component, adjunctive modality, and the intervention as a whole (i.e., comprised of components and adjunctive modalities) may vary in how it is delivered, for example, the duration of each session (and the use of each component within a session), the intensity of the intervention (e.g., minimal vs. maximal extension of range of motion, fewer vs. more repetitions), and the overall duration of the program (e.g., 1 week vs. 2 months). Further complicating the types of (p)rehabilitation, the overall intervention (or the various components) may be delivered by different professionals, ancillary trained personnel, or the patients (or their caregivers) themselves.

Given the wide range of potential activities that may fall within the concept of (p)rehabilitation, it is challenging to determine even what counts as a form of (p)rehabilitation. Based on the primary interests of the nominating health systems, we focus on structured interventions that involve health professionals or other trained individuals. Thus, for the purpose of this review, we defined ( $p$ )rehabilitation to be active, structured physical activities designed to attain measurable goals of improving impairments and movement-related function as defined by the ICF. ${ }^{38}$ The intervention must be delivered, supervised, and/or monitored by a healthcare professional or other trained individual, and the patient must be actively involved (i.e., not simply a passive recipient). Pharmaceutical and over-the counter interventions were outside the scope of interest.

Another important aspect of (p)rehabilitation is the setting in which it may be delivered. These vary widely, and examples include the acute hospital setting (i.e., immediately postoperative), a skilled nursing facility, outpatient rehabilitation facility, the patient's home, or a local community center or gym. Interventions may also be delivered virtually, by Internet, teleconference, Web app, or mobile device app. Various factors contribute to the choice of the (p)rehabilitation setting, including patient needs, caregiver support, and the specific components employed. Cost of the (p)rehabilitation services, which is largely determined by setting, plays a major role in decisions about which services to provide. In the inpatient setting, "accelerated" rehabilitation after TKA has been associated with reduced length of stay in the acute-hospital setting. ${ }^{39}$ The impact of setting of (p)rehabilitation programs on patient outcomes is unclear, with some research suggesting facility-based rehabilitation does not provide better recovery compared to home-based programs for uncomplicated TKA and THA patients, ${ }^{40}$ and other evidence suggesting early outpatient rehabilitation may lead to more rapid gains in function, strength, and reduced pain in the short term. ${ }^{23}$ Insurance status may influence patient preference for setting of care, such that patients under public insurance may be more likely to utilize home-based rehabilitation programs (supervised or unsupervised) over more resource-intensive facility-based rehabilitation programs (including inpatient rehabilitation and outpatient-based sessions). ${ }^{40}$ Although facility-based programs are more likely to have skilled, supervised personnel deliver services, evidence on the added benefit of a supervised program remains unclear. ${ }^{41,42}$

The total direct costs of care for TKA and THA episodes include the preoperative period (for assessment and, if used, prehabilitation), operative (acute) and postoperative (post-acute) periods, with as much as 40 percent of the cost occurring after discharge $(70 \%$ of which are from post-acute care facilities). ${ }^{43}$ Both operative and postoperative costs (for the health systems, payers, and patients) vary greatly throughout the United States without notable differences in outcomes. As much as a sixfold difference has been observed in cost of care (from the perspective of the health system) for patients discharged to various types of post-acute care, ${ }^{44}$ 
despite similarities in patient characteristics, readmission, and complication rates. Total joint replacement of the lower extremity (both THA and TKA) was the most prevalent clinical episode participating in the Centers for Medicare \& Medicaid Services bundled payment model. ${ }^{9}$ As major joint replacement surgeries and bundled payment models become more prevalent, ${ }^{45}$ many health systems and payers are working to understand how best to implement the most effective and also most cost-effective care for patients receiving TKA or THA without compromising their outcomes. $^{46-48}$

\section{Purpose of the Review}

This systematic review assesses prehabilitation and rehabilitation for patients who are undergoing (or have undergone) elective, unilateral, total knee or hip replacement surgery for osteoarthritis. Specifically, the review addresses:

- The benefits and harms of preoperative active structured physical activity programs (and specific components) for TKA (Key Question [KQ] 1) and THA (KQ 3)

- The comparative benefits and harms of postoperative active structured physical activity programs (and specific components) for TKA (KQ 2) and THA (KQ 4).

As the evidence base for rehabilitation is more established, the objective of KQs 2 and 4 are focused on the comparative effectiveness of different rehabilitation programs (i.e., which programs and/or components and methods by which they are delivered work best to improve outcomes). As the evidence base for prehabilitation is less established, the objective of KQs 1 and 3 is to determine the benefit of prehabilitation in general (prehabilitation vs. no prehabilitation) as well as the comparative effectiveness of different prehabilitation programs (i.e., which programs and/or components and method in which they are delivered work best to improve outcomes).

The intended audience for this systematic review includes healthcare systems, guideline developers, orthopedic surgeons, physical therapists and other rehabilitation professionals and providers of care for patients who have undergone (or will undergo) TKA or THA for osteoarthritis and are considering (p)rehabilitation, as well as patients and their caregivers. It is expected that the findings will inform individual professional practice and health system decision making for ( $\mathrm{p}$ )rehabilitation care surrounding total knee or hip replacement surgery. 


\section{Methods}

\section{Review Approach}

The Brown Evidence-based Practice Center conducted this systematic review (SR) based on the Agency for Healthcare Research and Quality (AHRQ) Methods Guide for Effectiveness and Comparative Effectiveness Reviews (available at https://effectivehealthcare.ahrq.gov/topics/cermethods-guide/overview). This SR also reports in accordance with the Preferred Items for Reporting in Systematic Reviews and Meta-Analyses (PRISMA), ${ }^{49}$ A Measurement Tool to Assess Systematic Reviews (AMSTAR 2), ${ }^{50}$ and any relevant extension statements.

A more detailed version of the SR methodology used can be found in Appendix A.

The topic of this report and preliminary Key Questions (KQs) arose through a process involving the nominator (AHRQ's Learning Health System Panel), a panel of Key Informants (KI), a Technical Expert Panel (TEP), the public, and AHRQ. The TEP provided high-level content and methodological expertise throughout development of the review protocol. The final protocol was posted on the Effective Health Care website at https://effectivehealthcare.ahrq.gov/products/major-joint-replacement/protocol on July 14, 2020, and revised and reposted on October 6, 2020. We submitted the protocol for registration in PROSPERO in July 2019. On August 16, 2020, PROSPERO published the protocol with registration number CRD42020199102.

\section{Key Questions}

KQ 1: What are the effects, comparative effects, and harms of (preoperative) prehabilitation services (and specific components) for patients with osteoarthritis undergoing elective, unilateral total knee replacement surgery on patient-reported outcomes, performance-based outcomes, and healthcare utilization?

$K Q$ 2: What are the effects, comparative effects, and harms of (postoperative) rehabilitation services (and specific components) for patients with osteoarthritis undergoing elective, unilateral total knee replacement surgery on patient-reported outcomes, performance-based outcomes, and healthcare utilization?

KQ 3: What are the effects, comparative effects, and harms of (preoperative) prehabilitation services (and specific components) for patients with osteoarthritis undergoing elective, unilateral total hip replacement surgery on patient-reported outcomes, performance-based outcomes, and healthcare utilization? 
KQ 4: What are the effects, comparative effects, and harms of (postoperative) rehabilitation services (and specific components) for patients with osteoarthritis undergoing elective, unilateral total hip replacement surgery on patient-reported outcomes, performance-based outcomes, and healthcare utilization?

For all KQs:

Subquestion a: Do the effects, comparative effects, and harms vary by patient factors, such as age, sex, race/ethnicity, socioeconomic status, body mass index, and comorbidities?

Subquestion b: Do the effects, comparative effects, and harms vary by surgical factors, such as surgical procedure, type of implant, perioperative protocol, type of hospital, and length of hospital stay?

Subquestion c: Do the effects, comparative effects, and harms vary by setting of active structured physical activity programs?

\section{Contextual Question}

Contextual Question: What are the major direct and indirect cost factors for the various aspects of rehabilitation and prehabilitation around major joint replacement surgery, including such factors as personnel, setting overhead, materials, and training?

\section{Analytic Framework}

Based on discussions with KIs and TEP, we developed an analytic framework (Appendix A Figure A-1) capturing the key elements of all KQs in one figure. It graphically lays out the populations, interventions, outcomes, and modifiers as they pertain to all KQs and, where applicable, specific KQs.

\section{Study Selection}

Literature searches were conducted in MEDLINE ${ }^{\circledR}$ (via PubMed), PsycINFO ${ }^{\circledR}$, Embase $^{\circledR}$, The Cochrane Register of Clinical Trials, CINAHL ${ }^{\circledR}$, and Scopus ${ }^{\circledR}$, restricted to 2005 through May 3, 2021. The time restriction was included to account for temporal trends related to changes in surgical techniques, implants, anesthesia, and, in particular, postoperative protocols (e.g., enhanced recovery after surgery protocol and rapid hospital discharge) more commonly employed since about $2000 .^{51}$ While there is no clear cutoff date to mark a practice changing shift in care, 2005 was selected as a reasonable date before which the KIs agreed that studies would be less generalizable to contemporary practice. 
Table 1 presents the major eligibility criteria for each KQ. More detailed criteria are presented in Appendix A. We included randomized controlled trials (RCT) and nonrandomized comparative studies (NRCS) with appropriate adjustment for confounding factors.

Table 1. Study eligibility criteria for Key Questions

\begin{tabular}{|c|c|}
\hline Eligibility Categories & Criteria \\
\hline Population & $\begin{array}{l}\text { Adult patients undergoing (or planning to undergo) elective non-revision, unilateral total } \\
\text { knee or hip replacement surgery for primary osteoarthritis } \\
\text { KQs } 1 \text { \& 3: Patients for whom the decision has been made to have TKA/THA surgery }\end{array}$ \\
\hline Intervention/Comparator & 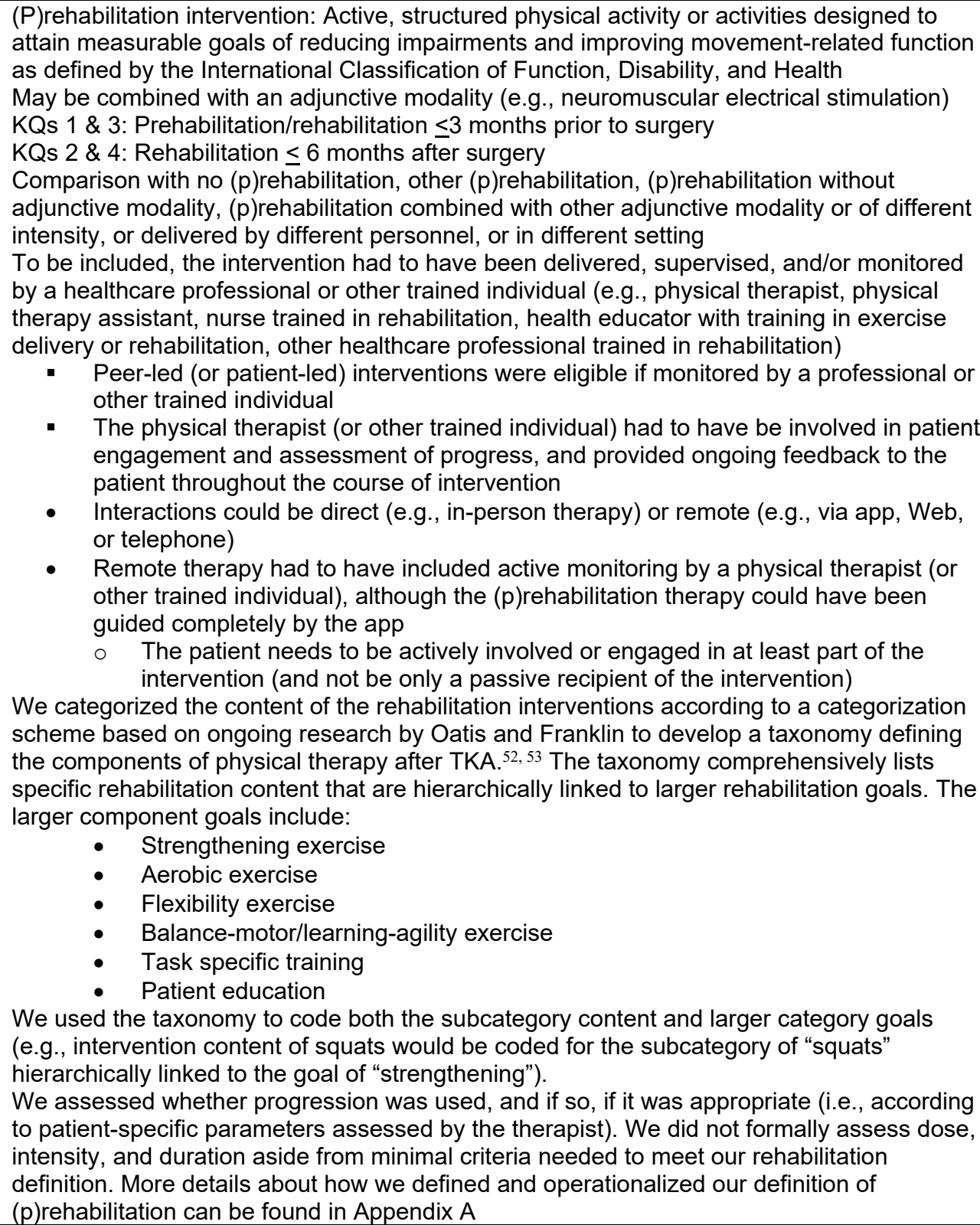 \\
\hline
\end{tabular}




\begin{tabular}{|c|c|}
\hline Eligibility Categories & Criteria \\
\hline Outcomes & 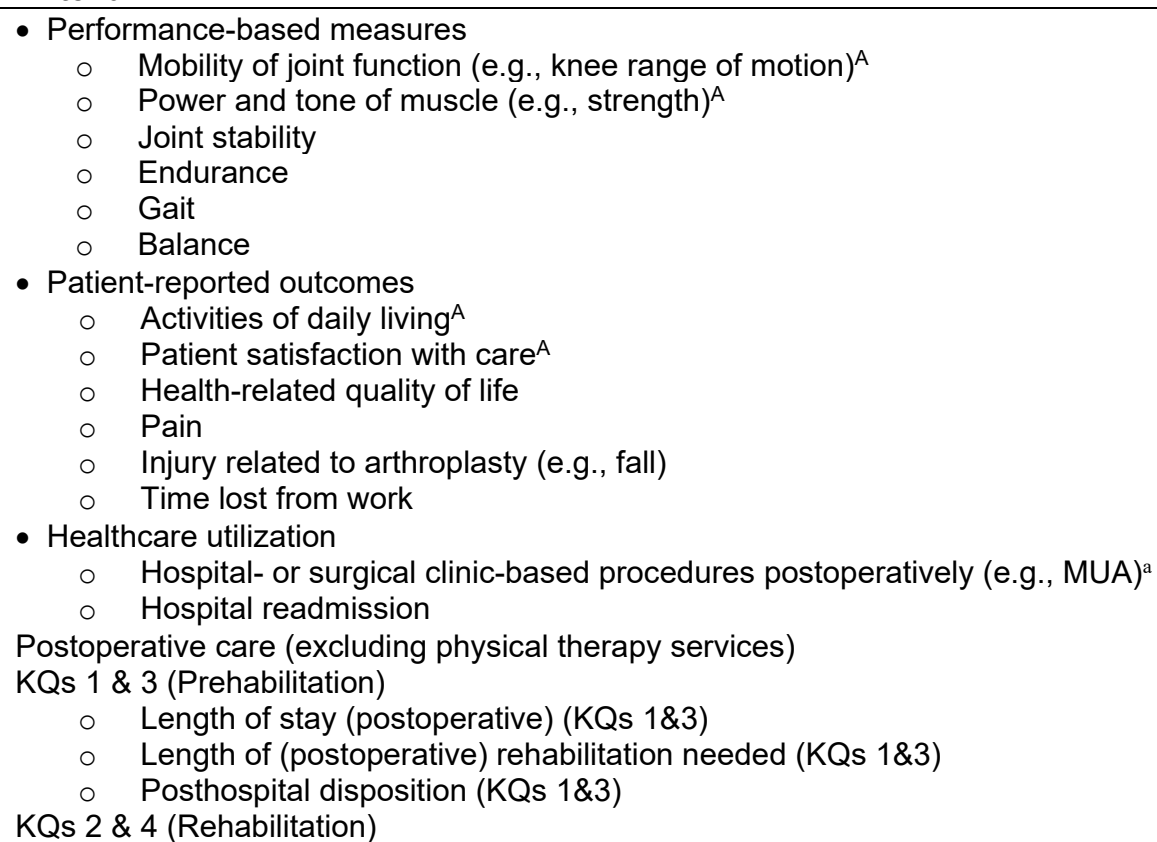 \\
\hline Design & $\begin{array}{l}\text { RCT: } N \geq 20 / a m^{B} \\
\text { NRCS: Restrict to studies that use analytic methods to minimize selection bias } \\
\quad N \geq 20^{B, C} \\
\text { Cost-effectiveness analyses }\end{array}$ \\
\hline
\end{tabular}

Abbreviations: $\mathrm{KQ}=$ Key Question, MUA $=$ manipulation under anesthesia, $\mathrm{NRCS}=$ nonrandomized comparative study, $\mathrm{RCT}=$ randomized controlled trial, THA $=$ total hip arthroplasty, TKA $=$ total knee arthroplasty

${ }^{\text {A }}$ Denotes important/priority outcomes that are included in strength of evidence tables.

${ }^{B}$ Minimum sample size cutoffs were employed to restrict the evidence base to studies that were more likely to be powered to detect differences in outcomes between arms while also being balanced for important baseline characteristics (in RCTs; otherwise, sufficient sample to allow for adjustment of baseline characteristics in NRCSs). The sample size of 20 or more per arm is not a concrete benchmark for achieving these aims, but rather, was chosen in consideration of the evidence base and with input from stakeholders.

${ }^{\mathrm{C}}$ Restricted to studies that use modeling or other analytic methods to minimize selection bias (due to inherent differences between people who receive one or the other intervention) or that restrict study eligibility criteria such that comparisons being made are between patients with similar presentations.

\section{Risk of Bias Assessment}

We evaluated each study for risk of bias and methodological quality. Because we included different study designs, we incorporated items from three different commonly used tools and tailored the set of items for each study design.

For RCTs, we used all the items from the Cochrane Risk of Bias tool, ${ }^{54}$ focusing on issues related to randomization and allocation concealment methodology; blinding of patients, study personnel/care providers, objective outcome assessors, and subjective outcome assessors; incomplete outcome data; selective outcome reporting; and other issues that could be related to bias. We supplemented the Cochrane Risk of Bias tool with items from the National Heart, Lung, and Blood Institute (NHLBI) tool that pertain to the adequacy of descriptions of study eligibility criteria, interventions, and outcomes. ${ }^{55}$

For NRCSs, we used the specific sections of Risk of Bias In Non-randomised Studies - of Interventions (ROBINS-I) tool ${ }^{56}$ that pertain to confounding and selection bias. ROBINS-I requires the identification of specific confounders of interest for the systematic review. For the 
purpose of assessing for the presence of potential confounding in studies, we considered demographics (such as age, sex, race/ethnicity), socioeconomic status, caregiver support, body mass index, comorbidities, prior arthroplasty of contralateral joint, narcotic use, preoperative symptoms/status (e.g., severity of symptoms including pain, impaired function, restricted movement, physical activity, frailty), surgical factors (e.g., surgical procedure or protocol, type of implant), and hospital type for all KQs. Additionally, for KQs 2 and 4 related to postoperative care, we considered length of hospital stay as an additional potential confounder.

\section{Data Synthesis and Analysis}

We had planned to summarize the evidence both qualitatively and, when feasible, quantitatively (via a network meta-analysis across the programs, focusing on comparisons of different components). However, due to the heterogeneity of interventions (i.e., almost completely unique content in (p)rehabilitation intervention and comparator arms, delivered at different times, in different settings, and by different personnel) and the lack of consistency of outcomes (e.g., different scales and metrics reported), meaningful statistical meta-analyses were not feasible, and we summarized the evidence only qualitatively.

Each study included in the systematic review is described in summary and evidence tables presenting study design features, study participant characteristics, descriptions of interventions, outcome results, and risk of bias/methodological quality. Summary tables briefly describe the studies and their findings.

For all KQs, we compared interventions with their comparators for their effects (grouping related interventions and comparisons as feasible), using post mean differences in continuous outcome data (i.e., difference in follow-up mean between groups) or net mean differences (i.e., difference-in-difference, or the between-intervention comparison of within-intervention changes). As there were not sufficient studies reporting sufficiently similar outcomes to explore the association of the specific intervention factors (components, personnel, setting), using statistical methods, we sought to explore associations narratively across studies by considering each of the factors as a 'lens' of potential impact when looking at the evidence.

\section{Grading the Strength of the Body of Evidence}

We summarized the strength of evidence (SoE), addressing each major comparison for each KQ. As the evidence base for KQs 1, 3, and 4 was relatively small, we summarized the evidence base for these questions as a whole. For KQ 2 we summarized the evidence base for acuterehabilitation and post-acute rehabilitation for total knee arthroplasty (TKA). We graded the strength of the body of evidence as per the Agency for Healthcare Research and Quality (AHRQ) Methods Guide on assessing SoE. ${ }^{57,58}$ Further details are provided in the Methods Appendix A. We assessed SoE for each of the important patient and clinical outcome categories (starred outcomes in Eligibility Criteria outcome lists). We determined the relative importance of the outcomes with input from the TEP. The outcome categories for which SoE was assessed include activities of daily living; patient satisfaction with care; quality of life; mobility of joint function (e.g., knee range of motion); power of muscle (e.g., strength); hospital- or surgical clinic-based procedures postoperatively (e.g., need for manipulation under anesthesia); and injury related to therapy intervention. Additionally, for KQs 1 and 3 related to prehabilitation, we included: length of stay (postoperative) and posthospital disposition (e.g., to home, outpatient, skilled nursing facility, "subacute" rehabilitation, "acute" inpatient rehabilitation). The prioritized outcome domains are consistent with the "core outcome sets" recommended by Osteoarthritis 
Research Society International (OARSI) ${ }^{59}$ and the Total Joint Arthroplasty and Outcome Measures (TJAOM) ${ }^{60}$ toolkits, published in 2013 and 2014, respectively. Core outcome sets are agreed minimum sets of outcomes that should be reported in research in a given topic area. ${ }^{61}$

For each SoE assessment, we considered the number of studies, their designs and limitations (i.e., risk of bias and overall methodological quality), the directness of the evidence to the KQs (direct/indirect), the consistency (consistent/inconsistent) of study results, the precision (precise/imprecise) of any estimates of effect, the likelihood of reporting bias, other limitations, and the overall findings across studies. We also assessed the extent to which different (p)rehabilitation interventions were replicated within each KQ (replicated/not replicated interventions). Based on these assessments, we assigned a SoE rating as being either high, moderate, low, or insufficient evidence to estimate an effect.

Outcomes with highly imprecise estimates, highly inconsistent findings across studies, or with data from only one study (or in some cases, where a small number of all unique interventions contributed evidence to the outcome) were deemed to have insufficient evidence to allow for a conclusion (with the exception that a particularly large and generalizable single study could provide at least low SoE). This approach is consistent with the concept that for imprecise evidence "any estimate of effect is very uncertain," the definition of Very Low quality evidence per the Grading of Recommendations, Assessment, Development, and Evaluations (GRADE) approach. ${ }^{62}$ Outcomes for which we could make a conclusion but have limited confidence (i.e., it would not be unexpected for future studies to alter conclusions) due to limited number of studies, inconsistent findings, and/or poor replication of interventions were given low a SoE.

We summarized the data sources, basic study characteristics, and each SoE dimensional rating in an "Evidence Profile" table. This table details our reasoning for arriving at the overall SoE rating.

\section{Assessing Applicability}

For each KQ (or specific subquestion), we assessed the applicability of the included studies to adults having TKA or total hip arthroplasty (THA) in the United States. Applicability was primarily based on the studies' eligibility criteria and their included participants, specifically related to such factors as age, sex, and frailty, or comorbidities. We also considered operative procedures used and perioperative and postoperative care in relation to their applicability to contemporary practice, although these details were inconsistently reported.

\section{Addressing the Contextual Question}

Based on data and input garnered during our systematic review of the KQs, we answer the Contextual Question in a narrative format. In particular, we assessed the cost-effectiveness analyses regarding information about direct and indirect costs. We did not systematically extract or review eligible studies, create summary tables, or assess the strength of evidence for the Contextual Question. 


\section{Contextual Question}

The evidence was insufficient to comment on the costs, both indirect and direct, associated with prehabilitation and rehabilitation programs. Of particular note, we found no costeffectiveness analyses regarding prehabilitation or rehabilitation. Despite this, many studies noted cost as a justification for their study question - particularly studies of rehabilitation after total knee arthroplasty and total hip arthroplasty that evaluated the safety and effectiveness of rehabilitation delivered in lower resource settings or by lower resource personnel (as compared with interventions delivered in higher resource settings or by higher resource personnel. 


\section{Results}

\section{Literature Search Results}

The electronic literature search yielded 22,361 unique citations. A total of 83 unique primary studies met criteria. Appendix B provides a list of excluded studies. Appendix Figure C-1 summarizes the results of the search and screening processes. The 83 included studies were reported in 98 articles that were published between 2005 (based on our eligibility criteria) and 2021. The 83 studies comprised 78 randomized controlled trials (RCTs) and 5 nonrandomized comparative studies (NRCSs). The 83 included studies enrolled a total of 14,533 patients: 78 RCTs with 8,397 patients (ranging from 20 to 212 patients each) and 5 NRCSs with 6,156 patients (ranging from 68 to 1,213 patients each).

Table 2 summarizes the number of studies that addressed each Key Question (KQ), by study design. In general, there was a larger evidence base for total knee arthroplasty (TKA) compared with total hip arthroplasty (THA) and for prehabilitation compared with rehabilitation. Seventeen percent of the studies (13/78) addressed KQ 1 (prehabilitation for TKA). All studies addressing KQ 1 were RCTs. Sixty-three percent of the studies (49/78) addressed KQ 2 (rehabilitation for TKA) which was further categorized into rehabilitation in the acute phase after TKA (initiated within 2 weeks of surgery) ( $\mathrm{n}=18$ studies; $15 \mathrm{RCTs}, 3 \mathrm{NRCSs}$ ) and the post-acute phase (initiated 2 weeks or later following surgery) ( $\mathrm{n}=31$ studies; 30 RCTs, 1 NRCS). Eight percent of studies (6/78) addressed KQ3. All studies addressing KQ 3 were RCTs. Finally, 18 percent of the studies (14/78) addressed KQ 4 (prehabilitation for THA) (13 RCTS, 1 NRCS). These were further categorized into rehabilitation in the acute phase further THA (4 RCTs) and the postacute phase (9 RCTs, 1 NRCS).

Table 2. Number of studies addressing each Key Question, by study design

\begin{tabular}{|l|l|l|l|l|l|}
\hline Design & KQ 1 & KQ 2 & KQ 3 & KQ 4 & Total \\
\hline Randomized controlled trials & 13 & 49 & 6 & 14 & $78^{*}$ \\
\hline Nonrandomized comparative studies & 0 & 4 & 0 & 1 & 5 \\
\hline Total & 13 & 53 & 6 & 15 & $83^{*}$ \\
\hline
\end{tabular}

* Some randomized controlled trials addressed multiple Key Questions (KQs): 1 study included for both KQs 1 and 2, 1 study included for both KQs 1 and 3, 2 studies included for both KQs 2 and 4)

For all 83 studies, Appendix Tables C-1 to C-4 summarize the design, arm, and patient characteristics, intervention details and risk of bias assessments (separate sub-tables by study design. as risk of bias assessments for RCTs and NRCSs are reported separately). Further details about the literature search, included studies, and excluded studies (with reasons for their exclusion) are in Appendixes A and B.

\section{Description of Included Evidence}

Detailed findings, including tables for study designs, arms, and sample characteristics; and risk of bias are in the Results Appendix C. For each KQ, we grouped and reported outcomes under four larger categories: Body structure and function outcomes; Activity and participation outcomes; Other patient-reported outcomes; and Healthcare-utilization outcomes. The first three of these categories (and the outcomes that comprised within them) were informed by the International Classification of Functioning, Disability, and Health framework as operationalized by a group of US- and Canadian-based TKA and THA experts (clinicians, researchers, and 
patients). Where relevant, we call attention to specific appendix table numbers in the relevant subsections.

\section{Key Question 1: Prehabilitation for Total Knee Arthroplasty}

\section{Key Points}

- Compared with no prehabilitation, prehabilitation prior to TKA may reduce length of hospital stays and increase strength but may lead to comparable outcomes of pain, range of motion, and activities of daily living (ADL) after TKA (low strength of evidence [SoE] for all).

- Prehabilitation prior to TKA may not increase the risk of harms (low SoE).

- There is insufficient evidence regarding the impact of prehabilitation on quality of life (QoL) or need for postoperative procedures.

- There is no evidence on patient satisfaction with care after prehabilitation or the impact of prehabilitation on posthospital disposition.

- Given the heterogeneity of interventions and outcomes across studies, there is insufficient evidence on the effectiveness of specific prehabilitation intervention components at the level of goals (e.g., strength, flexibility) or presence of specific exercise components to address these goals.

- There is insufficient evidence on how the effect of prehabilitation programs may vary by patient, surgical, or setting factors.

- There is no evidence on the cost effectiveness of prehabilitation for TKA.

\section{Findings Pertaining to Prehabilitation for Total Knee Arthroplasty}

We found 13 eligible studies, all RCTs, that compared some version of a prehabilitation intervention to no prehabilitation (defined variably as "usual care", "activities as usual", or some form of minimal patient education). We rated seven of these 13 studies to be at overall high risk of bias, mostly related to lack of blinding of participants, study personnel, and/or outcome assessors and unclear methods of how random sequences were generated and/or concealed from patients. We rated the remaining six RCTs to be at overall moderate risk of bias mostly related to lack of blinding of participants, study personnel, and/or outcome assessors.

The 13 RCTs enrolled between 45 and 243 participants each. Most ( $n=9 ; 69 \%$ ) were conducted in Europe. Two trials were conducted in the United States, one in Malaysia, and one in Taiwan. Two RCTs (Topp 2009 and Villadsen 2014) ${ }^{63,64}$ were funded in part by companies that produce rehabilitation and orthopedic equipment. The average ages of participants were similar across studies, ranging from 63 to 72 years. The percentage of women in the studies varied between 27 and 82 percent. Average body mass indices (BMIs) ranged from 27 to 33 $\mathrm{kg} / \mathrm{m}^{2}$. In a subset of three studies that reported data, prior contralateral TKA ranged from 10 to 30 percent. Appendix Tables C-1.1, C-1.2, and C-1.3 include the full data for all 13 RCTs.

\section{Prehabilitation Versus No Prehabilitation}

Thirteen RCTs, reported in 15 articles, ${ }^{63-76}$ compared prehabilitation to no prehabilitation in a total of 1,328 patients who would undergo TKA (summarized in Figure 1). Only two specific prehabilitation interventions were evaluated by more than one study (two studies each) ${ }^{64,67,68,71}$; the remaining nine studies evaluated unique prehabilitation interventions comprised of varying 
goals and exercise components (as coded by our taxonomy) in different combinations, delivered in varying settings (by different modalities) by diverse personnel. Huber 2015 and Villadsen 2014 evaluated the neuromuscular training program (NEMEX-TJR) (targeting strength, aerobic endurance, flexibility, balance, task-specific training, and patient education) to patient education alone. ${ }^{a}$ Huang 2012 and Matassi 2014 evaluated a prehabilitation program targeting strength, flexibility, and patient education goals with a no prehabilitation control.

Prehabilitation interventions were initiated between 2 and 12 weeks prior to the scheduled TKA. Most prehabilitation interventions in these 13 RCTs included components to target strength $(n=11 / 13$ prehabilitation arms) and flexibility $(n=10 / 13)$, followed by components to address task-specific training $(n=7 / 13)$, balance-motor-learning-agility $(n=5 / 13)$, and patient education $(n=5 / 13)$. Aerobic exercise was present in only two of prehabilitation programs. Specific exercise components within prehabilitation goal components varied across programs. Only one study included an adjunctive modality (acupuncture) in combination with the prehabilitation program as compared with no prehabilitation. ${ }^{74}$ While one other study also assessed the effect of acupuncture, it was assessed alone as a single modality compared with standard rehabilitation or home exercise. Because the modality (acupuncture) did not meet our eligibility criteria of co-occurring with a prehabilitation program, the specific acupuncture arm is not discussed further. ${ }^{76}$ Six studies reported some form of progression (i.e., any change in exercise type, repetition, or load consistent with an increase in exercise intensity or difficulty), of which three were assessed by clinical experts on our team as appropriate (i.e., the progression was based on patient-specific or individualized parameters as opposed to a universal, time-based progression). ${ }^{64,65,68,69}$ No study compared prehabilitation with versus without progression.

Interventions were delivered by physical therapists in nine of the 13 studies. In other studies, the intervention was delivered by research personnel (Topp 2009), no one (i.e., unsupervised self-guided home component) (Huang 2012, Matassi 2014, Topp 2009), or this information was not reported (Valtonen 2015). Except in Matassi 2014, the prehabilitation interventions had at least part of the program delivered to patients in-person: six in outpatient rehabilitation facilities, three in both outpatient and home environments (with either some remote support or a selfguided component), one exclusively at home, one at an aquatic center, and one in an unclear setting. Matassi 2014 was designed to be self-guided at home.

Specific codes for intervention (and control arm, where present) goals and exercises, use of progression (and assessment of appropriateness), and details on personnel, mode of delivery, and setting are detailed in Tables 3.1 and 3.2 and Appendix Table C-2.3.

The heterogeneity of the included prehabilitation interventions (varying content, use and appropriateness of progression, and personnel, setting, and timing of intervention delivery) made it challenging to identify meaningful groupings of similar studies to synthesize. In the absence of meaningful clusters of similar studies, we opted to summarize all prehabilitation studies together but contextualize interpretations of individual study results with details about the specific form of prehabilitation evaluated in those studies. We report outcomes under the four following outcome categories: body structure and function; activity and participation; other patientreported; and healthcare utilization. Given intervention heterogeneity, we determined that metaanalysis was not warranted (i.e., average result would not have been interpretable/meaningful) and instead summarize results narratively.

\footnotetext{
${ }^{a}$ For the purpose of our coding, we determined that the intervention in Huber 2014 met coding criteria for patient education but Villadsen 2014 did not. For the purpose of this Key Question, however, the intervention and comparison of interest in the two studies seemed sufficiently similar to compare.
} 
Figure 1. Overview of studies of prehabilitation and no prehabilitation interventions for total knee arthroplasty
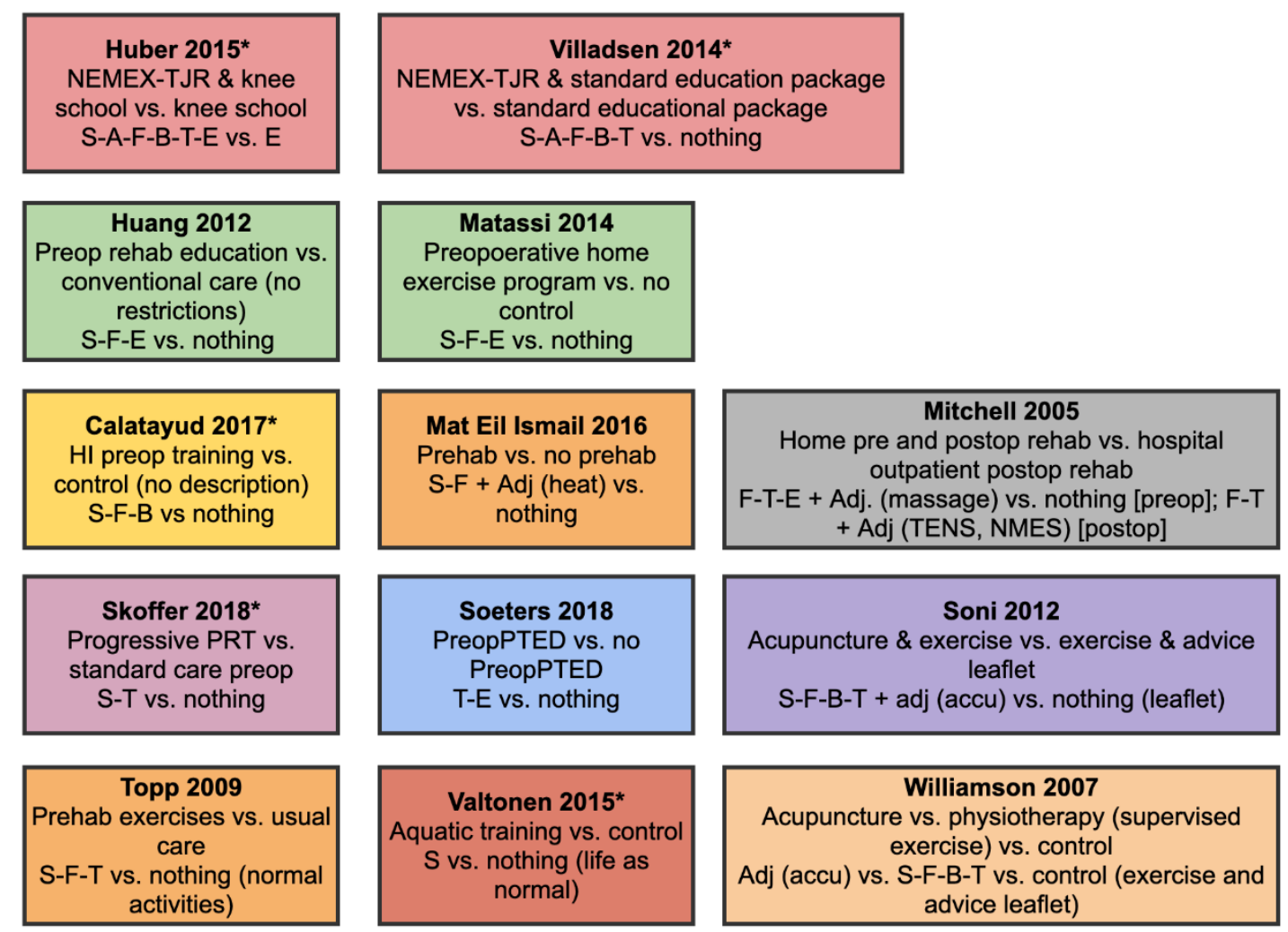

\begin{tabular}{c} 
Williamson 2007 \\
Acupuncture vs. physiotherapy (supervised \\
exercise) vs. control \\
Adj (accu) vs. S-F-B-T vs. control (exercise and \\
advice leaflet) \\
\hline
\end{tabular}

Figure presents studies $(\mathrm{n}=13)$ that evaluated prehabilitation programs for total knee arthroplasty versus various controls (predominately no active control with the exception of Mitchell 2005 which evaluated prehabilitation and postoperative rehabilitation combined vs. postoperative rehabilitation alone and did not include any active rehabilitation in the control arm during preoperative phase of the study). Study arms are described using study descriptors followed by goal components coded by the review team using the Oatis and Franklin taxonomy. The color is added for visual display and does not provide unique information.

Abbreviations: $\mathrm{Adj}=$ adjunctive, $\mathrm{A}=$ aerobic exercise, $\mathrm{B}=$ Balance-motor/learning-agility exercise, $\mathrm{E}=$ patient education, $\mathrm{F}=$ flexibility exercise, $\mathrm{HI}=$ high intensity, $\mathrm{NEMEX}=$ neuromuscular exercise, prehab $=$ prehabilitation, preop $=$ preoperative, postop $=$ postoperative, $\mathrm{RT}=$ resistance training, $\mathrm{S}=$ strengthening exercise, $\mathrm{T}=$ task-specific training, TENS $=$ transcutaneous electrical nerve stimulation, $\mathrm{TJR}=$ total joint replacement

* Intervention included progression which was deemed appropriate. 
Table 3.1. Goal components strength, aerobic, and flexibility and their specific exercise components for prehabilitation interventions versus no prehabilitation for total knee arthroplasty

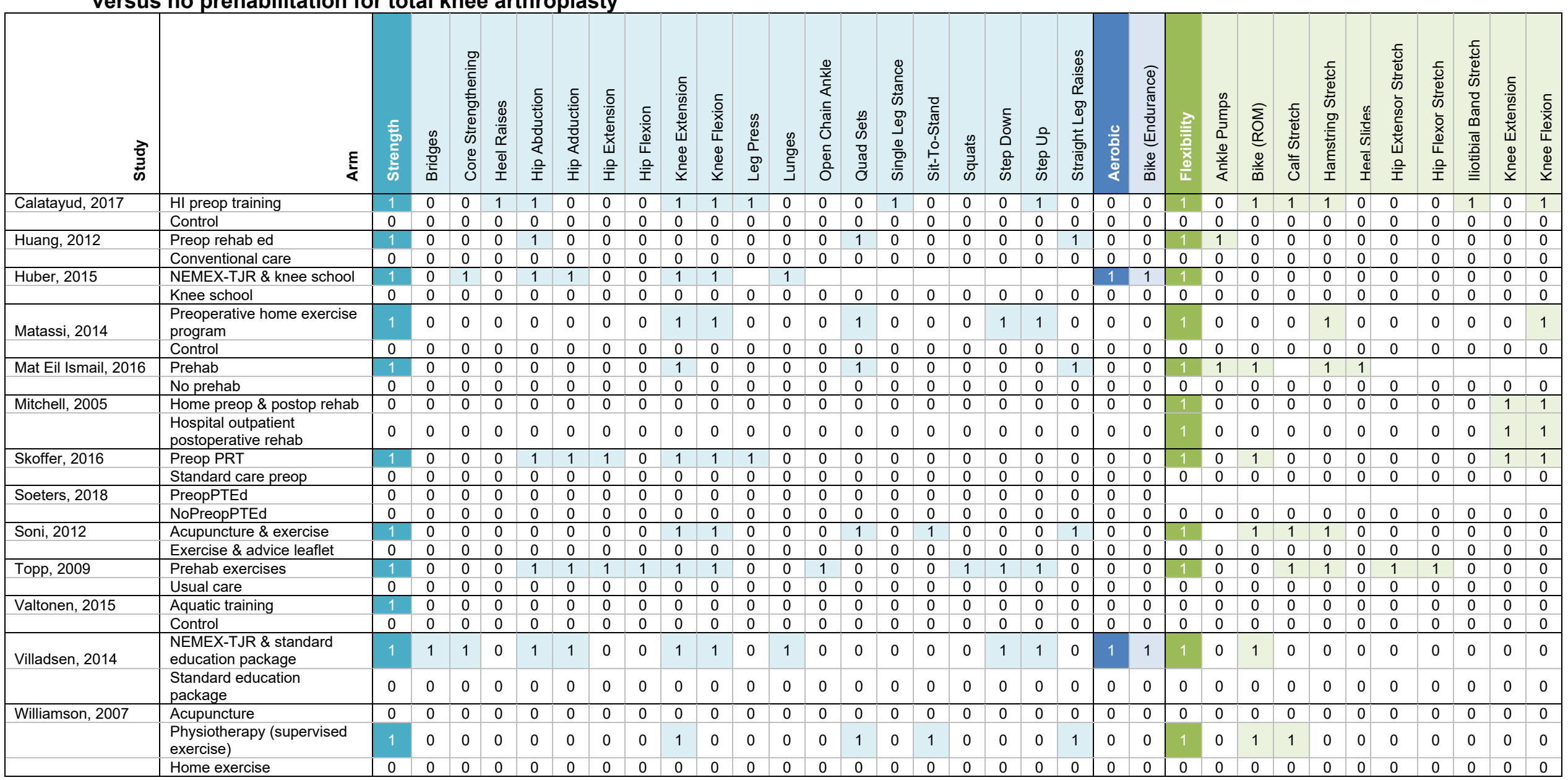

See Table 3.2 for goal components balance-motor-learning-agility, task specific training, patient education, and adjunctive modalities. The color is added for visual display and does not provide unique information.

$1=$ presence of component, $0=$ absence of component

Abbreviations: ed = education, $\mathrm{HI}=$ high intensity, NEMEX-TJR = neuromuscular training program, $\mathrm{PRT}=$ progressive resistive training; preop = preoperative; rehab =

rehabilitation, $\mathrm{ROM}=$ range of motion 
Table 3.2. Goal components balance-motor-learning-agility, task specific training, patient education, and adjunctive modalities and their specific exercise components for prehabilitation interventions versus no prehabilitation for total knee arthroplasty

\begin{tabular}{|c|c|c|c|c|c|c|c|c|c|c|c|c|c|c|c|c|c|c|c|c|c|c|c|c|c|c|}
\hline $\begin{array}{l}\overrightarrow{\bar{\partial}} \\
\text { के }\end{array}$ & $\frac{\xi}{\dot{z}}$ & 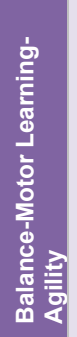 & 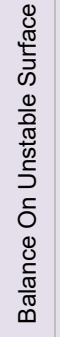 & 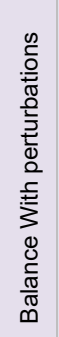 & $\begin{array}{l}\frac{c}{3} \\
0 \\
0 \\
\stackrel{0}{0} \\
\dot{\omega}\end{array}$ & 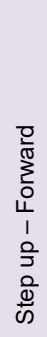 & 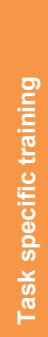 & 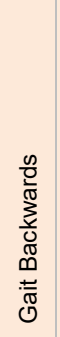 & 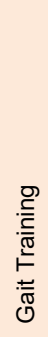 & 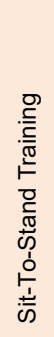 & 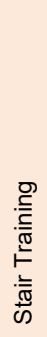 & 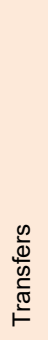 & 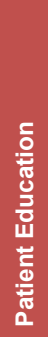 & 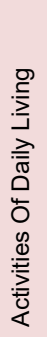 & 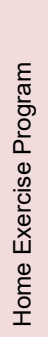 & 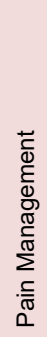 & 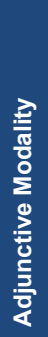 & 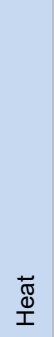 & 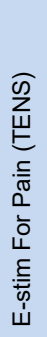 & 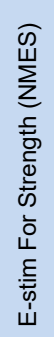 & $\begin{array}{l}\mathbb{D} \\
\mathbb{D} \\
\mathscr{D} \\
\mathscr{D} \\
\Sigma\end{array}$ & 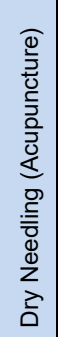 & 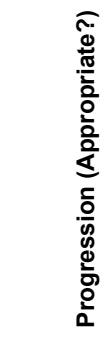 & 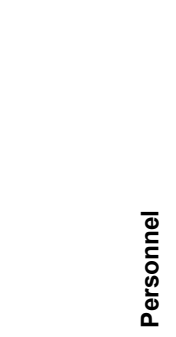 & 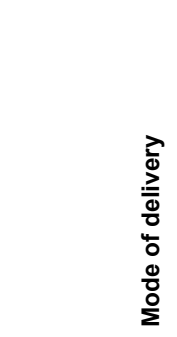 & 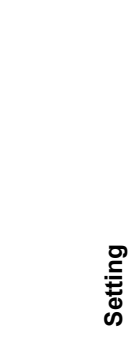 \\
\hline \multirow[t]{2}{*}{ Calatayud, 2017} & HI preop training & 1 & 1 & 0 & 0 & 0 & 0 & 0 & 0 & 0 & 0 & 0 & 0 & 0 & 0 & 0 & 0 & 0 & 0 & 0 & 0 & 0 & $\mathrm{Y}(\mathrm{Y})$ & PT & 1 & $\mathrm{O}$ \\
\hline & Control & 0 & 0 & 0 & 0 & 0 & 0 & 0 & 0 & 0 & 0 & 0 & 0 & 0 & 0 & 0 & 0 & 0 & 0 & 0 & 0 & 0 & $\mathrm{~N}$ & NA & NA & NA \\
\hline \multirow[t]{2}{*}{ Huang, 2012} & Preop rehab ed & 0 & 0 & 0 & 0 & 0 & 0 & 0 & 0 & 0 & 0 & 0 & 1 & 1 & 1 & 0 & 0 & 0 & 0 & 0 & 0 & 0 & $\mathrm{~N}$ & PT; None & $\mathrm{I} ; \mathrm{R}^{\mathrm{A}} ; \mathrm{SG}$ & $\mathrm{O} ; \mathrm{H}$ \\
\hline & Conventional care & 0 & 0 & 0 & 0 & 0 & 0 & 0 & 0 & 0 & 0 & 0 & 0 & 0 & 0 & 0 & 0 & 0 & 0 & 0 & 0 & 0 & $\mathrm{~N}$ & NA & NA & NA \\
\hline \multirow[t]{2}{*}{ Huber, 2015} & NEMEX-TJR \& knee school & 1 & 0 & 1 & 1 & 1 & 1 & 1 & 1 & 1 & 1 & & 1 & 0 & 0 & 1 & 0 & 0 & 0 & 0 & 0 & 0 & $\mathrm{Y}(\mathrm{Y})$ & PT & $\mathrm{I}$ & $\mathrm{O}$ \\
\hline & Knee school & 0 & 0 & 0 & 0 & 0 & 0 & 0 & 0 & 0 & 0 & 0 & 1 & 0 & 0 & 1 & 0 & 0 & 0 & 0 & 0 & 0 & $\mathrm{~N}$ & PT & I & $\mathrm{O}$ \\
\hline \multirow[t]{2}{*}{ Matassi, 2014} & $\begin{array}{l}\text { Preoperative home exercise } \\
\text { program }\end{array}$ & 0 & 0 & 0 & 0 & 0 & 0 & 0 & 0 & 0 & 0 & 0 & 1 & 0 & 1 & 0 & 0 & 0 & 0 & 0 & 0 & 0 & $\mathrm{~N}$ & None & SG & $\mathrm{H}$ \\
\hline & Control & 0 & 0 & 0 & 0 & 0 & 0 & 0 & 0 & 0 & 0 & 0 & 0 & 0 & 0 & 0 & 0 & 0 & 0 & 0 & 0 & 0 & $\mathrm{~N}$ & NA & NA & NA \\
\hline Mat Eil Ismail, & Prehab & 0 & 0 & 0 & 0 & 0 & 0 & 0 & 0 & 0 & 0 & 0 & 0 & 0 & 0 & 0 & 1 & 1 & 0 & 0 & 0 & 0 & $\mathrm{~N}$ & NR & NR & NR \\
\hline 2016 & No prehab & 0 & 0 & 0 & 0 & 0 & 0 & 0 & 0 & 0 & 0 & 0 & 0 & 0 & 0 & 0 & 0 & 0 & 0 & 0 & 0 & 0 & $\mathrm{~N}$ & NA & NA & NA \\
\hline \multirow[t]{2}{*}{ Skoffer, 2016} & Preop PRT & 0 & 0 & 0 & 0 & 0 & 0 & 0 & 0 & 0 & 0 & 0 & 0 & 0 & 0 & 0 & 0 & 0 & 0 & 0 & 0 & 0 & $\mathrm{Y}(\mathrm{N})$ & PT & 1 & $\mathrm{O}$ \\
\hline & Standard care preop & 0 & 0 & 0 & 0 & 0 & 0 & 0 & 0 & 0 & 0 & 0 & 0 & 0 & 0 & 0 & 0 & 0 & 0 & 0 & 0 & 0 & $\mathrm{~N}$ & NA & NA & NA \\
\hline \multirow[t]{2}{*}{ Soeters, 2018} & PreopPTEd & 0 & 0 & 0 & 0 & 0 & 1 & & & 1 & 1 & 1 & 1 & 1 & 0 & 0 & 0 & 0 & 0 & 0 & 0 & 0 & $\mathrm{~N}$ & PT & 1 & NR \\
\hline & NoPreopPTEd & 0 & 0 & 0 & 0 & 0 & 0 & 0 & 0 & 0 & 0 & 0 & 0 & 0 & 0 & 0 & 0 & 0 & 0 & 0 & 0 & 0 & $\mathrm{~N}$ & NA & NA & NA \\
\hline \multirow[t]{2}{*}{ Soni, 2012} & Acupuncture \& exercise & 1 & 1 & 0 & 0 & 0 & 1 & 0 & 0 & 0 & 1 & 0 & 0 & 0 & 0 & 0 & 1 & 0 & 0 & 0 & 0 & 1 & $\mathrm{~N}$ & PT & $\mathrm{I}$ & $\mathrm{O}$ \\
\hline & Exercise \& advice leaflet & 0 & 0 & 0 & 0 & 0 & 0 & 0 & 0 & 0 & 0 & 0 & 0 & 0 & 0 & 0 & 0 & 0 & 0 & 0 & 0 & 0 & $\mathrm{~N}$ & NA & NA & NA \\
\hline \multirow{2}{*}{ Topp, 2009} & Prehab exercises & 0 & 0 & 0 & 0 & 0 & 1 & 0 & 0 & 0 & 0 & 0 & 0 & 0 & 0 & 0 & 0 & 0 & 0 & 0 & 0 & 0 & $\mathrm{~N}$ & Other ${ }^{B} ;$ None & I; SG & $\mathrm{O} ; \mathrm{H}$ \\
\hline & Usual care & 0 & 0 & 0 & 0 & 0 & & 0 & 0 & 0 & 0 & 0 & 0 & 0 & 0 & 0 & 0 & 0 & 0 & 0 & 0 & 0 & $\mathrm{~N}$ & NA & NA & NA \\
\hline \multirow[t]{2}{*}{ Valtonen, 2015} & Aquatic training & 0 & 0 & 0 & 0 & 0 & 0 & 0 & 0 & 0 & 0 & 0 & 0 & 0 & 0 & 0 & 0 & 0 & 0 & 0 & 0 & 0 & $\mathrm{Y}(\mathrm{N})$ & NR & 1 & Other ${ }^{C}$ \\
\hline & Control & 0 & 0 & 0 & 0 & 0 & 0 & 0 & 0 & 0 & 0 & 0 & 0 & 0 & 0 & 0 & 0 & 0 & 0 & 0 & 0 & 0 & $\mathrm{~N}$ & NA & NA & NA \\
\hline \multirow[t]{2}{*}{ Villadsen, 2014} & $\begin{array}{l}\text { NEMEX-TJR \& standard } \\
\text { education package }\end{array}$ & 1 & 0 & 0 & 0 & 0 & 1 & 1 & 1 & 1 & 0 & 0 & 0 & 0 & 0 & 0 & 0 & 0 & 0 & 0 & 0 & 0 & $Y(Y)$ & PT & 1 & $\mathrm{O}$ \\
\hline & $\begin{array}{l}\text { Standard education } \\
\text { package }\end{array}$ & 0 & 0 & 0 & 0 & 0 & 0 & 0 & 0 & 0 & 0 & 0 & 0 & 0 & 0 & 0 & 0 & 0 & 0 & 0 & 0 & 0 & $\mathrm{~N}$ & NA & NA & NA \\
\hline \multirow[t]{3}{*}{ Williamson, 2007} & Acupuncture & 0 & 0 & 0 & 0 & 0 & 0 & 0 & 0 & 0 & 0 & 0 & 0 & 0 & 0 & 0 & 1 & 0 & 0 & 0 & 0 & 1 & $\mathrm{~N}$ & PT & 1 & $\mathrm{O}$ \\
\hline & $\begin{array}{l}\text { Physiotherapy (supervised } \\
\text { exercise) }\end{array}$ & 1 & 1 & 0 & 0 & 0 & 1 & 0 & 0 & 0 & 1 & 0 & 0 & 0 & 0 & 0 & 0 & 0 & 0 & 0 & 0 & 0 & $\mathrm{~N}$ & PT & 1 & $\mathrm{O}$ \\
\hline & Home exercise & 0 & 0 & 0 & 0 & 0 & 0 & 0 & 0 & 0 & 0 & 0 & 0 & 0 & 0 & 0 & 0 & 0 & 0 & 0 & 0 & 0 & $\mathrm{~N}$ & None & None & NA \\
\hline
\end{tabular}

See Table 3.1 for goal components strength, aerobic, and flexibility. The color is added for visual display and does not provide unique information.

$1=$ presence of component, $0=$ absence of component 
Abbreviations: ed = education, $\mathrm{H}=$ home, $\mathrm{HI}=$ high intensity, $\mathrm{I}=$ in-person; $\mathrm{NA}=$ not applicable, NEMEX-TJR = neuromuscular training program, $\mathrm{O}=$ outpatient physiotherapy center, PreopPTEd $=$ Preoperative physical therapy education, $\mathrm{PRT}=$ progressive resistive training; preop $=$ preoperative, prehab $=$ prehabilitation, $\mathrm{R}=$ remote, rehab $=$ rehabilitation, $\mathrm{SG}=$ self-guided.

A Remote via telephone

${ }^{B}$ Research personnel

${ }^{\mathrm{C}}$ Aquatic center 


\section{Body Structure and Function Outcomes}

Ten RCTs (Calatayud 2017, Huber 2015, Matassi 2014, Mitchell 2005, Skoffer 2016, Soni 2012, Topp 2009, Valtonen 2015, Villadsen 2014, Williamson 2007) reported on body structure and function outcomes comparing prehabilitation to no prehabilitation (Tables 4 to 9). The outcome domains included: Symptoms, Pain, Range of motion, Muscle strength, Energy and vigor, and Emotional functioning.

\section{Symptoms}

Five RCTs (Calatayud 2017, Huber 2015, Mitchell 2005, Skoffer 2016, Villadsen 2014) reported on physical well-being as continuous measures using the stiffness component of the Western Ontario and McMaster Universities Osteoarthritis Index (WOMAC; scores 0 to 8; lower score indicates reduced stiffness) and the symptoms component of the Knee Injury and Osteoarthritis Outcome Score (KOOS; scores 0-100; higher is better) score at 3 months after surgery (Table 4).

Four studies reported comparable scores between prehabilitation and control groups 3 months after TKA. One RCT (Calatayud 2017) reported statistically significant improvements in patients experience of stiffness among patients randomized to high intensity preoperative training at 3 month follow-up compared to control (mean difference [MD] $-0.9,95 \%$ confidence interval $[\mathrm{CI}]-1.3$ to -0.6$)$.

\section{Pain}

Nine RCTs reported pain data (Calatayud 2017, Huber 2015, Mitchell 2005, Skoffer 2016, Soni 2012, Topp 2009, Valtonen 2015, Villadsen 2014, Williamson 2007). Seven studies reported no significant differences between groups with respect to pain. Two studies (Calatayud 2017, Valtonen 2015) reported that prehabilitation was associated with significantly less pain than no prehabilitation (Table 5).

Huber 2015 and Villadsen 2014 evaluated a similar prehabilitation neuromuscular training program (NEMEX-TJR) combined with education, which was compared with an education control. Huber 2015 reported pain data using three different scores: the pain/discomfort component of EuroQol-5D (EQ-5D) (for each component 0 to 3, higher is worse), the pain component of KOOS (for each component: 0-100; higher is better), and the pain component of 36-Item Short Form Health Survey (SF-36) (for each component: 0-100; higher is better). Villadsen 2014 reported data using the pain component of the KOOS. Both studies of NEMEXTJR found no difference in pain between prehabilitation and control groups at 3 or 12 months after surgery.

The remaining eight studies evaluated different prehabilitation training programs and reported pain data using the visual analog scale (VAS), pain components of the WOMAC, KOOS, and SF-36 at 3 months after surgery. Of these, only Calatayud 2017 and Valtonen 2015 reported reduced pain associated with their various prehabilitation programs. Calatayud 2017 (high intensity preoperative training) reported data using a VAS (0 to 10; higher is worse) and pain component of the KOOS (for each component: 0-100; higher is better). At 3 months after surgery, patients randomized to the high intensity preoperative training had lower pain scores on the VAS (MD $-1.5,95 \% \mathrm{CI}-1.9$ to -1.1 ) and the pain component of the WOMAC (MD - 0.9, $95 \% \mathrm{CI}-1.5$ to -0.14$)$ compared to patients who did not receive prehabilitation training. Valtonen 2015 (aquatic training) reported data using a VAS (1 to 10; higher is worse). At 3 
months of followup, patients randomized to aquatic training reported a $58 \%$ decrease in pain ( $\mathrm{p}=0.001$; MD not reported) compared to patients who continued life as usual.

All other studies reported comparable pain scores between prehabilitation and control groups at 3 months after TKA.

\section{Range of Motion}

Four RCTs (Calatayud 2017, Huber 2015, Matassi 2014, Skoffer 2016) reported range of motion (ROM) data from various outcome measures, including active and passive knee ROM for extension and flexion of the knee joint. In several cases, whether ROM was active or passive was not specified. Studies measured ROM in degrees using goniometry. With the exception of one study (Matassi 2014), all ROM data was reported at 3 months after knee surgery (Table 6).

Two RCTs (Calatayud 2017 and Matassi 2014) reported that prehabilitation was associated with improvements in ROM of the knee joint compared to no prehabilitation. Calatayud 2017 reported significant improvements in active knee extension (MD -5.6, 95\% CI -6.9 to -4.3) and active knee flexion (MD 4.8, 95\% CI 0.2 to 9.5) in patients randomized to high intensity preoperative training compared to patients who did not receive prehabilitation training at 3 months follow-up. Matassi 2014 reported significant improvements in knee extension (MD and active/passive unspecified; $p=0.032$ ) in patients randomized to preoperative home exercise compared to control at 12 months after surgery but no significant between group difference in active or passive knee flexion.

The two other studies reported comparable range of motion between prehabilitation and control groups at 3 months after TKA.

\section{Muscle Strength}

Four RCTs (Calatayud 2017, Huber 2015, Skoffer 2016, Topp 2009) reported on muscle strength data from various outcome measures, including isometric and isokinetic knee extension and knee flexion strength. Studies measured strength in Newtons $(\mathrm{N})$, kilograms $(\mathrm{kg})$, or torque normalized to body weight $(\mathrm{Nm} / \mathrm{kg}$ ), using either a hand-held pull gauge or a dynamometer (for each, higher values indicate greater strength). All strength data was reported at 3 months following knee surgery (Table 7).

Two RCTs (Calatayud 2017 and Skoffer 2016) reported that prehabilitation was associated with greater improvements in muscle strength of the affected knee compared to no prehabilitation. Calatayud 2017 reported significant improvements in isometric knee extension (MD 8.5, 95\% CI 4.8 to 12.1) and knee flexion (MD 5.0, 95\% CI 4.3 to 5.7) in patients randomized to high intensity preoperative training compared to patients who did not receive prehabilitation training at 3 months follow-up. Skoffer 2016 reported significant improvements in isometric (MD 0.2, 95\% CI 0.1 to 0.3 ) and isokinetic knee extension (MD $0.295 \%$ CI 0.06 to 0.34 ), as well as isometric (MD $0.1,95 \%$ CI 0.001 to 0.2 ) and isokinetic knee flexion (MD 0.1, 95\% CI -0.01 to 0.21 ) in patients randomized to preoperative progressive resistance training compared to control at 3 months after surgery.

The two other studies reported comparable muscle strength between prehabilitation and control groups at 3 months after TKA. 


\section{Energy and Vigor}

Two RCTs (Huber 2015 and Mitchell 2005) reported on vigor using the vitality component of the SF-36 (scores 0 to 100, higher is better) (Table 8) and found no significant differences between prehabilitation and control at 3 months after surgery.

\section{Emotional Functioning}

Two RCTs (Huber 2015 and Mitchell 2005) reported on emotional functioning data from mental health, emotional role functioning and social functioning component scales of the SF-36 (scores 0 to 100, higher is better) and found no significant differences between prehabilitation and control at 3 months after surgery (Table 9). 
Table 4. Prehabilitation versus no prehabilitation for total knee arthroplasty - continuous outcomes, symptoms

\begin{tabular}{|c|c|c|c|c|c|c|c|c|c|}
\hline $\begin{array}{l}\text { Study, Year, PMID, } \\
\text { Country }\end{array}$ & Outcome Measurement & $\begin{array}{l}\text { Overall } \\
\text { RoB }\end{array}$ & $\begin{array}{l}\text { Time } \\
\text { Point }^{\mathrm{A}}\end{array}$ & Prehab, N & $\begin{array}{l}\text { Prehab, } \\
\text { Mean (SD) }\end{array}$ & $\begin{array}{l}\text { Control, } \\
\text { N }\end{array}$ & $\begin{array}{l}\text { Control } \\
\text { Mean (SD) }\end{array}$ & Effect Size $(95 \% \mathrm{Cl})$ & $\begin{array}{l}\text { Reported } \\
\text { p-Value }\end{array}$ \\
\hline $\begin{array}{l}\text { Calatayud, 2017, } \\
\text { 26768606, } \\
\text { Spain }\end{array}$ & WOMAC: Stiffness (0-8) & Moderate & $3 \mathrm{mo}$ & 22 & $\begin{array}{l}2.2(95 \% \mathrm{Cl} \\
2.0,2.5)\end{array}$ & 22 & $\begin{array}{l}3.2(95 \% \mathrm{Cl} \\
2.9,3.4)\end{array}$ & $-0.9(-1.3,-0.6)$ & $<0.05$ \\
\hline $\begin{array}{l}\text { Huber, 2015, } \\
\text { 25925404, } \\
\text { Switzerland }\end{array}$ & KOOS: Symptoms $(0-100)$ & High & $3 \mathrm{mo}$ & 21 & NR & 20 & NR & $4.6(-5.0,14.2)$ & NR \\
\hline $\begin{array}{l}\text { Mitchell, 2005, } \\
\text { 15869558, UK }\end{array}$ & WOMAC: Stiffness (0-8) & High & $3 \mathrm{mo}$ & 57 & $3.5(1.4)$ & 57 & $3.6(2.1)$ & $\begin{array}{l}\text { Adj MD -0.2 (-0.9, } \\
0.4)\end{array}$ & 0.496 \\
\hline $\begin{array}{l}\text { Skoffer, 2016, } \\
\text { 26713665, } \\
\text { Denmark }\end{array}$ & KOOS: Symptoms $(0-100)$ & Moderate & $3 \mathrm{mo}$ & 29 & $72.8(16.4)$ & 21 & $71.9(11.4)$ & $0.9(-4.6,6.4)^{\mathrm{B}}$ & $\overline{N R}$ \\
\hline $\begin{array}{l}\text { Villadsen, 2014, } \\
\text { 23661494, } \\
\text { Denmark }\end{array}$ & KOOS: Symptoms (0-100) & Moderate & $3 \mathrm{mo}$ & 84 & NR & 81 & NR & $-6.0(-13.4,1.5)$ & 0.12 \\
\hline
\end{tabular}

Statistically significant effect sizes are in bold text. In cases where calculated effect size confidence intervals were not-statistically significant but reported p-values were, we deferred to reported p-values and still bolded results.

Abbreviations: Adj $\mathrm{MD}=$ adjusted mean difference $\mathrm{CI}=$ confidence interval, $\mathrm{KOOS}=$ Knee injury and osteoarthritis outcome score, mo $=$ month, $\mathrm{NR}=$ not reported, $\mathrm{PMID}=$ PubMed identifier, Prehab = prehabilitation, RoB = risk of bias, SD = standard deviation, SF-36 = 36-Item Short Form survey, WOMAC = Western Ontario and McMaster

Universities Osteoarthritis Index

A Time from surgery

B Calculated 
Table 5. Prehabilitation versus no prehabilitation for total knee arthroplasty - continuous outcomes, pain

\begin{tabular}{|c|c|c|c|c|c|c|c|c|c|}
\hline $\begin{array}{l}\text { Study, Year, } \\
\text { PMID, Country }\end{array}$ & Outcome Measurement & $\begin{array}{l}\text { Overall } \\
\text { RoB }\end{array}$ & $\begin{array}{l}\text { Time } \\
\text { Point }^{A}\end{array}$ & $\begin{array}{l}\text { Prehab, } \\
\text { N }\end{array}$ & $\begin{array}{l}\text { Prehab, } \\
\text { Mean (SD) }\end{array}$ & Control, N & $\begin{array}{l}\text { Control } \\
\text { Mean (SD) }\end{array}$ & Effect Size $(95 \% \mathrm{CI})$ & $\begin{array}{l}\text { Reported } \\
\text { p-Value }\end{array}$ \\
\hline \multirow{2}{*}{$\begin{array}{l}\text { Calatayud, 2017, } \\
26768606, \\
\text { Spain }\end{array}$} & VAS $(0-10)$ & Moderate & $3 \mathrm{mo}$ & 22 & $\begin{array}{l}1.4(95 \% \mathrm{Cl} \\
1.1,1.7)\end{array}$ & 22 & $\begin{array}{l}2.9(95 \% \mathrm{Cl} \\
2.5,3.2)\end{array}$ & $-1.5(-1.9,-1.1)$ & $<0.05$ \\
\hline & WOMAC: Pain (0-20) & Moderate & $3 \mathrm{mo}$ & 22 & $\begin{array}{l}2.9(95 \% \mathrm{Cl} \\
2.5,3.3) \\
\end{array}$ & 22 & $\begin{array}{l}3.8(95 \% \mathrm{Cl} \\
3.4,4.2) \\
\end{array}$ & $-0.9(-1.5,-0.14)$ & $<0.05$ \\
\hline \multirow{7}{*}{$\begin{array}{l}\text { Huber, 2015, } \\
25925404, \\
\text { Switzerland }\end{array}$} & $\begin{array}{l}\text { EQ-5D: Pain/discomfort (1- } \\
\text { 3) }\end{array}$ & High & $3 \mathrm{mo}$ & 21 & NR & 20 & NR & $-0.0(-0.5,0.3)$ & NR \\
\hline & $\begin{array}{l}\text { EQ-5D: Pain/discomfort (1- } \\
\text { 3) }\end{array}$ & High & $12 \mathrm{mo}$ & 21 & NR & 20 & NR & $-0.1(-0.4,0.3)$ & NR \\
\hline & KOOS: Pain (0-100) & High & $3 \mathrm{mo}$ & 21 & NR & 20 & NR & $-3.3(-13.5,6.8)$ & NR \\
\hline & EQ-5D (VAS) (0-100) & High & $3 \mathrm{mo}$ & 21 & NR & 20 & NR & $1.2(-8.4,10.8)$ & NR \\
\hline & KOOS: Pain (0-100) & High & $12 \mathrm{mo}$ & 21 & NR & 20 & NR & $2.3(-8.5,13.0)$ & NR \\
\hline & SF-36: Bodily pain (0-100) & High & $3 \mathrm{mo}$ & 21 & NR & 20 & NR & $-3.4(-15.5,8.7)$ & NR \\
\hline & SF-36: Bodily pain (0-100) & High & $12 \mathrm{mo}$ & 21 & NR & 20 & NR & $4.9(-7.8,17.7)$ & NR \\
\hline \multirow{2}{*}{$\begin{array}{l}\text { Mitchell, 2005, } \\
\text { 15869558, UK }\end{array}$} & SF-36: Bodily pain (0-100) & High & $3 \mathrm{mo}$ & 57 & $46.6(20.6)$ & 57 & $48.5(26.8)$ & Adj MD -3.4 (-12.0, 5.2) & 0.432 \\
\hline & WOMAC: Pain (0-20) & High & $3 \mathrm{mo}$ & 57 & $6.8(3.7)$ & 57 & $6.9(4.3)$ & Adj MD $-0.5(-2.0,1.0)$ & 0.530 \\
\hline \multirow{4}{*}{$\begin{array}{l}\text { Skoffer, 2016, } \\
26713665, \\
\text { Denmark }\end{array}$} & VAS: Current pain (0-10) & High & $3 \mathrm{mo}$ & 29 & $1.0(1.7)$ & 21 & $1.1(1.3)$ & $-0.1(-0.7,0.5)^{\mathrm{B}}$ & NR \\
\hline & VAS: Average pain ${ }^{C}(0-10)$ & High & $3 \mathrm{mo}$ & 29 & $1.4(1.6)$ & 21 & $1.5(1.1)$ & $-0.1(-0.6,0.4)^{\mathrm{B}}$ & NR \\
\hline & VAS: Worst pain ${ }^{\mathrm{C}}(0-10)$ & High & $3 \mathrm{mo}$ & 29 & $2.6(2.6)$ & 21 & $2.4(1.9)$ & $0.2(-0.7,1.1)^{\mathrm{B}}$ & NR \\
\hline & KOOS: Pain (0-100) & High & $3 \mathrm{mo}$ & 29 & $78.1(16.3)$ & 21 & $79.9(14.2)$ & $-1.8(-7.8,4.2)^{\mathrm{B}}$ & NR \\
\hline $\begin{array}{l}\text { Soni, 2012, } \\
\text { 22914302, UK }\end{array}$ & VAS $(0-10)$ & Moderate & $3 \mathrm{mo}$ & 28 & $3.9(3)$ & 28 & $4.7(2.8)$ & $-0.8(-2.6,1.1)$ & NR \\
\hline \multirow{4}{*}{$\begin{array}{l}\text { Topp, 2009, } \\
\text { 19695525, USA }\end{array}$} & VAS: Ascend stairs (0-10) & High & $3 \mathrm{mo}$ & 26 & $1.33(0.31)$ & 28 & $1.26(0.30)$ & $0.07(-0.09,0.23)^{\mathrm{B}}$ & NR \\
\hline & VAS: Descend stairs (0-10) & High & $3 \mathrm{mo}$ & 26 & $1.42(0.37)$ & 28 & $1.45(0.35)$ & $-0.03(-0.22,0.16)^{B}$ & NR \\
\hline & VAS: Sit-to-stand $(0-10)^{\mathrm{D}}$ & High & $3 \mathrm{mo}$ & 26 & $1.62(0.29)$ & 28 & $1.06(0.28)$ & $0.56(0.41,0.71)^{\mathrm{B}}$ & NR \\
\hline & VAS: 6-min walk (0-10) & High & $3 \mathrm{mo}$ & 26 & $1.53(0.34)$ & 28 & $1.38(0.33)$ & $0.15(-0.03,0.33)^{\mathrm{B}}$ & NR \\
\hline $\begin{array}{l}\text { Valtonen, 2015, } \\
\text { CN-01126383, } \\
\text { Finland }\end{array}$ & VAS $(0-10)$ & High & $3 \mathrm{mo}$ & 31 & NR & 24 & NR & $-58 \%$ & 0.001 \\
\hline \multirow{2}{*}{$\begin{array}{l}\text { Villadsen, 2014, } \\
\text { 23661494, } \\
\text { Denmark }\end{array}$} & KOOS: Pain (0-100) & Moderate & $3 \mathrm{mo}$ & 84 & NR & 81 & NR & $-5.5(-13.0,2.9)$ & 0.1556 \\
\hline & EQ-5D (VAS) (0-100) & Moderate & $3 \mathrm{mo}$ & 84 & NR & 81 & NR & $2.8(-4.8,10.4)$ & 0.4684 \\
\hline $\begin{array}{l}\text { Williamson, } \\
2007, \\
17604311, \text { UK }\end{array}$ & VAS $(0-10)$ & High & $3 \mathrm{mo}$ & 23 & $3.86(2.59)$ & $19^{\mathrm{E}}$ & $3.95(2.59)$ & $-0.09(-1.71,1.53)$ & NR \\
\hline
\end{tabular}

Statistically significant effect sizes are in bold text. In cases where calculated effect size confidence intervals were not-statistically significant but reported p-values were, we deferred to reported p-values and still bolded results. 
Abbreviations: Adj $\mathrm{MD}=$ adjusted mean difference, $\mathrm{CI}=$ confidence interval, $\mathrm{EQ}-5 \mathrm{D}=$ EuroQol-5 dimensions, $\mathrm{KOOS}=\mathrm{Knee}$ injury and osteoarthritis outcome score, min $=$ minute, $\mathrm{mo}=$ month, $\mathrm{NR}=$ not reported, PMID $=$ PubMed identifier, RoB $=$ risk of bias, $\mathrm{SD}=$ standard deviation, SF-36 = 36-item short form health survey, WOMAC $=$ Western Ontario and McMaster Universities Osteoarthritis Index, VAS = visual analog scale.

A Time from surgery

B Calculated

${ }^{\mathrm{C}}$ During the past 14 days

D Repetitions in 30 seconds

${ }^{\mathrm{E}}$ Control arm sample size is uncertain from study report. Study was retained despite potential sample size of control being less than 20

Table 6. Prehabilitation versus no prehabilitation for total knee arthroplasty - continuous outcomes, range of motion

\begin{tabular}{|c|c|c|c|c|c|c|c|c|c|}
\hline $\begin{array}{l}\text { Study, Year, } \\
\text { PMID, Country }\end{array}$ & Outcome Measurement & Overall RoB & $\begin{array}{l}\text { Time } \\
\text { Point }\end{array}$ & $\begin{array}{l}\text { Prehab, } \\
\text { N }\end{array}$ & $\begin{array}{l}\text { Prehab, Mean } \\
\text { (SD) }\end{array}$ & $\begin{array}{l}\text { Control, } \\
\text { N }\end{array}$ & $\begin{array}{l}\text { Control, Mean } \\
\text { (SD) }\end{array}$ & $\begin{array}{l}\text { Effect Size } \\
(95 \% \mathrm{Cl})\end{array}$ & $\begin{array}{l}\text { Reported } \\
\text { p-Value }\end{array}$ \\
\hline \multirow{2}{*}{$\begin{array}{l}\text { Calatayud,2017, } \\
\text { 26768606, } \\
\text { Spain }\end{array}$} & $\begin{array}{l}\text { Active Knee ROM: Extension } \\
\left(_{(\text {deg })^{\mathrm{B}}}\right.\end{array}$ & Moderate & $3 \mathrm{mo}$ & 22 & $\begin{array}{l}8.2(95 \% \mathrm{Cl} \\
7.2,9.3)\end{array}$ & 22 & $\begin{array}{l}13.9(95 \% \mathrm{Cl} \\
12.8,14.9)\end{array}$ & $-5.6(-6.9,-4.3)$ & $<0.05$ \\
\hline & Active Knee ROM: Flexion (deg) & Moderate & $3 \mathrm{mo}$ & 22 & $\begin{array}{l}101.2(95 \% \mathrm{Cl} \\
97.8,104.7)\end{array}$ & 22 & $\begin{array}{l}96.4(95 \% \mathrm{Cl} \\
92.9,99.9)\end{array}$ & $4.8(0.2,9.5)$ & $<0.05$ \\
\hline \multirow{2}{*}{$\begin{array}{l}\text { Huber, 2015, } \\
25925404, \\
\text { Switzerland }\end{array}$} & $\begin{array}{l}\text { Knee ROM (active/passive } \\
\text { unspecified): Extension (deg) }\end{array}$ & High & $3 \mathrm{mo}$ & 21 & NR & 20 & NR & $1.4(-1.8 .4 .5)$ & NR \\
\hline & $\begin{array}{l}\text { Knee ROM (active/passive } \\
\text { unspecified): Flexion (deg) }\end{array}$ & High & $3 \mathrm{mo}$ & 21 & NR & 20 & NR & $-3.9(-10.2,2.4)$ & NR \\
\hline \multirow{3}{*}{$\begin{array}{l}\text { Matassi, 2014, } \\
23271039, \\
\text { Belgium }\end{array}$} & $\begin{array}{l}\text { Knee ROM (active/passive } \\
\text { unspecified): Extension (deg) }\end{array}$ & Moderate & $12 \mathrm{mo}$ & 61 & $1.00(\mathrm{NR})$ & 61 & $0.68(\mathrm{NR})$ & NR & 0.032 \\
\hline & Active Knee ROM: Flexion (deg) & Moderate & $12 \mathrm{mo}$ & 61 & $118.3(\mathrm{NR})$ & 61 & $118.7(\mathrm{NR})$ & NR & ns \\
\hline & $\begin{array}{l}\text { Passive Knee ROM: Flexion } \\
\text { (deg) }\end{array}$ & Moderate & $12 \mathrm{mo}$ & 61 & 120.5 (NR) & 61 & 120.4 (NR) & NR & ns \\
\hline \multirow{4}{*}{$\begin{array}{l}\text { Skoffer, 2016, } \\
\text { 26713665, } \\
\text { Denmark }\end{array}$} & $\begin{array}{l}\text { Active Knee ROM: Extension } \\
\text { (deg) }\end{array}$ & Moderate & $3 \mathrm{mo}$ & 29 & $3.3(2.8)$ & 21 & $4.3(2.4)$ & $-1.00(-2.45,0.45)$ & 0.089 \\
\hline & $\begin{array}{l}\text { Passive Knee ROM: Extension } \\
\text { (deg) }\end{array}$ & Moderate & $3 \mathrm{mo}$ & 29 & $1.2(2.3)$ & 21 & $1.7(2.4)$ & $-0.5(-1.4,0.4)^{\mathrm{C}}$ & 0.207 \\
\hline & Active Knee ROM: Flexion (deg) & Moderate & $3 \mathrm{mo}$ & 29 & $113.0(14.8)$ & 21 & $112.5(7.81)$ & $0.5(-5.84,6.84)$ & 0.995 \\
\hline & $\begin{array}{l}\text { Passive Knee ROM: Flexion } \\
\text { (deg) }\end{array}$ & Moderate & $3 \mathrm{mo}$ & 29 & $118.7(15.7)$ & 21 & $118.3(8.0)$ & $0.4(-4.6,5.4)^{\mathrm{C}}$ & 0.678 \\
\hline
\end{tabular}

Statistically significant effect sizes are in bold text. In cases where calculated effect size confidence intervals were not-statistically significant but reported p-values were, we deferred to reported p-values and still bolded results.

Abbreviations: $\mathrm{CI}=$ confidence interval, $\mathrm{deg}=$ degree, $\mathrm{mo}=$ month, $\mathrm{N}=$ number, $\mathrm{NR}=$ not reported, $\mathrm{PMID}=\mathrm{PubMed}$ identifier, $\mathrm{RoB}=$ risk of bias, $\mathrm{ROM}=$ range of motion, $\mathrm{SD}=$ standard deviation.

A Time from surgery

${ }^{B}$ Measured with goniometer

${ }^{\mathrm{C}}$ Calculated 
Table 7. Prehabilitation versus no prehabilitation for total knee arthroplasty - continuous outcomes, muscle strength

\begin{tabular}{|c|c|c|c|c|c|c|c|c|c|}
\hline $\begin{array}{l}\text { Study, Year, } \\
\text { PMID, Country }\end{array}$ & Outcome Measurement & $\begin{array}{l}\text { Overall } \\
\text { RoB }\end{array}$ & $\begin{array}{l}\text { Time } \\
\text { Point }^{A}\end{array}$ & $\begin{array}{l}\text { Prehab, } \\
\text { N }\end{array}$ & $\begin{array}{l}\text { Prehab, Mean } \\
\text { (SD) }\end{array}$ & $\begin{array}{l}\text { Control, } \\
\text { N }\end{array}$ & $\begin{array}{l}\text { Control, Mean } \\
\text { (SD) }\end{array}$ & $\begin{array}{l}\text { Effect Size } \\
(95 \% \mathrm{Cl})\end{array}$ & $\begin{array}{l}\text { Reported } \\
\text { p-Value }\end{array}$ \\
\hline \multirow{2}{*}{$\begin{array}{l}\text { Calatayud, 2017, } \\
\text { 26768606, } \\
\text { Spain }\end{array}$} & $\begin{array}{l}\text { Strength: Isometric knee extension } \\
(\mathrm{kg})^{\mathrm{B}}\end{array}$ & Moderate & $3 \mathrm{mo}$ & 22 & $\begin{array}{l}22.8(95 \% \mathrm{Cl} \\
19.7,25.9)\end{array}$ & 22 & $\begin{array}{l}14.3(95 \% \mathrm{Cl} \\
11.1,17.5)\end{array}$ & $8.5(4.8,12.1)$ & $<0.05$ \\
\hline & $\begin{array}{l}\text { Strength: Isometric knee flexion } \\
(\mathrm{kg})\end{array}$ & Moderate & $3 \mathrm{mo}$ & 22 & $\begin{array}{l}9.4(95 \% \mathrm{Cl} \\
8.8,9.9)\end{array}$ & 22 & $\begin{array}{l}4.4(95 \% \mathrm{Cl} \\
3.8,5.0)\end{array}$ & $5.0(4.3,5.7)$ & $<0.05$ \\
\hline \multirow{3}{*}{$\begin{array}{l}\text { Huber, 2015, } \\
25925404, \\
\text { Switzerland }\end{array}$} & $\begin{array}{l}\text { Strength: Isometric knee extension } \\
(\mathrm{N})^{\mathrm{C}}\end{array}$ & High & $3 \mathrm{mo}$ & 21 & NR & 20 & NR & $-3.5(-52.7,45.6)$ & NR \\
\hline & $\begin{array}{l}\text { Strength: Isometric knee flexion } \\
\text { (N) }\end{array}$ & High & $3 \mathrm{mo}$ & 21 & NR & 20 & NR & $\begin{array}{l}-12.7(-36.2 \\
10.8)\end{array}$ & NR \\
\hline & Strength: Knee-bending/30s ${ }^{\mathrm{D}}$ & High & $3 \mathrm{mo}$ & 21 & NR & 20 & NR & $-3.3(-7.4,0.8)$ & NR \\
\hline \multirow{4}{*}{$\begin{array}{l}\text { Skoffer, 2016, } \\
\text { 26713665, } \\
\text { Denmark }\end{array}$} & $\begin{array}{l}\text { Strength: Isometric peak knee } \\
\text { extension }(\mathrm{Nm} / \mathrm{kg})^{\mathrm{B}}\end{array}$ & Moderate & $3 \mathrm{mo}$ & 29 & $1.0(0.3)$ & 21 & $0.8(0.3)$ & $0.2(0.1,0.3)^{E}$ & $<0.001$ \\
\hline & $\begin{array}{l}\text { Strength: Isokinetic peak knee } \\
\text { extension }(\mathrm{Nm} / \mathrm{kg})\end{array}$ & Moderate & $3 \mathrm{mo}$ & 29 & $0.9(0.3)$ & 21 & $0.7(0.2)$ & $0.2(0.06,0.34)^{\mathrm{E}}$ & 0.002 \\
\hline & $\begin{array}{l}\text { Strength: Isometric peak knee } \\
\text { flexion }(\mathrm{Nm} / \mathrm{kg})\end{array}$ & Moderate & $3 \mathrm{mo}$ & 29 & $0.7(0.3)$ & 21 & $0.6(0.2)$ & $0.1(0.001,0.2)^{\mathrm{E}}$ & 0.042 \\
\hline & $\begin{array}{l}\text { Strength: Isokinetic peak knee } \\
\text { flexion }(\mathrm{Nm} / \mathrm{kg})\end{array}$ & Moderate & $3 \mathrm{mo}$ & 29 & $0.5(0.2)$ & 21 & $0.4(0.2)$ & $0.1(-0.01,0.21)^{\mathrm{E}}$ & 0.002 \\
\hline $\begin{array}{l}\text { Topp, 2009, } \\
\text { 19695525, } \\
\text { USA }\end{array}$ & $\begin{array}{l}\text { Strength: Knee peak extension } \\
(\mathrm{Nm} / \mathrm{kg})^{\mathrm{B}}\end{array}$ & High & $3 \mathrm{mo}$ & 26 & $62.27(\mathrm{SE}=4.81)$ & 28 & $\begin{array}{l}60.23 \\
(S E=5.00)\end{array}$ & $\begin{array}{l}2.04(-11.56 \\
15.64)^{\mathrm{E}}\end{array}$ & NR \\
\hline
\end{tabular}

Statistically significant effect sizes are in bold text. In cases where calculated effect size confidence intervals were not-statistically significant but reported p-values were, we deferred to reported p-values and still bolded results.

Abbreviations: $\mathrm{CI}=$ confidence interval, $\mathrm{kg}=$ kilogram, $\mathrm{N}=$ Newton, $\mathrm{Nm} / \mathrm{kg}=$ torque normalized to body weight, $\mathrm{NR}=$ not reported, $\mathrm{PMID}=\mathrm{PubMed}$ identifier, $\mathrm{RoB}=$ risk of bias, $\mathrm{s}=$ second, $\mathrm{SD}=$ standard deviation, $\mathrm{SE}=$ standard error

A Time from surgery

B Measured with a dynamometer

${ }^{\mathrm{C}}$ Measured with a hand-held pull gauge

Deasure rapid alternation between concentric and eccentric function, maximum number of knee-bending in 30 seconds (higher is better function)

${ }^{\mathrm{E}}$ Calculated

Table 8. Prehabilitation versus no prehabilitation for total knee arthroplasty - continuous outcomes, energy and vigor

\begin{tabular}{|l|l|l|l|l|l|l|l|l|}
$\begin{array}{l}\text { Study, Year, } \\
\text { PMID, Country }\end{array}$ & $\begin{array}{l}\text { Outcome } \\
\text { Measurement }\end{array}$ & $\begin{array}{l}\text { Overall } \\
\text { RoB }\end{array}$ & $\begin{array}{l}\text { Time } \\
\text { Point }^{\mathrm{A}}\end{array}$ & $\begin{array}{l}\text { Prehab, } \\
\text { N }\end{array}$ & $\begin{array}{l}\text { Prehab, Mean } \\
\text { (SD) }\end{array}$ & $\begin{array}{l}\text { Control, } \\
\text { N }\end{array}$ & $\begin{array}{l}\text { Control, Mean } \\
\text { (SD) }\end{array}$ & $\begin{array}{l}\text { Reported } \\
\text { p-Value }\end{array}$ \\
\hline $\begin{array}{l}\text { Huber, 2015, } \\
\begin{array}{l}25925404, \\
\text { Switzerland }\end{array}\end{array}$ & SF-36: Vitality (0-100) & High & $3 \mathrm{mo}$ & 21 & NR & 20 & NR \\
\hline $\begin{array}{l}\text { Mitchell, 2005, } \\
\text { 15869558, UK }\end{array}$ & SF-36: Vitality (0-100) & High & $3 \mathrm{mo}$ & 57 & $50.7(19.5)$ & 57 & $-8.3(-20.0,3.3)$ \\
\hline
\end{tabular}

Statistically significant effect sizes are in bold text. In cases where calculated effect size confidence intervals were not-statistically significant but reported p-values were, we deferred to reported p-values and still bolded results. 
Abbreviations: $\mathrm{CI}=$ confidence interval, $\mathrm{NR}=$ not reported, $\mathrm{PMID}=$ PubMed identifier, $\mathrm{RoB}=$ risk of bias, $\mathrm{SF}-36=36$-Item short form survey, $\mathrm{SD}=$ standard deviation.

A Time from surgery

Table 9. Prehabilitation versus no prehabilitation for total knee arthroplasty - continuous outcomes, emotional functioning (stress/coping)

\begin{tabular}{|c|c|c|c|c|c|c|c|c|c|}
\hline $\begin{array}{l}\text { Study, Year, } \\
\text { PMID, Country }\end{array}$ & Outcome Measurement & $\begin{array}{l}\text { Overall } \\
\text { RoB }\end{array}$ & $\begin{array}{l}\text { Time } \\
\text { Point }^{\mathrm{A}}\end{array}$ & $\begin{array}{l}\text { Prehab, } \\
\text { N }\end{array}$ & $\begin{array}{l}\text { Prehab, Mean } \\
\text { (SD) }\end{array}$ & $\begin{array}{l}\text { Control, } \\
\text { N }\end{array}$ & $\begin{array}{l}\text { Control, } \\
\text { Mean (SD) }\end{array}$ & $\begin{array}{l}\text { Effect Size } \\
(95 \% \mathrm{Cl})\end{array}$ & $\begin{array}{l}\text { Reported } \\
\text { p-Value }\end{array}$ \\
\hline \multirow{3}{*}{$\begin{array}{l}\text { Huber, 2015, } \\
25925404, \\
\text { Switzerland }\end{array}$} & $\begin{array}{l}\text { SF-36: Emotional role functioning } \\
(0-100)\end{array}$ & High & $3 \mathrm{mo}$ & 21 & NR & 20 & NR & $-10.2(-34.0,13.5)$ & NR \\
\hline & SF-36: Social functioning $(0-100)$ & High & $3 \mathrm{mo}$ & 21 & NR & 20 & NR & $-1.6(-13.7,10.5)$ & NR \\
\hline & SF-36: Mental health $(0-100)$ & High & $3 \mathrm{mo}$ & 21 & NR & 20 & NR & $-3.0(-12.2,6.1)$ & NR \\
\hline \multirow[t]{3}{*}{$\begin{array}{l}\text { Mitchell, 2005, } \\
\text { 15869558, UK }\end{array}$} & $\begin{array}{l}\text { SF-36: Emotional role functioning } \\
(0-100)\end{array}$ & High & $3 \mathrm{mo}$ & 57 & $48.0(46.7)$ & 57 & $45.6(44.8)$ & $\begin{array}{l}\text { Adj MD } 4.1(-10.9 \\
19.0)^{\mathrm{B}}\end{array}$ & 0.592 \\
\hline & SF-36: Social functioning $(0-100)$ & High & $3 \mathrm{mo}$ & 57 & $64.1(26.6)$ & 57 & $60.8(33.1)$ & $\begin{array}{l}\text { Adj MD } 6.7(-3.4 \\
16.7)\end{array}$ & 0.193 \\
\hline & SF-36: Mental health $(0-100)$ & High & $3 \mathrm{mo}$ & 57 & $68.0(20.4)$ & 57 & $71.2(20.0)$ & $\begin{array}{l}\text { Adj MD -2.9 (-9.3, } \\
3.5)\end{array}$ & 0.368 \\
\hline
\end{tabular}

Statistically significant effect sizes are in bold text. In cases where calculated effect size confidence intervals were not-statistically significant but reported p-values were, we deferred to reported p-values and still bolded results.

Abbreviations: $\mathrm{Adj}=$ adjusted, $\mathrm{CI}=$ confidence interval, $\mathrm{MD}=$ mean difference, $\mathrm{mo}=$ month, $\mathrm{NR}=$ not reported, $\mathrm{PMID}=\mathrm{PubMed}$ identifier, $\mathrm{RoB}=$ risk of bias, $\mathrm{SD}=$ standard deviation, SF-36 = 36-Item short form survey.

A Time from surgery

${ }^{B}$ Regression coefficient represents the effect on post-operative quality of life due to the presence of intervention after adjusting for pre-operative quality of life and pre-operative waiting time 


\section{Activity and Participation Outcomes}

Nine RCTs (Calatayud 2017, Huber 2015, Mitchell 2005, Skoffer 2016, Soni 2012, Topp 2009, Valtonen 2015, Villadsen 2014, Williamson 2007) reported on patient-reported outcomes comparing prehabilitation to no prehabilitation (Tables 10 to 16). The outcome domains included: Physical function and activities of daily living, repeat sit-to-stand test, balance, walking speed, walking distance, stair ascent and descent, and timed up and go.

\section{Physical Function and Activities of Daily Living}

Eight RCTs (Calatayud 2017, Huber 2015, Matassi 2014, Mitchell 2005, Skoffer 2016, Soni 2012, Villadsen 2014, Williamson 2007) reported on physical function and ADL using six different measurement instruments (Table 10). Seven studies found no significant differences between prehabilitation and control at 3 to 12 months after surgery.

Calatayud 2017 reported function using the physical component of the SF-36 (0-100, higher is better) and the physical function component of the WOMAC ( 0 to 68 , lower is better) and found significant improvements in function on both measures (SF-36: MD 2.7, 95\% CI 1.3 to 4.1; WOMAC: $-3.9,95 \% \mathrm{CI}-5.2$ to -2.7 ) among patients randomized to high intensity preoperative training compared to patients who did not receive prehabilitation training at 3 months follow-up.

\section{Repeated Sit-To-Stand Test}

Four RCTs (Huber 2015, Skoffer 2016, Topp 2009, Valtonen 2015) reported on three different outcomes measurements from repeated sit-to-stand tests (Table 11). Two studies reported the outcome measurement of the number of sit-to-stands completed in 30 seconds (higher is better), one study reported the amount of time to complete 5 sit-to-stands (in seconds; lower is better), and another study reported the number of times to complete an unspecified number of sit-to-stands (lower is better). Two studies reported improved performance in this test associated with prehabilitation compared to no prehabilitation, while the other two found no difference. Skoffer 2016 reported the number of sit-to stands performed in 30 seconds and found patients randomized to preoperative progressive resistance training performed significantly better than patients randomized to control (MD 3.3, 95\% CI 0.76 to 5.84) at 3 months after surgery. Valtonen 2015 reported the time to complete an unspecified number of sit-to-stands and found a

$15 \%$ decrease in the sit-to-stand time ( $\mathrm{MD}=$ not reported; $\mathrm{p}=0.003$ ) among patients randomized to aquatic training at 3 months after surgery.

The two other studies reported comparable sit-to-stand time (Huber 2015) and number of sitto-stands (Topp 2009) for their prehabilitation compared to control at 3 months follow-up.

\section{Balance}

One RCT (Calatayud 2017) reported static balance data from three different outcomes measurements of the Romberg Test: the anteroposterior range of center of pressure (COP) (millimeters [mm], lower COP indicates better balance); COP area (millimeters [mm], lower $\mathrm{COP}$ area indicates better balance); and medial lateral range of COP $(\mathrm{mm}$, lower COP indicates better balance) (Table 12). Tests were conducted with eyes open and closed using the NedSVE/IBV force platform. (Note that only results for eyes open were extracted.) Calatayud 2017 reported significant improvements in two of the three balance measures (anteroposterior range of COP; MD -3.2 $(-4.0,-2.4)$ and COP area: $-7.4(-12.3,-2.4)$ among patients 
randomized to the high intensity preoperative training compared to control at 3 months after surgery. The other balance measure (medial lateral range of COP) favored the prehabilitation group but was not significant (MD $-0.5,95 \% \mathrm{CI}-1.1$ to 0.2 ).

\section{Walking Speed}

Five RCTs (Huber 2015, Skoffer 2016, Soni 2012, Soni 2012, Valtonen 2015, Williamson 2007), reported on four different outcomes measurements of walking speed: the 50, 20, and 10 meter walk tests, and maximal walking speed (metric not specified) (Table 13).

Two studies reported prehabilitation was associated with improved performance of walking speed compared to control, while three studies reported no difference between groups. Skoffer 2016 reported findings from the 10-meter walk test and found patients randomized to preoperative progressive resistance training took significantly less time to complete 10-meter walk test compared to the control group at 3 months after surgery ( $M D-0.6$ seconds, $\mathrm{P}=0.216$ ). Valtonen 2015 reported maximal walking speed and observed a 15 percent increase in walking speed among patients randomized to aquatic training compared to control at 3 months after surgery $(\mathrm{P}=0.005)$.

The three other studies reported no significant difference between groups in terms of walking speed.

\section{Walking Distance}

Two RCTs (Skoffer 2016 and Topp 2009) reported data on walking distance using the 6minute walk test (6MWT), which measures the maximal walking distance covered in 6 minutes. Neither study observed significant differences in walking distance between prehabilitation and control at 3 months after surgery (Table 14).

\section{Stair Ascent and Descent}

Two RCTs (Calatayud 2017 and Topp 2009) reported data on stair tests from three different stair climb tests outcome measurements: four flights of stair ascend/descend (seconds, smaller is better); stair climb test to ascent flight of 22 steps (seconds, smaller is better); stair climb test to descend a flight of 22 steps (seconds, smaller is better) (Table 15). Calatayud 2017 reported data on the time it took patients to ascend and descend four flights of stairs and found improved performance of the stair test among patients randomized to the high intensity preoperative training compared to control at 3 months after surgery. Topp 2009 observed no significant differences between prehabilitation and no prehabilitation groups.

\section{Timed Up and Go}

Three RCTs (Calatayud 2017, Huber 2015, and Skoffer 2016) reported data on the Timed Up and Go (TUG) test (Table 16). TUG is designed to assess lower extremity mobility and function. It requires patients to stand from a chair, walk to a line 3 meters away, turn and walk back, turn and sit down in the chair (measured in seconds [s], smaller is better). Two RCTs (Calatayud 2017 and Skoffer 2016) reported improved performance of the TUG associated with their prehabilitation programs (high intensity preoperative training and progressive resistance training, respectively) compared to control at 3 months after surgery. Topp 2015 found no significant differences in the performance of the TUG test between prehabilitation and no prehabilitation groups at 3 months after surgery. 
Table 10. Prehabilitation versus no prehabilitation for total knee arthroplasty - continuous outcomes, physical function and activities of daily living

\begin{tabular}{|c|c|c|c|c|c|c|c|c|c|}
\hline $\begin{array}{l}\text { Study, Year, } \\
\text { PMID, Country }\end{array}$ & Outcome Measurement & $\begin{array}{l}\text { Overall } \\
\text { RoB }\end{array}$ & $\begin{array}{l}\text { Time } \\
\text { Point }^{A}\end{array}$ & $\begin{array}{l}\text { Prehab, } \\
\text { N }\end{array}$ & $\begin{array}{l}\text { Prehab, Mean } \\
\text { (SD) }\end{array}$ & $\begin{array}{l}\text { Control, } \\
\text { N }\end{array}$ & $\begin{array}{l}\text { Control Mean } \\
\text { (SD) }\end{array}$ & $\begin{array}{l}\text { Effect Size }(95 \% \\
\mathrm{CI})\end{array}$ & $\begin{array}{l}\text { Reported } p \text { - } \\
\text { Value }\end{array}$ \\
\hline \multirow{2}{*}{$\begin{array}{l}\text { Calatayud, 2017, } \\
26768606, \\
\text { Spain }\end{array}$} & $\begin{array}{l}\text { SF-36: Physical component (0- } \\
100)\end{array}$ & Moderate & $3 \mathrm{mo}$ & 22 & $\begin{array}{l}55.7(95 \% \mathrm{Cl} \\
54.6,56.8)\end{array}$ & 22 & $\begin{array}{l}53(95 \% \mathrm{Cl} \\
51.9,54.1)\end{array}$ & $2.7(1.3,4.1)$ & $<0.001$ \\
\hline & $\begin{array}{l}\text { WOMAC: Physical function (0- } \\
68 \text { ) }\end{array}$ & Moderate & $3 \mathrm{mo}$ & 22 & $\begin{array}{l}18.8(95 \% \mathrm{Cl} \\
17.8,19.7)\end{array}$ & 22 & $\begin{array}{l}22.7(95 \% \mathrm{Cl} \\
21.7,23.7)\end{array}$ & $-3.9(-5.2,-2.7)$ & $<0.05$ \\
\hline \multirow{7}{*}{$\begin{array}{l}\text { Huber, 2015, } \\
\text { 25925404, } \\
\text { Switzerland }\end{array}$} & EQ-5D: Self-care (1-3) & High & $3 \mathrm{mo}$ & 21 & NR & 20 & NR & $0.1(-0.1,0.3)$ & NR \\
\hline & EQ-5D: Usual activities (1-3) & High & $3 \mathrm{mo}$ & 21 & NR & 20 & NR & $0.0(-0.4,0.4)$ & NR \\
\hline & EQ-5D: Mobility (1-3) & High & $3 \mathrm{mo}$ & 21 & NR & 20 & NR & $-0.1(-0.4,0.2)$ & NR \\
\hline & KOOS: ADL (0-100) & High & $3 \mathrm{mo}$ & 21 & NR & 20 & NR & $-4.9(-16.3,6.5)$ & NR \\
\hline & KOOS: Sport/rec (0-100) & High & $3 \mathrm{mo}$ & 21 & NR & 20 & NR & $1.0(-19.9,21.8)$ & NR \\
\hline & $\begin{array}{l}\text { SF-36: Physical role functioning } \\
(0-100)\end{array}$ & High & $3 \mathrm{mo}$ & 21 & NR & 20 & NR & $-3.2(-32.2,25.9)$ & NR \\
\hline & $\begin{array}{l}\text { SF-36: Physical functioning (0- } \\
100)\end{array}$ & High & $3 \mathrm{mo}$ & 21 & NR & 20 & NR & $-6.6(-8.5,17.5)$ & NR \\
\hline \multirow{2}{*}{$\begin{array}{l}\text { Matassi, 2014, } \\
23271039, \\
\text { Belgium }\end{array}$} & Knee Society Score: Knee score & Moderate & $12 \mathrm{mo}$ & 61 & NR & 61 & NR & NR & ns \\
\hline & $\begin{array}{l}\text { Knee Society Score: Function } \\
\text { score }\end{array}$ & Moderate & $12 \mathrm{mo}$ & 61 & NR & 61 & NR & NR & ns \\
\hline \multirow[t]{3}{*}{$\begin{array}{l}\text { Mitchell, 2005, } \\
\text { 15869558, UK }\end{array}$} & $\begin{array}{l}\text { SF-36: Physical role functioning } \\
(0-100)\end{array}$ & High & $3 \mathrm{mo}$ & 57 & $27.6(37.1)$ & 57 & $23.2(36.2)$ & $\begin{array}{l}\text { Adj MD } 7.8(-5.6 \\
21.2)\end{array}$ & 0.249 \\
\hline & SF-36: Physical function $(0-100)$ & High & $3 \mathrm{mo}$ & 57 & $41.6(22.2)$ & 57 & $43.3(27.6)$ & $\begin{array}{l}\text { Adj MD } 2.5(-6.3 \text {, } \\
11.3)\end{array}$ & 0.579 \\
\hline & $\begin{array}{l}\text { WOMAC: Physical function (0- } \\
68)\end{array}$ & High & $3 \mathrm{mo}$ & 57 & $24.9(13.4)$ & 57 & $26.4(14.9)$ & $\begin{array}{l}\text { Adj MD -1.0 (- } \\
5.9,3.8)\end{array}$ & 0.677 \\
\hline \multirow{2}{*}{$\begin{array}{l}\text { Skoffer, 2016, } \\
\text { 26713665, } \\
\text { Denmark }\end{array}$} & KOOS: ADL (0-100) & Moderate & $3 \mathrm{mo}$ & 29 & $82.9(11.7)$ & 21 & $78.2(12.9)$ & $4.7(-0.3,9.7)^{\mathrm{B}}$ & NR \\
\hline & KOOS: Sport/rec (0-100) & Moderate & $3 \mathrm{mo}$ & 29 & $50.2(28.4)$ & 21 & $40(22.5)$ & $10.2(0.2,20.2)^{B}$ & NR \\
\hline $\begin{array}{l}\text { Soni, 2012, } \\
\text { 22914302, UK }\end{array}$ & Oxford knee score $(0-48)$ & Moderate & $3 \mathrm{mo}$ & 20 & $27.4(10)$ & 21 & $25.1(10.6)$ & $-2.2(-8.7,4.3)$ & NR \\
\hline \multirow{2}{*}{$\begin{array}{l}\text { Villadsen, 2014, } \\
\text { 23661494, } \\
\text { Denmark }\end{array}$} & KOOS: ADL (0-100) & Moderate & $3 \mathrm{mo}$ & 84 & NR & 81 & NR & $-5.6(-12.9,1.8)$ & 0.1371 \\
\hline & KOOS: Sport/rec (0-100) & Moderate & $3 \mathrm{mo}$ & 84 & NR & 81 & NR & $-5.6(-15.6,4.5)$ & 0.2779 \\
\hline $\begin{array}{l}\text { Williamson, 2007, } \\
\text { 17604311,UK }\end{array}$ & Oxford knee score $(0-48)$ & High & $3 \mathrm{mo}$ & 23 & $28.3(9.78)$ & $19^{\mathrm{C}}$ & $26.7(7.45)$ & $1.61(-3.91,7.13)$ & NR \\
\hline
\end{tabular}

Statistically significant effect sizes are in bold text. In cases where calculated effect size confidence intervals were not-statistically significant but reported p-values were, we deferred to reported p-values and still bolded results. 
Abbreviations: $\mathrm{Adj}=$ adjusted, $\mathrm{ADL}=$ activities of daily living, $\mathrm{CI}=$ confidence interval, $\mathrm{EQ}-5 \mathrm{D}=$ EuroQual, $\mathrm{KOOS}=\mathrm{Knee}$ injury and osteoarthritis outcome score, $\mathrm{MD}=$ mean difference, $\mathrm{mo}=$ month, $\mathrm{NR}=$ not reported, $\mathrm{ns}=$ not significant, $\mathrm{PMID}=$ PubMed identifier, rec $=$ recreation, $\mathrm{RoB}=$ risk of bias, $\mathrm{SD}=\mathrm{standard}$ deviation, $\mathrm{SF}-36=36-\mathrm{Item}$ short form survey, WOMAC $=$ Western Ontario and McMaster Universities Osteoarthritis Index.

A Time from surgery

B Calculated

${ }^{\mathrm{C}}$ Control arm sample size is uncertain from study report. Study was retained despite potential sample size of control being less than 20

Table 11. Prehabilitation versus no prehabilitation for total knee arthroplasty - continuous outcomes, repeated stand test (sit-to-stand)

\begin{tabular}{|c|c|c|c|c|c|c|c|c|c|}
\hline $\begin{array}{l}\text { Study, Year, PMID, } \\
\text { Country }\end{array}$ & Outcome Measurement & $\begin{array}{l}\text { Overall } \\
\text { RoB }\end{array}$ & $\begin{array}{l}\text { Time } \\
\text { Point }^{\mathrm{A}}\end{array}$ & $\begin{array}{l}\text { Prehab, } \\
\text { N }\end{array}$ & $\begin{array}{l}\text { Prehab, Mean } \\
\text { (SD) }\end{array}$ & $\begin{array}{l}\text { Control, } \\
\text { N }\end{array}$ & $\begin{array}{l}\text { Control Mean } \\
\text { (SD) }\end{array}$ & $\begin{array}{l}\text { Effect Size }(95 \% \\
\text { CI) }\end{array}$ & $\begin{array}{l}\text { Reported } p- \\
\text { Value }\end{array}$ \\
\hline $\begin{array}{l}\text { Huber, 2015, } \\
25925404, \text { Switzerland }\end{array}$ & $\begin{array}{l}\text { Chair stand test: Time to } \\
\text { complete } 5 \text { sit-to-stands (s) }\end{array}$ & High & $3 \mathrm{mo}$ & 21 & NR & 20 & NR & $2.0(-1.8,5.8)$ & NR \\
\hline $\begin{array}{l}\text { Skoffer, 2016, } \\
26713665 \text {, Denmark }\end{array}$ & $\begin{array}{l}\text { Chair stand test: Total sit-to- } \\
\text { stands in } 30 \mathrm{~s}(\mathrm{n})\end{array}$ & Moderate & $3 \mathrm{mo}$ & 29 & $14.7(4.7)$ & 21 & $11.0(4.4)$ & $3.3(0.76,5.84)^{B}$ & 0.001 \\
\hline $\begin{array}{l}\text { Topp, 2009, } \\
\text { 19695525, USA }\end{array}$ & $\begin{array}{l}\text { Chair stand test: Total sit-to- } \\
\text { stands in } 30 \mathrm{~s}(\mathrm{n})\end{array}$ & High & $3 \mathrm{mo}$ & 26 & $\begin{array}{l}12.87 \\
(\mathrm{SE}=0.82)\end{array}$ & 28 & $\begin{array}{l}11.25 \\
(\mathrm{SE}=0.79)\end{array}$ & $1.62(-0.73,3.97)^{\mathrm{B}}$ & NR \\
\hline $\begin{array}{l}\text { Valtonen, 2015, } \\
\text { CN-01126383, Finland }\end{array}$ & $\begin{array}{l}\text { Chair stand test: Time to } \\
\text { complete unspecified number } \\
\text { of sit-to-stands (NR) }\end{array}$ & High & $3 \mathrm{mo}$ & 31 & NR & 24 & NR & $\begin{array}{l}15 \% \text { decrease in } \\
\text { sit-to-stand time }\end{array}$ & 0.003 \\
\hline
\end{tabular}

Statistically significant effect sizes are in bold text. In cases where calculated effect size confidence intervals were not-statistically significant but reported p-values were, we deferred to reported p-values and still bolded results.

Abbreviations: $\mathrm{CI}=$ confidence interval, $\mathrm{mo}=$ month, $\mathrm{NR}=$ not reported, $\mathrm{PMID}=$ PubMed identifier, $\mathrm{RoB}=$ risk of bias, $\mathrm{SD}=$ standard deviation, $\mathrm{SE}=$ standard error.

A Time from surgery

${ }^{B}$ Calculated

Table 12. Prehabilitation versus no prehabilitation for total knee arthroplasty - continuous outcomes, balance

\begin{tabular}{|c|c|c|c|c|c|c|c|c|c|}
\hline $\begin{array}{l}\text { Study, Year, } \\
\text { PMID, Country }\end{array}$ & Outcome Measurement & $\begin{array}{l}\text { Overall } \\
\text { RoB }\end{array}$ & $\begin{array}{l}\text { Time } \\
\text { Point }^{A}\end{array}$ & $\begin{array}{l}\text { Prehab, } \\
\text { N }\end{array}$ & $\begin{array}{l}\text { Prehab, Mean } \\
\text { (SD) }\end{array}$ & $\begin{array}{l}\text { Control, } \\
\mathrm{N}\end{array}$ & $\begin{array}{l}\text { Control Mean } \\
\text { (SD) }\end{array}$ & $\begin{array}{l}\text { Effect Size }(95 \% \\
\mathrm{Cl})\end{array}$ & $\begin{array}{l}\text { Reported p- } \\
\text { Value }\end{array}$ \\
\hline \multirow[t]{3}{*}{$\begin{array}{l}\text { Calatayud, 2017, } \\
\text { 26768606, } \\
\text { Spain }\end{array}$} & $\begin{array}{l}\text { Romberg test (eyes open): } \\
\text { Anteroposterior range of COP } \\
(\mathrm{mm})\end{array}$ & Moderate & $3 \mathrm{mo}$ & 22 & $\begin{array}{l}17(95 \% \mathrm{Cl} \\
16.4,17.6)\end{array}$ & 22 & $\begin{array}{l}20.2(95 \% \mathrm{Cl} \\
19.6,20.9)\end{array}$ & $-3.2(-4.0,-2.4)$ & NR \\
\hline & $\begin{array}{l}\text { Romberg test (eyes open): COP } \\
\text { area }\end{array}$ & Moderate & $3 \mathrm{mo}$ & 22 & $\begin{array}{l}42.1(95 \% \mathrm{Cl} \\
38.4,45.7)\end{array}$ & 22 & $\begin{array}{l}49.4(95 \% \mathrm{Cl} \\
45.6,53.3)\end{array}$ & $-7.4(-12.3,-2.4)$ & NR \\
\hline & $\begin{array}{l}\text { Romberg test (eyes open): } \\
\text { Medial lateral range of COP }(\mathrm{mm})\end{array}$ & Moderate & $3 \mathrm{mo}$ & 22 & $\begin{array}{l}14.7(95 \% \mathrm{Cl} \\
14.2,15.1)\end{array}$ & 22 & $\begin{array}{l}15.1(95 \% \mathrm{Cl} \\
14.7,15.6)\end{array}$ & $-0.5(-1.1,0.2)$ & NR \\
\hline
\end{tabular}

Statistically significant effect sizes are in bold text. In cases where calculated effect size confidence intervals were not-statistically significant but reported p-values were, we deferred to reported p-values and still bolded results.

Abbreviations: $\mathrm{CI}=$ confidence interval, $\mathrm{COP}=$ center of pressure, $\mathrm{mm}=$ milimeter, $\mathrm{mo}=$ month, $\mathrm{NR}=$ not reported, $\mathrm{PMID}=\mathrm{PubMed}$ identifier, $\mathrm{RoB}=$ risk of bias, $\mathrm{SD}=$ standard deviation.

A Time from surgery 
Table 13. Prehabilitation versus no prehabilitation for total knee arthroplasty - continuous outcomes, walking speed

\begin{tabular}{|c|c|c|c|c|c|c|c|c|c|}
\hline $\begin{array}{l}\text { Study, Year, } \\
\text { PMID, Country }\end{array}$ & Outcome Measurement & $\begin{array}{l}\text { Overall } \\
\text { RoB }\end{array}$ & $\begin{array}{l}\text { Time } \\
\text { Point }^{A}\end{array}$ & $\begin{array}{l}\text { Prehab, } \\
\text { N }\end{array}$ & $\begin{array}{l}\text { Prehab, Mean } \\
\text { (SD) }\end{array}$ & $\begin{array}{l}\text { Control, } \\
\mathrm{N}\end{array}$ & $\begin{array}{l}\text { Control Mean } \\
\text { (SD) }\end{array}$ & $\begin{array}{l}\text { Effect Size }(95 \% \\
\mathrm{Cl})\end{array}$ & $\begin{array}{l}\text { Reported p- } \\
\text { Value }\end{array}$ \\
\hline $\begin{array}{l}\text { Huber, 2015, } \\
25925404, \\
\text { Switzerland }\end{array}$ & $20-m$ walk test ${ }^{B}$ & High & $3 \mathrm{mo}$ & 21 & NR & 20 & NR & $-0.5(-2.0,1.0)$ & NR \\
\hline $\begin{array}{l}\text { Skoffer, 2016, } \\
\text { 26713665, } \\
\text { Denmark }\end{array}$ & 10-m walk test (s) & Moderate & $3 \mathrm{mo}$ & 29 & $7.1(1.5)$ & 21 & $7.7(1.2)$ & $-0.6(-1.1,-0.1)^{\mathrm{C}}$ & 0.216 \\
\hline $\begin{array}{l}\text { Soni, 2012, } \\
\text { 22914302, } \\
\text { UK }\end{array}$ & 50-m walk test (s) & Moderate & $3 \mathrm{mo}$ & 20 & $64.1(44.7)$ & 21 & $55.0(18.4)$ & $9.1(-12.7,31.0)$ & NR \\
\hline $\begin{array}{l}\text { Valtonen, 2015, } \\
\text { CN-01126383, } \\
\text { Finland }\end{array}$ & Maximal walking speed (NR) & High & $3 \mathrm{mo}$ & 31 & NR & 24 & NR & $\begin{array}{l}15 \% \text { increase in } \\
\text { walking speed }\end{array}$ & 0.005 \\
\hline $\begin{array}{l}\text { Williamson, 2007, } \\
\text { 17604311, UK }\end{array}$ & $50-\mathrm{m}$ walk test (s) & High & $3 \mathrm{mo}$ & 23 & $46.6(11.4)$ & $19^{\mathrm{D}}$ & $44.1(6.91)$ & $2.51(-3.48,8.51)$ & $\mathrm{NR}$ \\
\hline
\end{tabular}

Statistically significant effect sizes are in bold text. In cases where calculated effect size confidence intervals were not-statistically significant but reported p-values were, we deferred to reported p-values and still bolded results.

Abbreviations: $\mathrm{CI}=$ confidence interval, $\mathrm{mo}=$ month, $\mathrm{NR}=$ not reported, $\mathrm{PMID}=$ PubMed identifier, $\mathrm{RoB}=$ risk of bias, $\mathrm{s}=\mathrm{second}, \mathrm{SD}=\mathrm{standard}$ deviation.

A Time from surgery

${ }^{B}$ Study reported that the test measures the time it takes to walk 20 meters at the participant's usual walking pace, and the number of steps that they take to walk 20 meters. Study

did not specify what scale was used for reported outcome (i.e., seconds vs. steps)

${ }^{\mathrm{C}}$ Calculated

${ }^{\mathrm{D}}$ Control arm sample size is uncertain from study report. Study was retained despite potential sample size of control being less than 20

Table 14. Prehabilitation versus no prehabilitation for total knee arthroplasty - continuous outcomes, walking distance

\begin{tabular}{|c|c|c|c|c|c|c|c|c|c|}
\hline $\begin{array}{l}\text { Study, Year, } \\
\text { PMID, Country }\end{array}$ & Outcome Measurement & $\begin{array}{l}\text { Overall } \\
\text { RoB }\end{array}$ & $\begin{array}{l}\text { Time } \\
\text { Point }^{A}\end{array}$ & $\begin{array}{l}\text { Prehab, } \\
\text { N }\end{array}$ & $\begin{array}{l}\text { Prehab, Mean } \\
\text { (SD) }\end{array}$ & $\begin{array}{l}\text { Control, } \\
\text { N }\end{array}$ & $\begin{array}{l}\text { Control Mean } \\
\text { (SD) }\end{array}$ & $\begin{array}{l}\text { Effect Size }(95 \% \\
\mathrm{Cl})\end{array}$ & $\begin{array}{l}\text { Reported } \\
\text { p-Value }\end{array}$ \\
\hline $\begin{array}{l}\text { Skoffer, 2016, } \\
\text { 26713665, } \\
\text { Denmark }\end{array}$ & 6MWT (m) & Moderate & $3 \mathrm{mo}$ & 29 & $449(94)$ & 21 & $433(74)$ & $16(-17,49)^{\mathrm{B}}$ & 0.208 \\
\hline $\begin{array}{l}\text { Topp, 2009, } \\
\text { 19695525, USA }\end{array}$ & 6MWT (m) & High & $3 \mathrm{mo}$ & 26 & 1337 (SE=58) & 28 & $1365(\mathrm{SE}=56)$ & $\begin{array}{l}-28(-194.28, \\
138.28)^{\mathrm{B}}\end{array}$ & NR \\
\hline
\end{tabular}

Statistically significant effect sizes are in bold text. In cases where calculated effect size confidence intervals were not-statistically significant but reported p-values were, we deferred to reported p-values and still bolded results.

Abbreviations: $6 \mathrm{MWT}=$ six-minute walk test $\mathrm{CI}=$ confidence interval, $\mathrm{mo}=$ month, $\mathrm{m}=$ meter, $\mathrm{NR}=$ not reported, $\mathrm{PMID}=\mathrm{PubMed}$ identifier, $\mathrm{RoB}=$ risk of bias, $\mathrm{SD}=$ standard deviation, $\mathrm{SE}=$ standard error.

A Time from surgery

${ }^{B}$ Calculated 
Table 15. Prehabilitation versus no prehabilitation for total knee arthroplasty - continuous outcomes, stair ascent/descent

\begin{tabular}{|c|c|c|c|c|c|c|c|c|c|}
\hline $\begin{array}{l}\text { Study, Year, } \\
\text { PMID, Country }\end{array}$ & Outcome Measurement & $\begin{array}{l}\text { Overall } \\
\text { RoB }\end{array}$ & $\begin{array}{l}\text { Time } \\
\text { Point }^{A}\end{array}$ & $\begin{array}{l}\text { Prehab, } \\
\text { N }\end{array}$ & $\begin{array}{l}\text { Prehab, Mean } \\
\text { (SD) }\end{array}$ & $\begin{array}{l}\text { Control, } \\
\mathrm{N}\end{array}$ & $\begin{array}{l}\text { Control Mean } \\
\text { (SD) }\end{array}$ & $\begin{array}{l}\text { Effect Size } \\
(95 \% \mathrm{Cl})\end{array}$ & $\begin{array}{l}\text { Reported p- } \\
\text { Value }\end{array}$ \\
\hline $\begin{array}{l}\text { Calatayud, } \\
2017, \\
26768606, \\
\text { Spain }\end{array}$ & $\begin{array}{l}\text { Stair climb test: Ascend } \\
\text { descend and descend flight of } 4 \\
\text { stairs (s) }\end{array}$ & Moderate & $3 \mathrm{mo}$ & 22 & $\begin{array}{l}7.9(95 \% \mathrm{Cl} \\
7.2,8.5)\end{array}$ & 22 & $\begin{array}{l}12.1(95 \% \mathrm{Cl} \\
11.5,12.8)\end{array}$ & $-4.2(-5.1,-3.4)$ & $<0.05$ \\
\hline \multirow{2}{*}{$\begin{array}{l}\text { Topp, } \\
2009 \text {, } \\
19695525, \\
\text { USA }\end{array}$} & $\begin{array}{l}\text { Stair climb test: Ascend flight of } \\
22 \text { stairs (s) }\end{array}$ & High & $3 \mathrm{mo}$ & 26 & $8.44(\mathrm{SE}=0.77)$ & 28 & $7.45(\mathrm{SE}=0.77)$ & $\begin{array}{l}0.99(-1.26 \\
3.24)^{\mathrm{B}}\end{array}$ & NR \\
\hline & $\begin{array}{l}\text { Stair climb test: Descend flight } \\
\text { of } 22 \text { stairs (s) }\end{array}$ & High & $3 \mathrm{mo}$ & 26 & $8.6(\mathrm{SE}=1.06)$ & 28 & $8.06(\mathrm{SE}=1.06)$ & $\begin{array}{l}0.54(-2.56 \\
3.64)^{\mathrm{B}}\end{array}$ & NR \\
\hline
\end{tabular}

Statistically significant effect sizes are in bold text. In cases where calculated effect size confidence intervals were not-statistically significant but reported p-values were, we deferred to reported p-values and still bolded results.

Abbreviations: $\mathrm{CI}=$ confidence interval, $\mathrm{mo}=$ month, $\mathrm{NR}=$ not reported, $\mathrm{PMID}=$ PubMed identifier, $\mathrm{RoB}=$ risk of bias, $\mathrm{s}=\mathrm{second}, \mathrm{SD}=\mathrm{standard}$ deviation, $\mathrm{SE}=$ standard error

A Time from surgery

B Calculated

Table 16. Prehabilitation versus no prehabilitation for total knee arthroplasty - continuous outcomes, Timed Up and Go

\begin{tabular}{|c|c|c|c|c|c|c|c|c|}
\hline $\begin{array}{l}\text { Study, Year, PMID, } \\
\text { Country }\end{array}$ & Overall RoB & Time Point ${ }^{A}$ & Prehab, N & Prehab, Mean (SD) & $\begin{array}{l}\text { Control, } \\
\mathrm{N}\end{array}$ & Control Mean (SD) & Effect Size $(95 \% \mathrm{Cl})$ & $\begin{array}{l}\text { Reported p- } \\
\text { Value }\end{array}$ \\
\hline $\begin{array}{l}\text { Calatayud, 2017, } \\
\text { 26768606, Spain }\end{array}$ & Moderate & $3 \mathrm{mo}$ & 22 & $7.0(95 \% \mathrm{Cl} 6.7,7.3)$ & 22 & $8.7(95 \% \mathrm{Cl} 8.3,9.1)$ & $-1.7(-2.1,-1.3)$ & $<0.05$ \\
\hline $\begin{array}{l}\text { Huber, 2015, } \\
\text { 25925404, Switzerland }\end{array}$ & High & $3 \mathrm{mo}$ & 21 & NR & 20 & $\mathrm{NR}$ & $1.6(-0.1,3.3)$ & $\mathrm{NR}$ \\
\hline $\begin{array}{l}\text { Skoffer,2016, } \\
\text { 26713665, Denmark }\end{array}$ & Moderate & $3 \mathrm{mo}$ & 29 & $7.9(2.3)$ & 21 & $8.9(2.1)$ & $-1.0(-1.9 .-0.1)^{\mathrm{B}}$ & 0.05 \\
\hline
\end{tabular}

Statistically significant effect sizes are in bold text. In cases where calculated effect size confidence intervals were not-statistically significant but reported p-values were, we deferred to reported p-values and still bolded results.

Abbreviations: $\mathrm{CI}=$ confidence interval, $\mathrm{mo}=$ month, $\mathrm{NR}=$ not reported, $\mathrm{PMID}=$ PubMed identifier, $\mathrm{RoB}=$ risk of bias, $\mathrm{s}=\mathrm{second}, \mathrm{SD}=$ standard deviation.

A Time from surgery

${ }^{B}$ Calculated 


\section{Other Patient-Reported Outcomes}

Seven RCTs (Calatayud 2017, Huber 2015, Mitchell 2005, Skoffer 2016, Soni 2012, Villadsen 2014, Williamson 2007) reported data on other patient-reported outcomes not captured by body structure and function or activity and participation domains comparing prehabilitation to no prehabilitation (Tables 17 and 18). Outcome domains included: QoL, patient satisfaction with care, and patient global assessments.

\section{Quality of Life}

Three RCTs (Huber 2015, Skoffer 2016, Villadsen 2014) reported data on QoL, using two different measurement instruments. These studies reported data using the KOOS (for each component 0-100; higher is better). One study (Skoffer 2018) also reported a study-specific assessment of QoL, in which patients were asked to rate their QoL on a scale from 0 (worse QoL imaginable) to 100 (best QoL imaginable) (Table 17).

Two studies (Huber 2015 and Villadsen 20214) reported comparable QoL scores between prehabilitation and control groups at 3 months after TKA. Skoffer 2016 reported QoL using the QoL component of the KOOS (scores 0-100; higher is better), as well as a study-specific scale (scores 0-100; higher is better), and reported significant improvement in QoL among patients randomized to progressive resistance training compared to control using the study-specific outcome measure at 3 months after surgery (MD 10.3, 95\% CI 2.8 to 17.8) but there was no significant difference observed between groups on the QoL component of the KOOS.

\section{Patient Satisfaction With Care}

No RCTs reported data on satisfaction with care.

\section{Patient Global Assessments}

Five RCTs (Calatayud 2017, Huber 2015, Mitchell 2005, Villadsen 2014, Williamson 2007) provided data on patients self-reported global assessment of their health using three different measurement instruments: the total score of the WOMAC (score 0-96; smaller is better); the general health component of the SF-36 (scores 0-100, higher is better), and the EQ-5D index (01) (Table 18).

Only one RCT (Calatayud 2017) reported that prehabilitation was associated with improvements in patients' global assessment of health. Calatayud 2017 reported data on patients' global health assessment using the total WOMAC score and found significant improvements among patients randomized to the high intensity preoperative training group compared to control (MD-5.8, 95\% CI-7.6 to -3.9 ) at 3 months after surgery.

All other studies reported comparable findings in patients' global health assessment scores among prehabilitation and control groups at 3 months after surgery. 
Table 17. Prehabilitation versus no prehabilitation for total knee arthroplasty - continuous outcomes, health-related quality of life

\begin{tabular}{|c|c|c|c|c|c|c|c|c|c|}
\hline $\begin{array}{l}\text { Study, Year, } \\
\text { PMID, Country }\end{array}$ & Outcome Measurement & $\begin{array}{l}\text { Overall } \\
\text { RoB }\end{array}$ & $\begin{array}{l}\text { Time } \\
\text { Point }^{\mathrm{A}}\end{array}$ & $\begin{array}{l}\text { Prehab, } \\
\text { N }\end{array}$ & $\begin{array}{l}\text { Prehab, } \\
\text { Mean (SD) }\end{array}$ & Control, N & $\begin{array}{l}\text { Control Mean } \\
\text { (SD) }\end{array}$ & Effect Size $(95 \% \mathrm{CI})$ & $\begin{array}{l}\text { Reported p- } \\
\text { Value }\end{array}$ \\
\hline $\begin{array}{l}\text { Huber, 2015, } \\
\text { 25925404, } \\
\text { Switzerland }\end{array}$ & KOOS: QoL $(0-100)$ & High & $3 \mathrm{mo}$ & 21 & NR & 20 & NR & $-5.9(-18.5,6.8)$ & NR \\
\hline \multirow{2}{*}{$\begin{array}{l}\text { Skoffer, 2016, } \\
\text { 26713665, } \\
\text { Denmark }\end{array}$} & KOOS: QoL (0-100) & Moderate & $3 \mathrm{mo}$ & 29 & $66.2(18.9)$ & 21 & $61.9(16.6)$ & $4.3(-2.7,11.3)^{\mathrm{B}}$ & NR \\
\hline & $\begin{array}{l}\text { Study-specific QoL scale } \\
(0-100)\end{array}$ & Moderate & $3 \mathrm{mo}$ & 29 & $86.7(10.5)$ & 21 & $76.4(20.1)$ & $10.3(2.8,17.8)^{\mathrm{B}}$ & NR \\
\hline $\begin{array}{l}\text { Villadsen, 2014, } \\
\text { 23661494, } \\
\text { Denmark }\end{array}$ & KOOS: QoL $(0-100)$ & Moderate & $3 \mathrm{mo}$ & 84 & 23 & 81 & 19 & $-4.6(-12.9,3.6)$ & 0.2666 \\
\hline
\end{tabular}

Statistically significant effect sizes are in bold text. In cases where calculated effect size confidence intervals were not-statistically significant but reported p-values were, we deferred to reported p-values and still bolded results.

Abbreviations: $\mathrm{CI}=$ confidence interval, $\mathrm{KOOS}=$ Knee injury and osteoarthritis outcome score, $\mathrm{mo}=$ month, $\mathrm{NR}=$ not reported, $\mathrm{PMID}=\mathrm{PubMed}$ identifier, $\mathrm{QoL}=$ quality of life, $\mathrm{RoB}=$ risk of bias, $\mathrm{SD}=$ standard deviation.

A Time from surgery

B Calculated

${ }^{\mathrm{C}}$ Health-related quality of life was recorded on a rating scale from 0 (worse health-related quality of life imaginable) to 100 (best health-related quality of life imaginable) 
Table 18. Prehabilitation versus no prehabilitation for total knee arthroplasty - continuous outcomes, patient global assessment

\begin{tabular}{|c|c|c|c|c|c|c|c|c|c|}
\hline $\begin{array}{l}\text { Study, Year, } \\
\text { PMID, Country }\end{array}$ & Outcome Measurement & $\begin{array}{l}\text { Overall } \\
\text { RoB }\end{array}$ & $\begin{array}{l}\text { Time } \\
\text { Point }^{\mathrm{A}}\end{array}$ & $\begin{array}{l}\text { Prehab, } \\
\text { N }\end{array}$ & $\begin{array}{l}\text { Prehab, } \\
\text { Mean (SD) }\end{array}$ & $\begin{array}{l}\text { Control, } \\
\text { N }\end{array}$ & $\begin{array}{l}\text { Control Mean } \\
\text { (SD) }\end{array}$ & Effect Size $(95 \% \mathrm{CI})$ & $\begin{array}{l}\text { Reported p- } \\
\text { Value }\end{array}$ \\
\hline $\begin{array}{l}\text { Calatayud, 2017, } \\
\text { 26768606, Spain }\end{array}$ & WOMAC: Total (0-96) & Moderate & $3 \mathrm{mo}$ & 22 & $\begin{array}{l}25(95 \% \mathrm{Cl} \\
23.5,26.4)\end{array}$ & 22 & $\begin{array}{l}30.7(95 \% \mathrm{Cl} \\
29.2,32.2)\end{array}$ & $-5.8(-7.6,-3.9)$ & $<0.05$ \\
\hline $\begin{array}{l}\text { Huber, 2015, } \\
25925404, \\
\text { Switzerland }\end{array}$ & $\begin{array}{l}\text { SF-36: General health (0- } \\
100)\end{array}$ & High & $3 \mathrm{mo}$ & 21 & NR & 20 & NR & $-2.8(-12.0,6.3)$ & NR \\
\hline $\begin{array}{l}\text { Mitchell, 2005, } \\
\text { 15869558, UK }\end{array}$ & $\begin{array}{l}\text { SF-36: General health (0- } \\
100)\end{array}$ & High & $3 \mathrm{mo}$ & 57 & $61.0(23.4)$ & 57 & $61.0(22.9)$ & $\begin{array}{l}\text { Adj MD -0.2 (-7.0, } \\
6.7)\end{array}$ & 0.964 \\
\hline $\begin{array}{l}\text { Villadsen, 2014, } \\
\text { 23661494, } \\
\text { Denmark }\end{array}$ & EQ-5D index (0-1) & Moderate & $3 \mathrm{mo}$ & 84 & NR & 81 & NR & $-0.06(-0.13,0.01)$ & 0.0781 \\
\hline $\begin{array}{l}\text { Williamson, 2007, } \\
17604311, \text { UK }\end{array}$ & WOMAC: Total (0-96) & High & $3 \mathrm{mo}$ & 23 & $26(17.7)$ & 19 & $24.6(16.8)$ & $1.33(-9.53,12.18)$ & NR \\
\hline
\end{tabular}

Statistically significant effect sizes are in bold text. In cases where calculated effect size confidence intervals were not-statistically significant but reported p-values were, we deferred to reported p-values and still bolded results.

Abbreviations: Adj $\mathrm{MD}=$ adjusted mean difference $\mathrm{CI}=$ confidence interval, $\mathrm{EQ}-5 \mathrm{D}=$ EuroQual, $\mathrm{KOOS}=$ Knee injury and osteoarthritis outcome score, mo $=$ month, $\mathrm{NR}=$ not reported, PMID $=$ PubMed identifier, RoB $=$ risk of bias, $\mathrm{SD}=$ standard deviation, $\mathrm{SF}-36=36$-Item short form survey, VAS $=$ visual analogue scale, $\mathrm{WOMAC}=\mathrm{Western}$ Ontario and McMaster Universities Osteoarthritis Index.

A Time from surgery 


\section{Healthcare Utilization Outcomes}

Five RCTs (Calatayud 2017, Huang 2012, Matassi 2014, Soeters 2018, Williamson 2007) reported on healthcare utilization outcomes comparing prehabilitation to no prehabilitation (Tables 19 to 21). Outcome domains included: length of stay, need for postoperative procedures, and other healthcare utilization outcomes.

\section{Length of Stay}

Five RCTs (Calatayud 2017, Huang 2012, Matassi 2014, Soeters 2018, Williamson 2007) provided data on length of stay (LOS) (mean days; smaller is better). While all studies reported reduced LOS among patients who were randomized to prehabilitation compared to control, only three studies were significant (Calatayud 2017: MD -1.9, 95\% CI -2.49 to -1.31; Huang 2012; MD $-1(95 \% \mathrm{CI}-1.8$ to 0.2 [based on calculated group data from report; study reported pvalue $=0.027])$, Matassi $2014 \mathrm{MD}-0.8(95 \% \mathrm{CI}-1.58$ to -0.02$)($ Table 19$)$.

\section{Need for Postoperative Procedures}

One RCT (Matassi 2014) reported data on need for postoperative procedures. Matassi 2014 reported on the number of patients reporting stiff knee who went on to receive manipulation under anesthesia and found no significant difference between prehabilitation and control groups (Table 20).

\section{Other Healthcare Utilization Outcomes}

One RCT (Soeters 2018) reported data on additional healthcare utilization outcome not captured above, including time to post-acute physical therapy discharge criteria (number of days, smaller is better) and the number of outpatients physical therapy sessions required (number of sessions, smaller is better). Readiness to discharge from physical therapy was defined as the ability to 1) independently transfer in and out of bed, a chair, and a toilet seat; 2) independently ambulate approximately 150 feet; 3 ) independently negotiate stairs; and 4) be independent with a home exercise program and activities of daily living. Soeters 2018 reported patients randomized to prehabilitation were more likely to meet physical therapists discharge criteria and require fewer outpatient therapy sessions, as compared to the control group (Table 21).

No RCT reported specific data on costs.

\section{Cost-Effectiveness}

We found no studies comparing the cost-effectiveness of prehabilitation with no prehabilitation.

\section{Harms From Prehabilitation}

Six RCTs (Huber 2015, Matassi 2014, Skoffer 2016, Soni 2012, Villadsen 2014, Williamson 2007) reported data on harms from participation in the prehabilitation intervention. All data was reported narratively and the severity of harms was low. Matassi 2014 reported exercise-related complaints in two patients: one patient developed knee pain and needed to stop the home exercise program and another patient develop ipsilateral adductor tendinitis (although there were no adductor-specific exercises in the program). Huber 2015, Skoffer 2016, Soni 2012, Villadsen 2014, Williamson 2007 reported no harms related to prehabilitation intervention. 
Table 19. Prehabilitation versus no prehabilitation for total knee arthroplasty - continuous outcomes, length of stay

\begin{tabular}{|c|c|c|c|c|c|c|c|c|c|}
\hline $\begin{array}{l}\text { Study, Year, } \\
\text { PMID, Country }\end{array}$ & Outcome & $\begin{array}{l}\text { Overall } \\
\text { RoB }\end{array}$ & $\begin{array}{l}\text { Time } \\
\text { Point }\end{array}$ & Prehab, N & Prehab (SD) & Control, N & $\begin{array}{l}\text { Control, } \\
\text { Mean (SD) }\end{array}$ & Effect Size $(95 \%$ CI) & $\begin{array}{l}\text { Reported p- } \\
\text { Value }\end{array}$ \\
\hline $\begin{array}{l}\text { Calatayud, 2017, } \\
26768606, \text { Spain }\end{array}$ & Length of stay (d) & Moderate & NA & 22 & $4.5(0.9)$ & 22 & $6.4(1.1)$ & $-1.9(-2.49,-1.31)^{\mathrm{B}}$ & $<0.001$ \\
\hline $\begin{array}{l}\text { Huang, 2012, } \\
22480863, \text { Taiwan }\end{array}$ & Length of stay (d) & High & NA & 126 & $\begin{array}{l}7 \text { (5) Range (5, } \\
10)\end{array}$ & 117 & $\begin{array}{l}8(1) \text { Range } \\
(5,12)\end{array}$ & $-1(-1.8,0.2)^{B}$ & 0.027 \\
\hline $\begin{array}{l}\text { Matassi, 2014, } \\
23271039, \\
\text { Belgium }\end{array}$ & Length of stay (d) & Moderate & NA & 61 & $9.1(2.1)$ & 61 & $9.9(2.3)$ & $-0.8(-1.58,-0.02)^{\mathrm{B}}$ & 0.011 \\
\hline $\begin{array}{l}\text { Soeters, 2018, } \\
\text { 29529614, USA }\end{array}$ & Length of stay (d) & Moderate & NA & 32 & $\begin{array}{l}2.7(95 \% \mathrm{Cl} \\
2.4,3.0)\end{array}$ & 31 & $\begin{array}{l}3.0(95 \% \mathrm{Cl} \\
2.7,3.3)\end{array}$ & NR & 0.161 \\
\hline $\begin{array}{l}\text { Williamson, 2007, } \\
\text { 17604311, UK }\end{array}$ & Length of stay (d) & Moderate & NA & 23 & $6.49(1.99)$ & $19^{\mathrm{C}}$ & $6.6(2.62)$ & $-0.12(-1.11,0.88)$ & 0.0984 \\
\hline
\end{tabular}

Statistically significant effect sizes are in bold text. In cases where calculated effect size confidence intervals were not-statistically significant but reported p-values were, we deferred to reported p-values and still bolded results.

Abbreviations: $\mathrm{CI}=$ confidence interval, $\mathrm{d}=$ day, $\mathrm{LOS}=$ length of stay, $\mathrm{NR}=$ not reported, $\mathrm{PMID}=\mathrm{PubMed}$ identifier, $\mathrm{RoB}=$ risk of bias, $\mathrm{SD}=$ standard deviation .

A Time from surgery

B Calculated

${ }^{\mathrm{C}}$ Control arm sample size is uncertain from study report. Study was retained despite potential sample size of control being less than 20

Table 20. Prehabilitation versus no prehabilitation for total knee arthroplasty - categorical outcomes, need for postoperative procedures

\begin{tabular}{|c|c|c|c|c|c|c|c|c|c|}
\hline $\begin{array}{l}\text { Study, Year, } \\
\text { PMID, Country }\end{array}$ & $\begin{array}{l}\text { Outcome } \\
\text { Measurement }\end{array}$ & $\begin{array}{l}\text { Overall } \\
\text { RoB }\end{array}$ & $\begin{array}{l}\text { Time } \\
\text { Point }^{A}\end{array}$ & Prehab, N & $\begin{array}{l}\text { Prehab, } \\
\text { Mean } \\
\text { (SD) }\end{array}$ & Control, N & Control Mean (SD) & $\begin{array}{l}\text { Effect Size } \\
(95 \% \mathrm{Cl})\end{array}$ & $\begin{array}{l}\text { Reported } \\
\text { p-Value }\end{array}$ \\
\hline $\begin{array}{l}\text { Matassi, 2014, } \\
\text { 23271039, } \\
\text { Belgium }\end{array}$ & $\begin{array}{l}\text { Need for } \\
\text { postoperative } \\
\text { procedures: Stiff } \\
\text { knee requiring } \\
\text { MUA }\end{array}$ & Moderate & $12 \mathrm{mo}$ & 61 & $5(8.2 \%)$ & 61 & $3(4.9 \%)$ & $\begin{array}{l}1.73(0.39 \\
7.57)\end{array}$ & NR \\
\hline
\end{tabular}

Statistically significant effect sizes are in bold text. In cases where calculated effect size confidence intervals were not-statistically significant but reported p-values were, we deferred to reported p-values and still bolded results.

Abbreviations: $\mathrm{CI}=$ confidence interval, $\mathrm{d}=$ day, $\mathrm{MUA}=$ manipulation under anesthesia, $\mathrm{NR}=$ not reported, $\mathrm{PMID}=\mathrm{PubMed}$ identifier, $\mathrm{RoB}=$ risk of bias, $\mathrm{SD}=$ standard deviation.

A Time from surgery 
Table 21. Prehabilitation versus no prehabilitation for total knee arthroplasty - continuous outcomes, other healthcare utilization outcomes

Study, Year,

PMID, Country

Outcome

Measurement

Overall

RoB

Time

Prehab, N

Prehab,

Control, N

Control Mean (SD)

Effect Size

Reported

Soeters, 2018

29529614, USA

\begin{tabular}{|l|l|l|l|}
\hline $\begin{array}{l}\text { Met discharge } \\
\text { criteria from } \\
\text { physical therapy }\end{array}$ & Moderate & NA & 32 \\
\hline $\begin{array}{l}\text { Outpatient physical } \\
\text { therapy sessions } \\
(\mathrm{n})\end{array}$ & Moderate & NA & 32 \\
\hline
\end{tabular}

(SD)

29529614, USA

(n)

Statistically significant effect sizes are in bold text. In cases where calculated effect size confidence intervals were not-statistically significant but reported p-values were, we deferred to reported p-values and still bolded results.

Abbreviations: $\mathrm{CI}=$ confidence interval, $\mathrm{n}=$ number, $\mathrm{NR}=$ not reported, $\mathrm{PMID}=$ PubMed identifier, $\mathrm{RoB}=$ risk of bias, $\mathrm{SD}=$ standard deviation

A Time from surgery 


\section{Heterogeneity of Treatment Effects (Subgroup Differences)}

No studies reported subgroup analyses or more specifically, formally analyzed possible heterogeneity of treatment effects, such as statistical tests for whether the comparative effect of rehabilitation versus its various comparators differed in one subgroup of patients versus another (e.g., patients with higher vs. lower measures of strength, flexibility, function, etc. at baseline).

\section{Applicability}

Studies were conducted across the globe (two in the United States) using diverse interventions employed in diverse healthcare settings. While the relative effect of the interventions on clinical outcomes (and harms) from non-US-based studies are likely applicable to the U.S. context, findings pertaining to healthcare system or resources (such as costs or comparisons of inpatient vs. outpatient rehabilitation) are likely country- and healthcare systemspecific. The sex of participants varied widely across studies ranging from 27 to 82 percent of participants being female. The average age of patients ages ranged from 63 to 72 years and the average BMIs ranged from 27 to $33 \mathrm{~kg} / \mathrm{m}^{2}$ (thus, in all studies, most patients were obese, but in several, many to most were morbidly obese). Most studies did not report whether patients had undergone previous contralateral replacement surgery; of those that did, proportions were low (less than 25\%). As such, the conclusions in this KQ are likely most applicable to middle-toolder-aged adults in high-income countries who are receiving their first total TKA for osteoarthritis.

\section{Summary of Comparison of Prehabilitation Versus No Prehabilitation for Patients Undergoing Total Knee Arthroplasty}

Table 22 summarizes the evidence for the comparison of prehabilitation versus no prehabilitation for TKA. We focus on the outcomes we prioritized in discussion with stakeholders.

There is low to insufficient SoE for all conclusions. Prehabilitation may result in increased strength and reduced length of hospital stay following TKA and may not lead to increased harms. Based on the evidence available, there is no evidence of a difference between prehabilitation and no prehabilitation in terms of pain, range of motion, and ADL. Additionally, there is insufficient evidence of the impact of prehabilitation on QoL, posthospital disposition or need for postoperative procedures (such as manipulation under anesthesia). No studies reported evidence on patients' satisfaction with prehabilitation as compared with no prehabilitation and posthospital disposition. 
Table 22. Evidence profile: Prehabilitation versus no prehabilitation for total knee arthroplasty

\begin{tabular}{|c|c|c|c|c|c|c|c|c|c|}
\hline $\begin{array}{l}\text { Outcome } \\
\text { Category }\end{array}$ & Outcome & $\begin{array}{l}\text { N Studies } \\
\text { (Participants) }\end{array}$ & RoB & Consistency & Precision & Directness & $\begin{array}{l}\text { Intervention } \\
\text { Replication }\end{array}$ & SoE & Conclusions \\
\hline \multirow[t]{3}{*}{$\begin{array}{l}\text { Body structure } \\
\text { and function }\end{array}$} & Pain & $9(725)$ & High & Consistent & Precise & Direct & $\begin{array}{l}2 \text { studies evaluated a } \\
\text { similar intervention; } \\
\text { remainder unique }\end{array}$ & Low & Similar pain \\
\hline & $\begin{array}{l}\text { Range of } \\
\text { motion }\end{array}$ & $4(448)$ & Moderate & Consistent & Precise & Direct & All unique & Low & Similar ROM \\
\hline & Strength & $4(257)$ & Moderate & Consistent & Precise & Direct & All unique & Low & $\begin{array}{l}\text { Increased } \\
\text { strength }\end{array}$ \\
\hline $\begin{array}{l}\text { Activity and } \\
\text { participation }\end{array}$ & ADLs & $6(636)$ & Moderate & Inconsistent & Precise & Direct & All unique & Low & Similar ADL \\
\hline \multirow[t]{2}{*}{$\begin{array}{l}\text { Other } \\
\text { outcomes }\end{array}$} & $\begin{array}{l}\text { Satisfaction } \\
\text { with care }\end{array}$ & 0 & NA & NA & NA & NA & NA & Insufficient & No evidence \\
\hline & QoL & $3(356)$ & Moderate & Inconsistent & Precise & Direct & All unique & Insufficient & No conclusion \\
\hline \multirow{3}{*}{$\begin{array}{l}\text { Healthcare } \\
\text { utilization }\end{array}$} & Length of stay & $5(485)$ & Moderate & Consistent & Precise & Direct & All unique & Low & Reduced LOS \\
\hline & $\begin{array}{l}\text { Posthospital } \\
\text { disposition }\end{array}$ & 0 & NA & NA & NA & NA & NA & Insufficient & No evidence \\
\hline & $\begin{array}{l}\text { Need for } \\
\text { postoperative } \\
\text { procedures }\end{array}$ & $1(122)$ & Moderate & $\begin{array}{l}\text { Consistency } \\
\text { unknown } \\
\text { (single study) }\end{array}$ & Precise & Direct & NA (single study) & Insufficient & No conclusion \\
\hline Harms & $\begin{array}{l}\text { Harms from } \\
\text { prehabilitation }\end{array}$ & $6(474)$ & Moderate & Consistent & Precise & Direct & All unique & Low & $\begin{array}{l}\text { No increased } \\
\text { harm }\end{array}$ \\
\hline
\end{tabular}

Abbreviations: ADLs = activities of daily living, $\mathrm{LOS}=$ length of stay, NA = not applicable, $\mathrm{QoL}=$ quality of life, $\mathrm{ROM}=$ range of motion, RoB $=$ risk of bias, $\mathrm{SoE}=$ strength of evidence 


\section{Key Question 2: Rehabilitation for Total Knee Arthroplasty}

\section{Key Points}

- Rehabilitation delivered in the acute phase after TKA may result in increased strength (low SoE).

- There is low SoE of no difference between the various programs of rehabilitation initiated in the acute and post-acute period and their comparators in terms of in pain, ROM, or ADL (low SoE for all).

- There is low SoE of no difference in satisfaction with care among patients who received rehabilitation in the acute phase compared with various less intensive controls or in QoL among patients who received rehabilitation in the post-acute phase compared with comparators (low SoE for both).

- No studies reported evidence on the risk of harms due to acute rehabilitation (insufficient evidence); harms from post-acute rehabilitation were low and comparable among groups (low SoE).

- There is insufficient evidence on the impact of acute rehabilitation on QoL, post-acute rehabilitation on satisfaction with care, and both acute and post-acute rehabilitation on the need for postoperative procedures.

- Given the heterogeneity of interventions and outcomes across studies, there is insufficient evidence on the effectiveness of specific rehabilitation intervention components at the level of goals (e.g., strength, flexibility) or presence of specific exercise components to address these goals.

- There is insufficient evidence on how the effect of rehabilitation programs may vary by patient, surgical, or setting factors.

- There is no evidence on the cost effectiveness of rehabilitation for TKA.

\section{Findings Pertaining to Rehabilitation for Total Knee Arthroplasty}

We found 53 studies (49 RCTs and 4 NRCSs) reported in 61 articles that evaluated the effectiveness of rehabilitation among patients who had undergone TKA. ${ }^{70,77-136}$ All studies were unique in terms of rehabilitation component goals and specific exercise components employed to address these goals; no rehabilitation intervention was evaluated by more than one study. Across studies, rehabilitation interventions were delivered in varying settings (by different modalities), by diverse personnel, at varying intensity, and at various points during the rehabilitation period. The heterogeneity of the interventions (and their comparators) made it challenging to identify meaningful groupings of similar studies to synthesize. In the absence of meaningful clusters of similar intervention/comparator studies, we opted to summarize the rehabilitation studies within two main groups of when the intervention/comparator rehabilitation content was initiated: in the acute and post-acute phases after surgery. As expert consensus is unclear regarding the definition of the different phases of rehabilitation following surgery, we defined these time periods using our best judgement. Acute phase was defined as rehabilitation initiated within 2 weeks of surgery and post-acute rehabilitation was defined as rehabilitation initiated 2 weeks or more after surgery. We report outcomes under the four following outcome categories: body structure and function; activity and participation; other patient-reported; and healthcare utilization. Given intervention heterogeneity, we determined that meta-analysis was not warranted; a summarized, 
average result would not have been interpretable or meaningful. Instead we summarize results narratively.

We coded 21 studies (18 RCTs and 3 NRCSs) as being delivered within an acute rehabilitation phase and 32 studies (31 RCTs and 1 NRCS) as being delivered within a postacute rehabilitation phase. Of the acute rehabilitation studies, 4 RCTs evaluated novel (hypothesized better) rehabilitation programs vs. standard care (variously defined) or alternative rehabilitation programs, 4 RCTs evaluated comparatively similar rehabilitation programs delivered with varying intensity and/or timing, 6 studies (3 RCTs, 3 NRCSs) evaluated comparatively similar rehabilitation programs delivered in different settings or by different personnel, and 7 RCTs evaluated comparatively similar rehabilitation programs with or without an adjunctive modality (Figure 2).

Among the 32 post-acute rehabilitation studies, 17 RCTs evaluated novel (hypothesized better) rehabilitation programs vs. standard care (variously defined) or alternative rehabilitation program, 1 RCT evaluated two active programs hypothesized to be similar effects, 1 NRCS evaluated a comparatively similar rehabilitation program delivered with varying intensity/timing, 6 RCTs evaluated comparatively similar rehabilitation programs delivered in different settings or by different personnel, and 7 RCTs evaluated comparatively similar rehabilitation programs with or without an adjunctive modality (Figure 3 ).

We rated 19 of the 49 RCTs to be at overall high risk of bias, mostly related to lack of blinding of participants, study personnel, and/or outcome assessors and unclear methods of how random sequences were generated and/or concealed from patients. We rated the remaining 30 RCTs to be at overall moderate risk of bias, mostly related to lack of blinding of participants, study personnel, and/or outcome assessors. We rated all four NRCSs to be at moderate risk of bias based on their non-randomized design; all four NRCSs reported data that was adjusted for important confounders using appropriate methods as per our inclusion criteria.

The 53 studies enrolled between 41 and 2,426 participants each. Studies were conducted across the globe, mostly commonly in the Europe $(n=25)$, followed by Asia $(n=13)$, North America $(n=9)$, and Australia $(n=6)$. Of the studies that reported funding information, three RCTs (DeJong 2020, den Hertog 2012, Piqueras 2013, Rockstroh 2010) were funded in part by industry that produce medical equipment or telecommunication technology. The average ages of

participants varied across studies, ranging from 54 and 79 years. The percentage of women in the studies varied across studies, from 27 to 100 percent. Average BMIs ranged from 27 to $35 \mathrm{~kg} / \mathrm{m}^{2}$. In a subset of six studies that reported data, prior contralateral TKA ranged from 13 to 43 percent. Appendix Tables C-2.1, C-2.2, C-2.3, and C-2.4 include the full data for all 53 studies.

\section{Acute-Phase Rehabilitation}

Twenty-one studies evaluated acute-phase rehabilitation in 6,049 patients (summarized in Figure 2). Acute-phase rehabilitation interventions were initiated in-hospital from immediately post-op to 2 weeks after surgery. Three acute-phase studies did not provide data to code the rehabilitation interventions (Chan 2018, Naylor 2017, Padgett 2018). The focus of these three studies was the setting to which patients were discharged. All remaining 18 acute-phase studies included some form of active rehabilitation in all 36 arms and included exercises to address the goal component of flexibility $(n=36 / 36)$. Most studies also included exercises address the goal component of strength $(n=32 / 36)$, task-specific training $(n=32 / 36)$, patient education $(n=15 / 30)$ and balance-motor-learning-agility $(n=12 / 36)$. Aerobic exercise was not commonly targeted in 
acute-phase rehabilitation interventions $(n=6 / 36)$. Specific exercise components within rehabilitation goal components varied across programs. Nine studies included an adjunctive modality in combination with the rehabilitation program: two (Li 2014 and Li 2017) with the same adjunctive modalities delivered to both study arms and seven (Avramidis 2011, Eymir 2020, Stevens-Lapsley 2012, Tsukada 2018, Jin 2018, Rockstroh 2010, Zapparoli 2020) where the added benefit of adjunctive therapy was the question of interest and the adjuvant modality varied between arms. The adjunctive modalities evaluated in these seven studies included neuromuscular electrical stimulation (NMES; $n=3$ RCTs); transcutaneous electrical nerve stimulation (TENS; $n=1$ RCT), biofeedback ( $n=1$ RCT), motor imagery $(n=1$ RCT), and continuous passive motion (vs. active-heel slides which was the comparison of interest for this review) ( $\mathrm{n}=1$ RCT). Eight studies reported some form of exercise progression, of which three were assessed by clinical experts on our team as appropriate (Buhagiar 2017, Eymir 2020, Stevens-Lapsley 2012). One study (Piquerras 2013) compared acute-phase virtual rehabilitation with progression to acute-phase conventional rehabilitation without progression.

Acute-phase rehabilitation interventions were delivered by physical therapists in 13 of the 18 studies. In other studies, the intervention was delivered by research personnel (Jin 2018) or was not reported (den Hertog 2012, Li 2017, Naylor 2017, Padgett 2018, Li 2014, Avramidis 2011, Rockstroh 2010). All acute-phase rehabilitation interventions were delivered to patients inperson (although, two studies that compared discharge destinations did not report the mode of delivery for each discharge destination). In addition to in-person delivery, three studies had a self-guided home-based component (Buhagiar 2017, Eymir 2020, Stevens-Lapsley 2012), and one study had a remote (via app or telephone) component (Piqueras 2013). Ten studies were delivered exclusively in an inpatient rehabilitation setting (den Hertog 2012, Sattler 2019, Iwakiri 2020, Lenssen 2006, Li 2017, Li 2014, Tsukada 2018, Jin 2018, Rockstroh 2010, Zapparoli 2020).

Three acute-phase rehabilitation interventions were compared in different settings. Harmer 2009 compared rehabilitation delivered in a swimming pool (combined with home-based therapy) to land-based rehabilitation delivered in the outpatient and home setting. Buhagiar 2017 compared an acute rehabilitation program delivered in the hospital to one delivered at home. Piqueras 2013 compared an identical program delivered in the acute and outpatient setting.

Three acute-phase rehabilitation studies (Chan 2018, Naylor 2017, and Padgett 2018) reported the destination to which patients were discharged (e.g., home, community hospital, long term care facility) and not the setting in which rehabilitation was provided, per se.

Specific codes for intervention (and control arm, where present) goals and exercises, use of progression (and assessment of appropriateness), and details on personnel, mode of delivery, and setting are detailed in Tables 23.1 and 23.2 and Appendix C-2.2. 
Figure 2. Overview of studies of acute-phase rehabilitation interventions and various controls for total knee arthroplasty

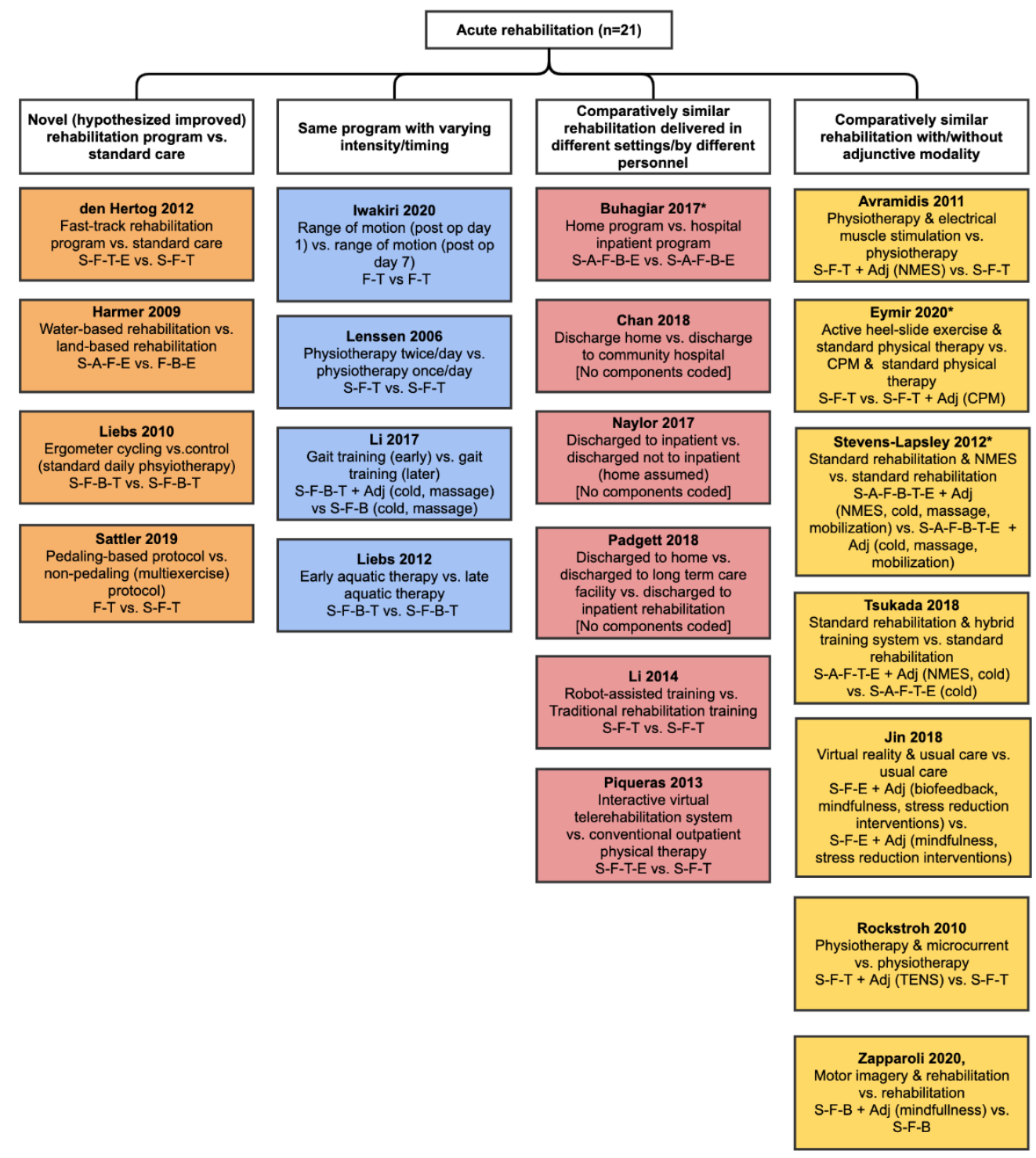


Figure presents categorization of studies $(\mathrm{n}=21)$ that evaluated acute rehabilitation programs for total knee arthroplasty. The first column lists novel (more intensive) programs compared with different programs (first group hypothesized to be better); the second column lists studies with comparatively similar rehabilitation programs in both arms that were delivered with different timing or intensity (first group hypothesized to be better); the third column lists studies with comparatively similar rehabilitation programs delivered in different settings or by different personnel (i.e., shift in resources providing care; groups hypothesized to be comparable); the fourth column lists studies with rehabilitation interventions comparing a rehabilitation program and an adjunctive modality vs. the same rehabilitation program alone (first group hypothesized to be better). Studies are defined using arm descriptors first and component coding second. The different colors are added to visually separate the columns and do not provide unique information.

Abbreviations: $\mathrm{Adj}=$ adjunctive, $\mathrm{A}=$ aerobic exercise, $\mathrm{B}=$ balance-motor/learning-agility exercise, $\mathrm{E}=$ patient education, $\mathrm{F}=$ flexibility exercise, $\mathrm{NMES}=$ neuromuscular electrical stimulation, $\mathrm{S}=$ strengthening exercise, $\mathrm{T}=$ task-specific training.

* Intervention included progression which was deemed appropriate. 
Table 23.1. Goal components strength, aerobic, and flexibility and their specific exercise components for acute-rehabilitation intervention versus various controls for total knee arthroplasty

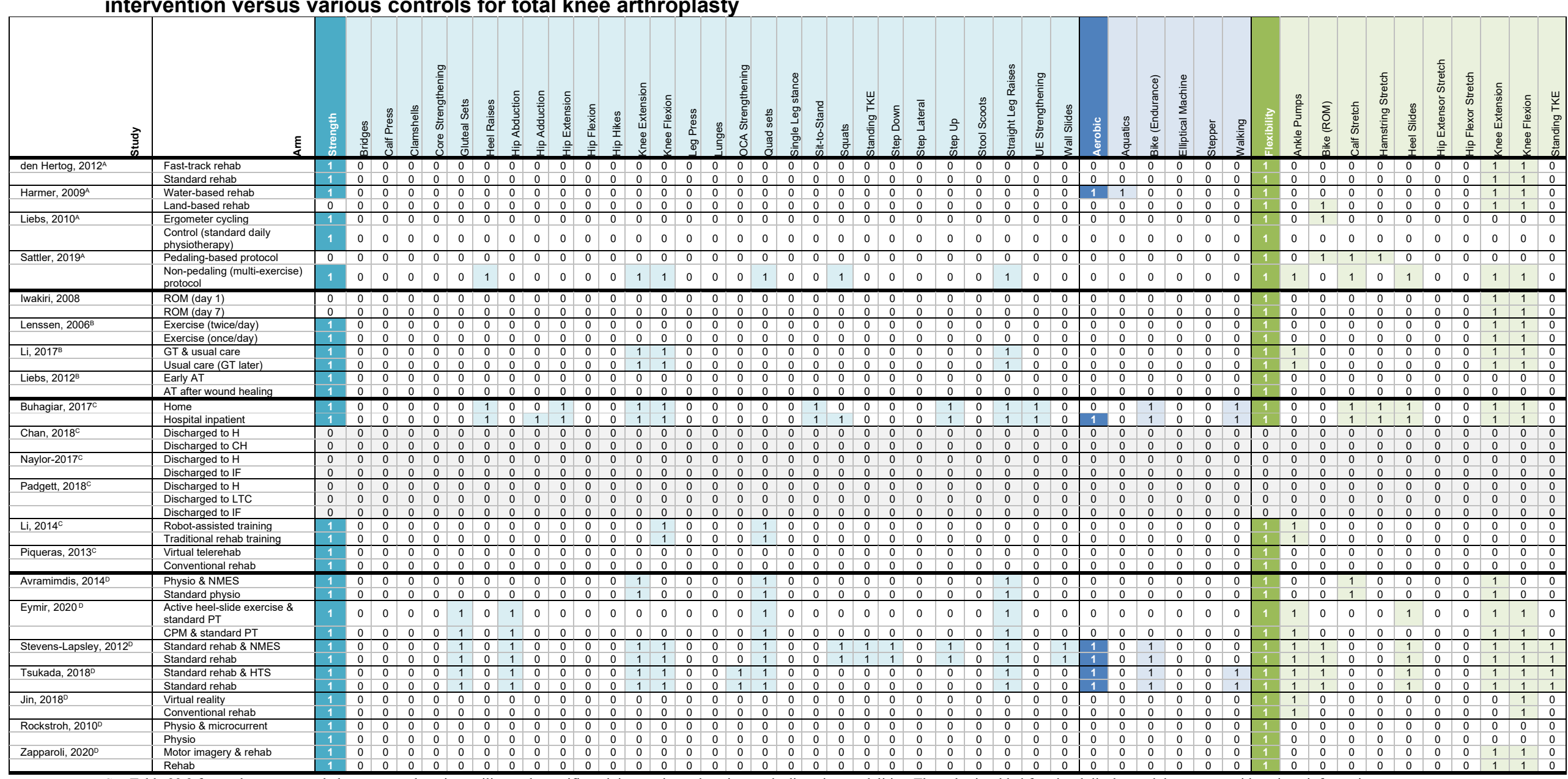

See Table 23.2 for goal components balance-motor-learning-agility, task specific training, patient education, and adjunctive modalities. The color is added for visual display and does not provide unique information.

$1=$ presence of component, $0=$ absence of component

Abbreviations: $\mathrm{AT}=$ aquatic therapy, $\mathrm{CH}=$ community hospital, $\mathrm{ex}=$ exercise, $\mathrm{GT}=$ gait training, $\mathrm{H}=$ home, $\mathrm{HTS}=$ hybrid training system, $\mathrm{IF}=$ inpatient facility, $\mathrm{LTC}=$ long term care facility, $\mathrm{OCA}=\mathrm{open}$ chain ankle, $\mathrm{UP}=$ upper extremity, physio = physiotherapy, rehab $=$ rehabilitation, ROM $=$ range of motion, TKE $=$ terminal knee extension

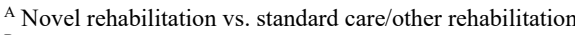

${ }^{\mathrm{C}}$ Similar rehabilitation delivered in different setting/by different personnel

${ }^{\mathrm{B}}$ Similar rehabilitation with varying intensity/timing

${ }^{\mathrm{D}}$ Similar rehabilitation with/without adjuvant modality 
Table 23.2. Goal components balance-motor-learning-agility, task specific training, patient education, and adjunctive modalities and their specific exercise components for acute-rehabilitation intervention versus various controls for total knee arthroplasty

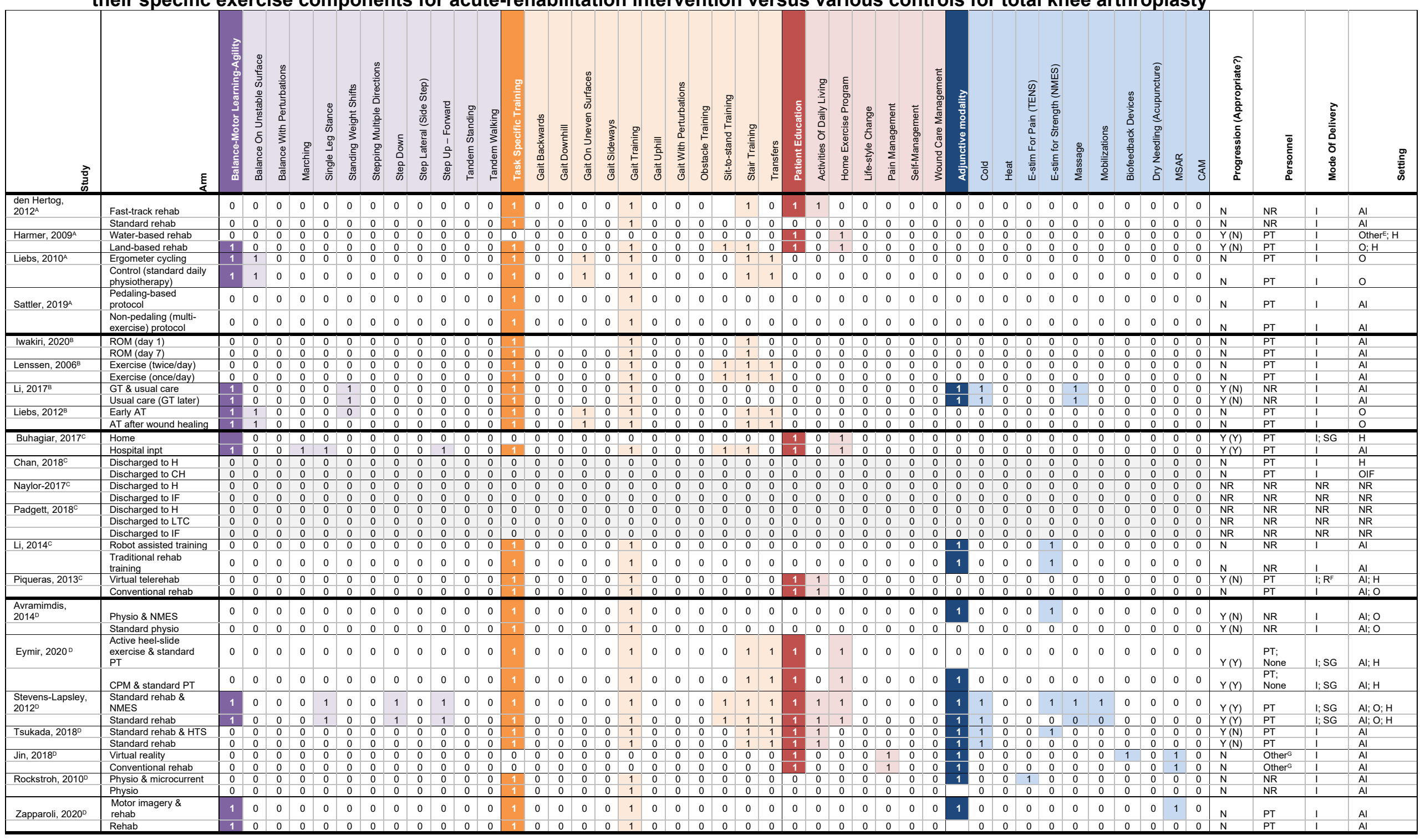


See Table 23.1 for goal components strength, aerobic, and flexibility. The color is added for visual display and does not provide unique information.

$1=$ presence of component, $0=$ absence of component

Abbreviations: $\mathrm{AI}=$ acute inpatient, $\mathrm{AT}=$ aquatic therapy, $\mathrm{CAM}=$ complementary and alternative therapies, $\mathrm{GT}=$ gait training, $\mathrm{H}=$ home, $\mathrm{HTS}=$ hybrid training system, I = in-person; inpt = inpatient, IF = inpatient facility, LTC = long term care facility, MSAR = mindfulness, stress/anxiety reduction, $\mathrm{NA}=$ not applicable, NMES = neuromuscular electrical stimulation, $\mathrm{O}=$ outpatient physiotherapy center, $\mathrm{OIF}=$ other inpatient facility, $\mathrm{PRT}=$ progressive resistive training; preop = preoperative; $\mathrm{PT}=$ physical therapist, $\mathrm{R}=$ remote, tele = telephone, $\mathrm{TENS}=$ transcutaneous electrical nerve stimulation, rehab $=$ rehabilitation, $\mathrm{SG}=$ self-guided

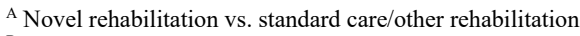

${ }^{\mathrm{B}}$ Similar rehabilitation with varying intensity/timing

${ }^{\mathrm{C}}$ Similar rehabilitation delivered in different setting/by different personnel

D Similar rehabilitation with/without adjuvant modality

${ }^{\mathrm{E}}$ Pool

${ }^{\mathrm{F}}$ Remote via app or telephone

${ }^{\mathrm{G}}$ Research personnel

\section{Body Structure and Function Outcomes Following Acute Rehabilitation}

Fourteen studies reported on body structure and function outcomes following acute rehabilitation compared with various controls: three studies (Harmer 2009, Liebs 2010, Sattler 2019) comparing novel acute rehabilitation programs with various comparators (less intensive rehabilitation or no care); four studies (Iwakiri 2020, Lenssen 2006, Li 2017, Liebs 2012) comparing acute rehabilitation programs with different timing and/or intensity; two studies (Buhagiar 2017 and Piqueras 2013) comparing acute rehabilitation programs delivered in different settings or by different personnel; and five studies (Avramidis 2011, Eymir 2020 Rockstroh 2010, Stevens-Lapsley 2012, Tsukada 2020) comparing acute rehabilitation programs with or without an adjunctive modality (Tables 24 to 28). The outcome domains included: symptoms, pain, range of motion, muscle strength, and emotional functioning.

\section{Symptoms}

Four studies (Harmer 2009, Liebs 2010, Lenssen 2006, Liebs 2012) reported data on symptoms using the stiffness component of the WOMAC (scores 0 to 8; lower score indicates reduced stiffness; Liebs 2010 and 2012 used a 0 to 100 score) and observed no differences between groups at follow-up ranging from 3 to 12 months after TKA (Table 24).

\section{Pain}

Twelve studies reported pain data (Harmer 2009, Liebs 2010, Sattler 2019, Lenssen 2006, Liebs 2012, Buhagiar 2017, Li 2015, Piqueras 2013, Rockstroh 2010, Eymir 2020, StevensLapsley 2012, Tsukada 2020) using three different measurement instruments (the pain component of the WOMAC, EQ-5D VAS, and VAS) (Table 25). Most studies $(n=10)$ found no difference in pain data between comparison groups. Two studies (Li 2017 and Rockstroh 2010) reported reduced pain in their respective intervention groups. Li 2017 reported pain on a VAS (010 , lower is better) and found that patients randomized to gait training and usual care reported significantly lower pain compared with patients randomized to usual care at 6 months after TKA (MD -2.4, 95\% CI -2.7 to -2.2). Rockstroh 2010 found reduced pain on the VAS scale among patients randomized to physiotherapy with adjunctive microcurrent therapy versus physiotherapy alone (MD 2.0, 95\% CI 1.4 to 2.6) at 3 months following TKA. 


\section{Range of Motion}

Nine studies (Harmer 2009, Sattler 2019, Iwakiri 2020, Lenssen 2006, Li 2017, Piqueras 2013, Buhagiar 2017, Eymir 2020, Stevens Lapsley 2012) reported ROM data from various outcome measures, including active and passive knee ROM for extension and flexion of the knee joint (Table 26). In several cases, whether active or passive ROM was measured was not specified. Where reported, studies measured ROM in degrees using goniometry. Seven studies reported comparable ROM between arms at follow-up measured between 3 and 24 months after TKA. Sattler 2019 reported improved knee flexion ROM at 4 months among patients randomized to the pedaling-based protocol (i.e., ROM exercise was a core component of the intervention) compared with patients randomized to the non-pedaling multi-exercise protocol (MD 2.7, 95\% CI 2.6 to 7.9) at 4 months after TKA. Li 2017 also reported improved knee extension and flexion among patients randomized to early gait training and usual care compared with usual care alone at 6 months after TKA.

\section{Muscle Strength}

Three studies (Piqueras 2013, Stevens Lapsley 2012, Tsukada 2020) reported data on muscle strength using various outcome measures, including isometric and isokinetic knee extension and knee flexion strength, quadricep and hamstring strength and torque, and percent quadriceps activation (Table 27). Studies measured strength in Newtons (N), kilograms (kg), or torque normalized to body weight $(\mathrm{Nm} / \mathrm{kg})$, usually with a dynamometer. All studies reported significant improvement for at least one measure of strength with the intervention rehabilitation arm. Piquera 2013 reported hamstring and quadricep strength data and found patients randomized to virtual rehabilitation achieved comparable functional improvements in strength as the conventional outpatient physical therapy group (as hypothesized by the non-inferiority design) and, in fact, the virtual rehabilitation demonstrated greater improvements in quadricep strength (MD not reported; $p=0.018$ ) at 3 months after TKA. Stevens Lapsley 2012 compared the added effect of adjunctive NMES combined with standard rehabilitation and found improved strength as measured by normalized quadricep torque and normalized hamstring torque at 1 year after TKA in the rehabilitation and adjunctive therapy group compared to rehabilitation alone. Tsukada 2020 compared the added effect of an NMES hybrid training system combined with standard rehabilitation and found improved strength in terms of isometric knee extension $(p<0.01)$ but not isometric knee flexion compared to standard rehabilitation at 3 months after TKA.

\section{Energy and Vigor}

No acute rehabilitation studies reported data on energy or vigor.

\section{Emotional Functioning}

Two studies (Avramidis 2011 and Stevens Lapsley 2012) reported on emotional functioning data from the mental health component scale of the SF-36 (scores 0 to 100, higher is better). Although Avramidis 2011 reported significant improvements among patients randomized to adjunctive TENS in combination with physiotherapy compared to physiotherapy alone at 3 months, differences were no longer significant at 12 months after surgery. Stevens Lapsley found no significant differences between groups who received physiotherapy combined with adjunctive NMES versus physiotherapy alone at 12 months after surgery (Table 28). 
Table 24. Acute rehabilitation versus various controls for total knee arthroplasty - continuous outcomes, symptoms

\begin{tabular}{|c|c|c|c|c|c|c|c|c|c|c|c|}
\hline $\begin{array}{l}\text { Study, Year, } \\
\text { PMID, } \\
\text { Country }\end{array}$ & Arm 1 & Arm 2 & $\begin{array}{l}\text { Overall } \\
\text { RoB }\end{array}$ & Outcome Measurement & $\begin{array}{l}\text { Time } \\
\text { Point }^{\mathrm{A}}\end{array}$ & $\begin{array}{l}\text { N Arm } \\
1\end{array}$ & $\begin{array}{l}\text { Arm 1, } \\
\text { Mean (SD) }\end{array}$ & $\begin{array}{l}\text { N Arm } \\
2\end{array}$ & $\begin{array}{l}\text { Arm 2, } \\
\text { Mean (SD) }\end{array}$ & $\begin{array}{l}\text { Effect Size } \\
(95 \% \mathrm{Cl})\end{array}$ & $\begin{array}{l}\text { Reported } \\
\text { p-Value }\end{array}$ \\
\hline $\begin{array}{l}\text { Harmer, 2009, } \\
\text { 19177536, } \\
\text { Australia }\end{array}$ & $\begin{array}{l}\text { Water-based } \\
\text { rehabilitation }\end{array}$ & $\begin{array}{l}\text { Land-based } \\
\text { rehabilitation }\end{array}$ & Moderate & WOMAC: Stiffness (0-8) & $6 \mathrm{mo}$ & 53 & $0.97(\mathrm{NR})$ & 49 & 0.86 (NR) & NR & NR \\
\hline $\begin{array}{l}\text { Lenssen, } \\
2006, \\
16942627, \\
\text { Netherlands }\end{array}$ & $\begin{array}{l}\text { Physiotherapy } \\
\text { [twice daily } \\
\text { (40 min/day)] }\end{array}$ & $\begin{array}{l}\text { Physiotherapy } \\
\text { [once daily ( } 20 \\
\mathrm{~min} / \text { day)] }\end{array}$ & Moderate & WOMAC: Stiffness (0-8) & $3 \mathrm{mo}$ & 21 & $6.1(1.2)$ & 22 & $6.5(1.1)$ & $-0.4(-1.04,0.32)$ & NR \\
\hline $\begin{array}{l}\text { Liebs, 2010, } \\
20360503\end{array}$ & $\begin{array}{l}\text { Ergometer } \\
\text { cycling }\end{array}$ & Control & Moderate & WOMAC: Stiffness (0-100) & $24 \mathrm{mo}$ & 52 & $23(22.8)$ & 66 & $17.4(17.3)$ & NR & 0.235 \\
\hline $\begin{array}{l}\text { Liebs, 2012, } \\
22196125\end{array}$ & $\begin{array}{l}\text { Early Aquatic } \\
\text { therapy }\end{array}$ & $\begin{array}{l}\text { Late Aquatic } \\
\text { therapy }\end{array}$ & Moderate & WOMAC: Stiffness (0-100) & $24 \mathrm{mo}$ & 66 & $15.2(14.1)$ & 69 & $20.4(21.7)$ & $0.28(\mathrm{NR})$ & 0.347 \\
\hline
\end{tabular}

Statistically significant effect sizes are in bold text. In cases where calculated effect size confidence intervals were not-statistically significant but reported p-values were, we deferred to reported p-values and still bolded results.

Abbreviations: $\mathrm{Adj}=$ adjusted, $\mathrm{CI}=$ confidence interval, $\mathrm{MD}=$ mean difference, $\mathrm{mo}=$ month, $\min =$ minutes, $\mathrm{NR}=$ not reported, $\mathrm{PMID}=\mathrm{PubMed}$ identifier, $\mathrm{RoB}=$ risk of bias, $\mathrm{SD}=$ standard deviation, WOMAC $=$ Western Ontario and McMaster Universities Osteoarthritis Index.

A Time from surgery

Table 25. Acute rehabilitation versus various controls for total knee arthroplasty - continuous outcomes, pain

\begin{tabular}{|c|c|c|c|c|c|c|c|c|c|c|c|}
\hline $\begin{array}{l}\text { Study, Year, } \\
\text { PMID, Country }\end{array}$ & Arm 1 & Arm 2 & $\begin{array}{l}\text { Overall } \\
\text { RoB }\end{array}$ & $\begin{array}{l}\text { Outcome } \\
\text { Measurement }\end{array}$ & $\begin{array}{l}\text { Time } \\
\text { Point }^{A}\end{array}$ & $\begin{array}{l}\text { N Arm } \\
1\end{array}$ & $\begin{array}{l}\text { Arm 1, Mean } \\
\text { (SD) }\end{array}$ & $\begin{array}{l}\text { N Arm } \\
2\end{array}$ & $\begin{array}{l}\text { Arm 2, Mean } \\
\text { (SD) }\end{array}$ & $\begin{array}{l}\text { Effect Size }(95 \% \\
\mathrm{Cl})\end{array}$ & $\begin{array}{l}\text { Reported } \\
\text { p-Value }\end{array}$ \\
\hline \multirow[t]{2}{*}{$\begin{array}{l}\text { Buhagiar, 2017, } \\
\text { 28291891, } \\
\text { Australia }\end{array}$} & $\begin{array}{l}\text { Hospital } \\
\text { Inpatient } \\
\text { Rehabilitation }\end{array}$ & Home Program & Moderate & $\begin{array}{l}\text { EQ-5D (VAS) } \\
(0-100)\end{array}$ & $6.5 \mathrm{mo}$ & 79 & $\begin{array}{l}78.8(95 \% \\
\mathrm{Cl} 75.3 \\
82.3)\end{array}$ & 80 & $\begin{array}{l}80.2(95 \% \mathrm{Cl} \\
76.7,83.8)\end{array}$ & $-1.41(-6.42,3.60)$ & NR \\
\hline & $\begin{array}{l}\text { Hospital } \\
\text { Inpatient } \\
\text { Rehabilitation }\end{array}$ & Home Program & Moderate & $\begin{array}{l}\text { EQ-5D (VAS) } \\
(0-100)\end{array}$ & $12 \mathrm{mo}$ & 79 & $\begin{array}{l}76.9(95 \% \\
\text { Cl } 73.4, \\
80.4)\end{array}$ & 77 & $\begin{array}{l}77.4(95 \% \mathrm{Cl} \\
73.8,81)\end{array}$ & $-0.50(-5.53,4.52)$ & NR \\
\hline \multirow[t]{2}{*}{$\begin{array}{l}\text { Eymir, 2020, } \\
32778907 \\
\text { Turkey }\end{array}$} & $\begin{array}{l}\text { Standard } \\
\text { Physiotherapy } \\
\text { plus Active heel- } \\
\text { slide exercise } \\
\text { (AHSE) }\end{array}$ & $\begin{array}{l}\text { Physiotherapy } \\
\text { plus CPM }\end{array}$ & High & $\begin{array}{l}\text { VAS (0-10): } \\
\text { Activity }\end{array}$ & $3 \mathrm{mo}$ & 55 & $1.5(2.3)$ & 58 & $1.0(1.9)$ & $0.6(0.8,3.0)$ & NS \\
\hline & $\begin{array}{l}\text { Standard } \\
\text { Physiotherapy } \\
\text { plus Active heel- } \\
\text { slide exercise } \\
\text { (AHSE) }\end{array}$ & $\begin{array}{l}\text { Physiotherapy } \\
\text { plus CPM }\end{array}$ & High & $\begin{array}{l}\text { VAS (0-10): } \\
\text { Rest }\end{array}$ & $3 \mathrm{mo}$ & 55 & $0.8(1.5)$ & 58 & $1.0(2.0)$ & $1.9(1.4,2.4)$ & NS \\
\hline \multirow{2}{*}{$\begin{array}{l}\text { Harmer, 2009, } \\
\text { 19177536, } \\
\text { Australia }\end{array}$} & $\begin{array}{l}\text { Water-based } \\
\text { rehabilitation }\end{array}$ & $\begin{array}{l}\text { Land-based } \\
\text { rehabilitation }\end{array}$ & Moderate & $\begin{array}{l}\text { WOMAC: Pain } \\
(0-20)\end{array}$ & $6 \mathrm{mo}$ & 53 & 1.69 (NR) & 49 & 1.89 (NR) & NR & NR \\
\hline & $\begin{array}{l}\text { Water-based } \\
\text { rehabilitation }\end{array}$ & $\begin{array}{l}\text { Land-based } \\
\text { rehabilitation }\end{array}$ & Moderate & VAS $(0-10)$ & $6 \mathrm{mo}$ & 53 & $0.76(\mathrm{NR})$ & 49 & 0.67 (NR) & $0.5(0.2,1.1)$ & NR \\
\hline
\end{tabular}




\begin{tabular}{|c|c|c|c|c|c|c|c|c|c|c|c|}
\hline $\begin{array}{l}\text { Study, Year, } \\
\text { PMID, Country }\end{array}$ & Arm 1 & Arm 2 & $\begin{array}{l}\text { Overall } \\
\text { RoB }\end{array}$ & $\begin{array}{l}\text { Outcome } \\
\text { Measurement }\end{array}$ & $\begin{array}{l}\text { Time } \\
\text { Point }^{\mathrm{A}}\end{array}$ & $\begin{array}{l}\text { N Arm } \\
1\end{array}$ & $\begin{array}{l}\text { Arm 1, Mean } \\
\text { (SD) }\end{array}$ & $\begin{array}{l}\text { N Arm } \\
2\end{array}$ & $\begin{array}{l}\text { Arm 2, Mean } \\
\text { (SD) }\end{array}$ & $\begin{array}{l}\text { Effect Size }(95 \% \\
\mathrm{Cl})\end{array}$ & $\begin{array}{l}\text { Reported } \\
\text { p-Value }\end{array}$ \\
\hline \multirow[t]{2}{*}{$\begin{array}{l}\text { Lenssen, 2006, } \\
16942627, \\
\text { Netherlands }\end{array}$} & $\begin{array}{l}\text { Physiotherapy } \\
\text { [twice daily(40 } \\
\text { mins/day)] }\end{array}$ & $\begin{array}{l}\text { Physiotherapy } \\
\text { [once daily(20 } \\
\text { mins/day)] }\end{array}$ & Moderate & $\begin{array}{l}\text { WOMAC: Pain } \\
(0-20)\end{array}$ & $3 \mathrm{mo}$ & 21 & $15.2(3.0)$ & 22 & $16.2(2.4)$ & $-1(-2.7,0.7)$ & NR \\
\hline & $\begin{array}{l}\text { Physiotherapy } \\
\text { [twice daily(40 } \\
\text { mins/day)] }\end{array}$ & $\begin{array}{l}\text { Physiotherapy } \\
\text { [once daily(20 } \\
\text { mins/day)] }\end{array}$ & Moderate & $\begin{array}{l}\text { VAS (0-10): } \\
\text { Last } 24 \mathrm{hrs}\end{array}$ & $3 \mathrm{mo}$ & 21 & $1.3(1.9)$ & 22 & $0.8(1.5)$ & $0.5(-0.6,1.6)$ & NR \\
\hline $\begin{array}{l}\text { Li, 2017, CN- } \\
01084888, \\
\text { China }\end{array}$ & $\begin{array}{l}\text { Gait training \& } \\
\text { usual care }\end{array}$ & Usual care & Moderate & Pain-VAS & $6 \mathrm{mo}$ & 43 & $0.51(0.74)$ & 43 & $2.93(0.88)$ & $-2.4(-2.7,-2.2)^{B}$ & NR \\
\hline $\begin{array}{l}\text { Liebs, 2010, } \\
20360503 \text {, } \\
\text { Germany }\end{array}$ & $\begin{array}{l}\text { Ergometer } \\
\text { cycling }\end{array}$ & Control & Moderate & $\begin{array}{l}\text { WOMAC: Pain } \\
(0-20)\end{array}$ & $24 \mathrm{mo}$ & 66 & $14.3(17.7)$ & 52 & $11.1(14.4)$ & NR & 0.278 \\
\hline $\begin{array}{l}\text { Liebs, 2012, } \\
22196125\end{array}$ & $\begin{array}{l}\text { Early Aquatic } \\
\text { therapy }\end{array}$ & $\begin{array}{l}\text { Late Aquatic } \\
\text { therapy }\end{array}$ & Moderate & $\begin{array}{l}\text { WOMAC: Pain } \\
(0-20)\end{array}$ & $24 \mathrm{mo}$ & 66 & $9.6(11.9)$ & 69 & $15.2(19.2)$ & 0.35 (NR, NR) & 0.097 \\
\hline $\begin{array}{l}\text { Piqueras, 2013, } \\
\text { 23474735, } \\
\text { Spain }\end{array}$ & $\begin{array}{l}\text { Interactive virtual } \\
\text { telerehabilitation } \\
\text { system }\end{array}$ & $\begin{array}{l}\text { Conventional } \\
\text { outpatient } \\
\text { physical therapy }\end{array}$ & Moderate & VAS (NR) & $3 \mathrm{mo}$ & 68 & NR (NR) & 65 & NR (NR) & NR & 0.284 \\
\hline $\begin{array}{l}\text { Rockstroh, } \\
2010, \\
20533147, \\
\text { Germany }\end{array}$ & $\begin{array}{l}\text { Physiotherapy \& } \\
\text { microcurrent }\end{array}$ & Physiotherapy & High & VAS (NR) & $3 \mathrm{mo}$ & 37 & $\begin{array}{l}\text { Median } \\
(\text { IQR) } 0(0,1)\end{array}$ & 41 & $\begin{array}{l}\text { Median (IQR) } \\
2(0,3)\end{array}$ & $2.0(1.4,2.6)^{B}$ & $<0.001$ \\
\hline $\begin{array}{l}\text { Sattler, 2019, } \\
\text { 30994586, } \\
\text { Australia }\end{array}$ & $\begin{array}{l}\text { Pedaling-based } \\
\text { protocol }\end{array}$ & $\begin{array}{l}\text { Non-pedaling } \\
\text { (multi-exercise) } \\
\text { protocol }\end{array}$ & Moderate & $\begin{array}{l}\text { EQ-5D (VAS) } \\
(0-100)\end{array}$ & $4 \mathrm{mo}$ & 28 & Median (90) & 28 & Median (8.8) & NR & NR \\
\hline $\begin{array}{l}\text { Stevens } \\
\text { Lapsley, 2012, } \\
22095207, \\
\text { USA }\end{array}$ & $\begin{array}{l}\text { Standard } \\
\text { rehabilitation \& } \\
\text { NMES }\end{array}$ & $\begin{array}{l}\text { Standard } \\
\text { rehabilitation }\end{array}$ & High & $\begin{array}{l}\text { VAS }(0-10): \\
\text { Pain while } \\
\text { resting }\end{array}$ & $12 \mathrm{mo}$ & 25 & $0.6(1.4)$ & 30 & $0.4(1.5)$ & $0.2(-0.3,0.7)^{\mathrm{B}}$ & NR \\
\hline $\begin{array}{l}\text { Tsukada, 2020, } \\
31723080, \\
\text { Japan }\end{array}$ & $\begin{array}{l}\text { Standard } \\
\text { rehabilitation \& } \\
\text { hybrid training } \\
\text { system }\end{array}$ & $\begin{array}{l}\text { Standard } \\
\text { rehabilitation }\end{array}$ & High & VAS $(0-10)$ & $3 \mathrm{mo}$ & 20 & 21 (NR) & 20 & 18 (NR) & NR & ns \\
\hline
\end{tabular}

Statistically significant effect sizes are in bold text. In cases where calculated effect size confidence intervals were not-statistically significant but reported p-values were, we deferred to reported p-values and still bolded results.

Abbreviations: $\mathrm{Adj}=$ adjusted, $\mathrm{CI}=$ confidence interval, $\mathrm{EQ}-5 \mathrm{D}=$ EuroQol-5D, $\mathrm{KOOS}=$ Knee injury and osteoarthritis outcome score, $\mathrm{MD}=$ mean difference, $\mathrm{mo}=\mathrm{month}, \mathrm{NR}=$ not reported, $\mathrm{PMID}=$ PubMed identifier, $\mathrm{RoB}=$ risk of bias, $\mathrm{SD}=$ standard deviation, $\mathrm{VAS}=$ visual analog scale, WOMAC $=$ Western Ontario and McMaster Universities

Osteoarthritis Index.

A Time from surgery

B Calculated 
Table 26. Acute rehabilitation versus various controls for total knee arthroplasty - continuous outcomes, range of motion

\begin{tabular}{|c|c|c|c|c|c|c|c|c|c|c|c|}
\hline $\begin{array}{l}\text { Study, Year, } \\
\text { PMID, Country }\end{array}$ & Arm 1 & Arm 2 & $\begin{array}{l}\text { Overall } \\
\text { RoB }\end{array}$ & $\begin{array}{l}\text { Outcome } \\
\text { Measurement }\end{array}$ & $\begin{array}{l}\text { Time } \\
\text { Point }^{\mathrm{A}}\end{array}$ & $\begin{array}{l}\text { N Arm } \\
1\end{array}$ & $\begin{array}{l}\text { Arm 1, Mean } \\
\text { (SD) }\end{array}$ & $\begin{array}{l}\text { N Arm } \\
2\end{array}$ & $\begin{array}{l}\text { Arm 2, Mean } \\
\text { (SD) }\end{array}$ & $\begin{array}{l}\text { Effect Size } \\
(95 \% \mathrm{Cl})\end{array}$ & $\begin{array}{l}\text { Reported } \\
\text { p-Value }\end{array}$ \\
\hline $\begin{array}{l}\text { Buhagiar, } \\
2017, \\
28291891, \\
\text { Australia }\end{array}$ & $\begin{array}{l}\text { Hospital } \\
\text { Inpatient } \\
\text { Rehabilitation }\end{array}$ & Home Program & Moderate & $\begin{array}{l}\text { Knee ROM } \\
\text { (active/passive } \\
\text { unspecified): } \\
\text { Flexion } \geq 100 \\
\text { degrees }^{B}\end{array}$ & $6.5 \mathrm{mo}$ & 80 & 66 events & 80 & 62 events & $\begin{array}{l}1.29(0.59, \\
2.84)^{\mathrm{C}}\end{array}$ & NR \\
\hline $\begin{array}{l}\text { Eymir, } \\
2020, \\
32778907 \\
\text { Turkey } \\
\end{array}$ & $\begin{array}{l}\text { Standard } \\
\text { Physiotherapy } \\
\text { plus Active heel- } \\
\text { slide exercise }\end{array}$ & $\begin{array}{l}\text { Physiotherapy } \\
\text { plus CPM }\end{array}$ & High & $\begin{array}{l}\text { Knee ROM } \\
\text { (active/passive } \\
\text { unspecified): } \\
\text { Flexion (deg) }\end{array}$ & $3 \mathrm{mo}$ & 55 & $110.0(11.8)$ & 58 & $109.1(13.0)$ & $-0.9(-4.1,2.3)$ & NS \\
\hline \multirow[t]{2}{*}{$\begin{array}{l}\text { Harmer, 2009, } \\
\text { 19177536, } \\
\text { Australia }\end{array}$} & $\begin{array}{l}\text { Water-based } \\
\text { rehabilitation }\end{array}$ & $\begin{array}{l}\text { Land-based } \\
\text { rehabilitation }\end{array}$ & Moderate & $\begin{array}{l}\text { Passive Knee } \\
\text { ROM: Extension } \\
\text { (deg) }\end{array}$ & $6 \mathrm{mo}$ & 53 & 0.86 (NR) & 49 & $1.71(\mathrm{NR})$ & NR & NR \\
\hline & $\begin{array}{l}\text { Water-based } \\
\text { rehabilitation }\end{array}$ & $\begin{array}{l}\text { Land-based } \\
\text { rehabilitation }\end{array}$ & Moderate & $\begin{array}{l}\text { Passive Knee } \\
\text { ROM: Flexion } \\
\text { (deg) }\end{array}$ & $6 \mathrm{mo}$ & 53 & 104.04 (NR) & 49 & 105.18 (NR) & NR & NR \\
\hline \multirow[t]{6}{*}{$\begin{array}{l}\text { Iwakiri, 2020, } \\
32373475 \text {, } \\
\text { Japan }\end{array}$} & ROM day 1 & ROM day 7 & High & $\begin{array}{l}\text { Knee ROM } \\
\text { (active/passive } \\
\text { unspecified: } \\
\text { Extension (deg) }\end{array}$ & $3 \mathrm{mo}$ & 55 & 1.20 (NR) & 54 & 2.45 (NR) & NR & NS \\
\hline & ROM day 1 & ROM day 7 & High & $\begin{array}{l}\text { Knee ROM } \\
\text { (active/passive } \\
\text { unspecified: } \\
\text { Extension (deg) }\end{array}$ & $12 \mathrm{mo}$ & 55 & 0 (NR) & 54 & 0 (NR) & NR & NS \\
\hline & ROM day 1 & ROM day 7 & High & $\begin{array}{l}\text { Knee ROM } \\
\text { (active/passive } \\
\text { unspecified: } \\
\text { Extension (deg) }\end{array}$ & $24 \mathrm{mo}$ & 55 & 0 (NR) & 54 & 0 (NR) & NR & NS \\
\hline & ROM day 1 & ROM day 7 & High & $\begin{array}{l}\text { Knee ROM } \\
\text { (active/passive } \\
\text { unspecified: } \\
\text { Flexion (deg) }\end{array}$ & $3 \mathrm{mo}$ & 55 & $125.82(\mathrm{NR})$ & 54 & $123.21(\mathrm{NR})$ & NR & NS \\
\hline & ROM day 1 & ROM day 7 & High & $\begin{array}{l}\text { Knee ROM } \\
\text { (active/passive } \\
\text { unspecified: } \\
\text { Flexion (deg) }\end{array}$ & $12 \mathrm{mo}$ & 55 & 124.41 (NR) & 54 & 119.84 (NR) & NR & NS \\
\hline & ROM day 1 & ROM day 7 & High & $\begin{array}{l}\text { Knee ROM } \\
\text { (active/passive } \\
\text { unspecified: } \\
\text { Flexion (deg) }\end{array}$ & $24 \mathrm{mo}$ & 55 & 124.74 (NR) & 54 & 119.84 (NR) & NR & NS \\
\hline \multirow[t]{2}{*}{$\begin{array}{l}\text { Lenssen, 2006, } \\
\text { 16942627, } \\
\text { Netherlands }\end{array}$} & $\begin{array}{l}\text { Physiotherapy } \\
\text { [twice daily(40 } \\
\text { mins/day)] }\end{array}$ & $\begin{array}{l}\text { Physiotherapy } \\
\text { [once daily(20 } \\
\text { mins/day)] }\end{array}$ & Moderate & $\begin{array}{l}\text { Passive knee } \\
\text { ROM: Extension } \\
\text { (deg) }\end{array}$ & $3 \mathrm{mo}$ & 21 & $3.8(4.3)$ & 22 & $5.5(4.6)$ & $-1.7(-4.5,1.03)$ & NR \\
\hline & $\begin{array}{l}\text { Physiotherapy } \\
\text { [twice daily(40 } \\
\text { mins/day)] }\end{array}$ & $\begin{array}{l}\text { Physiotherapy } \\
\text { [once daily(20 } \\
\text { mins/day)] }\end{array}$ & Moderate & $\begin{array}{l}\text { Passive knee } \\
\text { ROM: Flexion } \\
\text { (deg) }\end{array}$ & $3 \mathrm{mo}$ & 21 & $36.6(17.9)$ & 22 & $32.1(18.4)$ & $4.5(-6.8,15.6)$ & NR \\
\hline
\end{tabular}




\begin{tabular}{|c|c|c|c|c|c|c|c|c|c|c|c|}
\hline $\begin{array}{l}\text { Study, Year, } \\
\text { PMID, Country }\end{array}$ & Arm 1 & Arm 2 & $\begin{array}{l}\text { Overall } \\
\text { RoB }\end{array}$ & $\begin{array}{l}\text { Outcome } \\
\text { Measurement }\end{array}$ & $\begin{array}{l}\text { Time } \\
\text { Point }^{\mathrm{A}}\end{array}$ & $\begin{array}{l}\text { N Arm } \\
1\end{array}$ & $\begin{array}{l}\text { Arm 1, Mean } \\
\text { (SD) }\end{array}$ & $\begin{array}{l}\text { N Arm } \\
2\end{array}$ & $\begin{array}{l}\text { Arm 2, Mean } \\
\text { (SD) }\end{array}$ & $\begin{array}{l}\text { Effect Size } \\
(95 \% \mathrm{CI})\end{array}$ & $\begin{array}{l}\text { Reported } \\
\text { p-Value }\end{array}$ \\
\hline & $\begin{array}{l}\text { Physiotherapy } \\
\text { [twice daily(40 } \\
\text { mins/day)] }\end{array}$ & $\begin{array}{l}\text { Physiotherapy } \\
\text { [once daily(20 } \\
\text { mins/day)] }\end{array}$ & Moderate & $\begin{array}{l}\text { Active knee ROM: } \\
\text { Extension (deg) }\end{array}$ & $3 \mathrm{mo}$ & 21 & $5.3(5.1)$ & 22 & $8.3(5.5)$ & $-3(-6,7.03)$ & NR \\
\hline & $\begin{array}{l}\text { Physiotherapy } \\
\text { [twice daily(40 } \\
\text { mins/day)] }\end{array}$ & $\begin{array}{l}\text { Physiotherapy } \\
\text { [once daily(20 } \\
\text { mins/day)] }\end{array}$ & Moderate & $\begin{array}{l}\text { Active knee ROM: } \\
\text { Flexion (deg) }\end{array}$ & $3 \mathrm{mo}$ & 21 & $103.7(13)$ & 22 & $105.1(15)$ & $-1.4(-10.0,7.3)$ & NR \\
\hline $\begin{array}{l}\text { Li, 2017, CN- } \\
\text { 01084888, } \\
\text { China }\end{array}$ & $\begin{array}{l}\text { Gait training } \\
\text { \&usual care }\end{array}$ & Usual care & Moderate & $\begin{array}{l}\text { Knee ROM: } \\
\text { Extension and } \\
\text { flexion }\end{array}$ & $6 \mathrm{mo}$ & 43 & $135.14(7.19)$ & 43 & $94.84(2.77)$ & $\begin{array}{l}40.3(38.4 \\
42.2)^{\mathrm{B}}\end{array}$ & NR \\
\hline \multirow[t]{2}{*}{$\begin{array}{l}\text { Piqueras, 2013, } \\
\text { 23474735, } \\
\text { Spain }\end{array}$} & $\begin{array}{l}\text { Interactive virtual } \\
\text { telerehabilitation } \\
\text { system }\end{array}$ & $\begin{array}{l}\text { Conventional } \\
\text { outpatient } \\
\text { physical therapy }\end{array}$ & Moderate & $\begin{array}{l}\text { Active knee ROM: } \\
\text { Extension (deg) }\end{array}$ & $3 \mathrm{mo}$ & 68 & NR (NR) & 65 & NR (NR) & NR & 0.478 \\
\hline & $\begin{array}{l}\text { Interactive virtual } \\
\text { telerehabilitation } \\
\text { system }\end{array}$ & $\begin{array}{l}\text { Conventional } \\
\text { outpatient } \\
\text { physical therapy }\end{array}$ & Moderate & $\begin{array}{l}\text { Active knee ROM: } \\
\text { Flexion (deg) }\end{array}$ & $3 \mathrm{mo}$ & 68 & NR (NR) & 65 & NR (NR) & NR & 0.193 \\
\hline $\begin{array}{l}\text { Sattler, 2019, } \\
\text { 30994586, } \\
\text { Australia }\end{array}$ & $\begin{array}{l}\text { Pedaling-based } \\
\text { protocol }\end{array}$ & $\begin{array}{l}\text { Non-pedaling } \\
\text { (multi-exercise) } \\
\text { protocol }\end{array}$ & High & $\begin{array}{l}\text { Knee ROM } \\
\text { (active/passive } \\
\text { unspecified): } \\
\text { Flexion (deg) }\end{array}$ & $4 \mathrm{mo}$ & 28 & $113.0 \pm 10.4$ & 28 & $110.4(9.1)$ & 2.7 (2.6 to 7.9$)$ & 0.310 \\
\hline \multirow[t]{2}{*}{$\begin{array}{l}\text { Stevens } \\
\text { Lapsley,2012, } \\
\text { 22095207, USA }\end{array}$} & $\begin{array}{l}\text { Standard } \\
\text { rehabilitation \& } \\
\text { NMES }\end{array}$ & $\begin{array}{l}\text { Standard } \\
\text { rehabilitation }\end{array}$ & Moderate & $\begin{array}{l}\text { Active Knee } \\
\text { ROM: Extension }\end{array}$ & $12 \mathrm{mo}$ & 25 & $-2(3.5)$ & 30 & $-1.4(3.4)$ & $-0.6(-1.9,0.7)^{\mathrm{C}}$ & NR \\
\hline & $\begin{array}{l}\text { Standard } \\
\text { rehabilitation \& } \\
\text { NMES }\end{array}$ & $\begin{array}{l}\text { Standard } \\
\text { rehabilitation }\end{array}$ & Moderate & $\begin{array}{l}\text { Active Knee } \\
\text { ROM: Flexion }\end{array}$ & $12 \mathrm{mo}$ & 25 & $119.4(6.3)$ & 30 & $117(9.1)$ & $2.4(-0.5,5.3)^{C}$ & NR \\
\hline
\end{tabular}

Statistically significant effect sizes are in bold text. In cases where calculated effect size confidence intervals were not-statistically significant but reported p-values were, we deferred to reported p-values and still bolded results.

Abbreviations: Adj $\mathrm{MD}=$ adjusted mean difference, $\mathrm{CI}=$ confidence interval, $\mathrm{CPM}=$ continuous passive motion, deg $=\mathrm{degree}, \mathrm{mo}=\mathrm{month}, \mathrm{NMES}=$ neuromuscular electric stimulation, $\mathrm{NR}=$ not reported, $\mathrm{PMID}=$ PubMed identifier, $\mathrm{RoB}=$ risk of bias, $\mathrm{ROM}=$ range of motion, $\mathrm{SD}=$ standard deviation

A Time from surgery

${ }^{B}$ Categorical outcome

${ }^{\mathrm{C}}$ Calculated

Table 27. Acute rehabilitation versus various controls for total knee arthroplasty - continuous outcomes, muscle strength

\begin{tabular}{|c|c|c|c|c|c|c|c|c|c|c|c|}
\hline $\begin{array}{l}\text { Study, Year, } \\
\text { PMID, Country }\end{array}$ & Arm 1 & Arm 2 & $\begin{array}{l}\text { Overall } \\
\text { RoB }\end{array}$ & $\begin{array}{l}\text { Outcome } \\
\text { Measurement }\end{array}$ & $\begin{array}{l}\text { Time } \\
\text { Point }^{\mathrm{A}}\end{array}$ & $\begin{array}{l}\text { N Arm } \\
1\end{array}$ & $\begin{array}{l}\text { Arm 1, Mean } \\
\text { (SD) }\end{array}$ & $\begin{array}{l}\text { N Arm } \\
2\end{array}$ & $\begin{array}{l}\text { Arm 2, Mean } \\
\text { (SD) }\end{array}$ & $\begin{array}{l}\text { Effect Size } \\
(95 \% \mathrm{Cl})\end{array}$ & $\begin{array}{l}\text { Reported } \\
\text { p-Value }\end{array}$ \\
\hline $\begin{array}{l}\text { Piqueras, 2013, } \\
23474735, \\
\text { Spain }\end{array}$ & $\begin{array}{l}\text { Interactive virtual } \\
\text { telerehabilitation } \\
\text { system }\end{array}$ & $\begin{array}{l}\text { Conventional } \\
\text { outpatient } \\
\text { physical } \\
\text { therapy }\end{array}$ & Moderate & $\begin{array}{l}\text { Strength: } \\
\text { Hamstring (kg) }\end{array}$ & $3 \mathrm{mo}$ & 68 & NR (NR) & 65 & NR & NR & 0.349 \\
\hline
\end{tabular}




\begin{tabular}{|c|c|c|c|c|c|c|c|c|c|c|c|}
\hline $\begin{array}{l}\text { Study, Year, } \\
\text { PMID, Country }\end{array}$ & Arm 1 & Arm 2 & $\begin{array}{l}\text { Overall } \\
\text { RoB }\end{array}$ & $\begin{array}{l}\text { Outcome } \\
\text { Measurement }\end{array}$ & $\begin{array}{l}\text { Time } \\
\text { Point }^{\mathrm{A}}\end{array}$ & $\begin{array}{l}\text { N Arm } \\
1\end{array}$ & $\begin{array}{l}\text { Arm 1, Mean } \\
\text { (SD) }\end{array}$ & $\begin{array}{l}\text { N Arm } \\
2\end{array}$ & $\begin{array}{l}\text { Arm 2, Mean } \\
\text { (SD) }\end{array}$ & $\begin{array}{l}\text { Effect Size } \\
(95 \% \mathrm{Cl})\end{array}$ & $\begin{array}{l}\text { Reported } \\
\text { p-Value }\end{array}$ \\
\hline & $\begin{array}{l}\text { Interactive virtual } \\
\text { telerehabilitation } \\
\text { system }\end{array}$ & $\begin{array}{l}\text { Conventional } \\
\text { outpatient } \\
\text { physical } \\
\text { therapy }\end{array}$ & Moderate & $\begin{array}{l}\text { Strength: } \\
\text { Quadriceps } \\
(\mathrm{kg})\end{array}$ & $3 \mathrm{mo}$ & 68 & NR (NR) & 65 & NR & NR & 0.018 \\
\hline \multirow[t]{9}{*}{$\begin{array}{l}\text { Stevens } \\
\text { Lapsley,2012, } \\
\text { 22095207, USA }\end{array}$} & $\begin{array}{l}\text { Standard } \\
\text { rehabilitation \& NMES }\end{array}$ & $\begin{array}{l}\text { Standard } \\
\text { rehabilitation }\end{array}$ & Moderate & $\begin{array}{l}\text { Strength: } \\
\text { Quadriceps } \\
\text { femoris torque }^{B} \\
(\mathrm{Nm} / \mathrm{kg})\end{array}$ & $3 \mathrm{mo}$ & 30 & $1.42(0.52)$ & 29 & $1.20(0.42)$ & $\begin{array}{l}0.22(-0.02 \\
0.46)\end{array}$ & $\leq 0.05$ \\
\hline & $\begin{array}{l}\text { Standard } \\
\text { rehabilitation \& NMES }\end{array}$ & $\begin{array}{l}\text { Standard } \\
\text { rehabilitation }\end{array}$ & Moderate & $\begin{array}{l}\text { Strength: } \\
\text { Quadriceps } \\
\text { femoris torque }^{B} \\
(\mathrm{Nm} / \mathrm{kg})\end{array}$ & $6 \mathrm{mo}$ & 31 & $1.51(0.48)$ & 27 & $1.39(0.44)$ & $\begin{array}{l}0.12(-0.12 \\
0.36)^{\mathrm{C}}\end{array}$ & NR \\
\hline & $\begin{array}{l}\text { Standard } \\
\text { rehabilitation \& NMES }\end{array}$ & $\begin{array}{l}\text { Standard } \\
\text { rehabilitation }\end{array}$ & Moderate & $\begin{array}{l}\text { Strength: } \\
\text { Quadriceps } \\
\text { femoris torque }^{B} \\
(\mathrm{Nm} / \mathrm{kg})\end{array}$ & $12 \mathrm{mo}$ & 30 & $1.66(0.52)$ & 25 & $1.50(0.43)$ & $\begin{array}{l}0.16(-0.09 \\
0.41)^{\mathrm{C}}\end{array}$ & $\leq 0.05$ \\
\hline & $\begin{array}{l}\text { Standard } \\
\text { rehabilitation \& NMES }\end{array}$ & $\begin{array}{l}\text { Standard } \\
\text { rehabilitation }\end{array}$ & Moderate & $\begin{array}{l}\text { Strength: } \\
\text { Hamstring } \\
\text { torque }(\mathrm{Nm} / \mathrm{kg})\end{array}$ & $3 \mathrm{mo}$ & 29 & $0.73(0.21)$ & 30 & $0.65(0.24)$ & $\begin{array}{l}0.08(-0.03 \\
0.19)^{\mathrm{C}}\end{array}$ & NR \\
\hline & $\begin{array}{l}\text { Standard } \\
\text { rehabilitation \& NMES }\end{array}$ & $\begin{array}{l}\text { Standard } \\
\text { rehabilitation }\end{array}$ & Moderate & $\begin{array}{l}\text { Strength: } \\
\text { Hamstring } \\
\text { torque }(\mathrm{Nm} / \mathrm{kg})\end{array}$ & $6 \mathrm{mo}$ & 31 & $0.79(0.25)$ & 27 & $0.72(0.25)$ & $0.07(-0.06,0.2)^{C}$ & NR \\
\hline & $\begin{array}{l}\text { Standard } \\
\text { rehabilitation \& NMES }\end{array}$ & $\begin{array}{l}\text { Standard } \\
\text { rehabilitation }\end{array}$ & Moderate & $\begin{array}{l}\text { Strength: } \\
\text { Hamstring } \\
\text { torque }(\mathrm{Nm} / \mathrm{kg})\end{array}$ & $12 \mathrm{mo}$ & 30 & $0.83(0.25)$ & 24 & $0.72(0.29)$ & $\begin{array}{l}0.11(-0.04 \\
0.26)^{\mathrm{c}}\end{array}$ & $\leq 0.05$ \\
\hline & $\begin{array}{l}\text { Standard } \\
\text { rehabilitation \& NMES }\end{array}$ & $\begin{array}{l}\text { Standard } \\
\text { rehabilitation }\end{array}$ & Moderate & $\begin{array}{l}\text { Quadriceps } \\
\text { activation (\%) }\end{array}$ & $3 \mathrm{mo}$ & 30 & $86.5(12.9)$ & 29 & $85.4(11.5)$ & $1.1(-5.13,7.33)$ & NR \\
\hline & $\begin{array}{l}\text { Standard } \\
\text { rehabilitation \& NMES }\end{array}$ & $\begin{array}{l}\text { Standard } \\
\text { rehabilitation }\end{array}$ & Moderate & $\begin{array}{l}\text { Quadriceps } \\
\text { activation (\%) }\end{array}$ & $6 \mathrm{mo}$ & 31 & $88.4(10.1)$ & 26 & $84.2(10.0)$ & $4.2(-1.04,9.44)^{C}$ & NR \\
\hline & $\begin{array}{l}\text { Standard } \\
\text { rehabilitation \& NMES }\end{array}$ & $\begin{array}{l}\text { Standard } \\
\text { rehabilitation }\end{array}$ & Moderate & $\begin{array}{l}\text { Quadriceps } \\
\text { activation (\%) }\end{array}$ & $12 \mathrm{mo}$ & 30 & $87.6(9.2)$ & 23 & $85.9(11.9)$ & $1.7(-3.87,7.27)^{\mathrm{C}}$ & NR \\
\hline \multirow[t]{2}{*}{$\begin{array}{l}\text { Tsukada, 2020, } \\
\text { 31723080, } \\
\text { Japan }\end{array}$} & $\begin{array}{l}\text { Standard } \\
\text { rehabilitation \& hybrid } \\
\text { training system }\end{array}$ & $\begin{array}{l}\text { Standard } \\
\text { rehabilitation }\end{array}$ & Moderate & $\begin{array}{l}\text { Strength: } \\
\text { Isometric knee } \\
\text { extension }(\mathrm{N})\end{array}$ & $3 \mathrm{mo}$ & 20 & $184(\mathrm{NR})$ & 20 & $155(\mathrm{NR})$ & NR & ns \\
\hline & $\begin{array}{l}\text { Standard } \\
\text { rehabilitation \& hybrid } \\
\text { training system }\end{array}$ & $\begin{array}{l}\text { Standard } \\
\text { rehabilitation }\end{array}$ & Moderate & $\begin{array}{l}\text { Strength: } \\
\text { Isometric knee } \\
\text { flexion }(\mathrm{N})\end{array}$ & $3 \mathrm{mo}$ & 20 & 102 (NR) & 20 & 98 (NR) & NR & $<0.01$ \\
\hline
\end{tabular}

Statistically significant effect sizes are in bold text. In cases where calculated effect size confidence intervals were not-statistically significant but reported p-values were, we deferred to reported p-values and still bolded results.

Abbreviations: $\mathrm{CI}=$ confidence interval, $\mathrm{N}=$ Newton, $\mathrm{Nm}=$ peak torque, $\mathrm{NR}=$ not reported, $\mathrm{kg}=$ kilogram, $\mathrm{PMID}=\mathrm{PubMed}$ identifier, $\mathrm{RoB}=$ risk of bias, $\mathrm{SD}=$ standard deviation, $\mathrm{SE}=$ standard error.

A Time from surgery

B Normalized to body weight for all strength outcomes

${ }^{\mathrm{C}}$ Calculated 
Table 28. Acute rehabilitation versus various controls for total knee arthroplasty - continuous outcomes, emotional functioning (stress/coping)

\begin{tabular}{|c|c|c|c|c|c|c|c|c|c|c|c|}
\hline $\begin{array}{l}\text { Study, Year, } \\
\text { PMID, Country }\end{array}$ & Arm 1 & Arm 2 & Overall RoB & $\begin{array}{l}\text { Outcome } \\
\text { Measurement }\end{array}$ & $\begin{array}{l}\text { Time } \\
\text { Point }^{A}\end{array}$ & $\begin{array}{l}\text { N Arm } \\
1\end{array}$ & $\begin{array}{l}\text { Arm 1, } \\
\text { Mean } \\
\text { (SD) } \\
\end{array}$ & $\begin{array}{l}\text { N Arm } \\
2\end{array}$ & $\begin{array}{l}\text { Arm 2, } \\
\text { Mean (SD) }\end{array}$ & $\begin{array}{l}\text { Effect Size } \\
(95 \% \mathrm{Cl})\end{array}$ & $\begin{array}{l}\text { Reported } \\
\text { p-Value }\end{array}$ \\
\hline \multirow{2}{*}{$\begin{array}{l}\text { Avramidis, } \\
2011, \\
21410130, \\
\text { Greece }\end{array}$} & $\begin{array}{l}\text { TENS plus } \\
\text { Physiotherapy }\end{array}$ & Physiotherapy & Moderate & $\begin{array}{l}\text { SF-36: Mental health } \\
(0-100)\end{array}$ & $3 \mathrm{mo}$ & 12 & $53.51(4.2)$ & 19 & $49.2(4.23)$ & $4.3(2.1,6.5)^{B}$ & $<0.001$ \\
\hline & $\begin{array}{l}\text { TENS plus } \\
\text { Physiotherapy }\end{array}$ & Physiotherapy & Moderate & $\begin{array}{l}\text { SF-36: Mental health } \\
(0-100)\end{array}$ & $12 \mathrm{mo}$ & 15 & $\begin{array}{l}50.49 \\
(5.32) \\
\end{array}$ & 21 & $50.1(3.69)$ & $0.4(2.0,2.7)^{\mathrm{B}}$ & 0.694 \\
\hline $\begin{array}{l}\text { Stevens } \\
\text { Lapsley, 2012, } \\
\text { 22095207,USA }\end{array}$ & $\begin{array}{l}\text { Standard } \\
\text { rehabilitation \& } \\
\text { NMES }\end{array}$ & $\begin{array}{l}\text { Standard } \\
\text { rehabilitation }\end{array}$ & Moderate & $\begin{array}{l}\text { SF-36: Mental health } \\
(0-100)\end{array}$ & $12 \mathrm{mo}$ & 25 & $57.8(4.4)$ & 30 & $54.8(6.9)$ & $3(0.8,5.2)^{B}$ & NR \\
\hline
\end{tabular}

22095207,USA NMES

deferred to reported p-values and still bolded results.

Abbreviations: $\mathrm{CI}=$ confidence interval, $\mathrm{NR}=$ not reported, $\mathrm{PMID}=$ PubMed identifier, RoB $=$ risk of bias, $\mathrm{SF}-36=36-\mathrm{Item}$ short form survey, $\mathrm{SD}=$ standard deviation, TENS $=$ transcutaneous electrical nerve stimulation.

A Time from surgery

B Calculated 


\section{Activity and Participation Outcomes Following Acute-Rehabilitation}

Fifteen studies in total reported on activity and participation outcomes following acute rehabilitation compared to various controls: three studies (Harmer 2009, Liebs 2010, Sattler 2019) compared novel acute rehabilitation programs with various comparators (less intensive rehabilitation or no care); two studies (Lenssen 2006, Liebs 2012) compared acute rehabilitation programs with different timing and/or intensity; five studies (Buhagiar 2017, Chan 2018, Naylor 2017, Li 2014, Piqueras 2013) compared acute rehabilitation programs delivered in different settings or by different personnel, and three studies (Avramidis 2011, Eymir 2020, StevensLapsley 2012, Tsukada 2020, Zapparoli 2020) compared acute rehabilitation programs with or without an adjunctive modality (Tables 29 to 33). Outcome domains included: physical function and activities of daily living, transfers, balance, mobility, and timed up and go.

\section{Physical Function and Activities of Daily Living}

Eleven RCTs (Harmer 2009, Liebs 2010, Sattler 2019, Lenssen 2006, Liebs 2012, Buhagiar 2017, Chan 2018, Naylor 2017, Avramidis 2011, Eymir 2020, Stevens Lapsley 2012) reported data on patient-reported physical function and ADLs using various outcome measures (Table 29) at follow-ups between 3 months and 2 years after TKA surgery. Eight studies found no difference between groups in terms of patient-reported function and ADL; three studies (Harmer 2009, Chan 2018 and Avramidis 2011) reported significant differences between groups. Harmer 2009 reported physical function data using the function component of the WOMAC (0-68, lower is better) and found patients randomized to water-based rehabilitation reported significantly greater improvements in physical function compared to patients randomized to land-based rehabilitation $(\mathrm{p}=0.04)$. Chan 2018 reported physical function and ADL data using the ADL Oxford Knee score (scores 0 to 48, higher is better), the physical component of the SF-36 (0-100, higher is better), and the function component of the Knee Society Clinical Rating System (0-100, higher is better) and observed that while patients discharged home had lower ADL measured on the Oxford Knee Score, they had higher physical function as rated on the SF-36 and Knee Society Rating Scales. Avramidis 2011 also reported physical function and ADL using the physical component of the SF-36 and function component of the Knee Society Clinical Rating System and found that patients randomized to physiotherapy combined with adjunctive TENS reported better knee function on both scales at 3 and 12 months after TKA.

\section{Transfers}

Two studies reported data on transfers (Li 2014 and Tsukada 2020). Tsukada 2020 observed no differences between groups at follow-up 3 months; Li 2014 found patients randomized to robassisted training had improved performance of transfers (based on 10-minute sitting-standing times) at 6 and 12 months after TKA (Li 2014) (Table 30).

\section{Balance}

Two studies reported balance data. Li 2014 reported balance data from the Berg Balance Scale and found significant difference between compared groups at 6 or 12 months after TKA among patients randomized to robot-assisted training compared to those who received traditional rehabilitation training (Table 31). Zapparoli 2020 reported significantly fewer falls/near fall in the past year among patients randomized to specific motor imagery and rehabilitation compared to rehabilitation alone at 2 years following surgery. 


\section{Mobility}

Seven studies (Harmer 2009, Sattler 2019, Buhagiar 2017, Li 2014, Eymir 2020, Stevens Lapsley 2012, and Tsukada 2020) reported on various outcome measures of mobility including the 6-minute walk test, 10- and 15-meter walk tests, stair climb tests, Functional Ambulation Category system, and Iowa Ambulation Velocity Scale. Five studies reported no difference in mobility among groups at follow-up ranging from 3 to 12 months after TKA (Table 32). Li 2014 reported improved performance of the six-minute walk test (6MWT) among patients randomized to robot-assisted training compared to control at both 6 months (MD 63.6, 95\% CI 48.4 to 78.8) and 12 months (MD 73.4, 95\% CI 60.4 to 86.4) after TKA, but no difference in Functional Ambulation Category. Stevens Lapsley reported improved performance of the 6MWT (meters, larger is better; MD 46.8, $\mathrm{p}<0.05$ ) and stair climb test (seconds, smaller is better; MD -3.3, $\mathrm{p}<0.05$ ) among patients randomized to standard rehabilitation with adjunctive NMES compared with standard rehabilitation alone.

\section{Timed Up and Go}

Five studies (Sattler 2019, Piqueras 2013, Eymir 2020, Stevens Lapsley 2012, Tsukada 2020) reported data on the TUG test. Sattler 2019, Eymir 2020, Tsukada 2020 reported no difference in in the performance of the TUG (Table 33). Piqueras 2013 and Stevens Lapsley reported significantly improved performance of the TUG among patients receiving interactive virtual rehabilitation (vs. conventional outpatient physical therapy) and standard rehabilitation plus adjunctive NMES (vs. standard rehabilitation alone), respectively. 
Table 29. Acute rehabilitation versus various controls for total knee arthroplasty - continuous outcomes, physical function and activities of daily living

\begin{tabular}{|c|c|c|c|c|c|c|c|c|c|c|c|}
\hline $\begin{array}{l}\text { Study, } \\
\text { Year, PMID, } \\
\text { Country }\end{array}$ & Arm 1 & Arm 2 & $\begin{array}{l}\text { Overall } \\
\text { RoB }\end{array}$ & Outcome Measurement & $\begin{array}{l}\text { Time } \\
\text { Point }^{\mathrm{A}}\end{array}$ & $\begin{array}{l}\text { N Arm } \\
1\end{array}$ & $\begin{array}{l}\text { Arm 1, } \\
\text { Mean (SD) }\end{array}$ & $\begin{array}{l}\text { N Arm } \\
2\end{array}$ & $\begin{array}{l}\text { Arm 2, } \\
\text { Mean (SD) }\end{array}$ & $\begin{array}{l}\text { Effect Size } \\
(95 \% \mathrm{Cl})\end{array}$ & $\begin{array}{l}\text { Reported } \\
\text { p-Value }\end{array}$ \\
\hline \multirow{4}{*}{$\begin{array}{l}\text { Avramidis, } \\
2011, \\
21410130, \\
\text { Greece }\end{array}$} & $\begin{array}{l}\text { TENS plus } \\
\text { Physiotherapy }\end{array}$ & Physiotherapy & Moderate & Knee Society Score: Function & $3 \mathrm{mo}$ & 12 & $\begin{array}{l}150.94 \\
(14.26)\end{array}$ & 19 & $\begin{array}{l}141.74 \\
(12.38)\end{array}$ & $9.2(2.0,16.4)^{\mathrm{B}}$ & 0.003 \\
\hline & $\begin{array}{l}\text { TENS plus } \\
\text { Physiotherapy }\end{array}$ & Physiotherapy & Moderate & Knee Society Score: Function & $12 \mathrm{mo}$ & 15 & $\begin{array}{l}159.63 \\
(12.69) \\
\end{array}$ & 21 & $\begin{array}{l}156.40 \\
(12.11) \\
\end{array}$ & $3.2(2.7,9.1)^{\mathrm{B}}$ & 0.349 \\
\hline & $\begin{array}{l}\text { TENS plus } \\
\text { Physiotherapy }\end{array}$ & Physiotherapy & Moderate & $\begin{array}{l}\text { SF-36: Physical component } \\
(0-100)\end{array}$ & $3 \mathrm{mo}$ & 12 & $46.6(5.13)$ & 19 & $37.63(6.43)$ & $9(6.1,11.9)^{\mathrm{B}}$ & $<0.001$ \\
\hline & $\begin{array}{l}\text { TENS plus } \\
\text { Physiotherapy }\end{array}$ & Physiotherapy & Moderate & $\begin{array}{l}\text { SF-36: Physical component } \\
(0-100)\end{array}$ & $12 \mathrm{mo}$ & 15 & $53.9(4.26)$ & 21 & $47.37(3.84)$ & $6.5(4.6,8.5)^{\mathrm{B}}$ & $<0.001$ \\
\hline \multirow{2}{*}{$\begin{array}{l}\text { Buhagiar, } \\
2017, \\
28291891, \\
\text { Australia }\end{array}$} & $\begin{array}{l}\text { Hospital } \\
\text { Inpatient } \\
\text { Rehabilitation }\end{array}$ & $\begin{array}{l}\text { Home } \\
\text { Program }\end{array}$ & Moderate & Oxford knee score (0-48) & $6.5 \mathrm{mo}$ & 79 & $\begin{array}{l}36.9(95 \% \\
\mathrm{Cl} 35,38.7)\end{array}$ & 80 & $\begin{array}{l}34.8(95 \% \\
\mathrm{Cl} 32.9 \\
36.7)\end{array}$ & $\begin{array}{l}0.54(- \\
2.26,3.33)\end{array}$ & NR \\
\hline & $\begin{array}{l}\text { Hospital } \\
\text { Inpatient } \\
\text { Rehabilitation }\end{array}$ & $\begin{array}{l}\text { Home } \\
\text { Program }\end{array}$ & Moderate & Oxford knee score $(0-48)$ & $12 \mathrm{mo}$ & 79 & $\begin{array}{l}36.5(95 \% \\
\mathrm{Cl} 34.6, \\
38.4)\end{array}$ & 77 & $\begin{array}{l}37(95 \% \mathrm{Cl} \\
35.2,38.9)\end{array}$ & $\begin{array}{l}2.06(-0.59 \\
4.71)\end{array}$ & NR \\
\hline \multirow[t]{6}{*}{$\begin{array}{l}\text { Chan, 2018, } \\
\text { 29372260, } \\
\text { Singapore }\end{array}$} & $\begin{array}{l}\text { Discharge to } \\
\text { home }\end{array}$ & $\begin{array}{l}\text { Discharge to } \\
\text { community } \\
\text { hospitals }\end{array}$ & Moderate & Oxford knee score $(0-48)$ & $6 \mathrm{mo}$ & 967 & $\begin{array}{l}19.6(95 \% \\
\text { Cl 19.3, } \\
20.0)\end{array}$ & 98 & $\begin{array}{l}21.5(95 \% \\
\mathrm{Cl} 20.3 \\
22.6)\end{array}$ & $1.8(0.6,3.0)$ & 0.003 \\
\hline & $\begin{array}{l}\text { Discharge to } \\
\text { home }\end{array}$ & $\begin{array}{l}\text { Discharge to } \\
\text { community } \\
\text { hospitals }\end{array}$ & Moderate & Oxford knee score $(0-48)$ & $24 \mathrm{mo}$ & 801 & $\begin{array}{l}18.5(95 \% \\
\mathrm{Cl} 18.1 \\
18.9)\end{array}$ & 78 & $\begin{array}{l}22.0(95 \% \\
\mathrm{Cl} 20.9, \\
23.2)\end{array}$ & $-3.5(-4.8,-2.3)$ & $<0.0001$ \\
\hline & $\begin{array}{l}\text { Discharge to } \\
\text { home }\end{array}$ & $\begin{array}{l}\text { Discharge to } \\
\text { community } \\
\text { hospitals }\end{array}$ & Moderate & $\begin{array}{l}\text { SF-36: Physical functioning (0- } \\
100)\end{array}$ & $6 \mathrm{mo}$ & 967 & $\begin{array}{l}66.9(95 \% \\
\mathrm{Cl} 65.6, \\
68.2)\end{array}$ & 98 & $\begin{array}{l}59.1(95 \% \\
\text { Cl 55.0, } \\
63.1)\end{array}$ & $7.8(3.5,12.0)$ & 0.0004 \\
\hline & $\begin{array}{l}\text { Discharge to } \\
\text { home }\end{array}$ & $\begin{array}{l}\text { Discharge to } \\
\text { community } \\
\text { hospitals }\end{array}$ & Moderate & $\begin{array}{l}\text { SF-36: Physical functioning (0- } \\
100)\end{array}$ & $24 \mathrm{mo}$ & 801 & $\begin{array}{l}69.5(95 \% \\
\mathrm{Cl} 67.9, \\
71.0)\end{array}$ & 78 & $\begin{array}{l}57.2(95 \% \\
\text { Cl } 52.2, \\
62.1)\end{array}$ & $12.3(7.1,17.5)$ & $<0.0001$ \\
\hline & $\begin{array}{l}\text { Discharge to } \\
\text { home }\end{array}$ & $\begin{array}{l}\text { Discharge to } \\
\text { community } \\
\text { hospitals }\end{array}$ & Moderate & $\begin{array}{l}\text { Knee Society Clinical Rating } \\
\text { System: Function domain }\end{array}$ & $6 \mathrm{mo}$ & 967 & $\begin{array}{l}71.0(95 \% \\
\mathrm{Cl} 69.9 \\
72.1)\end{array}$ & 98 & $\begin{array}{l}62.3(95 \% \\
\mathrm{Cl} 58.8 \\
65.9)\end{array}$ & $8.7(4.9,12.4)$ & $<0.0001$ \\
\hline & $\begin{array}{l}\text { Discharge to } \\
\text { home }\end{array}$ & $\begin{array}{l}\text { Discharge to } \\
\text { community } \\
\text { hospitals }\end{array}$ & Moderate & $\begin{array}{l}\text { Knee Society Clinical Rating } \\
\text { System: Function domain }\end{array}$ & $24 \mathrm{mo}$ & 801 & $\begin{array}{l}73.9(95 \% \\
\mathrm{Cl} 72.6 \\
75.2)\end{array}$ & 78 & $\begin{array}{l}60.9(95 \% \\
\text { Cl } 56.7 \\
65.1)\end{array}$ & $\begin{array}{l}-13.0(- \\
17.4,08.6)\end{array}$ & $<0.0001$ \\
\hline \multirow{3}{*}{$\begin{array}{l}\text { Eymir, } \\
2020 \text {, } \\
32778907 \\
\text { Turkey }\end{array}$} & $\begin{array}{l}\text { Standard } \\
\text { physiotherapy } \\
\& \text { AHS }\end{array}$ & $\begin{array}{l}\text { Physiotherapy } \\
\text { \& CPM }\end{array}$ & High & $\begin{array}{l}\text { lowa Level of Assistant Scale: } \\
\text { Total }^{C}\end{array}$ & $3 \mathrm{mo}$ & 55 & $20.7(2.1)$ & 58 & $20.3(2.0)$ & $-0.4(-0.9,0.1)$ & $\mathrm{ns}$ \\
\hline & $\begin{array}{l}\text { Standard } \\
\text { physiotherapy } \\
\& \text { AHS }\end{array}$ & $\begin{array}{l}\text { Physiotherapy } \\
\text { \& CPM }\end{array}$ & High & $\begin{array}{l}\text { lowa Level of Assistant Scale: } \\
\text { Supine to sit }\end{array}$ & $3 \mathrm{mo}$ & 55 & $5.7(0.6)$ & 58 & $5.7(0.6)$ & $0(-0.2,0.2)$ & $\mathrm{ns}$ \\
\hline & $\begin{array}{l}\text { Standard } \\
\text { physiotherapy } \\
\& \text { AHS }\end{array}$ & $\begin{array}{l}\text { Physiotherapy } \\
\text { \& CPM }\end{array}$ & High & $\begin{array}{l}\text { lowa Level of Assistant Scale: } \\
\text { Sit to stand }\end{array}$ & $3 \mathrm{mo}$ & 55 & $5.3(0.6)$ & 58 & $5.2(0.7)$ & $0.4(0.2,0.6)$ & ns \\
\hline
\end{tabular}




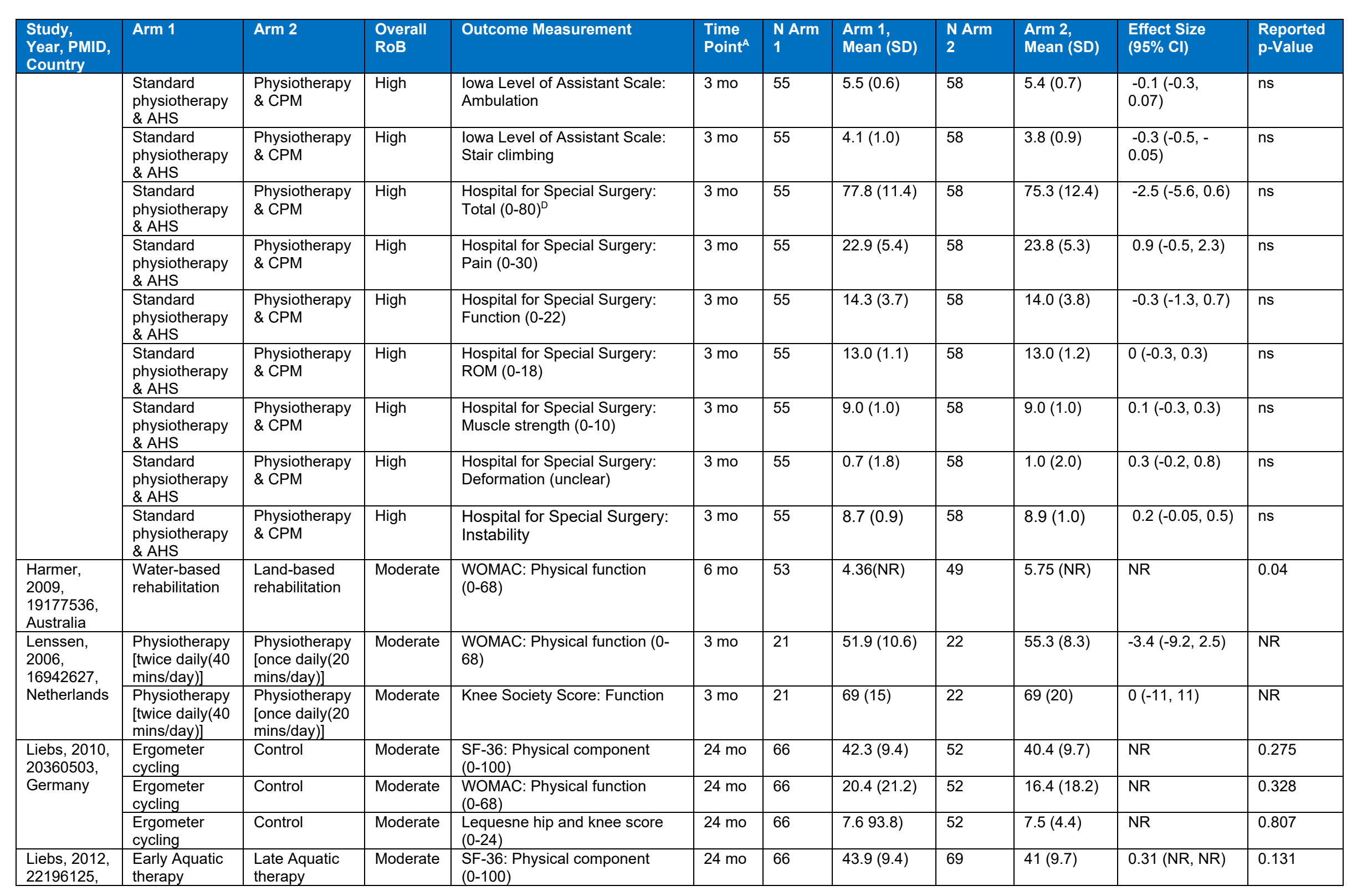




\begin{tabular}{|c|c|c|c|c|c|c|c|c|c|c|c|}
\hline $\begin{array}{l}\text { Study, } \\
\text { Year, PMID, } \\
\text { Country }\end{array}$ & Arm 1 & Arm 2 & $\begin{array}{l}\text { Overall } \\
\text { RoB }\end{array}$ & Outcome Measurement & $\begin{array}{l}\text { Time } \\
\text { Point }^{\mathrm{A}}\end{array}$ & $\begin{array}{l}\text { N Arm } \\
1\end{array}$ & $\begin{array}{l}\text { Arm 1, } \\
\text { Mean (SD) }\end{array}$ & $\begin{array}{l}\text { N Arm } \\
2\end{array}$ & $\begin{array}{l}\text { Arm 2, } \\
\text { Mean (SD) }\end{array}$ & $\begin{array}{l}\text { Effect Size } \\
(95 \% \mathrm{Cl})\end{array}$ & $\begin{array}{l}\text { Reportec } \\
\text { p-Value }\end{array}$ \\
\hline \multirow[t]{2}{*}{ Germany } & $\begin{array}{l}\text { Early Aquatic } \\
\text { therapy }\end{array}$ & $\begin{array}{l}\text { Late Aquatic } \\
\text { therapy }\end{array}$ & Moderate & $\begin{array}{l}\text { WOMAC: Physical function } \\
(0-68)\end{array}$ & $24 \mathrm{mo}$ & 66 & $13.8(13.6)$ & 69 & $20.7(21.3)$ & 0.39 (NR, NR) & 0.117 \\
\hline & $\begin{array}{l}\text { Early Aquatic } \\
\text { therapy }\end{array}$ & $\begin{array}{l}\text { Late Aquatic } \\
\text { therapy }\end{array}$ & Moderate & $\begin{array}{l}\text { Lequesne hip and knee score } \\
(0-24)\end{array}$ & $24 \mathrm{mo}$ & 66 & $6.8(3.8)$ & 69 & $7.4(3.8)$ & 0.15 (NR, NR) & 0.361 \\
\hline \multirow{2}{*}{$\begin{array}{l}\text { Naylor, } \\
2017, \\
28899328, \\
\text { Australia }\end{array}$} & $\begin{array}{l}\text { Inpatient } \\
\text { rehabilitation }\end{array}$ & $\begin{array}{l}\text { No inpatient } \\
\text { rehabilitation }\end{array}$ & High & Oxford knee score $(0-48)$ & $3 \mathrm{mo}$ & 129 & $\begin{array}{l}\text { Median } \\
(\text { IQR) } 40 \\
(34,43) \\
\end{array}$ & 129 & $\begin{array}{l}\text { Median } \\
(\text { IQR) } 40 \\
(34,43) \\
\end{array}$ & $0(-1.2,1.2)^{\mathrm{B}}$ & NR \\
\hline & $\begin{array}{l}\text { Inpatient } \\
\text { rehabilitation }\end{array}$ & $\begin{array}{l}\text { No inpatient } \\
\text { rehabilitation }\end{array}$ & High & Oxford knee score (0-48) & $12 \mathrm{mo}$ & 129 & $\begin{array}{l}\text { Median } \\
\text { (IQR) 44 } \\
(42,45)\end{array}$ & 129 & $\begin{array}{l}\text { Median } \\
(\text { IQR) } 44 \\
(42,46)\end{array}$ & $0(-0.4,0.4)^{\mathrm{B}}$ & NR \\
\hline $\begin{array}{l}\text { Sattler, } \\
2019, \\
30994586, \\
\text { Australia }\end{array}$ & $\begin{array}{l}\text { Pedaling- } \\
\text { based } \\
\text { protocol }\end{array}$ & $\begin{array}{l}\text { Non-pedaling } \\
\text { (multi- } \\
\text { exercise) } \\
\text { protocol }\end{array}$ & Moderate & Oxford knee score (0-48) & $4 \mathrm{mo}$ & 28 & $39.3(6.1)$ & 28 & $37.6(4.8)$ & $1.7(-0.4,3.8)^{\mathrm{B}}$ & NR \\
\hline $\begin{array}{l}\text { Stevens } \\
\text { Lapsley, } \\
2012, \\
22095207, \\
\text { USA }\end{array}$ & $\begin{array}{l}\text { Standard } \\
\text { rehabilitation } \\
\text { \& NMES }\end{array}$ & $\begin{array}{l}\text { Standard } \\
\text { rehabilitation }\end{array}$ & Moderate & $\begin{array}{l}\text { SF-36: Physical component } \\
(0-100)\end{array}$ & $12 \mathrm{mo}$ & 25 & $52.6(2.9)$ & 30 & $50.7(7.4)$ & $1.9(-0.4,4.2)^{\mathrm{B}}$ & NR \\
\hline
\end{tabular}

Statistically significant effect sizes are in bold text. In cases where calculated effect size confidence intervals were not-statistically significant but reported p-values were, we deferred to reported p-values and still bolded results.

Abbreviations: $\mathrm{ACSE}=$ active heal slide exercise, $\mathrm{CI}=$ confidence interval, $\mathrm{CPM}=$ continuous passive motion, $\mathrm{IQR}=$ interquartile range, mo $=$ month, $\mathrm{NMES}=$ neuromuscular electrical stimulation, $\mathrm{NR}=$ not reported, $\mathrm{PMID}=$ PubMed identifier, $\mathrm{RoB}=$ risk of bias, $\mathrm{SD}=$ standard deviation, $\mathrm{SF}-36=36$-Item short form survey, $\mathrm{WOMAC}=\mathrm{Western}$ Ontario and McMaster Universities Osteoarthritis Index.

A Time from surgery

B Calculated

C The Iowa Level of Assistance Scale was used to assess four functional activities (moving from the supine position to the sitting position, rising from the sitting position, walking $4.57 \mathrm{~m}$, and ascending and descending stairs for three steps). Activities are scored between 0-6 (6=independence). The total score is the sum of the four activity scores.

D Total Hospital for Special Surgery score is obtained by summing all the item scores and subtracting scores related to walking aids, loss of knee extension and varus/valgus deformity. 
Table 30. Acute rehabilitation versus various controls for total knee arthroplasty - continuous outcomes, transfers

\begin{tabular}{|c|c|c|c|c|c|c|c|c|c|c|c|}
\hline $\begin{array}{l}\text { Study, Year, } \\
\text { PMID, Country }\end{array}$ & Arm 1 & Arm 2 & $\begin{array}{l}\text { Overall } \\
\text { RoB }\end{array}$ & $\begin{array}{l}\text { Outcome } \\
\text { Measurement }\end{array}$ & $\begin{array}{l}\text { Time } \\
\text { Point }^{A}\end{array}$ & $\begin{array}{l}\text { N Arm } \\
1\end{array}$ & $\begin{array}{l}\text { Arm 1, Mean } \\
\text { (SD) }\end{array}$ & $\begin{array}{l}\text { N Arm } \\
2\end{array}$ & $\begin{array}{l}\text { Arm 2, Mean } \\
\text { (SD) }\end{array}$ & $\begin{array}{l}\text { Effect Size } \\
(95 \% \mathrm{Cl})\end{array}$ & $\begin{array}{l}\text { Reported } \\
\text { p-Value }\end{array}$ \\
\hline \multirow{2}{*}{$\begin{array}{l}\text { Li, 2014, } \\
23412304, \\
\text { China }\end{array}$} & $\begin{array}{l}\text { Robot-assisted } \\
\text { training }\end{array}$ & Control (CPM) & High & $\begin{array}{l}10 \mathrm{~m} \text { sitting } \\
\text { standing time(s) }\end{array}$ & $6 \mathrm{mo}$ & 30 & $8.7(1.7)$ & 30 & $11.1(1.9)$ & $\begin{array}{l}-2.4(-3.0,- \\
1.8)^{\mathrm{B}}\end{array}$ & $<0.05$ \\
\hline & $\begin{array}{l}\text { Robot-assisted } \\
\text { training }\end{array}$ & Control (CPM) & High & $\begin{array}{l}\text { 10m sitting } \\
\text { standing time(s) }\end{array}$ & $12 \mathrm{mo}$ & 30 & $8.7(1.4)$ & 30 & $11.5(2.1)$ & $\begin{array}{l}-2.8(-3.5,-2.1) \\
B\end{array}$ & $<0.05$ \\
\hline $\begin{array}{l}\text { Tsukada, 2020, } \\
\text { 31723080, } \\
\text { Japan }\end{array}$ & $\begin{array}{l}\text { Standard } \\
\text { rehabilitation \& } \\
\text { hybrid training } \\
\text { system }\end{array}$ & $\begin{array}{l}\text { Standard } \\
\text { rehabilitation }\end{array}$ & Moderate & $\begin{array}{l}10 \mathrm{~m} \text { sitting } \\
\text { standing time (s) }\end{array}$ & $3 \mathrm{mo}$ & 20 & 9 (NR) & 20 & $11(\mathrm{NR})$ & NR & ns \\
\hline
\end{tabular}

Statistically significant effect sizes are in bold text. In cases where calculated effect size confidence intervals were not-statistically significant but reported p-values were, we deferred to reported p-values and still bolded results.

Abbreviations: $\mathrm{CI}=$ confidence interval, $\mathrm{CPM}=$ continuous passive motion, $\mathrm{mo}=$ month, $\mathrm{NR}=$ not reported, $\mathrm{ns}=$ not significant, $\mathrm{PMID}=\mathrm{PubMed}$ identifier, $\mathrm{RoB}=$ risk of bias, $\mathrm{s}$ $=$ second, $\mathrm{SD}=$ standard deviation, $\mathrm{SE}=$ standard error.

A Time from surgery

B Calculated

Table 31. Acute rehabilitation versus various controls for total knee arthroplasty - continuous outcomes, balance

\begin{tabular}{|c|c|c|c|c|c|c|c|c|c|c|c|}
\hline $\begin{array}{l}\text { Study, Year, } \\
\text { PMID, Country }\end{array}$ & Arm 1 & Arm 2 & $\begin{array}{l}\text { Overall } \\
\text { RoB }\end{array}$ & $\begin{array}{l}\text { Outcome } \\
\text { Measurement }\end{array}$ & $\begin{array}{l}\text { Time } \\
\text { Point }^{A}\end{array}$ & N Arm & $\begin{array}{l}\text { Arm 1, Mean } \\
\text { (SD) }\end{array}$ & $\begin{array}{l}\text { N Arm } \\
2\end{array}$ & $\begin{array}{l}\text { Arm 2, Mean } \\
\text { (SD) }\end{array}$ & $\begin{array}{l}\text { Effect Size }(95 \% \\
\mathrm{CI})\end{array}$ & $\begin{array}{l}\text { Reported p- } \\
\text { Value }\end{array}$ \\
\hline \multirow[t]{2}{*}{$\begin{array}{l}\mathrm{Li}, 2014, \\
23412304, \\
\text { China }\end{array}$} & $\begin{array}{l}\text { Robot-assisted } \\
\text { training }\end{array}$ & $\begin{array}{l}\text { Traditional } \\
\text { rehabilitation } \\
\text { training }\end{array}$ & High & $\begin{array}{l}\text { Berg Balance } \\
\text { Scale }\end{array}$ & $6 \mathrm{mo}$ & 30 & $53.9(1.9)$ & 30 & $50.2(2.2)$ & $3.7(3,4.4)^{B}$ & $<0.05$ \\
\hline & $\begin{array}{l}\text { Robot-assisted } \\
\text { training }\end{array}$ & $\begin{array}{l}\text { Traditional } \\
\text { rehabilitation } \\
\text { training }\end{array}$ & High & $\begin{array}{l}\text { Berg Balance } \\
\text { Scale }\end{array}$ & $12 \mathrm{mo}$ & 30 & $54.5(1.7)$ & 30 & $49.9(2.4)$ & $4.6(3.8,5.4)^{B}$ & $<0.05$ \\
\hline $\begin{array}{l}\text { Zapparoli, } \\
2020 \text {, } \\
32488010 \\
\text { Italy }\end{array}$ & $\begin{array}{l}\text { Motor imagery \& } \\
\text { rehabilitation }\end{array}$ & Rehabilitation & High & $\begin{array}{l}\text { Falls/near falls } \\
\text { in the past } 12 \\
\text { mo }\end{array}$ & $24 \mathrm{mo}$ & 24 & NR & 24 & NR & $\begin{array}{l}\text { Exponential effect } \\
\text { size }(95 \% \mathrm{Cl}): 1.75 \\
(1.31,2.29) \\
\\
\text { Standardized mean } \\
\text { difference effect } \\
\text { size }(95 \% \mathrm{Cl}): 0.96 \\
(0.39,1.72)\end{array}$ & $<0.001$ \\
\hline
\end{tabular}

Statistically significant effect sizes are in bold text. In cases where calculated effect size confidence intervals were not-statistically significant but reported p-values were, we deferred to reported $\mathrm{p}$-values and still bolded results.

Abbreviations: $\mathrm{CI}=$ confidence interval, $\mathrm{mo}=$ month, $\mathrm{NR}=$ not reported, $\mathrm{ns}=$ not significant, $\mathrm{PMID}=\mathrm{PubMed}$ identifier, $\mathrm{RoB}=$ risk of bias, $\mathrm{SD}=$ standard deviation .

A Time from surgery

B Calculated 
Table 32. Acute rehabilitation versus various controls for total knee arthroplasty - continuous outcomes, mobility

\begin{tabular}{|c|c|c|c|c|c|c|c|c|c|c|c|}
\hline $\begin{array}{l}\text { Study, Year, } \\
\text { PMID, Country }\end{array}$ & Arm 1 & Arm 2 & $\begin{array}{l}\text { Overall } \\
\text { RoB }\end{array}$ & $\begin{array}{l}\text { Outcome } \\
\text { Measurement }\end{array}$ & $\begin{array}{l}\text { Time } \\
\text { Point }^{\mathrm{A}}\end{array}$ & $\begin{array}{l}\text { N Arm } \\
1\end{array}$ & $\begin{array}{l}\text { Arm 1, Mean } \\
\text { (SD) }\end{array}$ & $\begin{array}{l}\text { N Arm } \\
2\end{array}$ & $\begin{array}{l}\text { Arm 2, } \\
\text { Mean (SD) }\end{array}$ & $\begin{array}{l}\text { Effect Size } \\
(95 \% \mathrm{Cl})\end{array}$ & $\begin{array}{l}\text { Reported } \\
\text { p-Value }\end{array}$ \\
\hline \multirow[t]{3}{*}{$\begin{array}{l}\text { Buhagiar, 2017, } \\
\text { 28291891, } \\
\text { Australia }\end{array}$} & Home program & $\begin{array}{l}\text { Hospital inpatient } \\
\text { rehabilitation }\end{array}$ & Moderate & 6MWT (m) & $12 \mathrm{mo}$ & 80 & $\begin{array}{l}408.8(95 \% \\
\mathrm{Cl} 371.6 \\
438)\end{array}$ & 79 & $\begin{array}{l}391.2(95 \% \\
\text { Cl 358.1, } \\
424.4)\end{array}$ & $\begin{array}{l}13.54(-13.61, \\
40.69,)\end{array}$ & NR \\
\hline & Home program & $\begin{array}{l}\text { Hospital inpatient } \\
\text { rehabilitation }\end{array}$ & Moderate & $15 \mathrm{~m}$ walk test $(\mathrm{s})$ & $6.5 \mathrm{mo}$ & 80 & $\begin{array}{l}12.0(95 \% \mathrm{Cl} \\
10.9,13.1)\end{array}$ & 79 & $\begin{array}{l}12.5(95 \% \\
\text { Cl } 11.4 \\
13.6)\end{array}$ & $\begin{array}{l}0.50(-2.01, \\
1.01)\end{array}$ & NR \\
\hline & Home program & $\begin{array}{l}\text { Hospital inpatient } \\
\text { rehabilitation }\end{array}$ & Moderate & $15 \mathrm{~m}$ walk test (s) & $12 \mathrm{mo}$ & 77 & $\begin{array}{l}12.7(95 \% \mathrm{Cl} \\
11.6 \text { to } 13.8)\end{array}$ & 79 & $\begin{array}{l}12.3(95 \% \\
\mathrm{Cl} 11.2 \\
13.4) \\
\end{array}$ & $\begin{array}{l}-0.42(-1.10 \\
1.94)\end{array}$ & NR \\
\hline \multirow{2}{*}{$\begin{array}{l}\text { Eymir, } \\
2020, \\
32778907 \\
\text { Turkey }\end{array}$} & $\begin{array}{l}\text { Standard } \\
\text { physiotherapy \& } \\
\text { AHS }\end{array}$ & $\begin{array}{l}\text { Physiotherapy \& } \\
\text { CPM }\end{array}$ & High & $\begin{array}{l}\text { lowa Ambulation } \\
\text { Velocity Scale } \\
\text { (s) } D^{\mathrm{B}}\end{array}$ & $3 \mathrm{mo}$ & 55 & $17.2(14.1)$ & 58 & $23.3(15.6)$ & $6.1(2.2,9.9)$ & ns \\
\hline & $\begin{array}{l}\text { Standard } \\
\text { physiotherapy \& } \\
\text { AHS }\end{array}$ & $\begin{array}{l}\text { Physiotherapy \& } \\
\text { CPM }\end{array}$ & High & 10MWT (m) & $3 \mathrm{mo}$ & 55 & $12.9(9.9)$ & 58 & $17.6(11.6)$ & $4.7(1.8,7.5)$ & ns \\
\hline \multirow{2}{*}{$\begin{array}{l}\text { Harmer, 2009, } \\
19177536, \\
\text { Australia }\end{array}$} & $\begin{array}{l}\text { Water-based } \\
\text { rehabilitation }\end{array}$ & $\begin{array}{l}\text { Land-based } \\
\text { rehabilitation }\end{array}$ & Moderate & 6MWT (m) & $6 \mathrm{mo}$ & 53 & 407.24 (NR) & 49 & $\begin{array}{l}407.24 \\
(\mathrm{NR})\end{array}$ & NR & ns \\
\hline & $\begin{array}{l}\text { Water-based } \\
\text { rehabilitation }\end{array}$ & $\begin{array}{l}\text { Land-based } \\
\text { rehabilitation }\end{array}$ & Moderate & $\begin{array}{l}\text { Stair climb power } \\
(\mathrm{W})^{\mathrm{C}}\end{array}$ & $6 \mathrm{mo}$ & 53 & 164.35 (NR) & 49 & $\begin{array}{l}146.76 \\
(\mathrm{NR})\end{array}$ & NR & NR \\
\hline \multirow{4}{*}{$\begin{array}{l}\mathrm{Li}, 2014, \\
23412304, \\
\text { China }\end{array}$} & $\begin{array}{l}\text { Robot-assisted } \\
\text { training }\end{array}$ & Control & High & 6MWT (m) & $6 \mathrm{mo}$ & 30 & $668(46.3)$ & 30 & $604.4(36.9)$ & $\begin{array}{l}63.6(48.4, \\
78.8)^{\mathrm{D}}\end{array}$ & $<0.05$ \\
\hline & $\begin{array}{l}\text { Robot-assisted } \\
\text { training }\end{array}$ & Control & High & 6MWT (m) & $12 \mathrm{mo}$ & 30 & $681.9(37.7)$ & 30 & $608.5(34.8)$ & $\begin{array}{l}73.4(60.4, \\
86.4)^{\mathrm{D}} \\
\end{array}$ & $<0.05$ \\
\hline & $\begin{array}{l}\text { Robot-assisted } \\
\text { training }\end{array}$ & Control & High & $\begin{array}{l}\text { FunctioNRI } \\
\text { ambulation } \\
\text { category }(0-5) \\
\end{array}$ & $6 \mathrm{mo}$ & 30 & $5(\mathrm{NR})$ & 30 & $5(\mathrm{NR})$ & NR & NR \\
\hline & $\begin{array}{l}\text { Robot-assisted } \\
\text { training }\end{array}$ & Control & High & $\begin{array}{l}\text { FunctioNRI } \\
\text { ambulation } \\
\text { category }(0-5)\end{array}$ & $12 \mathrm{mo}$ & 30 & $5(\mathrm{NR})$ & 30 & 5 (NR) & NR & NR \\
\hline \multirow[t]{2}{*}{$\begin{array}{l}\text { Sattler, 2019, } \\
\text { 30994586, } \\
\text { Australia }\end{array}$} & $\begin{array}{l}\text { Pedaling-based } \\
\text { protocol }\end{array}$ & $\begin{array}{l}\text { Non-pedaling } \\
\text { (multi-exercise] } \\
\text { protocol }\end{array}$ & Moderate & 6MWT (m) & $4 \mathrm{mo}$ & 28 & $514.0(78.5)$ & 28 & $488.3(89.7)$ & $25.7(19.5,70.8$ & 0.259 \\
\hline & $\begin{array}{l}\text { Pedaling-based } \\
\text { protocol }\end{array}$ & $\begin{array}{l}\text { Non-pedaling } \\
\text { (multi-exercise] } \\
\text { protocol }\end{array}$ & Moderate & $\begin{array}{l}10 \mathrm{~m} \text { walk test } \\
(\mathrm{m} / \mathrm{s})\end{array}$ & $4 \mathrm{mo}$ & 28 & $1.54(0.24)$ & 28 & $1.50(0.25)$ & $\begin{array}{l}0.04(-0.01 \\
0.12)\end{array}$ & 0.592 \\
\hline \multirow[t]{2}{*}{$\begin{array}{l}\text { Stevens } \\
\text { Lapsley,2012, } \\
\text { 22095207, USA }\end{array}$} & $\begin{array}{l}\text { Standard } \\
\text { rehabilitation \& } \\
\text { NMES }\end{array}$ & $\begin{array}{l}\text { Standard } \\
\text { rehabilitation }\end{array}$ & Moderate & 6MWT (m) & $12 \mathrm{mo}$ & 25 & $524.6(81.6)$ & 30 & $477.8(94)$ & $46.8(0.4,93.2)^{D}$ & $<0.05$ \\
\hline & $\begin{array}{l}\text { Standard } \\
\text { rehabilitation \& } \\
\text { NMES }\end{array}$ & $\begin{array}{l}\text { Standard } \\
\text { rehabilitation }\end{array}$ & Moderate & $\begin{array}{l}\text { Stair climb test } \\
(s)^{E}\end{array}$ & $12 \mathrm{mo}$ & 25 & $11.5(4.3)$ & 30 & $14.8(9.3)$ & $-3.3(-7,0.4)^{\mathrm{D}}$ & $<0.05$ \\
\hline $\begin{array}{l}\text { Tsukada, 2020, } \\
\text { 31723080, } \\
\text { Japan }\end{array}$ & $\begin{array}{l}\text { Standard } \\
\text { rehabilitation \& } \\
\text { hybrid training } \\
\text { system }\end{array}$ & $\begin{array}{l}\text { Standard } \\
\text { rehabilitation }\end{array}$ & Moderate & $\begin{array}{l}\text { Stair climb test } \\
(\mathrm{s})^{\mathrm{F}}\end{array}$ & $3 \mathrm{mo}$ & 20 & $13(\mathrm{NR})$ & 20 & 16 (NR) & NR & ns \\
\hline
\end{tabular}

Statistically significant effect sizes are in bold text. 
Abbreviations: $\mathrm{AHS}=$ active heel slides, $\mathrm{CI}=$ confidence interval, $\mathrm{mo}=$ month, $\mathrm{m}=$ meter, $\mathrm{NR}=$ not reported, $\mathrm{PMID}=\mathrm{PubMed}$ identifier, $\mathrm{RoB}=$ risk of bias, $\mathrm{s}=\mathrm{second}, \mathrm{SD}=$ standard deviation, $\mathrm{SE}=$ standard error, $\mathrm{W}=$ watt.

A Time from surgery

B Speed at a distance of $13.4 \mathrm{~m}$

${ }^{\mathrm{C}}$ The time required to ascend 18 stairs (flights of 8 to 10 stairs, separated by a small landing) as fast as possible using handrails and walking aids as required. Stair climb power was calculated using this time, combined with patient's body mass index, total stair height, and ascent time.

D Calculated

D Speed at a distance of $13.4 \mathrm{~m}$

E Defined as the total time to ascend a flight of stairs, turn around and descend

F Defined as the total time to ascend 18 stairs as quickly as possible

Table 33. Acute rehabilitation versus various controls for total knee arthroplasty - continuous outcomes, Timed Up and Go

\begin{tabular}{|c|c|c|c|c|c|c|c|c|c|c|c|}
\hline $\begin{array}{l}\text { Study, Year, } \\
\text { PMID, Country }\end{array}$ & Arm 1 & Arm 2 & $\begin{array}{l}\text { Overall } \\
\text { RoB }\end{array}$ & $\begin{array}{l}\text { Outcome } \\
\text { Measurement }\end{array}$ & $\begin{array}{l}\text { Time } \\
\text { Point }^{\mathrm{A}}\end{array}$ & $\begin{array}{l}\text { N Arm } \\
1\end{array}$ & $\begin{array}{l}\text { Arm 1, Mean } \\
\text { (SD) }\end{array}$ & $\begin{array}{l}\text { N Arm } \\
2\end{array}$ & $\begin{array}{l}\text { Arm 2, Mean } \\
\text { (SD) }\end{array}$ & $\begin{array}{l}\text { Effect Size }(95 \% \\
\mathrm{Cl})\end{array}$ & $\begin{array}{l}\text { Reported } \\
\text { p-Value }\end{array}$ \\
\hline $\begin{array}{l}\text { Eymir, } \\
2020 \text {, } \\
32778907 \\
\text { Turkey }\end{array}$ & $\begin{array}{l}\text { Standard } \\
\text { physiotherapy \& } \\
\text { AHS }\end{array}$ & $\begin{array}{l}\text { Physiotherapy \& } \\
\text { CPM }\end{array}$ & High & TUG (s) & $3 \mathrm{mo}$ & 55 & $15.2(13.1)$ & 58 & $22.8(18.4)$ & $7.6(3.3,11.8)$ & ns \\
\hline $\begin{array}{l}\text { Piqueras, 2013, } \\
23474735, \\
\text { Spain }\end{array}$ & $\begin{array}{l}\text { Interactive virtual } \\
\text { telerehabilitation } \\
\text { system }\end{array}$ & $\begin{array}{l}\text { Conventional } \\
\text { outpatient } \\
\text { physical therapy }\end{array}$ & Moderate & TUG (s) & $3 \mathrm{mo}$ & 68 & NR (NR) & 65 & NR (NR) & NR & 0.020 \\
\hline $\begin{array}{l}\text { Sattler, 2019, } \\
\text { 30994586, } \\
\text { Australia }\end{array}$ & $\begin{array}{l}\text { Pedaling-based } \\
\text { protocol }\end{array}$ & $\begin{array}{l}\text { Non-pedaling } \\
\text { (multi-exercise) } \\
\text { protocol }\end{array}$ & Moderate & TUG (s) & $4 \mathrm{mo}$ & 28 & $6.9(1.3)$ & 28 & $7.1(1.3)$ & $-0.2(-0.7,0.3)^{\mathrm{B}}$ & NR \\
\hline $\begin{array}{l}\text { Stevens } \\
\text { Lapsley,2012, } \\
\text { 22095207,USA }\end{array}$ & $\begin{array}{l}\text { Standard } \\
\text { rehabilitation \& } \\
\text { NMES }\end{array}$ & $\begin{array}{l}\text { Standard } \\
\text { rehabilitation }\end{array}$ & Moderate & TUG (s) & $12 \mathrm{mo}$ & 25 & $6.7(1.7)$ & 30 & $8.3(2.8)$ & $-1.6(-2.5,-0.7)^{B}$ & $\overline{N R}$ \\
\hline $\begin{array}{l}\text { Tsukada, 2020, } \\
31723080, \\
\text { Japan }\end{array}$ & $\begin{array}{l}\text { Standard } \\
\text { rehabilitation \& } \\
\text { hybrid training } \\
\text { system }\end{array}$ & $\begin{array}{l}\text { Standard } \\
\text { rehabilitation }\end{array}$ & Moderate & TUG (s) & $3 \mathrm{mo}$ & 20 & 9 (NR) & 20 & 11 (NR) & NR & ns \\
\hline
\end{tabular}

Statistically significant effect sizes are in bold text.

Abbreviations: $\mathrm{CI}=$ confidence interval, $\mathrm{CPM}=$ continuous passive motion, mo $=$ month, $\mathrm{NR}=$ not reported, $\mathrm{PMID}=\mathrm{PubMed}$ identifier, $\mathrm{RoB}=$ risk of bias, $\mathrm{s}=$ second, $\mathrm{SD}=$ standard deviation, TUG = timed up and go test.

A Time from surgery

B Calculated 


\section{Other Patient-Reported Outcomes Following Acute Rehabilitation}

Ten studies in total reported other patient-reported outcomes following acute rehabilitation compared to various control: two studies (den Hertog 2012, Sattler 2019) compared novel acute rehabilitation programs to various comparators (less intensive rehabilitation or no care); two studies (Lenssen 2006, Liebs 2012) compared acute rehabilitation programs with different timing and/or intensity; three studies (Buhagiar 2017, Chan 2018, Padgett 2018) compared acute rehabilitation programs delivered in different settings or by different personnel, and three studies (Stevens-Lapsley 2012, Jin 2018, Rockstroh 2010) compared acute rehabilitation programs with or without an adjunctive modality (Tables 34 to 37). Outcome domains included: QoL, patient satisfaction with care, and patient global assessments.

\section{Health-Related Quality of Life}

Rockstroh 2010 reported QoL using the Oswestry Disability Index (0-100, lower is better) and found patients randomized to physiotherapy combined with adjunctive microcurrent (TENS) reported clinically significant improvements compared with patients randomized to physiotherapy alone at 3 months after TKA (Table 34).

\section{Patient Satisfaction With Care}

Three studies (Liebs 2010, Buhagiar 2017, Lenssen, 2006) reported data on satisfaction with care. While Liebs 2010 and Lenssen 2006 found no differences between rehabilitation arms (Table 35), Buhagiar 2017 found patients randomized to hospital inpatient rehabilitation reported significantly higher satisfaction with care compared with patients randomized to the home program (MD 8.9\%, 95\% CI 3.0 to 13.9 ).

\section{Patient Global Assessments}

Ten studies (den Hertog 2012, Sattler 2019, Iwakiri 2020, Lenssen 2006, Liebs 2012, Chan 2018, Padgett 2018, Buhagiar 2017, Stevens Lapsley 2012, Jin 2018) provided data on patients' self-reported global health assessment using nine different measurement instruments assessed between 3 and 24 months after TKA surgery (Table 36). Studies reported mixed findings: five studies reported comparable results among groups (Sattler 2019, Iwakiri 2020, Liebs 2012, Padgett 2018, Buhagiar, 2017) and five studies reported significant differences among groups (den Hertog, 2012, Lenssen, 2006, Chan, 2018, Stevens Lapsley, 2012, Jin, 2018). den Hertog reported global patient assessment data using the WOMAC index (0-10, lower is better) and the Knee Society Score (KSS; 0-100, higher is better) and found significant improvements for both measures among patients randomized to the fast-track rehabilitation in the acute period compared with standard care $(\mathrm{p}=0.002$ for WOMAC index, $\mathrm{p}=0.003$ for KSS).

Lenssen 2006 reported global patient assessment data using the KSS and total WOCAC score (0-96, lower is better) and found no difference on the KSS but improvements on the WOMAC associated with twice daily physiotherapy in the acute period (compared with once daily) (MD $-4.6,95 \% \mathrm{CI}-138$ to -3.9$)$.

Chan 2018 reported global patient assessment data using the KSS among patients discharged to home compared with community hospitals using an NRCS design observed patients discharged home had significantly improved compared with patients discharged to community hospitals at 2 years after TKA (MD 4.4, 95\% CI 1.4 to 7.3 ).

Stevens Lapsley 2012 reported patient global assessment data using the global rating scale of perceived knee function ( $0-100$, higher is better) and found greater improvements among patients 
randomized to adjunctive NMES and standard rehabilitation compared with patients randomized to standard rehabilitation alone (MD 8.3, 95\% CI 2.5 to 14.1) at 12 months after TKA.

Jin 2018 reported patient global assessment using the total WOMAC score and found significant improvements among patients randomized to virtual reality and usual care compared with usual care alone at both 3 months (MD $-3.9,95 \% \mathrm{CI}-5.6$ to -2.2$)$ and 6 months (MD -4.8 , $95 \%$ CI -6.1 to -3.4 ) after TKA. 
Table 34. Acute rehabilitation versus various controls for total knee arthroplasty - continuous outcomes, quality of life

\begin{tabular}{|c|c|c|c|c|c|c|c|c|c|c|c|}
\hline $\begin{array}{l}\text { Study, Year, } \\
\text { PMID, Country }\end{array}$ & Arm 1 & Arm 2 & $\begin{array}{l}\text { Overall } \\
\text { RoB }\end{array}$ & $\begin{array}{l}\text { Outcome } \\
\text { Measurement }\end{array}$ & $\begin{array}{l}\text { Time } \\
\text { Point }^{\mathrm{A}} \\
\end{array}$ & $\begin{array}{l}\text { N Arm } \\
1 \\
\end{array}$ & $\begin{array}{l}\text { Arm 1, Mean } \\
\text { (SD) }\end{array}$ & $\begin{array}{l}\text { N Arm } \\
2\end{array}$ & $\begin{array}{l}\text { Arm 2, Mean } \\
\text { (SD) }\end{array}$ & $\begin{array}{l}\text { Effect Size }(95 \% \\
\text { CI) }\end{array}$ & $\begin{array}{l}\text { Reported } \\
\text { p-Value }\end{array}$ \\
\hline $\begin{array}{l}\text { Rockstroh, } \\
2010, \\
20533147, \\
\text { Germany }\end{array}$ & $\begin{array}{l}\text { Physiotherapy \& } \\
\text { microcurrent }\end{array}$ & Physiotherapy & High & $\begin{array}{l}\text { QoL } \\
\text { (Oswestry } \\
\text { score) }\end{array}$ & $3 \mathrm{mo}$ & 37 & $\begin{array}{l}\text { Median } \\
\text { (IQR) } 91 \text { (81 } \\
, 91)\end{array}$ & 41 & $\begin{array}{l}\text { Median (IQR) } \\
78(57,87)\end{array}$ & $-13(-19.0,-7.02)^{B}$ & $<0.001$ \\
\hline
\end{tabular}

Statistically significant effect sizes are in bold text. In cases where calculated effect size confidence intervals were not-statistically significant but reported p-values were, we deferred to reported p-values and still bolded results.

Abbreviations: $\mathrm{CI}=$ confidence interval, $\mathrm{IQR}=$ interquartile range, $\mathrm{mo}=$ month, $\mathrm{NR}=$ not reported, $\mathrm{PMID}=\mathrm{PubMed}$ identifier, $\mathrm{QoL}=\mathrm{quality}$ of life, $\mathrm{RoB}=$ risk of bias, $\mathrm{SD}=$ standard deviation.

A Time from surgery

B Calculated

Table 35. Acute rehabilitation versus various controls for total knee arthroplasty - continuous outcomes, patient satisfaction with care

\begin{tabular}{|c|c|c|c|c|c|c|c|c|c|c|c|}
\hline $\begin{array}{l}\text { Study, Year, } \\
\text { PMID, Country }\end{array}$ & Arm 1 & Arm 2 & $\begin{array}{l}\text { Overall } \\
\text { RoB }\end{array}$ & $\begin{array}{l}\text { Outcome } \\
\text { Measurement }\end{array}$ & $\begin{array}{l}\text { Time } \\
\text { Point }^{\mathrm{A}}\end{array}$ & $\begin{array}{l}\text { N Arm } \\
1\end{array}$ & $\begin{array}{l}\text { Arm 1, Mean } \\
\text { (SD) }\end{array}$ & $\begin{array}{l}\text { N Arm } \\
2\end{array}$ & $\begin{array}{l}\text { Arm 2, Mean } \\
\text { (SD) }\end{array}$ & $\begin{array}{l}\text { Effect Size }(95 \% \\
\mathrm{Cl})\end{array}$ & $\begin{array}{l}\text { Reported } \\
\text { p-Value }\end{array}$ \\
\hline $\begin{array}{l}\text { Buhagiar, 2017, } \\
\text { 28291891, } \\
\text { Australia }\end{array}$ & $\begin{array}{l}\text { Hospital } \\
\text { Inpatient } \\
\text { Rehabilitation }\end{array}$ & Home Program & Moderate & $\begin{array}{l}\text { Patient } \\
\text { satisfaction } \\
\text { with care }\end{array}$ & NR & 81 & $\begin{array}{l}91.9(95 \% \\
\text { Cl 87.6, } \\
96.1)\end{array}$ & 84 & $\begin{array}{l}82.9(95 \% \mathrm{Cl} \\
78.7,87.2)\end{array}$ & $8.9(3.0,14.9)$ & NR \\
\hline $\begin{array}{l}\text { Lenssen, 2006, } \\
\text { 16942627, } \\
\text { Netherlands }\end{array}$ & $\begin{array}{l}\text { Physiotherapy } \\
\text { [twice daily(40 } \\
\text { mins/day)] }\end{array}$ & $\begin{array}{l}\text { Physiotherapy } \\
\text { [once daily(20 } \\
\text { mins/day)] }\end{array}$ & Moderate & $\begin{array}{l}\text { Patient } \\
\text { satisfaction } \\
\text { with care }\end{array}$ & $3 \mathrm{mo}$ & 21 & $8.7(1.6)$ & 22 & $9.4(0.9)$ & $-0.7(-1.5,0.15)$ & NR \\
\hline $\begin{array}{l}\text { Liebs, 2010, } \\
\text { 20360503, } \\
\text { Germany }\end{array}$ & $\begin{array}{l}\text { Ergometer } \\
\text { cycling }\end{array}$ & Control & Moderate & $\begin{array}{l}\text { Patient } \\
\text { satisfaction } \\
\text { with care }\end{array}$ & $24 \mathrm{mo}$ & 66 & $53(80 \%)^{\mathrm{B}}$ & 52 & $39(75 \%)$ & $\begin{array}{l}\text { RR: } 1.359 \text { (0.57- } \\
3.26)\end{array}$ & 0.490 \\
\hline
\end{tabular}

Statistically significant effect sizes are in bold text. In cases where calculated effect size confidence intervals were not-statistically significant but reported p-values were, we deferred to reported p-values and still bolded results.

Abbreviations: $\mathrm{CI}=$ confidence interval, $\mathrm{KOOS}=$ Knee injury and osteoarthritis outcome score, $\mathrm{mo}=$ month, $\mathrm{NR}=$ not reported, $\mathrm{PMID}=\mathrm{PubMed}$ identifier, $\mathrm{RoB}=$ risk of bias, $\mathrm{RR}=$ relative risk $\mathrm{SD}=$ standard deviation

A Time from surgery

${ }^{\mathrm{B}}$ Reported as number of patients responding (\%) ‘very satisfied' with results of TKA 
Table 36. Acute rehabilitation versus various controls for total knee arthroplasty - continuous outcomes, patient global assessment

\begin{tabular}{|c|c|c|c|c|c|c|c|c|c|c|c|}
\hline $\begin{array}{l}\text { Study, Year, } \\
\text { PMID, Country }\end{array}$ & Arm 1 & Arm 2 & $\begin{array}{l}\text { Overall } \\
\text { RoB }\end{array}$ & $\begin{array}{l}\text { Outcome } \\
\text { Measurement }\end{array}$ & $\begin{array}{l}\text { Time } \\
\text { Point }^{\mathrm{A}}\end{array}$ & N Arm & $\begin{array}{l}\text { Arm 1, Mean } \\
\text { (SD) }\end{array}$ & $\begin{array}{l}\text { N Arm } \\
2\end{array}$ & $\begin{array}{l}\text { Arm 2, Mean } \\
\text { (SD) }\end{array}$ & $\begin{array}{l}\text { Effect Size } \\
(95 \% \mathrm{Cl})\end{array}$ & $\begin{array}{l}\text { Reported } \\
\text { p-Value }\end{array}$ \\
\hline \multirow[t]{4}{*}{$\begin{array}{l}\text { Buhagiar, 2017, } \\
\text { 28291891, } \\
\text { Australia }\end{array}$} & $\begin{array}{l}\text { Hospital } \\
\text { Inpatient } \\
\text { Rehabilitation }\end{array}$ & Home Program & Moderate & KOOS (0-100) & $6.5 \mathrm{mo}$ & 79 & $\begin{array}{l}75.7(95 \% \\
\mathrm{Cl} 71.7 \\
79.9)\end{array}$ & 80 & $\begin{array}{l}73.7(95 \% \mathrm{Cl} \\
69.7,77.7)\end{array}$ & $1.99(-3.68,7.67)$ & NR \\
\hline & $\begin{array}{l}\text { Hospital } \\
\text { Inpatient } \\
\text { Rehabilitation }\end{array}$ & Home Program & Moderate & KOOS (0-100) & $12 \mathrm{mo}$ & 79 & $\begin{array}{l}76.4(95 \% \\
\mathrm{Cl} 72.4 \\
80.4)\end{array}$ & 77 & $\begin{array}{l}77(95 \% \mathrm{Cl} \\
73,81)\end{array}$ & $\begin{array}{l}-2.95(-8.74, \\
2.84)\end{array}$ & NR \\
\hline & $\begin{array}{l}\text { Hospital } \\
\text { Inpatient } \\
\text { Rehabilitation }\end{array}$ & Home Program & Moderate & EQ-5D index: 0-1 & $6.5 \mathrm{mo}$ & 79 & $\begin{array}{l}0.74(95 \% \\
\text { Cl 0.70, } \\
0.78)\end{array}$ & 80 & $\begin{array}{l}0.72(95 \% \mathrm{Cl} \\
0.68,0.77)\end{array}$ & $\begin{array}{l}-0.01(-0.07 \\
0.05)\end{array}$ & NR \\
\hline & $\begin{array}{l}\text { Hospital } \\
\text { Inpatient } \\
\text { Rehabilitation }\end{array}$ & Home Program & Moderate & EQ-5D index: 0-1 & $12 \mathrm{mo}$ & 79 & $\begin{array}{l}0.70(95 \% \\
\text { Cl } 0.66 \\
0.75)\end{array}$ & 77 & $\begin{array}{l}0.73(95 \% \mathrm{Cl} \\
0.69,0.78)\end{array}$ & $\begin{array}{l}0.02(-0.04, \\
0.08)\end{array}$ & NR \\
\hline \multirow[t]{2}{*}{$\begin{array}{l}\text { Chan, 2018, } \\
\text { 29372260, } \\
\text { Singapore }\end{array}$} & $\begin{array}{l}\text { Discharge to } \\
\text { home }\end{array}$ & $\begin{array}{l}\text { Discharge to } \\
\text { community } \\
\text { hospitals }\end{array}$ & Moderate & $\begin{array}{l}\text { Knee Society } \\
\text { Clinical Rating } \\
\text { System: Knee } \\
\text { domain }(0-100)\end{array}$ & $6 \mathrm{mo}$ & 967 & $\begin{array}{l}84.6(95 \% \\
\mathrm{Cl} 83.8 \\
85.4)\end{array}$ & 98 & $\begin{array}{l}82.2(95 \% \mathrm{Cl} \\
79.7,84.7)\end{array}$ & $-2.4(-5.1,0.2)$ & 0.0712 \\
\hline & $\begin{array}{l}\text { Discharge to } \\
\text { home }\end{array}$ & $\begin{array}{l}\text { Discharge to } \\
\text { community } \\
\text { hospitals }\end{array}$ & Moderate & $\begin{array}{l}\text { Knee Society } \\
\text { Clinical Rating } \\
\text { System: Knee } \\
\text { domain }(0-100)\end{array}$ & $24 \mathrm{mo}$ & 801 & $\begin{array}{l}85.1(95 \% \\
\mathrm{Cl} 84.2, \\
86.0)\end{array}$ & 78 & $\begin{array}{l}80.7(95 \% \mathrm{Cl} \\
77.9,83.5)\end{array}$ & $4.4(1.4,7.3)$ & 0.0035 \\
\hline \multirow{2}{*}{$\begin{array}{l}\text { den Hertog, } \\
2012, \\
22643801, \\
\text { Germany }\end{array}$} & $\begin{array}{l}\text { Fast-track } \\
\text { rehabilitation }\end{array}$ & Standard care & Moderate & $\begin{array}{l}\text { WOMAC index (0- } \\
10)\end{array}$ & $3 \mathrm{mo}$ & 74 & NR & 73 & NR & NR & 0.002 \\
\hline & $\begin{array}{l}\text { Fast-track } \\
\text { rehabilitation }\end{array}$ & Standard care & Moderate & $\begin{array}{l}\text { Knee society } \\
\text { score }(0-100)^{B}\end{array}$ & $3 \mathrm{mo}$ & 74 & NR & 73 & NR & NR & 0.0003 \\
\hline \multirow{3}{*}{$\begin{array}{l}\text { Iwakiri, } \\
2020, \\
32373475, \\
\text { Japan }\end{array}$} & ROM day 1 & ROM day 7 & High & $\begin{array}{l}\text { WOMAC: Total } \\
(0-96)^{C}\end{array}$ & $3 \mathrm{mo}$ & 55 & $19.1(16.8)$ & 54 & $15.0(10.3)$ & $-4.1(-8.0,-0.2)$ & 0.35 \\
\hline & ROM day 1 & ROM day 7 & High & $\begin{array}{l}\text { WOMAC: Total } \\
(0-96)^{C}\end{array}$ & $1 \mathrm{y}$ & 55 & $15.6(16.3)$ & 54 & $13.4(8.6)$ & $-2.2(-5.9,1.5)$ & 0.56 \\
\hline & ROM day 1 & ROM day 7 & High & $\begin{array}{l}\text { WOMAC: Total } \\
(0-96)^{C}\end{array}$ & $2 \mathrm{yrs}$ & 55 & $13.3(13.1)$ & 54 & $12.5(12.7)$ & $-0.8(-4.2,2.6)$ & 0.76 \\
\hline \multirow{2}{*}{$\begin{array}{l}\text { Jin, 2018, CN- } \\
01617489, \\
\text { China }\end{array}$} & $\begin{array}{l}\text { Virtual reality \& } \\
\text { usual care }\end{array}$ & Usual care & High & $\begin{array}{l}\text { WOMAC: Total } \\
(0-96)\end{array}$ & $3 \mathrm{mo}$ & 33 & $25.79(4.20)$ & 33 & $29.67(5.55)$ & $-3.9(-5.6,-2.2)^{\mathrm{D}}$ & 0.002 \\
\hline & $\begin{array}{l}\text { Virtual reality \& } \\
\text { usual care }\end{array}$ & Usual care & High & $\begin{array}{l}\text { WOMAC: Total } \\
(0-96)\end{array}$ & $6 \mathrm{mo}$ & 33 & $21.58(4.19)$ & 33 & $26.33(3.85)$ & $-4.8(-6.1,-3.4)^{\mathrm{D}}$ & 0 \\
\hline \multirow[t]{2}{*}{$\begin{array}{l}\text { Lenssen, 2006, } \\
\text { 16942627, } \\
\text { Netherlands }\end{array}$} & $\begin{array}{l}\text { Physiotherapy } \\
\text { [twice daily(40 } \\
\text { mins/day)] }\end{array}$ & $\begin{array}{l}\text { Physiotherapy } \\
\text { [once daily(20 } \\
\text { mins/day)] }\end{array}$ & Moderate & $\begin{array}{l}\text { WOMAC: Total } \\
(0-96)\end{array}$ & $3 \mathrm{mo}$ & 21 & $73.4(14.9)$ & 22 & $78.0(11.3)$ & $-4.6(-138,-3.9)$ & NR \\
\hline & $\begin{array}{l}\text { Physiotherapy } \\
\text { [twice daily(40 } \\
\text { mins/day)] }\end{array}$ & $\begin{array}{l}\text { Physiotherapy } \\
\text { [once daily(20 } \\
\text { mins/day)] }\end{array}$ & Moderate & $\begin{array}{l}\text { Knee society } \\
\text { score (knee) }\end{array}$ & $3 \mathrm{mo}$ & 21 & $80(17)$ & 22 & $80(18)$ & $0(-11.3,11.3)$ & NR \\
\hline $\begin{array}{l}\text { Liebs, 2012, } \\
\text { 22196125, } \\
\text { Germany }\end{array}$ & $\begin{array}{l}\text { Early Aquatic } \\
\text { therapy }\end{array}$ & $\begin{array}{l}\text { Late Aquatic } \\
\text { therapy }\end{array}$ & Moderate & SF-6D & $24 \mathrm{mo}$ & 66 & $0.721(0.119)$ & 69 & $0.703(0.135)$ & 0.14 (NR, NR) & 0.298 \\
\hline $\begin{array}{l}\text { Padgett, 2018, } \\
\text { 29352683, USA }\end{array}$ & $\begin{array}{l}\text { Inpatient } \\
\text { rehabilitation }\end{array}$ & $\begin{array}{l}\text { Discharge } \\
\text { Home }\end{array}$ & Moderate & SF-12 (0-100) & $24 \mathrm{mo}$ & 1213 & NR (NR) & 1213 & NR (NR) & NR & ns \\
\hline
\end{tabular}




\begin{tabular}{|c|c|c|c|c|c|c|c|c|c|c|c|}
\hline $\begin{array}{l}\text { Study, Year, } \\
\text { PMID, Country }\end{array}$ & Arm 1 & Arm 2 & $\begin{array}{l}\text { Overall } \\
\text { RoB }\end{array}$ & $\begin{array}{l}\text { Outcome } \\
\text { Measurement }\end{array}$ & $\begin{array}{l}\text { Time } \\
\text { Point }^{\mathrm{A}}\end{array}$ & $\begin{array}{l}\text { N Arm } \\
1\end{array}$ & $\begin{array}{l}\text { Arm 1, Mean } \\
\text { (SD) }\end{array}$ & $\begin{array}{l}\text { N Arm } \\
2\end{array}$ & $\begin{array}{l}\text { Arm 2, Mean } \\
\text { (SD) }\end{array}$ & $\begin{array}{l}\text { Effect Size } \\
(95 \% \mathrm{CI})\end{array}$ & $\begin{array}{l}\text { Reported } \\
\text { p-Value }\end{array}$ \\
\hline & $\begin{array}{l}\text { Inpatient } \\
\text { rehabilitation }\end{array}$ & $\begin{array}{l}\text { Skilled nursing } \\
\text { facility }\end{array}$ & Moderate & SF-12 (0-100) & $24 \mathrm{mo}$ & 1213 & NR (NR) & 492 & NR (NR) & NR & ns \\
\hline $\begin{array}{l}\text { Sattler, 2019, } \\
\text { 30994586, } \\
\text { Australia }\end{array}$ & $\begin{array}{l}\text { Pedaling-based } \\
\text { protocol }\end{array}$ & $\begin{array}{l}\text { Non-pedaling } \\
\text { (multi-exercise) } \\
\text { protocol }\end{array}$ & Moderate & EQ-5D (5-15) & $4 \mathrm{mo}$ & 28 & Median (6.0) & 28 & Median (7.0) & NR & NR \\
\hline $\begin{array}{l}\text { Stevens } \\
\text { Lapsley, 2012, } \\
\text { 22095207, USA }\end{array}$ & $\begin{array}{l}\text { Standard } \\
\text { rehabilitation \& } \\
\text { NMES }\end{array}$ & $\begin{array}{l}\text { Standard } \\
\text { rehabilitation }\end{array}$ & Moderate & $\begin{array}{l}\text { Global rating } \\
\text { scale of perceived } \\
\text { knee function }(0- \\
100)\end{array}$ & $12 \mathrm{mo}$ & 25 & $95.6(5.7)$ & 30 & $87.3(15)$ & $8.3(2.5,14.1)^{\mathrm{D}}$ & NR \\
\hline
\end{tabular}

Statistically significant effect sizes are in bold text. In cases where calculated effect size confidence intervals were not-statistically significant but reported p-values were, we deferred to reported p-values and still bolded results.

Abbreviations: $\mathrm{CI}=$ confidence interval, $\mathrm{EQ}-5 \mathrm{D}=$ EuroQual, $\mathrm{KOOS}=$ Knee injury and osteoarthritis outcome score, $\mathrm{mo}=$ month, $\mathrm{NMES}=$ neuromuscular electrical stimulation, $\mathrm{NR}=$ not reported, $\mathrm{ns}=$ not significant, $\mathrm{PMID}=$ PubMed identifier, $\mathrm{RoB}=$ risk of bias, $\mathrm{ROM}=$ range of motion, $\mathrm{SD}=\mathrm{standard}$ deviation, $\mathrm{ROM}=$ range of $\mathrm{motion}, \mathrm{SF}-12=12-$ item short form survey, SF-36 = 36-item short form survey, SF-6D = short-form six-dimension, WOMAC = Western Ontario and McMaster Universities Osteoarthritis Index.

A Time from surgery

B Scale combines assessment of function with pain, stability, range of motion, and muscle power and thus combines patient-reported with clinic-assessed outcomes

${ }^{C}$ Study did not report scale or interpretation for WOMAC total score. Low values are not consistent with the WOMAC score (0-96) where higher is better and may reflect the within-group change from baseline (which was also lower than expected: 38.3 [SD 20.9] for ROM Day 1 group vs. 43.7 [SD 16.1] for ROM Day 7 group).

D Calculated 


\section{Healthcare Utilization Outcomes Following Acute Rehabilitation}

Two studies (Harmer 2009 and Buhagiar, 2017) reported on healthcare-utilization outcomes following acute rehabilitation compared with various controls (Tables 37 and 38). Outcome domains included: need for postoperative procedures and other healthcare-utilization outcomes.

\section{Need for Postoperative Procedures}

Two studies (Harmer 2009 and Buhagiar 2017) reported data on the need for postoperative procedures after acute rehabilitation following TKA surgery and observed few events in which patient needed manipulation under anesthesia (MUA) to address stiff knee, with the proportions of patients requiring MUA comparable among groups (Table 37).

\section{Other Healthcare Utilization Outcomes}

Three studies reported other healthcare utilization data (Liebs 2010, Liebs 2012, and Buhagiar 2017), including the number of patients readmitted for limited ROM, time lost from work, and the number of outpatient physical therapy sessions required. Studies reported no significant differences between groups in these measures (Table 38).

Costs (indirect or direct) associated with rehabilitation programs were rarely reported. Sattler 2019 evaluated a pedaling-based protocol and reported purchasing pedals (total not reported) at a fixed cost (in the United States) of \$35 each. The pedals were loaned to trial participants who were asked to return them for future use. Buhagiar 2017 collected direct and indirect costs associated with the hospital inpatient and home-based rehabilitation programs but did not report them.

\section{Cost-Effectiveness}

We found no studies that compared the cost-effectiveness of acute rehabilitation with various comparators. Buhagiar 2017 had planned a cost-effectiveness analysis to complement their RCT of inpatient versus home rehabilitation if inpatient therapy was shown to be superior but did not conduct the analysis after findings were found to be comparable between groups with respect to patient-reported (quality of life, pain, function) and performance-based (6MWT) outcome measures.

\section{Harms From Rehabilitation}

No study reported adverse events from participating in the acute rehabilitation programs.

\section{Summary of Comparison of Acute Rehabilitation Versus Various Controls for Total Knee Arthroplasty}

Tables 39 summarize the evidence for the comparison of acute rehabilitation versus various comparators. We focus on the outcomes we prioritized in discussion with stakeholders.

There is low to insufficient SoE for all conclusions. Compared with various controls (usually less intensive active rehabilitation controls), rehabilitation in the acute phase after TKA may result in increased strength (low SoE) and comparable outcomes of pain, ROM, ADL and satisfaction with care (low SoE). There is insufficient evidence on the impact of acute rehabilitation on QoL and the need for postoperative procedures (such as manipulation under anesthesia). No studies reported evidence on harms associated with acute rehabilitation. 
Table 37. Acute rehabilitation versus various controls for total knee arthroplasty - categorical outcomes, need for postoperative procedures

\begin{tabular}{|c|c|c|c|c|c|c|c|c|c|c|c|}
\hline $\begin{array}{l}\text { Study, Year, } \\
\text { PMID, Country }\end{array}$ & Arm 1 & Arm 2 & $\begin{array}{l}\text { Overall } \\
\text { RoB }\end{array}$ & $\begin{array}{l}\text { Outcome } \\
\text { Measurement }\end{array}$ & $\begin{array}{l}\text { Time } \\
\text { Point }^{\mathrm{A}}\end{array}$ & $\begin{array}{l}\text { N Arm } \\
1\end{array}$ & $\begin{array}{l}\text { N Events } \\
\text { Arm } 2(\%)\end{array}$ & $\begin{array}{l}\text { N Arm } \\
2\end{array}$ & $\begin{array}{l}\text { N Events } \\
\text { Arm } 2(\%)\end{array}$ & $\begin{array}{l}\text { Effect Size }(95 \% \\
\text { Cl) }\end{array}$ & $\begin{array}{l}\text { Reported } \\
\text { p-Value }\end{array}$ \\
\hline $\begin{array}{l}\text { Buhagiar, 2017, } \\
\text { 28291891, } \\
\text { Australia }\end{array}$ & $\begin{array}{l}\text { Hospital } \\
\text { Inpatient } \\
\text { Rehabilitation }\end{array}$ & Home Program & Moderate & MUA & $12 \mathrm{mo}$ & 79 & $4(5 \%)$ & 77 & $3(4 \%)$ & $1.30(0.30,5.62)^{\mathrm{B}}$ & NR \\
\hline $\begin{array}{l}\text { Harmer, 2009, } \\
\text { 19177536, } \\
\text { Australia }\end{array}$ & $\begin{array}{l}\text { Water-based } \\
\text { rehabilitation }\end{array}$ & $\begin{array}{l}\text { Land-based } \\
\text { rehabilitation }\end{array}$ & Moderate & MUA & $6 \mathrm{mo}$ & 53 & $2(4 \%)$ & 49 & $2(4 \%)$ & $0.92(0.14,6.31)^{\mathrm{B}}$ & NR \\
\hline
\end{tabular}

Statistically significant effect sizes are in bold text. In cases where calculated effect size confidence intervals were not-statistically significant but reported p-values were, we

deferred to reported p-values and still bolded results.

Abbreviations: $\mathrm{CI}=$ confidence interval, $\mathrm{mo}=$ month, $\mathrm{MUA}=$ manipulation under anesthesia, $\mathrm{NR}=$ not reported, $\mathrm{PMID}=\mathrm{PubMed}$ identifier, $\mathrm{RoB}=$ risk of bias, $\mathrm{ROM}=$ range of motion.

A Time from surgery

B Calculated

Table 38. Acute rehabilitation versus various controls for total knee arthroplasty - continuous outcomes, other healthcare utilization outcomes

\begin{tabular}{|c|c|c|c|c|c|c|c|c|c|c|c|}
\hline $\begin{array}{l}\text { Study, Year, } \\
\text { PMID, Country }\end{array}$ & Arm 1 & Arm 2 & $\begin{array}{l}\text { Overall } \\
\text { RoB }\end{array}$ & $\begin{array}{l}\text { Outcome } \\
\text { Measurement }\end{array}$ & $\begin{array}{l}\text { Time } \\
\text { Point }^{A}\end{array}$ & $\begin{array}{l}\text { N Arm } \\
1\end{array}$ & $\begin{array}{l}\text { Arm 1, Mean } \\
\text { (SD) }\end{array}$ & $\begin{array}{l}\text { N Arm } \\
2\end{array}$ & $\begin{array}{l}\text { Arm 2, Mean } \\
\text { (SD) }\end{array}$ & $\begin{array}{l}\text { Effect Size } \\
(95 \% \mathrm{Cl})\end{array}$ & $\begin{array}{l}\text { Reported } \\
\text { p-Value }\end{array}$ \\
\hline \multirow[t]{2}{*}{$\begin{array}{l}\text { Buhagiar, 2017, } \\
\text { 28291891, } \\
\text { Australia }\end{array}$} & $\begin{array}{l}\text { Hospital Inpatient } \\
\text { Rehabilitation }\end{array}$ & $\begin{array}{l}\text { Home } \\
\text { Program }\end{array}$ & Moderate & $\begin{array}{l}\text { Time lost from work } \\
\text { (time to return to } \\
\text { work) (week) }\end{array}$ & $\mathrm{NA}$ & 81 & $\begin{array}{l}7.57(95 \% \mathrm{Cl} \\
4.86,10.25)\end{array}$ & 84 & $\begin{array}{l}7.80(95 \% \mathrm{Cl} \\
5.54,10.06)\end{array}$ & $\begin{array}{l}-0.23(-3.76 \\
3.30)\end{array}$ & NR \\
\hline & $\begin{array}{l}\text { Hospital Inpatient } \\
\text { Rehabilitation }\end{array}$ & $\begin{array}{l}\text { Home } \\
\text { Program }\end{array}$ & Moderate & $\begin{array}{l}\text { Outpatient physical } \\
\text { therapy sessions }\end{array}$ & $\mathrm{NA}$ & 81 & $\begin{array}{l}3.02(95 \% \mathrm{Cl} \\
2.75,3.3)\end{array}$ & 84 & $\begin{array}{l}3.07(95 \% \mathrm{Cl} \\
2.81,3.34)\end{array}$ & $\begin{array}{l}-0.05(-0.43, \\
0.33)\end{array}$ & NR \\
\hline $\begin{array}{l}\text { Liebs, 2010, } \\
\text { 20360503, } \\
\text { Germany }\end{array}$ & Ergometer cycling & Control & Moderate & $\begin{array}{l}\text { Admitted to } \\
\text { hospital for limited } \\
\text { range of motion }\end{array}$ & $24 \mathrm{mo}$ & 66 & $1(2 \%)$ & 52 & $0(0)$ & $\begin{array}{l}1.88(0.06 \\
55.02)^{\mathrm{B}}\end{array}$ & NR \\
\hline $\begin{array}{l}\text { Liebs, 2012, } \\
\text { 22196125, } \\
\text { Germany }\end{array}$ & $\begin{array}{l}\text { Early Aquatic } \\
\text { therapy }\end{array}$ & $\begin{array}{l}\text { Late Aquatic } \\
\text { therapy }\end{array}$ & Moderate & $\begin{array}{l}\text { Readmitted to } \\
\text { hospital for limited } \\
\text { range of motion }\end{array}$ & $3 \mathrm{mo}$ & 21 & $2(10 \%)$ & 22 & $1(5 \%)$ & $\begin{array}{l}2.10(0.20 \\
21.42)^{\mathrm{B}}\end{array}$ & NR \\
\hline
\end{tabular}

Statistically significant effect sizes are in bold text. In cases where calculated effect size confidence intervals were not-statistically significant but reported p-values were, we deferred to reported p-values and still bolded results.

Abbreviations: $\mathrm{CI}=$ confidence interval, $\mathrm{mo}=$ month, $\mathrm{NA}=$ not applicable, $\mathrm{NR}=$ not reported, $\mathrm{PMID}=\mathrm{PubMed}$ identifier, $\mathrm{RoB}=$ risk of bias, $\mathrm{ROM}=$ range of motion.

\footnotetext{
A Time from surgery

${ }^{B}$ Calculated
} 
Table 39. Evidence profile: Acute rehabilitation versus various controls for total knee arthroplasty

\begin{tabular}{|c|c|c|c|c|c|c|c|c|c|}
\hline $\begin{array}{l}\text { Outcome } \\
\text { Category }\end{array}$ & Outcome & $\begin{array}{l}\text { N Studies } \\
\text { (Participants) }\end{array}$ & RoB & Consistency & Precision & Directness & $\begin{array}{l}\text { Intervention } \\
\text { Replication }\end{array}$ & SoE & Conclusions \\
\hline \multirow{3}{*}{$\begin{array}{l}\text { Body structure } \\
\text { and function }\end{array}$} & Pain & $12(1115)$ & Moderate & Consistent & Precise & Direct & All unique & Low & Similar pain \\
\hline & ROM & $9(857)$ & Moderate & Consistent & Precise & Direct & All unique & Low & Similar ROM \\
\hline & Strength & $3(232)$ & Moderate & Consistent & Precise & Direct & All unique & Low & $\begin{array}{l}\text { Increased } \\
\text { strength }\end{array}$ \\
\hline $\begin{array}{l}\text { Activity and } \\
\text { participation }\end{array}$ & ADLs & $11(2055)$ & Moderate & Inconsistent & Precise & Direct & All unique & Low & Similar ADL \\
\hline \multirow[t]{2}{*}{$\begin{array}{l}\text { Other patient- } \\
\text { reported } \\
\text { outcomes }\end{array}$} & $\begin{array}{l}\text { Satisfaction } \\
\text { with care }\end{array}$ & $3(326)$ & Moderate & Inconsistent & Precise & Direct & All unique & Low & $\begin{array}{l}\text { Similar } \\
\text { satisfaction } \\
\text { with care }\end{array}$ \\
\hline & QoL & $1(78)$ & High & $\begin{array}{l}\text { Consistency } \\
\text { unknown (single } \\
\text { study) }\end{array}$ & Precise & Direct & NA (single study) & Insufficient & No conclusion \\
\hline $\begin{array}{l}\text { Healthcare } \\
\text { utilization }\end{array}$ & $\begin{array}{l}\text { Need for } \\
\text { postoperative } \\
\text { procedures }\end{array}$ & $2(258)$ & Moderate & Consistent & Precise & Direct & Both unique & Insufficient & No conclusion \\
\hline Harms & $\begin{array}{l}\text { Harms from } \\
\text { rehabilitation }\end{array}$ & 0 & $\mathrm{NA}$ & NA & NA & NA & NA & Insufficient & No evidence \\
\hline
\end{tabular}

Abbreviations: ADLs = activities of daily living, $\mathrm{QoL}=$ quality of life, $\mathrm{NA}=$ not applicable, $\mathrm{QoL}=$ quality of life, $\mathrm{RoB}=$ risk of bias, $\mathrm{ROM}=$ range of motion, $\mathrm{SoE}=$ strength of evidence. 


\section{Post-Acute Phase Rehabilitation}

There were 32 studies that evaluated post-acute phase rehabilitation in 5,484 patients (summarized in Figure 3). Post-acute phase rehabilitation interventions were typically initiated post-discharge between 2 and 8 weeks following TKA surgery. The interventions subsequently continued up to 6 months. One study defined itself as both acute and post-acute as it initiated rehabilitation 4 days after surgery but continued for 11 weeks (Bade 2017); we categorized this study as post-acute.

One study (Andersen 2018), which focused on the comparison of the setting of rehabilitation and personnel delivering it, did not provide data to code the rehabilitation interventions. We coded rehabilitation content in the remaining 30 studies. Most studies had some form of active comparison, even when described as "usual" or "standard care". Studies varied in their definitions of standard practice and in some cases usual care was no further rehabilitation (or was not reported). We were able to code rehabilitation content in 57 of the 66 arms of the 31 studies. Most rehabilitation interventions included exercises to address the goal components of strength (27/31 studies; 49/66 arms) and flexibility (25/31 studies; 44/66 arms), followed by task-specific training (21/31 studies; 34/66 arms), patient education (20/31 studies; 29/66 arms), and balancemotor-learning-agility (17/31 studies; 25/66 arms). Only half the studies included exercises targeted at aerobic endurance (15/31 studies; 24/66 arms).

We found 14 studies that included an adjunctive modality in combination with the rehabilitation program (i.e., heat, cold, NMES, TENS, biofeedback devices, dry needling, massage, mobilization, mindfulness/stress reducing activities, and complementary and alternative therapies). Of these, six RCTs were designed to evaluate the added benefit of an adjunctive therapy (NMES: 2 RCTs; biofeedback: 1 RCT; dry needling: 1 RCT; and Tai chi: 1 $\mathrm{RCT}$ ). We found 24 studies that reported some form of progression, of which 16 were assessed by clinical experts on our team as appropriate in at least one arm of the study. One study compared a high-intensity progressive rehabilitation versus low-intensity rehabilitation without progression (Bade 2017). Eight other studies had appropriate progression coded in one arm and no progression in the comparison arm (Brun-Olsen 2013, Fransen 2017, Lenguerrand 2020, Liao 2020, Madsen 2013, Bily 2016, Hamilton 2020, Piva 2019).

Post-acute rehabilitation interventions were delivered by physical therapists in 28 of the 32 studies. Four remaining studies did not report who delivered the rehabilitation intervention (Li 2015, Demircioglu 2015, Shanb 2014, Li 2019). Three studies had either additional personnel deliver one arm of the intervention (an athletic trainer for one arm in Piva 2019, a Tai Chi instructor for one arm in $\mathrm{Li} 2019$ ) or a component of the intervention in combination with the physical therapist (a psychologist delivered the cognitive-behavioral component in Cai 2018).

Most of the post-acute rehabilitation interventions were delivered to patients in-person $(n=30)$. Li 2015 had no in-person component and compared remote Patient Education (by telephone, once a month for 6 months) to promote physical activity to no Patient Education. Moutzouri 2018 compared two patient self-guided interventions intended to be performed by patients independently at home (focal sensorimotor exercise training vs. functional exercise training). Several studies had self-guided components in addition to in-person supervised rehabilitation in either or both arms or compared some form of supervised rehabilitation to selfguided rehabilitation in the comparison arm. Four studies included some form of remote rehabilitation, delivered either by telephone ( $\mathrm{n}=2$ studies), video ( $\mathrm{n}=1$ study), or Web (1 study). 
Interventions were delivered in various settings (and often, in combinations of settings). Ten studies evaluated one or both rehabilitation arms in outpatient settings exclusively (Artz 2017, Bruun-Olsen 2013, DeJong 2020, Lenguerrand 2020, Liao 2015, Bily 2016, Pua 2017, Petterson 2009, Shanb 2014, Petersen 2018). Four studies evaluated one or both rehabilitation arms at home exclusively (Li 2015, Minns Lowe, 2012, Moutzouri, 2018, Moffet, 2015). Six studies evaluated rehabilitation programs delivered both in both outpatient and home settings in one or both arms (Bade 2017, Fransen 2017, Heikkila 2017, Liao 2020 Madsen 2013, Piva 2017) and six studies evaluated rehabilitation programs that were delivered in the outpatient setting in one arm and to patients in their home in the other arm (Vuorenmaa 2014, Andersen 2018, Hamilton 2020, Mitchell 2005, Tousignant 2011, Demircioglu 2015). In one study the intervention was delivered in the acute and outpatient setting in both study arms (Schache, 2019) and one study was delivered in the acute, outpatient, and home setting in both study arms (Kauppila 2009). In one study the intervention was delivered in a non-acute inpatient facility in both study arms (Monticone 2013). In one study the intervention was delivered in a gym setting (for groupclasses) in one arm, compared with an outpatient setting in the other arm (Piva 2019). Two studies did not report the setting in which the rehabilitation was delivered (Cai 2018 and $\mathrm{Li}$ 2019).

Specific codes for intervention (and control arm, where present) goals and exercises, use of progression (and assessment of appropriateness), and details on personnel, mode of delivery, and setting are detailed in Tables 40 and 41 and Appendix C-2.2. 
Figure 3. Overview of studies of post-acute phase rehabilitation interventions for total knee arthroplasty

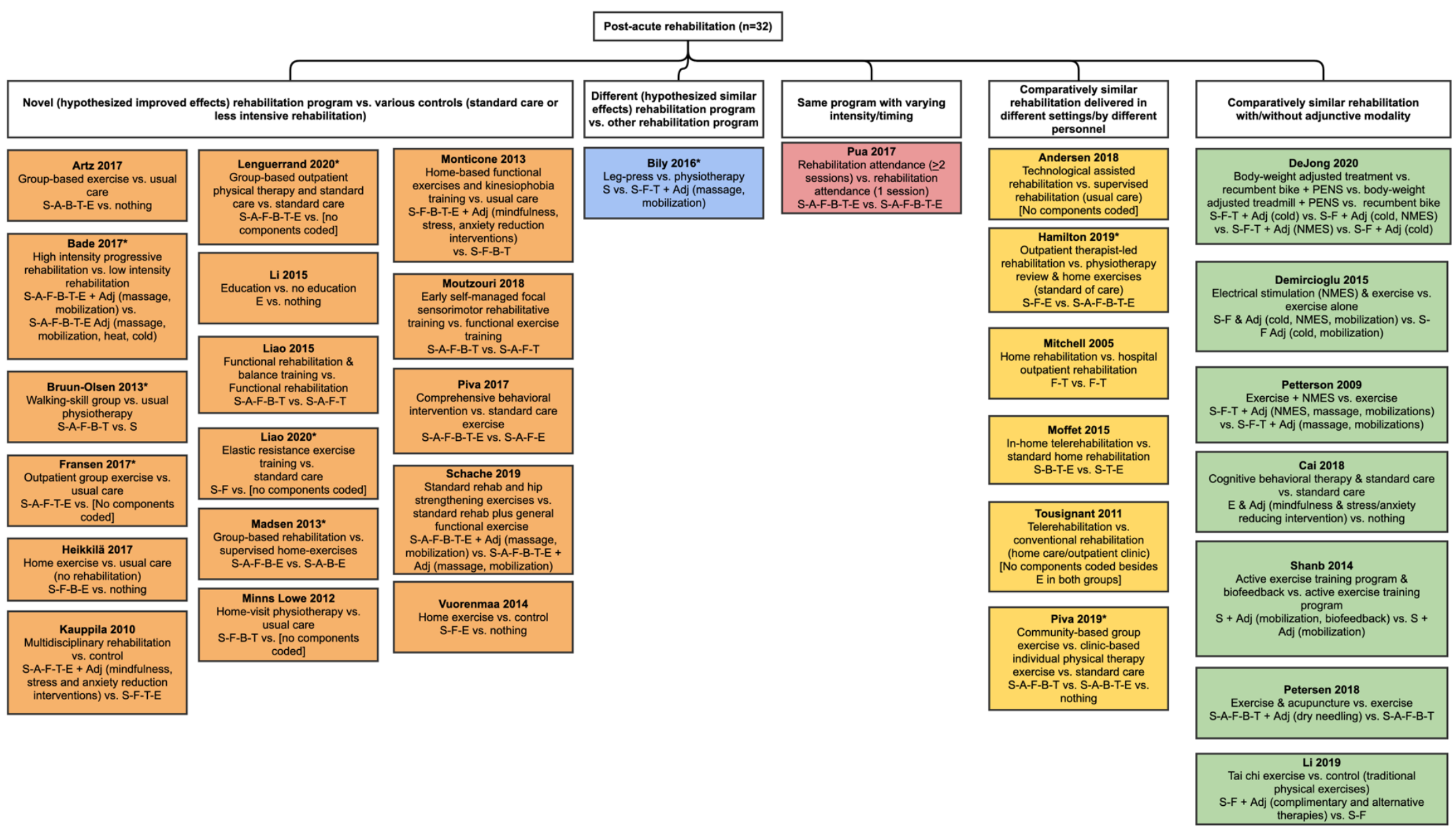

Figure presents categorization of studies ( $\mathrm{n}=32$ ) that evaluated post rehabilitation programs for TKA. The first column lists a novel (more intensive) program compared to a different program (first group hypothesized to be better); the second colunn lists a single study comparing two different rehabilitation programs hypothesized to be equivalent; the third column lists a single study with comparatively similar rehabilitation programs in both arms that were delivered with different timing or intensity (first group hypothesized to be better); the fourth column lists studies with comparatively similar rehabilitation programs delivered in different settings or by different personnel (i.e., shift in resources providing care; groups hypothesized to be comparable); the fifth column lists studies with rehabilitation interventions comparing a rehabilitation program and an adjunctive modality vs. the same rehabilitation program alone (first group hypothesized to be better). Studies are defined using arm descriptors first and component coding second. The different colors are added to visually separate the columns and do not provide unique information.

Abbreviations: Adj $=$ adjunctive, $\mathrm{A}=$ aerobic exercise, $\mathrm{B}=$ balance-motor/learning-agility exercise, $\mathrm{E}=$ patient education, $\mathrm{F}=$ flexibility exercise, $\mathrm{S}=$ strengthening exercise, $\mathrm{T}=$ task-specific training

* Intervention included progression which was deemed appropriate 
Table 40.1. Goal components strength, aerobic, and flexibility and their specific exercise components for post-acute-rehabilitation interventions (part 1 ) versus various controls for total knee arthroplasty

\begin{tabular}{|c|c|c|c|c|c|c|c|c|c|c|c|c|c|c|c|c|c|c|c|c|c|c|c|c|c|c|c|c|c|c|c|c|c|c|c|c|c|c|c|c|c|c|c|c|}
\hline $\begin{array}{l}\overline{\vec{D}} \\
\bar{\omega}\end{array}$ & hasolo & 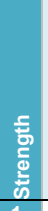 & & & 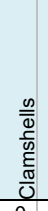 & 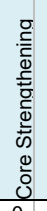 & 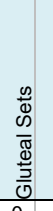 & 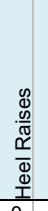 & 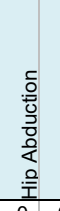 & 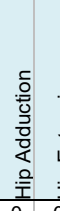 & 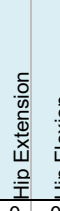 & & & 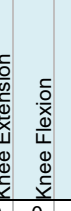 & 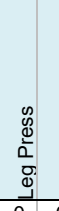 & $\begin{array}{l}0 \\
0 \\
0 \\
\\
\end{array}$ & 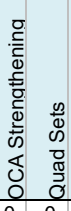 & 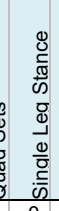 & 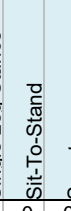 & & 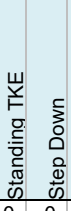 & 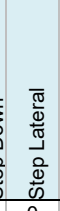 & 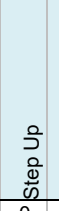 & $\begin{array}{l}\frac{n}{0} \\
0 \\
\omega \\
\overline{0} \\
i \\
i\end{array}$ & 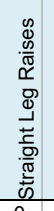 & 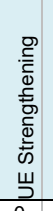 & 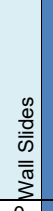 & $\begin{array}{l}\frac{2}{0} \\
\frac{0}{0} \\
\frac{1}{4}\end{array}$ & $\begin{array}{l}0 \\
\frac{3}{0} \\
\frac{\pi}{2} \\
\frac{3}{4}\end{array}$ & 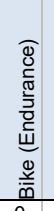 & 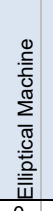 & $\begin{array}{l}\text { o. } \\
\text { o. } \\
\text { is }\end{array}$ & 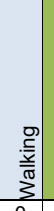 & $\begin{array}{l}\frac{\overline{\bar{z}}}{\overline{\frac{0}{\alpha}}} \\
\frac{0}{\bar{l}} \\
\end{array}$ & 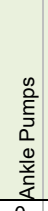 & 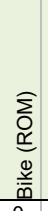 & 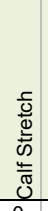 & 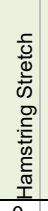 & 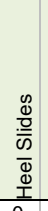 & 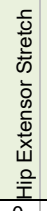 & 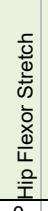 & 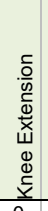 & 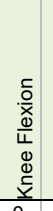 & 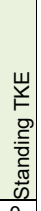 \\
\hline Bade, 2017 & \begin{tabular}{|l} 
HI prog rehab \\
Hill
\end{tabular} & $\frac{0}{1}$ & 0 & 0 & $\frac{0}{1}$ & 0 & $\frac{0}{1}$ & $\frac{0}{1}$ & $\begin{array}{l}0 \\
1\end{array}$ & $\begin{array}{cc}0 & 0 \\
1 & 1\end{array}$ & $\begin{array}{ll}0 & 0 \\
1 & 1\end{array}$ & $\begin{array}{ll}0 & 0 \\
1 & 0\end{array}$ & $\begin{array}{ll}0 & 0 \\
0 & 1\end{array}$ & $\begin{array}{l}0 \\
1\end{array}$ & \begin{tabular}{l|l}
0 & \\
1
\end{tabular} & $\begin{array}{ll}0 & 0 \\
1 & 0\end{array}$ & $\begin{array}{c}0 \\
0\end{array}$ & $\begin{array}{l}0 \\
0\end{array}$ & $\begin{array}{cc}0 & 0 \\
1 & 1\end{array}$ & $\begin{array}{ll}0 & 0 \\
1 & 0\end{array}$ & $\begin{array}{ll}0 & 0 \\
0 & 1\end{array}$ & 0 & $\frac{0}{1}$ & 0 & $\frac{0}{1}$ & $\begin{array}{l}0 \\
0\end{array}$ & $\frac{0}{1}$ & $\frac{0}{1}$ & $\begin{array}{l}0 \\
0\end{array}$ & $\frac{0}{1}$ & $\begin{array}{l}0 \\
0\end{array}$ & $\begin{array}{l}0 \\
0\end{array}$ & $\frac{0}{1}$ & $\frac{0}{1}$ & $\frac{0}{1}$ & $\frac{0}{1}$ & 1 & $\frac{0}{1}$ & $\frac{0}{1}$ & $\begin{array}{l}0 \\
0\end{array}$ & 0 & $\frac{0}{1}$ & $\frac{0}{1}$ & 0 \\
\hline & & - & 0 & 0 & 1 & 0 & 1 & 1 & 0 & 0 & & 0 & $0_{0}+2$ & 1 & 0 & 0 & 1 & 1 & 1 & & 10 & & & & 1 & 0 & 0 & 1 & 0 & 1 & 0 & 0 & 1 & 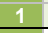 & 1 & 1 & 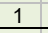 & 1 & 1 & 0 & 0 & & 1 & \\
\hline Bruun-Olsen, 2013 & Wa & 1 & 0 & 0 & 0 & 0 & 0 & 0 & 0 & $0 \quad 0$ & \begin{tabular}{l|l}
0 & 0 \\
\end{tabular} & $\begin{array}{l}0 \\
\end{array}$ & 0 & 0 & 0 & 1 & \begin{tabular}{l|l|}
0 & 0 \\
\end{tabular} & 1 & $\begin{array}{ll}11 \\
\end{array}$ & $\overline{0}$ & $\begin{array}{ll}0 & 1 \\
\end{array}$ & & & & 0 & 0 & 0 & 1 & 0 & 0 & 0 & 0 & 1 & 1 & 0 & 0 & 1 & 1 & 0 & 0 & & & & \\
\hline & Usual p p & 1 & 0 & 0 & 0 & 0 & 0 & 0 & 0 & $0 \quad 0$ & $\begin{array}{ll}0 & 0 \\
\end{array}$ & $\begin{array}{ll}0 & 0 \\
\end{array}$ & 0 & 0 & 0 & $\begin{array}{ll}0 & 0 \\
\end{array}$ & \begin{tabular}{l|l}
0 & 0 \\
\end{tabular} & 0 & $\begin{array}{ll}0 & 0 \\
\end{array}$ & 0 & $\begin{array}{ll}0 & 0 \\
\end{array}$ & 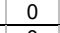 & 0 & 0 & 0 & 0 & 0 & 0 & 0 & 0 & 0 & 0 & 0 & 1 & 0 & 0 & 0 & 0 & 0 & 0 & 0 & r & 0 & \\
\hline Cai, 2018 & |CBT for kinesiop & 0 & 0 & 0 & 0 & 0 & 0 & 0 & 0 & 0 & 0 & 0 & b & 1 & 0 & 0 & \begin{tabular}{l|l}
0 & 0 \\
\end{tabular} & 0 & $\begin{array}{ll}0 & 0 \\
0\end{array}$ & 0 & $\begin{array}{ll}0 & 0 \\
\end{array}$ & 0 & & & 0 & 0 & 0 & 0 & 0 & 0 & 0 & 0 & 0 & 0 & 0 & 0 & 0 & 0 & 0 & 0 & 0 & 0 & 0 & \\
\hline & Stan & 0 & 0 & 0 & 0 & 0 & 0 & 0 & 0 & 0 & 0 & 0 & & 0 & 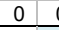 & 0 & \begin{tabular}{l|l}
0 & 0 \\
\end{tabular} & n & 0 & 0 & $0=0$ & 0 & & & 0 & 0 & 0 & 0 & 0 & 0 & 0 & 0 & 0 & 0 & 0 & 0 & 0 & 0 & 0 & 0 & 0 & 0 & 0 & \\
\hline Fransen, 2017 & |O- 9 & 1 & 1 & 0 & 0 & 0 & 0 & 1 & 1 & 0 & 100 & $\begin{array}{l}0 \\
0\end{array}$ & 1 & 0 & 0 & 0 & \begin{tabular}{l|l|}
0 & 0 \\
\end{tabular} & 0 & 1 & & 0 & & & & & R & 1 & 1 & 0 & 1 & 0 & 0 & & & 0 & & & & & & & & & \\
\hline & Usu & 0 & 0 & 0 & 0 & 0 & 0 & 0 & 0 & c & $\begin{array}{ll}0 & 0 \\
& 0\end{array}$ & $\begin{array}{ll}0 & 0 \\
\end{array}$ & 0 & $\Omega^{2}+2$ & 0 & 0 & \begin{tabular}{l|l}
0 & 0 \\
\end{tabular} & 0 & 0 & 0 & 0 & 0 & & & & 0 & 0 & 0 & 0 & 0 & & 0 & 0 & 0 & 0 & & 1 & 0 & & & & & & \\
\hline Heikkila, 2017 & Exerci & 1 & 0 & 0 & 0 & 0 & 0 & & 0 & 0 & \begin{tabular}{l|l}
0 & 0 \\
\end{tabular} & $\begin{array}{ll}0 & 0 \\
& 0\end{array}$ & 0 & 1 & 0 & 0 & \begin{tabular}{l|l}
0 & 1 \\
\end{tabular} & 0 & 11 & 1 & $\begin{array}{ll}0 & 0 \\
\end{array}$ & 1 & & & & & 1 & 0 & 0 & 0 & 0 & 0 & & 1 & 0 & 1 & 1 & 1 & 0 & 0 & 1 & 0 & 0 & \\
\hline & Co & 0 & 0 & 0 & 0 & 0 & 0 & 0 & 0 & 0 & \begin{tabular}{l|l}
0 & 0 \\
\end{tabular} & 0 & 5 & 0 & 0 & 0 & \begin{tabular}{l|l|}
0 & 0 \\
\end{tabular} & 0 & 0 & 0 & $\begin{array}{ll}0 & 0 \\
\end{array}$ & 0 & & & & 0 & 0 & 0 & 0 & 0 & 0 & 0 & 0 & 0 & 0 & 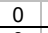 & 0 & 0 & 0 & 5 & 0 & 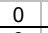 & 0 & \\
\hline Kauppila, 2009 & Mul & 1 & 0 & 0 & 0 & 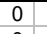 & 0 & & 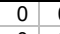 & & ( & 0 & & & & & 0 & 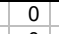 & 0 & & 0 & 0 & & & & & 0 & 1 & 1 & 0 & 0 & 0 & 1 & 1 & 0 & 0 & 0 & 0 & 0 & 0 & & & & \\
\hline & & 1 & & 0 & & 0 & & & & & & & & & & & 0 & & & & & & & & & & & 0 & & & & & & & & & & & & & & & & \\
\hline Lenguerrand, 2020 & Grp- & 1 & 0 & 0 & 0 & 0 & 0 & 0 & 0 & 0 & \begin{tabular}{l|l}
0 & 0 \\
\end{tabular} & 0 & 1 & 1 & 0 & $\overline{0}$ & 0 & 0 & & 0 & 0 & ( & & & & & & 1 & & & & & 1 & 1 & 0 & 1 & & 1 & c & & & & & \\
\hline & Us & 0 & 0 & 0 & 0 & 0 & 0 & & 0 & 0 & $\begin{array}{ll}0 & 0 \\
\end{array}$ & 0 & 0 & 0 & 0 & 0 & 0 & 0 & 0 & 0 & $\begin{array}{ll}0 & 0 \\
\end{array}$ & 0 & & & & & & 0 & 0 & 0 & & & 0 & 0 & 0 & 0 & 0 & 0 & 0 & & & & 0 & \\
\hline Li, 2015 & Ed & 0 & 0 & 0 & 0 & 0 & 0 & 0 & 0 & 0 & 0 & 0 & 5 & 0 & 0 & 0 & 0 & 0 & $\begin{array}{ll}0 & 0 \\
\end{array}$ & 0 & $\begin{array}{ll}0 & 0 \\
\end{array}$ & 0 & & & 0 & 0 & 0 & 0 & 0 & 0 & & 0 & 0 & 0 & 0 & 0 & 0 & 0 & 0 & 0 & 0 & 0 & ces & \\
\hline & Non & 0 & 0 & 0 & 0 & 0 & 0 & 0 & 0 & 0 & $\begin{array}{ll}0 & 0 \\
\end{array}$ & 0 & 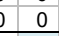 & 0 & 0 & 0 & 0 & 0 & $\begin{array}{ll}0 & 0 \\
\end{array}$ & 0 & 0 & 0 & & & & 0 & 0 & 0 & 0 & 0 & & 0 & 50 & 0 & 0 & 0 & 0 & 2 & 0 & 0 & 0 & 0 & ces & \\
\hline & & 1 & 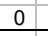 & 0 & 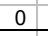 & 2 & 0 & & 1 & 2 & & & & 1 & 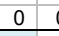 & 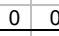 & 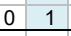 & 0 & & & & & & & & & & 1 & & & & & & 1 & & & & & & & & & & \\
\hline Liao, 2020 & El & 1 & 0 & 0 & 0 & 0 & 0 & & 1 & 1 & 1 & 0 & 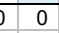 & 1 & 1 & 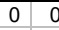 & 0 & 0 & 5 & 0 & 0 & c & & & & 1 & 0 & 0 & 0 & & & 0 & 0 & $0^{\circ}+2$ & 0 & 0 & 0 & 0 & & & & & 0 & \\
\hline & & 0 & 0 & 0 & 0 & 0 & 0 & & 0 & & \begin{tabular}{l|l}
0 & 0 \\
\end{tabular} & 0 & & -1 & 0 & 0 & 0 & 0 & \begin{tabular}{l|l}
0 & 0 \\
\end{tabular} & 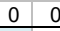 & 0 & c & & & & & 0 & 0 & & & & & 0 & & 0 & & 0 & 0 & 0 & & & & & \\
\hline Madsen, 2013 & Grou & 1 & 0 & 0 & 0 & 1 & 0 & 0 & 0 & $\begin{array}{lll}0 & 0\end{array}$ & \begin{tabular}{l|l}
0 & 0 \\
\end{tabular} & $\begin{array}{ll}0 & 0\end{array}$ & 51 & 1 & $\begin{array}{ll}1 & \\
\end{array}$ & \begin{tabular}{l|l}
0 & 0 \\
\end{tabular} & \begin{tabular}{l|l}
0 & 0 \\
\end{tabular} & 0 & $\begin{array}{ll}0 & 1 \\
\end{array}$ & 1 & $\begin{array}{ll}0 & 0 \\
\end{array}$ & 0 & 0 & 0 & 0 & 1 & 0 & 1 & 0 & 1 & 0 & 0 & 1 & 1 & 0 & 1 & 0 & 0 & 0 & 0 & 0 & 0 & 0 & \\
\hline & & 1 & 0 & 0 & 0 & 0 & 0 & 0 & 0 & 0 & \begin{tabular}{l|l}
0 & 0
\end{tabular} & $\begin{array}{ll}0 & 0\end{array}$ & 0 & 0 & 0 & 0 & \begin{tabular}{l|l}
0 & 0
\end{tabular} & 0 & 00 & 0 & $\begin{array}{ll}0 & 0\end{array}$ & 0 & 0 & 0 & 0 & 0 & 0 & 1 & 0 & 1 & 0 & 0 & 1 & 0 & 0 & 0 & 0 & 0 & 0 & 0 & 0 & 0 & 0 & \\
\hline Minns Lowe, 2012 & Home- & 1 & 0 & 0 & 0 & 0 & 0 & 1 & 0 & 0 & \begin{tabular}{l|l}
0 & 0 \\
\end{tabular} & $\begin{array}{l}0 \\
0\end{array}$ & 0 & 0 & 0 & 0 & 0 & 1 & 1 & 1 & 0 & 0 & 1 & 0 & 0 & 0 & 1 & 0 & 0 & 0 & 0 & 0 & 0 & 1 & 0 & 0 & 1 & 1 & 0 & 0 & 0 & 0 & 1 & \\
\hline & & 0 & 0 & 0 & 0 & 0 & 0 & & 0 & & 0 & 0 & & 0 & s & c & 0 & 0 & & 0 & 0 & c & & & & & & 0 & & & & & 0 & 0 & $\underline{n}$ & & & $=0$ & & & & & 0 & \\
\hline Monticone, 2013 & Ex & 1 & 0 & 0 & & 0 & & & & & 0 & 0 & 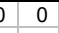 & & & 0 & 0 & 5 & & & 0 & c & & & 0 & 0 & 0 & 0 & 0 & 0 & 0 & 0 & & & 0 & 1 & 0 & 0 & 0 & 0 & 0 & 0 & 0 & \\
\hline & & 1 & 0 & 0 & 0 & 0 & 0 & 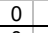 & 0 & 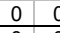 & $\begin{array}{ll}0 & 0 \\
\end{array}$ & $\underline{0}$ & 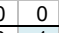 & & 0 & 0 & \begin{tabular}{l|l}
0 & 0 \\
\end{tabular} & 0 & $\begin{array}{ll}0 & 0 \\
\end{array}$ & 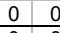 & 0 & c & & & & 0 & 0 & 0 & 0 & & & 0 & 0 & 1 & 0 & & & 0 & & 0 & 0 & 0 & 0 & \\
\hline Moutzouri, 2018 & FSE & 1 & 0 & 0 & 0 & 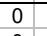 & Th & 0 & S & 0 & 0 & 0 & 1 & 0 & 0 & 0 & & & & & n & $\pi$ & & & & & & & & & & & & & & & 0 & & & & & & & \\
\hline & & & 0 & 0 & & 0 & & & & & & & & & & & & & & & & & & & & & & & & & & & & & & & & & & & & & & \\
\hline Piva, 2017 & CE & $=$ & 0 & 0 & 0 & 0 & 0 & 0 & 1 & 0 & 1 & 0 & 1 & 1 & 0 & 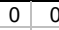 & 0 & 0 & 1 & 1 & 0 & $\mathrm{c}$ & & 0 & 0 & 0 & 0 & 1 & 0 & 0 & 5 & 5 & 1 & 1 & 0 & 1 & 1 & 1 & 0 & 0 & 0 & 1 & 1 & \\
\hline & & 1 & 0 & 0 & 0 & 0 & 0 & 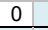 & 1 & 0 & 10 & 0 & _t & _t & 0 & 0 & \begin{tabular}{l|l|}
0 & 0 \\
\end{tabular} & 0 & \begin{tabular}{l|l}
0 & 0 \\
\end{tabular} & 0 & $\begin{array}{ll}0 & 0 \\
\end{array}$ & 0 & & 0 & 0 & 0 & 0 & & 0 & 0 & 0 & 0 & 1 & & 0 & 1 & 1 & 1 & 0 & 0 & 0 & 1 & 1 & \\
\hline Schache, 2019 & Stand & 1 & 0 & 0 & 0 & 0 & 0 & 1 & 1 & 01 & \begin{tabular}{l|l}
1 & 0 \\
\end{tabular} & $\begin{array}{ll}0 & 1 \\
\end{array}$ & 10 & 1 & 1 & \begin{tabular}{l|l}
0 & 0 \\
\end{tabular} & \begin{tabular}{l|l|}
0 & 1 \\
\end{tabular} & 0 & \begin{tabular}{l|l}
0 & 1 \\
\end{tabular} & 10 & $\begin{array}{ll}0 & 1 \\
\end{array}$ & 1 & 1 & 0 & 0 & 0 & 0 & 1 & 0 & 1 & 0 & 0 & 0 & 1 & 0 & 0 & 1 & 0 & 0 & 1 & 0 & 1 & 1 & \\
\hline & eral & 1 & 0 & 0 & 0 & 0 & 0 & 1 & 0 & 0 & \begin{tabular}{l|l}
0 & 0
\end{tabular} & $\begin{array}{ll}0 & 0\end{array}$ & 0 & 1 & 1 & \begin{tabular}{l|l}
0 & 0
\end{tabular} & \begin{tabular}{|l|l}
0 & 1
\end{tabular} & 0 & \begin{tabular}{l|l}
0 & 1
\end{tabular} & 10 & \begin{tabular}{l|l}
0 & 1
\end{tabular} & 0 & 1 & 0 & 0 & 0 & 0 & 1 & 0 & 1 & 0 & 0 & 0 & 1 & 0 & 0 & 1 & 0 & 0 & 1 & 0 & 1 & 1 & \\
\hline orenmaa, 2014 & & 1 & 0 & 0 & 0 & 0 & 0 & 1 & 0 & 0 & 0 & 0 & 1 & 1 & 0 & 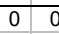 & 0 & 0 & 1 & 1 & 0 & $c$ & 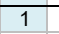 & & 0 & & 1 & & & & 0 & 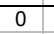 & 0 & 1 & 0 & 1 & 1 & 1 & $c$ & 0 & & & & \\
\hline & Contr & 0 & 0 & 0 & 0 & 0 & 0 & & 0 & 0 & \begin{tabular}{l|l}
0 & 0 \\
\end{tabular} & $\begin{array}{ll}0 & 0 \\
\end{array}$ & $\begin{array}{l}0 \\
0\end{array}$ & 0 & 0 & 0 & \begin{tabular}{l|l}
0 & 0 \\
\end{tabular} & 0 & \begin{tabular}{l|l}
0 & 0 \\
\end{tabular} & $\begin{array}{ll}0 & 0 \\
\end{array}$ & $\begin{array}{ll}0 & 0 \\
\end{array}$ & 0 & 0 & 0 & 0 & 0 & 0 & 0 & 0 & 0 & 0 & 0 & & & & 0 & & & 0 & 0 & 0 & 0 & 0 & \\
\hline
\end{tabular}

This table represents the first half of the studies assessing post-acute rehabilitation interventions (part 1). All studies in this table assessed novel interventions hypothesized to improve effects compared with controls. See Tables 41 . and 41.2 for remainder of the studies assessing post-acute rehabilitation interventions (part 2). See
for part 1 studies. The color is added for visual display and does not provide unique information.

$1=$ presence of component, $0=$ absence of component

Abbreviations. $\mathrm{BT}=$ balance training, $\mathrm{CBI}=$ comprehensive behavioral intervention, $\mathrm{ed}=$ education, ex $=$ exercise, $\mathrm{fxn}=$ function, fxtl $=$ functional, $\mathrm{FSET}=$ focal sensorimotor exercise training, grp $=$ group, $\mathrm{HAT}=$ hip abduction training, $\mathrm{HI}=$ high intensity, $\mathrm{LI}=$ low intensity, $\mathrm{O}=$ outpatient, $\mathrm{PA}=$ physical activity, physio = physiotherapy, rehab = rehabilitation, $\mathrm{ROM}=$ range of motion, $\mathrm{TKE}=$ terminal $\mathrm{knee}$ extension 
Table 40.2. Goal components balance-motor-learning-agility, task specific training, patient education, and adjunctive modalities and their specific exercise components for post-acute-rehabilitation interventions (part 1) versus various controls for total knee arthroplasty

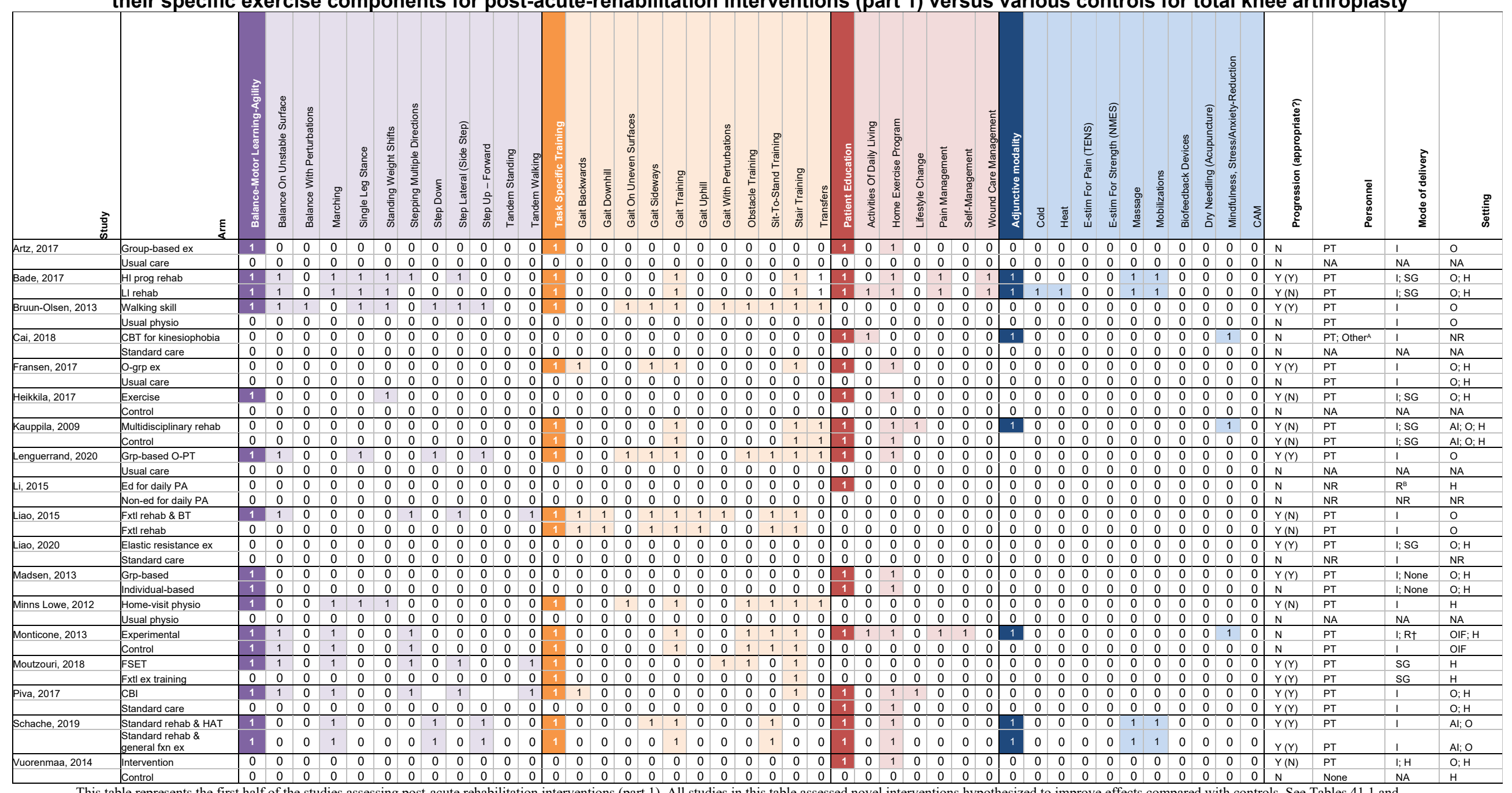

This table represents the first half of the studies assessing post-acute rehabilitation interventions (part 1). All studies in this table assessed novel interventions hypothesized to improve effects compared with controls. See Tables 41.1 and 41.2 for remainder of the studies assessing post-acute rehabilitation interventions (part 2). See Table 40.1 for goal components strength, aerobic, and flexibility for part 1 studies. The color is added for visual display and does not provid unique information. The color is added for visual display and does not provide unique information. $1=$ presence of component, $0=$ absence of component 
Abbreviations: $\mathrm{AI}=$ acute in-patient; $\mathrm{BT}=$ balance training, $\mathrm{CBI}=$ comprehensive behavioral intervention, $\mathrm{ex}=$ exercise, $\mathrm{fxn}=$ function, fxtl $=$ functional,, $\mathrm{FSET}=$ focal sensorimotor exercise training, grp $=$ group, $\mathrm{HAT}=$ hip abduction training, $\mathrm{HI}=$ high intensity, $\mathrm{H}=$ home, $\mathrm{I}=$ in-person, $\mathrm{G}=$ gym/other community center, $\mathrm{NA}=$ not applicable, $\mathrm{NMES}=$ neuromuscular electrical stimulation, $\mathrm{NR}=$ not reported, $\mathrm{O}=$ outpatient physiotherapy center; OIF $=$ other inpatient facility, PENS = patterned electrical neuromuscular stimulation, $\mathrm{PT}=$ physical therapist $\mathrm{R}=$ remote; rehab $=$ rehabilitation; $\mathrm{SG}=$ self-guided; tele $=$ telephone; TENS $=$ transcutaneous electrical nerve stimulation, video $=$ videoconference

A Psychologist

${ }^{\mathrm{B}}$ Remote via telephone

Table 41.1. Goal components strength, aerobic, and flexibility and their specific exercise components for post-acute-rehabilitation interventions (part 2) versus various controls for total knee arthroplasty

\begin{tabular}{|c|c|c|c|c|c|c|c|c|c|c|c|c|c|c|c|c|c|c|c|c|c|c|c|c|c|c|c|c|c|c|c|c|c|c|c|c|c|c|c|c|c|c|c|c|c|c|}
\hline $\begin{array}{l}\overrightarrow{2} \\
\overrightarrow{0}\end{array}$ & $\frac{\xi}{4}$ & & & 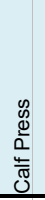 & $\begin{array}{l}\frac{\omega}{\overline{0}} \\
\frac{5}{\omega} \\
\frac{5}{0} \\
\frac{\omega}{0} \\
\end{array}$ & 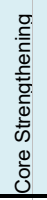 & $\begin{array}{l}\frac{n}{0} \\
\infty \\
\frac{1}{\pi} \\
\frac{\Phi}{10} \\
\frac{3}{0} \\
\end{array}$ & 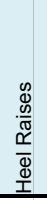 & 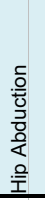 & 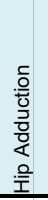 & 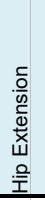 & 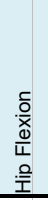 & 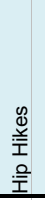 & 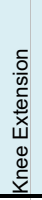 & 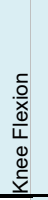 & 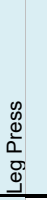 & 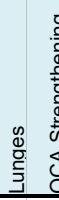 & 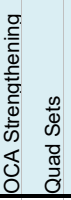 & 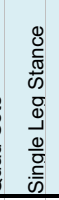 & 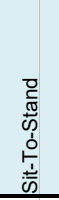 & $\begin{array}{l}\frac{n}{0} \\
\frac{\infty}{\tilde{\omega}} \\
\stackrel{0}{\infty} \\
\end{array}$ & 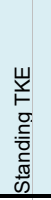 & 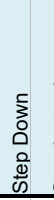 & 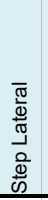 & 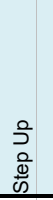 & 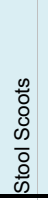 & 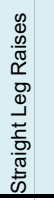 & 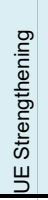 & $\begin{array}{l}\frac{\infty}{0} \\
\frac{0}{0} \\
\frac{5}{\bar{w}} \\
3 \\
\end{array}$ & $\begin{array}{l}\frac{0}{0} \\
\frac{0}{0} \\
\end{array}$ & $\begin{array}{l}\stackrel{0}{0} \\
\stackrel{0}{\frac{\pi}{3}} \\
\frac{3}{0} \\
\end{array}$ & 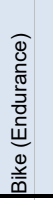 & 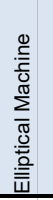 & 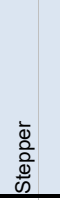 & $\begin{array}{r}\frac{0}{5} \\
\frac{5}{\pi / 0} \\
3 \\
\end{array}$ & $\begin{array}{l}\frac{1}{\overline{0}} \\
\frac{0}{\frac{0}{2}} \\
\frac{0}{11} \\
\end{array}$ & 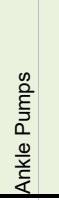 & $\begin{array}{l}\widehat{\Sigma} \\
\underline{0} \\
\underline{\underline{x}} \\
\stackrel{\Xi}{\mathbf{m}} \\
\overline{\mathbf{m}}\end{array}$ & 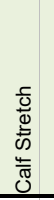 & 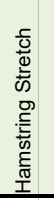 & 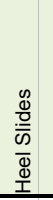 & 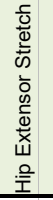 & 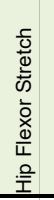 & 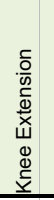 & 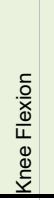 & 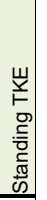 \\
\hline Bily, 2016 & Leg press group & 1 & 0 & 0 & 0 & 0 & $\overline{0}$ & 0 & $\overline{0}$ & 0 & 0 & 0 & 0 & 0 & 0 & 1 & \begin{tabular}{l|l}
0 & 0 \\
\end{tabular} & $\begin{array}{ll}0 & 0 \\
\end{array}$ & 0 & 0 & 0 & 0 & 0 & 0 & 0 & 0 & 0 & 0 & 0 & 0 & 0 & 0 & 0 & 0 & 0 & 0 & 0 & 0 & 0 & 0 & 0 & 0 & 0 & 0 & 0 & 0 \\
\hline Pua, $2017^{8}$ & $\frac{p h}{>2}$ & $\frac{1}{1}$ & 0 & 0 & $\frac{0}{0}$ & 0 & $\frac{0}{n}$ & $\frac{0}{0}$ & $\frac{1}{0}$ & 0 & 0 & 0 & $\frac{0}{n}$ & $\frac{1}{1}$ & $\frac{1}{n}$ & $\frac{0}{1}$ & $\begin{array}{ll}0 & 0 \\
0 & 0\end{array}$ & $\begin{array}{ll}0 & 1 \\
0 & 1 \\
\end{array}$ & $\frac{0}{0}$ & 0 & 1 & 0 & 0 & 0 & 1 & 0 & 1 & 0 & 0 & $\frac{0}{1}$ & 0 & 0 & 0 & 0 & 0 & & 0 & 1 & 0 & 0 & 0 & 0 & & 0 & 0 & \\
\hline rua, $20 T^{\circ}$ & $\begin{array}{l}\text { 2 renab session } \\
1 \text { rehab session }\end{array}$ & 1 & 0 & 0 & 0 & 0 & 0 & 0 & 0 & 0 & 0 & 0 & 0 & 1 & 0 & 1 & $\begin{array}{ll}0 & 0 \\
0 & 0\end{array}$ & $\begin{array}{cc}0 & 1 \\
0 & 1\end{array}$ & 0 & 1 & 0 & 0 & 1 & 0 & 1 & 0 & 1 & 0 & 1 & 1 & 0 & 0 & 0 & 1 & 0 & & 0 & 1 & 0 & 1 & 0 & 0 & 0 & 1 & 1 & \\
\hline & No rehabilitation attendance & 0 & 0 & 0 & 0 & 0 & 0 & 0 & 0 & 0 & 0 & 0 & 0 & 0 & 0 & 0 & $0 \quad 0$ & $\begin{array}{ll}0 & 0 \\
0\end{array}$ & 0 & 0 & 0 & 0 & 0 & 0 & & & 0 & 0 & 0 & 0 & 0 & 0 & 0 & 0 & 0 & 0 & 0 & 0 & 0 & 0 & 0 & 0 & 0 & 0 & 0 & \\
\hline & Supervi & $\frac{0}{1}$ & $\frac{0}{0}$ & $\frac{0}{0}$ & $\begin{array}{l}0 \\
0\end{array}$ & 0 & $\frac{0}{0}$ & 0 & $\begin{array}{l}0 \\
0\end{array}$ & 0 & 0 & $\begin{array}{l}0 \\
0\end{array}$ & 0 & $\begin{array}{l}0 \\
1\end{array}$ & 0 & $\frac{0}{1}$ & \begin{tabular}{l|l}
0 & 0 \\
0 & 0
\end{tabular} & $\begin{array}{ll}0 & 0 \\
0 & 1\end{array}$ & $\frac{0}{0}$ & $\begin{array}{l}0 \\
0\end{array}$ & 0 & $\frac{0}{0}$ & $\frac{0}{0}$ & 0 & & 0 & $\frac{0}{1}$ & 0 & 0 & 1 & 0 & 0 & 0 & $\frac{0}{0}$ & & 0 & $\frac{0}{0}$ & 0 & 0 & 0 & $\frac{0}{0}$ & $\frac{0}{0}$ & $\frac{0}{0}$ & 0 & $\frac{0}{1}$ & \\
\hline & $\begin{array}{l}\text { Pupartithnt therapisit-led renab } \\
\text { Physio review home exercises }\end{array}$ & 1 & 0 & 0 & 0 & 0 & 0 & & 0 & 0 & 0 & & 0 & 0 & 0 & & $\begin{array}{ll}0 & 0 \\
0\end{array}$ & $\begin{array}{ll}0 & 0 \\
0\end{array}$ & 0 & 0 & 0 & 0 & 0 & 0 & & 0 & 0 & 0 & 0 & 0 & 0 & 0 & 0 & 0 & 0 & & 0 & 0 & 0 & 0 & 0 & 0 & 0 & 0 & 0 & \\
\hline Mitchell, $2005^{\mathrm{C}}$ & Home & 0 & 0 & 0 & 0 & 0 & 0 & 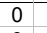 & 0 & 0 & 0 & 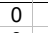 & 0 & 0 & 0 & 0 & 0 & $\begin{array}{ll}0 & 0 \\
\end{array}$ & 0 & 0 & R & 0 & 0 & 0 & 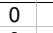 & 0 & 0 & 0 & 0 & 0 & 0 & 0 & 0 & 0 & 0 & 1 & 0 & 0 & 0 & 0 & 0 & 0 & 0 & 1 & 1 & \\
\hline & Outpatient & 0 & 0 & 0 & 0 & 0 & 0 & 0 & 0 & 0 & & 0 & & 0 & 0 & 0 & 0 & $\begin{array}{ll}0 & 0 \\
\end{array}$ & 0 & 0 & & 0 & & & & & 0 & 0 & 0 & 0 & 0 & 0 & 0 & 0 & 0 & & 0 & 0 & 0 & 0 & 0 & 0 & 0 & 1 & 1 & \\
\hline Moffet, $2015^{\circ}$ & Telerehabilitation & 1 & 0 & 0 & 0 & 0 & & 0 & 0 & 0 & 0 & 0 & 0 & 0 & 0 & 0 & 0 & $\begin{array}{ll}0 & 0 \\
\end{array}$ & 0 & 0 & 0 & 0 & 0 & & & 0 & 0 & 0 & 0 & 0 & 0 & 0 & 0 & 0 & 0 & 0 & 0 & 0 & 0 & 0 & 0 & 0 & 0 & 0 & 0 & \\
\hline & & 1 & 0 & 0 & 0 & 0 & & 0 & 0 & 0 & 0 & 0 & 0 & 0 & 0 & & 0 & 0 & 0 & 0 & 0 & 0 & & & & & & & & 0 & 0 & & & u & 0 & 0 & 0 & 0 & 0 & 0 & 0 & 0 & 0 & 0 & 0 & \\
\hline Tousignant, $2011^{\mathrm{C}}$ & & 0 & 0 & 0 & 0 & 0 & 0 & 5 & 0 & 0 & 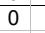 & 0 & & 0 & 0 & 0 & 0 & 0 & 0 & 0 & 0 & 0 & & & & & & & & 0 & 0 & & & & 0 & 0 & 0 & 0 & 0 & 0 & 0 & 0 & 0 & 0 & 0 & \\
\hline & & 0 & 0 & 0 & 0 & 0 & & & & & & & & 0 & & & & & & & & & & & & & & & & & & & & & & 0 & & & & & & & & & & \\
\hline Piva, $2019^{\mathrm{c}}$ & Clinic-bas & 1 & 0 & 0 & 0 & 0 & 0 & 0 & 0 & 0 & 1 & 1 & 0 & 1 & 1 & 0 & 0 & 0 & 0 & 0 & 1 & 0 & & & & & & & & & & & & & & 0 & 0 & & & & & & 0 & & & \\
\hline & grp exercise & 1 & 0 & 0 & 0 & 0 & 0 & 0 & 0 & 0 & 0 & 0 & 0 & 0 & 0 & 0 & 0 & $0 \quad 0$ & 0 & 0 & 0 & 0 & 0 & 0 & & & & & 0 & & 0 & 0 & & 0 & 0 & & 0 & 0 & 0 & 0 & 0 & 0 & 0 & 0 & 0 & \\
\hline & Usual car & 0 & 0 & 0 & 0 & 0 & & 0 & 0 & 0 & 0 & 0 & 0 & 0 & 0 & 0 & 0 & 0 & 0 & 0 & 0 & 0 & 0 & & & & & & 0 & 0 & 0 & & & 0 & 0 & 0 & 0 & 0 & 0 & 0 & 0 & 0 & 0 & 0 & 0 & \\
\hline DeJong, $2020^{\circ}$ & Body-w & 1 & 0 & 0 & 0 & 0 & 0 & 0 & 0 & 0 & 0 & 0 & $\pi$ & 0 & 0 & 0 & \begin{tabular}{l|l}
0 & 0 \\
\end{tabular} & \begin{tabular}{l|l}
0 & 0
\end{tabular} & 0 & 0 & 0 & 0 & 0 & 0 & 0 & 0 & 0 & 0 & 0 & 0 & 0 & 0 & 0 & 0 & 0 & - & 0 & 0 & 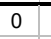 & 0 & 0 & 0 & 0 & 0 & 0 & \\
\hline & djusted Treadmill \& & 1 & 0 & 0 & 0 & 0 & 0 & 0 & 0 & 0 & 0 & 0 & 0 & 0 & 0 & 0 & 0 & $\begin{array}{ll}0 & 0\end{array}$ & 0 & 0 & 0 & 0 & 0 & 0 & 0 & 0 & 0 & 0 & 0 & 0 & 0 & 0 & 0 & 0 & 0 & 1 & 0 & 0 & 0 & 0 & 0 & 0 & 0 & 0 & 0 & \\
\hline & & 1 & 0 & 0 & 0 & 0 & 0 & 0 & 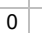 & 0 & 0 & 0 & 0 & 0 & 0 & 0 & 0 & 0 & 0 & 0 & 0 & 0 & $\underline{0}$ & 0 & $\underline{0}$ & 0 & $\underline{0}$ & 0 & 0 & 0 & 0 & 0 & 0 & 0 & 0 & & 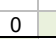 & 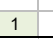 & 0 & 0 & 0 & 0 & 0 & 0 & 0 & \\
\hline Demircioglu, 2015 & MES & $\because$ & 0 & 0 & 0 & 0 & 0 & 0 & 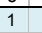 & 1 & 0 & & 0 & 1 & 0 & 0 & r & 1 & 0 & & 0 & & 0 & & & & & & & & 0 & 0 & 0 & 0 & 0 & & 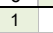 & 0 & 0 & 0 & 0 & 0 & 0 & 1 & 1 & \\
\hline & & 1 & 0 & 0 & & 0 & & 0 & & 1 & 0 & 0 & 0 & 1 & 0 & 0 & 0 & 1 & 0 & 0 & 0 & 1 & 0 & 0 & & & & 0 & 0 & 0 & 0 & 0 & 0 & 0 & 0 & & 1 & 0 & 0 & 0 & 0 & 0 & 0 & 1 & 1 & \\
\hline Petterson, $2009^{\circ}$ & Ex & 1 & 0 & 0 & 0 & 0 & 0 & 0 & 1 & 0 & 0 & 0 & 0 & 1 & 1 & 0 & 1 & $\begin{array}{lll}0 & 1 \\
\end{array}$ & 0 & 0 & 0 & 1 & 1 & & & 0 & 1 & 0 & 1 & 0 & 0 & 0 & 0 & 0 & 0 & & 0 & 1 & 0 & 0 & 0 & 0 & 0 & 1 & 1 & \\
\hline & Exercis & 1 & 0 & 0 & 0 & 0 & 0 & 0 & 1 & 0 & 0 & 0 & 0 & 1 & 1 & 0 & 1 & $\begin{array}{ll}0 & 1 \\
\end{array}$ & 0 & 0 & 0 & 1 & 1 & 0 & & 0 & & 0 & 1 & 0 & 0 & 0 & 0 & 0 & 0 & & 0 & 1 & 0 & 0 & 0 & 0 & 0 & 1 & 1 & \\
\hline Shanb, $2014^{\circ}$ & & -5 & 0 & 0 & 0 & 0 & & 0 & 1 & 0 & 0 & 0 & 0 & 1 & 1 & 0 & ( & ( & 0 & 0 & 年 & 1 & 0 & & & & & 0 & 0 & 0 & 0 & 0 & 0 & 0 & & 0 & 0 & 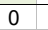 & 0 & 0 & 0 & 0 & 0 & 0 & 0 & \\
\hline & & $e^{2}$ & 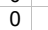 & 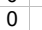 & 0 & 0 & 0 & 0 & & 0 & 0 & & 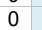 & & 1 & 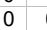 & & 1 & 0 & 0 & & & & & & & & & 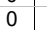 & 0 & & & 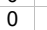 & & 0 & 0 & & & & & & & & & & \\
\hline Petersen, $2018^{\circ}$ & & 1 & 0 & 0 & 0 & 0 & 0 & 0 & 0 & 0 & 0 & 0 & 0 & 0 & 0 & 0 & 0 & 0 & 0 & 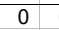 & 0 & 0 & 0 & & & & & & & & 0 & & & 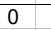 & 0 & & 0 & 0 & 0 & & & & & & & \\
\hline & & & 0 & 0 & 0 & 0 & 0 & 0 & & 0 & & & & 0 & 0 & & 0 & 0 & 0 & & & & & & & & & & & & 0 & & & 0 & 0 & & 0 & 0 & 0 & 0 & 0 & 0 & 0 & 0 & 0 & \\
\hline Li, 2019 & Sta & 1 & 0 & 5 & 0 & 0 & 0 & 0 & 0 & 0 & 0 & 0 & $\overline{0}$ & 0 & 0 & 0 & 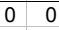 & 0 & 0 & 0 & 0 & & 0 & & & & & & 0 & 0 & 0 & 0 & 0 & 0 & 0 & & 0 & 0 & 0 & 0 & 1 & 0 & 0 & 0 & 0 & \\
\hline & Tai chi & 1 & 0 & 0 & 0 & 0 & 0 & 0 & 0 & 0 & 0 & 0 & 0 & 0 & 0 & 0 & $\begin{array}{ll}0 & 0 \\
\end{array}$ & $\begin{array}{ll}0 & 1 \\
\end{array}$ & 0 & 0 & 0 & 0 & 0 & 0 & 0 & 0 & 1 & 0 & 0 & 0 & 0 & 0 & 0 & 0 & 0 & & 0 & 0 & 0 & 0 & 1 & 0 & 0 & 0 & 0 & 0 \\
\hline
\end{tabular}

41.2 for goal components balance-motor-learning-agility, task specific training, patient education, and adjunctive modalities for part 2 studies. The color is added for visual display and does not provide unique information.

$1=$ presence of component, $0=$ absence of component 
Abbreviations: NMES $=$ neuromuscular electrical stimulation, rehab $=$ rehabilitation, $\mathrm{ROM}=$ range of motion, $\mathrm{PT}=$ physiotherapy, $\mathrm{TKE}=$ terminal knee extension.

${ }^{\text {A }}$ Different rehabilitation program (hypothesized similar)

${ }^{\mathrm{B}}$ Similar rehabilitation with varying intensity/timing

Similar rehabilitation with/without adjuvant modality

Table 41.2. Goal components balance-motor-learning-agility, task specific training, patient education, and adjunctive modalities and their specific exercise components for post-acute-rehabilitation interventions (part 2) versus various controls for total knee arthroplasty

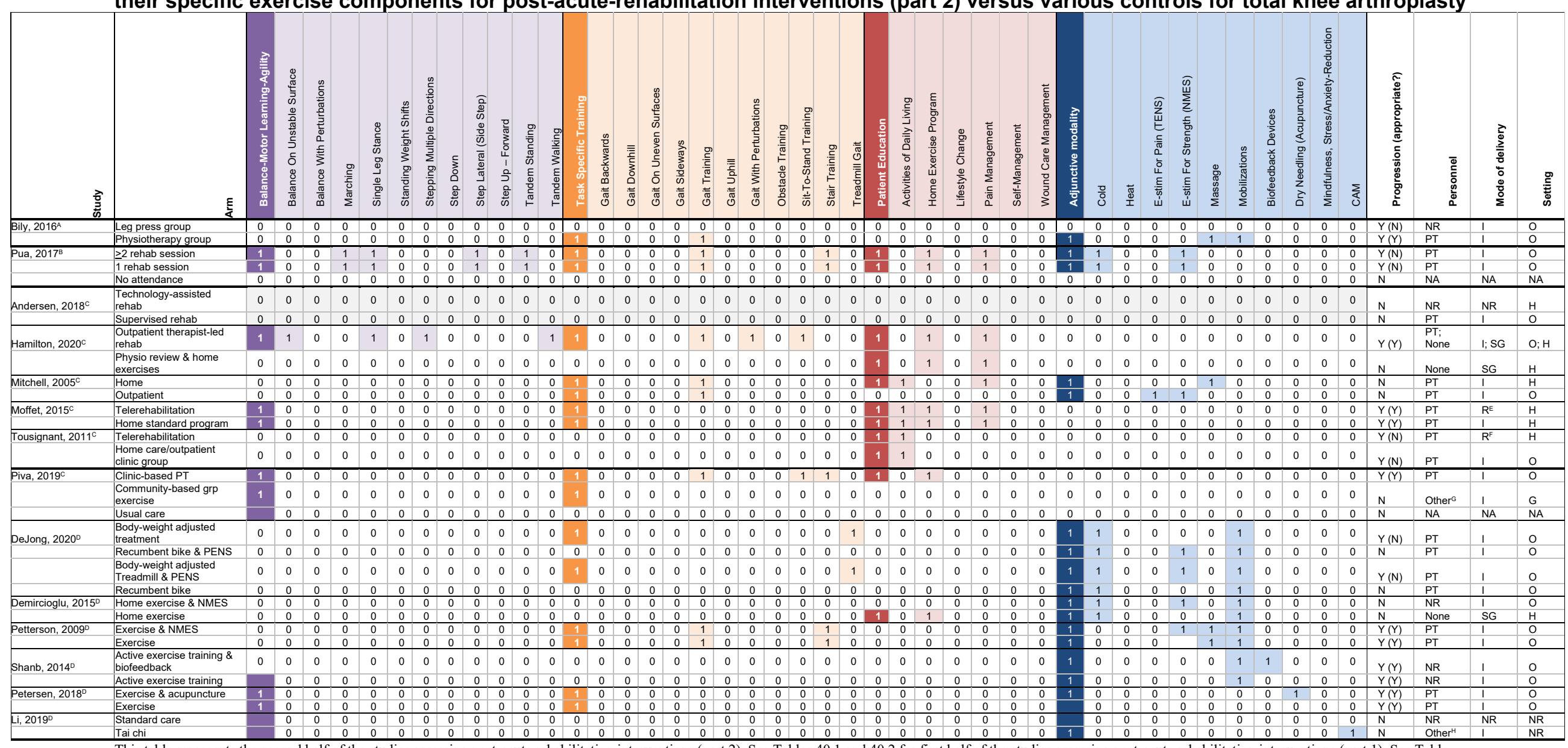

This table represents the second half of the studies assessing post-acute rehabilitation interventions (part 2). See Tables 40.1 and 40.2 for first half of the studies assessing post-acute rehabilitation interventions (part 1). See Table 41.2 for goal components balance-motor-learning-agility, task specific training, patient education, and adjunctive modalities for part 2 studies. The color is added for visual display and does not provide unique information. The color is added for visual display and does not provide unique information. 


\section{$1=$ presence of component, $2=$ absence of component}

Abbreviations: $\mathrm{AI}=$ acute in-patient; $\mathrm{CAM}=$ complementary and alternative therapies, $\mathrm{I}=$ in-person; $\mathrm{G}=$ gym $/$ other community center; $\mathrm{H}=$ home; $\mathrm{NA}=$ not applicable, $\mathrm{NR}=$ not reported, $\mathrm{NMES}=$ neuromuscular electrical stimulation, $\mathrm{O}=$ outpatient physiotherapy center, $\mathrm{OIF}=$ other inpatient facility, $\mathrm{PT}=$ physical therapist, $\mathrm{R}=$ remote; rehab $=$ rehabilitation, $\mathrm{SG}=$ self-guided; tele = telephone; TENS $=$ transcutaneous electrical nerve stimulation

${ }^{\AA}$ Different rehabilitation program (hypothesized similar)

${ }^{B}$ Similar rehabilitation with varying intensity/timing

${ }^{\mathrm{C}}$ Similar rehabilitation delivered in different setting/by different personnel

${ }^{\mathrm{D}}$ Similar rehabilitation with/without adjuvant modality

${ }^{\mathrm{E}}$ Remote via video

G Athletic trainer

${ }^{\mathrm{H}}$ Tai Chi instructors 


\section{Body Structure and Function Outcomes Following Post-Acute Rehabilitation}

We found 26 studies that reported on body structure and function outcomes following postacute rehabilitation, with various comparators: 17 studies (Artz 2017, Bade 2017, Bruun-Olsen 2013, Cai 2018, Fransen 2017, Heikkilä 2017, Kauppila 2010, Lenguerrand 2020, Li 2015, Liao 2015, Liao 220, Madsen 2013, Minns Lowe 2012, Monticone 2013, Moutzouri 2018, Schache 2019, Vuorenmaa 2014) compared novel rehabilitation programs with various comparators (less intensive rehabilitation or no care); one study (Bily 2016) compared different (hypothesized equivalent) rehabilitation programs, no studies compared rehabilitation programs with different timing and/or intensity; four studies (Andersen 2018, Mitchell 2005, Moffet 2015, Tousignant 2011) compared rehabilitation programs delivered in different settings or by different personnel, and five studies (Demircioglu 2015, Li 2019, Petterson 2009, Peterson 2018, Shanb 2014) compared rehabilitation programs with or without an adjunctive modality (Tables 42 to 47). The outcome domains included: symptoms, pain, range of motion, muscle strength, energy and vigor, and emotional functioning.

\section{Symptoms}

Eleven studies (Artz 2017, Bruun-Olsen 2013, Lenguerrand 2020, Minns Lowe 2012, Monticone 2013, Schache 2019, Mitchell 2005, Moffet 2015, DeJong 2020, Demircioglu 2015, Petersen 2018) reported data on symptoms using the stiffness component of the WOMAC and the symptoms component of the KOOS (Table 42). Most ( $=9$ ) studies observed no differences between groups at follow-up ranging from 3 to 12 months after TKA. Monticone 2013 reported symptoms using the symptoms component of the KOOS (0-100, larger is better) and found that patients randomized to home-based functional exercises and kinesiophobia training reported experiencing fewer symptoms than patients randomized to usual care (a less intensive rehabilitation exercise program without kinesiophobia training) at 6 and 12 months after TKA. Demircioglu 2015 reported symptoms using the stiffness component of the WOMAC (0-100, larger is worse) and found that patients randomized to exercise and adjunctive NMES reported experiencing fewer symptoms than patients randomized to exercise alone at 3 months after TKA (MD $-5.0,95 \%$ CI -9.5 to -0.5 ).

\section{Pain}

We found pain data reported in 22 studies (Artz 2017, Cai 2018, Bruun-Olsen 2013, Fransen 2017, Heikkilä 2017, Lenguerrand 2020, Li 2015, Liao 2015, Liao 2020, Minns Lowe 2012, Monticone 2013, Moutzouri 2018, Schache 2019, Vuorenmaa 2014, Bily 2016, Mitchell 2005, Moffet 2015, DeJong 2020, Demircioglu 2015, Li 2019, Petersen 2018, Petterson 2009) (Table 43), using six different outcome measures assessed between 3 and 12 months after surgery. Ten of the 13 studies that compared a novel (hypothesized better) rehabilitation program with various comparators found no difference in pain measures between groups. In three studies (Liao 2020, Monticone 2013, Moutzouri 2018) the novel rehabilitation program was associated with significant reductions in pain. Liao 2020 reported pain data using the pain component of the WOMAC (0-20; lower is better) and found that patients randomized to elastic resistance exercise training reported significantly reduced pain compared with patients randomized to standard care (conservative physical therapy without any resistance training) $(\mathrm{p}=0.001)$ at 4 months after TKA. Monticone 2013 reported pain data using the pain component of the KOOS (0-100; higher is 
better) and a VAS (0-10; lower is better) and found that patients randomized to home-based functional exercises and kinesiophobia training reported significantly reduced pain at 6 and 12 months after TKA on both KOOS (12 months MD: 9.6, 95\% CI 4.6 to 14.5) and VAS (12 months MD: $-1.0,95 \% \mathrm{CI}-1.6$ to -0.5$)$ measures. Moutzouri 2018 reported pain data using a VAS (0-10, lower is better) and found that patients randomized to early self-managed focal sensorimotor exercise training reported significantly reduced pain compared with those randomized to function exercise training at 3.5 months after TKA (MD -1.7, 95\% CI -2 to $-1.4)$.

Bily 2016 (evaluating two rehabilitation programs hypothesized to be equivalent) found no difference between patients randomized to the leg press group compared with patients randomized to the physiotherapy group at 3 months follow-up.

Two studies assessed the impact of the setting of rehabilitation on measures of pain (Mitchell 2005: hospital vs. home; Moffet 2015: in-home telerehabilitation versus standard rehabilitation) and found no differences between groups at 3 and 4 months after TKA, respectively.

Finally, three of the four studies that assessed the impact of different rehabilitation programs with and without adjunctive therapy found no difference between compared groups. Two studies (Cai 2018 and Demircioglu 2015) reported reduced pain in the adjunctive therapy groups. Cai 2018 reported pain data using two measures: the VAS (0-10, lower is better) and the Pain Catastrophizing Scale (0-52, lower is better) and found that patients randomized to the cognitive behavior therapy and standard care arm reported reduced pain (net mean difference [NMD] $-0.57,95 \% \mathrm{CI}-0.9$ to -0.2 ) and pain catastrophizing (NMD $-7.7,95 \% \mathrm{CI}-9.3$ to -6.1 ) at 6 months after TKA. Demircioglu 2015 reported pain data using the pain component of the WOMAC (0-20 but appeared to convert to 0-100, smaller is better) compared exercise with NMES to exercise alone found reduced pain among patients who received the adjunctive NMES (MD $-5.7,95 \% \mathrm{CI}-11.3$ to -0.1 ).

\section{Range of Motion}

We found 15 studies (Bade 2017, Bruun-Olsen 2013, Fransen 2017, Kauppila 2010, Madsen 2013, Moutzouri 2018, Schache 2019, Vuorenmaa 2014, Bily 2016, Demircioglu 2015, Li 2019, Petterson 2009, Andersen 2018, Moffet 2015, Tousignant 2011) that reported range of motion (ROM) data from various outcome measures, including active and passive knee ROM for extension and flexion of the knee joint (Table 44). In several cases, whether the measure was active or passive ROM was not specified. Where reported, studies measured ROM in degrees using goniometry. Most studies ( $\mathrm{n}=13$ ) reported comparable ROM between arms at follow-up measured between 3 and 12 months after TKA across the various interventions and comparisons. Moutzouri 2018 showed significant improvements in active knee ROM flexion among patients randomized to early self-managed focal sensorimotor exercise training compared with patients randomized to functional exercise training. Li 2019 found significant differences between groups: extension ROM, but not flexion ROM, (whether active or passive not specified) was significantly increased among patients randomized to Tai Chi compared with patients randomized to rehabilitation at 3 months after TKA.

\section{Muscle Strength}

We found 14 studies (Bade, 2017, Bruun-Olsen, 2013, Fransen, 2017, Heikkilä, 2017, Kauppila, 2010, Madsen, 2013, Minns Lowe, 2012, Moutzouri, 2018, Schache, 2019, Vuorenmaa, 2014, Bily, 2016, Moffet, 2015, Petterson, 2009, Shanb, 2014) reported strength 
data from various outcome measures including isometric and isokinetic knee extension and knee flexion strength, and quadricep and hamstring strength and torque, among others (Table 45).

Studies typically used a dynamometer to measure strength and presented strength data variably (Newtons [N], kilograms [kg], torque normalized to body weight $[\mathrm{Nm} / \mathrm{kg}]$ and others; for each, higher values indicate greater strength). One study reported strength using a composite scale, the Index of Muscle Function designed to assess strength and function.

Seven of the 10 studies that compared patients randomized to a novel (hypothesized better) rehabilitation program with various comparators found no difference in strength measures between groups. In three studies (Heikkilä, 2017, Moutzouri 2018, and Vuorenmaa 2014) the novel rehabilitation program was associated with significant increases in strength (in at least one measure reported). Heikkilä 2017 reported patients randomized to home-based rehabilitation showed increased knee extension (MD 70, 95\% CI 33 to 108) and knee flexion strength (MD 30, 95\% CI 17 to 43 ) compared with patients randomized to usual care (no additional rehabilitation after discharge) at 14 months after TKA. Moutzouri 2018 reported that patients randomized to early self-managed focal sensorimotor exercise training had improved peak force $(\mathrm{N})$ compared with patients randomized to functional exercise training (MD 12.1, 95\% CI 3.9 to 20.2).

Vuorenmaa 2014 reported patients randomized to home exercise had significantly improved isometric knee flexion (but not extension) (MD NR; $\mathrm{P}=0.009$ ) compared with patients randomized to control (no additional guidance after baseline measurements). Of the remaining four studies (that compared rehabilitation in different settings or with or without an adjunctive modality) only one study (Shanb 2014) reported improved strength. Shanb 2014 reported patients randomized to active exercise training and biofeedback had significantly improved performance of quadricep isometric peak torque at 4 months following surgery $(\mathrm{P}=0.01)$

\section{Energy and Vigor}

One study (Mitchell 2005) reported on vigor using the vitality component of the SF-36 (scores 0 to 100, higher is better) (Table 46) and found no significant differences between groups at 3 months after surgery.

\section{Emotional Functioning}

Eight studies (Bade 2017, Fransen 2017, Schache 2019, Vuorenmaa 2014, Mitchell 2005, Demircioglu 2015, Li 2019, Petterson 2009) reported on emotional functioning data from the mental health, emotional role functioning and social functioning component scales of the SF-36 (scores 0 to 100 , higher is better) (Table 47). Most studies $(n=7)$ reported comparable results among groups on measures of emotional functioning between 3 and 12 months after TKA. One study (Li 2009) reported significant improvements in mental health among patients randomized to adjunctive Tai Chi compared with rehabilitation alone at 3 months after TKA $(p=0.03)$. 
Table 42. Post-acute rehabilitation versus various controls for total knee arthroplasty - continuous outcomes, symptoms

\begin{tabular}{|c|c|c|c|c|c|c|c|c|c|c|c|}
\hline $\begin{array}{l}\text { Study, Year, } \\
\text { PMID, Country }\end{array}$ & Arm 1 & Arm 2 & $\begin{array}{l}\text { Overall } \\
\text { RoB }\end{array}$ & $\begin{array}{l}\text { Outcome } \\
\text { Measurement }\end{array}$ & $\begin{array}{l}\text { Time } \\
\text { Point }^{\mathrm{A}}\end{array}$ & N Arm & $\begin{array}{l}\text { Arm 1, Mean } \\
\text { (SD) }\end{array}$ & $\begin{array}{l}\text { N Arm } \\
2\end{array}$ & $\begin{array}{l}\text { Arm 2, Mean } \\
\text { (SD) }\end{array}$ & $\begin{array}{l}\text { Effect Size } \\
(95 \% \mathrm{Cl})\end{array}$ & $\begin{array}{l}\text { Reported } \\
\text { p-Value }\end{array}$ \\
\hline \multirow[t]{2}{*}{$\begin{array}{l}\text { Artz, 2017, } \\
27068368, U K\end{array}$} & $\begin{array}{l}\text { Group-based } \\
\text { exercise }\end{array}$ & Usual care & High & $\begin{array}{l}\text { KOOS: } \\
\text { Symptoms (0- } \\
100)\end{array}$ & $3 \mathrm{mo}$ & 19 & $59.6(16.4)$ & 12 & $54.8(16.9)$ & $4.8(-3.9,13.5)^{\mathrm{B}}$ & NR \\
\hline & $\begin{array}{l}\text { Group-based } \\
\text { exercise }\end{array}$ & Usual care & High & $\begin{array}{l}\text { KOOS: } \\
\text { Symptoms (0- } \\
100)\end{array}$ & $6 \mathrm{mo}$ & 21 & $58.4(18.9)$ & 15 & $56.7(14.3)$ & $1.7(-6,9.4)^{\mathrm{B}}$ & NR \\
\hline $\begin{array}{l}\text { Bruun-Olsen, } \\
\text { 2013, 23614370, } \\
\text { Norway }\end{array}$ & $\begin{array}{l}\text { Walking-skill } \\
\text { group }\end{array}$ & $\begin{array}{l}21 \text { individual } \\
\text { physiotherapy } \\
\text { sessions }\end{array}$ & Moderate & $\begin{array}{l}\text { KOOS: } \\
\text { Symptoms (0- } \\
100)\end{array}$ & $9 \mathrm{mo}$ & 29 & $52(18)$ & 28 & $73(21)$ & $\operatorname{adj} 2(-9,13)$ & NR \\
\hline \multirow[t]{3}{*}{$\begin{array}{l}\text { DeJong, } \\
2020 \\
32360105 \\
\text { USA }\end{array}$} & $\begin{array}{l}\text { Body Weight- } \\
\text { Adjustable } \\
\text { Treadmill \& } \\
\text { PENS }\end{array}$ & $\begin{array}{l}\text { Recumbent } \\
\text { Bike \& PENS }\end{array}$ & High & $\begin{array}{l}\text { KOOS: } \\
\text { Symptoms (0- } \\
100)\end{array}$ & $6 \mathrm{mo}$ & 70 & $84.3(18.7)$ & 78 & $81.1(16.2)$ & $-3.2(-7.2,0.8)$ & NR \\
\hline & $\begin{array}{l}\text { Body Weight- } \\
\text { Adjustable } \\
\text { Treadmill \& } \\
\text { PENS }\end{array}$ & $\begin{array}{l}\text { Body Weight- } \\
\text { Adjustable } \\
\text { Treadmill }\end{array}$ & High & $\begin{array}{l}\text { KOOS: } \\
\text { Symptoms (0- } \\
100)\end{array}$ & $6 \mathrm{mo}$ & 70 & $84.3(18.7)$ & 76 & $83.3(19.8)$ & $-1.0(-5.5,3.4)$ & NR \\
\hline & $\begin{array}{l}\text { Body Weight- } \\
\text { Adjustable } \\
\text { Treadmill \& } \\
\text { PENS }\end{array}$ & $\begin{array}{l}\text { Recumbent } \\
\text { Bike/Usual } \\
\text { Care }\end{array}$ & High & $\begin{array}{l}\text { KOOS: } \\
\text { Symptoms (0- } \\
100)\end{array}$ & $6 \mathrm{mo}$ & 70 & $84.3(18.7)$ & 74 & $87.0(12.2)$ & $2.7(-1.1,6.5)$ & NR \\
\hline $\begin{array}{l}\text { Demircioglu, 2015, } \\
\text { 26355656, Turkey }\end{array}$ & NMES \& exercise & Exercise & High & $\begin{array}{l}\text { WOMAC: } \\
\text { Stiffness }(0-100)\end{array}$ & $3 \mathrm{mo}$ & 30 & $42.9(12.6)$ & 30 & $47.9(12.3)$ & $-5(-9.5,-0.5)^{B}$ & NR \\
\hline $\begin{array}{l}\text { Lenguerrand, } \\
2020,31033232 \text {, } \\
\text { UK }\end{array}$ & $\begin{array}{l}\text { Physical therapy } \\
\text { and standard } \\
\text { care }\end{array}$ & Standard care & Moderate & $\begin{array}{l}\text { KOOS: } \\
\text { Symptoms (0- } \\
100)\end{array}$ & $12 \mathrm{mo}$ & 74 & $77(17)$ & 64 & $77(20)$ & $0(-4.5,45)^{\mathrm{B}}$ & 0.377 \\
\hline \multirow[t]{3}{*}{$\begin{array}{l}\text { Minns Lowe, } \\
\text { 2012, 22180446, } \\
\text { UK }\end{array}$} & $\begin{array}{l}\text { Home-visit } \\
\text { physiotherapy }\end{array}$ & Usual care & High & $\begin{array}{l}\text { KOOS: } \\
\text { Symptoms (0- } \\
100)\end{array}$ & $3 \mathrm{mo}$ & 46 & $\begin{array}{l}\text { Media (IQR) } \\
67.9(29)\end{array}$ & 47 & $\begin{array}{l}\text { Media (IQR) } \\
71.4(29)\end{array}$ & NR & NR \\
\hline & $\begin{array}{l}\text { Home-visit } \\
\text { physiotherapy }\end{array}$ & Usual care & High & $\begin{array}{l}\text { KOOS: } \\
\text { Symptoms (0- } \\
100)\end{array}$ & $6 \mathrm{mo}$ & 42 & $\begin{array}{l}\text { Media (IQR) } \\
76.8(21)\end{array}$ & 44 & $\begin{array}{l}\text { Media (IQR) } \\
71.4(29)\end{array}$ & NR & NR \\
\hline & $\begin{array}{l}\text { Home-visit } \\
\text { physiotherapy }\end{array}$ & Usual care & High & $\begin{array}{l}\text { KOOS: } \\
\text { Symptoms (0- } \\
100)\end{array}$ & $12 \mathrm{mo}$ & 44 & $\begin{array}{l}\text { Media (IQR) } \\
82.1(18)\end{array}$ & 48 & $\begin{array}{l}\text { Media (IQR) } \\
78.8(31)\end{array}$ & NR & NR \\
\hline $\begin{array}{l}\text { Mitchell, 2005, } \\
\text { 15869558, UK }\end{array}$ & Hospital & Home & High & $\begin{array}{l}\text { WOMAC: } \\
\text { Stiffness }(0-100)\end{array}$ & $4 \mathrm{mo}$ & 57 & $3.6(2.1)$ & 57 & $3.5(1.4)$ & $\begin{array}{l}\text { Adj MD -0.2 (- } \\
0.9,0.4)\end{array}$ & 0.496 \\
\hline \multirow[t]{2}{*}{$\begin{array}{l}\text { Moffet, 2015, } \\
26178888, \\
\text { Canada }\end{array}$} & $\begin{array}{l}\text { In-home } \\
\text { Telerehabilitation }\end{array}$ & $\begin{array}{l}\text { Standard } \\
\text { home } \\
\text { rehabilitation }\end{array}$ & Moderate & $\begin{array}{l}\text { KOOS: } \\
\text { Symptoms (0- } \\
100)\end{array}$ & $4 \mathrm{mo}$ & 100 & 71.9 (NR) & 98 & 74.8 (NR) & $-2.6(-7,1.8)$ & NR \\
\hline & $\begin{array}{l}\text { In-home } \\
\text { Telerehabilitation }\end{array}$ & $\begin{array}{l}\text { Standard } \\
\text { home } \\
\text { rehabilitation }\end{array}$ & Moderate & $\begin{array}{l}\text { WOMAC: } \\
\text { Stiffness }(0-100)\end{array}$ & $4 \mathrm{mo}$ & 100 & 72.1 (NR) & 98 & $71(\mathrm{NR})$ & $-0.7(-5.2,6.5)$ & NR \\
\hline
\end{tabular}




\begin{tabular}{|c|c|c|c|c|c|c|c|c|c|c|c|}
\hline $\begin{array}{l}\text { Study, Year, } \\
\text { PMID, Country }\end{array}$ & Arm 1 & Arm 2 & $\begin{array}{l}\text { Overall } \\
\text { RoB }\end{array}$ & $\begin{array}{l}\text { Outcome } \\
\text { Measurement }\end{array}$ & $\begin{array}{l}\text { Time } \\
\text { Point }^{\mathrm{A}}\end{array}$ & $\begin{array}{l}\text { N Arm } \\
1\end{array}$ & $\begin{array}{l}\text { Arm 1, Mean } \\
\text { (SD) }\end{array}$ & $\begin{array}{l}\text { N Arm } \\
2\end{array}$ & $\begin{array}{l}\text { Arm 2, Mean } \\
\text { (SD) }\end{array}$ & $\begin{array}{l}\text { Effect Size } \\
(95 \% \mathrm{CI})\end{array}$ & $\begin{array}{l}\text { Reported } \\
\text { p-Value }\end{array}$ \\
\hline \multirow[t]{2}{*}{$\begin{array}{l}\text { Monticone, 2013, } \\
\text { 23063624, Italy }\end{array}$} & $\begin{array}{l}\text { Home-based } \\
\text { functional } \\
\text { exercises and } \\
\text { kinesiophobia } \\
\text { training }\end{array}$ & Usual care & Moderate & $\begin{array}{l}\text { KOOS: } \\
\text { Symptoms (0- } \\
100)\end{array}$ & $6 \mathrm{mo}$ & 55 & NR (NR) & 55 & NR (NR) & $\begin{array}{l}13.1(8.44, \\
17.76)\end{array}$ & NR \\
\hline & $\begin{array}{l}\text { Home-based } \\
\text { functional } \\
\text { exercises and } \\
\text { kinesiophobia } \\
\text { training }\end{array}$ & Usual care & Moderate & $\begin{array}{l}\text { KOOS: } \\
\text { Symptoms (0- } \\
100)\end{array}$ & $12 \mathrm{mo}$ & 55 & NR (NR) & 55 & NR (NR) & $\begin{array}{l}10.59(6.0, \\
15.18)\end{array}$ & NR \\
\hline $\begin{array}{l}\text { Petersen, 2018, } \\
\text { 29294078, } \\
\text { Netherlands }\end{array}$ & $\begin{array}{l}\text { Exercise \& } \\
\text { acupuncture }\end{array}$ & Exercise & Moderate & $\begin{array}{l}\text { KOOS: } \\
\text { Symptoms (0- } \\
100)\end{array}$ & $3 \mathrm{mo}$ & 82 & $\begin{array}{l}\mathrm{N} \text { with } \\
\text { success (\%) } \\
46(55)\end{array}$ & 83 & $\begin{array}{l}\mathrm{N} \text { with } \\
\text { success }(\%) \\
50(60)\end{array}$ & $\begin{array}{l}\text { RR 0.92 (0.71, } \\
1.19)\end{array}$ & 0.53 \\
\hline $\begin{array}{l}\text { Schache, 2019, } \\
\text { 31208916, } \\
\text { Australia }\end{array}$ & $\begin{array}{l}\text { Standard } \\
\text { rehabilitation and } \\
\text { hip strengthening } \\
\text { exercises }\end{array}$ & $\begin{array}{l}\text { Standard } \\
\text { rehabilitation } \\
\text { plus general } \\
\text { functional } \\
\text { exercise }\end{array}$ & Moderate & $\begin{array}{l}\text { KOOS: } \\
\text { Symptoms (0- } \\
100)\end{array}$ & $6.5 \mathrm{mo}$ & 48 & $82(13)$ & 48 & 79 (14) & $2(-4,9)$ & NR \\
\hline
\end{tabular}

Statistically significant effect sizes are in bold text. In cases where calculated effect size confidence intervals were not-statistically significant but reported p-values were, we deferred to reported p-values and still bolded results.

Abbreviations: Adj MD = adjusted mean difference $\mathrm{CI}=$ confidence interval, $\mathrm{KOOS}=$ Knee injury and osteoarthritis outcome score, mo $=$ month, $\mathrm{NR}=$ not reported, $\mathrm{PENS}=$ patterned electrical neuromuscular stimulation, $\mathrm{PMID}=$ PubMed identifier, $\mathrm{RR}=$ relative risk; RoB $=$ risk of bias, $\mathrm{SD}=\mathrm{standard}$ deviation, $\mathrm{WOMAC}=\mathrm{Western}$ Ontario and McMaster Universities Osteoarthritis Index.

A Time from surgery

${ }^{B}$ Calculated

Table 43. Post-acute rehabilitation versus various controls for total knee arthroplasty - continuous outcomes, pain

\begin{tabular}{|c|c|c|c|c|c|c|c|c|c|c|c|}
\hline $\begin{array}{l}\text { Study, Year, } \\
\text { PMID, Country }\end{array}$ & Arm 1 & Arm 2 & $\begin{array}{l}\text { Overall } \\
\text { RoB }\end{array}$ & $\begin{array}{l}\text { Outcome } \\
\text { Measurement }\end{array}$ & $\begin{array}{l}\text { Time } \\
\text { Point }^{\mathrm{A}}\end{array}$ & $\begin{array}{l}\text { N Arm } \\
1\end{array}$ & $\begin{array}{l}\text { Arm 1, Mean } \\
\text { (SD) }\end{array}$ & $\begin{array}{l}\text { N Arm } \\
2\end{array}$ & $\begin{array}{l}\text { Arm 2, Mean } \\
\text { (SD) }\end{array}$ & $\begin{array}{l}\text { Effect Size } \\
(95 \% \mathrm{CI})\end{array}$ & $\begin{array}{l}\text { Reported } \\
\text { p-Value }\end{array}$ \\
\hline \multirow[t]{4}{*}{$\begin{array}{l}\text { Artz, 2017, } \\
27068368, \text { UK }\end{array}$} & $\begin{array}{l}\text { Group-based } \\
\text { exercise }\end{array}$ & Usual care & High & $\begin{array}{l}\text { KOOS: Pain (0- } \\
100)\end{array}$ & $3 \mathrm{mo}$ & 19 & $74.1(19.9)$ & 12 & $19(74.1)$ & $\begin{array}{l}55.1(16.9 \\
93.3)^{\mathrm{B}}\end{array}$ & NR \\
\hline & $\begin{array}{l}\text { Group-based } \\
\text { exercise }\end{array}$ & Usual care & High & $\begin{array}{l}\text { KOOS: Pain (0- } \\
100)\end{array}$ & $6 \mathrm{mo}$ & 21 & $78.6(25.9)$ & 15 & $70.9(27.1)$ & $\begin{array}{l}7.7(-4.9 \\
20.3)^{\mathrm{B}}\end{array}$ & NR \\
\hline & $\begin{array}{l}\text { Group-based } \\
\text { exercise }\end{array}$ & Usual care & High & VAS (NR) & $3 \mathrm{mo}$ & 19 & $3.5(3.1)$ & 12 & $3.6(2.2)$ & $\begin{array}{l}-0.1(-1.4, \\
1.2)^{\mathrm{B}}\end{array}$ & NR \\
\hline & $\begin{array}{l}\text { Group-based } \\
\text { exercise }\end{array}$ & Usual care & High & VAS (NR) & $6 \mathrm{mo}$ & 21 & $2.9(3.4)$ & 15 & $3.9(3.6)$ & $-1(-2.7,0.7)^{\mathrm{B}}$ & NR \\
\hline \multirow[t]{2}{*}{$\begin{array}{l}\text { Bruun-Olsen, } \\
2013 \text {, } \\
23614370, \\
\text { Norway }\end{array}$} & $\begin{array}{l}\text { Walking-skill } \\
\text { group }\end{array}$ & $\begin{array}{l}15 \text { individual } \\
\text { physiotherapy } \\
\text { sessions }\end{array}$ & Moderate & $\begin{array}{l}\text { KOOS: Pain (0- } \\
100)\end{array}$ & $9 \mathrm{mo}$ & 29 & $82(21)$ & 28 & $74(23)$ & Adj $0(-9,10)$ & NR \\
\hline & Leg-press group & $\begin{array}{l}\text { Physiotherapy } \\
\text { group }\end{array}$ & High & $\begin{array}{l}\text { VAS (0-100): } \\
\text { During activity }\end{array}$ & $3 \mathrm{mo}$ & 26 & $2.7(0.45)$ & 29 & $2.3(0.41)$ & $0.4(0.2,0.6)^{\mathrm{B}}$ & 0.17 \\
\hline
\end{tabular}




\begin{tabular}{|c|c|c|c|c|c|c|c|c|c|c|c|}
\hline $\begin{array}{l}\text { Study, Year, } \\
\text { PMID, Country }\end{array}$ & Arm 1 & Arm 2 & $\begin{array}{l}\text { Overall } \\
\text { RoB }\end{array}$ & $\begin{array}{l}\text { Outcome } \\
\text { Measurement }\end{array}$ & $\begin{array}{l}\text { Time } \\
\text { Point }^{\mathrm{A}}\end{array}$ & $\begin{array}{l}\text { N Arm } \\
1\end{array}$ & $\begin{array}{l}\text { Arm 1, Mean } \\
\text { (SD) }\end{array}$ & $\begin{array}{l}\text { N Arm } \\
2\end{array}$ & $\begin{array}{l}\text { Arm 2, Mean } \\
\text { (SD) }\end{array}$ & $\begin{array}{l}\text { Effect Size } \\
(95 \% \mathrm{Cl})\end{array}$ & $\begin{array}{l}\text { Reported } \\
\text { p-Value }\end{array}$ \\
\hline $\begin{array}{l}\text { Bily, 2016, } \\
26763947 \text {, } \\
\text { Austria }\end{array}$ & Leg-press group & $\begin{array}{l}\text { Physiotherapy } \\
\text { group }\end{array}$ & High & $\begin{array}{l}\text { VAS }(0-100): \text { At } \\
\text { rest }^{\mathrm{D}}\end{array}$ & $3 \mathrm{mo}$ & 26 & $1.3(0.36)$ & 29 & $1.1(0.31)$ & $0.2(0.1,0.3)^{\mathrm{B}}$ & 0.51 \\
\hline \multirow[t]{2}{*}{$\begin{array}{l}\text { Cai, 2018, } \\
29239772, \\
\text { China }\end{array}$} & $\begin{array}{l}\text { Cognitive } \\
\text { behavioral } \\
\text { therapy \& } \\
\text { standard care }\end{array}$ & Standard care & Moderate & VAS $(0-10)$ & $6 \mathrm{mo}$ & 50 & $5.63(0.73)$ & 50 & $6.27(0.86)$ & $\begin{array}{l}\text { NMD -0.57 (- } \\
0.9,-0.2)^{\mathrm{B}}\end{array}$ & 0.080 \\
\hline & $\begin{array}{l}\text { Cognitive } \\
\text { behavioral } \\
\text { therapy \& } \\
\text { standard care }\end{array}$ & Standard care & Moderate & $\begin{array}{l}\text { Pain } \\
\text { catastrophizing } \\
\text { scale }(0-52)\end{array}$ & $6 \mathrm{mo}$ & 50 & $23.34(3.82)$ & 50 & $30.40(4.34)$ & $\begin{array}{l}\text { NMD -7.7 (- } \\
9.3,-6.1)^{\mathrm{B}}\end{array}$ & $<.001$ \\
\hline \multirow[t]{3}{*}{$\begin{array}{l}\text { DeJong, } \\
2020, \\
32360105 \\
\text { USA }\end{array}$} & $\begin{array}{l}\text { Body Weight- } \\
\text { Adjustable } \\
\text { Treadmill \& } \\
\text { PENS }\end{array}$ & $\begin{array}{l}\text { Recumbent Bike } \\
\text { \& PENS }\end{array}$ & High & $\begin{array}{l}\text { KOOS: Pain (0- } \\
100)\end{array}$ & $6 \mathrm{mo}$ & 70 & $86.7(18.4)$ & 78 & $83.8(18.5)$ & $-2.9(-7.1,1.3)$ & NR \\
\hline & $\begin{array}{l}\text { Body Weight- } \\
\text { Adjustable } \\
\text { Treadmill \& } \\
\text { PENS }\end{array}$ & $\begin{array}{l}\text { Body Weight- } \\
\text { Adjustable } \\
\text { Treadmill }\end{array}$ & High & $\begin{array}{l}\text { KOOS: Pain (0- } \\
100)\end{array}$ & $6 \mathrm{mo}$ & 70 & $86.7(18.4)$ & 76 & $87.6(18.6)$ & $0.9(-3.3,5.1)$ & NR \\
\hline & $\begin{array}{l}\text { Body Weight- } \\
\text { Adjustable } \\
\text { Treadmill \& } \\
\text { PENS }\end{array}$ & $\begin{array}{l}\text { Recumbent } \\
\text { Bike/Usual Care }\end{array}$ & High & $\begin{array}{l}\text { KOOS: Pain (0- } \\
100)\end{array}$ & $6 \mathrm{mo}$ & 70 & $86.7(18.4)$ & 74 & $89.9(18.6)$ & $3.2(-1.0,7.4)$ & NR \\
\hline \multirow{2}{*}{$\begin{array}{l}\text { Demircioglu, } \\
2015, \\
26355656, \\
\text { Turkey }\end{array}$} & $\begin{array}{l}\text { NMES \& } \\
\text { exercise }\end{array}$ & Exercise & High & $\begin{array}{l}\text { WOMAC: Pain (0- } \\
\text { 20) }\end{array}$ & $3 \mathrm{mo}$ & 30 & $42.8(16.8)$ & 30 & $48.5(14.2)$ & $\begin{array}{l}-5.7(-11.3,- \\
0.1)^{\mathrm{B}}\end{array}$ & NR \\
\hline & $\begin{array}{l}\text { NMES \& } \\
\text { exercise }\end{array}$ & Exercise & High & VAS $(0-10)$ & $3 \mathrm{mo}$ & 30 & $8.4(0.6)$ & 30 & $3.5(0.6)$ & $4.9(4.7,5.1)^{B}$ & NR \\
\hline $\begin{array}{l}\text { Fransen, 2017, } \\
27868384, \\
\text { Australia }\end{array}$ & $\begin{array}{l}\text { Outpatient } \\
\text { exercise group }\end{array}$ & Usual care & Moderate & $\begin{array}{l}\text { WOMAC: Pain (0- } \\
\text { 20) }\end{array}$ & $12 \mathrm{mo}$ & 179 & $2.6(0.2)$ & 169 & $2.5(0.2)$ & $-0.1(-0.7,0.5)$ & 0.71 \\
\hline \multirow{2}{*}{$\begin{array}{l}\text { Heikkilä, 2017, } \\
28119232, \\
\text { Finland }\end{array}$} & Home exercise & Control & High & VAS $(0-100)^{\mathrm{E}}$ & $2 \mathrm{mo}$ & 53 & $22(20)$ & 55 & $27(22)$ & $\begin{array}{l}-5(-10.6 \\
0.6)^{\mathrm{B}}\end{array}$ & NR \\
\hline & Home exercise & Control & High & VAS $(0-100)^{\mathrm{E}}$ & $14 \mathrm{mo}$ & 50 & $12(21)$ & 52 & $15(20)$ & $-3(-8.6,2.6)^{\mathrm{B}}$ & NR \\
\hline $\begin{array}{l}\text { Lenguerrand, } \\
2020 \text {, } \\
31033232 \text {, UK }\end{array}$ & $\begin{array}{l}\text { Physical therapy } \\
\text { and standard } \\
\text { care }\end{array}$ & Standard care & Moderate & $\begin{array}{l}\text { KOOS: Pain (0- } \\
100)\end{array}$ & $12 \mathrm{mo}$ & 66 & $83(20)$ & 57 & $81(21)$ & $2(-3.2,7.2)^{\mathrm{B}}$ & 0.111 \\
\hline \multirow{2}{*}{$\begin{array}{l}\text { Li, 2015, CN- } \\
01084888, \\
\text { China }\end{array}$} & Education & No education & High & VAS (NR) & $3 \mathrm{mo}$ & 25 & NR & 25 & NR & No difference & NR \\
\hline & Education & No education & High & VAS (NR) & $6 \mathrm{mo}$ & 25 & NR & 25 & NR & No difference & NR \\
\hline $\begin{array}{l}\text { Li, 2019, } \\
31003647, \\
\text { China }\end{array}$ & Tai chi exercise & Control & Moderate & $\begin{array}{l}\text { WOMAC: Pain (0- } \\
20)\end{array}$ & $3 \mathrm{mo}$ & 54 & $9.1(2)$ & 53 & $9.3(1.9)$ & $\begin{array}{l}-0.2(-0.7 \\
0.3)^{\mathrm{B}}\end{array}$ & 0.07 \\
\hline $\begin{array}{l}\text { Liao, 2015, } \\
\text { 25552523, } \\
\text { Taiwan }\end{array}$ & $\begin{array}{l}\text { Functional plus } \\
\text { balance } \\
\text { rehabilitation }\end{array}$ & $\begin{array}{l}\text { Functional } \\
\text { rehabilitation }\end{array}$ & High & $\begin{array}{l}\text { WOMAC: Pain (0- } \\
\text { 20) }\end{array}$ & $32 \mathrm{w}$ & 65 & $1.9(1.2)$ & 65 & $1.6(1.0)$ & $\begin{array}{l}0.3(0.03 \\
0.6)^{\mathrm{B}}\end{array}$ & NR \\
\hline
\end{tabular}




\begin{tabular}{|c|c|c|c|c|c|c|c|c|c|c|c|}
\hline $\begin{array}{l}\text { Study, Year, } \\
\text { PMID, Country }\end{array}$ & Arm 1 & Arm 2 & $\begin{array}{l}\text { Overall } \\
\text { RoB }\end{array}$ & $\begin{array}{l}\text { Outcome } \\
\text { Measurement }\end{array}$ & $\begin{array}{l}\text { Time } \\
\text { Point }^{\mathrm{A}}\end{array}$ & $\begin{array}{l}\text { N Arm } \\
1\end{array}$ & $\begin{array}{l}\text { Arm 1, Mean } \\
\text { (SD) }\end{array}$ & $\begin{array}{l}\text { N Arm } \\
2\end{array}$ & $\begin{array}{l}\text { Arm 2, Mean } \\
\text { (SD) }\end{array}$ & $\begin{array}{l}\text { Effect Size } \\
(95 \% \mathrm{Cl})\end{array}$ & $\begin{array}{l}\text { Reported } \\
\text { p-Value }\end{array}$ \\
\hline $\begin{array}{l}\text { Liao, } 2020, \\
31687984, \\
\text { Taiwan }\end{array}$ & $\begin{array}{l}\text { Elastic } \\
\text { resistance } \\
\text { exercise training }\end{array}$ & Standard care & Moderate & $\begin{array}{l}\text { WOMAC: Pain (0- } \\
20)\end{array}$ & $4 \mathrm{mo}$ & 30 & $2.97(1.59)$ & 30 & $4.48(1.39)$ & $-1.5(-2,-1)^{B}$ & 0.001 \\
\hline \multirow[t]{3}{*}{$\begin{array}{l}\text { Minns Lowe, } \\
2012 \text {, } \\
22180446 \text {, UK }\end{array}$} & $\begin{array}{l}\text { Home-visit } \\
\text { physiotherapy }\end{array}$ & Usual care & High & $\begin{array}{l}\text { KOOS: Pain (0- } \\
100)\end{array}$ & $3 \mathrm{mo}$ & 46 & $\begin{array}{l}\text { Median } \\
(\text { IQR) } 69.1 \\
(28) \\
\end{array}$ & 47 & $\begin{array}{l}\text { Median } \\
(\text { IQR) } 72.2 \\
(29) \\
\end{array}$ & NR & NR \\
\hline & $\begin{array}{l}\text { Home-visit } \\
\text { physiotherapy }\end{array}$ & Usual care & High & $\begin{array}{l}\text { KOOS: Pain (0- } \\
100)\end{array}$ & $6 \mathrm{mo}$ & 42 & $\begin{array}{l}\text { Median } \\
(\text { IQR) } 75(25)\end{array}$ & 43 & $\begin{array}{l}\text { Median } \\
(\text { IQR) } 75(31)\end{array}$ & NR & NR \\
\hline & $\begin{array}{l}\text { Home-visit } \\
\text { physiotherapy }\end{array}$ & Usual care & High & $\begin{array}{l}\text { KOOS: Pain (0- } \\
100)\end{array}$ & $12 \mathrm{mo}$ & 44 & $\begin{array}{l}\text { Median } \\
\text { (IQR) } 80.6 \\
(36)\end{array}$ & 48 & $\begin{array}{l}\text { Median } \\
\text { (IQR) } 90.3 \\
(33)\end{array}$ & NR & NR \\
\hline \multirow[t]{2}{*}{$\begin{array}{l}\text { Mitchell, 2005, } \\
\text { 15869558, UK }\end{array}$} & Hospital & Home & High & $\begin{array}{l}\text { SF-36: Bodily pain } \\
(0-100)\end{array}$ & $4 \mathrm{mo}$ & 57 & $48.5(26.8)$ & 57 & $46.6(20.6)$ & $\begin{array}{l}\text { Adj MD -3.4 (- } \\
12.0,5.2)\end{array}$ & 0.432 \\
\hline & Hospital & Home & High & $\begin{array}{l}\text { WOMAC: Pain (0- } \\
\text { 20) }\end{array}$ & $4 \mathrm{mo}$ & 57 & $6.9(4.3)$ & 57 & $6.8(3.7)$ & $\begin{array}{l}\text { Adj MD -0.5 (- } \\
2.0,1.0)\end{array}$ & 0.53 \\
\hline \multirow{2}{*}{$\begin{array}{l}\text { Moffet, 2015, } \\
26178888, \\
\text { Canada }\end{array}$} & $\begin{array}{l}\text { In-home } \\
\text { Telerehabilitation }\end{array}$ & $\begin{array}{l}\text { Standard home } \\
\text { rehabilitation }\end{array}$ & Moderate & $\begin{array}{l}\text { KOOS: Pain (0- } \\
100)\end{array}$ & $4 \mathrm{mo}$ & 100 & 78.1 (NR) & 98 & 80.1 (NR) & $-1.8(-6.2,2.5)$ & NR \\
\hline & $\begin{array}{l}\text { In-home } \\
\text { Telerehabilitation }\end{array}$ & $\begin{array}{l}\text { Standard home } \\
\text { rehabilitation }\end{array}$ & Moderate & $\begin{array}{l}\text { WOMAC: Pain (0- } \\
\text { 20) }\end{array}$ & $4 \mathrm{mo}$ & 100 & 82.8 (NR) & 98 & 84 (NR) & $-0.7(-4.8,3.4)$ & NR \\
\hline \multirow[t]{4}{*}{$\begin{array}{l}\text { Monticone, } \\
2013, \\
23063624, \text { Italy }\end{array}$} & $\begin{array}{l}\text { Home-based } \\
\text { functional } \\
\text { exercises and } \\
\text { kinesiophobia } \\
\text { training }\end{array}$ & Usual care & Moderate & $\begin{array}{l}\text { KOOS: Pain (0- } \\
100)\end{array}$ & $6 \mathrm{mo}$ & 55 & NR & 55 & NR & $\begin{array}{l}\text { 10.34 (4.34, } \\
16.35)\end{array}$ & NR \\
\hline & $\begin{array}{l}\text { Home-based } \\
\text { functional } \\
\text { exercises and } \\
\text { kinesiophobia } \\
\text { training }\end{array}$ & Usual care & Moderate & $\begin{array}{l}\text { KOOS: Pain (0- } \\
100)\end{array}$ & $12 \mathrm{mo}$ & 55 & NR & 55 & NR & $\begin{array}{l}9.56(4.58, \\
14.54)\end{array}$ & NR \\
\hline & $\begin{array}{l}\text { Home-based } \\
\text { functional } \\
\text { exercises and } \\
\text { kinesiophobia } \\
\text { training }\end{array}$ & Usual care & Moderate & VAS (0-10) & $6 \mathrm{mo}$ & 55 & NR & 55 & NR & $-1.5(-2,-1)$ & NR \\
\hline & $\begin{array}{l}\text { Home-based } \\
\text { functional } \\
\text { exercises and } \\
\text { kinesiophobia } \\
\text { training }\end{array}$ & Usual care & Moderate & VAS (0-10) & $12 \mathrm{mo}$ & 55 & NR & 55 & NR & $\begin{array}{l}-1.0(-1.6,- \\
0.5)\end{array}$ & NR \\
\hline $\begin{array}{l}\text { Moutzouri, } \\
2018, \\
29473481, \\
\text { Greece }\end{array}$ & $\begin{array}{l}\text { Early self- } \\
\text { managed focal } \\
\text { sensorimotor } \\
\text { exercise training }\end{array}$ & $\begin{array}{l}\text { Functional } \\
\text { exercise training }\end{array}$ & Moderate & VAS (0-10) & $3.5 \mathrm{mo}$ & 25 & $0.7(0.7)$ & 26 & $2.4(0.8)$ & $-1.7(-2,-1.4)^{\mathrm{B}}$ & NR \\
\hline $\begin{array}{l}\text { Petersen, 2018, } \\
\text { 29294078, } \\
\text { Netherlands }\end{array}$ & $\begin{array}{l}\text { Exercise \& } \\
\text { acupuncture }\end{array}$ & Exercise & High & $\begin{array}{l}\text { KOOS: Pain (0- } \\
100)\end{array}$ & $3 \mathrm{mo}$ & 82 & $\begin{array}{l}\mathrm{N} \text { with } \\
\text { success (\%) } \\
56(67) \\
\end{array}$ & 83 & $\begin{array}{l}\mathrm{N} \text { with } \\
\text { success }(\%) \\
60(73)\end{array}$ & $\begin{array}{l}\text { RR } 0.73(1.09 \text {, } \\
0.89)\end{array}$ & 0.259 \\
\hline
\end{tabular}




\begin{tabular}{|c|c|c|c|c|c|c|c|c|c|c|c|}
\hline $\begin{array}{l}\text { Study, Year, } \\
\text { PMID, Country }\end{array}$ & Arm 1 & Arm 2 & $\begin{array}{l}\text { Overall } \\
\text { RoB }\end{array}$ & $\begin{array}{l}\text { Outcome } \\
\text { Measurement }\end{array}$ & $\begin{array}{l}\text { Time } \\
\text { Point }^{\mathrm{A}}\end{array}$ & $\begin{array}{l}\text { N Arm } \\
1\end{array}$ & $\begin{array}{l}\text { Arm 1, Mean } \\
\text { (SD) }\end{array}$ & $\begin{array}{l}\text { N Arm } \\
2\end{array}$ & $\begin{array}{l}\text { Arm 2, Mean } \\
\text { (SD) }\end{array}$ & $\begin{array}{l}\text { Effect Size } \\
(95 \% \mathrm{Cl})\end{array}$ & $\begin{array}{l}\text { Reported } \\
\text { p-Value }\end{array}$ \\
\hline \multirow{2}{*}{$\begin{array}{l}\text { Petterson, } \\
2009 \text {, } \\
19177542 \text {, USA }\end{array}$} & $\begin{array}{l}\text { Exercise \& } \\
\text { NMES group }\end{array}$ & Exercise & High & KOS: Pain $(0-5)^{F}$ & $3 \mathrm{mo}$ & 92 & 1.08 (NR) & 78 & 1.11 (NR) & NR & NR \\
\hline & $\begin{array}{l}\text { Exercise \& } \\
\text { NMES group }\end{array}$ & Exercise & High & KOS: Pain (0-5) & $12 \mathrm{mo}$ & 61 & 0.89 (NR) & 68 & 0.82 (NR) & NR & NR \\
\hline \multirow[t]{3}{*}{$\begin{array}{l}\text { Schache, 2019, } \\
\text { 31208916, } \\
\text { Australia }\end{array}$} & $\begin{array}{l}\text { Standard } \\
\text { rehabilitation and } \\
\text { hip } \\
\text { strengthening } \\
\text { exercises }\end{array}$ & $\begin{array}{l}\text { Standard } \\
\text { rehabilitation plus } \\
\text { general functional } \\
\text { exercise }\end{array}$ & Moderate & $\begin{array}{l}\text { KOOS: Pain (0- } \\
100)\end{array}$ & $6.5 \mathrm{mo}$ & 48 & $87(11)$ & 48 & $71(15)$ & $1(-5,8)$ & NR \\
\hline & $\begin{array}{l}\text { Standard } \\
\text { rehabilitation and } \\
\text { hip } \\
\text { strengthening } \\
\text { exercises }\end{array}$ & $\begin{array}{l}\text { Standard } \\
\text { rehabilitation plus } \\
\text { general functional } \\
\text { exercise }\end{array}$ & Moderate & $\begin{array}{l}\text { KOOS: Pain (0- } \\
100)\end{array}$ & $6.5 \mathrm{mo}$ & 48 & $73(19)$ & 48 & $70(21)$ & $3(-6,13)$ & NR \\
\hline & $\begin{array}{l}\text { Standard } \\
\text { rehabilitation and } \\
\text { hip } \\
\text { strengthening } \\
\text { exercises }\end{array}$ & $\begin{array}{l}\text { Standard } \\
\text { rehabilitation plus } \\
\text { general functional } \\
\text { exercise }\end{array}$ & Moderate & VAS (0-10) & $6.5 \mathrm{mo}$ & 48 & $0(1)$ & 48 & $1(0)$ & $0(-1,1)$ & NR \\
\hline $\begin{array}{l}\text { Vuorenmaa, } \\
2014, \\
24241606, \\
\text { Finland }\end{array}$ & Home exercise & Control & Moderate & $\begin{array}{l}\text { WOMAC: Pain (0- } \\
\text { 20) }\end{array}$ & $12 \mathrm{mo}$ & 53 & 38 (NR) & 55 & $37(\mathrm{NR})$ & NR & NR \\
\hline
\end{tabular}

Statistically significant effect sizes are in bold text. In cases where calculated effect size confidence intervals were not-statistically significant but reported p-values were, we deferred to reported p-values and still bolded results.

Abbreviations: Adj MD = adjusted mean difference, $\mathrm{CI}=$ confidence interval, $\mathrm{EQ}-5 \mathrm{D}=$ EuroQuol, $\mathrm{KOS}=\mathrm{Knee}$ Outcome Survey, $\mathrm{KOOS}=\mathrm{Knee}$ injury and osteoarthritis outcome score, $\min =$ minute, $\mathrm{mo}=$ month, NMES $=$ neuromuscular electrical stimulation, $\mathrm{NR}=$ not reported, $\mathrm{PENS}=$ patterned electrical neuromuscular stimulation, $\mathrm{PMID}=\mathrm{PubMed}$ identifier, $\mathrm{RR}=$ relative risk, $\mathrm{RoB}=$ risk of bias, $\mathrm{SD}=$ standard deviation, $\mathrm{SF}-36=36$-item short form survey, WOMAC $=$ Western Ontario and McMaster Universities Osteoarthritis Index, VAS = visual analogue scale, $\mathrm{w}=$ weeks.

A Time from surgery

${ }^{B}$ Calculated

C During last 48 hours

D Before the functional activity

${ }^{\mathrm{E}}$ Knee pain during loading

${ }^{F}$ Measured with question from the Knee Outcome Survey (designed for Activities of Daily Living) on pain "How does pain affect the function of your knee during daily activities?" Scores ranged from 0 (pain prevents me from all activities) to 5 (pain has no effect on daily activities). 
Table 44. Post-acute rehabilitation versus various controls for total knee arthroplasty - continuous outcomes, range of motion

\begin{tabular}{|c|c|c|c|c|c|c|c|c|c|c|c|}
\hline $\begin{array}{l}\text { Study, Year, } \\
\text { PMID, Country }\end{array}$ & Arm 1 & Arm 2 & $\begin{array}{l}\text { Overall } \\
\text { RoB }\end{array}$ & $\begin{array}{l}\text { Outcome } \\
\text { Measurement }\end{array}$ & $\begin{array}{l}\text { Time } \\
\text { Point }^{\mathrm{A}}\end{array}$ & $\begin{array}{l}\text { N Arm } \\
1\end{array}$ & $\begin{array}{l}\text { Arm 1, Mean } \\
\text { (SD) }\end{array}$ & $\begin{array}{l}\text { N Arm } \\
2\end{array}$ & $\begin{array}{l}\text { Arm 2, Mean } \\
\text { (SD) }\end{array}$ & $\begin{array}{l}\text { Effect Size } \\
(95 \% \mathrm{Cl})\end{array}$ & $\begin{array}{l}\text { Reported } \\
\text { p-Value }\end{array}$ \\
\hline \multirow{2}{*}{$\begin{array}{l}\text { Andersen, } \\
\text { 2018, CN- } \\
\text { 01647420, } \\
\text { Denmark }\end{array}$} & $\begin{array}{l}\text { Technological } \\
\text { assisted } \\
\text { rehabilitation } \\
\end{array}$ & $\begin{array}{l}\text { Supervised } \\
\text { rehabilitation }\end{array}$ & High & $\begin{array}{l}\text { Active knee ROM: } \\
\text { Extension and flexion } \\
\text { (deg) }\end{array}$ & $6 \mathrm{mo}$ & NR & NR (NR) & NR & NR (NR) & $<10 \% \mathrm{MD}$ & NR \\
\hline & $\begin{array}{l}\text { Technological } \\
\text { assisted } \\
\text { rehabilitation }\end{array}$ & $\begin{array}{l}\text { Supervised } \\
\text { rehabilitation }\end{array}$ & High & $\begin{array}{l}\text { Active knee ROM: } \\
\text { Extension and } \\
\text { flexion(deg) }\end{array}$ & $12 \mathrm{mo}$ & NR & NR (NR) & NR & NR (NR) & $<10 \% \mathrm{MD}$ & NR \\
\hline \multirow[t]{6}{*}{$\begin{array}{l}\text { Bade, } 2017, \\
27813347, \text { USA }\end{array}$} & $\begin{array}{l}\text { High-intensity } \\
\text { progressive } \\
\text { rehabilitation }\end{array}$ & $\begin{array}{l}\text { Low-intensity } \\
\text { rehabilitation }\end{array}$ & Moderate & $\begin{array}{l}\text { Active knee ROM: } \\
\text { Extension (deg) }\end{array}$ & $3 \mathrm{mo}$ & 77 & $-0.09(2.97)$ & 76 & $0.06(2.37)$ & $-0.2(-0.8,0.5)^{\mathrm{B}}$ & NR \\
\hline & $\begin{array}{l}\text { High-intensity } \\
\text { progressive } \\
\text { rehabilitation }\end{array}$ & $\begin{array}{l}\text { Low-intensity } \\
\text { rehabilitation }\end{array}$ & Moderate & $\begin{array}{l}\text { Active knee ROM: } \\
\text { Extension (deg) }\end{array}$ & $6 \mathrm{mo}$ & 71 & $-1.38(1.66)$ & 71 & $-0.90(2.62)$ & $-0.5(-1.0,0.1)^{\mathrm{B}}$ & NR \\
\hline & $\begin{array}{l}\text { High-intensity } \\
\text { progressive } \\
\text { rehabilitation } \\
\end{array}$ & $\begin{array}{l}\text { Low-intensity } \\
\text { rehabilitation }\end{array}$ & Moderate & $\begin{array}{l}\text { Active knee ROM: } \\
\text { Extension (deg) }\end{array}$ & $12 \mathrm{mo}$ & 71 & $-2.18(2.43)$ & 67 & $-1.76(2.28)$ & $-0.4(-1,0.1)^{\mathrm{B}}$ & NR \\
\hline & $\begin{array}{l}\text { High-intensity } \\
\text { progressive } \\
\text { rehabilitation }\end{array}$ & $\begin{array}{l}\text { Low-intensity } \\
\text { rehabilitation }\end{array}$ & Moderate & $\begin{array}{l}\text { Active knee ROM: } \\
\text { Flexion (deg) }\end{array}$ & $3 \mathrm{mo}$ & 77 & $123.79(9.1)$ & 76 & $123.71(8.97)$ & $0.1(-1.9,2.1)^{\mathrm{B}}$ & NR \\
\hline & $\begin{array}{l}\text { High-intensity } \\
\text { progressive } \\
\text { rehabilitation }\end{array}$ & $\begin{array}{l}\text { Low-intensity } \\
\text { rehabilitation }\end{array}$ & Moderate & $\begin{array}{l}\text { Active knee ROM: } \\
\text { Flexion (deg) }\end{array}$ & $6 \mathrm{mo}$ & 71 & $127.10(6.57)$ & 71 & $127.45(7.88)$ & $-0.4(-2.1,1.4)^{\mathrm{B}}$ & NR \\
\hline & $\begin{array}{l}\text { High-intensity } \\
\text { progressive } \\
\text { rehabilitation }\end{array}$ & $\begin{array}{l}\text { Low-intensity } \\
\text { rehabilitation }\end{array}$ & Moderate & $\begin{array}{l}\text { Active knee ROM: } \\
\text { Flexion (deg) }\end{array}$ & $12 \mathrm{mo}$ & 71 & $129.28(8.89)$ & 67 & $128.27(8.61)$ & $1(-1.1,3.1)^{\mathrm{B}}$ & NR \\
\hline \multirow[t]{2}{*}{$\begin{array}{l}\text { Bily, 2016, } \\
26763947, \\
\text { Austria }\end{array}$} & Leg-press group & $\begin{array}{l}\text { Physiotherapy } \\
\text { group }\end{array}$ & High & $\begin{array}{l}\text { Active knee ROM: } \\
\text { Extension and flexion } \\
\text { (deg) }\end{array}$ & $3 \mathrm{mo}$ & 26 & $114.1(2.36)$ & 29 & $111.2(1.58)$ & NR & 0.09 \\
\hline & Leg-press group & $\begin{array}{l}\text { Physiotherapy } \\
\text { group }\end{array}$ & High & $\begin{array}{l}\text { Passive knee ROM: } \\
\text { Extension and } \\
\text { flexion(deg) }\end{array}$ & $3 \mathrm{mo}$ & 26 & $116.2(2.46)$ & 29 & $112.8(1.51)$ & $3.4(2.6,4.2)^{\mathrm{B}}$ & 0.30 \\
\hline $\begin{array}{l}\text { Bruun-Olsen, } \\
2013, \\
23614370, \\
\text { Norway }\end{array}$ & $\begin{array}{l}\text { Walking-skill } \\
\text { group }\end{array}$ & $\begin{array}{l}\text { Usual } \\
\text { physiotherapy }\end{array}$ & Moderate & $\begin{array}{l}\text { Active knee ROM: } \\
\text { Extension and flexion: } \\
\text { (deg) }\end{array}$ & $9 \mathrm{mo}$ & 29 & $118(7)$ & 28 & $114(17)$ & adj $1(-4,7)$ & NR \\
\hline \multirow{2}{*}{$\begin{array}{l}\text { Demircioglu, } \\
2015, \\
26355656, \\
\text { Turkey }\end{array}$} & $\begin{array}{l}\text { NMES \& } \\
\text { exercise }\end{array}$ & Exercise & High & $\begin{array}{l}\text { Passive knee ROM: } \\
\text { Extension (deg) }\end{array}$ & $3 \mathrm{mo}$ & 30 & $-0.3(1.3)$ & 30 & $-0.5(1.5)$ & $0.2(-0.3,0.7)^{\mathrm{B}}$ & NR \\
\hline & $\begin{array}{l}\text { NMES \& } \\
\text { exercise }\end{array}$ & Exercise & High & $\begin{array}{l}\text { Passive knee ROM: } \\
\text { Flexion (deg) }\end{array}$ & $3 \mathrm{mo}$ & 30 & $113.2(7.7)$ & 30 & $110.5(7.9)$ & $2.7(-0.1,5.5)^{\mathrm{B}}$ & NR \\
\hline \multirow{2}{*}{$\begin{array}{l}\text { Fransen, 2017, } \\
27868384, \\
\text { Australia }\end{array}$} & $\begin{array}{l}\text { Outpatient } \\
\text { exercise group }\end{array}$ & Usual care & Moderate & $\begin{array}{l}\text { Active knee ROM: } \\
\text { Extension (deg) }\end{array}$ & $12 \mathrm{mo}$ & 112 & $-1.6(0.4)$ & 98 & $\begin{array}{l}\text { Mean (SE) - } \\
2.7(0.4)\end{array}$ & NR & NR \\
\hline & $\begin{array}{l}\text { Outpatient } \\
\text { exercise group }\end{array}$ & Usual care & Moderate & $\begin{array}{l}\text { Active knee ROM: } \\
\text { Flexion (deg) }\end{array}$ & $12 \mathrm{mo}$ & 112 & $109.2(0.9)$ & 98 & $\begin{array}{l}\text { Mean (SE) } \\
109.6(1)\end{array}$ & NR & NR \\
\hline
\end{tabular}




\begin{tabular}{|c|c|c|c|c|c|c|c|c|c|c|c|}
\hline $\begin{array}{l}\text { Study, Year, } \\
\text { PMID, Country }\end{array}$ & Arm 1 & Arm 2 & $\begin{array}{l}\text { Overall } \\
\text { RoB }\end{array}$ & $\begin{array}{l}\text { Outcome } \\
\text { Measurement }\end{array}$ & $\begin{array}{l}\text { Time } \\
\text { Point }^{\mathrm{A}}\end{array}$ & $\begin{array}{l}\text { N Arm } \\
1\end{array}$ & $\begin{array}{l}\text { Arm 1, Mean } \\
\text { (SD) }\end{array}$ & $\begin{array}{l}\text { N Arm } \\
2\end{array}$ & $\begin{array}{l}\text { Arm 2, Mean } \\
\text { (SD) }\end{array}$ & $\begin{array}{l}\text { Effect Size } \\
(95 \% \mathrm{CI})\end{array}$ & $\begin{array}{l}\text { Reported } \\
\text { p-Value }\end{array}$ \\
\hline \multirow[t]{2}{*}{$\begin{array}{l}\text { Kauppila, 2010, } \\
\text { 20354057, } \\
\text { Finland }\end{array}$} & $\begin{array}{l}\text { Multidisciplinary } \\
\text { rehabilitation } \\
\text { group }\end{array}$ & Control & Moderate & $\begin{array}{l}\text { Active knee ROM: } \\
\text { Flexion (deg) }\end{array}$ & $6 \mathrm{mo}$ & 36 & $\begin{array}{l}\text { Lower limit } \\
\text { mean }(S D) \\
\text { upper limit } \\
\text { mean }(S D) \\
(6,36,3)\end{array}$ & 38 & $\begin{array}{l}\text { Lower limit } \\
\text { mean(SD); } \\
\text { upper limit } \\
\text { mean(SD) }(6 \\
, 36,3)\end{array}$ & NR & NR \\
\hline & $\begin{array}{l}\text { Multidisciplinary } \\
\text { rehabilitation } \\
\text { group }\end{array}$ & Control & Moderate & $\begin{array}{l}\text { Active knee ROM: } \\
\text { Flexion (deg) }\end{array}$ & $12 \mathrm{mo}$ & 36 & $\begin{array}{l}\text { Lower limit } \\
\text { mean(SD); } \\
\text { upper limit } \\
\text { mean(SD) } \\
(5,45,4)\end{array}$ & 38 & $\begin{array}{l}\text { Lower limit } \\
\text { mean }(S D) ; \\
\text { upper limit } \\
\text { mean }(S D) \\
(4,44,4)\end{array}$ & NR & NR \\
\hline \multirow[t]{2}{*}{$\begin{array}{l}\mathrm{Li}, 2019, \\
31003647, \\
\text { China }\end{array}$} & Tai chi & Control & Moderate & $\begin{array}{l}\text { Knee ROM } \\
\text { (active/passive } \\
\text { unspecified): } \\
\text { Extension (deg) }\end{array}$ & $3 \mathrm{mo}$ & 54 & $1.5(0.3)$ & 53 & $1.9(0.2)$ & $\begin{array}{l}-0.4(-0.5,- \\
0.3)^{\mathrm{B}}\end{array}$ & 0.59 \\
\hline & Tai chi & Control & Moderate & $\begin{array}{l}\text { Knee ROM } \\
\text { (active/passive } \\
\text { unspecified): Flexion } \\
\text { (deg) }\end{array}$ & $3 \mathrm{mo}$ & 54 & $112.1(14.8)$ & 53 & 110 (12.9) & $2.1(-1.6,5.8)^{\mathrm{B}}$ & 0.62 \\
\hline \multirow[t]{2}{*}{$\begin{array}{l}\text { Madsen, 2013, } \\
\text { 23651717, } \\
\text { Denmark }\end{array}$} & $\begin{array}{l}\text { Group-based } \\
\text { rehabilitation }\end{array}$ & $\begin{array}{l}\text { Supervised } \\
\text { home-exercises }\end{array}$ & High & $\begin{array}{l}\text { Active Knee ROM: } \\
\text { Flexion and extension } \\
\text { (deg) }\end{array}$ & $3 \mathrm{mo}$ & 36 & NR (NR) & 34 & NR (NR) & NR & 0.9 \\
\hline & $\begin{array}{l}\text { Group-based } \\
\text { rehabilitation }\end{array}$ & $\begin{array}{l}\text { Supervised } \\
\text { home-exercises }\end{array}$ & High & $\begin{array}{l}\text { Knee ROM flexion } \\
\text { and extension (deg) }\end{array}$ & $6 \mathrm{mo}$ & 36 & NR (NR) & 32 & NR (NR) & NR & 0.5 \\
\hline \multirow[t]{2}{*}{$\begin{array}{l}\text { Moffet, 2015, } \\
\text { 26178888, } \\
\text { Canada }\end{array}$} & $\begin{array}{l}\text { In-home } \\
\text { Telerehabilitation }\end{array}$ & $\begin{array}{l}\text { Standard home } \\
\text { rehabilitation }\end{array}$ & Moderate & $\begin{array}{l}\text { Knee ROM } \\
\text { (active/passive } \\
\text { unspecified): } \\
\text { Extension (deg) }\end{array}$ & $4 \mathrm{mo}$ & 100 & $-3.4(\mathrm{NR})$ & 98 & $-3.6(N R)$ & $0.01(-1,1)$ & NR \\
\hline & $\begin{array}{l}\text { In-home } \\
\text { Telerehabilitation }\end{array}$ & $\begin{array}{l}\text { Standard home } \\
\text { rehabilitation }\end{array}$ & Moderate & $\begin{array}{l}\text { Knee ROM } \\
\text { (active/passive } \\
\text { unspecified): Flexion } \\
\text { (deg) }\end{array}$ & $4 \mathrm{mo}$ & 100 & 112.4 (NR) & 98 & 111.5 (NR) & $1.1(-2.1,4.3)$ & NR \\
\hline \multirow[t]{2}{*}{$\begin{array}{l}\text { Moutzouri, } \\
2018, \\
29473481 \\
\text { Greece }\end{array}$} & $\begin{array}{l}\text { Early self- } \\
\text { managed focal } \\
\text { sensorimotor } \\
\text { exercise training }\end{array}$ & $\begin{array}{l}\text { Functional } \\
\text { exercise } \\
\text { training }\end{array}$ & Moderate & $\begin{array}{l}\text { Active knee ROM } \\
\text { extension: (deg) }\end{array}$ & $3.5 \mathrm{mo}$ & 25 & $0.2(1.1)$ & 26 & $-1.6(0.9)$ & $1.8(1.4,2.2)^{\mathrm{B}}$ & ns \\
\hline & $\begin{array}{l}\text { Early self- } \\
\text { managed focal } \\
\text { sensorimotor } \\
\text { exercise training }\end{array}$ & $\begin{array}{l}\text { Functional } \\
\text { exercise } \\
\text { training }\end{array}$ & Moderate & $\begin{array}{l}\text { Active knee ROM } \\
\text { flexion: (deg) }\end{array}$ & $3.5 \mathrm{mo}$ & 25 & $107.3(6.9)$ & 26 & $103.7(6.9)$ & $3.6(0.9,6.3)^{B}$ & $<0.005$ \\
\hline \multirow{4}{*}{$\begin{array}{l}\text { Petterson, } \\
2009, \\
19177542, \text { USA }\end{array}$} & $\begin{array}{l}\text { Exercise \& } \\
\text { NMES group }\end{array}$ & Exercise & High & $\begin{array}{l}\text { Active knee ROM: } \\
\text { Extension (deg) }\end{array}$ & $3 \mathrm{mo}$ & 92 & $1.8(\mathrm{NR})$ & 78 & 2.0 (NR) & NR & NR \\
\hline & $\begin{array}{l}\text { Exercise \& } \\
\text { NMES group }\end{array}$ & Exercise & High & $\begin{array}{l}\text { Active knee ROM: } \\
\text { Extension (deg) }\end{array}$ & $12 \mathrm{mo}$ & 61 & 0.4 (NR) & 68 & $0.3(\mathrm{NR})$ & NR & NR \\
\hline & $\begin{array}{l}\text { Exercise \& } \\
\text { NMES group }\end{array}$ & Exercise & High & $\begin{array}{l}\text { Active knee ROM: } \\
\text { Flexion (deg) }\end{array}$ & $3 \mathrm{mo}$ & 92 & 114.7 (NR) & 78 & 115.2 (NR) & NR & NR \\
\hline & $\begin{array}{l}\text { Exercise \& } \\
\text { NMES group }\end{array}$ & Exercise & High & $\begin{array}{l}\text { Active knee ROM: } \\
\text { Flexion (deg) }\end{array}$ & $12 \mathrm{mo}$ & 61 & 119 (NR) & 68 & 120.9 (NR) & NR & NR \\
\hline
\end{tabular}




\begin{tabular}{|c|c|c|c|c|c|c|c|c|c|c|c|}
\hline $\begin{array}{l}\text { Study, Year, } \\
\text { PMID, Country }\end{array}$ & Arm 1 & Arm 2 & $\begin{array}{l}\text { Overall } \\
\text { RoB }\end{array}$ & $\begin{array}{l}\text { Outcome } \\
\text { Measurement }\end{array}$ & $\begin{array}{l}\text { Time } \\
\text { Point }^{\mathrm{A}}\end{array}$ & $\begin{array}{l}\text { N Arm } \\
1\end{array}$ & $\begin{array}{l}\text { Arm 1, Mean } \\
\text { (SD) }\end{array}$ & $\begin{array}{l}\text { N Arm } \\
2\end{array}$ & $\begin{array}{l}\text { Arm 2, Mean } \\
\text { (SD) }\end{array}$ & $\begin{array}{l}\text { Effect Size } \\
(95 \% \mathrm{Cl})\end{array}$ & $\begin{array}{l}\text { Reported } \\
\text { p-Value }\end{array}$ \\
\hline \multirow[t]{2}{*}{$\begin{array}{l}\text { Schache, 2019, } \\
31208916, \\
\text { Australia }\end{array}$} & $\begin{array}{l}\text { Standard } \\
\text { rehabilitation and } \\
\text { hip } \\
\text { strengthening } \\
\text { exercises }\end{array}$ & $\begin{array}{l}\text { Standard } \\
\text { rehabilitation } \\
\text { plus general } \\
\text { functional } \\
\text { exercise }\end{array}$ & Moderate & $\begin{array}{l}\text { Knee ROM extension } \\
\text { (deg) }\end{array}$ & $6.5 \mathrm{mo}$ & 48 & $0(1)$ & 48 & $0(2)$ & $-1(-3,2)$ & NR \\
\hline & $\begin{array}{l}\text { Standard } \\
\text { rehabilitation and } \\
\text { hip } \\
\text { strengthening } \\
\text { exercises }\end{array}$ & $\begin{array}{l}\text { Standard } \\
\text { rehabilitation } \\
\text { plus general } \\
\text { functional } \\
\text { exercise }\end{array}$ & Moderate & $\begin{array}{l}\text { Knee ROM flexion } \\
\text { (deg) }\end{array}$ & $6.5 \mathrm{mo}$ & 48 & $121(6)$ & 48 & $118(9)$ & $1(-4,6)$ & NR \\
\hline \multirow[t]{2}{*}{$\begin{array}{l}\text { Tousignant, } \\
2011, \\
21398389, \\
\text { Canada }\end{array}$} & Telerehabilitation & $\begin{array}{l}\text { Home } \\
\text { care/outpatient } \\
\text { clinic }\end{array}$ & Moderate & $\begin{array}{l}\text { Knee ROM } \\
\text { (active/passive } \\
\text { unspecified): } \\
\text { Extension (deg) }\end{array}$ & $4 \mathrm{mo}$ & 21 & $-2.1(\mathrm{NR})$ & 20 & $-1.8(\mathrm{NR})$ & NR & NR \\
\hline & Telerehabilitation & $\begin{array}{l}\text { Home } \\
\text { care/outpatient } \\
\text { clinic }\end{array}$ & Moderate & $\begin{array}{l}\text { Knee ROM } \\
\text { (active/passive } \\
\text { unspecified): Flexion } \\
\text { (deg) }\end{array}$ & $4 \mathrm{mo}$ & 21 & $115.2(\mathrm{NR})$ & 20 & $109.7(\mathrm{NR})$ & NR & NR \\
\hline \multirow{4}{*}{$\begin{array}{l}\text { Vuorenmaa, } \\
2014, \\
24241606, \\
\text { Finland }\end{array}$} & Home exercise & Control & Moderate & $\begin{array}{l}\text { Active knee ROM: } \\
\text { Extension deficit (deg) }\end{array}$ & $12 \mathrm{mo}$ & 53 & $14.9(\mathrm{NR})$ & 55 & $14.3(\mathrm{NR})$ & NR & NR \\
\hline & Home exercise & Control & Moderate & $\begin{array}{l}\text { Passive knee ROM } \\
\text { Extension deficit (deg) }\end{array}$ & $12 \mathrm{mo}$ & 53 & 8.7 (NR) & 55 & $7.8(\mathrm{NR})$ & NR & NR \\
\hline & Home exercise & Control & Moderate & $\begin{array}{l}\text { Active knee ROM: } \\
\text { Flexion (deg) }\end{array}$ & $12 \mathrm{mo}$ & 53 & $113.4(\mathrm{NR})$ & 55 & $114.8(\mathrm{NR})$ & NR & 0.98 \\
\hline & Home exercise & Control & Moderate & $\begin{array}{l}\text { Passive knee ROM: } \\
\text { Flexion (deg) }\end{array}$ & $12 \mathrm{mo}$ & 53 & $117.2(\mathrm{NR})$ & 55 & $116.9(\mathrm{NR})$ & NR & 0.86 \\
\hline
\end{tabular}

Statistically significant effect sizes are in bold text. In cases where calculated effect size confidence intervals were not-statistically significant but reported p-values were, we deferred to reported p-values and still bolded results.

Abbreviations: Adj MD = adjusted mean difference, $\mathrm{CI}=$ confidence interval, $\mathrm{deg}=\mathrm{deg}, \mathrm{mo}=$ month, $\mathrm{ns}=$ not significant, $\mathrm{NR}=$ not reported, $\mathrm{PMID}=\mathrm{PubMed}$ identifier, RoB $=$ risk of bias, $\mathrm{ROM}=$ range of motion, $\mathrm{SD}=$ standard deviation.

\footnotetext{
A Time from surgery

${ }^{B}$ Calculated
} 
Table 45. Post-acute rehabilitation versus various controls for total knee arthroplasty - continuous outcomes, muscle strength and

\begin{tabular}{|c|c|c|c|c|c|c|c|c|c|c|c|}
\hline $\begin{array}{l}\text { Study, Year, } \\
\text { PMID, Country }\end{array}$ & Arm 1 & Arm 2 & $\begin{array}{l}\text { Overall } \\
\text { RoB }\end{array}$ & $\begin{array}{l}\text { Outcome } \\
\text { Measurement }\end{array}$ & $\begin{array}{l}\text { Time } \\
\text { Point }^{\mathrm{A}}\end{array}$ & $\begin{array}{l}\text { N Arm } \\
1\end{array}$ & $\begin{array}{l}\text { Arm 1, } \\
\text { Mean } \\
\text { (SD) }\end{array}$ & $\begin{array}{l}\text { N Arm } \\
2\end{array}$ & $\begin{array}{l}\text { Arm 2, } \\
\text { Mean } \\
\text { (SD) }\end{array}$ & $\begin{array}{l}\text { Effect Size } \\
(95 \% \mathrm{Cl})\end{array}$ & $\begin{array}{l}\text { Reportec } \\
\text { p-Value }\end{array}$ \\
\hline \multirow[t]{9}{*}{$\begin{array}{l}\text { Bade, 2017, } \\
\text { 27813347, USA }\end{array}$} & $\begin{array}{l}\text { High-intensity } \\
\text { progressive } \\
\text { rehabilitation }\end{array}$ & $\begin{array}{l}\text { Low-intensity } \\
\text { rehabilitation }\end{array}$ & Moderate & $\begin{array}{l}\text { Strength: Isometric } \\
\text { peak contraction of } \\
\text { the hamstring }(\mathrm{Nm} / \mathrm{kg})\end{array}$ & $3 \mathrm{mo}$ & 77 & $0.76(0.28)$ & 76 & $0.74(0.26)$ & $0.02(-0.04,0.1)^{\mathrm{B}}$ & NR \\
\hline & $\begin{array}{l}\text { High-intensity } \\
\text { progressive } \\
\text { rehabilitation }\end{array}$ & $\begin{array}{l}\text { Low-intensity } \\
\text { rehabilitation }\end{array}$ & Moderate & $\begin{array}{l}\text { Strength: Isometric } \\
\text { peak contraction of } \\
\text { the hamstring }(\mathrm{Nm} / \mathrm{kg})\end{array}$ & $6 \mathrm{mo}$ & 71 & $0.8(0.29)$ & 71 & $0.8(0.27)$ & $0(-0.1,0.1)^{\mathrm{B}}$ & NR \\
\hline & $\begin{array}{l}\text { High-intensity } \\
\text { progressive } \\
\text { rehabilitation }\end{array}$ & $\begin{array}{l}\text { Low-intensity } \\
\text { rehabilitation }\end{array}$ & Moderate & $\begin{array}{l}\text { Strength: Isometric } \\
\text { peak contraction of } \\
\text { the hamstring }(\mathrm{Nm} / \mathrm{kg})\end{array}$ & $12 \mathrm{mo}$ & 70 & $0.84(0.31)$ & 67 & $0.85(0.29)$ & $-0.01(-0.1,0.1)^{\mathrm{B}}$ & NR \\
\hline & $\begin{array}{l}\text { High-intensity } \\
\text { progressive } \\
\text { rehabilitation }\end{array}$ & $\begin{array}{l}\text { Low-intensity } \\
\text { rehabilitation }\end{array}$ & Moderate & $\begin{array}{l}\text { Strength: Isometric } \\
\text { peak contraction of } \\
\text { the quadricep }(\mathrm{Nm} / \mathrm{kg})\end{array}$ & $3 \mathrm{mo}$ & 77 & $1.21(0.42)$ & 76 & $1.15(0.4)$ & $0.03(-0.1,0.2)^{B}$ & NR \\
\hline & $\begin{array}{l}\text { High-intensity } \\
\text { progressive } \\
\text { rehabilitation }\end{array}$ & $\begin{array}{l}\text { Low-intensity } \\
\text { rehabilitation }\end{array}$ & Moderate & $\begin{array}{l}\text { Strength: Isometric } \\
\text { peak contraction of } \\
\text { the quadricep }(\mathrm{Nm} / \mathrm{kg})\end{array}$ & $6 \mathrm{mo}$ & 71 & $1.35(0.46)$ & 71 & $1.35(0.4)$ & $0(-0.1,0.1)^{\mathrm{B}}$ & NR \\
\hline & $\begin{array}{l}\text { High-intensity } \\
\text { progressive } \\
\text { rehabilitation }\end{array}$ & $\begin{array}{l}\text { Low-intensity } \\
\text { rehabilitation }\end{array}$ & Moderate & $\begin{array}{l}\text { Strength: Isometric } \\
\text { peak contraction of } \\
\text { the quadricep }(\mathrm{Nm} / \mathrm{kg})\end{array}$ & $12 \mathrm{mo}$ & 70 & $1.42(0.47)$ & 67 & $1.43(0.44)$ & $-0.01(-0.1,0.1)^{\mathrm{B}}$ & NR \\
\hline & $\begin{array}{l}\text { High-intensity } \\
\text { progressive } \\
\text { rehabilitation }\end{array}$ & $\begin{array}{l}\text { Low-intensity } \\
\text { rehabilitation }\end{array}$ & Moderate & $\begin{array}{l}\text { Quadriceps activation } \\
(\%)\end{array}$ & $3 \mathrm{mo}$ & 67 & $\begin{array}{l}82.77 \\
(10.78)\end{array}$ & 63 & $\begin{array}{l}79.94 \\
(13.78)\end{array}$ & $2.8(-0.2,5.9)^{\mathrm{B}}$ & NR \\
\hline & $\begin{array}{l}\text { High-intensity } \\
\text { progressive } \\
\text { rehabilitation }\end{array}$ & $\begin{array}{l}\text { Low-intensity } \\
\text { rehabilitation }\end{array}$ & Moderate & $\begin{array}{l}\text { Quadriceps activation } \\
(\%)\end{array}$ & $6 \mathrm{mo}$ & 61 & $\begin{array}{l}80.87 \\
(12.01)\end{array}$ & 62 & $\begin{array}{l}82.92 \\
(9.55)\end{array}$ & $-2.1(-4.8,0.7)^{\mathrm{B}}$ & NR \\
\hline & $\begin{array}{l}\text { High-intensity } \\
\text { progressive } \\
\text { rehabilitation }\end{array}$ & $\begin{array}{l}\text { Low-intensity } \\
\text { rehabilitation }\end{array}$ & Moderate & $\begin{array}{l}\text { Quadriceps activation } \\
(\%)\end{array}$ & $12 \mathrm{mo}$ & 62 & $\begin{array}{l}83.39 \\
(11.73)\end{array}$ & 59 & $\begin{array}{l}83.73 \\
(10.12)\end{array}$ & $-0.3(-3.1,2.4)^{\mathrm{B}}$ & NR \\
\hline \multirow[t]{2}{*}{$\begin{array}{l}\text { Bily, 2016, } \\
26763947, \\
\text { Austria }\end{array}$} & Leg-press group & $\begin{array}{l}\text { Physiotherapy } \\
\text { group }\end{array}$ & High & $\begin{array}{l}\text { Strength: Isometric } \\
\text { peak knee extension } \\
(\mathrm{Nm} / \mathrm{kg})\end{array}$ & $3 \mathrm{mo}$ & 26 & $1.0(0.09)$ & 29 & $0.9(0.06)$ & NR & 0.16 \\
\hline & Leg-press group & $\begin{array}{l}\text { Physiotherapy } \\
\text { group }\end{array}$ & High & $\begin{array}{l}\text { Strength: Leg press } \\
(\mathrm{N} / \mathrm{kg})\end{array}$ & $3 \mathrm{mo}$ & 26 & $10.3(1.06)$ & 29 & $9.1(0.7)$ & NR & 0.19 \\
\hline $\begin{array}{l}\text { Bruun-Olsen, } \\
2013, \\
23614370, \\
\text { Norway }\end{array}$ & $\begin{array}{l}\text { Walking-skill } \\
\text { group }\end{array}$ & $\begin{array}{l}27 \text { individual } \\
\text { physiotherapy } \\
\text { sessions }\end{array}$ & Moderate & $\begin{array}{l}\text { Strength/function: } \\
\text { Index of muscle } \\
\text { function }\end{array}$ & $9 \mathrm{mo}$ & 29 & $11(7)$ & 28 & $12(7)$ & Adj MD -1 $(-3,2)$ & NR \\
\hline \multirow{2}{*}{$\begin{array}{l}\text { Fransen, 2017, } \\
27868384, \\
\text { Australia }\end{array}$} & $\begin{array}{l}\text { Outpatient } \\
\text { exercise group }\end{array}$ & Usual care & Moderate & $\begin{array}{l}\text { Strength: Isometric } \\
\text { knee extension }(\mathrm{Nm})\end{array}$ & $12 \mathrm{mo}$ & 112 & $77.2(3.3)$ & 98 & $\begin{array}{l}\text { Mean (SE) } \\
74.6(3.4)\end{array}$ & NR & NR \\
\hline & $\begin{array}{l}\text { Outpatient } \\
\text { exercise group }\end{array}$ & Usual care & Moderate & $\begin{array}{l}\text { Strength: Isometric } \\
\text { knee flexion }(\mathrm{Nm})\end{array}$ & $12 \mathrm{mo}$ & 112 & $42.8(2.3)$ & 98 & $\begin{array}{l}\text { Mean (SE) } \\
44.3(2.5)\end{array}$ & NR & NR \\
\hline \multirow{2}{*}{$\begin{array}{l}\text { Heikkilä, 2017, } \\
\text { 28119232, } \\
\text { Finland }\end{array}$} & Home exercise & Control & High & $\begin{array}{l}\text { Strength: Isometric } \\
\text { knee extension }(\mathrm{N})\end{array}$ & $14 \mathrm{mo}$ & 53 & $350(130)$ & 55 & $280(150)$ & $70(32.5,107.5)^{B}$ & NR \\
\hline & Home exercise & Control & High & $\begin{array}{l}\text { Strength: Isometric } \\
\text { knee flexion }(\mathrm{N})\end{array}$ & $14 \mathrm{mo}$ & 53 & $150(50)$ & 55 & $120(50)$ & $30(16.7,43.3)^{B}$ & NR \\
\hline
\end{tabular}




\begin{tabular}{|c|c|c|c|c|c|c|c|c|c|c|c|}
\hline $\begin{array}{l}\text { Study, Year, } \\
\text { PMID, Country }\end{array}$ & Arm 1 & Arm 2 & $\begin{array}{l}\text { Overall } \\
\text { RoB }\end{array}$ & $\begin{array}{l}\text { Outcome } \\
\text { Measurement }\end{array}$ & $\begin{array}{l}\text { Time } \\
\text { Point }^{\mathrm{A}}\end{array}$ & $\begin{array}{l}\text { N Arm } \\
1\end{array}$ & $\begin{array}{l}\text { Arm 1, } \\
\text { Mean } \\
\text { (SD) }\end{array}$ & $\begin{array}{l}\text { N Arm } \\
2\end{array}$ & $\begin{array}{l}\text { Arm 2, } \\
\text { Mean } \\
\text { (SD) }\end{array}$ & $\begin{array}{l}\text { Effect Size } \\
(95 \% \mathrm{CI})\end{array}$ & $\begin{array}{l}\text { Reported } \\
\text { p-Value }\end{array}$ \\
\hline \multirow[t]{8}{*}{$\begin{array}{l}\text { Kauppila, 2010, } \\
20354057, \\
\text { Finland }\end{array}$} & $\begin{array}{l}\text { Multidisciplinary } \\
\text { rehabilitation } \\
\text { group }\end{array}$ & Control & Moderate & $\begin{array}{l}\text { Strength: Peak torque } \\
\text { extension }(\mathrm{Nm})^{\mathrm{D}}\end{array}$ & $6 \mathrm{mo}$ & 29 & $88.8(25)$ & 33 & $93.8(30.4)$ & $-5(-14.8,4.8)^{\mathrm{B}}$ & NR \\
\hline & $\begin{array}{l}\text { Multidisciplinary } \\
\text { rehabilitation } \\
\text { group }\end{array}$ & Control & Moderate & $\begin{array}{l}\text { Strength: Peak torque } \\
\text { extension }(\mathrm{Nm})^{\mathrm{D}}\end{array}$ & $12 \mathrm{mo}$ & 29 & $98(28.1)$ & 32 & $99.2(39.1)$ & $-1.2(-13.4,11)^{\mathrm{B}}$ & NR \\
\hline & $\begin{array}{l}\text { Multidisciplinary } \\
\text { rehabilitation } \\
\text { group }\end{array}$ & Control & Moderate & $\begin{array}{l}\text { Strength: Relative } \\
\text { peak torque extension } \\
(\mathrm{Nm} / \mathrm{kg})\end{array}$ & $6 \mathrm{mo}$ & 28 & $1.15(0.44)$ & 29 & $1.14(0.39)$ & $0.01(-0.1,0.2)^{\mathrm{B}}$ & NR \\
\hline & $\begin{array}{l}\text { Multidisciplinary } \\
\text { rehabilitation } \\
\text { group }\end{array}$ & Control & Moderate & $\begin{array}{l}\text { Strength: Relative } \\
\text { peak torque extension } \\
(\mathrm{Nm} / \mathrm{kg})\end{array}$ & $12 \mathrm{mo}$ & 29 & $1.23(0.43)$ & 32 & $1.21(0.49)$ & $0.02(-0.1,0.2)^{\mathrm{B}}$ & NR \\
\hline & $\begin{array}{l}\text { Multidisciplinary } \\
\text { rehabilitation } \\
\text { group }\end{array}$ & Control & Moderate & $\begin{array}{l}\text { Strength: Relative } \\
\text { peak torque flexion } \\
(\mathrm{Nm} / \mathrm{kg})\end{array}$ & $6 \mathrm{mo}$ & 28 & $0.80(0.27)$ & 29 & $0.83(0.24)$ & $-0.03(-0.1,0.1)^{B}$ & NR \\
\hline & $\begin{array}{l}\text { Multidisciplinary } \\
\text { rehabilitation } \\
\text { group }\end{array}$ & Control & Moderate & $\begin{array}{l}\text { Strength: Relative } \\
\text { peak torque flexion } \\
(\mathrm{Nm} / \mathrm{kg})\end{array}$ & $12 \mathrm{mo}$ & 29 & $0.85(0.3)$ & 32 & $0.78(0.21)$ & $0.1(-0.03,0.2)^{\mathrm{B}}$ & NR \\
\hline & $\begin{array}{l}\text { Multidisciplinary } \\
\text { rehabilitation } \\
\text { group }\end{array}$ & Control & Moderate & $\begin{array}{l}\text { Strength: Hamstring/ } \\
\text { quadricep ratio } \\
\text { (proportion) }\end{array}$ & $6 \mathrm{mo}$ & 29 & $0.74(0.24)$ & 33 & $0.77(0.22)$ & $-0.03(-0.1,0.1)^{B}$ & NR \\
\hline & $\begin{array}{l}\text { Multidisciplinary } \\
\text { rehabilitation } \\
\text { group }\end{array}$ & Control & Moderate & $\begin{array}{l}\text { Strength: Hamstring/ } \\
\text { quadricep ratio } \\
\text { (proportion) }\end{array}$ & $12 \mathrm{mo}$ & 29 & $0.70(0.18)$ & 31 & $0.79(0.71)$ & $-0.1(-0.3,0.1)^{B}$ & NR \\
\hline \multirow[t]{4}{*}{$\begin{array}{l}\text { Madsen, 2013, } \\
\text { 23651717, } \\
\text { Denmark }\end{array}$} & $\begin{array}{l}\text { Group-based } \\
\text { rehabilitation }\end{array}$ & $\begin{array}{l}\text { Supervised home- } \\
\text { exercises }\end{array}$ & High & $\begin{array}{l}\text { Strength: Asymmetry } \\
\text { leg extensor power } \\
(\mathrm{W} / \mathrm{kg})^{\mathrm{E}}\end{array}$ & $3 \mathrm{mo}$ & 36 & NR (NR) & 34 & NR (NR) & NR & 0.1 \\
\hline & $\begin{array}{l}\text { Group-based } \\
\text { rehabilitation }\end{array}$ & $\begin{array}{l}\text { Supervised home- } \\
\text { exercises }\end{array}$ & High & $\begin{array}{l}\text { Strength: Asymmetry } \\
\text { leg extensor power } \\
(\mathrm{W} / \mathrm{kg})^{\mathrm{E}}\end{array}$ & $6 \mathrm{mo}$ & 36 & NR (NR) & 32 & NR (NR) & NR & 0.5 \\
\hline & $\begin{array}{l}\text { Group-based } \\
\text { rehabilitation }\end{array}$ & $\begin{array}{l}\text { Supervised home- } \\
\text { exercises }\end{array}$ & High & $\begin{array}{l}\text { Strength: Peak force } \\
(\mathrm{W} / \mathrm{kg})\end{array}$ & $3 \mathrm{mo}$ & 36 & NR (NR) & 34 & NR (NR) & NR & 0.2 \\
\hline & $\begin{array}{l}\text { Group-based } \\
\text { rehabilitation }\end{array}$ & $\begin{array}{l}\text { Supervised home- } \\
\text { exercises }\end{array}$ & High & $\begin{array}{l}\text { Strength: Peak force } \\
(\mathrm{W} / \mathrm{kg})\end{array}$ & $6 \mathrm{mo}$ & 36 & NR (NR) & 32 & NR (NR) & NR & 0.1 \\
\hline \multirow[t]{2}{*}{$\begin{array}{l}\text { Minns Lowe, } \\
2012 \text {, } \\
22180446, \text { UK }\end{array}$} & $\begin{array}{l}\text { Home-visit } \\
\text { physiotherapy }\end{array}$ & Usual care & High & $\begin{array}{l}\text { Strength: Leg } \\
\text { extension power } \\
(\mathrm{W} / \mathrm{kg})\end{array}$ & $3 \mathrm{mo}$ & 42 & $\begin{array}{l}\text { Median } \\
0.7 \text { (NR) }\end{array}$ & 39 & $\begin{array}{l}\text { Median } \\
0.72(N R)\end{array}$ & NR & NR \\
\hline & $\begin{array}{l}\text { Home-visit } \\
\text { physiotherapy }\end{array}$ & Usual care & High & $\begin{array}{l}\text { Strength: Leg } \\
\text { extension power } \\
(\mathrm{W} / \mathrm{kg})\end{array}$ & $12 \mathrm{mo}$ & 38 & $\begin{array}{l}\text { Median } \\
0.87(N R)\end{array}$ & 42 & $\begin{array}{l}\text { Median } \\
0.87(N R)\end{array}$ & NR & NR \\
\hline \multirow[t]{2}{*}{$\begin{array}{l}\text { Moffet, 2015, } \\
\text { 26178888, } \\
\text { Canada }\end{array}$} & $\begin{array}{l}\text { In-home } \\
\text { Telerehabilitation }\end{array}$ & $\begin{array}{l}\text { Standard home } \\
\text { rehabilitation }\end{array}$ & Moderate & $\begin{array}{l}\text { Strength: Isokinetic } \\
\text { knee extension at } 30 \\
\text { deg flexion }(\mathrm{Nm})\end{array}$ & $4 \mathrm{mo}$ & 100 & 74.6 (NR) & 98 & 76.4 (NR) & $0.4(-9.7,10.4)$ & NR \\
\hline & $\begin{array}{l}\text { In-home } \\
\text { Telerehabilitation }\end{array}$ & $\begin{array}{l}\text { Standard home } \\
\text { rehabilitation }\end{array}$ & Moderate & $\begin{array}{l}\text { Strength: Isokinetic } \\
\text { knee extension at } 60 \\
\text { deg flexion }(\mathrm{Nm})\end{array}$ & $4 \mathrm{mo}$ & 100 & $\begin{array}{l}105.4 \\
\text { (NR) }\end{array}$ & 98 & $\begin{array}{l}105.7 \\
(N R)\end{array}$ & $-1.1(-7.9,5.7)$ & NR \\
\hline
\end{tabular}




\begin{tabular}{|c|c|c|c|c|c|c|c|c|c|c|c|}
\hline $\begin{array}{l}\text { Study, Year, } \\
\text { PMID, Country }\end{array}$ & Arm 1 & Arm 2 & $\begin{array}{l}\text { Overall } \\
\text { RoB }\end{array}$ & $\begin{array}{l}\text { Outcome } \\
\text { Measurement }\end{array}$ & $\begin{array}{l}\text { Time } \\
\text { Point }^{\mathrm{A}}\end{array}$ & $\begin{array}{l}\text { N Arm } \\
1\end{array}$ & $\begin{array}{l}\text { Arm 1, } \\
\text { Mean } \\
\text { (SD) }\end{array}$ & $\begin{array}{l}\text { N Arm } \\
2\end{array}$ & $\begin{array}{l}\text { Arm 2, } \\
\text { Mean } \\
\text { (SD) }\end{array}$ & $\begin{array}{l}\text { Effect Size } \\
(95 \% \mathrm{Cl})\end{array}$ & $\begin{array}{l}\text { Reported } \\
\text { p-Value }\end{array}$ \\
\hline & $\begin{array}{l}\text { In-home } \\
\text { Telerehabilitation }\end{array}$ & $\begin{array}{l}\text { Standard home } \\
\text { rehabilitation }\end{array}$ & Moderate & $\begin{array}{l}\text { Strength: Isokinetic } \\
\text { knee flexion at } 30 \text { deg } \\
\text { flexion }(\mathrm{Nm})\end{array}$ & $4 \mathrm{mo}$ & 100 & $74.6(\mathrm{NR})$ & 98 & 76.4 (NR) & $-1.1(-7.9,5.7)$ & NR \\
\hline & $\begin{array}{l}\text { In-home } \\
\text { Telerehabilitation }\end{array}$ & $\begin{array}{l}\text { Standard home } \\
\text { rehabilitation }\end{array}$ & Moderate & $\begin{array}{l}\text { Strength: Isokinetic } \\
\text { knee flexion at } 60 \text { deg } \\
\text { flexion }(\mathrm{Nm})\end{array}$ & $4 \mathrm{mo}$ & 100 & $\begin{array}{l}105.4 \\
(N R)\end{array}$ & 98 & $\begin{array}{l}105.7 \\
(\mathrm{NR})\end{array}$ & $0.4(-9.7,10.4)$ & NR \\
\hline $\begin{array}{l}\text { Moutzouri, } \\
2018, \\
29473481, \\
\text { Greece }\end{array}$ & $\begin{array}{l}\text { Early self- } \\
\text { managed focal } \\
\text { sensorimotor } \\
\text { exercise training }\end{array}$ & $\begin{array}{l}\text { Functional } \\
\text { exercise training }\end{array}$ & Moderate & $\begin{array}{l}\text { Strength: Peak force } \\
\text { (N) }\end{array}$ & $\begin{array}{l}3.5 \\
\mathrm{mo}^{\mathrm{F}}\end{array}$ & 25 & $67.5(17.4)$ & 26 & $55.4(23.5)$ & $12.1(3.9,20.2)^{B}$ & NR \\
\hline \multirow[t]{2}{*}{$\begin{array}{l}\text { Petterson, } \\
2009, \\
19177542, \text { USA }\end{array}$} & $\begin{array}{l}\text { Exercise \& } \\
\text { NMES group }\end{array}$ & Exercise & High & $\begin{array}{l}\text { Normalized maximum } \\
\text { voluntary isometric } \\
\text { contraction }\left(\mathrm{N} / \mathrm{kg} / \mathrm{m}^{2}\right)\end{array}$ & $3 \mathrm{mo}$ & 92 & $\begin{array}{l}17.35 \\
\text { (NR) }\end{array}$ & 78 & $\begin{array}{l}19.05 \\
(\mathrm{NR})\end{array}$ & NR & NR \\
\hline & $\begin{array}{l}\text { Exercise \& } \\
\text { NMES group }\end{array}$ & Exercise & High & $\begin{array}{l}\text { Normalized maximum } \\
\text { voluntary isometric } \\
\text { contraction }\left(\mathrm{N} / \mathrm{kg} / \mathrm{m}^{2}\right)\end{array}$ & $12 \mathrm{mo}$ & 61 & $\begin{array}{l}20.60 \\
(N R)\end{array}$ & 68 & $\begin{array}{l}22.64 \\
(N R)\end{array}$ & NR & NR \\
\hline \multirow[t]{2}{*}{$\begin{array}{l}\text { Schache, 2019, } \\
\text { 31208916, } \\
\text { Australia }\end{array}$} & $\begin{array}{l}\text { Standard } \\
\text { rehabilitation and } \\
\text { hip } \\
\text { strengthening } \\
\text { exercises }\end{array}$ & $\begin{array}{l}\text { Standard } \\
\text { rehabilitation plus } \\
\text { general functional } \\
\text { exercise }\end{array}$ & Moderate & $\begin{array}{l}\text { Strength: Quadricep } \\
\left(\mathrm{N} / \mathrm{kg} / \mathrm{m}^{2}\right)^{\mathrm{G}}\end{array}$ & $6 \mathrm{mo}$ & 48 & $4.0(1.6)$ & 48 & $4.1(1.7)$ & $0.0(-0.5,0.5)$ & NR \\
\hline & $\begin{array}{l}\text { Standard } \\
\text { rehabilitation and } \\
\text { hip } \\
\text { strengthening } \\
\text { exercises }\end{array}$ & $\begin{array}{l}\text { Standard } \\
\text { rehabilitation plus } \\
\text { general functional } \\
\text { exercise }\end{array}$ & Moderate & $\begin{array}{l}\text { Strength: Quadricep } \\
\left(\mathrm{N} / \mathrm{kg} / \mathrm{m}^{2}\right)\end{array}$ & $6 \mathrm{mo}$ & 48 & $\begin{array}{l}6.6 \\
(3.0)\end{array}$ & 48 & $\begin{array}{l}6.9 \\
(3.4)\end{array}$ & $-0.4(-1.6,0.8)$ & NR \\
\hline \multirow[t]{2}{*}{$\begin{array}{l}\text { Shanb, 2014, } \\
\text { CN-01041112, } \\
\text { Saudi Arabia }\end{array}$} & $\begin{array}{l}\text { Active exercise } \\
\text { training program } \\
\& \text { biofeedback }\end{array}$ & $\begin{array}{l}\text { Active exercise } \\
\text { training program }\end{array}$ & High & $\begin{array}{l}\text { Central activation } \\
\text { ratio }^{H}(0-1)\end{array}$ & $4 \mathrm{mo}$ & 21 & $0.89(0.04)$ & 24 & $0.93(0.03)$ & $\begin{array}{l}-0.04(-0.1,- \\
0.03)^{\mathrm{B}}\end{array}$ & 0.97 \\
\hline & $\begin{array}{l}\text { Active exercise } \\
\text { training program } \\
\& \text { biofeedback }\end{array}$ & $\begin{array}{l}\text { Active exercise } \\
\text { training program }\end{array}$ & High & $\begin{array}{l}\text { Strength: Quadriceps } \\
\text { isometric peak torque }\end{array}$ & $4 \mathrm{mo}$ & 21 & $2.31(0.66)$ & 24 & $2.3(0.32)$ & $0.01(-0.2,0.3)^{\mathrm{B}}$ & 0.01 \\
\hline \multirow{2}{*}{$\begin{array}{l}\text { Vuorenmaa, } \\
2014, \\
24241606, \\
\text { Finland } \\
\end{array}$} & Home exercise & Control & Moderate & $\begin{array}{l}\text { Strength: Isometric } \\
\text { knee extension }(\mathrm{Kg})\end{array}$ & $12 \mathrm{mo}$ & 53 & 33.3 (NR) & 55 & 27.9 (NR) & NR & 0.50 \\
\hline & Home exercise & Control & Moderate & $\begin{array}{l}\text { Strength: Isometric } \\
\text { knee flexion }(\mathrm{Kg})\end{array}$ & $12 \mathrm{mo}$ & 53 & 14.7 (NR) & 55 & 12.5 (NR) & NR & 0.009 \\
\hline
\end{tabular}

Statistically significant effect sizes are in bold text. In cases where calculated effect size confidence intervals were not-statistically significant but reported p-values were, we deferred to reported p-values and still bolded results.

Abbreviations: Adj MD = adjusted mean difference, $\mathrm{CI}=$ confidence interval, $\mathrm{N}=$ Newton, $\mathrm{Nm}=$ Newton meters, $\mathrm{NR}=$ not reported, $\mathrm{PMID}=\mathrm{PubMed}$ identifier, RoB $=$ risk of bias, $\mathrm{SD}=$ standard deviation, $\mathrm{SE}=$ standard error.

A Time from surgery

B Calculated

${ }^{\mathrm{C}}$ Functional test comprising of 13 items evaluating muscle strength, balance and endurance in lying, sitting and standing positions, sum score is 40 , best is 0 
D Measured with the Lido Active Multijoint Rehabilitation System

E Measured using the Nottingham Leg Extensor Power Rig

F Defined as 14 weeks

G Normalized to body mass index

${ }^{\mathrm{H}}$ Larger is better; central activation ratio of 1.0 indicates complete activation of the muscle with no increase of the maximal voluntary force being detected during the electrical stimulation

Table 46. Post-acute rehabilitation versus various controls for total knee arthroplasty - continuous outcomes, energy and vigor

\begin{tabular}{|c|c|c|c|c|c|c|c|c|c|c|c|}
\hline $\begin{array}{l}\text { Study, Year, } \\
\text { PMID, Country }\end{array}$ & Arm 1 & Arm 2 & Overall RoB & $\begin{array}{l}\text { Outcome } \\
\text { Measurement }\end{array}$ & $\begin{array}{l}\text { Time } \\
\text { Point }^{\mathrm{A}}\end{array}$ & $\begin{array}{l}\text { N Arm } \\
1\end{array}$ & $\begin{array}{l}\text { Arm 1, Mean } \\
\text { (SD) }\end{array}$ & $\begin{array}{l}\text { N Arm } \\
2\end{array}$ & $\begin{array}{l}\text { Arm 2, Mean } \\
\text { (SD) }\end{array}$ & $\begin{array}{l}\text { Effect Size }(95 \% \\
\mathrm{CI})\end{array}$ & $\begin{array}{l}\text { Reported } \\
\text { p-Value }\end{array}$ \\
\hline $\begin{array}{l}\text { Mitchell, 2005, } \\
\text { 15869558, UK }\end{array}$ & Home & Hospital & High & SF-36: Vitality $(0-100)$ & $3 \mathrm{mo}$ & 57 & $50.7(19.5)$ & 57 & $48.2(23.7)$ & $3.4(-3.5,10.3)$ & 0.33 \\
\hline
\end{tabular}

Statistically significant effect sizes are in bold text. In ca

deferred to reported p-values and still bolded results.

Abbreviations: $\mathrm{CI}=$ confidence interval, $\mathrm{mo}=$ month, $\mathrm{NR}=$ not reported, PMID $=$ PubMed identifier, RoB $=$ risk of bias, $\mathrm{SF}-36=36-\mathrm{Item}$ short form survey, $\mathrm{SD}=$ standard deviation.

A Time from surgery

Table 47. Post-acute rehabilitation versus various controls for total knee arthroplasty - continuous outcomes, emotional functioning

\begin{tabular}{|c|c|c|c|c|c|c|c|c|c|c|c|}
\hline $\begin{array}{l}\text { Study, Year, } \\
\text { PMID, Country }\end{array}$ & Arm 1 & Arm 2 & $\begin{array}{l}\text { Overall } \\
\text { RoB }\end{array}$ & Outcome Measurement & $\begin{array}{l}\text { Time } \\
\text { Point }^{A}\end{array}$ & $\begin{array}{l}\text { N Arm } \\
1\end{array}$ & $\begin{array}{l}\text { Arm 1, } \\
\text { Mean (SD) }\end{array}$ & $\begin{array}{l}\text { N Arm } \\
2\end{array}$ & $\begin{array}{l}\text { Arm 2, } \\
\text { Mean (SD) }\end{array}$ & $\begin{array}{l}\text { Effect Size } \\
(95 \% \mathrm{CI})\end{array}$ & $\begin{array}{l}\text { Reported } \\
\text { p-Value }\end{array}$ \\
\hline \multirow[t]{3}{*}{$\begin{array}{l}\text { Bade, 2017, } \\
27813347 \text {, USA }\end{array}$} & $\begin{array}{l}\text { High-intensity } \\
\text { progressive } \\
\text { rehabilitation }\end{array}$ & $\begin{array}{l}\text { Low-intensity } \\
\text { rehabilitation }\end{array}$ & Moderate & $\begin{array}{l}\text { SF 12: Mental health } \\
(0-100)\end{array}$ & $3 \mathrm{mo}$ & 75 & $56.73(7.29)$ & 75 & $57.05(6.86)$ & $\begin{array}{l}-0.3(-1.9 \\
1.3)^{\mathrm{B}}\end{array}$ & $>0.05$ \\
\hline & $\begin{array}{l}\text { High-intensity } \\
\text { progressive } \\
\text { rehabilitation }\end{array}$ & $\begin{array}{l}\text { Low-intensity } \\
\text { rehabilitation }\end{array}$ & Moderate & $\begin{array}{l}\text { SF 12: Mental health } \\
(0-100)\end{array}$ & $6 \mathrm{mo}$ & 71 & $55.76(7.26)$ & 68 & $56.64(6.20)$ & $\begin{array}{l}-0.9(-2.5, \\
0.7)^{\mathrm{B}}\end{array}$ & $>0.05$ \\
\hline & $\begin{array}{l}\text { High-intensity } \\
\text { progressive } \\
\text { rehabilitation }\end{array}$ & $\begin{array}{l}\text { Low-intensity } \\
\text { rehabilitation }\end{array}$ & Moderate & $\begin{array}{l}\text { SF 12: Mental health } \\
(0-100)\end{array}$ & $12 \mathrm{mo}$ & 67 & $55.76(6.48)$ & 61 & $57.83(3.58)$ & $\begin{array}{l}-2.1(-3.4,- \\
0.7)^{\mathrm{B}}\end{array}$ & $>0.05$ \\
\hline $\begin{array}{l}\text { Demircioglu, } \\
2015, \\
26355656, \\
\text { Turkey }\end{array}$ & $\begin{array}{l}\text { NMES \& } \\
\text { exercise }\end{array}$ & Exercise & High & $\begin{array}{l}\text { SF-36: Mental health } \\
(0-100)\end{array}$ & $3 \mathrm{mo}$ & 30 & $65.1(12.1)$ & 30 & $60.9(15.8)$ & $\begin{array}{l}4.2(-0.9 \\
9.3)^{\mathrm{B}}\end{array}$ & NR \\
\hline $\begin{array}{l}\text { Fransen, 2017, } \\
27868384, \\
\text { Australia }\end{array}$ & $\begin{array}{l}\text { Outpatient } \\
\text { exercise group }\end{array}$ & Usual care & Moderate & $\begin{array}{l}\text { SF-36: Mental health } \\
(0-100)\end{array}$ & $12 \mathrm{mo}$ & 179 & $54.3(0.7)$ & 169 & Mean 53.1 & NR & NR \\
\hline $\begin{array}{l}\text { Li, 2019, } \\
31003647, \\
\text { China }\end{array}$ & $\begin{array}{l}\text { Tai chi chuan } \\
\text { exercise }\end{array}$ & Control & Moderate & $\begin{array}{l}\text { SF-36: Mental health } \\
(0-100)\end{array}$ & $3 \mathrm{mo}^{\mathrm{C}}$ & 54 & $58.5(1.5)$ & 53 & $54.1(1.7)$ & $\begin{array}{l}4.4(4.0 \\
4.8)^{B}\end{array}$ & 0.03 \\
\hline $\begin{array}{l}\text { Mitchell, 2005, } \\
\text { 15869558, UK }\end{array}$ & Hospital & Home & High & $\begin{array}{l}\text { SF-36: Emotional role } \\
\text { functioning } \\
(0-100)\end{array}$ & $3 \mathrm{mo}$ & 57 & $45.6(44.8)$ & 57 & $48.0(46.7)$ & $\begin{array}{l}\text { Adj MD } 4.1 \\
(-10.9 \\
19.0)\end{array}$ & 0.592 \\
\hline
\end{tabular}




\begin{tabular}{|c|c|c|c|c|c|c|c|c|c|c|c|}
\hline $\begin{array}{l}\text { Study, Year, } \\
\text { PMID, Country }\end{array}$ & Arm 1 & Arm 2 & $\begin{array}{l}\text { Overall } \\
\text { RoB }\end{array}$ & Outcome Measurement & $\begin{array}{l}\text { Time } \\
\text { Point }^{\mathrm{A}}\end{array}$ & $\begin{array}{l}\text { N Arm } \\
1\end{array}$ & $\begin{array}{l}\text { Arm 1, } \\
\text { Mean (SD) }\end{array}$ & $\begin{array}{l}\text { N Arm } \\
2\end{array}$ & $\begin{array}{l}\text { Arm 2, } \\
\text { Mean (SD) }\end{array}$ & $\begin{array}{l}\text { Effect Size } \\
(95 \% \mathrm{Cl})\end{array}$ & $\begin{array}{l}\text { Reported } \\
\text { p-Value }\end{array}$ \\
\hline & Hospital & Home & High & $\begin{array}{l}\text { SF-36: Social functioning } \\
(0-100)\end{array}$ & $3 \mathrm{mo}$ & 57 & $60.8(33.1)$ & 57 & $64.1(26.6)$ & $\begin{array}{l}\text { Adj MD } 6.7 \\
(-3.4,16.7)\end{array}$ & 0.193 \\
\hline & Hospital & Home & High & $\begin{array}{l}\text { SF-36: Mental health } \\
(0-100)\end{array}$ & $3 \mathrm{mo}$ & 57 & $71.2(20.0)$ & 57 & $68.0(20.4)$ & $\begin{array}{l}\text { Adj MD -2.9 } \\
(-9.3,3.5)\end{array}$ & 0.368 \\
\hline \multirow{2}{*}{$\begin{array}{l}\text { Petterson, } \\
2009 \text {, } \\
19177542 \text {, USA }\end{array}$} & $\begin{array}{l}\text { Exercise \& } \\
\text { NMES group }\end{array}$ & Exercise & High & $\begin{array}{l}\text { SF-36: Mental health } \\
(0-100)\end{array}$ & $3 \mathrm{mo}$ & 92 & 56.77 (NR) & 78 & 57.17 (NR) & NR & NR \\
\hline & $\begin{array}{l}\text { Exercise \& } \\
\text { NMES group }\end{array}$ & Exercise & High & $\begin{array}{l}\text { SF-36: Mental health } \\
(0-100)\end{array}$ & $12 \mathrm{mo}$ & 61 & $57.16(\mathrm{NR})$ & 68 & $56.63(\mathrm{NR})$ & NR & NR \\
\hline $\begin{array}{l}\text { Schache, 2019, } \\
31208916, \\
\text { Australia }\end{array}$ & $\begin{array}{l}\text { Standard } \\
\text { rehabilitation \& } \\
\text { hip } \\
\text { strengthening } \\
\text { exercises }\end{array}$ & $\begin{array}{l}\text { Standard } \\
\text { rehabilitation \& } \\
\text { general } \\
\text { functional } \\
\text { exercise }\end{array}$ & Moderate & $\begin{array}{l}\text { SF-36: Mental health } \\
(0-100)\end{array}$ & $6.5 \mathrm{mo}$ & 48 & $57(6)$ & 48 & $55(8)$ & $1(-4,5)$ & NR \\
\hline $\begin{array}{l}\text { Vuorenmaa, } \\
2014, \\
24241606, \\
\text { Finland } \\
\end{array}$ & Home exercise & Control & Moderate & $\begin{array}{l}\text { SF-36: Mental health } \\
(0-100)\end{array}$ & $12 \mathrm{mo}$ & 53 & 47 (NR) & 55 & 48 (NR) & NR & NR \\
\hline
\end{tabular}

Statistically significant effect sizes are in bold text. In cases where calculated effect size confidence intervals were not-statistically significant but reported p-values were, we deferred to reported p-values and still bolded results.

Abbreviations: $\mathrm{CI}=$ confidence interval, $\mathrm{NR}=$ not reported, $\mathrm{PMID}=$ PubMed identifier, $\mathrm{RoB}=$ risk of bias, $\mathrm{SF}-12=12$-item short form survey, SF-36 $=36$-item short form survey, $\mathrm{SD}=$ standard deviation.

A Time from surgery

B Calculated

${ }^{\mathrm{C}}$ Defined as 14 weeks 


\section{Activity and Participation Outcomes Following Post-Acute Rehabilitation}

We found 27 studies in total reported on activity and participation outcomes following postacute rehabilitation compared with various comparators: 17 studies (Artz 2017, Bade 2017, Bruun-Olsen 2013, Cai 2018, Fransen 2017, Heikkilä 2017, Kauppila 2010, Lenguerrand 2020, Li 2015, Liao 2015, Liao 220, Madsen 2013, Minns Lowe 2012, Monticone 2013, Moutzouri 2018, Schache 2019, Vuorenmaa 2014) compared novel rehabilitation programs to various comparators (less intensive rehabilitation or no care); one study (Bily 2016) compared different (hypothesized equivalent) rehabilitation programs, no studies compared rehabilitation programs with different timing/intensities; four studies (Andersen 2018, Mitchell 2005, Moffet 2015, Tousignant 2011) compared rehabilitation programs delivered in different settings or by different personnel, and four studies (DeJong 2020, Demircioglu 2015, Petterson 2009, Peterson 2018, Li 2019) compared rehabilitation programs with or without an adjunctive modality (Tables 48 to 52). Outcomes included: physical function and activities of daily living, transfers, balance, mobility, and timed up and go.

\section{Physical Function and Activities of Daily Living}

We found 22 studies (Artz 2017, Fransen 2017, Lenguerrand 2020, Liao 2015, Liao 2020, Madsen 2013, Minns Lowe 2012, Monticone 2013, Moutzouri 2018, Piva 2017, Schache 2019, Vuorenmaa 2014, Pua 2017, Hamilton 2020, Mitchell 2005, Moffet 2015, Piva 2019, DeJong 2020, Demircioglu 2015, Li 2019, Petersen 2018, Petterson 2009) that reported data on patientreported physical function and ADLs using various outcome measures (Table 48) at follow-up times between 3 and 12 months after TKA surgery.

Fifteen studies reported no difference in patient-reported function and ADL measures between groups. Seven studies (Artz 2017, Liao 2015, Liao 2020, Minns Lowe 2012, Moutzouri 2018, Hamilton 2020, Li 2019) found significant differences between groups, though the direction of effect was not consistent whether the intervention favored more or less intensive forms of rehabilitation.

Artz 2018 reported function and ADL data using five scales (UCLA Activity Scale, ADL component of the KOOS, Sports and recreation component of the KOOS, the Lower Extremity Functional Scale [LEFS], and the Activities-specific balance confidence scale) and observed no differences between groups on all scales at 3 and 6 months after TKA with the exception of the LEFS (0-80, higher is better, minimal clinically important difference [MCID] 9). Based on the LEFS scale, the Artz 2017 feasibility study reported patients randomized to group-exercise had clinically and statistically significant improvement in function compared with patients randomized to usual care (referral to physiotherapy as needed) (MD 12.8, 95\% CI 3.6 to 22.0).

Liao 2015 reported function using the functional component of the WOMAC $(0-48$, higher is worse) and found patients randomized to functional and balance rehabilitation reported worse function compared with patients randomized to functional rehabilitation alone (MD 6.2, 95\% CI 4.3 to 8.1 ) at 32 weeks follow-up.

Liao 2020 reported function data using the function component of the WOMAC (0-68, higher is worse) and found patients randomized to elastic resistance exercise training reported better function than those randomized to standard care (consisting of conservative rehabilitation without any resistance exercise training) (MD $-8.6,95 \% \mathrm{CI}-10.4$ to -6.7 ). 
Minns Lowe 2012 reported ADL using the Oxford Knee Score (0-48, higher is better) and ADL component of the KOOS (0-100, higher is better) and found patients randomized to home care reported comparable ADL with patients randomized to usual care (dissemination of exercise booklet and referral to outpatient physiotherapy as needed) based on the Oxford Knee Score at 3, 6 , and 12 months following surgery and the ADL component of the KOOS as 12 months following surgery, but exhibited greater improvement in the ADL component of the KOOS early at 3 months after TKA (MD -7.1, 95\% CI -12.1 to -2.1 ), and 6 months (MD $-6.0,95 \% \mathrm{CI}$ -11.8 to -0.2 ).

Moutzouri 2018 reported the ADL component of the Knee Outcome Survey (distinct from the KOOS; 0-48, higher is better) and found that patients randomized to early self-managed focal sensorimotor exercise training reported better ADL performance than those randomized to functional exercise training (MD 19.0, 95\% CI 15.4 to 22.6).

Li 2019 reported function using the physical component of the SF-36 (0-100, higher is better) and the function component of the WOMAC (0-68, higher is worse) and found patients randomized to Tai Chi and rehabilitation reported more improved function compared to patients randomized to traditional rehabilitation exercises alone (physical component of the SF-36: MD 9.0, 95\% CI 8.5 to 9.5; function component of WOMAC: MD $-6.1,95 \% \mathrm{CI}-7.1$ to -5.1 ).

Hamilton 2020 reported ADL using the Oxford Knee Score and found patients randomized to therapist-led rehabilitation reported comparable ADL to patients randomized to home-based rehabilitation at 6 and 12 months following surgery despite early benefits in favor of home-based rehabilitation observed at 3 months.

\section{Transfers}

Seven studies (Liao 2015, Liao 2020, Madsen 2013, Minns Lowe 2012, Piva 2017, Schache 2019, Andersen 2018) reported transfer data using either the 30-second Timed Chair Stand Test (number of repetitions, larger is better) or time to complete five sit-to-stands on the Chair Stand Test (seconds, smaller is better) (Table 49). Most studies $(n=5)$ reported comparable sit-to-stand performance among groups with the exception of Liao 2015 and Liao 2020. Liao 2015 reported that patients randomized to functional plus balance rehabilitation completed significantly more sit-to-stands compared with those randomized to functional rehabilitation alone (MD - 1, 95\% CI -1.4 to -0.6 ). Liao 2020 reported that patients randomized to elastic resistance exercise training completed significantly more sit-to-stands compared with those randomized to standard care (MD 3.1, 95\% CI 2.0 to 4.1 ).

\section{Balance}

Five studies (Bruun-Olsen 2013, Liao 2020, Madsen 2013, Piva 2017, Schache 2019) reported on five difference outcome measurements of static and dynamic balance (Table 50). Most studies reported comparable balance between groups 3 to 9 months after TKA. Liao 2020 reported balance using the Forward Reach Test (measured in centimeters, larger is better) and Single-Leg Stance (measured in seconds, larger is better) and found significantly improved balance on both measures among patients randomized to the elastic resistance training exercise group compared with patients randomized to standard care.

\section{Mobility}

Sixteen studies (Bade 2017, Bruun-Olsen 2013, Fransen 2017, Heikkilä 2017, Kauppila 2010, Liao 2015, Madsen 2013, Minns Lowe 2012, Piva 2017, Schache 2019, Vuorenmaa 2014, 
Bily 2016, Andersen 2018, Moffet 2015, Petterson 2009, Li 2019) reported on various outcome measures of mobility including the 6MWT, 10-, 15- and 40-meter walk tests, stair climb tests among others (Table 51). Most studies $(n=11)$ reported no difference in mobility among groups at follow-up times ranging from 3 to 12 months after TKA.

Five studies reported significant differences between groups for at least one mobility outcome (Bruun-Olsen 2013, Heikkilä 2017, Liao 2015, Piva 2017, Li 2019). Bruun-Olsen 2013 reported three measures of mobility (Figure eight test, 6MWT, and Stair climb test) and found groups were comparable in their performance of the measures with the exception of the 6MWT (meters, larger is better) where patients randomized to the walking-skill group were able to walk further than patients randomized to the usual physiotherapy group at 9 months after TKA (adjusted MD 44, 95\% CI 8 to 80).

Heikkilä 2017 reported four measures of mobility (cadence at maximal and normal walking speed; velocity at maximal and normal walking speed) and found patients randomized to home exercise had improved cadence at maximal walking speed (steps/minute, larger is better, MD 7.7 95\% CI 3.5 to 11.9) and velocity at normal walking speed (meters/seconds, larger is better, MD $0.2,95 \%$ CI 0.04 to 0.3 ) compared to control (no intervention after discharge) at 14 months; other measures were comparable between groups.

Liao 2015 reported mobility data using the stair climb test (seconds, shorter is better) and gait speed (meters/second, larger is better) and found patients randomized to functional and balance rehabilitation had improved mobility as assessed on both measures (stair climb test: MD -2.5, 95\% CI -3.2 to -1.7 ; gait speed: MD $0.16,95 \%$ CI 0.07 to 0.26 ) compared with patients randomized to functional rehabilitation alone at 32 weeks after TKA surgery.

Piva 2017 reported mobility data using three measures and found comparable performance between groups based on self-selected gait speed and stair climb test but observed less improvement on the 6MWT among patients randomized to the comprehensive behavioral intervention (including rehabilitation) compared with the standard of care exercise program (MD $-45.4,95 \% \mathrm{CI}-87.2$ to -3.6$)$ at 6 months after TKA.

Li 2019 reported mobility using the 6MWT and found improved mobility among patients randomized to adjunctive Tai Chi compared with patients who received standard rehabilitation alone (MD 37.9, 95\% CI 24.6 to 51.2) at 3 months after TKA.

\section{Timed Up and Go}

Ten studies (Bade 2017, Liao 2020, Moutzouri 2018, Schache 2019, Vuorenmaa 2014, Bily 2016, Andersen 2018, Hamilton 2020, Demircioglu 2015, Petterson 2009) reported data on the TUG (Table 52). Most studies $(n=9)$ reported comparable performance of the TUG test between groups. One study (Liao 2020) reported significant improvement in the performance of the TUG test (seconds, smaller is better) among patients randomizing to elastic resistance training compared with those randomized to standard care $(\mathrm{MD}-3.2,95 \% \mathrm{CI}-4.0$ to -2.3$)$ at 4 months after surgery. 
Table 48. Post-acute rehabilitation versus various controls for total knee arthroplasty - continuous outcomes, physical function and activities of daily living

\begin{tabular}{|c|c|c|c|c|c|c|c|c|c|c|c|}
\hline $\begin{array}{l}\text { Study, Year, } \\
\text { PMID, Country }\end{array}$ & Arm 1 & Arm 2 & $\begin{array}{l}\text { Overall } \\
\text { RoB }\end{array}$ & $\begin{array}{l}\text { Outcome } \\
\text { Measurement }\end{array}$ & $\begin{array}{l}\text { Time } \\
\text { Point }^{\mathrm{A}}\end{array}$ & $\begin{array}{l}\text { N Arm } \\
1\end{array}$ & $\begin{array}{l}\text { Arm 1, } \\
\text { Mean } \\
\text { (SD) }\end{array}$ & $\begin{array}{l}\text { N Arm } \\
2\end{array}$ & $\begin{array}{l}\text { Arm 2, } \\
\text { Mean } \\
\text { (SD) }\end{array}$ & $\begin{array}{l}\text { Effect Size } \\
(95 \% \mathrm{Cl})\end{array}$ & $\begin{array}{l}\text { Reported } \\
\text { p-Value }\end{array}$ \\
\hline \multirow[t]{10}{*}{$\begin{array}{l}\text { Artz, 2017, } \\
27068368, \text { UK }\end{array}$} & $\begin{array}{l}\text { Group-based } \\
\text { exercise }\end{array}$ & Usual care & High & UCLA activity scale & $3 \mathrm{mo}$ & 19 & $4.9(1.7)$ & 12 & $4.3(1.1)$ & $0.6(-0.1,1.3)^{\mathrm{B}}$ & NR \\
\hline & $\begin{array}{l}\text { Group-based } \\
\text { exercise }\end{array}$ & Usual care & High & UCLA activity scale & $6 \mathrm{mo}$ & 21 & $5.2(1.5)$ & 15 & $4.5(1.9)$ & $0.7(-0.1,1.5)^{\mathrm{B}}$ & NR \\
\hline & $\begin{array}{l}\text { Group-based } \\
\text { exercise }\end{array}$ & Usual care & High & KOOS: ADL (0-100) & $3 \mathrm{mo}$ & 19 & $81.2(15.9)$ & 12 & $76.1(18.5)$ & $5.1(-4.2,14.4)^{B}$ & NR \\
\hline & $\begin{array}{l}\text { Group-based } \\
\text { exercise }\end{array}$ & Usual care & High & KOOS: ADL (0-100) & $6 \mathrm{mo}$ & 21 & $79.6(23.4)$ & 15 & $73.5(26.4)$ & $6.1(-5.9,18.1)^{B}$ & NR \\
\hline & $\begin{array}{l}\text { Group-based } \\
\text { exercise }\end{array}$ & Usual care & High & $\begin{array}{l}\text { KOOS: Sport/rec (0- } \\
100)^{\mathrm{C}}\end{array}$ & $3 \mathrm{mo}$ & 19 & $39.2(29.4)$ & 12 & $27.9(20.2)$ & $\begin{array}{l}11.3(-1.1, \\
23.7)^{\mathrm{B}} \\
\end{array}$ & NR \\
\hline & $\begin{array}{l}\text { Group-based } \\
\text { exercise }\end{array}$ & Usual care & High & $\begin{array}{l}\text { KOOS: Sport/rec (0- } \\
100)^{\mathrm{C}}\end{array}$ & $6 \mathrm{mo}$ & 21 & $46.3(35.4)$ & 15 & $37.1(25.7)$ & $9.2(-5,23.4)^{B}$ & NR \\
\hline & $\begin{array}{l}\text { Group-based } \\
\text { exercise }\end{array}$ & Usual care & High & LEFS & $3 \mathrm{mo}$ & 19 & $55.8(15.6)$ & 12 & $48.8(17.4)$ & $7(-1.8,15.8)^{B}$ & NR \\
\hline & $\begin{array}{l}\text { Group-based } \\
\text { exercise }\end{array}$ & Usual care & High & LEFS & $6 \mathrm{mo}$ & 21 & $57.8(15.2)$ & 15 & $45.0(20.8)$ & $\begin{array}{l}12.8(3.6 \\
22.0)^{\mathrm{B}} \\
\end{array}$ & NR \\
\hline & $\begin{array}{l}\text { Group-based } \\
\text { exercise }\end{array}$ & Usual care & High & $\begin{array}{l}\text { Activities-specific } \\
\text { balance confidence } \\
\text { scale }^{\mathrm{D}}\end{array}$ & $3 \mathrm{mo}$ & 19 & $84.3(15.2)$ & 12 & $79.0(19.4)$ & $5.3(-4.3,14.9)^{B}$ & NR \\
\hline & $\begin{array}{l}\text { Group-based } \\
\text { exercise }\end{array}$ & Usual care & High & $\begin{array}{l}\text { Activities-specific } \\
\text { balance confidence } \\
\text { scale }^{\mathrm{D}}\end{array}$ & $6 \mathrm{mo}$ & 21 & $84.1(17.3)$ & 15 & $80.7(19.8)$ & $3.4(-5.6,12.4)^{B}$ & NR \\
\hline \multirow[t]{5}{*}{$\begin{array}{l}\text { DeJong, } \\
2020, \\
32360105 \\
\text { USA }\end{array}$} & $\begin{array}{l}\text { Body Weight- } \\
\text { Adjustable } \\
\text { Treadmill \& } \\
\text { PENS }\end{array}$ & $\begin{array}{l}\text { Recumbent Bike } \\
\text { \& PENS }\end{array}$ & High & KOOS: ADL (0-100) & $6 \mathrm{mo}$ & 70 & $90.7(17.2)$ & 78 & $88.9(15.2)$ & $-1.8(-5.5,1.9)$ & NR \\
\hline & $\begin{array}{l}\text { Body Weight- } \\
\text { Adjustable } \\
\text { Treadmill \& } \\
\text { PENS }\end{array}$ & $\begin{array}{l}\text { Body Weight- } \\
\text { Adjustable } \\
\text { Treadmill }\end{array}$ & High & KOOS: ADL (0-100) & $6 \mathrm{mo}$ & 70 & $90.7(17.2)$ & 76 & $90.5(17.2)$ & $-0.2(-4.1,3.7)$ & NR \\
\hline & $\begin{array}{l}\text { Body Weight- } \\
\text { Adjustable } \\
\text { Treadmill \& } \\
\text { PENS }\end{array}$ & $\begin{array}{l}\text { Recumbent } \\
\text { Bike/Usual Care }\end{array}$ & High & KOOS: ADL (0-100) & $6 \mathrm{mo}$ & 70 & $90.7(17.2)$ & 74 & $92.8(8.1)$ & $2.1(-1.8,6.0)$ & NR \\
\hline & $\begin{array}{l}\text { Body Weight- } \\
\text { Adjustable } \\
\text { Treadmill \& } \\
\text { PENS }\end{array}$ & $\begin{array}{l}\text { Recumbent Bike } \\
\text { \& PENS }\end{array}$ & High & $\begin{array}{l}\text { KOOS: Sport/rec (0- } \\
100)\end{array}$ & $6 \mathrm{mo}$ & 70 & $53.3(26.4)$ & 78 & $49.9(29.6)$ & $-3.4(-9.8,3.0)$ & NR \\
\hline & $\begin{array}{l}\text { Body Weight- } \\
\text { Adjustable } \\
\text { Treadmill \& } \\
\text { PENS }\end{array}$ & $\begin{array}{l}\text { Body Weight- } \\
\text { Adjustable } \\
\text { Treadmill }\end{array}$ & High & $\begin{array}{l}\text { KOOS: Sport/rec (0- } \\
100)\end{array}$ & $6 \mathrm{mo}$ & 70 & $53.3(26.4)$ & 76 & $50.0(28.2)$ & $-3.3(-9.5,2.9)$ & NR \\
\hline
\end{tabular}




\begin{tabular}{|c|c|c|c|c|c|c|c|c|c|c|c|}
\hline $\begin{array}{l}\text { Study, Year, } \\
\text { PMID, Country }\end{array}$ & Arm 1 & Arm 2 & $\begin{array}{l}\text { Overall } \\
\text { RoB }\end{array}$ & $\begin{array}{l}\text { Outcome } \\
\text { Measurement }\end{array}$ & $\begin{array}{l}\text { Time } \\
\text { Point }^{\mathrm{A}}\end{array}$ & $\begin{array}{l}\text { N Arm } \\
1\end{array}$ & $\begin{array}{l}\text { Arm 1, } \\
\text { Mean } \\
\text { (SD) }\end{array}$ & $\begin{array}{l}\text { N Arm } \\
2\end{array}$ & $\begin{array}{l}\text { Arm 2, } \\
\text { Mean } \\
\text { (SD) }\end{array}$ & $\begin{array}{l}\text { Effect Size } \\
(95 \% \mathrm{Cl})\end{array}$ & $\begin{array}{l}\text { Reported } \\
\text { p-Value }\end{array}$ \\
\hline & $\begin{array}{l}\text { Body Weight- } \\
\text { Adjustable } \\
\text { Treadmill \& } \\
\text { PENS }\end{array}$ & $\begin{array}{l}\text { Recumbent } \\
\text { Bike/Usual Care }\end{array}$ & High & $\begin{array}{l}\text { KOOS: Sport/rec (0- } \\
100)\end{array}$ & $6 \mathrm{mo}$ & 70 & $53.3(26.4)$ & 74 & $55.9(25.5)$ & $2.6(-3.4,8.6)$ & NR \\
\hline \multirow{2}{*}{$\begin{array}{l}\text { Demircioglu, } \\
2015, \\
26355656, \\
\text { Turkey } \\
\end{array}$} & $\begin{array}{l}\text { NMES \& } \\
\text { exercise }\end{array}$ & Exercise & High & $\begin{array}{l}\text { SF-12: Physical } \\
\text { component }\end{array}$ & $3 \mathrm{mo}$ & 30 & $68.5(14.8)$ & 30 & $67.8(14.5)$ & $0.7(-4.5,5.9)^{\mathrm{B}}$ & NR \\
\hline & $\begin{array}{l}\text { NMES \& } \\
\text { exercise }\end{array}$ & Exercise & High & $\begin{array}{l}\text { WOMAC: Physical } \\
\text { function }(0-68)\end{array}$ & $3 \mathrm{mo}$ & 30 & $44.5(12.5)$ & 30 & $48.8(16.5)$ & $-4.3(-9.6,1.0)^{B}$ & NR \\
\hline \multirow{2}{*}{$\begin{array}{l}\text { Fransen, 2017, } \\
27868384, \\
\text { Australia }\end{array}$} & $\begin{array}{l}\text { Outpatient } \\
\text { exercise group }\end{array}$ & Usual care & Moderate & $\begin{array}{l}\text { QoL: SF-12 (physical } \\
\text { component) }\end{array}$ & $12 \mathrm{mo}$ & 179 & $42.7(0.6)$ & 169 & $\begin{array}{l}\text { Mean (SE) } \\
43.2(0.6)\end{array}$ & NR & NR \\
\hline & $\begin{array}{l}\text { Outpatient } \\
\text { exercise group }\end{array}$ & Usual care & Moderate & $\begin{array}{l}\text { WOMAC: Physical } \\
\text { function }(0-68)\end{array}$ & $12 \mathrm{mo}$ & 179 & $11.3(0.7)$ & 169 & $\begin{array}{l}\text { Mean (SE) } \\
10.4(0.7)\end{array}$ & NR & 0.71 \\
\hline \multirow[t]{3}{*}{$\begin{array}{l}\text { Hamilton, 2020, } \\
\text { 33051212,UK }\end{array}$} & Therapist led & $\begin{array}{l}\text { Home-based } \\
\text { exercises }\end{array}$ & Moderate & $\begin{array}{l}\text { Oxford knee score (0- } \\
48)\end{array}$ & $3 \mathrm{mo}^{\mathrm{E}}$ & 154 & $3.97(2.46)$ & 150 & $4.44(2.41)$ & $1.60(0.05,3.16)$ & 0.04 \\
\hline & Therapist led & $\begin{array}{l}\text { Home-based } \\
\text { exercises }\end{array}$ & Moderate & $\begin{array}{l}\text { Oxford knee score (0- } \\
48)\end{array}$ & $6 \mathrm{mo}$ & 150 & $\begin{array}{l}32.12 \\
(8.81)\end{array}$ & 151 & $\begin{array}{l}30.34 \\
(8.75) \\
\end{array}$ & $\begin{array}{l}1.70(- \\
0.11,3.51)\end{array}$ & 0.07 \\
\hline & Therapist led & $\begin{array}{l}\text { Home-based } \\
\text { exercises }\end{array}$ & Moderate & $\begin{array}{l}\text { Oxford knee score (0- } \\
48)\end{array}$ & $12 \mathrm{mo}$ & 148 & $\begin{array}{l}33.55 \\
(10.06)\end{array}$ & 156 & $\begin{array}{l}31.57 \\
(9.68)\end{array}$ & $\begin{array}{l}1.91(- \\
0.18,3.99)\end{array}$ & 0.07 \\
\hline \multirow[t]{3}{*}{$\begin{array}{l}\text { Lenguerrand, } \\
2020 \text {, } \\
31033232 \text {, UK }\end{array}$} & $\begin{array}{l}\text { Physical therapy } \\
\text { and standard } \\
\text { care }\end{array}$ & Standard care & Moderate & KOOS: ADL (0-100) & $12 \mathrm{mo}$ & 66 & $82(17)$ & 57 & $81(20)$ & $1(-3.7,5.7)^{\mathrm{B}}$ & 0.291 \\
\hline & $\begin{array}{l}\text { Physical therapy } \\
\text { and standard } \\
\text { care }\end{array}$ & Standard care & Moderate & $\begin{array}{l}\text { KOOS: Sport/rec (0- } \\
100)\end{array}$ & $12 \mathrm{mo}$ & 61 & $\begin{array}{l}\text { Median } \\
(\text { IQR) } 45 \\
(25,75) \\
\end{array}$ & 55 & \begin{tabular}{|l} 
Median \\
$($ IQR) 45 \\
$(25,65)$ \\
\end{tabular} & NR & 0.199 \\
\hline & $\begin{array}{l}\text { Physical therapy } \\
\text { and standard } \\
\text { care }\end{array}$ & Standard care & Moderate & LEFS & $12 \mathrm{mo}$ & 81 & $56(19)$ & 83 & $53(18)$ & $3(-1.0,7.0)^{\mathrm{B}}$ & NR \\
\hline \multirow{2}{*}{$\begin{array}{l}\text { Li, 2019, } \\
31003647, \\
\text { China }\end{array}$} & Tai chi exercise & Control & Moderate & $\begin{array}{l}\text { SF-36: Physical } \\
\text { component }(0-100)\end{array}$ & $3 \mathrm{mo}^{\mathrm{E}}$ & 54 & $54.2(1.5)$ & 53 & $45.2(1.9)$ & $9.0(8.5,9.5)^{B}$ & 0.01 \\
\hline & Tai chi exercise & Control & Moderate & $\begin{array}{l}\text { WOMAC: Physical } \\
\text { function }(0-68)\end{array}$ & $3 \mathrm{mo}$ & 54 & $35.5(3.2)$ & 53 & $41.6(4.1)$ & $\begin{array}{l}-6.1(-7.1,- \\
5.1)^{\mathrm{B}}\end{array}$ & 0.03 \\
\hline $\begin{array}{l}\text { Liao, 2015, } \\
\text { 25552523, } \\
\text { Taiwan }\end{array}$ & $\begin{array}{l}\text { Functional plus } \\
\text { balance } \\
\text { rehabilitation }\end{array}$ & $\begin{array}{l}\text { Functional } \\
\text { rehabilitation }\end{array}$ & High & $\begin{array}{l}\text { WOMAC: Physical } \\
\text { function }(0-68)\end{array}$ & $32 w$ & 65 & $28.6(8.1)$ & 65 & $22.4(7.9)$ & $6.2(4.3,8.1)^{B}$ & NR \\
\hline $\begin{array}{l}\text { Liao, 2020, } \\
31687984, \\
\text { Taiwan }\end{array}$ & $\begin{array}{l}\text { Elastic } \\
\text { resistance } \\
\text { exercise training }\end{array}$ & Standard care & Moderate & $\begin{array}{l}\text { WOMAC: Physical } \\
\text { function }(0-68)\end{array}$ & $4 \mathrm{mo}$ & 30 & $\begin{array}{l}13.17 \\
(3.78)\end{array}$ & 30 & $\begin{array}{l}21.72 \\
(6.06)\end{array}$ & $\begin{array}{l}-8.6(-10.4,- \\
6.7)^{\mathrm{B}}\end{array}$ & $<0.001$ \\
\hline \multirow{4}{*}{$\begin{array}{l}\text { Madsen, 2013, } \\
\text { 23651717, } \\
\text { Denmark }\end{array}$} & $\begin{array}{l}\text { Group-based } \\
\text { rehabilitation }\end{array}$ & $\begin{array}{l}\text { Supervised home- } \\
\text { exercises }\end{array}$ & High & $\begin{array}{l}\text { Oxford knee score (0- } \\
48)\end{array}$ & $3 \mathrm{mo}$ & 36 & NR (NR) & 34 & NR (NR) & NR & 0.7 \\
\hline & $\begin{array}{l}\text { Group-based } \\
\text { rehabilitation }\end{array}$ & $\begin{array}{l}\text { Supervised home- } \\
\text { exercises }\end{array}$ & High & $\begin{array}{l}\text { Oxford knee score (0- } \\
48)\end{array}$ & $6 \mathrm{mo}$ & 36 & NR (NR) & 32 & NR (NR) & NR & 0.7 \\
\hline & $\begin{array}{l}\text { Group-based } \\
\text { rehabilitation }\end{array}$ & $\begin{array}{l}\text { Supervised home- } \\
\text { exercises }\end{array}$ & High & $\begin{array}{l}\text { SF-36: Physical } \\
\text { function }(0-100)\end{array}$ & $3 \mathrm{mo}$ & 36 & NR (NR) & 34 & NR (NR) & NR & 0.7 \\
\hline & $\begin{array}{l}\text { Group-based } \\
\text { rehabilitation }\end{array}$ & $\begin{array}{l}\text { Supervised home- } \\
\text { exercises }\end{array}$ & High & $\begin{array}{l}\text { SF-36: Physical } \\
\text { function }(0-100)\end{array}$ & $6 \mathrm{mo}$ & 36 & NR (NR) & 32 & NR (NR) & NR & 0.5 \\
\hline
\end{tabular}




\begin{tabular}{|c|c|c|c|c|c|c|c|c|c|c|c|}
\hline $\begin{array}{l}\text { Study, Year, } \\
\text { PMID, Country }\end{array}$ & Arm 1 & Arm 2 & $\begin{array}{l}\text { Overall } \\
\text { RoB }\end{array}$ & $\begin{array}{l}\text { Outcome } \\
\text { Measurement }\end{array}$ & $\begin{array}{l}\text { Time } \\
\text { Point }\end{array}$ & $\begin{array}{l}\text { N Arm } \\
1\end{array}$ & $\begin{array}{l}\text { Arm 1, } \\
\text { Mean } \\
\text { (SD) }\end{array}$ & $\begin{array}{l}\text { N Arm } \\
2\end{array}$ & $\begin{array}{l}\text { Arm 2, } \\
\text { Mean } \\
\text { (SD) }\end{array}$ & $\begin{array}{l}\text { Effect Size } \\
(95 \% \mathrm{Cl})\end{array}$ & $\begin{array}{l}\text { Reported } \\
\text { p-Value }\end{array}$ \\
\hline \multirow[t]{9}{*}{$\begin{array}{l}\text { Minns Lowe, } \\
2012 \text {, } \\
22180446, \text { U }\end{array}$} & $\begin{array}{l}\text { Home-visit } \\
\text { physiotherapy }\end{array}$ & Usual care & High & $\begin{array}{l}\text { Oxford knee score (0- } \\
48)\end{array}$ & $3 \mathrm{mo}$ & 46 & $\begin{array}{l}\text { Media } \\
\text { (IQR) } 33.5 \\
(13)\end{array}$ & 47 & $\begin{array}{l}\text { Median } \\
(\text { IQR) } 34 \\
(12)\end{array}$ & $\begin{array}{l}\text { Median diff }-2.2 \\
(-6.4,2)\end{array}$ & 0.3 \\
\hline & $\begin{array}{l}\text { Home-visit } \\
\text { physiotherapy }\end{array}$ & Usual care & High & $\begin{array}{l}\text { Oxford knee score (0- } \\
48)\end{array}$ & $6 \mathrm{mo}$ & 42 & $\begin{array}{l}\text { Median } \\
(\text { IQR) } 36 \\
(12) \\
\end{array}$ & 44 & $\begin{array}{l}\text { Median } \\
\text { (IQR) } 36 \\
(13)\end{array}$ & $\begin{array}{l}\text { Median diff - } \\
0.05(-4.6,4.5)\end{array}$ & 0.98 \\
\hline & $\begin{array}{l}\text { Home-visit } \\
\text { physiotherapy }\end{array}$ & Usual care & High & $\begin{array}{l}\text { Oxford knee score (0- } \\
48)\end{array}$ & $12 \mathrm{mo}$ & 46 & $\begin{array}{l}\text { Median } \\
\text { (IQR) } 40 \\
(10) \\
\end{array}$ & 48 & $\begin{array}{l}\text { Median } \\
(\text { IQR) } 38.5 \\
(12) \\
\end{array}$ & $\begin{array}{l}\text { Median diff } 0.2 \\
(-3.8,4.2)\end{array}$ & 0.94 \\
\hline & $\begin{array}{l}\text { Home-visit } \\
\text { physiotherapy }\end{array}$ & Usual care & High & KOOS: ADL (0-100) & $3 \mathrm{mo}$ & 42 & $\begin{array}{l}\text { Median } \\
(\text { IQR) } 69.9 \\
(21)\end{array}$ & 44 & $\begin{array}{l}\text { Median } \\
\text { (IQR) } 75 \\
(31)\end{array}$ & $\begin{array}{l}-7.1(-12.1,- \\
2.1)^{\mathrm{B}}\end{array}$ & NR \\
\hline & $\begin{array}{l}\text { Home-visit } \\
\text { physiotherapy }\end{array}$ & Usual care & High & KOOS: ADL (0-100) & $6 \mathrm{mo}$ & 41 & $\begin{array}{l}\text { Median } \\
(\text { IQR) } 78.1 \\
(26)\end{array}$ & 41 & $\begin{array}{l}\text { Median } \\
(\text { IQR)72.1 } \\
(34)\end{array}$ & $\begin{array}{l}-6.0(-11.8,- \\
0.2)^{\mathrm{B}}\end{array}$ & NR \\
\hline & $\begin{array}{l}\text { Home-visit } \\
\text { physiotherapy }\end{array}$ & Usual care & High & KOOS: ADL (0-100) & $12 \mathrm{mo}$ & 41 & $\begin{array}{l}\text { Median } \\
(\text { IQR) } 85.3 \\
(21) \\
\end{array}$ & 46 & $\begin{array}{l}\text { Median } \\
(\text { IQR) } 89.4 \\
(23) \\
\end{array}$ & $4.1(-0.3,8.4)^{B}$ & NR \\
\hline & $\begin{array}{l}\text { Home-visit } \\
\text { physiotherapy }\end{array}$ & Usual care & High & $\begin{array}{l}\text { KOOS: Sport/rec (0- } \\
100)\end{array}$ & $3 \mathrm{mo}$ & 18 & $\begin{array}{l}\text { Median } \\
\text { (IQR) } 41.7 \\
(25) \\
\end{array}$ & 22 & $\begin{array}{l}\text { Median } \\
\text { (IQR) } 31.7 \\
(57) \\
\end{array}$ & NR & NR \\
\hline & $\begin{array}{l}\text { Home-visit } \\
\text { physiotherapy }\end{array}$ & Usual care & High & $\begin{array}{l}\text { KOOS: Sport/rec (0- } \\
100)\end{array}$ & $6 \mathrm{mo}$ & 28 & $\begin{array}{l}\text { Median } \\
\text { (IQR) } 50 \\
(44)\end{array}$ & 30 & $\begin{array}{l}\text { Media } \\
\text { (IQR) } 35 \\
(35)\end{array}$ & NR & NR \\
\hline & $\begin{array}{l}\text { Home-visit } \\
\text { physiotherapy }\end{array}$ & Usual care & High & $\begin{array}{l}\text { KOOS: Sport/rec (0- } \\
100)\end{array}$ & $12 \mathrm{mo}$ & 33 & $\begin{array}{l}\text { Median } \\
\text { (IQR) } 60 \\
(41)\end{array}$ & 35 & $\begin{array}{l}\text { Media } \\
(\text { IQR) } 50 \\
(55)\end{array}$ & NR & NR \\
\hline \multirow[t]{3}{*}{$\begin{array}{l}\text { Mitchell, 2005, } \\
\text { 15869558, UK }\end{array}$} & Hospital & Home & High & $\begin{array}{l}\text { QoL: SF-36 (physical } \\
\text { component) }\end{array}$ & $3 \mathrm{mo}$ & 57 & $43.3(27.6)$ & 57 & $41.6(22.2)$ & $\begin{array}{l}\text { Adj MD } 2.5(- \\
6.3,11.3)\end{array}$ & 0.579 \\
\hline & Hospital & Home & High & $\begin{array}{l}\text { SF-36: Physical } \\
\text { function }(0-100)\end{array}$ & $3 \mathrm{mo}$ & 57 & $23.2(36.2)$ & 57 & $27.6(37.1)$ & $\begin{array}{l}\text { Adj MD } 7.8(- \\
5.6,21.2)\end{array}$ & 0.249 \\
\hline & Hospital & Home & High & $\begin{array}{l}\text { WOMAC: Physical } \\
\text { function }(0-68)\end{array}$ & $3 \mathrm{mo}$ & 57 & $24.4(14.9)$ & 57 & $24.9(13.4)$ & $\begin{array}{l}\text { Adj Md -1.0 (- } \\
5.9,3.8)\end{array}$ & 0.677 \\
\hline \multirow{3}{*}{$\begin{array}{l}\text { Moffet, 2015, } \\
26178888, \\
\text { Canada }\end{array}$} & $\begin{array}{l}\text { In-home } \\
\text { Telerehabilitation }\end{array}$ & $\begin{array}{l}\text { Standard home } \\
\text { rehabilitation }\end{array}$ & Moderate & KOOS: ADL (0-100) & $4 \mathrm{mo}$ & 100 & 84.2 (NR) & 98 & 85.7 (NR) & $-0.8(-4.7,3)$ & NR \\
\hline & $\begin{array}{l}\text { In-home } \\
\text { Telerehabilitation }\end{array}$ & $\begin{array}{l}\text { Standard home } \\
\text { rehabilitation }\end{array}$ & Moderate & $\begin{array}{l}\text { KOOS: Sport/rec (0- } \\
100)\end{array}$ & $4 \mathrm{mo}$ & 100 & 29.8 (NR) & 98 & 30.9 (NR) & $-1.9(-8.8,5)$ & NR \\
\hline & $\begin{array}{l}\text { In-home } \\
\text { Telerehabilitation }\end{array}$ & $\begin{array}{l}\text { Standard home } \\
\text { rehabilitation }\end{array}$ & Moderate & $\begin{array}{l}\text { WOMAC: Physical } \\
\text { function }(0-68)\end{array}$ & $4 \mathrm{mo}$ & 100 & 83.9 (NR) & 98 & 84.9 (NR) & $-0.1(-3.9,3.7)$ & NR \\
\hline \multirow[t]{2}{*}{$\begin{array}{l}\text { Monticone, } \\
2013 \text {, } \\
23063624, \text { Italy }\end{array}$} & $\begin{array}{l}\text { Home-based } \\
\text { functional } \\
\text { exercises and } \\
\text { kinesiophobia } \\
\text { training }\end{array}$ & Usual care & Moderate & KOOS: ADL (0-100) & $6 \mathrm{mo}$ & 55 & NR & 55 & NR & $\begin{array}{l}14.22(8.35 \\
20.08)\end{array}$ & NR \\
\hline & $\begin{array}{l}\text { Home-based } \\
\text { functional }\end{array}$ & Usual care & Moderate & KOOS: ADL (0-100) & $12 \mathrm{mo}$ & 55 & NR & 55 & NR & $\begin{array}{l}\text { 11.84 (6.79, } \\
16.89)\end{array}$ & NR \\
\hline
\end{tabular}




\begin{tabular}{|c|c|c|c|c|c|c|c|c|c|c|c|}
\hline $\begin{array}{l}\text { Study, Year, } \\
\text { PMID, Country }\end{array}$ & Arm 1 & Arm 2 & $\begin{array}{l}\text { Overall } \\
\text { RoB }\end{array}$ & $\begin{array}{l}\text { Outcome } \\
\text { Measurement }\end{array}$ & $\begin{array}{l}\text { Time } \\
\text { Point }^{\mathrm{A}}\end{array}$ & $\begin{array}{l}\text { N Arm } \\
1\end{array}$ & $\begin{array}{l}\text { Arm 1, } \\
\text { Mean } \\
\text { (SD) }\end{array}$ & $\begin{array}{l}\text { N Arm } \\
2\end{array}$ & $\begin{array}{l}\text { Arm 2, } \\
\text { Mean } \\
\text { (SD) }\end{array}$ & $\begin{array}{l}\text { Effect Size } \\
(95 \% \mathrm{CI})\end{array}$ & $\begin{array}{l}\text { Reported } \\
\text { p-Value }\end{array}$ \\
\hline & $\begin{array}{l}\text { exercises and } \\
\text { kinesiophobia } \\
\text { training }\end{array}$ & & & & & & & & & & \\
\hline & $\begin{array}{l}\text { Home-based } \\
\text { functional } \\
\text { exercises and } \\
\text { kinesiophobia } \\
\text { training }\end{array}$ & Usual care & Moderate & $\begin{array}{l}\text { KOOS: Sport/rec (0- } \\
100)\end{array}$ & $6 \mathrm{mo}$ & 55 & NR & 55 & NR & $\begin{array}{l}\text { 13.31 (5.81, } \\
20.79)\end{array}$ & NR \\
\hline & $\begin{array}{l}\text { Home-based } \\
\text { functional } \\
\text { exercises and } \\
\text { kinesiophobia } \\
\text { training }\end{array}$ & Usual care & Moderate & $\begin{array}{l}\text { KOOS: Sport/rec (0- } \\
100)\end{array}$ & $12 \mathrm{mo}$ & 55 & NR & 55 & NR & $\begin{array}{l}10.69(2.79 \\
18.62)\end{array}$ & NR \\
\hline $\begin{array}{l}\text { Moutzouri, } \\
2018, \\
29473481, \\
\text { Greece }\end{array}$ & $\begin{array}{l}\text { Early self- } \\
\text { managed focal } \\
\text { sensorimotor } \\
\text { exercise training }\end{array}$ & $\begin{array}{l}\text { Functional } \\
\text { exercise training }\end{array}$ & Moderate & KOS: ADL (0-100) & $3.5 \mathrm{mo}$ & 25 & $79.6(9)$ & 26 & $60.6(9.3)$ & $\begin{array}{l}19.0(15.4, \\
22.6)^{\mathrm{B}}\end{array}$ & NR \\
\hline \multirow[t]{2}{*}{$\begin{array}{l}\text { Piva, 2017, } \\
\text { 28217891, USA }\end{array}$} & $\begin{array}{l}\text { Comprehensive } \\
\text { behavioral } \\
\text { intervention }\end{array}$ & $\begin{array}{l}\text { Standard of care } \\
\text { exercise }\end{array}$ & Moderate & $\begin{array}{l}\text { SF-36: Physical } \\
\text { function }(0-100)\end{array}$ & $6 \mathrm{mo}$ & 21 & $76.7(16.1)$ & 20 & $70.3(24.2)$ & $6.4(-2.9,15.7)^{B}$ & NR \\
\hline & $\begin{array}{l}\text { Comprehensive } \\
\text { behavioral } \\
\text { intervention }\end{array}$ & $\begin{array}{l}\text { Standard of care } \\
\text { exercise }\end{array}$ & Moderate & $\begin{array}{l}\text { WOMAC: Physical } \\
\text { function }(0-68)\end{array}$ & $6 \mathrm{mo}$ & 21 & $11.8(6.7)$ & 20 & $12.8(10.8)$ & $-1(-5.1,3.1)^{\mathrm{B}}$ & \\
\hline \multirow[t]{5}{*}{$\begin{array}{l}\text { Piva, 2019, } \\
\text { 30794296, USA }\end{array}$} & $\begin{array}{l}\text { Clinic-based } \\
\text { group exercise }\end{array}$ & Standard care & Moderate & $\begin{array}{l}\text { Canadian } \\
\text { occupational } \\
\text { performance } \\
\text { measure: } \\
\text { Performance }(0-10)\end{array}$ & $3 \mathrm{mo}$ & 90 & $6.5(1.7)$ & 44 & $5.4(1.7)$ & $1.3(0.7,0.6$ & NR \\
\hline & $\begin{array}{l}\text { Clinic-based } \\
\text { group exercise }\end{array}$ & Standard care & Moderate & $\begin{array}{l}\text { Canadian } \\
\text { occupational } \\
\text { performance } \\
\text { measure: } \\
\text { Performance }(0-10)\end{array}$ & $6 \mathrm{mo}$ & 88 & $6.8(1.9)$ & 45 & $6.0(1.6)$ & $0.7(0.1,1.2)$ & NR \\
\hline & $\begin{array}{l}\text { Clinic-based } \\
\text { group exercise }\end{array}$ & Standard care & Moderate & $\begin{array}{l}\text { Canadian } \\
\text { occupational } \\
\text { performance } \\
\text { measure: Satisfaction } \\
(0-10)\end{array}$ & $3 \mathrm{mo}$ & 90 & $6.6(1.8)$ & 44 & $5.0(2.0)$ & $0.7(0.1,1.4)$ & NR \\
\hline & $\begin{array}{l}\text { Clinic-based } \\
\text { group exercise }\end{array}$ & Standard care & Moderate & $\begin{array}{l}\text { Canadian } \\
\text { occupational } \\
\text { performance } \\
\text { measure: Satisfaction } \\
(0-10)\end{array}$ & $6 \mathrm{mo}$ & 89 & $6.8(2.1)$ & 45 & $5.7(1.9)$ & $0.8(0.1,1.5)$ & NR \\
\hline & $\begin{array}{l}\text { Community- } \\
\text { based group } \\
\text { exercise }\end{array}$ & Standard care & Moderate & $\begin{array}{l}\text { Canadian } \\
\text { occupational } \\
\text { performance }\end{array}$ & $3 \mathrm{mo}$ & 87 & $6.0(1.8)$ & 44 & $5.4(1.7)$ & $1.3(0.7,0.6$ & NR \\
\hline
\end{tabular}




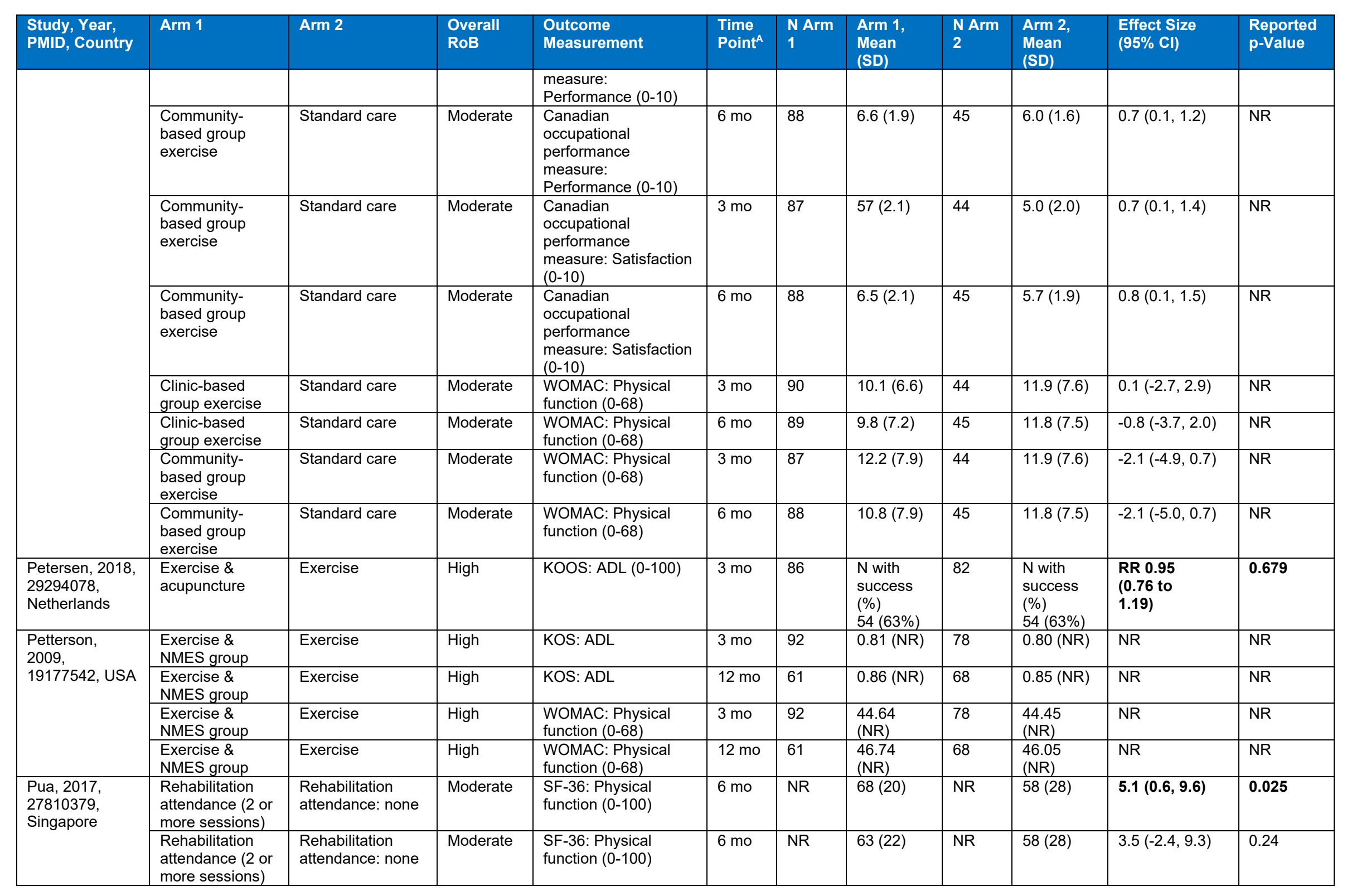




\begin{tabular}{|c|c|c|c|c|c|c|c|c|c|c|c|}
\hline $\begin{array}{l}\text { Study, Year, } \\
\text { PMID, Country }\end{array}$ & Arm 1 & Arm 2 & $\begin{array}{l}\text { Overall } \\
\text { RoB }\end{array}$ & $\begin{array}{l}\text { Outcome } \\
\text { Measurement }\end{array}$ & $\begin{array}{l}\text { Time } \\
\text { Point }^{\mathrm{A}}\end{array}$ & $\begin{array}{l}\text { N Arm } \\
1\end{array}$ & $\begin{array}{l}\text { Arm 1, } \\
\text { Mean } \\
\text { (SD) }\end{array}$ & $\begin{array}{l}\text { N Arm } \\
2\end{array}$ & $\begin{array}{l}\text { Arm 2, } \\
\text { Mean } \\
\text { (SD) }\end{array}$ & $\begin{array}{l}\text { Effect Size } \\
(95 \% \mathrm{Cl})\end{array}$ & $\begin{array}{l}\text { Reported } \\
\text { p-Value }\end{array}$ \\
\hline \multirow[t]{3}{*}{$\begin{array}{l}\text { Schache, 2019, } \\
31208916, \\
\text { Australia }\end{array}$} & $\begin{array}{l}\text { Standard } \\
\text { rehabilitation and } \\
\text { hip } \\
\text { strengthening } \\
\text { exercises }\end{array}$ & $\begin{array}{l}\text { Standard } \\
\text { rehabilitation plus } \\
\text { general functional } \\
\text { exercise }\end{array}$ & Moderate & KOOS: ADL (0-100) & $6.5 \mathrm{mo}$ & 48 & $90(11)$ & 48 & $88(13)$ & $3(-5,11)$ & NR \\
\hline & $\begin{array}{l}\text { Standard } \\
\text { rehabilitation and } \\
\text { hip } \\
\text { strengthening } \\
\text { exercises }\end{array}$ & $\begin{array}{l}\text { Standard } \\
\text { rehabilitation plus } \\
\text { general functional } \\
\text { exercise }\end{array}$ & Moderate & LEFS & $6.5 \mathrm{mo}$ & 48 & $53(12)$ & 48 & $54(12)$ & $-2(-7.0,3.0)$ & NR \\
\hline & $\begin{array}{l}\text { Standard } \\
\text { rehabilitation and } \\
\text { hip } \\
\text { strengthening } \\
\text { exercises }\end{array}$ & $\begin{array}{l}\text { Standard } \\
\text { rehabilitation plus } \\
\text { general functional } \\
\text { exercise }\end{array}$ & Moderate & $\begin{array}{l}\text { SF-12: Physical } \\
\text { component }\end{array}$ & $6.5 \mathrm{mo}$ & 48 & $47(8)$ & 48 & $46(9)$ & $1(-3,5)$ & NR \\
\hline \multirow{2}{*}{$\begin{array}{l}\text { Vuorenmaa, } \\
2014, \\
\text { 24241606, } \\
\text { Finland }\end{array}$} & Home exercise & Control & Moderate & $\begin{array}{l}\text { SF-36: Physical } \\
\text { function }(0-100)\end{array}$ & $12 \mathrm{mo}$ & 53 & $23(\mathrm{NR})$ & 55 & 27 (NR) & NR & NR \\
\hline & Home exercise & Control & Moderate & $\begin{array}{l}\text { WOMAC: Physical } \\
\text { function }(0-68)\end{array}$ & $12 \mathrm{mo}$ & 53 & 44 (NR) & 55 & 40 (NR) & NR & NR \\
\hline
\end{tabular}

Statistically significant effect sizes are in bold text. In cases where calculated effect size confidence intervals were not-statistically significant but reported p-values were, we deferred to reported p-values and still bolded results.

Abbreviations: Adj MD = adjusted mean difference, $\mathrm{ADL}=$ activities of daily living, $\mathrm{CI}=$ confidence interval, $\mathrm{EQ}-5 \mathrm{D}=\mathrm{EuroQual}, \mathrm{KOS}=\mathrm{Knee}$ outcome survey, $\mathrm{KOOS}=\mathrm{Knee}$ injury and osteoarthritis outcome score, LEFS $=$ Lower Extremity Functional Scale, mo $=$ month, NR $=$ not reported, PMID $=$ PubMed identifier, rec $=$ recreation, RR $=$ relative risk, $\mathrm{RoB}=$ risk of bias, $\mathrm{SD}=$ standard deviation, SF-36 $=36$-Item short form survey, WOMAC $=$ Western Ontario and McMaster Universities Osteoarthritis Index.

A Time from surgery

${ }^{B}$ Calculated

${ }^{\mathrm{C}}$ We included the sports and recreation component of the KOOS in the table of physical function as it was most related to other domains. Given it assesses function beyond activities of daily living though, it was not included in our assessment of the evidence of ADLs for the evidence profile

D This scale is patient-reported and distinct from the other performance-based measures, but relates to patient's confidence about balance specifically and was therefore included here

${ }^{\mathrm{E}}$ Defined as 14 weeks 
Table 49. Post-acute rehabilitation versus various controls for total knee arthroplasty - continuous outcomes, transfers

\begin{tabular}{|c|c|c|c|c|c|c|c|c|c|c|c|}
\hline $\begin{array}{l}\text { Study, Year, } \\
\text { PMID, } \\
\text { Country }\end{array}$ & Arm 1 & Arm 2 & $\begin{array}{l}\text { Overall } \\
\text { RoB }\end{array}$ & $\begin{array}{l}\text { Outcome } \\
\text { Measurement }\end{array}$ & $\begin{array}{l}\text { Time } \\
\text { Point }^{\mathrm{A}}\end{array}$ & $\begin{array}{l}\text { N Arm } \\
1\end{array}$ & $\begin{array}{l}\text { Arm 1, Mean } \\
\text { (SD) }\end{array}$ & $\begin{array}{l}\text { N Arm } \\
2\end{array}$ & $\begin{array}{l}\text { Arm 2, Mean } \\
\text { (SD) }\end{array}$ & $\begin{array}{l}\text { Effect Size } \\
(95 \% \mathrm{Cl})\end{array}$ & $\begin{array}{l}\text { Reported } \\
\text { p-Value }\end{array}$ \\
\hline \multirow[t]{2}{*}{$\begin{array}{l}\text { Andersen, } \\
2018, \mathrm{CN}- \\
01647420 \\
\text { Denmark }\end{array}$} & $\begin{array}{l}\text { Technological } \\
\text { assisted } \\
\text { rehabilitation }\end{array}$ & $\begin{array}{l}\text { Supervised } \\
\text { rehabilitation }\end{array}$ & High & $\begin{array}{l}\text { Chair stand test } \\
\text { (timed or number of } \\
\text { repetitions } \\
\text { unspecified) }\end{array}$ & $6 \mathrm{mo}$ & NR & NR (NR) & NR & NR & $<10 \%(\mathrm{MD})$ & ns \\
\hline & $\begin{array}{l}\text { Technological } \\
\text { assisted } \\
\text { rehabilitation }\end{array}$ & $\begin{array}{l}\text { Supervised } \\
\text { rehabilitation }\end{array}$ & High & $\begin{array}{l}\text { Chair stand test } \\
\text { (timed or number of } \\
\text { repetitions } \\
\text { unspecified) }\end{array}$ & $12 \mathrm{mo}$ & NR & NR (NR) & NR & NR & $<10 \%(\mathrm{MD})$ & ns \\
\hline $\begin{array}{l}\text { Liao, 2015, } \\
\text { 25552523, } \\
\text { Taiwan }\end{array}$ & $\begin{array}{l}\text { Functional plus } \\
\text { balance } \\
\text { rehabilitation }\end{array}$ & $\begin{array}{l}\text { Functional } \\
\text { rehabilitation }\end{array}$ & High & $\begin{array}{l}\text { Chair stand test: } \\
\text { Total sit-to-stands in } \\
30 \mathrm{~s}(\mathrm{n})\end{array}$ & $32 w$ & 65 & $8.7(1.7)$ & 65 & $7.7(1.7)$ & $1(0.6,1.4)^{B}$ & $<0.001$ \\
\hline $\begin{array}{l}\text { Liao, 2020, } \\
\text { 31687984, } \\
\text { Taiwan }\end{array}$ & $\begin{array}{l}\text { Elastic resistance } \\
\text { exercise training }\end{array}$ & Standard care & Moderate & $\begin{array}{l}\text { Chair stand test: } \\
\text { Total sit-to-stands in } \\
30 \text { s (n) }\end{array}$ & $4 \mathrm{mo}$ & 30 & $17.67(2.92)$ & 30 & $14.60(2.86)$ & $3.1(2.0,4.1)^{\mathrm{B}}$ & 0.001 \\
\hline \multirow{4}{*}{$\begin{array}{l}\text { Madsen, } \\
2013, \\
23651717, \\
\text { Denmark }\end{array}$} & $\begin{array}{l}\text { Group-based } \\
\text { rehabilitation }\end{array}$ & $\begin{array}{l}\text { Supervised } \\
\text { home-exercises }\end{array}$ & High & $\begin{array}{l}\text { Chair stand test: } \\
\text { Time to complete } 5 \\
\text { sit-to-stands (s) }\end{array}$ & $3 \mathrm{mo}$ & 36 & NR (NR) & 34 & NR (NR) & NR & 0.2 \\
\hline & $\begin{array}{l}\text { Group-based } \\
\text { rehabilitation }\end{array}$ & $\begin{array}{l}\text { Supervised } \\
\text { home-exercises }\end{array}$ & High & $\begin{array}{l}\text { Chair stand test: } \\
\text { Time to complete } 5 \\
\text { sit-to-stands (s) }\end{array}$ & $6 \mathrm{mo}$ & 36 & NR (NR) & 32 & NR (NR) & NR & 0.1 \\
\hline & $\begin{array}{l}\text { Group-based } \\
\text { rehabilitation }\end{array}$ & $\begin{array}{l}\text { Supervised } \\
\text { home-exercises }\end{array}$ & High & $\begin{array}{l}\text { Chair stand test: } \\
\text { Total sit-to-stands in } \\
30 \mathrm{~s}(\mathrm{n})\end{array}$ & $3 \mathrm{mo}$ & 36 & NR (NR) & 34 & NR (NR) & NR & 0.8 \\
\hline & $\begin{array}{l}\text { Group-based } \\
\text { rehabilitation }\end{array}$ & $\begin{array}{l}\text { Supervised } \\
\text { home-exercises }\end{array}$ & High & $\begin{array}{l}\text { Chair stand test: } \\
\text { Total sit-to-stands in } \\
30 \text { s (n) }\end{array}$ & $6 \mathrm{mo}$ & 36 & NR (NR) & 32 & NR (NR) & NR & 0.2 \\
\hline \multirow[t]{3}{*}{$\begin{array}{l}\text { Minns Lowe, } \\
2012, \\
22180446, \\
\text { UK }\end{array}$} & $\begin{array}{l}\text { Home-visit } \\
\text { physiotherapy }\end{array}$ & Usual care & High & $\begin{array}{l}\text { Chair stand test: } \\
\text { Total sit-to-stands in } \\
30 \mathrm{~s}(\mathrm{n})\end{array}$ & $3 \mathrm{mo}$ & 43 & $\begin{array}{l}\text { Median } \\
(\text { IQR) } 7(4)\end{array}$ & 43 & $\begin{array}{l}\text { Median } \\
(\text { IQR) } 7(6)\end{array}$ & $\begin{array}{l}\text { Median } \\
\text { difference } \\
0.56(0.44,- \\
0.9) \\
\end{array}$ & 0.2 \\
\hline & $\begin{array}{l}\text { Home-visit } \\
\text { physiotherapy }\end{array}$ & Usual care & High & $\begin{array}{l}\text { Chair stand test: } \\
\text { Total sit-to-stands in } \\
30 \mathrm{~s}(\mathrm{n})\end{array}$ & $6 \mathrm{mo}$ & NR & NR & NR & NR & NR & NR \\
\hline & $\begin{array}{l}\text { Home-visit } \\
\text { physiotherapy }\end{array}$ & Usual care & High & $\begin{array}{l}\text { Chair stand test: } \\
\text { Total sit-to-stands in } \\
30 \mathrm{~s}(\mathrm{n})\end{array}$ & $12 \mathrm{mo}$ & 40 & $\begin{array}{l}\text { Media (IQR) } \\
7(8)\end{array}$ & 43 & $\begin{array}{l}\text { Media (IQR) } \\
8(6)\end{array}$ & $\begin{array}{l}\text { Median } \\
\text { difference }-0.2 \\
(-1.8,1.5) \\
\end{array}$ & 0.85 \\
\hline $\begin{array}{l}\text { Piva, 2017, } \\
28217891, \\
\text { USA }\end{array}$ & $\begin{array}{l}\text { Comprehensive } \\
\text { behavioral } \\
\text { intervention }\end{array}$ & $\begin{array}{l}\text { Standard of } \\
\text { care exercise }\end{array}$ & Moderate & $\begin{array}{l}\text { Chair stand test: } \\
\text { Time to complete } 5 \\
\text { sit-to-stands (s) }\end{array}$ & $6 \mathrm{mo}$ & 21 & $12.2(2.8)$ & 20 & $13.7(7.5)$ & $\begin{array}{l}-1.5(-4.4 \\
1.4)^{\dagger}\end{array}$ & NR \\
\hline $\begin{array}{l}\text { Schache, } \\
2019, \\
31208916, \\
\text { Australia }\end{array}$ & $\begin{array}{l}\text { Standard } \\
\text { rehabilitation \& hip } \\
\text { strengthening } \\
\text { exercises }\end{array}$ & $\begin{array}{l}\text { Standard } \\
\text { rehabilitation \& } \\
\text { general } \\
\text { functional } \\
\text { exercise }\end{array}$ & Moderate & $\begin{array}{l}\text { Chair stand test: } \\
\text { Total sit-to-stands in } \\
30 \mathrm{~s}(\mathrm{n})\end{array}$ & $6.5 \mathrm{mo}$ & 48 & $15(4)$ & 48 & $15(5)$ & $0(-2,1)$ & NR \\
\hline
\end{tabular}


Statistically significant effect sizes are in bold text. In cases where calculated effect size confidence intervals were not-statistically significant but reported p-values were, we deferred to reported p-values and still bolded results.

Abbreviations: $\mathrm{CI}=$ confidence interval, $\mathrm{CST}=$ chair stand test, $\mathrm{IQR}=$ interquartile range, $\mathrm{MD}=$ mean difference, $\mathrm{mo}=$ month, $\mathrm{n}=$ number, $\mathrm{NR}=$ not reported, $\mathrm{PMID}=\mathrm{PubMed}$ identifier, $\mathrm{RoB}=$ risk of bias, $\mathrm{SD}=$ standard deviation, $\mathrm{SE}=$ standard error.

A Time from surgery

${ }^{\mathrm{B}}$ Calculated

Table 50. Post-acute rehabilitation versus various controls for total knee arthroplasty - continuous outcomes, balance

\begin{tabular}{|c|c|c|c|c|c|c|c|c|c|c|c|}
\hline $\begin{array}{l}\text { Study, Year, } \\
\text { PMID, Country }\end{array}$ & Arm 1 & Arm 2 & $\begin{array}{l}\text { Overall } \\
\text { RoB }\end{array}$ & $\begin{array}{l}\text { Outcome } \\
\text { Measurement }\end{array}$ & $\begin{array}{l}\text { Time } \\
\text { Point }^{\mathrm{A}}\end{array}$ & $\begin{array}{l}\text { N Arm } \\
1\end{array}$ & $\begin{array}{l}\text { Arm 1, Mean } \\
\text { (SD) }\end{array}$ & $\begin{array}{l}\text { N Arm } \\
2\end{array}$ & $\begin{array}{l}\text { Arm 2, } \\
\text { Mean (SD) }\end{array}$ & $\begin{array}{l}\text { Effect Size } \\
(95 \% \mathrm{Cl})\end{array}$ & $\begin{array}{l}\text { Reported } \\
\text { p-Value }\end{array}$ \\
\hline $\begin{array}{l}\text { Bruun-Olsen, } \\
2013, \\
23614370, \\
\text { Norway }\end{array}$ & $\begin{array}{l}\text { Walking-skill } \\
\text { group }\end{array}$ & $\begin{array}{l}\text { Usual } \\
\text { physiotherapy } \\
\text { care }\end{array}$ & Moderate & $\begin{array}{l}\text { Balance: Time stands } \\
\text { (s) }\end{array}$ & $9 \mathrm{mo}$ & 29 & $29(7)$ & 28 & $32(13)$ & $\begin{array}{l}\text { Adj MD -2(-7, } \\
\text { 3) }\end{array}$ & NR \\
\hline \multirow{2}{*}{$\begin{array}{l}\text { Liao, 2020, } \\
31687984, \\
\text { Taiwan }\end{array}$} & $\begin{array}{l}\text { Elastic } \\
\text { resistance } \\
\text { exercise training } \\
\end{array}$ & Standard care & Moderate & $\begin{array}{l}\text { Balance: Forward reach } \\
\text { test }(\mathrm{cm})\end{array}$ & $4 \mathrm{mo}$ & 30 & $24.23(6.99)$ & 30 & $18.34(5.69)$ & $5.9(3.6,8.2)^{B}$ & 0.004 \\
\hline & $\begin{array}{l}\text { Elastic } \\
\text { resistance } \\
\text { exercise training }\end{array}$ & Standard care & Moderate & $\begin{array}{l}\text { Balance: Single-leg } \\
\text { stance (s) }\end{array}$ & $4 \mathrm{mo}$ & 30 & $18.84(5.73)$ & 30 & $13.87(7.58)$ & $5(2.5,7.4)^{\mathrm{B}}$ & 0.004 \\
\hline \multirow{2}{*}{$\begin{array}{l}\text { Madsen, 2013, } \\
\text { 23651717, } \\
\text { Denmark }\end{array}$} & $\begin{array}{l}\text { Group-based } \\
\text { rehabilitation }\end{array}$ & $\begin{array}{l}\text { Supervised } \\
\text { home- } \\
\text { exercises }\end{array}$ & High & Balance: Tandem test ${ }^{C}$ & $3 \mathrm{mo}$ & 36 & NR (NR) & 34 & NR (NR) & NR & 0.2 \\
\hline & $\begin{array}{l}\text { Group-based } \\
\text { rehabilitation }\end{array}$ & $\begin{array}{l}\text { Supervised } \\
\text { home- } \\
\text { exercises }\end{array}$ & High & Balance: Tandem test ${ }^{C}$ & $6 \mathrm{mo}$ & 36 & NR (NR) & 32 & NR (NR) & NR & 0.5 \\
\hline $\begin{array}{l}\text { Piva, 2017, } \\
\text { 28217891, USA }\end{array}$ & $\begin{array}{l}\text { Comprehensive } \\
\text { behavioral } \\
\text { intervention }\end{array}$ & $\begin{array}{l}\text { Standard of } \\
\text { care exercise }\end{array}$ & Moderate & $\begin{array}{l}\text { Balance: Single-leg } \\
\text { stance }^{\mathrm{D}}\end{array}$ & $6 \mathrm{mo}$ & 21 & $16.1(9.6)$ & 20 & $17.4(9.8)$ & $-1.3(-5.5,2.9)^{\mathrm{B}}$ & NR \\
\hline $\begin{array}{l}\text { Schache, 2019, } \\
31208916, \\
\text { Australia }\end{array}$ & $\begin{array}{l}\text { Standard } \\
\text { rehabilitation and } \\
\text { hip } \\
\text { strengthening } \\
\text { exercises }\end{array}$ & $\begin{array}{l}\text { Standard } \\
\text { rehabilitation } \\
\text { plus general } \\
\text { functional } \\
\text { exercise }\end{array}$ & Moderate & Balance: Step test & $6.5 \mathrm{mo}$ & 48 & $17(4)$ & 48 & $18(5)$ & $-1(-3,1)$ & NR \\
\hline
\end{tabular}

Statistically significant effect sizes are in bold text. In cases where calculated effect size confidence intervals were not-statistically significant but reported p-values were, we deferred to reported p-values and still bolded results.

Abbreviations: Adj $\mathrm{MD}=$ adjusted mean difference, $\mathrm{CI}=$ confidence interval, $\mathrm{cm}=$ centimeter, $\mathrm{mo}=$ month, $\mathrm{NR}=$ not reported, $\mathrm{PMID}=\mathrm{PubMed}$ identifier, RoB $=$ risk of bias, $\mathrm{s}$ $=$ seconds, $\mathrm{SD}=$ standard deviation

A Time from surgery

B Calculated

C 10 seconds each in side-by-side, semi-tandem and tandem stand

${ }^{D}$ Measures the time that participants balanced on 1 leg while keeping their hands on the wais 
Table 51. Post-acute rehabilitation versus various controls for total knee arthroplasty - continuous outcomes, mobility

\begin{tabular}{|c|c|c|c|c|c|c|c|c|c|c|c|}
\hline $\begin{array}{l}\text { Study, Year, } \\
\text { PMID, Country }\end{array}$ & Arm 1 & Arm 2 & $\begin{array}{l}\text { Overall } \\
\text { RoB }\end{array}$ & $\begin{array}{l}\text { Outcome } \\
\text { Measurement }\end{array}$ & $\begin{array}{l}\text { Time } \\
\text { Point }^{A}\end{array}$ & $\begin{array}{l}\text { N Arm } \\
1\end{array}$ & $\begin{array}{l}\text { Arm 1, } \\
\text { Mean (SD) }\end{array}$ & $\begin{array}{l}\text { N Arm } \\
2\end{array}$ & $\begin{array}{l}\text { Arm 2, } \\
\text { Mean (SD) }\end{array}$ & $\begin{array}{l}\text { Effect Size } \\
(95 \% \mathrm{CI})\end{array}$ & $\begin{array}{l}\text { Reported } \\
\text { p-Value }\end{array}$ \\
\hline \multirow{2}{*}{$\begin{array}{l}\text { Andersen, 2018, } \\
\text { CN-01647420, } \\
\text { Denmark }\end{array}$} & $\begin{array}{l}\text { Technological } \\
\text { assisted rehabilitation }\end{array}$ & $\begin{array}{l}\text { Supervised } \\
\text { rehabilitation }\end{array}$ & High & $\begin{array}{l}\text { 10-m walk test } \\
\text { (s) }\end{array}$ & $6 \mathrm{mo}$ & NR & NR (NR) & NR & NR (NR) & $<10 \%(\mathrm{MD})$ & ns \\
\hline & $\begin{array}{l}\text { Technological } \\
\text { assisted rehabilitation }\end{array}$ & $\begin{array}{l}\text { Supervised } \\
\text { rehabilitation }\end{array}$ & High & $\begin{array}{l}\text { 10-m walk test } \\
\text { (s) }\end{array}$ & $12 \mathrm{mo}$ & NR & NR (NR) & NR & NR (NR) & $<10 \%(\mathrm{MD})$ & ns \\
\hline \multirow[t]{6}{*}{$\begin{array}{l}\text { Bade, 2017, } \\
27813347, \text { USA }\end{array}$} & $\begin{array}{l}\text { High-intensity } \\
\text { progressive } \\
\text { rehabilitation }\end{array}$ & $\begin{array}{l}\text { Low-intensity } \\
\text { rehabilitation }\end{array}$ & Moderate & 6MWT (m) & $3 \mathrm{mo}$ & 77 & $493.7(92.4)$ & 76 & $478.7(82.7)$ & $\begin{array}{l}15(-4.7 \\
34.7)^{\mathrm{B}}\end{array}$ & NR \\
\hline & $\begin{array}{l}\text { High-intensity } \\
\text { progressive } \\
\text { rehabilitation }\end{array}$ & $\begin{array}{l}\text { Low-intensity } \\
\text { rehabilitation }\end{array}$ & Moderate & 6MWT (m) & $6 \mathrm{mo}$ & 71 & $520.3(91.1)$ & 71 & $511.7(77.7)$ & $\begin{array}{l}8.6(-11.2 \\
28.4)^{\mathrm{B}}\end{array}$ & NR \\
\hline & $\begin{array}{l}\text { High-intensity } \\
\text { progressive } \\
\text { rehabilitation }\end{array}$ & $\begin{array}{l}\text { Low-intensity } \\
\text { rehabilitation }\end{array}$ & Moderate & 6MWT (m) & $12 \mathrm{mo}$ & 69 & $531.7(98.9)$ & 67 & $513.6(78.4)$ & $\begin{array}{l}18.1(-3.3 \\
39.5)^{\mathrm{B}}\end{array}$ & NR \\
\hline & $\begin{array}{l}\text { High-intensity } \\
\text { progressive } \\
\text { rehabilitation }\end{array}$ & $\begin{array}{l}\text { Low-intensity } \\
\text { rehabilitation }\end{array}$ & Moderate & Stair climb test ${ }^{\mathrm{C}}$ & $3 \mathrm{mo}$ & 77 & $13.02(4.62)$ & 76 & $13.60(3.58)$ & $\begin{array}{l}-0.6(-1.5 \\
0.4)^{\mathrm{B}}\end{array}$ & NR \\
\hline & $\begin{array}{l}\text { High-intensity } \\
\text { progressive } \\
\text { rehabilitation }\end{array}$ & $\begin{array}{l}\text { Low-intensity } \\
\text { rehabilitation }\end{array}$ & Moderate & Stair climb test ${ }^{C}$ & $6 \mathrm{mo}$ & 71 & $11.78(4.29)$ & 71 & $12.15(3.3)$ & $\begin{array}{l}-0.4(-1.3 \\
0.5)^{\mathrm{B}}\end{array}$ & NR \\
\hline & $\begin{array}{l}\text { High-intensity } \\
\text { progressive } \\
\text { rehabilitation }\end{array}$ & $\begin{array}{l}\text { Low-intensity } \\
\text { rehabilitation }\end{array}$ & Moderate & Stair climb test ${ }^{\mathrm{C}}$ & $12 \mathrm{mo}$ & 70 & $11.40(3.62)$ & 67 & $11.77(3.15)$ & $\begin{array}{l}-0.4(-1.2 \\
0.4)^{\mathrm{B}}\end{array}$ & NR \\
\hline $\begin{array}{l}\text { Bily, 2016, } \\
\text { 26763947, Austria }\end{array}$ & Leg-press group & $\begin{array}{l}\text { Physiotherapy } \\
\text { group }\end{array}$ & High & $\begin{array}{l}\text { Stair climb test } \\
(\mathrm{s})^{\mathrm{C}}\end{array}$ & $3 \mathrm{mo}$ & 26 & $12.8(0.74)$ & 29 & $14.8(1.03)$ & $-2(-2.3,-1.7)^{\mathrm{B}}$ & 0.29 \\
\hline \multirow[t]{3}{*}{$\begin{array}{l}\text { Bruun-Olsen, } \\
\text { 2013, 23614370, } \\
\text { Norway }\end{array}$} & Walking-skill group & $\begin{array}{l}\text { Usual } \\
\text { physiotherapy } \\
\text { group }\end{array}$ & Moderate & $\begin{array}{l}\text { Figure eight test } \\
\text { (steps) }\end{array}$ & $9 \mathrm{mo}$ & 29 & $9(11)$ & 28 & $12(12)$ & Adj $-4(-8,1)$ & NR \\
\hline & Walking-skill group & $\begin{array}{l}\text { Usual } \\
\text { physiotherapy } \\
\text { group }\end{array}$ & Moderate & 6MWT (m) & $9 \mathrm{mo}$ & 29 & $492(90)$ & 28 & $425(93)$ & Adj $44(8,80)$ & 0.02 \\
\hline & Walking-skill group & $\begin{array}{l}\text { Usual } \\
\text { physiotherapy } \\
\text { group }\end{array}$ & Moderate & $\begin{array}{l}\text { Stair climb test } \\
(\mathrm{s})^{\mathrm{D}}\end{array}$ & $9 \mathrm{mo}$ & 29 & $14(8)$ & 28 & $15(7)$ & Adj $0(-4,4)$ & NR \\
\hline \multirow{2}{*}{$\begin{array}{l}\text { Fransen, 2017, } \\
27868384, \\
\text { Australia }\end{array}$} & $\begin{array}{l}\text { Outpatient exercise } \\
\text { group }\end{array}$ & Usual care & Moderate & $\begin{array}{l}\text { 50-foot walk } \\
\text { speed (s) }\end{array}$ & $12 \mathrm{mo}$ & 179 & $1.6(0)$ & 169 & 1.6; SE (0) & NR & NR \\
\hline & $\begin{array}{l}\text { Outpatient exercise } \\
\text { group }\end{array}$ & Usual care & Moderate & $\begin{array}{l}\text { Stair climb } \\
\text { power }(W)^{E}\end{array}$ & $12 \mathrm{mo}$ & 179 & $278(9)$ & 169 & 279; SE (9) & NR & NR \\
\hline \multirow[t]{3}{*}{$\begin{array}{l}\text { Heikkilä, 2017, } \\
\text { 28119232, Finland }\end{array}$} & Home exercise & Control & High & $\begin{array}{l}\text { Cadence: } \\
\text { Maximal } \\
\text { (Steps/min) }\end{array}$ & $14 \mathrm{mo}$ & 53 & $141.4(16.5)$ & 55 & $133.7(14.9)$ & $\begin{array}{l}7.7(3.5 \\
11.9)^{\mathrm{B}}\end{array}$ & NR \\
\hline & Home exercise & Control & High & $\begin{array}{l}\text { Cadence: } \\
\text { Normal } \\
\text { (Steps/min) }\end{array}$ & $14 \mathrm{mo}$ & 53 & $120.9(21.4)$ & 55 & $116.8(11.2)$ & $4.1(-0.9,9.1)^{B}$ & NR \\
\hline & Home exercise & Control & High & $\begin{array}{l}\text { Walking velocity: } \\
\text { Maximal }(\mathrm{m} / \mathrm{s})\end{array}$ & $14 \mathrm{mo}$ & 53 & $1.24(0.37)$ & 55 & $1.18(0.28)$ & $\begin{array}{l}0.1(-0.03 \\
0.2)^{\mathrm{B}}\end{array}$ & NR \\
\hline
\end{tabular}




\begin{tabular}{|c|c|c|c|c|c|c|c|c|c|c|c|}
\hline $\begin{array}{l}\text { Study, Year, } \\
\text { PMID, Country }\end{array}$ & Arm 1 & Arm 2 & $\begin{array}{l}\text { Overall } \\
\text { RoB }\end{array}$ & $\begin{array}{l}\text { Outcome } \\
\text { Measurement }\end{array}$ & $\begin{array}{l}\text { Time } \\
\text { Point }^{\mathrm{A}}\end{array}$ & $\begin{array}{l}\text { N Arm } \\
1\end{array}$ & $\begin{array}{l}\text { Arm 1, } \\
\text { Mean (SD) }\end{array}$ & $\begin{array}{l}\text { N Arm } \\
2\end{array}$ & $\begin{array}{l}\text { Arm 2, } \\
\text { Mean (SD) }\end{array}$ & $\begin{array}{l}\text { Effect Size } \\
(95 \% \mathrm{Cl})\end{array}$ & $\begin{array}{l}\text { Reported } \\
\text { p-Value }\end{array}$ \\
\hline & Home exercise & Control & High & $\begin{array}{l}\text { Walking velocity: } \\
\text { Normal }(\mathrm{m} / \mathrm{s})\end{array}$ & $14 \mathrm{mo}$ & 53 & $1.67(0.40)$ & 55 & $1.52(0.41)$ & $\begin{array}{l}0.2(0.04 \\
0.3)^{\mathrm{B}}\end{array}$ & NR \\
\hline \multirow[t]{6}{*}{$\begin{array}{l}\text { Kauppila, 2010, } \\
\text { 20354057, Finland }\end{array}$} & $\begin{array}{l}\text { Multidisciplinary } \\
\text { rehabilitation group }\end{array}$ & Control & Moderate & $\begin{array}{l}\text { 15-m walk test } \\
\text { (s) }\end{array}$ & $6 \mathrm{mo}$ & 36 & $13.4(2.4)$ & 39 & $13.3(2.5)$ & $0.1(-0.7,0.9)^{\mathrm{B}}$ & NR \\
\hline & $\begin{array}{l}\text { Multidisciplinary } \\
\text { rehabilitation group }\end{array}$ & Control & Moderate & $\begin{array}{l}\text { 15-m walk test } \\
\text { (s) }\end{array}$ & $12 \mathrm{mo}$ & 36 & $13.8(3.6)$ & 37 & $13.7(2.9)$ & $0.1(-1,1.2)^{\mathrm{B}}$ & 0.3 \\
\hline & $\begin{array}{l}\text { Multidisciplinary } \\
\text { rehabilitation group }\end{array}$ & Control & Moderate & $\begin{array}{l}\text { Stair climb test: } \\
\text { Ascend (s) }\end{array}$ & $6 \mathrm{mo}$ & 36 & $11(5.6)$ & 36 & $9.6(3.4)$ & $1.4(-0.2,3)^{\mathrm{B}}$ & NR \\
\hline & $\begin{array}{l}\text { Multidisciplinary } \\
\text { rehabilitation group }\end{array}$ & Control & Moderate & $\begin{array}{l}\text { Stair climb test: } \\
\text { Ascend (s) }\end{array}$ & $12 \mathrm{mo}$ & 36 & $10.3(3.7)$ & 34 & $10(4.1)$ & $0.3(-1,1.6)^{\mathrm{B}}$ & 0.5 \\
\hline & $\begin{array}{l}\text { Multidisciplinary } \\
\text { rehabilitation group }\end{array}$ & Control & Moderate & $\begin{array}{l}\text { Stair climb test: } \\
\text { Descend (s) }\end{array}$ & $6 \mathrm{mo}$ & 36 & $10.7(5)$ & 36 & $10.5(4.1)$ & $0.2(-1.3,1.7)^{\mathrm{B}}$ & NR \\
\hline & $\begin{array}{l}\text { Multidisciplinary } \\
\text { rehabilitation group }\end{array}$ & Control & Moderate & $\begin{array}{l}\text { Stair climb test: } \\
\text { Descend (s) }\end{array}$ & $12 \mathrm{mo}$ & 36 & $10.7(5.3)$ & 33 & $10.7(5)$ & $0(-1.7,1.7)^{\mathrm{B}}$ & 0.2 \\
\hline $\begin{array}{l}\text { Li, 2019, } \\
\text { 31003647, China }\end{array}$ & Tai chi exercise & Control & Moderate & 6MWT (m) & $3 \mathrm{mo}^{\mathrm{K}}$ & 54 & $467.1(51.4)$ & 53 & $429.2(47.5)$ & $\begin{array}{l}37.9(24.6 \\
51.2)^{\mathrm{B}}\end{array}$ & 0.01 \\
\hline \multirow[t]{2}{*}{$\begin{array}{l}\text { Liao, 2015, } \\
\text { 25552523, Taiwan }\end{array}$} & $\begin{array}{l}\text { Functional plus } \\
\text { balance rehabilitation }\end{array}$ & $\begin{array}{l}\text { Functional } \\
\text { rehabilitation }\end{array}$ & High & $\begin{array}{l}\text { Stair climb test } \\
(\mathrm{s})^{\mathrm{F}}\end{array}$ & $32 w$ & 65 & $12.2(1.8)$ & 65 & $14.5(2.5)$ & $\begin{array}{l}-2.5(-3.2 \\
-1.7)\end{array}$ & $<0.001$ \\
\hline & $\begin{array}{l}\text { Functional plus } \\
\text { balance rehabilitation }\end{array}$ & $\begin{array}{l}\text { Functional } \\
\text { rehabilitation }\end{array}$ & High & $\begin{array}{l}\text { Gait speed } \\
(\mathrm{m} / \mathrm{sec})\end{array}$ & $4 \mathrm{mo}$ & 30 & $1.42(0.28)$ & 30 & $1.25(0.30)$ & $\begin{array}{l}0.16(0.07 \\
0.26)\end{array}$ & $<0.01$ \\
\hline \multirow{2}{*}{$\begin{array}{l}\text { Madsen, 2013, } \\
\text { 23651717, } \\
\text { Denmark }\end{array}$} & $\begin{array}{l}\text { Group-based } \\
\text { rehabilitation }\end{array}$ & $\begin{array}{l}\text { Supervised home- } \\
\text { exercises }\end{array}$ & High & $\begin{array}{l}\text { Walking velocity } \\
(\mathrm{NR})^{\mathrm{G}}\end{array}$ & $3 \mathrm{mo}$ & 36 & $0.32(0.21)$ & 34 & $0.3(0.2)$ & NR & 0.7 \\
\hline & $\begin{array}{l}\text { Group-based } \\
\text { rehabilitation }\end{array}$ & $\begin{array}{l}\text { Supervised home- } \\
\text { exercises }\end{array}$ & High & $\begin{array}{l}\text { Walking velocity } \\
(\mathrm{NR})^{\mathrm{G}}\end{array}$ & $6 \mathrm{mo}$ & 36 & $0.40(0.22)$ & 32 & $0.36(0.22)$ & NR & 0.5 \\
\hline \multirow[t]{2}{*}{$\begin{array}{l}\text { Minns Lowe, } \\
\text { 2012, 22180446, } \\
\text { UK }\end{array}$} & $\begin{array}{l}\text { Home-visit } \\
\text { physiotherapy }\end{array}$ & Usual care & High & $\begin{array}{l}10-\mathrm{m} \text { walk test } \\
\text { (s) }\end{array}$ & $3 \mathrm{mo}$ & 42 & Median 9.9 & 43 & $\begin{array}{l}\text { Median } \\
10.3\end{array}$ & $\begin{array}{l}\text { Median } \\
\text { difference }-0.4 \\
(-1.6,1.3)\end{array}$ & 0.55 \\
\hline & $\begin{array}{l}\text { Home-visit } \\
\text { physiotherapy }\end{array}$ & Usual care & High & $\begin{array}{l}\text { 10-m walk test } \\
\text { (s) }\end{array}$ & $12 \mathrm{mo}$ & 40 & Median 9.2 & 43 & Median 9.1 & $\begin{array}{l}\text { Median } \\
\text { difference }-0.2 \\
(-1.5,1.2)\end{array}$ & 0.8 \\
\hline \multirow{2}{*}{$\begin{array}{l}\text { Moffet, 2015, } \\
26178888, \\
\text { Canada }\end{array}$} & $\begin{array}{l}\text { In-home } \\
\text { Telerehabilitation }\end{array}$ & $\begin{array}{l}\text { Standard home } \\
\text { rehabilitation }\end{array}$ & Moderate & 6MWT (m) & $4 \mathrm{mo}$ & 100 & $396.3(\mathrm{NR})$ & 98 & 407.5 (NR) & $\begin{array}{l}-7.4(-27.8 \\
13.1)\end{array}$ & NR \\
\hline & $\begin{array}{l}\text { In-home } \\
\text { Telerehabilitation }\end{array}$ & $\begin{array}{l}\text { Standard home } \\
\text { rehabilitation }\end{array}$ & Moderate & $\begin{array}{l}\text { Stair climb test } \\
(\mathrm{s})^{\mathrm{F}}\end{array}$ & $4 \mathrm{mo}$ & 100 & 29.9 (NR) & 98 & 26.6 (NR) & $-1.2(-4.8,2.4)$ & NR \\
\hline \multirow[t]{4}{*}{$\begin{array}{l}\text { Petterson, 2009, } \\
\text { 19177542, USA }\end{array}$} & $\begin{array}{l}\text { Exercise \& NMES } \\
\text { group }\end{array}$ & Exercise & High & $6 \mathrm{MWT}(\mathrm{m})$ & $3 \mathrm{mo}$ & 76 & $530(\mathrm{NR})$ & 92 & $535(\mathrm{NR})$ & NR & NR \\
\hline & $\begin{array}{l}\text { Exercise \& NMES } \\
\text { group }\end{array}$ & Exercise & High & 6MWT (m) & $12 \mathrm{mo}$ & 68 & 545 (NR) & 81 & 554 (NR) & NR & NR \\
\hline & $\begin{array}{l}\text { Exercise \& NMES } \\
\text { group }\end{array}$ & Exercise & High & $\begin{array}{l}\text { Stair climb test } \\
(\mathrm{s})^{\mathrm{F}}\end{array}$ & $3 \mathrm{mo}$ & 76 & 14.28 (NR) & 92 & 12.78 (NR) & NR & NR \\
\hline & $\begin{array}{l}\text { Exercise \& NMES } \\
\text { group }\end{array}$ & Exercise & High & $\begin{array}{l}\text { Stair climb test } \\
(\mathrm{s})^{\mathrm{F}}\end{array}$ & $12 \mathrm{mo}$ & 68 & $13.62(\mathrm{NR})$ & 81 & 11.75 (NR) & NR & NR \\
\hline $\begin{array}{l}\text { Piva, 2017, } \\
\text { 28217891, USA }\end{array}$ & $\begin{array}{l}\text { Comprehensive } \\
\text { behavioral } \\
\text { intervention }\end{array}$ & $\begin{array}{l}\text { Standard of care } \\
\text { exercise }\end{array}$ & Moderate & 6MWT (m) & $6 \mathrm{mo}$ & 21 & $472.6(86.5)$ & 20 & $518(103.3)$ & $\begin{array}{l}-45.4(-87.2,- \\
3.6)^{B}\end{array}$ & NR \\
\hline
\end{tabular}




\begin{tabular}{|c|c|c|c|c|c|c|c|c|c|c|c|}
\hline $\begin{array}{l}\text { Study, Year, } \\
\text { PMID, Country }\end{array}$ & Arm 1 & Arm 2 & $\begin{array}{l}\text { Overall } \\
\text { RoB }\end{array}$ & $\begin{array}{l}\text { Outcome } \\
\text { Measurement }\end{array}$ & $\begin{array}{l}\text { Time } \\
\text { Point }^{\mathrm{A}}\end{array}$ & $\begin{array}{l}\text { N Arm } \\
1\end{array}$ & $\begin{array}{l}\text { Arm 1, } \\
\text { Mean (SD) }\end{array}$ & $\begin{array}{l}\text { N Arm } \\
2\end{array}$ & $\begin{array}{l}\text { Arm 2, } \\
\text { Mean (SD) }\end{array}$ & $\begin{array}{l}\text { Effect Size } \\
(95 \% \mathrm{CI})\end{array}$ & $\begin{array}{l}\text { Reported } \\
\text { p-Value }\end{array}$ \\
\hline & $\begin{array}{l}\text { Comprehensive } \\
\text { behavioral } \\
\text { intervention }\end{array}$ & $\begin{array}{l}\text { Standard of care } \\
\text { exercise }\end{array}$ & Moderate & $\begin{array}{l}\text { Self-selected } \\
\text { gait speed }(\mathrm{m} / \mathrm{s})\end{array}$ & $6 \mathrm{mo}$ & 21 & $1.14(0.16)$ & 20 & $1.18(0.24)$ & $\begin{array}{l}-0.04(-0.1 \\
0.1)^{\mathrm{B}}\end{array}$ & NR \\
\hline & $\begin{array}{l}\text { Comprehensive } \\
\text { behavioral } \\
\text { intervention }\end{array}$ & $\begin{array}{l}\text { Standard of care } \\
\text { exercise }\end{array}$ & Moderate & $\begin{array}{l}\text { Stair climb test } \\
(\mathrm{s})^{\mathrm{H}}\end{array}$ & $6 \mathrm{mo}$ & 21 & $14.3(4.1)$ & 20 & $15.6(7.4)$ & $\begin{array}{l}-1.3(-4.1 \\
1.5)^{\mathrm{B}}\end{array}$ & NR \\
\hline \multirow[t]{3}{*}{$\begin{array}{l}\text { Schache, 2019, } \\
31208916, \\
\text { Australia }\end{array}$} & $\begin{array}{l}\text { Standard } \\
\text { rehabilitation \& hip } \\
\text { strengthening } \\
\text { exercises }\end{array}$ & $\begin{array}{l}\text { Standard } \\
\text { rehabilitation \& } \\
\text { general functional } \\
\text { exercise }\end{array}$ & Moderate & $\begin{array}{l}\text { 40m-fast-paced } \\
\text { walk test (s) }\end{array}$ & $6.5 \mathrm{mo}$ & 48 & $29(9)$ & 48 & $29(10)$ & $0(-15,16)$ & NR \\
\hline & $\begin{array}{l}\text { Standard } \\
\text { rehabilitation \& hip } \\
\text { strengthening } \\
\text { exercises }\end{array}$ & $\begin{array}{l}\text { Standard } \\
\text { rehabilitation \& } \\
\text { general functional } \\
\text { exercise }\end{array}$ & Moderate & 6MWT (m) & $6.5 \mathrm{mo}$ & 48 & $474(106)$ & 48 & $477(128)$ & $\begin{array}{l}-3(-36.5 \\
30.5)^{\mathrm{B}}\end{array}$ & NR \\
\hline & $\begin{array}{l}\text { Standard } \\
\text { rehabilitation \& hip } \\
\text { strengthening } \\
\text { exercises }\end{array}$ & $\begin{array}{l}\text { Standard } \\
\text { rehabilitation \& } \\
\text { general functional } \\
\text { exercise }\end{array}$ & Moderate & $\begin{array}{l}\text { Stair climb test } \\
(\mathrm{s})^{\prime}\end{array}$ & $6.5 \mathrm{mo}$ & 48 & $7(2)$ & 48 & $7(2)$ & $-2(-5,1)$ & NR \\
\hline $\begin{array}{l}\text { Vuorenmaa, 2014, } \\
24241606, \text { Finland }\end{array}$ & Home exercise & Control & Moderate & $\begin{array}{l}\text { Maximal walking } \\
\text { speed }(\mathrm{m} / \mathrm{s})^{\mathrm{J}}\end{array}$ & $12 \mathrm{mo}$ & 53 & $1.04(\mathrm{NR})$ & 55 & $1.18(\mathrm{NR})$ & NR & NR \\
\hline
\end{tabular}

Statistically significant effect sizes are in bold text. In cases where calculated effect size confidence intervals were not-statistically significant but reported p-values were, we deferred to reported p-values and still bolded results. If p-values were not significant, we did not bold our calculated confidence intervals, even if significant.

Abbreviations: $6 \mathrm{MWT}=$ six-minute walk test $\mathrm{CI}=$ confidence interval, $\mathrm{MD}=$ mean difference, $\mathrm{mo}=$ month, $\mathrm{m}=$ meter, $\mathrm{NR}=$ not reported, $\mathrm{ns}=$ not significant, $\mathrm{PMID}=\mathrm{PubMed}$ identifier, $\mathrm{RoB}=$ risk of bias, $\mathrm{SD}=$ standard deviation, $\mathrm{SE}=$ standard error, $\mathrm{W}=$ watt.

A Time from surgery

${ }^{B}$ Calculated

${ }^{\mathrm{C}}$ Defined as the total time to ascend and descend flight of 12 stairs

${ }^{D}$ Defined as the total time to ascend and descend a flight of 16 stairs

${ }^{E}$ Calculated from time to perform stair climb test, number of stairs, stair height, and body weight.

${ }^{\mathrm{F}}$ Specifics of the stair climb test not defined

${ }^{\mathrm{G}}$ Measured during a 10-m walk test. Unit not reported, likely $\mathrm{m} / \mathrm{s}$

${ }^{\mathrm{H}}$ Defined as the total time to ascend and descend a flight of 11 stairs

${ }^{\mathrm{I}}$ Defined as the time taken to ascend four steps in seconds

${ }^{\mathrm{J}}$ Measured using GAITRite Walkway System (CIR Systems Inc., Sparta, USA). Participants were instructed to walk barefoot as rapidly as possible. The participants started

walking from a point 2 meters in front of the mat and stopped at a point 2 meters beyond the mat

${ }^{\mathrm{K}}$ Defined as 14 weeks 
Table 52. Post-acute rehabilitation versus various controls for total knee arthroplasty - continuous outcomes, Timed Up and Go

\begin{tabular}{|c|c|c|c|c|c|c|c|c|c|c|}
\hline $\begin{array}{l}\text { Study, Year, } \\
\text { PMID, Country }\end{array}$ & Arm 1 & Arm 2 & $\begin{array}{l}\text { Overall } \\
\text { RoB }\end{array}$ & $\begin{array}{l}\text { Time } \\
\text { Point }^{\mathrm{A}}\end{array}$ & $\begin{array}{l}\text { N Arm } \\
1\end{array}$ & $\begin{array}{l}\text { Arm 1, Mean } \\
\text { (SD) }\end{array}$ & $\begin{array}{l}\text { N Arm } \\
2\end{array}$ & $\begin{array}{l}\text { Arm 2, Mean } \\
\text { (SD) }\end{array}$ & $\begin{array}{l}\text { Effect Size }(95 \% \\
\text { Cl) }\end{array}$ & $\begin{array}{l}\text { Reported p- } \\
\text { Value }\end{array}$ \\
\hline \multirow{2}{*}{$\begin{array}{l}\text { Andersen, 2018, } \\
\text { CN-01647420, } \\
\text { Denmark }\end{array}$} & $\begin{array}{l}\text { Technological } \\
\text { assisted rehabilitation }\end{array}$ & $\begin{array}{l}\text { Supervised } \\
\text { rehabilitation }\end{array}$ & High & $6 \mathrm{mo}$ & NR & NR & NR & NR & $<10 \%(\mathrm{MD})$ & ns \\
\hline & $\begin{array}{l}\text { Technological } \\
\text { assisted rehabilitation }\end{array}$ & $\begin{array}{l}\text { Supervised } \\
\text { rehabilitation }\end{array}$ & High & $12 \mathrm{mo}$ & NR & NR & NR & NR & $<10 \%(\mathrm{MD})$ & ns \\
\hline \multirow[t]{3}{*}{$\begin{array}{l}\text { Bade, 2017, } \\
27813347, \text { USA }\end{array}$} & $\begin{array}{l}\text { High-intensity } \\
\text { progressive } \\
\text { rehabilitation }\end{array}$ & $\begin{array}{l}\text { Low-intensity } \\
\text { rehabilitation }\end{array}$ & Moderate & $3 \mathrm{mo}$ & 77 & $7.58(1.82)$ & 76 & $7.98(1.58)$ & $-0.4(-0.8,-0.01)^{\mathrm{B}}$ & NR \\
\hline & $\begin{array}{l}\text { High-intensity } \\
\text { progressive } \\
\text { rehabilitation }\end{array}$ & $\begin{array}{l}\text { Low-intensity } \\
\text { rehabilitation }\end{array}$ & Moderate & $6 \mathrm{mo}$ & 71 & $7.33(1.6)$ & 71 & $7.48(1.45)$ & $-0.2(-0.5,0.2)^{\mathrm{B}}$ & NR \\
\hline & $\begin{array}{l}\text { High-intensity } \\
\text { progressive } \\
\text { rehabilitation }\end{array}$ & $\begin{array}{l}\text { Low-intensity } \\
\text { rehabilitation }\end{array}$ & Moderate & $12 \mathrm{mo}$ & 71 & $7.36(1.77)$ & 67 & $7.44(1.50)$ & $-0.1(-0.5,0.3)^{\mathrm{B}}$ & NR \\
\hline $\begin{array}{l}\text { Bily, 2016, } \\
26763947, \text { Austria }\end{array}$ & Leg-press group & Physiotherapy group & High & $3 \mathrm{mo}$ & 26 & $7.3(0.32)$ & 29 & $8.1(0.41)$ & $-0.8(-0.9,-0.7)^{\mathrm{B}}$ & $0.29^{C}$ \\
\hline $\begin{array}{l}\text { Demircioglu, 2015, } \\
\text { 26355656, Turkey }\end{array}$ & NMES \& exercise & Exercise & High & $3 \mathrm{mo}$ & 30 & $12.3(2.1)$ & 30 & $12.9(1.9)$ & $-0.6(-1.3,0.1)^{\mathrm{B}}$ & NR \\
\hline $\begin{array}{l}\text { Hamilton, 2020, } \\
\text { 33051212,UK }\end{array}$ & Therapist led & $\begin{array}{l}\text { Home-based } \\
\text { exercises }\end{array}$ & Moderate & $3 \mathrm{mo}^{\mathrm{D}}$ & 143 & $14.65(38.0)$ & 143 & $22.5(77.2)$ & $4.64(-14.25,4.96)$ & 0.34 \\
\hline $\begin{array}{l}\text { Liao, 2020, } \\
\text { 31687984, Taiwan }\end{array}$ & $\begin{array}{l}\text { Elastic resistance } \\
\text { exercise training }\end{array}$ & Standard care & Moderate & $4 \mathrm{mo}$ & 30 & $9.13(1.13)$ & 30 & $12.32(2.71)$ & $-3.2(-4.0,-2.3)^{\mathrm{B}}$ & 0.002 \\
\hline $\begin{array}{l}\text { Moutzouri, 2018, } \\
\text { 29473481, Greece }\end{array}$ & $\begin{array}{l}\text { Early self-managed } \\
\text { focal sensorimotor } \\
\text { exercise training }\end{array}$ & $\begin{array}{l}\text { Functional exercise } \\
\text { training }\end{array}$ & Moderate & $3.5 \mathrm{mo}$ & 25 & $8.1(1.7)$ & 26 & $12.4(2.5)$ & $-4.3(-5.2,-3.4)^{\mathrm{B}}$ & NR \\
\hline \multirow[t]{2}{*}{$\begin{array}{l}\text { Petterson, 2009, } \\
\text { 19177542, USA }\end{array}$} & $\begin{array}{l}\text { Exercise \& NMES } \\
\text { group }\end{array}$ & Exercise & High & $3 \mathrm{mo}$ & 92 & $8.02(\mathrm{NR})$ & 78 & 8.29 (NR) & NR & NR \\
\hline & $\begin{array}{l}\text { Exercise \& NMES } \\
\text { group }\end{array}$ & Exercise & High & $12 \mathrm{mo}$ & 61 & 7.68 (NR) & 68 & 8.07 (NR) & NR & NR \\
\hline $\begin{array}{l}\text { Schache, 2019, } \\
31208916, \\
\text { Australia }\end{array}$ & $\begin{array}{l}\text { Standard } \\
\text { rehabilitation and hip } \\
\text { strengthening } \\
\text { exercises }\end{array}$ & $\begin{array}{l}\text { Standard } \\
\text { rehabilitation plus } \\
\text { general functional } \\
\text { exercise }\end{array}$ & Moderate & $6.5 \mathrm{mo}$ & 48 & $8(2)$ & 48 & $8(3)$ & $-2(-6,2)$ & NR \\
\hline $\begin{array}{l}\text { Vuorenmaa, 2014, } \\
\text { 24241606, Finland }\end{array}$ & Home exercise & Control & Moderate & $12 \mathrm{mo}$ & 53 & 9.18 (NR) & 55 & 10.33 (NR) & NR & NR \\
\hline
\end{tabular}

Statistically significant effect sizes are in bold text. In cases where calculated effect size confidence intervals were not-statistically significant but reported p-values were, we deferred to reported p-values and still bolded results.

Abbreviations: $\mathrm{CI}=$ confidence interval, $\mathrm{MD}=$ mean difference, $\mathrm{mo}=$ month, $\mathrm{NMES}=$ neuromuscular electrical stimulation, $\mathrm{NR}=$ not reported, $\mathrm{ns}=$ not significant, $\mathrm{PMID}=$ PubMed identifier, $\mathrm{RoB}=$ risk of bias, $\mathrm{s}=$ second, $\mathrm{SD}=$ standard deviation

A Time from surgery

B Calculated

C Two-way repeated-measure analysis of variance adjusted for time and group was not significant

D Defined as 14 weeks 


\section{Other Patient-Reported Outcomes Following Post-Acute Rehabilitation}

We found 14 studies in total that reported other patient-reported outcomes following postacute rehabilitation compared with various comparators: seven studies (Artz 2017, Bade 2017, Bruun-Olsen 2013, Kauppila 2010, Lenguerrand 2020, Minns Lowe 2012, Monticone 2013) compared novel rehabilitation programs to various comparators (less intensive rehabilitation or no care); one study (Bily 2016) compared different (hypothesized equivalent) rehabilitation programs, no studies compared rehabilitation programs with different timing and/or intensities; four studies (Andersen 2018, Mitchell 2005, Moffet 2015, Piva 2019) compared rehabilitation programs delivered in different settings or by different personnel, and two studies (Demircioglu 2015, Peterson 2018) compared rehabilitation programs with or without an adjunctive modality (Tables 53 to 55). Outcomes included: quality of life, patient satisfaction with care, and patient global assessments.

\section{Quality of Life}

Twelve studies (Artz, 2017, Bruun-Olsen, 2013, Kauppila, 2010, Lenguerrand, 2020, Minns Lowe, 2012, Monticone, 2013, Andersen, 2018, Moffet, 2015, Piva, 2019, DeJong 2020, Demircioglu, 2015, Petersen, 2018) QoL using the QoL component of the KOOS and the total SF-36 (Table 53). Most studies $(\mathrm{n}=10)$ reported comparable QoL among rehabilitation arms at follow-up, ranging between 3 and 12 months following TKA surgery. Two studies (Monticone 2013 and Andersen 2018) reported improved QoL in one group. Monticone 2013 reported the QoL component of the KOOS (scores 0-100; higher is better) and found that patients randomized to the home-based functional exercises and kinesiophobia training reported improved QoL than those randomized to usual care at 6 and 12 months after TKA. Andersen 2018 also reported data on the QoL component of the KOOS and found that patients randomized to technological assisted rehabilitation had improved QoL compared to patients randomized to usual care at 6 months (difference of 12.2\%; P NR) after TKA but not at 12 months.

\section{Patient Satisfaction With Care}

One study (Moffet 2015) reported data on satisfaction with care and found no differences between patients randomized to in-home telerehabilitation compared with standard home rehabilitation (Table 54).

\section{Patient Global Assessments}

Ten studies (Artz 2017, Bade 2017, Kauppila 2010, Bily 2016, DeJong 2020, Demircioglu 2015, Mitchell 2005, Piva 2019) provided data on patients' self-reported global assessment of their health using five different measurement instruments assessed between 3 and 12 months after TKA surgery (Table 55). Most studies $(n=9)$ reported comparable results between groups with the exception of Artz 2017 and Demircioglu 2015. Artz 2017 reported significant improvements in the Measure yourself medical outcome profile (scores 0-6; lower is better, MCID 0.5-1.0) among patients randomized to group-based exercise compared to usual care at 3 months following surgery (MD $-0.9,95 \% \mathrm{CI}-1.5$ to -0.3 ) but not at 6 months. Demircioglu 2015 reported improved overall WOMAC outcomes (0-96, lower is better) among patients randomized to rehabilitation with adjunctive NMES compared with rehabilitation alone at 3 months after TKA (MD $-4.9,95 \% \mathrm{CI}-9.5$ to -0.3 ). 
Table 53. Post-acute rehabilitation versus various controls for total knee arthroplasty - continuous outcomes, quality of life

\begin{tabular}{|c|c|c|c|c|c|c|c|c|c|c|c|}
\hline $\begin{array}{l}\text { Study, Year, } \\
\text { PMID, Country }\end{array}$ & Arm 1 & Arm 2 & $\begin{array}{l}\text { Overall } \\
\text { RoB }\end{array}$ & $\begin{array}{l}\text { Outcome } \\
\text { Measurement }\end{array}$ & $\begin{array}{l}\text { Time } \\
\text { Point }^{\mathrm{A}}\end{array}$ & $\begin{array}{l}\text { N Arm } \\
1\end{array}$ & $\begin{array}{l}\text { Arm 1, Mean } \\
\text { (SD) }\end{array}$ & $\begin{array}{l}\text { N Arm } \\
2\end{array}$ & $\begin{array}{l}\text { Arm 2, } \\
\text { Mean (SD) }\end{array}$ & $\begin{array}{l}\text { Effect Size } \\
(95 \% \mathrm{Cl})\end{array}$ & $\begin{array}{l}\text { Reported } \\
\text { p-Value }\end{array}$ \\
\hline \multirow[t]{2}{*}{$\begin{array}{l}\text { Andersen, } \\
\text { 2018, CN- } \\
\text { 01647420, } \\
\text { Denmark }\end{array}$} & $\begin{array}{l}\text { Technological } \\
\text { assisted } \\
\text { rehabilitation }\end{array}$ & $\begin{array}{l}\text { Supervised } \\
\text { rehabilitation }\end{array}$ & High & $\begin{array}{l}\text { KOOS: QoL (0- } \\
100)\end{array}$ & $6 \mathrm{mo}$ & NR & $\mathrm{NR}(\mathrm{NR})$ & NR & NR (NR) & $\begin{array}{l}12.2 \% \text { (in favor } \\
\text { of } \\
\text { technological } \\
\text { assisted } \\
\text { rehabilitation) }\end{array}$ & NR \\
\hline & $\begin{array}{l}\text { Technological } \\
\text { assisted } \\
\text { rehabilitation }\end{array}$ & $\begin{array}{l}\text { Supervised } \\
\text { rehabilitation }\end{array}$ & High & $\begin{array}{l}\text { KOOS: QoL (0- } \\
100)\end{array}$ & $12 \mathrm{mo}$ & NR & NR (NR) & NR & NR (NR) & $\begin{array}{l}<10 \% \\
\text { difference } \\
\text { between } \\
\text { groups }\end{array}$ & NR \\
\hline \multirow[t]{2}{*}{$\begin{array}{l}\text { Artz, 2017, } \\
27068368, U K\end{array}$} & $\begin{array}{l}\text { Group-based } \\
\text { exercise }\end{array}$ & Usual care & High & $\begin{array}{l}\text { KOOS: QoL (0- } \\
100)\end{array}$ & $3 \mathrm{mo}$ & 19 & $52.4(27.1)$ & 12 & $36.1(17.3)$ & $\begin{array}{l}16.3(5.1 \\
27.5)^{\mathrm{B}}\end{array}$ & NR \\
\hline & $\begin{array}{l}\text { Group-based } \\
\text { exercise }\end{array}$ & Usual care & High & $\begin{array}{l}\text { KOOS: QoL (0- } \\
100)\end{array}$ & $6 \mathrm{mo}$ & 21 & $61.5(32.3)$ & 15 & $45.1(29.2)$ & $\begin{array}{l}16.4(2.1, \\
30.7)^{\mathrm{B}}\end{array}$ & NR \\
\hline $\begin{array}{l}\text { Bruun-Olsen, } \\
2013 \text {, } \\
23614370, \\
\text { Norway }\end{array}$ & $\begin{array}{l}\text { Walking-skill } \\
\text { group }\end{array}$ & $\begin{array}{l}17 \text { individual } \\
\text { physiotherapy } \\
\text { sessions }\end{array}$ & Moderate & $\begin{array}{l}\text { KOOS: QoL (0- } \\
100)\end{array}$ & $9 \mathrm{mo}$ & 29 & $72(24)$ & 28 & $62(26)$ & adj $5(-7,17)$ & NR \\
\hline \multirow[t]{3}{*}{$\begin{array}{l}\text { DeJong, } \\
2020, \\
32360105 \\
\text { USA }\end{array}$} & $\begin{array}{l}\text { Body Weight- } \\
\text { Adjustable } \\
\text { Treadmill \& } \\
\text { PENS }\end{array}$ & $\begin{array}{l}\text { Recumbent Bike } \\
\text { \& PENS }\end{array}$ & High & $\begin{array}{l}\text { KOOS: QoL (0- } \\
100)\end{array}$ & $6 \mathrm{mo}$ & 70 & $77.1(22.0)$ & 78 & $73.2(22.9)$ & $-3.9(-9.0,1.2)$ & NR \\
\hline & $\begin{array}{l}\text { Body Weight- } \\
\text { Adjustable } \\
\text { Treadmill \& } \\
\text { PENS }\end{array}$ & $\begin{array}{l}\text { Body Weight- } \\
\text { Adjustable } \\
\text { Treadmill }\end{array}$ & High & $\begin{array}{l}\text { KOOS: QoL (0- } \\
100)\end{array}$ & $6 \mathrm{mo}$ & 70 & $77.1(22.0)$ & 76 & $76.1(24.3)$ & $1.0(-6.3,4.3)$ & NR \\
\hline & $\begin{array}{l}\text { Body Weight- } \\
\text { Adjustable } \\
\text { Treadmill \& } \\
\text { PENS }\end{array}$ & $\begin{array}{l}\text { Recumbent } \\
\text { Bike/Usual Care }\end{array}$ & High & $\begin{array}{l}\text { KOOS: QoL (0- } \\
100)\end{array}$ & $6 \mathrm{mo}$ & 70 & $77.1(22.0)$ & 74 & $77.9(21.6)$ & $0.8(-4.2,5.8)$ & NR \\
\hline $\begin{array}{l}\text { Demircioglu, } \\
2015, \\
26355656, \\
\text { Turkey }\end{array}$ & $\begin{array}{l}\text { NMES \& } \\
\text { exercise }\end{array}$ & Exercise & High & SF-36 (0-100) & $3 \mathrm{mo}$ & 30 & $68(11.6)$ & 30 & $67.8(15.6)$ & $0.2(-4.8,5.2)^{\mathrm{B}}$ & NR \\
\hline $\begin{array}{l}\text { Kauppila, 2010, } \\
\text { 20354057, } \\
\text { Finland }\end{array}$ & $\begin{array}{l}\text { Multidisciplinary } \\
\text { rehabilitation } \\
\text { group }\end{array}$ & Control & Moderate & $\begin{array}{l}\text { HRQoL-15D } \\
\text { score }\end{array}$ & $1 \mathrm{y}$ & 36 & NR (NR) & 39 & NR (NR) & NR & $>0.05$ \\
\hline $\begin{array}{l}\text { Lenguerrand, } \\
2020 \text {, } \\
31033232 \text {, UK }\end{array}$ & $\begin{array}{l}\text { Physical therapy } \\
\text { and standard } \\
\text { care }\end{array}$ & Standard care & Moderate & $\begin{array}{l}\text { KOOS: QoL (0- } \\
100)\end{array}$ & $12 \mathrm{mo}$ & 72 & $\begin{array}{l}\text { Median (IQR) } \\
75(50,94)\end{array}$ & 69 & $\begin{array}{l}\text { Median } \\
(\text { IQR) } 63 \\
(56,88) \\
\end{array}$ & NR & 0.264 \\
\hline \multirow[t]{2}{*}{$\begin{array}{l}\text { Minns Lowe, } \\
2012 \text {, } \\
22180446 \text {, UK }\end{array}$} & $\begin{array}{l}\text { Home-visit } \\
\text { physiotherapy }\end{array}$ & Usual care & High & $\begin{array}{l}\text { KOOS: QoL (0- } \\
100)\end{array}$ & $3 \mathrm{mo}$ & 46 & $\begin{array}{l}\text { Median (IQR) } \\
53.1(25)\end{array}$ & 47 & $\begin{array}{l}\text { Media } \\
\text { (IQR) } 56.3 \\
(31)\end{array}$ & $3.2(-2.8,9.2)^{B}$ & NR \\
\hline & $\begin{array}{l}\text { Home-visit } \\
\text { physiotherapy }\end{array}$ & Usual care & High & $\begin{array}{l}\text { KOOS: QoL (0- } \\
100)\end{array}$ & $6 \mathrm{mo}$ & 42 & $\begin{array}{l}\text { Median (IQR) } \\
59.4(31)\end{array}$ & 43 & $\begin{array}{l}\text { Media } \\
\text { (IQR) } 59.4 \\
(41)\end{array}$ & $0(-8.1,8.1)^{\mathrm{B}}$ & NR \\
\hline
\end{tabular}




\begin{tabular}{|c|c|c|c|c|c|c|c|c|c|c|c|}
\hline $\begin{array}{l}\text { Study, Year, } \\
\text { PMID, Country }\end{array}$ & Arm 1 & Arm 2 & $\begin{array}{l}\text { Overall } \\
\text { RoB }\end{array}$ & $\begin{array}{l}\text { Outcome } \\
\text { Measurement }\end{array}$ & $\begin{array}{l}\text { Time } \\
\text { Point }^{\mathrm{A}}\end{array}$ & $\begin{array}{l}\text { N Arm } \\
1\end{array}$ & $\begin{array}{l}\text { Arm 1, Mean } \\
\text { (SD) }\end{array}$ & $\begin{array}{l}\text { N Arm } \\
2\end{array}$ & $\begin{array}{l}\text { Arm 2, } \\
\text { Mean (SD) }\end{array}$ & $\begin{array}{l}\text { Effect Size } \\
(95 \% \mathrm{CI})\end{array}$ & $\begin{array}{l}\text { Reported } \\
\text { p-Value }\end{array}$ \\
\hline & $\begin{array}{l}\text { Home-visit } \\
\text { physiotherapy }\end{array}$ & Usual care & High & $\begin{array}{l}\text { KOOS: QoL (0- } \\
100)\end{array}$ & $12 \mathrm{mo}$ & 44 & $\begin{array}{l}\text { Median (IQR) } \\
63(43)\end{array}$ & 48 & $\begin{array}{l}\text { Media } \\
\text { (IQR) } 62.5 \\
(42) \\
\end{array}$ & $-0.5(-9.6,8.6)$ & NR \\
\hline $\begin{array}{l}\text { Moffet, 2015, } \\
\text { 26178888, } \\
\text { Canada }\end{array}$ & $\begin{array}{l}\text { In-home } \\
\text { Telerehabilitation }\end{array}$ & $\begin{array}{l}\text { Standard home } \\
\text { rehabilitation }\end{array}$ & Moderate & $\begin{array}{l}\text { KOOS: QoL (0- } \\
100)\end{array}$ & $4 \mathrm{mo}$ & 100 & 69 (NR) & 98 & $69.5(\mathrm{NR})$ & $-0.4(-6.8,6.1)$ & NR \\
\hline \multirow[t]{2}{*}{$\begin{array}{l}\text { Monticone, } \\
2013 \text {, } \\
23063624 \text {, Italy }\end{array}$} & $\begin{array}{l}\text { Home-based } \\
\text { functional } \\
\text { exercises and } \\
\text { kinesiophobia } \\
\text { training }\end{array}$ & Usual care & Moderate & $\begin{array}{l}\text { KOOS: QoL (0- } \\
100)\end{array}$ & $6 \mathrm{mo}$ & 55 & NR & 55 & NR & $\begin{array}{l}10.81(3.01, \\
18.61)\end{array}$ & NR \\
\hline & $\begin{array}{l}\text { Home-based } \\
\text { functional } \\
\text { exercises and } \\
\text { kinesiophobia } \\
\text { training }\end{array}$ & Usual care & Moderate & $\begin{array}{l}\text { KOOS: QoL (0- } \\
100)\end{array}$ & $12 \mathrm{mo}$ & 55 & NR & 55 & NR & $\begin{array}{l}12.27(4.80, \\
19.74)\end{array}$ & NR \\
\hline $\begin{array}{l}\text { Petersen, 2018, } \\
\text { 29294078, } \\
\text { Netherlands }\end{array}$ & $\begin{array}{l}\text { Exercise \& } \\
\text { acupuncture }\end{array}$ & Exercise & Moderate & $\begin{array}{l}\text { KOOS: QoL (0- } \\
100)\end{array}$ & $3 \mathrm{mo}$ & 82 & $\begin{array}{l}\text { N with } \\
\text { success (\%) } \\
33(39)\end{array}$ & 83 & $\begin{array}{l}\text { N with } \\
\text { success } \\
\text { (\%) } 31(37)\end{array}$ & $\begin{array}{l}\text { RR 1.05 (0.72, } \\
1.55)\end{array}$ & 0.797 \\
\hline \multirow[t]{4}{*}{$\begin{array}{l}\text { Piva, 2019, } \\
\text { 30794296, USA }\end{array}$} & $\begin{array}{l}\text { Community- } \\
\text { based group } \\
\text { exercise }\end{array}$ & Standard care & Moderate & SF-36 (0-100) & $3 \mathrm{mo}$ & 90 & $45(9)$ & 44 & $44(8)$ & $0.7(-1.8,3.2)$ & NR \\
\hline & $\begin{array}{l}\text { Clinic-based } \\
\text { group exercise }\end{array}$ & Standard care & Moderate & SF-36 (0-100) & $3 \mathrm{mo}$ & 87 & $45(8)$ & 44 & $44(8)$ & $2.3(-0.2,4.7)$ & NR \\
\hline & $\begin{array}{l}\text { Community- } \\
\text { based group } \\
\text { exercise }\end{array}$ & Standard care & Moderate & SF-36 (0-100) & $6 \mathrm{mo}$ & 89 & $46(9)$ & 45 & $44(10)$ & $0.9(-2.0,3.7)$ & NR \\
\hline & $\begin{array}{l}\text { Clinic-based } \\
\text { group exercise }\end{array}$ & Standard care & Moderate & SF-36 (0-100) & $6 \mathrm{mo}$ & 88 & $45(9)$ & 45 & $44(10)$ & $3.4(0.5,6.2)$ & NR \\
\hline
\end{tabular}

Statistically significant effect sizes are in bold text. In cases where calculated effect size confidence intervals were not-statistically significant but reported p-values were, we deferred to reported p-values and still bolded results.

Abbreviations: $\mathrm{CI}=$ confidence interval, $\mathrm{HRQoL}-15 \mathrm{D}=15$-dimensional instrument of health-related quality of life, IQR $=$ interquartile range, $\mathrm{KOOS}=\mathrm{Knee}$ injury and osteoarthritis outcome score, $\mathrm{mo}=$ month, $\mathrm{NR}=$ not reported, $\mathrm{PMID}=$ PubMed identifier, $\mathrm{QoL}=$ quality of life, $\mathrm{RoB}=$ risk of bias, $\mathrm{SF}-36=36$-item short form survey, $\mathrm{SD}=$ standard deviation.

\footnotetext{
A Time from surgery

${ }^{\mathrm{B}}$ Calculated
} 
Table 54. Post-acute rehabilitation versus various controls for total knee arthroplasty - continuous outcomes, satisfaction with care

\begin{tabular}{|c|c|c|c|c|c|c|c|c|c|c|c|}
\hline $\begin{array}{l}\text { Study, Year, } \\
\text { PMID, Country }\end{array}$ & Arm 1 & Arm 2 & $\begin{array}{l}\text { Overall } \\
\text { RoB }\end{array}$ & $\begin{array}{l}\text { Outcome } \\
\text { Measurement }\end{array}$ & $\begin{array}{l}\text { Time } \\
\text { Point }^{A}\end{array}$ & $\begin{array}{l}\text { N Arm } \\
1\end{array}$ & $\begin{array}{l}\text { Arm 1, Mean } \\
\text { (SD) }\end{array}$ & $\begin{array}{l}\text { N Arm } \\
2\end{array}$ & $\begin{array}{l}\text { Arm 2, Mean } \\
\text { (SD) }\end{array}$ & $\begin{array}{l}\text { Effect Size }(95 \% \\
\mathrm{Cl})\end{array}$ & $\begin{array}{l}\text { Reported } \\
\text { p-Value }\end{array}$ \\
\hline $\begin{array}{l}\text { Moffet, 2015, } \\
\text { 26178888, } \\
\text { Canada }\end{array}$ & $\begin{array}{l}\text { In-home } \\
\text { Telerehabilitation }\end{array}$ & $\begin{array}{l}\text { Standard home } \\
\text { rehabilitation }\end{array}$ & Moderate & $\begin{array}{l}\text { Health care } \\
\text { satisfaction } \\
\text { questionnaire }\end{array}$ & $4 \mathrm{mo}$ & 98 & $90.3(9.9)$ & 82 & $89.3(9.6)$ & $-1.0(-3.02,1.02)^{\mathrm{B}}$ & 0.34 \\
\hline
\end{tabular}

Abbreviations: $\mathrm{CI}=$ confidence interval, $\mathrm{mo}=$ month, $\mathrm{PMID}=$ PubMed identifier, $\mathrm{RoB}=$ risk of bias, $\mathrm{SD}=$ standard deviation.

A Time from surgery

B Calculated

Table 55. Post-acute rehabilitation versus various controls for total knee arthroplasty - continuous outcomes, patient global

assessment

\begin{tabular}{|c|c|c|c|c|c|c|c|c|c|c|c|}
\hline $\begin{array}{l}\text { Study, Year, } \\
\text { PMID, Country }\end{array}$ & Arm 1 & Arm 2 & $\begin{array}{l}\text { Overall } \\
\text { RoB }\end{array}$ & $\begin{array}{l}\text { Outcome } \\
\text { Measurement }\end{array}$ & $\begin{array}{l}\text { Time } \\
\text { Point }^{\mathrm{A}}\end{array}$ & $\begin{array}{l}\text { N Arm } \\
1\end{array}$ & $\begin{array}{l}\text { Arm 1, } \\
\text { Mean (SD) }\end{array}$ & $\begin{array}{l}\text { N Arm } \\
2\end{array}$ & $\begin{array}{l}\text { Arm 2, } \\
\text { Mean (SD) }\end{array}$ & $\begin{array}{l}\text { Effect Size } \\
(95 \% \mathrm{CI})\end{array}$ & $\begin{array}{l}\text { Reported } \\
\text { p-Value }\end{array}$ \\
\hline \multirow[t]{2}{*}{$\begin{array}{l}\text { Artz, 2017, } \\
27068368, U K\end{array}$} & $\begin{array}{l}\text { Group-based } \\
\text { exercise }\end{array}$ & Usual care & High & $\begin{array}{l}\text { Measure yourself } \\
\text { medical outcome profile }\end{array}$ & $3 \mathrm{mo}$ & 19 & $1.9(1.3)$ & 12 & $2.8(0.9)$ & $\begin{array}{l}-0.9(-1.5,- \\
0.3)^{\mathrm{B}}\end{array}$ & NR \\
\hline & $\begin{array}{l}\text { Group-based } \\
\text { exercise }\end{array}$ & Usual care & High & $\begin{array}{l}\text { Measure yourself } \\
\text { medical outcome profile }\end{array}$ & $6 \mathrm{mo}$ & 21 & $1.9(1.4)$ & 15 & $2.4(1.3)$ & $\begin{array}{l}-0.5(-1.1 \\
0.1)^{\mathrm{B}}\end{array}$ & NR \\
\hline \multirow[t]{3}{*}{$\begin{array}{l}\text { Bade, 2017, } \\
27813347, \text { USA }\end{array}$} & $\begin{array}{l}\text { High-intensity } \\
\text { progressive } \\
\text { rehabilitation }\end{array}$ & $\begin{array}{l}\text { Low-intensity } \\
\text { rehabilitation }\end{array}$ & Moderate & WOMAC: Total (0-96) & $3 \mathrm{mo}$ & 72 & $14.49(8.98)$ & 75 & $14.55(8.38)$ & $\begin{array}{l}-0.1(-2.1 \\
1.9)^{\mathrm{B}}\end{array}$ & NR \\
\hline & $\begin{array}{l}\text { High-intensity } \\
\text { progressive } \\
\text { rehabilitation }\end{array}$ & $\begin{array}{l}\text { Low-intensity } \\
\text { rehabilitation }\end{array}$ & Moderate & WOMAC: Total (0-96) & $6 \mathrm{mo}$ & 66 & $8.97(7.27)$ & 67 & $10.60(9.45)$ & $\begin{array}{l}-1.6(-3.7 \\
0.4)^{\mathrm{B}}\end{array}$ & NR \\
\hline & $\begin{array}{l}\text { High-intensity } \\
\text { progressive } \\
\text { rehabilitation }\end{array}$ & $\begin{array}{l}\text { Low-intensity } \\
\text { rehabilitation }\end{array}$ & Moderate & WOMAC: Total (0-96) & $12 \mathrm{mo}$ & 62 & $6.69(7.75)$ & 62 & $7.16(6.28)$ & $\begin{array}{l}-0.5(-2.2 \\
1.3)^{\mathrm{B}}\end{array}$ & NR \\
\hline $\begin{array}{l}\text { Bily, 2016, } \\
\text { 26763947, } \\
\text { Austria }\end{array}$ & Leg-press group & $\begin{array}{l}\text { Physiotherapy } \\
\text { group }\end{array}$ & High & WOMAC: Total (0-96) & $3 \mathrm{mo}$ & 26 & $2.3(0.28)$ & 29 & $2.0(0.18)$ & $\begin{array}{l}0.3(0.2 \\
0.4)^{\mathrm{B}}\end{array}$ & 0.26 \\
\hline \multirow[t]{3}{*}{$\begin{array}{l}\text { DeJong, } \\
2020, \\
32360105 \\
\text { USA }\end{array}$} & $\begin{array}{l}\text { Body Weight- } \\
\text { Adjustable } \\
\text { Treadmill \& } \\
\text { PENS }\end{array}$ & $\begin{array}{l}\text { Recumbent } \\
\text { Bike \& PENS }\end{array}$ & High & KOOS: Total $(0-100)$ & $6 \mathrm{mo}$ & 70 & $78.4(17.8)$ & 78 & $75.4(17.7)$ & $\begin{array}{l}-3.0(-7.1 \\
1.1)\end{array}$ & NR \\
\hline & $\begin{array}{l}\text { Body Weight- } \\
\text { Adjustable } \\
\text { Treadmill \& } \\
\text { PENS }\end{array}$ & $\begin{array}{l}\text { Body Weight- } \\
\text { Adjustable } \\
\text { Treadmill }\end{array}$ & High & KOOS: Total $(0-100)$ & $6 \mathrm{mo}$ & 70 & $78.4(17.8)$ & 76 & $77.5(18.3)$ & $\begin{array}{l}-0.9(-5.0 \\
3.2)\end{array}$ & NR \\
\hline & $\begin{array}{l}\text { Body Weight- } \\
\text { Adjustable } \\
\text { Treadmill \& } \\
\text { PENS }\end{array}$ & $\begin{array}{l}\text { Recumbent } \\
\text { Bike/Usual } \\
\text { Care }\end{array}$ & High & KOOS: Total $(0-100)$ & $6 \mathrm{mo}$ & 70 & $78.4(17.8)$ & 74 & $80.7(13.2)$ & $\begin{array}{l}2.3(-1.4 \\
6.0)\end{array}$ & NR \\
\hline $\begin{array}{l}\text { Demircioglu, } \\
2015, \\
26355656, \\
\text { Turkey } \\
\end{array}$ & $\begin{array}{l}\text { NMES \& } \\
\text { exercise }\end{array}$ & Exercise & High & WOMAC: Total (0-96) & $3 \mathrm{mo}$ & 30 & $42.3(11.3)$ & 30 & $47.2(13.8)$ & $\begin{array}{l}-4.9(-9.5,- \\
0.3)^{B}\end{array}$ & NR \\
\hline
\end{tabular}




\begin{tabular}{|c|c|c|c|c|c|c|c|c|c|c|c|}
\hline $\begin{array}{l}\text { Study, Year, } \\
\text { PMID, Country }\end{array}$ & Arm 1 & Arm 2 & $\begin{array}{l}\text { Overall } \\
\text { RoB }\end{array}$ & $\begin{array}{l}\text { Outcome } \\
\text { Measurement }\end{array}$ & $\begin{array}{l}\text { Time } \\
\text { Point }^{\mathrm{A}}\end{array}$ & $\begin{array}{l}\text { N Arm } \\
1\end{array}$ & $\begin{array}{l}\text { Arm 1, } \\
\text { Mean (SD) }\end{array}$ & $\begin{array}{l}\text { N Arm } \\
2\end{array}$ & $\begin{array}{l}\text { Arm 2, } \\
\text { Mean (SD) }\end{array}$ & $\begin{array}{l}\text { Effect Size } \\
(95 \% \mathrm{CI})\end{array}$ & $\begin{array}{l}\text { Reported } \\
\text { p-Value }\end{array}$ \\
\hline $\begin{array}{l}\text { Kauppila, 2010, } \\
\text { 20354057, } \\
\text { Finland }\end{array}$ & $\begin{array}{l}\text { Multidisciplinary } \\
\text { rehabilitation } \\
\text { group }\end{array}$ & Control & Moderate & WOMAC: Total (0-96) & $12 \mathrm{mo}$ & 36 & NR (NR) & 39 & NR (NR) & NR & NR \\
\hline \multirow[t]{2}{*}{$\begin{array}{l}\text { Mitchell, 2005, } \\
\text { 15869558, UK }\end{array}$} & Hospital & Home & High & SF-6D (0.3-1.0) & $3 \mathrm{mo}$ & 57 & $0.56(0.12)$ & 57 & $0.57(0.09)$ & $\begin{array}{l}\text { Adj MD } \\
0.002(- \\
0.034,0.039)\end{array}$ & 0.894 \\
\hline & Hospital & Home & High & SF-36 (0-100) & $3 \mathrm{mo}$ & 57 & $61.0(22.9)$ & 57 & $61.0(23.4)$ & $\begin{array}{l}\text { Adj MD -0.2 } \\
(-7.0,6.7)\end{array}$ & 0.964 \\
\hline \multirow[t]{4}{*}{$\begin{array}{l}\text { Piva, 2019, } \\
\text { 30794296, USA }\end{array}$} & $\begin{array}{l}\text { Community- } \\
\text { based group } \\
\text { exercise }\end{array}$ & Standard care & Moderate & PROMIS & $3 \mathrm{mo}$ & 90 & $45(5)$ & 44 & $45(5)$ & $\begin{array}{l}0.5(-1.0 \\
1.9)\end{array}$ & NR \\
\hline & $\begin{array}{l}\text { Community- } \\
\text { based group } \\
\text { exercise }\end{array}$ & Standard care & Moderate & PROMIS & $6 \mathrm{mo}$ & 89 & $45(5)$ & 45 & $44(5)$ & $\begin{array}{l}1.4(-0.1 \\
2.9)\end{array}$ & NR \\
\hline & $\begin{array}{l}\text { Clinic-based } \\
\text { group exercise }\end{array}$ & Standard care & Moderate & PROMIS & $3 \mathrm{mo}$ & 87 & $45(5)$ & 44 & $45(5)$ & $\begin{array}{l}1.0(-0.4, \\
2.5)\end{array}$ & NR \\
\hline & $\begin{array}{l}\text { Clinic-based } \\
\text { group exercise }\end{array}$ & Standard care & Moderate & PROMIS & $6 \mathrm{mo}$ & 88 & $45(5)$ & 45 & $44(5)$ & $2.1(0.7,3.6)$ & NR \\
\hline
\end{tabular}

Statistically significant effect sizes are in bold text. In cases where calculated effect size confidence intervals were not-statistically significant but reported p-values were, we deferred to reported p-values and still bolded results.

Abbreviations: Adj MD = adjusted mean difference $\mathrm{CI}=$ confidence interval, $\mathrm{KOOS}=$ Knee injury and osteoarthritis outcome score, mo $=$ month, $\mathrm{NMES}=$ neuromuscular electrical stimulation, $\mathrm{NR}=$ not reported, $\mathrm{PENS}=$ patterned electrical neuromuscular stimulation, $\mathrm{PMID}=$ PubMed identifier, $\mathrm{PROMIS}=$ patient-reported outcomes measurement information system, $\mathrm{RoB}=$ risk of bias, $\mathrm{SD}=$ standard deviation, $\mathrm{SF}-36=36$-Item short form survey, $\mathrm{SF}-6 \mathrm{D}=$ short-form six-dimension, WOMAC $=$ Western Ontario and McMaster Universities Osteoarthritis Index.

A Time from surgery
B Calculated 


\section{Healthcare Utilization Outcomes Following Post-Acute Rehabilitation}

Three studies (Bade 2017, Hamilton 2020, Mitchel 2005)) reported on healthcare-utilization outcomes following acute rehabilitation compared to various control. Outcomes included: need for postoperative procedures and other healthcare-utilization outcomes.

\section{Need for Postoperative Procedures}

One study (Bade 2017) reported data on the need for postoperative procedures after rehabilitation following TKA surgery and observed few events in which patient needed MUA to address stiff knee, with comparable proportions of patients requiring MUA between groups (Table 56).

\section{Other Healthcare Utilization Outcomes}

Cost data (indirect or direct) associated with rehabilitation programs were rarely reported. Hamilton 2020 (UK-based) compared outpatient therapist-led rehabilitation with physiotherapy review and home exercises. Both programs operated within the constraints of standard UK National Health Service (NHS) resources allotted for TKA rehabilitation (equivalent to about six sessions). Mitchell 2005, also UK-based, reported no significant difference in the total NHS costs per patient between groups, but the costs associated with the home physiotherapy program were significantly more expensive compared with hospital outpatient (MD £137, 95\% CI £113 to $£ 160$; approximately $\$ 217,95 \%$ CI $\$ 179$ to $\$ 253$ US dollars during study time period).

\section{Cost-Effectiveness}

We found no studies comparing the cost-effectiveness of rehabilitation with various comparators. Lenguerrand 2020 (comparing group-based outpatient physical therapy and standard care vs. standard care alone) has planned to publish a cost-effectiveness analysis separately which was not identified by our searches.

\section{Harms From Rehabilitation}

Among studies that reported on harms, most $(n=10 / 17)$ reported no adverse events associated with the diverse rehabilitation programs (Bily 2016, Heikkila 2017, Kauppila 2010, Lenguerrand 2020, Li 2015, Liao 2020, Minns Lowe 2012, Moutzouri 2018, Petterson 2009, Petersen 2018) (Table 57). Of the seven studies (Bade 2017, Fransen 2017, Piva 2017, Vuorenmaa 2014, Moffet 2015, Piva 2019, Li 2019) reported data on harms, harms were of low severity (e.g., falls, musculoskeletal injuries, stiffness), uncommon (0 to $6 \%$ for all outcomes with the exception of Piva which reported $13 \%$ for arthralgia/joint pain for patients receiving clinic-based care), and comparable between groups. 
Table 56. Post-acute rehabilitation versus various controls for total knee arthroplasty - need for postoperative procedures

\begin{tabular}{|c|c|c|c|c|c|c|c|c|c|}
\hline $\begin{array}{l}\text { Study, Year, } \\
\text { PMID, Country }\end{array}$ & Arm 1 & Arm 2 & Overall RoB & $\begin{array}{l}\text { Outcome } \\
\text { Measurement }\end{array}$ & Time Point ${ }^{A}$ & $\begin{array}{l}\text { Arm } 1 \mathrm{n} / \mathrm{N} \\
(\%)\end{array}$ & $\begin{array}{l}\text { Arm } 2 \\
\text { n/N (\%) }\end{array}$ & OR $(95 \% \mathrm{CI})$ & $\begin{array}{l}\text { Reported } \\
\text { p-Value }\end{array}$ \\
\hline $\begin{array}{l}\text { Bade, } \\
2017, \\
27813347, \\
\text { USA }\end{array}$ & $\begin{array}{l}\text { High-intensity } \\
\text { progressive } \\
\text { rehabilitation }\end{array}$ & $\begin{array}{l}\text { Low-intensity } \\
\text { rehabilitation }\end{array}$ & Moderate & Knee manipulation & $12 \mathrm{mo}$ & $3 / 84$ & $1 / 78$ & $2.85(0.29,28.01)^{A}$ & NR \\
\hline
\end{tabular}

USA

deferred to reported p-values and still bolded results.

Abbreviations: $\mathrm{CI}=$ confidence interval, $\mathrm{mo}=$ month, $\mathrm{NR}=$ not reported, $\mathrm{OR}=$ odds ratio, $\mathrm{PMID}=\mathrm{PubMed}$ identifier, $\mathrm{RoB}=$ risk of bias .

A Calculated

Table 57. Post-acute rehabilitation versus various controls for total knee arthroplasty - harms

\begin{tabular}{|c|c|c|c|c|c|c|c|c|c|}
\hline $\begin{array}{l}\text { Study, Year, } \\
\text { PMID, } \\
\text { Country }\end{array}$ & Arm 1 & Arm 2 & $\begin{array}{l}\text { Overall } \\
\text { RoB }\end{array}$ & $\begin{array}{l}\text { Outcome } \\
\text { Measurement }\end{array}$ & $\begin{array}{l}\text { Time } \\
\text { Point }^{\mathrm{A}}\end{array}$ & $\begin{array}{l}\text { Arm } 1 \mathrm{n} / \mathrm{N} \\
(\%)\end{array}$ & $\begin{array}{l}\text { Arm } 2 n / N \\
(\%)\end{array}$ & OR $(95 \% \mathrm{CI})$ & $\begin{array}{l}\text { Reported p- } \\
\text { Value }\end{array}$ \\
\hline \multirow{3}{*}{$\begin{array}{l}\text { Bade, } \\
2017 \\
27813347 \\
\text { USA }\end{array}$} & $\begin{array}{l}\text { High-intensity } \\
\text { progressive } \\
\text { rehabilitation }\end{array}$ & $\begin{array}{l}\text { Low-intensity } \\
\text { rehabilitation }\end{array}$ & Moderate & Musculoskeletal injuries & $12 \mathrm{mo}$ & $0 / 84(0 \%)$ & $1 / 78(1 \%)$ & $0.46(0.02,13.94)^{\mathrm{B}}$ & NR \\
\hline & $\begin{array}{l}\text { High-intensity } \\
\text { progressive } \\
\text { rehabilitation } \\
\end{array}$ & $\begin{array}{l}\text { Low-intensity } \\
\text { rehabilitation }\end{array}$ & Moderate & Restricted knee ROM & $12 \mathrm{mo}$ & $3 / 84(4 \%)$ & $3 / 78(4 \%)$ & $0.93(0.18,4.73)^{\mathrm{B}}$ & NR \\
\hline & $\begin{array}{l}\text { High-intensity } \\
\text { progressive } \\
\text { rehabilitation }\end{array}$ & $\begin{array}{l}\text { Low-intensity } \\
\text { rehabilitation }\end{array}$ & Moderate & Fall & $12 \mathrm{mo}$ & $1 / 84(1 \%)$ & $3 / 78(4 \%)$ & $0.30(0.03,2.96)^{B}$ & NR \\
\hline $\begin{array}{l}\text { Li, 2019, } \\
31003647, \\
\text { China }\end{array}$ & Tai Chi & Control & Moderate & Fall & $3 \mathrm{mo}$ & $0 / 54(0 \%)$ & $3 / 53(6 \%)$ & $0.16(0.01,3.19)^{\mathrm{B}}$ & NR \\
\hline \multirow{2}{*}{$\begin{array}{l}\text { Moffet, 2015, } \\
\text { 26178888, } \\
\text { Canada }\end{array}$} & $\begin{array}{l}\text { In-home } \\
\text { Telerehabilitation }\end{array}$ & $\begin{array}{l}\text { Standard home } \\
\text { rehabilitation }\end{array}$ & Moderate & Fall & $4 \mathrm{mo}$ & $1 / 101(1 \%)$ & $0 / 104(0 \%)$ & $2.06(0.07,60.71)^{\mathrm{B}}$ & NR \\
\hline & $\begin{array}{l}\text { In-home } \\
\text { Telerehabilitation }\end{array}$ & $\begin{array}{l}\text { Standard home } \\
\text { rehabilitation }\end{array}$ & Moderate & $\begin{array}{l}\text { Wound bleeding (during } \\
\text { flexion exercises) }\end{array}$ & $4 \mathrm{mo}$ & $1 / 101(1 \%)$ & $0 / 104(0 \%)$ & $2.06(0.07,60.71)^{\mathrm{B}}$ & NR \\
\hline \multirow{6}{*}{$\begin{array}{l}\text { Piva, } \\
2019, \\
30794296, \\
\text { USA }\end{array}$} & $\begin{array}{l}\text { Community-based } \\
\text { group exercise }\end{array}$ & Standard care & Moderate & Arthralgia & $4 \mathrm{mo}$ & $7 / 96(1 \%)$ & $1 / 48(1 \%)$ & $3.70(0.44,30.95)^{\mathrm{B}}$ & NR \\
\hline & $\begin{array}{l}\text { Community-based } \\
\text { group exercise }\end{array}$ & Standard care & Moderate & Back Pain & $4 \mathrm{mo}$ & $2 / 96(2 \%)$ & $0 / 48(0 \%)$ & $2.02(0.09,45.71)^{B}$ & NR \\
\hline & $\begin{array}{l}\text { Community-based } \\
\text { group exercise }\end{array}$ & Standard care & Moderate & Fall & $4 \mathrm{mo}$ & $1 / 96(1 \%)$ & $1 / 48(2 \%)$ & $0.49(0.03,8.09)^{\mathrm{B}}$ & NR \\
\hline & $\begin{array}{l}\text { Community-based } \\
\text { group exercise }\end{array}$ & Standard care & Moderate & Myalgia & $4 \mathrm{mo}$ & $1 / 96(1 \%)$ & $0 / 48(0 \%)$ & $1.00(0.03,30.35)^{\mathrm{B}}$ & NR \\
\hline & $\begin{array}{l}\text { Community-based } \\
\text { group exercise }\end{array}$ & Standard care & Moderate & $\begin{array}{l}\text { Other musculoskeletal } \\
\text { and connective tissue }\end{array}$ & $4 \mathrm{mo}$ & $0 / 96(0 \%)$ & $0 / 48(0 \%)$ & $0.50(0.01,25.46)^{\mathrm{B}}$ & NR \\
\hline & Clinic based & Standard care & Moderate & Arthralgia/joint pain & $4 \mathrm{mo}$ & $\begin{array}{l}12 / 96 \\
(13 \%)\end{array}$ & $1 / 48(2 \%)$ & $6.71(0.85,53.26)^{\mathrm{B}}$ & NR \\
\hline
\end{tabular}




\begin{tabular}{|c|c|c|c|c|c|c|c|c|c|}
\hline $\begin{array}{l}\text { Study, Year, } \\
\text { PMID, } \\
\text { Country }\end{array}$ & Arm 1 & Arm 2 & $\begin{array}{l}\text { Overall } \\
\text { RoB }\end{array}$ & $\begin{array}{l}\text { Outcome } \\
\text { Measurement }\end{array}$ & $\begin{array}{l}\text { Time } \\
\text { Point }^{\mathrm{A}}\end{array}$ & $\begin{array}{l}\text { Arm } 1 \mathrm{n} / \mathrm{N} \\
(\%)\end{array}$ & $\begin{array}{l}\text { Arm } 2 n / N \\
(\%)\end{array}$ & OR $(95 \% \mathrm{CI})$ & $\begin{array}{l}\text { Reported p- } \\
\text { Value }\end{array}$ \\
\hline & Clinic based & Standard care & Moderate & Back Pain & $4 \mathrm{mo}$ & $1 / 96(1 \%)$ & $0 / 48(0 \%)$ & $1.00(0.03,30.35)^{\mathrm{B}}$ & NR \\
\hline & Clinic based & Standard care & Moderate & $\begin{array}{l}\text { Injury related to } \\
\text { arthroplasty-Fall }\end{array}$ & $4 \mathrm{mo}$ & $0 / 96(0 \%)$ & $1 / 48(2 \%)$ & $0.25(0.01,7.47)^{\mathrm{B}}$ & NR \\
\hline & Clinic based & Standard care & Moderate & Myalgia & $4 \mathrm{mo}$ & $0 / 96(0 \%)$ & $0 / 48(0 \%)$ & $0.50(0.01,25.46)^{\mathrm{B}}$ & NR \\
\hline & Clinic based & Standard care & Moderate & $\begin{array}{l}\text { Other musculoskeletal } \\
\text { and connective tissue }\end{array}$ & $4 \mathrm{mo}$ & $5 / 96(5 \%)$ & $0 / 48(0 \%)$ & $5.22(0.28,97.58)^{B}$ & NR \\
\hline
\end{tabular}

Statistically significant effect sizes are in bold text. In cases where calculated effect size confidence intervals were not-statistically significant but reported p-values were, we deferred to reported p-values and still bolded results.

Abbreviations: $\mathrm{CI}=$ confidence interval, $\mathrm{mo}=$ month, $\mathrm{NR}=$ not reported, $\mathrm{OR}=$ odds ratio, $\mathrm{PMID}=\mathrm{PubMed}$ identifier, $\mathrm{RoB}=$ risk of bias, $\mathrm{ROM}=$ range of motion

\footnotetext{
A Time from surgery

${ }^{B}$ Calculated
} 


\section{Heterogeneity of Treatment Effects (Subgroup Differences)}

No studies reported subgroup analyses or more specifically, formally analyzed possible heterogeneity of treatment effects, i.e., statistical tests for whether the comparative effect of rehabilitation versus its various comparators differed in one subgroup of patients versus another (e.g., patients with higher vs. lower measures of strength, flexibility, function, etc. at baseline).

\section{Applicability}

Studies were conducted across the globe (seven in the United States) using diverse interventions employed in diverse healthcare settings. While the relative effect of the interventions on clinical outcomes (and harms) from non-U.S.-based studies are likely applicable to the U.S. context, findings pertaining to healthcare system or resources (such as costs or comparisons of inpatient vs. outpatient rehabilitation) are likely country and healthcare system specific. The sex of participants varied widely across studies, ranging from 27 to 100 percent of participants being female. The average age of patients ages ranged from 56 to 79 years and the average BMIs ranged from 27 to $35 \mathrm{~kg} / \mathrm{m}^{2}$ (thus, in all studies, most patients were obese, but in several, many to most were morbidly obese). Most studies did not report whether patients had undergone previous contralateral replacement surgery; of those that did, proportions were low (less than $30 \%$ ). As such, the conclusions in this KQ are likely most applicable to middle-toolder-aged adults in high-income countries who are receiving their first total TKA for osteoarthritis.

\section{Summary of Comparison of Rehabilitation Versus Various Controls for Total Knee Arthroplasty}

Table 58 summarizes the evidence for the comparison of post-acute rehabilitation versus various comparators. We focus on the outcomes we prioritized in discussion with stakeholders.

There is low to insufficient SoE for all conclusions. Compared with various controls (usually less intensive active rehabilitation controls), rehabilitation in the post-acute phase may result in comparable outcomes of pain, ROM, strength, ADL, and QoL (low SoE). There is insufficient evidence on the impact of post-acute rehabilitation on satisfaction with care and need for postoperative procedures. There is low SoE that there is no difference between post-acute rehabilitation and various comparators in harms related to the intervention. 
Table 58. Evidence profile: Post-acute rehabilitation versus various controls for total knee arthroplasty

\begin{tabular}{|c|c|c|c|c|c|c|c|c|c|}
\hline $\begin{array}{l}\text { Outcome } \\
\text { Category }\end{array}$ & Outcome & $\begin{array}{l}\text { N Studies } \\
\text { (Participants) }\end{array}$ & RoB & Consistency & Precision & Directness & $\begin{array}{l}\text { Interventio } \\
\text { n } \\
\text { Replication }\end{array}$ & SoE & Conclusions \\
\hline \multirow{3}{*}{$\begin{array}{l}\text { Body structure } \\
\text { and function }\end{array}$} & Pain & $22(2478)$ & Moderate & Consistent & Precise & Direct & All unique & Low & Similar pain \\
\hline & ROM & $15(1487)$ & Moderate & Consistent & Precise & Direct & All unique & Low & Similar ROM \\
\hline & Strength & $14(1464)$ & Moderate & Consistent & Precise & Direct & All unique & Low & Similar strength \\
\hline $\begin{array}{l}\text { Activity and } \\
\text { participation }\end{array}$ & ADLs & $22(2657)$ & Moderate & Inconsistent & Precise & Direct & All unique & Low & Similar ADL \\
\hline \multirow[t]{2}{*}{$\begin{array}{l}\text { Other patient- } \\
\text { reported } \\
\text { outcomes }\end{array}$} & Satisfaction with care & $1(180)$ & Moderate & $\begin{array}{l}\text { Consistency } \\
\text { unknown } \\
\text { (single study) }\end{array}$ & Precise & Direct & $\begin{array}{l}\text { NA (single } \\
\text { study) }\end{array}$ & Insufficient & No conclusion \\
\hline & QoL & $12(1208)$ & Moderate & Consistent & Precise & Direct & All unique & Low & Similar QoL \\
\hline $\begin{array}{l}\text { Healthcare } \\
\text { utilization }\end{array}$ & $\begin{array}{l}\text { Need for postoperative } \\
\text { procedures }\end{array}$ & $1(162)$ & Moderate & $\begin{array}{l}\text { Consistency } \\
\text { unknown } \\
\text { (single study) }\end{array}$ & Precise & Direct & $\begin{array}{l}\text { NA (single } \\
\text { study) }\end{array}$ & Insufficient & No conclusion \\
\hline Harms & Harms from rehabilitation & $17(2333)$ & Moderate & NA & Precise & Direct & All unique & Low & Similar harms \\
\hline
\end{tabular}

Abbreviations: ADLs = activities of daily living, $\mathrm{QoL}=$ quality of life, $\mathrm{NA}=$ not applicable, $\mathrm{QoL}=$ quality of life, $\mathrm{RoB}=$ risk of bias, $\mathrm{ROM}=$ range of motion, $\mathrm{SoE}=\mathrm{strength}$ of evidence. 


\section{Key Question 3: Prehabilitation for Total Hip Arthroplasty}

\section{Key Points}

- For patients undergoing THA, there is insufficient evidence of a difference between prehabilitation and no prehabilitation for outcomes of pain, strength, ADLs, QoL, length of stay, or posthospital disposition.

- There was no evidence on satisfaction with care or risk of harms due to prehabilitation.

- There is insufficient evidence on the effectiveness of specific prehabilitation intervention components at the level of goals (e.g., strength, flexibility, etc.) or presence of specific exercise components to address these goals.

- There is insufficient evidence on modification of prehabilitation effects by patient, surgical, or setting factors.

- There is no evidence on the cost effectiveness of prehabilitation compared with no prehabilitation.

\section{Findings Pertaining to Prehabilitation for Total Hip Arthroplasty}

We found six eligible studies, all RCTs, that compared some version of a prehabilitation intervention to no prehabilitation (summarized in Figure 4). No prehabilitation was defined variably and generally included either specification of no additional care (e.g., "no therapy" [Bitterli 2011], ${ }^{137}$ "no additional care" (Vukomanović 2008), 138 "care as usual" (HolsgaardLarsen 2020, Pour 2007), ${ }^{139,140}$ or dissemination of information on surgical and postoperative expectations (Pour 2007, Rooks 2006, Soeters 2018) ${ }^{73,140,141}$ Of note, Pour 2007 and HolsgaardLarsen 2020 noted their dissemination of information as usual care, although this was not defined as usual care in other studies. We rated two of the RCTs to be at overall high risk of bias, mostly related to lack of blinding of participants, study personnel, and/or outcome assessors and unclear methods of how random sequences were generated and/or concealed from patients. We rated the remaining four trials to be at overall moderate risk of bias mostly related to lack of blinding of participants, study personnel, and/or outcome assessors.

The six RCTs enrolled between 45 and 94 participants each. Three were conducted in the United States and three in Europe. One study reported information on funding and was supported by industry (a medical technology company). ${ }^{140}$ The average ages of participants were similar across studies, ranging from 56 to 74 years. The percentage of women in the studies varied from 25 to 80 percent. Average BMI ranged from 26 to $30 \mathrm{~kg} / \mathrm{m}^{2}$. No trials reported information on the proportion of patients who had undergone prior contralateral THA. Appendix Tables C-3.1, C-3.2, and C-3.3 include the full data for all six RCTs.

\section{Prehabilitation Versus No Prehabilitation}

Six RCTs, reported in six articles (Bitterli 2011, Holsgaard-Larsen 2020, Pour 2007, Rooks 2006, Soeters 2018, Vukomanović 2008) compared prehabilitation to no prehabilitation in a total of 425 patients who subsequently underwent THA. Only two studies had somewhat similar interventions (Soeters 2018, Vukomanović 2008); the remaining four studies evaluated unique prehabilitation interventions comprised of varying goals and exercise components (as coded by our taxonomy), delivered in a variety of settings (by different modalities) by diverse (or no) personnel. Soeters 2018 and Vukomanović 2008 evaluated a preoperative physical therapy 
program (targeting task-specific training, and patient education goals) with a no prehabilitation control.

Prehabilitation interventions were initiated between 2 and 10 weeks prior to the scheduled THA (in four trials). Pour 2007 and Vukomanović 2008 did not report when participants began their prehabilitation programs.

The six trials did not have consistent component goals for the evaluated prehabilitation interventions. Exercises to target strength and task specific training were included in three trials. Components to target patient education were also included in three trials. Exercises to target aerobic endurance and flexibility were only included in one trial. No prehabilitation interventions included components of balance-motor-learning-agility. Specific exercise components within prehabilitation goal components varied across programs. No study included adjunctive modalities in combination with the prehabilitation programs.

Two studies reported some form of progression, of which one (Holsgaard-Larsen 2020) was assessed by clinical experts on our team as appropriate. No study compared prehabilitation with versus without progression.

Interventions were delivered by physical therapists in five of the six studies. Bitterli 2011 included one group where prehabilitation comprised an unsupervised self-guided home component (i.e., no one supervised) and a second group who received an in-person training component without a report of who delivered the intervention. Reported settings included home (Bitterli 2011), an outpatient rehabilitation facility (Pour 2007; Holsgaard-Larsen 2020), and a community fitness center (Rooks 2006). Two studies did not report where prehabilitation was delivered.

Specific codes for intervention (and control arm, where present) goals and exercises, use of progression (and assessment of appropriateness), and details on personnel, mode of delivery, and setting are detailed in Table 59 and Appendix Table C-3.2. 
Figure 4. Overview of studies of prehabilitation interventions for total hip arthroplasty
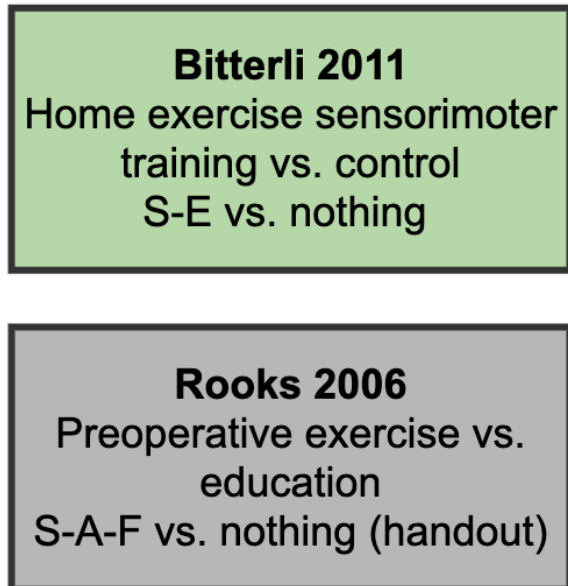
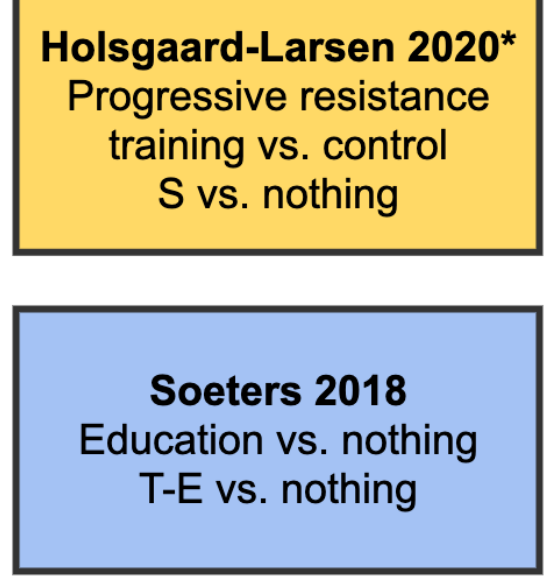
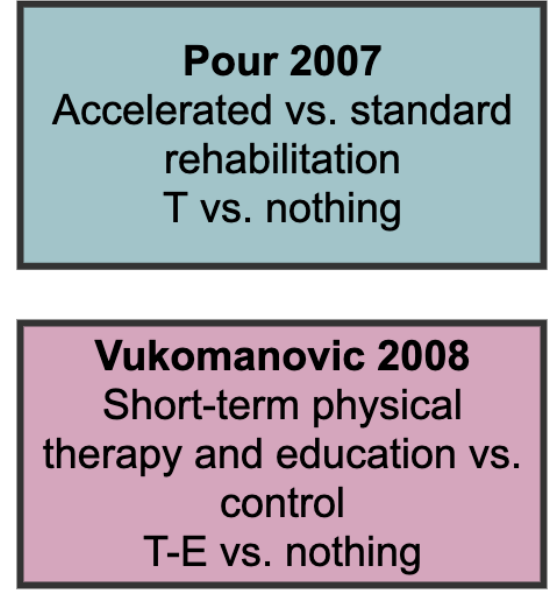

Figure presents studies $(n=6)$ that evaluated prehabilitation programs for total hip arthroplasty versus nothing (defined by the study and the goal components coded by the Oatis and Franklin taxonomy). The color is added for visual display and does not provide unique information.

Abbreviations: $\mathrm{Adj}=$ adjunctive, $\mathrm{A}=$ aerobic exercise, $\mathrm{B}=$ Balance-motor/Learning-agility exercise, $\mathrm{E}=$ patient education, $\mathrm{F}=$ flexibility exercise, $\mathrm{S}=$ strengthening exercise, $\mathrm{T}=$ task-specific training.

* Intervention included progression which was deemed appropriate. 
Table 59. Goal components and their specific exercise components for prehabilitation interventions versus no prehabilitation for total hip arthroplasty

\begin{tabular}{|c|c|c|c|c|c|c|c|c|c|c|c|c|c|c|c|c|c|c|c|c|c|c|c|c|c|c|c|c|c|c|c|}
\hline त्रे & $\frac{\xi}{<}$ & $\begin{array}{l}\text { 형 } \\
\text { 혛 }\end{array}$ & 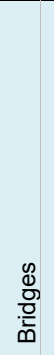 & 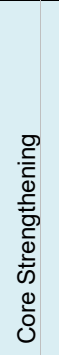 & 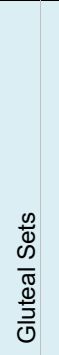 & $\begin{array}{l}\check{O} \\
\stackrel{0}{0} \\
\frac{3}{0} \\
\frac{0}{4} \\
\stackrel{0}{I}\end{array}$ & 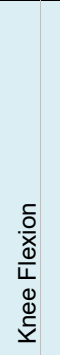 & 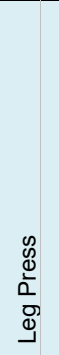 & $\begin{array}{l}\infty \\
\frac{n}{0} \\
\infty \\
0 \\
0 \\
0 \\
0 \\
0\end{array}$ & 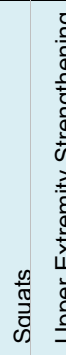 & 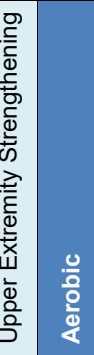 & 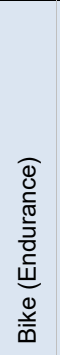 & 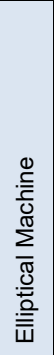 & $\frac{3}{\frac{2}{2}}$ & $\begin{array}{l}\frac{\infty}{2} \\
\frac{\xi}{3} \\
\frac{0}{2} \\
\frac{0}{2} \\
\frac{1}{2}\end{array}$ & 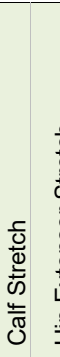 & 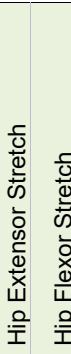 & 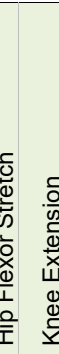 & 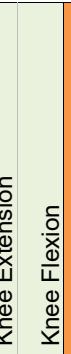 & 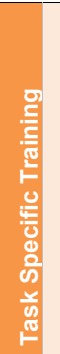 & 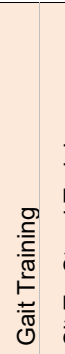 & 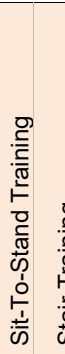 & 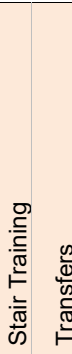 & 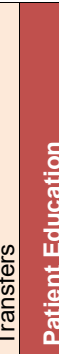 & 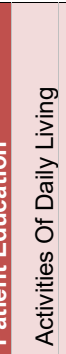 & 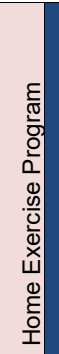 & 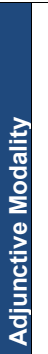 & 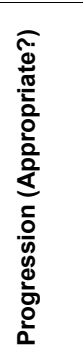 & 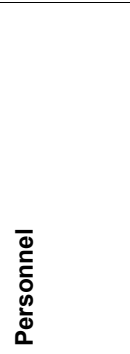 & 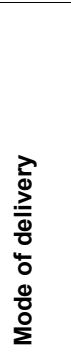 & $\begin{array}{l}\text { D } \\
\text { के } \\
\text { के }\end{array}$ \\
\hline \multirow[t]{2}{*}{ Bitterli, 2011} & Preop exercise & 1 & 1 & 0 & 1 & 1 & 1 & 0 & 1 & 10 & 0 & 0 & 0 & 00 & $\begin{array}{ll}0 & 0 \\
\end{array}$ & $\begin{array}{ll}0 & 0\end{array}$ & \begin{tabular}{l|l}
0 & 0
\end{tabular} & 0 & 0 & 0 & 0 & $\begin{array}{ll}0 & 0 \\
\end{array}$ & \begin{tabular}{l|l}
0 & 0
\end{tabular} & 1 & 0 & 1 & 0 & $\mathrm{~N}$ & NR; None & I; SG & $\mathrm{H}$ \\
\hline & No therapy & 0 & 0 & 0 & 0 & 0 & 0 & 0 & 0 & $\begin{array}{ll}0 & 0 \\
\end{array}$ & 0 & 0 & 0 & 00 & $\begin{array}{ll}0 & 0 \\
\end{array}$ & $\begin{array}{ll}0 & 0 \\
\end{array}$ & $\begin{array}{ll}0 & 0 \\
\end{array}$ & 0 & 0 & 0 & $0 \quad \mathrm{c}-1$ & $\begin{array}{ll}0 & 0 \\
\end{array}$ & $\begin{array}{ll}0 & 0 \\
\end{array}$ & 0 & 0 & 0 & 0 & $\mathrm{~N}$ & NA & NA & NA \\
\hline \multirow{2}{*}{$\begin{array}{l}\text { Holsgaard-Larsen } \\
2020\end{array}$} & Preop PRT & 1 & 0 & 0 & 0 & 0 & 0 & 0 & 0 & 00 & 0 & 0 & 0 & 00 & $\begin{array}{ll}0 & 0 \\
\end{array}$ & $0 \quad 0$ & 0 & 0 & 0 & 0 & 0 & $0 \quad 0$ & $\begin{array}{ll}0 & 0\end{array}$ & 0 & 0 & 0 & 0 & $\mathrm{Y}(\mathrm{Y})$ & PT & 1 & 0 \\
\hline & Control & 0 & 0 & 0 & 0 & 0 & 0 & 0 & 0 & $\begin{array}{ll}0 & 0 \\
\end{array}$ & 0 & 0 & 0 & 00 & $\begin{array}{ll}0 & 0 \\
\end{array}$ & $\begin{array}{ll}0 & 0 \\
\end{array}$ & $\begin{array}{ll}0 & 0 \\
\end{array}$ & 0 & 0 & 0 & $0 \quad 0$ & $\begin{array}{ll}0 & 0 \\
\end{array}$ & $\begin{array}{ll}0 & 0 \\
\end{array}$ & 0 & 0 & 0 & 0 & $\mathrm{~N}$ & NA & NA & NA \\
\hline \multirow[t]{2}{*}{ Pour, 2007} & Accelerated reh & 0 & 0 & 0 & 0 & 0 & 0 & 0 & 0 & $\begin{array}{ll}0 & 0 \\
\end{array}$ & 0 & 0 & 0 & 00 & $\begin{array}{ll}0 & 0\end{array}$ & $0 \quad 0$ & $0 \quad 0$ & 0 & 0 & 1 & 10 & $\begin{array}{ll}0 & 0\end{array}$ & $\begin{array}{ll}0 & 0\end{array}$ & 0 & 0 & 0 & 0 & $\mathrm{~N}$ & PT & I & 0 \\
\hline & Standard rehab & 0 & 0 & 0 & 0 & 0 & 0 & 0 & 0 & $0 \quad 0$ & 0 & 0 & 0 & 00 & $0 \quad 0$ & $0 \quad 0$ & $0 \quad 0$ & 0 & 0 & 0 & 0 & $0 \quad 0$ & $0 \quad 0$ & 0 & 0 & 0 & 0 & $\mathrm{~N}$ & NA & NA & NA \\
\hline \multirow[t]{2}{*}{ Rooks, 2006} & Preop exercise & 1 & 0 & 1 & 0 & 0 & 0 & 1 & 0 & \begin{tabular}{l|l}
0 & 1
\end{tabular} & 1 & 1 & 1 & 11 & $\begin{array}{ll}1 & 1 \\
\end{array}$ & $\begin{array}{ll}1 & 1 \\
\end{array}$ & $\begin{array}{ll}1 & 1\end{array}$ & 1 & 1 & 0 & 0 & \begin{tabular}{l|l}
0 & 0 \\
\end{tabular} & \begin{tabular}{l|l}
0 & 0
\end{tabular} & 0 & 0 & 0 & 0 & $\mathrm{Y}(\mathrm{N})$ & PT & 1 & G \\
\hline & Preop ed & 0 & 0 & 0 & 0 & 0 & 0 & 0 & 0 & 00 & 0 & 0 & 0 & 00 & $0 \quad 0$ & $0 \quad 0$ & $0 \quad 0$ & 0 & 0 & 0 & 0 & $0 \quad 0$ & $0 \quad 0$ & 0 & 0 & 0 & 0 & $\mathrm{~N}$ & NA & NA & NA \\
\hline \multirow[t]{2}{*}{ Soeters, 2018} & PreopPTEd & 0 & 0 & 0 & 0 & 0 & 0 & 0 & 0 & \begin{tabular}{l|l}
0 & 0 \\
\end{tabular} & 0 & 0 & 0 & 00 & \begin{tabular}{l|l}
0 & 0 \\
\end{tabular} & \begin{tabular}{l|l}
0 & 0 \\
\end{tabular} & \begin{tabular}{l|l}
0 & 0
\end{tabular} & 0 & 0 & 1 & 0 & $\begin{array}{ll}1 & 1\end{array}$ & \begin{tabular}{|l|l|}
1 & 1
\end{tabular} & 1 & 1 & 0 & 0 & $\mathrm{~N}$ & PT & 1 & NR \\
\hline & NoPreopPTEd & 0 & 0 & 0 & 0 & 0 & 0 & 0 & 0 & 00 & 0 & 0 & 0 & 00 & $0 \quad 0$ & $0 \quad 0$ & $0 \quad 0$ & 0 & 0 & 0 & 0 & $0 \quad 0$ & $0 \quad 0$ & 0 & 0 & 0 & 0 & $\mathrm{~N}$ & NA & NA & NA \\
\hline \multirow[t]{2}{*}{ Vukomanović, 2008} & Preop PT \& ed & 0 & 0 & 0 & 0 & 0 & 0 & 0 & 0 & 00 & 0 & 0 & 0 & 00 & 0 & $0 \quad 0$ & $0 \quad 0$ & 0 & 0 & 1 & 1 & $\begin{array}{ll}1 & 1\end{array}$ & 11 & 1 & 1 & 1 & 0 & $\mathrm{~N}$ & PT & & $\mathrm{NR}$ \\
\hline & Control & 0 & 0 & 0 & 0 & 0 & 0 & 0 & 0 & 00 & 0 & 0 & 0 & 00 & $0 \quad 0$ & $0 \quad 0$ & $0 \quad 0$ & 0 & 0 & 0 & $0 \mathrm{c}-\mathrm{r}-\mathrm{c}$ & $0 \quad 0$ & $0 \quad 0$ & 0 & 0 & 0 & 0 & $\mathrm{~N}$ & NA & NA & NA \\
\hline
\end{tabular}

The color is added for visual display and does not provide unique information.

$1=$ presence of component, $0=$ absence of component

Abbreviations: $\mathrm{AI}=$ acute in-patient; ed = education, $\mathrm{I}=$ in-person, $\mathrm{G}=$ gym/other community center, $\mathrm{H}=$ home, $\mathrm{NA}=$ not applicable, $\mathrm{NR}=$ not reported, $\mathrm{O}=$ outpatient physiotherapy center, $\mathrm{PT}=$ physical therapy, preop = preoperative; $\mathrm{PRT}=$ progression resistance training, rehab $=$ rehabilitation 
The heterogeneity of the included prehabilitation interventions (varying content, use and appropriateness of progression, and personnel, setting, and timing of intervention delivery) made it challenging to identify meaningful groupings of similar studies to synthesize. In the absence of meaningful clusters of similar studies, we opted to summarize all prehabilitation studies together but contextualize interpretations of individual study results with details about the specific form of prehabilitation evaluated in those studies. We report outcomes under the four following outcome categories: body structure and function; activity and participation; other patientreported; and healthcare-utilization. Given intervention heterogeneity, we determined that metaanalysis was not warranted (i.e., average result would not have been interpretable/meaningful) and instead summarize results narratively.

\section{Body Structure and Function Outcomes}

Three RCTs (Bitterli 2011, Holsgaard-Larsen, 2020, Rooks 2006) reported on body structure and function outcomes comparing prehabilitation with no prehabilitation (Tables 60 to 65). The outcomes included: symptoms, pain, range of motion, muscle strength, energy and vigor, and emotional functioning.

\section{Symptoms}

One study (Holsgaard-Larsen 2020) reported data on symptoms using the symptoms component of the Hip Disability Osteoarthritis Outcome Score (HOOS; 0 to 100; higher is better) (Table 60) and observed no difference between groups 12 months after THA.

\section{Pain}

Two studies (Holsgaard-Larsen 2020 and Rooks 2006) reported data on symptoms using the pain components of the HOOS (0 to 100; higher is better), SF-36 (for each component: 0-100; higher is better) and the WOMAC ( 0 to 20 , smaller is better) and observed no differences between groups at 6 and 12 months after THA (Table 61).

\section{Range of Motion}

One study (Holsgaard-Larsen 2020) reported data on knee extension and hip extension and observed no differences as 12-month follow-up (Table 62).

\section{Muscle Strength}

Rooks 2006 reported on muscle strength data using the one-repetition maximum leg press test, which is designed to assess lower-extremity strength (Table 63). During the test, resistance is systematically increased until patients maximum voluntary muscle force is achieved. Higher values indicate greater strength. Rooks 2006 reported no statistically (or clinically) significant difference between prehabilitation and control groups 6 months after THA (Rooks 2006).

\section{Energy and Vigor}

Bitterli 2011 reported on vigor using the vitality component of the SF-36 (scores 0 to 100, higher is better) (Table 64). The trial found a statistically significant improvement in vitality among patients randomized to the preoperative home exercise sensorimotor training program compared with those who received no preoperative therapy $(\mathrm{P}<0.05)$ at 12 months after surgery. The authors reported that Cohen's $d=0.65$, but did not provide data to allow estimation of the MD. 


\section{Emotional Functioning}

Bitterli 2011 reported on emotional functioning data from mental health scale of the SF-36 (scores 0 to 100, higher is better) and found a statistically significant improvement in mental health among patients randomized to the pre-operative home exercise sensorimotor training program compared with those who received no preoperative therapy (Cohen's $d=0.75 ; \mathrm{P}<0.05$ ) at 12 months after surgery (Table 65). No further data were reported. 
Table 60. Prehabilitation versus no prehabilitation for total hip arthroplasty - continuous outcomes, symptoms

\begin{tabular}{|l|l|l|l|l|l|l|l|l|}
\hline $\begin{array}{l}\text { Study, Year, } \\
\text { PMID, } \\
\text { Country }\end{array}$ & Outcome Measurement & Overall RoB & $\begin{array}{l}\text { Time } \\
\text { Point }\end{array}$ & $\begin{array}{l}\text { Prehab, } \\
\text { N }\end{array}$ & $\begin{array}{l}\text { Prehab, } \\
\text { Mean (SD) }\end{array}$ & $\begin{array}{l}\text { Control, } \\
\text { N }\end{array}$ & $\begin{array}{l}\text { Control, } \\
\text { Mean (SD) }\end{array}$ & $\begin{array}{l}\text { Effect Size } \\
\text { (95\% CI) }\end{array}$ \\
\hline $\begin{array}{l}\text { Holsgaard- } \\
\text { Larsen, } \\
\begin{array}{l}\text { 2020, } \\
\text { 32376477, } \\
\text { Denmark }\end{array}\end{array}$ & HOOS: Symptoms (0-100) & Moderate & 12 mos & 40 & NR & 40 & NR & NMD $4.9(-12.7,2.8)$ \\
\hline
\end{tabular}

Statistically significant effect sizes are in bold text. In cases where calculated effect size confidence intervals were not-statistically significant but reported p-values were, we deferred to reported p-values and still bolded results.

Abbreviations: $\mathrm{CI}=$ confidence interval, $\mathrm{mo}=$ month, $\mathrm{NMD}=$ net mean difference, $\mathrm{NR}=$ not reported, $\mathrm{PMID}=\mathrm{PubMed}$ identifier, $\mathrm{RoB}=$ risk of bias, $\mathrm{SD}=$ standard deviation, HOOS = Hip disability and osteoarthritis outcome

A Time from surgery

Table 61. Prehabilitation versus no prehabilitation for total hip arthroplasty - continuous outcomes, pain

\begin{tabular}{|c|c|c|c|c|c|c|c|c|c|}
\hline $\begin{array}{l}\text { Study, Year, } \\
\text { PMID, } \\
\text { Country }\end{array}$ & Outcome Measurement & Overall RoB & $\begin{array}{l}\text { Time } \\
\text { Point }^{A}\end{array}$ & $\begin{array}{l}\text { Prehab, } \\
\text { N }\end{array}$ & $\begin{array}{l}\text { Prehab, } \\
\text { Mean (SD) }\end{array}$ & $\begin{array}{l}\text { Control, } \\
\text { N }\end{array}$ & $\begin{array}{l}\text { Control, } \\
\text { Mean (SD) }\end{array}$ & $\begin{array}{l}\text { Effect Size } \\
(95 \% \mathrm{CI})\end{array}$ & $\begin{array}{l}\text { Reported } \\
\text { p-Value }\end{array}$ \\
\hline $\begin{array}{l}\text { Holsgaard- } \\
\text { Larsen, } \\
2020 \text {, } \\
32376477 \text {, } \\
\text { Denmark }\end{array}$ & HOOS: Pain (0-100) & Moderate & $12 \mathrm{mos}$ & 40 & NR & 40 & NR & NMD $0.5(-6.7,7.7)$ & 0.89 \\
\hline \multirow[t]{2}{*}{$\begin{array}{l}\text { Rooks, 2006, } \\
17013852 \text {, USA }\end{array}$} & SF-36: bodily pain $(0,100)$ & Moderate & $6 \mathrm{mo}$ & 25 & $79.6(21.2)$ & 24 & $77.4(16.3)$ & MD $2.2(-8.36,12.76)^{\mathrm{B}}$ & NR \\
\hline & WOMAC: pain $(0,20)$ & Moderate & $6 \mathrm{mo}$ & 25 & $1.1(1.7)$ & 24 & $1.0(1.2)$ & NMD $0.9(-0.59,2.39)^{B}$ & NR \\
\hline
\end{tabular}

Statistically significant effect sizes are in bold text. In cases where calculated effect size confidence intervals were not-statistically significant but reported p-values were, we deferred to reported p-values and still bolded results.

Abbreviations: $\mathrm{CI}=$ confidence interval, $\mathrm{mo}=$ month, $\mathrm{HOOS}=$ Hip disability and osteoarthritis outcome, $\mathrm{NMD}=$ net mean difference, $\mathrm{NR}=$ not reported, $\mathrm{PMID}=\mathrm{PubMed}$ identifier, RoB $=$ risk of bias, $\mathrm{SD}=$ standard deviation, $\mathrm{SF}-36=36$-Item short form survey, WOMAC = Western Ontario and McMaster Universities Osteoarthritis Index

A Time from surgery

${ }^{B}$ Calculated 
Table 62. Prehabilitation versus various controls for total hip arthroplasty - continuous outcomes, range of motion

\begin{tabular}{|c|c|c|c|c|c|c|c|c|c|}
\hline $\begin{array}{l}\text { Study, Year, } \\
\text { PMID, } \\
\text { Country }\end{array}$ & $\begin{array}{l}\text { Outcome } \\
\text { Measurement }\end{array}$ & $\begin{array}{l}\text { Overall } \\
\text { RoB }\end{array}$ & $\begin{array}{l}\text { Time } \\
\text { Point }^{A}\end{array}$ & $\begin{array}{l}\text { Prehab, } \\
\text { N }\end{array}$ & Prehab, Mean (SD) & $\begin{array}{l}\text { Control, } \\
\text { N }\end{array}$ & $\begin{array}{l}\text { Control, Mean } \\
\text { (SD) }\end{array}$ & $\begin{array}{l}\text { Effect Size } \\
(95 \% \mathrm{Cl})\end{array}$ & $\begin{array}{l}\text { Reported p- } \\
\text { Value }\end{array}$ \\
\hline \multirow{2}{*}{$\begin{array}{l}\text { Holsgaard- } \\
\text { Larsen, } \\
2020 \text {, } \\
32376477, \\
\text { Denmark }\end{array}$} & Knee extension & Moderate & $12 \mathrm{mo}$ & 40 & NR & 40 & NR & $\begin{array}{l}\text { NMD 0.10 } \\
(0.02,0.22)\end{array}$ & 0.088 \\
\hline & Hip extension & Moderate & $12 \mathrm{mo}$ & 40 & NR & 40 & NR & $\begin{array}{l}\text { NMD 0.09 } \\
(-0.05,0.22)\end{array}$ & 0.23 \\
\hline
\end{tabular}

Statistically significant effect sizes are in bold text. In cases where calculated effect size confidence intervals were not-statistically significant but reported p-values were, we deferred to reported p-values and still bolded results.

Abbreviations: $\mathrm{CI}=$ confidence interval, $\mathrm{kg}=$ kilogram, $\mathrm{mo}=$ month, $\mathrm{NMD}=$ net mean difference, $\mathrm{NR}=$ not reported, $\mathrm{PMID}=\mathrm{PubMed}$ identifier, $\mathrm{RoB}=$ risk of bias, $\mathrm{SD}=$ standard deviation

A Time from surgery

Table 63. Prehabilitation versus various controls for total hip arthroplasty - continuous outcomes, muscle strength

\begin{tabular}{|l|l|l|l|l|l|l|l|l|}
\hline $\begin{array}{l}\text { Study, Year, } \\
\text { PMID, } \\
\text { Country }\end{array}$ & $\begin{array}{l}\text { Outcome } \\
\text { Measurement }\end{array}$ & Overall RoB & $\begin{array}{l}\text { Time } \\
\text { Point }\end{array}$ & Prehab, N & $\begin{array}{l}\text { Prehab, } \\
\text { Mean (SD) }\end{array}$ & $\begin{array}{l}\text { Control, } \\
\text { N }\end{array}$ & $\begin{array}{l}\text { Control, } \\
\text { Mean (SD) }\end{array}$ & $\begin{array}{l}\text { Effect Size } \\
(95 \% \text { CI) }\end{array}$ \\
\hline $\begin{array}{l}\text { Rooks, 2006, } \\
17013852, \text { USA }\end{array}$ & $\begin{array}{l}\text { 1-repetition maximum } \\
(\mathrm{kg})\end{array}$ & Moderate & $6 \mathrm{mo}$ & 25 & $99(37)$ & 24 & $117(51)$ & $\begin{array}{l}\text { NMD -1 (-27.29, } \\
25.29)^{B}\end{array}$ \\
\hline
\end{tabular}

Statistically significant effect sizes are in bold text. In cases where calculated effect size confidence intervals were not-statistically significant but reported p-values were, we deferred to reported p-values and still bolded results.

Abbreviations: $\mathrm{CI}=$ confidence interval, $\mathrm{kg}=$ kilogram, $\mathrm{mo}=$ month, $\mathrm{NMD}=$ net mean difference, $\mathrm{NR}=$ not reported, $\mathrm{PMID}=\mathrm{PubMed}$ identifier, $\mathrm{RoB}=$ risk of bias, $\mathrm{SD}=$ standard deviation

A Time from surgery

${ }^{\mathrm{B}}$ Calculated

Table 64. Prehabilitation versus no prehabilitation for total hip arthroplasty - continuous outcomes, energy and vigor

\begin{tabular}{|l|l|l|l|l|l|l|l|l|l|}
$\begin{array}{l}\text { Study, Year, } \\
\text { PMID, } \\
\text { Country }\end{array}$ & Outcome Measurement & Overall RoB & $\begin{array}{l}\text { Time } \\
\text { Point }\end{array}$ & $\begin{array}{l}\text { Prehab, } \\
\text { N }\end{array}$ & $\begin{array}{l}\text { Prehab, } \\
\text { Mean (SD) }\end{array}$ & $\begin{array}{l}\text { Control, } \\
\text { N }\end{array}$ & $\begin{array}{l}\text { Control, } \\
\text { Mean (SD) } \\
\text { Value }\end{array}$ & $\begin{array}{l}\text { Effect Size } \\
\text { (95\% CI) }\end{array}$ \\
\hline $\begin{array}{l}\text { Bitterli, 2011, } \\
\text { 21630176, } \\
\text { Switzerland }\end{array}$ & SF-36: vitality (NR, NR) & Moderate & 12 mo & 41 & NR & 39 & NR & Cohen's d 0.65 \\
\hline
\end{tabular}

Statistically significant effect sizes are in bold text. In cases where calculated effect size confidence intervals were not-statistically significant but reported p-values were, we deferred to reported p-values and still bolded results.

Abbreviations: $\mathrm{CI}=$ confidence interval, $\mathrm{NR}=$ not reported, $\mathrm{PMID}=$ PubMed identifier, RoB $=$ risk of bias, $\mathrm{SF}-36=36-\mathrm{Item}$ short form survey, $\mathrm{SD}=$ standard deviation 
A Time from surgery

Table 65. Prehabilitation versus no prehabilitation for total hip arthroplasty - continuous outcomes, emotional functioning

(stress/coping)

Study, Year,

PMID,

Outcome Measurement

Overall RoB

Time

Prehab,

Prehab,
Mean (SD)

Contr

Control,
Control,
Mean (SD)

Effect Size

Country

SF-36: mental health (0-100)

Moderate

$12 \mathrm{mo}$

41

NR

\begin{tabular}{l|l}
39 & NR
\end{tabular}

\section{Cohen's d 0.75}

Reported p-

21630176 ,

Switzerland

Statistically significant effect sizes are in bold text. In cases where calculated effect size confidence intervals were not-statistically significant but reported p-values were, we deferred to reported p-values and still bolded results.

Abbreviations: $\mathrm{CI}=$ confidence interval, $\mathrm{NR}=$ not reported, $\mathrm{PMID}=$ PubMed identifier, $\mathrm{RoB}=$ risk of bias, $\mathrm{SF}-36=36$-Item short form survey, $\mathrm{SD}=$ standard deviation.

A Time from surgery 


\section{Activity and Participation Outcomes}

Three RCTs (Bitterli 2006, Holsgaard-Larsen 2020, Rooks 2006) reported on activity and participation outcomes, comparing prehabilitation to no prehabilitation (Tables 66 to 70 ). The outcomes included: patient-reported physical function, transfers, balance, mobility, and timed up and go.

\section{Patient-Reported Physical Function and Activities of Daily Living}

Rooks 2006, Bitterli 2006, and Holsgaard-Larsen 2020 reported on patient-reported physical function on $\mathrm{ADL}$ using three different measurement instruments and found no differences between groups between 6 and 12 months after THA (Table 66).

\section{Transfers}

One study (Holsgaard-Larsen 2020) reported data on transfers using the 5-time sit-to-stand test and observed no differences between groups at 12 months (Table 67).

\section{Balance}

Two studies (Bitterli 2006, Rooks 2006) reported data on balance. Bitterli 2006 recorded data on balance (dynamic or static not specified) from the Biodex Balance System but reported only that there were no statistically significant differences at 12 month follow-up. Rooks 2006 reported data on dynamic balance using the functional reach test (distance in centimeters [cm], higher is better) (Table 68) and found that patients randomized to preoperative exercise demonstrated significantly further capacity to reach compared with control groups at 6 months after THA (NMD 5.9, 95\% CI 1.8 to 10.0 ).

\section{Mobility}

Holsgaard-Larsen 2020 reported data on mobility from two stair test measures and two walk test measures. The stair climb tests assessed the steps/second to ascend or descend a flight of stairs (more is better) and found significant differences in the performance of the stair tests in favor of the prehabilitation group compared with no prehabilitation (Table 69). The 25-meter walk tests assessed the speed (meters/second) to walk 25 meters at normal speed and maximum speed and found no difference between groups.

\section{Timed Up and Go}

Rooks 2006 reported data on the TUG test and found no statistically significant differences between groups at 6 months after THA (Table 70). 
Table 66. Prehabilitation versus no prehabilitation for total hip arthroplasty - continuous outcomes, patient-reported physical function and activities of daily living

\begin{tabular}{|c|c|c|c|c|c|c|c|c|c|}
\hline $\begin{array}{l}\text { Study, Year, } \\
\text { PMID, } \\
\text { Country }\end{array}$ & Outcome Measurement & Overall RoB & $\begin{array}{l}\text { Time } \\
\text { Point }^{A}\end{array}$ & $\begin{array}{l}\text { Prehab, } \\
\text { N }\end{array}$ & $\begin{array}{l}\text { Prehab, } \\
\text { Mean (SD) }\end{array}$ & $\begin{array}{l}\text { Control, } \\
\text { N }\end{array}$ & $\begin{array}{l}\text { Control, } \\
\text { Mean (SD) }\end{array}$ & $\begin{array}{l}\text { Effect Size } \\
(95 \% \mathrm{Cl})\end{array}$ & $\begin{array}{l}\text { Report } \\
\text { ed p- } \\
\text { Value }\end{array}$ \\
\hline \multirow{2}{*}{$\begin{array}{l}\text { Bitterli, 2011, } \\
21630176, \\
\text { Switzerland }\end{array}$} & $\begin{array}{l}\text { SF-36: physical functioning (0- } \\
100)\end{array}$ & Moderate & $12 \mathrm{mo}$ & 41 & $\begin{array}{l}80.0(\mathrm{SE} \\
\left.93.1^{\mathrm{B}}\right)\end{array}$ & 39 & $\begin{array}{l}85.0(\mathrm{SE} \\
\left.98.9^{\mathrm{B}}\right)\end{array}$ & $\begin{array}{l}\text { Cohen's d } 0.39 \\
\text { MD -5 }(-163.93,153.93)^{B}\end{array}$ & 0.39 \\
\hline & WOMAC $(0,68)$ & Moderate & $12 \mathrm{mo}$ & 41 & NR & 39 & NR & Cohen's d 0.08 & 0.47 \\
\hline \multirow{2}{*}{$\begin{array}{l}\text { Holsgaard- } \\
\text { Larsen, } \\
2020, \\
32376477, \\
\text { Denmark }\end{array}$} & HOOS: Sport/rec (0-100) & Moderate & $12 \mathrm{mo}$ & 40 & NR & 40 & NR & NMD $6.2(-3.2,15.6)$ & 0.20 \\
\hline & HOOS: ADL (0-100) & Moderate & $12 \mathrm{mo}$ & 40 & NR & 40 & NR & NMD $2.6(-4.2,9.8)$ & 0.44 \\
\hline $\begin{array}{l}\text { Rooks, 2006, } \\
17013852, \text { USA }\end{array}$ & $\begin{array}{l}\text { SF-36: role limitation physical } \\
(0,100)\end{array}$ & Moderate & $6 \mathrm{mo}$ & 25 & $83.0(35.2)$ & 24 & $86.5(24.4)$ & NMD -2.4 $(-23.02,18.22)^{B}$ & NR \\
\hline $\begin{array}{l}\text { Rooks, 2006, } \\
17013852 \text {, USA }\end{array}$ & $\begin{array}{l}\text { SF-36: physical functioning (0- } \\
100)\end{array}$ & Moderate & $6 \mathrm{mo}$ & 25 & $81.7(18.1)$ & 24 & $76.6(18.6)$ & NMD $8.9(-1.31,19.11)^{B}$ & NR \\
\hline $\begin{array}{l}\text { Rooks, 2006, } \\
17013852, \text { USA }\end{array}$ & WOMAC: function (0-68) & Moderate & $6 \mathrm{mo}$ & 25 & $5.4(5.8)$ & 24 & $5.3(5.4)$ & NMD $0.8(-5.06,6.66)^{B}$ & NR \\
\hline
\end{tabular}

Statistically significant effect sizes are in bold text. In cases where calculated effect size confidence intervals were not-statistically significant but reported p-values were, we deferred to reported p-values and still bolded results.

Abbreviations: $\mathrm{ADL}=$ activities of daily living, $\mathrm{CI}=$ confidence interval, $\mathrm{HOOS}=$ Hip Disability Osteoarthritis Outcome $\mathrm{Score}, \mathrm{mo}=\mathrm{month}, \mathrm{NMD}=$ net $\mathrm{mean}$ difference, $\mathrm{NR}=$ not reported, $\mathrm{PMID}=$ PubMed identifier, $\mathrm{rec}=$ recreation, $\mathrm{RoB}=$ risk of bias, $\mathrm{SD}=$ standard deviation, $\mathrm{SF}-36=36$-Item short form survey, $\mathrm{WOMAC}=\mathrm{Western}$ Ontario and McMaster Universities Osteoarthritis Index.

A Time from surgery

${ }^{B}$ Calculated

Table 67. Prehabilitation versus no prehabilitation for total hip arthroplasty - continuous outcomes, transfers

\begin{tabular}{|l|l|l|l|l|l|l|l|l|}
\hline $\begin{array}{l}\text { Study, Year, } \\
\text { PMID, } \\
\text { Country }\end{array}$ & $\begin{array}{l}\text { Outcome } \\
\text { Measurement }\end{array}$ & Overall RoB & $\begin{array}{l}\text { Time } \\
\text { Point }\end{array}$ & $\begin{array}{l}\text { Prehab, } \\
\text { N }\end{array}$ & $\begin{array}{l}\text { Prehab, } \\
\text { Mean (SD) }\end{array}$ & $\begin{array}{l}\text { Control, } \\
\text { N }\end{array}$ & $\begin{array}{l}\text { Control, } \\
\text { Mean (SD) }\end{array}$ & $\begin{array}{l}\text { Effect Size } \\
\text { (95\% Cl) } \\
\text { p-Value }\end{array}$ \\
\hline $\begin{array}{l}\text { Holsgaard-Larsen, } \\
\text { 2020, 32376477, Denmark }\end{array}$ & $\begin{array}{l}\text { Sit-to-stand (5 } \\
\text { times) (s) }\end{array}$ & Moderate & 12 mo & 40 & NR & 40 & NR & $0.7(-1.0,4.3)$ \\
\hline
\end{tabular}

Statistically significant effect sizes are in bold text. In cases where calculated effect size confidence intervals were not-statistically significant but reported p-values were, we deferred to reported p-values and still bolded results.

Abbreviations: $\mathrm{CI}=$ confidence interval, $\mathrm{mo}=$ month, $\mathrm{NR}=$ not reported, $\mathrm{PMID}=$ PubMed identifier, $\mathrm{RoB}=$ risk of bias, $\mathrm{s}=\mathrm{second}, \mathrm{SD}=\mathrm{standard}$ deviation, $\mathrm{SE}=$ standard error

A Time from surgery 
Table 68. Prehabilitation versus no prehabilitation for total hip arthroplasty - continuous outcomes, balance

\begin{tabular}{|c|c|c|c|c|c|c|c|c|c|c|}
\hline $\begin{array}{l}\text { Study, } \\
\text { Year, } \\
\text { PMID, } \\
\text { Country }\end{array}$ & Outcome & Overall RoB & $\begin{array}{l}\text { Outcome } \\
\text { Measurement }\end{array}$ & $\begin{array}{l}\text { Time } \\
\text { point }^{\mathrm{A}}\end{array}$ & $\begin{array}{l}\text { Prehab, } \\
\text { N }\end{array}$ & $\begin{array}{l}\text { Prehab, } \\
\text { Mean (SD) }\end{array}$ & $\begin{array}{l}\text { Control, } \\
\text { N }\end{array}$ & $\begin{array}{l}\text { Control, } \\
\text { Mean (SD) }\end{array}$ & $\begin{array}{l}\text { Effect Size } \\
(95 \% \mathrm{Cl})\end{array}$ & $\begin{array}{l}\text { Reported } \\
\text { p-Value }\end{array}$ \\
\hline $\begin{array}{l}\text { Rooks, } \\
2006, \\
17013852, \\
\text { USA }\end{array}$ & Balance & Moderate & $\begin{array}{l}\text { Functional reach } \\
\text { (cm) }\end{array}$ & $6 \mathrm{mo}$ & 25 & $33.5(5.2)$ & 24 & $31.4(7.1)$ & NMD $5.9(1.83,9.97)^{B}$ & $<0.05$ \\
\hline
\end{tabular}

Statistically significant effect sizes are in bold text. In cases where calculated effect size confidence intervals were not-statistically significant but reported p-values were, we deferred to reported p-values and still bolded results.

Abbreviations: $\mathrm{CI}=$ confidence interval, $\mathrm{cm}=$ centimeter, $\mathrm{mo}=$ month, $\mathrm{NMD}=$ net mean difference, $\mathrm{PMID}=\mathrm{PubMed}$ identifier, $\mathrm{RoB}=$ risk of bias, $\mathrm{SD}=$ standard deviation.

A Time from surgery

${ }^{B}$ Calculated

Table 69. Prehabilitation versus no prehabilitation for total hip arthroplasty - continuous outcomes, mobility

\begin{tabular}{|c|c|c|c|c|c|c|c|c|c|}
\hline $\begin{array}{l}\text { Study, Year, } \\
\text { PMID, } \\
\text { Country }\end{array}$ & Outcome Measurement & $\begin{array}{l}\text { Overall } \\
\text { RoB }\end{array}$ & $\begin{array}{l}\text { Time } \\
\text { Point }^{\mathrm{A}}\end{array}$ & $\begin{array}{l}\text { Prehab, } \\
\text { N }\end{array}$ & $\begin{array}{l}\text { Prehab, } \\
\text { Mean (SD) }\end{array}$ & $\begin{array}{l}\text { Control, } \\
\mathrm{N}\end{array}$ & $\begin{array}{l}\text { Control, } \\
\text { Mean (SD) }\end{array}$ & $\begin{array}{l}\text { Effect Size } \\
(95 \% \mathrm{CI})\end{array}$ & $\begin{array}{l}\text { Reported } \\
\text { p-Value }\end{array}$ \\
\hline \multirow{4}{*}{$\begin{array}{l}\text { Holsgaard- } \\
\text { Larsen, } \\
2020 \text {, } \\
32376477, \\
\text { Denmark }\end{array}$} & $\begin{array}{l}\text { Stair climb test: ascending stairs } \\
\text { (steps/s) }\end{array}$ & Moderate & $12 \mathrm{mo}$ & 40 & NR & 40 & NR & $1.3(0.3,2.3)$ & 0.0011 \\
\hline & $\begin{array}{l}\text { Stair climb test: descending stairs } \\
\text { (steps/s) }\end{array}$ & Moderate & $12 \mathrm{mo}$ & 40 & NR & 40 & NR & $1.6(0.3,2.9)$ & 0.017 \\
\hline & $\begin{array}{l}\text { 25-m walk test (normal speed) } \\
(\mathrm{m} / \mathrm{s})\end{array}$ & Moderate & $12 \mathrm{mo}$ & 40 & NR & 40 & NR & $1.4(-0.07,2.8)$ & 0.062 \\
\hline & $25-\mathrm{m}$ walk test (max speed) $(\mathrm{m} / \mathrm{s})$ & Moderate & $12 \mathrm{mo}$ & 40 & NR & 40 & NR & $0.9(-0.4,2.2)$ & 0.17 \\
\hline
\end{tabular}

Statistically significant effect sizes are in bold text. In cases where calculated effect size confidence intervals were not-statistically significant but reported p-values were, we deferred to reported p-values and still bolded results.

Abbreviations: $\mathrm{CI}=$ confidence interval, $\mathrm{m}=$ meter, $\mathrm{mo}=$ month, $\mathrm{NR}=$ not reported, $\mathrm{PMID}=$ PubMed identifier, $\mathrm{RoB}=$ risk of bias, $\mathrm{SD}=$ standard deviation, $\mathrm{s}=$ seconds

A Time from surgery 
Table 70. Prehabilitation versus no prehabilitation for total hip arthroplasty - continuous outcomes, Timed Up and Go

\begin{tabular}{|l|l|l|l|l|l|l|l|l|l|}
\hline $\begin{array}{l}\text { Study, } \\
\text { Year, } \\
\text { PMID, } \\
\text { Country }\end{array}$ & Outcome Measurement & $\begin{array}{l}\text { Overall } \\
\text { RoB }\end{array}$ & $\begin{array}{l}\text { Time } \\
\text { point }\end{array}$ & $\begin{array}{l}\text { Prehab, } \\
\text { N }\end{array}$ & $\begin{array}{l}\text { Prehab, } \\
\text { Mean (SD) }\end{array}$ & $\begin{array}{l}\text { Control, } \\
\text { N }\end{array}$ & $\begin{array}{l}\text { Control, } \\
\text { Mean (SD) }\end{array}$ & $\begin{array}{l}\text { Effect Size } \\
\text { (95\% CI) } \\
\text { p-Value }\end{array}$ \\
\hline $\begin{array}{l}\text { Rooks, } \\
\begin{array}{l}1706, \\
\text { USA }\end{array}\end{array}$ & TUG (s) & Moderate & 6 mo & 25 & $9.76(1.29)$ & 24 & $9.41(1.46)$ & NMD -1.04 (-2.4, 0.32) \\
\hline
\end{tabular}

USA

Statistically significant effect sizes are in bold text. In cases where calculated effect size confidence intervals were not-statistically significant but reported p-values were, we deferred to reported p-values and still bolded results.

Abbreviations: $\mathrm{CI}=$ confidence interval, $\mathrm{NMD}=$ net mean difference, $\mathrm{NR}=$ not reported, $\mathrm{PMID}=\mathrm{PubMed}$ identifier, $\mathrm{RoB}=$ risk of bias, $\mathrm{mo}=$ month $, \mathrm{s}=\mathrm{second}, \mathrm{SD}=\mathrm{standard}$ deviation, $\mathrm{TUG}=$ timed up and go.

A Time from surgery

${ }^{B}$ Calculated 


\section{Other Patient-Reported Outcomes}

One study reported on other patient-reported outcomes, comparing prehabilitation with no prehabilitation (Holsgaard-Larsen 2020) (Table 71). The outcome domain included: quality of life.

\section{Health-Related Quality of Life}

One study (Holsgaard-Larsen 2020) reported the QoL component of HOOS and observed no differences between groups at 12 months follow-up after THA (Table 71). 
Table 71. Prehabilitation versus no prehabilitation for total hip arthroplasty - continuous outcomes, quality of life

\begin{tabular}{|c|c|c|c|c|c|c|c|c|c|}
\hline $\begin{array}{l}\text { Study, Year, } \\
\text { PMID, } \\
\text { Country }\end{array}$ & $\begin{array}{l}\text { Outcome } \\
\text { Measurement }\end{array}$ & Overall RoB & $\begin{array}{l}\text { Time } \\
\text { Point }^{A}\end{array}$ & Prehab, N & $\begin{array}{l}\text { Prehab, } \\
\text { Mean (SD) }\end{array}$ & Control, N & $\begin{array}{l}\text { Control, } \\
\text { Mean (SD) }\end{array}$ & $\begin{array}{l}\text { Effect Size } \\
(95 \% \mathrm{CI})\end{array}$ & $\begin{array}{l}\text { Reported p- } \\
\text { Value }\end{array}$ \\
\hline $\begin{array}{l}\text { Holsgaard-Larsen, } \\
2020 \text {, } \\
32376477, \\
\text { Denmark }\end{array}$ & $\begin{array}{l}\text { HOOS: QoL (0- } \\
100)\end{array}$ & Moderate & $12 \mathrm{mo}$ & 40 & NR & 40 & NR & $0.2(-8.9,9.3)$ & 0.97 \\
\hline
\end{tabular}

Statistically significant effect sizes are in bold text. In cases where calculated effect size confidence intervals were not-statistically significant but reported p-values were, we deferred to reported p-values and still bolded results.

Abbreviations: $\mathrm{CI}=$ confidence interval, $\mathrm{HOOS}=$ Hip disability and osteoarthritis outcome score, $\mathrm{mo}=$ month, $\mathrm{NR}=$ not reported, PMID $=$ PubMed identifier, $\mathrm{QoL}=$ quality of life, $\mathrm{RoB}=$ risk of bias, $\mathrm{SD}=$ standard deviation.

A Time from surgery 


\section{Healthcare Utilization Outcomes}

Four RCTs (Bitterli 2011, Pour 2007, Soeters 2018, Vukomanović) reported on healthcareutilization outcomes comparing prehabilitation to no prehabilitation (Tables 72 and 73). The outcomes included: length of stay and other healthcare utilization outcomes.

\section{Length of Stay}

Four RCTs (Bitterli 2011, Pour 2007, Soeters 2018, Vukomanović 2008) provided data on LOS (mean days; smaller is better) (Table 72). Three studies reported no significant differences in LOS between prehabilitation and control groups (Bitterli 2011, Soeters 2018 [the calculated effect size confidence interval was significant, but the reported p-value was not], Vukomanović 2008), but the fourth trial (Pour 2007) reported only that LOS was significantly reduced among patients who were randomized to prehabilitation compared with control (MD not reported; $\mathrm{P}=0.001)$.

\section{Other Healthcare Utilization Outcomes}

Three RCTs (Pour 2007, Soeters 2018, and Vukomanović 2008) reported data on discharge disposition, time to post-acute physical therapy discharge criteria (number of days, smaller is better) and the number of outpatient physical therapy sessions required (number of sessions, smaller is better) (Table 73). Pour 2007 reported patients randomized to the accelerated rehabilitation group (which comprised prehabilitation) were more likely to be discharged home (odds ratio [OR] 3.73, 95\% CI 1.23, 11.32), and less likely to be discharged to a skilled rehabilitation facility (OR $0.26,95 \%$ CI 0.08 to 0.78 ), as compared with patients who received standard rehabilitation (i.e., no prehabilitation).

Soeters 2018 defined readiness to discharge from physical therapy as the ability to 1) independently transfer in and out of bed, a chair, and a toilet seat; 2) independently ambulate approximately 150 feet; 3) independently negotiate stairs; and 4) be independent with a home exercise program and activities of daily living. They reported patients randomized to prehabilitation were more likely to meet physical therapists discharge criteria and require fewer outpatient therapy sessions, as compared with the control group.

Vukomanović 2008 also reported that patients randomized to prehabilitation had fewer outpatient physical therapy sessions compared with those who received no prehabilitation.

No RCT reported specific data on costs. Soeters 2018 noted that local resources may limited the uptake of their program despite "the negligible associated costs" they observed.

\section{Cost-Effectiveness}

We found no studies comparing the cost-effectiveness of prehabilitation with no prehabilitation.

\section{Harms From Prehabilitation}

No RCT reported data on harms from prehabilitation. 
Table 72. Prehabilitation versus no prehabilitation for total hip arthroplasty - continuous outcomes, length of stay

\begin{tabular}{|c|c|c|c|c|c|c|c|c|}
\hline $\begin{array}{l}\text { Study, Year, } \\
\text { PMID, } \\
\text { Country }\end{array}$ & $\begin{array}{l}\text { Outcome } \\
\text { Measurement }\end{array}$ & $\begin{array}{l}\text { Overall } \\
\text { RoB }\end{array}$ & $\begin{array}{l}\text { Prehab, } \\
\text { N }\end{array}$ & $\begin{array}{l}\text { Prehab, Mean } \\
\text { (SD) }\end{array}$ & Control, N & Control, Mean (SD) & $\begin{array}{l}\text { Effect Size } \\
(95 \% \mathrm{Cl})\end{array}$ & $\begin{array}{l}\text { Reported p- } \\
\text { Value }\end{array}$ \\
\hline $\begin{array}{l}\text { Bitterli, 2011, } \\
\text { 21630176, } \\
\text { Switzerland }\end{array}$ & Length of stay (d) & Moderate & 41 & $\begin{array}{l}14.6(2.5), \\
\text { Range (11-23) }\end{array}$ & 39 & 14.6 (2.6), Range (8-22) & $0(-1.12,1.12)^{A}$ & NR \\
\hline $\begin{array}{l}\text { Pour, 2007, } \\
\text { 17768187, USA }\end{array}$ & Length of stay (d) & High & 46 & 4.2, Range (3-8) & 48 & 3.5 , Range (2-5) & NR & 0.001 \\
\hline $\begin{array}{l}\text { Soeters, 2018, } \\
29529614, \text { USA }\end{array}$ & Length of stay (d) & Moderate & 31 & $\begin{array}{l}3.1 \text { (SE: } 0.26 \dagger) \\
95 \% \mathrm{Cl} 2.6,3.6\end{array}$ & 32 & $\begin{array}{l}4.1\left(\mathrm{SE}: 0.26^{\mathrm{A}}\right) \\
95 \% \mathrm{Cl} 3.6,4.6\end{array}$ & MD -1 $(-1.72,-0.28)^{A}$ & 0.15 \\
\hline $\begin{array}{l}\text { Vukomanović, } \\
2008, \\
18499950, \\
\text { Serbia }\end{array}$ & Length of stay (d) & High & 20 & $9.8(2.4)$ & 20 & $10.2(1.7)$ & $\mathrm{MD}-0.4(-1.56,0.76)^{\mathrm{A}}$ & 0.67 \\
\hline
\end{tabular}

Statistically significant effect sizes are in bold text. In cases where calculated effect size confidence intervals were not-statistically significant but reported p-values were, we deferred to reported p-values and still bolded results.

Abbreviations: $\mathrm{CI}=$ confidence interval, $\mathrm{d}=$ day, $\mathrm{MD}=$ mean difference, $\mathrm{mo}=$ months, $\mathrm{NR}=$ not reported, $\mathrm{PMID}=\mathrm{PubMed}$ identifier, RoB $=$ risk of bias, $\mathrm{SD}=$ standard deviation, $\mathrm{SE}=$ standard error

A Calculated 
Table 73. Prehabilitation versus no prehabilitation for total hip arthroplasty - continuous outcomes, other healthcare utilization outcomes

\begin{tabular}{|c|c|c|c|c|c|c|c|c|c|}
\hline $\begin{array}{l}\text { Study, Year, } \\
\text { PMID, } \\
\text { Country }\end{array}$ & $\begin{array}{l}\text { Outcome } \\
\text { Measurement }\end{array}$ & $\begin{array}{l}\text { Overall } \\
\text { RoB }\end{array}$ & $\begin{array}{l}\text { Time } \\
\text { Point }^{A}\end{array}$ & $\begin{array}{l}\text { Prehab, } \\
\text { N }\end{array}$ & $\begin{array}{l}\text { Prehab, Mean } \\
\text { (SD) }\end{array}$ & $\begin{array}{l}\text { Control, } \\
\text { N }\end{array}$ & $\begin{array}{l}\text { Control, Mean } \\
\text { (SD) }\end{array}$ & $\begin{array}{l}\text { Effect Size } \\
(95 \% \mathrm{CI})\end{array}$ & $\begin{array}{l}\text { Reported p- } \\
\text { Value }\end{array}$ \\
\hline \multirow[t]{2}{*}{$\begin{array}{l}\text { Pour, 2007, } \\
\text { 17768187, USA }\end{array}$} & $\begin{array}{l}\text { Discharge } \\
\text { disposition - home } \\
\text { (n) }\end{array}$ & High & NA & 46 & $41^{\mathrm{B}}$ & 48 & $33^{\mathrm{B}}$ & OR $3.73(1.23,11.32)^{\mathrm{C}}$ & NR \\
\hline & $\begin{array}{l}\text { Discharge } \\
\text { disposition - skilled } \\
\text { rehabilitation facility } \\
\text { (n) }\end{array}$ & High & $\mathrm{NA}$ & 46 & $5^{\mathrm{B}}$ & 48 & $15^{\mathrm{B}}$ & OR $0.26(0.08,0.78)^{C}$ & NR \\
\hline \multirow[t]{2}{*}{$\begin{array}{l}\text { Soeters, 2018, } \\
\text { 29529614, USA }\end{array}$} & $\begin{array}{l}\text { Met PT discharge } \\
\text { criteria (d) }\end{array}$ & Moderate & $\mathrm{NA}$ & 31 & $\begin{array}{l}1.3,95 \% \mathrm{Cl} \\
0.8,1.8\end{array}$ & 32 & $\begin{array}{l}2.3,95 \% \mathrm{Cl} 2.0 . \\
2.9\end{array}$ & $-1(-1.68,-0.32)^{C}$ & $<0.001$ \\
\hline & $\begin{array}{l}\text { Outpatient physical } \\
\text { therapy sessions } \\
\text { (PT visits) }\end{array}$ & Moderate & NA & 31 & $\begin{array}{l}3.1,95 \% \mathrm{Cl} \\
2.6,3.6\end{array}$ & 32 & $\begin{array}{l}4.1,95 \% \mathrm{Cl} 3.6, \\
4.6\end{array}$ & $-1(-1.72,-0.28)^{C}$ & 0.001 \\
\hline $\begin{array}{l}\text { Vukomanović, } \\
2008, \\
18499950, \\
\text { Serbia }\end{array}$ & $\begin{array}{l}\text { Outpatient physical } \\
\text { therapy sessions } \\
\text { (service utilization - } \\
\text { classes needed with } \\
\text { the therapist) }\end{array}$ & High & NA & 20 & $5.2(2.35)$ & 20 & $6.85(1.14)$ & $-1.65(-2.68,-0.62)^{C}$ & 0.02 \\
\hline
\end{tabular}

Statistically significant effect sizes are in bold text. In cases where calculated effect size confidence intervals were not-statistically significant but reported p-values were, we deferred to reported p-values and still bolded results.

Abbreviations: $\mathrm{CI}=$ confidence interval, $\mathrm{d}=$ day, $\mathrm{MD}=$ mean difference, $\mathrm{NR}=$ not reported, $\mathrm{n}=$ number, $\mathrm{PMID}=\mathrm{PubMed}$ identifier, $\mathrm{PT}=$ physical therapy, $\mathrm{RoB}=$ risk of bias, $\mathrm{SD}=$ standard deviation.

A Time from surgery

в Categorical outcome; number of patients

${ }^{\mathrm{C}}$ Calculated 


\section{Heterogeneity of Treatment Effects (Subgroup Differences)}

No studies reported subgroup analyses or more specifically, formally analyzed possible heterogeneity of treatment effects, such as statistical tests for whether the comparative effect of rehabilitation versus its various comparators differed in one subgroup of patients versus another (e.g., patients with higher vs. lower measures of strength, flexibility, function, etc. at baseline).

\section{Applicability}

Studies were conducted across the globe (three in the United States) using diverse interventions employed in diverse healthcare settings. While the relative effect of the interventions on clinical outcomes (and harms) from non-U.S.-based studies are likely applicable to the U.S. context, findings pertaining to healthcare system or resources are likely country and healthcare system specific. The sex of participants varied widely across studies ranging from 29 to 80 percent of participants being female. The average age of patients ages ranged from 56 to 74 years, and the average BMIs ranged from 26 to $30 \mathrm{~kg} / \mathrm{m}^{2}$ (thus, in all studies, most patients were obese, but in several, many to most were morbidly obese). No study reported whether patients had undergone previous contralateral replacement surgery. As such, the conclusions in this KQ are likely most applicable to middle-to-older-aged adults in high-income countries who are receiving their first total THA for osteoarthritis.

\section{Summary of Comparison of Prehabilitation Versus No Prehabilitation for Patients Undergoing Total Hip Arthroplasty}

Table 74 summarizes the evidence for the comparison of prehabilitation versus no prehabilitation for THA. We focus on the outcomes we prioritized in discussion with stakeholders.

There is insufficient evidence whether there is a difference between prehabilitation and no prehabilitation for pain, strength, ADLs, QoL, LOS, or posthospital disposition. No studies reported evidence on satisfaction with care, or risk of harms due to prehabilitation. 
Table 74. Evidence profile: Prehabilitation versus no prehabilitation for total hip arthroplasty

\begin{tabular}{|c|c|c|c|c|c|c|c|c|c|}
\hline $\begin{array}{l}\text { Outcome } \\
\text { Category }\end{array}$ & Outcome & $\begin{array}{l}\text { N Studies } \\
\text { (Participants) }\end{array}$ & RoB & Consistency & Precision & Directness & $\begin{array}{l}\text { Intervention } \\
\text { Replication }\end{array}$ & SoE & Conclusions \\
\hline \multirow{2}{*}{$\begin{array}{l}\text { Body structure } \\
\text { and function }\end{array}$} & Pain & $2(129)$ & Moderate & Consistent & Precise & Direct & Both unique & Insufficient & No conclusion \\
\hline & Strength & $1(49)$ & Moderate & $\begin{array}{l}\text { Consistency unknown } \\
\text { (single study) }\end{array}$ & Precise & Direct & NA (single study) & Insufficient & No conclusion \\
\hline $\begin{array}{l}\text { Activity and } \\
\text { participation }\end{array}$ & ADLs & $3(209)$ & Moderate & Consistent & Precise & Direct & All unique & Insufficient & No conclusion \\
\hline \multirow[t]{2}{*}{ Other outcomes } & $\begin{array}{l}\text { Satisfaction } \\
\text { with care }\end{array}$ & 0 & NA & NA & NA & NA & NA & Insufficient & No evidence \\
\hline & QoL & $1(80)$ & Moderate & $\begin{array}{l}\text { Consistency unknown } \\
\text { (single study) }\end{array}$ & Precise & Direct & NA (single study) & Insufficient & No conclusion \\
\hline \multirow{2}{*}{$\begin{array}{l}\text { Healthcare } \\
\text { utilization }\end{array}$} & Length of stay & $4(277)$ & High & Inconsistent & Precise & Direct & All unique & Insufficient & No conclusion \\
\hline & $\begin{array}{l}\text { Posthospital } \\
\text { disposition }\end{array}$ & $1(94)$ & High & $\begin{array}{l}\text { Consistency unknown } \\
\text { (single study) }\end{array}$ & NA & Direct & NA (single study) & Insufficient & No conclusion \\
\hline Harms & $\begin{array}{l}\text { Harms from } \\
\text { prehabilitation }\end{array}$ & 0 & NA & NA & NA & NA & NA & Insufficient & No evidence \\
\hline
\end{tabular}

Abbreviations: ADLs = activities of daily living, $\mathrm{QoL}=$ health-related quality of life, $\mathrm{NA}=$ not applicable, $\mathrm{QoL}=$ quality of life, RoB $=$ risk of bias, SoE $=$ strength of evidence. 


\section{Key Question 4: Rehabilitation for Total Hip Arthroplasty}

\section{Key Points}

- There is low SoE of no difference between the various programs of rehabilitation and their comparators in terms of pain, strength, ADL, or QoL (low SoE for all), and insufficient evidence on the impact of rehabilitation on hip range of motion.

- There is low SoE that there is no difference among rehabilitation programs with respect to harms from the rehabilitation interventions (low SoE).

- There is insufficient evidence for whether there is a difference between rehabilitation and various comparators on hip range of motion and satisfaction with care

- Given the heterogeneity of interventions and outcomes across studies, there is insufficient evidence on the effectiveness of specific rehabilitation intervention components at the level of goals (e.g., strength, flexibility, etc.) or presence of specific exercise components to address these goals.

- There is insufficient evidence on heterogeneity of treatment effect of rehabilitation programs by patient, surgical, or setting factors.

- There is no evidence on the cost effectiveness of rehabilitation for THA.

\section{Findings Pertaining to Rehabilitation for Total Hip Arthroplasty}

We found 15 studies (14 RCTs and one NRCS) reported in 20 articles that evaluated the effectiveness of rehabilitation among patients who had undergone THA (summarized in Figure 5). ${ }^{104,105,142-159}$ No rehabilitation intervention was evaluated by more than one study. Across studies, rehabilitation interventions were delivered in varying settings (by different modalities), by diverse personnel, at varying intensity, and at various points during the rehabilitation period.

The heterogeneity of the interventions (and their comparators, as almost all comparison groups had active rehabilitation content) made it challenging to identify meaningful groupings of similar studies to synthesize. In the absence of meaningful clusters of similar intervention/comparator studies, we opted to summarize the rehabilitation studies within two main groups of when the intervention/comparator rehabilitation content was initiated: in the acute and post-acute phases after surgery. As expert consensus is unclear in its definition of the different phases of rehabilitation following surgery (despite its perceived importance), we defined these time periods using our best judgement. Acute phase was defined as rehabilitation initiated immediately post-op to within the two weeks following surgery and post-acute rehabilitation was defined as rehabilitation initiated two to eight weeks following surgery. Within these two groups, we summarize the diverse rehabilitation studies together but contextualize interpretations of individual study results with details about the specific form of rehabilitation evaluated (in the intervention and comparator groups) in those studies. We report outcomes under the four following outcome categories: body structure and function; activity and participation; other patient-reported; and healthcare utilization. Given intervention heterogeneity, we determined that meta-analysis was not warranted (i.e., average result would not have been interpretable/meaningful) and instead summarize results narratively.

We coded four studies (4 RCTs) as being delivered within an acute rehabilitation phase and 11 studies (10 RCTs and 1 NRCS) as being delivered within a post-acute rehabilitation phase. Of the acute rehabilitation studies, two RCTs evaluated novel (hypothesized better) rehabilitation 
programs versus less intensive rehabilitation programs and two RCTs evaluated similar programs with varying timing of delivery. Of the post-acute rehabilitation studies, six RCTs evaluated novel (hypothesized better) rehabilitation programs versus standard care (variously defined) or alternative rehabilitation programs, and five studies (4 RCTs and 1 NRCS) evaluated comparatively similar rehabilitation programs delivered in different settings or by different personnel.

We rated four of the $14 \mathrm{RCTs}$ to at overall high risk of bias, mostly related to lack of blinding of participants, study personnel, and/or outcome assessors and unclear methods of how random sequences were generated and/or concealed from patients. We rated nine of the remaining RCTs to be at overall moderate risk of bias, mostly related to lack of blinding of participants, study personnel, and/or outcome assessors. One RCT was rated to be at overall low risk of bias. We rated the one NRCS to be at moderate risk of bias on the basis of their non-randomized design, although, all extracted data from this study were adjusted for important confounders using appropriate methods, as per our eligibility criteria.

The 15 studies enrolled between 54 and 280 participants each. Studies were conducted across the globe, mostly commonly in Europe $(n=10)$, followed by Australia $(n=3)$, with two studies from the United States. Of the studies that reported funding information, only one study was funded by industry (Medacta USA, Inc., which specializes in orthopedic products). The average ages of participants were similar across studies, ranging from 50 to 60 years. The percentage of women in the studies varied across studies, from 21 to 70 percent. Average BMIs ranged from 25 to $30 \mathrm{~kg} / \mathrm{m}^{2}$. Only one study (Mikkelsen 2014) reported the proportion of patients who had undergone prior contralateral THA (25\%). Appendix Tables C-4.1, C-4.2, C-4.3, and C-4.4 include the full data for all 14 studies.

Most rehabilitation interventions included exercises to address the goal components of strength (14/15 studies; 21/29 arms) and flexibility (11/15 studies; 15/29 arms), followed by task-specific training (8/15 studies; 13/29 arms), patient education (7/15 studies; 11/29 arms), and balance-motor-learning-agility (5/15 studies; 7/29 arms). Few studies included exercises targeted at aerobic endurance (3/15 studies; 3/29 arms).

Three of the studies included an adjunctive modality in combination with the rehabilitation program, including modalities of massage and mindfulness/stress reducing activities. In all cases, the adjunctive modalities were not the intervention component of interest in the study. We found seven studies that reported some form of progression, of which five were assessed by clinical experts on our team as appropriate in at least one arm of the study. One acute-phase rehabilitation study (Mikkelsen 2014) compared an early supervised rehabilitation program with appropriate progression to unsupervised home-based exercise without appropriate progression and one post-acute rehabilitation study (Coulter 2017) compared an unsupervised home-based physiotherapy program without appropriate progression to a supervised physiotherapy intervention with appropriate progression.

Rehabilitation interventions were delivered by physical therapists in 11 of the 15 studies. The remaining four studies did not report who delivered the rehabilitation intervention (Beck 2019, Giaquinto 2010, Lyp 2016, Naylor 2018). Three of these latter studies also had a self-guided component of the intervention where no personnel were with the patients while they performed their rehabilitation at home. No studies reported the use of additional or non-physical therapist personnel to deliver any interventions.

Fourteen of the 15 studies delivered the rehabilitation interventions to patients in-person for at least one of the study arms. Five studies had self-guided components in which patients 
performed self-directed exercise and one study evaluated rehabilitation delivered remotely via telephone. Interventions were delivered in varying settings (and often, combinations of settings). Six studies evaluated one or both rehabilitation arms in acute inpatient (Smith, 2009) or outpatient settings, exclusively (Beck, 2019, Giaquinto 2010, Heiberg 2012, Liebs 2010, Liebs 2012, Lyp 2016, Monticone 2014, Winther 2018). Three studies compared rehabilitation programs delivered in outpatient and home settings versus home alone (Austin 2017, Coulter 2017, Nelson 2020, Rao 2021). One study evaluated a rehabilitation program initiated in a fitness center followed by home exercise compared to home exercises alone (Mikkelsen 2014). Naylor 2018 compared discharge to inpatient versus no inpatient care but did not report the specifics of the type of setting for each arm.

Specific codes for intervention (and control arm, where present) goals and exercises, use of progression (and assessment of appropriateness), and details on personnel, mode of delivery, and setting are detailed in Table 75.1 and 75.2 and Appendix C-4.2. 
Figure 5. Overview of studies of acute and post-acute rehabilitation interventions versus various controls for total hip arthroplasty
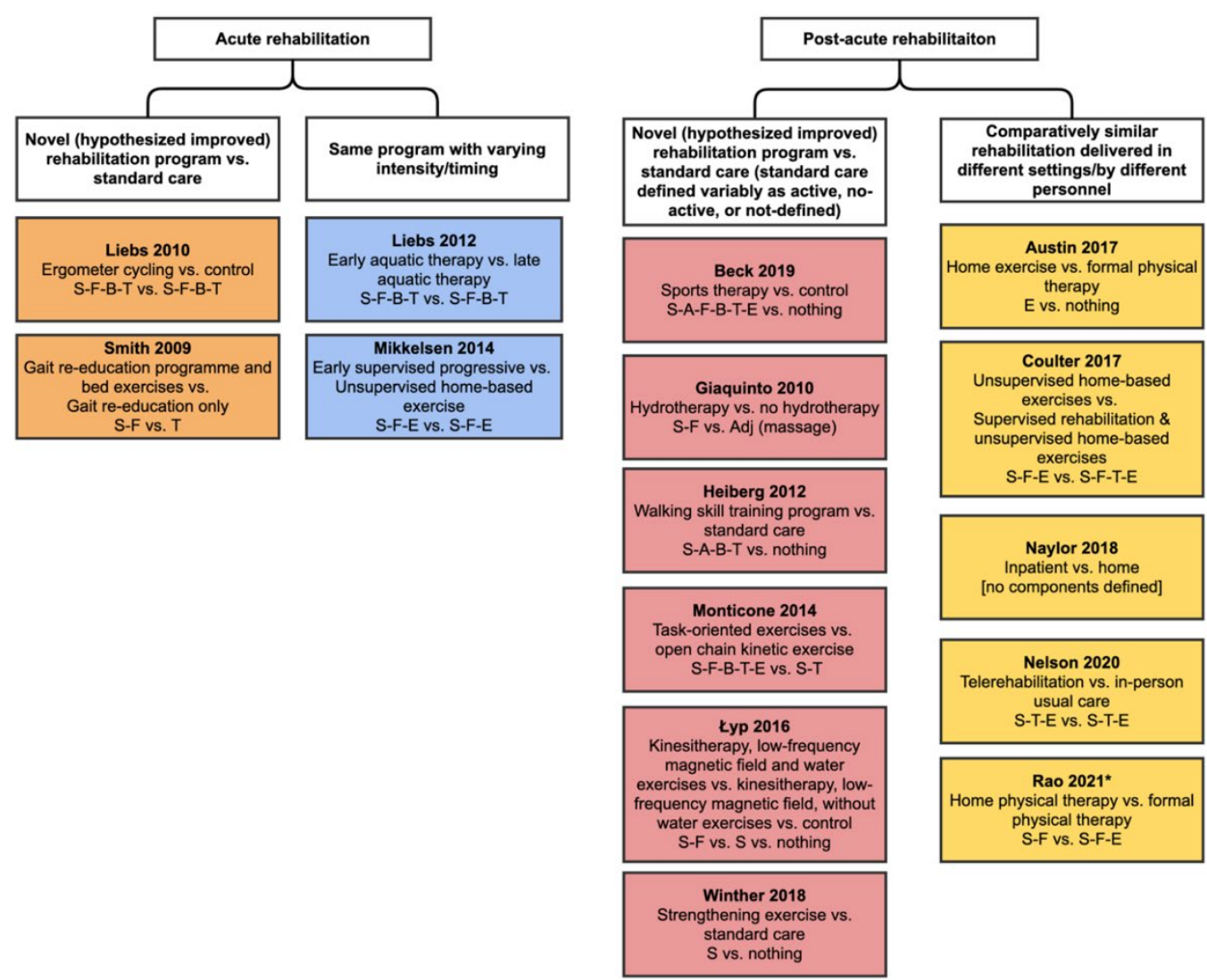

Figure presents categorization of KQ 4 studies that evaluated acute and post-acute rehabilitation programs for THA. The first column lists a novel (more intensive) acute rehabilitation program compared to a different program (first group hypothesized to be better); the second column lists studies with comparatively similar acute rehabilitation programs in both arms that were delivered with different timing or intensity (first group hypothesized to be better); the third column lists a novel (more intensive) post-acute rehabilitation program compared to a different program (first group hypothesized to be better); the fourth column lists studies with comparatively similar rehabilitation programs delivered in different settings or by different personnel

Abbreviations: Adj $=$ adjunctive, $\mathrm{A}=$ aerobic exercise, $\mathrm{B}=$ balance-motor-learning-agility exercise, $\mathrm{E}=$ patient education, $\mathrm{F}=$ flexibility exercise, $\mathrm{S}=$ strengthening exercise, $\mathrm{T}=$ task-specific training, THA = total hip arthroplasty.

* Intervention included progression which was deemed appropriate 
Table 75.1. Goal components strength, aerobic, and flexibility and their specific exercise components for rehabilitation interventions versus various controls for total hip arthroplasty

\begin{tabular}{|c|c|c|c|c|c|c|c|c|c|c|c|c|c|c|c|c|c|c|c|c|c|c|c|c|c|c|c|c|}
\hline 总 & $\frac{\xi}{\xi}$ & 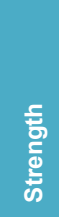 & $\begin{array}{l}0 \\
\frac{0}{0} \\
\frac{0}{0}\end{array}$ & 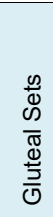 & 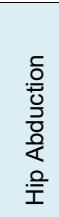 & 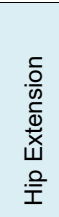 & $\begin{array}{l}\overline{ } \\
\frac{0}{X} \\
\frac{0}{\amalg !} \\
\stackrel{0}{\bar{I}}\end{array}$ & 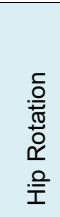 & 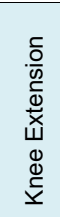 & 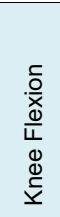 & $\begin{array}{l}\mathscr{W} \\
\Phi \\
0 \\
0 \\
\Phi \\
\end{array}$ & $\begin{array}{l}\mathscr{\infty} \\
\stackrel{\Xi}{\Xi} \\
\Xi\end{array}$ & 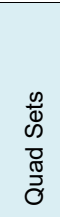 & 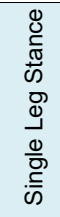 & 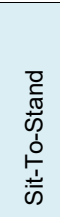 & $\begin{array}{l}\frac{n}{\pi} \\
\frac{0}{0} \\
\infty\end{array}$ & $\begin{array}{l}\text { ô } \\
\text { Q } \\
\text { बे } \\
\text { के }\end{array}$ & $\begin{array}{l}\text { गे } \\
\text { @े } \\
\text { ஸे }\end{array}$ & $\begin{array}{l}\frac{0}{0} \\
\frac{0}{\circ} \\
\frac{0}{8}\end{array}$ & 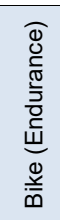 & 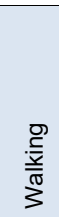 & 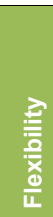 & 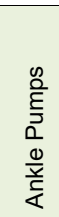 & 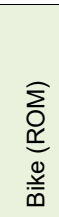 & 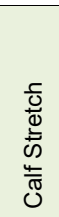 & $\begin{array}{l}\mathscr{D} \\
\stackrel{0}{\overline{0}} \\
\bar{\Phi} \\
\bar{\Phi}\end{array}$ & 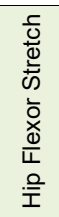 & 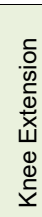 \\
\hline \multirow[t]{2}{*}{ Austin, 2017} & Home exercise & 0 & 0 & 0 & 0 & 0 & 0 & 0 & 0 & 0 & 0 & 0 & 0 & 0 & 0 & 0 & 0 & 0 & 0 & 0 & 0 & 0 & 0 & 0 & 0 & 0 & 0 & 0 \\
\hline & Formal PT & 0 & 0 & 0 & 0 & 0 & 0 & 0 & 0 & 0 & 0 & 0 & 0 & 0 & 0 & 0 & 0 & 0 & 0 & 0 & 0 & 0 & 0 & 0 & 0 & 0 & 0 & 0 \\
\hline \multirow[t]{2}{*}{ Beck, 2019} & Sport rehab & 1 & 0 & 0 & 0 & 0 & 0 & 0 & 0 & 0 & 0 & 0 & 0 & 0 & 0 & 1 & 0 & 0 & 1 & 1 & 1 & 1 & 0 & 0 & 0 & 0 & 1 & 0 \\
\hline & Control & 0 & 0 & 0 & 0 & 0 & 0 & 0 & 0 & 0 & 0 & 0 & 0 & 0 & 0 & 0 & 0 & 0 & 0 & 0 & 0 & 0 & 0 & 0 & 0 & 0 & 0 & 0 \\
\hline \multirow[t]{2}{*}{ Coulter, 2017} & Home PT & 1 & 0 & 0 & 0 & 0 & 0 & 0 & 0 & 0 & 0 & 0 & 0 & 0 & 0 & 0 & 0 & 0 & 0 & 0 & 0 & 1 & 0 & 0 & 0 & 0 & 0 & 0 \\
\hline & Supervised PT & 1 & 1 & 0 & 1 & 1 & 0 & 0 & 1 & 0 & 0 & 0 & 0 & 0 & 1 & 0 & 0 & 1 & 1 & 0 & 0 & 1 & 0 & 1 & 0 & 0 & 0 & 0 \\
\hline \multirow[t]{2}{*}{ Giaquinto, 2010} & Hydrotherapy & 1 & 0 & 0 & 0 & 0 & 0 & 0 & 0 & 0 & 0 & 0 & 0 & 0 & 0 & 0 & 0 & 0 & 0 & 0 & 0 & 1 & 0 & 0 & 0 & 0 & 0 & 0 \\
\hline & Land-based rehab & 0 & 0 & 0 & 0 & 0 & 0 & 0 & 0 & 0 & 0 & 0 & 0 & 0 & 0 & 0 & 0 & 0 & 0 & 0 & 0 & 0 & 0 & 0 & 0 & 0 & 0 & 0 \\
\hline \multirow[t]{2}{*}{ Heiberg, 2012} & Walking skill training & 1 & 0 & 0 & 0 & 0 & 0 & 0 & 0 & 0 & 0 & 1 & & 1 & 1 & 1 & 1 & 1 & 1 & 0 & 1 & 1 & 0 & 0 & 1 & 0 & 0 & 0 \\
\hline & Control (no PT) & 0 & 0 & 0 & 0 & 0 & 0 & 0 & 0 & 0 & 0 & 0 & 0 & 0 & 0 & 0 & 0 & 0 & 0 & 0 & 0 & 0 & 0 & 0 & 0 & 0 & 0 & 0 \\
\hline \multirow[t]{2}{*}{ Liebs, 2010} & Cycling & 1 & 0 & 0 & 0 & 0 & 0 & 0 & 0 & 0 & 0 & 0 & 0 & 0 & 0 & 0 & 0 & 0 & 0 & 0 & 0 & 1 & 0 & 1 & 0 & 0 & 0 & 0 \\
\hline & No cycling & 1 & 0 & 0 & 0 & 0 & 0 & 0 & 0 & 0 & 0 & 0 & 0 & 0 & 0 & 0 & 0 & 0 & 0 & 0 & 0 & 1 & 0 & 0 & 0 & 0 & 0 & 0 \\
\hline \multirow[t]{2}{*}{ Liebs, 2012} & Early AT & 1 & 0 & 0 & 0 & 0 & 0 & 0 & 0 & 0 & 0 & 0 & 0 & 0 & 0 & 0 & 0 & 0 & 0 & 0 & 0 & 1 & 0 & 0 & 0 & 0 & 0 & 0 \\
\hline & AT after wound healing & 1 & 0 & 0 & 0 & 0 & 0 & 0 & 0 & 0 & 0 & 0 & 0 & 0 & 0 & 0 & 0 & 0 & 0 & 0 & 0 & 1 & 0 & 0 & 0 & 0 & 0 & 0 \\
\hline \multirow[t]{3}{*}{ Lyp, 2016} & Group I & 1 & 0 & 0 & 0 & 0 & 0 & 0 & 0 & 0 & 0 & 0 & 0 & 0 & 0 & 0 & 0 & 0 & 0 & 0 & 0 & 1 & 0 & 0 & 0 & 0 & 0 & 0 \\
\hline & Group II & 1 & 0 & 0 & 0 & 0 & 0 & 0 & 0 & 0 & 0 & 0 & 0 & 0 & 0 & 0 & 0 & 0 & 0 & 0 & 0 & 0 & 0 & 0 & 0 & 0 & 0 & 0 \\
\hline & Group V & & 0 & 0 & 0 & 0 & 0 & 0 & 0 & 0 & 0 & 0 & 0 & 0 & 0 & 0 & 0 & 0 & 0 & 0 & 0 & 0 & 0 & 0 & 0 & 0 & 0 & 0 \\
\hline \multirow[t]{2}{*}{ Mikkelsen, 2014} & Early supervised PRT & 1 & 0 & 0 & 1 & 1 & 1 & 0 & 1 & 1 & 1 & 0 & 0 & 0 & 0 & 0 & 0 & 0 & 0 & 0 & 0 & 1 & 0 & 1 & 0 & 0 & 0 & 0 \\
\hline & Control & 1 & 0 & 0 & 1 & 1 & 1 & 0 & 1 & 1 & 0 & 0 & 0 & 0 & 0 & 0 & 0 & 0 & 0 & 0 & 0 & 0 & 0 & 0 & 0 & 0 & 0 & 0 \\
\hline \multirow[t]{2}{*}{ Monticone, 2014} & Intervention ${ }^{A}$ & 1 & 0 & 0 & 0 & 1 & 1 & 0 & 0 & 1 & 0 & 0 & 0 & 0 & 0 & 0 & 0 & 0 & 0 & 0 & 0 & 1 & 0 & 1 & 0 & 0 & 0 & 0 \\
\hline & Control $^{B}$ & 1 & 0 & 0 & 1 & 1 & 1 & 1 & 1 & 1 & 0 & 0 & 1 & 0 & 0 & 0 & 0 & 0 & 0 & 0 & 0 & 0 & 0 & 0 & 0 & 0 & 0 & 0 \\
\hline \multirow[t]{2}{*}{ Naylor, 2018} & Inpatient rehab & 0 & 0 & 0 & 0 & 0 & 0 & 0 & 0 & 0 & 0 & 0 & 0 & 0 & 0 & 0 & 0 & 0 & 0 & 0 & 0 & 0 & 0 & 0 & 0 & 0 & 0 & 0 \\
\hline & No inpatient rehab & 0 & 0 & 0 & 0 & 0 & 0 & 0 & 0 & 0 & 0 & 0 & 0 & 0 & 0 & 0 & 0 & 0 & 0 & 0 & 0 & 0 & 0 & 0 & 0 & 0 & 0 & 0 \\
\hline \multirow[t]{2}{*}{ Nelson, 2020} & Telerehabilitation & 1 & 0 & 0 & 1 & 1 & 1 & 0 & 1 & 0 & 0 & 0 & 0 & 0 & 0 & 0 & 0 & 0 & 0 & 0 & 0 & 0 & 0 & 0 & 0 & 0 & 0 & 0 \\
\hline & Control & 1 & 0 & 0 & 1 & 1 & 1 & 0 & 1 & 0 & 0 & 0 & 0 & 0 & 0 & 0 & 0 & 0 & 0 & 0 & 0 & 0 & 0 & 0 & 0 & 0 & 0 & 0 \\
\hline \multirow[t]{2}{*}{ Rao, 2021} & Home PT & 1 & 1 & 0 & 1 & 0 & 0 & 0 & 0 & 0 & 0 & 0 & 0 & 0 & 0 & 0 & 0 & 0 & 0 & 0 & 0 & 1 & 0 & 0 & 0 & 0 & 0 & 1 \\
\hline & Formal PT & 1 & 0 & 0 & 0 & 0 & 0 & 0 & 0 & 0 & 0 & 0 & 0 & 0 & 0 & 0 & 0 & 0 & 0 & 0 & 0 & 1 & 0 & 0 & 0 & 0 & 0 & 0 \\
\hline \multirow{2}{*}{ Smith, 2009} & Gait re-ed \& bed ex & 1 & 0 & 1 & 0 & 0 & 1 & 0 & 0 & 0 & 0 & 0 & 1 & 0 & 0 & 0 & 0 & 0 & 0 & 0 & 0 & 1 & 1 & 0 & 0 & 1 & 0 & 0 \\
\hline & Gait re-ed & 0 & 0 & 0 & 0 & 0 & 0 & 0 & 0 & 0 & 0 & 0 & 0 & 0 & 0 & 0 & 0 & 0 & 0 & 0 & 0 & 0 & 0 & 0 & 0 & 0 & 0 & 0 \\
\hline \multirow{2}{*}{ Winther, 2018} & Maximal ST & 1 & 0 & 0 & 1 & 0 & 0 & 0 & 0 & 0 & 1 & 0 & 0 & 0 & 0 & 0 & 0 & 0 & 0 & 0 & 0 & 0 & 0 & 0 & 0 & 0 & 0 & 0 \\
\hline & Conventional PT & 0 & 0 & 0 & 0 & 0 & 0 & 0 & 0 & 0 & 0 & 0 & 0 & 0 & 0 & 0 & 0 & 0 & 0 & 0 & 0 & 0 & 0 & 0 & 0 & 0 & 0 & 0 \\
\hline
\end{tabular}

See Table 75.2 for goal components Balance-motor-learning-agility, task specific training, patient education, and adjunctive modalities. The color is added for visual display and does not provide unique information.

$1=$ presence of component, $0=$ absence of component

Group I: kinesiotherapy, low-frequency magnetic field and water exercises; Group II: undergoing kinesiotherapy and low-frequency magnetic field, without water exercises; Group

$\mathrm{V}$ : awaiting rehabilitation

Abbreviations: $\mathrm{PRT}=$ progressive resistance training, $\mathrm{PT}=$ physical therapy, rehab $=$ rehabilitation; $\mathrm{ROM}=$ range of motion, $\mathrm{ST}=$ strength training 
A Task-oriented exercises; encouragement to abandon walking aids.

B Open kinetic chain exercises

Table 75.2. Goal components balance-motor-learning-agility, task specific training, patient education, and adjunctive modalities and their specific exercise components for rehabilitation interventions versus various controls for total hip arthroplasty

\begin{tabular}{|c|c|c|c|c|c|c|c|c|c|c|c|c|c|c|c|c|c|c|c|c|c|c|c|c|c|c|c|}
\hline $\begin{array}{l}\text { 훌 } \\
\text { के }\end{array}$ & $\underline{\xi}$ & 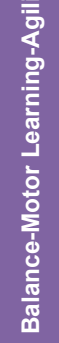 & 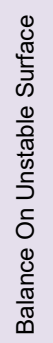 & 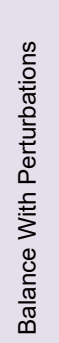 & 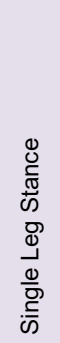 & 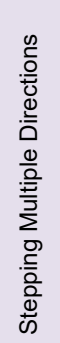 & 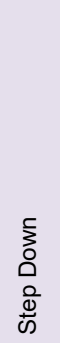 & 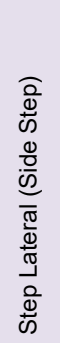 & 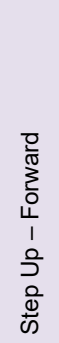 & 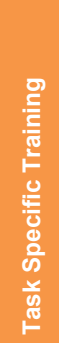 & 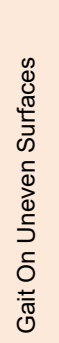 & 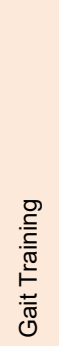 & 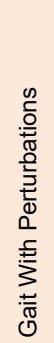 & 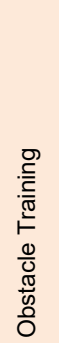 & 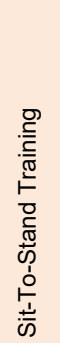 & 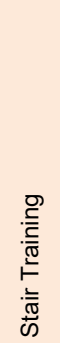 & 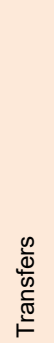 & 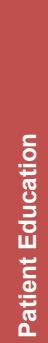 & 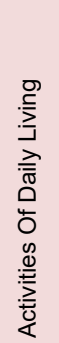 & 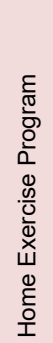 & 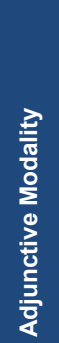 & 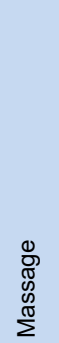 & 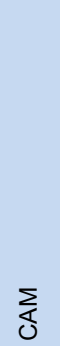 & 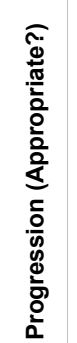 & 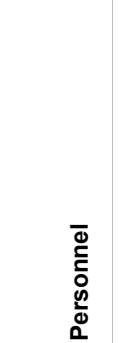 & 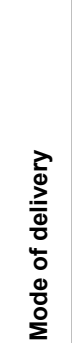 & 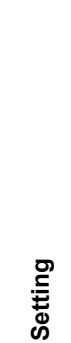 \\
\hline \multirow[t]{2}{*}{ Austin, 2017} & Home exercise & 0 & 0 & 0 & 0 & 0 & 0 & 0 & 0 & 0 & 0 & 0 & 0 & 0 & 0 & 0 & 0 & 1 & 0 & 1 & 0 & 0 & 0 & $\mathrm{~N}$ & None & SG & $\mathrm{H}$ \\
\hline & Formal PT & 0 & 0 & 0 & 0 & 0 & 0 & 0 & 0 & 0 & 0 & 0 & 0 & 0 & 0 & 0 & 0 & 0 & 0 & 0 & 0 & 0 & 0 & $\mathrm{~N}$ & PT & 1 & $\mathrm{O} ; \mathrm{H}$ \\
\hline \multirow[t]{2}{*}{ Beck, 2019} & Sport rehab & 1 & 1 & 0 & 0 & 0 & 0 & 0 & 0 & 1 & 0 & 1 & 0 & 0 & 0 & 0 & 0 & 1 & 0 & 0 & 1 & 1 & 0 & $\mathrm{~N}$ & NR & 1 & 0 \\
\hline & Control & 0 & 0 & 0 & 0 & 0 & 0 & 0 & 0 & 0 & 0 & 0 & 0 & 0 & 0 & 0 & 0 & 0 & 0 & 0 & 0 & 0 & 0 & $\mathrm{~N}$ & NA & NA & NA \\
\hline \multirow[t]{2}{*}{ Coulter, 2017} & Home PT & 0 & 0 & 0 & 0 & 0 & 0 & 0 & 0 & 0 & 0 & 0 & 0 & 0 & 0 & 0 & 0 & 1 & 0 & 1 & 0 & 0 & 0 & $\mathrm{Y}(\mathrm{N})$ & None & SG & $\mathrm{H}$ \\
\hline & Supervised PT & 0 & 0 & 0 & 0 & 0 & 0 & 0 & 0 & 1 & 0 & 1 & 0 & 0 & 1 & 1 & 0 & 1 & 0 & 1 & 0 & 0 & 0 & $Y(Y)$ & PT; None & I; SG & $\mathrm{O} ; \mathrm{H}$ \\
\hline \multirow[t]{2}{*}{ Giaquinto, 2010} & Hydrotherapy & 0 & 0 & 0 & 0 & 0 & 0 & 0 & 0 & 0 & 0 & 0 & 0 & 0 & 0 & 0 & 0 & 0 & 0 & 0 & 0 & 0 & 0 & $\mathrm{~N}$ & NR & I & OIF \\
\hline & Land-based rehab & 0 & 0 & 0 & 0 & 0 & 0 & 0 & 0 & 0 & 0 & 0 & 0 & 0 & 0 & 0 & 0 & 0 & 0 & 0 & 1 & 1 & 0 & $\mathrm{~N}$ & NR & 1 & OIF \\
\hline \multirow{2}{*}{ Heiberg, 2012} & Walking skill training & 1 & 1 & 1 & 1 & 0 & 1 & 1 & 1 & 1 & 1 & 0 & 1 & 1 & 0 & 1 & 0 & 0 & 0 & 0 & 0 & 0 & 0 & $\mathrm{Y}(\mathrm{Y})$ & PT & 1 & $\mathrm{O}$ \\
\hline & Control (no PT) & 0 & 0 & 0 & 0 & 0 & 0 & 0 & 0 & 0 & 0 & 0 & 0 & 0 & 0 & 0 & 0 & 0 & 0 & 0 & 0 & 0 & 0 & $\mathrm{~N}$ & NA & NA & NA \\
\hline \multirow[t]{2}{*}{ Liebs, 2010} & Cycling & 1 & 1 & 0 & 0 & 0 & 0 & 0 & 0 & 1 & 1 & 1 & 0 & 0 & 0 & 1 & 1 & 0 & 0 & 0 & 0 & 0 & 0 & $\mathrm{~N}$ & PT & 1 & $\mathrm{O}$ \\
\hline & No cycling & 1 & 1 & 0 & 0 & 0 & 0 & 0 & 0 & 1 & 1 & 1 & 0 & 0 & 0 & 1 & 1 & 0 & 0 & 0 & 0 & 0 & 0 & $\mathrm{~N}$ & PT & i & $\mathrm{O}$ \\
\hline \multirow[t]{2}{*}{ Liebs, 2012} & Early AT & 1 & 1 & 0 & 0 & 0 & 0 & 0 & 0 & 1 & 1 & 1 & 0 & 0 & 0 & 1 & 1 & 0 & 0 & 0 & 0 & 0 & 0 & $\mathrm{~N}$ & PT & i & $\mathrm{O}$ \\
\hline & AT after wound healing & 1 & 1 & 0 & 0 & 0 & 0 & 0 & 0 & 1 & 1 & 1 & 0 & 0 & 0 & 1 & 1 & 0 & 0 & 0 & 0 & 0 & 0 & $\mathrm{~N}$ & PT & i & 0 \\
\hline \multirow[t]{3}{*}{ Lyp, 2016} & Group I & 0 & 0 & 0 & 0 & 0 & 0 & 0 & 0 & 0 & 0 & 0 & 0 & 0 & 0 & 0 & 0 & 0 & 0 & 0 & 1 & 0 & 1 & $\mathrm{~N}$ & NR & i & $\mathrm{O}$ \\
\hline & Group II & 0 & 0 & 0 & 0 & 0 & 0 & 0 & 0 & 0 & 0 & 0 & 0 & 0 & 0 & 0 & 0 & 0 & 0 & 0 & 1 & 0 & 1 & $\mathrm{~N}$ & NR & i & 0 \\
\hline & Group V & 0 & 0 & 0 & 0 & 0 & 0 & 0 & 0 & 0 & 0 & 0 & 0 & 0 & 0 & 0 & 0 & 0 & 0 & 0 & 0 & 0 & 0 & $\mathrm{~N}$ & NA & NA & NA \\
\hline \multirow[t]{2}{*}{ Mikkelsen, 2014} & Early supervised PRT & 0 & 0 & 0 & 0 & 0 & 0 & 0 & 0 & 0 & 0 & 0 & 0 & 0 & 0 & 0 & 0 & 1 & 0 & 1 & 0 & 0 & 0 & $\mathrm{Y}(\mathrm{Y})$ & PT; None & I;SG & $\mathrm{G} ; \mathrm{H}$ \\
\hline & Control & 0 & 0 & 0 & 0 & 0 & 0 & 0 & 0 & 0 & 0 & 0 & 0 & 0 & 0 & 0 & 0 & 1 & 0 & 1 & 0 & 0 & 0 & $Y(N)$ & None & SG & $\mathrm{H}$ \\
\hline \multirow{2}{*}{ Monticone, 2014} & Intervention ${ }^{A}$ & 1 & 1 & 0 & 0 & 1 & 0 & 0 & 0 & 1 & 0 & 1 & 1 & 1 & 1 & 1 & & 1 & 1 & 0 & 0 & 0 & 0 & $\mathrm{Y}(\mathrm{N})$ & PT & 1 & OIF \\
\hline & Control $^{B}$ & 0 & 0 & 0 & 0 & 0 & 0 & 0 & 0 & 1 & 0 & 1 & 0 & 0 & 0 & 0 & 0 & 0 & 0 & 0 & 0 & 0 & 0 & $\mathrm{~N}$ & PT & i & OIF \\
\hline \multirow{2}{*}{ Naylor, 2018} & Inpatient rehab & 0 & 0 & 0 & 0 & 0 & 0 & 0 & 0 & 0 & 0 & 0 & 0 & 0 & 0 & 0 & 0 & 0 & 0 & 0 & 0 & 0 & 0 & NR & NR & NR & NR \\
\hline & No inpatient rehab & 0 & 0 & 0 & 0 & 0 & 0 & 0 & 0 & 0 & 0 & 0 & 0 & 0 & 0 & 0 & 0 & 0 & 0 & 0 & 0 & 0 & 0 & NR & NR & NR & NR \\
\hline \multirow[t]{2}{*}{ Nelson, 2020} & Telerehabilitation & 0 & 0 & 0 & 0 & 0 & 0 & 0 & 0 & 1 & 0 & 1 & 0 & 0 & 0 & 0 & 0 & 1 & 0 & 1 & 0 & 0 & 0 & $\mathrm{Y}(\mathrm{N})$ & PT & $\mathrm{R}^{\mathrm{c}}$ & $\mathrm{H}$ \\
\hline & Control & 0 & 0 & 0 & 0 & 0 & 0 & 0 & 0 & 1 & 0 & 1 & 0 & 0 & 0 & 0 & 0 & 1 & 0 & 1 & 0 & 0 & 0 & $Y(N)$ & PT & SG; I & $\mathrm{O} ; \mathrm{H}$ \\
\hline \multirow[t]{2}{*}{ Rao, 2021} & Home PT & 0 & 0 & 0 & 0 & 0 & 0 & 0 & 0 & 0 & 0 & 0 & 0 & 0 & 0 & 0 & 0 & 1 & 0 & 1 & 0 & 0 & 0 & $Y(N)$ & None & SG & $\mathrm{H}$ \\
\hline & Formal PT & 0 & 0 & 0 & 0 & 0 & 0 & 0 & 0 & 0 & 0 & 0 & 0 & 0 & 0 & 0 & 0 & 1 & 0 & 1 & 0 & 0 & 0 & $Y(Y)$ & PT; None & I; SG & $\mathrm{O} ; \mathrm{H}$ \\
\hline \multirow[t]{2}{*}{ Smith, 2009} & Gait re-ed \& bed ex & 0 & 0 & 0 & 0 & 0 & 0 & 0 & 0 & 1 & 0 & 1 & 0 & 0 & 0 & 1 & 0 & 0 & 0 & 0 & 0 & 0 & 0 & $\mathrm{Y}(\mathrm{Y})$ & PT & 1 & $\mathrm{Al}$ \\
\hline & Gait re-ed & 0 & 0 & 0 & 0 & 0 & 0 & 0 & 0 & 1 & 0 & 1 & 0 & 0 & 0 & 1 & 0 & 0 & 0 & 0 & 0 & 0 & 0 & $Y(Y)$ & PT & i & $\mathrm{Al}$ \\
\hline \multirow{2}{*}{ Winther, 2018} & Maximal ST & 0 & 0 & 0 & 0 & 0 & 0 & 0 & 0 & 0 & 0 & 0 & 0 & 0 & 0 & 0 & 0 & 0 & 0 & 0 & 0 & 0 & 0 & $\mathrm{Y}(\mathrm{Y})$ & PT & I & $\mathrm{O}$ \\
\hline & Conventional PT & 0 & 0 & 0 & 0 & 0 & 0 & 0 & 0 & 0 & 0 & 0 & 0 & 0 & 0 & 0 & 0 & 0 & 0 & 0 & 0 & 0 & 0 & $\mathrm{~N}$ & PT & i & $\mathrm{O}$ \\
\hline
\end{tabular}

See Table 75.1 for goal components strength, aerobic, and flexibility. The color is added for visual display and does not provide unique information.The color is added for visual display and does not provide unique information. 
Group I: kinesiotherapy, low-frequency magnetic field and water exercises; Group II: undergoing kinesiotherapy and low-frequency magnetic field, without water exercises; Group V: awaiting rehabilitation

Abbreviations: $\mathrm{CAM}=$ complementary and alternative therapies, ed = education, exercises, $\mathrm{I}=$ in-person; $\mathrm{G}=$ gym/other community center; $\mathrm{H}=$ home; $\mathrm{O}=$ outpatien physiotherapy center; OIF = other inpatient facility; PRT PT = physical therapy, rehab = rehabilitation; $\mathrm{SG}=$ self-guided; $\mathrm{ST}=$ strength training .

A Task-oriented exercises; encouragement to abandon walking aids

B Open kinetic chain exercises

${ }^{\mathrm{C}}$ Remote via telephone 


\section{Body Structure and Function Outcomes}

Twelve studies in total reported on body structure and function outcomes following rehabilitation compared to various controls: one study (Liebs 2010) compared novel acute rehabilitation programs to control; two studies (Liebs 2012, Mikkelsen 2014) compared acute rehabilitation programs with different timing and/or intensity; four studies (Beck 2019, Giaquinto 2010, Heiberg 2012, Monticone 2014) compared novel post-acute rehabilitation programs to various comparators (less intensive rehabilitation or no care); and four studies (Austin 2017, Coulter 2017, Naylor 2018, Nelson 2020, Rao 2021) compared rehabilitation programs delivered in different settings or by different personnel (Tables 76 to 81). The outcomes included: symptoms, pain, range of motion, muscle strength, energy and vigor, and emotional functioning.

\section{Symptoms}

Nine studies (Beck 2019, Giaquinto 2010, Heiberg 2012, Liebs 2010, Liebs 2012, Mikkelsen 2014, Monticone 2014, Nelson 2020, Rao 2021) reported data on symptoms using the stiffness component of the WOMAC (0 to 8; lower score indicates reduced stiffness; Beck 2019, Liebs 2012, and Monticone 2014 converted to a 0 to 100 score) and the symptoms component of the HOOS (0 to 100; higher is better) (Table 76). Seven studies observed no differences between groups at follow-up ranging from 6 months to 5 years post-THA. Liebs 2010 reported reduced stiffness among patients randomized to ergometer cycling compared with control (calculated MD $-5.295 \%$ CI -11.08 to $0.068 ; \mathrm{P}=0.047$ ) and Monticone 2014 reported reduced stiffness among patients randomized to task-oriented exercises compared with open chain kinetic exercises (calculated MD -9.0, 95\% CI -13.6, -4.4; $\mathrm{P}=0.037$ ).

\section{Pain}

Eleven studies reported pain data (Beck 2019, Giaquinto 2010, Heiberg 2012, Liebs 2010, Liebs 2012, Łyp, 2016, Mikkelsen 2014, Monticone 2014, Naylor 2018, Nelson 2020, Rao 2021) using six different measurement instruments (the pain component of the HOOS, the pain component of the WOMAC, the bodily pain component of the SF-36, the EQ-5D VAS, the VAS, and the Latinen scale measuring pain intensity) (Table 77). Most studies $(n=8)$ found no difference in pain data between comparison groups. Three studies (Beck 2019, Giaquinto 2010, Monticone 2014) reported reduced pain in their respective intervention groups. Beck 2019 reported pain data using the VAS, the pain component of WOMAC, and EQ-5D VAS. They found no difference between groups on two scales at 6 and 12 months after THA, but pain on the WOMAC scale was significantly better in the sport therapy group compared with the control group at 12 months follow-up $(\mathrm{p}=0.023)$. Giaquinto 2010 reported pain data using the pain component of the WOMAC only and found patients randomized to hydrotherapy had significantly reduced pain compared with patients randomized to no hydrotherapy ("land therapy" followed by scar mobility massage) at 6 months after THA $(\mathrm{p}<0.01)$. Monticone 2014 reported pain data using the pain component of the WOMAC ( 0 to 100 , lower is better), the bodily pain component of the SF-36 (0 to 100, higher is better), and the VAS and found patients randomized to task-oriented exercises reported significantly less pain compared to patients randomized to open-chain kinetic exercises at 12 months after THA on the WOMAC and SF-36 scales but not VAS. 


\section{Range of Motion}

Three studies (Heiberg 2012, Łyp, 2016, Winther 2020) reported ROM data from various outcome measures to assess ROM of the hip extensors, flexors, abductors and adductors. Two studies found no significant differences between arms; Lyp 2016 found significant improvements in active ROM of the hip in both groups that received kinesiotherapy (the therapeutic and corrective application of passive and active movements, such as massage, and of exercise) with or without water exercises (Table 78).

\section{Muscle Strength}

Five studies (Beck 2019, Heiberg 2012, Łyp, 2016, Mikkelsen 2014, Nelson 2020) reported strength data from various outcome measures to assess strength of the hip extensors, flexors, abductors and adductors (Table 79). Studies used a dynamometer to measure strength and presented strength data variably (Newton-meters [Nm], kilograms [kg], Watts [W]; for each, higher values indicate greater strength). One study reported strength using a composite scale, the Index of muscle function, which was designed to assess strength and function. Studies reported no significant differences between groups at follow-up ranging from 6 to 15 months after THA.

\section{Energy and Vigor}

Monticone 2014 reported on vigor using the vitality component of the SF-36 (scores 0 to 100 , higher is better) (Table 80). The study found a statistically significant improvement in vitality among patients randomized to task-oriented exercises compared to patients randomized to open-chain kinetic exercises (MD 8.5, 95\% CI 3.9 to 13.1).

\section{Emotional and Social Functioning}

Four studies (Austin 2017, Coulter 2017, Monticone 2014, Nelson 2020) reported on emotional and social functioning data from the mental health, social function, and emotional role scales of the SF-36. Studies reported comparable findings among groups on all scales at 6 to 12 months after THA (Table 81). 
Table 76. Rehabilitation versus various controls for total hip arthroplasty - continuous outcomes, symptoms

\begin{tabular}{|c|c|c|c|c|c|c|c|c|c|c|c|}
\hline $\begin{array}{l}\text { Study, Year, } \\
\text { PMID, } \\
\text { Country }\end{array}$ & Arm 1 & Arm 2 & $\begin{array}{l}\text { Overall } \\
\text { RoB }\end{array}$ & $\begin{array}{l}\text { Outcome } \\
\text { Measurement }\end{array}$ & $\begin{array}{l}\text { Time } \\
\text { Point }^{A}\end{array}$ & $\begin{array}{l}\text { Arm 1, } \\
\text { N }\end{array}$ & $\begin{array}{l}\text { Arm 1, Mean } \\
\text { (SD) }\end{array}$ & Arm 2, & $\begin{array}{l}\text { Arm 2, Mean } \\
\text { (SD) }\end{array}$ & $\begin{array}{l}\text { Effect Size } \\
(95 \% \mathrm{CI})\end{array}$ & $\begin{array}{l}\text { Reported } \\
\text { p-Value }\end{array}$ \\
\hline \multirow[t]{2}{*}{$\begin{array}{l}\text { Beck, 2019, } \\
30782304\end{array}$} & Sports therapy & Control & High & $\begin{array}{l}\text { WOMAC: Stiffness } \\
(0-100)\end{array}$ & $6 \mathrm{mo}$ & 63 & $\begin{array}{l}\text { Median (Q1, } \\
\text { Q3) } 87.5 \text { (70, } \\
\text { 100) }\end{array}$ & 51 & $\begin{array}{l}\text { Median (Q1, } \\
\text { Q3) } \\
87.5(70,100)\end{array}$ & NR & NR \\
\hline & Sports therapy & Control & High & $\begin{array}{l}\text { WOMAC: Stiffness } \\
(0-100)\end{array}$ & $12 \mathrm{mo}$ & 57 & $\begin{array}{l}\text { Median (Q1, } \\
\text { Q3) } 87.5(75, \\
100)\end{array}$ & 41 & $\begin{array}{l}\text { Median (Q1, } \\
\text { Q3) } \\
100(75,100)\end{array}$ & NR & NR \\
\hline $\begin{array}{l}\text { Giaquinto, } \\
2010, \\
19282040\end{array}$ & Hydrotherapy & $\begin{array}{l}\text { No } \\
\text { hydrotherapy }\end{array}$ & Low & $\begin{array}{l}\text { WOMAC: Stiffness } \\
(0-8)\end{array}$ & $6 \mathrm{mo}$ & 31 & Median 0 & 33 & Median 1 & NR & NR \\
\hline \multirow{2}{*}{$\begin{array}{l}\text { Heiberg, } \\
2012, \\
22170790\end{array}$} & $\begin{array}{l}\text { Walking skill } \\
\text { training program }\end{array}$ & Standard care & Moderate & $\begin{array}{l}\text { HOOS: Symptoms } \\
(0-100)\end{array}$ & $12 \mathrm{mo}$ & 35 & $\begin{array}{l}86(95 \% \mathrm{Cl}: \\
82,89)\end{array}$ & 33 & $\begin{array}{l}87 \text { (95\% Cl: } \\
84,91)\end{array}$ & $-2(-6,3)$ & NR \\
\hline & $\begin{array}{l}\text { Walking skill } \\
\text { training program }\end{array}$ & Standard care & Moderate & $\begin{array}{l}\text { HOOS: Symptoms } \\
(0-100)\end{array}$ & $5 y$ & 30 & $\begin{array}{l}\text { Mean }(95 \% \mathrm{Cl}) \\
84(79,89)\end{array}$ & 30 & $\begin{array}{l}\text { Mean }(95 \% \\
\mathrm{Cl}) \\
88(83,92)\end{array}$ & $\begin{array}{l}-4(-10.73 \\
2.73)^{\mathrm{B}}\end{array}$ & NR \\
\hline $\begin{array}{l}\text { Liebs, } 2010 \text {, } \\
20360503\end{array}$ & Ergometer cycling & Control & Moderate & $\begin{array}{l}\text { WOMAC: Stiffness } \\
(0-100)\end{array}$ & $24 \mathrm{mo}$ & 74 & $13.4(17.9)$ & 88 & $18.6(20.3)$ & $\begin{array}{l}-5.2(-11.08 \\
0.68)^{\mathrm{B}}\end{array}$ & 0.047 \\
\hline $\begin{array}{l}\text { Liebs, 2012, } \\
22196125\end{array}$ & $\begin{array}{l}\text { Early aquatic } \\
\text { therapy }\end{array}$ & $\begin{array}{l}\text { Late aquatic } \\
\text { therapy }\end{array}$ & Moderate & $\begin{array}{l}\text { WOMAC: Stiffness } \\
(0-100)\end{array}$ & $24 \mathrm{mo}$ & 100 & $20.8(23.4)$ & 110 & $16.9(18.2)$ & $\begin{array}{l}3.9(-1.81, \\
9.61)^{\mathrm{B}}\end{array}$ & 0.552 \\
\hline \multirow[t]{2}{*}{$\begin{array}{l}\text { Mikkelsen, } \\
2014, \\
25305374\end{array}$} & $\begin{array}{l}\text { Early supervised } \\
\text { progressive } \\
\text { resistance training }\end{array}$ & $\begin{array}{l}\text { Unsupervised } \\
\text { home-based } \\
\text { exercise }\end{array}$ & Moderate & $\begin{array}{l}\text { HOOS: Symptoms } \\
(0-100)\end{array}$ & $6 \mathrm{mo}$ & 32 & $85(15)$ & 30 & $86.2(13)$ & $\begin{array}{l}-1.2(-6.1, \\
3.7)^{\mathrm{B}}\end{array}$ & NR \\
\hline & $\begin{array}{l}\text { Early supervised } \\
\text { progressive } \\
\text { resistance training }\end{array}$ & $\begin{array}{l}\text { Unsupervised } \\
\text { home-based } \\
\text { exercise }\end{array}$ & Moderate & $\begin{array}{l}\text { HOOS: Symptoms } \\
(0-100)\end{array}$ & $12 \mathrm{mo}$ & 32 & $90.7(11)$ & 30 & $90(14)$ & $0.7(-3.8,5.2)^{\mathrm{B}}$ & NR \\
\hline $\begin{array}{l}\text { Monticone, } \\
2014, \\
24459172 \\
\end{array}$ & $\begin{array}{l}\text { Task-oriented } \\
\text { exercises }\end{array}$ & $\begin{array}{l}\text { Open chain } \\
\text { kinetic } \\
\text { exercises }\end{array}$ & Moderate & $\begin{array}{l}\text { WOMAC: Stiffness } \\
(0-100)\end{array}$ & $12 \mathrm{mo}$ & 50 & $19.8(12.4)$ & 50 & $28.8(18.8)$ & $\begin{array}{l}-9.0(-13.6,- \\
4.4)^{\mathrm{B}}\end{array}$ & 0.037 \\
\hline $\begin{array}{l}\text { Nelson, } \\
2020, \\
32026820 \\
\end{array}$ & Telerehabilitation & $\begin{array}{l}\text { In-person usual } \\
\text { care }\end{array}$ & Moderate & $\begin{array}{l}\text { HOOS: Symptoms } \\
(0-100)\end{array}$ & $6 \mathrm{mo}$ & 34 & $88(13)$ & 35 & $88(12)$ & $0(-4.2,4.2)^{\mathrm{B}}$ & NR \\
\hline $\begin{array}{l}\text { Rao, } \\
2021, \\
33863614 \\
\text { USA }\end{array}$ & $\begin{array}{l}\text { Home physical } \\
\text { therapy }\end{array}$ & $\begin{array}{l}\text { Formal physical } \\
\text { therapy }\end{array}$ & High & $\begin{array}{l}\text { HOOS: Symptoms } \\
(0-100)\end{array}$ & $6 \mathrm{mo}$ & 70 & $86.8(14.1)$ & 66 & $85.0(16.2)$ & $-1.8(-5.4,1.8)$ & 0.506 \\
\hline
\end{tabular}

Statistically significant effect sizes are in bold text. In cases where calculated effect size confidence intervals were not-statistically significant but reported p-values were, we deferred to reported p-values and still bolded results.

Abbreviations: $\mathrm{CI}=$ confidence interval, $\mathrm{HOOS}=$ Hip disability and osteoarthritis outcome score, $\mathrm{mo}=$ month, $\mathrm{NR}=$ not reported, $\mathrm{PMID}=\mathrm{PubMed}$ identifier, RoB $=$ risk of bias, $\mathrm{SD}=$ standard deviation, WOMAC $=$ Western Ontario and McMaster Universities Osteoarthritis Index

A Time from surgery

B Calculated 
Table 77. Rehabilitation versus various controls for total hip arthroplasty - continuous outcomes, pain

\begin{tabular}{|c|c|c|c|c|c|c|c|c|c|c|c|}
\hline $\begin{array}{l}\text { Study, Year, } \\
\text { PMID, } \\
\text { Country }\end{array}$ & Arm 1 & Arm 2 & $\begin{array}{l}\text { Overall } \\
\text { RoB }\end{array}$ & $\begin{array}{l}\text { Outcome } \\
\text { Measurement }\end{array}$ & $\begin{array}{l}\text { Time } \\
\text { Point }^{A}\end{array}$ & $\begin{array}{l}\text { Arm 1, } \\
\text { N }\end{array}$ & $\begin{array}{l}\text { Arm 1, Mean } \\
\text { (SD) }\end{array}$ & Arm 2, & $\begin{array}{l}\text { Control, Mean } \\
\text { (SD) }\end{array}$ & $\begin{array}{l}\text { Effect Size } \\
(95 \% \mathrm{CI})\end{array}$ & $\begin{array}{l}\text { Reported } \\
\text { p-Value }\end{array}$ \\
\hline \multirow[t]{6}{*}{$\begin{array}{l}\text { Beck, 2019, } \\
30782304\end{array}$} & Sports therapy & Control & High & VAS $(0-10)$ & $6 \mathrm{mo}$ & 63 & $\begin{array}{l}\text { Median }(\mathrm{Q} 1, \mathrm{Q} 3) \\
1(0,2)\end{array}$ & 51 & $\begin{array}{l}\text { Median (Q1, Q3) } \\
1(0,2)\end{array}$ & NR & 1.00 \\
\hline & Sports therapy & Control & High & VAS (0-10) & $12 \mathrm{mo}$ & 57 & $\begin{array}{l}\text { Median (Q1, Q3) } \\
0(0,1)\end{array}$ & 41 & $\begin{array}{l}\text { Median (Q1, Q3) } \\
0(0,2)\end{array}$ & NR & 0.882 \\
\hline & Sports therapy & Control & High & $\begin{array}{l}\text { WOMAC: Pain } \\
(0-100)\end{array}$ & $6 \mathrm{mo}$ & 63 & $\begin{array}{l}\text { Median (Q1, Q3) } \\
95(90,100)\end{array}$ & 51 & $\begin{array}{l}\text { Median (Q1, Q3) } \\
92.5(90,100)\end{array}$ & NR & 1.00 \\
\hline & Sports therapy & Control & High & $\begin{array}{l}\text { WOMAC: Pain } \\
(0-100)\end{array}$ & $12 \mathrm{mo}$ & 57 & $\begin{array}{l}\text { Median }(\mathrm{Q} 1, \mathrm{Q} 3) \\
\text { MD } 100(95,100)\end{array}$ & 41 & $\begin{array}{l}\text { Median (Q1, Q3) } \\
\text { MD } 95(90,100)\end{array}$ & NR & 0.023 \\
\hline & Sports therapy & Control & High & $\begin{array}{l}\text { EQ-5D (VAS) (0- } \\
100)\end{array}$ & $6 \mathrm{mo}$ & 63 & $\begin{array}{l}\text { Median (Q1, Q3) } \\
85(80,90)\end{array}$ & 51 & $\begin{array}{l}\text { Median (Q1, Q3) } \\
85(75,90)\end{array}$ & NR & 1.00 \\
\hline & Sports therapy & Control & High & $\begin{array}{l}\text { EQ-5D (VAS) (0- } \\
100)\end{array}$ & $12 \mathrm{mo}$ & 57 & $\begin{array}{l}\text { Median (Q1, Q3) } \\
90(80,90)\end{array}$ & 41 & $\begin{array}{l}\text { Median (Q1, Q3) } \\
85(70,90)\end{array}$ & NR & 1.00 \\
\hline $\begin{array}{l}\text { Giaquinto, } \\
2010, \\
19282040\end{array}$ & Hydrotherapy & $\begin{array}{l}\text { No } \\
\text { hydrotherapy }\end{array}$ & Low & $\begin{array}{l}\text { WOMAC: Pain } \\
(0-20)\end{array}$ & $6 \mathrm{mo}$ & 31 & Median 0 & 33 & Median 3 & NR & $<0.01$ \\
\hline \multirow[t]{2}{*}{$\begin{array}{l}\text { Heiberg, 2012, } \\
22170790\end{array}$} & $\begin{array}{l}\text { Walking skill } \\
\text { training program }\end{array}$ & Standard care & Moderate & $\begin{array}{l}\text { HOOS: Pain (0- } \\
100)\end{array}$ & $12 \mathrm{mo}$ & 35 & $\begin{array}{l}\text { Mean }(95 \% \mathrm{Cl}) \\
94(91,96)\end{array}$ & 33 & $\begin{array}{l}\text { Mean }(95 \% \mathrm{Cl}) \\
94(92,97)\end{array}$ & $-1(-4,3)$ & ns \\
\hline & $\begin{array}{l}\text { Walking skill } \\
\text { training program }\end{array}$ & Standard care & Moderate & $\begin{array}{l}\text { HOOS: Pain (0- } \\
100)\end{array}$ & $5 y$ & 30 & $\begin{array}{l}\text { Mean }(95 \% \mathrm{Cl}) \\
92(87,96) \\
\text { SE } 2.30^{*}\end{array}$ & 30 & $\begin{array}{l}\text { Mean }(95 \% \mathrm{Cl}) \\
95(91,98) \\
\text { SE } 1.79^{*}\end{array}$ & $\begin{array}{l}-3(-8.71 \\
2.71)^{\mathrm{B}}\end{array}$ & NR \\
\hline $\begin{array}{l}\text { Liebs, 2010, } \\
20360503\end{array}$ & $\begin{array}{l}\text { Ergometer } \\
\text { cycling }\end{array}$ & Control & Moderate & $\begin{array}{l}\text { WOMAC: Pain } \\
(0-100)\end{array}$ & $24 \mathrm{mo}$ & 74 & $54.3(25.7)$ & 88 & $6.2(11.3)$ & $\begin{array}{l}48.1(41.8 \\
54.41)^{\mathrm{B}}\end{array}$ & 0.076 \\
\hline $\begin{array}{l}\text { Liebs, 2012, } \\
22196125\end{array}$ & $\begin{array}{l}\text { Early aquatic } \\
\text { therapy }\end{array}$ & $\begin{array}{l}\text { Late aquatic } \\
\text { therapy }\end{array}$ & Moderate & $\begin{array}{l}\text { WOMAC: Pain } \\
(0-100)\end{array}$ & $24 \mathrm{mo}$ & 100 & $12.2(17.2)$ & 110 & $9.9(14.4)$ & $\begin{array}{l}2.3(-2.01 \\
6.61)^{\mathrm{B}}\end{array}$ & 0.839 \\
\hline \multirow[t]{3}{*}{$\begin{array}{l}\text { Łyp, } 2016, \\
27455419\end{array}$} & $\begin{array}{l}\text { Kinesiotherapy, } \\
\text { low-frequency } \\
\text { magnetic field } \\
\text { and water } \\
\text { exercises }\end{array}$ & Control & High & VAS $(0-10)$ & $\begin{array}{l}14.8 \\
\mathrm{mo}\end{array}$ & NR & $1.9(\mathrm{NR})$ & NR & 3.9 (NR) & NR & NR \\
\hline & $\begin{array}{l}\text { Kinesiotherapy, } \\
\text { low-frequency } \\
\text { magnetic field, } \\
\text { without water } \\
\text { exercises }\end{array}$ & Control & High & VAS (0-10) & $\begin{array}{l}14.8 \\
\text { mo }\end{array}$ & NR & $3.3(\mathrm{NR})$ & NR & 3.9 (NR) & NR & NR \\
\hline & $\begin{array}{l}\text { Kinesiotherapy, } \\
\text { low-frequency } \\
\text { magnetic field } \\
\text { and water } \\
\text { exercises }\end{array}$ & Control & High & $\begin{array}{l}\text { Pain intensity: } \\
\text { Laitinen scale }\end{array}$ & $\begin{array}{l}14.8 \\
\mathrm{mo}\end{array}$ & NR & 0.2 (NR) & NR & $1.1(\mathrm{NR})$ & NR & NR \\
\hline
\end{tabular}




\begin{tabular}{|c|c|c|c|c|c|c|c|c|c|c|c|}
\hline $\begin{array}{l}\text { Study, Year, } \\
\text { PMID, } \\
\text { Country }\end{array}$ & Arm 1 & Arm 2 & $\begin{array}{l}\text { Overall } \\
\text { RoB }\end{array}$ & $\begin{array}{l}\text { Outcome } \\
\text { Measurement }\end{array}$ & $\begin{array}{l}\text { Time } \\
\text { Point }^{A}\end{array}$ & $\begin{array}{l}\text { Arm 1, } \\
\mathrm{N}\end{array}$ & $\begin{array}{l}\text { Arm 1, Mean } \\
\text { (SD) }\end{array}$ & $\begin{array}{l}\text { Arm 2, } \\
\text { N }\end{array}$ & $\begin{array}{l}\text { Control, Mean } \\
\text { (SD) }\end{array}$ & $\begin{array}{l}\text { Effect Size } \\
(95 \% \mathrm{Cl})\end{array}$ & $\begin{array}{l}\text { Reported } \\
\text { p-Value }\end{array}$ \\
\hline & $\begin{array}{l}\text { Kinesiotherapy, } \\
\text { low-frequency } \\
\text { magnetic field, } \\
\text { without water } \\
\text { exercises }\end{array}$ & Control & High & $\begin{array}{l}\text { Pain intensity: } \\
\text { Laitinen scale }\end{array}$ & $\begin{array}{l}14.8 \\
\mathrm{mo}\end{array}$ & NR & $0.7(\mathrm{NR})$ & NR & $1.1(\mathrm{NR})$ & NR & NR \\
\hline \multirow[t]{2}{*}{$\begin{array}{l}\text { Mikkelsen, } \\
2014, \\
25305374\end{array}$} & $\begin{array}{l}\text { Early supervised } \\
\text { progressive } \\
\text { resistance } \\
\text { training }\end{array}$ & $\begin{array}{l}\text { Unsupervised } \\
\text { home-based } \\
\text { exercise }\end{array}$ & Moderate & $\begin{array}{l}\text { HOOS: Pain (0- } \\
\text { 100) }\end{array}$ & $6 \mathrm{mo}$ & 32 & $91.7(10)$ & 30 & $91.4(13)$ & $\begin{array}{l}0.3(-3.9 \\
4.5)^{\mathrm{B}}\end{array}$ & NR \\
\hline & $\begin{array}{l}\text { Early supervised } \\
\text { progressive } \\
\text { resistance } \\
\text { training }\end{array}$ & $\begin{array}{l}\text { Unsupervised } \\
\text { home-based } \\
\text { exercise }\end{array}$ & Moderate & $\begin{array}{l}\text { HOOS: Pain (0- } \\
100)\end{array}$ & $12 \mathrm{mo}$ & 32 & $94(8)$ & 30 & $92.2(14)$ & $\begin{array}{l}1.8(-2.5 \\
6.1)^{\mathrm{B}}\end{array}$ & NR \\
\hline \multirow[t]{3}{*}{$\begin{array}{l}\text { Monticone, } \\
2014, \\
24459172\end{array}$} & $\begin{array}{l}\text { Task-oriented } \\
\text { exercises }\end{array}$ & $\begin{array}{l}\text { Open chain } \\
\text { kinetic } \\
\text { exercises }\end{array}$ & Moderate & $\begin{array}{l}\text { WOMAC: Pain } \\
(0-100)\end{array}$ & $12 \mathrm{mo}$ & 50 & $25.2(16.1)$ & 50 & $34.9(18.7)$ & $\begin{array}{l}-9.7(-14.6,- \\
4.8)^{\mathrm{B}}\end{array}$ & NR \\
\hline & $\begin{array}{l}\text { Task-oriented } \\
\text { exercises }\end{array}$ & $\begin{array}{l}\text { Open chain } \\
\text { kinetic } \\
\text { exercises }\end{array}$ & Moderate & $\begin{array}{l}\text { SF-36: Bodily } \\
\text { pain }(0-100)\end{array}$ & $12 \mathrm{mo}$ & 50 & $79.8(26.1)$ & 50 & $63.9(25.2)$ & $\begin{array}{l}15.9(8.8, \\
23.0)^{\mathrm{B}}\end{array}$ & NR \\
\hline & $\begin{array}{l}\text { Task-oriented } \\
\text { exercises }\end{array}$ & $\begin{array}{l}\text { Open chain } \\
\text { kinetic } \\
\text { exercises }\end{array}$ & Moderate & VAS $(0-10)$ & $12 \mathrm{mo}$ & 50 & $0.8(1.3)$ & 50 & $1.4(2.6)$ & $\begin{array}{l}-0.6(-1.2 \\
0.02)^{\mathrm{B}}\end{array}$ & NR \\
\hline $\begin{array}{l}\text { Naylor, 2018, } \\
30021552\end{array}$ & $\begin{array}{l}\text { In-patient } \\
\text { rehabilitation }\end{array}$ & $\begin{array}{l}\text { No in-patient } \\
\text { rehabilitation }\end{array}$ & Moderate & $\begin{array}{l}\text { EuroQol VAS (0- } \\
100)\end{array}$ & $12 \mathrm{mo}$ & 123 & $\begin{array}{l}\text { Median (Q1, Q3) } \\
85(75,95)\end{array}$ & 123 & $\begin{array}{l}\text { Median (Q1, Q3) } \\
90(80,95)\end{array}$ & $\begin{array}{l}\text { Median } \\
\text { difference } \\
(\mathrm{Q} 1, \mathrm{Q} 3) 5(- \\
10,15)\end{array}$ & 0.09 \\
\hline \multirow[t]{2}{*}{$\begin{array}{l}\text { Nelson, 2020, } \\
32026820\end{array}$} & Telerehabilitation & $\begin{array}{l}\text { In-person usual } \\
\text { care }\end{array}$ & Moderate & $\begin{array}{l}\text { HOOS: Pain (0- } \\
100)\end{array}$ & $6 \mathrm{mo}$ & 34 & $92(12)$ & 35 & $89(13)$ & $3(-1.2,7.2)^{\mathrm{B}}$ & NR \\
\hline & Telerehabilitation & $\begin{array}{l}\text { In-person usual } \\
\text { care }\end{array}$ & Moderate & $\begin{array}{l}\text { EQ-5D: VAS (0- } \\
100)\end{array}$ & $6 \mathrm{mo}$ & 34 & $82(14)$ & 35 & $81(18)$ & $1(-4.4,6.4)^{\mathrm{B}}$ & NR \\
\hline $\begin{array}{l}\text { Rao, } \\
2021, \\
33863614 \\
\text { USA }\end{array}$ & $\begin{array}{l}\text { Home physical } \\
\text { therapy }\end{array}$ & $\begin{array}{l}\text { Formal physical } \\
\text { therapy }\end{array}$ & High & HOOS pain & $6 \mathrm{mo}$ & 70 & $87.6(15.4)$ & 66 & $85.2(18.6)$ & $\begin{array}{l}-2.4(-6.5 \\
1.7)\end{array}$ & 0.427 \\
\hline
\end{tabular}

Statistically significant effect sizes are in bold text. In cases where calculated effect size confidence intervals were not-statistically significant but reported p-values were, we deferred to reported p-values and still bolded results.

Abbreviations: $\mathrm{CI}=$ confidence interval, $\mathrm{EQ}-5 \mathrm{D}=$ EuroQuol, $\mathrm{HOOS}=$ Hip disability and osteoarthritis outcome score, mo $=$ month, $\mathrm{NR}=$ not reported, $\mathrm{PMID}=\mathrm{PubMed}$ identifier, $\mathrm{Q}=$ quartile, $\mathrm{RoB}=$ risk of bias, $\mathrm{SD}=$ standard deviation, WOMAC = Western Ontario and McMaster Universities Osteoarthritis Index, VAS = visual analogue scale,

A Time from surgery

${ }^{\text {B }}$ Calculated 
Table 78. Rehabilitation versus various controls for total hip arthroplasty - continuous outcomes, range of motion

\begin{tabular}{|c|c|c|c|c|c|c|c|c|c|c|c|}
\hline $\begin{array}{l}\text { Study, Year, } \\
\text { PMID, } \\
\text { Country }\end{array}$ & Arm 1 & Arm 2 & $\begin{array}{l}\text { Overall } \\
\text { RoB }\end{array}$ & $\begin{array}{l}\text { Outcome } \\
\text { Measurement }\end{array}$ & $\begin{array}{l}\text { Time } \\
\text { Point* }\end{array}$ & $\begin{array}{l}\text { Arm } \\
1, N\end{array}$ & $\begin{array}{l}\text { Arm 1, Mean } \\
\text { (SD) }\end{array}$ & Arm 2, & $\begin{array}{l}\text { Control, Mean } \\
\text { (SD) }\end{array}$ & $\begin{array}{l}\text { Effect Size } \\
(95 \% \mathrm{Cl})\end{array}$ & $\begin{array}{l}\text { Reported } \\
\text { p-Value }\end{array}$ \\
\hline \multirow[t]{4}{*}{$\begin{array}{l}\text { Heiberg, 2012, } \\
22170790\end{array}$} & $\begin{array}{l}\text { Walking skill } \\
\text { training program }\end{array}$ & Standard care & Moderate & $\begin{array}{l}\text { Active ROM in } \\
\text { hip: Flexion (deg) }\end{array}$ & $12 \mathrm{mo}$ & 35 & $\begin{array}{l}\text { Mean }(95 \% \mathrm{Cl}) \\
95(91,98)\end{array}$ & 33 & $\begin{array}{l}\text { Mean }(95 \% \mathrm{Cl}) \\
94(90,98)\end{array}$ & $1(-5,6)$ & ns \\
\hline & $\begin{array}{l}\text { Walking skill } \\
\text { training program }\end{array}$ & Standard care & Moderate & $\begin{array}{l}\text { Active ROM in } \\
\text { hip: Flexion (deg) }\end{array}$ & $5 y$ & 30 & $\begin{array}{l}\text { Mean }(95 \% \mathrm{Cl}) \\
104(99,109) \\
\text { SE } 2.55^{\mathrm{B}}\end{array}$ & 30 & $\begin{array}{l}\text { Mean }(95 \% \mathrm{Cl}) \\
100(94,106) \\
\text { SE } 3.06^{\mathrm{B}}\end{array}$ & $4(-3.81,11.81)^{B}$ & NR \\
\hline & $\begin{array}{l}\text { Walking skill } \\
\text { training program }\end{array}$ & Standard care & Moderate & $\begin{array}{l}\text { Active ROM in } \\
\text { hip: Extension } \\
\text { (deg) }\end{array}$ & $12 \mathrm{mo}$ & 35 & $\begin{array}{l}\text { Mean }(95 \% \mathrm{Cl}) \\
0(-2,1)\end{array}$ & 33 & $\begin{array}{l}\text { Mean }(95 \% \mathrm{Cl}) \\
-1(-3,0)\end{array}$ & $1(-1,3)$ & ns \\
\hline & $\begin{array}{l}\text { Walking skill } \\
\text { training program }\end{array}$ & Standard care & Moderate & $\begin{array}{l}\text { Active ROM in } \\
\text { hip: Abduction } \\
\text { (deg) }\end{array}$ & $12 \mathrm{mo}$ & 35 & $\begin{array}{l}\text { Mean }(95 \% \mathrm{Cl}) \\
25(23,27)\end{array}$ & 33 & $\begin{array}{l}\text { Mean }(95 \% \mathrm{Cl}) \\
25(23,27)\end{array}$ & $0(-3,3)$ & ns \\
\hline \multirow[t]{4}{*}{$\begin{array}{l}\text { Łyp, 2016, } \\
27455419\end{array}$} & $\begin{array}{l}\text { Kinesiotherapy, } \\
\text { low-frequency } \\
\text { magnetic field } \\
\text { and water } \\
\text { exercises }\end{array}$ & Control & High & $\begin{array}{l}\text { Active ROM in } \\
\text { hip: Unspecified } \\
\text { (deg) }\end{array}$ & $\begin{array}{l}14.8 \\
\mathrm{mo}\end{array}$ & NR & 205.6 (NR) & NR & 127.0 (NR) & NR & $0.001^{\mathrm{C}}$ \\
\hline & $\begin{array}{l}\text { Kinesiotherapy, } \\
\text { low-frequency } \\
\text { magnetic field, } \\
\text { without water } \\
\text { exercises }\end{array}$ & Control & High & $\begin{array}{l}\text { Active ROM in } \\
\text { hip: Unspecified } \\
\text { (deg) }\end{array}$ & $\begin{array}{l}14.8 \\
\mathrm{mo}\end{array}$ & NR & $197.6(\mathrm{NR})$ & NRN & 161.0 (NR) & NR & 0.001 \\
\hline & $\begin{array}{l}\text { Kinesiotherapy, } \\
\text { low-frequency } \\
\text { magnetic field } \\
\text { and water } \\
\text { exercises }\end{array}$ & Control & High & $\begin{array}{l}\text { Active ROM in } \\
\text { hip: Unspecified } \\
\text { (deg) }\end{array}$ & $\begin{array}{l}14.8 \\
\mathrm{mo}\end{array}$ & NR & 149.9 (NR) & NR & 127.0 (NR) & NR & 0.001 \\
\hline & $\begin{array}{l}\text { Kinesiotherapy, } \\
\text { low-frequency } \\
\text { magnetic field, } \\
\text { without water } \\
\text { exercises }\end{array}$ & Control & High & $\begin{array}{l}\text { Active ROM in } \\
\text { hip: Unspecified } \\
\text { (deg) }\end{array}$ & $\begin{array}{l}14.8 \\
\text { mo }\end{array}$ & NR & 187.5 (NR) & NR & 161.0 (NR) & NR & 0.001 \\
\hline $\begin{array}{l}\text { Winther, 2020, } \\
31977324\end{array}$ & $\begin{array}{l}\text { Strengthening } \\
\text { exercise }\end{array}$ & Standard care & Moderate & $\begin{array}{l}\text { Hip abduction } \\
\text { (deg) }\end{array}$ & $12 \mathrm{mo}$ & 22 & $12(4)$ & 22 & $12(4)$ & $0(-1.7,1.7)^{\mathrm{B}}$ & NR \\
\hline
\end{tabular}

Statistically significant effect sizes are in bold text. In cases where calculated effect size confidence intervals were not-statistically significant but reported p-values were, we

deferred to reported p-values and still bolded results.

Abbreviations: $\mathrm{CI}=$ confidence interval, $\mathrm{deg}=\mathrm{deg}, \mathrm{mo}=$ month, $\mathrm{NR}=$ not reported, $\mathrm{ns}=$ not significant, $\mathrm{PMID}=\mathrm{PubMed}$ identifier, $\mathrm{RoB}=$ risk of bias, $\mathrm{ROM}=$ range of motion, $\mathrm{SD}=$ standard deviation.

A Time from surgery

B Calculated

${ }^{\mathrm{C}}$ Noted as $\mathrm{p}<0.001$ for hip range of motion in general 
Table 79. Rehabilitation versus various controls for total hip arthroplasty - continuous outcomes, muscle strength

\begin{tabular}{|c|c|c|c|c|c|c|c|c|c|c|c|}
\hline $\begin{array}{l}\text { Study, Year, } \\
\text { PMID, } \\
\text { Country }\end{array}$ & Arm 1 & Arm 2 & $\begin{array}{l}\text { Overall } \\
\text { RoB }\end{array}$ & $\begin{array}{l}\text { Outcome } \\
\text { Measurement }\end{array}$ & $\begin{array}{l}\text { Time } \\
\text { Point }^{A}\end{array}$ & $\begin{array}{l}\text { Arm 1, } \\
\mathrm{N}\end{array}$ & $\begin{array}{l}\text { Arm 1, Mean } \\
\text { (SD) }\end{array}$ & $\begin{array}{l}\text { Arm 2, } \\
\text { N }\end{array}$ & $\begin{array}{l}\text { Arm 2, Mean } \\
\text { (SD) }\end{array}$ & $\begin{array}{l}\text { Effect } \\
\text { Size } \\
(95 \% \mathrm{Cl})\end{array}$ & $\begin{array}{l}\text { Reported } \\
\text { p-Value }\end{array}$ \\
\hline \multirow[t]{8}{*}{$\begin{array}{l}\text { Beck, 2019, } \\
30782304\end{array}$} & Sports therapy & Control & High & $\begin{array}{l}\text { Strength capacity: } \\
\text { Isokinetic extension } \\
(\mathrm{J} / \mathrm{kg})\end{array}$ & $6 \mathrm{mo}$ & 63 & $\begin{array}{l}\text { Median (Q1, Q3) } \\
0.69(0.44,0.96)\end{array}$ & 51 & $\begin{array}{l}\text { Median (Q1, Q3) } \\
0.55(0.06,0.79)\end{array}$ & NR & 1.00 \\
\hline & Sports therapy & Control & High & $\begin{array}{l}\text { Strength capacity: } \\
\text { Isokinetic extension } \\
(\mathrm{J} / \mathrm{kg})\end{array}$ & $12 \mathrm{mo}$ & 57 & $\begin{array}{l}\text { Median (Q1, Q3) } \\
0.86(0.46,1.11)\end{array}$ & 41 & $\begin{array}{l}\text { Median (Q1, Q3) } \\
0.93(0.42,1.07)\end{array}$ & NR & 1.00 \\
\hline & Sports therapy & Control & High & $\begin{array}{l}\text { Strength capacity: } \\
\text { Isokinetic flexion } \\
(\mathrm{J} / \mathrm{kg})\end{array}$ & $6 \mathrm{mo}$ & 63 & $\begin{array}{l}\text { Median }(\mathrm{Q} 1, \mathrm{Q} 3) \\
0.31(0.19,0.43)\end{array}$ & 51 & $\begin{array}{l}\text { Median (Q1, Q3) } \\
0.26(0.09,0.35)\end{array}$ & NR & 1.00 \\
\hline & Sports therapy & Control & High & $\begin{array}{l}\text { Strength capacity: } \\
\text { Isokinetic flexion } \\
(\mathrm{J} / \mathrm{kg})\end{array}$ & $12 \mathrm{mo}$ & 57 & $\begin{array}{l}\text { Median (Q1, Q3) } \\
0.55(0.31,0.69)\end{array}$ & 41 & $\begin{array}{l}\text { Median (Q1, Q3) } \\
0.42(0.31,0.58)\end{array}$ & NR & 0.616 \\
\hline & Sports therapy & Control & High & $\begin{array}{l}\text { Strength capacity: } \\
\text { Isokinetic abduction } \\
(\mathrm{J} / \mathrm{kg})\end{array}$ & $6 \mathrm{mo}$ & 63 & $\begin{array}{l}\text { Median (Q1, Q3) } \\
0.18(0.09,0.26)\end{array}$ & 51 & $\begin{array}{l}\text { Median (Q1, Q3) } \\
0.13(0.04,0.17)\end{array}$ & NR & 0.112 \\
\hline & Sports therapy & Control & High & $\begin{array}{l}\text { Strength capacity: } \\
\text { Isokinetic abduction } \\
(\mathrm{J} / \mathrm{kg})\end{array}$ & $12 \mathrm{mo}$ & 57 & $\begin{array}{l}\text { Median (Q1, Q3) } \\
0.23(0.14,0.35)\end{array}$ & 41 & $\begin{array}{l}\text { Median (Q1, Q3) } \\
0.19(0.15,0.28)\end{array}$ & NR & 1.00 \\
\hline & Sports therapy & Control & High & $\begin{array}{l}\text { Strength capacity: } \\
\text { Isokinetic adduction } \\
(\mathrm{J} / \mathrm{kg})\end{array}$ & $6 \mathrm{mo}$ & 63 & $\begin{array}{l}\text { Median (Q1, Q3) } \\
0.17(0.09,0.29)\end{array}$ & 51 & $\begin{array}{l}\text { Median (Q1, Q3) } \\
0.14(0.03,0.24)\end{array}$ & NR & 1.00 \\
\hline & Sports therapy & Control & High & $\begin{array}{l}\text { Strength capacity: } \\
\text { Isokinetic adduction } \\
(\mathrm{J} / \mathrm{kg})\end{array}$ & $12 \mathrm{mo}$ & 57 & $\begin{array}{l}\text { Median (Q1, Q3) } \\
0.23(0.14,0.35)\end{array}$ & 41 & $\begin{array}{l}\text { Median (Q1, Q3) } \\
0.19(0.15,0.28)\end{array}$ & NR & 1.00 \\
\hline $\begin{array}{l}\text { Heiberg, } \\
2012, \\
22170790 \\
\end{array}$ & $\begin{array}{l}\text { Walking skill } \\
\text { training program }\end{array}$ & Standard care & Moderate & $\begin{array}{l}\text { Index of muscle } \\
\text { function }{ }^{B}\end{array}$ & $12 \mathrm{mo}$ & 35 & $\begin{array}{l}\text { Mean }(95 \% \mathrm{Cl}) \\
7(6,9)\end{array}$ & 33 & $\begin{array}{l}\text { Mean }(95 \% \mathrm{Cl}) \\
10(8,11)\end{array}$ & $-2(-5,0)$ & ns \\
\hline \multirow[t]{3}{*}{$\begin{array}{l}\text { Łyp, 2016, } \\
2745541\end{array}$} & $\begin{array}{l}\text { Kinesiotherapy, } \\
\text { low-frequency } \\
\text { magnetic field and } \\
\text { water exercises }\end{array}$ & Control & High & $\begin{array}{l}\text { Strength: Extensors } \\
\text { and abductors }(\mathrm{Nm})^{\mathrm{C}}\end{array}$ & $\begin{array}{l}14.8 \\
\mathrm{mo}^{\mathrm{D}}\end{array}$ & NR & 81.8 (NR) & NR & 48.8 (NR) & NR & NR \\
\hline & $\begin{array}{l}\text { Kinesiotherapy, } \\
\text { low-frequency } \\
\text { magnetic field and } \\
\text { water exercises }\end{array}$ & $\begin{array}{l}\text { Kinesiotherapy, } \\
\text { low-frequency } \\
\text { magnetic field, } \\
\text { without water } \\
\text { exercises }\end{array}$ & High & $\begin{array}{l}\text { Strength: Extensors } \\
\text { and abductors }(\mathrm{Nm})^{\mathrm{E}}\end{array}$ & $14.8 \mathrm{mo}$ & NR & 83.6 (NR) & NR & 67.5 (NR) & NR & NR \\
\hline & $\begin{array}{l}\text { Kinesiotherapy, } \\
\text { low-frequency } \\
\text { magnetic field, } \\
\text { without water } \\
\text { exercises }\end{array}$ & Control & High & $\begin{array}{l}\text { Strength: Extensors } \\
\text { and abductors }(\mathrm{Nm})\end{array}$ & $14.8 \mathrm{mo}$ & NR & 67.3 (NR) & NR & 48.8 (NR) & NR & NR \\
\hline
\end{tabular}




\begin{tabular}{|c|c|c|c|c|c|c|c|c|c|c|c|}
\hline $\begin{array}{l}\text { Study, Year, } \\
\text { PMID, } \\
\text { Country }\end{array}$ & Arm 1 & Arm 2 & $\begin{array}{l}\text { Overall } \\
\text { RoB }\end{array}$ & $\begin{array}{l}\text { Outcome } \\
\text { Measurement }\end{array}$ & $\begin{array}{l}\text { Time } \\
\text { Point }^{A}\end{array}$ & $\begin{array}{l}\text { Arm 1, } \\
\text { N }\end{array}$ & $\begin{array}{l}\text { Arm 1, Mean } \\
\text { (SD) }\end{array}$ & $\begin{array}{l}\text { Arm 2, } \\
\text { N }\end{array}$ & $\begin{array}{l}\text { Arm 2, Mean } \\
\text { (SD) }\end{array}$ & $\begin{array}{l}\text { Effect } \\
\text { Size } \\
(95 \% \mathrm{Cl})\end{array}$ & $\begin{array}{l}\text { Reported } \\
\text { p-Value }\end{array}$ \\
\hline & $\begin{array}{l}\text { Kinesiotherapy, } \\
\text { low-frequency } \\
\text { magnetic field and } \\
\text { water exercises }\end{array}$ & $\begin{array}{l}\text { Kinesiotherapy, } \\
\text { low-frequency } \\
\text { magnetic field, } \\
\text { without water } \\
\text { exercises }\end{array}$ & & $\begin{array}{l}\text { Strength: Extensors } \\
\text { and abductors }(\mathrm{Nm})\end{array}$ & $14.8 \mathrm{mo}$ & NR & 68.7 (NR) & NR & 67.5 (NR) & NR & NR \\
\hline \multirow[t]{3}{*}{$\begin{array}{l}\text { Mikkelsen, } \\
2014, \\
25305374\end{array}$} & $\begin{array}{l}\text { Early supervised } \\
\text { progressive } \\
\text { resistance training }\end{array}$ & $\begin{array}{l}\text { Unsupervised } \\
\text { home-based } \\
\text { exercise }\end{array}$ & Moderate & $\begin{array}{l}\text { Strength: Peak } \\
\text { abduction strength } \\
(\mathrm{Nm} / \mathrm{kg})\end{array}$ & $6 \mathrm{mo}$ & 32 & $1.08(0.3)$ & 30 & $1.15(0.3)$ & $\begin{array}{l}-0.1(- \\
0.2 \\
0.03)^{F}\end{array}$ & NR \\
\hline & $\begin{array}{l}\text { Early supervised } \\
\text { progressive } \\
\text { resistance training }\end{array}$ & $\begin{array}{l}\text { Unsupervised } \\
\text { home-based } \\
\text { exercise }\end{array}$ & Moderate & Strength: Hip flexion & $6 \mathrm{mo}$ & \begin{tabular}{|l|}
32 \\
\end{tabular} & $1.33(0.3)$ & 30 & $1.41(0.4)$ & $\begin{array}{l}-0.1(- \\
0.2 \\
0.05)^{\mathrm{F}}\end{array}$ & NR \\
\hline & $\begin{array}{l}\text { Early supervised } \\
\text { progressive } \\
\text { resistance training }\end{array}$ & $\begin{array}{l}\text { Unsupervised } \\
\text { home-based } \\
\text { exercise }\end{array}$ & Moderate & $\begin{array}{l}\text { Leg extension power } \\
(\mathrm{W} / \mathrm{kg}))\end{array}$ & $6 \mathrm{mo}$ & 32 & $2.04(0.7)$ & 30 & $1.97(0.6)$ & $\begin{array}{l}0.1(-0.2, \\
0.3)^{\mathrm{F}}\end{array}$ & NR \\
\hline \multirow{6}{*}{$\begin{array}{l}\text { Nelson, } \\
2020, \\
32026820\end{array}$} & Telerehabilitation & $\begin{array}{l}\text { In-person usual } \\
\text { care }\end{array}$ & Moderate & $\begin{array}{l}\text { Strength: Hip } \\
\text { extension }(\mathrm{kg})\end{array}$ & $6 \mathrm{mo}$ & 34 & $8.6(2.9)$ & 35 & $8.1(2.7)$ & $\begin{array}{l}0.5(-0.4, \\
1.4)\end{array}$ & NR \\
\hline & Telerehabilitation & $\begin{array}{l}\text { In-person usual } \\
\text { care }\end{array}$ & Moderate & $\begin{array}{l}\text { Strength: Hip } \\
\text { adduction }(\mathrm{kg})\end{array}$ & $6 \mathrm{mo}$ & 34 & $4.5(1.4)$ & 35 & $4.1(1.4)$ & $\begin{array}{l}0.4(-0.1 \\
0.9)^{\mathrm{F}}\end{array}$ & NR \\
\hline & Telerehabilitation & $\begin{array}{l}\text { In-person usual } \\
\text { care }\end{array}$ & Moderate & $\begin{array}{l}\text { Strength: Hip } \\
\text { abduction }(\mathrm{kg})\end{array}$ & $6 \mathrm{mo}$ & 34 & $4.9(2.0)$ & 35 & $4.5(1.8)$ & $\begin{array}{l}0.4(-0.2, \\
1.0)^{\mathrm{F}}\end{array}$ & NR \\
\hline & Telerehabilitation & $\begin{array}{l}\text { In-person usual } \\
\text { care }\end{array}$ & Moderate & $\begin{array}{l}\text { Strength: Hip } \\
\text { internal rotator }(\mathrm{kg})\end{array}$ & $6 \mathrm{mo}$ & 34 & $4(1.5)$ & 35 & $3.9(1.3)$ & $\begin{array}{l}0.1(-0.4, \\
0.6)^{\mathrm{F}}\end{array}$ & NR \\
\hline & Telerehabilitation & $\begin{array}{l}\text { In-person usual } \\
\text { care }\end{array}$ & Moderate & $\begin{array}{l}\text { Strength: Hip } \\
\text { external rotator }(\mathrm{kg})\end{array}$ & $6 \mathrm{mo}$ & 34 & $2.3(1)$ & 35 & $2(0.8)$ & $\begin{array}{l}0.3(- \\
0.01 \\
0.6)^{F}\end{array}$ & NR \\
\hline & Telerehabilitation & $\begin{array}{l}\text { In-person usual } \\
\text { care }\end{array}$ & Moderate & $\begin{array}{l}\text { Strength: Hip flexion } \\
(\mathrm{kg})\end{array}$ & $6 \mathrm{mo}$ & 34 & $7.2(2.5)$ & 35 & $7(1.9)$ & $\begin{array}{l}0.2(-0.6, \\
1)^{\mathrm{F}}\end{array}$ & NR \\
\hline
\end{tabular}

Statistically significant effect sizes are in bold text. In cases where calculated effect size confidence intervals were not-statistically significant but reported p-values were, we deferred to reported p-values and still bolded results.

Abbreviations: $\mathrm{CI}=$ confidence interval, $\mathrm{J}=$ joule, $\mathrm{kg}=$ kilogram, $\mathrm{N}=$ Newton, $\mathrm{Nm}=$ peak torque, $\mathrm{NR}=$ not reported, $\mathrm{PMID}=\mathrm{PubMed}$ identifier, $\mathrm{RoB}=$ risk of bias, $\mathrm{SD}=$ standard deviation, $\mathrm{SE}=$ standard error, $\mathrm{W}=$ watt.

A Time from surgery

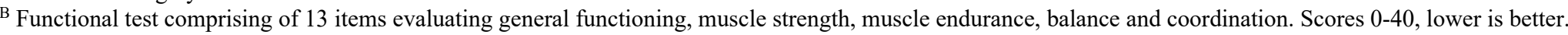

${ }^{C}$ Subgroup of cemented participants

D Study reported the average time between surgery and follow-up was 14.8 months (range 14.2 to 14.8 )

E Subgroup of uncemented participants

F Calculated 
Table 80. Rehabilitation versus various controls for total hip arthroplasty - continuous outcomes, energy and vigor

\begin{tabular}{|c|c|c|c|c|c|c|c|c|c|c|c|}
\hline $\begin{array}{l}\text { Study, Year, } \\
\text { PMID, } \\
\text { Country }\end{array}$ & Arm 1 & Arm 2 & $\begin{array}{l}\text { Overall } \\
\text { RoB }\end{array}$ & $\begin{array}{l}\text { Outcome } \\
\text { Measurement }\end{array}$ & $\begin{array}{l}\text { Time } \\
\text { Point }^{\mathrm{A}}\end{array}$ & $\begin{array}{l}\text { Arm 1, } \\
\mathrm{N}\end{array}$ & $\begin{array}{l}\text { Arm 1, Mean } \\
\text { (SD) }\end{array}$ & $\begin{array}{l}\text { Arm } \\
2, N\end{array}$ & $\begin{array}{l}\text { Arm 2, Mean } \\
\text { (SD) }\end{array}$ & $\begin{array}{l}\text { Effect Size } \\
(95 \% \mathrm{Cl})\end{array}$ & $\begin{array}{l}\text { Reported } \\
\text { p-Value }\end{array}$ \\
\hline $\begin{array}{l}\text { Monticone, } \\
2014, \\
24459172\end{array}$ & $\begin{array}{l}\text { Task-oriented } \\
\text { exercises }\end{array}$ & $\begin{array}{l}\text { Open chain } \\
\text { kinetic } \\
\text { exercises }\end{array}$ & Moderate & $\begin{array}{l}\text { SF-36: Vitality (0- } \\
100)\end{array}$ & $12 \mathrm{mo}$ & 50 & $66.9(17)$ & 50 & $58.4(15.9)$ & $8.5(3.9,13.1)^{B}$ & NR \\
\hline
\end{tabular}

Abbreviations: $\mathrm{CI}=$ confidence interval, $\mathrm{NR}=$ not reported, $\mathrm{PMID}=$ PubMed identifier, RoB $=$ risk of bias, $\mathrm{SF}-36=36$-Item short form survey, $\mathrm{SD}=$ standard deviation.

A Time from surgery

B Calculated

Table 81. Rehabilitation versus various controls for total hip arthroplasty - continuous outcomes, emotional functioning (stress/coping)

\begin{tabular}{|c|c|c|c|c|c|c|c|c|c|c|c|}
\hline $\begin{array}{l}\text { Study, Year, } \\
\text { PMID, } \\
\text { Country }\end{array}$ & Arm 1 & Arm 2 & $\begin{array}{l}\text { Overall } \\
\text { RoB }\end{array}$ & $\begin{array}{l}\text { Outcome } \\
\text { Measurement }\end{array}$ & $\begin{array}{l}\text { Time } \\
\text { Point }\end{array}$ & $\begin{array}{l}\text { Arm } 1 \\
\mathrm{~N}\end{array}$ & $\begin{array}{l}\text { Arm 1, Mean } \\
\text { (SD) }\end{array}$ & Arm 2, & $\begin{array}{l}\text { Arm 2, Mean } \\
\text { (SD) }\end{array}$ & $\begin{array}{l}\text { Effect Size } \\
(95 \% \mathrm{CI})\end{array}$ & $\begin{array}{l}\text { Reported } \\
\text { p-Value }\end{array}$ \\
\hline $\begin{array}{l}\text { Austin, 2017, } \\
28419032\end{array}$ & Home exercise & $\begin{array}{l}\text { Formal } \\
\text { Physical } \\
\text { Therapy }\end{array}$ & Moderate & $\begin{array}{l}\text { SF-36: mental } \\
\text { health }(0-100)\end{array}$ & $6-12 \mathrm{mo}$ & 52 & NR & 52 & NR & NR & 0.70 \\
\hline $\begin{array}{l}\text { Coulter, 2017, } \\
28506775\end{array}$ & $\begin{array}{l}\text { Unsupervised } \\
\text { home-based } \\
\text { exercises }\end{array}$ & $\begin{array}{l}\text { Supervised } \\
\text { rehabilitation \& } \\
\text { unsupervised } \\
\text { home-based } \\
\text { exercises }\end{array}$ & High & $\begin{array}{l}\text { SF-36: mental } \\
\text { health }(0-100)\end{array}$ & $6 \mathrm{mo}$ & 56 & $\begin{array}{l}\text { Mean }(95 \% \\
\mathrm{Cl}) \\
81.10(74.94, \\
87.25)\end{array}$ & 42 & $\begin{array}{l}\text { Mean }(95 \% \\
\mathrm{Cl}) \\
78.60(71.75 \\
85.47)\end{array}$ & $\begin{array}{l}2.5(-6.72 \\
11.72)^{\mathrm{B}}\end{array}$ & NR \\
\hline \multirow[t]{3}{*}{$\begin{array}{l}\text { Monticone, } \\
2014, \\
24459172\end{array}$} & $\begin{array}{l}\text { Task-oriented } \\
\text { exercises }\end{array}$ & $\begin{array}{l}\text { Open chain } \\
\text { kinetic } \\
\text { exercises }\end{array}$ & Moderate & $\begin{array}{l}\text { SF-36: social } \\
\text { function }(0-100)\end{array}$ & $12 \mathrm{mo}$ & 50 & $84.4(20.5)$ & 50 & $76.7(27)$ & $7.7(0.9,14.5)^{\mathrm{B}}$ & NR \\
\hline & $\begin{array}{l}\text { Task-oriented } \\
\text { exercises }\end{array}$ & $\begin{array}{l}\text { Open chain } \\
\text { kinetic } \\
\text { exercises }\end{array}$ & Moderate & $\begin{array}{l}\text { SF-36: emotional } \\
\text { role }(0-100)\end{array}$ & $12 \mathrm{mo}$ & 50 & $83(31.5)$ & 50 & $73.5(31)$ & $9.5(0.8,18.2)^{\mathrm{B}}$ & NR \\
\hline & $\begin{array}{l}\text { Task-oriented } \\
\text { exercises }\end{array}$ & $\begin{array}{l}\text { Open chain } \\
\text { kinetic } \\
\text { exercises }\end{array}$ & Moderate & $\begin{array}{l}\text { SF-36: mental } \\
\text { health }(0-100)\end{array}$ & $12 \mathrm{mo}$ & 50 & $74(16.6)$ & 50 & $66.2(19.3)$ & $7.8(2.8,12.8)^{\mathrm{B}}$ & NR \\
\hline $\begin{array}{l}\text { Nelson, } 2020 \text {, } \\
32026820\end{array}$ & Telerehabilitation & $\begin{array}{l}\text { In-person usual } \\
\text { care }\end{array}$ & Moderate & $\begin{array}{l}\text { SF-12: mental } \\
\text { health }(0-100)\end{array}$ & $6 \mathrm{mo}$ & 34 & $53(10)$ & 35 & $55(10)$ & $-2(-5.3,1.3)^{\mathrm{B}}$ & NR \\
\hline
\end{tabular}

Statistically significant effect sizes are in bold text. In cases where calculated effect size confidence intervals were not-statistically significant but reported p-values were, we deferred to reported p-values and still bolded results.

Abbreviations: $\mathrm{CI}=$ confidence interval, $\mathrm{NR}=$ not reported, $\mathrm{PMID}=$ PubMed identifier, RoB $=$ risk of bias, $\mathrm{SF}-12=12$-item short form survey, $\mathrm{SF}-36=36$-item short form survey, $\mathrm{SD}=$ standard deviation.

A Time from surgery

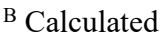




\section{Activity and Participation Outcomes}

Thirteen studies reported on activity and participation outcomes following rehabilitation compared to various control: two studies (Liebs 2010, Smith 2009) compared novel acute rehabilitation programs with control; two studies (Liebs 2012, Mikkelsen 2014) compared acute rehabilitation programs with different timing and/or intensity; five studies (Beck 2019, Giaquinto 2010, Heiberg 2012, Monticone 2014, Winther 2018) compared novel post-acute rehabilitation programs with various comparators (less intensive rehabilitation or no care); and four studies (Austin 2017, Coulter 2017, Nelson 2020, Rao 2021) compared rehabilitation programs delivered in different settings or by different personnel (Tables 82 to 85). Outcomes included: physical function and activities of daily living, transfers, mobility, and timed up and go.

\section{Physical Function and Activities of Daily Living}

Twelve studies (Austin 2017, Beck 2019, Coulter 2017, Giaquinto 2010, Heiberg 2012, Liebs 2010, Liebs 2012, Mikkelsen 2014, Monticone 2014, Nelson 2020, Rao 2021, Smith 2009) reported data on patient-reported physical function and ADLs, using various outcome measures (Table 82) at follow-ups between 6 months and 5 years after THA surgery. Eight studies found no difference between groups in terms of patient-reported function and ADL; four studies (Giaquinto 2010, Heiberg 2012, Liebs 2010, Monticone 2014) reported significant differences between groups. Giaquinto 2010 evaluated physical function data using the function component of the WOMAC (0-68, lower is better) and found that patients randomized to hydrotherapybased rehabilitation reported significantly greater improvements compared with patients randomized to no hydrotherapy (median difference $-14 ; \mathrm{P}<0.01$ ). Heiberg 2012 reported activity using the ADL and sports and recreation scales of the HOOS and the UCLA activity scale and found significant improvements on the UCLA activity score (1-10, higher is better) among patients randomized to walking skill program compared with patients randomized to standard care at 5 years after THA, but no differences between groups on the other scales. (Note that the study reported no between group differences; however, our calculated effect size suggested results were significant: MD 1, 95\% CI 0.18 to 1.82.) Liebs 2010 reported physical function using the physical component scales of the SF-36 (0 to 100, higher is better) and WOMAC (0 to 100 , lower is better) and found significant improvements on both scales among patients randomized to ergometer cycling compared with control (SF-36: MD 4.5, 95\% CI 1.56 to 7.44; WOMAC: MD $-5.7,95 \%$ CI -10.4 to -1.0$)$. Monticone 2014 reported function data using the Functional Independence Measure, the physical role and physical functioning components of the SF-36, and the function component of the WOMAC and found significant improvements among patients randomized to task-oriented exercises on all measures at 12 months after THA compared with patients randomized to open chain kinetic exercises.

\section{Transfers}

Two studies (Heiberg 2012 and Mikkelsen 2014) reported data on transfers using the 30second timed Chair stand test and observed no differences between groups at 6 months (Mikkelsen 2014) and 5-year (Heiberg 2012) follow-up after THA (Table 83)

\section{Mobility}

Four studies (Heiberg 2012, Mikkelsen 2014, Nelson 2020, Winther 2020) reported various outcome measures of mobility including the 6MWT, 40-meter fast paced walk test, stair climb 
tests, step test, figure eight test, speed in a 5-meter walkway, and step length. Studies reported no differences between groups at follow-up times ranging from 6 months to 5 years after THA (Table 84).

\section{Timed Up and Go}

Two studies (Coulter 2017 and Nelson 2020) reported data on the TUG test. Coulter 2017 reported no significant differences between groups. Nelson 2020 reported patients randomized to telerehabilitation performed the TUG test significantly quicker than patients randomized to inperson usual care (MD $-1.5,95 \% \mathrm{CI}-2.4$ to -0.6 ) (Table 85 ) 
Table 82. Rehabilitation versus various controls for total hip arthroplasty - continuous outcomes, physical function and activities of daily living

\begin{tabular}{|c|c|c|c|c|c|c|c|c|c|c|c|}
\hline $\begin{array}{l}\text { Study, Year, } \\
\text { PMID, } \\
\text { Country }\end{array}$ & Arm 1 & Arm 2 & $\begin{array}{l}\text { Overall } \\
\text { RoB }\end{array}$ & $\begin{array}{l}\text { Outcome } \\
\text { Measurement }\end{array}$ & $\begin{array}{l}\text { Time } \\
\text { Point }^{A}\end{array}$ & $\begin{array}{l}\text { Arm } \\
1, N\end{array}$ & $\begin{array}{l}\text { Arm 1, Mean } \\
\text { (SD) }\end{array}$ & $\begin{array}{l}\text { Arm 2, } \\
\text { N }\end{array}$ & $\begin{array}{l}\text { Arm 2, Mean } \\
\text { (SD) }\end{array}$ & $\begin{array}{l}\text { Effect Size } \\
(95 \% \mathrm{Cl})\end{array}$ & $\begin{array}{l}\text { Reported } \\
\text { p-Value }\end{array}$ \\
\hline $\begin{array}{l}\text { Austin, 2017, } \\
28419032\end{array}$ & $\begin{array}{l}\text { Home } \\
\text { exercise }\end{array}$ & $\begin{array}{l}\text { Formal physical } \\
\text { therapy }\end{array}$ & Moderate & SF-36: PCS (0-100) & $6-12 \mathrm{mo}$ & 52 & NR & 52 & NR & NR & 0.90 \\
\hline \multirow[t]{4}{*}{$\begin{array}{l}\text { Beck, 2019, } \\
30782304\end{array}$} & Sports therapy & Control & High & $\begin{array}{l}\text { WOMAC: ADL (0- } \\
100)\end{array}$ & $6 \mathrm{mo}$ & 63 & $\begin{array}{l}\text { Median (Q1, Q3) } \\
92.7(85.2,95.6)\end{array}$ & 51 & $\begin{array}{l}\text { Median (Q1, Q3) } \\
92.6(89.7,95.6)\end{array}$ & $\begin{array}{l}\text { Median difference } \\
0.03\end{array}$ & 1.00 \\
\hline & Sports therapy & Control & High & $\begin{array}{l}\text { WOMAC: ADL (0- } \\
100)\end{array}$ & $12 \mathrm{mo}$ & 57 & $\begin{array}{l}\text { Median (Q1, Q3) } \\
95.6(89.7,100)\end{array}$ & 41 & $\begin{array}{l}\text { Median (Q1, Q3) } \\
95.6(92.7,97.1)\end{array}$ & $\begin{array}{l}\text { Median difference } \\
0.11\end{array}$ & 1.00 \\
\hline & Sports therapy & Control & High & $\begin{array}{l}\text { UCLA activity scale } \\
(1-10)\end{array}$ & $6 \mathrm{mo}$ & 63 & $\begin{array}{l}\text { Median (Q1, Q3) } \\
7(6,7)\end{array}$ & 51 & $\begin{array}{l}\text { Median (Q1, Q3) } \\
7(6,7)\end{array}$ & $\begin{array}{l}\text { Median difference } \\
0.14\end{array}$ & 1.00 \\
\hline & Sports therapy & Control & High & $\begin{array}{l}\text { UCLA activity scale } \\
(1-10)\end{array}$ & $12 \mathrm{mo}$ & 57 & $\begin{array}{l}\text { Median (Q1, Q3) } \\
7(7,7)\end{array}$ & 41 & $\begin{array}{l}\text { Median (Q1, Q3) } \\
7(6,7)\end{array}$ & $\begin{array}{l}\text { Median difference } \\
0.18\end{array}$ & 0.378 \\
\hline $\begin{array}{l}\text { Coulter, 2017, } \\
28506775\end{array}$ & $\begin{array}{l}\text { Unsupervised } \\
\text { home-based } \\
\text { exercises }\end{array}$ & $\begin{array}{l}\text { Supervised } \\
\text { rehabilitation \& } \\
\text { unsupervised } \\
\text { home-based } \\
\text { exercises }\end{array}$ & High & SF-36: PCS (0-100) & $26 w$ & 56 & $\begin{array}{l}\text { Mean }(95 \% \mathrm{Cl}) \\
71.40(63.76 \\
79.03) \text { SE } 3.90^{\star}\end{array}$ & 42 & $\begin{array}{l}\text { Mean }(95 \% \mathrm{Cl}) \\
68.50(60.05 \\
77.02) \text { SE } 4.33^{*}\end{array}$ & $\begin{array}{l}\text { MD 2.9 (-8.52, } \\
14.32)^{\mathrm{B}}\end{array}$ & ns \\
\hline $\begin{array}{l}\text { Giaquinto, } \\
2010, \\
19282040\end{array}$ & Hydrotherapy & $\begin{array}{l}\text { No } \\
\text { hydrotherapy }\end{array}$ & Low & $\begin{array}{l}\text { WOMAC: function (0- } \\
68 \text { ) }\end{array}$ & $6 \mathrm{mo}$ & 31 & Median 4 & 33 & Median 18 & NR & $<0.01$ \\
\hline \multirow[t]{5}{*}{$\begin{array}{l}\text { Heiberg, } \\
2012, \\
22170790\end{array}$} & $\begin{array}{l}\text { Walking skill } \\
\text { training } \\
\text { program }\end{array}$ & Standard care & Moderate & HOOS: ADL (0-100) & $12 \mathrm{mo}$ & 35 & $\begin{array}{l}\text { Mean }(95 \% \mathrm{Cl}) \\
92(90,95)\end{array}$ & 33 & $\begin{array}{l}\text { Mean }(95 \% \mathrm{Cl}) \\
91(88,94)\end{array}$ & $1(-3,5)$ & ns \\
\hline & $\begin{array}{l}\text { Walking skill } \\
\text { training } \\
\text { program }\end{array}$ & Standard care & Moderate & HOOS: ADL (0-100) & $5 y$ & 30 & $\begin{array}{l}\text { Mean }(95 \% \mathrm{Cl}) \\
90(87,94) \\
\text { SE } 1.79^{*}\end{array}$ & 30 & $\begin{array}{l}\text { Mean }(95 \% \mathrm{Cl}) \\
93(89,97) \\
\text { SE } 2.04^{*}\end{array}$ & $\begin{array}{l}\text { MD -3 (-8.32, } \\
2.32)^{\mathrm{B}}\end{array}$ & NR \\
\hline & $\begin{array}{l}\text { Walking skill } \\
\text { training } \\
\text { program }\end{array}$ & Standard care & Moderate & $\begin{array}{l}\text { HOOS: sport/rec (0- } \\
\text { 100) }\end{array}$ & $12 \mathrm{mo}$ & 35 & $\begin{array}{l}\text { Mean }(95 \% \mathrm{Cl}) \\
79(73,86)\end{array}$ & 33 & $\begin{array}{l}\text { Mean }(95 \% \mathrm{Cl}) \\
78(72,84)\end{array}$ & $1(-8,10)$ & ns \\
\hline & $\begin{array}{l}\text { Walking skill } \\
\text { training } \\
\text { program }\end{array}$ & Standard care & Moderate & $\begin{array}{l}\text { HOOS: sport/rec (0- } \\
100)\end{array}$ & $5 y$ & 30 & $\begin{array}{l}\text { Mean }(95 \% \mathrm{Cl}) \\
75(69,82) \\
\text { SE } 3.32\end{array}$ & 30 & $\begin{array}{l}\text { Mean }(95 \% \mathrm{Cl}) \\
82(75,90) \\
\text { SE } 3.83\end{array}$ & $\begin{array}{l}\text { MD -7 (-16.94, } \\
2.94)^{B}\end{array}$ & NR \\
\hline & $\begin{array}{l}\text { Walking skill } \\
\text { training } \\
\text { program }\end{array}$ & Standard care & Moderate & $\begin{array}{l}\text { UCLA activity scale } \\
(1-10)\end{array}$ & $5 y$ & 30 & $8(1.3)$ & 30 & $7(1.9)$ & MD $1(0.18,1.82)^{B}$ & ns \\
\hline \multirow[t]{2}{*}{$\begin{array}{l}\text { Liebs, 2010, } \\
20360503\end{array}$} & $\begin{array}{l}\text { Ergometer } \\
\text { cycling }\end{array}$ & Control & Moderate & SF-36: PCS (0-100) & $24 \mathrm{mo}$ & 74 & $48.9(9)$ & 88 & $44.4(10.1)$ & $4.5(1.56,7.44)^{B}$ & 0.004 \\
\hline & $\begin{array}{l}\text { Ergometer } \\
\text { cycling }\end{array}$ & Control & Moderate & $\begin{array}{l}\text { WOMAC: function (0- } \\
100)\end{array}$ & $24 \mathrm{mo}$ & 74 & $9.0(13.9)$ & 88 & $14.7(16.7)$ & $\begin{array}{l}-5.7(-10.41,- \\
0.99)^{\mathrm{B}}\end{array}$ & 0.019 \\
\hline $\begin{array}{l}\text { Liebs, 2012, } \\
22196125\end{array}$ & $\begin{array}{l}\text { Early aquatic } \\
\text { therapy }\end{array}$ & $\begin{array}{l}\text { Late Aquatic } \\
\text { therapy (after } \\
\text { wound healing }\end{array}$ & Moderate & SF-36: PCS (0-100) & $24 \mathrm{mo}$ & 100 & $45.1(11.1)$ & 110 & $45.2(9.7)$ & $-0.1(-2.93,2.73)^{\mathrm{B}}$ & 0.808 \\
\hline
\end{tabular}




\begin{tabular}{|c|c|c|c|c|c|c|c|c|c|c|c|}
\hline $\begin{array}{l}\text { Study, Year, } \\
\text { PMID, } \\
\text { Country }\end{array}$ & Arm 1 & Arm 2 & $\begin{array}{l}\text { Overall } \\
\text { RoB }\end{array}$ & $\begin{array}{l}\text { Outcome } \\
\text { Measurement }\end{array}$ & $\begin{array}{l}\text { Time } \\
\text { Point }\end{array}$ & $\begin{array}{l}\text { Arm } \\
1, N\end{array}$ & $\begin{array}{l}\text { Arm 1, Mean } \\
\text { (SD) }\end{array}$ & $\begin{array}{l}\text { Arm 2, } \\
\text { N }\end{array}$ & $\begin{array}{l}\text { Arm 2, Mean } \\
\text { (SD) }\end{array}$ & $\begin{array}{l}\text { Effect Size } \\
(95 \% \mathrm{Cl})\end{array}$ & $\begin{array}{l}\text { Reported } \\
\text { p-Value }\end{array}$ \\
\hline & $\begin{array}{l}\text { Early aquatic } \\
\text { therapy }\end{array}$ & $\begin{array}{l}\text { Late Aquatic } \\
\text { therapy (after } \\
\text { wound healing }\end{array}$ & Moderate & $\begin{array}{l}\text { WOMAC: function (0- } \\
100)\end{array}$ & $24 \mathrm{mo}$ & 100 & $15.6(18.1)$ & 110 & $14.1(14.5)$ & $\begin{array}{l}\text { MD 1.5 (-2.96, } \\
5.96)^{\mathrm{B}}\end{array}$ & 0.825 \\
\hline \multirow[t]{4}{*}{$\begin{array}{l}\text { Mikkelsen, } \\
2014, \\
25305374\end{array}$} & $\begin{array}{l}\text { Early } \\
\text { supervised } \\
\text { progressive } \\
\text { resistance } \\
\text { training }\end{array}$ & $\begin{array}{l}\text { Unsupervised } \\
\text { home-based } \\
\text { exercise }\end{array}$ & Moderate & HOOS: ADL (0-100) & $6 \mathrm{mo}$ & 32 & $90.4(11)$ & 30 & $91.7(10)$ & $-1.3(-5,2.4)^{\mathrm{B}}$ & NR \\
\hline & $\begin{array}{l}\text { Early } \\
\text { supervised } \\
\text { progressive } \\
\text { resistance } \\
\text { training } \\
\end{array}$ & $\begin{array}{l}\text { Unsupervised } \\
\text { home-based } \\
\text { exercise }\end{array}$ & Moderate & HOOS: ADL (0-100) & $12 \mathrm{mo}$ & 32 & $93.4(8)$ & 30 & $92.1(12)$ & $1.3(-2.5,5.1)^{B}$ & NR \\
\hline & $\begin{array}{l}\text { Early } \\
\text { supervised } \\
\text { progressive } \\
\text { resistance } \\
\text { training }\end{array}$ & $\begin{array}{l}\text { Unsupervised } \\
\text { home-based } \\
\text { exercise }\end{array}$ & Moderate & $\begin{array}{l}\text { HOOS: sport/rec (0- } \\
100)\end{array}$ & $6 \mathrm{mo}$ & 32 & $80.1(17)$ & 30 & $83.7(17)$ & $-3.6(-9.6,2.4)^{B}$ & NR \\
\hline & $\begin{array}{l}\text { Early } \\
\text { supervised } \\
\text { progressive } \\
\text { resistance } \\
\text { training }\end{array}$ & $\begin{array}{l}\text { Unsupervised } \\
\text { home-based } \\
\text { exercise }\end{array}$ & Moderate & $\begin{array}{l}\text { HOOS: sport/rec (0- } \\
\text { 100) }\end{array}$ & $12 \mathrm{mo}$ & 32 & $81.9(20)$ & 30 & $92.8(19)$ & $-10.9(-17.8,-4.0)^{\mathrm{B}}$ & NR \\
\hline \multirow[t]{4}{*}{$\begin{array}{l}\text { Monticone, } \\
2014, \\
24459172\end{array}$} & $\begin{array}{l}\text { Task-oriented } \\
\text { exercises }\end{array}$ & $\begin{array}{l}\text { Open chain } \\
\text { kinetic exercise }\end{array}$ & Moderate & $\begin{array}{l}\text { Functional } \\
\text { independence } \\
\text { measure }(18-126)\end{array}$ & $12 \mathrm{mo}$ & 50 & $117.9(10.3)$ & 50 & $104.7(14)$ & $13.2(9.7,16.7)^{B}$ & NR \\
\hline & $\begin{array}{l}\text { Task-oriented } \\
\text { exercises }\end{array}$ & $\begin{array}{l}\text { Open chain } \\
\text { kinetic exercise }\end{array}$ & Moderate & $\begin{array}{l}\text { SF-36: physical } \\
\text { functioning }(0-100)\end{array}$ & $12 \mathrm{mo}$ & 50 & $73.1(20.8)$ & 50 & $60.9(18.3)$ & $12.2(6.7,17.7)^{\mathrm{B}}$ & NR \\
\hline & $\begin{array}{l}\text { Task-oriented } \\
\text { exercises }\end{array}$ & $\begin{array}{l}\text { Open chain } \\
\text { kinetic exercise }\end{array}$ & Moderate & $\begin{array}{l}\text { SF-36: physical role } \\
(0-100)\end{array}$ & $12 \mathrm{mo}$ & 50 & $76.1(33.3)$ & 50 & $51.1(45.1)$ & $25(13.8,36.2)^{B}$ & NR \\
\hline & $\begin{array}{l}\text { Task-oriented } \\
\text { exercises }\end{array}$ & $\begin{array}{l}\text { Open chain } \\
\text { kinetic exercise }\end{array}$ & Moderate & $\begin{array}{l}\text { WOMAC: function (0- } \\
100)\end{array}$ & $12 \mathrm{mo}$ & 50 & $20.0(11.1)$ & 50 & $30.6(14.9)$ & $-10.6(-14.3,-6.9)^{B}$ & NR \\
\hline \multirow[t]{3}{*}{$\begin{array}{l}\text { Nelson, 2020, } \\
32026820\end{array}$} & $\begin{array}{l}\text { Telerehabilitati } \\
\text { on }\end{array}$ & $\begin{array}{l}\text { In-person usual } \\
\text { care }\end{array}$ & Moderate & HOOS: ADL (0-100) & $6 \mathrm{mo}$ & 34 & $91(10)$ & 35 & $88(11)$ & $3(-0.5,6.5)^{\mathrm{B}}$ & NR \\
\hline & $\begin{array}{l}\text { Telerehabilitati } \\
\text { on }\end{array}$ & $\begin{array}{l}\text { In-person usual } \\
\text { care }\end{array}$ & Moderate & $\begin{array}{l}\text { HOOS: sport/rec (0- } \\
100)\end{array}$ & $6 \mathrm{mo}$ & 34 & $85(18)$ & 35 & $80(19)$ & $5(-1.2,11.2)^{B}$ & NR \\
\hline & $\begin{array}{l}\text { Telerehabilitati } \\
\text { on }\end{array}$ & $\begin{array}{l}\text { In-person usual } \\
\text { care }\end{array}$ & Moderate & SF-12: PCS (0-100) & $6 \mathrm{mo}$ & 34 & $47(10)$ & 35 & $43(10)$ & $4(0.7,7.3)^{B}$ & NR \\
\hline \multirow{2}{*}{$\begin{array}{l}\text { Rao, } \\
2021, \\
33863614 \\
\text { USA }\end{array}$} & $\begin{array}{l}\text { Home } \\
\text { physical } \\
\text { therapy }\end{array}$ & $\begin{array}{l}\text { Formal physical } \\
\text { therapy }\end{array}$ & High & HOOS: ADL (0-100) & $6 \mathrm{mo}$ & 70 & $89.0(14.5)$ & 66 & $87.3(15.6)$ & $-4.0(-7.7,-0.3)$ & 0.517 \\
\hline & $\begin{array}{l}\text { Home } \\
\text { physical } \\
\text { therapy }\end{array}$ & $\begin{array}{l}\text { Formal physical } \\
\text { therapy }\end{array}$ & High & $\begin{array}{l}\text { HOOS: sport/rec (0- } \\
100)\end{array}$ & $6 \mathrm{mo}$ & 70 & $80.3(20.6)$ & 66 & $74.8(24.3)$ & $-5.5(-10.9,-0.1)$ & 0.167 \\
\hline
\end{tabular}




\begin{tabular}{|c|c|c|c|c|c|c|c|c|c|c|c|}
\hline $\begin{array}{l}\text { Study, Year, } \\
\text { PMID, } \\
\text { Country }\end{array}$ & Arm 1 & Arm 2 & $\begin{array}{l}\text { Overall } \\
\text { RoB }\end{array}$ & $\begin{array}{l}\text { Outcome } \\
\text { Measurement }\end{array}$ & $\begin{array}{l}\text { Time } \\
\text { Point }\end{array}$ & $\begin{array}{l}\text { Arm } \\
1, N\end{array}$ & $\begin{array}{l}\text { Arm 1, Mean } \\
\text { (SD) }\end{array}$ & $\begin{array}{l}\text { Arm 2, } \\
\mathrm{N}\end{array}$ & $\begin{array}{l}\text { Arm 2, Mean } \\
\text { (SD) }\end{array}$ & $\begin{array}{l}\text { Effect Size } \\
(95 \% \mathrm{Cl})\end{array}$ & $\begin{array}{l}\text { Reported } \\
\text { p-Value }\end{array}$ \\
\hline \multirow[t]{5}{*}{$\begin{array}{l}\text { Smith, 2009, } \\
19876883\end{array}$} & $\begin{array}{l}\text { Gait re- } \\
\text { education } \\
\text { program \& } \\
\text { bed exercises }\end{array}$ & $\begin{array}{l}\text { Gait re- } \\
\text { education } \\
\text { program only }\end{array}$ & Moderate & $\begin{array}{l}\text { lowa level of } \\
\text { assistance scale }\end{array}$ & $12 \mathrm{mo}$ & 30 & $\begin{array}{l}\text { Median (Q1, Q3) } \\
0(0,0)\end{array}$ & 30 & $\begin{array}{l}\text { Median (Q1, Q3) } \\
0(0,3)\end{array}$ & NR & 0.2093 \\
\hline & $\begin{array}{l}\text { Gait re- } \\
\text { education } \\
\text { program \& } \\
\text { bed exercises }\end{array}$ & $\begin{array}{l}\text { Gait re- } \\
\text { education } \\
\text { program only }\end{array}$ & Moderate & $\begin{array}{l}\text { lowa level of } \\
\text { assistance scale }\end{array}$ & $12 \mathrm{mo}^{\mathrm{C}}$ & 23 & $\begin{array}{l}\text { Median (Q1, Q3) } \\
0(0,2)\end{array}$ & 28 & $\begin{array}{l}\text { Median (Q1, Q3) } \\
0(0,3.5)\end{array}$ & NR & 0.4296 \\
\hline & $\begin{array}{l}\text { Gait re- } \\
\text { education } \\
\text { program \& } \\
\text { bed exercises }\end{array}$ & $\begin{array}{l}\text { Gait re- } \\
\text { education } \\
\text { program only }\end{array}$ & Moderate & $\begin{array}{l}\text { lowa level of } \\
\text { assistance scale }\end{array}$ & $12 \mathrm{mo}^{\mathrm{D}}$ & 7 & $\begin{array}{l}\text { Median (Q1, Q3) } \\
0(0,0)\end{array}$ & 2 & $\begin{array}{l}\text { Median (Q1, Q3) } \\
0(0,0)\end{array}$ & NR & 1.000 \\
\hline & $\begin{array}{l}\text { Gait re- } \\
\text { education } \\
\text { program \& } \\
\text { bed exercises }\end{array}$ & $\begin{array}{l}\text { Gait re- } \\
\text { education } \\
\text { program only }\end{array}$ & Moderate & $\begin{array}{l}\text { lowa level of } \\
\text { assistance scale }\end{array}$ & $12 \mathrm{mo}^{\mathrm{E}}$ & 22 & $\begin{array}{l}\text { Median (Q1, Q3) } \\
0(0,0)\end{array}$ & 24 & $\begin{array}{l}\text { Median (Q1, Q3) } \\
0(0,3.5)\end{array}$ & NR & 0.3170 \\
\hline & $\begin{array}{l}\text { Gait re- } \\
\text { education } \\
\text { program \& } \\
\text { bed exercises }\end{array}$ & $\begin{array}{l}\text { Gait re- } \\
\text { education } \\
\text { program only }\end{array}$ & Moderate & $\begin{array}{l}\text { lowa level of } \\
\text { assistance scale }\end{array}$ & $12 \mathrm{mo}^{\mathrm{F}}$ & 8 & $\begin{array}{l}\text { Median (Q1, Q3) } \\
0(0,0)\end{array}$ & 6 & $\begin{array}{l}\text { Median (Q1, Q3) } \\
0(0,0)\end{array}$ & NR & 0.9156 \\
\hline
\end{tabular}

Statistically significant effect sizes are in bold text. In cases where calculated effect size confidence intervals were not-statistically significant but reported p-values were, we deferred to reported p-values and still bolded results.

Abbreviations: $\mathrm{CI}=$ confidence interval, $\mathrm{HOOS}=$ Hip disability and osteoarthritis outcome score, mo $=$ month, $\mathrm{NR}=$ not reported, $\mathrm{PCS}=$ physical component scale, $\mathrm{PMID}=$ PubMed identifier, $\mathrm{rec}=$ recreation, $\mathrm{RoB}=$ risk of bias, $\mathrm{SD}=$ standard deviation, SF-36 = 36-Item short form survey, WOMAC $=$ Western Ontario and McMaster Universities Osteoarthritis Index.

A Time from surgery

B Calculated

C Subgroup: Cemented

D Subgroup: Uncemented

E Subgroup: Posterior

F Subgroup: Anterolateral 
Table 83. Rehabilitation versus various controls for total hip arthroplasty - continuous outcomes, transfers

\begin{tabular}{|c|c|c|c|c|c|c|c|c|c|c|c|}
\hline $\begin{array}{l}\text { Study, Year, } \\
\text { PMID, } \\
\text { Country }\end{array}$ & Arm 1 & Arm 2 & $\begin{array}{l}\text { Overall } \\
\text { RoB }\end{array}$ & $\begin{array}{l}\text { Outcome } \\
\text { Measurement }\end{array}$ & $\begin{array}{l}\text { Time } \\
\text { Point }^{\mathrm{A}}\end{array}$ & $\begin{array}{l}\text { Arm 1, } \\
\mathrm{N}\end{array}$ & $\begin{array}{l}\text { Arm 1, } \\
\text { Mean (SD) }\end{array}$ & $\begin{array}{l}\text { Arm 2, } \\
\mathrm{N}\end{array}$ & $\begin{array}{l}\text { Arm 2, Mean } \\
\text { (SD) }\end{array}$ & $\begin{array}{l}\text { Effect Size } \\
(95 \% \mathrm{Cl})\end{array}$ & $\begin{array}{l}\text { Reported } \\
\text { p-Value }\end{array}$ \\
\hline $\begin{array}{l}\text { Heiberg, } \\
2012, \\
22170790 \\
\end{array}$ & $\begin{array}{l}\text { Walking skill training } \\
\text { program }\end{array}$ & Standard care & Moderate & 30s Chair stand test & $5 y$ & 30 & $12(3.7)$ & 30 & $12(4.6)$ & $0(-2.11,2.11)^{B}$ & ns \\
\hline $\begin{array}{l}\text { Mikkelsen, } \\
2014, \\
25305374\end{array}$ & $\begin{array}{l}\text { Early supervised } \\
\text { progressive } \\
\text { resistance training }\end{array}$ & $\begin{array}{l}\text { Unsupervised } \\
\text { home-based } \\
\text { exercise }\end{array}$ & Moderate & 30s Chair stand test & $6 \mathrm{mo}$ & 32 & $15.47(4.5)$ & 30 & $15.07(5.1)$ & $0.4(-1.3,2.1)^{B}$ & NR \\
\hline
\end{tabular}

Statistically significant effect sizes are in bold text. In cases where calculated effect size confidence intervals were not-statistically significant but reported p-values were, we deferred to reported p-values and still bolded results.

Abbreviations: $\mathrm{CI}=$ confidence interval, $\mathrm{mo}=$ month, $\mathrm{NR}=$ not reported, $\mathrm{ns}=$ not significant, $\mathrm{PMID}=\mathrm{PubMed}$ identifier, $\mathrm{RoB}=$ risk of bias, $\mathrm{s}=$ second, $\mathrm{SD}=$ standard deviation .

A Time from surgery

${ }^{\mathrm{B}}$ Calculated

Table 84. Rehabilitation versus various controls for total hip arthroplasty - continuous outcomes, mobility

\begin{tabular}{|c|c|c|c|c|c|c|c|c|c|c|c|}
\hline $\begin{array}{l}\text { Study, Year, } \\
\text { PMID, } \\
\text { Country }\end{array}$ & Arm 1 & Arm 2 & $\begin{array}{l}\text { Overall } \\
\text { RoB }\end{array}$ & $\begin{array}{l}\text { Outcome } \\
\text { Measurement }\end{array}$ & $\begin{array}{l}\text { Time } \\
\text { Point }\end{array}$ & $\begin{array}{l}\text { Arm 1, } \\
\mathrm{N}\end{array}$ & $\begin{array}{l}\text { Arm 1, Mean } \\
\text { (SD) }\end{array}$ & $\begin{array}{l}\text { Arm 2, } \\
\text { N }\end{array}$ & $\begin{array}{l}\text { Arm 2, Mean } \\
\text { (SD) }\end{array}$ & $\begin{array}{l}\text { Effect Size } \\
(95 \% \mathrm{Cl})\end{array}$ & $\begin{array}{l}\text { Reported } \\
\text { p-Value }\end{array}$ \\
\hline \multirow{5}{*}{$\begin{array}{l}\text { Heiberg, } \\
2012, \\
22170790\end{array}$} & $\begin{array}{l}\text { Walking skill } \\
\text { training program }\end{array}$ & $\begin{array}{l}\text { Standard } \\
\text { care }\end{array}$ & Moderate & 6MWT & $12 \mathrm{mo}$ & 35 & $\begin{array}{l}535 ; 95 \% \mathrm{Cl} \\
(516,555)\end{array}$ & 33 & $\begin{array}{l}483 ; 95 \% \mathrm{Cl} \\
(463,503)\end{array}$ & $52(24,80)$ & $<0.001$ \\
\hline & $\begin{array}{l}\text { Walking skill } \\
\text { training program }\end{array}$ & $\begin{array}{l}\text { Standard } \\
\text { care }\end{array}$ & Moderate & 6MWT & $5 y$ & 30 & $\begin{array}{l}524 ; 95 \% \mathrm{Cl} \\
(483,564)\end{array}$ & 30 & $\begin{array}{l}53095 \% \mathrm{Cl} \\
(487,573)\end{array}$ & $\begin{array}{l}-6.33(-186.67 \\
174.00)^{\mathrm{B}}\end{array}$ & NR \\
\hline & $\begin{array}{l}\text { Walking skill } \\
\text { training program }\end{array}$ & $\begin{array}{l}\text { Standard } \\
\text { care }\end{array}$ & Moderate & Stair climb test & $12 \mathrm{mo}$ & 35 & $\begin{array}{l}10 ; 95 \% \mathrm{Cl} \\
(9,11)\end{array}$ & 33 & $\begin{array}{l}12 ; 95 \% \mathrm{Cl} \\
(11,13)\end{array}$ & $-1(-3,0)$ & ns \\
\hline & $\begin{array}{l}\text { Walking skill } \\
\text { training program }\end{array}$ & $\begin{array}{l}\text { Standard } \\
\text { care }\end{array}$ & Moderate & Stair climb test & $5 y$ & 30 & $\begin{array}{l}13 ; 95 \% \mathrm{Cl} \\
(11,15)\end{array}$ & 30 & $\begin{array}{l}13 ; 95 \% \mathrm{Cl} \\
(11,15)\end{array}$ & $0(-2.83,2.83)^{\mathrm{B}}$ & NR \\
\hline & $\begin{array}{l}\text { Walking skill } \\
\text { training program }\end{array}$ & $\begin{array}{l}\text { Standard } \\
\text { care }\end{array}$ & Moderate & Figure eight test & $12 \mathrm{mo}$ & 35 & $\begin{array}{l}7 ; 95 \% \mathrm{Cl}(5, \\
8)\end{array}$ & 33 & $\begin{array}{l}895 \% \mathrm{Cl}(7, \\
10)\end{array}$ & $-1(-3,1)$ & ns \\
\hline $\begin{array}{l}\text { Mikkelsen, } \\
2014, \\
25305374\end{array}$ & $\begin{array}{l}\text { Early supervised } \\
\text { progressive } \\
\text { resistance training }\end{array}$ & $\begin{array}{l}\text { Unsupervised } \\
\text { home-based } \\
\text { exercise }\end{array}$ & Moderate & $\begin{array}{l}\text { 40-m-fast-paced walk } \\
\text { test }\end{array}$ & $6 \mathrm{mo}$ & 32 & $10.81(2.8)$ & 30 & $11.02(2.6)$ & $-0.2(-1.2,0.7)^{\mathrm{B}}$ & NR \\
\hline \multirow[t]{2}{*}{$\begin{array}{l}\text { Nelson, 2020, } \\
32026820\end{array}$} & Telerehabilitation & $\begin{array}{l}\text { In-person } \\
\text { usual care }\end{array}$ & Moderate & Step test & $6 \mathrm{mo}$ & 34 & $15.2(4)$ & 35 & $13.6(4.6)$ & $1.6(0.2,3.0)^{\mathrm{B}}$ & NR \\
\hline & Telerehabilitation & $\begin{array}{l}\text { In-person } \\
\text { usual care }\end{array}$ & Moderate & Stair climb test & $6 \mathrm{mo}$ & 32 & $9.07(3.0)$ & 30 & $9.03(2.8)$ & $0.04(-1,1.1)^{\mathrm{B}}$ & NR \\
\hline \multirow[t]{3}{*}{$\begin{array}{l}\text { Winther, 2020, } \\
31977324\end{array}$} & $\begin{array}{l}\text { Strengthening } \\
\text { exercise }\end{array}$ & $\begin{array}{l}\text { Standard } \\
\text { care }\end{array}$ & Moderate & 6MWT & $12 \mathrm{mo}$ & 22 & $627(96)$ & 22 & $628(110)$ & $\begin{array}{l}-1(-44.3 \\
42.3)^{\mathrm{B}} \\
\end{array}$ & NR \\
\hline & $\begin{array}{l}\text { Strengthening } \\
\text { exercise }\end{array}$ & $\begin{array}{l}\text { Standard } \\
\text { care }\end{array}$ & Moderate & $\begin{array}{l}\text { Speed in the 5-m } \\
\text { walkway }\end{array}$ & $6 \mathrm{mo}$ & 26 & 1.33 (NR) & 21 & 1.38 (NR) & NR & NR \\
\hline & $\begin{array}{l}\text { Strengthening } \\
\text { exercise }\end{array}$ & $\begin{array}{l}\text { Standard } \\
\text { care }\end{array}$ & Moderate & $\begin{array}{l}\text { Speed in the 5-m } \\
\text { walkway }\end{array}$ & $12 \mathrm{mo}$ & 22 & 1.38 (NR) & 22 & 1.42 (NR) & NR & NR \\
\hline
\end{tabular}




\begin{tabular}{|c|c|c|c|c|c|c|c|c|c|c|c|}
\hline $\begin{array}{l}\text { Study, Year, } \\
\text { PMID, } \\
\text { Country }\end{array}$ & Arm 1 & Arm 2 & $\begin{array}{l}\text { Overall } \\
\text { RoB }\end{array}$ & $\begin{array}{l}\text { Outcome } \\
\text { Measurement }\end{array}$ & $\begin{array}{l}\text { Time } \\
\text { Point }\end{array}$ & $\begin{array}{l}\text { Arm 1, } \\
\text { N }\end{array}$ & $\begin{array}{l}\text { Arm 1, Mean } \\
\text { (SD) }\end{array}$ & $\begin{array}{l}\text { Arm 2, } \\
\text { N }\end{array}$ & $\begin{array}{l}\text { Arm 2, Mean } \\
\text { (SD) }\end{array}$ & $\begin{array}{l}\text { Effect Size } \\
(95 \% \mathrm{Cl})\end{array}$ & $\begin{array}{l}\text { Reported } \\
\text { p-Value }\end{array}$ \\
\hline & $\begin{array}{l}\text { Strengthening } \\
\text { exercise }\end{array}$ & Standard care & Moderate & Step length & $6 \mathrm{mo}$ & 21 & 69.7 (NR) & 21 & 71.4 (NR) & NR & NR \\
\hline & $\begin{array}{l}\text { Strengthening } \\
\text { exercise }\end{array}$ & Standard care & Moderate & Step length & $12 \mathrm{mo}$ & 22 & 71.6 (NR) & 22 & 72.5 (NR) & NR & NR \\
\hline
\end{tabular}

Statistically significant effect sizes are in bold text. In cases where calculated effect size confidence intervals were not-statistically significant but reported p-values were, we deferred to reported p-values and still bolded results.

Abbreviations: $6 \mathrm{MWT}=$ six-minute walk test, $\mathrm{CI}=$ confidence interval, $\mathrm{m}=$ meter, $\mathrm{mo}=$ month, $\mathrm{m}=$ meter, $\mathrm{NR}=$ not reported, $\mathrm{ns}=$ not significant, $\mathrm{PMID}=\mathrm{PubMed}$ identifier, $\mathrm{RoB}=$ risk of bias, $\mathrm{SD}=$ standard deviation, $\mathrm{y}=$ year.

A Time from surgery

${ }^{B}$ Calculated

Table 85. Rehabilitation versus various controls for total hip arthroplasty - continuous outcomes, Timed Up and Go

\begin{tabular}{|c|c|c|c|c|c|c|c|c|c|c|c|}
\hline $\begin{array}{l}\text { Study, Year, } \\
\text { PMID, } \\
\text { Country }\end{array}$ & Arm 1 & Arm 2 & $\begin{array}{l}\text { Outcome } \\
\text { Measurement }\end{array}$ & $\begin{array}{l}\text { Overall } \\
\text { RoB }\end{array}$ & $\begin{array}{l}\text { Time } \\
\text { Point }\end{array}$ & $\begin{array}{l}\text { Arm } \\
1, N\end{array}$ & $\begin{array}{l}\text { Arm 1, } \\
\text { Mean (SD) }\end{array}$ & $\begin{array}{l}\text { Arm 2, } \\
\text { N }\end{array}$ & $\begin{array}{l}\text { Arm 2, Mean } \\
\text { (SD) }\end{array}$ & $\begin{array}{l}\text { Effect Size } \\
(95 \% \mathrm{Cl})\end{array}$ & $\begin{array}{l}\text { Reported } \\
\text { p-Value }\end{array}$ \\
\hline $\begin{array}{l}\text { Coulter, 2017, } \\
28506775\end{array}$ & $\begin{array}{l}\text { Unsupervised } \\
\text { home-based } \\
\text { exercises }\end{array}$ & $\begin{array}{l}\text { Supervised } \\
\text { rehabilitation \& } \\
\text { unsupervised } \\
\text { home-based } \\
\text { exercises }\end{array}$ & TUG (s) & High & $6 \mathrm{mo}$ & 56 & $\begin{array}{l}8.2 ; 95 \% \mathrm{Cl} \\
(6.9,9.4)\end{array}$ & 42 & $\begin{array}{l}8.9 ; 95 \% \mathrm{Cl} \\
(7.38,10.43)\end{array}$ & $\begin{array}{l}-0.74(-6.65, \\
5.17)^{\mathrm{B}}\end{array}$ & NR \\
\hline $\begin{array}{l}\text { Nelson, 2020, } \\
32026820\end{array}$ & Telerehabilitation & $\begin{array}{l}\text { In-person usual } \\
\text { care }\end{array}$ & TUG (s) & Moderate & $6 \mathrm{mo}$ & 34 & $7.9(2.3)$ & 35 & $9.4(3)$ & $\begin{array}{l}-1.5(-2.4,- \\
0.6)^{\mathrm{B}}\end{array}$ & NR \\
\hline
\end{tabular}

Statistically significant effect sizes are in bold text. In cases where calculated effect size confidence intervals were not-statistically significant but reported p-values were, we deferred to reported p-values and still bolded results.

Abbreviations: $\mathrm{CI}=$ confidence interval, $\mathrm{mo}=$ month, $\mathrm{NR}=$ not reported, $\mathrm{PMID}=$ PubMed identifier, $\mathrm{RoB}=$ risk of bias, $\mathrm{s}=\mathrm{second}, \mathrm{SD}=\mathrm{standard}$ deviation, $\mathrm{TUG}=$ timed up and go.

A Time from surgery

${ }^{B}$ Calculated 


\section{Other Patient-Reported Outcomes}

Thirteen studies in total reported on other patient-reported outcomes following rehabilitation compared with various controls: one study (Liebs 2010) compared novel acute rehabilitation programs with control; two studies (Liebs 2012, Mikkelsen 2014) compared acute rehabilitation programs with different timing and/or intensity; four studies (Beck 2019, Heiberg 2012, Monticone 2014, Winther 2018) compared novel post-acute rehabilitation programs with various comparators (less intensive rehabilitation or no care); and four studies (Austin 2017, Coulter 2017, Naylor 2018, Nelson 2020, Rao 2021) compared rehabilitation programs delivered in different settings or by different personnel (Tables 86-88). Outcomes include: quality of life, satisfaction with care, and patient global assessments.

\section{Health-Related Quality of Life}

Four studies (Heiber 2012, Mikkelsen 2014, Nelson 2020, Rao 2021) reported the QoL component of HOOS and observed no differences between groups at follow-up times between 6 months and 5 years after THA (Table 86)

\section{Patient Satisfaction With Care}

Two studies (Liebs 2010 and Liebs 2012) reported data on satisfaction with care and found no differences between rehabilitation arms (Table 87).

\section{Patient Global Assessments}

Ten studies (Austin 2017, Beck 2019, Coulter 2017, Heiberg 2012, Liebs 2010, Liebs 2012, Monticone 2014, Naylor 2018, Smith 2009, Winther 2020) provided data on patients' selfreported global assessment of their health using nine different measurement instruments (Table 88). The studies mostly observed no differences between groups at follow-up times between 6 months and 5 years after THA. Beck 2019 found a significant difference in favor of sports therapy compared with control on the EQ-5D scale at 6-month follow-up, but this difference was not sustained at the 12-month follow-up. 
Table 86. Rehabilitation versus various controls for total hip arthroplasty - continuous outcomes, quality of life

\begin{tabular}{|c|c|c|c|c|c|c|c|c|c|c|c|}
\hline $\begin{array}{l}\text { Study, Year, } \\
\text { PMID, } \\
\text { Country }\end{array}$ & Arm 1 & Arm 2 & $\begin{array}{l}\text { Overall } \\
\text { RoB }\end{array}$ & $\begin{array}{l}\text { Outcome } \\
\text { Measurement }\end{array}$ & $\begin{array}{l}\text { Time } \\
\text { Point }^{\mathrm{A}}\end{array}$ & $\begin{array}{l}\text { Arm 1, } \\
\text { N }\end{array}$ & $\begin{array}{l}\text { Arm 1, Mean } \\
\text { (SD) }\end{array}$ & Arm 2, & $\begin{array}{l}\text { Arm 2, Mean } \\
\text { (SD) }\end{array}$ & $\begin{array}{l}\text { Effect Size } \\
(95 \% \mathrm{Cl})\end{array}$ & $\begin{array}{l}\text { Reported } \\
\text { p-Value }\end{array}$ \\
\hline \multirow[t]{2}{*}{$\begin{array}{l}\text { Heiberg, 2012, } \\
22170790\end{array}$} & $\begin{array}{l}\text { Walking skill } \\
\text { training program }\end{array}$ & Standard care & Moderate & $\begin{array}{l}\text { HOOS: QoL (0- } \\
100)\end{array}$ & $12 \mathrm{mo}$ & 35 & $\begin{array}{l}81 ; 95 \% \mathrm{Cl} \\
(76,86)\end{array}$ & 33 & $\begin{array}{l}83 ; 95 \% \mathrm{Cl}(78, \\
88)\end{array}$ & $-2(-9,5)$ & ns \\
\hline & $\begin{array}{l}\text { Walking skill } \\
\text { training program }\end{array}$ & Standard care & Moderate & $\begin{array}{l}\text { HOOS: QoL (0- } \\
100)\end{array}$ & $5 y$ & 30 & $\begin{array}{l}85 ; 95 \% \mathrm{Cl} \\
(80,90)\end{array}$ & 30 & $\begin{array}{l}84 ; 95 \% \mathrm{Cl}(78, \\
90)\end{array}$ & $1(-6.81,8.81)^{\mathrm{B}}$ & NR \\
\hline \multirow[t]{2}{*}{$\begin{array}{l}\text { Mikkelsen, } \\
2014, \\
25305374\end{array}$} & $\begin{array}{l}\text { Early supervised } \\
\text { progressive } \\
\text { resistance } \\
\text { training } \\
\end{array}$ & $\begin{array}{l}\text { Unsupervised } \\
\text { home-based } \\
\text { exercise }\end{array}$ & Moderate & $\begin{array}{l}\text { HOOS: QoL (0- } \\
100)\end{array}$ & $6 \mathrm{mo}$ & 32 & $83.8(18)$ & 30 & $86.7(17)$ & $-2.9(-9.1,3.3)^{\mathrm{B}}$ & NR \\
\hline & $\begin{array}{l}\text { Early supervised } \\
\text { progressive } \\
\text { resistance } \\
\text { training }\end{array}$ & $\begin{array}{l}\text { Unsupervised } \\
\text { home-based } \\
\text { exercise }\end{array}$ & Moderate & $\begin{array}{l}\text { HOOS: QoL (0- } \\
100)\end{array}$ & $12 \mathrm{mo}$ & 32 & $86.7(16)$ & 30 & $86(20)$ & $0.7(-5.8,7.2)^{\mathrm{B}}$ & NR \\
\hline $\begin{array}{l}\text { Nelson, } 2020 \text {, } \\
32026820\end{array}$ & Telerehabilitation & $\begin{array}{l}\text { In-person usual } \\
\text { care }\end{array}$ & Moderate & $\begin{array}{l}\text { HOOS: QoL (0- } \\
100)\end{array}$ & $6 \mathrm{mo}$ & 34 & $79(22)$ & 35 & $77(21)$ & $2(-5.2,9.2)^{B}$ & NR \\
\hline $\begin{array}{l}\text { Rao, } \\
2021, \\
33863614 \\
\text { USA }\end{array}$ & $\begin{array}{l}\text { Home physical } \\
\text { therapy }\end{array}$ & $\begin{array}{l}\text { Formal physical } \\
\text { therapy }\end{array}$ & High & $\begin{array}{l}\text { HOOS: QoL (0- } \\
100)\end{array}$ & $6 \mathrm{mo}$ & 70 & $74.3(21.9)$ & 66 & $69.6(27.9)$ & $-4.7(-10.8,1.4)$ & 0.294 \\
\hline
\end{tabular}

Statistically significant effect sizes are in bold text. In cases where calculated effect size confidence intervals were not-statistically significant but reported p-values were, we deferred to reported p-values and still bolded results.

Abbreviations: $\mathrm{CI}=$ confidence interval, $\mathrm{HOOS}=$ Hip disability and osteoarthritis outcome score, mo $=$ month, $\mathrm{NR}=$ not reported, $\mathrm{ns}=$ not significant, $\mathrm{PMID}=\mathrm{PubMed}$ identifier $\mathrm{QoL}=$ quality of life, $\mathrm{RoB}=$ risk of bias, $\mathrm{SD}=$ standard deviation, $\mathrm{y}=$ year.

A Time from surgery

${ }^{B}$ Calculated

Table 87. Rehabilitation versus various controls for total hip arthroplasty - categorical outcomes, satisfaction with care

\begin{tabular}{|c|c|c|c|c|c|c|c|c|c|}
\hline $\begin{array}{l}\text { Study, Year, } \\
\text { PMID, } \\
\text { Country }\end{array}$ & Arm 1 & Arm 2 & $\begin{array}{l}\text { Overall } \\
\text { RoB }\end{array}$ & Outcome Measurement & $\begin{array}{l}\text { Time } \\
\text { Point }^{A}\end{array}$ & Arm $1 \mathrm{n} / \mathrm{N}(\%)$ & Arm 2 n/N (\%) & Effect Size $(95 \% \mathrm{CI})$ & $\begin{array}{l}\text { Reported } \\
\text { p-Value }\end{array}$ \\
\hline $\begin{array}{l}\text { Liebs, } 2010, \\
20360503\end{array}$ & Ergometer cycling & Control & Moderate & Patient satisfaction with care & $24 \mathrm{mo}$ & $69 / 74(93 \%)$ & $76 / 88(86 \%)$ & RR $2.18(0.73,6.49)$ & 0.155 \\
\hline $\begin{array}{l}\text { Liebs, 2012, } \\
22196125\end{array}$ & $\begin{array}{l}\text { Early aquatic } \\
\text { therapy }\end{array}$ & $\begin{array}{l}\text { Late aquatic } \\
\text { therapy }\end{array}$ & Moderate & Patient satisfaction with care & $24 \mathrm{mo}$ & $88 / 100(88 \%)$ & $97 / 110(88 \%)$ & RR $1.02(0.44,2.35)$ & 0.968 \\
\hline
\end{tabular}

Abbreviations: $\mathrm{CI}=$ confidence interval, $\mathrm{MD}=$ mean difference, $\mathrm{NMD}=$ net mean difference, $\mathrm{NR}=$ not reported, NA $=$ not applicable, $\mathrm{NS}=$ not significant, $\mathrm{PMID}=\mathrm{PubMed}$ identifier, $\mathrm{RR}=$ relative risk, $\mathrm{QOL}=$ quality of life, $\mathrm{d}=$ days, $\mathrm{mo}=$ months, $\mathrm{SD}=$ standard deviation.

A Time from surgery 
Table 88. Rehabilitation versus various controls for total hip arthroplasty - continuous outcomes, patient global assessment

\begin{tabular}{|c|c|c|c|c|c|c|c|c|c|c|c|}
\hline $\begin{array}{l}\text { Study, Year, } \\
\text { PMID, } \\
\text { Country }\end{array}$ & Arm 1 & Arm 2 & $\begin{array}{l}\text { Overall } \\
\text { RoB }\end{array}$ & $\begin{array}{l}\text { Outcome } \\
\text { Measurement }\end{array}$ & $\begin{array}{l}\text { Time } \\
\text { Point }^{A}\end{array}$ & $\begin{array}{l}\text { Arm 1, } \\
\text { N }\end{array}$ & $\begin{array}{l}\text { Arm 1, Mean } \\
\text { (SD) }\end{array}$ & $\begin{array}{l}\text { Arm 2, } \\
\text { N }\end{array}$ & $\begin{array}{l}\text { Arm 2, Mean } \\
\text { (SD) }\end{array}$ & $\begin{array}{l}\text { Effect Size } \\
(95 \% \mathrm{Cl})\end{array}$ & $\begin{array}{l}\text { Reported } \\
p-V \\
\text { alue }\end{array}$ \\
\hline \multirow[t]{2}{*}{$\begin{array}{l}\text { Austin, } 2017 \text {, } \\
28419032\end{array}$} & $\begin{array}{l}\text { Home } \\
\text { exercise }\end{array}$ & $\begin{array}{l}\text { Formal } \\
\text { Physical } \\
\text { Therapy }\end{array}$ & Moderate & $\begin{array}{l}\text { WOMAC: total (0- } \\
100)\end{array}$ & $6-12 \mathrm{mo}$ & 52 & NR & 52 & NR & NR & 0.80 \\
\hline & $\begin{array}{l}\text { Home } \\
\text { exercise }\end{array}$ & $\begin{array}{l}\text { Formal } \\
\text { Physical } \\
\text { Therapy }\end{array}$ & Moderate & $\begin{array}{l}\text { Harris hip score (0- } \\
100)^{\mathrm{B}}\end{array}$ & $6-12 \mathrm{mo}$ & 52 & NR & 52 & NR & NR & 0.82 \\
\hline \multirow[t]{6}{*}{$\begin{array}{l}\text { Beck, 2019, } \\
30782304\end{array}$} & Sport therapy & Control & High & EQ-5D (NR) & $6 \mathrm{mo}$ & 63 & $\begin{array}{l}\text { Median (Q1, Q3) } \\
1(0.89,1)\end{array}$ & 51 & $\begin{array}{l}\text { Median (Q1, Q3) } \\
0.89(0.89,1) \\
\end{array}$ & NR & 0.036 \\
\hline & Sport therapy & Control & High & EQ-5D (NR) & $12 \mathrm{mo}$ & 57 & $\begin{array}{l}\text { Median (Q1, Q3) } \\
1(0.89,1)\end{array}$ & 41 & $\begin{array}{l}\text { Median (Q1, Q3) } \\
1(0.89,1)\end{array}$ & NR & 0.891 \\
\hline & Sport therapy & Control & High & $\begin{array}{l}\text { WOMAC: total (0- } \\
100)\end{array}$ & $6 \mathrm{mo}$ & 63 & $\begin{array}{l}\text { Median (Q1, Q3) } \\
92.7(86.5,95.8)\end{array}$ & 51 & $\begin{array}{l}\text { Median (Q1, Q3) } \\
92.7(85.4,96.9) \\
\end{array}$ & NR & 1.00 \\
\hline & Sport therapy & Control & High & $\begin{array}{l}\text { WOMAC: total (0- } \\
100)\end{array}$ & $12 \mathrm{mo}$ & 57 & $\begin{array}{l}\text { Median (Q1, Q3) } \\
95.8(91.7,100) \\
\end{array}$ & 41 & $\begin{array}{l}\text { Median (Q1, Q3) } \\
95.8(88.5,96.9)\end{array}$ & NR & 1.00 \\
\hline & Sport therapy & Control & High & $\begin{array}{l}\text { Harris hip score (0- } \\
100)\end{array}$ & $6 \mathrm{mo}$ & 63 & $\begin{array}{l}\text { Median (Q1, Q3) } \\
93(86,96)\end{array}$ & 51 & $\begin{array}{l}\text { Median (Q1, Q3) } \\
95(88,96)\end{array}$ & NR & 1.00 \\
\hline & Sport therapy & Control & High & $\begin{array}{l}\text { Harris hip score }(0- \\
100)\end{array}$ & $12 \mathrm{mo}$ & 57 & $\begin{array}{l}\text { Median (Q1, Q3) } \\
96(93,98)\end{array}$ & 41 & $\begin{array}{l}\text { Median (Q1, Q3) } \\
96(90,97)\end{array}$ & NR & 1.00 \\
\hline $\begin{array}{l}\text { Coulter, 2017, } \\
28506775\end{array}$ & $\begin{array}{l}\text { Unsupervised } \\
\text { home-based } \\
\text { exercises }\end{array}$ & $\begin{array}{l}\text { Supervised } \\
\text { rehabilitation \& } \\
\text { unsupervised } \\
\text { home-based } \\
\text { exercises }\end{array}$ & High & $\begin{array}{l}\text { WOMAC: total (0- } \\
100)\end{array}$ & $6 \mathrm{mo}$ & 56 & $\begin{array}{l}\text { Mean }(95 \% \mathrm{Cl}) \\
18.4(11.88, \\
24.88) \\
\text { SE 3.32* }\end{array}$ & 42 & $\begin{array}{l}\text { Mean }(95 \% \mathrm{Cl}) \\
19.70(12.46, \\
26.98) \\
\text { SE 3.70* }\end{array}$ & $\begin{array}{l}-1.3(-11.04 \\
8.44)^{\mathrm{C}}\end{array}$ & ns \\
\hline $\begin{array}{l}\text { Heiberg, } \\
2012, \\
22170790 \\
\end{array}$ & $\begin{array}{l}\text { Walking skill } \\
\text { training } \\
\text { program }\end{array}$ & Standard care & Moderate & $\begin{array}{l}\text { Harris hip score (0- } \\
100)\end{array}$ & $12 \mathrm{mo}$ & 35 & $\begin{array}{l}\text { Mean }(95 \% \mathrm{Cl}) \\
96(93,98)\end{array}$ & 33 & $\begin{array}{l}\text { Mean }(95 \% \mathrm{Cl}) \\
92(90,95)\end{array}$ & $3(-1,7)$ & ns \\
\hline $\begin{array}{l}\text { Liebs, 2010, } \\
20360503\end{array}$ & $\begin{array}{l}\text { Ergometer } \\
\text { cycling }\end{array}$ & Control & Moderate & SF-6D & $24 \mathrm{mo}$ & 74 & $0.746(0.128)$ & 88 & $0.729(0.135)$ & $\begin{array}{l}0.02(-0.02, \\
0.06)^{\mathrm{C}}\end{array}$ & 0.13 \\
\hline $\begin{array}{l}\text { Liebs, } 2012, \\
22196125\end{array}$ & $\begin{array}{l}\text { Early aquatic } \\
\text { therapy }\end{array}$ & $\begin{array}{l}\text { Late aquatic } \\
\text { therapy }\end{array}$ & Moderate & SF-6D & $24 \mathrm{mo}$ & 100 & $0.730(0.146)$ & 110 & $0.744(0.122)$ & $\begin{array}{l}\text { MD -0.01 (- } \\
0.05,0.02)^{\mathrm{C}}\end{array}$ & 0.213 \\
\hline $\begin{array}{l}\text { Monticone, } \\
2014, \\
24459172 \\
\end{array}$ & $\begin{array}{l}\text { Task-oriented } \\
\text { exercises }\end{array}$ & $\begin{array}{l}\text { Open chain } \\
\text { kinetic } \\
\text { exercises }\end{array}$ & Moderate & $\begin{array}{l}\text { SF-36: General } \\
\text { health }(0-100)\end{array}$ & $12 \mathrm{mo}$ & 50 & $72.1(18.9)$ & 50 & $57.7(16.8)$ & $\begin{array}{l}14.4(9.4 \\
19.4)^{\mathrm{C}}\end{array}$ & NR \\
\hline $\begin{array}{l}\text { Naylor, 2018, } \\
30021552\end{array}$ & $\begin{array}{l}\text { In-patient } \\
\text { rehabilitation }\end{array}$ & $\begin{array}{l}\text { No in-patient } \\
\text { rehabilitation }\end{array}$ & Moderate & $\begin{array}{l}\text { Oxford hip score } \\
(12-60)^{\mathrm{D}}\end{array}$ & $12 \mathrm{mo}$ & 123 & $\begin{array}{l}\text { Median (Q1, Q3) } \\
48(46,48)\end{array}$ & 123 & $\begin{array}{l}\text { Median (Q1, Q3) } \\
48(46,48)\end{array}$ & $\begin{array}{l}\text { Median } \\
\text { difference } \\
(\mathrm{Q} 1, \mathrm{Q} 3) 0(- \\
1,1)\end{array}$ & 0.91 \\
\hline \multirow{2}{*}{$\begin{array}{l}\text { Rao, } \\
2021, \\
33863614 \\
\text { USA }\end{array}$} & $\begin{array}{l}\text { Home } \\
\text { physical } \\
\text { therapy }\end{array}$ & $\begin{array}{l}\text { Formal physical } \\
\text { therapy }\end{array}$ & High & SF-12: PCS & $6 \mathrm{mo}$ & 70 & $47.2(10.7)$ & 66 & $46.6(12.2)$ & $\begin{array}{l}-0.6(-3.4 \\
2.2)\end{array}$ & 0.644 \\
\hline & $\begin{array}{l}\text { Home } \\
\text { physical } \\
\text { therapy }\end{array}$ & $\begin{array}{l}\text { Formal physical } \\
\text { therapy }\end{array}$ & High & SF-12: MCS & $6 \mathrm{mo}$ & 70 & $54.9(8.0)$ & 66 & $52.3(8.9)$ & $\begin{array}{l}-2.6(-4.6,- \\
0.6)\end{array}$ & 0.087 \\
\hline
\end{tabular}




\begin{tabular}{|c|c|c|c|c|c|c|c|c|c|c|c|}
\hline $\begin{array}{l}\text { Study, Year, } \\
\text { PMID, } \\
\text { Country }\end{array}$ & Arm 1 & Arm 2 & $\begin{array}{l}\text { Overall } \\
\text { RoB }\end{array}$ & $\begin{array}{l}\text { Outcome } \\
\text { Measurement }\end{array}$ & $\begin{array}{l}\text { Time } \\
\text { Point }^{\mathrm{A}}\end{array}$ & $\begin{array}{l}\text { Arm 1, } \\
\text { N }\end{array}$ & $\begin{array}{l}\text { Arm 1, Mean } \\
\text { (SD) }\end{array}$ & $\begin{array}{l}\text { Arm 2, } \\
\text { N }\end{array}$ & $\begin{array}{l}\text { Arm 2, Mean } \\
\text { (SD) }\end{array}$ & $\begin{array}{l}\text { Effect Size } \\
(95 \% \mathrm{Cl})\end{array}$ & $\begin{array}{l}\text { Reported } \\
\text { p-V } \\
\text { alue }\end{array}$ \\
\hline \multirow[t]{5}{*}{$\begin{array}{l}\text { Smith, 2009, } \\
19876883\end{array}$} & $\begin{array}{l}\text { Gait re- } \\
\text { education } \\
\text { program \& } \\
\text { bed exercises }\end{array}$ & $\begin{array}{l}\text { Gait re- } \\
\text { education } \\
\text { program only }\end{array}$ & Moderate & SF-12 (0-100) & $12 \mathrm{mo}$ & 30 & $\begin{array}{l}\text { Median (Q1, Q3) } \\
32(30,34)\end{array}$ & 30 & $\begin{array}{l}\text { Median (Q1, Q3) } \\
33(31,34)\end{array}$ & $\begin{array}{l}\text { Median } \\
\text { difference } \\
(95 \% \mathrm{Cl}) 0(- \\
1,2)\end{array}$ & 0.4282 \\
\hline & $\begin{array}{l}\text { Gait re- } \\
\text { education } \\
\text { program \& } \\
\text { bed exercises }\end{array}$ & $\begin{array}{l}\text { Gait re- } \\
\text { education } \\
\text { program only }\end{array}$ & Moderate & SF-12 (0-100) & $12 \mathrm{mo}^{\mathrm{E}}$ & 30 & $\begin{array}{l}\text { Median (Q1, Q3) } \\
32(31,33)\end{array}$ & 30 & $\begin{array}{l}\text { Median (Q1, Q3) } \\
33(31,34)\end{array}$ & $\begin{array}{l}\text { Median } \\
\text { difference } \\
(95 \% \mathrm{Cl}) 0(- \\
1,2)\end{array}$ & 0.288 \\
\hline & $\begin{array}{l}\text { Gait re- } \\
\text { education } \\
\text { program \& } \\
\text { bed exercises }\end{array}$ & $\begin{array}{l}\text { Gait re- } \\
\text { education } \\
\text { program only }\end{array}$ & Moderate & SF-12 (0-100) & $12 \mathrm{mo}^{\mathrm{F}}$ & 30 & $\begin{array}{l}\text { Median (Q1, Q3) } \\
30(29,34)\end{array}$ & 30 & $\begin{array}{l}\text { Median (Q1, Q3) } \\
32.5(32,33)\end{array}$ & $\begin{array}{l}\text { Median } \\
\text { difference } \\
(95 \% \mathrm{Cl}) 2 \\
(\mathrm{NR}, \mathrm{NR})\end{array}$ & 0.7649 \\
\hline & $\begin{array}{l}\text { Gait re- } \\
\text { education } \\
\text { program \& } \\
\text { bed exercises }\end{array}$ & $\begin{array}{l}\text { Gait re- } \\
\text { education } \\
\text { program only }\end{array}$ & Moderate & SF-12 (0-100) & $12 \mathrm{mo}^{\mathrm{G}}$ & 30 & $\begin{array}{l}\text { Median (Q1, Q3) } \\
32.5(31,34)\end{array}$ & 30 & $\begin{array}{l}\text { Median (Q1, Q3) } \\
32(32,33)\end{array}$ & $\begin{array}{l}\text { Median } \\
\text { difference } \\
(95 \% \mathrm{Cl}) 1(- \\
1,3)\end{array}$ & 0.3193 \\
\hline & $\begin{array}{l}\text { Gait re- } \\
\text { education } \\
\text { program \& } \\
\text { bed exercises }\end{array}$ & $\begin{array}{l}\text { Gait re- } \\
\text { education } \\
\text { program only }\end{array}$ & Moderate & SF-12 (0-100) & $12 \mathrm{mo}^{\mathrm{H}}$ & 30 & $\begin{array}{l}\text { Median (Q1, Q3) } \\
31.5(29.5,33)\end{array}$ & 30 & $\begin{array}{l}\text { Median (Q1, Q3) } \\
32(32,33)\end{array}$ & $\begin{array}{l}\text { Median } \\
\text { difference } \\
(95 \% \mathrm{Cl}) 1(- \\
1,3)\end{array}$ & 0.3193 \\
\hline \multirow[t]{2}{*}{$\begin{array}{l}\text { Winther, 2020, } \\
31977324\end{array}$} & $\begin{array}{l}\text { Strengthening } \\
\text { exercise }\end{array}$ & Standard care & Moderate & $\begin{array}{l}\text { Harris hip score (0- } \\
100)\end{array}$ & $12 \mathrm{mo}$ & 22 & $95(7)$ & 22 & $93(9)$ & $2(-1.4,5.4)^{C}$ & NR \\
\hline & $\begin{array}{l}\text { Strengthening } \\
\text { exercise }\end{array}$ & Standard care & Moderate & $\begin{array}{l}\text { HOOS: Short form } \\
(0-100)\end{array}$ & $12 \mathrm{mo}$ & 22 & $8(10)$ & 22 & $8(10)$ & $0(-4.2,4.2)^{\mathrm{C}}$ & NR \\
\hline
\end{tabular}

Statistically significant effect sizes are in bold text. In cases where calculated effect size confidence intervals were not-statistically significant but reported p-values were, we deferred to reported p-values and still bolded results.

Abbreviations: $\mathrm{CI}=$ confidence interval, $\mathrm{EQ}-5 \mathrm{D}=$ EuroQual, $\mathrm{mo}=$ month, $\mathrm{MCS}=$ mental component scale, $\mathrm{NR}=$ not reported, $\mathrm{PCS}=$ physical component scale, $\mathrm{PMID}=\mathrm{PubMed}$ identifier, RoB $=$ risk of bias, $\mathrm{SD}=$ standard deviation, $\mathrm{SF}-6 \mathrm{D}=$ short-form six-dimension, $\mathrm{SF}-12=12$-item short form survey, $\mathrm{SF}-36=36$-item short form survey, WOMAC = Western Ontario and McMaster Universities Osteoarthritis Index.

A Time from surgery

${ }^{\mathrm{B}}$ Measure combines domains of pain, function, absence of deformity, and range of motion

${ }^{\mathrm{C}}$ Calculated

${ }^{\mathrm{D}}$ Measure combines pain and function

E Subgroup of cemented participants

${ }^{\mathrm{F}}$ Subgroup of uncemented participants

${ }^{\mathrm{G}}$ Subgroup of posterior participants

${ }^{\mathrm{H}}$ Subgroup of anterolateral participants 


\section{Healthcare Utilization Outcomes}

Few studies reported on healthcare-utilization outcomes following rehabilitation compared to various control. Liebs 2010 and Liebs 2012 reported on the number of participants who needed to be readmitted to hospital due to various adverse events within three months of THA and found rates low (4 to 10\%, including post-surgical adverse events) and comparable between groups.

\section{Costs}

Naylor 2018 compared the costs of an Australian-based inpatient versus no inpatient care and reported that median rehabilitation provider charges were 10 times higher for individuals who received inpatient rehabilitation compared with those who did not (median difference \$7582 (95\% CI $\$ 5649$ to $\$ 10,249, \mathrm{p}<0.001)$.

\section{Cost-Effectiveness}

We found no studies that compared the cost-effectiveness of rehabilitation with various comparators for THA.

\section{Harms From Rehabilitation}

Four studies (reported the occurrence of adverse events associated with the rehabilitation programs (e.g., pain, falls). Of the studies that reported data on adverse events (Liebs 2010, Liebs 2012, Mikkelsen 2014, Monticone 2014), the proportion of patients experiencing harms due to participation in the rehabilitation interventions were low ( 0 to $18 \%$; highest for "transitory pain worsening"), of low severity, and comparable between groups (Table 89). 
Table 89. Rehabilitation versus various controls for total hip arthroplasty - categorical outcomes, harms from rehabilitation

\begin{tabular}{|c|c|c|c|c|c|c|c|c|c|}
\hline $\begin{array}{l}\text { Study, Year, } \\
\text { PMID, } \\
\text { Country }\end{array}$ & Arm 1 & Arm 2 & $\begin{array}{l}\text { Overall } \\
\text { RoB }\end{array}$ & Outcome Measurement & $\begin{array}{l}\text { Time } \\
\text { Point }\end{array}$ & Arm 1, n/N & Arm 2, n/N & Effect Size $(95 \% \mathrm{Cl})$ & $\begin{array}{l}\text { Reported p- } \\
\text { Value }\end{array}$ \\
\hline $\begin{array}{l}\text { Liebs, } 2010 \\
20360503\end{array}$ & Ergometer cycling & Control & Moderate & Readmitted to hospital & $\begin{array}{l}\text { Within } 3 \\
\text { mo }\end{array}$ & $5 / 74(7 \%)^{\mathrm{B}}$ & $5 / 88(6 \%)^{\mathrm{C}}$ & RR $1.19(0.36,3.95)^{D}$ & NR \\
\hline $\begin{array}{l}\text { Liebs, } 2012, \\
22196125\end{array}$ & Early aquatic group & Late aquatic group & Moderate & Readmitted to hospital & $\begin{array}{l}\text { Within } 3 \\
\text { mo }\end{array}$ & $\begin{array}{l}10 / 100 \\
(10 \%)^{E}\end{array}$ & $4 / 110(4 \%)^{F}$ & RR $2.75(0.89,8.49)^{D}$ & NR \\
\hline $\begin{array}{l}\text { Mikkelsen, } \\
2014, \\
25305374\end{array}$ & $\begin{array}{l}\text { Early supervised } \\
\text { progressive } \\
\text { resistance training }\end{array}$ & $\begin{array}{l}\text { Unsupervised home- } \\
\text { based training }\end{array}$ & Moderate & $\begin{array}{l}\text { Knee pain in contralateral } \\
\text { leg }\end{array}$ & $12 \mathrm{mo}$ & $1 / 32(3 \%)$ & $0 / 30(0 \%)$ & $1.88(0.07,53.88)^{\mathrm{D}}$ & NR \\
\hline \multirow{2}{*}{$\begin{array}{l}\text { Monticone, } \\
2014, \\
24459172\end{array}$} & $\begin{array}{l}\text { Task-oriented } \\
\text { exercises }\end{array}$ & $\begin{array}{l}\text { Open chain kinetic } \\
\text { exercises }\end{array}$ & Moderate & Transitory pain worsening & $12 \mathrm{mo}$ & $8 / 50(16 \%)$ & $9 / 50(18 \%)$ & $0.89(0.37,2.12)^{\mathrm{D}}$ & NR \\
\hline & $\begin{array}{l}\text { Task-oriented } \\
\text { exercises }\end{array}$ & $\begin{array}{l}\text { Open chain kinetic } \\
\text { exercises }\end{array}$ & Moderate & Falls & $12 \mathrm{mo}$ & $2 / 50(4 \%)$ & $3 / 50(6 \%)$ & $0.67(0.12,3.82)^{\mathrm{D}}$ & NR \\
\hline
\end{tabular}

Statistically significant effect sizes are in bold text. In cases where calculated effect size confidence intervals were not-statistically significant but reported p-values were, we deferred to reported p-values and still bolded results.

Abbreviations: $\mathrm{CI}=$ confidence interval, $\mathrm{NR}=$ not reported, $\mathrm{PMID}=$ PubMed identifier, $\mathrm{RR}=$ relative risk, $\mathrm{mo}=$ month, $\mathrm{SD}=$ standard deviation

A Time from surgery

B Due to dislocation of the hip, other hip problems, fracture of lumbar vertebrae, cardiovascular problems, and overall pain in one patient each

${ }^{\mathrm{C}}$ Due to dislocation of the hip, acute coronary syndrome, hematoma revision, appendicitis, and an unknown reason in one each

D Calculated

${ }^{\mathrm{E}}$ Due to dislocation of hip, wound dehiscence, thrombosis $\mathrm{n}=2$, intestinal perforation, shunt revision, supervision after fall, abscess, appendicitis, pneumonia

${ }^{\mathrm{F}}$ Due to dislocation of hip, wound dehiscence, pulmonary embolism, pulmonary edema 


\section{Heterogeneity of Treatment Effects (Subgroup Differences)}

Two studies (Lyp 2016 and Smith 2009) formally analyzed possible heterogeneity of treatment effects, reporting statistical tests for whether the comparative effect of rehabilitation versus its various comparators differed in among subgroups. Lyp 2016 found that the type of prosthesis used (cemented or uncemented) did not impact the relative effects of rehabilitation on ROM or strength. Smith 2009 reported results by subgroups who received cemented or uncemented prosthesis and who had posterior or anterolateral surgical approaches. The study found no significant difference in outcomes across these subgroups, although sample sizes were small and likely lacked sufficient power for subgroup analyses. No study formally analyzed possible heterogeneity of treatment effects due to patients' baseline risk (e.g., patients with higher vs. lower measures of strength, flexibility, function, at baseline).

\section{Applicability}

Studies were conducted across the globe (two in the United States) using diverse interventions employed in diverse healthcare settings. While the relative effect of the interventions on clinical outcomes (and harms) from non-U.S.-based studies are likely applicable to the U.S. context, findings pertaining to healthcare system or resources (such as costs or comparisons of inpatient vs. outpatient rehabilitation) are likely country and healthcare system specific. The sex of participants varied widely across studies, ranging from 21 to 70 percent of participants being female. The average age of patients ages ranged from 54 to 71 years and the average BMIs ranged from 25 to $30 \mathrm{~kg} / \mathrm{m}^{2}$ (thus, in all studies, most patients were obese). Most studies did not report whether patients had undergone previous contralateral replacement surgery; of one study that did, the proportion was low (less than $25 \%$ ). As such, the conclusions in this KQ are likely most applicable to middle-to-older-aged adults in high-income countries who are receiving their first total TKA for osteoarthritis.

\section{Summary of Comparison of Rehabilitation Versus Various Controls for Total Hip Arthroplasty}

Table 90 summarizes the evidence for the comparison of rehabilitation versus various comparators and rehabilitation. We focus on the outcomes we prioritized in discussion with stakeholders.

While some studies found significant effects for outcomes, most studies did not and given the heterogeneity of interventions and lack of replication of unique rehabilitation programs, we determined that there is low to insufficient SoE for all conclusions. Specifically, there is low SoE of no difference between the various programs of rehabilitation (compared with no rehabilitation, lesser intensive rehabilitation, rehabilitation at different timing, and rehabilitation provided in different settings) following THA in terms of pain, strength, ADL, and QoL. There is insufficient evidence on the impact of rehabilitation on ROM and satisfaction with care. There is low SoE that there is no difference among rehabilitation programs with respect to harms related to rehabilitation. 
Table 90. Evidence profile: Rehabilitation versus various controls for total hip arthroplasty

\begin{tabular}{|c|c|c|c|c|c|c|c|c|c|}
\hline $\begin{array}{l}\text { Outcome } \\
\text { Category }\end{array}$ & Outcome & $\begin{array}{l}\text { N Studies } \\
\text { (Participants) }\end{array}$ & RoB & Consistency & Precision & Directness & $\begin{array}{l}\text { Intervention } \\
\text { Replication }\end{array}$ & SoE & Conclusions \\
\hline \multirow{3}{*}{$\begin{array}{l}\text { Body } \\
\text { structure and } \\
\text { function }\end{array}$} & Pain & $11(1297)$ & Moderate & Consistent & Precise & Direct & All unique & Low & Similar pain \\
\hline & ROM & $3(178)$ & Moderate & Inconsistent & Precise & Direct & All unique & Insufficient & No conclusion \\
\hline & Strength & $5(370)$ & Moderate & Consistent & Precise & Direct & All unique & Low & $\begin{array}{l}\text { Similar } \\
\text { strength }\end{array}$ \\
\hline $\begin{array}{l}\text { Activity and } \\
\text { participation }\end{array}$ & ADLs & $12(1247)$ & Moderate & Consistent & Precise & Direct & All unique & Low & Similar ADL \\
\hline \multirow[t]{2}{*}{$\begin{array}{l}\text { Other } \\
\text { outcomes }\end{array}$} & $\begin{array}{l}\text { Satisfaction } \\
\text { with care }\end{array}$ & $2(372)$ & Moderate & Consistent & Precise & Direct & All unique & Insufficient & No conclusion \\
\hline & QoL & $4(335)$ & Moderate & Consistent & Precise & Direct & All unique & Low & Similar QoL \\
\hline $\begin{array}{l}\text { Healthcare } \\
\text { utilization }\end{array}$ & $\begin{array}{l}\text { Need for } \\
\text { postoperative } \\
\text { procedures }\end{array}$ & 0 & NA & NA & NA & NA & NA & Insufficient & No evidence \\
\hline Harms & $\begin{array}{l}\text { Harms from } \\
\text { prehabilitation }\end{array}$ & 4 (534) & Moderate & Consistent & Precise & Direct & All unique & Low & Similar harms \\
\hline
\end{tabular}

Abbreviations: ADLs = activities of daily living, $\mathrm{QoL}=$ health-related quality of life, $\mathrm{NA}=$ not applicable, $\mathrm{QoL}=\mathrm{quality}$ of life, RoB $=$ risk of bias, $\mathrm{ROM}=$ range of motion, SoE $=$ strength of evidence. 


\section{Discussion}

\section{Findings in Relation to the Decisional Dilemmas}

Despite a large overall body of evidence ( 83 studies), mostly from randomized controlled trials (RCTs), for the four Key Questions (KQs), the evidence was sparse due to the extensive heterogeneity of interventions and outcomes.

For healthcare services deciding whether to offer prehabilitation (rehabilitation provided prior to surgery) to patients about to undergo total knee arthroplasty (TKA) for osteoarthritis, the evidence suggests that prehabilitation is comparable to no prehabilitation in terms of pain, range of motion (ROM), and activities of daily living (ADL). Prehabilitation may lead to increased strength and reduced length of hospital stay following TKA although there is no or insufficient evidence on patient satisfaction with care, posthospital disposition, and need for postoperative procedures, precluding conclusions on these outcomes. Of the studies that reported data on harms, there was no evidence increased harms among patients who participated in prehabilitation interventions compared to those who did not.

Although the largest number of studies addressed the comparative effectiveness of postoperative rehabilitation interventions versus a variety of comparators for patients who have undergone TKA for osteoarthritis, the evidence does not clearly establish whether any of the TKA rehabilitation programs (or attributes of programs, including exercise components, personnel, or setting) are more or less effective relative to each other. The pervasive diversity of rehabilitation programs that have been studied has resulted in an actual sparsity of evidence for any particular program or program attribute. In addition, the high degree of inconsistency in which outcomes have been evaluated and reported further precludes definitive conclusions. Nevertheless, there is some evidence to suggest that the diverse rehabilitation interventions were associated with comparable improvements in pain, ROM, strength (although some improvements in strength were observed following acute rehabilitation), and ADL. Compared with a variety of (usually less intensive) comparators, there is also some evidence to suggest that rehabilitation programs offered in the acute phase (initiated within 2 weeks of surgery) lead to similar satisfaction with care and rehabilitation programs delivered in the post-acute phase leads to similar quality of life (QoL). In contrast, the evidence is insufficient to establish the impact of rehabilitation on QoL (for acute rehabilitation), satisfaction with care (for post-acute rehabilitation), and the need for postoperative procedures (for both acute and post-acute rehabilitation). The evidence suggests that the risks of harms from post-acute rehabilitation interventions are low and comparable across groups; no studies reported evidence on harms from acute rehabilitation interventions.

The evidence informing the question of whether to offer prehabilitation to patients about to undergo total hip arthroplasty (THA) for osteoarthritis was the most limited across the four KQs. There was no or insufficient evidence for all patient-reported, performance-based, healthcare-utilization outcomes, and harms, precluding conclusions for the outcomes.

For patients undergoing rehabilitation following THA for osteoarthritis, there is some evidence to suggest that there is no difference between various rehabilitation interventions and less active (or no) rehabilitation interventions following THA in terms of pain, strength, ADLs or QoL. There was no or insufficient evidence for whether there is a difference between rehabilitation and various comparators in terms of hip range of motion or satisfaction with care, precluding conclusions for these outcomes. In terms of adverse events, the evidence suggests that 
the risks of harms from rehabilitation interventions are low (and of low severity) and are comparable across groups.

The most reassuring finding pertained to the safety of the prehabilitation and rehabilitation interventions. While the relative effectiveness of diverse programs for diverse outcomes remains uncertain, we found no evidence of increased harm from patients' participation in prehabilitation or rehabilitation programs prior to or after their TKA or THA, respectively.

Evidence was insufficient to comment on the costs, both indirect and direct, associated with prehabilitation and rehabilitation programs. Of particular note, we found no cost-effectiveness analyses regarding prehabilitation or rehabilitation. Despite this, many studies noted cost as a justification for their study question — particularly studies of rehabilitation after TKA and THA that evaluated the safety and effectiveness of rehabilitation delivered in lower-resource settings or by lower-resource personnel compared with higher-resource settings or personnel.

There was insufficient evidence of specific outcomes to explore heterogeneity of treatment effect of rehabilitation programs by patient, surgical, or setting factors. Thus, there is no evidence to help determine which patients, which specific surgeries, or which types of facilities would most benefit from rehabilitation programs (or any specific components of rehabilitation).

\section{Strengths and Limitations}

\section{Strengths and Limitations of the Evidence Base}

Although there have been numerous RCTs published on the topic of rehabilitation after TKA and THA (and to a lesser extent prehabilitation prior to TKA or THA), the evidence specific to almost all questions of interest is remarkably sparse. With the exception of three rehabilitation interventions evaluated in two pairs of studies each, every study evaluated a different (p)rehabilitation intervention comprised of different exercise goals and specific exercise components, delivered with varying timing and/or intensity, by different personnel, in different settings.

Adding to the complexity of diverse interventions, studies reported highly diverse outcomes - including not only different outcome domains, but also different scales and metrics within shared outcome domains (for example, different scores or tools or adapted versions of existing tools) - making it difficult to learn across studies. Studies were typically small to medium (with only 20 to 60 patients). The small sample sizes might have resulted in underpowered studies and, thus, misleadingly statistically nonsignificant findings. This is especially important for KQs 2 and 4 which compared various forms of active interventions (and thus would require a much larger sample to demonstrate an advantage of a likely smaller relative effect size). Relatively few studies directly assessed the setting of the intervention (i.e., evaluating the empirical effect of similar programs delivered in different settings). Among the 11 RCTs and 5 appropriately adjusted nonrandomized comparative studies (NRCSs) that did evaluate setting, all addressed postoperative rehabilitation. Most of these studies poorly reported the details of the rehabilitation programs (or did not report intervention content at all) making it difficult to compare with other studies. Many studies compared different intensity interventions conducted in different settings, making it difficult to disentangle the unique impact of setting from the intervention content (among other factors).

Progression is considered to be an important feature of well-designed (p)rehabilitation interventions to ensure programs of exercise are continuously adapted to specific patientperformance and do not become static. Yet, progression was rarely reported in our sample of 
studies, and where it was, was often deemed inappropriate by our clinician experts (i.e., patients were not progressed based on patient-specific parameters).

Across study designs, data were rarely reported for subgroups based on factors, such as surgical approach (posterior vs. anterior) or prosthesis affixing method (cement vs. no cement) and never reported within patient subgroups or setting subgroups. We were therefore unable to conclude whether there was (or was not) heterogeneity of treatment effects based on patient, surgical setting, or other characteristics.

We assessed most of the comparative studies (RCTs and NRCSs) to be at overall moderate or high risk of bias, primarily because participants, care providers, and/or outcome assessors were not blinded and because of uncertain or high-risk methods of randomization. While blinding of participants (i.e., patients) and care providers (i.e., physical therapist) will almost always be impossible in studies addressing the (p)rehabilitation KQs in this systematic review (SR), lack of blinding can still lead to bias and should therefore still be considered in interpreting results. Moreover, although for subjective patient-reported outcomes, such as pain and activities of daily living, it may be impossible to blind the outcome assessors (i.e., patients), it is possible to blind the outcome assessors (e.g., rehabilitation assistants) for objective outcomes of physical performance. The NRCSs were usually considered to be at moderate risk of confounding. In general, reporting of interventions and outcomes varied widely, ranging from thoroughly to poorly described.

\section{Strengths and Limitations of the Systematic Review Process}

We followed contemporary standards for conducting SRs, including multiple stakeholder engagement in KQ development and refinement and careful adherence to recommended methods for literature searching, screening, data extraction, risk of bias assessment, qualitative synthesis, quantitative synthesis (deemed inappropriate in this case), and strength of evidence (SoE) assessment. The SR was narrowly focused on the population of patients who have undergone (or are planning to undergo) total, unilateral TKA or THA due to osteoarthritis but broad inclusive to the range of (p)rehabilitation interventions evaluated (and diversity of their reporting) in this population.

During protocol development, we prioritized outcomes (and timing of outcomes) in consultation with panels of Key Informants and Technical Experts and in keeping with published core outcome sets for performance-based outcomes and patient- and performance-based outcomes pertinent to patients undergoing TKA or THA. ${ }^{59,60}$ However, many of the prioritized outcomes were either not reported in included studies or were reported in an insufficient number of studies to allow conclusions from our narrative synthesis (or to support meta-analysis or metaregression). Unreported or rarely reported patient-reported outcomes included QoL and satisfaction with care. Unreported or rarely reported performance-based outcomes (especially in studies of THA) included range of motion and strength. Healthcare-utilization outcomes were rarely reported across all studies. Eighty-one studies were excluded for reporting only short-term patient-reported and performance-based outcomes (i.e., $<3$ months for TKA and $<6$ months for THA). While these cutoffs were informed by stakeholder input, it may be considered a limitation for considering the evidence of early post-operative improvements in patient-reported and performance-based outcomes following TKA and THA.

One of the largest challenges we faced in this SR was that many factors were of interest that may affect outcomes and were combined in such diverse ways that it was difficult to disentangle their relative contribution and identify trends. We attempted to apply a systematic organization to 
both the heterogeneity of the interventions and the outcomes using the most contemporary approaches and evidence in the field, as informed by our technical and clinical experts. We hoped to conduct a network meta-analysis across the programs, focusing on comparisons of different intervention components. However, the described heterogeneity of interventions and the lack of consistency of reported outcome precluded meaningful quantitative syntheses.

We believe a strength and novel feature of this SR was our detailed coding of the "active ingredients" of the (p)rehabilitation interventions using a comprehensive taxonomy developed by Oatis and Franklin. ${ }^{52}$ For each intervention and comparator evaluated, we coded the specific exercises included in the program and goals these exercises sought to address (e.g., strength, flexibility). Systematic and standardized coding of the content of (p)rehabilitation interventions offers an important step forward in synthesizing (p)rehabilitation evidence by establishing a common language of the active ingredients contained within interventions to compare across studies. Such codes can be used decision makers to guide the replication of successful individual studies of interest and design future promising studies. While we were unable to link these codes to intervention outcomes (due to the large number of intervention components combined with the sparse reporting of specific outcomes across studies), the thorough process of coding each program provided insights into the general composition of (p)rehabilitation programs studied and what components were more or less commonly employed across studies. It also revealed the vast creativity and heterogeneity of the (p)rehabilitation interventions and the lack of reporting or standardization of "standard care" comparators. Prior to synthesizing results, we used these codes, combined with expert input to make sensible groupings of studies within KQs.

Despite the value of using this taxonomy, it was not without its limitations. The Oatis and Franklin taxonomy was designed to code interventions delivered to patients after TKA by physical therapists or physical therapy assistants in the outpatient or home health settings. As such, the taxonomy was excellent in capturing the interventions delivered in these settings and by these providers but faced certain challenges when applied to both TKA and THA, in a variety of settings, with a variety of providers (including patient-led). For example, some of the exercises used for the hip were not, or only crudely, reflected in the TKA-based taxonomy. As another example, some of the studies of interest included acute and/or inpatient rehabilitation. Thus, exercises that are only appropriate to these acute and/or inpatient rehabilitation settings were not always reflected in the taxonomy (e.g., open chain ankle strengthening exercises).

There were two aspects of the (p)rehabilitation programs, timing and intensity/dosage, that were not well-captured in this review. Regarding timing, the KQs separate prehabilitation from rehabilitation, which captures one aspect of timing. However, the extraction plan in capturing other aspects of timing within each KQ was limited, particularly for rehabilitation. For example, some studies investigated rehabilitation programs of equal length and in the same setting (e.g., 8 week outpatient program), yet started at different time points post-rehabilitation (e.g., at 1 week vs. 12 weeks). Rehabilitation at these stages target different goals and are not likely meaningful in comparison, yet this difference in the programs is masked in the current analysis. Future reviews should extract timing information in case it is meaningful for interpretation. Intensity and dosage, in any form (e.g., challenge of the exercise, duration of the session in minutes, duration of the program in days/weeks, number of sessions), were not captured in this review. Codes represented the presence or absence of the active ingredients and did not represent their dosage (i.e., an exercise only needed to be performed once in the program to be coded as present). This presents a major limitation in synthesizing the results to answer the KQs. In extracting and coding data according to the protocol, the investigators observed high variation 
with respect to intensity among the included studies which may have led to the observed variation in results. For example, some studies used limited or no resistance with strengthening exercises (with and without progressions), while other studies were specifically prescribed resistance and progression consistent with recommendations for increasing muscle strength. Some studies included two supervised sessions with rehabilitation personnel, while others included over 30 supervised sessions with rehabilitation personnel. Some studies reported 10minute intervention sessions while others reported 90-minute intervention sessions. Some studies investigated 2-week programs, while others investigated 16-week programs. This variation in intensity and dosage is not evident in this evidence synthesis yet may be an important factor driving outcomes. Given the high amount of variation in the other aspects of the extracted information, these details would only contribute to increased heterogeneity in addition to the heterogeneity already noted.

Finally, use pharmacological agents (particularly as adjunctive to (p)rehabilitation) may represent an important confounding variable but were considered beyond the scope of this review.

\section{Applicability}

Most studies in this SR were conducted outside the United States, particularly in Europe and high-or middle-income East Asian countries; a smaller portion of studies were conducted in Australia and North America, with only 14 studies conducted in the United States, While the relative effect of the interventions on clinical outcomes (and harms) from non-U.S.-based studies are likely applicable to the U.S. context, findings pertaining to healthcare system or resources (such as costs or comparisons of inpatient vs. outpatient rehabilitation) are likely country and healthcare system specific. For example, the relatively short inpatient stays in the United States (compared with other countries) may reduce the generalizability to the United States of comparisons of different settings conducted in other countries.

The sex ratios varied widely across studies, ranging from 27 to 100 percent of participants being female. The average age of patients ages ranged from 50 to 79 years and the average body mass indexes ranged from 25 to $35 \mathrm{~kg} / \mathrm{m}^{2}$ (thus, in all studies, most patients were obese, but in several, many to most were morbidly obese). Most studies did not report whether patients had undergone previous contralateral replacement surgery; of those that did, proportions were low (less than 25\%). As such, the conclusions in this SR are likely most applicable to middle-toolder-aged adults in high-income countries who are receiving their first total TKA or THA for osteoarthritis.

The applicability of the findings may also be limited to the specific interventions that have been studied and the particular healthcare settings in which they are intended to (or could feasibly) be delivered. Several interventions were resource intensive in terms of technology, personnel, or setting whereas others were designed to be less intensive in materials or human resources. Beyond resources, variation of rehabilitation practice - including terminology and outcomes of measurement - is well established, and thus application of novel interventions to novel settings may be challenging, especially if the details of interventions and how they are operationalized are poorly described.

\section{Implications for Clinical Practice}

The evidence base for the KQ addressing prehabilitation for TKA is limited. While there is some evidence that prehabilitation may lead to reduced lengths of stay in the acute post-operative 
period as compared to no prehabilitation, there is no clear indication that it leads to improvements in patient-reported, performance-based, or other healthcare-utilization outcomes. Follow-up for outcomes ranged from 3 to 12 months. Thus, the decision of whether or not to provide prehabilitation (or for patients, to participate in prehabilitation) appears to still largely be based on its hypothesized ability to increase patient function and capacity prior to surgery with the aim of quicker recovery afterwards. More evidence is needed to discern the relative effect of diverse prehabilitation programs to each other or specific components or methods of delivery of prehabilitation programs. As no studies provided evidence on patient satisfaction with receiving prehabilitation, it is difficult to determine the degree to which patients value receiving prehabilitation care to support their TKA recovery.

The evidence base for rehabilitation following TKA was largest, based on the number of studies included. Despite this, the evidence for the comparative value of specific rehabilitation interventions with their specific methods of delivery (timing, setting, personnel, progression) over comparator rehabilitation interventions was weak, due to sparsity of similar intervention/comparison/outcome combinations within group of studies. Follow-up for outcomes ranged from 3 to 24 months. There is some evidence to suggest that strength-based programs delivered in the acute phase after surgery, combined with adjunctive neuromuscular electrical stimulation may lead to reduced pain and increased strength. ${ }^{64,68,69}$ The evidence supporting other rehabilitation programs (or specific components and how they are delivered) is inconclusive.

The evidence base for prehabilitation prior to THA was smallest, and like prehabilitation prior to TKA, showed no clear evidence to inform decisions on whether or not to offer (or participate in) prehabilitation programs, or what components of prehabilitation following THA may be more or less effective. Follow-up ranged from 6 to 12 months. No study reported patient satisfaction with prehabilitation for THA.

Similar to rehabilitation following THA, there is limited evidence of the comparative effectiveness of diverse rehabilitation programs following THA with respect to patient-reported, performance-based or healthcare-utilization outcomes and no evidence of what specific attributes of interventions (e.g., component goals or specific exercises, personnel, setting) are associated with better or worse outcomes. Follow-up ranged from 6 to 60 months.

In the absence of definitive evidence, stakeholders may want to use other decision-making factors to decide whether or not to implement prehabilitation or what specific rehabilitation program to implement. We have provided detailed extraction of content of these programs (overlaid with a standardized taxonomy) which decision makers may consider in conjunction with our extraction of outcomes. For example, decision makers considering (p)rehabilitation programs can identify studies in our review that are similar to programs that are being offered in their context and explore how these studies have performed on their prioritized outcomes or consider components of similar interventions that could be added to their own. Additionally, health systems could look at programs that are feasible to consider for implementation based on their available resources.

\section{Implications for Research}

Further research is needed to address various questions set out by this SR to determine the comparative effectiveness of diverse prehabilitation and rehabilitation interventions delivered in various settings by various personnel. More than just the conduct of large, well-executed RCTs, further work is needed by funders, researchers, and clinicians to orchestrate an overall 
program of rehabilitation research that supports more efficient and synergistic learning across studies: namely through standardized labeling, defining, and reporting of the various (p)rehabilitation interventions and consistent use of a core set of patient-reported, performancebased, and healthcare-utilization outcomes. Such work is crucial if we are to efficiently build a body of rehabilitation evidence that truly seeks to enable evidence-based decision making about which interventions should be offered to adults undergoing TKA or THA for osteoarthritis to achieve the best clinical outcomes, reduce avoidable complications or joint failures, and be costand resource-effective for the health system, patients, and their caregivers.

For interventions, we found that the taxonomy of exercises created by Oatis and Franklin, which grouped interventions within goal components, offers a feasible foundation for a categorization scheme to design, implement, and report (p)rehabilitation interventions. ${ }^{52}$ Consistent use of an agreed-upon taxonomy, adopted by the field, would greatly enhance the validity and efficiency of subsequent evidence syntheses of these studies, and ideally lend itself to more robust quantitative syntheses, such as multivariable meta-regression to estimate the independent effects of specific components. ${ }^{160}$

Regarding outcomes, we are aware of core performance-based outcome sets for many outcomes ${ }^{59}$ but not for patient-reported or healthcare-utilization outcomes. Despite restricting our evidence to contemporary studies (since_2005), we found that reported outcomes were highly heterogenous and often did not include core outcomes. Further work is needed to define what core outcomes are essential to evaluation of (p)rehabilitation after TKA and THA and ideally that such outcomes become expected standards for funding of studies and publishing of their results. Many of the studies included in this review had moderate to high risk of bias, in part due to lack of blinding of clinicians, patients, and outcome assessors. While it is difficult to blind clinicians and patients in (p)rehabilitation studies, threats to bias may be minimized by improved blinding of outcomes. Specifically, studies may blind outcome assessors (for performance-based outcomes) and analysts (for all outcome). Thus, in addition to considering what core outcomes should be included in studies, stakeholders (including funders) should consider standards for how such core outcomes should be collected and analyzed to minimize threats to bias.

Finally, for reporting, future studies may consider using the Template for Intervention Description and Replication (TIDieR) checklist to ensure comprehensive reporting of all intervention elements (who, what, where, how, etc.). ${ }^{161}$ Lenguerrand 2020 used the TIDIER checklist, which greatly enhanced our ability to code the intervention content for this study. Transparent and comprehensive reporting of rehabilitation interventions, including any active (p)rehabilitation components delivered to comparator arms, will greatly improve both the capacity of practitioners to apply (effective) interventions in practice and support more robust and informative evidence synthesis. Related to interventions, outcomes, and reporting, future studies (and reviews) should consider being more explicit in considering the therapeutic validity of interventions and matching outcomes to theoretically linked intervention content. ${ }^{162}$

Given the absence of evidence on costs across all KQs (despite many studies noting cost as a justification for their study question), future studies should consider collecting information on the direct and indirect costs of (p)rehabilitation for TKA and THA and consider conducting a CEA analysis alongside effectiveness analyses. Jette et al. make similar calls for action in rehabilitation research for TKA and note additional implications such as need for coordinated multisite programs of research to facilitate larger sample sizes, replication studies to enhance investigation of promising studies, and interventions grounded in theory, among others. ${ }^{163}$ 
Despite being an important feature of well-designed (p)rehabilitation interventions, progression was often not reported in our sample of studies, and where it was, was often deemed inappropriate by our clinician experts. Future studies should report whether progression was performed or not and if performed, on what patient parameters progression was based.

An important question remains regarding which patients might most benefit from (p)rehabilitation or from any specific component of therapy. However, there is sparse evidence to address this question. Future studies should be large enough, and thus sufficiently powered, to adequately address a priori aspects of heterogeneity of treatment effect.

\section{Conclusions}

Although we found a large body of evidence, we were able to make only a few specific conclusions in this SR, all of which were based on insufficient or low SoE. Generally speaking, there is low SoE that various forms of prehabilitation and rehabilitation may result in comparable pain, ROM, and strength; and insufficient SoE for ADLs, patient satisfaction, and need for postoperative procedures. There is low SoE that prehabilitation may lead to reduced length of stay for following TKA and low SoE that adverse events (where reported) due to (p)rehabilitation are low and comparable among groups. Future research, ideally large RCTs, is needed to compare the relative effectiveness of well specified ( $p$ )rehabilitation interventions, delivered by varying personnel, in different settings, on a core set of patient-reported, performance-based, and healthcare-utilization outcomes. 


\section{References}

1. National Center for Chronic Disease Prevention and Health Promotion (NCCDPHP). Arthritis. Atlanta, GA: National Center for Chronic Disease Prevention and Health Promotion; 2019. https://www.cdc.gov/chronicdisease/resourc es/publications/factsheets/arthritis.htm. Accessed on June 16, 2020.

2. Ethgen $O$, Bruyère $O$, Richy $F$, et al. Healthrelated quality of life in total hip and total knee arthroplasty. A qualitative and systematic review of the literature. J Bone Joint Surg Am. 2004 May;86(5):963-74. doi: 10.2106/00004623-200405000-00012. PMID: 15118039.

3. Rissanen $\mathrm{P}$, Aro S, Slätis $\mathrm{P}$, et al. Health and quality of life before and after hip or knee arthroplasty. J Arthroplasty. 1995 Apr;10(2):169-75. doi: 10.1016/s08835403(05)80123-8. PMID: 7798097.

4. March LM, Cross MJ, Lapsley H, et al. Outcomes after hip or knee replacement surgery for osteoarthritis. A prospective cohort study comparing patients' quality of life before and after surgery with age-related population norms. Med J Aust. 1999 Sep 6;171(5):235-8. PMID: 10495753.

5. Quinn RH, Murray J, Pezold R, et al. Management of Osteoarthritis of the Hip. J Am Acad Orthop Surg. 2018 Oct 15;26(20):e434-e6. doi: 10.5435/jaaos-d-18-00351. PMID: 30134309 .

6. Mizner RL, Petterson SC, Stevens JE, et al. Preoperative quadriceps strength predicts functional ability one year after total knee arthroplasty. J Rheumatol. 2005 Aug;32(8):1533-9. PMID: 16078331.

7. Whitehurst MA, Johnson BL, Parker CM, et al. The benefits of a functional exercise circuit for older adults. J Strength Cond Res. 2005 Aug;19(3):647-51. doi: 10.1519/r-14964.1. PMID: 16095420.

8. Singh JA. Epidemiology of knee and hip arthroplasty: a systematic review. Open Orthop J. 2011 Mar 16;5:80-5. doi: 10.2174/1874325001105010080. PMID: 21584277 .
9. Cavanaugh AM, Rauh MJ, Thompson CA, et al. Rehabilitation After Total Knee Arthroplasty: Do Racial Disparities Exist? J Arthroplasty. 2020 Mar;35(3):683-9. doi: 10.1016/j.arth.2019.10.048. PMID: 31801659.

10. . Projected volume of primary and revision total joint arthroplasty in the United States, 20302060. American Academy of Orthopaedic Surgeons 2018 Annual Meeting (New Orleans, LA); 2018.

11. Campbell P, Shen FW, McKellop H. Biologic and tribologic considerations of alternative bearing surfaces. Clin Orthop Relat Res. 2004 Jan(418):98-111. doi: 10.1097/00003086-200401000-00017. PMID: 15043100.

12. D'Antonio JA, Sutton K. Ceramic materials as bearing surfaces for total hip arthroplasty. J Am Acad Orthop Surg. 2009 Feb;17(2):638. PMID: 19202119.

13. Jacobs CA, Christensen CP, Greenwald AS, et al. Clinical performance of highly cross-linked polyethylenes in total hip arthroplasty. J Bone Joint Surg Am. 2007 Dec;89(12):2779-86. doi: 10.2106/jbjs.G.00043. PMID: 18056513.

14. López-López JA, Humphriss RL, Beswick AD, et al. Choice of implant combinations in total hip replacement: systematic review and network meta-analysis. Bmj. 2017 Nov 2;359:j4651. doi: 10.1136/bmj.j4651. PMID: 29097396.

15. Meneghini RM, Hallab NJ, Jacobs JJ. The biology of alternative bearing surfaces in total joint arthroplasty. Instr Course Lect. 2005;54:481-93. PMID: 15948473.

16. Rajpura A, Kendoff D, Board TN. The current state of bearing surfaces in total hip replacement. Bone Joint J. 2014 Feb;96b(2):147-56. doi: 10.1302/0301620x.96b2.31920. PMID: 24493177. 
17. Bragdon CR, Doerner M, Martell J, et al. The 2012 John Charnley Award: Clinical multicenter studies of the wear performance of highly crosslinked remelted polyethylene in THA. Clin Orthop Relat Res. 2013 Feb;471(2):393-402. doi: 10.1007/s11999012-2604-0. PMID: 23070661.

18. Currier BH, Currier JH, Franklin KJ, et al. Comparison of Wear and Oxidation in Retrieved Conventional and Highly CrossLinked UHMWPE Tibial Inserts. J Arthroplasty. 2015 Dec;30(12):2349-53. doi: 10.1016/j.arth.2015.06.014. PMID: 26143237.

19. Prock-Gibbs H, Pumilia CA, Meckmongkol T, et al. Incidence of Osteolysis and Aseptic Loosening Following Metal-on-Highly Cross-Linked Polyethylene Hip Arthroplasty: A Systematic Review of Studies with Up to 15-Year Follow-up. J Bone Joint Surg Am. 2021 Apr 21;103(8):728-40. doi: 10.2106/jbjs.20.01086. PMID: 33411465 .

20. Galmiche R, Poitras S, Dobransky J, et al. Does surgical approach influence mid- to longterm patient-reported outcomes after primary total hip replacement? A comparison of the 3 main surgical approaches. Can J Surg. 2020 Apr 17;63(22):E181-e9. doi: 10.1503/cjs.008919. PMID: 32302085.

21. Moretti VM, Post ZD. Surgical Approaches for Total Hip Arthroplasty. Indian J Orthop. 2017 Jul-Aug;51(4):368-76. doi: 10.4103/ortho.IJOrtho_317_16. PMID: 28790465.

22. Putananon C, Tuchinda H, Arirachakaran A, et al. Comparison of direct anterior, lateral, posterior and posterior-2 approaches in total hip arthroplasty: network meta-analysis. Eur J Orthop Surg Traumatol. 2018 Feb;28(2):255-67. doi: 10.1007/s00590-0172046-1. PMID: 28956180.

23. Christensen JC, Paxton RJ, Baym C, et al. Benefits of direct patient discharge to outpatient physical therapy after total knee arthroplasty. Disabil Rehabil. 2020 Mar;42(5):660-6. doi: 10.1080/09638288.2018.1505968. PMID: 30616406 .
24. Ewen AM, Stewart S, St Clair Gibson A, et al. Post-operative gait analysis in total hip replacement patients-a review of current literature and meta-analysis. Gait Posture. 2012 May;36(1):1-6. doi: 10.1016/j.gaitpost.2011.12.024. PMID: 22410129.

25. Jensen C, Aagaard P, Overgaard S. Recovery in mechanical muscle strength following resurfacing vs standard total hip arthroplasty - a randomised clinical trial. Osteoarthritis Cartilage. 2011 Sep;19(9):1108-16. doi: 10.1016/j.joca.2011.06.011. PMID: 21749928.

26. Kolk S, Minten MJ, van Bon GE, et al. Gait and gait-related activities of daily living after total hip arthroplasty: a systematic review. Clin Biomech (Bristol, Avon). 2014 Jun;29(6):705-18. doi: 10.1016/j.clinbiomech.2014.05.008. PMID: 24951319.

27. Maffiuletti NA, Bizzini M, Widler K, et al. Asymmetry in quadriceps rate of force development as a functional outcome measure in TKA. Clin Orthop Relat Res. 2010 Jan;468(1):191-8. doi: 10.1007/s11999-009-0978-4. PMID: 19597897.

28. Valtonen A, Pöyhönen T, Heinonen A, et al. Muscle deficits persist after unilateral knee replacement and have implications for rehabilitation. Phys Ther. 2009 Oct;89(10):1072-9. doi: 10.2522/ptj.20070295. PMID: 19713269.

29. Bourne RB, Chesworth BM, Davis AM, et al. Patient satisfaction after total knee arthroplasty: who is satisfied and who is not? Clin Orthop Relat Res. 2010 Jan;468(1):57-63. doi: 10.1007/s11999-0091119-9. PMID: 19844772.

30. Doma K, Grant A, Morris J. The Effects of Balance Training on Balance Performance and Functional Outcome Measures Following Total Knee Arthroplasty: A Systematic Review and Meta-Analysis. Sports Med. 2018 Oct;48(10):2367-85. doi: 10.1007/s40279-018-0964-7. PMID: 30117054. 
31. Wu JQ, Mao LB, Wu J. Efficacy of exercise for improving functional outcomes for patients undergoing total hip arthroplasty: A metaanalysis. Medicine (Baltimore). 2019 Mar;98(10):e14591. doi: 10.1097/md.0000000000014591. PMID: 30855443 .

32. Snow R, Granata J, Ruhil AV, et al. Associations between preoperative physical therapy and post-acute care utilization patterns and cost in total joint replacement. J Bone Joint Surg Am. 2014 Oct 1;96(19):e165. doi: 10.2106/jbjs.M.01285. PMID: 25274793.

33. Fortin PR, Clarke AE, Joseph L, et al. Outcomes of total hip and knee replacement: preoperative functional status predicts outcomes at six months after surgery. Arthritis Rheum. 1999 Aug;42(8):1722-8. doi: 10.1002/15290131(199908)42:8<1722::Aidanr22>3.0.Co;2-r. PMID: 10446873.

34. Lingard EA, Katz JN, Wright EA, et al. Predicting the outcome of total knee arthroplasty. J Bone Joint Surg Am. 2004 Oct;86(10):2179-86. doi: 10.2106/00004623-200410000-00008. PMID: 15466726.

35. Peer MA, Rush R, Gallacher PD, et al. Presurgery exercise and post-operative physical function of people undergoing knee replacement surgery: A systematic review and meta-analysis of randomized controlled trials. J Rehabil Med. 2017 Apr 6;49(4):30415. doi: 10.2340/16501977-2210. PMID: 28352936.

36. Moyer R, Ikert K, Long K, et al. The Value of Preoperative Exercise and Education for Patients Undergoing Total Hip and Knee Arthroplasty: A Systematic Review and Meta-Analysis. JBJS Rev. 2017 Dec;5(12):e2. doi: 10.2106/jbjs.Rvw.17.00015. PMID: 29232265.

37. Sharma R, Ardebili MA, Abdulla IN. Does Rehabilitation before Total Knee Arthroplasty Benefit Postoperative Recovery? A Systematic Review. Indian J Orthop. 2019 Jan-Feb;53(1):138-47. doi: 10.4103/ortho.IJOrtho_643_17. PMID: 30905994 .
38. International Classification of Functioning, Disability and Health (ICF). Geneva, Switzerland: World Health Organization; 2018. https://www.who.int/classifications/icf/en/. Accessed on April 30, 2020.

39. Henderson KG, Wallis JA, Snowdon DA. Active physiotherapy interventions following total knee arthroplasty in the hospital and inpatient rehabilitation settings: a systematic review and meta-analysis. Physiotherapy. 2018 Mar;104(1):25-35. doi: 10.1016/j.physio.2017.01.002. PMID: 28802773.

40. Naylor JM, Hart A, Harris IA, et al. Variation in rehabilitation setting after uncomplicated total knee or hip arthroplasty: a call for evidence-based guidelines. BMC Musculoskelet Disord. 2019 May 15;20(1):214. doi: 10.1186/s12891-0192570-8. PMID: 31092230.

41. Hansen S, Aaboe J, Mechlenburg I, et al. Effects of supervised exercise compared to nonsupervised exercise early after total hip replacement on patient-reported function, pain, health-related quality of life and performance-based function - a systematic review and meta-analysis of randomized controlled trials. Clin Rehabil. 2019 Jan;33(1):13-23. doi: 10.1177/0269215518791213. PMID: 30073856 .

42. Sattler LN, Hing WA, Vertullo CJ. What is the evidence to support early supervised exercise therapy after primary total knee replacement? A systematic review and metaanalysis. BMC Musculoskelet Disord. 2019 Jan 29;20(1):42. doi: 10.1186/s12891-0192415-5. PMID: 30696416.

43. Bozic KJ, Ward L, Vail TP, et al. Bundled payments in total joint arthroplasty: targeting opportunities for quality improvement and cost reduction. Clin Orthop Relat Res. 2014 Jan;472(1):188-93. doi: 10.1007/s1 1999-013-3034-3. PMID: 23649225.

44. Haas DA, Kaplan RS. Variation in the cost of care for primary total knee arthroplasties. Arthroplast Today. 2017 Mar;3(1):33-7. doi: 10.1016/j.artd.2016.08.001. PMID: 28378004 . 
45. Services CfMM. Comprehensive Care for Joint Replacement Model. CMS.gov; 2021. https://innovation.cms.gov/innovationmodels/cjr. Accessed on 06/25/21.

46. Weeks WB, Schoellkopf WJ, Ballard DJ, et al. Episode-of-Care Characteristics and Costs for Hip and Knee Replacement Surgery in Hospitals Belonging to the High Value Healthcare Collaborative Compared With Similar Hospitals in the Same Health Care Markets. Med Care. 2017 Jun;55(6):583-9. doi: 10.1097/mlr.0000000000000710. PMID: 28319584.

47. Chandra A, Dalton MA, Holmes J. Large increases in spending on postacute care in Medicare point to the potential for cost savings in these settings. Health Aff (Millwood). 2013 May;32(5):864-72. doi: 10.1377/hlthaff.2012.1262. PMID: 23650319.

48. Ponnusamy KE, Naseer Z, El Dafrawy MH, et al. Post-Discharge Care Duration, Charges, and Outcomes Among Medicare Patients After Primary Total Hip and Knee Arthroplasty. J Bone Joint Surg Am. 2017 Jun 7;99(11):e55. doi: 10.2106/jbjs.16.00166. PMID: 28590385.

49. Liberati A, Altman DG, Tetzlaff J, et al. The PRISMA statement for reporting systematic reviews and meta-analyses of studies that evaluate healthcare interventions: explanation and elaboration. Bmj. $2009 \mathrm{Jul}$ 21;339:b2700. doi: 10.1136/bmj.b2700. PMID: 19622552.

50. Shea BJ, Reeves BC, Wells G, et al. AMSTAR 2: a critical appraisal tool for systematic reviews that include randomised or nonrandomised studies of healthcare interventions, or both. Bmj. $2017 \mathrm{Sep}$ 21;358:j4008. doi: 10.1136/bmj.j4008. PMID: 28935701.

51. Zhu S, Qian W, Jiang C, et al. Enhanced recovery after surgery for hip and knee arthroplasty: a systematic review and meta-analysis. Postgrad Med J. 2017 Dec;93(1106):736-42. doi: 10.1136/postgradmedj-2017-134991. PMID: 28751437.

52. Franklin PD. Defining Components of Physical Therapy Achieving Maximum Function after TKR. Northwestern University at Chicago, Chicago, IL, United States; 2019.
53. Oatis CA, Johnson JK, DeWan T, et al. Characteristics of Usual Physical Therapy Post-Total Knee Replacement and Their Associations With Functional Outcomes. Arthritis Care Res (Hoboken). 2019 Sep;71(9):1171-7. doi: 10.1002/acr.23761. PMID: 30281207.

54. Higgins JP, Altman DG, Gøtzsche PC, et al. The Cochrane Collaboration's tool for assessing risk of bias in randomised trials. Bmj. 2011 Oct 18;343:d5928. doi: 10.1136/bmj.d5928. PMID: 22008217.

55. National Heart, Lung, and Blood Institute. Study Quality Assessment Tools.; 2019. https://www.nhlbi.nih.gov/healthtopics/study-quality-assessment-tools. Accessed on January 23, 2020.

56. Sterne JA, Hernán MA, Reeves BC, et al. ROBINS-I: a tool for assessing risk of bias in non-randomised studies of interventions. Bmj. 2016 Oct 12;355:i4919. doi: 10.1136/bmj.i4919. PMID: 27733354.

57. Berkman ND, Lohr KN, Ansari M, et al. AHRQ Methods for Effective Health Care

Grading the Strength of a Body of Evidence When Assessing Health Care Interventions for the Effective Health Care Program of the Agency for Healthcare Research and Quality: An Update. Methods Guide for Effectiveness and Comparative Effectiveness Reviews. Rockville (MD): Agency for Healthcare Research and Quality (US); 2008.

58. Berkman ND, Lohr KN, Ansari MT, et al. Grading the strength of a body of evidence when assessing health care interventions: an EPC update. J Clin Epidemiol. 2015 Nov;68(11):1312-24. doi: 10.1016/j.jclinepi.2014.11.023. PMID: 25721570 .

59. Dobson F, Hinman RS, Roos EM, et al. OARSI recommended performance-based tests to assess physical function in people diagnosed with hip or knee osteoarthritis. Osteoarthritis Cartilage. 2013 Aug;21(8):1042-52. doi: 10.1016/j.joca.2013.05.002. PMID: 23680877. 
60. Westby MD, Brittain A, Backman CL. Expert consensus on best practices for post-acute rehabilitation after total hip and knee arthroplasty: a Canada and United States Delphi study. Arthritis Care Res (Hoboken). 2014 Mar;66(3):411-23. doi: 10.1002/acr.22164. PMID: 24023047.

61. Williamson PR, Altman DG, Blazeby JM, et al. Developing core outcome sets for clinical trials: issues to consider. Trials. $2012 \mathrm{Aug}$ 6;13:132. doi: 10.1186/1745-6215-13-132. PMID: 22867278.

62. Atkins D, Best D, Briss PA, et al. Grading quality of evidence and strength of recommendations. Bmj. 2004 Jun 19;328(7454):1490. doi: 10.1136/bmj.328.7454.1490. PMID: 15205295 .

63. Topp R, Swank AM, Quesada PM, et al. The effect of prehabilitation exercise on strength and functioning after total knee arthroplasty. Pm r. 2009 Aug;1(8):729-35. doi: 10.1016/j.pmrj.2009.06.003. PMID: 19695525.

64. Villadsen A, Overgaard S, Holsgaard-Larsen A, et al. Postoperative effects of neuromuscular exercise prior to hip or knee arthroplasty: a randomised controlled trial. Ann Rheum Dis. 2014 Jun;73(6):1130-7. doi: 10.1136/annrheumdis-2012-203135. PMID: 23661494.

65. Calatayud J, Casaña J, Ezzatvar Y, et al. Highintensity preoperative training improves physical and functional recovery in the early post-operative periods after total knee arthroplasty: a randomized controlled trial. Knee Surg Sports Traumatol Arthrosc. 2017 Sep;25(9):2864-72. doi: 10.1007/s00167016-3985-5. PMID: 26768606.

66. Casaña J, Calatayud J, Ezzatvar Y, et al. Preoperative high-intensity strength training improves postural control after TKA: randomized-controlled trial. Knee Surg Sports Traumatol Arthrosc. 2019 Apr;27(4):1057-66. doi: 10.1007/s00167018-5246-2. PMID: 30361758.
67. Huang SW, Chen PH, Chou YH. Effects of a preoperative simplified home rehabilitation education program on length of stay of total knee arthroplasty patients. Orthop Traumatol Surg Res. 2012 May;98(3):25964. doi: 10.1016/j.otsr.2011.12.004. PMID: 22480863.

68. Huber EO, de Bie RA, Roos EM, et al. Effect of pre-operative neuromuscular training on functional outcome after total knee replacement: a randomized-controlled trial. BMC Musculoskelet Disord. 2013 May 3;14:157. doi: 10.1186/1471-2474-14-157. PMID: 23641782.

69. Huber EO, Roos EM, Meichtry A, et al. Effect of preoperative neuromuscular training (NEMEX-TJR) on functional outcome after total knee replacement: an assessor-blinded randomized controlled trial. BMC Musculoskelet Disord. 2015 Apr 25;16:101. doi: 10.1186/s12891-015-0556-8. PMID: 25925404.

70. Mitchell C, Walker J, Walters S, et al. Costs and effectiveness of pre- and post-operative home physiotherapy for total knee replacement: randomized controlled trial. J Eval Clin Pract. 2005 Jun;11(3):283-92. doi: 10.1111/j.1365-2753.2005.00535.x. PMID: 15869558 .

71. Matassi F, Duerinckx J, Vandenneucker H, et al. Range of motion after total knee arthroplasty: the effect of a preoperative home exercise program. Knee Surg Sports Traumatol Arthrosc. 2014 Mar;22(3):703-9. doi: 10.1007/s00167-012-2349-z. PMID: 23271039.

72. Skoffer B, Maribo T, Mechlenburg I, et al. Efficacy of Preoperative Progressive Resistance Training on Postoperative Outcomes in Patients Undergoing Total Knee Arthroplasty. Arthritis Care Res (Hoboken). 2016 Sep;68(9):1239-51. doi: 10.1002/acr.22825. PMID: 26713665.

73. Soeters R, White PB, Murray-Weir M, et al. Preoperative Physical Therapy Education Reduces Time to Meet Functional Milestones After Total Joint Arthroplasty. Clin Orthop Relat Res. 2018 Jan;476(1):408. doi: 10.1007/s11999.0000000000000010. PMID: 29529614. 
74. Soni A, Joshi A, Mudge N, et al. Supervised exercise plus acupuncture for moderate to severe knee osteoarthritis: a small randomised controlled trial. Acupunct Med. 2012 Sep;30(3):176-81. doi: 10.1136/acupmed-2012-010128. PMID: 22914302.

75. Valtonen A, Pöyhönen T, Manninen M, et al. Effects of preoperative aquatic resistance training on knee pain, mobility limitation and muscle impairments in people with latestage knee osteoarthritis. Physiotherapy (united kingdom). 2015;101:eS1568-eS9. doi: 10.1016/j.physio.2015.03.1570. PMID: CN-01126383.

76. Williamson L, Wyatt MR, Yein K, et al. Severe knee osteoarthritis: a randomized controlled trial of acupuncture, physiotherapy (supervised exercise) and standard management for patients awaiting knee replacement. Rheumatology (Oxford). 2007 Sep;46(9):1445-9. doi:

10.1093/rheumatology/kem119. PMID: 17604311.

77. Andersen HH. Technological assisted rehabilitation following total knee joint replacement. a randomised controlled noninferiority trial. Annals of the rheumatic diseases. 2018;77:1823-4. doi: 10.1136/annrheumdis-2018-eular.6484. PMID: CN-01647420.

78. Artz N, Dixon S, Wylde V, et al. Comparison of group-based outpatient physiotherapy with usual care after total knee replacement: a feasibility study for a randomized controlled trial. Clin Rehabil. 2017 Apr;31(4):487-99. doi: 10.1177/0269215516642503. PMID: 27068368.

79. Avramidis K, Karachalios T, Popotonasios K, et al. Does electric stimulation of the vastus medialis muscle influence rehabilitation after total knee replacement? Orthopedics. 2011 Mar 11;34(3):175. doi: 10.3928/01477447-20110124-06. PMID: 21410130 .

80. Bade MJ, Struessel T, Dayton M, et al. Early High-Intensity Versus Low-Intensity Rehabilitation After Total Knee Arthroplasty: A Randomized Controlled Trial. Arthritis Care Res (Hoboken). 2017 Sep;69(9):1360-8. doi: 10.1002/acr.23139. PMID: 27813347.
81. Bily W, Franz C, Trimmel L, et al. Effects of Leg-Press Training With Moderate Vibration on Muscle Strength, Pain, and Function After Total Knee Arthroplasty: A Randomized Controlled Trial. Arch Phys Med Rehabil. 2016 Jun;97(6):857-65. doi: 10.1016/j.apmr.2015.12.015. PMID: 26763947.

82. Bruun-Olsen V, Heiberg KE, Wahl AK, et al. The immediate and long-term effects of a walking-skill program compared to usual physiotherapy care in patients who have undergone total knee arthroplasty (TKA): a randomized controlled trial. Disabil Rehabil. 2013;35(23):2008-15. doi: 10.3109/09638288.2013.770084. PMID: 23614370 .

83. Buhagiar MA, Naylor JM, Harris IA, et al. Effect of Inpatient Rehabilitation vs a Monitored Home-Based Program on Mobility in Patients With Total Knee Arthroplasty: The HIHO Randomized Clinical Trial. Jama. 2017 Mar 14;317(10):1037-46. doi: 10.1001/jama.2017.1224. PMID: 28291891.

84. Buhagiar MA, Naylor JM, Harris IA, et al. Hospital Inpatient versus HOme-based rehabilitation after knee arthroplasty (The HIHO study): study protocol for a randomized controlled trial. Trials. 2013 Dec 17;14:432. doi: 10.1186/1745-6215-14432. PMID: 24341348.

85. Cai L, Gao H, Xu H, et al. Does a Program Based on Cognitive Behavioral Therapy Affect Kinesiophobia in Patients Following Total Knee Arthroplasty? A Randomized, Controlled Trial With a 6-Month FollowUp. Journal of Arthroplasty. 2018;33(3):704-10. doi: 10.1016/j.arth.2017.10.035. PMID: 127919407. Language: English. Entry Date: 20181111. Revision Date: 20181109. Publication Type: journal article. Journal Subset: Biomedical.

86. Chan HY, Sultana R, Yeo SJ, et al. Comparison of outcome measures from different pathways following total knee arthroplasty. Singapore Med J. 2018 Sep;59(9):476-86. doi: 10.11622/smedj.2018011. PMID: 29372260 . 
87. Demircioglu DT, Paker N, Erbil E, et al. The effect of neuromuscular electrical stimulation on functional status and quality of life after knee arthroplasty: a randomized controlled study. J Phys Ther Sci. 2015 Aug;27(8):2501-6. doi: 10.1589/jpts.27.2501. PMID: 26355656.

88. den Hertog A, Gliesche K, Timm J, et al. Pathway-controlled fast-track rehabilitation after total knee arthroplasty: a randomized prospective clinical study evaluating the recovery pattern, drug consumption, and length of stay. Arch Orthop Trauma Surg. 2012 Aug;132(8):1153-63. doi: 10.1007/s00402-012-1528-1. PMID: 22643801.

89. Fransen M, Nairn L, Bridgett L, et al. Post-Acute Rehabilitation After Total Knee Replacement: A Multicenter Randomized Clinical Trial Comparing Long-Term Outcomes. Arthritis Care Res (Hoboken). 2017 Feb;69(2):192-200. doi: 10.1002/acr.23117. PMID: 27868384.

90. Hamilton DF, Beard DJ, Barker KL, et al. Targeting rehabilitation to improve outcomes after total knee arthroplasty in patients at risk of poor outcomes: randomised controlled trial. bmj. 2020;371.

91. Harmer AR, Naylor JM, Crosbie J, et al. Landbased versus water-based rehabilitation following total knee replacement: a randomized, single-blind trial. Arthritis Rheum. 2009 Feb 15;61(2):184-91. doi: 10.1002/art.24420. PMID: 19177536.

92. Heikkilä A, Sevander-Kreus N, Häkkinen A, et al. Effect of total knee replacement surgery and postoperative 12 month home exercise program on gait parameters. Gait Posture. 2017 Mar;53:92-7. doi: 10.1016/j.gaitpost.2017.01.004. PMID: 28119232.

93. Jin C, Feng Y, Ni Y, et al. Virtual reality intervention in postoperative rehabilitation after total knee arthroplasty: a prospective and randomized controlled clinical trial. International journal of clinical and experimental medicine. 2018;11(6):6119-24. PMID: CN-01617489.
94. Kauppila AM, Kyllönen E, Ohtonen P, et al. Multidisciplinary rehabilitation after primary total knee arthroplasty: a randomized controlled study of its effects on functional capacity and quality of life. Clin Rehabil. 2010 May;24(5):398-411. doi: 10.1177/0269215509346089. PMID: 20354057.

95. Kauppila AM, Sintonen H, Aronen P, et al. Economic evaluation of multidisciplinary rehabilitation after primary total knee arthroplasty based on a randomized controlled trial. Arthritis Care Res (Hoboken). 2011 Mar;63(3):335-41. doi: 10.1002/acr.20398. PMID: 21080347.

96. Lenguerrand E, Artz N, Marques E, et al. Effect of Group-Based Outpatient Physical Therapy on Function After Total Knee Replacement: Results From a Multicenter Randomized Controlled Trial. Arthritis Care Res (Hoboken). 2020 Jun;72(6):768-77. doi: 10.1002/acr.23909. PMID: 31033232.

97. Lenssen AF, Crijns YH, Waltjé EM, et al. Efficiency of immediate postoperative inpatient physical therapy following total knee arthroplasty: an RCT. BMC Musculoskelet Disord. 2006 Aug 31;7:71. doi: 10.1186/1471-2474-7-71. PMID: 16942627.

98. Li J, Wu T, Xu Z, et al. A pilot study of post-total knee replacement gait rehabilitation using lower limbs robot-assisted training system. Eur J Orthop Surg Traumatol. 2014 Feb;24(2):203-8. doi: 10.1007/s00590-0121159-9. PMID: 23412304.

99. Li L, Cheng S, Wang G, et al. Tai chi chuan exercises improve functional outcomes and quality of life in patients with primary total knee arthroplasty due to knee osteoarthritis. Complement Ther Clin Pract. 2019 May;35:121-5. doi: 10.1016/j.ctcp.2019.02.003. PMID: 31003647.

100. Li L, Wang Z, Yin MH, et al. Effect of early gait training on the functional rehabilitation after total knee arthroplasty. Chinese journal of tissue engineering research. 2017;21(27):4288-93. doi: 10.3969/j.issn.2095-4344.2017.27.005. PMID: CN-01419703. 
101. Li Z, Jiang L, Lin J. The effect of education for daily physical activity level recovery of osteoarthritis patients after total knee arthroplasty. A prospective randomized controlled clinical trial using accelerometry. Osteoarthritis and cartilage. 2015;23:A373. PMID: CN-01084888.

102. Liao CD, Lin LF, Huang YC, et al. Functional outcomes of outpatient balance training following total knee replacement in patients with knee osteoarthritis: a randomized controlled trial. Clin Rehabil. 2015 Sep;29(9):855-67. doi: 10.1177/0269215514564086. PMID: 25552523.

103. Liao CD, Tsauo JY, Chiu YS, et al. Effects of Elastic Resistance Exercise After Total Knee Replacement on Muscle Mass and Physical Function in Elderly Women With Osteoarthritis: A Randomized Controlled Trial. Am J Phys Med Rehabil. 2020 May;99(5):381-9. doi: 10.1097/phm.0000000000001344. PMID: 31687984.

104. Liebs TR, Herzberg W, Rüther W, et al. Ergometer cycling after hip or knee replacement surgery: a randomized controlled trial. J Bone Joint Surg Am. 2010 Apr;92(4):814-22. doi: 10.2106/jbjs.H.01359. PMID: 20360503.

105. Liebs TR, Herzberg W, Rüther W, et al. Multicenter randomized controlled trial comparing early versus late aquatic therapy after total hip or knee arthroplasty. Arch Phys Med Rehabil. 2012 Feb;93(2):192-9. doi: 10.1016/j.apmr.2011.09.011. PMID: 22196125.

106. Madsen M, Larsen K, Madsen IK, et al. Late group-based rehabilitation has no advantages compared with supervised homeexercises after total knee arthroplasty. Dan Med J. 2013 Apr;60(4):A4607. PMID: 23651717.

107. Minns Lowe CJ, Barker KL, Holder R, et al. Comparison of postdischarge physiotherapy versus usual care following primary total knee arthroplasty for osteoarthritis: an exploratory pilot randomized clinical trial. Clin Rehabil. 2012 Jul;26(7):629-41. doi: 10.1177/0269215511427749. PMID: 22180446.
108. Moffet H, Tousignant M, Nadeau S, et al. Patient Satisfaction with In-Home Telerehabilitation After Total Knee Arthroplasty: Results from a Randomized Controlled Trial. Telemed J E Health. 2017 Feb;23(2):80-7. doi: 10.1089/tmj.2016.0060. PMID: 27529575.

109. Moffet H, Tousignant M, Nadeau S, et al. InHome Telerehabilitation Compared with Face-to-Face Rehabilitation After Total Knee Arthroplasty: A Noninferiority Randomized Controlled Trial. J Bone Joint Surg Am. 2015 Jul 15;97(14):1129-41. doi: 10.2106/jbjs.N.01066. PMID: 26178888.

110. Monticone M, Ferrante S, Rocca B, et al. Homebased functional exercises aimed at managing kinesiophobia contribute to improving disability and quality of life of patients undergoing total knee arthroplasty: a randomized controlled trial. Arch Phys Med Rehabil. 2013 Feb;94(2):231-9. doi: 10.1016/j.apmr.2012.10.003. PMID: 23063624.

111. Moutzouri M, Coutts F, Gliatis J, et al. Early initiation of home-based sensori-motor training improves muscle strength, activation and size in patients after knee replacement: a secondary analysis of a controlled clinical trial. BMC Musculoskelet Disord. 2019 May 17;20(1):231. doi: 10.1186/s12891-019-2575-3. PMID: 31101039.

112. Moutzouri M, Gleeson N, Coutts F, et al. Early self-managed focal sensorimotor rehabilitative training enhances functional mobility and sensorimotor function in patients following total knee replacement: a controlled clinical trial. Clin Rehabil. 2018 Jul;32(7):888-98. doi: 10.1177/0269215518757291. PMID: 29473481.

113. Naylor JM, Hart A, Mittal R, et al. The value of inpatient rehabilitation after uncomplicated knee arthroplasty: a propensity score analysis. Med J Aust. 2017 Sep 18;207(6):250-5. doi: 10.5694/mja16.01362. PMID: 28899328. 
114. Padgett DE, Christ AB, Joseph AD, et al. Discharge to Inpatient Rehab Does Not Result in Improved Functional Outcomes Following Primary Total Knee Arthroplasty. J Arthroplasty. 2018 Jun;33(6):1663-7. doi: 10.1016/j.arth.2017.12.033. PMID: 29352683.

115. Petersen T, Hautopp H, Duus B, et al. No Effect of Acupuncture as Adjunctive Therapy for Patients with Total Knee Replacement: A Randomized Controlled Trial. Pain Med. 2018 Jun 1;19(6):1280-9. doi: 10.1093/pm/pnx317. PMID: 29294078.

116. Petterson SC, Mizner RL, Stevens JE, et al. Improved function from progressive strengthening interventions after total knee arthroplasty: a randomized clinical trial with an imbedded prospective cohort. Arthritis Rheum. 2009 Feb 15;61(2):174-83. doi: 10.1002/art.24167. PMID: 19177542.

117. Piqueras M, Marco E, Coll M, et al. Effectiveness of an interactive virtual telerehabilitation system in patients after total knee arthoplasty: a randomized controlled trial. J Rehabil Med. 2013 Apr;45(4):392-6. doi: 10.2340/165019771119. PMID: 23474735.

118. Piva SR, Almeida GJ, Gil AB, et al. Effect of Comprehensive Behavioral and Exercise Intervention on Physical Function and Activity Participation After Total Knee Replacement: A Pilot Randomized Study. Arthritis Care Res (Hoboken). 2017 Dec;69(12):1855-62. doi: 10.1002/acr.23227. PMID: 28217891.

119. Piva SR, Moore CG, Schneider M, et al. A randomized trial to compare exercise treatment methods for patients after total knee replacement: protocol paper. BMC Musculoskelet Disord. 2015 Oct 16;16:303. doi: 10.1186/s12891-015-0761-5. PMID: 26474988.

120. Piva SR, Schneider MJ, Moore CG, et al. Effectiveness of Later-Stage Exercise Programs vs Usual Medical Care on Physical Function and Activity After Total Knee Replacement: A Randomized Clinical Trial. JAMA Netw Open. 2019 Feb 1;2(2):e190018. doi: 10.1001/jamanetworkopen.2019.0018. PMID: 30794296.
121. Pua YH, Seah FJ, Poon CL, et al. Association between rehabilitation attendance and physical function following discharge after total knee arthroplasty: prospective cohort study. Osteoarthritis Cartilage. 2017 Apr;25(4):462-9. doi: 10.1016/j.joca.2016.10.020. PMID: 27810379.

122. Rockstroh G, Schleicher W, Krummenauer F. [Effectiveness of microcurrent therapy as a constituent of post-hospital rehabilitative treatment in patients after total knee alloarthroplasty - a randomized clinical trial]. Rehabilitation (Stuttg). 2010 Jun;49(3):173-9. doi: 10.1055/s-00291246152. PMID: 20533147.

123. Sattler LN, Hing WA, Vertullo CJ. PedalingBased Protocol Superior to a 10-Exercise, Non-Pedaling Protocol for Postoperative Rehabilitation After Total Knee Replacement: A Randomized Controlled Trial. J Bone Joint Surg Am. 2019 Apr 17;101(8):688-95. doi: 10.2106/jbjs.18.00898. PMID: 30994586.

124. Schache MB, McClelland JA, Webster KE. Does the addition of hip strengthening exercises improve outcomes following total knee arthroplasty? A study protocol for a randomized trial. BMC Musculoskelet Disord. 2016 Jun 13;17:259. doi: 10.1186/s12891-016-1104-x. PMID: 27295978.

125. Schache MB, McClelland JA, Webster KE. Incorporating hip abductor strengthening exercises into a rehabilitation program did not improve outcomes in people following total knee arthroplasty: a randomised trial. J Physiother. 2019 Jul;65(3):136-43. doi: 10.1016/j.jphys.2019.05.008. PMID: 31208916.

126. Shanb AS, Youssef EF. Effects of adding biofeedback training to active exercises after total knee arthroplasty. Journal of musculoskeletal research. 2014;17(1). doi: 10.1142/S0218957714500018. PMID: CN01041112.

127. Simpson AH, Hamilton DF, Beard DJ, et al. Targeted rehabilitation to improve outcome after total knee replacement (TRIO): study protocol for a randomised controlled trial. Trials. 2014 Feb 1;15:44. doi: 10.1186/1745-6215-15-44. PMID: 24484541. 
128. Stevens-Lapsley JE, Balter JE, Wolfe P, et al. Early neuromuscular electrical stimulation to improve quadriceps muscle strength after total knee arthroplasty: a randomized controlled trial. Phys Ther. 2012

Feb;92(2):210-26. doi: 10.2522/ptj.20110124. PMID: 22095207.

129. Tousignant M, Moffet H, Boissy P, et al. A randomized controlled trial of home telerehabilitation for post-knee arthroplasty. J Telemed Telecare. 2011;17(4):195-8. doi: 10.1258/jtt.2010.100602. PMID: 21398389.

130. Tsukada Y, Matsuse H, Shinozaki N, et al. Combined application of electrically stimulated antagonist muscle contraction and volitional muscle contraction prevents muscle strength weakness and promotes physical function recovery after total knee arthroplasty: A randomized controlled trial. Kurume Medical Journal. 2018;65(4):14554. doi: 10.2739/kurumemedj.MS654007.

131. Vuorenmaa M, Ylinen J, Piitulainen K, et al. Efficacy of a 12-month, monitored home exercise programme compared with normal care commencing 2 months after total knee arthroplasty: a randomized controlled trial. J Rehabil Med. 2014 Feb;46(2):166-72. doi: 10.2340/16501977-1242. PMID: 24241606.

132. Wylde V, Artz N, Marques E, et al. Effectiveness and cost-effectiveness of outpatient physiotherapy after knee replacement for osteoarthritis: study protocol for a randomised controlled trial. Trials. 2016 Jun 13;17(1):289. doi: 10.1186/s13063-016-1418-x. PMID: 27296366.

133. DeJong G, Hsieh CJ, Vita MT, et al. Innovative Devices Did Not Provide Superior Total Knee Arthroplasty Outcomes in PostOperative Rehabilitation: Results From a Four-Arm Randomized Clinical Trial. J Arthroplasty. 2020 Aug;35(8):2054-65. doi: 10.1016/j.arth.2020.03.048. PMID: 32360105 .

134. Eymir M, Erduran M, Ünver B. Active heelslide exercise therapy facilitates the functional and proprioceptive enhancement following total knee arthroplasty compared to continuous passive motion. Knee Surg Sports Traumatol Arthrosc. 2020 Aug 10. doi: 10.1007/s00167-020-06181-4. PMID: 32778907 .
135. Iwakiri K, Ohta Y, Shibata Y, et al. Initiating range of motion exercises within 24 hours following total knee arthroplasty affects the reduction of postoperative pain: A randomized controlled trial. Asia Pac J Sports Med Arthrosc Rehabil Technol. 2020 Jul;21:11-6. doi: 10.1016/j.asmart.2020.03.003. PMID: 32373475 .

136. Zapparoli L, Sacheli LM, Seghezzi S, et al. Motor imagery training speeds up gait recovery and decreases the risk of falls in patients submitted to total knee arthroplasty. Sci Rep. 2020 Jun 2;10(1):8917. doi: 10.1038/s41598-020-65820-5. PMID: 32488010 .

137. Bitterli R, Sieben JM, Hartmann M, et al. Presurgical sensorimotor training for patients undergoing total hip replacement: a randomised controlled trial. Int J Sports Med. 2011 Sep;32(9):725-32. doi: 10.1055/s-0031-1271696. PMID: 21630176.

138. Vukomanović A, Popović Z, Durović A, et al. The effects of short-term preoperative physical therapy and education on early functional recovery of patients younger than 70 undergoing total hip arthroplasty. Vojnosanit Pregl. 2008 Apr;65(4):291-7. doi: 10.2298/vsp0804291v. PMID: 18499950.

139. Holsgaard-Larsen A, Hermann A, Zerahn B, et al. Effects of progressive resistance training prior to total HIP arthroplasty - a secondary analysis of a randomized controlled trial. Osteoarthritis Cartilage. 2020

Aug;28(8):1038-45. doi: 10.1016/j.joca.2020.04.010. PMID: 32376477.

140. Pour AE, Parvizi J, Sharkey PF, et al. Minimally invasive hip arthroplasty: what role does patient preconditioning play? J Bone Joint Surg Am. 2007 Sep;89(9):1920-7. doi: 10.2106/jbjs.F.01153. PMID: 17768187.

141. Rooks DS, Huang J, Bierbaum BE, et al. Effect of preoperative exercise on measures of functional status in men and women undergoing total hip and knee arthroplasty. Arthritis Rheum. 2006 Oct 15;55(5):700-8. doi: 10.1002/art.22223. PMID: 17013852. 
142. Austin MS, Urbani BT, Fleischman AN, et al. Formal Physical Therapy After Total Hip Arthroplasty Is Not Required: A Randomized Controlled Trial. J Bone Joint Surg Am. 2017 Apr 19;99(8):648-55. doi: 10.2106/jbjs.16.00674. PMID: 28419032.

143. Beck H, Beyer F, Gering F, et al. Sports Therapy Interventions Following Total Hip Replacement: A Randomized Controlled Trial. Deutsches Aerzteblatt International. 2019;116(1/2):1-8. doi: 10.3238/arztebl.2019.0001. PMID: 134820207. Language: English. Entry Date: In Process. Revision Date: 20190223. Publication Type: Article. Journal Subset: Biomedical.

144. Coulter C, Perriman DM, Neeman TM, et al. Supervised or Unsupervised Rehabilitation After Total Hip Replacement Provides Similar Improvements for Patients: A Randomized Controlled Trial. Arch Phys Med Rehabil. 2017 Nov;98(11):2253-64. doi: 10.1016/j.apmr.2017.03.032. PMID: 28506775 .

145. Giaquinto S, Ciotola E, Dall'armi V, et al. Hydrotherapy after total hip arthroplasty: a follow-up study. Arch Gerontol Geriatr. 2010 Jan-Feb;50(1):92-5. doi: 10.1016/j.archger.2009.02.005. PMID: 19282040 .

146. Heiberg KE, Bruun-Olsen V, Ekeland A, et al. Effect of a walking skill training program in patients who have undergone total hip arthroplasty: Followup one year after surgery. Arthritis Care Res (Hoboken). 2012 Mar;64(3):415-23. doi: 10.1002/acr.20681. PMID: 22170790.

147. Heiberg KE, Figved W. Exercise, recovery of physical functioning, and prediction of physical activity after total hip arthroplasty. 5-year follow-up of a rct. Annals of the rheumatic diseases. 2015;74:1318-9. doi: 10.1136/annrheumdis-2015-eular.3078. PMID: CN-01128862.

148. Heiberg KE, Figved W. Physical Functioning and Prediction of Physical Activity After Total Hip Arthroplasty: Five-Year Followup of a Randomized Controlled Trial. Arthritis Care Res (Hoboken). 2016 Apr;68(4):45462. doi: 10.1002/acr.22679. PMID: 26239078 .
149. Łyp M, Kaczor R, Cabak A, et al. A Water Rehabilitation Program in Patients with Hip Osteoarthritis Before and After Total Hip Replacement. Med Sci Monit. 2016 Jul 25;22:2635-42. doi: 10.12659/msm.896203. PMID: 27455419

150. Mikkelsen LR, Mechlenburg I, Søballe K, et al. Effect of early supervised progressive resistance training compared to unsupervised home-based exercise after fasttrack total hip replacement applied to patients with preoperative functional limitations. A single-blinded randomised controlled trial. Osteoarthritis Cartilage. 2014 Dec;22(12):2051-8. doi: 10.1016/j.joca.2014.09.025. PMID: 25305374.

151. Monticone M, Ambrosini E, Rocca B, et al. Task-oriented exercises and early full weight-bearing contribute to improving disability after total hip replacement: a randomized controlled trial. Clin Rehabil. 2014 Jul;28(7):658-68. doi: 10.1177/0269215513519342. PMID: 24459172.

152. Naylor JM, Hart A, Mittal R, et al. The effectiveness of inpatient rehabilitation after uncomplicated total hip arthroplasty: a propensity score matched cohort. BMC Musculoskelet Disord. 2018 Jul 18;19(1):236. doi: 10.1186/s12891-0182134-3. PMID: 30021552.

153. Nelson M, Bourke M, Crossley K, et al. Telerehabilitation Versus Traditional Care Following Total Hip Replacement: A Randomized Controlled Trial Protocol. JMIR Res Protoc. 2017 Mar 2;6(3):e34. doi: 10.2196/resprot.7083. PMID: 28254734.

154. Nelson M, Bourke M, Crossley K, et al. Telerehabilitation is non-inferior to usual care following total hip replacement - a randomized controlled non-inferiority trial. Physiotherapy. 2020 Jun;107:19-27. doi: 10.1016/j.physio.2019.06.006. PMID: 32026820 .

155. Rao BM, Cieslewicz TJ, Sochacki KR, et al. Worse Preoperative Pain and Higher Activity Levels Predict Patient Choice of Formal Physical Therapy After Primary Anterior Total Hip Arthroplasty. J Arthroplasty. 2021 Mar 16. doi: 10.1016/j.arth.2021.03.027. PMID: 33863614. 
156. Smith TO, Mann CJ, Clark A, et al. Bed exercises following total hip replacement: 1 year follow-up of a single-blinded randomised controlled trial. Hip Int. 2009 Jul-Sep;19(3):268-73. doi: 10.1177/112070000901900314. PMID: 19876883.

157. Smith TO, Mann CJV, Clark A, et al. Bed exercises following total hip replacement: a randomised controlled trial. Physiotherapy. 2008;94(4):286-91. PMID: 105615532. Language: English. Entry Date: 20090227. Revision Date: 20150819. Publication Type: Journal Article.

158. Winther SB, Foss OA, Husby OS, et al. A randomized controlled trial on maximal strength training in 60 patients undergoing total hip arthroplasty. Acta Orthop. 2018 Jun;89(3):295-301. doi: 10.1080/17453674.2018.1441362. PMID: 29493347.

159. Winther SB, Foss OA, Klaksvik J, et al. Increased Muscle Strength Limits Postural Sway During Daily Living Activities in Total Hip Arthroplasty Patients. Am J Phys Med Rehabil. 2020 Jul;99(7):608-12. doi: 10.1097/phm.0000000000001382. PMID: 31977324.
160. Ivers N, Tricco AC, Trikalinos TA, et al. Seeing the forests and the trees--innovative approaches to exploring heterogeneity in systematic reviews of complex interventions to enhance health system decision-making: a protocol. Syst Rev. 2014 Aug 12;3:88. doi: 10.1186/2046-4053-3-88. PMID: 25115289.

161. Hoffmann TC, Glasziou PP, Boutron I, et al. Better reporting of interventions: template for intervention description and replication (TIDieR) checklist and guide. Bmj. 2014 Mar 7;348:g1687. doi: 10.1136/bmj.g1687. PMID: 24609605.

162. Hoogeboom TJ, Oosting E, Vriezekolk JE, et al. Therapeutic validity and effectiveness of preoperative exercise on functional recovery after joint replacement: a systematic review and meta-analysis. PLoS One. 2012;7(5):e38031. doi: 10.1371/journal.pone.0038031. PMID: 22675429.

163. Jette DU, Hunter SJ, Jette AM. Overcoming Research Challenges to Improve Clinical Practice Guideline Development. Phys Ther. 2020 Oct 30;100(11):1889-90. doi: 10.1093/ptj/pzaa153. PMID: 33125083. 


\section{Abbreviations and Acronyms}

6MWT

ADL

AHRQ

AMSTAR-2

BMI

CI

CINAHL

COI

COP

CPG

CPM

EPC

EQ-5D

GRADE

HOOS

ICF

IQR

KI

KOS

KOOS

KQ

LEFS

MCID

MD

MUA

NHLBI

NMD

NMES

NRCS

OARSI

OR

PENS

PMID

PROMIS

PRT

QoL

$\mathrm{RCT}$

RoB

ROM

ROBINS-I

RR

SD

SE

SF-6D
Six-minute walk test activities of daily living Agency for Healthcare Research and Quality

A Measurement Tool to Assess Systematic Reviews

body mass index

confidence interval

Cumulative Index to Nursing and Allied Health Literature

conflicts of interest

center of pressure

clinical practice guideline

continuous passive motion

Evidence-based Practice Center

EuroQol-5 dimensions

Grading of Recommendations, Assessment, Development, and

Evaluations

Hip Disability Osteoarthritis Outcome Score

International Classification of Functioning, Disability and Health

interquartile range

Key Informant

Knee Outcome Survey

Knee injury and Osteoarthritis Outcome Score

Key Question

Lower Extremity Functional Scale

minimal clinically important difference

mean difference

manipulation under anesthesia

National Heart, Lung, and Blood Institute

net mean difference

neuromuscular electrical stimulation

nonrandomized comparative study

Osteoarthritis Research Society International

odds ratio

patterned electrical neuromuscular stimulation

PubMed identifier

Patient-Reported Outcomes Measurement Information System

progressive resistive training

quality of life

randomized controlled trial

risk of bias

range of motion

Risk of Bias in Nonrandomized Studies of Interventions

relative risk

standard deviation

standard error

Short-form six-dimension 


$\begin{array}{ll}\text { SF-12 } & \text { 12-Item Short Form Health Survey } \\ \text { SF-36 } & \text { 36-Item Short Form Health Survey } \\ \text { SoE } & \text { strength of evidence } \\ \text { SR } & \text { systematic review } \\ \text { SRDR } & \text { Systematic Review Data Repository } \\ \text { TEP } & \text { Technical expert panel } \\ \text { TENS } & \text { transcutaneous electrical nerve stimulation } \\ \text { TJAOM } & \text { Total Joint Arthroplasty and Outcome Measures } \\ \text { TIDieR } & \text { Template for Intervention Description and Replications } \\ \text { TKA } & \text { total knee arthroplasty } \\ \text { THA } & \text { total hip arthroplasty } \\ \text { TUG } & \text { Timed Up and Go test } \\ \text { TOO } & \text { Task order officer } \\ \text { U.K. } & \text { United Kingdom } \\ \text { U.S. } & \text { United States } \\ \text { VAS } & \text { Visual Analog Scale } \\ \text { WOMAC } & \text { Western Ontario and McMaster Universities Osteoarthritis Index }\end{array}$




\section{Appendix A. Methods}

\section{Analytic Framework}

Figure A-1. Analytic framework for KQs 1-4: Different types of prehabilitation or rehabilitation for knee or hip replacement surgery

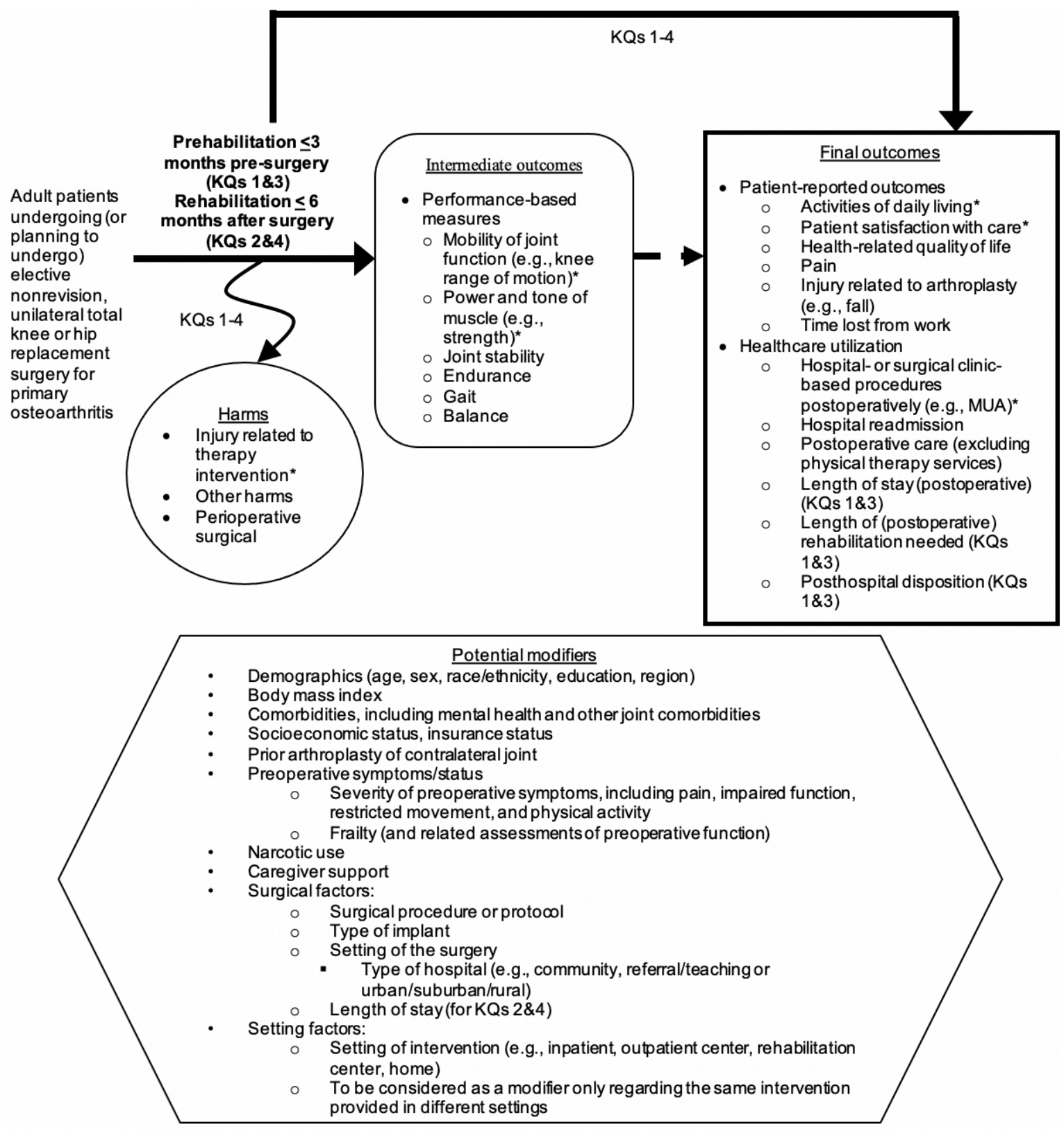

Abbreviations: KQ = Key Question, MUA = manipulation under anesthesia.

* Denotes important/priority outcomes that are included in Strength of Evidence tables (Tables 22, 39, 58, 74, and 90 in the Main Report). 


\section{Literature Search}

We searched for primary studies in MEDLINE (via PubMed), PsycINFO, Embase, the Cochrane Register of Clinical Trials, CINAHL, and Scopus. Duplicate citations were removed prior to screening. We did not employ any language restrictions to the search but included filters to remove nonhuman studies and articles that are not primary studies. Upon discussion with Key Informants and a Technical Expert Panel (TEP), we restricted studies to those published during or after 2005 to ensure the body of evidence is consistent with contemporary surgical and rehabilitation practices. We included MeSH or Emtree terms, along with free-text words, related to arthroplasty, knee replacement, hip replacement, total knee, total hip, rehabilitation, prehabilitation, physical therapy, physiotherapy, postoperative care. The searches were independently peer reviewed.

We also ran a search of the ClinicalTrials.gov registry for ongoing studies, unpublished study protocols, and unpublished study results. The reference lists of relevant existing systematic reviews were screened for additional eligible studies. Additional articles suggested to us from any source, including peer and public review, were screened applying identical eligibility criteria. Non-English language articles were screened and data extracted by readers of the relevant languages. All eligible studies were in languages extractable by the research team.

\section{Database Search Strategies}

\section{PubMed $1 / 1 / 2005$ to $5 / 3 / 2021$}

((arthroplast* or hip replacement* or knee replacement* or joint replacement* or total hip or total knee or total joint*) OR "Arthroplasty, Replacement, Hip"[Mesh] OR "Arthroplasty, Replacement, Knee"[Mesh] OR (("Arthroplasty"[Mesh] or arthroplasty or replacement) and (knee or hip)))

AND

((pre-hab* or prehab*) OR "Arthroplasty, Replacement, Knee/rehabilitation"[Mesh] OR "Arthroplasty, Replacement, Hip/rehabilitation"[Mesh] OR ((presurg* or preoperativ* or presurg* or pre-operativ* or early or home) and (rehab or rehabilitate or rehabilitation or re-hab* or "Rehabilitation"[Mesh] or "Physical Therapy Modalities"[Mesh] or "physical therapy" or physiotherapy*)) OR ("Preoperative Care/methods"[Mesh] OR "Preoperative Care/rehabilitation"[Mesh] ) OR ((before or prior to) and (arthroplast* or hip replacement* or knee replacement* or joint replacement* or total hip or total knee or total joint*) and (rehab or rehabilitate or rehabilitation or re-hab* or intervention* or recovery)) OR (("Preoperative Care"[MESH] OR "Preoperative Period" [MESH]) and (rehab or rehabilitate or rehabilitation or re-hab* or "Rehabilitation"[Mesh] or "Physical Therapy Modalities"[Mesh] or "physical therapy" or physiotherapy*)) OR ("Postoperative Period"'[Mesh] and (rehab or rehabilitate or rehabilitation or re-hab*)) OR ((postsurg* or post-surg* or postoperativ* or post-operativ*) AND (rehab or rehabilitate or rehabilitation or re-hab* or "Rehabilitation"[Mesh] or "Physical Therapy Modalities"[Mesh] or "physical therapy" or physiotherapy*)) OR ((after or post) AND (arthroplast* or hip replacement* or knee replacement* or joint replacement* or total hip or total knee or total joint*) AND (rehab or rehabilitate or rehabilitation or re-hab* or "Rehabilitation"[Mesh] or "Physical Therapy Modalities"[Mesh] or "physical therapy" or physiotherapy*)))

AND 
("Cohort Studies"[Mesh] OR cohort OR "Clinical Trial" [Publication Type] OR (follow-up or followup) OR longitudinal OR "Placebos"[Mesh] OR placebo* OR "Research Design"[Mesh] OR "Evaluation Study" [Publication Type] OR "Comparative Study" [Publication Type] OR ((comparative or Intervention) AND study) OR pretest* OR posttest* OR prepost* OR "before and after" OR interrupted time* OR time serie* OR intervention* OR ((quasi-experiment* OR quasiexperiment* OR quasi or experimental) and (method or study or trial or design*)) OR "real world" OR "real-world" OR "Case-Control Studies"[Mesh] OR (case and control) OR "Random Allocation"[Mesh] OR "Clinical Trial" [Publication Type] OR "Double-Blind Method"[Mesh] OR "Single-Blind Method"[Mesh] OR random* OR "Placebos"[Mesh] OR placebo OR ((clinical OR controlled) and trial*) OR ((singl* or doubl* or trebl* or tripl*) and (blind* or mask*)) OR rct OR crossover OR cross-over OR cross-over OR RCT OR "Randomized Controlled Trial" [Publication Type] OR systematic[sb] OR meta-analysis[pt] OR meta-analysis as topic[mh] OR meta-analysis[mh] OR meta analy* OR metanaly* OR metaanaly* OR met analy* OR (systematic AND (review* OR overview*)) OR "Review Literature as Topic"[Mesh] OR cochrane[tiab] OR embase[tiab] OR (psychlit[tiab] or psyclit[tiab]) OR (psychinfo[tiab] or psycinfo[tiab]) OR (cinahl[tiab] OR cinhal[tiab] OR "cumulative index to nursing and allied health") OR science citation index[tiab] OR ibids[tiab] OR "international bibliographic information on dietary supplements" OR cancerlit[tiab] OR reference list*[tiab] OR bibliograph*[tiab] OR hand-search*[tiab] OR relevant journals[tiab] OR manual search*[tiab] OR ((selection OR inclusion OR exclusion) AND criteria[tiab]) OR data extraction[tiab] OR relevant journals OR "Systematic Review" [Publication Type])

NOT

("address"[pt] or "autobiography"[pt] or "bibliography"[pt] or "biography"[pt] or "case reports" [pt] or "comment" [pt] or "congress"[pt] or "dictionary" [pt] or "directory" [pt] or "festschrift"[pt] or "historical article"[pt] or "interview"[pt] or "lecture"[pt] or "legal case"[pt] or "legislation"[pt] or "news"[pt] or "newspaper article"[pt] or "patient education handout"[pt] or "periodical index"[pt] or "comment on" or ("Animals"[Mesh] NOT "Humans"[Mesh]) OR rats[tw] or rat $[\mathrm{tw}]$ or cow[tw] or cows[tw] or chicken*[tw] or horse[tw] or horses[tw] or mice $[\mathrm{tw}]$ or mouse[tw] or bovine $[\mathrm{tw}]$ or sheep $[\mathrm{tw}]$ or ovine[tw] or murinae[tw] or cats[tw] or cat $[\mathrm{tw}]$ or $\operatorname{dog}[\mathrm{tw}]$ or $\operatorname{dogs}[\mathrm{tw}]$ or rodent $[\mathrm{tw}]$ )

\section{CINAHL/PsycINFO 1/1/2005 to 5/3/2021}

(arthroplast* or hip replacement* or knee replacement* or joint replacement* or total hip or total knee or total joint* or ((arthroplasty or replacement) and (knee or hip)))

AND

(pre-hab* or prehab* or ((presurg* or preoperativ* or pre-surg* or pre-operativ* or early or home) and (rehab or rehabilitate or rehabilitation or re-hab* or "physical therapy" or physiotherapy*)) or ((before or prior to) and (arthroplast* or hip replacement* or knee replacement* or joint replacement* or total hip or total knee or total joint*) and (rehab or rehabilitate or rehabilitation or re-hab* or intervention* or recovery)) or ((postsurg* or postsurg* or postoperativ* or post-operativ*) and (rehab or rehabilitate or rehabilitation or re-hab* or "physical therapy" or physiotherapy*)) OR ((after or post) AND (arthroplast* or hip replacement* or knee replacement* or joint replacement* or total hip or total knee or total joint*) AND (rehab or rehabilitate or rehabilitation or re-hab* or "physical therapy" or physiotherapy*))) 


\section{Cochrane Databases 1/1/2005 to 5/3/2021}

\#1 MeSH descriptor: [Arthroplasty, Replacement, Hip] explode all trees

\#2 MeSH descriptor: [Arthroplasty, Replacement, Knee] explode all trees

\#3 (arthroplast* or hip replacement* or knee replacement* or joint replacement* or total hip or total knee or total joint*)

\#4 ((arthroplasty or replacement) and (knee or hip))

\#5 \#1 OR \#2 OR \#3 OR \#4

\#6 (pre-hab* or prehab* OR ((presurg* or preoperativ* or pre-surg* or pre-operativ* or early or home) and (rehab or rehabilitate or rehabilitation or re-hab* or "physical therapy" or physiotherapy*)) OR ((before or prior to) and (arthroplast* or hip replacement* or knee replacement* or joint replacement* or total hip or total knee or total joint*) and (rehab or rehabilitate or rehabilitation or re-hab* or intervention* or recovery)) OR ((postsurg* or post-surg* or postoperativ* or post-operativ*) AND (rehab or rehabilitate or rehabilitation or re-hab* or "physical therapy" or physiotherapy*)) OR ((after or post) AND (arthroplast* or hip replacement* or knee replacement* or joint replacement* or total hip or total knee or total joint*) AND (rehab or rehabilitate or rehabilitation or re-hab* or "physical therapy" or physiotherapy*)))

\#7 \#5 AND \#6

\section{Embase 1/1/2005 to 5/3/2021}

\#5 \#3 AND \#4 AND ([article]/lim OR [article in press]/lim) AND [2005-2020]/py

\#4 'pre hab*' OR prehab* OR ((presurg* OR preoperativ* OR 'pre surg*' OR 'pre operativ*' OR early OR home) AND (rehab OR rehabilitate OR rehabilitation OR 're hab*' OR 'physical therapy' OR physiotherapy*)) OR ((before OR prior) AND to AND (((()(arthroplast* OR hip) AND replacement* OR knee) AND replacement* OR joint) AND replacement* OR total) AND hip OR total) AND knee OR total) AND joint* AND (rehab OR rehabilitate OR rehabilitation OR 're hab*' OR intervention* OR recovery)) OR ((postsurg* OR 'post surg*' OR postoperativ* OR 'post operativ*') AND (rehab OR rehabilitate OR rehabilitation OR 're hab*' OR 'physical therapy' OR physiotherapy*)) OR ((after OR post) AND ((()((arthroplast* OR hip) AND replacement* OR knee) AND replacement* OR joint) AND replacement* OR total) AND hip OR total) AND knee OR total) AND joint* AND (rehab OR rehabilitate OR rehabilitation OR 're hab*' OR 'physical therapy' OR physiotherapy*))

\#3 \#1 OR \#2

\#2 (hip OR knee) AND replacement

\#1 'arthropathy'/exp OR 'arthropathy' AND (knee OR hip) 


\section{Inclusion/Exclusion Criteria Details}

\section{Study Eligibility Criteria for All Key Questions}

\section{Population(s)}

- Adults ( $\geq 18$ years old) undergoing (or planning to undergo) total hip or knee replacement surgery

○ for primary osteoarthritis

- elective (nonemergent) surgery

$\circ$ primary surgery (not revision)

o unilateral TJR

- Exclude: Studies where $\geq 10 \%$ of patients underwent total knee or hip replacement surgery:

$\circ$ for partial joint replacement

○ for causes other than primary osteoarthritis (e.g., cancer, trauma, rheumatoid arthritis)

$\circ$ for emergency surgery

$\circ$ for revision joint replacement

○ bilateral TJR (simultaneous in both joints)

- N.B. Studies that reported stratified or subgroup analyses of the population of interest were included if they meet the other eligibility criteria (e.g., if they included unilateral and bilateral surgeries but reported data specific to unilateral)

- Did not exclude based on prior surgeries to other joints (including contralateral hip or knee)

\section{Intervention(s):}

- Active, structured physical activity or activities designed to attain measurable goals of reducing impairments and improving movement-related function as defined by the International Classification of Functioning, Disability and Health (ICF)

- Any movement-related physical goal including improvements beyond the basal (or baseline) state in: mobility and stability of joint function (including flexibility and range of motion), movement control, power and tone of muscles (including strength), gait, endurance; along with the related goal of reducing pain.

- Interventions need to be sufficiently described to be replicable by a therapist or other professional. The exception to this was rehabilitation interventions delivered in different settings (inpatient vs. outpatient), which we included even if there was not sufficient detail about their (p)rehabilitation interventions (and noted such in our coding).

- Single or multiple components. For multicomponent interventions, the goals of the intervention criteria refer to the overall intervention, not necessarily to each individual component. We categorized the content of the rehabilitation interventions according to a categorization scheme based on ongoing research by Oatis and Franklin to develop a taxonomy defining the components of physical therapy after TKR. ${ }^{1,2}$ The taxonomy comprehensively lists specific rehabilitation content that are hierarchically linked to larger rehabilitation goals. The larger component goals include:

- Strengthening exercise 
- Aerobic exercise

- Flexibility exercise

- Balance-motor/learning-agility exercise

- Task specific training

- Patient education

- We used the taxonomy to code both the subcategory content and larger category goals (e.g., intervention content of squats would be coded for the subcategory of "squats" hierarchically linked to the goal of "strengthening").

- Exclude: Continuous passive motion (CPM) was not included as there is strong evidence, summarized in an existing systematic review, ${ }^{3}$ that that component is ineffective.

- The intervention had to have been delivered, supervised, and/or monitored by a healthcare professional or other trained individual (e.g., physical therapist, physical therapy assistant, nurse trained in rehabilitation, health educator with training in exercise delivery or rehabilitation, other healthcare professional trained in rehabilitation)

- Peer-led (or patient-led) interventions were eligible if monitored by a professional or other trained individual

- The physical therapist (or other trained individual) had to have been involved in patient engagement and assessment of progress, and provided ongoing feedback to the patient throughout the course of intervention

- This interaction could have been direct (e.g., in-person therapy) or remote (e.g., via app, Web, or telephone)

- Remote therapy had to have included active monitoring by a physical therapist (or other trained individual), although the (p)rehabilitation therapy could have been guided completely by the app

- The patient needed to be actively involved or engaged in at least part of the intervention (and not be only a passive recipient of the intervention)

- Interventions evaluating the combined benefit an intervention defined above with an adjunctive modality were also included.

Adjunctive modalities are either passively applied to patients and/or do not (on their own) have the direct goals of reducing impairments or improving movement-related function but are used to help other components achieve these goals. Examples of therapies that were considered adjunctive modalities if combined with an intervention meeting criterion above included:

- Neuromuscular electrical stimulation (NMES)

- Transcutaneous electrical nerve stimulation (TENS)

- Manual therapy (e.g., therapeutic massage, passive range of motion)

- Biofeedback devices

- Cryotherapy (or other thermal therapies)

$\circ \quad$ Dry needling

- Mindfulness, stress/anxiety-reduction interventions

- Complementary and alternative therapies (excluding ingested, inhaled, or transcutaneous treatments) 
- Modalities had to have been sufficiently described to be replicable by a therapist or other professional

- Exclude: Interventions that were not active, structured physical activities delivered by a healthcare professional or other trained individual, including devices not designed to be used primarily during active therapy; for example:

- Splinting, bracing, taping

- One-time distribution of information

- Assistive devices (e.g., crutches vs. canes or walkers)

- Exclude: Interventions (as a whole) without specific goals (e.g., unsupervised swimming, walking, cycling, hiking).

- Exclude: Interventions (as a whole) without active engagement of the healthcare professional (e.g., only set-up and removal of intervention without monitoring, or healthcare professional engagement only to measure pre- and post-intervention outcome measures).

- Exclude: Surgical or hospital process-improvement interventions (e.g., early mobilizations, enhanced recovery after surgery [ERAS], care managers, pre-anesthesia protocols)

- Exclude: Pharmaceutical (or over-the-counter) treatments (although, allowed as part of an overall intervention)

\section{Comparator(s):}

- No active, structured physical activity, as defined above

- Allow "usual care" only if the intervention arm includes well-defined components or adjunctive modalities plus the same "usual care"

- Other active structured physical activity (or set of activities)

- Other adjunctive modality

- Different duration (or intensity) of intervention

- Different providers

- Different setting

- Exclude: no comparison (or comparison with only pre-intervention state)

Outcomes: $(*$ denotes important/priority outcomes that were included in Strength of Evidence tables)

- Patient-reported outcomes

- Activities of daily living*

- Patient satisfaction with care*

- Quality of life (QoL)*

- Pain

○ Injury related to arthroplasty (e.g., fall)

- Time lost from work

- Measures that combined these outcome domains (e.g., Hip disability/Knee injury and osteoarthritis outcome score [HOOS/KOOS])

- Performance-based outcomes

○ Mobility of joint function (e.g., knee range of motion)*

$\circ$ Power and tone of muscle (e.g., strength)*

$\circ$ Joint stability 
- Endurance

○ Gait

- Balance

- Measures that combined these domains (e.g., timed-up-and-go [TUG], stair climb test)

- Healthcare utilization

- Hospital- or surgical clinic-based procedures postoperatively (e.g., need for manipulation under anesthesia)*

- Hospital readmission

- Postoperative care (excluding physical therapy services)

- Harms

○ Injury related to therapy intervention*

O Other harms related to therapy intervention

\section{Modifiers/Subgroups of interest:}

- Patient factors:

○ Demographics (age, sex, race/ethnicity, education, region)

- Body mass index

- Comorbidities, including mental health and other joint comorbidities

- Socioeconomic status, insurance status

- Prior arthroplasty of contralateral joint

- Preoperative symptoms/status

- Severity of preoperative symptoms, including pain, impaired function, restricted movement, and physical activity

- Frailty (and related assessments of preoperative function)

- Narcotic use

○ Caregiver support (outside of (p)rehabilitation)

- Surgical factors:

- Surgical procedure

- Perioperative protocols (e.g., enhanced recovery after surgery)

○ Type of implant

○ Setting of surgery

- Type of hospital (e.g., community, referral/teaching, or urban/suburban/rural)

- Setting factors:

- Setting of intervention (e.g., inpatient, outpatient center, rehabilitation center, home)

- Was considered as a modifier only regarding the same intervention provided in different settings

\section{Timing:}

- $\quad$ Study publication date $\geq 2005$

- $\geq 50 \%$ of surgeries occurred after 2005

- Outcomes 
○ Patient-reported and performance-based outcomes ${ }^{\mathrm{a}}$

- $\geq 3$ months postoperative for KQ 1 and 2 (TKA)

- $\geq 6$ months postoperative for KQ 3 and 4 (THA)

○ Healthcare utilization outcomes

- Perioperative for KQ 1 and 3 (prehabilitation)

- $\leq 3$ months

- For prehabilitation, starting at the initiation of intervention

- Harms: duration of ( $p$ )rehabilitation intervention

\section{Setting:}

- Any setting, including:

- Acute inpatient (postoperative)

O Other inpatient facility (e.g., skilled nursing facility)

$\circ$ Physical therapy/rehabilitation facility (outpatient)

○ Home

- Gym or other community center

o Other

\section{Design:}

- $\quad \mathrm{RCTs}, \mathrm{N} \geq 20$ per group

- $\mathrm{NRCS}, \mathrm{N} \geq 20$ per group, with or without adjustment for confounders

- Prospective or retrospective (as long as there was a clear, specific intervention)

- Parallel or series comparisons (i.e., "pre-post" studies that evaluate different cohorts of patients receiving vs. not receiving an intervention before and after a change in available (p)rehabilitation services)

- Cost-effectiveness (and related) analyses (for relevant QoL data, as available)

- Exclude: noncomparative (single group) studies (i.e., where all received the same intervention and there is no comparison intervention)

- Exclude: crossover studies (where the same individual receives more than one intervention in series)

- Exclude: case reports or series; case-control studies \{Davis, 2011 \#523\}

\section{Additional Criteria for KQs 1 and 3 (Prehabilitation)}

\section{Population:}

- Patients in whom the decision has been made to have a joint replacement surgery

- Exclude: Patients who are trying to avoid or delay surgery

\footnotetext{
${ }^{\text {a }}$ Time point cutoffs for outcomes were informed through stakeholder feedback which resonated with literature noting a lag in recovery immediately after TKA/THA. Specifically, for postoperative outcomes (for both prehabilitation and rehabilitation), stakeholders agreed that short-term outcomes (less than 3 months for TKA and 6 months for THA) were too early to see functional- and patient-reported improvements and suggested that these outcomes are likely to be influenced by other patient and surgical factors, in additional to any (p)rehabilitation received. The exception was short term post-operative healthcare utilization outcomes following prehabilitation (e.g., length of stay, discharge disposition).
} 


\section{Interventions:}

- Delivered within 3 months prior to surgery

- Exclude: Preoperative interventions designed to reduce symptoms or prevent or delay surgery; i.e., interventions not designed to be prehabilitation for planned surgery

\section{Outcomes (in addition to those listed above for all KQs):}

- Healthcare utilization

- Length of stay (postoperative)*

- Posthospital disposition (e.g., to home, outpatient, skilled nursing facility, "subacute" rehabilitation, "acute" inpatient rehabilitation)*

$\circ$ Length of (postoperative) rehabilitation needed

- Harms

○ Perioperative surgical complications

\section{Additional Criteria for KQs 2 and 4 (Postoperative Rehabilitation)}

Interventions:

- Delivered within 6 months following surgery

Potential Modifiers:

- Length of hospital stay

\section{Screening Process}

Citations from all searches were de-duplicated and then entered into Abstrackr software (http://abstrackr.cebm.brown.edu/) to enable title and abstract screening. The team conducted multiple rounds of pilot screening. During each pilot round, all researchers screened the same 100 abstracts and discussed conflicts, with the goal of training the team in the nuances of the eligibility criteria and refining them as needed. After the pilot rounds, we screened abstracts in duplicate. Conflicts were discussed by the team (during the initial period of abstract screening) and then resolved by the Project Lead. The Abstrackr software has machine learning capabilities to predict the likelihood of relevance of each citation. Daily, the list of unscreened abstracts was sorted so that most potentially relevant articles are presented first. After the software suggested that no remaining unscreened abstracts were likely to be relevant (when the predictor value was $<0.40$ for all unscreened abstract), we single screened all remaining abstracts.

Potentially relevant citations were retrieved in full text and entered into an evidence map in Google Sheets for rescreening and data collection. We collected basic study information regarding Key Question (KQ) addressed, study design, sample size, timing of outcome reporting, and rejection reasons. Rejection reasons were confirmed by a senior researcher.

\section{Data Extraction and Data Management}

For all KQs, data for study elements other than intervention details, were extracted directly into the Systematic Review Data Repository (SRDR) at https://srdrplus.ahrq.gov/public data? $\mathrm{id}=2965 \&$ type $=$ project. We created a combined data extraction form for all KQs. We extracted information on study characteristics, eligibility criteria, participant characteristics, intervention and comparator details, outcome definitions, and results (including mean scale score for each arm, effect sizes, and P values). Study- and outcome-level risk of bias assessment was conducted during data extraction within SRDR. 
We extracted intervention details for all KQs using an extraction form in Excel. We extracted information on the goals of the exercise(s) and where reported and specific exercises used to address these goals. We also extracted information on whether the (p)rehabilitation was progressed (i.e., changed over time) and if so, whether it was appropriate (i.e., according to patient-specific parameters assessed by the therapist) that was assessed by a clinical expert on our team. Finally, we extracted information about who delivered the intervention, (personnel), how (mode of delivery), and where (setting). Details follow.

\section{Coding Interventions}

\section{Guiding Principles and Assumptions}

- We assumed that some studies may describe interventions vaguely, that is, by the goal of the intervention (e.g., strengthening exercises) rather than the specific content components being delivered to achieve that goal (e.g., squats to promote muscle strength).

- We understood that some specific components of (p)rehabilitation interventions may target multiple (p)rehabilitation goals (e.g., step downs may have the goal of improving strength and balance).

- We assumed that the effects of interventions as defined by their i) goals and ii) specific content components are of interest to decision-makers to understand impact of (p)rehabilitation interventions from different categorization perspectives and given the limitations of varying reporting detail.

- We assumed that identifying the gaps in describing (p)rehabilitation interventions according to both their i) goals and ii) specific content components is of interest to decision-makers to identify areas for improving the design and reporting of primary studies

- We assumed that refining linkage of i) goals and ii) specific content components is of interest to decision-makers to improve intervention design and professional practice (e.g., understand what specific components are most/least frequently used to achieve certain goals and lead to most/least change in outcomes).

\section{Coding Process and Taxonomy}

We used Oatis/Franklin's hierarchical taxonomy ${ }^{1}$ to code both the intervention goal and specific content components, as feasible.

We coded interventions:

- Per large categories largely defined by the goal/aim of the intervention ( $n=6$ components)

○ Strengthening

- Aerobic

- Flexibility

- Balance-motor/Learning-agility

- Task specific training

- Patient education (see note below)

- Note that while we coded patient education, the intervention (as a whole) had to meet the criteria of an active, structured rehabilitation program. Thus, patient education alone would not be eligible. 
- Per smaller subcategories nested within the large categories (that are not all distinct and may target multiple goals/aims) ( $\mathrm{n}=91$ specific content components)

- Strengthening ( $\mathrm{n}=62$ components)

- Aerobic ( $\mathrm{n}=9$ components)

$\circ \quad$ Flexibility ( $\mathrm{n}=17$ components)

$\circ$ Balance-motor/Learning-agility ( $\mathrm{n}=17$ components)

$\circ$ Task specific training $(\mathrm{n}=17$ components)

- Patient education ( $n=6$ components)

- Each study was independently coded by two investigators, one with expertise in rehabilitation interventions (LT) and the other with expertise in multicomponent interventions (KJK)

- Each investigator reviewed the content of the intervention and:

- Sought to match the content to a specific content component (i.e., subcategories). Where a match could be made, the investigator inserted a code ' 1 ' to indicate its presence in cell (otherwise ' 0 ' to indicate absence).

- Subsequently sought to match the specific content component to the higher category intervention goal. Determination of the goal of the specific content component was based on the hierarchical taxonomy and interpretation of how the component was used (e.g., description of the parameters used to implement it) and other contextualizing details of the text.

- The latter was especially important for specific content components capable of addressing multiple goals (e.g., 'step down' can address "strengthening" and "balance-motor learning-agility").

- Inserted article text used to justify any specific content component or goal codes in the cell for the larger goal category and indicated what specific content component the text was meant to justify.

- Descriptive content was used to justify coding where discrepancies arose and provided qualitative text for further consideration of the taxonomy.

- Both investigators met virtually to compare codes, identify disagreements, discuss and come to consensus, revising coding rules as necessary.

- Where conflicts remained, a third reviewer (DP) was engaged in group discussion until consensus was achieved.

\section{Additional General Principles}

The following principles were used to guide intervention coding:

a. The intervention of at least one arm of each included study needed to be sufficiently described to be replicable by a therapist or other professional.

a. Studies defining interventions as "rehabilitation" without further detail were excluded.

b. Studies defining interventions based on rehabilitation goals only (e.g., "strengthening exercises") were included and coded according to the goal, but not regarding the specific content component for which there was no information

b. We coded the rehabilitation i) goals and ii) specific content components of all study arms, regardless of arm label (e.g., control, "treatment as usual") if rehabilitation content and goals met the descriptions above. 


\section{Adjunctive Modalities and Intervention Modifiers}

In addition to coding primary intervention components (by goals and specific content components, above), we coded the presence of the following adjunctive modalities:

- Modalities

○ Cold

○ Heat

- Compression for edema

- E-stim for pain (TENS)

$\circ \quad$ E-stim for strength (NMES)

O Other modalities for pain

- Ultrasound

- Manual therapy (e.g., therapeutic massage, passive range of motion)

○ Contract-relax for knee flexion/extension ROM

- Hold-relax for knee flexion/extension ROM

- Massage for edema control

- Massage for scar mobility

- Massage/myofascial techniques for soft tissue

○ Mobilizations - Tibiofemoral

○ Mobilizations - Patellar

- Biofeedback devices

- Dry needling

- Mindfulness, stress/anxiety-reduction interventions

- Complementary and alternative therapies (excluding ingested or inhaled treatments)

\section{Intervention modifiers:}

- Progression. Study stated that progression was a part of the intervention (Code 1=yes; $0=$ no).

- Appropriate progression. Progression deemed appropriate based on parameters defined (Code $1=$ present; $0=$ absent).

- Personnel. The intervention had to have been delivered, supervised, and/or monitored by a healthcare professional or other trained individual. Peer-led (or patient-led) interventions are eligible if monitored by a professional or other trained individual. The physical/healthcare professional (or other trained individual) must be involved in patient engagement and assessment of progress, and must provide ongoing feedback to the patient throughout the course of intervention

- Mode of delivery. The interaction with the healthcare professional or other trained individual had to have been direct (e.g., in-person therapy) or remote (e.g., via app, Web, or telephone). Remote therapy must include active monitoring by a physical therapist (or other trained individual), although the (p)rehabilitation therapy may be guided completely by the app.

- Setting of intervention. Physical location in which the intervention was delivered (may overlap slightly with mode of delivery). We extracted all that applied

- Acute inpatient (postoperative)

O Other inpatient facility (e.g., skilled nursing facility)

○ Physical therapy/rehabilitation facility (outpatient)

○ Home 
- Gym or other community center

O Other (specify)

- Not reported

\section{Specific Coding Elements}

- MJR_id (number=unique ID for study as created by MJR review)

- $\quad$ source (text= file pdf name used and additional sources other than primary paper).

- Exclude (category=yes/no/maybe). If no or maybe, give reason in note

- Exclude_note (text $=$ specific text describing why intervention is/is not well specified)

Labels:

- arm_name (text=specific label for arm as written in article; each study arm extracted into a unique row)

- Ix_well_specified (binary $0=$ no; $1=y e s$; is the intervention as a whole well specified?) Code YES if: Intervention is sufficiently described to be replicable by a therapist or other professional in practice

- $\quad$ Code NO if: Intervention is generally not well specified

- Ix_well_specified_note (text =specific text describing why intervention is/is not well specified)

\section{Intervention i) Goal and ii) Specific Content}

For each arm evaluated in the study, determine:

1. Strengthening (binary $0=$ no; $1=$ yes)

Code YES (to strengthening goal) if intervention describes

- Strengthening exercise generally

- One or more of the specific content components below and coder interprets that component supports the strengthening goal (also code YES to the specific content binary $0=$ no; $1=$ yes)

- If position unclear code 1 (position unclear) for all relevant codes

1.1 Bridges One-legged (supine hip extension)

1.2 BridgesTwo-legged (supine hip extension)

1.3 Calf press (one-leg)

1.4 Calf press (two-legs)

1.5 Clamshells

1.6 Core strengthening

1.7 Deadlifts

1.8 Gluteal Sets

1.9 Heel raises - bilateral

1.10 Heel raises - unilateral

1.11 Hip abduction in sidelying

1.12 Hip abduction in standing

1.13 Hip abduction in supine

1.14 Hip adduction in sidelying 
1.15 Hip adduction in standing

1.16 Hip adduction in supine

1.17 Hip extension in sidelying

1.18 Hip extension in prone

1.19 Hip extension in standing

1.20 Hip flexion in sidelying

1.21 Hip flexion in sitting

1.22 Hip flexion in standing

1.23 Hip flexion in supine

1.24 Hip hikes in standing

1.25 Hip hikes in supine

1.26 Hip rotation external (lateral)

1.27 Hip rotation internal (medial)

1.28 Knee extension machine (one-leg)

1.29 Knee extension machine (two-legs)

1.30 Knee extension AAROM in sitting or supine (short- or long arc quad)

1.31 Knee extension in sitting or supine (long arc quad)

1.32 Knee extension in sitting or supine (short arc quad)

1.33 Knee flexion machine (Hamstring curl) one knee

1.34 Knee flexion machine (Hamstring curl) two knees

1.35 Knee flexion in prone

1.36 Knee flexion in sitting or supine

1.37 Knee flexion in standing

1.38 Leg Press (one leg)

1.39 Leg Press (two legs)

1.40 Leg Press (side lying)

1.41 Lunges

1.42 Lunges to side (lateral lunge)

1.43 Quad sets

1.44 Quadruped arm lift

1.45 Quadruped leg lift

1.46 Quadruped arm and leg lift

1.47 Single Leg Stance (SLS)

1.48 Sit-to-stand

1.49 Squats

1.50 Squats (one leg)

1.51 Standing TKE (terminal knee extension)

1.52 Step down

1.53 Step down laterally

1.54 Step lateral

1.55 Step up - forward

1.56 Step up - lateral

1.57 Stool scoots

1.58 Straight leg raise (SLR)

1.59 Toe raises

1.60 Upper extremity strengthening 
1.61 Wall slides

1.62 Wall slides - Lateral (hip AB and ADductors)

Code NO if:

- No mention of strengthening exercise goal generally

- No mention of specific content components interpreted as seeking to improve the strengthening goal

Strengthening note (text=text to support goal and specific content codes)

2. Aerobic (binary $0=$ no; $1=$ yes)

Code YES (to aerobic endurance goal) if intervention describes

- Aerobic exercise generally

- One or more of the specific content components below and coder interprets that component supports the aerobic endurance goal (also code YES to the specific content binary $0=$ no; $1=$ yes

2.1 Aquatics (water aerobics, water walking)

2.2 Bike (Endurance)

2.3 Elliptical machine

2.4 Jogging in place or overland

2.5 Rowing machine

2.6 Step-ups

2.7 Stepper (upright or sitting)

2.8 Treadmill walking

2.9 Walking

Code NO if:

- No mention of aerobic exercise goal generally

- No mention of specific content components interpreted as seeking to improve the aerobic endurance goal

Aerobic note $\quad($ text $=$ text to support goal and specific content codes $)$

3. Flexibility (binary $0=$ no; $1=$ yes)

Code YES (to flexibility goal) if intervention describes

- Flexibility exercise generally

- One or more of the specific content components below and coder interprets that component supports the flexibility goal (also code YES to the specific content binary $0=$ no; $1=$ yes)

- If position unclear code 1 (position unclear) for all relevant codes

3.1 Ankle pumps

3.2 Bike (ROM)

3.3 Calf stretch with knee bent

3.4 Calf stretch with knee straight 
3.5 Hamstring stretch in any position

3.6 Heel slides

3.7 Hip extensor stretch (knee to chest)

3.8 Hip flexor stretch

3.9 Iliotibial band (ITB) stretch in any position

3.10 Knee extension AROM

3.11 Knee extension PROM in supine

3.12 Knee extension PROM in prone

3.13 Knee flexion AROM

3.14 Knee flexion PROM in sitting or supine

3.15 Knee flexion AROM in any position (rectus femoris stretch)

3.16 Knee flexion PROM in prone (rectus femoris stretch)

3.17 Standing terminal knee extension

\section{Code NO if:}

- No mention of flexibility exercise goal generally

- No mention of specific content components interpreted as seeking to improve the flexibility goal

Flexibility note (text $=$ text to support goal and specific content codes)

4. Balance-Motor Learning-Agility (BMLA) (binary $0=$ no; $1=$ yes)

Code YES (to a BMLA goal) if intervention describes

- BMLA exercise generally

- One or more of the specific content components below and coder interprets that component supports the BMLA goal (also code YES to the specific content binary $0=$ no; $1=$ yes)

4.1 Balance in kneeling

4.2 Balance in quadruped

4.3 Balance on unstable surface

4.4 Balance with perturbations

4.5 Ladder drills

4.6 Marching

4.7 Quadruped

4.8 Single leg stance

4.9 Standing weight shifts

4.10 Stepping multiple directions (grapevine)

4.11 Step down

4.12 Step down laterally

4.13 Step lateral (side step)

4.14 Step up - forward

4.15 Step up - lateral

4.16 Tandem standing

4.17 Tandem walking 
Code NO if:

- No mention of BMLA goal generally

- No mention of specific content components interpreted as seeking to improve the a BMLA goal

Balance-Motor Learning-Agility note (text $=$ text to support goal and specific content codes)

5. Task specific training (binary $0=$ no; $1=$ yes)

Code YES (to task specific training goal) if intervention describes

- Task specific training generally

- One or more of the specific content components below and coder interprets that component supports the task specific training goal (also code YES to the specific content binary $0=$ no; $1=$ yes)

5.1 Car transfers

5.2 Deadlifts

5.3 Floor-to-sit or Floor-to-stand

5.4 Gait backwards

5.5 Gait downhill

5.6 Gait on uneven surfaces

5.7 Gait sideways

5.8 Gait training

5.9 Gait uphill

5.10 Gait with perturbations

5.11 Gait with resistance

5.12 Obstacle training

5.13 Sit-to-stand training

5.14 Sports specific training

5.15 Stair training

5.16 Treadmill gait

5.17 Treadmill gait (retro)

Code NO if:

- No mention of task specific training goal generally

- No mention of specific content components interpreted as seeking to improve the task specific training goal

Task specific training note (text $=$ text to support goal and specific content codes)

6. Patient education (binary $0=$ no; $1=$ yes)

Code YES (to patient education goal) if intervention describes

- Patient education generally 
- One or more of the specific content components below and coder interprets that component supports the patient education goal (also code YES to the specific content binary $0=$ no; $1=$ yes)
6.1 ADLs
6.2 Home exercise program (HEP)
6.3 Life-style change
6.4 Pain management
6.5 Self-management
6.6 Wound care management

Code NO if:

- No mention of patient education goal generally

- No mention of specific content components interpreted as seeking to improve the flexibility goal

Patient education note (text=text to support goal and specific content codes)

7. Adjunctive modalities (Binary $0=$ no; $1=$ yes)

Code YES (to each adjunctive modality as relevant) if intervention describes the presence of any of the following adjunctive modalities

- Modalities

7.1 Cold

7.2 Heat

7.3 Compression for edema

7.4 E-stim for pain (TENS)

7.5 E-stim for strength (NMES)

7.6 Other modalities for pain

7.7 Ultrasound

7.8 Manual therapy (e.g., therapeutic massage, passive range of motion)

7.9 Contract-relax for knee flexion/extension ROM

7.10 Hold-relax for knee flexion/extension ROM

7.11 Massage for edema control

7.12 Massage for scar mobility

7.13 Massage/myofascial techniques for soft tissue

7.14 Mobilizations - Tibiofemoral

7.15 Mobilizations - Patellar

7.16 Biofeedback devices

7.17 Dry needling

7.18 Mindfulness, stress/anxiety-reduction interventions

7.10 Complementary and alternative therapies (excluding ingested or inhaled treatments)

Code NO if: 
- $\quad$ No mention of using adjunctive modality(ies)

For each arm evaluated in the study, determine:

Effect modifiers

Progression (binary $0=$ no; $1=$ yes)

Code YES if:

- $\quad$ Study states that progression was a part of the intervention (Code $1=y e s ; 0=$ no).

May be progression by time and/or patient response.

Code NO if:

- The intervention does not mentioned progression of the intervention.

Progression_appropriate (binary $0=$ no; $1=$ yes)

Code YES if:

- The progression program is deemed appropriate based on parameters defined.

Code NO if:

- The progression program is not deemed appropriate based on parameters defined.

Code Unclear if:

- $\quad$ Not enough information to determine Yes or No.

Progression_note (text $=$ specific description of the details of progression)

\section{Personnel (categories)}

Indicate personnel who delivered the intervention from the following.

Select all that apply.

- Physical therapist

- Nurse

- Educator

- Peer

- Athletic trainer

- Exercise physiologist

- None (unsupervised)

- Other

Personnel_note (text=specific description of the details of the personnel delivering the intervention) 
Mode_of_delivery_ (categories)

Indicate mode of how the intervention was delivered.

Select all that apply.

- In-person therapy

- Remote via app

- Remote via web

- Remote via telephone

- $\quad$ Self-guided (unsupervised)

Mode_of_delivery_note (text=specific description of the details of how the intervention was delivered)

\section{Setting (categories)}

Select a prespecified category of where the intervention was delivered.

Select all that apply.

- Acute inpatient (postoperative)

- Other inpatient facility (e.g., skilled nursing facility)

- Physical therapy/rehabilitation facility (outpatient)

- Home

- Gym or other community center

- Other (specify)

- Not reported

Setting_note

(text $=$ specific description of where the intervention was delivered)

\section{Additional intervention comments}

Use to note content of interventions that you think may be relevant/we may want to be aware of but are not of sufficient information, or cross the threshold to warrant coding (e.g., provided supplemental information, esp. in control group, but not really sufficient to count as patient education)

\section{Concerns/queries}

Use to note other potential methodological concerns separate from the intervention

\section{Risk of Bias Assessment (Details)}

We evaluated each study for risk of bias and methodological quality. Because we included a variety of study designs, we incorporated items from three different existing commonly-used tools and tailored the set of items for each study design. The three tools were the Cochrane Risk of Bias Tool, ${ }^{4}$ the Risk of Bias in Nonrandomized Studies (ROBINS-I) Tool, ${ }^{5}$ and the National Heart, Lung, and Blood Institute (NHLBI) Quality Assessment Tool. ${ }^{6}$ 
For RCTs, we used all the items from the Cochrane Risk of Bias tool, ${ }^{4}$ focusing on issues related to randomization and allocation concealment methodology; blinding of patients, study personnel/care providers, objective outcome assessors, and subjective outcome assessors; incomplete outcome data; selective outcome reporting; and other issues that could be related to bias.

For NRCSs, we used the specific sections of ROBINS-I ${ }^{5}$ that pertain to confounding and selection bias. ROBINS-I requires the identification of specific confounders of interest for the systematic review. For the purpose of assessing for the presence of potential confounding in studies, we considered demographics (such as age, sex, race/ethnicity), socioeconomic status, caregiver support, body mass index (BMI), comorbidities, prior arthroplasty of contralateral joint, narcotic use, preoperative symptoms/status (e.g., severity of symptoms including pain, impaired function, restricted movement, physical activity, frailty), surgical factors (e.g., surgical procedure or protocol, type of implant), and hospital type for all KQs. Additionally, for KQs 2 and 4 related to postoperative care, we considered length of hospital stay as an additional potential confounder. For RCTs, we also supplemented the Cochrane Risk of Bias tool with items from the NHLBI tool that pertain to the adequacy of descriptions of study eligibility criteria, interventions, and outcomes. ${ }^{6}$

\section{Data Synthesis and Analysis (Details)}

We had planned to summarize the evidence both qualitatively and, when feasible, quantitatively (via a network meta-analysis across the programs, focusing on comparisons of different components). However, due to the heterogeneity of interventions (i.e., almost completely unique content in (p)rehabilitation intervention and comparator arms, delivered at different times, in different settings and by different personnel) and lack of consistency of outcome reported, meaningful statistical meta-analyses were not feasible, and we summarized the evidence only qualitatively.

Each study included in the systematic review is described in summary and evidence tables presenting study design features, study participant characteristics, descriptions of interventions, outcome results, and risk of bias/methodological quality. Summary tables briefly describe the studies and their findings.

For KQs 1-4, we compared interventions with their comparators for their effects (grouping related interventions and comparisons as feasible), using post mean differences in continuous outcome data (i.e., difference in follow-up mean between groups) or net mean differences (i.e., difference-in-difference, or the between-intervention comparison of within-intervention changes). As there was not sufficient studies reporting sufficiently similar outcomes to explore the association of the specific intervention factors (components, personnel, setting), using statistical methods, we sought to explore associations narratively across studies by considering each of the factors as a 'lens' of potential impact when looking at the evidence.

\section{Grading the Strength of the Body of Evidence (Details)}

We evaluated the strength of evidence (SoE) addressing each major conclusion for each KQ (and subquestion). We graded the SoE as per the Agency for Healthcare Research and Quality (AHRQ) Methods Guide. ${ }^{7,8}$

We assessed the SoE for key outcomes For each SoE assessment, we considered the number of studies, the study limitations (i.e., risk of bias and overall methodological quality), the directness of the evidence to the KQs, the consistency of study results, the precision of any 
estimates of effect, the likelihood of reporting bias, other limitations, and the overall findings across studies. We interpreted directness based on the proximity of the outcome to the clinical outcome of interest (i.e., intermediate) and whether the outcome was assessed among the individuals of interest vs. proxy. For example, a patient-reported outcome of function or a performance-based outcome of strength would both be considered direct. We interpreted precision based on the confidence intervals of the individual studies. This is considered appropriate in the GRADE methods "if a meta-analysis is infeasible or inappropriate", reviewers may consider the narrowness of the range of CIs or the significant level of p-values in the individual studies in the evidence base". ${ }^{8}$

Based on these assessments, we assigned a SoE rating as being either high, moderate, low, or insufficient to estimate an effect.

Outcomes with highly imprecise estimates, highly inconsistent findings across studies, or with data from only one study, were deemed to have insufficient evidence to allow a conclusion. This overall approach is consistent with the definition of Very Low quality evidence per GRADE defined as "any estimate of effect is very uncertain".

\section{Peer Review and Public Commentary}

Experts in fields of physiotherapy orthopaedic surgery, and individuals representing stakeholder and user communities (e.g., health systems, and guideline and policy development) were invited to provide external peer review of this systematic review; AHRQ and an associate editor also provided comments. Subsequent to peer review, the draft report was posted on the AHRQ website for 4 weeks to elicit public comment (May 10, 2021 to June 7, 2021). A disposition of comments table of public comments will be posted on the EHC website 3 months after the Agency posts the final systematic review. 


\section{References for Appendix A}

1. Franklin PD. Defining Components of Physical Therapy Achieving Maximum Function after TKR. Northwestern University at Chicago, Chicago, IL, United States; 2019.

2. Oatis CA, Johnson JK, DeWan T, et al. Characteristics of Usual Physical Therapy Post-Total Knee Replacement and Their Associations With Functional Outcomes. Arthritis Care Res (Hoboken). 2019 Sep;71(9):1171-7. doi: 10.1002/acr.23761. PMID: 30281207.

3. Yang X, Li GH, Wang HJ, et al. Continuous Passive Motion After Total Knee Arthroplasty: A Systematic Review and Meta-analysis of Associated Effects on Clinical Outcomes. Arch Phys Med Rehabil. 2019 Sep;100(9):1763-78. doi: 10.1016/j.apmr.2019.02.001. PMID: 30831093.

4. Higgins JP, Altman DG, Gøtzsche PC, et al. The Cochrane Collaboration's tool for assessing risk of bias in randomised trials. Bmj. 2011 Oct 18;343:d5928. doi: 10.1136/bmj.d5928. PMID: 22008217.

5. Sterne JA, Hernán MA, Reeves BC, et al. ROBINS-I: a tool for assessing risk of bias in nonrandomised studies of interventions. Bmj. 2016 Oct 12;355:i4919. doi: 10.1136/bmj.i4919. PMID: 27733354.

6. National Heart, Lung, and Blood Institute. Study Quality Assessment Tools.; 2019. https://www.nhlbi.nih.gov/health-topics/study-quality-assessment-tools. Accessed on January 23, 2020.

7. Berkman ND, Lohr KN, Ansari M, et al. AHRQ Methods for Effective Health Care

Grading the Strength of a Body of Evidence When Assessing Health Care Interventions for the Effective Health Care Program of the Agency for Healthcare Research and Quality: An Update. Methods Guide for Effectiveness and Comparative Effectiveness Reviews. Rockville (MD): Agency for Healthcare Research and Quality (US); 2008.

8. Berkman ND, Lohr KN, Ansari MT, et al. Grading the strength of a body of evidence when assessing health care interventions: an EPC update. J Clin Epidemiol. 2015 Nov;68(11):1312-24. doi: 10.1016/j.jclinepi.2014.11.023. PMID: 25721570.

9. Atkins D, Best D, Briss PA, et al. Grading quality of evidence and strength of recommendations. Bmj. 2004 Jun 19;328(7454):1490. doi: 10.1136/bmj.328.7454.1490. PMID: 15205295. 


\section{Appendix B. Excluded Studies}

Abbas C; Daher J. Pilot study: Post-operative rehabilitation pathway changes and implementation of functional closed kinetic chain exercise in total hip and total knee replacement patient.. Journal of bodywork and movement therapies. 29037634. D: NRCS not adjusted

Abbass Reslan H; Moustafa SM; Saghieh S; Sharara ES; Badr LK. Does intervention improve the outcomes of patients after total knee replacement surgery?. International journal of orthopaedic and trauma nursing. 30393030.

D: NRCS not adjusted

Actrn. Telerehabilitation for patients with total knee replacement.

http://www.who.int/trialsearch/Trial2.aspx?TrialID= ACTRN12606000346572. CN-01807385.

Protocol/study registration

Actrn. Early aquatic physiotherapy after total hip and total knee replacement surgery.

http://www.who.int/trialsearch/Trial2.aspx?TrialID= ACTRN12616000494437. CN-01860201.

Protocol/study registration

Actrn. The STEP study: a Pragmatic, randomized, controlled trial comparing two post operative management pathways in knee or hip replacement surgery.

http://www.who.int/trialsearch/Trial2.aspx?TrialID= ACTRN12616000696493. CN-01882269.

Protocol/study registration

Actrn. Face-to-face physiotherapy compared to a supported home exercise program for the management of musculoskeletal conditions: the REFORM trial.

http://www.who.int/trialsearch/Trial2.aspx?TrialID= ACTRN12619000065190. CN-01946979.

Protocol/study registration

Actrn. Biofeedback-assisted exercise in the rehabilitation of patients after total knee arthroplasty $\sqrt{ } \varnothing, C^{\prime \prime}$, Äú effects on functional outcomes and quality of life.

http://www.who.int/trialsearch/Trial2.aspx?TrialID= ACTRN12618001782224. CN-01947485.

Protocol/study registration

Actrn. A pilot study of a psychologically informed physiotherapy for people awaiting knee replacement surgery.

http://www.who.int/trialsearch/Trial2.aspx?TrialID= ACTRN12618001867280. CN-01948991.

Protocol/study registration
Actrn. The effects of adding neuromuscular electrical stimulation to standard inpatient rehabilitation on quadriceps strength and physical function in individuals with total knee replacement. http://www.who.int/trialsearch/Trial2.aspx?TrialID= ACTRN12610000601033. CN-01840019.

Protocol/study registration

Actrn. A Randomised Controlled Study on the Maxm Skate: a Lower Limb Rehabilitation Device for use following Total Knee Arthroplasty. http://www.who.int/trialsearch/Trial2.aspx?TrialID= ACTRN12616001081404. CN-01877939.

Protocol/study registration

Actrn. The HIHO 2 Study: hospital Inpatient versus Home-based Rehabilitation after Total Hip Replacement. http://www.who.int/trialsearch/Trial2.aspx?TrialID= ACTRN12617000848303. CN-01885965.

Protocol/study registration

Actrn. A pedaling-based three exercise protocol compared to a non-pedaling ten exercise protocol for immediate post-operative rehabilitation after total knee replacement in a randomized controlled trial. http://www.who.int/trialsearch/Trial2.aspx?TrialID= ACTRN12617000647336. CN-01895094.

Protocol/study registration

Adie, S.; Kwan, A.; Naylor, J. M.; Harris, I. A.; Mittal, R.. Cryotherapy following total knee replacement. Cochrane Database of Systematic Reviews. 22972114.

SR or guideline

Agostini M; Moja L; Banzi R; Pistotti V; Tonin P; Venneri A; Turolla A. Telerehabilitation and recovery of motor function: a systematic review and meta-analysis.. Journal of telemedicine and telecare. 25712109.

SR or guideline

Akbaba, Y. A.; Yeldan, I.; G ${ }^{\circ}$ ney, N.; $\sqrt{n} z d i n \sqrt{ }$ Bler, A. R.. The effects of rehabilitation program after total knee replacement on balance and functionality. Osteoarthritis \& Cartilage. 113582965. Language:. P: Not unilateral replacement (exclude bilateral replacement surgeries)

Alaca N; Atalay A; Guven Z. Comparison of the long-term effectiveness of progressive neuromuscular facilitation and continuous passive motion therapies after total knee arthroplasty.. Journal of physical therapy science. 26696702.

D: $\mathrm{N}<20$ per group 
Alghadir A; Iqbal ZA; Anwer S. Comparison of the effect of pre- and post-operative physical therapy versus post-operative physical therapy alone on pain and recovery of function after total knee arthroplasty.. Journal of physical therapy science. 27821929.

$\mathrm{T}$ : Timing outcome too early

Almeida, G. J.; Khoja, S. S.; Zelle, B. A.. Effect of prehabilitation in older adults undergoing total joint replacement: An Overview of Systematic Reviews. Curr Geriatr Rep. 33344110.

SR or guideline

Alnahdi, A. H.; Zeni, J. A.; Snyder-Mackler, L.. The effect of progressive strengthening programs on function and gait mechanics after unilateral total knee arthroplasty: a randomized clinical trial.

Osteoarthritis and cartilage.. CN-01033286.

$\mathrm{D}: \mathrm{N}<20$ per group

Alomar, J. A.; Catelani, M. B. C.; Smith, C. N.; Patterson, C. G.; Artman, T. M.; Piva, S. R.. Validity and Responsiveness of Floor Sitting-Rising Test in Post-Total Knee Arthroplasty: A Cohort Study. Arch Phys Med Rehabil. 32315648.

D: Single group/no comparison

Alomar, J. A.; Catelani, M. B. C.; Smith, C. N.; Patterson, C. G.; Artman, T. M.; Piva, S. R.. Validity and Responsiveness of Floor Sitting-Rising Test in Post $\bigvee_{\varnothing}, C_{C}$, , ÄúTotal Knee Arthroplasty: a Cohort Study. Archives of physical medicine and rehabilitation. CN-02120408.

Duplicate/No additional data

Alomar, Jehan A.; Catelani, Maria Beatriz C.; Smith, Clair N.; Patterson, Charity G.; Artman, Tamara M.; Piva, Sara R.. Validity and Responsiveness of Floor Sitting-Rising Test in Post, ÄiTotal Knee

Arthroplasty: A Cohort Study. Archives of Physical

Medicine \& Rehabilitation. 32315648.

Duplicate/No additional data

Alrawashdeh, W.; Eschweiler, J.; Migliorini, F.; El Mansy, Y.; Tingart, M.; Rath, B.. Benefit of Modified Rehabilitation Programs after Total Knee Arthroplasty: a systematic review and meta-analysis. J Rehabil Med. 33846757.

SR or guideline

Alsiri, N. F.; Alhadhoud, M. A.; Al-Mukaimi, A.; Palmer, S.. The effect of Mulligan's mobilization with movement following total knee arthroplasty: Protocol of a single-blind randomized controlled trial. Musculoskeletal Care. 33045126.

Protocol/study registration
Alsiri, N. F.,; Alhadhoud, M. A.,; Al-Mukaimi, A.; Palmer, S.. The effect of Mulligan's mobilization with movement following total knee arthroplasty: protocol of a single-blind randomized controlled trial. Musculoskeletal care. 33045126.

Duplicate/No additional data

Alsiri, Najla F.; Alhadhoud, Meshal A.; Al, ÄêMukaimi, Ali; Palmer, Shea. The effect of Mulligan's mobilization with movement following total knee arthroplasty: Protocol of a single, Äêblind randomized controlled trial. Musculoskeletal Care. 33045126.

Duplicate/No additional data

Amano, T.. Factors for Assessing the Effectiveness of Early Rehabilitation after Minimally Invasive Total Knee Arthroplasty: A Prospective Cohort Study. PLoS One. 27410385.

D: Single group/no comparison

Andermann I; Wehsarg FK. [Follow-up treatment of patients with prosthetic joint replacement].. Rehabilitation: Sozialmedizin, physikalische Medizin, Praventivmedizin; internationale Zeitschrift mit Zentralblatt. 6672913.

Full text unavailable

Anonymous. Post hip replacement walking training may improve physical ability. Aging Health. 104090676. Language:.

Full text unavailable

Anonymous. The effect of early application of electromyographic biofeedback to quadriceps femoris muscle on rehabilitation outcomes in patients with total knee arthroplasty. Turkish Journal of Physiotherapy Rehabilitation. 107984321. Language:.

Full text unavailable

Aprile I; Iacovelli C; Cruciani A; Simbolotti C; Loreti S; Galli G; Vulpiani MC; Padua L. Technological rehabilitation versus conventional rehabilitation following hip replacement: A prospective controlled study.. Journal of back and musculoskeletal rehabilitation. 31743982.

P: Not osteoarthritis (exclude cancer, trauma, rheumatoid arthritis)

Aprile I; Rizzo RS; Romanini E; De Santis F; Marsan S; Rinaldi G; Padua L. Group rehabilitation versus individual rehabilitation following knee and hip replacement: a pilot study with randomized, singleblind, cross-over design.. European journal of physical and rehabilitation medicine. 21666574. D: $\mathrm{N}<20$ per group 
Aprile, I.; Iacovelli, C.; Cruciani, A.; Simbolotti, C.; Loreti, S.; Galli, G.; Vulpiani, M. C.; Padua, L.. Technological rehabilitation versus conventional rehabilitation following hip replacement: a prospective controlled study. Journal of back and musculoskeletal rehabilitation. 31743982.

P: Not osteoarthritis (exclude cancer, trauma, rheumatoid arthritis)

Arienti, C.; Pollet, J.; Buraschi, R.; Piovanelli, B.; Villafa $_{ \pm}$e, J. H.; Galeri, S.; Negrini, S.. Fast-track rehabilitation after total knee arthroplasty reduces length of hospital stay: A prospective, case-control clinical trial. Turk J Phys Med Rehabil. 33364559. Duplicate/No additional data

Arienti, C.; Pollet, J.; Buraschi, R.; Piovanelli, B.; Villafa $\sqrt{ \pm e}$, J. H.; Galeri, S.; Negrini, S.. Fast-track rehabilitation after total knee arthroplasty reduces length of hospital stay: A prospective, case-control clinical trial. Turkish Journal of Physical Medicine and Rehabilitation. .

I: Not (p)rehabilitation

Arkin, L. C.; Reising, E.; Penoyer, D.; Talbert, S.. Impact of Bundled Care on Outcomes Following Elective Primary Total Hip or Total Knee Arthroplasty. Orthop Nurs. 31343631. I: Not (p)rehabilitation

Artz N; Elvers KT; Lowe CM; Sackley C; Jepson P; Beswick AD. Effectiveness of physiotherapy exercise following total knee replacement: systematic review and meta-analysis.. BMC musculoskeletal disorders. 25886975.

SR or guideline

Atabaki, S.; Farahani, M. A.; Haghani, S.. Effect of rehabilitation education on pain, knee stiffness and performance difficulty in patients undergoing knee replacement surgery: a randomized clinical trial. Journal of acute disease. CN-02073860.

$\mathrm{T}$ : Timing outcome too early

Aunger, J. A.; Greaves, C. J.; Davis, E. T.; Asamane, E. A.; Whittaker, A. C.; Greig, C. A.. A novel behavioural INTErvention to REduce Sitting Time in older adults undergoing orthopaedic surgery (INTEREST): results of a randomised-controlled feasibility study. Aging Clin Exp Res. 31975288. $\mathrm{D}: \mathrm{N}<20$ per group

Aunger, J. A.; Greaves, C. J.; Davis, E. T.; Greig, C. A.. A novel behavioural INTErvention to REduce Sitting Time in older adults undergoing orthopaedic surgery (INTEREST): protocol for a randomised controlled feasibility study. Pilot Feasibility Stud.
30997142.

$\mathrm{D}: \mathrm{N}<20$ per group

Avramidis K; Strike PW; Taylor PN; Swain ID. Effectiveness of electric stimulation of the vastus medialis muscle in the rehabilitation of patients after total knee arthroplasty.. Archives of physical medicine and rehabilitation. 14669193.

published before 2005

Aytekin E; Sukur E; Oz N; Telatar A; Eroglu Demir S; Sayiner Caglar N; Ozturkmen Y; Ozgonenel L. The effect of a 12 week prehabilitation program on pain and function for patients undergoing total knee arthroplasty: A prospective controlled study.. Journal of clinical orthopaedics and trauma. 30828206.

D: NRCS not adjusted

Aytekin E, E Sukur, N Oz, A Telatar, SE Demir, NS Caglar, Y Ozturkmen. The effect of a 12 week prehabilitation program on pain and function for patients undergoing total knee arthroplasty: a prospective controlled study. Journal of clinical orthopaedics and trauma. CN-01919783.

Duplicate/No additional data

Bade, M.; Struessel, T.; Paxton, R.; Winters, J.; Baym, C.; Stevens-Lapsley, J.. Performance on a Clinical Quadriceps Activation Battery Is Related to a Laboratory Measure of Activation and Recovery After Total Knee Arthroplasty. Arch Phys Med Rehabil. 28864244.

Duplicate/No additional data

Bade, M. J.; Christensen, J. C.; Zeni, J. A., Jr.; Christiansen, C. L.; Dayton, M. R.; Forster, J. E.; Cheuy, V. A.; Stevens-Lapsley, J. E.. Movement pattern biofeedback training after total knee arthroplasty: Randomized clinical trial protocol. Contemp Clin Trials. 32171937.

Protocol/study registration

Bade, Michael John. Improving strength and function after total knee arthroplasty. Unknown. .

Full text unavailable

Baek J; Park N; Lee B; Jee S; Yang S; Kang S. Effects of Repetitive Peripheral Magnetic Stimulation Over Vastus Lateralis in Patients After Hip Replacement Surgery.. Annals of rehabilitation medicine. 29560326.

P: Not osteoarthritis (exclude cancer, trauma, rheumatoid arthritis)

Bai, X.. [Clinical effects of comprehensive rehabilitation after minimally invasive total hip arthroplasty]. Zhongguo Gu Shang. 19594035. Full text unavailable 
Barad, S. J.; Howell, S. M.; Tom, J.. Is a shortened length of stay and increased rate of discharge to home associated with a low readmission rate and costeffectiveness after primary total knee arthroplasty?. Arthroplast Today. 29564377.

I: Not (p)rehabilitation

Barbay K. Research evidence for the use of preoperative exercise in patients preparing for total hip or total knee arthroplasty.. Orthopedic nursing. 19494760.

SR or guideline

Barber-Westin SD; Noyes FR. Aerobic Physical Fitness and Recreational Sports Participation After Total Knee Arthroplasty.. Sports health. 27644234. SR or guideline

Barker KL; Beard D; Price A; Toye F; Underwood M; Drummond A; Collins G; Dutton S; Campbell H; Kenealy N; Room J; Lamb SE. COmmunity-based Rehabilitation after Knee Arthroplasty (CORKA): study protocol for a randomised controlled trial.. Trials. 27737685.

Protocol/study registration

Barker KL; Newman MA; Hughes T; Sackley C; Pandit H; Kiran A; Murray DW. Recovery of function following hip resurfacing arthroplasty: a randomized controlled trial comparing an accelerated versus standard physiotherapy rehabilitation programme.. Clinical rehabilitation. 23576032. P: Not total relacement (exclude partial)

Barker, K. L.; Room, J.; Knight, R.; Dutton, S. J.; Toye, F.; Leal, J.; Kent, S.; Kenealy, N.; Schussel, M. M.; Collins, G.; Beard, D. J.; Price, A.; Underwood, M.; Drummond, A.; Cook, E.; Lamb, S. E.. Outpatient physiotherapy versus home-based rehabilitation for patients at risk of poor outcomes after knee arthroplasty: CORKA RCT. Health Technol Assess. 33250068. P: Not total relacement (exclude partial)

Barrois B; Gouin F; Ribinik P; Revel M; Rannou F. What is the interest of rehabilitation in physical medicine and functional rehabilitation ward after total hip arthroplasty? Elaboration of french clinical practice guidelines.. Annales de readaptation et de medecine physique : revue scientifique de la Societe francaise de reeducation fonctionnelle de readaptation et de medecine physique. 17850912 . SR or guideline

Barrois, B.; Ribinik, P.; Gougeon, F.; Rannou, F.; Revel, M.. What is the role of rehabilitation in a specialized center after total knee replacement? Elaboration of French recommendations for clinical practice. Annales de Readaptation et de Medecine Physique. .

Full text unavailable

Baulig C; Grams M; Rohrig B; Linck-Eleftheriadis S; Krummenauer F. Clinical outcome and cost effectiveness of inpatient rehabilitation after total hip and knee arthroplasty. A multi-centre cohort benchmarking study between nine rehabilitation departments in Rhineland-Palatinate (Western Germany).. European journal of physical and rehabilitation medicine. 26006080.

$\mathrm{T}$ : Timing outcome too early

Beaupre LA; Davies DM; Jones CA; Cinats JG. Exercise combined with continuous passive motion or slider board therapy compared with exercise only: a randomized controlled trial of patients following total knee arthroplasty.. Physical therapy. 11296803. published before 2005

Beaupre LA; Lier D; Davies DM; Johnston DB. The effect of a preoperative exercise and education program on functional recovery, health related quality of life, and health service utilization following primary total knee arthroplasty.. The Journal of rheumatology. 15170931. published before 2005

Beaupre LA; Masson EC; Luckhurst BJ; Arafah O; O'Connor GJ. A randomized pilot study of a comprehensive postoperative exercise program compared with usual care following primary total hip arthroplasty in subjects less than 65 years of age: feasibility, selection of outcome measures and timing of assessment. BMC musculoskeletal disorders. 24889213.

D: $\mathrm{N}<20$ per group

Beck H; Beyer F; Gering F; Gunther KP; Lutzner C; Walther A; Stiehler M. Sports Therapy Interventions Following Total Hip Replacement.. Deutsches Arzteblatt international. 30782304. Duplicate/No additional data

Bedekar N; Prabhu A; Shyam A; Sancheti K; Sancheti P. Comparative study of conventional therapy and additional yogasanas for knee rehabilitation after total knee arthroplasty.. International journal of yoga. 22869995. D: NRCS not adjusted

Benz T; Angst F; Oesch P; Hilfiker R; Lehmann S; Mueller Mebes C; Kramer E; Verra ML. Comparison of patients in three different rehabilitation settings after knee or hip arthroplasty: a natural observational, prospective study.. BMC musculoskeletal disorders. 
26497597.

D: NRCS not adjusted

Berge DJ; Dolin SJ; Williams AC; Harman R. Preoperative and post-operative effect of a pain management programme prior to total hip replacement: a randomized controlled trial.. Pain. 15275749 .

published before 2005

Bettger, J. P.; Green, C. L.; Holmes, D. N.; Chokshi, A.; Mather, R. C.; Hoch, B. T.; de Leon, A. J.; Aluisio, F.; Seyler, T. M.; Del Gaizo, D. J.; et al.. Effects of Virtual Exercise Rehabilitation In-Home Therapy Compared with Traditional Care After Total Knee Arthroplasty: VERITAS, a Randomized Controlled Trial. Journal of bone and joint surgery. American volume. CN-02005570. Duplicate/No additional data

Bhatia, S.; Karvannan, H.; Prem, V.. The effect of bio psychosocial model of rehabilitation on pain and quality of life after total knee replacement: a randomized controlled trial. Journal of arthroscopy and joint surgery. CN-02202836.

$\mathrm{D}: \mathrm{N}<20$ per group

Bini SA; Mahajan J. Clinical outcomes of remote asynchronous telerehabilitation are equivalent to traditional therapy following total knee arthroplasty: A randomized control study.. Journal of telemedicine and telecare. 26940798.

$\mathrm{D}: \mathrm{N}<20$ per group

Birch S; Stilling M; Mechlenburg I; Hansen TB. Effectiveness of a physiotherapist delivered cognitive-behavioral patient education for patients who undergoes operation for total knee arthroplasty: a protocol of a randomized controlled trial.. BMC musculoskeletal disorders. 28320421.

Protocol/study registration

Bistolfi A; Zanovello J; Ferracini R; Allisiardi F; Lioce E; Magistroni E; Berchialla P; Da Rold I; Massazza G. Evaluation of the Effectiveness of Neuromuscular Electrical Stimulation After Total Knee Arthroplasty: A Meta-Analysis.. American journal of physical medicine \& rehabilitation. 29016401.

SR or guideline

Biton, Danielle. Walking biomechanics of end stage knee OA and TKA patients. University of Louisville Doctoral Dissertation. .

$\mathrm{D}: \mathrm{N}<20$ per group

Blasco JM; Acosta-Ballester Y; Igual-Camacho C; Hernandez-Guillen D; Gomez MC; Roig-Casasus S; Puigcerver-Aranda P. Preferred Outcome Measures
Used in Randomized Clinical Trials of Total Knee Replacement Rehabilitation: A Systematic Review.. PM \& R : the journal of injury, function, and rehabilitation. 31883225 .

SR or guideline

Blasco JM; Igual-Camacho C; Blasco MC; AntonAnton V; Ortiz-Llueca A; Roig-Casasus S. The efficacy of virtual reality tools for total knee replacement rehabilitation: A systematic review.. Physiotherapy theory and practice. 31313607.

SR or guideline

Blasco JM; Igual-Camacho C; Roig-Casasus S. Inhome versus hospital preoperative balance and proprioceptive training in patients undergoing TKR; rationale, design, and method of a randomized controlled trial.. BMC musculoskeletal disorders. 29221471.

T: Timing outcome too early

Blasco, J. M.; Acosta-Ballester, Y.; MartinezGarrido, I.; Garcia-Molina, P.; Igual-Camacho, C.; Roig-Casasus, S.. The effects of preoperative balance training on balance and functional outcome after total knee replacement: a randomized controlled trial. Clinical rehabilitation. CN-01995859.

$\mathrm{T}$ : Timing outcome too early

Blasco, J. M.; Acosta-Ballester, Y.; MartinezGarrido, I.; Garcia-Molina, P.; Igual-Camacho, C.; Roig-Casasus, S.. The effects of preoperative balance training on balance and functional outcome after total knee replacement: a randomized controlled trial. Clin Rehabil. 31608677.

T: Timing outcome too early

Blasco, J. M.; Hern $\sqrt{ }{ }^{\circ}$ ndez-Guillen, D.; Dom $\sqrt{ } \neq$ nguez-Navarro, F.; Acosta-Ballester, Y.; Alakhdar-Mohmara, Y.; Roig-Casas $\sqrt{ } \int_{\mathrm{s}}$, S..

Sensorimotor training prior total knee arthroplasty and effects on functional outcome: A systematic review and meta-analysis. Gait Posture. 33711615. SR or guideline

Bohl, D. D.; Li, J.; Calkins, T. E.; Darrith, B.; Edmiston, T. A.; Nam, D.; Gerlinger, T. L.; Levine, B. R.; Della Valle, C. J.. Physical Therapy on Postoperative Day Zero Following Total Knee Arthroplasty: A Randomized, Controlled Trial of 394 Patients. J Arthroplasty. 30827716.

$\mathrm{O}$ : No outcomes of interest

Borgwardt L; Zerahn B; Bliddal H; Christiansen C; Sylvest J; Borgwardt A. Similar clinical outcome after unicompartmental knee arthroplasty using a conventional or accelerated care program: a randomized, controlled study of 40 patients.. Acta 
orthopaedica. 19513890.

P: Not total relacement (exclude partial)

Bourne, Robert B.. Physiotherapy exercise provides short-term functional benefit after total knee arthroplasty. Journal of Bone \& Joint Surgery, American Volume. .

SR or guideline

Brander, V.; Stulberg, S. D.. Rehabilitation after hipand knee-joint replacement: an experience- and evidence-based approach to care. American Journal of Physical Medicine \& Rehabilitation. 106165720.

Language:.

D: Not primary study (or SR or GL)

Brandes M; Wirsik N; Niehoff H; Heimsoth J; Mohring B. Impact of a tailored activity counselling intervention during inpatient rehabilitation after knee and hip arthroplasty - an explorative RCT.. BMC musculoskeletal disorders. 29960605.

P: Mixed TKA/THA with no clean subgroup analyses

Brennan GP; Fritz JM; Houck LT; Hunter SJ. Outpatient rehabilitation care process factors and clinical outcomes among patients discharged home following unilateral total knee arthroplasty.. The Journal of arthroplasty. 25765128.

D: Single group/no comparison

Bretzke, P.; Potempa, C.; Sauer, V.; Egermann, M.; Engels, S.. Treatment costs after knee replacement A comparison between the ambulatory and inpatient rehabilitation treatment. Gesundheitsokonomie und Qualitatsmanagement. .

I: Not well defined/no (p)rehabilitation content to define

Bretzke, Pascal; Potempa, Christoph; Sauer, Volker; Egermann, Martin; Engels, Stephan.

Behandlungskosten nach Knieprothesen-OP -- Ein Vergleich zwischen der ambulanten und station $\sqrt{ }$ \&ren Rehabilitationsbehandlung. Gesundheits $\sqrt{ } \partial$ konomie \& Qualit $\sqrt{ }$ 乌tsmanagement. 126208046. Language:. O: No outcomes of interest

Brosky, T.; Topp, R.; Finley, M.; Killian, C.; Pariser, D.; Brown, K.; Bloemer, G.; Stearns, Z.. Effects of prehabilitation on early rehabilitation outcomes following total knee arthroplasty in patients with knee osteoarthritis. Physiotherapy (united kingdom).. CN-01089314.

Full text unavailable

Brown, K.; Loprinzi, P. D.; Brosky, J. A.; Topp, R.. Prehabilitation influences exercise-related psychological constructs such as self-efficacy and outcome expectations to exercise. J Strength Cond
Res. 23588484.

$\mathrm{D}: \mathrm{N}<20$ per group

Browne JP; Bastaki H; Dawson J. What is the optimal time point to assess patient-reported recovery after hip and knee replacement? A systematic review and analysis of routinely reported outcome data from the English patient-reported outcome measures programme.. Health and quality of life outcomes. 23895227.

SR or guideline

Brunenberg DE; van Steyn MJ; Sluimer JC; Bekebrede LL; Bulstra SK; Joore MA. Joint recovery programme versus usual care: an economic evaluation of a clinical pathway for joint replacement surgery.. Medical care. 16166871.

I: Not (p)rehabilitation

Bugbee WD; Pulido PA; Goldberg T; D'Lima DD. Use of an Anti-Gravity Treadmill for Early Postoperative Rehabilitation After Total Knee Replacement: A Pilot Study to Determine Safety and Feasibility.. American journal of orthopedics (Belle Mead, N.J.). 27327921.

$\mathrm{D}: \mathrm{N}<20$ per group

Buhagiar MA; Naylor JM; Harris IA; Xuan W; Adie $\mathrm{S}$; Lewin A. Assessment of Outcomes of Inpatient or Clinic-Based vs Home-Based Rehabilitation After Total Knee Arthroplasty: A Systematic Review and Meta-analysis.. JAMA network open. 31026026. SR or guideline

Buker N; Akkaya S; Akkaya N; Gokalp O; Kavlak E; Ok N; Kiter AE; Kitis A. Comparison of effects of supervised physiotherapy and a standardized home program on functional status in patients with total knee arthroplasty: a prospective study.. Journal of physical therapy science. 25364104.

$\mathrm{D}: \mathrm{N}<20$ per group

Bulthuis Y; Drossaers-Bakker KW; Taal E; Rasker J; Oostveen J; van't Pad Bosch P; Oosterveld F; van de Laar M. Arthritis patients show long-term benefits from 3 weeks intensive exercise training directly following hospital discharge.. Rheumatology (Oxford, England). 17956917.

P: Not osteoarthritis (exclude cancer, trauma, rheumatoid arthritis)

Busato, Massimo; Quagliati, Cristian; Magri, Lara; Filippi, Alessandra; Sanna, Alberto; Branchini, Mirco; Marchand, Aurelie Marie; Stecco, Antonio. Fascial Manipulation Associated With Standard Care Compared to Only Standard Postsurgical Care for Total Hip Arthroplasty: A $\neg \nmid$ Randomized Controlled Trial. PM \& R: Journal of Injury, Function \& 
Rehabilitation. 120276437. Language:.

$\mathrm{T}$ : Timing outcome too early

Busso, C.; Castorina, G.; Di Monaco, M.; Rodriguez, D.; Mahdavi, H.; Balocco, S.; Trucco, M.; Conti, M.; Castagna, A.; Minetto, M. A.. Effectiveness of a home-based telerehabilitation system in patients after total hip arthroplasty: study protocol of a randomized controlled trial. Trials. 33054811.

Protocol/study registration

Cabilan CJ; Hines S; Munday J. The Impact of Prehabilitation on Postoperative Functional Status, Healthcare Utilization, Pain, and Quality of Life: A Systematic Review.. Orthopedic nursing. 27441877. SR or guideline

Cabilan CJ; Hines S; Munday J. The effectiveness of prehabilitation or preoperative exercise for surgical patients: a systematic review.. JBI database of systematic reviews and implementation reports. 26447015 .

SR or guideline

Cabilan, C. J.; Hines, Sonia; Munday, Judy. The Impact of Prehabilitation on Postoperative Functional Status, Healthcare Utilization, Pain, and Quality of Life. Orthopaedic Nursing. 117287521. Language:. SR or guideline

Cai, L.; Gao, H.; Xu, H.; Wang, Y.; Lyu, P.; Liu, Y.. Does a Program Based on Cognitive Behavioral Therapy Affect Kinesiophobia in Patients Following Total Knee Arthroplasty? A Randomized, Controlled Trial With a 6-Month Follow-Up. Journal of arthroplasty. CN-01440292.

Duplicate/No additional data

Campbell KJ; Louie PK; Bohl DD; Edmiston T; Mikhail C; Li J; Khorsand DA; Levine BR; Gerlinger TL. A Novel, Automated Text-Messaging System Is Effective in Patients Undergoing Total Joint Arthroplasty.. The Journal of bone and joint surgery. American volume. 30653044.

P: Mixed TKA/THA with no clean subgroup analyses

Campbell, T. M.; Ghaedi, B. B.; Ghogomu, E. T.; Westby, M.; Welch, V. A.. Effectiveness of stretching and bracing for the treatment of osteoarthritis-associated joint contractures prior to joint replacement: a systematic review protocol. BMJ Open. 31366647.

SR or guideline

Carmeli, E.; Sheklow, S. L.; Coleman, R.. A comparative study of organized class-based exercise programs versus individual home-based exercise programs for elderly patients following hip surgery.
Disabil Rehabil. 16882639.

T: Timing outcome too early

Castellano JJ; Rojas AM; Karia R; Hunter T; Slover J; Moroz A. A Randomized, Double-Blind, PlaceboControlled Study of Neuromuscular Electrical Stimulation (NMES) use for Recovery after Elective Total Hip Replacement Surgery.. Bulletin of the Hospital for Joint Disease (2013). 27815950. $\mathrm{D}: \mathrm{N}<20$ per group

Cavanaugh AM; Rauh MJ; Thompson CA; Alcaraz JE; Bird CE; Gilmer TP; LaCroix AZ. Rehabilitation After Total Knee Arthroplasty: Do Racial Disparities Exist?. The Journal of arthroplasty. 31801659. I: Not well defined/no (p)rehabilitation content to define

Cavill S; McKenzie K; Munro A; McKeever J; Whelan L; Biggs L; Skinner EH; Haines TP. The effect of prehabilitation on the range of motion and functional outcomes in patients following the total knee or hip arthroplasty: A pilot randomized trial.. Physiotherapy theory and practice. 27050325.

$\mathrm{D}: \mathrm{N}<20$ per group

Cheatham, Scott W.. Do Patient Factors and Prehabilitation Improve Outcomes After Total Knee Arthroplasty?. Topics in Geriatric Rehabilitation. 108032162. Language:.

SR or guideline

Chen AF; Stewart MK; Heyl AE; Klatt BA. Effect of immediate postoperative physical therapy on length of stay for total joint arthroplasty patients.. The Journal of arthroplasty. 22386604.

D: NRCS not adjusted

Chen B; Zimmerman JR; Soulen L; DeLisa JA. Continuous passive motion after total knee arthroplasty: a prospective study.. American journal of physical medicine \& rehabilitation. 10994883. published before 2005

Chen G; Gu RX; Xu DD. [The application of electroacupuncture to postoperative rehabilitation of total knee replacement].. Zhongguo zhen jiu = Chinese acupuncture \& moxibustion. 22734376. T: Timing outcome too early

Chen H; Li S; Ruan T; Liu L; Fang L. Is it necessary to perform prehabilitation exercise for patients undergoing total knee arthroplasty: meta-analysis of randomized controlled trials.. The Physician and sportsmedicine. 29125384.

Full text unavailable

Chen H; Du S-S; Fan L-J; Jiang j. Effects of combination therapy on the movement function of 
lower extremity after total knee arthroplasty in the elderly. Chinese journal of tissue engineering research. CN-01459729.

P: Not osteoarthritis (exclude cancer, trauma, rheumatoid arthritis)

Chen HW; Chen HM; Wang YC; Chen PY; Chien $\mathrm{CW}$. Association between rehabilitation timing and major complications of total knee arthroplatsy.. Journal of rehabilitation medicine. 22674241. I: Not well defined/no (p)rehabilitation content to define

Chen M; Li P; Lin F. Influence of structured telephone follow-up on patient compliance with rehabilitation after total knee arthroplasty.. Patient preference and adherence. 27042020.

I: Not (p)rehabilitation

Chen SR; Chen CS; Lin PC. The effect of educational intervention on the pain and rehabilitation performance of patients who undergo a total knee replacement.. Journal of clinical nursing. 24313941.

D: NRCS not adjusted

Chen, H. H.; Yeh, M. L.. A new multimedia-based instruction for hip arthroplasty in clinical practice. Comput Methods Programs Biomed. 16182405.

$\mathrm{T}$ : Timing outcome too early

Chen, X.; Li, X.; Zhu, Z.; Wang, H.; Yu, Z.; Bai, X.. Effects of progressive resistance training for early postoperative fast-track total hip or knee arthroplasty: A systematic review and meta-analysis. Asian J Surg. 33715964.

SR or guideline

Chen, Yu; Luo, Chunmei; Wang, Juan. Effectiveness of home rehabilitation exercise for patients after total knee replacement: meta analysis. Chinese Nursing Research. 129204068. Language:.

SR or guideline

Chen, Z.; Shen, Z.; Ye, X.; Xu, Y.; Liu, J.; Shi, X.; Chen, G.; Wu, J.; Chen, W.; Jiang, T.; Liu, W.; Xu, X.. Acupuncture for Rehabilitation After Total Knee Arthroplasty: A Systematic Review and MetaAnalysis of Randomized Controlled Trials. Front Med (Lausanne). 33553202.

SR or guideline

Chesham RA; Shanmugam S. Does preoperative physiotherapy improve postoperative, patient-based outcomes in older adults who have undergone total knee arthroplasty? A systematic review.. Physiotherapy theory and practice. 27736286. SR or guideline
ChiCtr. Comparison of rehabilitation effects of low stool assisted standardized home exercise program and supervised physiotherapy after total knee arthroplasty (TKA).

http://www.who.int/trialsearch/Trial2.aspx?TrialID= ChiCTR-IOR-17011264. CN-01885144.

Protocol/study registration

ChiCtr. Rehabilitation following Total Knee Replacement for Sarcopenic Obesity in Elderly. http:/www.who.int/trialsearch/Trial2.aspx?TrialID= ChiCTR-IPR-15006242. CN-01830124.

Protocol/study registration

ChiCtr. Effect of Additional Balance Training on Blood Biomachenical Value and Functional Fitness after Total Knee Replacement in Overweight and Obese Patients with Knee Osteoarthritis. http://www.who.int/trialsearch/Trial2.aspx?TrialID= ChiCTR-IPR-17012106. CN-01894361.

Protocol/study registration

ChiCtr. Intelligent and rehabilitation exercises reduced Postoperative Cognitive Dysfunction (POCD) on elderly patients with total hipreplacement surgery. http://www.who.int/trialsearch/Trial2.aspx?TrialID= ChiCTR-TRC-14004431. CN-01871791.

Protocol/study registration

ChiCtr. Tai chi chuan exercise improves postoperative rehabilitation in patients with primary total knee arthroplasty due to knee Osteoarthritis: a randomized controlled trial.

http://www.who.int/trialsearch/Trial2.aspx?TrialID= ChiCTR1800017510. CN-01908202.

Protocol/study registration

ChiCtr. The clinical research of transcutaneous electric nerve stimulation in the management of function recovery after total knee arthroplasty. http://www.who.int/trialsearch/Trial2.aspx?TrialID= ChiCTR1800016478. CN-01909073.

Protocol/study registration

ChiCtr. Clinical study for opposing needling method on analgesia and rehabilitation after unilateral total knee arthroplasty.

http://www.who.int/trialsearch/Trial2.aspx?TrialID= ChiCTR1800020297. CN-01947165.

Protocol/study registration

ChiCtr. Model construction and application of out-ofhospital rehabilitation management for empty nest elderly after total knee arthroplasty based on mobile APP: a randomized controlled trial. http://www.who.int/trialsearch/Trial2.aspx?TrialID= 
ChiCTR1900022046. CN-01973863.

Protocol/study registration

Chimenti CE; Ingersoll G. Comparison of home health care physical therapy outcomes following total knee replacement with and without subacute rehabilitation.. Journal of geriatric physical therapy (2001). 18171493.

D: NRCS not adjusted

Chisari, E.; Grosso, M. J.; Kozaily, E.; Nelson, C. L.; Sherman, M. B.; Courtney, P. M.. High Bundled Payment Costs Following Total Hip and Knee Arthroplasty in African Americans. J Arthroplasty. 32807564.

I: Not (p)rehabilitation

Chiung-Jui Su D; Yuan KS; Weng SF; Hong RB; Wu MP; Wu HM; Chou W. Can Early Rehabilitation after Total Hip Arthroplasty Reduce Its Major Complications and Medical Expenses? Report from a Nationally Representative Cohort.. BioMed research international. 26146625.

P: Not osteoarthritis (exclude cancer, trauma, rheumatoid arthritis)

Chou LN; Chen ML. Effects of Elastic Band Exercise on Lower Limb Rehabilitation of Elderly Patients Undergoing Total Knee Arthroplasty.. Rehabilitation nursing : the official journal of the Association of Rehabilitation Nurses. 30601799.

$\mathrm{T}$ : Timing outcome too early

Chow TP; Ng GY. Active, passive and proprioceptive neuromuscular facilitation stretching are comparable in improving the knee flexion range in people with total knee replacement: a randomized controlled trial.. Clinical rehabilitation. 20685725. T: Timing outcome too early

Christensen JC; Paxton RJ; Baym C; Forster JE; Dayton MR; Hogan CA; Stevens-Lapsley JE. Benefits of direct patient discharge to outpatient physical therapy after total knee arthroplasty.. Disability and rehabilitation. 30616406.

T: Timing outcome too early

Christiansen CL; Bade MJ; Davidson BS; Dayton MR; Stevens-Lapsley JE. Effects of Weight-Bearing Biofeedback Training on Functional Movement Patterns Following Total Knee Arthroplasty: A Randomized Controlled Trial.. The Journal of orthopaedic and sports physical therapy. 26207975. $\mathrm{D}: \mathrm{N}<20$ per group

Christiansen MB; Thoma LM; Master H; Schmitt LA; Pohlig R; White DK. A Physical TherapistAdministered Physical Activity Intervention After Total Knee Replacement: Protocol for a Randomized
Controlled Trial.. Physical therapy. 29608733. Duplicate/No additional data

Christiansen MB; Thoma LM; Master H; Mathews D; Schmitt LA; White DK. Preliminary findings of a novel physical therapist administered physical activity intervention after total knee replacement. Osteoarthritis and cartilage. CN-01623563.

$\mathrm{D}: \mathrm{N}<20$ per group

Christiansen, M.; Thoma, L.; Master, H.; Mathews, D.; Schmitt, L.; Ziegler, M.; White, D.. 1-year outcomes from a novel physical therapistadministered physical activity intervention after total knee replacement: a pilot study. Arthritis and rheumatology. CN-01787108.

$\mathrm{D}: \mathrm{N}<20$ per group

Christiansen, M. B.; Thoma, L. M.; Master, H.; Voinier, D.; Schmitt, L. A.; Ziegler, M. L.; LaValley, M. P.; White, D. K.. Feasibility and Preliminary Outcomes of a Physical Therapist-Administered Physical Activity Intervention After Total Knee Replacement. Arthritis care \& research. CN01937514.

$\mathrm{D}: \mathrm{N}<20$ per group

Christiansen, M. B.; Thoma, L. M.; Master, H.; Voinier, D.; Schmitt, L. A.; Ziegler, M. L.; LaValley, M. P.; White, D. K.. The Feasibility and Preliminary Outcomes of a Physical Therapist-Administered Physical Activity Intervention After Total Knee Replacement. Arthritis Care Res (Hoboken). 30908867.

$\mathrm{D}: \mathrm{N}<20$ per group

Christiansen, Meredith B.; Thoma, Louise M.; Master, Hiral; Schmitt, Laura A.; Pohlig, Ryan; White, Daniel K.. A Physical Therapist-Administered Physical Activity Intervention After Total Knee Replacement: Protocol for a Randomized Controlled Trial. Physical Therapy. 29608733. Protocol/study registration

Chughtai M; McGinn T; Bhave A; Khan S; Vashist M; Khlopas A; Mont MA. Innovative Multimodal Physical Therapy Reduces Incidence of Repeat Manipulation under Anesthesia in Post-Total Knee Arthroplasty Patients Who Had an Initial Manipulation under Anesthesia.. The journal of knee surgery. 27652686.

D: NRCS not adjusted

Chughtai M; Shah NV; Sultan AA; Solow M; Tiberi JV; Mehran N; North T; Moskal JT; Newman JM; Samuel LT; Bhave A; Mont MA. The role of prehabilitation with a telerehabilitation system prior to total knee arthroplasty.. Annals of translational 
medicine. 30963063.

D: NRCS not adjusted

Chughtai, M.; Newman, J. M.; Sultan, A. A.; Khlopas, A.; Navarro, S. M.; Bhave, A.; Mont, M. A.. The Role of Virtual Rehabilitation in Total Knee and Hip Arthroplasty. Surg Technol Int. 29566421. D: Not primary study (or SR or GL)

Cien, A.; Riggle, P. K.; Brazier, B. G.; Popovich, J. M.; Crawford, S.; Cochran, J. M.. Examining the use of the pressure modulated knee rehabilitation machine (PMKR) with traditional physical therapy versus traditional physical therapy alone following total knee arthroplasty: a randomized control study. Current orthopaedic practice. $\mathrm{CN}-02229354$. $\mathrm{T}$ : Timing outcome too early

Cien, Adam; Riggle, Patrick K.; Brazier, Brett G.; Popovich Jr, John M.; Crawford, Stan; Cochran, Jason M.. Examining the use of the pressure modulated knee rehabilitation machine (PMKR) with traditional physical therapy versus traditional physical therapy alone following total knee arthroplasty: a randomized control study. Current Orthopaedic Practice. 148634995. Language:. Duplicate/No additional data

Clode NJ; Perry MA; Wulff L. Does physiotherapy prehabilitation improve pre-surgical outcomes and influence patient expectations prior to knee and hip joint arthroplasty?. International journal of orthopaedic and trauma nursing. 29954717. D: NRCS not adjusted

Closa C; Mas MA; Santaeugenia SJ; Inzitari M; Ribera A; Gallofre M. Hospital-at-home Integrated Care Program for Older Patients With Orthopedic Processes: An Efficient Alternative to Usual Hospital-Based Care.. Journal of the American Medical Directors Association. 28578883.

P: Not osteoarthritis (exclude cancer, trauma, rheumatoid arthritis)

Closa, C.; Macul $(\mathbb{C}$, F.; Suso, S.; Flotats, G.; Perramon, A.. Home-based rehabilitation after total knee arthroplasty: a cost-effectiveness study.

Rehabilitacion. 107874294. Language:.

Full text unavailable

Coleman, G.; White, D. K.; Thoma, L. M.; Mathews, D.; Christiansen, M. B.; Schmitt, L. A.; Jakiela, J. T.; Master, H.. Does a physical therapist-administered physical activity intervention reduce sedentary time after total knee replacement: An exploratory study? Musculoskeletal Care. 32975020.

$\mathrm{D}: \mathrm{N}<20$ per group
Colibazzi, V.; Coladonato, A.; Zanazzo, M.;

Romanini, E.. Evidence based rehabilitation after hip arthroplasty. Hip Int. 33267691.

SR or guideline

Conley, C. E. W.; Mattacola, C. G.; Jochimsen, K. N.; Dressler, E. V.; Lattermann, C.; Howard, J. S.. A Comparison of Neuromuscular Electrical Stimulation Parameters for Postoperative Quadriceps Strength in Patients After Knee Surgery: A Systematic Review. Sports Health. 33428557.

SR or guideline

Cook L; Northgraves MJ; Fairhurst C; Ronaldson S; Torgerson DJ; Kent J; Reed M. Knee Replacement Bandaging Study (KReBS) evaluating the effect of a two-layer compression bandage system on knee function following total knee arthroplasty: study protocol for a randomised controlled trial.. Trials. 31068223.

Protocol/study registration

Coppola SM; Collins SM. Is physical therapy more beneficial than unsupervised home exercise in treatment of post surgical knee disorders? A systematic review.. The Knee. 18851916. SR or guideline

Correia FD; Nogueira A; Magalhaes I; Guimaraes J; Moreira M; Barradas I; Molinos M; Teixeira L; Tulha J; Seabra R; Lains J; Bento V. Medium-Term Outcomes of Digital Versus Conventional HomeBased Rehabilitation After Total Knee Arthroplasty: Prospective, Parallel-Group Feasibility Study.. JMIR rehabilitation and assistive technologies. 30816849. D: NRCS not adjusted

Correia FD; Nogueira A; Magalhaes I; Guimaraes J; Moreira M; Barradas I; Teixeira L; Tulha J; Seabra R; Lains J; Bento V. Home-based Rehabilitation With A Novel Digital Biofeedback System versus Conventional In-person Rehabilitation after Total Knee Replacement: a feasibility study.. Scientific reports. 30050087.

D: NRCS not adjusted

Coudeyre E; Jardin C; Givron P; Ribinik P; Revel M; Rannou F. Could preoperative rehabilitation modify postoperative outcomes after total hip and knee arthroplasty? Elaboration of French clinical practice guidelines.. Annales de readaptation et de medecine physique : revue scientifique de la Societe francaise de reeducation fonctionnelle de readaptation et de medecine physique. 17343953.

SR or guideline

Coudeyre, E.; Jardin, C.; Givron, P.; Ribinik, P.; Revel, M.; Rannou, F.. Could preoperative 
rehabilitation modify postoperative outcomes after total hip and $\neg \dagger$ knee arthroplasty? Elaboration of $\neg \dagger$ French clinical practice guidelines. Annales de Readaptation et de Medecine Physique. .

SR or guideline

Coulter CL; Scarvell JM; Neeman TM; Smith PN. Physiotherapist-directed rehabilitation exercises in the outpatient or home setting improve strength, gait speed and cadence after elective total hip replacement: a systematic review.. Journal of physiotherapy. 24287215.

SR or guideline

Coulter CL; Weber JM; Scarvell JM. Group physiotherapy provides similar outcomes for participants after joint replacement surgery as 1-to-1 physiotherapy: a sequential cohort study.. Archives of physical medicine and rehabilitation. 19801063. D: NRCS not adjusted

Coulter, C.; Perriman, D. M.; Neeman, T. M.; Smith, P. N.; Scarvell, J. M.. Supervised or Unsupervised Rehabilitation After Total Hip Replacement Provides Similar Improvements for Patients: a Randomized Controlled Trial. Archives of physical medicine and rehabilitation. CN-01417522.

Duplicate/No additional data

Crowe J; Henderson J. Pre-arthroplasty rehabilitation is effective in reducing hospital stay.. Canadian journal of occupational therapy. Revue canadienne d'ergotherapie. 12704972.

published before 2005

Ctri. exercise for hip muscle strengthening in total knee replacement surgery. http://www.who.int/trialsearch/Trial2.aspx?TrialID= CTRI/2017/08/009457. CN-01891619.

Protocol/study registration

Ctri. Effect of two different electrical stimulation on quadriceps for pain and function in patients after Total Knee Replacement. http://www.who.int/trialsearch/Trial2.aspx?TrialID= CTRI/2018/05/013611. CN-01895892.

Protocol/study registration

Ctri. A study to assess the effectiveness of a patient care protocol on patient post surgery recovery outcomes of patients undergoing knee or hip replacement surgery.

http://www.who.int/trialsearch/Trial2.aspx? TrialID= CTRI/2018/05/014240. CN-01905121.

Protocol/study registration

Ctri. Effect of proprioceptive neuromuscular facilitation stretching on balance, proprioception and functional abilities in unilateral knee arthroplasty.
http://www.who.int/trialsearch/Trial2.aspx?TrialID= CTRI/2019/05/019150. CN-01969217.

Protocol/study registration

Culliton SE; Bryant DM; MacDonald SJ; Hibbert KM; Chesworth BM. Effect of an e-Learning Tool on Expectations and Satisfaction Following Total Knee Arthroplasty: A Randomized Controlled Trial.. The Journal of arthroplasty. 29555496.

P: Not first replacement (exclude revision surgeries)

Czyzewska A; Glinkowski WM; Walesiak K; Krawczak K; Cabaj D; Gorecki A. Effects of preoperative physiotherapy in hip osteoarthritis patients awaiting total hip replacement.. Archives of medical science : AMS. 25395951.

D: NRCS not adjusted

D'Lima DD; Colwell CW Jr; Morris BA; Hardwick ME; Kozin F. The effect of preoperative exercise on total knee replacement outcomes.. Clinical orthopaedics and related research. 8620638 . published before 2005

Daltroy LH; Morlino CI; Eaton HM; Poss R; Liang $\mathrm{MH}$. Preoperative education for total hip and knee replacement patients.. Arthritis care and research : the official journal of the Arthritis Health Professions Association. 10030179.

P: Not osteoarthritis (exclude cancer, trauma, rheumatoid arthritis)

Dan, Mirela; Boca, Ioan-Cosmin. PERKS OF PHYSICAL THERAPY DUE TO A COMPLEX REHABILITATION PROGRAM AFTER TOTAL KNEE ARTHROPLASTY. Romanian Journal of Physical Therapy / Revista Romana de Kinetoterapie. 104139983. Language:.

Full text unavailable

das Nair R; Mhizha-Murira JR;; erson P; Carpenter H; Clarke S; Groves S; Leighton P; Scammell BE; Topcu G; Walsh DA; Lincoln NB. Home-based presurgical psychological intervention for knee osteoarthritis (HAPPiKNEES): a feasibility randomized controlled trial.. Clinical rehabilitation. 29424236.

D: $\mathrm{N}<20$ per group

Dauty M; Genty M; Ribinik P. Physical training in rehabilitation programs before and after total hip and knee arthroplasty.. Annales de readaptation et de medecine physique : revue scientifique de la Societe francaise de reeducation fonctionnelle de readaptation et de medecine physique. 17482710 . SR or guideline

Davidovitch RI; Anoushiravani AA; Feng JE; Chen KK; Karia R; Schwarzkopf R; Iorio R. Home Health 
Services Are Not Required for Select Total Hip Arthroplasty Candidates: Assessment and Supplementation With an Electronic Recovery Application.. The Journal of arthroplasty. 29588123. D: NRCS not adjusted

Davies DM; Johnston DW; Beaupre LA; Lier DA. Effect of adjunctive range-of-motion therapy after primary total knee arthroplasty on the use of health services after hospital discharge.. Canadian journal of surgery. Journal canadien de chirurgie. 12585791. published before 2005

Davila Castrodad IM; Recai TM; Abraham MM; Etcheson JI; Mohamed NS; Edalatpour A; Delanois RE. Rehabilitation protocols following total knee arthroplasty: a review of study designs and outcome measures.. Annals of translational medicine. 31728379 .

SR or guideline

Davis AM. Osteoarthritis year in review: rehabilitation and outcomes.. Osteoarthritis and cartilage. 22261405.

SR or guideline

Debbi, E. M.; Bernfeld, B.; Herman, A.; Salai, M.; Laufer, Y.; Wolf, A.. A Biomechanical Foot-Worn Device Improves Total Knee Arthroplasty Outcomes. J Arthroplasty. 30352770.

I: Not (p)rehabilitation

Dejong G; Horn SD; Smout RJ; Tian W; Putman K; Gassaway J. Joint replacement rehabilitation outcomes on discharge from skilled nursing facilities and inpatient rehabilitation facilities.. Archives of physical medicine and rehabilitation. 19651262. $\mathrm{T}$ : Timing outcome too early

DeJong G; Hsieh CH; Gassaway J; Horn SD; Smout RJ; Putman K; James R; Brown M; Newman EM; Foley MP. Characterizing rehabilitation services for patients with knee and hip replacement in skilled nursing facilities and inpatient rehabilitation facilities.. Archives of physical medicine and rehabilitation. 19651261.

T: Timing outcome too early

DeJong G; Tian W; Smout RJ; Horn SD; Putman K; Hsieh CH; Gassaway J; Smith P. Long-term outcomes of joint replacement rehabilitation patients discharged from skilled nursing and inpatient rehabilitation facilities.. Archives of physical medicine and rehabilitation. 19651264. P: Not unilateral replacement (exclude bilateral replacement surgeries)

DeJong G; Tian W; Smout RJ; Horn SD; Putman K; Smith P; Gassaway J; Davanzo JE. Use of rehabilitation and other health care services by patients with joint replacement after discharge from skilled nursing and inpatient rehabilitation facilities.. Archives of physical medicine and rehabilitation. 19651263.

P: Not unilateral replacement (exclude bilateral replacement surgeries)

Delanois R; Sodhi N; Acuna A; Doll K; Mont MA; Bhave A. Use of home neuromuscular electrical stimulation in the first 6 weeks improves function and reduces pain after primary total knee arthroplasty: a matched comparison.. Annals of translational medicine. 31728378.

$\mathrm{D}: \mathrm{N}<20$ per group

Denduluri, S. K.; Huddleston, J. I., 3rd; Amanatullah, D. F.. Preoperative Exercise Participation Reflects Patient Engagement and Predicts Earlier Patient Discharge and Less Gait Aid Dependence After Total Joint Arthroplasty. Orthopedics. 32602926.

I: Not (p)rehabilitation

Dennis, J.; Wylde, V.; Gooberman-Hill, R.; Blom, A. W.; Beswick, A. D.. Effects of presurgical interventions on chronic pain after total knee replacement: a systematic review and meta-analysis of randomised controlled trials. BMJ Open. 31964670.

SR or guideline

Di Monaco M; Castiglioni C. Which type of exercise therapy is effective after hip arthroplasty? A systematic review of randomized controlled trials. European journal of physical and rehabilitation medicine. 24172644.

SR or guideline

Di Monaco M; Vallero F; Tappero R; Cavanna A. Rehabilitation after total hip arthroplasty: a systematic review of controlled trials on physical exercise programs.. European journal of physical and rehabilitation medicine. 19238130.

SR or guideline

Dias Correia F; Nogueira A; Magalhaes I; Guimaraes J; Moreira M; Barradas I; Molinos M; Teixeira L; Pires J; Seabra R; Lains J; Bento V. Digital Versus Conventional Rehabilitation After Total Hip Arthroplasty: A Single-Center, Parallel-Group Pilot Study.. JMIR rehabilitation and assistive technologies. 31228176.

D: NRCS not adjusted

Doerfler D; Gurney B; Mermier C; Rauh M; Black L;;rews R. High-Velocity Quadriceps Exercises Compared to Slow-Velocity Quadriceps Exercises Following Total Knee Arthroplasty: A Randomized 
Clinical Study.. Journal of geriatric physical therapy (2001). 26428903.

D: $\mathrm{N}<20$ per group

Doiron-Cadrin P; Kairy D; Vendittoli PA; Lowry V; Poitras S; Desmeules F. Feasibility and preliminary effects of a tele-prehabilitation program and an inperson prehablitation program compared to usual care for total hip or knee arthroplasty candidates: a pilot randomized controlled trial.. Disability and rehabilitation. 30638076.

$\mathrm{D}: \mathrm{N}<20$ per group

Doiron-Cadrin P; Kairy D; Vendittoli PA; Lowry V; Poitras S; Desmeules F. Effects of a teleprehabilitation program or an in-person prehabilitation program in surgical candidates awaiting total hip or knee arthroplasty: Protocol of a pilot single blind randomized controlled trial.. Contemporary clinical trials communications. 29736482.

$\mathrm{D}: \mathrm{N}<20$ per group

Dom $\sqrt{ } \neq$ nguez-Navarro, F.; Silvestre-Mu $\sqrt{ \pm}_{\text {oz, A.; }}$.; Igual-Camacho, C.; $\mathrm{D} \vee \neq$ az-D $\sqrt{ } \neq \mathrm{az}, \mathrm{B}$.; Torrella, J. V.; Rodrigo, J.; Pay $\sqrt{ }^{\circ}$-Rubio, A.; Roig-Casas $\sqrt{ } \int_{\mathrm{s}}, \mathrm{S}$.; Blasco, J. M.. A randomized controlled trial assessing the effects of preoperative strengthening plus balance training on balance and functional outcome up to $1 \neg$ †year following total knee replacement. Knee Surg Sports Traumatol Arthrosc. 32342139.

$\mathrm{D}: \mathrm{N}<20$ per group

Doma K; Grant A; Morris J. The Effects of Balance Training on Balance Performance and Functional Outcome Measures Following Total Knee Arthroplasty: A Systematic Review and MetaAnalysis.. Sports medicine (Auckland, N.Z.). 30117054.

SR or guideline

Dominguez-Navarro F; Igual-Camacho C; SilvestreMunoz A; Roig-Casasus S; Blasco JM. Effects of balance and proprioceptive training on total hip and knee replacement rehabilitation: A systematic review and meta-analysis.. Gait \& posture. 29525292.

SR or guideline

Dominguez-Navarro, F.; Silvestre-Munoz, A.; IgualCamacho, C.; Diaz-Diaz, B.; Torrella, J. V.; Rodrigo, J.; Paya-Rubio, A.; Roig-Casasus, S.; Blasco, J. M.. A randomized controlled trial assessing the effects of preoperative strengthening plus balance training on balance and functional outcome up to $1 \sqrt{ }$ Ç year following total knee replacement. Knee surgery, sports traumatology, arthroscopy. 32342139.

Duplicate/No additional data
Donec, V.; Kri $\approx^{\circ} f$ çi $\sim^{\prime}$ nas, A.. The effectiveness of Kinesio Taping $\neg Æ$ after total knee replacement in early postoperative rehabilitation period. A randomized controlled trial. European journal of physical and rehabilitation medicine. CN-01116657. T: Timing outcome too early

Dong F; Li M, Liu J. Effect of department of orthopedics rehabilitation integrated mode on knee joint pain, function and quality of life in total knee arthroplasty. Biomedical research (india). CN01603168.

P: Not osteoarthritis (exclude cancer, trauma, rheumatoid arthritis)

Dowsey MM; Castle DJ; Knowles SR; Monshat K; Salzberg MR; Choong PF. The effect of mindfulness training prior to total joint arthroplasty on postoperative pain and physical function: study protocol for a randomised controlled trial.. Trials. 24899242. Protocol/study registration

Drks. Does an external compression tool influence postoperative swelling after total knee arthroplasty?. http://www.who.int/trialsearch/Trial2.aspx?TrialID= DRKS00010906. CN-01851920.

Protocol/study registration

Drks. The effectiveness of rehabilitation after a Total Hip Arthroplasty (THA): a comparison of Usual Care in the Netherlands versus Germany.

http://www.who.int/trialsearch/Trial2.aspx?TrialID= DRKS00011345. CN-01858260.

Protocol/study registration

Drks. Prehabilitation for patients with osteoarthritis of the hip or knee waiting for a total Joint replacement for hip or knee.

http://www.who.int/trialsearch/Trial2.aspx?TrialID= DRKS00009239. CN-01865886.

Protocol/study registration

Drks. Impact of a tailored activity counselling intervention during inpatient rehabilitation after knee and hip arthroplasty.

http://www.who.int/trialsearch/Trial2.aspx?TrialID= DRKS00012682. CN-01889666.

Protocol/study registration

Drks. A multi-stage randomised controlled study for the assessment of postoperative therapeutic procedures after total knee arthroplasty. http://www.who.int/trialsearch/Trial2.aspx?TrialID= DRKS00013617. CN-01898256.

Protocol/study registration

Drks. Effect of Mulligan $¥$ s MWM-Technique to improve knee flexion in total knee replacement patients in the rehabilitation phase - A randomized 
controlled trial.

http://www.who.int/trialsearch/Trial2.aspx?TrialID= DRKS00006180. CN-01798189.

Protocol/study registration

Du, X. L.; Fang, H. P.; Fang, Z.; Li, F.. Preventive effect of comprehensive rehabilitation nursing on early complications of total hip arthroplasty. Journal of clinical rehabilitative tissue engineering research. CN-01015965.

Full text unavailable

Eichler S; Rabe S; Salzwedel A; Muller S; Stoll J; Tilgner N; John M; Wegscheider K; Mayer F; Voller $\mathrm{H}$. Effectiveness of an interactive telerehabilitation system with home-based exercise training in patients after total hip or knee replacement: study protocol for a multicenter, superiority, no-blinded randomized controlled trial.. Trials. 28934966.

Protocol/study registration

Eichler S; Salzwedel A; Rabe S; Mueller S; Mayer F; Wochatz M; Hadzic M; John M; Wegscheider K; Voller H. The Effectiveness of Telerehabilitation as a Supplement to Rehabilitation in Patients After Total Knee or Hip Replacement: Randomized Controlled Trial.. JMIR rehabilitation and assistive technologies. 31697239.

P: Mixed TKA/THA with no clean subgroup analyses

Eisermann U; Haase I; Kladny B. Computer-aided multimedia training in orthopedic rehabilitation.. American journal of physical medicine \& rehabilitation. 15314531.

published before 2005

Evgeniadis, G.; Beneka, A.; Malliou, P.; Mavromoustakos, S.; Godolias, G.. Effects of pre- or postoperative therapeutic exercise on the quality of life, before and after total knee arthroplasty for osteoarthritis. Journal of Back \& Musculoskeletal Rehabilitation. 105687198. Language:.

D: $\mathrm{N}<20$ per group

Ewell, M.; Griffin, C.; Hull, J.. The use of focal knee joint cryotherapy to improve functional outcomes after total knee arthroplasty: review article. Pm r. 24534102.

SR or guideline

Falvey JR; Bade MJ; Forster JE; Burke RE; Jennings JM; Nuccio E; Stevens-Lapsley JE. Home-HealthCare Physical Therapy Improves Early Functional Recovery of Medicare Beneficiaries After Total Knee Arthroplasty.. The Journal of bone and joint surgery. American volume. 30334882.

T: Timing outcome too early
Fernandes L; Roos EM; Overgaard S; Villadsen A; Sogaard R. Supervised neuromuscular exercise prior to hip and knee replacement: 12-month clinical effect and cost-utility analysis alongside a randomised controlled trial.. BMC musculoskeletal disorders. 28061841.

P: Mixed TKA/THA with no clean subgroup analyses

Ferrara PE; Rabini A; Maggi L; Piazzini DB; Logroscino G; Magliocchetti G; Amabile E; Tancredi G; Aulisa AG; Padua L; Aprile I; Bertolini C. Effect of pre-operative physiotherapy in patients with endstage osteoarthritis undergoing hip arthroplasty.. Clinical rehabilitation. 18955429.

$\mathrm{D}: \mathrm{N}<20$ per group

Ferrara, P. E.; Rabini, A.; Aprile, I.; Maggi, L.; Piazzini, D. B.; Logroscino, G.; Lombi, G. M.; Amabile, E.; Tancredi, G.; Aulisa, A. G.; Padua, L.; Bertolini, C.. Effect of pre-operative physiotherapy in patients with end-stage osteoarthritis undergoing hip arthroplasty [corrected] [published erratum appears in CLIN REHABIL 2008 Dec;22(12):1137]. Clinical Rehabilitation. 105570096. Language:.

Duplicate/No additional data

ffs, R. B. R.. Physiotherapeutic treatment in people who underwent hip replacement surgery. http:/www.who.int/trialsearch/Trial2.aspx?TrialID= RBR-395ffs. CN-02071126.

Protocol/study registration

Ficklscherer, A.; Stapf, J.; Meissner, K. M.; Niethammer, T.; Lahner, M.; Wagenhauser, M.; Muller, P. E.; Pietschmann, M. F.. Testing the feasibility and safety of the Nintendo Wii gaming console in orthopedic rehabilitation: a pilot randomized controlled study. Arch Med Sci. 27904518.

$\mathrm{D}: \mathrm{N}<20$ per group

Fillingham YA; Darrith B; Lonner JH; Culvern C; Crizer M; Della Valle CJ. Formal Physical Therapy May Not Be Necessary After Unicompartmental Knee Arthroplasty: A Randomized Clinical Trial.. The Journal of arthroplasty. 29555497.

P: Not total relacement (exclude partial)

Fitz W; Shukla P; Li L; Scott RD. Early Regain of Function and Proprioceptive Improvement Following Knee Arthroplasty.. The archives of bone and joint surgery. 30637308.

T: Timing outcome too early

Fleischman AN; Crizer MP; Tarabichi M; Smith S; Rothman RH; Lonner JH; Chen AF. 2018 John N. Insall Award: Recovery of Knee Flexion With Unsupervised Home Exercise Is Not Inferior to 
Outpatient Physical Therapy After TKA: A Randomized Trial.. Clinical orthopaedics and related research. 30794229.

I: Not well defined/no (p)rehabilitation content to define

Florez-Garcia M; Garcia-Perez F; Curbelo R; PerezPorta I; Nishishinya B; Rosario Lozano MP; Carmona L. Efficacy and safety of home-based exercises versus individualized supervised outpatient physical therapy programs after total knee arthroplasty: a systematic review and meta-analysis.. Knee surgery, sports traumatology, arthroscopy : official journal of the ESSKA. 27401004.

SR or guideline

Freburger JK. An analysis of the relationship between the utilization of physical therapy services and outcomes of care for patients after total hip arthroplasty.. Physical therapy. 10792855. published before 2005

Frost H; Lamb SE; Robertson S. A randomized controlled trial of exercise to improve mobility and function after elective knee arthroplasty. Feasibility, results and methodological difficulties.. Clinical rehabilitation. 11911518.

published before 2005

Fu, M. C.; Samuel, A. M.; Sculco, P. K.; MacLean, C. H.; Padgett, D. E.; McLawhorn, A. S.. Discharge to Inpatient Facilities After Total Hip Arthroplasty Is Associated With Increased Postdischarge Morbidity. J Arthroplasty. 28455181.

I: Not (p)rehabilitation

Fujii T; Nishio N; Bandou M; Kitano S; Noboru M; Seko M; Inagaki Y; Tanaka Y. Do Individual Differences in Physical Therapists' Skills Affect Postoperative Results of Total Knee Arthroplasty? The journal of knee surgery. 31067587.

D: Single group/no comparison

Fung V; Ho A; Shaffer J; Chung E; Gomez M. Use of Nintendo Wii Fit in the rehabilitation of outpatients following total knee replacement: a preliminary randomised controlled trial..

Physiotherapy. 22898573.

T: Timing outcome too early

Fusakul, Y.; Thiengwittayaporn, S.; Anukoolkarn, K.; Aranyavalai, T.; Phonpichit, C.; Saensri, P.; Kaewprasert, M.. Therapeutic effects of low-level laser therapy in postoperative total knee arthroplasty surgery: a double-blinded randomized, placebocontrolled trial. Annals of the rheumatic diseases. CN-01065813.

T: Timing outcome too early
Fusco F; Turchetti G. Telerehabilitation after total knee replacement in Italy: cost-effectiveness and cost-utility analysis of a mixed telerehabilitationstandard rehabilitation programme compared with usual care.. BMJ open. 27188803.

O: No outcomes of interest

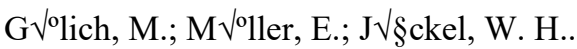

Rehabilitation after total hip and knee replacement (THR/TKR): results of a systematic guideline and literature search...Proceedings of the 9th Congress of European Federation for Research in Rehabilitation, Budapest, Hungary, 26-29 August 2007. International Journal of Rehabilitation Research. 105841256. Language:.

Full text unavailable

Galea MP; Levinger P; Lythgo N; Cimoli C; Weller R; Tully E; McMeeken J; Westh R. A targeted homeand center-based exercise program for people after total hip replacement: a randomized clinical trial.. Archives of physical medicine and rehabilitation. 18586222.

Duplicate/No additional data

Galea, M. P.; Levinger, P.; Lythgo, N.; Cimoli, C.; Weller, R.; Tully, E.; McMeeken, J.; Westh, R.. A targeted home- and center-based exercise program for people after total hip replacement: a randomized clinical trial [corrected] [published erratum appears in ARCH PHYS MED REHABIL 2009

Dec;90(12):2162]. Archives of Physical Medicine \& Rehabilitation. 105662976. Language:.

$\mathrm{D}: \mathrm{N}<20$ per group

Gao, Z. y; Wu, J. x; Lin, W. 1; Tuo, M.; Zhu, Q. x; Wei, L.; Wang, L.; Li, T. s. Comprehensive rehabilitation following total knee arthroplasty: a randomized controlled trial. Journal of clinical rehabilitative tissue engineering research. $\mathrm{CN}-$ 00903363.

Full text unavailable

Gavin JP; Immins T; Wainwright T. Stair negotiation as a rehabilitation intervention for enhancing recovery following total hip and knee replacement surgery.. International journal of orthopaedic and trauma nursing. 28302377.

SR or guideline

Gawel, Justina A.; Brown, Sarah E.; Collins, Jenna C.; McCallum, Christine. Does pre-operative physical therapy improve post-surgical outcomes of patients undergoing a total knee and/or total hip arthroplasty? A systematic review. Physiotherapy Practice \& Research. 104050171. Language:. SR or guideline 
Genet F; Mascard E; Coudeyre E; Revel M; Rannou F. The benefits of ambulatory physiotherapy after total knee replacement. Clinical practice recommendations.. Annales de readaptation et de medecine physique : revue scientifique de la Societe francaise de reeducation fonctionnelle de readaptation et de medecine physique. 17963973 . SR or guideline

Ghomrawi, H. M. K.. Does post-acute care placement affect outcomes for total hip replacement patients? University of Minnesota Doctoral Dissertation. . Full text unavailable

Gianola S; Stucovitz E; Castellini G; Mascali M; Vanni F; Tramacere I; Banfi G; Tornese D. Effects of early virtual reality-based rehabilitation in patients with total knee arthroplasty: A randomized controlled trial.. Medicine. 32049833.

$\mathrm{T}$ : Timing outcome too early

Giaquinto, S.; Ciotola, E.; Dall'Armi, V.; Margutti, F.. Hydrotherapy after total knee arthroplasty. A follow-up study. Arch Gerontol Geriatr. 19735951. Duplicate/No additional data

Gibson AJ; Shields N. Effects of Aquatic Therapy and Land-Based Therapy versus Land-Based Therapy Alone on Range of Motion, Edema, and Function after Hip or Knee Replacement: A Systematic Review and Meta-analysis.. Physiotherapy Canada. Physiotherapie Canada. 25931664.

SR or guideline

Gilbey HJ; Ackland TR; Wang AW; Morton AR; Trouchet T; Tapper J. Exercise improves early functional recovery after total hip arthroplasty.. Clinical orthopaedics and related research. 12616059. published before 2005

Gildone, A. A comparison of two rehabilitation protocols after total knee arthroplasty: Does flexion affect mobility and blood loss? Journal of Orthopaedics and Traumatology. PMC4874975. D: NRCS not adjusted

Gill SD; McBurney H. Does exercise reduce pain and improve physical function before hip or knee replacement surgery? A systematic review and metaanalysis of randomized controlled trials.. Archives of physical medicine and rehabilitation. 22960276.

SR or guideline

Gill SD; McBurney H; Schulz DL. Land-based versus pool-based exercise for people awaiting joing replacement surgery of the hip or knee: results of a randomized controlled trial. New Zealand Journal of Physiotherapy. 105266416. Language:.

P: Mixed TKA/THA with no clean subgroup analyses
Gocen Z; Sen A; Unver B; Karatosun V; Gunal I. The effect of preoperative physiotherapy and education on the outcome of total hip replacement: a prospective randomized controlled trial.. Clinical rehabilitation. 15180117.

published before 2005

Goffredo, M.; Pournajaf, S.; D'Elia, T. Sasso; Pellicciari, L.; Bertone, M.; Criscuolo, S.; Damiani, C.; Franceschini, M.. Does virtual reality rehabilitation influence postural control after total Knee replacement?. Gait \& Posture. 138888453. Language:.

D: $\mathrm{N}<20$ per group

Goodman, S. M.; Mehta, B. Y.; Mandl, L. A.; Szymonifka, J. D.; Finik, J.; Figgie, M. P.; NavarroMill $\sqrt{ }{ }^{\circ}$ n, I. Y.; Bostrom, M. P.; Parks, M. L.; Padgett, D. E.; McLawhorn, A. S.; Antao, V. C.; Yates, A. J.; Springer, B. D.; Lyman, S. L.; Singh, J. A..

Validation of the Hip Disability and Osteoarthritis Outcome Score and Knee Injury and Osteoarthritis Outcome Score Pain and Function Subscales for Use in Total Hip Replacement and Total Knee Replacement Clinical Trials. J Arthroplasty. 31952945.

I: Not (p)rehabilitation

Gotlin RS; Hershkowitz S; Juris PM; Gonzalez EG; Scott WN; Insall JN. Electrical stimulation effect on extensor lag and length of hospital stay after total knee arthroplasty.. Archives of physical medicine and rehabilitation. 8085929.

published before 2005

Goto K; Morishita T; Kamada S; Saita K; Fukuda H; Shiota E; Sankai Y; Inoue T. Feasibility of rehabilitation using the single-joint hybrid assistive limb to facilitate early recovery following total knee arthroplasty: A pilot study.. Assistive technology : the official journal of RESNA. 27689789. D: $\mathrm{N}<20$ per group

Grل§nicher, P.; St $\sqrt{\partial g g l, ~ T . ; ~ F u c e n t e s e, ~ S . ~ F . ; ~}$ Adelsberger, R.; Swanenburg, J.. Preoperative exercise in patients undergoing total knee arthroplasty: a pilot randomized controlled trial. Arch Physiother. 32774889.

D: $\mathrm{N}<20$ per group

Grasmick, Paige; Chughtai, Morad; Khlopas, Anton. Poster 45: Early Outpatient Physical Therapy May Improve Range, Äêof, ÄêMotion following Total Knee Arthroplasty. PM \& R: Journal of Injury, Function \& Rehabilitation. 133075448. Language:. D: NRCS not adjusted 
Gremeaux V; Renault J; Pardon L; Deley G; Lepers R; Casillas JM. Low-frequency electric muscle stimulation combined with physical therapy after total hip arthroplasty for hip osteoarthritis in elderly patients: a randomized controlled trial.. Archives of physical medicine and rehabilitation. 19061737. $\mathrm{D}: \mathrm{N}<20$ per group

Gremeaux, V.; Renault, J.; Pardon, L.; Deley, G.; Lepers, R.; Casillas, J. M.. Low-frequency electric muscle stimulation combined with physical therapy after total hip osteoarthritis in elderly patients: a randomized controlled trial. Isokinetics and exercise science. 19061737.

$\mathrm{D}: \mathrm{N}<20$ per group

Groot, L.; Gademan, M. G. J.; Peter, W. F.; van den Hout, W. B.; Verburg, H.; Vliet Vlieland, T. P. M.; Reijman, M.. Rationale and design of the PaTIO study: PhysiotherApeutic Treat-to-target Intervention after Orthopaedic surgery. BMC Musculoskelet Disord. 32795283.

Protocol/study registration

Grotle M; Garratt AM; Klokkerud M; Lochting I; Uhlig T; Hagen KB. What's in team rehabilitation care after arthroplasty for osteoarthritis? Results from a multicenter, longitudinal study assessing structure, process, and outcome.. Physical therapy. 20045807. D: Single group/no comparison

Gumaa, M.; Rehan Youssef, A.. Is Virtual Reality Effective in Orthopedic Rehabilitation? A Systematic Review and Meta-Analysis. Phys Ther. 31343702. SR or guideline

Gustavson AM; Wolfe P; Falvey JR; Eckhoff DG; Toth MJ; Stevens-Lapsley JE. Men and Women Demonstrate Differences in Early Functional Recovery After Total Knee Arthroplasty.. Archives of physical medicine and rehabilitation. 27063363. SR or guideline

Gwam CU; McGinnis T; Etcheson JI; George NE; Sultan AA; Delanois RE; Bhave A; Harwin SF; Mont MA. Use of Neuromuscular Electrical Stimulation During Physical Therapy May Reduce the Incidence of Arthrofibrosis After Total Knee Arthroplasty.. Surgical technology international. 29791704.

Full text unavailable

Haas R; O'Brien L; Bowles KA; Haines T. Effectiveness of a weekend physiotherapy service on short-term outcomes following hip and knee joint replacement surgery: a quasi-experimental study.. Clinical rehabilitation. 29865898.

P: Mixed TKA/THA with no clean subgroup analyses
Haas R; Sarkies M; Bowles KA; O'Brien L; Haines $T$. Early commencement of physical therapy in the acute phase following elective lower limb arthroplasty produces favorable outcomes: a systematic review and meta-analysis examining allied health service models.. Osteoarthritis and cartilage. 27224276.

SR or guideline

Haas, R.; Bowles, K. A.; O'Brien, L.; Haines, T.. The effect of transferring weekend physical therapy services from the acute to sub-acute setting in patients following hip and knee arthroplasty: a quasiexperimental study. Physiother Theory Pract. 32568601.

P: Mixed TKA/THA with no clean subgroup analyses

Hadamus, A.; Bialoszewski, D.; et al.. Assessment of the Effectiveness of Rehabilitation after Total Knee Replacement Surgery Using Sample Entropy and Classical Measures of Body Balance. Entropy (Basel). 33573057.

$\mathrm{T}$ : Timing outcome too early

Hadamus, A.; Bialoszewski, D.; Kowalska, A. J.; Urbaniak, E.; Wydra, K.; Boratynski, R.; Kobza, A.; Selegrat, M.; Marczynski, W.. There is little impact of balance training in Virtual Reality on selected balance parameters in patients after hip arthroplasty. Gait \& posture. CN-02086177.

I: Not well defined/no (p)rehabilitation content to define

Hadamus, A.; Bialoszewski, D.; Urbaniak, E.; Wydra, K.; Kowalska, A. J.; Boratynski, R.; Kobza, A.; Selegrat, M.; Marczynski, W.. Balance training in virtual reality in patients after hip replacement does not influence foot pressure distribution in static balance test. Gait \& posture. $\mathrm{CN}-02087563$. I: Not well defined/no (p)rehabilitation content to define

Hadamus, A.; Bialoszewski, D.; Wydra, K.; Kowalska, A. J.; Urbaniak, E.; Boratynski, R.; Kobza, A.; Selegrat, M.; Marczynski, W.. Balance training in virtual reality improves temporal gait parameters in patients after total hip replacement. Gait \& posture. CN-02086179.

I: Not well defined/no (p)rehabilitation content to define

Halil, Deniz. Manuelle Therapie nach Mulligan zur Verbesserung der

KnieflexionbeiPatientennachKnietotalendoprothese: Randomisierte kontrollierte Studie. Manuelle Therapie. 128068181. Language:.

D: $\mathrm{N}<20$ per group 
Hamilton, D.. Targeting physiotherapy to patients at risk of poor outcomes following total knee arthroplasty: the TRIO randomised controlled trial. Physiotherapy (united kingdom). CN-01793703. Duplicate/No additional data

Hamilton, D.; Beard, D.; Barker, K.; MacFarlane, G.; Murray, G.; Simpson, H.. Targeting physiotherapy to patients at risk of poor outcomes following total knee arthroplasty: the TRIO randomised controlled trial. Physiotherapy (united kingdom). CN-01793703. Duplicate/No additional data

Hamilton, D. F.; Loth, F. C.; MacDonald, D. J.; MacFarlane, G. J.; Beard, D. J.; Simpson, A. H. R.; Patton, J. T.; Howie, C. R.. Exploring variation in patient access of post-discharge physiotherapy following total hip and knee arthroplasty under a choice based system in the UK: an observational cohort study. BMJ Open. 30787073.

P: Not osteoarthritis (exclude cancer, trauma, rheumatoid arthritis)

Han AS; Nairn L; Harmer AR; Crosbie J; March L; Parker D; Crawford R; Fransen M. Early rehabilitation after total knee replacement surgery: a multicenter, noninferiority, randomized clinical trial comparing a home exercise program with usual outpatient care.. Arthritis care \& research. 25220488. $\mathrm{T}$ : Timing outcome too early

Hansen S; Aaboe J; Mechlenburg I; Overgaard S; Mikkelsen LR. Effects of supervised exercise compared to non-supervised exercise early after total hip replacement on patient-reported function, pain, health-related quality of life and performance-based function - a systematic review and meta-analysis of randomized controlled trials.. Clinical rehabilitation. 30073856.

SR or guideline

Hansen, Sebrina; Aaboe, Jens; Mechlenburg, Inger; Overgaard, S $\sqrt{ }$ Tren; Mikkelsen, Lone Ramer. Effects of supervised exercise compared to non-supervised exercise early after total hip replacement on patientreported function, pain, health-related quality of life and performance-based function, Äì a systematic review and meta-analysis of randomized controlled trials. Clinical Rehabilitation. 133798326. Language:. SR or guideline

Hardt S; Schulz MRG; Pfitzner T; Wassilew G; Horstmann H; Liodakis E; Weber-Spickschen TS. Improved early outcome after TKA through an appbased active muscle training programme-a randomized-controlled trial.. Knee surgery, sports traumatology, arthroscopy : official journal of the
ESSKA. 29589050.

T: Timing outcome too early

Harmelink KEM; Zeegers AVCM; Tonis TM; Hullegie W; Nijhuis-van der Sanden MWG; Staal JB. The effectiveness of the use of a digital activity coaching system in addition to a two-week homebased exercise program in patients after total knee arthroplasty: study protocol for a randomized controlled trial.. BMC musculoskeletal disorders. 28679400.

Protocol/study registration

Harnirattisai T; Johnson RA. Effectiveness of a behavioral change intervention in Thai elders after knee replacement.. Nursing research. 15778651. P: Not first replacement (exclude revision surgeries)

Hawke, L. J.; Shields, N.; Dowsey, M. M.; Choong, P. F. M.; Taylor, N. F.. Effectiveness of behavioural interventions on physical activity levels after hip or knee joint replacement: a systematic review. Disabil Rehabil. 31067136.

SR or guideline

Henderson KG; Wallis JA; Snowdon DA. Active physiotherapy interventions following total knee arthroplasty in the hospital and inpatient rehabilitation settings: a systematic review and metaanalysis.. Physiotherapy. 28802773.

SR or guideline

Hensman-Crook, A.. The effectiveness of physiotherapy intervention with home exercise programme versus patient directed home exercise programme following total knee replacement. Internal medicine journal. CN-00836694. P: Not osteoarthritis (exclude cancer, trauma, rheumatoid arthritis)

Herbold JA; Bonistall K; Walsh MB. Rehabilitation following total knee replacement, total hip replacement, and hip fracture: a case-controlled comparison.. Journal of geriatric physical therapy (2001). 22124414.

P: Not osteoarthritis (exclude cancer, trauma, rheumatoid arthritis)

Hermann A; Holsgaard-Larsen A; Zerahn B; Mejdahl S; Overgaard S. Preoperative progressive explosivetype resistance training is feasible and effective in patients with hip osteoarthritis scheduled for total hip arthroplasty--a randomized controlled trial..

Osteoarthritis and cartilage. 26285180.

$\mathrm{T}$ : Timing outcome too early

Hermann, A.; Holsgaard-Larsen, A.; Zerahn, B.; Mejdahl, S.; Overgaard, S.. Preoperative progressive explosive-type resistance training is feasible and 
effective in patients with hip osteoarthritis scheduled for total hip arthroplasty - a randomized controlled trial. Osteoarthritis and Cartilage. 26285180.

Duplicate/No additional data

Hermann, A.; Holsgaard-Larsen, A.; Zerahn, B.; Mejdahl, S.; Overgaard, S.. Preoperative effects of progressive explosive-type resistance training in patients with osteoarthritis scheduled for total hip arthroplasty-a prospective randomized clinical trial. Osteoarthritis and cartilage.. CN-01064055.

$\mathrm{T}$ : Timing outcome too early

Hershko E; Tauber C; Carmeli E. Biofeedback versus physiotherapy in patients with partial weightbearing.. American journal of orthopedics (Belle Mead, N.J.). 18587509.

P: Not osteoarthritis (exclude cancer, trauma, rheumatoid arthritis)

Hesse S; Werner C; Seibel H; von Frankenberg S; Kappel EM; Kirker S; Kading M. Treadmill training with partial body-weight support after total hip arthroplasty: a randomized controlled trial.. Archives of physical medicine and rehabilitation. 14669181. published before 2005

Hewitt B; Shakespeare D. Flexion vs. extension: a comparison of post-operative total knee arthroplasty mobilisation regimes.. The Knee. 11706693.

published before 2005

Hiraga Y; Hisano S; Nomiyama K; Hirakawa Y. Effects of using activity diary for goal setting in occupational therapy on reducing pain and improving psychological and physical performance in patients after total knee arthroplasty: A non-randomised controlled study.. Hong Kong journal of occupational therapy : HKJOT. 31217762.

D: NRCS not adjusted

Hiyama Y; Kamitani T; Wada O; Mizuno K; Yamada M. Effects of Group-Based Exercise on Range of Motion, Muscle Strength, Functional Ability, and Pain During the Acute Phase After Total Knee Arthroplasty: A Controlled Clinical Trial.. The Journal of orthopaedic and sports physical therapy. 27494052.

T: Timing outcome too early

Hol AM; van Grinsven S; Lucas C; van Susante JL; van Loon CJ. Partial versus unrestricted weight bearing after an uncemented femoral stem in total hip arthroplasty: recommendation of a concise rehabilitation protocol from a systematic review of the literature.. Archives of orthopaedic and trauma surgery. 20012073.

SR or guideline
Holsgaard-Larsen, A.; Hermann, A.; Zerahn, B.; Mejdahl, S.; Overgaard, S.. Postoperative effects of progressive resistance training prior to total hip arthroplasty e one year outcome of a randomized controlled trial. Osteoarthritis and cartilage. $\mathrm{CN}$ 01613457.

Duplicate/No additional data

Holsgaard-Larsen, A.; Hermann, A.; Zerahn, B.; Mejdahl, S.; Overgaard, S.. Effects of progressive resistance training prior to total HIP arthroplasty - a secondary analysis of a randomized controlled trial. Osteoarthritis Cartilage. 32376477.

Duplicate/No additional data

Hoogeboom TJ; Dronkers JJ; van den Ende CH; Oosting E; van Meeteren NL. Preoperative therapeutic exercise in frail elderly scheduled for total hip replacement: a randomized pilot trial.. Clinical rehabilitation. 20554640.

D: $\mathrm{N}<20$ per group

Hoogeboom TJ; Oosting E; Vriezekolk JE; Veenhof $\mathrm{C}$; Siemonsma PC; de Bie RA; van den Ende CH; van Meeteren NL. Therapeutic validity and effectiveness of preoperative exercise on functional recovery after joint replacement: a systematic review and meta-analysis.. PloS one. 22675429.

SR or guideline

Hoorntje, A.; Waterval-Witjes, S.; Koenraadt, K. L. M.; Kuijer, Ppfm; Blankevoort, L.; Kerkhoffs, Gmmj; van Geenen, R. C. I.. Goal Attainment Scaling Rehabilitation Improves Satisfaction with Work Activities for Younger Working Patients After Knee Arthroplasty: Results from the Randomized Controlled ACTION Trial. J Bone Joint Surg Am. 32453116.

P: Not total relacement (exclude partial)

Hoorntje, A.; Witjes, S.; Kuijer, Ppfm; Bussmann, J. B. J.; Horemans, H. L. D.; Kerkhoffs, Gmmj; van Geenen, R. C. I.; Koenraadt, K. L. M.. Does ActivityBased Rehabilitation With Goal Attainment Scaling Increase Physical Activity Among Younger Knee Arthroplasty Patients? Results From the Randomized Controlled ACTION Trial. Journal of arthroplasty. CN-02006891.

Duplicate/No additional data

Hoorntje, A.; Witjes, S.; Kuijer, Ppfm; Bussmann, J. B. J.; Horemans, H. L. D.; Kerkhoffs, Gmmj; van Geenen, R. C. I.; Koenraadt, K. L. M.. Does ActivityBased Rehabilitation With Goal Attainment Scaling Increase Physical Activity Among Younger Knee Arthroplasty Patients? Results From the Randomized Controlled ACTION Trial. J Arthroplasty. 31740103. P: Not total relacement (exclude partial) 
Hsieh, C. J.; DeJong, G.; Vita, M.; Zeymo, A.; Desale, S.. Effect of Outpatient Rehabilitation on Functional Mobility After Single Total Knee Arthroplasty: A Randomized Clinical Trial. JAMA Netw Open. 32940679.

$\mathrm{T}$ : Timing outcome too early

Hsu WH; Hsu WB; Shen WJ; Lin ZR; Chang SH; Hsu RW. Twenty-four-week hospital-based progressive resistance training on functional recovery in female patients post total knee arthroplasty.. The Knee. 30902510.

$\mathrm{D}: \mathrm{N}<20$ per group

Hsu WH; Hsu WB; Shen WJ; Lin ZR; Chang SH; Hsu RW. Circuit training enhances function in patients undergoing total knee arthroplasty: a retrospective cohort study.. Journal of orthopaedic surgery and research. 29052519.

$\mathrm{D}: \mathrm{N}<20$ per group

Huang P; He J; Zhang YM. [The mobile application of patient management in education and follow-up for patients following total knee arthroplasty].. Zhonghua yi xue za zhi. 28592069.

I: Not well defined/no (p)rehabilitation content to define

Huang TT; Sung CC; Wang WS; Wang BH. The effects of the empowerment education program in older adults with total hip replacement surgery.. Journal of advanced nursing. 28122161.

T: Timing outcome too early

Huang, S.; Kuo, M. L.; Yu, H. M.; Huang, C. H.; Shieh, W. Y.; Hsu, W. H.; Huang, Y. P.; Kuo, F. C.; Lee, M. S.. Clinical information and guidance shared via a patient infotainment system can reduce hospital stay and maintain 2 medical quality for total knee arthroplasty: A single-blinded quasi-randomised controlled trial. Int J Nurs Stud. 32105971.

I: Not (p)rehabilitation

Hughes K; Kuffner L; Dean B. Effect of weekend physical therapy treatment on postoperative length of stay following total hip and total knee arthroplasty.. Physiotherapy Canada. Physiotherapie Canada. 10130908.

Full text unavailable

Hui, L.. Hip prosthesis replacement and clinical rehabilitation treatment. Journal of Clinical Rehabilitative Tissue Engineering Research. . Full text unavailable

Hurkmans HL; Bussmann JB; Benda E; Verhaar JA; Stam HJ. Effectiveness of audio feedback for partial weight-bearing in and outside the hospital: a randomized controlled trial.. Archives of physical medicine and rehabilitation. 22325684.

$\mathrm{D}: \mathrm{N}<20$ per group

Hurkmans HL; Bussmann JB; Selles RW; Benda E; Stam HJ; Verhaar JA. The difference between actual and prescribed weight bearing of total hip patients with a trochanteric osteotomy: long-term vertical force measurements inside and outside the hospital.. Archives of physical medicine and rehabilitation. 17270518.

D: NRCS not adjusted

Husby VS; Foss OA; Husby OS; Winther SB. Randomized controlled trial of maximal strength training vs. standard rehabilitation following total knee arthroplasty.. European journal of physical and rehabilitation medicine. 28901118.

$\mathrm{D}: \mathrm{N}<20$ per group

Husby VS; Helgerud J; Bjorgen S; Husby OS; Benum P; Hoff J. Early maximal strength training is an efficient treatment for patients operated with total hip arthroplasty.. Archives of physical medicine and rehabilitation. 19801053.

D: $\mathrm{N}<20$ per group

Husby VS; Helgerud J; Bjorgen S; Husby OS; Benum P; Hoff J. Early postoperative maximal strength training improves work efficiency 6-12 months after osteoarthritis-induced total hip arthroplasty in patients younger than 60 years. American journal of physical medicine \& rehabilitation. 20134307.

$\mathrm{D}: \mathrm{N}<20$ per group

Hussain MS; Li J; Brindal E; van Kasteren Y; Varnfield M; Reeson A; Berkovsky S; Freyne J. Supporting the Delivery of Total Knee Replacements Care for Both Patients and Their Clinicians With a Mobile App and Web-Based Tool: Randomized Controlled Trial Protocol.. JMIR research protocols. 28249832.

Protocol/study registration

Husted, R. S.; Juhl, C.; Troelsen, A.; Thorborg, K.; Kallemose, T.; Rathleff, M. S.; Bandholm, T.. The relationship between prescribed pre-operative kneeextensor exercise dosage and effect on knee-extensor strength prior to and following total knee arthroplasty: a systematic review and meta-regression analysis of randomized controlled trials.

Osteoarthritis Cartilage. 32890744.

SR or guideline

Hutchinson AG; Gooden B; Lyons MC; Roe JP; O'Sullivan MD; Salmon LJ; Martina K; Pinczewski LA. Inpatient rehabilitation did not positively affect 6-month patient-reported outcomes after hip or knee 
arthroplasty.. ANZ journal of surgery. 30173415. D: NRCS not adjusted

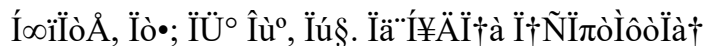

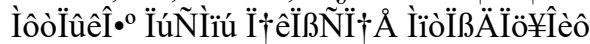

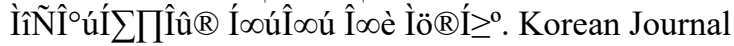
of Adult Nursing. 147878662.

T: Timing outcome too early

Ingram, Christian; Tingle, Casey. Preoperative PT linked with decreased need for post-acute care services following THA,TKA. Orthopedics Today. 107838875. Language:.

Full text unavailable

Irct20091124002769N. The effect of Rehabilitation Education in patients Who undergo a Total knee Replacement. http://www.who.int/trialsearch/Trial2.aspx?TrialID $=\mathrm{I}$ RCT20091124002769N6. CN-01906748.

Protocol/study registration

Irct2014111619980N. Effect of educational program on pain intensity and range of motion in patients undergoing joint replacement.

http://www.who.int/trialsearch/Trial2.aspx?TrialID=I RCT2014111619980N1. CN-01871089.

Protocol/study registration

Irct2017050933772N. Pilates-based Exercise after Total Hip Arthroplasty.

http://www.who.int/trialsearch/Trial2.aspx?TrialID=I RCT2017050933772N2. CN-01892274.

Protocol/study registration

Iskender, Mahinur Durmus; Bektas, Ozgur; Eren, Handan. Effect of preoperative in, Äêbed exercises and mobilization training on postoperative anxiety and mobilization level. Japan Journal of Nursing Science. 146362395. Language:.

T: Timing outcome too early

Isrctn. The effectiveness of adding arm exercise programme following hip replacement in the elderly. http://www.who.int/trialsearch/Trial2.aspx?TrialID=I SRCTN73197506. CN-01815054.

Protocol/study registration

Isrctn. Evaluation of I-ONE therapy in patients undergoing total knee arthoplasty.

http://www.who.int/trialsearch/Trial2.aspx?TrialID=I SRCTN98459871. CN-01817574.

Protocol/study registration

Isrctn. Optimising patient function following elective Total Hip Replacement (THR) surgery. http://www.who.int/trialsearch/Trial2.aspx?TrialID=I SRCTN13019951. CN-01847758.

Protocol/study registration
Isrctn. COmmunity-based Rehabilitation after Knee Arthroplasty (CORKA).

http://www.who.int/trialsearch/Trial2.aspx?TrialID=I SRCTN13517704. CN-01878442.

Protocol/study registration

Isrctn. The effect of the Mulligan mobilization with movement approach following knee replacement surgery.

http://www.who.int/trialsearch/Trial2.aspx?TrialID=I SRCTN13028992. CN-01947531.

Protocol/study registration

Isrctn. Knee rehabilitation: home visual feedback and video call follow up.

http://www.who.int/trialsearch/Trial2.aspx?TrialID=I SRCTN33424520. CN-01805430.

Protocol/study registration

Isrctn. Activity orientated rehabilitation following knee arthroplasty: feasibility study.

http://www.who.int/trialsearch/Trial2.aspx?TrialID=I SRCTN13579789. CN-01806744.

Protocol/study registration

Isrctn. Kneehab $\neg Æ$ pre and post total knee replacement surgery.

http://www.who.int/trialsearch/Trial2.aspx?TrialID=I SRCTN89785408. CN-01815908.

Protocol/study registration

Isrctn. Feasibility of a therapeutic exercise before total hip replacement.

http://www.who.int/trialsearch/Trial2.aspx?TrialID=I SRCTN09005050. CN-01834685.

Protocol/study registration

Isrctn. Efficiency of immediate postoperative inpatient physical therapy (PT) following total knee arthroplasty: a randomised controlled trial (RCT). http://www.who.int/trialsearch/Trial2.aspx?TrialID=I SRCTN49658674. CN-01854471.

Protocol/study registration

Isrctn. What are the effects of balance exercises in patients' following total knee replacement ability to perform every day life activities?.

http://www.who.int/trialsearch/Trial2.aspx?TrialID=I SRCTN12101643. CN-01868226.

Protocol/study registration

Isrctn. PEP-TALK: a study investigating whether having group discussions in addition to physiotherapy improves the amount of physical activity following hip and knee replacement. http://www.who.int/trialsearch/Trial2.aspx?TrialID=I SRCTN29770908. CN-01949054.

Protocol/study registration 
Iwakiri, K.; Ohta, Y.; Shibata, Y.; Minoda, Y.; Kobayashi, A.; Nakamura, H.. Initiating range of motion exercises within 24 hours following total knee arthroplasty affects the reduction of postoperative pain: a randomized controlled trial. Asia-pacific journal of sports medicine, arthroscopy, rehabilitation and technology. CN-02121688.

Duplicate/No additional data

Jacobson AF; Umberger WA; Palmieri PA; Alexander TS; Myerscough RP; Draucker CB; Steudte-Schmiedgen S; Kirschbaum C. Guided Imagery for Total Knee Replacement: A Randomized, Placebo-Controlled Pilot Study.. Journal of alternative and complementary medicine (New York, N.Y.). 27214055.

I: Not (p)rehabilitation

Jacofsky DJ; Kocisky S; Dixon D; Jacofsky MC. Secure tracks device improves functional recovery and pain after total knee arthroplasty: a prospective, randomized, pilot study.. Surgical technology international. 21082587.

Full text unavailable

Jaczewska-Bogacka J; Stolarczyk A. Improvement in Gait Pattern After Knee Arthroplasty Followed by Proprioceptive Neuromuscular Facilitation Physiotherapy.. Advances in experimental medicine and biology. 29594754.

D: NRCS not adjusted

Jahic D; Omerovic D; Tanovic AT; Dzankovic F; Campara MT. The Effect of Prehabilitation on Postoperative Outcome in Patients Following Primary Total Knee Arthroplasty.. Medical archives (Sarajevo, Bosnia and Herzegovina). 30814777. $\mathrm{D}: \mathrm{N}<20$ per group

Jakobsen TL; Kehlet H; Husted H; Petersen J; Bandholm T. Early progressive strength training to enhance recovery after fast-track total knee arthroplasty: a randomized controlled trial.. Arthritis care \& research. 25074397.

P: Not unilateral replacement (exclude bilateral replacement surgeries)

Jame Bozorgi AA; Ghamkhar L; Kahlaee AH; Sabouri H. The Effectiveness of Occupational Therapy Supervised Usage of Adaptive Devices on Functional Outcomes and Independence after Total Hip Replacement in Iranian Elderly: A Randomized Controlled Trial.. Occupational therapy international. 26625939.

T: Timing outcome too early

Jan MH; Hung JY; Lin JC; Wang SF; Liu TK; Tang PF. Effects of a home program on strength, walking speed, and function after total hip replacement.. Archives of physical medicine and rehabilitation. 15605331. published before 2005

Jansson, M. M.; Rantala, A.; Miettunen, J.; Puhto, A. P.; Pikkarainen, M.. The effects and safety of telerehabilitation in patients with lower-limb joint replacement: A systematic review and narrative synthesis. J Telemed Telecare. 32316837.

SR or guideline

Jarski RW; Loniewski EG; Williams J; Bahu A; Shafinia S; Gibbs K; Muller M. The effectiveness of osteopathic manipulative treatment as complementary therapy following surgery: a prospective, match-controlled outcome study.. Alternative therapies in health and medicine. 10979164.

Full text unavailable

Jayabalan, Prakash; Almeida, Gustavo J.; Wan, Huang; Sowa, Gwendolyn A.; Piva, Sara R.. THE INVESTIGATION OF CANDIDATE BIOMARKERS TO ASSESS THE EFFICACY OF A NOVEL REHABILITATION REGIMEN FOLLOWING TOTAL KNEE ARTHROPLASTY: A PILOT STUDY. American Journal of Physical Medicine \& Rehabilitation. 95897199. Language:. Full text unavailable

Jesudason C; Stiller K. Are bed exercises necessary following hip arthroplasty?. The Australian journal of physiotherapy. 12047205.

published before 2005

Jiang S; Xiang J; Gao X; Guo K; Liu B. The comparison of telerehabilitation and face-to-face rehabilitation after total knee arthroplasty: A systematic review and meta-analysis.. Journal of telemedicine and telecare. 28027679.

SR or guideline

Jogi P; Overend TJ; Spaulding SJ; Zecevic A; Kramer JF. Effectiveness of balance exercises in the acute post-operative phase following total hip and knee arthroplasty: A randomized clinical trial.. SAGE open medicine. 26770765.

T: Timing outcome too early

Jogi P; Zecevic A; Overend TJ; Spaulding SJ; Kramer JF. Force-plate analyses of balance following a balance exercise program during acute postoperative phase in individuals with total hip and knee arthroplasty: A randomized clinical trial.. SAGE open medicine. 27867519.

T: Timing outcome too early 
Jogi, Pankaj. Effectiveness Of Balance Exercises

Following Total Hip And Knee Joint Arthroplasty.

University of Western Ontario Doctoral

Dissertation. .

Full text unavailable

Johanson MA; Cohen BA; Snyder KH; McKinley AJ; Scott ML. Outcomes for aging adults following total hip arthroplasty in an acute rehabilitation facility versus a subacute rehabilitation facility: a pilot study.. Journal of geriatric physical therapy (2001). 20039586.

$\mathrm{D}: \mathrm{N}<20$ per group

Jones S; Alnaib M; Kokkinakis M; Wilkinson M; St Clair Gibson A; Kader D. Pre-operative patient education reduces length of stay after knee joint arthroplasty.. Annals of the Royal College of Surgeons of England. 21418755.

D: NRCS not adjusted

Jonsson, B.; Sigurdsson, E.; Siggeirsdottir, K.; Janssen, H.; Gudnason, V.; Matthasson, T.. Early discharge, education and home based rehabilitation reduces unit costs after total hip replacement: results of a cost analysis in a randomised study. The journal of bone and joint surgery (proceedings). $\mathrm{CN}$ 00626721.

P: Not osteoarthritis (exclude cancer, trauma, rheumatoid arthritis)

Jordan LR; Siegel JL; Olivo JL. Early flexion routine. An alternative method of continuous passive motion.. Clinical orthopaedics and related research. 7634672.

Full text unavailable

Jordan RW; Smith NA; Chahal GS; Casson C; Reed MR; Sprowson AP. Enhanced education and physiotherapy before knee replacement; is it worth it? A systematic review.. Physiotherapy. 24882691.

SR or guideline

Jorgensen PB; Bogh SB; Kierkegaard S; Sorensen H; Odgaard A; Soballe K; Mechlenburg I. The efficacy of early initiated, supervised, progressive resistance training compared to unsupervised, home-based exercise after unicompartmental knee arthroplasty: a single-blinded randomized controlled trial.. Clinical rehabilitation. 27029938.

P: Not total relacement (exclude partial)

Jorgensen, S. L.; Bohn, M. B.; Aagaard, P.; Mechlenburg, I.. Efficacy of low-load blood flow restricted resistance EXercise in patients with Knee osteoarthritis scheduled for total knee replacement (EXKnee): protocol for a multicentre randomised controlled trial. BMJ Open. 33004382.

Protocol/study registration

Jprn Umin. Effectiveness of therapeutic exercises or a shoe lift for functional leg length discrepancy after total hip arthroplasty.

http://www.who.int/trialsearch/Trial2.aspx?TrialID=J PRN-UMIN000009095. CN-01818643.

Protocol/study registration

Jprn Umin. The study of effective training method in early rehabilitation after knee arthroplasty: effects of quadriceps femoris muscle setting exercise with visual feedback.

http://www.who.int/trialsearch/Trial2.aspx?TrialID=J PRN-UMIN000026077. CN-01826399.

Protocol/study registration

Jprn Umin. The effects of pre-operative rehabilitation on clinical outcomes after knee arthroplasty: a randomized controlled trial.

http://www.who.int/trialsearch/Trial2.aspx?TrialID=J PRN-UMIN000024196. CN-01828995.

Protocol/study registration

Jprn Umin. Effect of difference of decubitus position in abducting the hip on function after hip replacement arthroplasty.

http://www.who.int/trialsearch/Trial2.aspx?TrialID=J PRN-UMIN000020378. CN-01837941.

Protocol/study registration

Jprn Umin. The effect of walking training by using anti-gravity tredmil after total knee arthroplasty. http://www.who.int/trialsearch/Trial2.aspx?TrialID =J PRN-UMIN000018872. CN-01881557.

Protocol/study registration

Jprn Umin. Effect of High-intensity and Short Term Preoperative Rehabilitation in TKA Patients. http://www.who.int/trialsearch/Trial2.aspx?TrialID=J PRN-UMIN000027198. CN-01888203.

Protocol/study registration

Jprn Umin. The effect of high velocity trunk exercise on gait ability of patients after total hip arthroplasty.

http://www.who.int/trialsearch/Trial2.aspx?TrialID $=\mathrm{J}$ PRN-UMIN000029392. CN-01891189.

Protocol/study registration

Jprn Umin. A study on a new rehabilitation program after total hip arthroplasty.

http://www.who.int/trialsearch/Trial2.aspx?TrialID =J PRN-UMIN000030073. CN-01893868.

Protocol/study registration

Jprn Umin. Benefits of self-calf massage for the prevention of the vein thromboembolism after total knee arthroplasty. 
http://www.who.int/trialsearch/Trial2.aspx?TrialID=J PRN-UMIN000031327. CN-01902820.

Protocol/study registration

Jprn Umin. Comparative study of physiotherapy program after total knee arthroplasty.

http://www.who.int/trialsearch/Trial2 aspx?TrialID=J PRN-UMIN000032977. CN-01910512.

Protocol/study registration

Jprn Umin. Efficacy of 4 weeks preoperative exercise with blood flow restriction on ischemia reperfusion injury after total knee arthroplasty. http://www.who.int/trialsearch/Trial2.aspx?TrialID=J PRN-UMIN000037981. CN-02070438.

Protocol/study registration

Jprn Umin. Effects of early starting of intensive functional rehabilitation on recovery of body function and activity in people undergoing total knee arthroplasty.

http://www.who.int/trialsearch/Trial2.aspx?TrialID=J PRN-UMIN000024029. CN-01829689.

Protocol/study registration

Jprn Umin. An Easy and Safe Training Method for Trunk Function Improves Mobility in Total Knee Arthroplasty Patients.

http://www.who.int/trialsearch/Trial2.aspx?TrialID=J PRN-UMIN000027909. CN-01888466.

Protocol/study registration

Judd DL; Cheuy VA; Forster JE; Christiansen CL; Stevens-Lapsley JE. Incorporating Specific Functional Strength Integration Techniques to Improve Functional Performance for Veterans After Total Hip Arthroplasty: Protocol for a Randomized Clinical Trial.. Physical therapy. 31392991.

Protocol/study registration

Judd, Dana L.. Improving rehabilitation outcomes after total hip arthroplasty. University of Colorado Doctoral Dissertation. .

$\mathrm{D}: \mathrm{N}<20$ per group

Jung JY; Cho JH; Chung SH. Acupuncture for postoperative pain following total knee arthroplasty: a systematic review protocol.. BMJ open. 26582406. SR or guideline

Kaczor, R.; Lyp, M.; Ogonowski, A.. Physiotherapy in the swimming pool patients after the alloplastic operation of the hip joint...Proceedings of the 10th Congress of the European Federation for Research in Rehabilitation, Riga, Latvia, 09-12 September 2009. International Journal of Rehabilitation Research.

105444997. Language:.

O: No outcomes of interest
Kadi, M. R.; Hepguler, S.; Dede, E.; Ozturk, C.; Aydogdu, S.; Aktuglu, K.; Ozkayin, N.; Atamaz, F. C.. Is electrotherapy effective in the management of pain, range of motion, quality of life, edema following total knee arthroplasty surgery?

Randomized controlled trial. Annals of the rheumatic diseases. CN-01729555.

T: Timing outcome too early

Kalron A; Tawil H; Peleg-Shani S; Vatine JJ. Effect of telerehabilitation on mobility in people after hip surgery: a pilot feasibility study.. International journal of rehabilitation research. Internationale Zeitschrift fur Rehabilitationsforschung. Revue internationale de recherches de readaptation. 29794545.

$\mathrm{D}: \mathrm{N}<20$ per group

Karaborklu Argut, S.; Celik, D.; Kilicoglu, O. I.. The Combination of Exercise and Manual Therapy Versus Exercise Alone in Total Knee Arthroplasty Rehabilitation: A Randomized Controlled Clinical Trial. Pm r. 33352007.

T: Timing outcome too early

Karaman A; Yuksel I; Kinikli GI; Caglar O. Do Pilates-based exercises following total knee arthroplasty improve postural control and quality of life?. Physiotherapy theory and practice. 28443790. $\mathrm{D}: \mathrm{N}<20$ per group

Karihtala, T.; Heinonen, A.; Manninen, M.; P $\sqrt{ } \partial y h \sqrt{ } \partial$ nen, T.; Sipil $\sqrt{ } \S$, S.; Valtonen, A.. Effects of preoperative group-based aquatic training on health related quality of life in persons with late stage knee osteoarthritis. Physiotherapy (united kingdom). CN01126478.

I: Not (p)rehabilitation

Karim, A.; Pulido, L.; Incavo, S.. Does Accelerated Physical Therapy After Elective Primary Hip and Knee Arthroplasty Facilitate Early Discharge?. American journal of orthopedics (Belle Mead, N.J.). . Full text unavailable

Kathrins B; Kathrins R; Marsico R; Frank M; Stevenson-Marshall B; Orozco F; Ong A. Comparison of day rehabilitation to skilled nursing facility for the rehabilitation for total knee arthroplasty.. American journal of physical medicine \& rehabilitation. 22854905.

D: NRCS not adjusted

Kelly MA; Finley M; Lichtman SW; Hyland MR; Edeer AO. Comparative Analysis of High-Velocity Versus Low-Velocity Exercise on Outcomes After Total Knee Arthroplasty: A Randomized Clinical Trial.. Journal of geriatric physical therapy (2001). 
26428900.

$\mathrm{D}: \mathrm{N}<20$ per group

Kelly MH; Ackerman RM. Total joint arthroplasty: a comparison of postacute settings on patient functional outcomes.. Orthopedic nursing. 11052054. published before 2005

Keswani A; Weiser MC; Shin J; Lovy AJ; Moucha CS. Discharge Destination After Revision Total Joint Arthroplasty: An Analysis of Postdischarge Outcomes and Placement Risk Factors.. The Journal of arthroplasty. 27172864.

I: Not (p)rehabilitation

Khan F; Ng L; Gonzalez S; Hale T; Turner-Stokes L. Multidisciplinary rehabilitation programmes following joint replacement at the hip and knee in chronic arthropathy.. The Cochrane database of systematic reviews. 18425906.

SR or guideline

Khazi, Z. M.. Risk factors for opioid use after total shoulder arthroplasty. J Shoulder Elbow Surg. 31495704.

P: Not MJR (hip/knee)

Khoziainova, S. S.; Kovlen, D. V.; Ponomarenko, G. N.; Abuseva, G. R.; Adkhamov, B. M.; Ishchuk, V. N.; Karpova, T. N.; Kondrina, E. F.; Konoplyankin, I. V.; Podberezkina, L. A.; Tolmachev, S. V.. [Physical therapy in the rehabilitation of patients after endoprosthetic replacement of major joints in the lower extremities: a scientometric analysis of evidence-based studies]. Vopr Kurortol Fizioter Lech Fiz Kult. 31880761.

Full text unavailable

Kim, B.; Lohman, E.; Yim, J.. Acupuncture-like Transcutaneous Electrical Nerve Stimulation for Pain, Function, and Biochemical Inflammation After Total Knee Arthroplasty. Altern Ther Health Med. 32088676.

$\mathrm{D}: \mathrm{N}<20$ per group

Kim, J. H.; Noh, J. W.; Kim, M. Y.; Lee, J. U.; Yang, S. M.; Kim, J.. Effect of orthopedic therapy on patients with total knee replacement for health science research. Toxicology and environmental health sciences. CN-01791482.

Full text unavailable

Kim, S.; Hsu, F. C.; Groban, L.; Williamson, J.; Messier, S.. A pilot study of aquatic prehabilitation in adults with knee osteoarthritis undergoing total knee arthroplasty - short term outcome. BMC Musculoskelet Disord. 33902505. Duplicate/No additional data
Kim, Sunghye; Hsu, Fang-Chi; Groban, Leanne; Williamson, Jeff; Messier, Stephen. A pilot study of aquatic prehabilitation in adults with knee osteoarthritis undergoing total knee arthroplasty ,Äì short term outcome. BMC Musculoskeletal Disorders. 33902505.

T: Timing outcome too early

Klement, M. R.; Rondon, A. J.; McEntee, R. M.; Kheir, M.; Austin, M. S.. Web-Based, Self-Directed Physical Therapy After Total Hip Arthroplasty Is Safe and Effective for Most, but Not All, Patients. J Arthroplasty. 30477966.

D: NRCS not adjusted

Kline PW; Melanson EL; Sullivan WJ; Blatchford PJ; Miller MJ; Stevens-Lapsley JE; Christiansen CL. Improving Physical Activity Through Adjunct Telerehabilitation Following Total Knee Arthroplasty: Randomized Controlled Trial Protocol.. Physical therapy. 30329126.

Protocol/study registration

Klugarova J; Klugar M; Gallo J; Mareckova J; Kelnarova Z. The effectiveness of inpatient physical therapy compared to outpatient physical therapy for older adults after total hip replacement in the postdischarge period: a systematic review protocol.. JBI database of systematic reviews and implementation reports. 26455842.

SR or guideline

Klugarova J; Klugar M; Mareckova J; Gallo J; Kelnarova Z. The effectiveness of inpatient physical therapy compared to outpatient physical therapy in older adults after total hip replacement in the postdischarge period: a systematic review.. JBI database of systematic reviews and implementation reports. 26878927.

SR or guideline

Klugarova, Jitka; Klugar, Miloslav; Gallo, Jiri; Mareckova, Jana; Kelnarova, Zuzana. The effectiveness of inpatient physical therapy compared to outpatient physical therapy for older adults after total hip replacement in the post-discharge period: a systematic review. JBI Database of Systematic Reviews \& Implementation Reports. 26878927. SR or guideline

Ko V; Naylor J; Harris I; Crosbie J; Yeo A; Mittal R. One-to-one therapy is not superior to group or homebased therapy after total knee arthroplasty: a randomized, superiority trial.. The Journal of bone and joint surgery. American volume. 24196464. P: Not unilateral replacement (exclude bilateral replacement surgeries) 
Ko, V. W. M.; Naylor, J. M.; Harris, I. A.; Yeo, A. E. T.; Crosbie, J.. Is centre-based rehabilitation superior to home-based rehabilitation after knee replacement? A single-blind, randomised controlled trial. Arthritis and rheumatism. CN-01003367.

P: Not unilateral replacement (exclude bilateral replacement surgeries)

Koksal I; Tahta M; Simsek ME; Dogan M; Bozkurt M. Efficacy of rapid recovery protocol for total knee arthroplasty: a retrospective study.. Acta orthopaedica et traumatologica turcica. 26312464. D: NRCS not adjusted

Kolber, Morey J.; Hanney, William J.; Lamb, Brittney M.; Trukman, Benjamin. Does Physical Therapy Visit Frequency Influence Acute Care Length of Stay Following Knee Arthroplasty?. Topics in Geriatric Rehabilitation. 108032161. Language:.

D: Not primary study (or SR or GL)

Kondo, Y.; Yoshida, Y.; Iioka, T.; Kataoka, H.; Sakamoto, J.; Hirase, T.; Honda, Y.; Nawata, A.; Okita, M.. Short-Term Effects of Isometric Quadriceps Muscle Exercise with Auditory and Visual Feedback on Pain, Physical Function, and Performance after Total Knee Arthroplasty: A Randomized Controlled Trial. J Knee Surg. 33241542 .

T: Timing outcome too early

Koneva, E. S.. [The effectiveness of gait rehabilitation in the patients following endoprosthetic hip replacement by means of the biofeedback-based hardware videoreconstruction of the walking stereotype]. Vopr Kurortol Fizioter Lech Fiz Kult. 26841525.

Full text unavailable

Koneva, E. S.; Lyadov, K. V.; Shapovalenko, T. V.; Zhukova, E. V.; Polushkin, V. G.. [The hardware techniques for the restoration of the gait stereotype in the patients following total hip replacement: the personalized approach]. Vopr Kurortol Fizioter Lech Fiz Kult. 29652043.

$\mathrm{T}$ : Timing outcome too early

Kotani, N.; Morishita, T.; Saita, K.; Kamada, S.; Maeyama, A.; Abe, H.; Yamamoto, T.; Shiota, E.; Inoue, T.. Feasibility of supplemental robot-assisted knee flexion exercise following total knee arthroplasty. J Back Musculoskelet Rehabil. 31561326. $\mathrm{D}: \mathrm{N}<20$ per group

Kramer JF; Speechley M; Bourne R; Rorabeck C; Vaz M. Comparison of clinic- and home-based rehabilitation programs after total knee arthroplasty.. Clinical orthopaedics and related research. 12771834. P: Not unilateral replacement (exclude bilateral replacement surgeries)

Krastanova, M. S.; Vacheva, D.; Mircheva, A.. A comparative analysis between the recovery results of patients with hip joint replacement in the period of early rehabilitation at home (13-45 days after surgery). Journal of IMAB - Annual Proceeding (Scientific Papers). .

P: Not osteoarthritis (exclude cancer, trauma, rheumatoid arthritis)

Krummenauer F; Gunther KP; Witzlebf WC. The incremental cost effectiveness of inpatient versus outpatient rehabilitation after total hip arthroplasty results of a pilot investigation.. European journal of medical research. 18558552.

$\mathrm{D}: \mathrm{N}<20$ per group

Kubo, Y.; Sugiyama, S.; Takachu, R.; Sugiura, T.; Sawada, M.; Kobori, K.; Kobori, M.. Effects of preoperative low-intensity training with slow movement on early quadriceps weakness after total knee arthroplasty in patients with knee osteoarthritis: a retrospective propensity score-matched study. BMC Sports Sci Med Rehabil. 33292439.

T: Timing outcome too early

Kuether, J.; Moore, A.; Kahan, J.; Martucci, J.; Messina, T.; Perreault, R.; Sembler, R.; Tarutis, J.; Zazulak, B.; Rubin, L. E.; O'Connor, M. I..

Telerehabilitation for Total Hip and Knee Arthroplasty Patients: A Pilot Series with High Patient Satisfaction. Hss j. 31624476.

P: Mixed TKA/THA with no clean subgroup analyses

Kuijer, Ppfm; van Haeren, M. M.; Daams, J. G.; Frings-Dresen, M. H. W.. Better return to work and sports after knee arthroplasty rehabilitation?. Occup Med (Lond). 30260436.

SR or guideline

Kumar PJ; McPherson EJ; Dorr LD; Wan Z; Baldwin K. Rehabilitation after total knee arthroplasty: a comparison of 2 rehabilitation techniques.. Clinical orthopaedics and related research. 8895624 .

published before 2005

Kwok IH; Paton B; Haddad FS. Does Pre-Operative Physiotherapy Improve Outcomes in Primary Total Knee Arthroplasty? - A Systematic Review.. The Journal of arthroplasty. 25913232.

SR or guideline

L Snell D; Hipango J; Sinnott KA; Dunn JA; Rothwell A; Hsieh CJ; DeJong G; Hooper G. Rehabilitation after total joint replacement: a scoping 
study.. Disability and rehabilitation. 28330380. SR or guideline

Labraca NS; Castro-Sanchez AM; MataranPenarrocha GA; Arroyo-Morales M; Sanchez-Joya Mdel M; Moreno-Lorenzo C. Benefits of starting rehabilitation within 24 hours of primary total knee arthroplasty: randomized clinical trial.. Clinical rehabilitation. 21382863.

I: Not (p)rehabilitation

Lang CE. Comparison of 6- and 7-day physical therapy coverage on length of stay and discharge outcome for individuals with total hip and knee arthroplasty.. The Journal of orthopaedic and sports physical therapy. 9653686.

published before 2005

Large KE; Page CJ; Brock K; Dowsey MM; Choong PF. Physiotherapy-led arthroplasty review clinic: a preliminary outcomes analysis.. Australian health review : a publication of the Australian Hospital Association. 25297119.

I: Not (p)rehabilitation

Larsen K; Hansen TB; Soballe K. Hip arthroplasty patients benefit from accelerated perioperative care and rehabilitation: a quasi-experimental study of 98 patients.. Acta orthopaedica. 18839368.

I: Not (p)rehabilitation

Larsen K; Hansen TB; Thomsen PB; Christiansen T; Soballe K. Cost-effectiveness of accelerated perioperative care and rehabilitation after total hip and knee arthroplasty.. The Journal of bone and joint surgery. American volume. 19339559.

$\mathrm{T}$ : Timing outcome too early

Larsen K; Hvass KE; Hansen TB; Thomsen PB; Soballe K. Effectiveness of accelerated perioperative care and rehabilitation intervention compared to current intervention after hip and knee arthroplasty. A before-after trial of 247 patients with a 3-month follow-up.. BMC musculoskeletal disorders. 18442380 .

O: No outcomes of interest

Larsen K; Sorensen OG; Hansen TB; Thomsen PB; Soballe K. Accelerated perioperative care and rehabilitation intervention for hip and knee replacement is effective: a randomized clinical trial involving 87 patients with 3 months of follow-up.. Acta orthopaedica. 18478482.

T: Timing outcome too early

Lee-Moloney, E.; Curley, A. E.; Cassells, M.; O'Rahilly, M.; Cassidy, E.. Does hydrotherapy improve functional outcomes post total hip replacement (THR)...Annual Conference of the Irish
Society of Chartered Physiotherapists. Physiotherapy Ireland. 105409099. Language:.

Full text unavailable

Lee, Y. K.; Kim, B. R.. Effects of an early eccentrically based rehabilitation after total knee arth-roplasty. Osteoporosis international. Conference: world congress on osteoporosis, osteoarthritis and musculoskeletal diseases, WCO-IOF-ESCEO 2016. Malaga spain. Conference start: 20160414. Conference end: 20160417. Conference publication: (var.pagings). $\mathrm{CN}-01266845$.

D: $\mathrm{N}<20$ per group

Lenguerrand, E.; Artz, N.; Marques, E.; Sanderson, E.; Lewis, K.; Murray, J.; Parwez, T.; Bertram, W; Beswick, A. D.; Burston, A.; et al.. Effect of GroupBased Outpatient Physical Therapy on Function After Total Knee Replacement: results From a Multicenter Randomized Controlled Trial. Arthritis care \& research. 31033232.

Duplicate/No additional data

Lenssen AF; Crijns YH; Waltje EM; Roox GM; van Steyn MJ; Geesink RJ; van den Brandt PA; de Bie RA. Effectiveness of prolonged use of continuous passive motion (CPM) as an adjunct to physiotherapy following total knee arthroplasty: design of a randomised controlled trial [ISRCTN85759656].. BMC musculoskeletal disorders. 16504087.

I: Not (p)rehabilitation

Leonard H. Live Music Therapy During Rehabilitation After Total Knee Arthroplasty: A Randomized Controlled Trial.. Journal of music therapy. 30770536.

$\mathrm{D}: \mathrm{N}<20$ per group

Li D; Yang Z; Kang P; Xie X. Home-Based Compared with Hospital-Based Rehabilitation Program for Patients Undergoing Total Knee Arthroplasty for Osteoarthritis: A Systematic Review and Meta-analysis of Randomized Controlled Trials.. American journal of physical medicine \& rehabilitation. 27584144.

SR or guideline

Li J; Song Y. Transcutaneous electrical nerve stimulation for postoperative pain control after total knee arthroplasty: A meta-analysis of randomized controlled trials.. Medicine. 28906393.

SR or guideline

Li, L. L.; Gan, Y. Y.; Zhang, L. N.; Wang, Y. B.; Zhang, F.; Qi, J. M.. The effect of post-discharge telephone intervention on rehabilitation following total hip replacement surgery. International journal of nursing sciences. CN-00998976. 
P: Not osteoarthritis (exclude cancer, trauma, rheumatoid arthritis)

Liang MH; Cullen KE; Larson MG; Schwartz JA; Robb-Nicholson C; Fossel AH; Roberge N; Poss R. Effects of reducing physical therapy services on outcomes in total joint arthroplasty.. Medical care. 3695647.

published before 2005

Liao CD; Liou TH; Huang YY; Huang YC. Effects of balance training on functional outcome after total knee replacement in patients with knee osteoarthritis: a randomized controlled trial.. Clinical rehabilitation. 23463772.

T: Timing outcome too early

Liao, C. D.; Chiu, Y. S.; Ku, J. W.; Huang, S. W.; Liou, T. H.. Effects of Elastic Resistance Exercise on Postoperative Outcomes Linked to the ICF Core Sets for Osteoarthritis after Total Knee Replacement in Overweight and Obese Older Women with Sarcopenia Risk: A Randomized Controlled Trial. J Clin Med. 32664548.

Protocol/study registration

Liao, C. D.; Tsauo, J. Y.; Chiu, Y. S.; Ku, J. W.; Huang, S. W.; Liou, T. H.. Effects of Elastic Resistance Exercise After Total Knee Replacement on Muscle Mass and Physical Function in Elderly Women With Osteoarthritis: a Randomized Controlled Trial. American journal of physical medicine \& rehabilitation. CN-02007538. Duplicate/No additional data

Licciardone JC; Stoll ST; Cardarelli KM; Gamber RG; Swift JN Jr; Winn WB. A randomized controlled trial of osteopathic manipulative treatment following knee or hip arthroplasty.. The Journal of the American Osteopathic Association. 15176518. P: Not osteoarthritis (exclude cancer, trauma, rheumatoid arthritis)

Liebs TR; Herzberg W; Ruther W; Russlies M; Hassenpflug J. Quality-Adjusted Life Years Gained by Hip and Knee Replacement Surgery and Its Aftercare.. Archives of physical medicine and rehabilitation. 26792619.

Duplicate/No additional data

Lilikakis AK; Gillespie B; Villar RN. The benefit of modified rehabilitation and minimally invasive techniques in total hip replacement.. Annals of the Royal College of Surgeons of England. 18634739. D: NRCS not adjusted

Lin CW; March L; Crosbie J; Crawford R; Graves S; Naylor J; Harmer A; Jan S; Bennell K; Harris I; Parker D; Moffet H; Fransen M. Maximum recovery after knee replacement--the MARKER study rationale and protocol.. BMC musculoskeletal disorders. 19534770.

Protocol/study registration

Lin YH; Lee SY; Su WR; Kao CC; Tai TW; Chen TB. Effects of nurse-led lower extremity strength training on knee function recovery in patients who underwent total knee replacement.. Journal of clinical nursing. 29603823.

I: Not (p)rehabilitation

Lin, C. W. C.; March, L.; Crosbie, J.; Crawford, R.; Graves, S.; Naylor, J.; Harmer, A.; Jan, S.; Bennell, K.; Harris, I.; Parker, D.; Moffet, H.; Fransen, M.. Maximum recovery after knee replacement the MARKER study rationale and protocol. BMC Musculoskeletal Disorders. .

Duplicate/No additional data

Lin, S. J.; Chen, S. H.; Chai, H. M.; Jan, M. H.; Jiang, C. C.. Effects of proprioceptive neuromuscular facilitation stretching technique on knee joint function in people following unilateral total knee arthroplasty. Physiotherapy (united kingdom).. CN01102774.

Full text unavailable

Lin, Yu-Hua; Lee, Su-Ying; Su, Wei-Ren; Kao, Chia-Chan; Tai, Ta-Wei; Chen, Tai-Been. Effects of nurse, Âêled lower extremity strength training on knee function recovery in patients who underwent total knee replacement. Journal of Clinical Nursing (John Wiley \& Sons, Inc.). 129933148. Language:. Duplicate/No additional data

Liptak MG; Theodoulou A; Kaambwa B; Saunders S; Hinrichs SW; Woodman RJ; Krishnan J. The safety, efficacy and cost-effectiveness of the Maxm Skate, a lower limb rehabilitation device for use following total knee arthroplasty: study protocol for a randomised controlled trial.. Trials. 30630494. Protocol/study registration

Liptak, M. G.; Theodoulou, A.; Hassell, T. D.; Hinrichs, S. W.; Saunders, S.; Quinn, S. J.; Krishnan, J.. A randomised controlled study protocol on the maxm skate; A lower limb rehabilitation device for use following total knee arthroplasty. Orthopaedic journal of sports medicine. Conference: 2016 combined australian knee society and new zealand knee and sport society meeting. Australia. CN01669680.

Protocol/study registration

Liu SC; Hou ZL; Tang QX; Qiao XF; Yang JH; Ji $\mathrm{QH}$. Effect of knee joint function training on joint functional rehabilitation after knee replacement.. 
Medicine. 29995759.

D: NRCS not adjusted

Liu, H.; Cong, H.; Chen, L.; Wu, H.; Yang, X.; Cao, Y.. Efficacy and Safety of Lower Limb Progressive Resistance Exercise for Patients With Total Knee Arthroplasty: A Meta-analysis of Randomized Controlled Trials. Arch Phys Med Rehabil. 32569586.

SR or guideline

Liu, M.; Jia, Y.; Shi, N.. Application value of an integrated treatment model of orthopedic rehabilitation in patients undergoing total knee arthroplasty. International journal of clinical and experimental medicine. $\mathrm{CN}-01651429$.

P: Not unilateral replacement (exclude bilateral replacement surgeries)

Liu, W.; Wu, Y. L.; Cong, R. J.; Fu, P. L.; Li, X. H.; $\mathrm{Wu}, \mathrm{H}$. S.. Controlled active motion and continuous passive motion are beneficial to function rehabilitation after total knee arthroplasty. Journal of clinical rehabilitative tissue engineering research. CN-01017193.

Full text unavailable

Liu, X. Z.; Liu, M. Z.; Hong, F.; Rong, X. J.. Application of cinesiateics in the rehabilitative treatment of knee joint after knee arthroplasty. Chinese Journal of Clinical Rehabilitation. . published before 2005

Liu, Y. J.; Xu, H. P.; Zhao, H.. Self-management approaches following total knee arthroplasty. Journal of clinical rehabilitative tissue engineering research. CN-00894731.

Full text unavailable

Liu, Z.; Huang, D.; Zhuo, D.. Research on early inpatient rehabilitation after cementless total hip arthroplasty. Chinese journal of rehabilitation medicine. CN-00613447.

$\mathrm{D}: \mathrm{N}<20$ per group

Lo, C. W. T.; Brodie, M. A.; Tsang, W. W. N.; Yan, C. H.; Lam, P. L.; Chan, C. M.; Lord, S. R.; Wong, A. Y. L.. Acceptability and feasibility of a community-based strength, balance, and Tai Chi rehabilitation program in improving physical function and balance of patients after total knee arthroplasty: study protocol for a pilot randomized controlled trial. Trials. 33573664.

Protocol/study registration

Lopez-Liria R; Padilla-Gongora D; CatalanMatamoros D; Rocamora-Perez P; Perez-de la Cruz $\mathrm{S}$; Fernandez-Sanchez M. Home-Based versus Hospital-Based Rehabilitation Program after Total
Knee Replacement.. BioMed research international. 25961017.

P: Not unilateral replacement (exclude bilateral replacement surgeries)

Lopez-Liria R; Vega-Ramirez F; Catalan-Matamoros D; Padilla Gongora D; Martinez-Cortes M; MesaRuiz A. [Home care rehabilitation and physiotherapy in knee prosthesis].. Anales del sistema sanitario de Navarra. 22552131.

SR or guideline

Losina E; Collins JE; Deshpande BR; Smith SR; Michl GL; Usiskin IM; Klara KM; Winter AR; Yang HY; Selzer F; Katz JN. Financial Incentives and Health Coaching to Improve Physical Activity Following Total Knee Replacement: A Randomized Controlled Trial.. Arthritis care \& research.

28732147.

I: Not (p)rehabilitation

Losina, E.; Collins, J. E.; Daigle, M. E.; DonnellFink, L. A.; Prokopetz, J. J.; Strnad, D.; Lerner, V.; Rome, B. N.; Ghazinouri, R.; Skoniecki, D. J.; Katz, J. N.; Wright, J.. The AViKA (Adding Value in Knee Arthroplasty) postoperative care navigation trial: rationale and design features. BMC Musculoskelet Disord. 24119081.

Protocol/study registration

Louw A; Diener I; Butler DS; Puentedura EJ. Preoperative education addressing postoperative pain in total joint arthroplasty: review of content and educational delivery methods.. Physiotherapy theory and practice. 23035767.

SR or guideline

Lowe CJ; Davies L; Sackley CM; Barker KL. Effectiveness of land-based physiotherapy exercise following hospital discharge following hip arthroplasty for osteoarthritis: an updated systematic review.. Physiotherapy. 25724323.

SR or guideline

Lysack C; Dama M; Neufeld S;;reassi E. A compliance and satisfaction with home exercise: a comparison of computer-assisted video instruction and routine rehabilitation practice.. Journal of allied health. 16032913.

D: $\mathrm{N}<20$ per group

Madara KC; Marmon A; Aljehani M; HunterGiordano A; Zeni J Jr; Raisis L. PROGRESSIVE REHABILITATION AFTER TOTAL HIP ARTHROPLASTY: A PILOT AND FEASIBILITY STUDY.. International journal of sports physical therapy. 31440408.

$\mathrm{D}: \mathrm{N}<20$ per group 
Mahomed NN; Davis AM; Hawker G; Badley E; Davey JR; Syed KA; Coyte PC; Gandhi R; Wright JG. Inpatient compared with home-based rehabilitation following primary unilateral total hip or knee replacement: a randomized controlled trial.. The Journal of bone and joint surgery. American volume. 18676897.

P: Mixed TKA/THA with no clean subgroup analyses

Maidment ZL; Hordacre BG; Barr CJ. Effect of weekend physiotherapy provision on physiotherapy and hospital length of stay after total knee and total hip replacement.. Australian health review : a publication of the Australian Hospital Association. 24804607.

Duplicate/No additional data

Maidment, Zoe L.; Hordacre, Brenton G.; Barr, Christopher J.. Effect of weekend physiotherapy provision on physiotherapy and hospital length of stay after total knee and total hip replacement. Australian Health Review. 103960171. Language:. D: NRCS not adjusted

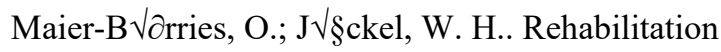
after hip and knee arthroplasty]. Rehabilitation. 23761209.

D: Not primary study (or SR or GL)

Mak JC; Fransen M; Jennings M; March L; Mittal R; Harris IA. Evidence-based review for patients undergoing elective hip and knee replacement.. ANZ journal of surgery. 23496209.

SR or guideline

Makimoto, K.; Fujita, K.; Konno, R.. Experience of patients following lower limb joint replacement: a systematic review protocol. JBI Database System Rev Implement Rep. 30024435.

I: Not (p)rehabilitation

Mallinson TR; Bateman J; Tseng HY; Manheim L; Almagor O; Deutsch A; Heinemann AW. A comparison of discharge functional status after rehabilitation in skilled nursing, home health, and medical rehabilitation settings for patients after lower-extremity joint replacement surgery.. Archives of physical medicine and rehabilitation. 21530718. P: Mixed TKA/THA with no clean subgroup analyses

Maniar RN; Baviskar JV; Singhi T; Rathi SS. To use or not to use continuous passive motion post-total knee arthroplasty presenting functional assessment results in early recovery.. The Journal of arthroplasty. 21752575.

I: Not (p)rehabilitation

Marchisio, A. E.; Ribeiro, T. A.; Umpierres, C. S. A.; GalvلÉo, L.; Rosito, R.; Macedo, C. A. S.; Galia, C.
R.. Accelerated rehabilitation versus conventional rehabilitation in total hip arthroplasty (ARTHA): a randomized double blinded clinical trial. Rev Col Bras Cir. 32844909.

$\mathrm{T}$ : Timing outcome too early

Marcu, I. R.; Patru, S.; Bighea, A.; Popescu, R. S.; Bumbea, A. M.. Effect of hydrotherapy vs. Conventional land-based exercise in patients with hiparthroplasty for osteoarthritis. Osteoporosis international.. $\mathrm{CN}-01058588$.

$\mathrm{D}: \mathrm{N}<20$ per group

Marcu, I. R.; Patru, S.; Matei, D.; Bighea, A. C.. Role of physical exercise in patients with knee arthroplasty for osteoarthritis. Osteoporosis international. CN01409681.

$\mathrm{T}$ : Timing outcome too early

Marusic U; Grospretre S; Paravlic A; Kovac S; Pisot $\mathrm{R}$; Taube W. Motor Imagery during Action Observation of Locomotor Tasks Improves Rehabilitation Outcome in Older Adults after Total Hip Arthroplasty.. Neural plasticity. 29755513. D: $\mathrm{N}<20$ per group

Masaracchio M; Hanney WJ; Liu X; Kolber M; Kirker K. Timing of rehabilitation on length of stay and cost in patients with hip or knee joint arthroplasty: A systematic review with metaanalysis.. PloS one. 28575058.

SR or guideline

Matei, D.; Bighea, A.; Patru, S.; Traistaru, R.; Popescu, R. S.. Effectiveness of rehabilitation program on lower limb functional status after knee arthroplasty. Osteoporosis international.. CN01060283.

$\mathrm{D}: \mathrm{N}<20$ per group

Matheis C; Stoggl T. Strength and mobilization training within the first week following total hip arthroplasty.. Journal of bodywork and movement therapies. 29861260.

D: $\mathrm{N}<20$ per group

Matsuse, H.; Nago, T.; Shinozaki, N.; Hashida, R.; Takano, Y.; Shiba, N.. Combined application of electrical stimulation and volitional contraction prevents muscle weakness. Archives of physical medicine and rehabilitation. CN-01420996.

$\mathrm{T}$ : Timing outcome too early

Mayer J; Bohn J; Gorlich P; Eberspacher H. [Mental gait training -- effectiveness of a therapy method in the rehabilitation after hip-replacement].. Zeitschrift fur Orthopadie und ihre Grenzgebiete. 16118757. Full text unavailable 
McAvoy, R.. Aquatic and land based therapy vs. land therapy on the outcome of total knee arthroplasty: a pilot randomized clinical trial. Journal of Aquatic Physical Therapy. 105364336. Language:.

$\mathrm{D}: \mathrm{N}<20$ per group

McDonald S; Hetrick S; Green S. Pre-operative education for hip or knee replacement.. The Cochrane database of systematic reviews. 14974019. published before 2005

McDonald S; Page MJ; Beringer K; Wasiak J; Sprowson A. Preoperative education for hip or knee replacement.. The Cochrane database of systematic reviews. 24820247.

SR or guideline

McGinn T; Chughtai M; Bhave A; Ali O; Mudaliar P; Khlopas A; Harwin SF; Mont MA. Innovative Multi-Modal Physical Therapy Reduces Incidence of Manipulation Under Anesthesia (MUA) in NonObese Primary Total Knee Arthroplasty.. Surgical technology international. 27608746.

Full text unavailable

McGregor AH; Rylands H; Owen A; Dore CJ; Hughes SP. Does preoperative hip rehabilitation advice improve recovery and patient satisfaction?. The Journal of arthroplasty. 15188105.

$\mathrm{D}: \mathrm{N}<20$ per group

McHugh, G.. The role of prehabilitation on the outcome of total knee arthroplasty: A randomized control trial. University College Dublin Doctoral Dissertation. .

Full text unavailable

McInnes J; Larson MG; Daltroy LH; Brown T; Fossel AH; Eaton HM; Shulman-Kirwan B; Steindorf S; Poss R; Liang MH. A controlled evaluation of continuous passive motion in patients undergoing total knee arthroplasty.. JAMA. 1512910.

published before 2005

McKay C; Prapavessis H; Doherty T. The effect of a prehabilitation exercise program on quadriceps strength for patients undergoing total knee arthroplasty: a randomized controlled pilot study.. PM \& R : the journal of injury, function, and rehabilitation. 22698852.

D: $\mathrm{N}<20$ per group

Medical Advisory Secretariat. Physiotherapy rehabilitation after total knee or hip replacement: an evidence-based analysis.. Ontario health technology assessment series. 23074477.

SR or guideline
Mehta B; Singh JA; Ho K; Parks M; Nelson C; D'Angelo D; Ibrahim SA. Race, Discharge Disposition, and Readmissions After Elective Hip Replacement: Analysis of a Large Regional Dataset.. Health equity. 31872168.

T: Timing outcome too early

Mehta, S. J.; Hume, E.; Troxel, A. B.; Reitz, C.; Norton, L.; Lacko, H.; McDonald, C.; Freeman, J.; Marcus, N.; Volpp, K. G.; Asch, D. A.. Effect of Remote Monitoring on Discharge to Home, Return to Activity, and Rehospitalization After Hip and Knee Arthroplasty: A Randomized Clinical Trial. JAMA Netw Open. 33346847.

I: Not (p)rehabilitation

Menon, N.; Turcotte, J. J.; Stone, A. H.; Adkins, A. L.; MacDonald, J. H.; King, P. J.. Outpatient, HomeBased Physical Therapy Promotes Decreased Length of Stay and Post-Acute Resource Utilization After Total Joint Arthroplasty. J Arthroplasty. 32340828. I: Not (p)rehabilitation

Mikami Y; Orita N; Yamasaki T; Kamijo Y; Kimura H; Adachi N. The Effect of Simultaneous Antigravity Treadmill Training and Electrical Muscle Stimulation After Total Hip Arthroplasty: Short Follow-Up Time.. Annals of rehabilitation medicine. 31499601. D: NRCS not adjusted

Mikashima Y; Takagi T; Tomatsu T; Horikoshi M; Ikari K; Momohara S. Efficacy of acupuncture during post-acute phase of rehabilitation after total knee arthroplasty.. Journal of traditional Chinese medicine $=$ Chung i tsa chih ying wen pan. 23427386 .

$\mathrm{T}$ : Timing outcome too early

Mikkelsen LR; Mikkelsen SS; Christensen FB. Early, intensified home-based exercise after total hip replacement--a pilot study.. Physiotherapy research international : the journal for researchers and clinicians in physical therapy. 22451440.

$\mathrm{T}$ : Timing outcome too early

Mikkelsen LR; Petersen MK; Soballe K; Mikkelsen $\mathrm{S}$; Mechlenburg I. Does reduced movement restrictions and use of assistive devices affect rehabilitation outcome after total hip replacement? A non-randomized, controlled study.. European journal of physical and rehabilitation medicine. 24476806. P: Not unilateral replacement (exclude bilateral replacement surgeries)

Mikkelsen, L. R.; Madsen, M. N.; Rathleff, M. S.; Thorborg, K.; Rossen, C. B.; Kallemose, T.; Bandholm, T.. Pragmatic home-based exercise after total hip arthroplasty-silkeborg: Protocol for a prospective cohort study (PHETHAS-1) [version 1; 
peer review: 3 approved]. F1000Research. . Protocol/study registration

Mikkelsen, L. R.; Mikkelsen, S. S.; Christensen, F. B.. Early, intensified home-based exercise after total hip replacement - A pilot study. Physiotherapy

Research International. .

Duplicate/No additional data

Mikkelsen, L. R.; Mikkelsen, S. S.; Christensen, F. B.. Effect of intensified home-based exercise after Total Hip Replacement - A clinical randomised controlled trial. Osteoarthritis and cartilage. CN01786464.

$\mathrm{T}$ : Timing outcome too early

Mikkelsen, L. R.; Petersen, A. K.; Mechlenburg, I.; Mikkelsen, S.; Soballe, K.; Bandholm, T.. Description of load progression and pain response during progressive resistance training early after total hip arthroplasty: secondary analyses from a randomized controlled trial. Clin Rehabil. 26851251. D: Single group/no comparison

Mikkelsen, L. R.; Petersen, A. K.; Soballe, K.; Mikkelsen, S. S.; Mechlenburg, I.. Asymmetry and pelvic movements 6 months after total hip replacement Secondary analyses from a randomized controlled trial. Acta Orthop Belg. 31677630. $\mathrm{D}: \mathrm{N}<20$ per group

Mikkelsen, Lone R.; Mikkelsen, $\mathrm{S} \sqrt{ }$ Пren S.; Christensen, Finn B.. Early, Intensified Home-based Exercise after Total Hip Replacement - A Pilot Study Early, Intensified Home-based Exercise after Total Hip Replacement - A Pilot Study. Physiotherapy Research International. 22451440.

Duplicate/No additional data

Minns Lowe CJ; Barker KL; Dewey M; Sackley CM. Effectiveness of physiotherapy exercise after knee arthroplasty for osteoarthritis: systematic review and meta-analysis of randomised controlled trials.. BMJ (Clinical research ed.). 17884861.

SR or guideline

Minns Lowe CJ; Barker KL; Dewey ME; Sackley CM. Effectiveness of physiotherapy exercise following hip arthroplasty for osteoarthritis: a systematic review of clinical trials.. BMC musculoskeletal disorders. 19653883.

SR or guideline

Mishra, Neha J.; Ganvir, Suvarna. Meta-Analysis on Physiotherapy Modalities Used in Patients of Total Knee Replacement for Pain Relief. Indian Journal of Physiotherapy \& Occupational Therapy. 132964890. Language:.

SR or guideline
Mitrovic D; Davidovic M; Erceg P; Marinkovic J. The effectiveness of supplementary arm and upper body exercises following total hip arthroplasty for osteoarthritis in the elderly: a randomized controlled trial.. Clinical rehabilitation. 27353247.

T: Timing outcome too early

Mockford BJ; Thompson NW; Humphreys P; Beverland DE. Does a standard outpatient physiotherapy regime improve the range of knee motion after primary total knee arthroplasty?. The Journal of arthroplasty. 18534481.

I: Not (p)rehabilitation

Moe, Rikke Helene; Dagfinrud, Hanne. A neuromuscular exercise program prior to hip or knee arthroplasty does not improve recovery of function three months after surgery. Journal of Physiotherapy (Elsevier). 103768282. Language:.

P: Mixed TKA/THA with no clean subgroup analyses

Moffet H; Collet JP; Shapiro SH; Paradis G; Marquis F; Roy L. Effectiveness of intensive rehabilitation on functional ability and quality of life after first total knee arthroplasty: A single-blind randomized controlled trial.. Archives of physical medicine and rehabilitation. 15083429 .

published before 2005

Moffet, H.; Tousignant, M.; Nadeau, S.; Merette, C.; Boissy, P.; Corriveau, H.; Marquis, F.; Cabana, F.; Ranger, P.; Belzile, E.; et al.. Evaluating the quality of an on-going clinical trial on the effectiveness of telerehabilitation service after knee arthroplasty: a one-year summary. Physiotherapy (united kingdom). CN-01089256.

Full text unavailable

Molla, Razieh Yousefian; Sadeghi, Heydar; Kahlaee, Amir Hossein. The Effect of Early Progressive Resistive Exercise Therapy on Balance Control of Patients With Total Knee Arthroplasty: A Randomized Controlled Trial. Topics in Geriatric Rehabilitation. 126207976. Language:.

P: Not unilateral replacement (exclude bilateral replacement surgeries)

Moller G; Goldie I; Jonsson E. Hospital care versus home care for rehabilitation after hip replacement.. International journal of technology assessment in health care. 1601598 .

published before 2005

Monaghan B; Cunningham P; Harrington P; Hing W; Blake C; O' Dohertya D; Cusack T. Randomised controlled trial to evaluate a physiotherapy-led functional exercise programme after total hip 
replacement.. Physiotherapy. 27126617.

T: Timing outcome too early

Monaghan B; Grant T; Hing W; Cusack T.

Functional exercise after total hip replacement

(FEATHER): a randomised control trial.. BMC musculoskeletal disorders. 23190932.

T: Timing outcome too early

Monaghan, B.; Blake, C.; Hing, W.; Cusack, T.. Functional exercise after total hip replacement (feather). Physiotherapy (united kingdom). CN01126440.

T: Timing outcome too early

Monaghan, B.; Caulfield, B.; O'Math $\sqrt{ }$ na, D. P.. Surface neuromusular electrical stimulation for quadriceps strengthening pre and post total knee replacement: a cochrane review. Physiotherapy (united kingdom). CN-01076246.

SR or guideline

Monaghan, B.; Cunningham, P.; Blake, C.; Hing, W.; Cusack, T.. A pilot RCT examining real-time ultrasound in the measurement of change in posterior gluteus medius thickness post total hip replacement. Physiotherapy (united kingdom).. CN-01163656. I: Not well defined/no (p)rehabilitation content to define

Monaghan, Brenda; Cusack, Tara. Effectiveness of delayed rehabilitation programmes in patients following total hip replacement. Physical Therapy Reviews. 104038990. Language:.

SR or guideline

Montgomery F; Eliasson M. Continuous passive motion compared to active physical therapy after knee arthroplasty: similar hospitalization times in a randomized study of 68 patients.. Acta orthopaedica Scandinavica. 8615108.

published before 2005

Moretti B; Notarnicola A; Moretti L; Setti S; De Terlizzi F; Pesce V; Patella V. I-ONE therapy in patients undergoing total knee arthroplasty: a prospective, randomized and controlled study.. BMC musculoskeletal disorders. 22672794.

$D: N<20$ per group

Morishima Y; Mizushima T; Yamauchi K; Morikawa M; Masuki S; Nose H. Effects of home-based interval walking training on thigh muscle strength and aerobic capacity in female total hip arthroplasty patients: a randomized, controlled pilot study.. PloS one. 25268505.

$\mathrm{D}: \mathrm{N}<20$ per group
Morri M; Vigna D; Raffa D; Donati DM; Benedetti MG. Effect of Game Based Balance Exercises on Rehabilitation After Knee Surgery: A Controlled Observational Study.. Journal of medical systems. 30980213.

P: Not osteoarthritis (exclude cancer, trauma, rheumatoid arthritis)

Moukarzel M; Di Rienzo F; Lahoud JC; Hoyek F; Collet C; Guillot A; Hoyek N. The therapeutic role of motor imagery during the acute phase after total knee arthroplasty: a pilot study.. Disability and rehabilitation. 29275638.

$\mathrm{D}: \mathrm{N}<20$ per group

Moukarzel, M.; Guillot, A.; Di Rienzo, F.; Hoyek, N.. The therapeutic role of motor imagery during the chronic phase after total knee arthroplasty: a pilot randomized controlled trial. Eur J Phys Rehabil Med. 31615192.

D: $\mathrm{N}<20$ per group

Moutzouri M; Gleeson N; Billis E; Panoutsopoulou I; Gliatis J. What is the effect of sensori-motor training on functional outcome and balance performance of patients' undergoing TKR? A systematic review.. Physiotherapy. 26712529.

SR or guideline

Moyer R; Ikert K; Long K; Marsh J. The Value of Preoperative Exercise and Education for Patients Undergoing Total Hip and Knee Arthroplasty: A Systematic Review and Meta-Analysis.. JBJS reviews. 29232265.

SR or guideline

Mueller, E.; Blatt, K.; G` ${ }^{\circ} l i c h, ~ M . ; ~ J \sqrt{ }$ §ckel, W. H.. Treatment schemes and guidelines for rehabilitation after Total Hip Replacement (THR): results of an international survey and a multicenter study in Germany...Proceedings of the 9th Congress of European Federation for Research in Rehabilitation, Budapest, Hungary, 26-29 August 2007. International Journal of Rehabilitation Research. 105841158.

Language:.

Full text unavailable

Muller E; Mittag O; Gulich M; Uhlmann A; Jackel WH. [Systematic literature analysis on therapies applied in rehabilitation of hip and knee arthroplasty: methods, results and challenges].. Die Rehabilitation. 19421937.

SR or guideline

Munajat M; Mohd Nordin NA; Mohamad Yahya NH; Zulkifly AH. Effects of low-intensity pulsed ultrasound on recovery of physical impairments, functional performance and quality of life after total 
knee arthroplasty: Protocol for a quasi-experimental study.. Medicine. 31490397.

$\mathrm{D}: \mathrm{N}<20$ per group

Munin MC; Rudy TE; Glynn NW; Crossett LS; Rubash HE. Early inpatient rehabilitation after elective hip and knee arthroplasty.. JAMA. 9515999. published before 2005

Muto, T.; Kanemura, N.; Takayanagi, K.; Ogawa, R.; Tanikawa, H.; Okuma, K.. Effects of multi-joint kinetics-chain exercise versus conventional exercise for patients with TKA: a randomized controlled trial. a 3-months research. Physiotherapy (united kingdom). CN-01126437.

$\mathrm{D}: \mathrm{N}<20$ per group

Nakamura, M.; Kise, C.; Hasegawa, S.; Misaki, S.. Effectiveness of early high-intensity balance training for early home life independence after total knee arthroplasty: a pseudo-randomized controlled trial. Phys Ther Res. 32850283.

P: Not unilateral replacement (exclude bilateral replacement surgeries)

Nakanowatari; Suzukamo, Y.; Izumi, S.. Specific exercises and heel lift improve functional and patientperceived leg length discrepancies after total hip arthroplasty: a randomized controlled trial.

Physiotherapy (united kingdom). CN-01126435. $\mathrm{D}: \mathrm{N}<20$ per group

Nakanowatari T PhD Pt; Suzukamo Y PhD; Izumi SI $\mathrm{PhD}$ Md. The Effectiveness of Specific Exercise Approach or Modifiable Heel Lift in the Treatment of Functional Leg Length Discrepancy in Early Postsurgery Inpatients after Total Hip Arthroplasty: A Randomized Controlled Trial with a PROBE design.. Physical therapy research. 28289580.

$\mathrm{D}: \mathrm{N}<20$ per group

Nankaku M; Ikeguchi R; Goto K; So K; Kuroda Y; Matsuda S. Hip external rotator exercise contributes to improving physical functions in the early stage after total hip arthroplasty using an anterolateral approach: a randomized controlled trial.. Disability and rehabilitation. 26750398.

Full text unavailable

Naylor JM; Crosbie J; Ko V. Is there a role for rehabilitation streaming following total knee arthroplasty? Preliminary insights from a randomized controlled trial.. Journal of rehabilitation medicine. 25437509.

P: Not unilateral replacement (exclude bilateral replacement surgeries)

Naylor JM; Ko V. Heart rate response and factors affecting exercise performance during home- or class-based rehabilitation for knee replacement recipients: lessons for clinical practice.. Journal of evaluation in clinical practice. 21114797.

$\mathrm{T}$ : Timing outcome too early

Nct. A Multidisciplinary Intervention in Total Knee Arthroplasty.

https://clinicaltrials.gov/show/NCT03771430. CN01701559.

Protocol/study registration

Nct. Evaluation of the Effect of Rehabilitation Sport After Total Hip Arthroplasty (THA). https://clinicaltrials.gov/show/NCT03584451. CN01625631.

Protocol/study registration

Nct. Personalized Prehabilitation. https://clinicaltrials.gov/show/NCT03601728. CN01625845.

Protocol/study registration

Nct. Community-based Rehabilitation and Fall

Prevention Program After Total Knee Arthroplasty. https://clinicaltrials.gov/show/NCT03615638. CN01626031.

Protocol/study registration

Nct. Kinesio $\neg Æ$ Tex Taping for Post Total Knee Replacement Patients.

https://clinicaltrials.gov/show/NCT03681106. CN01648424.

Protocol/study registration

Nct. Using a Smartphone App to Aid in Functional Mobility Return Following Total Knee Arthroplasty. https://clinicaltrials.gov/show/NCT03607461. CN01661612.

Protocol/study registration

Nct. Effects of Motor Imagery Intervention on Functional Recovery Following Total Knee Arthroplasty. https://clinicaltrials.gov/show/NCT03684148. CN01663596.

Protocol/study registration

Nct. The PATH Study to Promote Physical Activity After Knee Replacement. https://clinicaltrials.gov/show/NCT03768206. CN01795325.

Protocol/study registration

Nct. Action Observation in Knee Replacement. https://clinicaltrials.gov/show/NCT02707419. CN01556496.

Protocol/study registration

Nct. Evaluation of an Educational Program

Associated With Exercises (EDEX) Before Total 
Knee Arthroplasty.

https:/clinicaltrials.gov/show/NCT01671917. CN-

01537252.

Protocol/study registration

Nct. Home Rehabilitation in Patients After Primary Total Knee Arthroplasty.

https:/clinicaltrials.gov/show/NCT02409719. CN01505538.

Protocol/study registration

Nct. Total Hip Arthroplasty: fast Track Protocol is the Future?.

https:/clinicaltrials.gov/show/NCT03875976. CN-

01930942.

Protocol/study registration

Nct. Effects of Early Home-based Strength and Sensory-motor Training After THA on Functional Outcome and Patient Satisfaction. https:/clinicaltrials.gov/show/NCT04061993. CN01968039.

Protocol/study registration

Nct. Effectiveness of a Novel Gaming System on Post-operative Rehabilitation Outcomes After Total Knee Arthroplasty: a Pilot Study. https://clinicaltrials.gov/show/NCT04080401. CN01968376.

Protocol/study registration

Nct. Improving Physical Activity and Gait Symmetry After Total Knee Arthroplasty. https:/clinicaltrials.gov/show/NCT04090125. CN01975736.

Protocol/study registration

Nct. The Efficacy of Low-load Blood Flow

Restricted Resistance Before TKR.

https:/clinicaltrials.gov/show/NCT04081493. CN-

01984118 .

Protocol/study registration

Nct. Digital Patient Journey Solution for Patients Undergoing Elective Hip and Knee Arthroplasty Dueto Primary Osteoarthritis. https:/clinicaltrials.gov/show/NCT04083326. CN01984155 .

Protocol/study registration

Nct. The Whole Body Vibration Training for Total Knee arthroplasty-the Improvement of the Lower Limb. https://clinicaltrials.gov/show/NCT04107350. CN-01992324.

Protocol/study registration

Nct. PNF Stretching for TKA on ROM. https:/clinicaltrials.gov/show/NCT00797875. CN-
01485674.

Protocol/study registration

Nct. Effect of Physiotherapy After Total Knee

Replacement.

https://clinicaltrials.gov/show/NCT00807716. CN01485946.

Protocol/study registration

Nct. Walking Skill Training Program Effects in Patients With Total Hip Arthroplasty. https://clinicaltrials.gov/show/NCT00808483. CN01485965.

Protocol/study registration

Nct. Biomechanics of Gait Pattern Adaptation in Patients After Total Knee Arthroplasty. https://clinicaltrials.gov/show/NCT01412814. CN01488024.

Protocol/study registration

Nct. Exercises in the Post-operative Rehabilitation of THA. https://clinicaltrials.gov/show/NCT03208829. CN-01495452.

Protocol/study registration

Nct. Pre-operative Rehabilitation Exercise Program for Total Knee Arthroplasty. https://clinicaltrials.gov/show/NCT00493142. CN01498020.

Protocol/study registration

Nct. Hydrotherapy Versus Physiotherapy for Shortterm Rehabilitation After Primary TKR. https://clinicaltrials.gov/show/NCT00878358. CN01500364.

Protocol/study registration

Nct. Group Exercise After Hip Replacement Surgery. https://clinicaltrials.gov/show/NCT01253798. CN01502183.

Protocol/study registration

Nct. Efficacy of Preoperative Education and Miniinvasive Surgery for Total Hip Replacement. https://clinicaltrials.gov/show/NCT00449228. CN01514680 .

Protocol/study registration

Nct. Ambulation Versus Standing on Postoperative Day 0. https://clinicaltrials.gov/show/NCT02879188. CN-01520418.

Protocol/study registration

Nct. Fit-Joint: getting Fit for Hip or Knee

Replacement.

https://clinicaltrials.gov/show/NCT02885337. CN01520529.

Protocol/study registration 
Nct. Comparative Effectiveness of an Activityspecific Monitoring Device- StepRite. https:/clinicaltrials.gov/show/NCT02900781. CN01520802.

Protocol/study registration

Nct. Home PT vs FORCE PT.

https://clinicaltrials.gov/show/NCT02911389. CN01521014

Protocol/study registration

Nct. Virtual vs. Traditional Physical Therapy

Following Total Knee Replacement. https:/clinicaltrials.gov/show/NCT02914210. CN01521072.

Protocol/study registration

Nct. Improving Rehabilitation Outcomes After Total Hip Arthroplasty.

https://clinicaltrials.gov/show/NCT02920866. CN-

01521244.

Protocol/study registration

Nct. Effects of Dynamic Splinting on Knee Flexion Angle After Total Knee Arthroplasty: a Randomized Controlled Trial.

https://clinicaltrials.gov/show/NCT02928835. CN01521450

Protocol/study registration

Nct. Quadriceps Exercise Before Total Knee

Arthroplasty (The QUADX-1 Trial).

https://clinicaltrials.gov/show/NCT02931058. CN-

01521512

Protocol/study registration

Nct. Accelerated Rehabilitation in Hip Arthroplasty. https://clinicaltrials.gov/show/NCT02933632. CN01521572.

Protocol/study registration

Nct. The Effect of Isokinetic Strengthening Training. https://clinicaltrials.gov/show/NCT02938416. CN-

01521686.

Protocol/study registration

Nct. Effect of Downhill-uphill Walking Exercises on Functional Level and Muscle Strength in Patients With Knee Arthroplasty. https://clinicaltrials.gov/show/NCT03421938. CN01522640.

Protocol/study registration

Nct. Effectiveness of Acupuncture as an Adjunct to Rehabilitation After Knee Arthroplasty. https://clinicaltrials.gov/show/NCT00935155. CN01524727.

Protocol/study registration
Nct. Ergometer Cycling After Replacement of the Hip or Knee Joint.

https://clinicaltrials.gov/show/NCT00951990. CN01525159 .

Protocol/study registration

Nct. Effects of Kneehab 12-week Peri-operative

Total Knee Arthroplasty.

https://clinicaltrials.gov/show/NCT01096524. CN01528838 .

Protocol/study registration

Nct. Rehabilitation After Fast-track Total Knee

Arthroplasty.

https://clinicaltrials.gov/show/NCT01329081. CN01532806.

Protocol/study registration

Nct. Effectiveness of Exercise Programs Following Total Hip and Knee Joint Arthroplasty.

https://clinicaltrials.gov/show/NCT01555307. CN01536193.

Protocol/study registration

Nct. Independent Exercise Compared With Formal Rehabilitation Following Primary Total Knee

Replacement.

https://clinicaltrials.gov/show/NCT01826305. CN01541462.

Protocol/study registration

Nct. Influence of Manual Lymph Drainage During Hospitalization on Swelling, Function and Pain in Patients After Total Knee Replacement.

https://clinicaltrials.gov/show/NCT01657149. CN01536876.

Protocol/study registration

Nct. Targeted Rehabilitation to Improve Outcome After Knee Replacement- A Physiotherapy Study. https://clinicaltrials.gov/show/NCT01849445. CN01542068 .

Protocol/study registration

Nct. M'-Technique, Guided Imagery, or Standard of Care on Anxiety and Pain Pre- \& Post-operatively in Elective Joint Replacement Patients.

https://clinicaltrials.gov/show/NCT01874379. CN01542718.

Protocol/study registration

Nct. Effectiveness of Passive and Active ROM Exercises Following TKA. https://clinicaltrials.gov/show/NCT02062138. CN01543678.

Protocol/study registration

Nct. Neurocognitive Rehabilitation After Hip Replacement. 
https:/clinicaltrials.gov/show/NCT02231567. CN01548466.

Protocol/study registration

Nct. The Effect of a Telerehabilitaion Program on Gait and Balance in Patients After Hip Surgery. https:/clinicaltrials.gov/show/NCT02451085. CN01552278 .

Protocol/study registration

Nct. Microcurrent Stimulation Reduces Post-

Operative Swelling and Healing Time Following

Knee Replacement Surgery.

https:/clinicaltrials.gov/show/NCT02623660. CN-

01554237.

Protocol/study registration

Nct. Overcoming TWEAK Signaling to Restore Muscle and Mobility After Joint Replacement. https://clinicaltrials.gov/show/NCT02628795. CN01554357.

Protocol/study registration

Nct. Rehabilitation of Patients After THR - Based on Patients $\neg ¥$ Selfrated Health.

https://clinicaltrials.gov/show/NCT02644096. CN01554805.

Protocol/study registration

Nct. PT vs no PT Following THA.

https:/clinicaltrials.gov/show/NCT02687945. CN-

01555962 .

Protocol/study registration

Nct. Comparison Between Kinesiotaping and Cold Therapy After Total Knee Arthroplasty.

https://clinicaltrials.gov/show/NCT02747901. CN01557601.

Protocol/study registration

Nct. Measuring Every Day (MED) Study.

https:/clinicaltrials.gov/show/NCT03010605. CN-

01561035 .

Protocol/study registration

Nct. Transcutaneous Electrical Nerve Stimulation After Total Knee Arthroplasty.

https://clinicaltrials.gov/show/NCT03046225. CN01561866.

Protocol/study registration

Nct. Novel Pre-Surgery Exercise-Conditioning in Patients Waiting for Total Knee Arthroplasty (TKA). https:/clinicaltrials.gov/show/NCT03113032. CN01563393

Protocol/study registration

Nct. Effectiveness of Reduced Frequency Physical Therapy in Total Knee Arthroplasty. https://clinicaltrials.gov/show/NCT03302832. CN-
01564642.

Protocol/study registration

Nct. Movement Pattern Biofeedback Training After Total Knee Arthroplasty. https://clinicaltrials.gov/show/NCT03325062. CN01565256.

Protocol/study registration

Nct. interACTION: a Portable Joint Function

Monitoring and Training System for Remote

Rehabilitation Following TKA.

https://clinicaltrials.gov/show/NCT02646761. CN01584606.

Protocol/study registration

Nct. Effects of Specific Balance Training Prior TKR Surgery in the Early Postoperative Outcomes. https:/clinicaltrials.gov/show/NCT02995668. CN01585834.

Protocol/study registration

Nct. Lymphodreinage Integrated With Kinesio Tape in TKA Patients.

https://clinicaltrials.gov/show/NCT03452995. CN01589584.

Protocol/study registration

Nct. Effect of Functional Electrical Stimulation in Gluteus Medius in Rehabilitation After Total Hip Arthroplasty.

https://clinicaltrials.gov/show/NCT02861027. CN01592345.

Protocol/study registration

Nct. Action Observation in Hip Replacement. https://clinicaltrials.gov/show/NCT02861638. CN01592353.

Protocol/study registration

Nct. Maximal Strength Training in Patients

Undergoing Total Hip Arthroplasty.

https://clinicaltrials.gov/show/NCT02498093. CN01593269.

Protocol/study registration

Nct. Laser + Cryo-thermal Therapy Following Total Knee Replacement Surgery.

https://clinicaltrials.gov/show/NCT04183673. CN02010338.

Protocol/study registration

Nct. Physical Therapy Following Total Hip

Arthroplasty.

https://clinicaltrials.gov/show/NCT04199390. CN02011889.

Protocol/study registration

Nct. Electrical Stimulation After Total Knee

Arthroplasty. 
https:/clinicaltrials.gov/show/NCT00224913. CN02013080.

Protocol/study registration

Nct. Evaluation of Functional Rehabilitation in Patients Undergoing Physiotherapy After Total Hip Arthroplasty.

https://clinicaltrials.gov/show/NCT01491048. CN02018837.

Protocol/study registration

Nct. Preoperative Strength Training in Patients With Total Knee Arthroplasty. https:/clinicaltrials.gov/show/NCT01647243. CN02020205.

Protocol/study registration

Nct. The Difference Between Rehabilitation With or Without Strength Training After Total Knee Replacement. https://clinicaltrials.gov/show/NCT01351831. CN02023132.

Protocol/study registration

Nct. Effect of Pre-surgery Neuromuscular Physiotherapy (PT).

https:/clinicaltrials.gov/show/NCT00913575. CN02025169.

Protocol/study registration

Nct. The Feasibility of a Comprehensive Behavioral Intervention in Patient Post TKA.

https:/clinicaltrials.gov/show/NCT01799772. CN02026040.

Protocol/study registration

Nct. Effect of Cycle Ergometer in the Rehabilitation of Elderly Patients With Total Hip Arthroplasty. https:/clinicaltrials.gov/show/NCT01622465. CN02026777.

Protocol/study registration

Nct. Early Neuromuscular Electrical Stimulation For Quadriceps Muscle Activation Deficits Following Total Knee Replacement. https://clinicaltrials.gov/show/NCT00800254. CN02028949.

Protocol/study registration

Nct. The HIHO Study: hospital Inpatient vs Home Rehabilitation After Total Knee Replacement. https://clinicaltrials.gov/show/NCT01583153. CN02031557.

Protocol/study registration

Nct. In-home Versus Hospital Preoperative Training for Patients Undergoing Total Knee Replacement. https:/clinicaltrials.gov/show/NCT03100890. CN-
02044128 .

Protocol/study registration

Nct. Improving Function After Knee Arthroplasty

With Weight-Bearing Biofeedback.

https://clinicaltrials.gov/show/NCT01333189. CN02044690.

Protocol/study registration

Nct. Fast Track Total Hip Arthroplasty vs Standard Care. https:/clinicaltrials.gov/show/NCT04211987. CN-02053511.

Protocol/study registration

Nct. Insole-based Visual Biofeedback for Weightbearing in Total Hip Replacement.

https://clinicaltrials.gov/show/NCT04268082. CN02072613.

Protocol/study registration

Nct. Early Virtual Reality Based Home

Rehabilitation Program After Total Hip Arthroplasty. https://clinicaltrials.gov/show/NCT04221425. CN02079173.

Protocol/study registration

Nct. Backwards Walking Programme Following Hip and Knee Arthroplasty.

https://clinicaltrials.gov/show/NCT04247802. CN02079877.

Protocol/study registration

Nct. Does Rehabilitation After Total Hip and Knee Arthroplasty Work.

https://clinicaltrials.gov/show/NCT03750448. CN01701290.

Protocol/study registration

Nct. A Prospective Multicenter Longitudinal Cohort Study of the Mymobility Platform.

https://clinicaltrials.gov/show/NCT03737149. CN01794847.

Protocol/study registration

Nct. Study to Reduce Sitting in Older Adults Undergoing Hip or Knee Replacements. https://clinicaltrials.gov/show/NCT03740412. CN01918373.

Protocol/study registration

Nct. Postoperative Rehabilitation After Knee

Arthroplasty: anti-Gravity Treadmill.

https://clinicaltrials.gov/show/NCT03904030. CN01931184.

Protocol/study registration

Nct. Interprofessional Preoperative Geriatric Assessment for Older Arthroplasty Patients With Multimorbidity.

https://clinicaltrials.gov/show/NCT04001699. CN- 
01946313.

Protocol/study registration

Nct. PARQVE Prior to Total Knee Replacement. https://clinicaltrials.gov/show/NCT04017858. CN01953275.

Protocol/study registration

Nct. Is There an Alternative to Immediate Home Physical Therapy Following Total Knee Arthroplasty. https://clinicaltrials.gov/show/NCT04060251. CN01983534.

Protocol/study registration

Nct. Impact of Volunteerism in the Acute Setting. https://clinicaltrials.gov/show/NCT04063553. CN01983619 .

Protocol/study registration

Nct. Evaluation of Safety and Efficacy of ReHub in Patients Who Underwent Primary TKA (REHAPT). https://clinicaltrials.gov/show/NCT04155957. CN02001765.

Protocol/study registration

Nct. Usability and Effectiveness of ReHub in Patients After Total Hip Arthroplasty.

https://clinicaltrials.gov/show/NCT04176315. CN02010149.

Protocol/study registration

Nct. Early Rehabilitation After Total Hip

Replacement.

https://clinicaltrials.gov/show/NCT01214954. CN02023625.

Protocol/study registration

Nct. Preoperative Resistance Training in Patients Scheduled for Total Hip Arthroplasty. https://clinicaltrials.gov/show/NCT01164111. CN02029732.

Protocol/study registration

Nct. A Trial to Establish Realistic Patient Expectations of Total Knee Replacement. https://clinicaltrials.gov/show/NCT01732562. CN02032670.

Protocol/study registration

Nct. Comparison of Treatments Following Total Knee Replacement. https:/clinicaltrials.gov/show/NCT02237911. CN02042772.

Protocol/study registration

Nct. A Physical Therapist Administered Physical Activity Intervention After Total Knee Replacement. https://clinicaltrials.gov/show/NCT03228719. CN-
02047013.

Protocol/study registration

Nct. Active Versus Passive Motion Device Following Knee Replacement Surgery. https://clinicaltrials.gov/show/NCT02339831. CN01575712.

Protocol/study registration

Nct. Fascial Manipulation $\neg Æ$ Associated With Standard Care Versus Standard Post-surgical Care for Total Hip Arthroplasty.

https://clinicaltrials.gov/show/NCT02576028. CN02029844.

Protocol/study registration

Nct. Virtual Reality Rehabilitation in Patients With Total Knee Replacement. https://clinicaltrials.gov/show/NCT03454256. CN01483677.

Protocol/study registration

Nct. Effect of Preoperative Exercise in Patients Undergoing Total Knee Arthroplasty. https://clinicaltrials.gov/show/NCT03160534. CN01494334.

Protocol/study registration

Nct. Comparison of Physiotherapy Versus Home Exercise Following Hip Replacement Surgery. https://clinicaltrials.gov/show/NCT00175448. CN01497204.

Protocol/study registration

Nct. The Effect of Perioperative Neuromuscular Training on the Outcome of Total Knee Arthroplasty. https://clinicaltrials.gov/show/NCT00492674. CN01498012.

Protocol/study registration

Nct. Interactive Virtual Telerehabilitation After Total Knee Arthroplasty.

https://clinicaltrials.gov/show/NCT01604174. CN01503624.

Protocol/study registration

Nct. Effects of Virtual Reality Rehabilitation in Patients With Total Knee Arthroplasty. https://clinicaltrials.gov/show/NCT02413996. CN01505655.

Protocol/study registration

Nct. Muscle Function in Elderly Postoperative Patients.

https://clinicaltrials.gov/show/NCT00559780. CN01515743.

Protocol/study registration

Nct. The Impaction of Exercise Training on Bone Mineral Density in Patients After Total Knee 
Arthroplasty.

https://clinicaltrials.gov/show/NCT02928562. CN-

01521444 .

Protocol/study registration

Nct. A Telemedicine Solution for Remote Support of Rehabilitation, for Patients Undergoing, Total Hip Arthroplasty Surgery.

https://clinicaltrials.gov/show/NCT00969020. CN01525617.

Protocol/study registration

Nct. Progressive Rehabilitation Following Total

Knee Arthroplasty.

https://clinicaltrials.gov/show/NCT01537328. CN01535697.

Protocol/study registration

Nct. Controlled Study of Kneehab XP for Patients

Undergoing Total Knee Replacement.

https://clinicaltrials.gov/show/NCT01548040. CN-

01535979.

Protocol/study registration

Nct. Strength and Function Following Total Hip Arthroplasty.

https://clinicaltrials.gov/show/NCT01817010. CN01541194.

Protocol/study registration

Nct. Impact of Prehabilitation in Total Knee

Replacement.

https://clinicaltrials.gov/show/NCT01844934. CN-

01541939.

Protocol/study registration

Nct. Effective Rehabilitation of Patients Operated With Total Knee Arthroplasty.

https://clinicaltrials.gov/show/NCT01877733. CN-

01542816.

Protocol/study registration

Nct. Collaborative-care Intervention to Promote Physical Activity After Total Knee Arthroplasty. https://clinicaltrials.gov/show/NCT02075931. CN01544021.

Protocol/study registration

Nct. Unrestricted Rehabilitation Following Primary THA. https://clinicaltrials.gov/show/NCT02079467. CN-01544106.

Protocol/study registration

Nct. The Effects of End-of-range Grade A+ Mobilization Following Acute Primary TKA. https://clinicaltrials.gov/show/NCT02105857. CN01544814.

Protocol/study registration
Nct. Inpatient Versus Outpatient Rehabilitation After TKA. https://clinicaltrials.gov/show/NCT02120313. CN-01545214.

Protocol/study registration

Nct. Influences of Balance Training With a Dynamometric Platform in Total Knee Arthroplasty. https://clinicaltrials.gov/show/NCT02734225. CN01557239.

Protocol/study registration

Nct. Feasibility of the Hip Instructional

Prehabilitation Program for Enhanced Recovery (HIPPER). https://clinicaltrials.gov/show/NCT02969512. CN01560121.

Protocol/study registration

Nct. Effectiveness of a Psychological Intervention in Patients Undergoing Total Knee Arthroplasty. https://clinicaltrials.gov/show/NCT02988947. CN01560541.

Protocol/study registration

Nct. Digital Biofeedback System Versus

Conventional Home-based Rehabilitation After Total Hip Replacement Hip Replacement. https://clinicaltrials.gov/show/NCT03045549. CN01561849.

Protocol/study registration

Nct. Transcutaneous Electrical Nerve Stimulation After Total Hip Arthroplasty.

https://clinicaltrials.gov/show/NCT03046212. CN01561865.

Protocol/study registration

Nct. Rehabilitation for Total Knee Replacement: a Novel Biofeedback System Versus Conventional Home-based Rehabilitation. https://clinicaltrials.gov/show/NCT03047252. CN01561886.

Protocol/study registration

Nct. The Clinical Observation on Taping After Total Knee Replacement.

https://clinicaltrials.gov/show/NCT03340584. CN01565752 .

Protocol/study registration

Nct. Home Rehabilitation Using Interactive Device Versus Inpatient Rehabilitation in Total Knee Arthroplasty.

https://clinicaltrials.gov/show/NCT03476148. CN01567463.

Protocol/study registration

Nct. Prehabilitation Using Aquatic Exercise. https://clinicaltrials.gov/show/NCT02773745. CN- 
01581797.

Protocol/study registration

Nct. Early Postoperative Compex Rehab NMES Use for Total Knee Arthroplasty Patients.

https://clinicaltrials.gov/show/NCT01844193. CN01585304.

Protocol/study registration

Nct. Physiotherapy and Therapeutic Education After Total Knee Arthroplasty. https://clinicaltrials.gov/show/NCT03198247. CN01588803.

Protocol/study registration

Nct. Efficacy of Preoperative Muscle Training on Postoperative Orthopaedic Surgery Recovery. https://clinicaltrials.gov/show/NCT03483519. CN01589120.

Protocol/study registration

Nct. Evaluation of Gait Rehabilitation Robot of an End-Effector on Neuro-Muscular Pathway in Patients After Knee Arthroplasty. https://clinicaltrials.gov/show/NCT02962453. CN01594018

Protocol/study registration

Nct. Pre-operative One-on-One Physical Therapy Education Improves Postoperative Function and Patient Satisfaction After Total Joint Arthroplasty. https://clinicaltrials.gov/show/NCT02872337. CN01599272.

Protocol/study registration

Nct. Effectiveness of a Community-based Tai Chi Rehabilitation Program for Patients After Total Knee Arthroplasty.

https://clinicaltrials.gov/show/NCT03565380. CN01609883

Protocol/study registration

Nct. Better Before - Better After: prehabilitation Program for Older Patients Awaiting Total Hip Replacement. https://clinicaltrials.gov/show/NCT03602105. CN01661492.

Protocol/study registration

Nct. Virtual Reality for Post Operative Pain Management After Total Knee Arthroplasty. https://clinicaltrials.gov/show/NCT03665233. CN01663133.

Protocol/study registration

Nct. Gamification in Knee Replacement

Rehabilitation.

https://clinicaltrials.gov/show/NCT03717727. CN-
01664397.

Protocol/study registration

Nct. https://clinicaltrials.gov/show/NCT04291833. . CN-02088678.

Protocol/study registration

Negm AM; Kennedy CC; Ioannidis G; GajicVeljanoski O; Lee J; Thabane L; Adachi JD; Marr S; Lau A; Atkinson S; Petruccelli D; DeBeer J; Winemaker M; Avram V; Deheshi B; Williams D; Armstrong D; Lumb B; Panju A; Richardson J; Papaioannou A. Getting fit for hip and knee replacement: a protocol for the Fit-Joints pilot randomized controlled trial of a multi-modal intervention in frail patients with osteoarthritis.. Pilot and feasibility studies. 30038794.

Protocol/study registration

Negus JJ; Cawthorne DP; Chen JS; Scholes CJ; Parker DA; March LM. Patient outcomes using Wiienhanced rehabilitation after total knee replacement the TKR-POWER study.. Contemporary clinical trials. 25460343.

Protocol/study registration

Nelson, M.; Bourke, M.; Crossley, K.; Russell, T.. Telerehabilitation is non-inferior to usual care following total hip replacement $\sqrt{ }_{\varnothing}$, Ç“, Äù a randomized controlled non-inferiority trial. Physiotherapy (united kingdom). CN-02073436. Duplicate/No additional data

Nelson, M.; Bourke, M.; Crossley, K.; Russell, T.. Telerehabilitation is non-inferior to usual care following total hip replacement, Äî a randomized controlled non-inferiority trial. Physiotherapy (United Kingdom). .

Duplicate/No additional data

Nelson, M.; Bourke, M.; Crossley, K.; Russell, T.. Telerehabilitation is non-inferior to usual care following total hip replacement - a randomized controlled non-inferiority trial. Physiotherapy. 32026820.

Duplicate/No additional data

Neoh, E. C.; Tay, M. R. J.; Tan, J.; Lim, Y. T.; Sanchalika, A.; Chong, L. F.; Guoping, K. T.. Shortterm Impact of Peer Volunteers in Providing Inpatient Rehabilitative Exercises for Patients after Total Knee Arthroplasty. Arch Phys Med Rehabil. 33775656.

T: Timing outcome too early

Ni, S. H.; Jiang, W. T.; Guo, L.; Jin, Y. H.; Jiang, T. L.; Zhao, Y.; Zhao, J.. Cryotherapy on postoperative rehabilitation of joint arthroplasty. Knee Surg Sports 
Traumatol Arthrosc. 24928371.

SR or guideline

Nielsen PT; Rechnagel K; Nielsen SE. No effect of continuous passive motion after arthroplasty of the knee.. Acta orthopaedica Scandinavica. 3188866.

I: Not (p)rehabilitation

Nigam AK; Taylor DM; Valeyeva Z. Non-invasive interactive neurostimulation (InterX) reduces acute pain in patients following total knee replacement surgery: a randomised, controlled trial.. Journal of orthopaedic surgery and research. 21864362.

$\mathrm{T}$ : Timing outcome too early

Ntr. Rapid Recovery in patients undergoing total knee arthroplasty, first better than faster!. http://www.who.int/trialsearch/Trial2.aspx?TrialID= NTR3191. CN-01869147.

Protocol/study registration

Ntr. activity coach in TKA following a homebased exercise program.

http://www.who.int/trialsearch/Trial2.aspx?TrialID= NTR5109. CN-01877574.

Protocol/study registration

Ntr. ACTION trial: active again after knee arthroplasty!.

http://www.who.int/trialsearch/Trial2.aspx?TrialID= NTR5251. CN-01880571.

Protocol/study registration

Ntr. Physiotherapeutic Treat-to-target Intervention after Orthopaedic surgery; a cost-effectiveness study. http://www.who.int/trialsearch/Trial2.aspx?TrialID= NTR7129. CN-01896972.

Protocol/study registration

Ntr. Preoperative strength training for patients awaiting total knee arthroplasty.

http://www.who.int/trialsearch/Trial2.aspx?TrialID= NTR2278. CN-01863699.

Protocol/study registration

O'Brien J; Hamilton K; Williams A; Fell J; Mulford J; Cheney M; Wu S; Bird ML. Improving physical activity, pain and function in patients waiting for hip and knee arthroplasty by combining targeted exercise training with behaviour change counselling: study protocol for a randomised controlled trial.. Trials. 30086780 .

Protocol/study registration

Oatis CA; Li W; DiRusso JM; Hoover MJ; Johnston KK; Butz MK; Phillips AL; Nanovic KM; Cummings EC; Rosal MC; Ayers DC; Franklin PD. Variations in Delivery and Exercise Content of Physical Therapy Rehabilitation Following Total Knee Replacement
Surgery: A Cross-Sectional Observation Study.. International journal of physical medicine \& rehabilitation. 26594649.

D: $\mathrm{N}<20$ per group

Oh, H. T.; Hwangbo, G.. The effects of proprioception exercise with and without visual feedback on the pain and balance in patients after total knee arthroplasty. J Phys Ther Sci. 29410581. $\mathrm{D}: \mathrm{N}<20$ per group

Okoro T; Lemmey AB; Maddison P;;rew JG. An appraisal of rehabilitation regimes used for improving functional outcome after total hip replacement surgery.. Sports medicine, arthroscopy, rehabilitation, therapy $\&$ technology : SMARTT. 22313723.

SR or guideline

Okoro T; Whitaker R; Gardner A; Maddison P;;rew

JG; Lemmey A. Does an early home-based progressive resistance training program improve function following total hip replacement? Results of a randomized controlled study.. BMC musculoskeletal disorders. 27097833.

D: $\mathrm{N}<20$ per group

Okoro, T.; Morrison, V.; Maddison, P.; Lemmey, A.

B.;;rew, J. G.. An assessment of the impact of behavioural cognitions on function in patients partaking in a trial of early home-based progressive resistance training after total hip replacement surgery. Disabil Rehabil. 23480673.

D: $\mathrm{N}<20$ per group

Olsson LE; Karlsson J; Berg U; Karrholm J; Hansson E. Person-centred care compared with standardized care for patients undergoing total hip arthroplasty--a quasi-experimental study.. Journal of orthopaedic surgery and research. 25359278.

D: NRCS not adjusted

Onggo JR; Onggo JD; De Steiger R; Hau R. The Efficacy and Safety of Inpatient Rehabilitation Compared With Home Discharge After Hip or Knee Arthroplasty: A Meta-Analysis and Systematic Review.. The Journal of arthroplasty. 31053467. SR or guideline

Oosting E; Jans MP; Dronkers JJ; Naber RH; Dronkers-Landman CM; Appelman-de Vries SM; van Meeteren NL. Preoperative home-based physical therapy versus usual care to improve functional health of frail older adults scheduled for elective total hip arthroplasty: a pilot randomized controlled trial.. Archives of physical medicine and rehabilitation. 22365481.

D: $\mathrm{N}<20$ per group 
Oosting, E.; Hoogeboom, T.; Appelman, S.; Dronkers, J.; Van Meeteren, N.. Feasibility of an intensive therapeutic exercise program for frail elderly prior to total hip arthroplasty: two randomized pilot studies. Physiotherapy (united kingdom).. CN01163650 .

T: Timing outcome too early

Orndahl, C. M.; Perera, R. A.; Riddle, D. L.. Associations Between Physical Therapy Visits and Pain and Physical Function After Knee Arthroplasty: a Cross-Lagged Panel Analysis of People Who Catastrophize About Pain Prior to Surgery. Physical therapy. 32990311.

D: Single group/no comparison

Orndahl, C. M.; Perera, R. A.; Riddle, D. L.. Associations Between Physical Therapy Visits and Pain and Physical Function After Knee Arthroplasty: A Cross-Lagged Panel Analysis of People Who Catastrophize About Pain Prior to Surgery. Phys Ther. 32990311.

Duplicate/No additional data

Oskanian, T. L.; Solopova, I. A.; Grishin, A. A.; Sidorov, V. D.. Rehabilitation of patients after total endoprosthesis replacement of hip joint by the method of functional electrostimulation. Voprosy kurortologii, fizioterapii, i lechebno«ê fizichesko«ê kultury. .

O: No outcomes of interest

Osmond, Michael R.; Kumbhare, Dinesh. SecondOrder Peer Reviews of Clinically Relevant Articles for the Physiatrist: Effect of Inpatient Rehabilitation vs a Monitored Home-Based Program on Mobility in Patients With Total Knee Arthroplasty: The HIHO Randomized Clinical Trial. American Journal of Physical Medicine \& Rehabilitation. 128636542. Language:.

D: Not primary study (or SR or GL)

Ozdemir O; Tosun BU. Effects of Home Exercise Programmes During Home Visits After Hip Replacement: A Systematic Review.. Journal of the College of Physicians and Surgeons--Pakistan : JCPSP. 28292366.

SR or guideline

Pagnotta G; Rich E; Eckardt P; Lavin P; Burriesci R. The Effect of a Rapid Rehabilitation Program on Patients Undergoing Unilateral Total Knee Arthroplasty.. Orthopedic nursing. 28358773. P: Not osteoarthritis (exclude cancer, trauma, rheumatoid arthritis)

Pal S; Chughtai M; Sultan AA; Khlopas A; Sodhi N; George NE; Etcheson JI; Gwam CU; Newman JM;
Samuel LT; Bhave A; DaVanzo JE; Mont MA. Impact of Neuromuscular Electrical Stimulation (NMES) on 90-Day Episode Costs and Post-Acute Care Utilization in Total Knee Replacement Patients with Disuse Atrophy.. Surgical technology international. 29316600.

Full text unavailable

Panda, S.; Bali, S.; Kirubakaran, R.; Hagenberg, A.. Telerehabilitation and total knee arthroplasty: A systematic review and meta-analysis of randomised controlled trials. International Journal of Therapy \& Rehabilitation. 109824127. Language:.

SR or guideline

Papalia, R.; Campi, S.; Vorini, F.; Zampogna, B.; Vasta, S.; Papalia, G.; Fossati, C.; Torre, G.; Denaro, V.. The Role of Physical Activity and Rehabilitation Following Hip and Knee Arthroplasty in the Elderly. J Clin Med. 32397459.

SR or guideline

Paravlic, A. H.; Pisot, R.; Marusic, U.. Specific and general adaptations following motor imagery practice focused on muscle strength in total knee arthroplasty rehabilitation: A randomized controlled trial. PLoS One. 31412056.

D: $\mathrm{N}<20$ per group

Park KH; Song MR. The Effects of Postdischarge Telephone Counseling and Short Message Service on the Knee Function, Activities of Daily Living, and Life Satisfaction of Patients Undergoing Total Knee Replacement.. Orthopedic nursing. 28363197. $\mathrm{D}: \mathrm{N}<20$ per group

Park, T. Y.; Kim, H. J.; Lee, J. H.; Sunwoo, Y. Y.; Do, K. S.; Han, S. N.; Song, Y. K.; Chae, D. S.. Efficacy and safety of acupuncture treatment as an adjunctive therapy after knee replacement: Singlecenter, pragmatic, randomized, assessor blinded, pilot study. Medicine (Baltimore). 33725856.

Protocol/study registration

Pastora-Bernal JM; Martin-Valero R; Baron-Lopez FJ; Estebanez-Perez MJ. Evidence of Benefit of Telerehabitation After Orthopedic Surgery: A Systematic Review.. Journal of medical Internet research. 28455277.

SR or guideline

Pathak S; Ganduglia CM; Awad SS; Chan W; Swint JM; Morgan RO. Is Post-discharge Rehabilitation Timing Associated with 90-Day Readmission in Primary Total Joint Arthroplasty?. HSS journal : the musculoskeletal journal of Hospital for Special Surgery. 31624478.

I: Not (p)rehabilitation 
Paxton RJ; Forster JE; Miller MJ; Gerron KL; Stevens-Lapsley JE; Christiansen CL. A Feasibility Study for Improved Physical Activity After Total Knee Arthroplasty.. Journal of aging and physical activity. 28338406.

$\mathrm{D}: \mathrm{N}<20$ per group

Pearse, E. O.; Caldwell, B. F.; Lockwood, R. J.; Hollard, J.. Early mobilisation after conventional knee replacement may reduce the risk of postoperative venous thromboembolism. Journal of Bone \& Joint Surgery, British Volume. 105997415.

Language:.

I: Not (p)rehabilitation

Peer MA; Rush R; Gallacher PD; Gleeson N. Presurgery exercise and post-operative physical function of people undergoing knee replacement surgery: A systematic review and meta-analysis of randomized controlled trials.. Journal of rehabilitation medicine. 28352936.

SR or guideline

Pereira, L. C.; Jolles-Haeberli, B.. The effects of endof-range grade $\mathrm{A}+$ mobilisation following acute primary TKA. Swiss medical weekly. CN-01049724. P: Not MJR (hip/knee)

Pereira, Luis C.; Jolles, Brigitte M.. The effect of end-of-range grade $\mathrm{A}+$ knee mobilisation following acute primary total knee arthroplasty: A randomised controlled trial. International Journal of Therapy \& Rehabilitation. 111492251. Language:.

$\mathrm{D}: \mathrm{N}<20$ per group

Perez-Porta, I.. Eficacia de las alternativas de rehabilitacion tras el alta hospitalaria en pacientes intervenidos de protesis total de rodilla. Revision sistematica. Rehabilitacion. 121170375. Language:. SR or guideline

Pesce, V.; Notarnicola, A.; Setti, S.; Moretti, L.; Vicenti, G.; Moretti, B.. I-ONE therapy in patients undergoing total knee arthroplasty: results at 12 months. Journal of orthopaedics and traumatology. CN-01004048.

$\mathrm{D}: \mathrm{N}<20$ per group

Peter, Wilfred F.; Tilbury, Claire; Verdegaal, Susan H. M.; Onstenk, Ron; Vehmeijer, Stefan B.;

Vermeulen, Erik M.; van der Linden-van der Zwaag, Enrike M. J.; Nelissen, Rob G. H. H.; Vlieland, Thea P. M. Vliet. The provision of preoperative and postoperative physical therapy in elderly people with hip and knee osteoarthritis undergoing primary joint replacement surgery. Current Orthopaedic Practice. 113756973. Language:.

D: Single group/no comparison
Petersen MK; Madsen C;; ersen NT; Soballe K. Efficacy of multimodal optimization of mobilization and nutrition in patients undergoing hip replacement: a randomized clinical trial.. Acta anaesthesiologica Scandinavica. 16987366.

I: Not (p)rehabilitation

Pethe-Kania K; Opara JA; Kania D; Kidon Z; Lukaszewicz $\mathrm{T}$. The follow-up posturography in rehabilitation after total hip arthroplasty.. Acta of bioengineering and biomechanics. 28552931.

$\mathrm{T}$ : Timing outcome too early

Pfeufer D; Gililland J; Bocker W; Kammerlander C;; erson M; Krahenbuhl N; Pelt C. Training with biofeedback devices improves clinical outcome compared to usual care in patients with unilateral TKA: a systematic review.. Knee surgery, sports traumatology, arthroscopy : official journal of the ESSKA. 30334150.

SR or guideline

Pinsornsak, P.; Kanitnate, S.; Boontanapibul, K.. The effect of immediate post-operative knee range of motion photographs on post-operative range of motion after total knee arthroplasty : An assessorblinded randomized controlled clinical trial in sixty patients. Int Orthop. 33230607.

Duplicate/No additional data

Pinsornsak, P.; Kanitnate, S.; Boontanapibul, K.. The effect of immediate post-operative knee range of motion photographs on post-operative range of motion after total knee arthroplasty: an assessorblinded randomized controlled clinical trial in sixty patients. International orthopaedics. CN-02204921. I: Not (p)rehabilitation

Piqueras, Merc $\sqrt{ }($; Marco, Ester; Coll, Maria; Escalada, Ferran; Baliester, Alex; Cinca, Carme; Belmonte, Roser; Muniesa, Josep M..

EFFECTIVENESS OF AN INTERACTIVE VIRTUAL TELEREHABILITATION SYSTEM IN PATIENTS AFTER TOTAL KNEE ARTHROPLASTY: A RANDOMIZED CONTROLLED TRIAL. Journal of Rehabilitation Medicine (Stiftelsen Rehabiliteringsinformation). 108007373. Language:.

Duplicate/No additional data

Piva SR; Gil AB; Almeida GJ; DiGioia AM 3rd; Levison TJ; Fitzgerald GK. A balance exercise program appears to improve function for patients with total knee arthroplasty: a randomized clinical trial.. Physical therapy. 20378678.

$\mathrm{D}: \mathrm{N}<20$ per group 
Piva, S. R.; Almeida, G.; Gil, A. B.; Teixeira, P. E.; Fitzgerald, G. K.. Effectiveness and feasibility of a balance training program post total knee arthroplasty: pilot randomized trial. Journal of Orthopaedic \& Sports Physical Therapy. 105637167. Language:. Full text unavailable

Piva, S. R.; Farrokhi, S.; Almeida, G.; Fitzgerald, G. K.; Levison, T. J.; DiGioia, A. M.. Dose-Associated Changes in Gait Parameters in Response to Exercise Programs after Total Knee Arthroplasty: Secondary Analysis of Two Randomized Studies. Int J Phys Med Rehabil. 27019858.

D: Not primary study (or SR or GL)

Piva, S. R.; Schneider, M.; Moore-Patterson, C.; Catelani, M. B.; Gil, A.; Klatt, B.; DiGioia, A.; Almeida, G. J.; Khoja, S. S.; Sowa, G.; et al.. Randomized trial on exercise at late-stage after total knee replacement. Arthritis and rheumatology. CN01793073.

Full text unavailable

Pohl T; Brauner T; Wearing S; Stamer K; Horstmann $\mathrm{T}$. Effects of sensorimotor training volume on recovery of sensorimotor function in patients following lower limb arthroplasty.. BMC musculoskeletal disorders. 26286593.

P: Mixed TKA/THA with no clean subgroup analyses

Ponzio DY; Park AG; Bhat SB; Purtill JJ. Can We Reduce the Utilization of Home-Visiting Nurse Services After Primary Total Joint Arthroplasty?. The Journal of arthroplasty. 27113944.

D: NRCS not adjusted

Postel JM; Thoumie P; Missaoui B; Biau D; Ribinik P; Revel M; Rannou F. Continuous passive motion compared with intermittent mobilization after total knee arthroplasty. Elaboration of French clinical practice guidelines.. Annales de readaptation et de medecine physique : revue scientifique de la Societe francaise de reeducation fonctionnelle de readaptation et de medecine physique. 17412445 . I: Not (p)rehabilitation

Pozzi F; Snyder-Mackler L; Zeni J. Physical exercise after knee arthroplasty: a systematic review of controlled trials.. European journal of physical and rehabilitation medicine. 24172642.

SR or guideline

Pozzi F; White DK; Snyder-Mackler L; Zeni JA. Restoring physical function after knee replacement: a cross sectional comparison of progressive strengthening vs standard physical therapy.. Physiotherapy theory and practice. 29877749. D: NRCS not adjusted
Pritchard, K. T.; Baillargeon, J.; Raji, M. A.; Chou, L. N.; Downer, B.; Kuo, Y. F.. Association of Occupational and Physical Therapy With Duration of Prescription Opioid Use After Hip or Knee Arthroplasty: A Retrospective Cohort Study of Medicare Enrollees. Arch Phys Med Rehabil. 33617862.

I: Not well defined/no (p)rehabilitation content to define

Prusynski, R. A.; Gustavson, A. M.; Shrivastav, S. R.; Mroz, T. M.. Rehabilitation Intensity and Patient Outcomes in Skilled Nursing Facilities in the United States: A Systematic Review. Phys Ther. 33388761. SR or guideline

Prvu Bettger J; Green CL; Holmes DN; Chokshi A; Mather RC 3rd; Hoch BT; de Leon AJ; Aluisio F; Seyler TM; Del Gaizo DJ; Chiavetta J; Webb L; Miller V; Smith JM; Peterson ED. Effects of Virtual Exercise Rehabilitation In-Home Therapy Compared with Traditional Care After Total Knee Arthroplasty: VERITAS, a Randomized Controlled Trial.. The Journal of bone and joint surgery. American volume. 31743238.

P: Not osteoarthritis (exclude cancer, trauma, rheumatoid arthritis)

Pua YH; Ong PH. Association of early ambulation with length of stay and costs in total knee arthroplasty: retrospective cohort study.. American journal of physical medicine \& rehabilitation. 24879549.

I: Not (p)rehabilitation

Pua YH; Ong PH; Chong HC; Lo NN. Sunday physiotherapy reduces inpatient stay in knee arthroplasty: a retrospective cohort study.. Archives of physical medicine and rehabilitation. 21621663.

D: NRCS not adjusted

Quack, V.; Ippendorf, A. V.; Betsch, M.; Schenker, H.; Nebelung, S.; Rath, B.; Tingart, M.; L ${ }^{\circ}$ ring, C.. Multidisziplin $\sqrt{\text { \&re Rehabilitation und multimodale }}$ Fast-Track-Rehabilitation in der Knieendoprothetik: Schneller, besser, $\mathrm{g} \sqrt{ }{ }^{\circ}$ nstiger? Eine Umfrage und systematische Literaturrecherche. Rehabilitation. 26317840.

SR or guideline

$\mathrm{R} \sqrt{ } \geq$ denas-Mart $\sqrt{\neq} \neq$ nez, S.; Santos-Andr $\sqrt{ } \mathrm{C} s, \mathrm{~J}$. F.; Abril-Boren, C.; Usabiaga-Bernal, T.; Abouh-Lais, S.; Aguilar-Naranjo, J. J.. Effectiveness of a presurgery rehabilitation program in total knee arthroplasty. Rehabilitacion. 105750491. Language:. Full text unavailable 
Raaben M; Vogely HC; Blokhuis TJ. Real-time visual biofeedback to improve therapy compliance after total hip arthroplasty: A pilot randomized controlled trial.. Gait \& posture. 29413802.

I: Not (p)rehabilitation

Radulovic TN; Lazovic M; Jandric S; Bucma T; Cvjetkovic DD; Manojlovic S. The Effects of Continued Rehabilitation After Primary Knee Replacement.. Medical archives (Sarajevo, Bosnia and Herzegovina). 27147789.

I: Not well defined/no (p)rehabilitation content to define

Rajan RA; Pack Y; Jackson H; Gillies C; Asirvatham R. No need for outpatient physiotherapy following total knee arthroplasty: a randomized trial of 120 patients.. Acta orthopaedica Scandinavica. 15022811. published before 2005

Rakel, B. A.; Zimmerman, M. B.; Geasland, K.; Embree, J.; Clark, C. R.; Noiseux, N. O.; Callaghan, J. J.; Herr, K.; Walsh, D.; Sluka, K. A..

Transcutaneous electrical nerve stimulation for the control of pain during rehabilitation after total knee arthroplasty: A randomized, blinded, placebocontrolled trial. Pain. 25270585.

$\mathrm{T}$ : Timing outcome too early

Ramanathan, D.; Saleh, A.; Klika, A. K.; Higuera, C. A.; Barsoum, W. K.. The Use of Transcutaneous Electrical Nerve Stimulation After Total Knee Arthroplasty: A Prospective Randomized Controlled Trial. Surg Technol Int. 28537354.

Full text unavailable

Ramos de Souza Matos, Lilian Ramine; dos Santos, Renata Maria Eloi; Barros Medrado, Beatriz; Alencar Lessa, Eric; Mac $\sqrt{\mathrm{TM}}^{\mathrm{M}}$ do da Silva Bezerra, Naiara $\mathrm{K}{ }^{\circ}$ ssia; Menezes Silva Bonfim, Ester. Fisioterapia no $\mathrm{p} \sqrt{ } \geq \mathrm{s}$-operat $\sqrt{ } \geq$ rio de artroplastia de quadril: uma

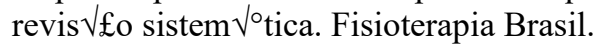
147990033. Language:.

SR or guideline

Rampazo-Lacativa MK; D'Elboux MJ. Effect of cycle ergometer and conventional exercises on rehabilitation of older patients with total hip arthroplasty: study protocol for randomized controlled trial.. Trials. 25873151.

Protocol/study registration

Rannou F; Coudeyre E; Ribinik P; Mace Y; Poiraudeau S; Revel M. Establishing recommendations for physical medicine and rehabilitation: the SOFMER methodology.. Annales de readaptation et de medecine physique : revue scientifique de la Societe francaise de reeducation fonctionnelle de readaptation et de medecine physique. 17306408.

SR or guideline

Raphael M; Jaeger M; van Vlymen J. Easily adoptable total joint arthroplasty program allows discharge home in two days.. Canadian journal of anaesthesia $=$ Journal canadien d'anesthesie. 21822756.

I: Not (p)rehabilitation

Reh J: Hwang T-H; Schmitz G; Effenberg AO. Dual mode gait sonification for rehabilitation after unilateral hip arthroplasty. Brain sciences. CN02081171.

D: $\mathrm{N}<20$ per group

Reilly KA; Beard DJ; Barker KL; Dodd CA; Price AJ; Murray DW. Efficacy of an accelerated recovery protocol for Oxford unicompartmental knee arthroplasty--a randomised controlled trial.. The Knee. 15994082.

P: Not total relacement (exclude partial)

Renkawitz T; Rieder T; Handel M; Koller M; Drescher J; Bonnlaender G; Grifka J. Comparison of two accelerated clinical pathways--after total knee replacement how fast can we really go?. Clinical rehabilitation. 20156984.

I: Not (p)rehabilitation

Rezzan, G.; Marinelli, G.; Marcato, F.; Melegati, G.; Tettamanti, A.; Gatti, R.. Efficacy of action observation pre-operative training in functional recovery after hip and knee prosthesis. Physiotherapy (united kingdom).. CN-01163624.

D: $\mathrm{N}<20$ per group

Rhon, D. I.; Snodgrass, S. J.; Cleland, J. A.; Greenlee, T. A.; Sissel, C. D.; Cook, C. E.. Comparison of Downstream Health Care Utilization, Costs, and Long-Term Opioid Use: Physical Therapist Management Versus Opioid Therapy Management After Arthroscopic Hip Surgery. Phys Ther. 29669080.

P: Not osteoarthritis (exclude cancer, trauma, rheumatoid arthritis)

Riddle DL; Keefe FJ; Ang D; J K; Dumenci L; Jensen MP; Bair MJ; Reed SD; Kroenke K. A phase III randomized three-arm trial of physical therapist delivered pain coping skills training for patients with total knee arthroplasty: the KASTPain protocol.. BMC musculoskeletal disorders. 22906061. Protocol/study registration 
Riggs RV; Roberts PS; Aronow H; Younan T. Joint replacement and hip fracture readmission rates: impact of discharge destination.. PM \& R : the journal of injury, function, and rehabilitation. 20869678.

I: Not (p)rehabilitation

Rindflesch A; Hake M; Haack R; Kleven A; McPartlin M; Strikwerda J; Hollman J. Evaluating Clinical Performance of Student Physical Therapists: A Comparison of Student- and Staff-Managed Patient Outcomes After Hip Arthroplasty.. Journal of allied health. 30194827.

T: Timing outcome too early

Rivard A; Warren S; Voaklander D; Jones A. The efficacy of pre-operative home visits for total hip replacement clients.. Canadian journal of occupational therapy. Revue canadienne d'ergotherapie. 14619762.

published before 2005

Robbins CE; Casey D; Bono JV; Murphy SB; Talmo CT; Ward DM. A multidisciplinary total hip arthroplasty protocol with accelerated postoperative rehabilitation: does the patient benefit?. American journal of orthopedics (Belle Mead, N.J.). 24730003. P: Not osteoarthritis (exclude cancer, trauma, rheumatoid arthritis)

Roberts JL; Pritchard AW; Williams M; Totton N; Morrison V; Din NU; Williams NH. Mixed methods process evaluation of an enhanced community-based rehabilitation intervention for elderly patients with hip fracture.. BMJ open. 30093516.

P: Not osteoarthritis (exclude cancer, trauma, rheumatoid arthritis)

Rodgers JA; Garvin KL; Walker CW; Morford D; Urban J; Bedard J. Preoperative physical therapy in primary total knee arthroplasty.. The Journal of arthroplasty. 9645522. published before 2005

Roig-Casasus S; Blasco JM; Lopez-Bueno L; BlascoIgual MC. Balance Training With a Dynamometric Platform Following Total Knee Replacement: A Randomized Controlled Trial.. Journal of geriatric physical therapy (2001). 28252471.

$\mathrm{D}: \mathrm{N}<20$ per group

Room, J.; Batting, M.; Barker, K. L.. Development of a functional rehabilitation intervention for post knee arthroplasty patients: COmmunity based Rehabilitation post Knee Arthroplasty (CORKA) trial. Physiotherapy. 32026846.

O: No outcomes of interest
Room, J.; Newman, M.; Batting, M.; Kenealy, N.; Barker, K.. Development of a functional rehabilitation intervention for post knee arthroplasty patients: COmmunity based Rehabilitation post Knee Arthroplasty (CORKA) trial...The Chartered Society of Physiotherapy UK Conference 2018, Birmingham, UK, 19-20 October 2018. Physiotherapy. 134150142. Language:.

Duplicate/No additional data

Rosal MC; Ayers D; Li W; Oatis C; Borg A; Zheng $\mathrm{H}$; Franklin P. A randomized clinical trial of a perioperative behavioral intervention to improve physical activity adherence and functional outcomes following total knee replacement.. BMC musculoskeletal disorders. 21981909.

Protocol/study registration

Russell TG; Buttrum P; Wootton R; Jull GA. Lowbandwidth telerehabilitation for patients who have undergone total knee replacement: preliminary results.. Journal of telemedicine and telecare. 14728759. published before 2005

Russell TG; Buttrum P; Wootton R; Jull GA. Internet-based outpatient telerehabilitation for patients following total knee arthroplasty: a randomized controlled trial.. The Journal of bone and joint surgery. American volume. 21248209.

$\mathrm{T}$ : Timing outcome too early

Russo LR; Benedetti MG; Mariani E; Roberti di Sarsina T; Zaffagnini S. The Videoinsight((R)) Method: improving early results following total knee arthroplasty.. Knee surgery, sports traumatology, arthroscopy : official journal of the ESSKA. 27161196.

I: Not well defined/no (p)rehabilitation content to define

Russo, Luciana; Benedetti, Maria; Mariani, Elisabetta; Roberti di Sarsina, Tommaso; Zaffagnini, Stefano; Russo, Luciana Rebecca; Benedetti, Maria Grazia. The Videoinsight $\neg Æ$ Method: improving early results following total knee arthroplasty. Knee Surgery, Sports Traumatology, Arthroscopy.

27161196.

Duplicate/No additional data

Ryan, T. G.; Ohlson, B.; Adams, R.. Post operative total knee replacement rehabilitation: a new method using music video. The journal of bone and joint surgery (proceedings). CN-00695969.

P: Not unilateral replacement (exclude bilateral replacement surgeries) 
Saleh, Khaled J.; Novicoff, Wendy M.. Starting rehabilitation within 24 hours after total knee arthroplasty was better than delaying to within 48 to 72 hours. Journal of Bone \& Joint Surgery, American Volume. 115365483. Language:.

D: Not primary study (or SR or GL)

Sano Y; Iwata A; Wanaka H; Matsui M; Yamamoto $\mathrm{S}$; Koyanagi J; Iwata H. An easy and safe training method for trunk function improves mobility in total knee arthroplasty patients: A quasi-randomized controlled trial.. PloS one. 30286130.

$\mathrm{T}$ : Timing outcome too early

Sarpong NO; Lakra A; Jennings E; Cooper HJ; Shah RP; Geller JA. Same-Day Physical Therapy

Following Total Knee Arthroplasty Leads to Improved Inpatient Physical Therapy Performance and Decreased Inpatient Opioid Consumption.. The Journal of arthroplasty. 31427131.

$\mathrm{T}$ : Timing outcome too early

Sattler LN; Hing WA; Vertullo CJ. What is the evidence to support early supervised exercise therapy after primary total knee replacement? A systematic review and meta-analysis.. BMC musculoskeletal disorders. 30696416.

SR or guideline

Saueressig, T.; Owen, P. J.; Zebisch, J.; Herbst, M.; Belavy, D. L.. Evaluation of Exercise Interventions and Outcomes After Hip Arthroplasty: A Systematic Review and Meta-analysis. JAMA Netw Open. 33635329.

SR or guideline

Saunders R; Seaman K; Ashford C; Sullivan T; McDowall J; Whitehead L; Ewens B; Pedler K; Gullick K. An eHealth Program for Patients Undergoing a Total Hip Arthroplasty: Protocol for a Randomized Controlled Trial.. JMIR research protocols. 29895515.

Protocol/study registration

Schega, L.; Bertram, D.; Folsch, C.; Hamacher, D.. The influence of visual feedback on the mental representation of gait in patients with THR: a new approach for an experimental rehabilitation strategy. Appl Psychophysiol Biofeedback. 24442243. D: $\mathrm{N}<20$ per group

Seeber GH; Wijnen A; Lazovic D; Bulstra SK; Dietz $\mathrm{G}$; van Lingen CP; Stevens M. Effectiveness of rehabilitation after a total hip arthroplasty: a protocol for an observational study for the comparison of usual care in the Netherlands versus Germany.. BMJ open. 28801413.

D: Not primary study (or SR or GL)
Shabbir, M.; Umar, B.; Ehsan, S.; Munir, S.; Bunin, U.; Sarfraz, K.. Comparison of functional training and strength training in improving knee extension lag after first four weeks of total knee replacement. Biomedical research (india). CN-01395172.

$\mathrm{T}$ : Timing outcome too early

Shah CK; Keswani A; Chi D; Sher A; Koenig KM; Moucha CS. Nonelective Primary Total Hip Arthroplasty: The Effect of Discharge Destination on Postdischarge Outcomes.. The Journal of arthroplasty. 28455179.

P: Not osteoarthritis (exclude cancer, trauma, rheumatoid arthritis)

Sharareh B; Le NB; Hoang MT; Schwarzkopf R. Factors determining discharge destination for patients undergoing total joint arthroplasty.. The Journal of arthroplasty. 24631127.

P: Mixed TKA/THA with no clean subgroup analyses

Sharma R; Ardebili MA; Abdulla IN. Does Rehabilitation before Total Knee Arthroplasty Benefit Postoperative Recovery? A Systematic Review.. Indian journal of orthopaedics. 30905994. SR or guideline

Sharma V; Morgan PM; Cheng EY. Factors influencing early rehabilitation after THA: a systematic review.. Clinical orthopaedics and related research. 19277807.

SR or guideline

Shi J; Wang S; Wei Y; Wu J; Chen F; Huang G; Chen J; Wei L; Jiang J; Xia J. Lower limb amputation and rehabilitation in total joint arthroplasties in the ipsilateral limb.. Prosthetics and orthotics international. 23924777.

SR or guideline

Shin HR; Park K; Seo J; An SH; Yeom SR; Kwon YD. Acupuncture for perioperative care of total hip arthroplasty: A systemic review of controlled trials and meta-analysis.. Medicine. 30985714.

SR or guideline

Shoemaker, M. J.; Gibson, C.; Saagman, S.. Preoperative exercise in individuals undergoing total knee arthroplasty: State of the evidence. Topics in Geriatric Rehabilitation. .

SR or guideline

Shoemaker, Michael J.; Gibson, Cortney; Saagman, Sarah. Preoperative Exercise in Individuals Undergoing Total Knee Arthroplasty. Topics in Geriatric Rehabilitation. 108032163. Language:. SR or guideline 
Shukla H; Nair SR; Thakker D. Role of telerehabilitation in patients following total knee arthroplasty: Evidence from a systematic literature review and meta-analysis.. Journal of telemedicine and telecare. 26843466.

SR or guideline

Siggeirsdottir K; Olafsson O; Jonsson H; Iwarsson S; Gudnason V; Jonsson BY. Short hospital stay augmented with education and home-based rehabilitation improves function and quality of life after hip replacement: randomized study of 50 patients with 6 months of follow-up.. Acta orthopaedica. 16195074.

Published before 2005

Sigurdsson E; Siggeirsdottir K; Jonsson H Jr; Gudnason V; Matthiasson T; Jonsson BY. Early discharge and home intervention reduces unit costs after total hip replacement: results of a cost analysis in a randomized study.. International journal of health care finance and economics. 18566886.

P: Not osteoarthritis (exclude cancer, trauma, rheumatoid arthritis)

Silkman Baker C; McKeon JM. Does preoperative rehabilitation improve patient-based outcomes in persons who have undergone total knee arthroplasty? A systematic review.. PM \& R : the journal of injury, function, and rehabilitation. 23093216.

SR or guideline

Silkman, Carrie; McKeon, Jennifer. Effects Of Preoperative Rehabilitation In Individuals Who Have Undergone Total Knee Arthroplasty: A Systematic Review. Medicine \& Science in Sports \& Exercise. 114660209. Language:.

SR or guideline

Simmons, Lucy; Smith, Toby. Effectiveness of preoperative physiotherapy-based programmes on outcomes following total knee arthroplasty: a systematic review and meta-analysis. Physical Therapy Reviews. 104302997. Language:.

SR or guideline

Simpson AHRW; Bell-Higgs A; Conaghan PG; Craig P; Hamilton DF; Hankey C; Keerie C; Kingsbury SR; Kinsella E; Leeds AR; Norrie J; Pandit HG; Ross HM; Simpson SA; Tuck C. Osteoarthritis Preoperative Package for care of Orthotics, Rehabilitation, Topical and oral agent Usage and Nutrition to Improve ouTcomes at a Year (OPPORTUNITY); a feasibility study protocol for a randomised controlled trial.. Trials. 32075663.

Protocol/study registration
Sindhu, Bijender; Sharma, Manoj; Biraynia, Raj K.. Comparison of Supervised Rehabilitation vs. Home Based Unsupervised Rehabilitation Programs after Total Knee Arthroplasty: A Pilot Study. Indian Journal of Physiotherapy \& Occupational Therapy. 103805069. Language:.

D: $\mathrm{N}<20$ per group

Sisak K; Darch R; Burgess LC; Middleton RG; Wainwright TW. A preoperative education class reduces length of stay for total knee replacement patients identified at risk of an extended length of stay.. Journal of rehabilitation medicine. 31512732. D: NRCS not adjusted

Skoffer B; Dalgas U; Mechlenburg I. Progressive resistance training before and after total hip and knee arthroplasty: a systematic review.. Clinical rehabilitation. 24994766.

SR or guideline

Skoffer B; Maribo T; Mechlenburg I; Korsgaard CG; Soballe K; Dalgas U. Efficacy of preoperative progressive resistance training in patients undergoing total knee arthroplasty: 12-month follow-up data from a randomized controlled trial.. Clinical rehabilitation. 31663369 .

D: Single group/no comparison

Skoffer, B.; Dalgas, U.; Maribo, T.; Soballe, K.; Mechlenburg, I.. No Exacerbation of Knee Joint Pain and Effusion Following Preoperative Progressive Resistance Training in Patients Scheduled for Total Knee Arthroplasty: Secondary Analyses From a Randomized Controlled Trial. Pm r. 29129608. D: Single group/no comparison

Skoffer, B.; Maribo, T.; Mechlenburg, I.; Korsgaard, C. G.; Soballe, K.; Dalgas, U.. Efficacy of preoperative progressive resistance training in patients undergoing total knee arthroplasty: 12-month follow-up data from a randomized controlled trial. Clinical rehabilitation. CN-02008879. Duplicate/No additional data

Skou, S. T. Cost-effectiveness of total knee replacement in addition to non-surgical treatment: A 2-year outcome from a randomised trial in secondary care in Denmark. BMJ Open. 31948990.

D: Single group/no comparison

Smith KC; Paltiel AD; Yang HY; Collins JE; Katz JN; Losina E. Cost-effectiveness of health coaching and financial incentives to promote physical activity after total knee replacement.. Osteoarthritis and cartilage. 30092263.

I: Not (p)rehabilitation 
Smith, T. O.; Parsons, S.; Fordham, B.; Ooms, A.; Dutton, S.; Hing, C.; Barber, V. S.; Png, M. E.; Lamb, S.. Behaviour change physiotherapy intervention to increase physical activity following hip and knee replacement (PEP-TALK): study protocol for a pragmatic randomised controlled trial. BMJ Open. 32690503.

Protocol/study registration

Smith, W. A.; Zucker-Levin, A.; Mihalko, W. M.; Williams, M.; Loftin, M.; Gurney, J. G.. A

Randomized Study of Exercise and Fitness Trackers in Obese Patients After Total Knee Arthroplasty. Orthop Clin North Am. 30477705.

D: Single group/no comparison

Snow R; Granata J; Ruhil AV; Vogel K; McShane M; Wasielewski R. Associations between preoperative physical therapy and post-acute care utilization patterns and cost in total joint replacement.. The Journal of bone and joint surgery. American volume. 25274793.

D: NRCS not adjusted

Soni, A.; Mudge, N.; Joshi, A.; Wyatt, M.; Williamson, L.. Severe knee osteoarthritis: a study of combined acupuncture and physiotherapy vs home exercise advice in patients awaiting total knee arthroplasty. Rheumatology. CN-01775946. Full text unavailable

Spalevic, M.; Lazovic, M.; Kocic, M.; Dimitrijevic, L.; Stankovic, I.; Savic, D.. The effects of preoperative physical therapy in total hip replacement surgery...Proceedings of the 10th Congress of the European Federation for Research in Rehabilitation, Riga, Latvia, 09-12 September 2009. International Journal of Rehabilitation Research. 105445003. Language:.

D: $\mathrm{N}<20$ per group

Spangehl, Mark J.. Is It Time for Telerehabilitation to Go Mainstream? Commentary on an article by $\mathrm{H} \sqrt{ }(\mathrm{I} / \sqrt{ } \circledast$ ne Moffet, PT, PhD, et al.: 'In-Home Telerehabilitation Compared with Face-to-Face Rehabilitation After Total Knee Arthroplasty. A Noninferiority Randomized Controlled Trial'. Journal of Bone \& Joint Surgery, American Volume. 109598475. Language:.

D: Not primary study (or SR or GL)

Spiegl, Claudia; Strutzenberger, Gerda; Schwameder, Hermann. EMG-Biofeedbacktraining bei Patienten mit Knieendoprothese. Zeitschrift fur Physiotherapeuten. 128635346. Language:. Full text unavailable
Stambough JB; Nunley RM; Curry MC; Steger-May $\mathrm{K}$; Clohisy JC. Rapid recovery protocols for primary total hip arthroplasty can safely reduce length of stay without increasing readmissions.. The Journal of arthroplasty. 25683296.

I: Not (p)rehabilitation

Stauber, Anja; Sch $\sqrt{ }{ }^{\circ} \sqrt{ }$ üler, Nadine; Palmdorf, Sarah; Sch $\sqrt{ }{ }^{\circ}$ rholz, Nina; Bruns, David; Osterbrink, J $\sqrt{ }{ }^{\circ}$ rgen; Nestler, Nadja. RECOVER-E - a mobile app for patients undergoing total knee or hip replacement: study protocol. BMC Musculoskeletal Disorders. 141544166. Language:.

Protocol/study registration

Stein, G.; Knoell, P.; Faymonville, C.; Kaulhausen, T.; Siewe, J.; Otto, C.; Eysel, P.; Zarghooni, K.. Whole body vibration compared to conventional physiotherapy in patients with gonarthrosis: a protocol for a randomized, controlled study. BMC Musculoskelet Disord. 20565956.

P: Not MJR (hip/knee)

Steinhilber B; Haupt G; Miller R; Boeer J; Grau S; Janssen P; Krauss I. Feasibility and efficacy of an 8week progressive home-based strengthening exercise program in patients with osteoarthritis of the hip and/or total hip joint replacement: a preliminary trial.. Clinical rheumatology. 22086491.

P: Not total relacement (exclude partial)

Stevens-Lapsley JE; Balter JE; Wolfe P; Eckhoff DG; Schwartz RS; Schenkman M; Kohrt WM. Relationship between intensity of quadriceps muscle neuromuscular electrical stimulation and strength recovery after total knee arthroplasty.. Physical therapy. 22652985.

$\mathrm{D}: \mathrm{N}<20$ per group

Stevens-Lapsley, Jennifer E.; Balter, Jaclyn E.; Wolfe, Pamela; Eckhoff, Donald G.; Schwartz, Robert S.; Schenkman, Margaret; Kohrt, Wendy M.. Relationship Between Intensity of Quadriceps Muscle Neuromuscular Electrical Stimulation and Strength Recovery After Total Knee

Arthroplasty...[corrected] [published erratum appears in PHYS THER 2012; 92(10):1362]. Physical

Therapy. 108096636. Language:.

$\mathrm{D}: \mathrm{N}<20$ per group

Stockton KA; Mengersen KA. Effect of multiple physiotherapy sessions on functional outcomes in the initial postoperative period after primary total hip replacement: a randomized controlled trial.. Archives of physical medicine and rehabilitation. 19801052. T: Timing outcome too early 
Stockton, K.; Rand, K.; Mengersen, K.. Fast tracking patients undergoing primary total hip replacement From research to clinical practice. Internal medicine journal. CN-01777574.

T: Timing outcome too early

Strom H; Huss K; Larsson S. Unrestricted weight bearing and intensive physiotherapy after uncemented total hip arthroplasty.. Scandinavian journal of surgery : SJS : official organ for the Finnish Surgical Society and the Scandinavian Surgical Society. 16579257.

D: $\mathrm{N}<20$ per group

Strom H; Nilsson O; Milbrink J; Mallmin H; Larsson S. Early migration pattern of the uncemented CLS stem in total hip arthroplasties.. Clinical orthopaedics and related research. 16936584 .

$\mathrm{D}: \mathrm{N}<20$ per group

Strom H; Nilsson O; Milbrink J; Mallmin H; Larsson $\mathrm{S}$. The effect of early weight bearing on migration pattern of the uncemented CLS stem in total hip arthroplasty.. The Journal of arthroplasty. 18078880. O: No outcomes of interest

Suetta C; Magnusson SP; Rosted A; Aagaard P; Jakobsen AK; Larsen LH; Duus B; Kjaer M. Resistance training in the early postoperative phase reduces hospitalization and leads to muscle hypertrophy in elderly hip surgery patients--a controlled, randomized study.. Journal of the American Geriatrics Society. 15571536. published before 2005

Suetta, C.;;ersen, J. L.; Dalgas, U.; Berget, J.; Koskinen, S.; Aagaard, P.; Magnusson, S. P.; Kjaer, M.. Resistance training induces qualitative changes in muscle morphology, muscle architecture, and muscle function in elderly postoperative patients. J Appl Physiol (1985). 18420714.

$\mathrm{D}: \mathrm{N}<20$ per group

Suh MJ; Kim BR; Kim SR; Han EY; Lee SY. Effects of Early Combined Eccentric-Concentric Versus Concentric Resistance Training Following Total Knee Arthroplasty.. Annals of rehabilitation medicine. 29201821.

P: Not unilateral replacement (exclude bilateral replacement surgeries)

Suksathien R; Suksathien Y. The effect of navigator on length of stay and rehabilitation for total hip arthroplasty patients.. Journal of the Medical Association of Thailand = Chotmaihet thangphaet. 24511719.

published before 2005
Svinoy OE; Bergland A; Risberg MA; Pripp AH; Hilde G. Better before-better after: efficacy of prehabilitation for older patients with osteoarthritis awaiting total hip replacement-a study protocol for a randomised controlled trial in South-Eastern

Norway.. BMJ open. 31892650.

Protocol/study registration

Swank AM; Kachelman JB; Bibeau W; Quesada PM; Nyland J; Malkani A; Topp RV. Prehabilitation before total knee arthroplasty increases strength and function in older adults with severe osteoarthritis.. Journal of strength and conditioning research. 21217530.

$\mathrm{T}$ : Timing outcome too early

Szots K; Konradsen H; Solgaard S; Ostergaard B. Telephone Follow-Up by Nurse After Total Knee Arthroplasty: Results of a Randomized Clinical Trial.. Orthopedic nursing. 27851679.

I: Not (p)rehabilitation

Szots, K.; Konradsen, H.; Solgaard, S.; Ostergaard, B.. Telephone follow-up by nurse following total knee arthroplasty - protocol for a randomized clinical trial (NCT 01771315). BMC Nurs. 24872728. I: Not (p)rehabilitation

Tanaka R; Hayashizaki T; Taniguchi R; Kobayashi J; Umehara T. Effect of an intensive functional rehabilitation program on the recovery of activities of daily living after total knee arthroplasty: A multicenter, randomized, controlled trial.. Journal of orthopaedic science : official journal of the Japanese Orthopaedic Association. 31182258.

$\mathrm{T}$ : Timing outcome too early

Tanaka Y; Oka H; Nakayama S; Ueno T; Matsudaira K; Miura T; Tanaka K; Tanaka S. Improvement of walking ability during postoperative rehabilitation with the hybrid assistive limb after total knee arthroplasty: A randomized controlled study.. SAGE open medicine. 28634540.

$\mathrm{D}: \mathrm{N}<20$ per group

Tanaka, R.; Hayashizaki, T.; Taniguchi, R.; Kobayashi, J.; Umehara, T.. Effect of an intensive functional rehabilitation program on the recovery of activities of daily living after total knee arthroplasty: a multicenter, randomized, controlled trial. Journal of orthopaedic science. CN-01963575.

Duplicate/No additional data

Tanaka, Ryo; Hayashizaki, Takuya; Taniguchi, Ryoji; Kobayashi, Jun; Umehara, Takuya. Effect of an intensive functional rehabilitation program on the recovery of activities of daily living after total knee arthroplasty: $\mathrm{A} \neg \nmid$ multicenter, randomized, controlled 
trial. Journal of Orthopaedic Science. 142208167. Language:.

Duplicate/No additional data

Tappen RM; Whitehead D; Folden SL; Hall R. Effect of a video intervention on functional recovery following hip replacement and hip fracture repair.. Rehabilitation nursing : the official journal of the Association of Rehabilitation Nurses. 14521003. P: Not osteoarthritis (exclude cancer, trauma, rheumatoid arthritis)

Tayrose G; Newman D; Slover J; Jaffe F; Hunter T; Bosco J 3rd. Rapid mobilization decreases length-ofstay in joint replacement patients.. Bulletin of the Hospital for Joint Disease (2013). 24151950. D: NRCS not adjusted

Tedesco, D.; Gori, D.; Desai, K. R.; Asch, S.; Carroll, I. R.; Curtin, C.; McDonald, K. M.; Fantini, M. P.; Hernandez-Boussard, T.. Drug-Free Interventions to Reduce Pain or Opioid Consumption After Total Knee Arthroplasty: A Systematic Review and Metaanalysis. JAMA Surg. 28813550.

SR or guideline

Temfemo A; Doutrellot PL; Ahmaidi S. [Early muscular strengthening after total hip arthroplasty: association of two models of rehabilitation].. Annales de readaptation et de medecine physique : revue scientifique de la Societe francaise de reeducation fonctionnelle de readaptation et de medecine physique. 18164092.

$\mathrm{T}$ : Timing outcome too early

Thiengwittayaporn, S.; Kangkano, N.; Krishnamra, N.; Charoenphandhu, N.. Comparison between diaryactuated rehabilitation program and conventional physical therapy on mobility and function following total knee arthroplasty. Osteoporosis international. CN-01409621.

P: Not osteoarthritis (exclude cancer, trauma, rheumatoid arthritis)

Tian W; DeJong G; Horn SD; Putman K; Hsieh CH; DaVanzo JE. Efficient rehabilitation care for joint replacement patients: skilled nursing facility or inpatient rehabilitation facility?. Medical decision making : an international journal of the Society for Medical Decision Making. 21487103.

$\mathrm{T}$ : Timing outcome too early

Timmers, T.; Janssen, L.; van der Weegen, W.; Das, D.; Marijnissen, W. J.; Hannink, G.; van der Zwaard, B. C.; Plat, A.; Thomassen, B.; Swen, J. W.; Kool, R. B.; Lambers Heerspink, F. O.. The Effect of an App for Day-to-Day Postoperative Care Education on Patients With Total Knee Replacement: Randomized
Controlled Trial. JMIR Mhealth Uhealth. 31638594. $\mathrm{T}$ : Timing outcome too early

Torisho C; Mohaddes M; Gustafsson K; Rolfson O. Minor influence of patient education and physiotherapy interventions before total hip replacement on patient-reported outcomes: an observational study of 30,756 patients in the Swedish Hip Arthroplasty Register.. Acta orthopaedica. 30994040.

I: Not (p)rehabilitation

Tousignant M; Boissy P; Moffet H; Corriveau H; Cabana F; Marquis F; Simard J. Patients' satisfaction of healthcare services and perception with in-home telerehabilitation and physiotherapists' satisfaction toward technology for post-knee arthroplasty: an embedded study in a randomized trial.. Telemedicine journal and e-health : the official journal of the American Telemedicine Association. 21492030. $\mathrm{T}$ : Timing outcome too early

Tousignant M; Moffet H; Nadeau S; Merette C; Boissy P; Corriveau H; Marquis F; Cabana F; Ranger P; Belzile EL; Dimentberg R. Cost analysis of inhome telerehabilitation for post-knee arthroplasty.. Journal of medical Internet research. 25840501. $\mathrm{O}$ : No outcomes of interest

Tousignant, M.; Moffet, H.; Boissy, P.; Corriveau, H.; Cabana, F.; Marquis, E.. Patients and physiotherapists satisfaction of in-home telerehabilittion for post-knee arthroplasty. Physiotherapy (united kingdom).. CN-01089231. D: Single group/no comparison

Tousignant, M.; Moffet, H.; Nadeau, S.; Merette, C.; Boissy, P.; Corriveau, H.; Marquis, F.; Cabana, F.; Ranger, P.; Belzile, E. L.; et al.. Is tele-rehabilitation an adequate economic alternative to conventional rehabilitation?. Physiotherapy (united kingdom).. CN-01163622.

Duplicate/No additional data

Tribe KL; Lapsley HM; Cross MJ; Courtenay BG; Brooks PM; March LM. Selection of patients for inpatient rehabilitation or direct home discharge following total joint replacement surgery: a comparison of health status and out-of-pocket expenditure of patients undergoing hip and knee arthroplasty for osteoarthritis.. Chronic illness. 17152453.

I: Not (p)rehabilitation

Trudelle-Jackson E; Smith SS. Effects of a late-phase exercise program after total hip arthroplasty: a randomized controlled trial.. Archives of physical 
medicine and rehabilitation. 15241750 .

$\mathrm{D}: \mathrm{N}<20$ per group

Tse BK; Walters TL; Howard SK; Kim TE; Memtsoudis SG; Sun EC; Kou A; Graham L; King R; Mariano ER. A matched case-control comparison of hospital costs and outcomes for knee replacement patients admitted postoperatively to acute care versus rehabilitation.. Journal of anesthesia. 28477230. D: $\mathrm{N}<20$ per group

Tse, B. K.; Walters, T. L.; Howard, S. K.; Kim, T. E.; Memtsoudis, S. G.; Sun, E. C.; Kou, A.; Graham, L.; King, R.; Mariano, E. R.. A matched case, Äìcontrol comparison of hospital costs and outcomes for knee replacement patients admitted postoperatively to acute care versus rehabilitation. Journal of Anesthesia. .

Duplicate/No additional data

Tsukada Y; Matsuse H; Shinozaki N; Takano Y; Nago T; Shiba N. Combined Application of Electrically Stimulated Antagonist Muscle Contraction and Volitional Muscle Contraction Prevents Muscle Strength Weakness and Promotes Physical Function Recovery After Total Knee Arthroplasty: A Randomized Controlled Trial.. The Kurume medical journal. 31723080.

Duplicate/No additional data

Tsukada, Y.; Matsuse, H.; Shinozaki, N.; Takano, Y.; Nago, T.; Shiba, N.. Combined Application of Electrically Stimulated Antagonist Muscle Contraction and Volitional Muscle Contraction Prevents Muscle Strength Weakness and Promotes Physical Function Recovery After Total Knee Arthroplasty: a Randomized Controlled Trial. The Kurume medical journal. CN-02009205.

Duplicate/No additional data

Tsukagoshi R; Tateuchi H; Fukumoto Y; Ibuki S; Akiyama H; So K; Kuroda Y; Okumura H; Ichihashi N. Functional performance of female patients more than 6 months after total hip arthroplasty shows greater improvement with weight-bearing exercise than with non-weight-bearing exercise. Randomized controlled trial.. European journal of physical and rehabilitation medicine. 25051209.

P: Not unilateral replacement (exclude bilateral replacement surgeries)

Tsukagoshi R; Tateuchi H; Fukumoto Y; Okumura $\mathrm{H}$; Ichihashi N. Stepping exercises improve muscle strength in the early postoperative phase after total hip arthroplasty: a retrospective study.. American journal of physical medicine \& rehabilitation. 22157435.

D: $\mathrm{N}<20$ per group
Tsukagoshi, R.; Tateuchi, H.; Fukumoto, Y.; Okumura, H.; Ichihashi, N.. Stepping exercise improves muscle strength in the early postoperative phase after total hip arthroplasty: a clinical controlled trial. Physiotherapy (united kingdom).. CN01102725 .

$\mathrm{D}: \mathrm{N}<20$ per group

Tungtrongjit Y; Weingkum P; Saunkool P. The effect of preoperative quadriceps exercise on functional outcome after total knee arthroplasty.. Journal of the Medical Association of Thailand = Chotmaihet thangphaet. 23451440.

Full text unavailable

Tyagi V; Huez C; Henderson S; Lukasiewicz A; Gardner E; Rubin L; Weis L. The effect of a preoperative education class on the rate of manipulation under anesthesia after total knee arthroplasty in a veterans population.. Arthroplasty today. 30186922.

I: Not (p)rehabilitation

Uesugi, Yuko; Hayashi, Shinya; Fujishiro, Takaaki; Kanzaki, Noriyuki; Nishiyama, Takayuki. Effectiveness of distance education intervention using video footage in postoperative patients after total hip arthroplasty. International Journal of Orthopaedic \& Trauma Nursing. 86812101. Language:.

$\mathrm{D}: \mathrm{N}<20$ per group

Umehara T; Tanaka R. Effective exercise intervention period for improving body function or activity in patients with knee osteoarthritis undergoing total knee arthroplasty: a systematic review and meta-analysis.. Brazilian journal of physical therapy. 29174345.

SR or guideline

Umpierres CS; Ribeiro TA; Marchisio AE; Galvao L; Borges IN; Macedo CA; Galia CR. Rehabilitation following total hip arthroplasty evaluation over short follow-up time: randomized clinical trial.. Journal of rehabilitation research and development. 25856757. $\mathrm{T}$ : Timing outcome too early

Unver B; Karatosun V; Gunal I; Angin S. Comparison of two different rehabilitation programmes for thrust plate prosthesis: a randomized controlled study.. Clinical rehabilitation. 14763723. published before 2005

Vadher K; Knight R; Barker KL; Dutton SJ. COmmunity-based Rehabilitation after Knee Arthroplasty (CORKA): statistical analysis plan for a randomised controlled trial.. Trials. 30454051.

Protocol/study registration 
Valdes Vilches, M.; Fernandez Ferreras, T.; Serra Tarragon, N.; Bujedo Pertejo, A.; San Segundo Mozo, R.; Molins Roca, J.. Feedback and neuromuscular electrical stimulation during an early phase of a rehabilitation programe after total knee arthroplasty. Trauma (Spain). .

Full text unavailable

Valkenet K; van de Port IG; Dronkers JJ; de Vries WR; Lindeman E; Backx FJ. The effects of preoperative exercise therapy on postoperative outcome: a systematic review.. Clinical rehabilitation. 21059667.

SR or guideline

Valtonen A; Poyhonen T; Sipila S; Heinonen A. Effects of aquatic resistance training on mobility limitation and lower-limb impairments after knee replacement.. Archives of physical medicine and rehabilitation. 20510971.

I: Too early (prehab) or late (rehab)

Valtonen A; Poyhonen T; Sipila S; Heinonen A. Maintenance of aquatic training-induced benefits on mobility and lower-extremity muscles among persons with unilateral knee replacement.. Archives of physical medicine and rehabilitation. 22133241. I: Too early (prehab) or late (rehab)

Valtonen, A.; P $\sqrt{ } \partial y h \sqrt{ } \partial$ nen, T.; Sipil $\sqrt{ } \S$, S.; Heinonen, A.. Maintenance of aquatic training-induced benefits in mobility and lower extremity muscles among persons with unilateral knee replacement. Physiotherapy (united kingdom). 22133241. $\mathrm{D}: \mathrm{N}<20$ per group

van Eeden, Florien M.; van Halewijn, Karlijn F.; Swart, Nynke M.; Festen, Dederieke A.; Bily, W.; Franz, C.; Trimmel, L.; Kasche, W.; Kern, H.; Loefler, S.; Zampieri, S.; Sarabon, N.; Cvecka, J.; Zenz, P.. Efficacy and Safety of Leg-Press Training With Moderate Vibration After Total Knee Arthroplasty Remains Unclear...Bily W, Franz C, Trimmel L, Loefler S, Cvecka J, Zampieri S, Kasche W, Sarabon N, Zenz P, Kern H. Effects of Leg-Press Training With Moderate Vibration on Muscle Strength, Pain, and Function After Total Knee Arthroplasty: A Randomized Controlled Trial. Arch Phys Med Rehabil. 2016 Jun;97(6):857-65. Archives of Physical Medicine \& Rehabilitation. 118999077. Language:.

D: Not primary study (or SR or GL)

Van Herck P; Vanhaecht K; Deneckere S; Bellemans J; Panella M; Barbieri A; Sermeus W. Key interventions and outcomes in joint arthroplasty clinical pathways: a systematic review.. Journal of evaluation in clinical practice. 20367814. I: Not (p)rehabilitation

van Leeuwen, D. M.; de Ruiter, C. J.; Nolte, P. A.; de Haan, A.. Preoperative Strength Training for Elderly Patients Awaiting Total Knee Arthroplasty. Rehabilitation Research \& Practice. 24693435. $\mathrm{D}: \mathrm{N}<20$ per group

Vasta, S.; Papalia, R.; Torre, G.; Vorini, F.; Papalia, G.; Zampogna, B.; Fossati, C.; Bravi, M.; Campi, S.; Denaro, V.. The Influence of Preoperative Physical

Activity on Postoperative Outcomes of Knee and Hip Arthroplasty Surgery in the Elderly: A Systematic Review. J Clin Med. 32244426.

SR or guideline

Vesterby MS; Pedersen PU; Laursen M; Mikkelsen S; Larsen J; Soballe K; Jorgensen LB. Telemedicine support shortens length of stay after fast-track hip replacement.. Acta orthopaedica. 28097941.

I: Not well defined/no (p)rehabilitation content to define

Villafane JH; Isgro M; Borsatti M; Berjano P; Pirali $\mathrm{C}$; Negrini S. Effects of action observation treatment in recovery after total knee replacement: a prospective clinical trial.. Clinical rehabilitation. 27068367.

$\mathrm{D}: \mathrm{N}<20$ per group

Villafane JH; Pirali C; Isgro M; Vanti C; Buraschi R; Negrini S. Effects of Action Observation Therapy in Patients Recovering From Total Hip Arthroplasty Arthroplasty: A Prospective Clinical Trial.. Journal of chiropractic medicine. 27857630.

D: $\mathrm{N}<20$ per group

Visschedijk, J.. Fear of falling in patients with hip fractures: prevalence and related psychological factors. J Am Med Dir Assoc. 23218746.

P: Not MJR (hip/knee)

Volpato HB; Szego P; Lenza M; Milan SL; Talerman $\mathrm{C}$; Ferretti M. Femoral quadriceps neuromuscular electrical stimulation after total knee arthroplasty: a systematic review.. Einstein (Sao Paulo, Brazil). 26537511.

SR or guideline

Wallis JA; Taylor NF. Pre-operative interventions (non-surgical and non-pharmacological) for patients with hip or knee osteoarthritis awaiting joint replacement surgery--a systematic review and metaanalysis.. Osteoarthritis and cartilage. 21959097. SR or guideline 
Wallis, J. A.; Taylor, N. F.. Pre-operative interventions (non-surgical and non-pharmacological) for patients with hip or knee osteoarthritis awaiting joint replacement surgery - a systematic review and meta-analysis. Osteoarthritis and cartilage. $\mathrm{CN}$ 01758144.

SR or guideline

Walsh MB; Herbold J. Outcome after rehabilitation for total joint replacement at IRF and SNF: a casecontrolled comparison.. American journal of physical medicine \& rehabilitation. 16357542.

$\mathrm{T}$ : Timing outcome too early

Wang AW; Gilbey HJ; Ackland TR. Perioperative exercise programs improve early return of ambulatory function after total hip arthroplasty: a randomized, controlled trial.. American journal of physical medicine \& rehabilitation. 12394990.

$\mathrm{D}: \mathrm{N}<20$ per group

Wang J; Tong Y; Jiang Y; Zhu H; Gao H; Wei R; Que X; Gao L. The effectiveness of extended care based on Internet and home care platform for orthopaedics after hip replacement surgery in China.. Journal of clinical nursing. 29851157.

P: Not osteoarthritis (exclude cancer, trauma, rheumatoid arthritis)

Wang L; Lee M; Zhang Z; Moodie J; Cheng D; Martin J. Does preoperative rehabilitation for patients planning to undergo joint replacement surgery improve outcomes? A systematic review and metaanalysis of randomised controlled trials.. BMJ open. 26839013.

SR or guideline

Wang X; Hunter DJ; Vesentini G; Pozzobon D; Ferreira ML. Technology-assisted rehabilitation following total knee or hip replacement for people with osteoarthritis: a systematic review and metaanalysis.. BMC musculoskeletal disorders. 31679511. SR or guideline

Wang, H. Y.; Yu, G. S.; Li, J. H.; Zhang, S. X.; Lin, Y. B.. An updated meta-analysis evaluating limb management after total knee arthroplasty-what is the optimal method?. J Orthop Surg Res. 30971262. SR or guideline

Wang, Q.; Lee, R. L.; Hunter, S.; Chan, S. W.. The effectiveness of internet-based telerehabilitation among patients after total joint arthroplasty: A systematic review and meta-analysis of randomised controlled trials. J Telemed Telecare. 33459120. SR or guideline

Wang, W. L.; Rondon, A. J.; Tan, T. L.; Wilsman, J.; Purtill, J. J.. Self-Directed Home Exercises vs
Outpatient Physical Therapy After Total Knee Arthroplasty: Value and Outcomes Following a Protocol Change. J Arthroplasty. 31178383.

D: NRCS not adjusted

Wang, X.; Hunter, D. J.; Robbins, S.; Capistrano, S.; Duong, V.; Melo, L.; Harris, A.; Ferreira, M..

Participatory health through behavioural engagement and disruptive digital technology for postoperative rehabilitation: protocol of the PATHway trial. BMJ Open. 33455931.

Protocol/study registration

Warner, S.; Ahmad, A.; Afzal, M. W.; Khan, S.; Aslam, M. M.; Gillani, S. A.. Comparison of routine physical therapy exercises with and without core stability exercises in total knee replacement patients. Rawal medical journal. CN-02244937.

$\mathrm{T}$ : Timing outcome too early

Warren M; Kozik J; Cook J; Prefontaine P; Ganley K. A Comparative Study to Determine Functional and Clinical Outcome Differences Between Patients Receiving Outpatient Direct Physical Therapy Versus Home Physical Therapy Followed by Outpatient Physical Therapy After Total Knee Arthroplasty.. Orthopedic nursing. 27851675.

$\mathrm{T}$ : Timing outcome too early

Wei, X. P.; Li, X. L.; Xing, X. Y.; Zhang, X. Y.. Daily living activities and effect evaluation following total hip replacement: community return visit. Journal of clinical rehabilitative tissue engineering research. CN-00803970.

Full text unavailable

Weidenhielm L; Mattsson E; Brostrom LA; WersallRobertsson E. Effect of preoperative physiotherapy in unicompartmental prosthetic knee replacement..

Scandinavian journal of rehabilitation medicine. 8465163.

Full text unavailable

Welsh, R. L.; Graham, J. E.; Karmarkar, A. M.; Leland, N. E.; Baillargeon, J. G.; Wild, D. L.; Ottenbacher, K. J.. Effects of Postacute Settings on Readmission Rates and Reasons for $\neg \nmid$ Readmission Following Total Knee Arthroplasty. Journal of the American Medical Directors Association. .

Duplicate/No additional data

Welsh, Rodney Laine; Graham, James E.; Karmarkar, Amol M.; Leland, Natalie E.; Baillargeon, Jacques G.; Wild, Dana L.; Ottenbacher, Kenneth J.. Effects of Postacute Settings on Readmission Rates and Reasons for Readmission Following Total Knee Arthroplasty. Journal of the American Medical Directors Association. 
122012072. Language:.

P: Not unilateral replacement (exclude bilateral replacement surgeries)

Westby, M. D.; Kennedy, D.; Jones, D. L.; Jones, A.; Doyle-Waters, M. M.; Backman, C. L.. Post-acute physiotherapy for primary total knee arthroplasty: a cochrane systematic review. Arthritis and rheumatism. CN-01781602.

SR or guideline

White PB; Carli AV; Meftah M; Ghazi N; Alexiades MM; Windsor RE; Ranawat AS. Patients Discharged to Inpatient Rehabilitation Facilities Undergo More Diagnostic Interventions With No Improvement in Outcomes.. Orthopedics. 30321438.

$\mathrm{T}$ : Timing outcome too early

White, S. C.; Lifeso, R. M.. Altering asymmetric limb loading after hip arthroplasty using real-time dynamic feedback when walking. Arch Phys Med Rehabil. 16213238.

$\mathrm{D}: \mathrm{N}<20$ per group

Wijnen A; Bouma SE; Seeber GH; van der Woude LHV; Bulstra SK; Lazovic D; Stevens M; van den Akker-Scheek I. The therapeutic validity and effectiveness of physiotherapeutic exercise following total hip arthroplasty for osteoarthritis: A systematic review.. PloS one. 29547670.

SR or guideline

Wijnen, A.; Hoogland, J.; Munsterman, T.; Gerritsma, C. L.; Dijkstra, B.; Zijlstra, W. P.; Dekker, J. S.; Annegarn, J.; Ibarra, F.; Slager, G. E.; Zijlstra, W.; Stevens, M.. Effectiveness of a Home-Based Rehabilitation Program After Total Hip Arthroplasty Driven by a Tablet App and Remote Coaching: Nonrandomized Controlled Trial Combining a Single-Arm Intervention Cohort With Historical Controls. JMIR Rehabil Assist Technol. 32338621. $\mathrm{D}: \mathrm{N}<20$ per group

Winther, S. B.; Foss, O. A.; Husby, O. S.; Wik, T. S.; Klaksvik, J.; Husby, V. S.. A randomized controlled trial on maximal strength training in 60 patients undergoing total hip arthroplasty: implementing maximal strength training into clinical practice. Acta orthopaedica. CN-01465748.

Duplicate/No additional data

Witjes, S.; Hoorntje, A.; Kuijer, P. P.; Koenraadt, K. L.; Blankevoort, L.; Kerkhoffs, G. M.; van Geenen, R. C.. Does Goal Attainment Scaling improve satisfaction regarding performance of activities of younger knee arthroplasty patients? Study protocol of the randomized controlled ACTION trial. BMC
Musculoskelet Disord. 26936270.

Protocol/study registration

Wojcik, B.; Jablonski, M.; Gebala, E.; Drelich, M.. A comparison of effectiveness of fascial relaxation and classic model of patients rehabilitation after hip joint endoprosthetics. Ortop Traumatol Rehabil.

22619101.

Full text unavailable

Wong J; Wong S; Nolde T; Yabsley RH. Effects of an experimental program on post-hospital adjustment of early discharged patients.. International journal of nursing studies. 2155881.

published before 2005

Worland RL; Arredondo J; Angles F; Lopez-Jimenez F; Jessup DE. Home continuous passive motion machine versus professional physical therapy following total knee replacement.. The Journal of arthroplasty. 9802665.

published before 2005

Wozniak-Czekierda W; Wozniak K; Hadamus A; Bialoszewski D. Use of Kinesiology Taping in Rehabilitation after Knee Arthroplasty: a Randomised Clinical Study.. Ortopedia, traumatologia, rehabilitacja. 29154230.

T: Timing outcome too early

Wu JQ; Mao LB; Wu J. Efficacy of exercise for improving functional outcomes for patients undergoing total hip arthroplasty: A meta-analysis.. Medicine. 30855443.

SR or guideline

Wu, J. Q.; Bao, H. W.; Mao, L. B.; Liu, L. F.; Li, Y. M.; Hou, J. Z.; Wu, C. H.; Zhou, Y. J.; Wang, Z.; Cheng, Y. X.; Wu, J.. Proprioceptive training on the recovery of total knee arthroplasty patients: A metaanalysis protocol of randomized controlled trial. Medicine (Baltimore). 33371137.

Protocol/study registration

Wylde V; Dennis J; Gooberman-Hill R; Beswick AD. Effectiveness of postdischarge interventions for reducing the severity of chronic pain after total knee replacement: systematic review of randomised controlled trials.. BMJ open. 29490967.

SR or guideline

Wylde V; Marques E; Artz N; Blom A; GoobermanHill R. Effectiveness and cost-effectiveness of a group-based pain self-management intervention for patients undergoing total hip replacement: feasibility study for a randomized controlled trial.. Trials. 24885915.

I: Not well defined/no (p)rehabilitation content to define 
Wylde, V.; Artz, N.; Dixon, S.; Marques, E.; Lenguerrand, E.; Blom, A.; Gooberman-Hill, R.. Effectiveness and cost-effectiveness of a group-based outpatient physiotherapy intervention following knee replacement for osteoarthritis: feasibility study for a randomised controlled trial. Osteoarthritis and cartilage. CN-01727063.

$\mathrm{O}$ : No outcomes of interest

Xiaolong, Y.; Jianzhong, W.. The effect of acupuncture during post-acute phase of rehabilitation after total knee arthroplasty. Annals of physical and rehabilitation medicine.. CN-01065983.

P: Not total relacement (exclude partial)

Xiong J; Li H; Li X; Wang L; Zhao P; Meng D; Wei ZX; Tian T. Electroacupuncture for postoperative pain management after total knee arthroplasty: Protocol for a systematic review and meta-analysis.. Medicine. 29489645.

SR or guideline

Yan, B. P.. Effects of early rehabilitation training and psychological intervention on physical and mental health of patients undergoing replacement of total hip. Chinese Journal of Clinical Rehabilitation. . P: Not osteoarthritis (exclude cancer, trauma, rheumatoid arthritis)

Yang TH; Yeh WL; Chen HY; Chen YF; Ni KC; Lee KH. Compare the Traditional Chinese Medicine manipulation with rehabilitation on inpatients after total knee arthroplasty.. The Journal of arthroplasty. 23602417.

$\mathrm{D}: \mathrm{N}<20$ per group

Yang X; Li GH; Wang HJ; Wang CY. Continuous Passive Motion After Total Knee Arthroplasty: A Systematic Review and Meta-analysis of Associated Effects on Clinical Outcomes.. Archives of physical medicine and rehabilitation. 30831093.

I: Not (p)rehabilitation

Yang, M.; Wang, H. L.; Qin, D. W.; Guo, X. F.. Gluteus maximus and gluteus medius exercise following elder total hip arthroplasty. Journal of clinical rehabilitative tissue engineering research. CN-01016098.

Full text unavailable

Yayac, M.; Moltz, R.; Pivec, R.; Lonner, J. H.; Courtney, P. M.; Austin, M. S.. Formal Physical Therapy Following Total Hip and Knee Arthroplasty Incurs Additional Cost Without Improving Outcomes. J Arthroplasty. 32674941.

P: Mixed TKA/THA with no clean subgroup analyses

Ye, X. p. Functional rehabilitation training following hip replacement. Journal of Clinical Rehabilitative
Tissue Engineering Research. .

Full text unavailable

Yeh ML; Chen HH; Liu PH. Effects of multimedia with printed nursing guide in education on selfefficacy and functional activity and hospitalization in patients with hip replacement.. Patient education and counseling. 15911196.

P: Not osteoarthritis (exclude cancer, trauma, rheumatoid arthritis)

Yildirim, Ahmet $\sqrt{\tilde{n} z g} \sqrt{ }^{\circ} \mathrm{r}$; $\sqrt{\text { ñKen, }} \sqrt{ }$ ñznur; $\sqrt{\tilde{n} K e n}$, $\sqrt{ }$ ñzdamar Fuad; $K \sqrt{ }$ ñSeo $f$ ûLu, Belma $F{ }^{{ }^{\circ}}$ sun; Sezer, Nebahat; UVáAner, Ahmet. Impact of Hospital Rehabilitation on Functional Outcomes and Quality of Life after Total Knee Arthroplasty. Turkish Journal of Physical Medicine \& Rehabilitation / Turkiye Fiziksel Tip ve Rehabilitasyon Dergisi. 110383805. Language:.

$\mathrm{T}$ : Timing outcome too early

Yim, S. J.; Min, K. D.; Lee, Y. K.; Kim, H. T.; Kang, H. K.. Efficacy of Physiotherapist after Total Knee Arthroplasty. The journal of korean knee society. CN-01046648.

P: Not unilateral replacement (exclude bilateral replacement surgeries)

Yousefian Molla, R.; Sadeghi, H.; Kahlaee, A. H.. The Effect of Early Progressive Resistive Exercise Therapy on Balance Control of Patients with Total Knee Arthroplasty. Topics in geriatric rehabilitation. CN-01428208.

T: Timing outcome too early

Yue C; Zhang X; Zhu Y; Jia Y; Wang H; Liu Y. Systematic Review of Three Electrical Stimulation Techniques for Rehabilitation After Total Knee Arthroplasty.. The Journal of arthroplasty. 29530519. SR or guideline

Zachwieja, E.; Theosmy, E. G.; Yacovelli, S. J.; Beatty, E. W.; McGrath, M. E.; Lonner, J. H.. WebBased Self-Directed Exercise Program Is CostEffective Compared to Formal Physical Therapy After Primary Total Knee Arthroplasty. J

Arthroplasty. 32423757.

D: NRCS not adjusted

Zech A; Hendrich S; Pfeifer K. Association Between Exercise Therapy Dose and Functional Improvements in the Early Postoperative Phase After Hip and Knee Arthroplasty: An Observational Study.. PM \& R : the journal of injury, function, and rehabilitation. 25892356.

D: Single group/no comparison

Zeni J Jr; Abujaber S; Flowers P; Pozzi F; SnyderMackler L. Biofeedback to promote movement 
symmetry after total knee arthroplasty: a feasibility study.. The Journal of orthopaedic and sports physical therapy. 23892267.

$\mathrm{D}: \mathrm{N}<20$ per group

Zeni Jr, Joseph; Zeni, Joseph, Jr.. Evidence supports supervised physical therapy programs after TKA.

Orthopedics. .

D: Not primary study (or SR or GL)

Zhang, W. C.; Xiao, D.. Efficacy of proprioceptive training on the recovery of total joint arthroplasty patients: a meta-analysis. J Orthop Surg Res.

33143719 .

SR or guideline

Zhao, X. Y.; Li, W. L.; Fang, W.. Clinical studies on postoperative rehabilitation for total hip replacement. Zhongguo gu shang $=$ China journal of orthopaedics and traumatology. .

D: Not primary study (or SR or GL)

Zhu, Y.; Feng, Y.; Peng, L.. Effect of transcutaneous electrical nerve stimulation for pain control after total knee arthroplasty: A systematic review and metaanalysis. J Rehabil Med. 28933513.

SR or guideline

Zietek P; Zietek J; Szczypior K; Safranow K. Effect of adding one 15-minute-walk on the day of surgery to fast-track rehabilitation after total knee arthroplasty: a randomized, single-blind study.. European journal of physical and rehabilitation medicine. 25230888.

I: Not (p)rehabilitation

[Unreadable]. [Unreadable]. Arak Medical University Journal. 148323156. Language:.

$\mathrm{D}: \mathrm{N}<20$ per group

[Unreadable]. [Unreadable]. Nursing of Integrated Traditional Chinese \& Western Medicine. .

Full text unavailable 


\section{Appendix C. Search Results, Study Design, Arm Details, Baseline Characteristics, and Risk of Bias Assessments}

\section{Search Results}

Figure C-1. Literature flow diagram

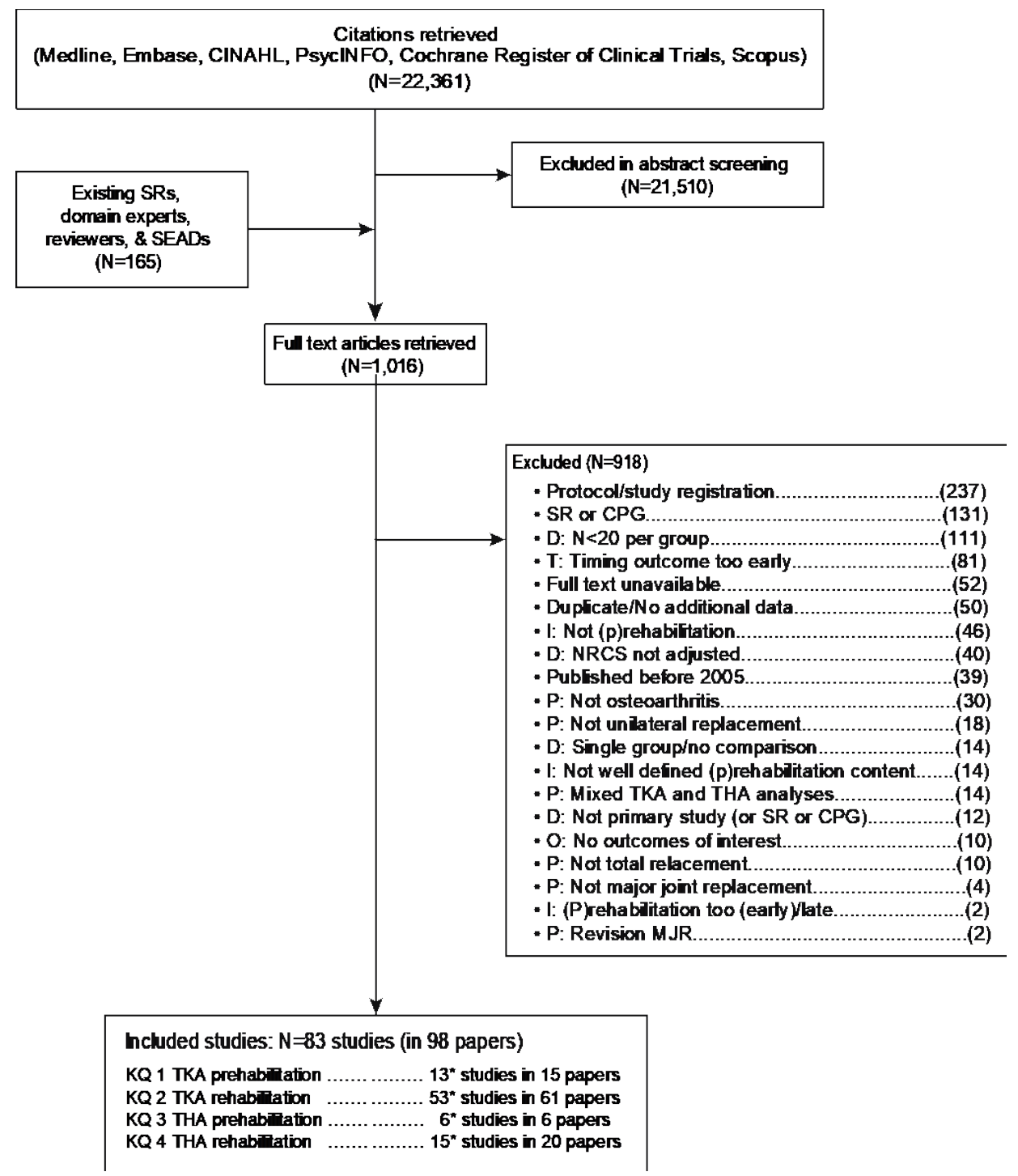

Abbreviations: $\mathrm{CPG}=$ clinical practice guideline, $\mathrm{D}=$ (study) design, $\mathrm{I}=($ study $)$ intervention, $\mathrm{KQ}=\mathrm{Key}$ Question, $\mathrm{MJR}=$ major joint replacement, $\mathrm{NRCS}=$ nonrandomized comparative study, $\mathrm{O}=$ (study) outcomes, $\mathrm{P}=$ (study) population, $\mathrm{SEAD}=$ supplemental evidence and data (request), $\mathrm{SR}=$ systematic review, $\mathrm{T}=($ outcome) timing, $\mathrm{THA}=$ total hip arthroplasty, $\mathrm{TKA}=$ total knee arthroplasty.

* 1 study included for both KQs 1 and 2, 1 study included for both KQs 1 and 3, 2 studies included for both KQs 2 and 4. 


\section{Included Studies}

\section{Key Question 1 (Prehabilitation for Total Knee Arthroplasty)}

1. Calatayud J, Casaña J, Ezzatvar Y, et al. High-intensity preoperative training improves physical and functional recovery in the early post-operative periods after total knee arthroplasty: a randomized controlled trial. Knee Surg Sports Traumatol Arthrosc. 2017 Sep;25(9):2864-72. doi: 10.1007/s00167-016-3985-5. PMID: 26768606.

2. Casaña J, Calatayud J, Ezzatvar Y, et al. Preoperative high-intensity strength training improves postural control after TKA: randomized-controlled trial. Knee Surg Sports Traumatol Arthrosc. 2019 Apr;27(4):1057-66. doi: 10.1007/s00167-018-5246-2. PMID: 30361758.

3. Huang SW, Chen PH, Chou YH. Effects of a preoperative simplified home rehabilitation education program on length of stay of total knee arthroplasty patients. Orthop Traumatol Surg Res. 2012 May;98(3):259-64. doi: 10.1016/j.otsr.2011.12.004. PMID: 22480863.

4. Huber EO, de Bie RA, Roos EM, et al. Effect of pre-operative neuromuscular training on functional outcome after total knee replacement: a randomized-controlled trial. BMC Musculoskelet Disord. 2013 May 3;14:157. doi: 10.1186/1471-2474-14-157. PMID: 23641782.

5. Huber EO, Roos EM, Meichtry A, et al. Effect of preoperative neuromuscular training (NEMEX-TJR) on functional outcome after total knee replacement: an assessor-blinded randomized controlled trial. BMC Musculoskelet Disord. 2015 Apr 25;16:101. doi: 10.1186/s12891-015-0556-8. PMID: 25925404.

6. Mat Eil Ismail MS, Sharifudin MA, Shokri AA, et al. Preoperative physiotherapy and short-term functional outcomes of primary total knee arthroplasty. Singapore Med J. 2016 Mar;57(3):138-43. doi: 10.11622/smedj.2016055. PMID: 26996450.

7. Matassi F, Duerinckx J, Vandenneucker H, et al. Range of motion after total knee arthroplasty: the effect of a preoperative home exercise program. Knee Surg Sports Traumatol Arthrosc. 2014 Mar;22(3):703-9. doi: 10.1007/s00167-012-2349-z. PMID: 23271039.

8. Mitchell C, Walker J, Walters S, et al. Costs and effectiveness of pre- and post-operative home physiotherapy for total knee replacement: randomized controlled trial. J Eval Clin Pract. 2005 Jun;11(3):283-92. doi: 10.1111/j.1365-2753.2005.00535.x. PMID: 15869558.

9. Skoffer B, Maribo T, Mechlenburg I, et al. Efficacy of Preoperative Progressive Resistance Training on Postoperative Outcomes in Patients Undergoing Total Knee Arthroplasty. Arthritis Care Res (Hoboken). 2016 Sep;68(9):1239-51. doi: 10.1002/acr.22825. PMID: 26713665.

10. Soeters R, White PB, Murray-Weir M, et al. Preoperative Physical Therapy Education Reduces Time to Meet Functional Milestones After Total Joint Arthroplasty. Clin Orthop Relat Res. 2018 Jan;476(1):40-8. doi: 10.1007/s11999.0000000000000010. PMID: 29529614.

11. Soni A, Joshi A, Mudge N, et al. Supervised exercise plus acupuncture for moderate to severe knee osteoarthritis: a small randomised controlled trial. Acupunct Med. 2012 Sep;30(3):176-81. doi: 10.1136/acupmed-2012-010128. PMID: 22914302.

12. Topp R, Swank AM, Quesada PM, et al. The effect of prehabilitation exercise on strength and functioning after total knee arthroplasty. Pm r. 2009 Aug;1(8):729-35. doi: 10.1016/j.pmrj.2009.06.003. PMID: 19695525.

13. Valtonen A, Pöyhönen T, Manninen M, et al. Effects of preoperative aquatic resistance training on knee pain, mobility limitation and muscle impairments in people with late-stage knee osteoarthritis. Physiotherapy (united kingdom). 2015;101:eS1568 - eS9. doi: 10.1016/j.physio.2015.03.1570. PMID: CN-01126383.

14. Villadsen A, Overgaard S, Holsgaard-Larsen A, et al. Postoperative effects of neuromuscular exercise prior to hip or knee arthroplasty: a randomised controlled trial. Ann Rheum Dis. 2014 Jun;73(6):1130-7. doi: 10.1136/annrheumdis-2012-203135. PMID: 23661494.

15. Williamson L, Wyatt MR, Yein K, et al. Severe knee osteoarthritis: a randomized controlled trial of acupuncture, physiotherapy (supervised exercise) and standard management for patients awaiting knee replacement. Rheumatology (Oxford). 2007 Sep;46(9):1445-9. doi: 10.1093/rheumatology/kem119. PMID: 17604311.

\section{Key Question 2 (Rehabilitation for Total Knee Arthroplasty)}

1. Andersen HH. Technological assisted rehabilitation following total knee joint replacement. a randomised controlled non-inferiority trial. Annals of the rheumatic diseases. 2018;77:1823 - 4. doi: 10.1136/annrheumdis2018-eular.6484. PMID: CN-01647420. 
2. Artz N, Dixon S, Wylde V, et al. Comparison of group-based outpatient physiotherapy with usual care after total knee replacement: a feasibility study for a randomized controlled trial. Clin Rehabil. 2017 Apr;31(4):487-99. doi: 10.1177/0269215516642503. PMID: 27068368.

3. Avramidis K, Karachalios T, Popotonasios K, et al. Does electric stimulation of the vastus medialis muscle influence rehabilitation after total knee replacement? Orthopedics. 2011 Mar 11;34(3):175. doi: 10.3928/01477447-20110124-06. PMID: 21410130.

4. Bade MJ, Struessel T, Dayton M, et al. Early High-Intensity Versus Low-Intensity Rehabilitation After Total Knee Arthroplasty: A Randomized Controlled Trial. Arthritis Care Res (Hoboken). 2017 Sep;69(9):1360-8. doi: 10.1002/acr.23139. PMID: 27813347.

5. Bily W, Franz C, Trimmel L, et al. Effects of Leg-Press Training With Moderate Vibration on Muscle Strength, Pain, and Function After Total Knee Arthroplasty: A Randomized Controlled Trial. Arch Phys Med Rehabil. 2016 Jun;97(6):857-65. doi: 10.1016/j.apmr.2015.12.015. PMID: 26763947.

6. Bruun-Olsen V, Heiberg KE, Wahl AK, et al. The immediate and long-term effects of a walking-skill program compared to usual physiotherapy care in patients who have undergone total knee arthroplasty (TKA): a randomized controlled trial. Disabil Rehabil. 2013;35(23):2008-15. doi: 10.3109/09638288.2013.770084. PMID: 23614370 .

7. Buhagiar MA, Naylor JM, Harris IA, et al. Effect of Inpatient Rehabilitation vs a Monitored Home-Based Program on Mobility in Patients With Total Knee Arthroplasty: The HIHO Randomized Clinical Trial. Jama. 2017 Mar 14;317(10):1037-46. doi: 10.1001/jama.2017.1224. PMID: 28291891.

8. Buhagiar MA, Naylor JM, Harris IA, et al. Hospital Inpatient versus HOme-based rehabilitation after knee arthroplasty (The HIHO study): study protocol for a randomized controlled trial. Trials. 2013 Dec 17;14:432. doi: 10.1186/1745-6215-14-432. PMID: 24341348.

9. Cai L, Gao H, Xu H, et al. Does a Program Based on Cognitive Behavioral Therapy Affect Kinesiophobia in Patients Following Total Knee Arthroplasty? A Randomized, Controlled Trial With a 6-Month Follow-Up. Journal of Arthroplasty. 2018;33(3):704-10. doi: 10.1016/j.arth.2017.10.035. PMID: 127919407. Language: English. Entry Date: 20181111. Revision Date: 20181109. Publication Type: journal article. Journal Subset: Biomedical.

10. Chan HY, Sultana R, Yeo SJ, et al. Comparison of outcome measures from different pathways following total knee arthroplasty. Singapore Med J. 2018 Sep;59(9):476-86. doi: 10.11622/smedj.2018011. PMID: 29372260.

11. DeJong G, Hsieh CJ, Vita MT, et al. Innovative Devices Did Not Provide Superior Total Knee Arthroplasty Outcomes in Post-Operative Rehabilitation: Results From a Four-Arm Randomized Clinical Trial. J Arthroplasty. 2020 Aug;35(8):2054-65. doi: 10.1016/j.arth.2020.03.048. PMID: 32360105.

12. Demircioglu DT, Paker N, Erbil E, et al. The effect of neuromuscular electrical stimulation on functional status and quality of life after knee arthroplasty: a randomized controlled study. J Phys Ther Sci. 2015 Aug;27(8):25016. doi: 10.1589/jpts.27.2501. PMID: 26355656.

13. den Hertog A, Gliesche K, Timm J, et al. Pathway-controlled fast-track rehabilitation after total knee arthroplasty: a randomized prospective clinical study evaluating the recovery pattern, drug consumption, and length of stay. Arch Orthop Trauma Surg. 2012 Aug;132(8):1153-63. doi: 10.1007/s00402-012-1528-1. PMID: 22643801.

14. Eymir M, Erduran M, Ünver B. Active heel-slide exercise therapy facilitates the functional and proprioceptive enhancement following total knee arthroplasty compared to continuous passive motion. Knee Surg Sports Traumatol Arthrosc. 2020 Aug 10. doi: 10.1007/s00167-020-06181-4. PMID: 32778907.

15. Fransen M, Nairn L, Bridgett L, et al. Post-Acute Rehabilitation After Total Knee Replacement: A Multicenter Randomized Clinical Trial Comparing Long-Term Outcomes. Arthritis Care Res (Hoboken). 2017 Feb;69(2):192200. doi: 10.1002/acr.23117. PMID: 27868384.

16. Hamilton DF, Beard DJ, Barker KL, et al. Targeting rehabilitation to improve outcomes after total knee arthroplasty in patients at risk of poor outcomes: randomised controlled trial. bmj. 2020;371.

17. Harmer AR, Naylor JM, Crosbie J, et al. Land-based versus water-based rehabilitation following total knee replacement: a randomized, single-blind trial. Arthritis Rheum. 2009 Feb 15;61(2):184-91. doi: 10.1002/art.24420. PMID: 19177536.

18. Heikkilä A, Sevander-Kreus N, Häkkinen A, et al. Effect of total knee replacement surgery and postoperative 12 month home exercise program on gait parameters. Gait Posture. 2017 Mar;53:92-7. doi: 10.1016/j.gaitpost.2017.01.004. PMID: 28119232.

19. Iwakiri K, Ohta Y, Shibata $Y$, et al. Initiating range of motion exercises within 24 hours following total knee arthroplasty affects the reduction of postoperative pain: A randomized controlled trial. Asia Pac J Sports Med Arthrosc Rehabil Technol. 2020 Jul;21:11-6. doi: 10.1016/j.asmart.2020.03.003. PMID: 32373475. 
20. Jin C, Feng Y, Ni Y, et al. Virtual reality intervention in postoperative rehabilitation after total knee arthroplasty: a prospective and randomized controlled clinical trial. International journal of clinical and experimental medicine. 2018;11(6):6119 - 24. PMID: CN-01617489.

21. Kauppila AM, Kyllönen E, Ohtonen P, et al. Multidisciplinary rehabilitation after primary total knee arthroplasty: a randomized controlled study of its effects on functional capacity and quality of life. Clin Rehabil. 2010 May;24(5):398-411. doi: 10.1177/0269215509346089. PMID: 20354057.

22. Kauppila AM, Sintonen H, Aronen P, et al. Economic evaluation of multidisciplinary rehabilitation after primary total knee arthroplasty based on a randomized controlled trial. Arthritis Care Res (Hoboken). 2011 Mar;63(3):33541. doi: 10.1002/acr.20398. PMID: 21080347.

23. Lenguerrand E, Artz N, Marques E, et al. Effect of Group-Based Outpatient Physical Therapy on Function After Total Knee Replacement: Results From a Multicenter Randomized Controlled Trial. Arthritis Care Res (Hoboken). 2020 Jun;72(6):768-77. doi: 10.1002/acr.23909. PMID: 31033232.

24. Lenssen AF, Crijns YH, Waltjé EM, et al. Efficiency of immediate postoperative inpatient physical therapy following total knee arthroplasty: an RCT. BMC Musculoskelet Disord. 2006 Aug 31;7:71. doi: 10.1186/14712474-7-71. PMID: 16942627.

25. Li J, Wu T, Xu Z, et al. A pilot study of post-total knee replacement gait rehabilitation using lower limbs robotassisted training system. Eur J Orthop Surg Traumatol. 2014 Feb;24(2):203-8. doi: 10.1007/s00590-012-1159-9. PMID: 23412304.

26. Li L, Cheng S, Wang G, et al. Tai chi chuan exercises improve functional outcomes and quality of life in patients with primary total knee arthroplasty due to knee osteoarthritis. Complement Ther Clin Pract. 2019 May;35:121-5. doi: 10.1016/j.ctcp.2019.02.003. PMID: 31003647.

27. Li L, Wang Z, Yin MH, et al. Effect of early gait training on the functional rehabilitation after total knee arthroplasty. Chinese journal of tissue engineering research. 2017;21(27):4288 - 93. doi: 10.3969/j.issn.20954344.2017.27.005. PMID: CN-01419703.

28. Li Z, Jiang L, Lin J. The effect of education for daily physical activity level recovery of osteoarthritis patients after total knee arthroplasty. A prospective randomized controlled clinical trial using accelerometry. Osteoarthritis and cartilage. 2015;23:A373. PMID: CN-01084888.

29. Liao CD, Lin LF, Huang YC, et al. Functional outcomes of outpatient balance training following total knee replacement in patients with knee osteoarthritis: a randomized controlled trial. Clin Rehabil. 2015 Sep;29(9):85567. doi: 10.1177/0269215514564086. PMID: 25552523.

30. Liao CD, Tsauo JY, Chiu YS, et al. Effects of Elastic Resistance Exercise After Total Knee Replacement on Muscle Mass and Physical Function in Elderly Women With Osteoarthritis: A Randomized Controlled Trial. Am J Phys Med Rehabil. 2020 May;99(5):381-9. doi: 10.1097/phm.0000000000001344. PMID: 31687984.

31. Liebs TR, Herzberg W, Rüther W, et al. Ergometer cycling after hip or knee replacement surgery: a randomized controlled trial. J Bone Joint Surg Am. 2010 Apr;92(4):814-22. doi: 10.2106/jbjs.H.01359. PMID: 20360503.

32. Liebs TR, Herzberg W, Rüther W, et al. Multicenter randomized controlled trial comparing early versus late aquatic therapy after total hip or knee arthroplasty. Arch Phys Med Rehabil. 2012 Feb;93(2):192-9. doi: 10.1016/j.apmr.2011.09.011. PMID: 22196125.

33. Madsen M, Larsen K, Madsen IK, et al. Late group-based rehabilitation has no advantages compared with supervised home-exercises after total knee arthroplasty. Dan Med J. 2013 Apr;60(4):A4607. PMID: 23651717.

34. Minns Lowe CJ, Barker KL, Holder R, et al. Comparison of postdischarge physiotherapy versus usual care following primary total knee arthroplasty for osteoarthritis: an exploratory pilot randomized clinical trial. Clin Rehabil. 2012 Jul;26(7):629-41. doi: 10.1177/0269215511427749. PMID: 22180446.

35. Mitchell C, Walker J, Walters S, et al. Costs and effectiveness of pre- and post-operative home physiotherapy for total knee replacement: randomized controlled trial. J Eval Clin Pract. 2005 Jun;11(3):283-92. doi: 10.1111/j.1365-2753.2005.00535.x. PMID: 15869558.

36. Moffet H, Tousignant M, Nadeau S, et al. Patient Satisfaction with In-Home Telerehabilitation After Total Knee Arthroplasty: Results from a Randomized Controlled Trial. Telemed J E Health. 2017 Feb;23(2):80-7. doi: 10.1089/tmj.2016.0060. PMID: 27529575.

37. Moffet H, Tousignant M, Nadeau S, et al. In-Home Telerehabilitation Compared with Face-to-Face Rehabilitation After Total Knee Arthroplasty: A Noninferiority Randomized Controlled Trial. J Bone Joint Surg Am. 2015 Jul 15;97(14):1129-41. doi: 10.2106/jbjs.N.01066. PMID: 26178888.

38. Monticone M, Ferrante S, Rocca B, et al. Home-based functional exercises aimed at managing kinesiophobia contribute to improving disability and quality of life of patients undergoing total knee arthroplasty: a randomized controlled trial. Arch Phys Med Rehabil. 2013 Feb;94(2):231-9. doi: 10.1016/j.apmr.2012.10.003. PMID: 23063624. 
39. Moutzouri M, Coutts F, Gliatis J, et al. Early initiation of home-based sensori-motor training improves muscle strength, activation and size in patients after knee replacement: a secondary analysis of a controlled clinical trial. BMC Musculoskelet Disord. 2019 May 17;20(1):231. doi: 10.1186/s12891-019-2575-3. PMID: 31101039.

40. Moutzouri M, Gleeson N, Coutts F, et al. Early self-managed focal sensorimotor rehabilitative training enhances functional mobility and sensorimotor function in patients following total knee replacement: a controlled clinical trial. Clin Rehabil. 2018 Jul;32(7):888-98. doi: 10.1177/0269215518757291. PMID: 29473481.

41. Naylor JM, Hart A, Mittal R, et al. The value of inpatient rehabilitation after uncomplicated knee arthroplasty: a propensity score analysis. Med J Aust. 2017 Sep 18;207(6):250-5. doi: 10.5694/mja16.01362. PMID: 28899328.

42. Padgett DE, Christ AB, Joseph AD, et al. Discharge to Inpatient Rehab Does Not Result in Improved Functional Outcomes Following Primary Total Knee Arthroplasty. J Arthroplasty. 2018 Jun;33(6):1663-7. doi: 10.1016/j.arth.2017.12.033. PMID: 29352683.

43. Petersen T, Hautopp H, Duus B, et al. No Effect of Acupuncture as Adjunctive Therapy for Patients with Total Knee Replacement: A Randomized Controlled Trial. Pain Med. 2018 Jun 1;19(6):1280-9. doi: 10.1093/pm/pnx317. PMID: 29294078.

44. Petterson SC, Mizner RL, Stevens JE, et al. Improved function from progressive strengthening interventions after total knee arthroplasty: a randomized clinical trial with an imbedded prospective cohort. Arthritis Rheum. 2009 Feb 15;61(2):174-83. doi: 10.1002/art.24167. PMID: 19177542.

45. Piqueras M, Marco E, Coll M, et al. Effectiveness of an interactive virtual telerehabilitation system in patients after total knee arthoplasty: a randomized controlled trial. J Rehabil Med. 2013 Apr;45(4):392-6. doi: 10.2340/16501977-1119. PMID: 23474735.

46. Piva SR, Almeida GJ, Gil AB, et al. Effect of Comprehensive Behavioral and Exercise Intervention on Physical Function and Activity Participation After Total Knee Replacement: A Pilot Randomized Study. Arthritis Care Res (Hoboken). 2017 Dec;69(12):1855-62. doi: 10.1002/acr.23227. PMID: 28217891.

47. Piva SR, Moore CG, Schneider M, et al. A randomized trial to compare exercise treatment methods for patients after total knee replacement: protocol paper. BMC Musculoskelet Disord. 2015 Oct 16;16:303. doi: 10.1186/s12891-015-0761-5. PMID: 26474988.

48. Piva SR, Schneider MJ, Moore CG, et al. Effectiveness of Later-Stage Exercise Programs vs Usual Medical Care on Physical Function and Activity After Total Knee Replacement: A Randomized Clinical Trial. JAMA Netw Open. 2019 Feb 1;2(2):e190018. doi: 10.1001/jamanetworkopen.2019.0018. PMID: 30794296.

49. Pua YH, Seah FJ, Poon CL, et al. Association between rehabilitation attendance and physical function following discharge after total knee arthroplasty: prospective cohort study. Osteoarthritis Cartilage. 2017 Apr;25(4):462-9. doi: 10.1016/j.joca.2016.10.020. PMID: 27810379.

50. Rockstroh G, Schleicher W, Krummenauer F. [Effectiveness of microcurrent therapy as a constituent of posthospital rehabilitative treatment in patients after total knee alloarthroplasty - a randomized clinical trial]. Rehabilitation (Stuttg). 2010 Jun;49(3):173-9. doi: 10.1055/s-0029-1246152. PMID: 20533147.

51. Sattler LN, Hing WA, Vertullo CJ. Pedaling-Based Protocol Superior to a 10-Exercise, Non-Pedaling Protocol for Postoperative Rehabilitation After Total Knee Replacement: A Randomized Controlled Trial. J Bone Joint Surg Am. 2019 Apr 17;101(8):688-95. doi: 10.2106/jbjs.18.00898. PMID: 30994586.

52. Schache MB, McClelland JA, Webster KE. Does the addition of hip strengthening exercises improve outcomes following total knee arthroplasty? A study protocol for a randomized trial. BMC Musculoskelet Disord. 2016 Jun 13;17:259. doi: 10.1186/s12891-016-1104-x. PMID: 27295978.

53. Schache MB, McClelland JA, Webster KE. Incorporating hip abductor strengthening exercises into a rehabilitation program did not improve outcomes in people following total knee arthroplasty: a randomised trial. J Physiother. 2019 Jul;65(3):136-43. doi: 10.1016/j.jphys.2019.05.008. PMID: 31208916.

54. Shanb AS, Youssef EF. Effects of adding biofeedback training to active exercises after total knee arthroplasty. Journal of musculoskeletal research. 2014;17(1). doi: 10.1142/S0218957714500018. PMID: CN-01041112.

55. Simpson AH, Hamilton DF, Beard DJ, et al. Targeted rehabilitation to improve outcome after total knee replacement (TRIO): study protocol for a randomised controlled trial. Trials. 2014 Feb 1;15:44. doi: 10.1186/1745-6215-15-44. PMID: 24484541.

56. Stevens-Lapsley JE, Balter JE, Wolfe P, et al. Early neuromuscular electrical stimulation to improve quadriceps muscle strength after total knee arthroplasty: a randomized controlled trial. Phys Ther. 2012 Feb;92(2):210-26. doi: 10.2522/ptj.20110124. PMID: 22095207.

57. Tousignant M, Moffet H, Boissy $\mathrm{P}$, et al. A randomized controlled trial of home telerehabilitation for post-knee arthroplasty. J Telemed Telecare. 2011;17(4):195-8. doi: 10.1258/jtt.2010.100602. PMID: 21398389.

58. Tsukada Y, Matsuse H, Shinozaki N, et al. Combined application of electrically stimulated antagonist muscle contraction and volitional muscle contraction prevents muscle strength weakness and promotes physical function 
recovery after total knee arthroplasty: A randomized controlled trial. Kurume Medical Journal. 2018;65(4):14554. doi: 10.2739/kurumemedj.MS654007.

59. Vuorenmaa M, Ylinen J, Piitulainen K, et al. Efficacy of a 12-month, monitored home exercise programme compared with normal care commencing 2 months after total knee arthroplasty: a randomized controlled trial. J Rehabil Med. 2014 Feb;46(2):166-72. doi: 10.2340/16501977-1242. PMID: 24241606.

60. Wylde V, Artz N, Marques E, et al. Effectiveness and cost-effectiveness of outpatient physiotherapy after knee replacement for osteoarthritis: study protocol for a randomised controlled trial. Trials. 2016 Jun 13;17(1):289. doi: 10.1186/s13063-016-1418-x. PMID: 27296366.

61. Zapparoli L, Sacheli LM, Seghezzi S, et al. Motor imagery training speeds up gait recovery and decreases the risk of falls in patients submitted to total knee arthroplasty. Sci Rep. 2020 Jun 2;10(1):8917. doi: 10.1038/s41598-02065820-5. PMID: 32488010.

\section{Key Question 3 (Prehabilitation for Total Hip Arthroplasty)}

1. Bitterli R, Sieben JM, Hartmann M, et al. Pre-surgical sensorimotor training for patients undergoing total hip replacement: a randomised controlled trial. Int J Sports Med. 2011 Sep;32(9):725-32. doi: 10.1055/s-00311271696. PMID: 21630176.

2. Holsgaard-Larsen A, Hermann A, Zerahn B, et al. Effects of progressive resistance training prior to total HIP arthroplasty - a secondary analysis of a randomized controlled trial. Osteoarthritis Cartilage. 2020 Aug;28(8):1038-45. doi: 10.1016/j.joca.2020.04.010. PMID: 32376477.

3. Pour AE, Parvizi J, Sharkey PF, et al. Minimally invasive hip arthroplasty: what role does patient preconditioning play? J Bone Joint Surg Am. 2007 Sep;89(9):1920-7. doi: 10.2106/jbjs.F.01153. PMID: 17768187.

4. Rooks DS, Huang J, Bierbaum BE, et al. Effect of preoperative exercise on measures of functional status in men and women undergoing total hip and knee arthroplasty. Arthritis Rheum. 2006 Oct 15;55(5):700-8. doi: 10.1002/art.22223. PMID: 17013852.

5. Soeters R, White PB, Murray-Weir M, et al. Preoperative Physical Therapy Education Reduces Time to Meet Functional Milestones After Total Joint Arthroplasty. Clin Orthop Relat Res. 2018 Jan;476(1):40-8. doi: 10.1007/s11999.000000000000010. PMID: 29529614.

6. Vukomanović A, Popović Z, Durović A, et al. The effects of short-term preoperative physical therapy and education on early functional recovery of patients younger than 70 undergoing total hip arthroplasty. Vojnosanit Pregl. 2008 Apr;65(4):291-7. doi: 10.2298/vsp0804291v. PMID: 18499950.

\section{Key Question 4 (Rehabilitation for Total Hip Arthroplasty)}

1. Austin MS, Urbani BT, Fleischman AN, et al. Formal Physical Therapy After Total Hip Arthroplasty Is Not Required: A Randomized Controlled Trial. J Bone Joint Surg Am. 2017 Apr 19;99(8):648-55. doi: 10.2106/jbjs.16.00674. PMID: 28419032.

2. Beck H, Beyer F, Gering F, et al. Sports Therapy Interventions Following Total Hip Replacement: A Randomized Controlled Trial. Deutsches Aerzteblatt International. 2019;116(1/2):1-8. doi: 10.3238/arztebl.2019.0001. PMID: 134820207. Language: English. Entry Date: In Process. Revision Date: 20190223. Publication Type: Article. Journal Subset: Biomedical.

3. Coulter C, Perriman DM, Neeman TM, et al. Supervised or Unsupervised Rehabilitation After Total Hip Replacement Provides Similar Improvements for Patients: A Randomized Controlled Trial. Arch Phys Med Rehabil. 2017 Nov;98(11):2253-64. doi: 10.1016/j.apmr.2017.03.032. PMID: 28506775.

4. Giaquinto S, Ciotola E, Dall'armi V, et al. Hydrotherapy after total hip arthroplasty: a follow-up study. Arch Gerontol Geriatr. 2010 Jan-Feb;50(1):92-5. doi: 10.1016/j.archger.2009.02.005. PMID: 19282040.

5. Heiberg KE, Bruun-Olsen V, Ekeland A, et al. Effect of a walking skill training program in patients who have undergone total hip arthroplasty: Followup one year after surgery. Arthritis Care Res (Hoboken). 2012 Mar;64(3):415-23. doi: 10.1002/acr.20681. PMID: 22170790.

6. Heiberg KE, Figved W. Exercise, recovery of physical functioning, and prediction of physical activity after total hip arthroplasty. 5-year follow-up of a rct. Annals of the rheumatic diseases. 2015;74:1318 - 9. doi: 10.1136/annrheumdis-2015-eular.3078. PMID: CN-01128862.

7. Heiberg KE, Figved W. Physical Functioning and Prediction of Physical Activity After Total Hip Arthroplasty: Five-Year Followup of a Randomized Controlled Trial. Arthritis Care Res (Hoboken). 2016 Apr;68(4):454-62. doi: 10.1002/acr.22679. PMID: 26239078. 
8. Liebs TR, Herzberg W, Rüther W, et al. Ergometer cycling after hip or knee replacement surgery: a randomized controlled trial. J Bone Joint Surg Am. 2010 Apr;92(4):814-22. doi: 10.2106/jbjs.H.01359. PMID: 20360503.

9. Liebs TR, Herzberg W, Rüther W, et al. Multicenter randomized controlled trial comparing early versus late aquatic therapy after total hip or knee arthroplasty. Arch Phys Med Rehabil. 2012 Feb;93(2):192-9. doi: 10.1016/j.apmr.2011.09.011. PMID: 22196125.

10. Łyp M, Kaczor R, Cabak A, et al. A Water Rehabilitation Program in Patients with Hip Osteoarthritis Before and After Total Hip Replacement. Med Sci Monit. 2016 Jul 25;22:2635-42. doi: 10.12659/msm.896203. PMID: 27455419.

11. Mikkelsen LR, Mechlenburg I, Søballe K, et al. Effect of early supervised progressive resistance training compared to unsupervised home-based exercise after fast-track total hip replacement applied to patients with preoperative functional limitations. A single-blinded randomised controlled trial. Osteoarthritis Cartilage. 2014 Dec;22(12):2051-8. doi: 10.1016/j.joca.2014.09.025. PMID: 25305374.

12. Monticone M, Ambrosini E, Rocca B, et al. Task-oriented exercises and early full weight-bearing contribute to improving disability after total hip replacement: a randomized controlled trial. Clin Rehabil. 2014 Jul;28(7):65868. doi: 10.1177/0269215513519342. PMID: 24459172.

13. Naylor JM, Hart A, Mittal R, et al. The effectiveness of inpatient rehabilitation after uncomplicated total hip arthroplasty: a propensity score matched cohort. BMC Musculoskelet Disord. 2018 Jul 18;19(1):236. doi: 10.1186/s12891-018-2134-3. PMID: 30021552.

14. Nelson M, Bourke M, Crossley K, et al. Telerehabilitation Versus Traditional Care Following Total Hip Replacement: A Randomized Controlled Trial Protocol. JMIR Res Protoc. 2017 Mar 2;6(3):e34. doi: 10.2196/resprot.7083. PMID: 28254734.

15. Nelson M, Bourke M, Crossley K, et al. Telerehabilitation is non-inferior to usual care following total hip replacement - a randomized controlled non-inferiority trial. Physiotherapy. 2020 Jun;107:19-27. doi: 10.1016/j.physio.2019.06.006. PMID: 32026820.

16. Rao BM, Cieslewicz TJ, Sochacki KR, et al. Worse Preoperative Pain and Higher Activity Levels Predict Patient Choice of Formal Physical Therapy After Primary Anterior Total Hip Arthroplasty. J Arthroplasty. 2021 Mar 16. doi: 10.1016/j.arth.2021.03.027. PMID: 33863614.

17. Smith TO, Mann CJ, Clark A, et al. Bed exercises following total hip replacement: 1 year follow-up of a singleblinded randomised controlled trial. Hip Int. 2009 Jul-Sep;19(3):268-73. doi: 10.1177/112070000901900314. PMID: 19876883.

18. Smith TO, Mann CJV, Clark A, et al. Bed exercises following total hip replacement: a randomised controlled trial. Physiotherapy. 2008;94(4):286-91. PMID: 105615532. Language: English. Entry Date: 20090227. Revision Date: 20150819. Publication Type: Journal Article.

19. Winther SB, Foss OA, Husby OS, et al. A randomized controlled trial on maximal strength training in 60 patients undergoing total hip arthroplasty. Acta Orthop. 2018 Jun;89(3):295-301. doi: 10.1080/17453674.2018.1441362. PMID: 29493347.

20. Winther SB, Foss OA, Klaksvik J, et al. Increased Muscle Strength Limits Postural Sway During Daily Living Activities in Total Hip Arthroplasty Patients. Am J Phys Med Rehabil. 2020 Jul;99(7):608-12. doi: 10.1097/phm.0000000000001382. PMID: 31977324. 


\section{Study Design Details and Arms, Risk of Bias}

\section{Key Question 1: Prehabilitation for Total Knee Arthroplasty}

Table C-1.1. KQ 1. Design details and arms

\begin{tabular}{|c|c|c|c|c|c|c|c|c|c|}
\hline $\begin{array}{l}\text { Study } \\
\text { Year, } \\
\text { PMID, } \\
\text { Country }\end{array}$ & $\begin{array}{l}\text { Funding } \\
\text { Source }\end{array}$ & $\begin{array}{l}\text { Risk of } \\
\text { Bias }\end{array}$ & Eligibility Criteria & Intervention ${ }^{B}$ & $\begin{array}{l}\mathrm{N}, \\
\text { Enrolled }\end{array}$ & $\begin{array}{l}\text { Mean Age, } \\
\text { Years (SD) }\end{array}$ & $\begin{array}{l}\text { Female, } \\
\%\end{array}$ & $\begin{array}{l}\text { Mean } \\
\text { BMIC (SD) }^{\text {C }}\end{array}$ & $\begin{array}{l}\text { Prior } \\
\text { Contralateral } \\
\text { Arthroplasty }\end{array}$ \\
\hline \multirow[t]{2}{*}{$\begin{array}{l}\text { Calatayud, } \\
2017 \\
26768606 \\
\text { Spain }\end{array}$} & $\begin{array}{l}\text { Non- } \\
\text { industry }\end{array}$ & Moderate & $\begin{array}{l}\text { INCLUSION: } \geq 60 \text { yo, diagnosed with advanced } \\
\text { idiopathic knee OA and scheduled for unilateral } \\
\text { TKA. EXCLUSION: Pain in the contralateral limb } \\
\text { (defined as maximum pain of } 4 \text { of } 10 \text { during daily } \\
\text { activities), hip or knee joint replacement in the } \\
\text { previous year, any medical condition in which } \\
\text { exercise was contraindicated, presence of any } \\
\text { disease that affected functional performance. }\end{array}$ & $\begin{array}{l}\text { High intensity preoperative training } \\
\text { Comp: S-F-B } \\
\text { AdjMod: - } \\
\text { Set: O }\end{array}$ & 25 & $66.8(4.8)$ & $72 \%$ & $32.3(4.2)$ & NR \\
\hline & No data & No data & No data & $\begin{array}{l}\text { Control } \\
\text { Comp: NR } \\
\text { AdjMod: NR } \\
\text { Set: NR } \\
\end{array}$ & 25 & $66.7(3.1)$ & $76 \%$ & $31.2(3.8)$ & NR \\
\hline \multirow[t]{2}{*}{$\begin{array}{l}\text { Huang, } \\
2012, \\
22480863, \\
\text { Taiwan }\end{array}$} & NR & High & $\begin{array}{l}\text { INCLUSION: Scheduled to undergo unilateral, } \\
\text { primary TKA for advanced OA, the ability to follow } \\
\text { rehabilitation program and an interval of } 4 \text { weeks } \\
\text { between enrolment and surgery. EXCLUSION: } \\
\text { Inflammatory arthritis (e.g., RA or psoriatic } \\
\text { arthritis), any medical condition in which a } \\
\text { moderate level of exercise was contraindicated } \\
\text { (e.g., heart failure or hypertension). }\end{array}$ & $\begin{array}{l}\text { Preoperative rehabilitation education } \\
\text { program } \\
\text { Comp: S-F-E } \\
\text { AdjMod: - } \\
\text { Set: O, H }\end{array}$ & 126 & $69.8(7.2)$ & $70 \%$ & $27.1(4.0)$ & NR \\
\hline & No data & No data & No data & $\begin{array}{l}\text { Conventional care } \\
\text { Comp: - } \\
\text { AdjMod: - } \\
\text { Set: - }\end{array}$ & 117 & $70.5(7.4)$ & $74 \%$ & $27.2(4.5)$ & NR \\
\hline \multirow[t]{2}{*}{$\begin{array}{l}\text { Huber, } \\
2015, \\
25925404, \\
\text { Switzerland }\end{array}$} & $\begin{array}{l}\text { Non- } \\
\text { industry }\end{array}$ & High & $\begin{array}{l}\text { INCLUSION: 55-90 yo, living at home, on waitlist } \\
\text { for primary TKR, sufficient time prior to surgery to } \\
\text { take (minimum } 8 \text { sessions of the training program. } \\
\text { EXCLUSION: Revision surgery, history of } \\
\text { inflammatory arthritis, cognitive impairments, } \\
\text { absence before or after surgery, inability to walk } \\
\text { at least } 3 \text { meters with or without a walking aid. }\end{array}$ & $\begin{array}{l}\text { Neuromuscular training program } \\
\text { (NEMEX-TJR) \& knee school } \\
\text { Comp: S-A-F-B-T-E } \\
\text { AdjMod: - } \\
\text { Set: O }\end{array}$ & 22 & $68.8(8.0)$ & $50 \%$ & $30.8(4.9)$ & NR \\
\hline & No data & No data & No data & $\begin{array}{l}\text { Knee school } \\
\text { Comp: E } \\
\text { AdjMod: - } \\
\text { Set: O } \\
\end{array}$ & 23 & $71.9(8.1)$ & $44 \%$ & $29.9(5.5)$ & NR \\
\hline
\end{tabular}




\begin{tabular}{|c|c|c|c|c|c|c|c|c|c|}
\hline $\begin{array}{l}\text { Study } \\
\text { Year, } \\
\text { PMID, } \\
\text { Country }\end{array}$ & $\begin{array}{l}\text { Funding } \\
\text { Source }\end{array}$ & $\begin{array}{l}\text { Risk of } \\
\text { Bias }\end{array}$ & Eligibility Criteria & Intervention ${ }^{B}$ & $\begin{array}{l}\mathrm{N}, \\
\text { Enrolled }\end{array}$ & $\begin{array}{l}\text { Mean Age, } \\
\text { Years (SD) }\end{array}$ & $\begin{array}{l}\text { Female, } \\
\%\end{array}$ & $\begin{array}{l}\text { Mean } \\
\text { BMIC }^{\mathrm{C}} \text { (SD) }\end{array}$ & $\begin{array}{l}\text { Prior } \\
\text { Contralateral } \\
\text { Arthroplasty }\end{array}$ \\
\hline \multirow[t]{2}{*}{$\begin{array}{l}\text { Matassi, } \\
2014 \\
23271039 \\
\text { Belgium }\end{array}$} & NR & Moderate & $\begin{array}{l}\text { INCLUSION: } 18-90 \text { yo, non-inflammatory OA, } \\
\text { scheduled primary unilateral TKA, moderate to } \\
\text { severe pain in the affected knee. EXCLUSION: } \\
\text { BMI > 35, physical activity needs less than } \\
\text { moderate, previous hip or knee replacement } \\
\text { surgery in the last } 6 \text { months, failed total or } \\
\text { unicondylar knee replacement of the affected } \\
\text { knee or high tibial osteotomy of the affected knee, } \\
\text { active local infection or systemic infection, } \\
\text { physical, emotional or neurological conditions that } \\
\text { would compromise the patients compliance with } \\
\text { the preoperative home exercise regime, } \\
\text { postoperative rehabilitation and follow-up, grade } \\
\text { three collateral ligament insufficiency, knee } \\
\text { flexion less than } 80, \text { fixed flexion deformity } \\
\text { greater then } 20, \text { varus or valgus align- ment } \\
\text { greater than } 10 \text { unless correctable to under } 10, \\
\text { immunosuppressive disorder, } \\
\text { immunosuppressive therapy or auto-immune } \\
\text { diseases including inflammatory arthritis, intra- } \\
\text { articular steroid infiltration in the affected knee } \\
\text { within } 6 \text { weeks of the baseline assessment, } \\
\text { recent fracture ( } 3 \text { months) of upper or lower } \\
\text { extremity, inability to understand the study } \\
\text { (dementia, language problem), physiotherapy for } \\
\text { the affected knee during the preceding } 6 \text { months. }\end{array}$ & $\begin{array}{l}\text { Preoperative home exercise program } \\
\text { Comp: S-F-E } \\
\text { AdjMod: - } \\
\text { Set: H }\end{array}$ & 61 & $66(7.2)$ & $54 \%$ & $29(4.3)$ & NR \\
\hline & No data & No data & No data & $\begin{array}{l}\text { Control } \\
\text { Comp: - } \\
\text { AdjMod: - } \\
\text { Set: - }\end{array}$ & 61 & $67(7.7)$ & $43 \%$ & $28(3.7)$ & NR \\
\hline \multirow[t]{2}{*}{$\begin{array}{l}\text { Mat Eil } \\
\text { Ismail, } \\
2016, \\
26996450 \\
\text { Malaysia }\end{array}$} & NR & High & $\begin{array}{l}\text { INCLUSION: > } 45 \text { yr, lived within a convenient } \\
\text { distance of the physiotherapy facility, had been } \\
\text { diagnosed with unilateral or bilateral primary knee } \\
\text { OA; and underwent unilateral TKA at HUSM. } \\
\text { EXCLUSION: Systemic inflammatory arthritis; } \\
\text { degenerative joint diseases involving the hip or } \\
\text { ankle joint or spine; medical comorbidities with an } \\
\text { inability to tolerate a moderate level of physical } \\
\text { exertion; premorbid knee joint stiffness; history of } \\
\text { cardiovascular accident; and cognitive, } \\
\text { psychological or language impairment. }\end{array}$ & $\begin{array}{l}\text { Prehabilitation } \\
\text { Comp: S-F } \\
\text { AdjMod: Heat } \\
\text { Set: NR }\end{array}$ & 24 & $62.4(\mathrm{NR})$ & $92 \%$ & NR & NR \\
\hline & No data & No data & No data & $\begin{array}{l}\text { No prehabilitation } \\
\text { Comp: - } \\
\text { AdjMod: - } \\
\text { Set: - }\end{array}$ & 26 & 64.3 (NR) & $81 \%$ & NR & NR \\
\hline
\end{tabular}




\begin{tabular}{|c|c|c|c|c|c|c|c|c|c|}
\hline $\begin{array}{l}\text { Study }^{\mathrm{A}} \text {, } \\
\text { Year, } \\
\text { PMID, } \\
\text { Country }\end{array}$ & $\begin{array}{l}\text { Funding } \\
\text { Source }\end{array}$ & $\begin{array}{l}\text { Risk of } \\
\text { Bias }\end{array}$ & Eligibility Criteria & Intervention ${ }^{B}$ & $\begin{array}{l}\mathbf{N}, \\
\text { Enrolled }\end{array}$ & $\begin{array}{l}\text { Mean Age, } \\
\text { Years (SD) }\end{array}$ & $\begin{array}{l}\text { Female, } \\
\%\end{array}$ & $\begin{array}{l}\text { Mean } \\
\text { BMIC }^{C} \text { (SD) }\end{array}$ & $\begin{array}{l}\text { Prior } \\
\text { Contralateral } \\
\text { Arthroplasty }\end{array}$ \\
\hline \multirow[t]{2}{*}{$\begin{array}{l}\text { Mitchell, } \\
2005, \\
15869558, \\
\text { UK }\end{array}$} & $\begin{array}{l}\text { Non- } \\
\text { industry }\end{array}$ & High & $\begin{array}{l}\text { INCLUSION: primary unilateral TKA for OA. } \\
\text { EXCLUSION: revision TKA, bilateral and } \\
\text { unicondylar knee replacements, TKA for trauma, } \\
\text { onset of serious comorbidity or terminal illness } \\
\text { since patient placed on the waiting list, } \\
\text { contralateral knee replacement within the } \\
\text { preceding } 12 \text { months. }\end{array}$ & $\begin{array}{l}\text { Home pre-operative and post- } \\
\text { operative rehabilitation } \\
\text { Comp: F-T-E } \\
\text { AdjMod: Massage } \\
\text { Set: H }\end{array}$ & 57 & $70.0(7.2)$ & $63 \%$ & NR & $16 \%$ \\
\hline & No data & No data & No data & $\begin{array}{l}\text { Hospital outpatient post-operative } \\
\text { rehabilitation } \\
\text { Comp: F-T } \\
\text { AdjMod: TENS, NMES } \\
\text { Set: O }\end{array}$ & 58 & $70.6(8.2)$ & $53 \%$ & NR & $26 \%$ \\
\hline \multirow[t]{2}{*}{$\begin{array}{l}\text { Skoffer, } \\
2016, \\
26713665, \\
\text { Denmark }\end{array}$} & $\begin{array}{l}\text { Non- } \\
\text { industry }\end{array}$ & Moderate & $\begin{array}{l}\text { INCLUSION: >18 yo, scheduled for primary } \\
\text { unilateral TKA, were radiographically and } \\
\text { clinically diagnosed with OA, residents in the } \\
\text { Aarhus municipality, able to transport themselves } \\
\text { to training. EXCLUSION: Heart disease or } \\
\text { uncontrolled hypertension, neuromuscular or } \\
\text { neurodegenerative conditions, unable to } \\
\text { comprehend the protocol instructions }\end{array}$ & $\begin{array}{l}\text { Preoperative progressive resistance } \\
\text { training } \\
\text { Comp: S-F } \\
\text { AdjMod: - } \\
\text { Set: O }\end{array}$ & 30 & $70.7(7.3)$ & $63 \%$ & $\begin{array}{l}30.0 \\
\text { Range } \\
(22.6 \\
42.5)\end{array}$ & $10 \%$ \\
\hline & No data & No data & No data & $\begin{array}{l}\text { Standard care preoperatively } \\
\text { Comp: - } \\
\text { AdjMod: - } \\
\text { Set: - }\end{array}$ & 29 & $70.1(6.4)$ & $59 \%$ & $\begin{array}{l}31.8 ; \\
\text { Range } \\
(24.3 \\
42.2) \\
\end{array}$ & $14 \%$ \\
\hline \multirow[t]{2}{*}{$\begin{array}{l}\text { Soeters, } \\
2018 \\
29529614 \\
\text { USA }\end{array}$} & NR & Moderate & $\begin{array}{l}\text { INCLUSION: } 18-85 \text { yo, scheduled for unilateral } \\
\text { THA or TKA, able to independently ambulate a } \\
\text { half a block or more with or without an assistive } \\
\text { device, able to independently perform } \\
\text { nonreciprocal stairs with or without assistive } \\
\text { devices, and planned to be discharged home after } \\
\text { surgery. EXCLUSION: Patients who did not } \\
\text { undergo scheduled surgery, underwent a } \\
\text { procedure other than primary TJA, or were } \\
\text { discharged to inpatient rehabilitation centers }\end{array}$ & $\begin{array}{l}\text { Preoperative physical therapy } \\
\text { education (PreopPTEd) } \\
\text { Comp: T-E } \\
\text { AdjMod: - } \\
\text { Set: NR }\end{array}$ & 32 & $\begin{array}{l}61(9) ; \\
\text { Range (37- } \\
98)^{\mathrm{D}}\end{array}$ & $44 \%$ & $\begin{array}{l}29(6) ; \\
\text { Range } \\
\text { (19-46) }\end{array}$ & NR \\
\hline & No data & No data & No data & $\begin{array}{l}\text { No preoperative physical therapy } \\
\text { education } \\
\text { Comp: - } \\
\text { AdjMod: - } \\
\text { Set: - }\end{array}$ & 31 & $\begin{array}{l}62(8) ; \\
\text { Range (45- } \\
85)\end{array}$ & $29 \%$ & $\begin{array}{l}29(6) ; \\
\text { Range } \\
\text { (17-48) }\end{array}$ & NR \\
\hline
\end{tabular}




\begin{tabular}{|c|c|c|c|c|c|c|c|c|c|}
\hline $\begin{array}{l}\text { Study }^{\mathrm{A}} \\
\text { Year, } \\
\text { PMID, } \\
\text { Country }\end{array}$ & $\begin{array}{l}\text { Funding } \\
\text { Source }\end{array}$ & $\begin{array}{l}\text { Risk of } \\
\text { Bias }\end{array}$ & Eligibility Criteria & Intervention ${ }^{B}$ & $\begin{array}{l}\mathrm{N}, \\
\text { Enrolled }\end{array}$ & $\begin{array}{l}\text { Mean Age, } \\
\text { Years (SD) }\end{array}$ & $\begin{array}{l}\text { Female, } \\
\%\end{array}$ & $\begin{array}{l}\text { Mean } \\
\text { BMIC }^{\mathrm{C}} \text { (SD) }\end{array}$ & $\begin{array}{l}\text { Prior } \\
\text { Contralateral } \\
\text { Arthroplasty }\end{array}$ \\
\hline \multirow[t]{2}{*}{$\begin{array}{l}\text { Soni, } \\
2012 \\
22914302 \\
\text { UK }\end{array}$} & $\begin{array}{l}\text { Non- } \\
\text { industry }\end{array}$ & Moderate & $\begin{array}{l}\text { INCLUSION: Listed for knee arthroplasty due to } \\
\text { OA who had unilateral or bilateral knee pain } \\
\text { lasting more than } 3 \text { months. EXCLUSION: } \\
\text { Anticoagulants or diagnosed as having a bleeding } \\
\text { diathesis, needle-phobic, allergic to metal, } \\
\text { experiencing any skin disease around the knee, } \\
\text { within } 3 \text { months of receiving an intra-articular } \\
\text { steroid injection, experiencing back or hip pain, } \\
\text { diagnosed as having RA, within } 12 \text { months of } \\
\text { receiving acupuncture or physiotherapy }\end{array}$ & $\begin{array}{l}\text { Acupuncture \& exercise } \\
\text { Comp: S-F-B-T } \\
\text { AdjMod: Acupuncture } \\
\text { Set: O }\end{array}$ & 28 & $66.9(9.8)$ & $54 \%$ & $31.4(4.2)$ & NR \\
\hline & No data & No data & No data & $\begin{array}{l}\text { Exercise \& advice leaflet } \\
\text { Comp: - } \\
\text { AdjMod: - } \\
\text { Set: - }\end{array}$ & 28 & $70.0(7.9)$ & $46 \%$ & $31.1(4.9)$ & NR \\
\hline \multirow[t]{2}{*}{$\begin{array}{l}\text { Topp, } \\
2009, \\
19695525 \\
\text { USA }\end{array}$} & $\begin{array}{l}\text { Non- } \\
\text { industry } \\
\& \\
\text { Industry }\end{array}$ & High & $\begin{array}{l}\text { INCLUSION: } \geq 50 \text { yo, scheduled for a unilateral } \\
\text { TKA, and did not meet standard exclusion criteria } \\
\text { for engaging in moderate intensity exercise. } \\
\text { EXCLUSION: NR }\end{array}$ & $\begin{array}{l}\text { Prehabilitation exercises } \\
\text { Comp: S-F-T } \\
\text { AdjMod: - } \\
\text { Set: O, H }\end{array}$ & 26 & $64.1(7.1)$ & $27 \%$ & $32.2(5.9)$ & NR \\
\hline & No data & No data & No data & $\begin{array}{l}\text { Usual care } \\
\text { Comp: - } \\
\text { AdjMod: - } \\
\text { Set: - }\end{array}$ & 28 & $63.5(6.7)$ & $36 \%$ & $32.0(6.1)$ & NR \\
\hline \multirow{2}{*}{$\begin{array}{l}\text { Valtonen, } \\
2015 \\
\text { CN- } \\
01126383 \\
\text { Finland }\end{array}$} & NR & High & $\begin{array}{l}\text { INCLUSION: Scheduled for unilateral TKA } \\
\text { EXCLUSION: NR }\end{array}$ & $\begin{array}{l}\text { Aquatic training } \\
\text { Comp: S } \\
\text { AdjMod: - } \\
\text { Set: Aquatic center }\end{array}$ & 31 & NR & NR & NR & NR \\
\hline & No data & No data & No data & $\begin{array}{l}\text { Control } \\
\text { Comp: - } \\
\text { AdjMod:- } \\
\text { Set: - }\end{array}$ & 24 & NR & NR & NR & NR \\
\hline \multirow[t]{2}{*}{$\begin{array}{l}\text { Villadsen, } \\
2014, \\
23661494, \\
\text { Denmark }\end{array}$} & $\begin{array}{l}\text { Non- } \\
\text { industry } \\
\text { and } \\
\text { Industry }\end{array}$ & Moderate & $\begin{array}{l}\text { INCLUSION: } \geq 18 \text { years, scheduled for primary } \\
\text { unilateral THA or TKA due to severe symptomatic } \\
\text { OA. EXCLUSION: current or previous fractures in } \\
\text { or adjacent to the joint, inflammatory arthritis and } \\
\text { comorbidity (e.g., severe heart disease and } \\
\text { neurological deficits) contraindicating exercise } \\
\text { and testing, scheduled for bilateral TJA }\end{array}$ & $\begin{array}{l}\text { Neuromuscular exercise (NEMEX- } \\
\text { TJR) \& standard education package } \\
\text { Comp: S-A-F-B-T } \\
\text { AdjMod: - } \\
\text { Set: O }\end{array}$ & 41 & $67.1(8.8)$ & $61 \%$ & $30.8(4.9)$ & NR \\
\hline & No data & No data & No data & $\begin{array}{l}\text { Standard education package } \\
\text { Comp: - } \\
\text { AdjMod: - } \\
\text { Set: - } \\
\end{array}$ & 40 & $65.1(9.0)$ & $60 \%$ & $33.4(5.8)$ & NR \\
\hline
\end{tabular}




\begin{tabular}{|c|c|c|c|c|c|c|c|c|c|}
\hline $\begin{array}{l}\text { Study } \\
\text { Year, } \\
\text { PMID, } \\
\text { Country }\end{array}$ & $\begin{array}{l}\text { Funding } \\
\text { Source }\end{array}$ & $\begin{array}{l}\text { Risk of } \\
\text { Bias }\end{array}$ & Eligibility Criteria & Intervention $^{B}$ & $\begin{array}{l}\mathrm{N}, \\
\text { Enrolled }\end{array}$ & $\begin{array}{l}\text { Mean Age, } \\
\text { Years (SD) }\end{array}$ & $\begin{array}{l}\text { Female, } \\
\%\end{array}$ & $\begin{array}{l}\text { Mean } \\
\text { BMIC }^{C} \text { (SD) }\end{array}$ & $\begin{array}{l}\text { Prior } \\
\text { Contralateral } \\
\text { Arthroplasty }\end{array}$ \\
\hline \multirow[t]{3}{*}{$\begin{array}{l}\text { Williamson, } \\
2007 \\
17604311 \\
\text { UK }\end{array}$} & $\begin{array}{l}\text { Non- } \\
\text { industry }\end{array}$ & Moderate & $\begin{array}{l}\text { INCLUSION: knee arthroplasty due to OA; with } \\
\text { unilateral or bilateral knee pain lasting }>3 \\
\text { months. EXCLUSION: taking anticoagulants; } \\
\text { within } 2 \text { months after receiving an intra-articular } \\
\text { steroid injection; experiencing back pain } \\
\text { associated with referred leg pain; suffering from } \\
\text { ipsilateral OA of the hip; suffering psoriasis or } \\
\text { other skin disease in the region of the knee; } \\
\text { suffering from RA; received acupuncture or } \\
\text { physiotherapy treatment in the previous year. }\end{array}$ & $\begin{array}{l}\text { Acupuncture } \\
\text { Comp: - } \\
\text { AdjMod: Acupuncture } \\
\text { Set: O }\end{array}$ & 60 & $72.4(7.7)$ & $55 \%$ & $30.9(6.0)$ & NR \\
\hline & No data & No data & No data & $\begin{array}{l}\text { Physiotherapy (supervised exercise) } \\
\text { Comp: S-F-B-T } \\
\text { AdjMod:- } \\
\text { Set: O }\end{array}$ & 60 & $70.0(8.8)$ & $52 \%$ & $32.8(5.7)$ & NR \\
\hline & No data & No data & No data & $\begin{array}{l}\text { Control } \\
\text { Comp: - } \\
\text { AdjMod: - } \\
\text { Set: - }\end{array}$ & 61 & $69.6(10)$ & $54 \%$ & $32.7(6.4)$ & NR \\
\hline
\end{tabular}

Abbreviations: $\mathrm{BMI}=\mathrm{H}=$ home, $\mathrm{NA}=$ not applicable, NMES $=$ neuromuscular electrical stimulation, $\mathrm{NR}=$ not reported, $\mathrm{O}=$ outpatient physiotherapy center, $\mathrm{OA}=$ osteoarthritis, $\mathrm{PMID}=$ PubMed identifier, RA = rheumatoid arthritis, $\mathrm{SD}=$ standard deviation, $\mathrm{SD}=$ standard deviation, TENS = transcutaneous electrical nerve stimulation, THA = total hip arthroplasty, TJA = total joint arthroplasty, TKA = total knee arthroplasty, yo = years old

A All randomized controlled trials, except as footnoted. There were no non-randomized comparative studies in Key Question 1.

B Including Components (Comp); Adjunctive modalities (AdjMod); and Setting (Set).

Components: $\mathrm{A}=$ aerobic exercise, $\mathrm{B}=$ Balance-motor/Learning-agility exercise, $\mathrm{E}=$ patient education, $\mathrm{F}=$ flexibility exercise, $\mathrm{S}=$ strengthening exercise, $\mathrm{T}=$ task-specific training.

C $\mathrm{kg} / \mathrm{m}^{2}$

D Reported age, gender, BMI data for total joint replacement population (TKA and THA) combined 
Table C-1.2. KQ 1. Prehabilitation component details

\begin{tabular}{|c|c|c|c|c|c|c|c|}
\hline $\begin{array}{l}\text { Study, } \\
\text { Year, } \\
\text { PMID, } \\
\text { Country }\end{array}$ & Intended Comparison & Arm & Components (Specific Exercises/Strategies) & $\begin{array}{l}\text { Progression } \\
\text { (Appropriate?) }\end{array}$ & Personnel & $\begin{array}{l}\text { Mode of } \\
\text { Delivery }\end{array}$ & Setting \\
\hline \multirow[t]{2}{*}{$\begin{array}{l}\text { Calatayud, } \\
2017 \\
26768606, \\
\text { Spain }\end{array}$} & $\begin{array}{l}\text { High intensity } \\
\text { preoperative training vs } \\
\text { control group } \\
\text { [undescribed] }\end{array}$ & $\begin{array}{l}\text { High intensity } \\
\text { preoperative training }\end{array}$ & $\begin{array}{l}\text { 1. Strength } \\
\text { 1.9 Heel raises - bilateral (calf raises) } \\
\text { 1.11 Hip abduction in sidelying (position unclear) } \\
\text { 1.12 Hip abduction in standing (position unclear) } \\
\text { 1.13 Hip abduction in supine (position unclear) } \\
\text { 1.31 Knee extension in sitting or supine (long arc quad) } \\
\text { (position unclear) } \\
\text { 1.32 Knee extension in sitting or supine (short arc quad) } \\
\text { (position unclear) } \\
\text { 1.35 Knee flexion in prone (position unclear) } \\
\text { 1.36 Knee flexion in sitting or supine (position unclear) } \\
\text { 1.37 Knee flexion in standing(position unclear) } \\
\text { 1.38 Leg press (one leg) (one or two legs unclear) } \\
\text { 1.39 Leg press (two legs) (one or two legs unclear) } \\
\text { 1.47 Single leg stance } \\
\text { 1.55 Step up - forward } \\
\text { 3. Flexibility } \\
\text { 3.2 Bike (ROM) } \\
\text { 3.3 Calf stretch with knee bent (soleus) (unclear bent or } \\
\text { straight) } \\
\text { 3.4 Calf stretch with knee straight (gastric) (unclear bent or } \\
\text { straight) } \\
\text { 3.9 lliotibial band stretch in any position } \\
\text { 3.15 Knee flexion AROM in any position (rectus femoris } \\
\text { stretch) } \\
\text { 3.16 Knee flexion PROM in prone (rectus femoris stretch) } \\
\text { 4. Balance-Motor Learning-Agility } \\
\text { 4.3 Balance on unstable surface }\end{array}$ & $\mathrm{Y}(\mathrm{Y})$ & $\begin{array}{l}\text { Physical } \\
\text { therapist }\end{array}$ & In-person & $\begin{array}{l}\text { Physical } \\
\text { therapy/rehabilitation } \\
\text { facility (outpatient) }\end{array}$ \\
\hline & No data & Control & $\begin{array}{l}\text { NR } \\
\text { [No description provided] }\end{array}$ & NR & NR & NR & NR \\
\hline \multirow[t]{2}{*}{$\begin{array}{l}\text { Huang, } \\
2012, \\
22480863, \\
\text { Taiwan }\end{array}$} & $\begin{array}{l}\text { Preoperative rehabilitation } \\
\text { education program vs. No } \\
\text { preoperative rehabilitation } \\
\text { education program } \\
\text { (conventional care) }\end{array}$ & $\begin{array}{l}\text { Preoperative } \\
\text { rehabilitation } \\
\text { education program }\end{array}$ & $\begin{array}{l}\text { 1. Strength (goal) } \\
\text { 1.11 Hip abduction in sidelying (position unclear) } \\
\text { 1.12 Hip abduction in standing (position unclear) } \\
\text { 1.13 Hip abduction in supine (position unclear) } \\
\text { 1.43 Quad sets } \\
\text { 1.58 Straight leg raise } \\
\text { 3. Flexibility } \\
\text { 3.1 Ankle pumps } \\
\text { 6. Patient education } \\
\text { 6.1 ADLs } \\
\text { 6.2 Home exercise program }\end{array}$ & $\mathrm{N}(\mathrm{NA})$ & $\begin{array}{l}\text { Physical } \\
\text { therapist; } \\
\text { None } \\
\text { (unsupervised) }\end{array}$ & $\begin{array}{l}\text { In-person; Self- } \\
\text { guided } \\
\text { (unsupervised); } \\
\text { Remote via } \\
\text { telephone }\end{array}$ & $\begin{array}{l}\text { Physical } \\
\text { therapy/rehabilitation } \\
\text { facility (outpatient); } \\
\text { Home }\end{array}$ \\
\hline & No data & $\begin{array}{l}\text { Conventional pre- } \\
\text { TKA care }\end{array}$ & $\begin{array}{l}\text { NA } \\
\text { [No prehab or education; usual activities and exercise not } \\
\text { prohibited] }\end{array}$ & NA & NA & NA & NA \\
\hline
\end{tabular}




\begin{tabular}{|c|c|c|c|c|c|c|c|}
\hline $\begin{array}{l}\text { Study, } \\
\text { Year, } \\
\text { PMID, } \\
\text { Country }\end{array}$ & Intended Comparison & Arm & Components (Specific Exercises/Strategies) & $\begin{array}{l}\text { Progression } \\
\text { (Appropriate?) }\end{array}$ & Personnel & $\begin{array}{l}\text { Mode of } \\
\text { Delivery }\end{array}$ & Setting \\
\hline \multirow[t]{2}{*}{$\begin{array}{l}\text { Huber, } \\
2015, \\
25925404, \\
\text { Switzerland }\end{array}$} & $\begin{array}{l}\text { Neuromuscular training } \\
\text { program (NEMEX-TJR) \& } \\
\text { knee school (education) } \\
\text { vs. Knee school } \\
\text { (education) }\end{array}$ & $\begin{array}{l}\text { Neuromuscular } \\
\text { training program } \\
\text { (NEMEX-TJR) \& } \\
\text { knee school } \\
\text { (education) }\end{array}$ & $\begin{array}{l}\text { 1. Strength } \\
\text { 1.6 Core strengthening } \\
\text { 1.12 Hip abduction in standing } \\
\text { 1.15 Hip adduction in standing } \\
\text { 1.31 Knee extension in sitting or supine (long arc quad) } \\
\text { 1.36 Knee flexion in sitting or supine } \\
\text { 1.41 Lunges } \\
\text { 1.42 Lunges to side (lateral lunge) } \\
\text { 2. Aerobic } \\
\text { 2.2 Bike (endurance) } \\
\text { 3. Flexibility } \\
\text { [specific exercises not defined] } \\
\text { 4. Balance-Motor Learning-Agility } \\
\text { 4.4 Balance with perturbations } \\
\text { 4.11 Step down } \\
\text { 4.14 Step up-forward } \\
\text { 5. Task specific training } \\
\text { 5.4 Gait backwards } \\
\text { 5.8 Gait training } \\
\text { 5.13 Sit-to-stand training } \\
\text { 5.15 Stair training } \\
\text { 6. Patient education } \\
\text { 6.4 Pain management } \\
\end{array}$ & $\mathrm{Y}(\mathrm{Y})$ & $\begin{array}{l}\text { Physical } \\
\text { therapist }\end{array}$ & In-person & $\begin{array}{l}\text { Physical } \\
\text { therapy/rehabilitation } \\
\text { facility (outpatient) }\end{array}$ \\
\hline & No data & $\begin{array}{l}\text { Knee school } \\
\text { (education) }\end{array}$ & $\begin{array}{l}\text { 6. Patient education } \\
\text { 6.4 Pain management }\end{array}$ & $\mathrm{N}(\mathrm{NA})$ & $\begin{array}{l}\text { Physical } \\
\text { therapist }\end{array}$ & In-person & $\begin{array}{l}\text { Physical } \\
\text { therapy/rehabilitation } \\
\text { facility (outpatient) }\end{array}$ \\
\hline \multirow[t]{2}{*}{$\begin{array}{l}\text { Matassi, } \\
2014, \\
23271039 \\
\text { Belgium }\end{array}$} & $\begin{array}{l}\text { Preoperative home } \\
\text { exercise program vs. } \\
\text { usual activity }\end{array}$ & $\begin{array}{l}\text { Preoperative home } \\
\text { exercise program }\end{array}$ & $\begin{array}{l}\text { 1. Strength } \\
\text { 1.31 Knee extension in sitting or supine (long arc quad) } \\
\text { 1.35 Knee flexion in prone } \\
\text { 1.43 Quad sets } \\
\text { 1.52 Step down } \\
\text { 1.55 Step up - forward } \\
\text { 3. Flexibility } \\
\text { 3.5 Hamstring stretch in any position } \\
\text { 3.15 Knee flexion AROM in any position (rectus femoris } \\
\text { stretch) } \\
\text { 6. Patient education } \\
\text { 6.2 Home exercise program }\end{array}$ & $\mathrm{N}(\mathrm{NA})$ & $\begin{array}{l}\text { None } \\
\text { (unsupervised) }\end{array}$ & $\begin{array}{l}\text { Self-guided } \\
\text { (unsupervised) }\end{array}$ & Home \\
\hline & No data & Control & $\begin{array}{l}\text { NA } \\
\text { [Instructed to continue usual activities until surgery] }\end{array}$ & NA & NA & NA & NA \\
\hline
\end{tabular}




\begin{tabular}{|c|c|c|c|c|c|c|c|}
\hline $\begin{array}{l}\text { Study, } \\
\text { Year, } \\
\text { PMID, } \\
\text { Country }\end{array}$ & Intended Comparison & Arm & Components (Specific Exercises/Strategies) & $\begin{array}{l}\text { Progression } \\
\text { (Appropriate?) }\end{array}$ & Personnel & $\begin{array}{l}\text { Mode of } \\
\text { Delivery }\end{array}$ & Setting \\
\hline \multirow[t]{2}{*}{$\begin{array}{l}\text { Mat Eil } \\
\text { Ismail, } \\
2016, \\
26996450, \\
\text { Malaysia }\end{array}$} & $\begin{array}{l}\text { Preoperative } \\
\text { physiotherapy vs. No } \\
\text { preoperative } \\
\text { physiotherapy }\end{array}$ & Prehabilitation & $\begin{array}{l}\text { 1. Strength } \\
\text { 1.31 Knee extension in sitting or supine (long arc quad) } \\
\text { 1.32 Knee extension in sitting or supine (short arc quad) } \\
\text { 1.43 Quad sets } \\
\text { 1.58 Straight leg raises } \\
\text { 3. Flexibility } \\
\text { 3.1 Ankle pumps } \\
\text { 3.2 Bike (ROM) } \\
\text { 3.5 Hamstring stretch in any position } \\
\text { 3.6 Heel slides } \\
\text { 7. Adjunctive modality } \\
\text { 7.2 Heat }\end{array}$ & $\mathrm{N}(\mathrm{NA})$ & NR & NR & NR \\
\hline & No data & No prehabiltation & $\begin{array}{l}\text { NA } \\
\text { [No additional care preoperative; same postoperative as the } \\
\text { intervention group] }\end{array}$ & $\mathrm{N}(\mathrm{NA})$ & NA & NA & NA \\
\hline \multirow[t]{2}{*}{$\begin{array}{l}\text { Mitchell, } \\
2005, \\
15869558, \\
\text { UK }\end{array}$} & $\begin{array}{l}\text { Pre- and post-operative } \\
\text { physiotherapy at home vs. } \\
\text { hospital outpatient post- } \\
\text { operative } \\
\text { physiotherapy }\end{array}$ & $\begin{array}{l}\text { Home pre-operative } \\
\text { and post-operative } \\
\text { rehabilitation }\end{array}$ & $\begin{array}{l}\text { 3. Flexibility } \\
\text { 3.10 Knee extension AROM (unclear) } \\
\text { 3.11 Knee extension PROM in supine (unclear) } \\
\text { 3.12 Knee extension PROM in prone (unclear) } \\
\text { 3.13 Knee flexion AROM (unclear) } \\
\text { 3.14 Knee flexion PROM in sitting or supine (unclear) } \\
\text { 3.15 Knee flexion AROM in any position (rectus femoris } \\
\text { stretch) (unclear) } \\
\text { 3.16 Knee flexion PROM in prone (rectus femoris stretch) } \\
\text { (unclear) } \\
\text { 5. Task specific training } \\
\text { 5.8 Gait training } \\
\text { 6. Patient education } \\
\text { 6.1 ADLs } \\
\text { 6.4 Pain management } \\
\text { 7. Adjunctive modality } \\
\text { 7.12 Massage/myofascial techniques for soft tissue } \\
\end{array}$ & $\mathrm{N}$ (NA) & $\begin{array}{l}\text { Physical } \\
\text { therapist }\end{array}$ & In-person & Home \\
\hline & No data & $\begin{array}{l}\text { Hospital outpatient } \\
\text { post-operative } \\
\text { rehabilitation }\end{array}$ & $\begin{array}{l}\text { 3. Flexibility } \\
\text { 3.10 Knee extension AROM (unclear) } \\
\text { 3.11 Knee extension PROM in supine (unclear) } \\
\text { 3.12 Knee extension PROM in prone (unclear) } \\
\text { 3.13 Knee flexion AROM (unclear) } \\
\text { 3.14 Knee flexion PROM in sitting or supine (unclear) } \\
\text { 3.15 Knee flexion AROM in any position (rectus femoris } \\
\text { stretch) (unclear) } \\
\text { 3.16 Knee flexion PROM in prone (rectus femoris stretch) } \\
\text { (unclear) } \\
\text { 5. Task specific training } \\
\text { 5.8 Gait training } \\
\text { 7. Adjunctive modality } \\
\text { 7.4 E-stim for pain (TENS) } \\
\text { 7.5 E-stim for strength (NMES) }\end{array}$ & $\mathrm{N}(\mathrm{NA})$ & $\begin{array}{l}\text { Physical } \\
\text { therapist }\end{array}$ & In-person & $\begin{array}{l}\text { Physical } \\
\text { therapy/rehabilitation } \\
\text { facility (outpatient) }\end{array}$ \\
\hline
\end{tabular}




\begin{tabular}{|c|c|c|c|c|c|c|c|}
\hline $\begin{array}{l}\text { Study, } \\
\text { Year, } \\
\text { PMID, } \\
\text { Country }\end{array}$ & Intended Comparison & Arm & Components (Specific Exercises/Strategies) & $\begin{array}{l}\text { Progression } \\
\text { (Appropriate?) }\end{array}$ & Personnel & $\begin{array}{l}\text { Mode of } \\
\text { Delivery }\end{array}$ & Setting \\
\hline \multirow[t]{2}{*}{$\begin{array}{l}\text { Skoffer, } \\
2016 \\
26713665 \\
\text { Denmark }\end{array}$} & $\begin{array}{l}\text { Preoperative and } \\
\text { postoperative progressive } \\
\text { resistance } \\
\text { training vs. postoperative } \\
\text { progressive resistance } \\
\text { training }^{B}\end{array}$ & $\begin{array}{l}\text { Preoperative } \\
\text { progressive } \\
\text { resistance training }\end{array}$ & $\begin{array}{l}\text { 1. Strength } \\
\text { 1.11 Hip abduction in sidelying (position unclear) } \\
\text { 1.12 Hip abduction in standing (position unclear) } \\
\text { 1.13 Hip abduction in supine (position unclear) } \\
\text { 1.14 Hip adduction in sidelying (position unclear) } \\
\text { 1.15 Hip adduction in standing (position unclear) } \\
\text { 1.16 Hip adduction in supine (position unclear) } \\
\text { 1.17 Hip extension in sidelying (position unclear) } \\
\text { 1.18 Hip extension in prone (position unclear) } \\
\text { 1.19 Hip extension in standing (position unclear) } \\
\text { 1.28 Knee extension machine (one-leg) } \\
\text { 1.33 Knee flexion machine (Hamstring curl) one knee } \\
\text { 1.38 Leg press (one leg) } \\
\text { 3. Flexibility } \\
\text { 3.2 Bike (ROM) } \\
\text { 3.10 Knee extension AROM (unclear) } \\
\text { 3.11 Knee extension PROM in supine (unclear) } \\
\text { 3.12 Knee extension PROM in prone (unclear) } \\
\text { 3.13 Knee flexion AROM (unclear) } \\
\text { 3.14 Knee flexion PROM in sitting or supine (unclear) } \\
\text { 3.15 Knee flexion AROM in any position (rectus femoris } \\
\text { stretch) (unclear) } \\
\text { 3.16 Knee flexion PROM in prone (rectus femoris stretch) } \\
\text { (unclear) }\end{array}$ & $\mathrm{Y}(\mathrm{N})$ & $\begin{array}{l}\text { Physical } \\
\text { therapist }\end{array}$ & In-person & $\begin{array}{l}\text { Physical } \\
\text { therapy/rehabilitation } \\
\text { facility (outpatient) }\end{array}$ \\
\hline & No data & $\begin{array}{l}\text { Standard care } \\
\text { preoperatively }\end{array}$ & $\begin{array}{l}\text { NA } \\
\text { [No training preoperatively; same postoperative progressive } \\
\text { resistance training as intervention group] }\end{array}$ & NA & NA & NA & NA \\
\hline \multirow[t]{2}{*}{$\begin{array}{l}\text { Soeters, } \\
2018 \\
29529614 \\
\text { USA }\end{array}$} & $\begin{array}{l}\text { One-on-one preoperative } \\
\text { physical therapy } \\
\text { education and microsite } \\
\text { vs. no preoperative } \\
\text { physical therapy } \\
\text { education }\end{array}$ & $\begin{array}{l}\text { Preoperative physical } \\
\text { therapy education } \\
\text { (PreopPTEd) }\end{array}$ & $\begin{array}{l}\text { 5. Task specific training } \\
\text { 5.1 Transfers } \\
\text { 5.13 Sit-to-stand training } \\
\text { 5.15 Stair training } \\
\text { 6. Patient education } \\
\text { 6.1 ADLs }\end{array}$ & & $\begin{array}{l}\text { Physical } \\
\text { therapist }\end{array}$ & In-person & $\begin{array}{l}\text { NR (Unclear if home } \\
\text { or outpatient) }\end{array}$ \\
\hline & No data & $\begin{array}{l}\text { No preoperative } \\
\text { physical therapy } \\
\text { education }\end{array}$ & $\begin{array}{l}\text { NA } \\
\text { [One preoperative group education class (usual care at the } \\
\text { institution); postoperatively received the same physical } \\
\text { therapy as the intervention group] }\end{array}$ & NA & NA & NA & NA \\
\hline
\end{tabular}




\begin{tabular}{|c|c|c|c|c|c|c|c|}
\hline $\begin{array}{l}\text { Study, } \\
\text { Year, } \\
\text { PMID, } \\
\text { Country }\end{array}$ & Intended Comparison & Arm & Components (Specific Exercises/Strategies) & $\begin{array}{l}\text { Progression } \\
\text { (Appropriate?) }\end{array}$ & Personnel & $\begin{array}{l}\text { Mode of } \\
\text { Delivery }\end{array}$ & Setting \\
\hline \multirow[t]{2}{*}{$\begin{array}{l}\text { Soni, } \\
2012, \\
22914302, \\
\text { UK }\end{array}$} & $\begin{array}{l}\text { Combined acupuncture } \\
\text { and physiotherapy vs. } \\
\text { standard care }\end{array}$ & $\begin{array}{l}\text { Acupuncture \& } \\
\text { exercise }\end{array}$ & $\begin{array}{l}\text { 1. Strength } \\
\text { 1.32 Knee extension in sitting or supine (short arc quad) } \\
\text { 1.36 Knee flexion in sitting or supine } \\
\text { 1.43 Quad sets } \\
\text { 1.48 Sit-to-stand } \\
\text { 1.58 Straight leg raise } \\
\text { 3. Flexibility } \\
\text { 3.2 Bike (ROM) } \\
\text { 3.3 Calf stretch with knee bent (soleus) (unclear knee bent or } \\
\text { straight) } \\
\text { 3.4 Calf stretch with knee straight (gastric) (unclear knee bent } \\
\text { or straight) } \\
\text { 4. Balance-Motor Learning-Agility } \\
\text { 4.3 Balance on unstable surface } \\
\text { 5. Task specific training } \\
\text { 5.15 Stair training } \\
\text { 7. Adjunctive modality } \\
\text { 7.16 Dry needling (acupuncture) }\end{array}$ & $\mathrm{N}(\mathrm{NA})$ & $\begin{array}{l}\text { Physical } \\
\text { therapist }\end{array}$ & In-person & $\begin{array}{l}\text { Physical } \\
\text { therapy/rehabilitation } \\
\text { facility (outpatient) }\end{array}$ \\
\hline & No data & $\begin{array}{l}\text { Exercise \& advice } \\
\text { leaflet }\end{array}$ & $\begin{array}{l}\text { NA } \\
\text { [Exercise and advice leaflet designed by the physiotherapy, } \\
\text { orthopedic, and rheumatology departments] }\end{array}$ & $\mathrm{N}(\mathrm{NA})$ & NA & NA & NA \\
\hline
\end{tabular}




\begin{tabular}{|c|c|c|c|c|c|c|c|}
\hline $\begin{array}{l}\text { Study, } \\
\text { Year, } \\
\text { PMID, } \\
\text { Country }\end{array}$ & Intended Comparison & Arm & Components (Specific Exercises/Strategies) & $\begin{array}{l}\text { Progression } \\
\text { (Appropriate?) }\end{array}$ & Personnel & $\begin{array}{l}\text { Mode of } \\
\text { Delivery }\end{array}$ & Setting \\
\hline \multirow[t]{2}{*}{$\begin{array}{l}\text { Topp, } \\
2009 \\
19695525 \\
\text { USA }\end{array}$} & $\begin{array}{l}\text { Prehabilitation vs. usual } \\
\text { care }\end{array}$ & $\begin{array}{l}\text { Prehabilitation } \\
\text { exercise }\end{array}$ & $\begin{array}{l}\text { 1. Strength } \\
\text { 1.11 Hip abduction in sidelying (position unclear) } \\
\text { 1.12 Hip abduction in standing (position unclear) } \\
\text { 1.13 Hip abduction in supine (position unclear) } \\
\text { 1.14 Hip adduction in sidelying (position unclear) } \\
\text { 1.15 Hip adduction in standing (position unclear) } \\
\text { 1.16 Hip adduction in supine (position unclear) } \\
\text { 1.17 Hip extension in sidelying (position unclear) } \\
\text { 1.18 Hip extension in prone (position unclear) } \\
\text { 1.19 Hip extension in standing (position unclear) } \\
\text { 1.20 Hip flexion in sidelying (position unclear) } \\
\text { 1.21 Hip flexion in sitting (position unclear) } \\
\text { 1.22 Hip flexion in standing (position unclear) } \\
\text { 1.31 Knee extension in sitting or supine (long arc quad) (long } \\
\text { or short arc unclear) } \\
\text { 1.32 Knee extension in sitting or supine (short arc quad) (long } \\
\text { or short arc unclear) } \\
\text { 1.35 Knee flexion in prone (position unclear) } \\
\text { 1.36 Knee flexion in sitting or supine (position unclear) } \\
\text { 1.37 Knee flexion in standing (position unclear) } \\
\text { 1.49 Squats } \\
\text { 1.52 Step down } \\
\text { 1.53 Step down laterally } \\
\text { 1.55 Step up - forward } \\
\text { 1.56 Step up - lateral } \\
\text { 1.63 Open chain ankle dorsiflexion/plantar } \\
\text { flexion/inversion/eversion } \\
\text { 3. Flexibility } \\
\text { 3.3 Calf stretch with knee bent (soleus) (unclear knees bent or } \\
\text { straight) } \\
\text { 3.4 Calf stretch with knee straight (gastric) (unclear knees } \\
\text { bent or straight) } \\
\text { 3.7 Hip extensor stretch (knee to chest) (unclear) } \\
\text { 3.8 Hip flexor stretch (iliopsoas) (unclear) } \\
\text { 5. Task specific training } \\
\text { [specific exercises not defined] }\end{array}$ & $\mathrm{N}(\mathrm{NA})$ & $\begin{array}{l}\text { Other } \\
\text { (research } \\
\text { personnel); } \\
\text { None } \\
\text { (unsupervised) }\end{array}$ & $\begin{array}{l}\text { In-person; Self- } \\
\text { guided } \\
\text { (unsupervised) }\end{array}$ & $\begin{array}{l}\text { Physical } \\
\text { therapy/rehabilitation } \\
\text { facility (outpatient); } \\
\text { Home }\end{array}$ \\
\hline & No data & Usual care & [Continue normal activities until the TKA] & $\mathrm{N}(\mathrm{NA})$ & NA & NA & NA \\
\hline \multirow{2}{*}{$\begin{array}{l}\text { Valtonen, } \\
2015, \\
\text { CN- } \\
01126383, \\
\text { Finland }\end{array}$} & $\begin{array}{l}\text { Preoperative progressive } \\
\text { aquatic resistance training } \\
\text { vs. Usual care } \\
\text { [Abstract only] }\end{array}$ & Aquatic training & $\begin{array}{l}\text { 1. Strength } \\
\text { [specific exercises not defined] }\end{array}$ & $\mathrm{Y}(\mathrm{N})$ & NR & In-person & $\begin{array}{l}\text { Other (aquatic } \\
\text { center) }\end{array}$ \\
\hline & No data & Control & $\begin{array}{l}\text { NA } \\
\text { [Continued life as normal] }\end{array}$ & NA & NA & NA & NA \\
\hline
\end{tabular}




\begin{tabular}{|c|c|c|c|c|c|c|c|}
\hline $\begin{array}{l}\text { Study, } \\
\text { Year, } \\
\text { PMID, } \\
\text { Country }\end{array}$ & Intended Comparison & Arm & Components (Specific Exercises/Strategies) & $\begin{array}{l}\text { Progression } \\
\text { (Appropriate?) }\end{array}$ & Personnel & $\begin{array}{l}\text { Mode of } \\
\text { Delivery }\end{array}$ & Setting \\
\hline \multirow[t]{2}{*}{$\begin{array}{l}\text { Villadsen, } \\
2014, \\
23661494 \\
\text { Denmark }\end{array}$} & $\begin{array}{l}\text { Preoperative } \\
\text { neuromuscular exercise } \\
\text { program plus standard } \\
\text { education vs. standard } \\
\text { education alone }\end{array}$ & $\begin{array}{l}\text { Neuromuscular } \\
\text { exercise (NEMEX- } \\
\text { TJR) \& standard } \\
\text { education package }\end{array}$ & $\begin{array}{l}\text { 1. Strength } \\
\text { 1.2 Bridges Two-legged (supine hip extension) } \\
\text { 1.6 Core strengthening } \\
\text { 1.12 Hip abduction in standing } \\
\text { 1.15 Hip adduction in standing } \\
\text { 1.31 Knee extension in sitting or supine (long arc quad) } \\
\text { 1.36 Knee flexion in sitting or supine } \\
\text { 1.41 Lunges } \\
\text { 1.42 Lunges to side (lateral lunge) } \\
\text { 1.52 Step down } \\
\text { 1.55 Step up - forward } \\
\text { 2. Aerobic } \\
\text { 2.2 Bike (Endurance) } \\
\text { 3. Flexibility } \\
\text { 3.2 Bike (ROM) } \\
\text { 4. Balance-Motor Learning-Agility } \\
\text { [specific exercises not defined] } \\
\text { 5. Task specific training } \\
\text { 5.4 Gait backwards } \\
\text { 5.8 Gait training } \\
\text { 5.13 Sit-to-stand training }\end{array}$ & $\mathrm{Y}(\mathrm{Y})$ & $\begin{array}{l}\text { Physical } \\
\text { therapist }\end{array}$ & In-person & $\begin{array}{l}\text { Physical } \\
\text { therapy/rehabilitation } \\
\text { facility (outpatient) }\end{array}$ \\
\hline & No data & $\begin{array}{l}\text { Standard education } \\
\text { package }\end{array}$ & $\begin{array}{l}\text { NA } \\
\text { [No limitations regarding exercise habits or seeking other } \\
\text { treatment] }\end{array}$ & NA & NA & NA & NA \\
\hline \multirow[t]{3}{*}{$\begin{array}{l}\text { Williamson, } \\
2007 \\
17604311 \\
\text { UK }\end{array}$} & $\begin{array}{l}\text { Preoperative acupuncture } \\
\text { vs. preoperative } \\
\text { physiotherapy (supervised } \\
\text { exercise) vs. standard } \\
\text { care }\end{array}$ & Acupuncture & $\begin{array}{l}\text { 7. Adjunctive modality } \\
\text { 7.16 Dry needling (acupuncture) }\end{array}$ & $\mathrm{N}(\mathrm{NA})$ & $\begin{array}{l}\text { Physical } \\
\text { therapist }\end{array}$ & In-person & $\begin{array}{l}\text { Physical } \\
\text { therapy/rehabilitation } \\
\text { facility (outpatient) }\end{array}$ \\
\hline & No data & $\begin{array}{l}\text { Physiotherapy } \\
\text { (supervised exercise) }\end{array}$ & $\begin{array}{l}\text { 1. Strength } \\
\text { 1.30 Knee extension AAROM in sitting or supine (short- or } \\
\text { long arc quad) (long or short unclear) } \\
\text { 1.31 Knee extension in sitting or supine (long arc quad) (long } \\
\text { or short unclear) } \\
\text { 1.43 Quad sets } \\
\text { 1.48 Sit-to-stand } \\
\text { 1.58 Straight leg raise } \\
\text { 3. Flexibility } \\
\text { 3.2 Bike (ROM) } \\
\text { 3.3 Calf stretch with knee bent (soleus) (unclear knees bent or } \\
\text { straight) } \\
\text { 3.4 Calf stretch with knee straight (gastric) (unclear knees } \\
\text { bent or straight) } \\
\text { 4. Balance-Motor Learning-Agility } \\
\text { 4.3 Balance on unstable surface } \\
\text { 5. Task specific training } \\
\text { 5.15 Stair training }\end{array}$ & $\mathrm{N}(\mathrm{NA})$ & $\begin{array}{l}\text { Physical } \\
\text { therapist }\end{array}$ & In-person & $\begin{array}{l}\text { Physical } \\
\text { therapy/rehabilitation } \\
\text { facility (outpatient) }\end{array}$ \\
\hline & No data & Control & $\begin{array}{l}\text { NA } \\
\text { [Exercise and advice leaflet] }\end{array}$ & $\mathrm{N}(\mathrm{NA})$ & $\begin{array}{l}\text { None } \\
\text { (unsupervised) }\end{array}$ & $\begin{array}{l}\text { None } \\
\text { (unsupervised) }\end{array}$ & Home \\
\hline
\end{tabular}


Abbreviations: AAROM = assisted active range of motion, $\mathrm{ADL}=$ activities of daily living, $\mathrm{AROM}=$ active range of motion, $\mathrm{NA}=$ not applicable, NMES $=$ neuromuscular electrical stimulation $\mathrm{NR}=$ not reported, $\mathrm{PROM}=$ passive range of motion, $\mathrm{ROM}=$ range of motion, $\mathrm{TENS}=$ transcutaneous electrical nerve stimulation, $\mathrm{TKA}=$ total knee arthroplasty.

A Where position was not specified, all positions were coded with a comment of 'position unclear'

B Only preoperative exercises were coded

Table C-1.3. KQ 1. Risk of bias assessment for primary studies - randomized controlled trials (RCTs)

\begin{tabular}{|c|c|c|c|c|c|c|c|c|c|c|c|c|}
\hline Study, Year, PMID & Random & Allocation & $\begin{array}{l}\text { Blinding, } \\
\text { Participants }\end{array}$ & $\begin{array}{l}\text { Blinding, } \\
\text { Providers }\end{array}$ & $\begin{array}{l}\text { Blinding, Outcome, } \\
\text { Obj / Subj }\end{array}$ & Dropout & $\begin{array}{l}\text { Reporting } \\
\text { Bias }\end{array}$ & Other & Population & Intervention & Outcomes & Overall RoB \\
\hline $\begin{array}{l}\text { Calatayud, 2017, } \\
26768606\end{array}$ & Low & Low & High & High & Unsure & Low & Low & Low & No & No & No & Moderate \\
\hline $\begin{array}{l}\text { Huang, 2012, } \\
22480863\end{array}$ & Unsure & Unsure & High & High & Low & Low & Low & Low & No & No & No & High \\
\hline $\begin{array}{l}\text { Huber, 2015, } \\
25925404\end{array}$ & Low & Low & High & High & Low & Low & High & High $^{A}$ & No & No & No & High \\
\hline $\begin{array}{l}\text { Matassi, } 2014, \\
23271039\end{array}$ & Low & Unsure & High & High & Low & Low & Low & Low & No & No & No & Moderate \\
\hline $\begin{array}{l}\text { Mat Eli Ismail, 2016, } \\
26996450\end{array}$ & High & Unsure & High & High & High & Low & Low & Low & No & No & No & High \\
\hline $\begin{array}{l}\text { Mitchell, 2005, } \\
15869558\end{array}$ & Low & High & High & High & Unsure & Low & Low & Low & No & No & No & High \\
\hline $\begin{array}{l}\text { Skoffer, 2016, } \\
26713665\end{array}$ & Low & Low & High & High & Low & Low & Low & $\operatorname{High}^{\mathrm{B}}$ & No & No & No & Moderate \\
\hline $\begin{array}{l}\text { Soeters, } 2018, \\
29529614\end{array}$ & Low & Low & High & Low & Low & Low & Low & Low & No & No & No & Moderate \\
\hline $\begin{array}{l}\text { Soni, 2012, } \\
22914302\end{array}$ & Low & Low & High & High & Low & Low & Low & Low & No & No & No & Moderate \\
\hline $\begin{array}{l}\text { Topp, 2009, } \\
19695525\end{array}$ & Unsure & Unsure & High & Low & Unsure & Low & Low & Low & No & No & No & High \\
\hline $\begin{array}{l}\text { Valtonen, 2015, No } \\
\text { PMID }\end{array}$ & Unsure & Unsure & Low & Low & Unsure & Unsure & Unsure & Low & No & Yes & Yes & High \\
\hline $\begin{array}{l}\text { Villadsen, 2014, } \\
23661494\end{array}$ & Low & Low & High & High & Low & High & Low & Low & No & No & No & High \\
\hline $\begin{array}{l}\text { Williamson, 2007, } \\
17604311\end{array}$ & Low & Low & High & Low & Low & Low & Low & Low & No & No & No & Moderate \\
\hline
\end{tabular}

PMID $=$ Obj = objective, PubMed Identifier, Subj = subjective

From the Cochrane Risk of Bias Tool (each item rated as Low, High, Unsure, or N/A). Ratings are color coded for emphasis only.

- Random: Random sequence generation (selection bias): Selection bias (biased allocation to interventions) due to inadequate generation of a randomized sequence;

- Allocation: Allocation concealment (selection bias): Selection bias (biased allocation to interventions) due to inadequate concealment of allocations prior to assignment;

- Blinding of participants (performance bias): Performance bias due to knowledge of the allocated interventions by participants during the study;

- Blinding of personnel/care providers (performance bias): Performance bias due to knowledge of the allocated interventions by personnel/care providers during the study;

- Blinding of outcome assessor (detection bias): Detection bias due to knowledge of the allocated interventions by outcome assessors;

- Dropout: Incomplete outcome data (attrition bias): Attrition bias due to amount, nature or handling of incomplete outcome data;

- Reporting Bias: Selective outcome reporting (outcome reporting bias): Bias arising from outcomes being selectively reported based on the direction and/or strength of the results;

- $\quad$ Other Bias: Bias due to problems not covered elsewhere in the table.

From the National Heart, Lung, and Blood Institute (NHLBI) Quality Assessment Tool (each item rated as No, Yes, or Unsure) 
- Population: Eligibility criteria not prespecified and clearly described: potentially related to selection bias;

- Intervention: Intervention not clearly described and delivered consistently: potentially related to performance bias

- Outcomes: Outcomes not prespecified, clearly defined, valid, reliable, and assessed consistently: potentially related to detection bias.

Overall risk of bias assessed as HIGH, MODERATE, or LOW.

A The study failed to recruit the sample size planned ( $\mathrm{n}=45$ recruited vs. $\mathrm{n}=80$ planned)

B The study failed to recruit the sample size planned ( $\mathrm{n}=59$ recruited vs. $\mathrm{n}=79$ planned) 


\section{Key Question 2: Rehabilitation for Total Knee Arthroplasty}

\begin{tabular}{|c|c|c|c|c|c|c|c|c|c|}
\hline $\begin{array}{l}\text { Study }{ }^{A} \text {, Year, } \\
\text { PMID, } \\
\text { Country }\end{array}$ & $\begin{array}{l}\text { Funding } \\
\text { Source }\end{array}$ & $\begin{array}{l}\text { Risk of } \\
\text { Bias }\end{array}$ & Eligibility Criteria & Intervention ${ }^{B}$ & $\begin{array}{l}\mathrm{N}, \\
\text { Enrolled }\end{array}$ & $\begin{array}{l}\text { Mean Age, } \\
\text { Years (SD) }\end{array}$ & $\begin{array}{l}\text { Female, } \\
\%\end{array}$ & $\begin{array}{l}\text { Mean } \\
\mathrm{BMI}^{\mathrm{C}} \\
\text { (SD) }\end{array}$ & $\begin{array}{l}\text { Prior } \\
\text { Contralateral } \\
\text { Athroplasty }\end{array}$ \\
\hline \multirow[t]{2}{*}{$\begin{array}{l}\text { Andersen, } \\
2018 \text {, } \\
\text { CN- } \\
01647420, \\
\text { Denmark }\end{array}$} & $\mathrm{NR}$ & High & $\begin{array}{l}\text { INCLUSION: Patients who } \\
\text { underwent TKR. EXCLUSION: NR }\end{array}$ & $\begin{array}{l}\text { Technological } \\
\text { assisted rehabilitation } \\
\text { Comp: - } \\
\text { AdjMod: - } \\
\text { Set: H }\end{array}$ & $\begin{array}{l}155 \text { (all } \\
\text { participant } \\
\text { s) }\end{array}$ & NR & NR & $\mathrm{NR}$ & NR \\
\hline & No data & No data & No data & $\begin{array}{l}\text { Supervised } \\
\text { rehabilitation } \\
\text { Comp: } 0 \\
\text { AdjMod: - } \\
\text { Set: } 0\end{array}$ & $\begin{array}{l}155 \text { (all } \\
\text { participant } \\
\text { s) }\end{array}$ & NR & NR & NR & NR \\
\hline \multirow[t]{2}{*}{$\begin{array}{l}\text { Artz, } \\
2017, \\
27068368, \\
\text { UK }\end{array}$} & $\begin{array}{l}\text { Non- } \\
\text { industry }\end{array}$ & High & $\begin{array}{l}\text { INCLUSION: Patients undergoing a } \\
\text { primary TKR for OA were eligible for } \\
\text { participation in the study. } \\
\text { EXCLUSION: Exclusion criteria } \\
\text { included: knee replacement for } \\
\text { conditions other than osteoarthritis, } \\
\text { revision knee surgery, inability to } \\
\text { participate in exercise for any } \\
\text { medical reason such as unstable } \\
\text { cardiovascular or cardio-respiratory } \\
\text { disease, diagnosis of severe } \\
\text { neurological disorders, inability to } \\
\text { provide informed consent. }\end{array}$ & $\begin{array}{l}6 \text { outpatient group- } \\
\text { based exercise } \\
\text { sessions } \\
\text { Comp: S-A-B-T-E } \\
\text { AdjMod: - } \\
\text { Set: } \text { O }\end{array}$ & 23 & $70.0(57,81)$ & $52 \%$ & NR & $\mathrm{NR}$ \\
\hline & No data & No data & No data & $\begin{array}{l}\text { Usual care } \\
\text { Comp: - } \\
\text { AdjMod: - } \\
\text { Set: - }\end{array}$ & 23 & $67.2(51,82)$ & $52 \%$ & NR & NR \\
\hline $\begin{array}{l}\text { Avramidis, } \\
2011, \\
21410130, \\
\text { Greece }\end{array}$ & NR & Moderate & $\begin{array}{l}\text { INCLUSION: good mental health, } \\
\text { unilateral knee osteoarthritis with } \\
\text { Kellgren- Lawrence radiographic } \\
\text { severity } 18 \text { 2, and age between } 60 \\
\text { and } 75 \text { years. EXCLUSION: } \\
\text { rheumatoid arthritis, symptomatic } \\
\text { osteoarthritis of other big joints of the } \\
\text { lower extremities, history of epilepsy, } \\
\text { a cardiac pacemaker, poor } \\
\text { understanding of the use of the } \\
\text { stimulator, and lesions of the skin } \\
\text { over the vastus medialis and lateral } \\
\text { part of the thigh. }\end{array}$ & $\begin{array}{l}\text { TENS plus } \\
\text { Physiotherapy } \\
\text { Comp: S-F-T } \\
\text { AdjMod: NMES } \\
\text { Set: I }\end{array}$ & 38 & $70.54(4.68)$ & $80 \%$ & $\begin{array}{l}27.38 \\
(2.65)\end{array}$ & NR \\
\hline
\end{tabular}




\begin{tabular}{|c|c|c|c|c|c|c|c|c|c|}
\hline \multirow[t]{2}{*}{$\begin{array}{l}\text { Study }{ }^{A} \text {, Year, } \\
\text { PMID, } \\
\text { Country }\end{array}$} & $\begin{array}{l}\text { Funding } \\
\text { Source }\end{array}$ & $\begin{array}{l}\text { Risk of } \\
\text { Bias }\end{array}$ & Eligibility Criteria & Intervention ${ }^{B}$ & $\begin{array}{l}\mathbf{N}, \\
\text { Enrolled }\end{array}$ & $\begin{array}{l}\text { Mean Age, } \\
\text { Years (SD) }\end{array}$ & $\begin{array}{l}\text { Female, } \\
\%\end{array}$ & $\begin{array}{l}\text { Mean } \\
\text { BMI }^{C} \\
(\mathrm{SD})\end{array}$ & $\begin{array}{l}\text { Prior } \\
\text { Contralateral } \\
\text { Athroplasty }\end{array}$ \\
\hline & No data & No data & No data & $\begin{array}{l}\text { Physiotherapy } \\
\text { Comp: S-F-T } \\
\text { AdjMod: - } \\
\text { Set: I }\end{array}$ & 38 & $70.66(3.73)$ & $83 \%$ & $\begin{array}{l}27.14 \\
(3.31)\end{array}$ & NR \\
\hline \multirow[t]{2}{*}{$\begin{array}{l}\text { Bade, } \\
2017, \\
27813347, \\
\text { USA }\end{array}$} & $\begin{array}{l}\text { Non- } \\
\text { industry }\end{array}$ & Moderate & $\begin{array}{l}\text { INCLUSION: Patients underwent a } \\
\text { primary, unilateral TKA secondary to } \\
\text { knee OA and were ages } 50-85 \\
\text { years. EXCLUSION: Current smoker, } \\
\text { current cancer treatment, } \\
\text { uncontrolled diabetes mellitus } \\
\text { (glycosylated haemoglobin } \\
\text { level .7.0), body mass index .40 } \\
\mathrm{kg} / \mathrm{m} 2, \text { neurologic, vascular, or } \\
\text { cardiac problems that limited } \\
\text { function, discharge to location other } \\
\text { than home after surgery (e.g., skilled } \\
\text { nursing facility), or severe contra- } \\
\text { lateral knee OA (pain level .5 of } 10 \\
\text { with stair climbing) or other } \\
\text { orthopaedic conditions that limited } \\
\text { function and necessitated alternative } \\
\text { concurrent intervention (e.g., severe } \\
\text { lumbar spinal stenosis, severe hip or } \\
\text { ankle OA). }\end{array}$ & $\begin{array}{l}\text { High-intensity } \\
\text { progressive } \\
\text { rehabilitation } \\
\text { Comp: S-A-F-B-T-E } \\
\text { AdjMod: Massage, } \\
\text { mobilization } \\
\text { Set: O, H }\end{array}$ & 84 & $63(8)$ & $54 \%$ & $31(5)$ & NR \\
\hline & No data & No data & No data & $\begin{array}{l}\text { Low-intensity } \\
\text { rehabilitation } \\
\text { Comp: S-A-F-B-T-E } \\
\text { AdjMod: Massage, } \\
\text { mobilization, heat, } \\
\text { cold } \\
\text { Set: O, H }\end{array}$ & 78 & $64(7)$ & $56 \%$ & $30(5)$ & NR \\
\hline
\end{tabular}




\begin{tabular}{|c|c|c|c|c|c|c|c|c|c|}
\hline $\begin{array}{l}\text { Study }{ }^{\mathrm{A}} \text {, Year, } \\
\text { PMID, } \\
\text { Country }\end{array}$ & $\begin{array}{l}\text { Funding } \\
\text { Source }\end{array}$ & $\begin{array}{l}\text { Risk of } \\
\text { Bias }\end{array}$ & Eligibility Criteria & Intervention ${ }^{B}$ & $\begin{array}{l}\mathbf{N}, \\
\text { Enrolled }\end{array}$ & $\begin{array}{l}\text { Mean Age, } \\
\text { Years (SD) }\end{array}$ & $\begin{array}{l}\text { Female, } \\
\%\end{array}$ & $\begin{array}{l}\text { Mean } \\
\text { BMIc } \\
\text { (SD) }\end{array}$ & $\begin{array}{l}\text { Prior } \\
\text { Contralateral } \\
\text { Athroplasty }\end{array}$ \\
\hline \multirow[t]{2}{*}{$\begin{array}{l}\text { Bily, } \\
2016, \\
26763947, \\
\text { Austria }\end{array}$} & $\begin{array}{l}\text { Non- } \\
\text { industry }\end{array}$ & High & $\begin{array}{l}\text { INCLUSION: Subjects scheduled for } \\
\text { their first TKA. EXCLUSION: Patients } \\
\text { who might face a high health risk } \\
\text { during maximum strength } \\
\text { measurement in the context of this } \\
\text { study. Other specific exclusion } \\
\text { criteria were body mass } \\
\text { index }>40 \mathrm{~kg} / \mathrm{m} 2 \text {; previous knee } \\
\text { replacement; pain in the contralateral } \\
\text { leg }>5 \text { VAS recent deep vein } \\
\text { thrombosis or any infection; } \\
\text { myopathy; neurologic, pulmonary, or } \\
\text { symptomatic cardiovascular } \\
\text { diseases; vertigo or impaired } \\
\text { cognitive function; recent or past } \\
\text { cancer; rheumatism; or any other } \\
\text { relevant limitations of the } \\
\text { musculoskeletal system }\end{array}$ & $\begin{array}{l}\text { Leg-press group } \\
\text { Comp: S } \\
\text { AdjMod: - } \\
\text { Set: O, H }\end{array}$ & 31 & $68.3(6.7)$ & $69 \%$ & $28(3.8)$ & NR \\
\hline & No data & No data & No data & $\begin{array}{l}\text { Physiotherapy group } \\
\text { Comp: S-F-T } \\
\text { AdjMod: Massage, } \\
\text { mobilization } \\
\text { Set: O, H }\end{array}$ & 31 & $64.9(6.0)$ & $65 \%$ & $\begin{array}{l}28.7 \\
(4.1)\end{array}$ & NR \\
\hline \multirow[t]{2}{*}{$\begin{array}{l}\text { Bruun-Olsen, } \\
2013, \\
23614370, \\
\text { Norway }\end{array}$} & $\begin{array}{l}\text { Non- } \\
\text { industry }\end{array}$ & Moderate & $\begin{array}{l}\text { INCLUSION: Elective primary TKA at } \\
\text { two local county hospitals in Norway. } \\
\text { OA of the knee according to } \\
\text { diagnostic criteria, residence close to } \\
\text { the hospitals so as to be able to } \\
\text { attend the training sessions. } \\
\text { EXCLUSION: Other walking } \\
\text { impairments than those related to } \\
\text { their operated knee, patients with } \\
\text { rheumatoid arthritis, severe } \\
\text { osteoarthritis in the hips or contra } \\
\text { lateral knee, neurological diseases, } \\
\text { dementia, as well as those with a } \\
\text { history of drug abuse }\end{array}$ & $\begin{array}{l}\text { Walking-skill group } \\
\text { Comp: S-A-F-B-T } \\
\text { AdjMod: - } \\
\text { Set: O }\end{array}$ & 29 & $68(8)$ & $62 \%$ & $28(6)$ & NR \\
\hline & No data & No data & No data & $\begin{array}{l}\text { Usual physiotherapy } \\
\text { care } \\
\text { Comp: S } \\
\text { AdjMod: - } \\
\text { Set: O }\end{array}$ & 28 & $69(10)$ & $50 \%$ & $29(5)$ & NR \\
\hline
\end{tabular}




\begin{tabular}{|c|c|c|c|c|c|c|c|c|c|}
\hline $\begin{array}{l}\text { Study }{ }^{\mathrm{A}} \text {, Year, } \\
\text { PMID, } \\
\text { Country }\end{array}$ & $\begin{array}{l}\text { Funding } \\
\text { Source }\end{array}$ & $\begin{array}{l}\text { Risk of } \\
\text { Bias }\end{array}$ & Eligibility Criteria & Intervention ${ }^{B}$ & $\begin{array}{l}\mathbf{N}, \\
\text { Enrolled }\end{array}$ & $\begin{array}{l}\text { Mean Age, } \\
\text { Years (SD) }\end{array}$ & $\begin{array}{l}\text { Female, } \\
\%\end{array}$ & $\begin{array}{l}\text { Mean } \\
\text { BMI } \\
\text { (SD) }\end{array}$ & $\begin{array}{l}\text { Prior } \\
\text { Contralateral } \\
\text { Athroplasty }\end{array}$ \\
\hline \multirow[t]{2}{*}{$\begin{array}{l}\text { Buhagiar, } \\
2017, \\
28291891, \\
\text { Australia }\end{array}$} & $\begin{array}{l}\text { Non- } \\
\text { industry }\end{array}$ & Moderate & $\begin{array}{l}\text { INCLUSION: >= } 40 \text { years with a } \\
\text { primary diagnosis of OA and to } \\
\text { undergo a primary, unilateral TKA. } \\
\text { EXCLUSION: Predisposition to be } \\
\text { discharged to an inpatient } \\
\text { rehabilitation facility due to lack of } \\
\text { social support (lack of an able } \\
\text { caregiver); having other major } \\
\text { coexisting physical impairments such } \\
\text { as hemiplegia or amputation; and } \\
\text { unable to perform a home exercise } \\
\text { program without support from } \\
\text { another person. }\end{array}$ & $\begin{array}{l}\text { Home Program } \\
\text { Comp: S-A-F-B-E } \\
\text { AdjMod: - } \\
\text { Set: H, self-guided }\end{array}$ & 81 & $66.9(9)$ & $68 \%$ & $34.8(7)$ & NR \\
\hline & No data & No data & No data & $\begin{array}{l}\text { Hospital Inpatient } \\
\text { Rehabilitation } \\
\text { Comp: S-A-F-B-E } \\
\text { AdjMod: - } \\
\text { Set: H }\end{array}$ & 84 & $66.9(8)$ & $69 \%$ & $34.7(7)$ & NR \\
\hline \multirow[t]{2}{*}{$\begin{array}{l}\text { Cai, } \\
2018, \\
29239772, \\
\text { China }\end{array}$} & NR & Moderate & $\begin{array}{l}\text { INCLUSION: post unilateral TKA for } \\
\text { knee OA, >45 years, high level of } \\
\text { kinesiophobia based on a score }>37 \\
\text { on the Tamps Scale for } \\
\text { Kinesiophobia (TSK). EXCLUSION: } \\
\text { neurologic disorder, psychiatric or } \\
\text { psychological disorder, prior knee } \\
\text { surgery, history of patellar luxation, } \\
\text { torn meniscus, or ligament injury, } \\
\text { scheduled for revision know } \\
\text { arthroplasty, had previously } \\
\text { participated in a CBT intervention. }\end{array}$ & $\begin{array}{l}\text { Cognitive behavioral } \\
\text { therapy (CBT) plus } \\
\text { standard care } \\
\text { Comp: E } \\
\text { AdjMod: mindfulness, } \\
\text { stress/anxiety- } \\
\text { reduction } \\
\text { interventions } \\
\text { Set: I }\end{array}$ & 50 & $65.26(8.30)$ & $64 \%$ & $\begin{array}{l}26.52 \\
(2.78)\end{array}$ & NR \\
\hline & No data & No data & No data & $\begin{array}{l}\text { Standard care } \\
\text { Comp: - } \\
\text { AdjMod: - } \\
\text { Set: - }\end{array}$ & 50 & $66.18(7.04)$ & $60 \%$ & $\begin{array}{l}26.63 \\
(4.74)\end{array}$ & NR \\
\hline $\begin{array}{l}\text { Chan, } \\
2018, \\
29372260, \\
\text { Singapore }^{\mathrm{D}}\end{array}$ & NR & Moderate & $\begin{array}{l}\text { INCLUSION: Patients who } \\
\text { underwent primary unilateral TKA } \\
\text { and had a primary diagnosis of OA. } \\
\text { EXCLUSION: Patients who } \\
\text { underwent revision TKA or } \\
\text { contralateral leg TKA within two } \\
\text { years of primary TKA, or did not } \\
\text { complete at least six months of } \\
\text { follow-up }\end{array}$ & $\begin{array}{l}\text { Discharge to home } \\
\text { Comp: - } \\
\text { AdjMod: - } \\
\text { Set: H }\end{array}$ & 103 & $67.2(7.8)$ & $78 \%$ & $\begin{array}{l}27.9 \\
(4.8)\end{array}$ & NR \\
\hline
\end{tabular}




\begin{tabular}{|c|c|c|c|c|c|c|c|c|c|}
\hline $\begin{array}{l}\text { Study }{ }^{\mathrm{A}} \text {, Year, } \\
\text { PMID, } \\
\text { Country }\end{array}$ & $\begin{array}{l}\text { Funding } \\
\text { Source }\end{array}$ & $\begin{array}{l}\text { Risk of } \\
\text { Bias }\end{array}$ & Eligibility Criteria & Intervention ${ }^{B}$ & $\begin{array}{l}\mathbf{N}, \\
\text { Enrolled }\end{array}$ & $\begin{array}{l}\text { Mean Age, } \\
\text { Years (SD) }\end{array}$ & $\begin{array}{l}\text { Female, } \\
\%\end{array}$ & $\begin{array}{l}\text { Mean } \\
\text { BMIc } \\
\text { (SD) }\end{array}$ & $\begin{array}{l}\text { Prior } \\
\text { Contralateral } \\
\text { Athroplasty }\end{array}$ \\
\hline & No data & No data & No data & $\begin{array}{l}\text { Discharge to } \\
\text { community hospitals } \\
\text { Comp: - } \\
\text { AdjMod: - } \\
\text { Set: I }\end{array}$ & 1,017 & $70.7(7.6)$ & $87 \%$ & $\begin{array}{l}27.3 \\
(5.0)\end{array}$ & NR \\
\hline $\begin{array}{l}\text { DeJong, } \\
2020, \\
32360105 \\
\text { USA }\end{array}$ & Industry & High & $\begin{array}{l}\text { INCLUSION: (1) had undergone an } \\
\text { elective unilateral TKA and initiated } \\
\text { outpatient PT } \leq 24 \text { days post-TKA; } \\
(2) \geq 40 \text { yo; and ( } 3 \text { ) weighed less than } \\
300 \text { pounds (due to the weight } \\
\text { limitation for the body weight- } \\
\text { adjustable treadmill). } \\
\text { EXCLUSION: (1) undergone a lower } \\
\text { extremity joint replacement } \\
\text { procedure, including a revision, } \\
\text { second, or bilateral TKA or THA <1 } \\
\text { yr prior to their current TKA; ( } 2) \\
\text { whose payer was workers' } \\
\text { compensation; ( } 3 \text { ) who were in } \\
\text { litigation related to injury or disease } \\
\text { associated with their current TKA; (4) } \\
\text { who were pregnant or may be } \\
\text { pregnant; (5) who had a medical } \\
\text { history of neurologic disorders, RA, } \\
\text { or gout (unless <6 mo since last } \\
\text { exacerbation or flare up and under } \\
\text { control medically); ( } 6 \text { ) who were } \\
\text { under active cancer treatment with } \\
\text { history of malignancy in either or } \\
\text { both lower extremities, or with } \\
\text { evidence of signs or symptoms of } \\
\text { cancer, chemotherapy, or radiation } \\
<1 \text { yr prior to their current TKA; ( } 7 \text { ) } \\
\text { who developed DVT post-TKA; ( } 8) \\
\text { who were unable to proceed or } \\
\text { continue the planned outpatient } \\
\text { program because of complications } \\
\text { such as wound infection related to } \\
\text { the TKA and severe orthostatic } \\
\text { hypotension; ( } 9 \text { ) who required } \\
\text { manipulation under anaesthesia } \\
\text { post-TKA; and (10) who received }>2 \\
\text { wks of other care in another post- } \\
\text { acute setting prior to outpatient PT. }\end{array}$ & $\begin{array}{l}\text { Body-weight adjusted } \\
\text { treatment } \\
\text { Comp: S-F-T } \\
\text { Adj: Cold } \\
\text { Set: O }\end{array}$ & 76 & $64.9(7.7)$ & $58 \%$ & $\begin{array}{l}31.2 \\
(6.4)\end{array}$ & 15.4 \\
\hline
\end{tabular}




\begin{tabular}{|c|c|c|c|c|c|c|c|c|c|}
\hline $\begin{array}{l}\text { Study }{ }^{\mathrm{A}} \text {, Year, } \\
\text { PMID, } \\
\text { Country }\end{array}$ & $\begin{array}{l}\text { Funding } \\
\text { Source }\end{array}$ & $\begin{array}{l}\text { Risk of } \\
\text { Bias }\end{array}$ & Eligibility Criteria & Intervention ${ }^{B}$ & $\begin{array}{l}\mathbf{N}, \\
\text { Enrolled }\end{array}$ & $\begin{array}{l}\text { Mean Age, } \\
\text { Years (SD) }\end{array}$ & $\begin{array}{l}\text { Female, } \\
\%\end{array}$ & $\begin{array}{l}\text { Mean } \\
\text { BMI } \\
\text { (SD) }\end{array}$ & $\begin{array}{l}\text { Prior } \\
\text { Contralateral } \\
\text { Athroplasty }\end{array}$ \\
\hline & No data & No data & No data & $\begin{array}{l}\text { Recumbent bike \& } \\
\text { PENS } \\
\text { Comp: S-F } \\
\text { Adj: Cold, NMES } \\
\text { Set: O }\end{array}$ & 78 & $62.9(8.0)$ & $64 \%$ & $\begin{array}{l}32.2 \\
(6.5)\end{array}$ & 15.6 \\
\hline & No data & No data & No data & $\begin{array}{l}\text { Body-weight adjusted } \\
\text { treadmill \& PENS } \\
\text { Comp: S-F-T } \\
\text { Adj: Cold, NMES } \\
\text { Set: O }\end{array}$ & 70 & $62.7(7.7)$ & $59 \%$ & $\begin{array}{l}31.4 \\
(5.7)\end{array}$ & 15.6 \\
\hline & No data & No data & No data & $\begin{array}{l}\text { Recumbent bike } \\
\text { Comp: S-F } \\
\text { Adj: Cold } \\
\text { Set: O } \\
\end{array}$ & 74 & $62.8(8.3)$ & $58 \%$ & $\begin{array}{l}31.5 \\
(5.8)\end{array}$ & 22.8 \\
\hline \multirow[t]{2}{*}{$\begin{array}{l}\text { Demircioglu, } \\
2015, \\
26355656 \\
\text { Turkey }\end{array}$} & NR & High & $\begin{array}{l}\text { INCLUSION: knee OA who were } \\
\text { admitted to the Orthopaedia and } \\
\text { Traumatology outpatient clinic for } \\
\text { TKA of the Istanbul Physical } \\
\text { Medicine and Rehabilitation Training } \\
\text { Hospital, Istanbul, Turkey between } \\
\text { 01-September-2006 and 01-April- } \\
\text { 2007. EXCLUSION: Symptomatic hip } \\
\text { osteoarthritis, concomitant cardiac or } \\
\text { internal diseases precluding surgical } \\
\text { treatment, a history of epilepsy, the } \\
\text { presence of a pacemaker, a skin } \\
\text { lesion over the quadriceps muscle } \\
\text { that required electrode application, } \\
\text { muscle atrophy, or severe cognitive } \\
\text { dysfunction. }\end{array}$ & $\begin{array}{l}\text { NMES \& exercise } \\
\text { Comp: S-F } \\
\text { AdjMod: Cold, NMES, } \\
\text { mobilizations } \\
\text { Set: O }\end{array}$ & 30 & $66.2(7.8)$ & $93 \%$ & $\begin{array}{l}29.1 \\
(3.9)\end{array}$ & NR \\
\hline & No data & No data & No data & $\begin{array}{l}\text { Exercise } \\
\text { Comp: S-F } \\
\text { AdjMod: cold, } \\
\text { mobilization } \\
\text { Set: H }\end{array}$ & 30 & $64.6(6.6)$ & $97 \%$ & $\begin{array}{l}30.1 \\
(4.6)\end{array}$ & NR \\
\hline $\begin{array}{l}\text { den Hertog, } \\
2012 \\
22643801 \\
\text { Germany }\end{array}$ & Industry & Moderate & $\begin{array}{l}\text { INCLUSION: Male and female } \\
\text { patients (age range } 4085 \text { years), } \\
\text { admitted for elective TKA. } \\
\text { EXCLUSION: Lack of cooperation } \\
\text { capability, American Society of } \\
\text { Anaesthesiologists (ASA) score [3, } \\
\text { rheumatoid arthritis, cancer co- } \\
\text { morbidity, alcohol or drug abuse, } \\
\text { previous major surgery on the } \\
\text { affected joint, neurologic or } \\
\text { psychiatric dis- ease, pregnancy, and } \\
\text { participation in other clinical studies }\end{array}$ & $\begin{array}{l}\text { Fast-track } \\
\text { rehabilitation program } \\
\text { Comp: S-F-T-E } \\
\text { AdjMod: - } \\
\text { Set: I }\end{array}$ & 74 & $68.25(7.91)$ & $73 \%$ & $\begin{array}{l}30.38 \\
(6.05)\end{array}$ & NR \\
\hline
\end{tabular}




\begin{tabular}{|c|c|c|c|c|c|c|c|c|c|}
\hline $\begin{array}{l}\text { Study }{ }^{A}, \text { Year, } \\
\text { PMID, } \\
\text { Country }\end{array}$ & $\begin{array}{l}\text { Funding } \\
\text { Source }\end{array}$ & $\begin{array}{l}\text { Risk of } \\
\text { Bias }\end{array}$ & Eligibility Criteria & Intervention $^{B}$ & $\begin{array}{l}\mathrm{N}, \\
\text { Enrolled }\end{array}$ & $\begin{array}{l}\text { Mean Age, } \\
\text { Years (SD) }\end{array}$ & $\begin{array}{l}\text { Female, } \\
\%\end{array}$ & $\begin{array}{l}\text { Mean } \\
\text { BMIc } \\
\text { (SD) }\end{array}$ & $\begin{array}{l}\text { Prior } \\
\text { Contralateral } \\
\text { Athroplasty }\end{array}$ \\
\hline & No data & No data & No data & $\begin{array}{l}\text { Standard care } \\
\text { Comp: S-F-T } \\
\text { AdjMod: - } \\
\text { Set: I }\end{array}$ & 73 & $66.58(8.21)$ & $69 \%$ & $\begin{array}{l}31.17 \\
(5.82)\end{array}$ & NR \\
\hline \multirow[t]{2}{*}{$\begin{array}{l}\text { Eymir, } \\
2020, \\
32778907 \\
\text { Turkey }\end{array}$} & NR & High & $\begin{array}{l}\text { INCLUSION: } \geq 30 y o \text { and were } \\
\text { scheduled for unilateral primary TKA. } \\
\text { EXCLUSION: required urgent } \\
\text { intervention, had a previous } \\
\text { orthopaedic, neurological, cardiac } \\
\text { disorder or surgery } \\
\text { that causes gait disturbance, had a } \\
\text { BMI } \\
\text { greater than } 40 \mathrm{~kg} / \mathrm{m}^{2} \text {, or were } \\
\text { planned for bilateral, revision or } \\
\text { cementless TKA surgery. }\end{array}$ & $\begin{array}{l}\text { Active heel-slide } \\
\text { exercise \& standard } \\
\text { physical therapy } \\
\text { Comp: S-F-T } \\
\text { Adj: - } \\
\text { Set: Al; H }\end{array}$ & 58 & $68.9(8.9)$ & $85 \%$ & $\begin{array}{l}31.6 \\
(4.5)\end{array}$ & NR \\
\hline & No data & No data & No data & $\begin{array}{l}\text { Continuous passive } \\
\text { motion \& standard } \\
\text { physical therapy } \\
\text { Comp: S-F-T } \\
\text { Adj: - } \\
\text { Set: Al; H }\end{array}$ & 55 & $68.9(8.3)$ & $91 \%$ & $\begin{array}{l}31.3 \\
(4.3)\end{array}$ & NR \\
\hline \multirow[t]{2}{*}{$\begin{array}{l}\text { Fransen, } \\
2017, \\
27868384, \\
\text { Australia }\end{array}$} & $\begin{array}{l}\text { Non- } \\
\text { industry }\end{array}$ & Moderate & $\begin{array}{l}\text { INCLUSION: } 4574 \text { years, } \\
\text { undergoing unilateral or bilateral } \\
\text { primary TKR, and able to be } \\
\text { discharged home from the } \\
\text { orthopedic ward. EXCLUSION: } \\
\text { Previous unicompartmental } \\
\text { replacement or tibial osteotomy on } \\
\text { the same knee, major comorbidity } \\
\text { precluding aerobic exercise at } 50 \\
60 \% \text { maximum heart rate, or a } \\
\text { diagnosis of rheumatoid arthritis or a } \\
\text { major neurologic condition. }\end{array}$ & $\begin{array}{l}\text { Outpatient group } \\
\text { exercise } \\
\text { Comp: S-A-F-T-E } \\
\text { AdjMod: - } \\
\text { Set: O, H }\end{array}$ & 210 & $64(6.5)$ & $54 \%$ & $\begin{array}{l}32.2 \\
(5.6)\end{array}$ & $13 \%$ \\
\hline & No data & No data & No data & $\begin{array}{l}\text { Usual care } \\
\text { Comp: - } \\
\text { AdjMod: - } \\
\text { Set: O, H }\end{array}$ & 212 & $65.2(6)$ & $52 \%$ & $\begin{array}{l}31.7 \\
(6.7)\end{array}$ & $17 \%$ \\
\hline
\end{tabular}




\begin{tabular}{|c|c|c|c|c|c|c|c|c|c|}
\hline $\begin{array}{l}\text { Study }{ }^{A} \text {, Year, } \\
\text { PMID, } \\
\text { Country }\end{array}$ & $\begin{array}{l}\text { Funding } \\
\text { Source }\end{array}$ & $\begin{array}{l}\text { Risk of } \\
\text { Bias }\end{array}$ & Eligibility Criteria & Intervention ${ }^{\mathrm{B}}$ & $\begin{array}{l}\mathbf{N}, \\
\text { Enrolled }\end{array}$ & $\begin{array}{l}\text { Mean Age, } \\
\text { Years (SD) }\end{array}$ & $\begin{array}{l}\text { Female, } \\
\%\end{array}$ & $\begin{array}{l}\text { Mean } \\
\text { BMIc } \\
\text { (SD) }\end{array}$ & $\begin{array}{l}\text { Prior } \\
\text { Contralateral } \\
\text { Athroplasty }\end{array}$ \\
\hline \multirow[t]{2}{*}{$\begin{array}{l}\text { Hamilton, } \\
2020, \\
33051212, \\
\text { UK }\end{array}$} & $\begin{array}{l}\text { Non- } \\
\text { industry }\end{array}$ & Moderate & $\begin{array}{l}\text { INCLUSION: have undergone a } \\
\text { primary TKA for OA, at risk for a poor } \\
\text { outcome (defined as Oxford knee } \\
\text { score } \leq 26 \\
\text { points completed at } 6 \text { wks } \\
\text { postoperative assessment). } \\
\text { EXCLUSION: unwilling to comply } \\
\text { rehabilitation protocols, } \\
\text { underwent arthroplasty purely for } \\
\text { pain relief (ie, those with no } \\
\text { expectation of mobilising } \\
\text { postoperatively),required complex } \\
\text { revision procedures, could not, } \\
\text { or were unwilling to, attend their local } \\
\text { outpatient department for } \\
\text { rehabilitation, or had already } \\
\text { received structured ongoing } \\
\text { outpatient physiotherapy at six } \\
\text { weeks post-surgery. }\end{array}$ & $\begin{array}{l}\text { Outpatient therapist- } \\
\text { led rehabilitation } \\
\text { Comp: S-F-E } \\
\text { AdjMod: - } \\
\text { Set: H }\end{array}$ & 163 & $66.8(6.49)$ & $59 \%$ & $\begin{array}{l}31.19 \\
(5.30)\end{array}$ & NR \\
\hline & No data & No data & No data & $\begin{array}{l}\text { Physiotherapy review } \\
\text { \& home exercises } \\
\text { (standard of care) } \\
\text { Comp: S-A-F-B-T-E } \\
\text { AdjMod: - } \\
\text { Set: O, H }\end{array}$ & 171 & $68.2(9.44)$ & $62 \%$ & $\begin{array}{l}31.50 \\
(6.18)\end{array}$ & NR \\
\hline \multirow[t]{2}{*}{$\begin{array}{l}\text { Harmer, } \\
2009, \\
19177536, \\
\text { Australia }\end{array}$} & $\begin{array}{l}\text { Non- } \\
\text { industry }\end{array}$ & Moderate & $\begin{array}{l}\text { INCLUSION: Primary TKR. } \\
\text { EXCLUSION: Postoperative deep } \\
\text { joint infection, bilateral joint surgery } \\
\text { or surgery planned for another joint } \\
\text { within } 6 \text { months, and documented } \\
\text { dementia or other neurologic } \\
\text { condition that precluded informed } \\
\text { consent }\end{array}$ & $\begin{array}{l}\text { Water-based } \\
\text { rehabilitation } \\
\text { Comp: S-A-F-E } \\
\text { AdjMod: - } \\
\text { Set: H }\end{array}$ & 53 & $68.7(9.1)$ & $57 \%$ & $\begin{array}{l}31.6 \\
(5.8)\end{array}$ & NR \\
\hline & No data & No data & No data & $\begin{array}{l}\text { Land-based } \\
\text { rehabilitation } \\
\text { Comp: F-B-E } \\
\text { AdjMod: - } \\
\text { Set: O, H }\end{array}$ & 49 & $67.8(6.3)$ & $57 \%$ & $30.6(5)$ & NR \\
\hline
\end{tabular}




\begin{tabular}{|c|c|c|c|c|c|c|c|c|c|}
\hline $\begin{array}{l}\text { Study }{ }^{A}, \text { Year, } \\
\text { PMID, } \\
\text { Country }\end{array}$ & $\begin{array}{l}\text { Funding } \\
\text { Source }\end{array}$ & $\begin{array}{l}\text { Risk of } \\
\text { Bias }\end{array}$ & Eligibility Criteria & Intervention $^{B}$ & $\begin{array}{l}\mathrm{N}, \\
\text { Enrolled }\end{array}$ & $\begin{array}{l}\text { Mean Age, } \\
\text { Years (SD) }\end{array}$ & $\begin{array}{l}\text { Female, } \\
\%\end{array}$ & $\begin{array}{l}\text { Mean } \\
\text { BMIc } \\
\text { (SD) }\end{array}$ & $\begin{array}{l}\text { Prior } \\
\text { Contralateral } \\
\text { Athroplasty }\end{array}$ \\
\hline \multirow[t]{2}{*}{$\begin{array}{l}\text { Heikkilä, } \\
2017, \\
28119232, \\
\text { Finland }\end{array}$} & NR & High & $\begin{array}{l}\text { INCLUSION: 1) diagnosed knee OA, } \\
\text { 2) primary arthroplasty of the knee in } \\
\text { question, and 3) age over } 18 \text { years. } \\
\text { EXCLUSION: ) other surgery for } \\
\text { lower limbs planned to be carried out } \\
\text { within } 12 \text { months, 2) dementia, 3) } \\
\text { other serious co-morbidities } \\
\text { preventing active training, and 4) } \\
\text { difficulty in visiting a physiotherapist } \\
\text { due to long travelling distance. }\end{array}$ & $\begin{array}{l}\text { Home exercise } \\
\text { Comp: S-F-B-E } \\
\text { AdjMod: - } \\
\text { Set: H, O }\end{array}$ & 51 & $69(8)$ & $57 \%$ & Missing & $28 \%$ \\
\hline & No data & No data & No data & $\begin{array}{l}\text { Control } \\
\text { Comp: - } \\
\text { AdjMod: - } \\
\text { Set: - }\end{array}$ & 53 & Missing? & $65 \%$ & $69(8)$ & $31 \%$ \\
\hline \multirow[t]{2}{*}{$\begin{array}{l}\text { Iwakiri, } \\
2020, \\
32373475 \\
\text { Japan }\end{array}$} & NR & High & $\begin{array}{l}\text { INCLUSION: Unilateral TKAs } \\
\text { EXCLUSION: Patients with renal } \\
\text { insufficiency, a history of cardiac } \\
\text { disease, deep vein thrombosis, or } \\
\text { surgery of the knee joint; patients } \\
\text { who were scheduled for } \\
\text { simultaneous or staged bilateral TKA } \\
\text { or for revision TKA. }\end{array}$ & $\begin{array}{l}\text { Range of motion } \\
\text { (post op day 1) } \\
\text { Comp: F-T } \\
\text { AdjMod: - } \\
\text { Set: Al; H }\end{array}$ & 55 & $75.0(7.3)$ & $82 \%$ & $\begin{array}{l}24.5 \\
(4.2)\end{array}$ & NR \\
\hline & No data & No data & No data & $\begin{array}{l}\text { Range of motion } \\
\text { (post op day 7) } \\
\text { Comp: F-T } \\
\text { AdjMod: - } \\
\text { Set: Al }\end{array}$ & 54 & $75.6(6.2)$ & $82 \%$ & $\begin{array}{l}25.2 \\
(3.7)\end{array}$ & NR \\
\hline \multirow[t]{2}{*}{$\begin{array}{l}\text { Jin, } \\
2018, \\
\text { CN- } \\
01617489 \\
\text { China }\end{array}$} & NR & High & $\begin{array}{l}\text { INCLUSION: OA, unilateral TKA for } \\
\text { the first time, informed content was } \\
\text { obtained. EXCLUSION: Overweight } \\
\text { (BMI>I= } 30 \mathrm{~kg} / \mathrm{m} 2) \text {, severe } \\
\text { osteoporosis, ligament injury or } \\
\text { periprosthetic fracture occurring } \\
\text { during TKA, Unstable vital signs, } \\
\text { complications of incision healing, or } \\
\text { clot formation in leg veins, Vision } \\
\text { loss, hearing loss, or functional } \\
\text { illiteracy. }\end{array}$ & $\begin{array}{l}\text { VR plus usual care } \\
\text { Comp: S-F-E } \\
\text { AdjMod: biofeedback, } \\
\text { mindfulness, stress } \\
\text { reduction } \\
\text { interventions } \\
\text { Set: I }\end{array}$ & 33 & $66.45(3.49)$ & $55 \%$ & $\begin{array}{l}24.52 \\
(2.27)\end{array}$ & NR \\
\hline & No data & No data & No data & $\begin{array}{l}\text { Usual care } \\
\text { Comp: S-F-E } \\
\text { AdjMod: mindfulness, } \\
\text { dstress reduction } \\
\text { interventions } \\
\text { Set: I }\end{array}$ & 33 & $66.30(4.41)$ & $61 \%$ & $\begin{array}{l}24.97 \\
(2.52)\end{array}$ & NR \\
\hline
\end{tabular}




\begin{tabular}{|c|c|c|c|c|c|c|c|c|c|}
\hline $\begin{array}{l}\text { Study }{ }^{A}, \text { Year, } \\
\text { PMID, } \\
\text { Country }\end{array}$ & $\begin{array}{l}\text { Funding } \\
\text { Source }\end{array}$ & $\begin{array}{l}\text { Risk of } \\
\text { Bias }\end{array}$ & Eligibility Criteria & Intervention ${ }^{B}$ & $\begin{array}{l}\mathrm{N}, \\
\text { Enrolled }\end{array}$ & $\begin{array}{l}\text { Mean Age, } \\
\text { Years (SD) }\end{array}$ & $\begin{array}{l}\text { Female, } \\
\%\end{array}$ & $\begin{array}{l}\text { Mean } \\
\text { BMIc } \\
\text { (SD) }\end{array}$ & $\begin{array}{l}\text { Prior } \\
\text { Contralateral } \\
\text { Athroplasty }\end{array}$ \\
\hline \multirow[t]{2}{*}{$\begin{array}{l}\text { Kauppila, } \\
2010, \\
20354057 \\
\text { Finland }\end{array}$} & $\begin{array}{l}\text { Non- } \\
\text { industry }\end{array}$ & Moderate & $\begin{array}{l}\text { INCLUSION: (1) diagnosis of primary } \\
\text { osteoarthritis of the knee; (2) } 6080 \\
\text { years of age; and (3) primary } \\
\text { unilateral total knee arthroplasty as a } \\
\text { scheduled procedure. EXCLUSION: } \\
\text { (1) severe cardiovascular or } \\
\text { pulmonary disease (New York Heart } \\
\text { Association III IV), (2) severe } \\
\text { dementia (Mini-Mental State } \\
\text { Examination 518), } 19 \text { (3) rheumatoid } \\
\text { arthritis, (4) primary total knee } \\
\text { arthroplasty scheduled as treatment } \\
\text { of an acute trauma of the knee, (5) } \\
\text { planned use of a special } \\
\text { endoprosthesis, and (6) major } \\
\text { postoperative complication as a } \\
\text { contraindication for intensive } \\
\text { rehabilitation }\end{array}$ & $\begin{array}{l}\text { Multidisciplinary } \\
\text { rehabilitation } \\
\text { Comp: S-A-F-T-E } \\
\text { AdjMod: mindfulness, } \\
\text { stress and anxiety } \\
\text { reduction } \\
\text { interventions } \\
\text { Set: I, O, H }\end{array}$ & 36 & $70.7(5.7)$ & $76 \%$ & $\begin{array}{l}32.9 \\
(6.8)\end{array}$ & $18.2 \%$ \\
\hline & No data & No data & No data & $\begin{array}{l}\text { Control } \\
\text { Comp: S-F-T-E } \\
\text { AdjMod: - } \\
\text { Set: I, O, H }\end{array}$ & 39 & $70.6(5.3)$ & $79 \%$ & $32(4.4)$ & $16.7 \%$ \\
\hline \multirow[t]{2}{*}{$\begin{array}{l}\text { Lenguerrand, } \\
2020 \text {, } \\
31033232 \text {, } \\
\text { UK }\end{array}$} & $\begin{array}{l}\text { Non- } \\
\text { industry }\end{array}$ & Moderate & $\begin{array}{l}\text { INCLUSION: National Health } \\
\text { Services patients >= } 18 \text { years who } \\
\text { are listed for primary TKR due to } \\
\text { OA.EXCLUSION: Patients listed for } \\
\text { TKR for reasons other than OA, } \\
\text { patients listed for revision TKR, } \\
\text { inability to participate in exercise for } \\
\text { medical reasons such as unstable } \\
\text { cardiovascular or severe } \\
\text { neurological conditions, unable or } \\
\text { unwilling to attend physiotherapy } \\
\text { classes after surgery, post-operative } \\
\text { complication(s) or interventions } \\
\text { within the first } 2 \text { weeks. }\end{array}$ & $\begin{array}{l}\text { Group-based } \\
\text { outpatient physical } \\
\text { therapy and standard } \\
\text { care } \\
\text { Comp: S-A-F-B-T-E } \\
\text { AdjMod: - } \\
\text { Set: O }\end{array}$ & 89 & $69(9)$ & $56 \%$ & NR & NR \\
\hline & No data & No data & No data & $\begin{array}{l}\text { Standard care } \\
\text { Comp: - } \\
\text { AdjMod: - } \\
\text { Set: NA }\end{array}$ & 91 & $69(9)$ & $54 \%$ & NR & NR \\
\hline $\begin{array}{l}\text { Lenssen, } \\
2006, \\
16942627, \\
\text { Netherlands }\end{array}$ & NR & Moderate & $\begin{array}{l}\text { INCLUSION: scheduled in the 'Joint } \\
\text { Care' program and signed an } \\
\text { informed consent form. } \\
\text { EXCLUSION: > } 85 \text { years, comorbidity } \\
\text { influencing gait, patients who did not } \\
\text { speak dutch }\end{array}$ & $\begin{array}{l}\text { Physiotherapy [twice } \\
\text { daily ( } 40 \text { mins/day)] } \\
\text { Comp: S-F-T } \\
\text { AdjMod: - } \\
\text { Set: I }\end{array}$ & 21 & $70(8.5)$ & $71 \%$ & NR & NR \\
\hline
\end{tabular}




\begin{tabular}{|c|c|c|c|c|c|c|c|c|c|}
\hline $\begin{array}{l}\text { Study }{ }^{A}, \text { Year, } \\
\text { PMID, } \\
\text { Country }\end{array}$ & $\begin{array}{l}\text { Funding } \\
\text { Source }\end{array}$ & $\begin{array}{l}\text { Risk of } \\
\text { Bias }\end{array}$ & Eligibility Criteria & Intervention $^{B}$ & $\begin{array}{l}\mathbf{N}, \\
\text { Enrolled }\end{array}$ & $\begin{array}{l}\text { Mean Age, } \\
\text { Years (SD) }\end{array}$ & $\begin{array}{l}\text { Female, } \\
\%\end{array}$ & $\begin{array}{l}\text { Mean } \\
\text { BMIc } \\
\text { (SD) }\end{array}$ & $\begin{array}{l}\text { Prior } \\
\text { Contralateral } \\
\text { Athroplasty }\end{array}$ \\
\hline & . & . & & $\begin{array}{l}\text { Physiotherapy [once } \\
\text { daily ( } 20 \text { mins/day)] } \\
\text { Comp: S-F-T } \\
\text { AdjMod: - } \\
\text { Set: I }\end{array}$ & 22 & $67(7)$ & $77 \%$ & NR & $\mathrm{NR}$ \\
\hline \multirow[t]{2}{*}{$\begin{array}{l}\mathrm{Li}, \\
2014, \\
23412304, \\
\text { China }\end{array}$} & $\begin{array}{l}\text { Non- } \\
\text { industry }\end{array}$ & High & $\begin{array}{l}\text { INCLUSION: (1) no neural or } \\
\text { muscular system disease, (2) } \\
\text { unilateral OA knee joint TKR, and (3) } \\
\text { able to walk safely and } \\
\text { independently and functional } \\
\text { ambulation (FAC) score C } 3 \text {. } \\
\text { EXCLUSION: (1) mental disease, } \\
\text { dementia, and intelligence } \\
\text { impairment before OA and a history } \\
\text { of cerebral organic disease and of } \\
\text { mental disorders (mini mental state } \\
\text { evaluation score } \backslash 23),(2) \\
\text { cardiopulmonary functional lability, } \\
\text { (3) serious cardiac and renal } \\
\text { dysfunction and hemopathicactive } \\
\text { peptic ulcer, and (4) other medical } \\
\text { and surgical diseases which may } \\
\text { lead to hemorrhage }\end{array}$ & $\begin{array}{l}\text { Robot-assisted } \\
\text { training } \\
\text { Comp: S-F-T } \\
\text { AdjMod: NMES } \\
\text { Set: I }\end{array}$ & $\begin{array}{l}60 \text { (all } \\
\text { participant } \\
\text { s) }\end{array}$ & NR & NR & NR & NR \\
\hline & No data & No data & No data & $\begin{array}{l}\text { Traditional } \\
\text { rehabilitation training } \\
\text { Comp: S-F-T } \\
\text { AdjMod: NMES } \\
\text { Set: I }\end{array}$ & & NR & NR & NR & NR \\
\hline \multirow[t]{2}{*}{$\begin{array}{l}\mathrm{Li}, \\
2015, \\
\mathrm{CN}- \\
\text { 01084888, } \\
\text { China }\end{array}$} & NR & High & $\begin{array}{l}\text { INCLUSION: Age between } 55 \text { and } \\
75 ; \text { the diagnosis was knee } \\
\text { osteoarthritis with the } \\
\text { Kellgren/Lawrence grade 4; the body } \\
\text { mass index (BMI) was less than 35; } \\
\text { affected by the unilateral knee OA } \\
\text { undergoing primary knee TKA; living } \\
\text { in Beijing. EXCLUSION: Infectious } \\
\text { joint diseases; hip joint disease or } \\
\text { ankle joint disease which affected } \\
\text { the daily physical activities; } \\
\text { comorbidities such as chronic } \\
\text { obstructive pulmonary disease which } \\
\text { affected the daily physical activities }\end{array}$ & $\begin{array}{l}\text { Functional plus } \\
\text { balance rehabilitation } \\
\text { Comp: E } \\
\text { AdjMod: - } \\
\text { Set: H }\end{array}$ & 25 & $71.43(6.33)$ & $75 \%$ & $\begin{array}{l}27.88 \\
(5.02)\end{array}$ & NR \\
\hline & No data & No data & No data & $\begin{array}{l}\text { No education } \\
\text { Comp: - } \\
\text { AdjMod: - } \\
\text { Set: - }\end{array}$ & 25 & $73.40(7.04)$ & $65 \%$ & $\begin{array}{l}26.97 \\
(4.15)\end{array}$ & NR \\
\hline
\end{tabular}




\begin{tabular}{|c|c|c|c|c|c|c|c|c|c|}
\hline $\begin{array}{l}\text { Study }{ }^{\mathrm{A}} \text {, Year, } \\
\text { PMID, } \\
\text { Country }\end{array}$ & $\begin{array}{l}\text { Funding } \\
\text { Source }\end{array}$ & $\begin{array}{l}\text { Risk of } \\
\text { Bias }\end{array}$ & Eligibility Criteria & Intervention ${ }^{B}$ & $\begin{array}{l}\mathrm{N}, \\
\text { Enrolled }\end{array}$ & $\begin{array}{l}\text { Mean Age, } \\
\text { Years (SD) }\end{array}$ & $\begin{array}{l}\text { Female, } \\
\%\end{array}$ & $\begin{array}{l}\text { Mean } \\
\text { BMIC } \\
\text { (SD) }\end{array}$ & $\begin{array}{l}\text { Prior } \\
\text { Contralateral } \\
\text { Athroplasty }\end{array}$ \\
\hline \multirow[t]{2}{*}{$\begin{array}{l}\text { Li, } \\
2017, \\
\text { CN- } \\
\text { 01419703, } \\
\text { China }\end{array}$} & NR & Moderate & $\begin{array}{l}\text { INCLUSION: (1) Patients undergoing } \\
\text { TKR for the first time with a unilateral } \\
\text { knee joint; (2) The surgery of the } \\
\text { patients was performed by the same } \\
\text { group of doctors and the same } \\
\text { anesthesiologist, and the same } \\
\text { manufacturer and the same material } \\
\text { prosthesis were selected; (3) Other } \\
\text { factors such as trauma, arthritis and } \\
\text { other diseases were excluded; (4) } \\
\text { Patients and their families indicate } \\
\text { good medical compliance and strong } \\
\text { willingness to participate. } \\
\text { EXCLUSION: (1) Patients with } \\
\text { sequelae of cerebrovascular } \\
\text { disease; (2) Mental illness and } \\
\text { intellectual disability cannot } \\
\text { cooperate; (3) Patients with severe } \\
\text { liver, kidney, heart, and lung } \\
\text { insufficiency, tumors; (4) TKR has } \\
\text { joint infection, joint tuberculosis or } \\
\text { acute, Chronic osteomyelitis, or } \\
\text { combined with serious medical } \\
\text { diseases that restrict walking, and } \\
\text { other joint diseases of the lower } \\
\text { extremities cause severe deformities } \\
\text { and restricted mobility }\end{array}$ & $\begin{array}{l}\text { Gait training \& usual } \\
\text { care } \\
\text { Comp: S-F-B-T } \\
\text { AdjMod: Cold, } \\
\text { massage for edema } \\
\text { control } \\
\text { Set: I }\end{array}$ & 24 & $76.33(5.28)$ & $56 \%$ & NR & NR \\
\hline & No data & No data & No data & $\begin{array}{l}\text { Usual care } \\
\text { Comp: S-F-B } \\
\text { AdjMod: Cold, } \\
\text { massage for edema } \\
\text { control } \\
\text { Set: I }\end{array}$ & 22 & $78.47(5.50)$ & $51 \%$ & NR & NR \\
\hline
\end{tabular}




\begin{tabular}{|c|c|c|c|c|c|c|c|c|c|}
\hline $\begin{array}{l}\text { Study }{ }^{A} \text {, Year, } \\
\text { PMID, } \\
\text { Country }\end{array}$ & $\begin{array}{l}\text { Funding } \\
\text { Source }\end{array}$ & $\begin{array}{l}\text { Risk of } \\
\text { Bias }\end{array}$ & Eligibility Criteria & Intervention ${ }^{\mathrm{B}}$ & $\begin{array}{l}\mathbf{N}, \\
\text { Enrolled }\end{array}$ & $\begin{array}{l}\text { Mean Age, } \\
\text { Years (SD) }\end{array}$ & $\begin{array}{l}\text { Female, } \\
\%\end{array}$ & $\begin{array}{l}\text { Mean } \\
\text { BMIc } \\
\text { (SD) }\end{array}$ & $\begin{array}{l}\text { Prior } \\
\text { Contralateral } \\
\text { Athroplasty }\end{array}$ \\
\hline \multirow[t]{2}{*}{$\begin{array}{l}\text { Li, } \\
2019, \\
31003647, \\
\text { China }\end{array}$} & $\begin{array}{l}\text { Non- } \\
\text { industry }\end{array}$ & Moderate & $\begin{array}{l}\text { INCLUSION: (a) clinical and } \\
\text { radiographic evidence diagnosed } \\
\text { with end- stage knee OA according } \\
\text { to the diagnosis criteria and } \\
\text { scheduled for primary unilateral TKA } \\
\text { surgery; (b) } 65-74 \text { years of age; (c) } \\
\text { no history of significant } \\
\text { cardiovascular, pulmonary, } \\
\text { metabolic, musculoskeletal, or other } \\
\text { chronic diseases; (d) fully informed } \\
\text { consent about the program; (e) a } \\
\text { partner to oversee the entire } \\
\text { exercise process to ensure safety; } \\
\text { and (f) a normally active lifestyle. } \\
\text { EXCLUSION: (a) a history of knee } \\
\text { infection, a lesion involving bilateral } \\
\text { knees, or any intra-articular } \\
\text { hyaluronic acid injections in the } 6 \\
\text { months prior to assessment; (b) } \\
\text { serious medical conditions that } \\
\text { limited his/her ability to safely } \\
\text { participate in either the TCC or } \\
\text { physical therapy programs; (c) } \\
\text { inability to walk at least } 150 \text { m in } 6 \\
\text { min due to some serious diseases } \\
\text { (e.g., epilepsy, diminished mental } \\
\text { capabilities); (d) previous experience } \\
\text { with TCC or exercised regularly with } \\
\text { other similar types of complementary } \\
\text { and alternative medicine such as qi } \\
\text { gong or yoga; or (e) inability to } \\
\text { complete the study (e.g., not } \\
\text { Chinese-speaking or intended to } \\
\text { move out of the region) }\end{array}$ & $\begin{array}{l}\text { Tai chi exercise } \\
\text { Comp: S-F } \\
\text { AdjMod: } \\
\text { complementary and } \\
\text { alternative therapies } \\
\text { Set: NR }\end{array}$ & 64 & $69.6(4.3)$ & $52 \%$ & $\begin{array}{l}23.7 \\
(3.6)\end{array}$ & NR \\
\hline & No data & No data & No data & $\begin{array}{l}\text { Control (traditional } \\
\text { physical exercises) } \\
\text { Comp: S-F } \\
\text { AdjMod: - } \\
\text { Set: NR }\end{array}$ & 65 & $68.5(3.5)$ & $55 \%$ & $\begin{array}{l}24.2 \\
(2.9)\end{array}$ & NR \\
\hline $\begin{array}{l}\text { Liao, } \\
2015, \\
25552523, \\
\text { Taiwan }\end{array}$ & $\begin{array}{l}\text { Non- } \\
\text { industry }\end{array}$ & High & INCLUSION: OA. EXCLUSION: NR & $\begin{array}{l}\text { Functional } \\
\text { rehabilitation \& } \\
\text { balance training } \\
\text { Comp: S-A-F-B-T } \\
\text { AdjMod: - } \\
\text { Set: O }\end{array}$ & 53 & $71.43(6.33)$ & $75 \%$ & $\begin{array}{l}27.88 \\
(5.02)\end{array}$ & NR \\
\hline
\end{tabular}




\begin{tabular}{|c|c|c|c|c|c|c|c|c|c|}
\hline $\begin{array}{l}\text { Study }{ }^{\mathrm{A}} \text {, Year, } \\
\text { PMID, } \\
\text { Country }\end{array}$ & $\begin{array}{l}\text { Funding } \\
\text { Source }\end{array}$ & $\begin{array}{l}\text { Risk of } \\
\text { Bias }\end{array}$ & Eligibility Criteria & Intervention ${ }^{B}$ & $\begin{array}{l}\mathrm{N}, \\
\text { Enrolled }\end{array}$ & $\begin{array}{l}\text { Mean Age, } \\
\text { Years (SD) }\end{array}$ & $\begin{array}{l}\text { Female, } \\
\%\end{array}$ & $\begin{array}{l}\text { Mean } \\
\text { BMIC } \\
\text { (SD) }\end{array}$ & $\begin{array}{l}\text { Prior } \\
\text { Contralateral } \\
\text { Athroplasty }\end{array}$ \\
\hline & No data & No data & No data & $\begin{array}{l}\text { Functional } \\
\text { rehabilitation } \\
\text { Comp: S-A-F-T } \\
\text { AdjMod: - } \\
\text { Set: O }\end{array}$ & 55 & $73.40(7.04)$ & $65 \%$ & $\begin{array}{l}26.97 \\
(4.15)\end{array}$ & NR \\
\hline \multirow[t]{2}{*}{$\begin{array}{l}\text { Liao, } \\
2020, \\
31687984, \\
\text { Taiwan }\end{array}$} & $\begin{array}{l}\text { Non- } \\
\text { industry }\end{array}$ & Moderate & $\begin{array}{l}\text { INCLUSION: (a) older women aged } \\
60 \text { and } 85 \text { yrs, (b) radiological } \\
\text { diagnosis of KOA (Kellgren and } \\
\text { Lawrence grade III or higher), and (c) } \\
\text { scheduled to undergo a primary } \\
\text { TKR. EXCLUSION: (a) uncontrolled } \\
\text { hypertension, (b) any cardiovascular } \\
\text { or pulmonary disease that would } \\
\text { prevent them from engaging in an } \\
\text { exercise study, and (c) neurological } \\
\text { or cognitive impairment that may } \\
\text { interfere with compliance with and } \\
\text { adherence to a home-based exercise } \\
\text { program. }\end{array}$ & $\begin{array}{l}\text { Elastic resistance } \\
\text { exercise training } \\
\text { Comp: S-F } \\
\text { AdjMod: - } \\
\text { Set: O, H }\end{array}$ & 28 & $72.22(7.75)$ & $100 \%$ & $\begin{array}{l}28.54 \\
(3.88)\end{array}$ & NR \\
\hline & No data & No data & No data & $\begin{array}{l}\text { Standard care } \\
\text { Comp: - } \\
\text { AdjMod: - } \\
\text { Set: NR }\end{array}$ & 27 & $69.79(6.72)$ & $100 \%$ & $\begin{array}{l}27.25 \\
(4.36)\end{array}$ & NR \\
\hline \multirow[t]{2}{*}{$\begin{array}{l}\text { Liebs, } \\
2010, \\
20360503, \\
\text { Germany }\end{array}$} & $\begin{array}{l}\text { Non- } \\
\text { industry }\end{array}$ & Moderate & $\begin{array}{l}\text { INCLUSION: Primary unilateral TKR } \\
\text { on an elective basis after a diagnosis } \\
\text { of OA or osteonecrosis. } \\
\text { EXCLUSION: History of septic } \\
\text { arthritis, a hip or knee fracture, an } \\
\text { intraoperative complication, revision } \\
\text { arthroplasty, RA, (6) lower extremity } \\
\text { amputation, a malignant tumor. } \\
\text { NB. Knee population reported here }\end{array}$ & $\begin{array}{l}\text { Ergometer cycling } \\
\text { Comp: S-F-B-T } \\
\text { AdjMod: - } \\
\text { Set: O }\end{array}$ & 85 & $69.7(8)$ & $62 \%$ & $\begin{array}{l}29.7 \\
(4.8)\end{array}$ & NR \\
\hline & No data & No data & No data & $\begin{array}{l}\text { Control } \\
\text { Comp: S-F-B-T } \\
\text { AdjMod: - } \\
\text { Set: O } \\
\end{array}$ & 74 & $69.9(7.8)$ & $52 \%$ & $\begin{array}{l}29.2 \\
(4.4)\end{array}$ & NR \\
\hline $\begin{array}{l}\text { Liebs, } \\
2012, \\
22196125, \\
\text { Germany }\end{array}$ & $\begin{array}{l}\text { Non- } \\
\text { industry }\end{array}$ & Moderate & $\begin{array}{l}\text { INCLUSION: Unilateral hip or knee } \\
\text { replacement surgery at participating } \\
\text { centers on an elective basis after } \\
\text { diagnosis of OA. EXCLUSION: } \\
\text { History of septic arthritis, hip or knee } \\
\text { fracture, intraoperative } \\
\text { complications, revision arthroplasty, } \\
\text { RA, amputations, malignancy }\end{array}$ & $\begin{array}{l}\text { Early aquatic therapy } \\
\text { Comp: S-F-B-T } \\
\text { AdjMod: - } \\
\text { Set: O }\end{array}$ & 66 & $68.5(8.6)$ & $61 \%$ & $29.3(5)$ & NR \\
\hline
\end{tabular}




\begin{tabular}{|c|c|c|c|c|c|c|c|c|c|}
\hline $\begin{array}{l}\text { Study }{ }^{\mathrm{A}} \text {, Year, } \\
\text { PMID, } \\
\text { Country }\end{array}$ & $\begin{array}{l}\text { Funding } \\
\text { Source }\end{array}$ & $\begin{array}{l}\text { Risk of } \\
\text { Bias }\end{array}$ & Eligibility Criteria & Intervention ${ }^{B}$ & $\begin{array}{l}\mathbf{N}, \\
\text { Enrolled }\end{array}$ & $\begin{array}{l}\text { Mean Age, } \\
\text { Years (SD) }\end{array}$ & $\begin{array}{l}\text { Female, } \\
\%\end{array}$ & $\begin{array}{l}\text { Mean } \\
\text { BMIc } \\
\text { (SD) }\end{array}$ & $\begin{array}{l}\text { Prior } \\
\text { Contralateral } \\
\text { Athroplasty }\end{array}$ \\
\hline & No data & No data & No data & $\begin{array}{l}\text { Late aquatic therapy } \\
\text { Comp: S-F-B-T } \\
\text { AdjMod: - } \\
\text { Set: O }\end{array}$ & 69 & $70.9(7.5)$ & $72 \%$ & $\begin{array}{l}29.3 \\
(4.6)\end{array}$ & NR \\
\hline \multirow[t]{2}{*}{$\begin{array}{l}\text { Madsen, } \\
2013, \\
23651717, \\
\text { Denmark }\end{array}$} & $\begin{array}{l}\text { Non- } \\
\text { industry }\end{array}$ & High & $\begin{array}{l}\text { INCLUSION: 1) age } 18 \text { years or } \\
\text { more, 2) primary TKA for } \\
\text { osteoarthritis, 3) patient living in one } \\
\text { of three municipalities, 4) patient } \\
\text { able to travel to the rehabilitation } \\
\text { centre independently. EXCLUSION: } \\
\text { 1) neuromuscular or } \\
\text { neurodegenerative diseases, 2) knee } \\
\text { infection after TKA or other major } \\
\text { complications (e.g. loosening or } \\
\text { embolism excluding superficial } \\
\text { thrombophlebitis), 3) problems } \\
\text { related to mobility, muscle strength } \\
\text { or excessive pain preventing the } \\
\text { patient from following the } \\
\text { rehabilitation program, 4) patient } \\
\text { unable to understand the instructions } \\
\text { due to dementia or language } \\
\text { problems }\end{array}$ & $\begin{array}{l}\text { Group-based } \\
\text { rehabilitation } \\
\text { Comp: S-A-F-B-E } \\
\text { AdjMod: } \\
\text { Set:O, H }\end{array}$ & 47 & $66.9(8.5)$ & $47 \%$ & NR & NR \\
\hline & No data & No data & No data & $\begin{array}{l}\text { Supervised home- } \\
\text { exercises } \\
\text { Comp: S-A-B-E } \\
\text { AdjMod: - } \\
\text { Set: O, H }\end{array}$ & 50 & $66.2(8.2)$ & $50 \%$ & NR & NR \\
\hline \multirow[t]{2}{*}{$\begin{array}{l}\text { Minns Lowe, } \\
2012, \\
22180446, \\
\text { UK }\end{array}$} & No industry & High & $\begin{array}{l}\text { INCLUSION: Patients undergoing } \\
\text { elective primary total knee } \\
\text { arthroplasty for OA. EXCLUSION: } \\
\text { Bilateral arthroplasty, planned } \\
\text { unicompartmental prosthesis, } \\
\text { minimally invasive surgery, planned } \\
\text { further joint surgery within } 12 \\
\text { months, inflammatory arthritis, } \\
\text { existing comorbidities preventing } \\
\text { participation in treatment }\end{array}$ & $\begin{array}{l}\text { Home-visit } \\
\text { physiotherapy } \\
\text { Comp: S-F-B-T } \\
\text { AdjMod: - } \\
\text { Set: H }\end{array}$ & 56 & $67.84(8.45)$ & $57 \%$ & $\begin{array}{l}31.32 \\
(6.28)\end{array}$ & $38.2 \%$ \\
\hline & No data & No data & No data & $\begin{array}{l}\text { Usual care } \\
\text { Comp: - } \\
\text { AdjMod:- } \\
\text { Set:- }\end{array}$ & 51 & $70.76(9.45)$ & $59 \%$ & $\begin{array}{l}29.27 \\
(5.82)\end{array}$ & $43.1 \%$ \\
\hline
\end{tabular}




\begin{tabular}{|c|c|c|c|c|c|c|c|c|c|}
\hline $\begin{array}{l}\text { Study }{ }^{A}, \text { Year, } \\
\text { PMID, } \\
\text { Country }\end{array}$ & $\begin{array}{l}\text { Funding } \\
\text { Source }\end{array}$ & $\begin{array}{l}\text { Risk of } \\
\text { Bias }\end{array}$ & Eligibility Criteria & Intervention $^{B}$ & $\begin{array}{l}\mathrm{N}, \\
\text { Enrolled }\end{array}$ & $\begin{array}{l}\text { Mean Age, } \\
\text { Years (SD) }\end{array}$ & $\begin{array}{l}\text { Female, } \\
\%\end{array}$ & $\begin{array}{l}\text { Mean } \\
\text { BMIc } \\
\text { (SD) }\end{array}$ & $\begin{array}{l}\text { Prior } \\
\text { Contralateral } \\
\text { Athroplasty }\end{array}$ \\
\hline \multirow[t]{2}{*}{$\begin{array}{l}\text { Mitchell, } \\
2005, \\
15869558, \\
\text { UK }\end{array}$} & $\begin{array}{l}\text { Non- } \\
\text { industry }\end{array}$ & High & $\begin{array}{l}\text { INCLUSION: primary unilateral TKA } \\
\text { for OA. EXCLUSION: revision TKA, } \\
\text { bilateral and unicondylar knee } \\
\text { replacements, TKA for trauma, onset } \\
\text { of serious comorbidity or terminal } \\
\text { illness since patient placed on the } \\
\text { waiting list, contralateral knee } \\
\text { replacement within the preceding } 12 \\
\text { months }\end{array}$ & $\begin{array}{l}\text { Home rehabilitation } \\
\text { Comp: F-T } \\
\text { AdjMod: Massage } \\
\text { Set: H }\end{array}$ & 80 & $70.0(7.2)$ & $63 \%$ & NR & $15.8 \%$ \\
\hline & No data & No data & No data & $\begin{array}{l}\text { Hospital rehabilitation } \\
\text { Comp: F-T } \\
\text { AdjMod: - } \\
\text { Set: O }\end{array}$ & 81 & $70.6(8.2)$ & $53 \%$ & NR & $26.4 \%$ \\
\hline \multirow[t]{2}{*}{$\begin{array}{l}\text { Moffet, } \\
2015, \\
26178888, \\
\text { Canada }\end{array}$} & $\begin{array}{l}\text { Non- } \\
\text { industry }\end{array}$ & Moderate & $\begin{array}{l}\text { INCLUSION: Waiting for a primary } \\
\text { TKA after a diagnosis of OA, } \\
\text { returning home after hospital } \\
\text { discharge, living in an area served by } \\
\text { high-speed Internet services (at least } \\
512 \mathrm{~kb} / \mathrm{s} \text { in upload), and living within } \\
\text { a 1-hour driving distance from the } \\
\text { treating hospital. EXCLUSION: } \\
\text { Health conditions that could interfere } \\
\text { with tests or the rehab program, } \\
\text { including other lower-limb surgery in } \\
\text { the last } 9 \text { months; were planning a } \\
\text { second lower-limb surgery within } 4 \\
\text { months; had cognitive or } \\
\text { collaboration problems; had major } \\
\text { post-op complications; or had } \\
\text { weight-bearing restrictions for a } \\
\text { period longer than } 2 \text { weeks after } \\
\text { surgery. }\end{array}$ & $\begin{array}{l}\text { In-home } \\
\text { telerehabilitation } \\
\text { Comp: S-B-T-E } \\
\text { AdjMod: - } \\
\text { Set: H }\end{array}$ & 104 & $65(8)$ & $58 \%$ & $34(7)$ & NR \\
\hline & No data & No data & No data & $\begin{array}{l}\text { Standard home } \\
\text { rehabilitation } \\
\text { Comp: S-T-E } \\
\text { AdjMod: - } \\
\text { Set: H }\end{array}$ & 101 & $67(8)$ & $45 \%$ & $33(6)$ & NR \\
\hline
\end{tabular}




\begin{tabular}{|c|c|c|c|c|c|c|c|c|c|}
\hline $\begin{array}{l}\text { Study }{ }^{A}, \text { Year, } \\
\text { PMID, } \\
\text { Country }\end{array}$ & $\begin{array}{l}\text { Funding } \\
\text { Source }\end{array}$ & $\begin{array}{l}\text { Risk of } \\
\text { Bias }\end{array}$ & Eligibility Criteria & Intervention ${ }^{B}$ & $\begin{array}{l}\mathbf{N}, \\
\text { Enrolled }\end{array}$ & $\begin{array}{l}\text { Mean Age, } \\
\text { Years (SD) }\end{array}$ & $\begin{array}{l}\text { Female, } \\
\%\end{array}$ & $\begin{array}{l}\text { Mean } \\
\text { BMIC } \\
(\mathrm{SD}) \\
\end{array}$ & $\begin{array}{l}\text { Prior } \\
\text { Contralateral } \\
\text { Athroplasty }\end{array}$ \\
\hline \multirow[t]{2}{*}{$\begin{array}{l}\text { Monticone, } \\
2013, \\
23063624, \\
\text { Italy }\end{array}$} & NR & Moderate & $\begin{array}{l}\text { INCLUSION: Primary TKA because } \\
\text { of knee OA performed } 7 \text { to } 10 \text { days } \\
\text { before admission to our rehabilitation } \\
\text { unit, a good understanding of Italian, } \\
\text { and aged }>50 \text { years. EXCLUSION: } \\
\text { Cognitive impairment and all other } \\
\text { causes of knee pain, such as } \\
\text { previous lower limb surgery, } \\
\text { infection, fracture, osteonecrosis or } \\
\text { malignancy, and systemic or } \\
\text { neuromuscular diseases. Any } \\
\text { subjects receiving compensation for } \\
\text { work-related disabilities or who had } \\
\text { previously participated in a cognitive- } \\
\text { behavioural intervention }\end{array}$ & $\begin{array}{l}\text { Home-based } \\
\text { functional exercises } \\
\text { and kinesiophobia } \\
\text { training } \\
\text { Comp: S-F-B-T-E } \\
\text { AdjMod: mindfulness, } \\
\text { stress, anxiety } \\
\text { reduction } \\
\text { interventions } \\
\text { Set: H, I }\end{array}$ & 55 & $67(6.1)$ & $66 \%$ & $28(3.4)$ & NR \\
\hline & No data & No data & No data & $\begin{array}{l}\text { Usual care } \\
\text { Comp: S-F-B-T } \\
\text { AdjMod: - } \\
\text { Set: I }\end{array}$ & 55 & $68(7.1)$ & $62 \%$ & $28.3(5)$ & NR \\
\hline \multirow[t]{2}{*}{$\begin{array}{l}\text { Moutzouri, } \\
2018, \\
29473481 \\
\text { Greece }\end{array}$} & $\begin{array}{l}\text { Non- } \\
\text { industry }\end{array}$ & Moderate & $\begin{array}{l}\text { INCLUSION: Participants were that } \\
\text { they had elected to undergo primary } \\
\text { unilateral total knee replacement as } \\
\text { a result of advanced osteoarthritis } \\
\text { and they had been ambulatory at the } \\
\text { time of surgery. EXCLUSION: (a) } \\
\text { neurological conditions; (b) vestibular } \\
\text { disorders that might affect balance; } \\
\text { (c) other lower extremity orthopaedic } \\
\text { problems }\end{array}$ & $\begin{array}{l}\text { Early self-managed } \\
\text { focal sensorimotor } \\
\text { rehabilitative training } \\
\text { Comp: S-A-F-B-T } \\
\text { AdjMod: - } \\
\text { Set: H }\end{array}$ & 26 & $71.3(5.3)$ & NR & NR & NR \\
\hline & No data & No data & No data & $\begin{array}{l}\text { Functional exercise } \\
\text { training } \\
\text { Comp: S-A-F-T } \\
\text { AdjMod: - } \\
\text { Set: H }\end{array}$ & 25 & $72.3(5.6)$ & NR & NR & NR \\
\hline $\begin{array}{l}\text { Naylor, } \\
2017, \\
28899328, \\
\text { Australia }\end{array}$ & $\begin{array}{l}\text { Non- } \\
\text { industry }\end{array}$ & High & $\begin{array}{l}\text { INCLUSION: awaiting arthroplasty } \\
\text { secondary to OA, primary TKA, } \\
\text { private insurance. EXCLUSION: } \\
\text { patients referred to inpatient } \\
\text { rehabilitation because of slow } \\
\text { progress, and those who had } \\
\text { conditions that would alter their } \\
\text { typical recovery and rehabilitation } \\
\text { pathway, such as simultaneous } \\
\text { bilateral surgeries, as well as those } \\
\text { who experienced a significant } \\
\text { complication within } 90 \text { days of } \\
\text { surgery }\end{array}$ & $\begin{array}{l}\text { Inpatient rehabilitation } \\
\text { Comp: - } \\
\text { AdjMod: - } \\
\text { Set: I }\end{array}$ & 185 & $68.9(8.9)$ & $37 \%$ & $\begin{array}{l}30.6 \\
(5.9)\end{array}$ & NR \\
\hline
\end{tabular}




\begin{tabular}{|c|c|c|c|c|c|c|c|c|c|}
\hline $\begin{array}{l}\text { Study }{ }^{\mathrm{A}} \text {, Year, } \\
\text { PMID, } \\
\text { Country }\end{array}$ & $\begin{array}{l}\text { Funding } \\
\text { Source }\end{array}$ & $\begin{array}{l}\text { Risk of } \\
\text { Bias }\end{array}$ & Eligibility Criteria & Intervention ${ }^{\mathrm{B}}$ & $\begin{array}{l}\mathbf{N}, \\
\text { Enrolled }\end{array}$ & $\begin{array}{l}\text { Mean Age, } \\
\text { Years (SD) }\end{array}$ & $\begin{array}{l}\text { Female, } \\
\%\end{array}$ & $\begin{array}{l}\text { Mean } \\
\text { BMI } \\
\text { (SD) }\end{array}$ & $\begin{array}{l}\text { Prior } \\
\text { Contralateral } \\
\text { Athroplasty }\end{array}$ \\
\hline & No data & No data & No data & $\begin{array}{l}\text { No inpatient } \\
\text { rehabilitation } \\
\text { Comp: - } \\
\text { AdjMod: - } \\
\text { Set: NR (assumed } \\
\text { home) }\end{array}$ & 147 & $67.2(7.3)$ & $51 \%$ & $\begin{array}{l}30.7 \\
(5.1)\end{array}$ & NR \\
\hline \multirow[t]{3}{*}{$\begin{array}{l}\text { Padgett, } \\
2018 \\
29352683 \\
\text { USA }^{D}\end{array}$} & NR & Moderate & $\begin{array}{l}\text { INCLUSION: Patients undergoing } \\
\text { primary unilateral TKA with a } \\
\text { diagnosis of osteoarthritis with or } \\
\text { without inflammatory disorders. } \\
\text { EXCLUSION: Patients with a } \\
\text { diagnosis of post-traumatic } \\
\text { osteoarthritis }\end{array}$ & $\begin{array}{l}\text { Discharged to home } \\
\text { Comp: - } \\
\text { AdjMod: - } \\
\text { Set: H }\end{array}$ & 1213 & NR & NR & NR & NR \\
\hline & No data & No data & No data & $\begin{array}{l}\text { Discharged to long } \\
\text { term care facility } \\
\text { Comp: - } \\
\text { AdjMod:- } \\
\text { Set: I }\end{array}$ & 1213 & NR & NR & NR & NR \\
\hline & No data & No data & No data & $\begin{array}{l}\text { Discharged to } \\
\text { inpatient rehabilitation } \\
\text { Comp: - } \\
\text { AdjMod:- } \\
\text { Set: I }\end{array}$ & 492 & NR & NR & NR & NR \\
\hline \multirow[t]{2}{*}{$\begin{array}{l}\text { Petersen, } \\
2018, \\
29294078, \\
\text { Netherlands }\end{array}$} & $\begin{array}{l}\text { Non- } \\
\text { industry }\end{array}$ & Moderate & $\begin{array}{l}\text { INCLUSION: Hemi, or total, knee } \\
\text { replacement and } 18 \text { years. } \\
\text { EXCLUSION: Serious medical } \\
\text { conditions that would influence } \\
\text { rehabilitation (i.e., hip dysfunction, } \\
\text { myocardial diseases, or inflammatory } \\
\text { arthritis), current use of } \\
\text { anticoagulants, infection }\end{array}$ & $\begin{array}{l}\text { Exercise \& } \\
\text { acupuncture } \\
\text { Comp: S-A-F-B-T } \\
\text { AdjMod: Dry needling } \\
\text { Set: O }\end{array}$ & 87 & $56(8)$ & $56 \%$ & NR & NR \\
\hline & No data & No data & No data & $\begin{array}{l}\text { Exercise } \\
\text { Comp: S-A-F-B-T } \\
\text { AdjMod: - } \\
\text { Set: O }\end{array}$ & 85 & $56(6.8)$ & $61 \%$ & NR & NR \\
\hline
\end{tabular}




\begin{tabular}{|c|c|c|c|c|c|c|c|c|c|}
\hline $\begin{array}{l}\text { Study }{ }^{\mathrm{A}} \text {, Year, } \\
\text { PMID, } \\
\text { Country }\end{array}$ & $\begin{array}{l}\text { Funding } \\
\text { Source }\end{array}$ & $\begin{array}{l}\text { Risk of } \\
\text { Bias }\end{array}$ & Eligibility Criteria & Intervention ${ }^{B}$ & $\begin{array}{l}\mathrm{N}, \\
\text { Enrolled }\end{array}$ & $\begin{array}{l}\text { Mean Age, } \\
\text { Years (SD) }\end{array}$ & $\begin{array}{l}\text { Female, } \\
\%\end{array}$ & $\begin{array}{l}\text { Mean } \\
\text { BMIC } \\
\text { (SD) }\end{array}$ & $\begin{array}{l}\text { Prior } \\
\text { Contralateral } \\
\text { Athroplasty }\end{array}$ \\
\hline \multirow[t]{2}{*}{$\begin{array}{l}\text { Petterson, } \\
2009, \\
19177542, \\
\text { USA }\end{array}$} & $\begin{array}{l}\text { Non- } \\
\text { industry }\end{array}$ & High & $\begin{array}{l}\text { INCLUSION: Ages } 50-85 \text { years } \\
\text { scheduled to undergo unilateral TKA. } \\
\text { EXCLUSION: 1) uncontrolled } \\
\text { hypertension, 2) diabetes, 3) body } \\
\text { mass index (BMI) } 40 \mathrm{~kg} / \mathrm{m} 2(20), 4) \\
\text { symptomatic OA in the contralateral } \\
\text { knee (defined as self-reported knee } \\
\text { pain }>4 \text { on a } 10 \text {-point verbal analog } \\
\text { scale), 5) other lower extremity } \\
\text { orthopedic problems limiting function, } \\
6 \text { ) neurologic impairment, or } 7 \text { ) a } \\
\text { residence outside of a } 20 \text {-mile radius } \\
\text { of the clinic }\end{array}$ & $\begin{array}{l}\text { Exercise \& NMES } \\
\text { Comp: S-F-T } \\
\text { AdjMod: NMES, } \\
\text { massage, } \\
\text { mobilizations } \\
\text { Set: O }\end{array}$ & 100 & $65.3(8.3)$ & $47 \%$ & $\begin{array}{l}29.67 \\
(4.85)\end{array}$ & NR \\
\hline & No data & No data & No data & $\begin{array}{l}\text { Exercise } \\
\text { Comp: S-F-T } \\
\text { AdjMod: Massage, } \\
\text { mobilizations } \\
\text { Set: O }\end{array}$ & 100 & $65.2(8.5)$ & $45 \%$ & $\begin{array}{l}29.99 \\
(3.90)\end{array}$ & NR \\
\hline \multirow[t]{2}{*}{$\begin{array}{l}\text { Piqueras, } \\
2013, \\
23474735, \\
\text { Spain }\end{array}$} & Industry & Moderate & $\begin{array}{l}\text { INCLUSION: Successful primary } \\
\text { TKA surgery; post-TKA active range } \\
\text { of motion: flexion } 80 \text { degrees and } \\
\text { extension } 10 \text { degrees, without signs } \\
\text { of stiffness; ability to walk with the } \\
\text { use of a walking aid. EXCLUSION: } \\
\text { Sensory, cognitive and/or praxic } \\
\text { impairment; concomitant medical } \\
\text { conditions that may influence the } \\
\text { rehabilitation process; discharge to } \\
\text { destination other than home; patients } \\
\text { with any local or systemic } \\
\text { complication (e.g. surgical wound } \\
\text { infection, suspicion of deep vein } \\
\text { thrombosis) in the } 3 \text { month follow- } \\
\text { up period }\end{array}$ & $\begin{array}{l}\text { Interactive virtual } \\
\text { telerehabilitation } \\
\text { system } \\
\text { Comp: S-F-T-E } \\
\text { AdjMod: - } \\
\text { Set: H }\end{array}$ & 72 & NR & $63 \%$ & NR & NR \\
\hline & No data & No data & No data & $\begin{array}{l}\text { Conventional } \\
\text { outpatient physical } \\
\text { therapy } \\
\text { Comp: S-F-T } \\
\text { AdjMod: - } \\
\text { Set: I, O }\end{array}$ & 70 & NR & $83 \%$ & NR & NR \\
\hline
\end{tabular}




\begin{tabular}{|c|c|c|c|c|c|c|c|c|c|}
\hline $\begin{array}{l}\text { Study }{ }^{\mathrm{A}} \text {, Year, } \\
\text { PMID, } \\
\text { Country }\end{array}$ & $\begin{array}{l}\text { Funding } \\
\text { Source }\end{array}$ & $\begin{array}{l}\text { Risk of } \\
\text { Bias }\end{array}$ & Eligibility Criteria & Intervention ${ }^{B}$ & $\begin{array}{l}\mathrm{N}, \\
\text { Enrolled }\end{array}$ & $\begin{array}{l}\text { Mean Age, } \\
\text { Years (SD) }\end{array}$ & $\begin{array}{l}\text { Female, } \\
\%\end{array}$ & $\begin{array}{l}\text { Mean } \\
\text { BMIC } \\
\text { (SD) }\end{array}$ & $\begin{array}{l}\text { Prior } \\
\text { Contralateral } \\
\text { Athroplasty }\end{array}$ \\
\hline \multirow[t]{2}{*}{$\begin{array}{l}\text { Piva, } \\
2017, \\
28217891, \\
\text { USA }\end{array}$} & $\begin{array}{l}\text { Non- } \\
\text { industry }\end{array}$ & Moderate & $\begin{array}{l}\text { INCLUSION: Subjects who were } \\
\text { ages } 50 \text { years, had unilateral TKR } \\
\text { done } 3 \text { to } 6 \text { months prior to starting } \\
\text { the study, had medical clearance } \\
\text { from the knee surgeon to participate } \\
\text { in the study, and were English } \\
\text { speakers. EXCLUSION: Bilateral or } \\
\text { revision TKR, previous hip or ankle } \\
\text { joint replacement, regular } \\
\text { participation in exercise programs } \\
\text { (>2 times a week), inability to } \\
\text { ambulate } 30 \text { meters without an } \\
\text { assistive device, } 2 \text { or more falls } \\
\text { within the past year, acute illness, } \\
\text { severe visual impairment, lower-leg } \\
\text { amputation, uncontrolled diabetes } \\
\text { mellitus, and other neurologic, } \\
\text { muscular, and cardiovascular } \\
\text { diseases that could confound the } \\
\text { results or prevent safe exercise } \\
\text { participation }\end{array}$ & $\begin{array}{l}\text { Comprehensive } \\
\text { behavioral } \\
\text { intervention } \\
\text { Comp: S-A-F-B-T-E } \\
\text { AdjMod: - } \\
\text { Set: O, H }\end{array}$ & 22 & $68.1(7.5)$ & $82 \%$ & $\begin{array}{l}31.2 \\
(3.6)\end{array}$ & $\mathrm{NR}$ \\
\hline & No data & No data & No data & $\begin{array}{l}\text { Standard care } \\
\text { exercise } \\
\text { Comp: S-A-F-E } \\
\text { AdjMod: - } \\
\text { Set: O, H }\end{array}$ & 22 & $68.3(5.5)$ & $59 \%$ & $\begin{array}{l}29.3 \\
(4.1)\end{array}$ & NR \\
\hline \multirow[t]{3}{*}{$\begin{array}{l}\text { Piva, } \\
2019, \\
30794296, \\
\text { USA }\end{array}$} & $\begin{array}{l}\text { Non- } \\
\text { industry }\end{array}$ & Moderate & $\begin{array}{l}\text { INCLUSION: Unilateral primary } \\
\text { TKR, >= } 60 \text { years, TKR } 2-4 \text { mo } \\
\text { before screening, moderate } \\
\text { functional limitations defined by a } \\
\text { WOMAC-PF >=9, medical clearance } \\
\text { to exercise. EXCLUSION: } \\
\text { Contraindications to exercise, } \\
\text { neuromuscular disorders of the lower } \\
\text { extremities, inability to independently } \\
\text { walk } 50 \mathrm{~m} \text {, terminal illness, intent to } \\
\text { undergo another TKR. }\end{array}$ & $\begin{array}{l}\text { Community-based } \\
\text { exercise group } \\
\text { Comp: S-A-F-B-T } \\
\text { AdjMod: - } \\
\text { Set: Gym, community } \\
\text { centre }\end{array}$ & 96 & $70(7)$ & $60 \%$ & $\begin{array}{l}31.3 \\
(6.3)\end{array}$ & NR \\
\hline & No data & No data & No data & $\begin{array}{l}\text { Clinic-based } \\
\text { individual physical } \\
\text { therapy exercise } \\
\text { Comp: S-A-B-T-E } \\
\text { AdjMod: - } \\
\text { Set: O }\end{array}$ & 96 & $69(6)$ & $62 \%$ & $\begin{array}{l}30.8 \\
(5.3)\end{array}$ & NR \\
\hline & No data & No data & No data & $\begin{array}{l}\text { Standard care } \\
\text { Comp: - } \\
\text { AdjMod: - } \\
\text { Set: - }\end{array}$ & 48 & $70(7)$ & $65 \%$ & $\begin{array}{l}31.5 \\
(5.1)\end{array}$ & NR \\
\hline
\end{tabular}




\begin{tabular}{|c|c|c|c|c|c|c|c|c|c|}
\hline $\begin{array}{l}\text { Study }{ }^{A} \text {, Year, } \\
\text { PMID, } \\
\text { Country }\end{array}$ & $\begin{array}{l}\text { Funding } \\
\text { Source }\end{array}$ & $\begin{array}{l}\text { Risk of } \\
\text { Bias }\end{array}$ & Eligibility Criteria & Intervention ${ }^{B}$ & $\begin{array}{l}\mathrm{N}, \\
\text { Enrolled }\end{array}$ & $\begin{array}{l}\text { Mean Age, } \\
\text { Years (SD) }\end{array}$ & $\begin{array}{l}\text { Female, } \\
\%\end{array}$ & $\begin{array}{l}\text { Mean } \\
\text { BMIc } \\
\text { (SD) }\end{array}$ & $\begin{array}{l}\text { Prior } \\
\text { Contralateral } \\
\text { Athroplasty }\end{array}$ \\
\hline \multirow[t]{3}{*}{$\begin{array}{l}\text { Pua, } \\
2017, \\
27810379, \\
\text { Singapore }^{\mathrm{D}}\end{array}$} & $\begin{array}{l}\text { Non- } \\
\text { industry }\end{array}$ & Moderate & $\begin{array}{l}\text { INCLUSION: Patients age }>/=50 \\
\text { years who underwent a primary TKA } \\
\text { and were discharged home non- } \\
\text { missing follow-up ( } 6 \text { months). } \\
\text { EXCLUSION: Patients who had a } \\
\text { history of rheumatoid arthritis and } \\
\text { patients with stroke or Parkinson's } \\
\text { disease }\end{array}$ & $\begin{array}{l}\text { Rehabilitation } \\
\text { attendance: two or } \\
\text { more sessions } \\
\text { Comp: S-A-F-B-T-E } \\
\text { AdjMod: cold, NMES } \\
\text { Set: O }\end{array}$ & 1386 & $\begin{array}{l}67.1(7.5) ; \\
\text { Median } \\
\text { SUBVALUE( } \\
66.8)\end{array}$ & $76 \%$ & $\begin{array}{l}27.3 \\
(4.4)\end{array}$ & $\mathrm{NR}$ \\
\hline & No data & No data & No data & $\begin{array}{l}\text { Rehabilitation } \\
\text { attendance: once } \\
\text { Comp: S-A-F-B-T-E } \\
\text { AdjMod: cold, NMES } \\
\text { Set: O }\end{array}$ & 86 & $\begin{array}{l}70(7.3) ; \\
\text { Median } \\
\text { SUBVALUE( } \\
71.2)\end{array}$ & $77 \%$ & $\begin{array}{l}28.5 \\
(4.9)\end{array}$ & NR \\
\hline & No data & No data & No data & $\begin{array}{l}\text { Rehabilitation } \\
\text { attendance: none } \\
\text { Comp: - } \\
\text { AdjMod: - } \\
\text { Set: - }\end{array}$ & 68 & $\begin{array}{l}70.8(8.1) ; \\
\text { Median } \\
\text { SUBVALUE( } \\
71.5)\end{array}$ & $74 \%$ & $27(4.9)$ & NR \\
\hline \multirow[t]{2}{*}{$\begin{array}{l}\text { Rockstroh, } \\
2010, \\
20533147, \\
\text { Germany }\end{array}$} & Industry & High & $\begin{array}{l}\text { INCLUSION: age <= 85; had TKA } \\
\text { and were treated with inpatient rehab } \\
\text { at the Klinik Bavaria Kreischa. } \\
\text { EXCLUSION: people with } \\
\text { thrombosis, cardiopulmonary } \\
\text { insufficiency, tumors, infections, skin } \\
\text { diseases, rheumatism, alcohol or } \\
\text { drug abuse, ongoing pension } \\
\text { request, patients on diuretics, a } \\
\text { reduced calorie diet, or lymphatic } \\
\text { drainage, or those with mental or } \\
\text { speech problems. }\end{array}$ & $\begin{array}{l}\text { Physiotherapy \& } \\
\text { microcurrent } \\
\text { Comp: S-F-T } \\
\text { AdjMod: TENS } \\
\text { Set: I }\end{array}$ & 44 & 60) & NR & NR & NR \\
\hline & No data & No data & No data & $\begin{array}{l}\text { Physiotherapy } \\
\text { Comp: S-F-T } \\
\text { AdjMod: - } \\
\text { Set: I }\end{array}$ & 45 & 57) & NR & NR & NR \\
\hline
\end{tabular}




\begin{tabular}{|c|c|c|c|c|c|c|c|c|c|}
\hline $\begin{array}{l}\text { Study }{ }^{A} \text {, Year, } \\
\text { PMID, } \\
\text { Country }\end{array}$ & $\begin{array}{l}\text { Funding } \\
\text { Source }\end{array}$ & $\begin{array}{l}\text { Risk of } \\
\text { Bias }\end{array}$ & Eligibility Criteria & Intervention ${ }^{\mathrm{B}}$ & $\begin{array}{l}\mathbf{N}, \\
\text { Enrolled }\end{array}$ & $\begin{array}{l}\text { Mean Age, } \\
\text { Years (SD) }\end{array}$ & $\begin{array}{l}\text { Female, } \\
\%\end{array}$ & $\begin{array}{l}\text { Mean } \\
\text { BMIc } \\
\text { (SD) }\end{array}$ & $\begin{array}{l}\text { Prior } \\
\text { Contralateral } \\
\text { Athroplasty }\end{array}$ \\
\hline \multirow[t]{2}{*}{$\begin{array}{l}\text { Sattler, } \\
2019, \\
30994586 \\
\text { Australia }\end{array}$} & NR & Moderate & $\begin{array}{l}\text { INCLUSION: Patients }>/=18 \text { years of } \\
\text { age who were scheduled to undergo } \\
\text { uni- lateral TKR for a primary } \\
\text { diagnosis of osteoarthritis were } \\
\text { eligible for inclusion. EXCLUSION: } \\
\text { Patients were excluded if they (1) } \\
\text { preoperatively planned to be } \\
\text { discharged to an inpatient } \\
\text { rehabilitation/hostel facility such that } \\
\text { the home exercise program could not } \\
\text { be completed independently, (2) } \\
\text { declined to participate, or (3) were } \\
\text { scheduled for a contralateral TKR } \\
\text { within } 4 \text { months of the initial } \\
\text { procedure. }\end{array}$ & $\begin{array}{l}\text { Pedaling-based } \\
\text { protocol } \\
\text { Comp: F-T } \\
\text { AdjMod: - } \\
\text { Set: NR }\end{array}$ & 30 & $66.0(8.7)$ & $40 \%$ & $\begin{array}{l}29.4 \\
(4.4)\end{array}$ & $\mathrm{NR}$ \\
\hline & No data & No data & No data & $\begin{array}{l}\text { Non-pedalling (multi- } \\
\text { exercise protocol) } \\
\text { Comp: S-F-T } \\
\text { AdjMod: - } \\
\text { Set: - }\end{array}$ & 30 & $66.8(6.7)$ & $27 \%$ & $\begin{array}{l}29.3 \\
(4.3)\end{array}$ & NR \\
\hline \multirow[t]{2}{*}{$\begin{array}{l}\text { Schache, } \\
2019 \\
31208916 \\
\text { Australia }\end{array}$} & NR & Moderate & $\begin{array}{l}\text { INCLUSION: >=50 years and had } \\
\text { undergone primary unilateral TKA for } \\
\text { end-stage OA in the previous } 2 \\
\text { weeks. EXCLUSION: Uncontrolled } \\
\text { cardiovascular disease or } \\
\text { uncontrolled diabetes; a history of } \\
\text { ipsilateral hip replacement, ipsilateral } \\
\text { hip OA or lateral hip pain; or } \\
\text { neurological or any other conditions } \\
\text { affecting strength or function of the } \\
\text { lower limbs. }\end{array}$ & $\begin{array}{l}\text { Standard rehab and } \\
\text { hip strengthening } \\
\text { exercises } \\
\text { Comp: S-A-F-B-T-E } \\
\text { AdjMod: massage, } \\
\text { mobilization } \\
\text { Set: I, O }\end{array}$ & 54 & $70(7)$ & $72 \%$ & $30(6)$ & NR \\
\hline & No data & No data & No data & $\begin{array}{l}\text { Standard rehab plus } \\
\text { general functional } \\
\text { exercise } \\
\text { Comp: S-A-F-B-T-E } \\
\text { AdjMod: massage, } \\
\text { mobilizations } \\
\text { Set: I, O } \\
\end{array}$ & 51 & $69(7)$ & $58 \%$ & $31(6)$ & NR \\
\hline $\begin{array}{l}\text { Shanb, } \\
2014, \\
\text { CN- } \\
01041112, \\
\text { Saudi Arabia }\end{array}$ & NR & High & $\begin{array}{l}\text { INCLUSION: } 58-67 \text { years, cemented } \\
\text { fixed and bore non constrained } \\
\text { prosthesis, BMI<30, moderate } \\
\text { activity. EXCLUSION: previous knee } \\
\text { surgery with post op complications }\end{array}$ & $\begin{array}{l}\text { Active exercise } \\
\text { training program \& } \\
\text { biofeedback } \\
\text { Comp: S } \\
\text { AdjMod: mobilization, } \\
\text { biofeedback devices } \\
\text { Set: O }\end{array}$ & $\begin{array}{l}50 \text { (all } \\
\text { participant } \\
\text { s) }\end{array}$ & $60.00(0.89)$ & $38 \%$ & $\begin{array}{l}25.28 \\
(0.44)\end{array}$ & NR \\
\hline
\end{tabular}




\begin{tabular}{|c|c|c|c|c|c|c|c|c|c|}
\hline $\begin{array}{l}\text { Study }{ }^{A} \text {, Year, } \\
\text { PMID, } \\
\text { Country }\end{array}$ & $\begin{array}{l}\text { Funding } \\
\text { Source }\end{array}$ & $\begin{array}{l}\text { Risk of } \\
\text { Bias }\end{array}$ & Eligibility Criteria & Intervention ${ }^{\mathrm{B}}$ & $\begin{array}{l}\text { N, } \\
\text { Enrolled }\end{array}$ & $\begin{array}{l}\text { Mean Age, } \\
\text { Years (SD) }\end{array}$ & $\begin{array}{l}\text { Female, } \\
\%\end{array}$ & $\begin{array}{l}\text { Mean } \\
\text { BMIc } \\
\text { (SD) }\end{array}$ & $\begin{array}{l}\text { Prior } \\
\text { Contralateral } \\
\text { Athroplasty }\end{array}$ \\
\hline & No data & No data & No data & $\begin{array}{l}\text { Active exercise } \\
\text { training program } \\
\text { Comp: S } \\
\text { AdjMod: mobilization } \\
\text { Set: O }\end{array}$ & & $60.6(5.08)$ & $30 \%$ & $\begin{array}{l}26.18 \\
(0.45)\end{array}$ & NR \\
\hline \multirow[t]{2}{*}{$\begin{array}{l}\text { Stevens- } \\
\text { Lapsley, } \\
2012, \\
22095207, \\
\text { USA }\end{array}$} & $\begin{array}{l}\text { Non- } \\
\text { industry }\end{array}$ & Moderate & $\begin{array}{l}\text { INCLUSION: } 50-85 \text { years. } \\
\text { EXCLUSION: Uncontrolled } \\
\text { hypertension, uncontrolled diabetes, } \\
\text { BMI > } 35 \mathrm{~kg} / \mathrm{m} 2 \text {, significant } \\
\text { neurologic impairments, contralateral } \\
\text { knee OA (as defined by pain > than } \\
4 / 10 \text { with activity), or other unstable } \\
\text { lower-extremity orthopaedic } \\
\text { conditions. }\end{array}$ & $\begin{array}{l}\text { Standard } \\
\text { rehabilitation \& NMES } \\
\text { Comp: S-A-F-B-T-E } \\
\text { AdjMod: cold, NMES, } \\
\text { massage, } \\
\text { mobilization } \\
\text { Set: I, H, O }\end{array}$ & 35 & $66.2(9.1)$ & $57 \%$ & $\begin{array}{l}27.1 \\
(4.9)\end{array}$ & NR \\
\hline & No data & No data & No data & $\begin{array}{l}\text { Standard } \\
\text { rehabilitation } \\
\text { Comp: S-A-F-B-T-E } \\
\text { AdjMod: cold, } \\
\text { massage, } \\
\text { mobilization } \\
\text { Set: I, H, O }\end{array}$ & 31 & $64.8(7.7)$ & $52 \%$ & $\begin{array}{l}31.2 \\
(4.2)\end{array}$ & NR \\
\hline \multirow[t]{2}{*}{$\begin{array}{l}\text { Tousignant, } \\
2011, \\
21398389, \\
\text { Canada }\end{array}$} & $\begin{array}{l}\text { Non- } \\
\text { industry }\end{array}$ & High & $\begin{array}{l}\text { INCLUSION: Patients who had TKA. } \\
\text { EXCLUSION: NR }\end{array}$ & $\begin{array}{l}\text { Home } \\
\text { telerehabilitation } \\
\text { Comp: E } \\
\text { AdjMod: - } \\
\text { Set: H }\end{array}$ & 21 & $66(10)$ & NR & NR & NR \\
\hline & No data & No data & No data & $\begin{array}{l}\text { Conventional } \\
\text { rehabilitation } \\
\text { Comp: E } \\
\text { AdjMod: - } \\
\text { Set: O }\end{array}$ & 20 & $66(13)$ & NR & NR & NR \\
\hline $\begin{array}{l}\text { Tsukada, } \\
2020, \\
31723080, \\
\text { Japan }\end{array}$ & $\begin{array}{l}\text { Non- } \\
\text { industry }\end{array}$ & Moderate & $\begin{array}{l}\text { INCLUSION: unilateral TKA for knee } \\
\text { OA, female, >50 years. } \\
\text { EXCLUSION: lower limb amputation, } \\
\text { lower limb surgery in the last } 3 \\
\text { months, inability to walk without a } \\
\text { cane or walker, inflammatory joint, } \\
\text { rheumatoid, psoriatic arthritis, } \\
\text { polymyalgia rheumatic, multiple } \\
\text { sclerosis, neurodegenerative } \\
\text { disorder, known neuropathy, } \\
\text { uncontrolled diabetes, currently } \\
\text { being treated for cancer, terminal } \\
\text { illness, h/o myocardial infarction, use } \\
\text { of supplemental oxygen, implanted } \\
\text { cardiac pacemaker }\end{array}$ & $\begin{array}{l}\text { Standard } \\
\text { rehabilitation \& } \\
\text { hybride training } \\
\text { system } \\
\text { Comp: S-A-F-T-E } \\
\text { AdjMod: cold, NMES } \\
\text { Set: I }\end{array}$ & 26 & $72.8(8.2)$ & - & $\begin{array}{l}27.0 \\
(4.7)\end{array}$ & NR \\
\hline
\end{tabular}




\begin{tabular}{|c|c|c|c|c|c|c|c|c|c|}
\hline $\begin{array}{l}\text { Study }{ }^{\mathrm{A}} \text {, Year, } \\
\text { PMID, } \\
\text { Country }\end{array}$ & $\begin{array}{l}\text { Funding } \\
\text { Source }\end{array}$ & $\begin{array}{l}\text { Risk of } \\
\text { Bias }\end{array}$ & Eligibility Criteria & Intervention $^{B}$ & $\begin{array}{l}\mathrm{N}, \\
\text { Enrolled }\end{array}$ & $\begin{array}{l}\text { Mean Age, } \\
\text { Years (SD) }\end{array}$ & $\begin{array}{l}\text { Female, } \\
\%\end{array}$ & $\begin{array}{l}\text { Mean } \\
\text { BMIC } \\
\text { (SD) }\end{array}$ & $\begin{array}{l}\text { Prior } \\
\text { Contralateral } \\
\text { Athroplasty }\end{array}$ \\
\hline & No data & No data & No data & $\begin{array}{l}\text { Standard } \\
\text { rehabilitation } \\
\text { Comp: S-A-F-T-E } \\
\text { AdjMod: cold } \\
\text { Set: I }\end{array}$ & 27 & $74.1(8.6)$ & - & $\begin{array}{l}27.2 \\
(4.6)\end{array}$ & $\mathrm{NR}$ \\
\hline \multirow[t]{2}{*}{$\begin{array}{l}\text { Vuorenmaa, } \\
2014, \\
\text { 24241606, } \\
\text { Finland }\end{array}$} & $\begin{array}{l}\text { Non- } \\
\text { industry }\end{array}$ & Moderate & $\begin{array}{l}\text { INCLUSION: (i) diagnosed knee OA; } \\
\text { (ii) primary arthroplasty of the knee in } \\
\text { question; and (iii) age over } 18 \text { years. } \\
\text { EXCLUSION: (i) other surgery for the } \\
\text { lower limbs planned to be performed } \\
\text { within } 12 \text { months; (ii) dementia; (iii) } \\
\text { fibro- myalgia; (iv) other serious co- } \\
\text { morbidities preventing active training; } \\
\text { and (v) difficulty visiting a } \\
\text { physiotherapist due to a long } \\
\text { travelling distance. }\end{array}$ & $\begin{array}{l}\text { Home exercise } \\
\text { Comp: S-F-E } \\
\text { AdjMod: - } \\
\text { Set: O, H }\end{array}$ & 53 & $69(8)$ & $57 \%$ & $31(5)$ & $28 \%$ \\
\hline & No data & No data & No data & $\begin{array}{l}\text { Control } \\
\text { Comp: - } \\
\text { AdjMod: - } \\
\text { Set: H }\end{array}$ & 55 & $69(9)$ & $65 \%$ & $31(6)$ & $31 \%$ \\
\hline \multirow[t]{2}{*}{$\begin{array}{l}\text { Zapparoli, } \\
2020, \\
32488010 \\
\text { Italy }\end{array}$} & $\begin{array}{l}\text { Non- } \\
\text { Industry }\end{array}$ & High & $\begin{array}{l}\text { INCLUSION: (1) age comprised } \\
\text { between } 45 \text { and } 80 \text { years old, (2) } \\
\text { being enrolled in the local residential } \\
\text { rehabilitation program. EXCLUSION: } \\
\text { (1) presence of neurologic or } \\
\text { neurodegenerative diseases, (2) on- } \\
\text { going psychopharmacological } \\
\text { treatments. }\end{array}$ & $\begin{array}{l}\text { Motor imagery \& } \\
\text { rehabilitation } \\
\text { Comp: S-F-B } \\
\text { AdjMod: Mindfulness } \\
\text { Set: Al }\end{array}$ & 24 & $66.2(8.0)$ & $46 \%$ & $\begin{array}{l}38.4 \\
(6.6)\end{array}$ & NR \\
\hline & No data & No data & No data & $\begin{array}{l}\text { Rehabilitation } \\
\text { Comp: S-F-B } \\
\text { AdjMod: - } \\
\text { Set: Al }\end{array}$ & 24 & $66.6(7.5)$ & $71 \%$ & $\begin{array}{l}31.4 \\
(6.7)\end{array}$ & NR \\
\hline
\end{tabular}

Abbreviations: $\mathrm{AI}=$ acute inpatient, $\mathrm{BMI}=$ body mass index, $\mathrm{DVT}=$ deep vein thrombosis, $\mathrm{H}=$ home, mo $=$ month, $\mathrm{NA}=$ not applicable, $\mathrm{NMES}=$ neuromuscular electrical stimulation, $\mathrm{NR}=$ not reported, $\mathrm{O}=$ outpatient physiotherapy center, $\mathrm{OA}=$ osteoarthritis, $\mathrm{PMID}=\mathrm{PubMed}$ identifier, $\mathrm{RA}=$ rheumatoid arthritis, $\mathrm{SD}=$ standard deviation, $\mathrm{SD}=$ standard deviation, TENS $=$ transcutaneous electrical nerve stimulation, THA $=$ total hip arthroplasty, TJA $=$ total joint arthroplasty, TKA $=$ total knee arthroplasty, wks $=$ weeks, yo $=$ years old, $\mathrm{yr}=$ year.

A All randomized controlled trials, except as footnoted.

B Including Components (Comp); Adjunctive modalities (AdjMod); and Setting (Set).

Components: $\mathrm{A}=$ aerobic exercise, $\mathrm{B}=$ Balance-motor/Learning-agility exercise, $\mathrm{E}=$ patient education, $\mathrm{F}=$ flexibility exercise, $\mathrm{S}=$ strengthening exercise, $\mathrm{T}=$ task-specific training.

C $\mathrm{kg} / \mathrm{m}^{2}$

D Non-randomized controlled study 
Table C-2.2. KQ 2. Rehabilitation component details

\begin{tabular}{|c|c|c|c|c|c|c|c|}
\hline $\begin{array}{l}\text { Study, Year, } \\
\text { PMID, } \\
\text { Country }\end{array}$ & $\begin{array}{l}\text { Intended } \\
\text { Comparison }\end{array}$ & Arm & Components (Specific Exercises/Strategies) & $\begin{array}{l}\text { Progression } \\
\text { (Appropriate?) }\end{array}$ & Personnel & $\begin{array}{l}\text { Mode of } \\
\text { Delivery }\end{array}$ & Setting \\
\hline \multirow[t]{2}{*}{$\begin{array}{l}\text { Andersen, } \\
\text { 2018, } \\
\text { CN-01647420, } \\
\text { Denmark }\end{array}$} & $\begin{array}{l}\text { Technological } \\
\text { assisted } \\
\text { rehabilitation vs. } \\
\text { supervised } \\
\text { rehabilitation (usual } \\
\text { care) [Abstract only] }\end{array}$ & $\begin{array}{l}\text { Technological } \\
\text { assisted } \\
\text { rehabilitation }\end{array}$ & $\begin{array}{l}\text { [specific goals and exercises not defined; } \\
\text { comparison of setting and method of delivery] }\end{array}$ & $0(\mathrm{NA})$ & Unclear & Unclear & Home \\
\hline & No data & $\begin{array}{l}\text { Supervised } \\
\text { rehabilitation }\end{array}$ & $\begin{array}{l}\text { [specific goals and exercises not defined; } \\
\text { comparison of setting and method of delivery] }\end{array}$ & 0 (NA) & $\begin{array}{l}\text { Physical } \\
\text { therapist }\end{array}$ & In-person & $\begin{array}{l}\text { Physical } \\
\text { therapy/rehabilitation } \\
\text { facility (outpatient) }\end{array}$ \\
\hline \multirow[t]{2}{*}{$\begin{array}{l}\text { Artz, } \\
2017 \\
27068368 \\
\text { UK }\end{array}$} & $\begin{array}{l}\text { Group-based } \\
\text { outpatient } \\
\text { physiotherapy vs. } \\
\text { usual care }\end{array}$ & $\begin{array}{l}\text { Group-based } \\
\text { exercise }\end{array}$ & $\begin{array}{l}\text { 1. Strength } \\
\text { [specific exercises not defined] } \\
\text { 2. Aerobic } \\
\text { [specific exercises not defined] } \\
\text { 4. Balance-Motor Learning-Agility } \\
\text { [specific exercises not defined] } \\
\text { 5. Task specific training } \\
\text { [specific exercises not defined] } \\
\text { 6. Patient education } \\
\text { 6.2 Home exercise program }\end{array}$ & 0 (NA) & $\begin{array}{l}\text { Physical } \\
\text { therapist }\end{array}$ & In-person & $\begin{array}{l}\text { Physical } \\
\text { therapy/rehabilitation } \\
\text { facility (outpatient) }\end{array}$ \\
\hline & No data & Usual care & $\begin{array}{l}\text { NA } \\
\text { [Routine post-operative care provided by the } \\
\text { health service including an exercise booklet and } \\
\text { individual referral to physiotherapy if indicated] }\end{array}$ & 0 (NA) & NA & NA & NA \\
\hline \multirow[t]{2}{*}{$\begin{array}{l}\text { Avramidis, } \\
2011 \\
21410130 \\
\text { Greece }\end{array}$} & $\begin{array}{l}\text { Electric stimulation } \\
\text { of the vastus } \\
\text { medialis muscle } \\
\text { and standard } \\
\text { physiotherapy vs. } \\
\text { physiotherapy only }\end{array}$ & $\begin{array}{l}\text { Physiotherapy \& } \\
\text { electrical muscle } \\
\text { stimulation }\end{array}$ & $\begin{array}{l}\text { 1. Strength } \\
\text { 1.31 Knee extension in sitting or supine (long arc } \\
\text { quad) (long or short unclear) } \\
\text { 1.32 Knee extension in sitting or supine (short arc } \\
\text { quad) (long or short unclear) } \\
\text { 1.43 Quad sets } \\
\text { 1.58 Straight leg raise } \\
\text { 3. Flexibility } \\
\text { 3.4 Calf stretch with knee straight (gastroc) } \\
\text { 3.11 Knee extension PROM in supine (position } \\
\text { unclear) } \\
\text { 3.12 Knee extension PROM in prone (position } \\
\text { unclear) } \\
\text { 5. Task specific training } \\
\text { 5.8 Gait training } \\
\text { 7. Adjunctive modality } \\
\text { 7.5 E-stim for strength (NMES) }\end{array}$ & $\mathrm{Y}(\mathrm{N})$ & NR & In-person & $\begin{array}{l}\text { Acute Inpatient; } \\
\text { Physical } \\
\text { therapy/rehabilitation } \\
\text { facility (outpatient) }\end{array}$ \\
\hline & No data & Physiotherapy & $\begin{array}{l}\text { 1. Strength } \\
\text { 1.31 Knee extension in sitting or supine (long arc } \\
\text { quad) (long or short unclear) } \\
\text { 1.32 Knee extension in sitting or supine (short arc } \\
\text { quad) (long or short unclear) } \\
\text { 1.43 Quad sets } \\
\text { 1.58 Straight leg raise } \\
\text { 3. Flexibility } \\
\text { 3.4 Calf stretch with knee straight (gastroc) } \\
\end{array}$ & $\mathrm{Y}(\mathrm{N})$ & NR & In-person & $\begin{array}{l}\text { Acute Inpatient; } \\
\text { Physical } \\
\text { therapy/rehabilitation } \\
\text { facility (outpatient) }\end{array}$ \\
\hline
\end{tabular}




\begin{tabular}{|c|c|c|c|c|c|c|c|}
\hline $\begin{array}{l}\text { Study, Year, } \\
\text { PMID, } \\
\text { Country }\end{array}$ & $\begin{array}{l}\text { Intended } \\
\text { Comparison }\end{array}$ & Arm & Components (Specific Exercises/Strategies) & $\begin{array}{l}\text { Progression } \\
\text { (Appropriate?) }\end{array}$ & Personnel & $\begin{array}{l}\text { Mode of } \\
\text { Delivery }\end{array}$ & Setting \\
\hline & & & $\begin{array}{l}\text { 3.11 Knee extension PROM in supine (position } \\
\text { unclear) } \\
3.12 \text { Knee extension PROM in prone (position } \\
\text { unclear) } \\
\text { 5. Task specific training } \\
\text { 5.8 Gait training }\end{array}$ & & & & \\
\hline $\begin{array}{l}\text { Bade, } \\
2017, \\
27813347, \\
\text { USA }\end{array}$ & $\begin{array}{l}\text { High-intensity } \\
\text { progressive } \\
\text { rehabilitation vs. } \\
\text { low-intensity } \\
\text { rehabilitation }\end{array}$ & $\begin{array}{l}\text { High-intensity } \\
\text { progressive } \\
\text { rehabilitation }\end{array}$ & 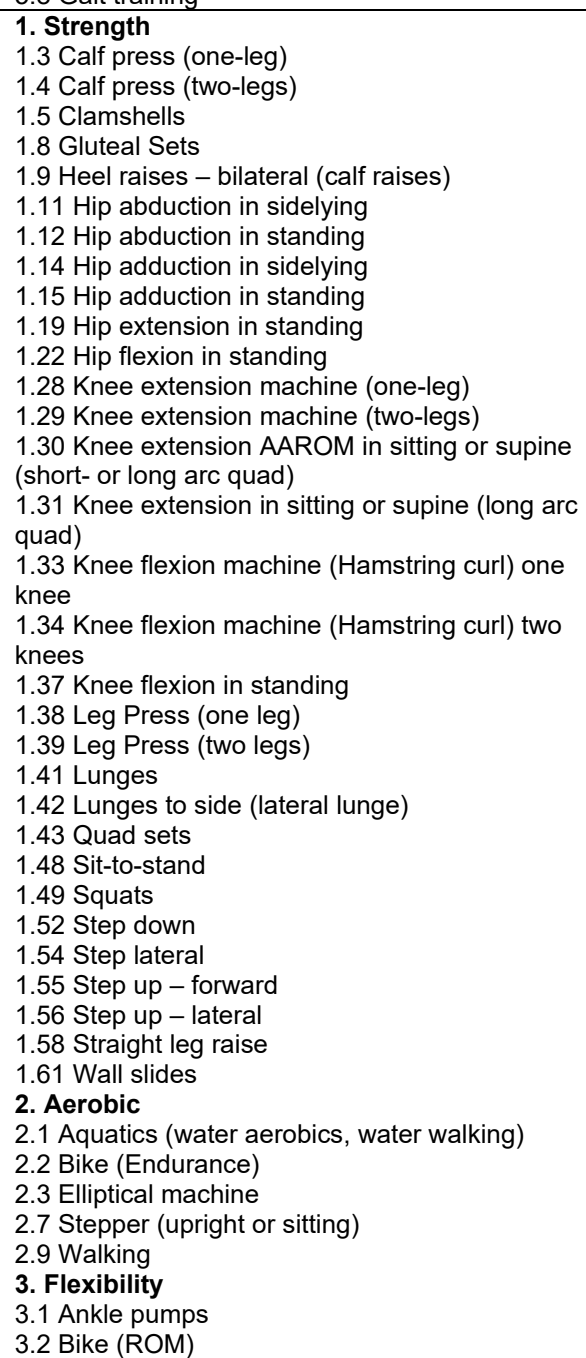 & $\mathrm{Y}(\mathrm{Y})$ & $\begin{array}{l}\text { Physical } \\
\text { therapist }\end{array}$ & In-person & $\begin{array}{l}\text { Physical } \\
\text { therapy/rehabilitation } \\
\text { facility (outpatient); } \\
\text { Home }\end{array}$ \\
\hline
\end{tabular}




\begin{tabular}{|c|c|c|c|c|c|c|c|}
\hline $\begin{array}{l}\text { Study, Year, } \\
\text { PMID, } \\
\text { Country }\end{array}$ & $\begin{array}{l}\text { Intended } \\
\text { Comparison }\end{array}$ & Arm & Components (Specific Exercises/Strategies) & $\begin{array}{l}\text { Progression } \\
\text { (Appropriate?) }\end{array}$ & Personnel & $\begin{array}{l}\text { Mode of } \\
\text { Delivery }\end{array}$ & Setting \\
\hline & & & $\begin{array}{l}\text { 3.4 Calf stretch with knee straight (gastroc) } \\
\text { 3.5 Hamstring stretch in any position } \\
\text { 3.6 Heel slides } \\
\text { 3.11 Knee extension PROM in supine (position } \\
\text { unclear) } \\
\text { 3.12 Knee extension PROM in prone (position } \\
\text { unclear) } \\
\text { 3.15 Knee flexion AROM in any position (rectus } \\
\text { femoris stretch) } \\
\text { 4. Balance-Motor Learning-Agility } \\
\text { 4.3 Balance on unstable surface } \\
\text { 4.6 Marching } \\
\text { 4.8 Single leg stance } \\
\text { 4.9 Standing weight shifts } \\
\text { 4.10 Stepping multiple directions (grapevine) } \\
\text { 4.13 Step lateral (side step) } \\
\text { 5. Task specific training } \\
\text { 5.1 Transfers } \\
\text { 5.8 Gait training } \\
\text { 5.15 Stair training } \\
\text { 6. Patient education } \\
\text { 6.2 Home exercise program } \\
\text { 6.4 Pain management } \\
\text { 6.6 Wound care management } \\
\text { 7. Adjunctive modality } \\
\text { 7.11 Massage for scar mobility } \\
\text { 7.13 Mobilizations - Tibiofemoral } \\
\text { 7.14 Mobilizations - Patellar }\end{array}$ & & & & \\
\hline & No data & $\begin{array}{l}\text { Low-intensity } \\
\text { rehabilitation }\end{array}$ & $\begin{array}{l}\text { 1. Strength } \\
\text { 1.5 Clamshells } \\
\text { 1.8 Gluteal Sets } \\
\text { 1.9 Heel raises - bilateral (calf raises) } \\
\text { 1.32 Knee extension in sitting or supine (short arc } \\
\text { quad) } \\
\text { 1.36 Knee flexion in sitting or supine } \\
\text { 1.37 Knee flexion in standing } \\
\text { 1.43 Quad sets } \\
\text { 1.47 Single leg stance } \\
\text { 1.48 Sit-to-stand } \\
\text { 1.49 Squats } \\
\text { 1.51 Standing terminal knee extension } \\
\text { 1.57 Stool scoots } \\
\text { 1.58 Straight leg raise } \\
\text { 2. Aerobic } \\
\text { 2.2 Bike (Endurance) } \\
\text { 2.9 Walking } \\
\text { 3. Flexibility } \\
\text { 3.1 Ankle pumps } \\
\text { 3.2 Bike (ROM) } \\
\text { 3.4 Calf stretch with knee straight (gastroc) } \\
\text { 3.5 Hamstring stretch in any position }\end{array}$ & $\mathrm{Y}(\mathrm{N})$ & $\begin{array}{l}\text { Physical } \\
\text { therapist }\end{array}$ & In-person & $\begin{array}{l}\text { Physical } \\
\text { therapy/rehabilitation } \\
\text { facility (outpatient); } \\
\text { Home }\end{array}$ \\
\hline
\end{tabular}




\begin{tabular}{|c|c|c|c|c|c|c|c|}
\hline $\begin{array}{l}\text { Study, Year, } \\
\text { PMID, } \\
\text { Country }\end{array}$ & $\begin{array}{l}\text { Intended } \\
\text { Comparison }\end{array}$ & Arm & Components (Specific Exercises/Strategies) & $\begin{array}{l}\text { Progression } \\
\text { (Appropriate?) }\end{array}$ & Personnel & $\begin{array}{l}\text { Mode of } \\
\text { Delivery }\end{array}$ & Setting \\
\hline & & & $\begin{array}{l}\text { 3.6 Heel slides } \\
\text { 3.11 Knee extension PROM in supine (position } \\
\text { unclear) } \\
\text { 3.12 Knee extension PROM in prone (position } \\
\text { unclear) } \\
\text { 3.15 Knee flexion AROM in any position (rectus } \\
\text { femoris stretch) } \\
\text { 4. Balance-Motor Learning-Agility } \\
\text { 4.3 Balance on unstable surface } \\
\text { 4.6 Marching } \\
\text { 4.8 Single leg stance } \\
\text { 4.9 Standing weight shifts } \\
\text { 5. Task specific training } \\
\text { 5.1 Transfers } \\
\text { 5.8 Gait training } \\
\text { 5.15 Stair training } \\
\text { 6. Patient education } \\
\text { 6.1 Activities of daily living } \\
\text { 6.2 Home exercise program } \\
\text { 6.4 Pain management } \\
\text { 6.6 Wound care management } \\
\text { 7. Adjunctive modality } \\
\text { 7.1 Cold } \\
\text { 7.2 Heat } \\
\text { 7.11 Massage for scar mobility } \\
\text { 7.13 Mobilizations - Tibiofemoral } \\
\text { 7.14 Mobilizations - Patellar }\end{array}$ & & & & \\
\hline \multirow[t]{2}{*}{$\begin{array}{l}\text { Bily, } \\
2016, \\
26763947, \\
\text { Austria }\end{array}$} & $\begin{array}{l}\text { Leg-press training } \\
\text { with moderate } \\
\text { vibration vs. } \\
\text { functional } \\
\text { physiotherapy }\end{array}$ & Leg-press & $\begin{array}{l}\text { 1. Strength } \\
\text { 1.38 Leg Press (one leg) }\end{array}$ & $\mathrm{Y}(\mathrm{N})$ & Unclear & In-person & $\begin{array}{l}\text { Physical } \\
\text { therapy/rehabilitation } \\
\text { facility (outpatient) }\end{array}$ \\
\hline & No data & Physiotherapy & $\begin{array}{l}\text { 1. Strength } \\
\text { 1.11 Hip abduction in sidelying } \\
\text { 1.12 Hip abduction in standing } \\
\text { 1.13 Hip abduction in supine } \\
\text { 1.31 Knee extension in sitting or supine (long arc } \\
\text { quad)(short or long unclear) } \\
\text { 1.32 Knee extension in sitting or supine (short arc } \\
\text { quad) (short or long unclear) } \\
\text { 1.35 Knee flexion in prone (position unclear) } \\
\text { 1.36 Knee flexion in sitting or supine (position } \\
\text { unclear) } \\
\text { 1.37 Knee flexion in standing (position unclear) } \\
\text { 1.43 Quad sets } \\
\text { 1.49 Squats } \\
\text { 1.55 Step up - forward } \\
\text { 1.58 Straight leg raise } \\
\text { 3. Flexibility } \\
\text { 3.2 Bike (ROM) }\end{array}$ & $\mathrm{Y}(\mathrm{Y})$ & $\begin{array}{l}\text { Physical } \\
\text { therapist }\end{array}$ & In-person & $\begin{array}{l}\text { Physical } \\
\text { therapy/rehabilitation } \\
\text { facility (outpatient) }\end{array}$ \\
\hline
\end{tabular}




\begin{tabular}{|c|c|c|c|c|c|c|c|}
\hline $\begin{array}{l}\text { Study, Year, } \\
\text { PMID, } \\
\text { Country }\end{array}$ & $\begin{array}{l}\text { Intended } \\
\text { Comparison }\end{array}$ & Arm & Components (Specific Exercises/Strategies) & $\begin{array}{l}\text { Progression } \\
\text { (Appropriate?) }\end{array}$ & Personnel & $\begin{array}{l}\text { Mode of } \\
\text { Delivery }\end{array}$ & Setting \\
\hline & & & $\begin{array}{l}\text { 5. Task specific training } \\
\text { 5.8 Gait training } \\
\text { 7. Adjunctive modality } \\
\text { 7.11 Massage for scar mobility } \\
\text { 7.13 Mobilizations - Tibiofemoral } \\
\text { 7.14 Mobilizations - Patellar }\end{array}$ & & & & \\
\hline \multirow[t]{2}{*}{$\begin{array}{l}\text { Bruun-Olsen, } \\
2013, \\
23614370, \\
\text { Norway }\end{array}$} & $\begin{array}{l}\text { Walking-skill } \\
\text { program vs. usual } \\
\text { physiotherapy care }\end{array}$ & $\begin{array}{l}\text { Walking-skill } \\
\text { group }\end{array}$ & $\begin{array}{l}\text { 1. Strength } \\
\text { 1.41 Lunges } \\
\text { 1.42 Lunges to side (lateral lunge) } \\
\text { 1.47 Single leg stance } \\
\text { 1.48 Sit-to-stand } \\
\text { 1.49 Squats } \\
\text { 1.52 Step down } \\
\text { 1.55 Step up - forward } \\
\text { 2. Aerobic } \\
\text { 2.9 Walking } \\
\text { 3. Flexibility } \\
\text { 3.3 Calf stretch with knee bent (soleus) (position } \\
\text { unclear) } \\
\text { 3.4 Calf stretch with knee straight (gastroc) } \\
\text { (position unclear) } \\
\text { 3.5 Hamstring stretch in any position } \\
\text { 3.16 Knee flexion PROM in prone (rectus femoris } \\
\text { stretch) } \\
\text { 4. Balance-Motor Learning-Agility } \\
\text { 4.3 Balance on unstable surface } \\
\text { 4.4 Balance with perturbations } \\
\text { 4.8 Single leg stance } \\
\text { 4.9 Standing weight shifts } \\
\text { 4.11 Step down } \\
\text { 4.13 Step lateral (side step) } \\
\text { 4.14 Step up - forward } \\
\text { 5. Task specific training } \\
\text { 5.1 Transfers } \\
\text { 5.6 Gait on uneven surfaces } \\
\text { 5.7 Gait sideways } \\
\text { 5.8 Gait training } \\
\text { 5.10 Gait with perturbations } \\
\text { 5.12 Obstacle training } \\
\text { 5.13 Sit-to-stand training } \\
\text { 5.15 Stair training }\end{array}$ & $\mathrm{Y}(\mathrm{Y})$ & $\begin{array}{l}\text { Physical } \\
\text { therapist }\end{array}$ & In-person & $\begin{array}{l}\text { Physical } \\
\text { therapy/rehabilitation } \\
\text { facility (outpatient) }\end{array}$ \\
\hline & No data & $\begin{array}{l}\text { Usual } \\
\text { physiotherapy } \\
\text { care }\end{array}$ & $\begin{array}{l}\text { 1. Strength } \\
\text { [specific exercises not defined] } \\
\text { [12 physiotherapy sessions by community } \\
\text { physiotherapists; surveys after physiotherapy } \\
\text { indicate that most exercises targeted range of } \\
\text { motion and strengthening and primarily occurred } \\
\text { in sitting] }\end{array}$ & $\mathrm{N}(\mathrm{NA})$ & $\begin{array}{l}\text { Physical } \\
\text { therapist }\end{array}$ & In-person & $\begin{array}{l}\text { Physical } \\
\text { therapy/rehabilitation } \\
\text { facility (outpatient) }\end{array}$ \\
\hline $\begin{array}{l}\text { Buhagiar, } \\
2017 \text {, }\end{array}$ & $\begin{array}{l}\text { Monitored home } \\
\text { program vs. }\end{array}$ & Home program & $\begin{array}{l}\text { 1. Strength } \\
1.9 \text { Heel raises - bilateral (calf raises) }\end{array}$ & $\mathrm{Y}(\mathrm{Y})$ & $\begin{array}{l}\text { Physical } \\
\text { therapist }\end{array}$ & $\begin{array}{l}\text { In-person; } \\
\text { Home }\end{array}$ & Home \\
\hline
\end{tabular}




\begin{tabular}{|c|c|c|c|c|c|c|c|}
\hline $\begin{array}{l}\text { Study, Year, } \\
\text { PMID, } \\
\text { Country }\end{array}$ & $\begin{array}{l}\text { Intended } \\
\text { Comparison }\end{array}$ & Arm & Components (Specific Exercises/Strategies) & $\begin{array}{l}\text { Progression } \\
\text { (Appropriate?) }\end{array}$ & Personnel & $\begin{array}{l}\text { Mode of } \\
\text { Delivery }\end{array}$ & Setting \\
\hline \multirow[t]{2}{*}{$\begin{array}{l}\text { 28291891, } \\
\text { Australia }\end{array}$} & $\begin{array}{l}\text { inpatient } \\
\text { rehabilitation plus } \\
\text { monitored home } \\
\text { program }\end{array}$ & & $\begin{array}{l}\text { 1.19 Hip extension in standing } \\
\text { 1.32 Knee extension in sitting or supine (short arc } \\
\text { quad) } \\
\text { 1.37 Knee flexion in standing } \\
\text { 1.48 Sit-to-stand } \\
\text { 1.55 Step up - forward } \\
\text { 1.58 Straight leg raise } \\
\text { 1.60 Upper extremity strengthening } \\
\text { 2. Aerobic } \\
\text { 2.2 Bike (Endurance) } \\
\text { 2.9 Walking } \\
\text { 3. Flexibility } \\
\text { 3.3 Calf stretch with knee bent (soleus) } \\
\text { 3.4 Calf stretch with knee straight (gastroc) } \\
\text { 3.5 Hamstring stretch in any position } \\
\text { 3.6 Heel slides } \\
\text { 3.10 Knee extension AROM } \\
\text { 3.14 Knee flexion PROM in sitting or supine } \\
\text { 4. Balance-Motor Learning-Agility } \\
\text { 4.6 Marching } \\
\text { 4.8 Single leg stance } \\
\text { 4.15 Step up - lateral } \\
\text { 5. Task specific training } \\
\text { 5.13 Sit-to-stand training } \\
\text { 5.15 Stair training } \\
\text { 5.16 Treadmill gait } \\
\text { 6. Patient education } \\
\text { 6.2 Home exercise program }\end{array}$ & & & $\begin{array}{l}\text { (monitored; } \\
\text { access to PT); } \\
\text { Self-guided }\end{array}$ & \\
\hline & No data & $\begin{array}{l}\text { Hospital } \\
\text { inpatient } \\
\text { rehabilitation }\end{array}$ & $\begin{array}{l}\text { 1. Strength } \\
\text { 1.9 Heel raises - bilateral (calf raises) } \\
\text { 1.15 Hip adduction in standing } \\
\text { 1.19 Hip extension in standing } \\
\text { 1.31 Knee extension in sitting or supine (long arc } \\
\text { quad) } \\
\text { 1.32 Knee extension in sitting or supine (short arc } \\
\text { quad) } \\
\text { 1.36 Knee flexion in sitting or supine } \\
\text { 1.37 Knee flexion in standing } \\
\text { 1.48 Sit-to-stand } \\
\text { 1.49 Squats } \\
\text { 1.55 Step up - forward } \\
\text { 1.56 Step up - lateral } \\
\text { 1.58 Straight leg raise } \\
\text { 1.60 Upper extremity strengthening } \\
\text { 2. Aerobic } \\
\text { 2.2 Bike (Endurance) } \\
\text { 2.8 Treadmill walking } \\
\text { 2.9 Walking } \\
\text { 3. Flexibility } \\
\text { 3.3 Calf stretch with knee bent (soleus) } \\
\text { 3.4 Calf stretch with knee straight (gastroc) } \\
\end{array}$ & $\mathrm{Y}(\mathrm{Y})$ & $\begin{array}{l}\text { Physical } \\
\text { therapist }\end{array}$ & $\begin{array}{l}\text { In-person; } \\
\text { Home }\end{array}$ & $\begin{array}{l}\text { Acute inpatient } \\
\text { (postoperative) }\end{array}$ \\
\hline
\end{tabular}




\begin{tabular}{|c|c|c|c|c|c|c|c|}
\hline $\begin{array}{l}\text { Study, Year, } \\
\text { PMID, } \\
\text { Country }\end{array}$ & $\begin{array}{l}\text { Intended } \\
\text { Comparison }\end{array}$ & Arm & Components (Specific Exercises/Strategies) & $\begin{array}{l}\text { Progression } \\
\text { (Appropriate?) }\end{array}$ & Personnel & $\begin{array}{l}\text { Mode of } \\
\text { Delivery }\end{array}$ & Setting \\
\hline & & & $\begin{array}{l}\text { 3.5 Hamstring stretch in any position } \\
\text { 3.6 Heel slides } \\
\text { 3.10 Knee extension AROM } \\
\text { 3.14 Knee flexion PROM in sitting or supine } \\
\text { 3.16 Knee flexion PROM in prone (rectus femoris } \\
\text { stretch) } \\
\text { 4. Balance-Motor Learning-Agility } \\
\text { 4.6 Marching } \\
\text { 4.8 Single leg stance } \\
\text { 4.15 Step up - lateral } \\
\text { 5. Task specific training } \\
\text { 5.13 Sit-to-stand training } \\
\text { 5.15 Stair training } \\
\text { 5.16 Treadmill gait } \\
\text { 6. Patient education } \\
\text { 6.2 Home exercise program }\end{array}$ & & & & \\
\hline \multirow[t]{2}{*}{$\begin{array}{l}\text { Cai, } \\
2018, \\
29239772, \\
\text { China }\end{array}$} & $\begin{array}{l}\text { Cognitive } \\
\text { behavioral therapy } \\
\text { vs. standard care }\end{array}$ & $\begin{array}{l}\text { Cognitive } \\
\text { behavioral } \\
\text { therapy \& } \\
\text { standard care }\end{array}$ & $\begin{array}{l}\text { 6. Patient education } \\
\text { 6.1 Activities of daily living } \\
\text { 7. Adjunctive modality } \\
\text { 7.17 Mindfulness, stress/anxiety-reduction } \\
\text { interventions }\end{array}$ & $\mathrm{N}(\mathrm{NA})$ & $\begin{array}{l}\text { Physical } \\
\text { therapist; Other } \\
\text { (Psychologist) }\end{array}$ & In-person & NR \\
\hline & No data & Standard care & $\begin{array}{l}\text { NA } \\
\text { [Standard care including pain management and } \\
\text { rehabilitative exercises] }\end{array}$ & $\mathrm{N}(\mathrm{NA})$ & NA & NA & NA \\
\hline \multirow[t]{2}{*}{$\begin{array}{l}\text { Chan, } \\
2018, \\
29372260 \\
\text { Singapore }^{\mathrm{A}}\end{array}$} & $\begin{array}{l}\text { Discharge to home } \\
\text { vs. discharge to } \\
\text { community } \\
\text { hospitals }\end{array}$ & $\begin{array}{l}\text { Discharge to } \\
\text { home }\end{array}$ & $\begin{array}{l}\text { [specific goals and exercises not defined; } \\
\text { comparison of setting] }\end{array}$ & $\mathrm{N}(\mathrm{NA})$ & $\begin{array}{l}\text { Physical } \\
\text { therapist }\end{array}$ & In-person & Home \\
\hline & No data & $\begin{array}{l}\text { Discharge to } \\
\text { community } \\
\text { hospitals }\end{array}$ & $\begin{array}{l}\text { [specific goals and exercises not defined; } \\
\text { comparison of setting] }\end{array}$ & $\mathrm{N}(\mathrm{NA})$ & $\begin{array}{l}\text { Physical } \\
\text { therapist }\end{array}$ & In-person & $\begin{array}{l}\text { Other inpatient } \\
\text { facility }\end{array}$ \\
\hline \multirow[t]{3}{*}{$\begin{array}{l}\text { DeJong, } \\
2020 \\
32360105 \\
\text { USA }\end{array}$} & $\begin{array}{l}\text { Body-weight } \\
\text { adjusted treatment } \\
\text { vs. recumbent bike } \\
\text { \& PENS vs. Body- } \\
\text { weight adjusted } \\
\text { treadmill \& PENS } \\
\text { vs. Recumbent } \\
\text { bike }\end{array}$ & $\begin{array}{l}\text { Body-weight } \\
\text { adjusted } \\
\text { treatment }\end{array}$ & $\begin{array}{l}\text { 1. Strength } \\
\text { [specific exercises not defined] } \\
\text { 3. Flexibility } \\
\text { 5. Task specific training } \\
\text { 5.16 Treadmill gait } \\
\text { 7. Adjunctive modality } \\
\text { 7.1 Cold } \\
\text { 7.13 Mobilizations - Tibiofemoral }\end{array}$ & $\mathrm{Y}(\mathrm{N})$ & $\begin{array}{l}\text { Physical } \\
\text { therapist }\end{array}$ & In-person & $\begin{array}{l}\text { Physical } \\
\text { therapy/rehabilitation } \\
\text { facility (outpatient) }\end{array}$ \\
\hline & No data & $\begin{array}{l}\text { Recumbent } \\
\text { bike \& PENS }\end{array}$ & $\begin{array}{l}\text { 1. Strength } \\
\text { [specific exercises not defined] } \\
\text { 3. Flexibility } \\
\text { 3.2 Bike (ROM) } \\
\text { 7. Adjunctive modality } \\
\text { 7.1 Cold } \\
\text { 7.5 E-stim for strength (NMES) } \\
\text { 7.13 Mobilizations - Tibiofemoral }\end{array}$ & $\mathrm{N}(\mathrm{NA})$ & $\begin{array}{l}\text { Physical } \\
\text { therapist }\end{array}$ & In-person & $\begin{array}{l}\text { Physical } \\
\text { therapy/rehabilitation } \\
\text { facility (outpatient) }\end{array}$ \\
\hline & No data & $\begin{array}{l}\text { Body-weight } \\
\text { adjusted }\end{array}$ & $\begin{array}{l}\text { 1. Strength } \\
\text { [specific exercises not defined] } \\
\text { 3. Flexibility }\end{array}$ & $\mathrm{Y}(\mathrm{N})$ & $\begin{array}{l}\text { Physical } \\
\text { therapist }\end{array}$ & In-person & $\begin{array}{l}\text { Physical } \\
\text { therapy/rehabilitation } \\
\text { facility (outpatient) }\end{array}$ \\
\hline
\end{tabular}




\begin{tabular}{|c|c|c|c|c|c|c|c|}
\hline $\begin{array}{l}\text { Study, Year, } \\
\text { PMID, } \\
\text { Country }\end{array}$ & $\begin{array}{l}\text { Intended } \\
\text { Comparison }\end{array}$ & Arm & Components (Specific Exercises/Strategies) & $\begin{array}{l}\text { Progression } \\
\text { (Appropriate?) }\end{array}$ & Personnel & $\begin{array}{l}\text { Mode of } \\
\text { Delivery }\end{array}$ & Setting \\
\hline & & $\begin{array}{l}\text { treadmill \& } \\
\text { PENS }\end{array}$ & $\begin{array}{l}\text { 5. Task specific training } \\
\text { 5.16 Treadmill gait } \\
\text { 7. Adjunctive modality } \\
\text { 7.1 Cold } \\
\text { 7.5 E-stim for strength (NMES) } \\
\text { 7.13 Mobilizations - Tibiofemoral }\end{array}$ & & & & \\
\hline & No data & $\begin{array}{l}\text { Recumbent } \\
\text { bike }\end{array}$ & $\begin{array}{l}\text { 1. Strength } \\
\text { [specific exercises not defined] } \\
\text { 3. Flexibility } \\
\text { 3.2 Bike (ROM) } \\
\text { 7. Adjunctive modality } \\
\text { 7.1 Cold } \\
\text { 7.13 Mobilizations - Tibiofemoral }\end{array}$ & $\mathrm{N}(\mathrm{NA})$ & $\begin{array}{l}\text { Physical } \\
\text { therapist }\end{array}$ & In-person & $\begin{array}{l}\text { Physical } \\
\text { therapy/rehabilitation } \\
\text { facility (outpatient) }\end{array}$ \\
\hline \multirow[t]{2}{*}{$\begin{array}{l}\text { Demircioglu, } \\
2015, \\
26355656, \\
\text { Turkey }\end{array}$} & $\begin{array}{l}\text { Neuromuscular } \\
\text { electrical stimulation } \\
\text { plus standard } \\
\text { rehabilitation } \\
\text { (exercise) vs. } \\
\text { standard } \\
\text { rehabilitation } \\
\text { (exercise) }\end{array}$ & $\begin{array}{l}\text { Electrical } \\
\text { stimulation } \\
\text { (NMES) \& } \\
\text { exercise }\end{array}$ & $\begin{array}{l}\text { 1. Strength } \\
\text { 1.11 Hip abduction in sidelying (position unclear) } \\
\text { 1.12 Hip abduction in standing (position unclear) } \\
\text { 1.13 Hip abduction in supine (position unclear) } \\
\text { 1.14 Hip adduction in sidelying (position unclear) } \\
\text { 1.15 Hip adduction in standing (position unclear) } \\
\text { 1.16 Hip adduction in supine (position unclear) } \\
\text { 1.30 Knee extension AAROM in sitting or supine } \\
\text { (short- or long arc quad) } \\
\text { 1.43 Quad sets } \\
\text { 1.51 Standing terminal knee extension } \\
\text { 1.58 Straight leg raises } \\
\text { 3. Flexibility } \\
\text { 3.1 Ankle pumps } \\
\text { 3.10 Knee extension AROM (extension/flexion } \\
\text { not specified; just ROM) } \\
\text { 3.13 Knee flexion AROM (extension/flexion not } \\
\text { specified; just ROM) } \\
\text { 7. Adjunctive modality } \\
\text { 7.1. Cold } \\
\text { 7.5 E-stim for strength (NMES) } \\
\text { 7.14 Mobilizations - Patellar }\end{array}$ & 0 (NA) & Unclear & $\begin{array}{l}\text { In-person for } \\
\text { NMES }\end{array}$ & $\begin{array}{l}\text { Physical } \\
\text { therapy/rehabilitation } \\
\text { facility (outpatient) }\end{array}$ \\
\hline & No data & Exercise & $\begin{array}{l}\text { 1. Strength } \\
\text { 1.11 Hip abduction in sidelying (position unclear) } \\
\text { 1.12 Hip abduction in standing (position unclear) } \\
\text { 1.13 Hip abduction in supine (position unclear) } \\
\text { 1.14 Hip adduction in sidelying (position unclear) } \\
\text { 1.15 Hip adduction in standing (position unclear) } \\
\text { 1.16 Hip adduction in supine (position unclear) } \\
\text { 1.30 Knee extension AAROM in sitting or supine } \\
\text { (short- or long arc quad) } \\
\text { 1.43 Quad sets } \\
\text { 1.51 Standing terminal knee extension } \\
\text { 1.58 Straight leg raises } \\
\text { 3. Flexibility } \\
\text { 3.1 Ankle pumps }\end{array}$ & $\mathrm{N}(\mathrm{NA})$ & $\begin{array}{l}\text { None } \\
\text { (unsupervised) }\end{array}$ & $\begin{array}{l}\text { Self-guided } \\
\text { (unsupervised) }\end{array}$ & Home \\
\hline
\end{tabular}




\begin{tabular}{|c|c|c|c|c|c|c|c|}
\hline $\begin{array}{l}\text { Study, Year, } \\
\text { PMID, } \\
\text { Country }\end{array}$ & $\begin{array}{l}\text { Intended } \\
\text { Comparison }\end{array}$ & Arm & Components (Specific Exercises/Strategies) & $\begin{array}{l}\text { Progression } \\
\text { (Appropriate?) }\end{array}$ & Personnel & $\begin{array}{l}\text { Mode of } \\
\text { Delivery }\end{array}$ & Setting \\
\hline & & & $\begin{array}{l}\text { 3.10 Knee extension AROM (extension/flexion } \\
\text { not specified; just ROM) } \\
\text { 3.13 Knee flexion AROM (extension/flexion not } \\
\text { specified; just ROM) } \\
\text { 6. Patient education } \\
\text { 6.2 Home exercise program } \\
\text { 7. Adjunctive modality } \\
\text { 7.1. Cold } \\
\text { 7.14 Mobilizations - Patellar }\end{array}$ & & & & \\
\hline \multirow[t]{2}{*}{$\begin{array}{l}\text { den Hertog, } \\
2012, \\
22643801 \\
\text { Germany }\end{array}$} & $\begin{array}{l}\text { Fast-track } \\
\text { rehabilitation (“Joint } \\
\text { Care” program) vs. } \\
\text { standard } \\
\text { rehabilitation }\end{array}$ & $\begin{array}{l}\text { Fast-track } \\
\text { rehabilitation } \\
\text { program }\end{array}$ & $\begin{array}{l}\text { 1. Strength } \\
\text { [specific exercises not defined] } \\
\text { 3. Flexibility } \\
\text { 3.11 Knee extension PROM in supine (position } \\
\text { unclear) } \\
\text { 3.12 Knee extension PROM in prone (position } \\
\text { unclear) } \\
\text { 3.14 Knee flexion PROM in sitting or supine } \\
\text { 5. Task specific training } \\
\text { 5.8 Gait training } \\
\text { 5.15 Stair training } \\
\text { 6. Patient education } \\
\text { 6.1 Activities of daily living }\end{array}$ & $\mathrm{N}(\mathrm{NA})$ & Unclear & In-person & Acute inpatient \\
\hline & No data & Standard care & $\begin{array}{l}\text { 1. Strength } \\
\text { [specific exercises not defined] } \\
\text { 3. Flexibility } \\
\text { 3.11 Knee extension PROM in supine (position } \\
\text { unclear) } \\
\text { 3.12 Knee extension PROM in prone (position } \\
\text { unclear) } \\
\text { 3.14 Knee flexion PROM in sitting or supine } \\
\text { 5. Task specific training } \\
\text { 5.8 Gait training } \\
\text { [Standard care consisted of similar exercises as } \\
\text { the fast-track group, but different timing after } \\
\text { surgery] }\end{array}$ & $\mathrm{N}(\mathrm{NA})$ & Unclear & In-person & Acute inpatient \\
\hline $\begin{array}{l}\text { Eymir, } \\
2020, \\
32778907 \\
\text { Turkey }\end{array}$ & $\begin{array}{l}\text { Active heel-slide } \\
\text { exercise \& } \\
\text { standard physical } \\
\text { therapy vs. }\end{array}$ & $\begin{array}{l}\text { Active heel- } \\
\text { slide exercise \& } \\
\text { standard } \\
\text { physical } \\
\text { therapy }\end{array}$ & $\begin{array}{l}\text { 1. Strength } \\
\text { 1.8 Gluteal Sets } \\
\text { 1.13 Hip abduction in supine } \\
\text { 1.43 Quad sets } \\
\text { 1.58 Straight leg raises } \\
\text { 3. Flexibility } \\
\text { 3.1 Ankle pumps } \\
\text { 3.6 Heel slides } \\
\text { 3.10 Knee extension AROM } \\
\text { 3.13 Knee flexion AROM } \\
\text { 5. Task specific training } \\
\text { 5.1 Transfers } \\
\text { 5.8 Gait training } \\
\text { 5.15 Stair training } \\
\text { 6. Patient education }\end{array}$ & $\mathrm{Y}(\mathrm{Y})$ & $\begin{array}{l}\text { Physical } \\
\text { therapist; None } \\
\text { (unsupervised) }\end{array}$ & $\begin{array}{l}\text { In-person; } \\
\text { self-guided }\end{array}$ & $\begin{array}{l}\text { Acute inpatient } \\
\text { home }\end{array}$ \\
\hline
\end{tabular}




\begin{tabular}{|c|c|c|c|c|c|c|c|}
\hline \multirow[t]{3}{*}{$\begin{array}{l}\text { Study, Year, } \\
\text { PMID, } \\
\text { Country }\end{array}$} & $\begin{array}{l}\text { Intended } \\
\text { Comparison }\end{array}$ & Arm & Components (Specific Exercises/Strategies) & $\begin{array}{l}\text { Progression } \\
\text { (Appropriate?) }\end{array}$ & Personnel & $\begin{array}{l}\text { Mode of } \\
\text { Delivery }\end{array}$ & Setting \\
\hline & & & 6.2 Home exercise program & & & & \\
\hline & No data & $\begin{array}{l}\text { Continuous } \\
\text { passive motion } \\
\text { \& standard } \\
\text { physical } \\
\text { therapy }\end{array}$ & $\begin{array}{l}\text { 1. Strength } \\
\text { 1.8 Gluteal Sets } \\
\text { 1.13 Hip abduction in supine } \\
\text { 1.43 Quad sets } \\
\text { 1.58 Straight leg raises } \\
\text { 3. Flexibility } \\
\text { 3.1 Ankle pumps } \\
\text { 3.10 Knee extension AROM } \\
\text { 3.13 Knee flexion AROM } \\
\text { 5. Task specific training } \\
\text { 5.1 Transfers } \\
\text { 5.8 Gait training } \\
\text { 5.15 Stair training } \\
\text { 6. Patient education } \\
\text { 6.2 Home exercise program } \\
\text { 7. Adjunctive modality }\end{array}$ & $\mathrm{Y}(\mathrm{Y})$ & $\begin{array}{l}\text { Physical } \\
\text { therapist; None } \\
\text { (unsupervised) }\end{array}$ & $\begin{array}{l}\text { In-person; } \\
\text { self-guided }\end{array}$ & $\begin{array}{l}\text { Acute inpatient; } \\
\text { home }\end{array}$ \\
\hline $\begin{array}{l}\text { Fransen, } \\
2017, \\
27868384, \\
\text { Australia }\end{array}$ & $\begin{array}{l}\text { Post-acute group } \\
\text { exercise program } \\
\text { vs. usual care }\end{array}$ & $\begin{array}{l}\text { Outpatient group } \\
\text { exercise }\end{array}$ & $\begin{array}{l}\text { 1. Strength } \\
\text { 1.2 Bridges Two-legged (supine hip extension) } \\
\text { 1.9 Heel raises - bilateral (calf raises) } \\
\text { 1.12 Hip abduction in standing } \\
\text { 1.19 Hip extension in standing } \\
\text { 1.31 Knee extension in sitting or supine (long arc } \\
\text { quad) } \\
\text { 1.32 Knee extension in sitting or supine (short arc } \\
\text { quad) } \\
\text { 1.41 Lunges } \\
\text { 1.48 Sit-to-stand } \\
\text { 1.49 Squats } \\
\text { 1.52 Step down } \\
\text { 1.54 Step lateral } \\
\text { 1.55 Step up - forward } \\
\text { 1.56 Step up - lateral } \\
\text { 1.61 Wall slides } \\
\text { 2. Aerobic } \\
\text { 2.2 Bike (Endurance) } \\
\text { 2.8 Treadmill walking } \\
\text { 3. Flexibility } \\
\text { 3.3 Calf stretch with knee bent (soleus) } \\
\text { 3.4 Calf stretch with knee straight (gastroc) } \\
\text { 3.5 Hamstring stretch in any position } \\
\text { 3.10 Knee extension AROM } \\
\text { 3.13 Knee flexion AROM } \\
\text { 5. Task specific training } \\
\text { 5.4 Gait backwards } \\
\text { 5.7 Gait sideways } \\
\text { 5.8 Gait training } \\
\text { 5.15 Stair training } \\
\text { 6. Patient education } \\
\text { 6.2 Home exercise program }\end{array}$ & $\mathrm{Y}(\mathrm{Y})$ & $\begin{array}{l}\text { Physical } \\
\text { therapist }\end{array}$ & In-person & $\begin{array}{l}\text { Physical } \\
\text { therapy/rehabilitation } \\
\text { facility (outpatient); } \\
\text { Home }\end{array}$ \\
\hline
\end{tabular}




\begin{tabular}{|c|c|c|c|c|c|c|c|}
\hline $\begin{array}{l}\text { Study, Year, } \\
\text { PMID, }\end{array}$ & $\begin{array}{l}\text { Intended } \\
\text { Comparison }\end{array}$ & Arm & Components (Specific Exercises/Strategies) & $\begin{array}{l}\text { Progression } \\
\text { (Appropriate?) }\end{array}$ & Personnel & $\begin{array}{l}\text { Mode of } \\
\text { Delivery }\end{array}$ & Setting \\
\hline & No data & Usual care & $\begin{array}{l}\text { [specific goals and exercises not defined] } \\
\text { [Able to access acute rehabilitation management } \\
\text { as provided by the hospital or recommended by } \\
\text { the orthopedic surgeon; } 85 \% \text { received at least } \\
\text { one face-to-face physiotherapy visit at some } \\
\text { point post-TKA] }\end{array}$ & $N(N A)$ & $\begin{array}{l}\text { Physical } \\
\text { therapist }\end{array}$ & In-person & $\begin{array}{l}\text { Physical } \\
\text { therapy/rehabilitation } \\
\text { facility (outpatient); } \\
\text { Home }\end{array}$ \\
\hline \multirow[t]{2}{*}{$\begin{array}{l}\text { Hamilton, } \\
2020 \\
33051212 \\
\text { UK }\end{array}$} & $\begin{array}{l}\text { Progressive } \\
\text { outpatient } \\
\text { physiotherapy vs. } \\
\text { single } \\
\text { physiotherapy } \\
\text { review and home } \\
\text { exercise } \\
\text { based intervention }\end{array}$ & $\begin{array}{l}\text { Outpatient } \\
\text { therapist-led } \\
\text { rehabilitation }\end{array}$ & $\begin{array}{l}\text { 1. Strength } \\
\text { 1.31 Knee extension in sitting or supine (long arc } \\
\text { quad) } \\
\text { 1.38 Leg Press (one leg) (unclear one or two } \\
\text { legs) } \\
\text { 1.39 Leg Press (two legs) (unclear one or two } \\
\text { legs) } \\
\text { 1.43 Quad sets } \\
\text { 1.50 Squats (one leg) } \\
\text { 1.55 Step up - forward } \\
\text { 1.56 Step up - lateral } \\
\text { 1.58 Straight leg raises } \\
\text { 2. Aerobic } \\
\text { 2.9 Walking } \\
\text { 3. Flexibility } \\
\text { 3.2 Bike (ROM) } \\
\text { 3.3 Calf stretch with knee bent (soleus) } \\
\text { 3.4 Calf stretch with knee straight (gastroc) } \\
\text { 3.5 Hamstring stretch in any position } \\
\text { 3.11 Knee extension PROM in supine } \\
\text { 3.13 Knee flexion AROM } \\
\text { 4. Balance-Motor Learning-Agility } \\
\text { 4.3 Balance on unstable surface } \\
\text { 4.8 Single leg stance } \\
\text { 4.10 Stepping multiple directions (grapevine) } \\
\text { 4.17 Tandem walking } \\
\text { 5. Task specific training } \\
\text { 5.8 Gait training } \\
\text { 5.10 Gait with perturbations } \\
\text { 5.13 Sit-to-stand training } \\
\text { 6. Patient education } \\
\text { 6.2 Home exercise program } \\
\text { 6.4 Pain management }\end{array}$ & $\mathrm{Y}(\mathrm{Y})$ & $\begin{array}{l}\text { Physical } \\
\text { therapist; None } \\
\text { (unsupervised) }\end{array}$ & $\begin{array}{l}\text { In-person; } \\
\text { Self-guided } \\
\text { (unsupervised) }\end{array}$ & $\begin{array}{l}\text { Physical } \\
\text { therapy/rehabilitation } \\
\text { facility (outpatient); } \\
\text { Home }\end{array}$ \\
\hline & No data & $\begin{array}{l}\text { Physiotherapy } \\
\text { review \& home } \\
\text { exercises } \\
\text { (standard of } \\
\text { care) }\end{array}$ & $\begin{array}{l}\text { 1. Strength } \\
\text { [specific exercises not defined] } \\
\text { 3. Flexibility } \\
\text { [specific exercises not defined] } \\
\text { 6. Patient education } \\
\text { 6.2 Home exercise program } \\
\text { 6.4 Pain management } \\
\end{array}$ & $\mathrm{N}(\mathrm{NA})$ & $\begin{array}{l}\text { None } \\
\text { (unsupervised) }\end{array}$ & $\begin{array}{l}\text { Self-guided } \\
\text { (unsupervised) }\end{array}$ & Home \\
\hline $\begin{array}{l}\text { Harmer, } \\
2009, \\
19177536 \\
\text { Australia } \\
\end{array}$ & $\begin{array}{l}\text { Water-based } \\
\text { rehabilitation vs. } \\
\text { land-based } \\
\text { rehabilitation } \\
\end{array}$ & $\begin{array}{l}\text { Water-based } \\
\text { rehabilitation }\end{array}$ & $\begin{array}{l}\text { 1. Strength } \\
\text { 2. Aerobic } \\
\text { 2.1 Aquatics (water aerobics, water walking) } \\
\text { 3. Flexibility }\end{array}$ & $\mathrm{Y}(\mathrm{N})$ & $\begin{array}{l}\text { Physical } \\
\text { therapist }\end{array}$ & In-person & Other (pool); Home \\
\hline
\end{tabular}




\begin{tabular}{|c|c|c|c|c|c|c|c|}
\hline $\begin{array}{l}\text { Study, Year, } \\
\text { PMID, } \\
\text { Country }\end{array}$ & $\begin{array}{l}\text { Intended } \\
\text { Comparison }\end{array}$ & Arm & Components (Specific Exercises/Strategies) & $\begin{array}{l}\text { Progression } \\
\text { (Appropriate?) }\end{array}$ & Personnel & $\begin{array}{l}\text { Mode of } \\
\text { Delivery }\end{array}$ & Setting \\
\hline & & & $\begin{array}{l}\text { 3.10 Knee extension AROM } \\
\text { 3.13 Knee flexion AROM } \\
\text { 6. Patient education } \\
\text { 6.2 Home exercise program }\end{array}$ & & & & \\
\hline & No data & $\begin{array}{l}\text { Land-based } \\
\text { rehabilitation }\end{array}$ & $\begin{array}{l}\text { 3. Flexibility } \\
\text { 3.2 Bike (ROM) } \\
\text { 3.10 Knee extension AROM } \\
\text { 3.13 Knee flexion AROM } \\
\text { 4. Balance-Motor Learning-Agility } \\
\text { [specific exercises not defined] } \\
\text { 5. Task specific training } \\
\text { 5.13 Sit-to-stand training } \\
\text { 5.15 Stair training } \\
\text { 5.16 Treadmill gait } \\
\text { 6. Patient education } \\
\text { 6.2 Home exercise program } \\
\end{array}$ & $\mathrm{Y}(\mathrm{N})$ & $\begin{array}{l}\text { Physical } \\
\text { therapist }\end{array}$ & In-person & $\begin{array}{l}\text { Physical } \\
\text { therapy/rehabilitation } \\
\text { facility (outpatient); } \\
\text { Home }\end{array}$ \\
\hline \multirow[t]{2}{*}{$\begin{array}{l}\text { Heikkilä, } \\
2017, \\
28119232, \\
\text { Finland }\end{array}$} & $\begin{array}{l}\text { One-year } \\
\text { progressive } \\
\text { postoperative home } \\
\text { exercise program } \\
\text { vs. usual care }\end{array}$ & Home exercise & $\begin{array}{l}\text { 1. Strength } \\
\text { 1.9 Heel raises - bilateral (calf raises) } \\
\text { 1.10 Heel raises - unilateral } \\
\text { 1.36 Knee flexion in sitting or supine } \\
\text { 1.43 Quad sets } \\
\text { 1.48 Sit-to-stand } \\
\text { 1.49 Squats } \\
\text { 1.54 Step lateral } \\
\text { 1.61 Wall slides } \\
\text { 3. Flexibility } \\
\text { 3.2 Bike (ROM) } \\
\text { 3.3 Calf stretch with knee bent (soleus) } \\
\text { 3.4 Calf stretch with knee straight (gastroc) } \\
\text { 3.5 Hamstring stretch in any position } \\
\text { 3.8 Hip flexor stretch (iliopsoas) } \\
\text { 4. Balance-Motor Learning-Agility } \\
\text { 4.9 Standing weight shifts } \\
\text { 6. Patient education } \\
\text { 6.2 Home exercise program }\end{array}$ & $\mathrm{Y}(\mathrm{N})$ & $\begin{array}{l}\text { Physical } \\
\text { therapist }\end{array}$ & $\begin{array}{l}\text { In-person; } \\
\text { Self-guided } \\
\text { (unsupervised) } \\
\text { - checked in at } \\
\text { check-in visits }\end{array}$ & $\begin{array}{l}\text { Home; Physical } \\
\text { therapy/rehabilitation } \\
\text { facility (outpatient) }\end{array}$ \\
\hline & No data & Control & $\begin{array}{l}\text { [no intervention after discharge] } \\
\text { [Usual care consisting of the acute rehabilitation } \\
\text { after surgery in the hospital and no additional } \\
\text { guidance after discharge] }\end{array}$ & $\mathrm{N}(\mathrm{NA})$ & NA & NA & NA \\
\hline $\begin{array}{l}\text { Iwakiri, } \\
2020 \\
32373475 \\
\text { Japan }\end{array}$ & $\begin{array}{l}\text { Range of motion } \\
\text { (post op day } 7 \text { ) vs. } \\
\text { range of motion } \\
\text { (post op day } 7 \text { ) }\end{array}$ & $\begin{array}{l}\text { Range of } \\
\text { motion (post op } \\
\text { day 1) }\end{array}$ & $\begin{array}{l}\text { 3. Flexibility } \\
\text { 3.10 Knee extension AROM } \\
\text { 3.11 Knee extension PROM in supine (position } \\
\text { unclear) } \\
\text { 3.12 Knee extension PROM in prone (position } \\
\text { unclear) } \\
\text { 3.13 Knee flexion AROM } \\
\text { 3.14 Knee flexion PROM in sitting or supine } \\
\text { (position unclear) } \\
3.15 \text { Knee flexion AROM in any position (rectus } \\
\text { femoris stretch) (position unclear) }\end{array}$ & $\mathrm{N}(\mathrm{NA})$ & $\begin{array}{l}\text { Physical } \\
\text { therapist }\end{array}$ & In-person & Acute inpatient \\
\hline
\end{tabular}




\begin{tabular}{|c|c|c|c|c|c|c|c|}
\hline $\begin{array}{l}\text { Study, Year, } \\
\text { PMID, } \\
\text { Country }\end{array}$ & $\begin{array}{l}\text { Intended } \\
\text { Comparison }\end{array}$ & Arm & Components (Specific Exercises/Strategies) & $\begin{array}{l}\text { Progression } \\
\text { (Appropriate?) }\end{array}$ & Personnel & $\begin{array}{l}\text { Mode of } \\
\text { Delivery }\end{array}$ & Setting \\
\hline & & & $\begin{array}{l}\text { 3.16 Knee flexion PROM in prone (rectus femoris } \\
\text { stretch) } \\
\text { 5. Task specific training } \\
\text { 5.8 Gait training } \\
\text { 5.15 Stair training }\end{array}$ & & & & \\
\hline & No data & $\begin{array}{l}\text { Range of motion } \\
\text { (post op day } 7 \text { ) }\end{array}$ & $\begin{array}{l}\text { 3. Flexibility } \\
\text { 3.10 Knee extension AROM } \\
\text { 3.11 Knee extension PROM in supine (position } \\
\text { unclear) } \\
\text { 3.12 Knee extension PROM in prone (position } \\
\text { unclear) } \\
\text { 3.13 Knee flexion AROM } \\
\text { 3.14 Knee flexion PROM in sitting or supine } \\
\text { (position unclear) } \\
\text { 3.15 Knee flexion AROM in any position (rectus } \\
\text { femoris stretch) (position unclear) } \\
\text { 3.16 Knee flexion PROM in prone (rectus femoris } \\
\text { stretch) } \\
\text { 5. Task specific training } \\
\text { 5.8 Gait training } \\
\text { 5.15 Stair training }\end{array}$ & $\mathrm{N}(\mathrm{NA})$ & $\begin{array}{l}\text { Physical } \\
\text { therapist }\end{array}$ & In-person & Acute inpatient \\
\hline \multirow[t]{2}{*}{$\begin{array}{l}\text { Jin, } \\
2018, \\
\text { CN-01617489, } \\
\text { China }\end{array}$} & $\begin{array}{l}\text { Virtual reality plus } \\
\text { conventional acute } \\
\text { rehabilitation care } \\
\text { vs. conventional } \\
\text { acute rehabilitation } \\
\text { care }\end{array}$ & $\begin{array}{l}\text { Virtual reality } \\
\text { plus usual care }\end{array}$ & $\begin{array}{l}\text { 1. Strength } \\
\text { [specific exercises not defined] } \\
\text { 3. Flexibility } \\
\text { 3.1 Ankle pumps } \\
\text { 3.14 Knee flexion PROM in sitting or supine } \\
\text { 6. Patient education } \\
\text { 6.4 Pain management } \\
\text { 7. Adjunctive modality } \\
\text { 7.15 Biofeedback devices } \\
\text { 7.17 Mindfulness, stress/anxiety-reduction } \\
\text { interventions }\end{array}$ & $\mathrm{N}(\mathrm{NA})$ & $\begin{array}{l}\text { Other } \\
\text { (Research } \\
\text { personnel) }\end{array}$ & In-person & $\begin{array}{l}\text { Acute inpatient } \\
\text { (postoperative) }\end{array}$ \\
\hline & No data & Usual care & $\begin{array}{l}\text { 1. Strength } \\
\text { [specific exercises not defined] } \\
\text { 3. Flexibility } \\
\text { 3.1 Ankle pumps } \\
\text { 3.14 Knee flexion PROM in sitting or supine } \\
\text { 6. Patient education } \\
\text { 6.4 Pain management } \\
\text { 7. Adjunctive modality } \\
\text { 7.17 Mindfulness, stress/anxiety-reduction } \\
\text { interventions }\end{array}$ & $\mathrm{N}(\mathrm{NA})$ & $\begin{array}{l}\text { Other } \\
\text { (Research } \\
\text { personnel) }\end{array}$ & In-person & $\begin{array}{l}\text { Acute inpatient } \\
\text { (postoperative) }\end{array}$ \\
\hline $\begin{array}{l}\text { Kauppila, } \\
2010 \\
20354057 \\
\text { Finland }\end{array}$ & $\begin{array}{l}\text { Multidisciplinary } \\
\text { rehabilitation } \\
\text { program vs. } \\
\text { conventional care }\end{array}$ & $\begin{array}{l}\text { Multidisciplinary } \\
\text { rehabilitation }\end{array}$ & $\begin{array}{l}\text { 1. Strength } \\
1.31 \text { Knee extension in sitting or supine (long arc } \\
\text { quad) } \\
1.36 \text { Knee flexion in sitting or supine } \\
\text { 1.37 Knee flexion in standing } \\
\text { 2. Aerobic } \\
\text { 2.1 Aquatics (water aerobics, water walking) } \\
\text { 2.9 Walking }\end{array}$ & $\mathrm{Y}(\mathrm{N})$ & $\begin{array}{l}\text { Physical } \\
\text { therapist }\end{array}$ & $\begin{array}{l}\text { In-person; } \\
\text { Self-guided } \\
\text { (unsupervised) }\end{array}$ & $\begin{array}{l}\text { Acute inpatient } \\
\text { (postoperative); } \\
\text { Physical } \\
\text { therapy/rehabilitation } \\
\text { facility (outpatient); } \\
\text { Home }\end{array}$ \\
\hline
\end{tabular}




\begin{tabular}{|c|c|c|c|c|c|c|c|}
\hline $\begin{array}{l}\text { Study, Year, } \\
\text { PMID, } \\
\text { Country }\end{array}$ & $\begin{array}{l}\text { Intended } \\
\text { Comparison }\end{array}$ & Arm & Components (Specific Exercises/Strategies) & $\begin{array}{l}\text { Progression } \\
\text { (Appropriate?) }\end{array}$ & Personnel & $\begin{array}{l}\text { Mode of } \\
\text { Delivery }\end{array}$ & Setting \\
\hline & & & $\begin{array}{l}\text { 3. Flexibility } \\
\text { [specific exercises not defined] } \\
\text { 5. Task specific training } \\
\text { 5.1 Transfers } \\
\text { 5.8 Gait training } \\
\text { 5.15 Stair training } \\
\text { 6. Patient education } \\
\text { 6.2 Home exercise program } \\
\text { 6.3 Life-style change } \\
\text { 7. Adjunctive modality } \\
\text { 7.17 Mindfulness, stress/anxiety-reduction } \\
\text { interventions }\end{array}$ & & & & \\
\hline & No data & Control & $\begin{array}{l}\text { 1. Strength } \\
\text { [specific exercises not defined] } \\
\text { 3. Flexibility } \\
\text { [specific exercises not defined] } \\
\text { 5. Task specific training } \\
\text { 5.1 Transfers } \\
\text { 5.8 Gait training } \\
\text { 5.15 Stair training } \\
\text { 6. Patient education } \\
\text { 6.2 Home exercise program } \\
\text { [Conventional care: pre-operative exercise } \\
\text { recommendations, acute exercise program for } \\
\text { lower extremity strength and joint mobility, and } \\
\text { supervised exercise program at 2-month visit] }\end{array}$ & $\mathrm{Y}(\mathrm{N})$ & $\begin{array}{l}\text { Physical } \\
\text { therapist }\end{array}$ & $\begin{array}{l}\text { In-person; } \\
\text { Self-guided } \\
\text { (unsupervised) }\end{array}$ & $\begin{array}{l}\text { Acute inpatient } \\
\text { (postoperative); } \\
\text { Physical } \\
\text { therapy/rehabilitation } \\
\text { facility (outpatient); } \\
\text { Home }\end{array}$ \\
\hline $\begin{array}{l}\text { Lenguerrand, } \\
2020 \text {, } \\
31033232, \\
\text { UK }\end{array}$ & $\begin{array}{l}\text { Group-based } \\
\text { outpatient physical } \\
\text { therapy sessions } \\
\text { plus usual care vs. } \\
\text { usual care }\end{array}$ & $\begin{array}{l}\text { Group-based } \\
\text { outpatient } \\
\text { physical therapy }\end{array}$ & $\begin{array}{l}\text { 1. Strength } \\
\text { 1.31 Knee extension in sitting or supine (long arc } \\
\text { quad) (unclear if long or short) } \\
\text { 1.32 Knee extension in sitting or supine (short arc } \\
\text { quad) (unclear if long or short) } \\
\text { 1.35 Knee flexion in prone (position unclear) } \\
\text { 1.36 Knee flexion in sitting or supine (position } \\
\text { unclear) } \\
\text { 1.41 Lunges } \\
\text { 1.49 Squats } \\
\text { 1.52 Step down } \\
\text { 1.55 Step up - forward } \\
\text { 2. Aerobic } \\
\text { 2.2 Bike (Endurance) } \\
\text { 2.8 Treadmill walking } \\
\text { 3. Flexibility } \\
\text { 3.2 Bike (ROM) } \\
\text { 3.5 Hamstring stretch in any position } \\
\text { 3.10 Knee extension AROM } \\
\text { 3.13 Knee flexion AROM } \\
\text { 3.15 Knee flexion AROM in any position (rectus } \\
\text { femoris stretch) } \\
\text { 4. Balance-Motor Learning-Agility } \\
\text { 4.3 Balance on unstable surface }\end{array}$ & $\mathrm{Y}(\mathrm{Y})$ & $\begin{array}{l}\text { Physical } \\
\text { therapist }\end{array}$ & In-person & $\begin{array}{l}\text { Physical } \\
\text { therapy/rehabilitation } \\
\text { facility (outpatient) }\end{array}$ \\
\hline
\end{tabular}




\begin{tabular}{|c|c|c|c|c|c|c|c|}
\hline $\begin{array}{l}\text { Study, Year, } \\
\text { PMID, } \\
\text { Country }\end{array}$ & $\begin{array}{l}\text { Intended } \\
\text { Comparison }\end{array}$ & Arm & Components (Specific Exercises/Strategies) & $\begin{array}{l}\text { Progression } \\
\text { (Appropriate?) }\end{array}$ & Personnel & $\begin{array}{l}\text { Mode of } \\
\text { Delivery }\end{array}$ & Setting \\
\hline & & & $\begin{array}{l}\text { 4.8 Single leg stance } \\
\text { 4.11 Step down } \\
\text { 4.14 Step up - forward } \\
\text { 5. Task specific training } \\
\text { 5.1. Transfers } \\
\text { 5.6 Gait on uneven surfaces } \\
\text { 5.7 Gait sideways } \\
\text { 5.8 Gait training } \\
\text { 5.12 Obstacle training } \\
\text { 5.13 Sit-to-stand training } \\
\text { 5.15 Stair training } \\
\text { 5.16 Treadmill gait } \\
\text { 6. Patient education } \\
\text { 6.2 Home exercise program }\end{array}$ & & & & \\
\hline & No data & Standard care & $\begin{array}{l}\text { NA } \\
\text { [Usual care: Advice on knee-specific and function } \\
\text { exercises and referral for outpatient PT as } \\
\text { needed] }\end{array}$ & $N(N A)$ & NA & NA & NA \\
\hline \multirow[t]{2}{*}{$\begin{array}{l}\text { Lenssen, } \\
2006, \\
16942627 \\
\text { Netherlands }\end{array}$} & $\begin{array}{l}\text { Two physical } \\
\text { therapy sessions } \\
\text { per day vs. one } \\
\text { physical therapy } \\
\text { session per day }\end{array}$ & $\begin{array}{l}\text { Physiotherapy } \\
\text { (twice daily; } 40 \\
\text { mins/day)] }\end{array}$ & $\begin{array}{l}\text { 1. Strength } \\
\text { [specific exercises not defined] } \\
\text { 3. Flexibility } \\
\text { 3.10 Knee extension AROM } \\
\text { 3.11 Knee extension PROM in supine } \\
\text { 3.12 Knee extension PROM in prone } \\
\text { 3.13 Knee flexion AROM } \\
\text { 3.14 Knee flexion PROM in sitting or supine } \\
\text { 3.15 Knee flexion AROM in any position (rectus } \\
\text { femoris stretch) } \\
\text { 3.16 Knee flexion PROM in prone (rectus femoris } \\
\text { stretch) } \\
\text { 5. Task specific training } \\
\text { 5.1 Transfers } \\
\text { 5.8 Gait training } \\
\text { 5.13 Sit-to-stand training } \\
\text { 5.15 Stair training }\end{array}$ & $\mathrm{N}(\mathrm{NA})$ & $\begin{array}{l}\text { Physical } \\
\text { therapist }\end{array}$ & In-person & Acute Inpatient \\
\hline & No data & $\begin{array}{l}\text { Physiotherapy } \\
\text { (once daily; } 20 \\
\text { mins/day)] }\end{array}$ & $\begin{array}{l}\text { 1. Strength } \\
\text { [specific exercises not defined] } \\
\text { 3. Flexibility } \\
\text { 3.10 Knee extension AROM } \\
\text { 3.11 Knee extension PROM in supine } \\
\text { 3.12 Knee extension PROM in prone } \\
\text { 3.13 Knee flexion AROM } \\
\text { 3.14 Knee flexion PROM in sitting or supine } \\
\text { 3.15 Knee flexion AROM in any position (rectus } \\
\text { femoris stretch) } \\
\text { 3.16 Knee flexion PROM in prone (rectus femoris } \\
\text { stretch) } \\
\text { 5. Task specific training } \\
\text { 5.1 Transfers } \\
\text { 5.8 Gait training }\end{array}$ & $\mathrm{N}(\mathrm{NA})$ & $\begin{array}{l}\text { Physical } \\
\text { therapist }\end{array}$ & In-person & Acute Inpatient \\
\hline
\end{tabular}




\begin{tabular}{|c|c|c|c|c|c|c|c|}
\hline $\begin{array}{l}\text { Study, Year, } \\
\text { PMID, } \\
\text { Country }\end{array}$ & $\begin{array}{l}\text { Intended } \\
\text { Comparison }\end{array}$ & Arm & Components (Specific Exercises/Strategies) & $\begin{array}{l}\text { Progression } \\
\text { (Appropriate?) }\end{array}$ & Personnel & $\begin{array}{l}\text { Mode of } \\
\text { Delivery }\end{array}$ & Setting \\
\hline & & & $\begin{array}{l}\text { 5.13 Sit-to-stand training } \\
\text { 5.15 Stair training }\end{array}$ & & & & \\
\hline \multirow[t]{2}{*}{$\begin{array}{l}\mathrm{Li}, \\
2014, \\
23412304, \\
\text { China }\end{array}$} & $\begin{array}{l}\text { Lower-limb robot } \\
\text { assisted training } \\
\text { system vs. } \\
\text { traditional } \\
\text { rehabilitation } \\
\text { training }\end{array}$ & $\begin{array}{l}\text { Robot-assisted } \\
\text { training }\end{array}$ & $\begin{array}{l}\text { 1. Strength } \\
\text { 1.36 Knee flexion in sitting or supine } \\
\text { 1.43 Quad sets } \\
\text { 3. Flexibility } \\
\text { 3.1 Ankle pumps } \\
\text { 5. Task specific training } \\
\text { 5.8 Gait training (robot) } \\
\text { 7. Adjunctive modality } \\
\text { 7.5 E-stim for strength (NMES) }\end{array}$ & $\mathrm{N}(\mathrm{NA})$ & NR & In-person & $\begin{array}{l}\text { Acute inpatient } \\
\text { (postoperative) }\end{array}$ \\
\hline & No data & $\begin{array}{l}\text { Traditional } \\
\text { rehabilitation } \\
\text { training }\end{array}$ & $\begin{array}{l}\text { 1. Strength } \\
\text { 1.36 Knee flexion in sitting or supine } \\
\text { 1.43 Quad sets } \\
\text { 3. Flexibility } \\
\text { 3.1 Ankle pumps } \\
\text { 5. Task specific training } \\
\text { 5.8 Gait training (assistive devices) } \\
\text { 7. Adjunctive modality } \\
\text { 7.5 E-stim for strength (NMES) }\end{array}$ & $\mathrm{N}(\mathrm{NA})$ & NR & In-person & $\begin{array}{l}\text { Acute inpatient } \\
\text { (postoperative) }\end{array}$ \\
\hline \multirow[t]{2}{*}{$\begin{array}{l}\mathrm{Li}, \\
2015, \\
\mathrm{CN}-01084888, \\
\text { China }\end{array}$} & $\begin{array}{l}\text { Education for daily } \\
\text { physical activity vs. } \\
\text { no education } \\
\text { [Abstract only] }\end{array}$ & Education & $\begin{array}{l}\text { 6. Patient education } \\
\text { [specific elements of education not defined] }\end{array}$ & $\mathrm{Y}(\mathrm{N})$ & NR & $\begin{array}{l}\text { Remote via } \\
\text { telephone }\end{array}$ & Home \\
\hline & No data & No education & $\begin{array}{l}\text { NA } \\
\text { [No additional education] }\end{array}$ & $\mathrm{Y}(\mathrm{N})$ & NR & NR & NR \\
\hline $\begin{array}{l}\mathrm{Li}, \\
2017, \\
\text { CN-01419703, } \\
\text { China }\end{array}$ & $\begin{array}{l}\text { Early gait training } \\
\text { vs. basic } \\
\text { rehabilitation }\end{array}$ & $\begin{array}{l}\text { Gait training \& } \\
\text { usual care }\end{array}$ & $\begin{array}{l}\text { 1. Strength } \\
\text { 1.32 Knee extension in sitting or supine (short arc } \\
\text { quad) (unclear long or short arc) } \\
\text { 1.33 Knee flexion machine (Hamstring curl) one } \\
\text { knee (unclear long or short arc) } \\
\text { 1.58 Straight leg raises } \\
\text { 3. Flexibility } \\
\text { 3.1 Ankle pumps } \\
\text { 3.10 Knee extension AROM } \\
\text { 3.11 Knee extension PROM in supine (position } \\
\text { unclear) } \\
\text { 3.12 Knee extension PROM in prone (position } \\
\text { unclear) } \\
\text { 3.13 Knee flexion AROM } \\
\text { 3.14 Knee flexion PROM in sitting or supine } \\
\text { 3.15 Knee flexion AROM in any position (rectus } \\
\text { femoris stretch) } \\
\text { 3.16 Knee flexion PROM in prone (rectus femoris } \\
\text { stretch) } \\
\text { 4. Balance-Motor Learning-Agility } \\
\text { 4.9 Standing weight shifts } \\
\text { 5. Task specific training } \\
\text { 5.8 Gait training } \\
\text { 7. Adjunctive modality }\end{array}$ & $\mathrm{Y}(\mathrm{N})$ & Unclear & In-person & Acute inpatient \\
\hline
\end{tabular}




\begin{tabular}{|c|c|c|c|c|c|c|c|}
\hline $\begin{array}{l}\text { Study, Year, } \\
\text { PMID, } \\
\text { Country }\end{array}$ & $\begin{array}{l}\text { Intended } \\
\text { Comparison }\end{array}$ & Arm & Components (Specific Exercises/Strategies) & $\begin{array}{l}\text { Progression } \\
\text { (Appropriate?) }\end{array}$ & Personnel & $\begin{array}{l}\text { Mode of } \\
\text { Delivery }\end{array}$ & Setting \\
\hline & & & $\begin{array}{l}\text { 7.1. Cold } \\
\text { 7.10 Massage for edema control }\end{array}$ & & & & \\
\hline & No data & $\begin{array}{l}\text { Usual care } \\
\text { (including gait } \\
\text { training but later) }\end{array}$ & $\begin{array}{l}\text { 1. Strength } \\
\text { 1.32 Knee extension in sitting or supine (short arc } \\
\text { quad) } \\
1.33 \text { Knee flexion machine (Hamstring curl) one } \\
\text { knee } \\
\text { 1.58 Straight leg raises } \\
\text { 3. Flexibility } \\
\text { 3.1 Ankle pumps } \\
\text { 3.10 Knee extension AROM } \\
\text { 3.11 Knee extension PROM in supine (position } \\
\text { unclear) } \\
\text { 3.12 Knee extension PROM in prone (position } \\
\text { unclear) } \\
\text { 3.13 Knee flexion AROM } \\
\text { 3.14 Knee flexion PROM in sitting or supine } \\
\text { 3.15 Knee flexion AROM in any position (rectus } \\
\text { femoris stretch) } \\
\text { 3.16 Knee flexion PROM in prone (rectus femoris } \\
\text { stretch) } \\
\text { 4. Balance-Motor Learning-Agility } \\
\text { 4.9 Standing weight shifts } \\
\text { 5. Task specific training } \\
\text { 5.8 Gait training } \\
\text { 7. Adjunctive modality } \\
\text { 7.1. Cold } \\
\text { 7.10 Massage for edema control }\end{array}$ & $\mathrm{Y}(\mathrm{N})$ & Unclear & In-person & Acute inpatient \\
\hline \multirow[t]{2}{*}{$\begin{array}{l}\text { Li, } \\
2019, \\
31003647, \\
\text { China }\end{array}$} & $\begin{array}{l}\text { Tai chi chuan vs. } \\
\text { Traditional physical } \\
\text { exercises }\end{array}$ & Tai chi exercise & $\begin{array}{l}\text { 1. Strength } \\
\text { 1.43 Quad sets } \\
\text { 1.58 Straight leg raises } \\
\text { 3. Flexibility } \\
\text { 3.6 Heel slides } \\
\text { 7. Adjunctive modality } \\
\text { 7.18 Complementary and alternative therapies } \\
\text { (Tai Chi) }\end{array}$ & $\mathrm{N}$ & Other & In-person & NR \\
\hline & No data & $\begin{array}{l}\text { Control } \\
\text { (traditional } \\
\text { physical } \\
\text { exercises) }\end{array}$ & $\begin{array}{l}\text { 1. Strength } \\
\text { 1.43 Quad sets } \\
\text { 1.58 Straight leg raises } \\
\text { 3. Flexibility } \\
\text { 3.6 Heel slides }\end{array}$ & $\mathrm{N}$ & NR & NR & NR \\
\hline $\begin{array}{l}\text { Liao, } \\
2015, \\
25552523, \\
\text { Taiwan }\end{array}$ & $\begin{array}{l}\text { General functional } \\
\text { rehabilitation plus } \\
\text { balance training vs. } \\
\text { general functional } \\
\text { rehabilitation alone }\end{array}$ & $\begin{array}{l}\text { Functional } \\
\text { rehabilitation \& } \\
\text { balance training }\end{array}$ & $\begin{array}{l}\text { 1. Strength } \\
\text { 1.11 Hip abduction in sidelying (position unclear) } \\
1.12 \mathrm{Hip} \text { abduction in standing (position unclear) } \\
1.13 \mathrm{Hip} \text { abduction in supine (position unclear) } \\
1.31 \mathrm{Knee} \text { extension in sitting or supine (long arc } \\
\text { quad) } \\
1.32 \mathrm{Knee} \text { extension in sitting or supine (short arc } \\
\text { quad) } \\
1.35 \mathrm{Knee} \text { flexion in prone }\end{array}$ & $\mathrm{Y}(\mathrm{N})$ & $\begin{array}{l}\text { Physical } \\
\text { therapist }\end{array}$ & In-person & $\begin{array}{l}\text { Physical } \\
\text { therapy/rehabilitation } \\
\text { facility (outpatient) }\end{array}$ \\
\hline
\end{tabular}




\begin{tabular}{|c|c|c|c|c|c|c|c|}
\hline $\begin{array}{l}\text { Study, Year, } \\
\text { PMID, } \\
\text { Country }\end{array}$ & $\begin{array}{l}\text { Intended } \\
\text { Comparison }\end{array}$ & Arm & Components (Specific Exercises/Strategies) & $\begin{array}{l}\text { Progression } \\
\text { (Appropriate?) }\end{array}$ & Personnel & $\begin{array}{l}\text { Mode of } \\
\text { Delivery }\end{array}$ & Setting \\
\hline & & & $\begin{array}{l}\text { 1.36 Knee flexion in sitting or supine } \\
\text { 1.37 Knee flexion in standing } \\
\text { 1.43 Quad sets } \\
\text { 2. Aerobic } \\
\text { 2.2 Bike (Endurance) } \\
\text { 2.8 Treadmill walking } \\
\text { 3. Flexibility } \\
\text { 3.1 Ankle pumps } \\
\text { 3.5 Hamstring stretch in any position } \\
\text { 3.10 Knee extension AROM (unclear) } \\
\text { 3.11 Knee extension PROM in supine (unclear) } \\
\text { 3.12 Knee extension PROM in prone (unclear) } \\
\text { 3.13 Knee flexion AROM (unclear) } \\
\text { 3.14 Knee flexion PROM in sitting or supine } \\
\text { (unclear) } \\
\text { 3.15 Knee flexion AROM in any position (rectus } \\
\text { femoris stretch) } \\
\text { 3.16 Knee flexion PROM in prone (rectus femoris } \\
\text { stretch) } \\
\text { 4. Balance-Motor Learning-Agility } \\
\text { 4.3 Balance on unstable surface } \\
\text { 4.10 Stepping multiple directions (grapevine) } \\
\text { 4.13 Step lateral (side step) } \\
\text { 4.17 Tandem walking } \\
\text { 5. Task specific training } \\
\text { 5.4 Gait backwards } \\
\text { 5.5 Gait downhill } \\
\text { 5.7 Gait sideways } \\
\text { 5.9 Gait uphill } \\
\text { 5.10 Gait with perturbations } \\
\text { 5.13 Sit-to-stand training } \\
\text { 5.15 Stair training } \\
\text { 5.16 Treadmill gait }\end{array}$ & & & & \\
\hline & No data & $\begin{array}{l}\text { Functional } \\
\text { rehabilitation }\end{array}$ & $\begin{array}{l}\text { 1. Strength } \\
\text { 1.11 Hip abduction in sidelying (position unclear) } \\
\text { 1.12 Hip abduction in standing (position unclear) } \\
\text { 1.13 Hip abduction in supine (position unclear) } \\
\text { 1.31 Knee extension in sitting or supine (long arc } \\
\text { quad) } \\
\text { 1.32 Knee extension in sitting or supine (short arc } \\
\text { quad) } \\
\text { 1.35 Knee flexion in prone } \\
\text { 1.36 Knee flexion in sitting or supine } \\
\text { 1.37 Knee flexion in standing } \\
\text { 1.43 Quad sets } \\
\text { 2. Aerobic } \\
\text { 2.2 Bike (Endurance) } \\
\text { 2.8 Treadmill walking } \\
\text { 3. Flexibility } \\
\text { 3.1 Ankle pumps } \\
\text { 3.5 Hamstring stretch in any position }\end{array}$ & $\mathrm{Y}(\mathrm{N})$ & $\begin{array}{l}\text { Physical } \\
\text { therapist }\end{array}$ & In-person & $\begin{array}{l}\text { Physical } \\
\text { therapy/rehabilitation } \\
\text { facility (outpatient) }\end{array}$ \\
\hline
\end{tabular}




\begin{tabular}{|c|c|c|c|c|c|c|c|}
\hline $\begin{array}{l}\text { Study, Year, } \\
\text { PMID, } \\
\text { Country }\end{array}$ & $\begin{array}{l}\text { Intended } \\
\text { Comparison }\end{array}$ & Arm & Components (Specific Exercises/Strategies) & $\begin{array}{l}\text { Progression } \\
\text { (Appropriate?) }\end{array}$ & Personnel & $\begin{array}{l}\text { Mode of } \\
\text { Delivery }\end{array}$ & Setting \\
\hline & & & $\begin{array}{l}\text { 3.10 Knee extension AROM (unclear) } \\
\text { 3.11 Knee extension PROM in supine (unclear) } \\
\text { 3.12 Knee extension PROM in prone (unclear) } \\
\text { 3.13 Knee flexion AROM (unclear) } \\
\text { 3.14 Knee flexion PROM in sitting or supine } \\
\text { (unclear) } \\
\text { 3.15 Knee flexion AROM in any position (rectus } \\
\text { femoris stretch) } \\
\text { 3.16 Knee flexion PROM in prone (rectus femoris } \\
\text { stretch) } \\
\text { 5. Task specific training } \\
\text { 5.4 Gait backwards } \\
\text { 5.5 Gait downhill } \\
\text { 5.7 Gait sideways } \\
\text { 5.9 Gait uphill } \\
\text { 5.13 Sit-to-stand training } \\
\text { 5.15 Stair training } \\
\text { 5.16 Treadmill gait }\end{array}$ & & & & \\
\hline \multirow[t]{2}{*}{$\begin{array}{l}\text { Liao, } \\
2020, \\
31687984, \\
\text { Taiwan }\end{array}$} & $\begin{array}{l}\text { Elastic resistance } \\
\text { exercise training vs. } \\
\text { standard care }\end{array}$ & $\begin{array}{l}\text { Elastic } \\
\text { resistance } \\
\text { exercise training }\end{array}$ & $\begin{array}{l}\text { 1. Strength } \\
\text { 1.11 Hip abduction in sidelying (position unclear) } \\
\text { 1.12 Hip abduction in standing (position unclear) } \\
\text { 1.13 Hip abduction in supine (position unclear) } \\
\text { 1.14 Hip adduction in sidelying (position unclear) } \\
\text { 1.15 Hip adduction in standing (position unclear) } \\
\text { 1.16 Hip adduction in supine (position unclear) } \\
\text { 1.17 Hip extension in sidelying (position unclear) } \\
\text { 1.18 Hip extension in prone (position unclear) } \\
\text { 1.19 Hip extension in standing (position unclear) } \\
\text { 1.20 Hip flexion in sidelying (position unclear) } \\
\text { 1.21 Hip flexion in sitting (position unclear) } \\
\text { 1.23 Hip flexion in supine (position unclear) } \\
\text { 1.22 Hip flexion in standing (position unclear) } \\
\text { 1.35 Knee flexion in prone } \\
\text { 1.36 Knee flexion in sitting or supine } \\
\text { 1.37 Knee flexion in standing } \\
\text { 1.38 Leg Press (one leg) } \\
\text { 1.39 Leg Press (two legs) } \\
\text { 1.60 Upper extremity strengthening } \\
\text { 3. Flexibility } \\
\text { [specific exercises not defined] }\end{array}$ & $\mathrm{Y}(\mathrm{Y})$ & $\begin{array}{l}\text { Physical } \\
\text { therapist }\end{array}$ & $\begin{array}{l}\text { In-person; } \\
\text { Self-guided } \\
\text { (unsupervised) }\end{array}$ & $\begin{array}{l}\text { Physical } \\
\text { therapy/rehabilitation } \\
\text { facility (outpatient); } \\
\text { Home }\end{array}$ \\
\hline & No data & Standard care & $\begin{array}{l}\text { NA } \\
\text { [Standard care consisted of knee osteoarthritis } \\
\text { education, pharmacologic therapy, and } \\
\text { conservative physical therapy without any } \\
\text { resistance exercise training (active and passive } \\
\text { range of motion, stretching, and functional } \\
\text { conditioning), and maintenance of usually activity } \\
\text { level] }\end{array}$ & NA & NR & In-person & NR \\
\hline
\end{tabular}




\begin{tabular}{|c|c|c|c|c|c|c|c|}
\hline $\begin{array}{l}\text { Study, Year, } \\
\text { PMID, } \\
\text { Country }\end{array}$ & $\begin{array}{l}\text { Intended } \\
\text { Comparison }\end{array}$ & Arm & Components (Specific Exercises/Strategies) & $\begin{array}{l}\text { Progression } \\
\text { (Appropriate?) }\end{array}$ & Personnel & $\begin{array}{l}\text { Mode of } \\
\text { Delivery }\end{array}$ & Setting \\
\hline \multirow[t]{2}{*}{$\begin{array}{l}\text { Liebs, } \\
2010, \\
20360503 \\
\text { Germany }\end{array}$} & $\begin{array}{l}\text { Ergometer cycling } \\
\text { plus standard } \\
\text { physiotherapy vs. } \\
\text { standard } \\
\text { physiotherapy alone }\end{array}$ & $\begin{array}{l}\text { Ergometer } \\
\text { cycling }\end{array}$ & $\begin{array}{l}\text { 1. Strength } \\
\text { [specific exercises not defined] } \\
\text { 3. Flexibility } \\
\text { 3.2 Bike (ROM) } \\
\text { 4. Balance-Motor Learning-Agility } \\
\text { 4.3 Balance on unstable surface } \\
\text { 5. Task specific training } \\
\text { 5.1 Transfers } \\
\text { 5.6 Gait on uneven surfaces } \\
\text { 5.8 Gait training } \\
\text { 5.15 Stair training }\end{array}$ & $\mathrm{N}(\mathrm{NA})$ & $\begin{array}{l}\text { Physical } \\
\text { therapist }\end{array}$ & In-person & $\begin{array}{l}\text { Physical } \\
\text { therapy/rehabilitation } \\
\text { facility (outpatient) }\end{array}$ \\
\hline & No data & $\begin{array}{l}\text { Control } \\
\text { (standard daily } \\
\text { physiotherapy) }\end{array}$ & $\begin{array}{l}\text { 1. Strength } \\
\text { [specific exercises not defined] } \\
\text { 3. Flexibility } \\
\text { [specific exercises not defined] } \\
\text { 4. Balance-Motor Learning-Agility } \\
\text { 4.3 Balance on unstable surface } \\
\text { 5. Task specific training } \\
\text { 5.1 Transfers } \\
\text { 5.6 Gait on uneven surfaces } \\
\text { 5.8 Gait training } \\
\text { 5.15 Stair training }\end{array}$ & $\mathrm{N}(\mathrm{NA})$ & $\begin{array}{l}\text { Physical } \\
\text { therapist }\end{array}$ & In-person & $\begin{array}{l}\text { Physical } \\
\text { therapy/rehabilitation } \\
\text { facility (outpatient) }\end{array}$ \\
\hline \multirow[t]{2}{*}{$\begin{array}{l}\text { Liebs, } \\
2012, \\
22196125, \\
\text { Germany }\end{array}$} & $\begin{array}{l}\text { Early aquatic } \\
\text { therapy vs. late } \\
\text { aquatic therapy }\end{array}$ & $\begin{array}{l}\text { Early Aquatic } \\
\text { therapy }\end{array}$ & $\begin{array}{l}\text { 1. Strength } \\
\text { [specific exercises not defined] } \\
\text { 3. Flexibility } \\
\text { [specific exercises not defined] } \\
\text { 4. Balance-Motor Learning-Agility } \\
\text { 4.3 Balance on unstable surface } \\
\text { 5. Task specific training } \\
\text { 5.1 Transfers } \\
\text { 5.6 Gait on uneven surfaces } \\
\text { 5.8 Gait training } \\
\text { 5.15 Stair training }\end{array}$ & $N(N A)$ & $\begin{array}{l}\text { Physical } \\
\text { therapist }\end{array}$ & In-person & $\begin{array}{l}\text { Physical } \\
\text { therapy/rehabilitation } \\
\text { facility (outpatient) }\end{array}$ \\
\hline & No data & $\begin{array}{l}\text { Late Aquatic } \\
\text { therapy (after } \\
\text { wound healing) }\end{array}$ & $\begin{array}{l}\text { 1. Strength } \\
\text { [specific exercises not defined] } \\
\text { 3. Flexibility } \\
\text { [specific exercises not defined] } \\
\text { 4. Balance-Motor Learning-Agility } \\
\text { 4.3 Balance on unstable surface } \\
\text { 5. Task specific training } \\
\text { 5.1 Transfers } \\
\text { 5.6 Gait on uneven surfaces } \\
\text { 5.8 Gait training } \\
\text { 5.15 Stair training }\end{array}$ & $\mathrm{N}(\mathrm{NA})$ & $\begin{array}{l}\text { Physical } \\
\text { therapist }\end{array}$ & In-person & $\begin{array}{l}\text { Physical } \\
\text { therapy/rehabilitation } \\
\text { facility (outpatient) }\end{array}$ \\
\hline $\begin{array}{l}\text { Madsen, } \\
2013, \\
23651717, \\
\text { Denmark }\end{array}$ & $\begin{array}{l}\text { Group-based } \\
\text { rehabilitation vs. } \\
\text { individual } \\
\text { supervised home } \\
\text { training }\end{array}$ & $\begin{array}{l}\text { Group-based } \\
\text { rehabilitation }\end{array}$ & $\begin{array}{l}\text { 1. Strength } \\
\text { 1.6 Core strengthening } \\
1.28 \text { Knee extension machine (one-leg) } \\
1.29 \text { Knee extension machine (two-legs) } \\
1.33 \text { Knee flexion machine (Hamstring curl) one } \\
\text { knee }\end{array}$ & $\mathrm{Y}(\mathrm{Y})$ & $\begin{array}{l}\text { Physical } \\
\text { therapist }\end{array}$ & $\begin{array}{l}\text { In-person; } \\
\text { None } \\
\text { (unsupervised) }\end{array}$ & $\begin{array}{l}\text { Physical } \\
\text { therapy/rehabilitation } \\
\text { facility (outpatient); } \\
\text { Home }\end{array}$ \\
\hline
\end{tabular}




\begin{tabular}{|c|c|c|c|c|c|c|c|}
\hline $\begin{array}{l}\text { Study, Year, } \\
\text { PMID, } \\
\text { Country }\end{array}$ & $\begin{array}{l}\text { Intended } \\
\text { Comparison }\end{array}$ & Arm & Components (Specific Exercises/Strategies) & $\begin{array}{l}\text { Progression } \\
\text { (Appropriate?) }\end{array}$ & Personnel & $\begin{array}{l}\text { Mode of } \\
\text { Delivery }\end{array}$ & Setting \\
\hline & & & $\begin{array}{l}\text { 1.34 Knee flexion machine (Hamstring curl) two } \\
\text { knees } \\
\text { 1.38 Leg Press (one leg) } \\
\text { 1.39 Leg Press (two legs) } \\
\text { 1.49 Squats } \\
\text { 1.60 Upper extremity strengthening } \\
\text { 2. Aerobic } \\
\text { 2.2 Bike (Endurance) } \\
\text { 2.9 Walking } \\
\text { 3. Flexibility } \\
\text { 3.2 Bike (ROM) } \\
\text { 4. Balance-Motor Learning-Agility } \\
\text { [specific exercises not defined] } \\
\text { 6. Patient education } \\
\text { 6.2 Home exercise program }\end{array}$ & & & & \\
\hline & No data & $\begin{array}{l}\text { Supervised } \\
\text { home-exercises }\end{array}$ & $\begin{array}{l}\text { 1. Strength } \\
\text { [specific exercises not defined] } \\
\text { 2. Aerobic } \\
\text { 2.2 Bike (Endurance) } \\
\text { 2.9 Walking } \\
\text { 4. Balance-Motor Learning-Agility } \\
\text { [specific exercises not defined] } \\
\text { 6. Patient education } \\
\text { 6.2 Home exercise program }\end{array}$ & $\mathrm{N}(\mathrm{N})$ & $\begin{array}{l}\text { Physical } \\
\text { therapist }\end{array}$ & $\begin{array}{l}\text { In-person } \\
\text { (planned } \\
\text { visits); None } \\
\text { (unsupervised) }\end{array}$ & $\begin{array}{l}\text { Physical } \\
\text { therapy/rehabilitation } \\
\text { facility (outpatient); } \\
\text { Home }\end{array}$ \\
\hline $\begin{array}{l}\text { Minns Lowe, } \\
2012 \\
22180446, \\
\text { UK }\end{array}$ & $\begin{array}{l}\text { Home visit } \\
\text { physiotherapy visits } \\
\text { vs. usual care }\end{array}$ & $\begin{array}{l}\text { Home-visit } \\
\text { physiotherapy }\end{array}$ & $\begin{array}{l}\text { 1. Strength } \\
\text { 1.9 Heel raises - bilateral (calf raises) } \\
\text { 1.10 Heel raises - unilateral } \\
\text { 1.47 Single leg stance } \\
\text { 1.48 Sit-to-stand } \\
\text { 1.49 Squats } \\
\text { 1.55 Step up - forward } \\
\text { 1.61 Wall slides } \\
\text { 3. Flexibility } \\
\text { 3.3 Calf stretch with knee bent (soleus) } \\
\text { 3.4 Calf stretch with knee straight (gastroc) } \\
\text { 3.5 Hamstring stretch in any position } \\
\text { 3.15 Knee flexion AROM in any position (rectus } \\
\text { femoris stretch) } \\
\text { 3.16 Knee flexion PROM in prone (rectus femoris } \\
\text { stretch) } \\
\text { 4. Balance-Motor Learning-Agility } \\
\text { 4.6 Marching } \\
\text { 4.8 Single leg stance } \\
\text { 4.9 Standing weight shifts } \\
\text { 5. Task specific training } \\
\text { 5.1 Transfers } \\
\text { 5.6 Gait on uneven surfaces } \\
\text { 5.8 Gait training } \\
\text { 5.12 Obstacle training } \\
\text { 5.13 Sit-to-stand training }\end{array}$ & $\mathrm{Y}(\mathrm{N})$ & $\begin{array}{l}\text { Physical } \\
\text { therapist }\end{array}$ & In-person & Home \\
\hline
\end{tabular}




\begin{tabular}{|c|c|c|c|c|c|c|c|}
\hline \multirow[t]{3}{*}{$\begin{array}{l}\text { Study, Year, } \\
\text { PMID, } \\
\text { Country }\end{array}$} & $\begin{array}{l}\text { Intended } \\
\text { Comparison }\end{array}$ & Arm & Components (Specific Exercises/Strategies) & $\begin{array}{l}\text { Progression } \\
\text { (Appropriate?) }\end{array}$ & Personnel & $\begin{array}{l}\text { Mode of } \\
\text { Delivery }\end{array}$ & Setting \\
\hline & & & 5.15 Stair training & & & & \\
\hline & No data & Usual care & $\begin{array}{l}\text { NA } \\
\text { [Usual care consisted of an advice booklet with } \\
\text { gait training and exercise advice; referral to } \\
\text { outpatient physiotherapy possible if recovery in } \\
\text { acute was slow or if not achieving recovery } \\
\text { targets at follow-up (less than } 20 \% \text { of patients)] }\end{array}$ & NA & NA & NA & NA \\
\hline \multirow[t]{2}{*}{$\begin{array}{l}\text { Mitchell, } \\
2005, \\
15869558, \\
\text { UKA }\end{array}$} & $\begin{array}{l}\text { Home pre-operative } \\
\text { and post-operative } \\
\text { physiotherapy vs. } \\
\text { usual outpatient } \\
\text { post-operative } \\
\text { physiotherapy }\end{array}$ & $\begin{array}{l}\text { Home } \\
\text { rehabilitation }\end{array}$ & $\begin{array}{l}\text { 3. Flexibility } \\
\text { 3.10 Knee extension AROM (unclear) } \\
\text { 3.11 Knee extension PROM in supine (unclear) } \\
\text { 3.12 Knee extension PROM in prone (unclear) } \\
\text { 3.13 Knee flexion AROM (unclear) } \\
\text { 3.14 Knee flexion PROM in sitting or supine } \\
\text { (unclear) } \\
\text { 3.15 Knee flexion AROM in any position (rectus } \\
\text { femoris stretch) (unclear) } \\
\text { 3.16 Knee flexion PROM in prone (rectus femoris } \\
\text { stretch) (unclear) } \\
\text { 5. Task specific training } \\
\text { 5.8 Gait training } \\
\text { 6. Patient education } \\
\text { 6.1 ADLs } \\
\text { 6.4 Pain management } \\
\text { 7. Adjunctive modality } \\
\text { 7.12 Massage/myofascial techniques for soft } \\
\text { tissue }\end{array}$ & $\mathrm{N}(\mathrm{NA})$ & $\begin{array}{l}\text { Physical } \\
\text { therapist }\end{array}$ & In-person & Home \\
\hline & No data & $\begin{array}{l}\text { Hospital } \\
\text { outpatient } \\
\text { rehabilitation }\end{array}$ & $\begin{array}{l}\text { 3. Flexibility } \\
\text { 3.10 Knee extension AROM (unclear) } \\
\text { 3.11 Knee extension PROM in supine (unclear) } \\
\text { 3.12 Knee extension PROM in prone (unclear) } \\
\text { 3.13 Knee flexion AROM (unclear) } \\
\text { 3.14 Knee flexion PROM in sitting or supine } \\
\text { (unclear) } \\
\text { 3.15 Knee flexion AROM in any position (rectus } \\
\text { femoris stretch) (unclear) } \\
\text { 3.16 Knee flexion PROM in prone (rectus femoris } \\
\text { stretch) (unclear) } \\
\text { 5. Task specific training } \\
\text { 5.8 Gait training } \\
\text { 7. Adjunctive modality } \\
\text { 7.4 E-stim for pain (TENS) } \\
\text { 7.5 E-stim for strength (NMES) }\end{array}$ & $\mathrm{N}(\mathrm{NA})$ & $\begin{array}{l}\text { Physical } \\
\text { therapist }\end{array}$ & In-person & $\begin{array}{l}\text { Physical } \\
\text { therapy/rehabilitation } \\
\text { facility (outpatient) }\end{array}$ \\
\hline $\begin{array}{l}\text { Moffet, } \\
2015, \\
26178888, \\
\text { Canada }\end{array}$ & $\begin{array}{l}\text { In-home } \\
\text { telerehabilitation vs. } \\
\text { face-to-face home } \\
\text { rehabilitation }\end{array}$ & $\begin{array}{l}\text { In-home } \\
\text { telerehabilitation }\end{array}$ & $\begin{array}{l}\text { 1. Strength } \\
\text { [specific exercises not defined] } \\
\text { 4. Balance-Motor Learning-Agility } \\
\text { [specific exercises not defined] } \\
\text { 5. Task specific training } \\
\text { [specific exercises not defined] } \\
\text { 6. Patient education } \\
\text { 6.1 Activities of daily living }\end{array}$ & $\mathrm{Y}(\mathrm{Y})$ & $\begin{array}{l}\text { Physical } \\
\text { therapist }\end{array}$ & $\begin{array}{l}\text { Remote via } \\
\text { videoconfence }\end{array}$ & Home \\
\hline
\end{tabular}




\begin{tabular}{|c|c|c|c|c|c|c|c|}
\hline $\begin{array}{l}\text { Study, Year, } \\
\text { PMID, } \\
\text { Country }\end{array}$ & $\begin{array}{l}\text { Intended } \\
\text { Comparison }\end{array}$ & Arm & Components (Specific Exercises/Strategies) & $\begin{array}{l}\text { Progression } \\
\text { (Appropriate?) }\end{array}$ & Personnel & $\begin{array}{l}\text { Mode of } \\
\text { Delivery }\end{array}$ & Setting \\
\hline & & & $\begin{array}{l}\text { 6.2 Home exercise program } \\
\text { 6.4 Pain management }\end{array}$ & & & & \\
\hline & No data & $\begin{array}{l}\text { Standard home } \\
\text { rehabilitation }\end{array}$ & $\begin{array}{l}\text { 1. Strength } \\
\text { [specific exercises not defined] } \\
\text { 4. Balance-Motor Learning-Agility } \\
\text { [specific exercises not defined] } \\
\text { 5. Task specific training } \\
\text { [specific exercises not defined] } \\
\text { 6. Patient education } \\
\text { 6.1 Activities of daily living } \\
\text { 6.2 Home exercise program } \\
\text { 6.4 Pain management }\end{array}$ & $\mathrm{Y}(\mathrm{Y})$ & $\begin{array}{l}\text { Physical } \\
\text { therapist }\end{array}$ & In-person & Home \\
\hline \multirow[t]{2}{*}{$\begin{array}{l}\text { Monticone, } \\
2013, \\
23063624, \\
\text { Italy }\end{array}$} & $\begin{array}{l}\text { Home-based } \\
\text { functional exercises } \\
\text { targeted at } \\
\text { managing } \\
\text { kinesiophobia vs. } \\
\text { general advice of } \\
\text { staying active }\end{array}$ & $\begin{array}{l}\text { Home-based } \\
\text { functional } \\
\text { exercises and } \\
\text { kinesiophobia } \\
\text { training }\end{array}$ & $\begin{array}{l}\text { 1. Strength } \\
\text { 1.37 Knee flexion in standing } \\
\text { 3. Flexibility } \\
\text { 3.2 Bike (ROM) } \\
\text { 4. Balance-Motor Learning-Agility } \\
\text { 4.3 Balance on unstable surface } \\
\text { 4.6 Marching } \\
\text { 4.10 Stepping multiple directions (grapevine) } \\
\text { 5. Task specific training } \\
\text { 5.8 Gait training } \\
\text { 5.12 Obstacle training } \\
\text { 5.13 Sit-to-stand training } \\
\text { 5.15 Stair training } \\
\text { 6. Patient education } \\
\text { 6.1 Activities of daily living } \\
\text { 6.2 Home exercise program } \\
\text { 6.4 Pain management } \\
\text { 6.5 Self-management } \\
\text { 7. Adjunctive modality } \\
\text { 7.17 Mindfulness, stress/anxiety-reduction } \\
\text { interventions }\end{array}$ & $\mathrm{N}(\mathrm{N})$ & $\begin{array}{l}\text { Physical } \\
\text { therapist }\end{array}$ & $\begin{array}{l}\text { In-person; } \\
\text { Remote via } \\
\text { telephone }\end{array}$ & $\begin{array}{l}\text { Other inpatient } \\
\text { facility (rehabilitation } \\
\text { centre); Home }\end{array}$ \\
\hline & No data & Usual care & $\begin{array}{l}\text { 1. Strength } \\
\text { 1.37 Knee flexion in standing } \\
\text { 3. Flexibility } \\
\text { 3.2 Bike (ROM) } \\
\text { 4. Balance-Motor Learning-Agility } \\
\text { 4.3 Balance on unstable surface } \\
\text { 4.6 Marching } \\
\text { 4.10 Stepping multiple directions (grapevine) } \\
\text { 5. Task specific training } \\
\text { 5.8 Gait training } \\
\text { 5.12 Obstacle training } \\
\text { 5.13 Sit-to-stand training } \\
\text { 5.15 Stair training }\end{array}$ & $\mathrm{N}(\mathrm{N})$ & $\begin{array}{l}\text { Physical } \\
\text { therapist }\end{array}$ & In-person & $\begin{array}{l}\text { Other inpatient } \\
\text { facility (rehabilitation } \\
\text { center) }\end{array}$ \\
\hline $\begin{array}{l}\text { Moutzouri, } \\
2018, \\
29473481, \\
\text { NR }\end{array}$ & $\begin{array}{l}\text { Early self-managed } \\
\text { focal sensorimotor } \\
\text { training vs. } \\
\text { functional }\end{array}$ & $\begin{array}{l}\text { Early self- } \\
\text { managed focal } \\
\text { sensorimotor } \\
\text { exercise training }\end{array}$ & $\begin{array}{l}\text { 1. Strength } \\
\text { 1.31 Knee extension in sitting or supine (long arc } \\
\text { quad) }\end{array}$ & $\mathrm{Y}(\mathrm{Y})$ & $\begin{array}{l}\text { Physical } \\
\text { therapist }\end{array}$ & $\begin{array}{l}\text { Self-guided } \\
\text { (unsupervised) }\end{array}$ & Home \\
\hline
\end{tabular}




\begin{tabular}{|c|c|c|c|c|c|c|c|}
\hline $\begin{array}{l}\text { Study, Year, } \\
\text { PMID, } \\
\text { Country }\end{array}$ & $\begin{array}{l}\text { Intended } \\
\text { Comparison }\end{array}$ & Arm & Components (Specific Exercises/Strategies) & $\begin{array}{l}\text { Progression } \\
\text { (Appropriate?) }\end{array}$ & Personnel & $\begin{array}{l}\text { Mode of } \\
\text { Delivery }\end{array}$ & Setting \\
\hline & exercise training & & $\begin{array}{l}\text { 1.32 Knee extension in sitting or supine (short arc } \\
\text { quad) } \\
\text { 1.48 Sit-to-stand } \\
\text { 1.61 Wall slides } \\
\text { 2. Aerobic } \\
\text { 2.2 Bike (Endurance) } \\
\text { 2.9 Walking } \\
\text { 3. Flexibility } \\
\text { 3.6 Heel slides } \\
\text { 3.10 Knee extension AROM (unclear) } \\
\text { 3.11 Knee extension PROM in supine (unclear) } \\
\text { 3.12 Knee extension PROM in prone (unclear) } \\
\text { 3.13 Knee flexion AROM (unclear) } \\
\text { 3.14 Knee flexion PROM in sitting or supine } \\
\text { (unclear) } \\
\text { 4. Balance-Motor Learning-Agility } \\
\text { 4.3 Balance on unstable surface } \\
\text { 4.6 Marching } \\
\text { 4.10 Stepping multiple directions (grapevine) } \\
\text { 4.13 Step lateral (side step) } \\
\text { 4.17 Tandem walking } \\
\text { 5. Task specific training } \\
\text { 5.10 Gait with perturbations } \\
\text { 5.12 Obstacle training } \\
\text { 5.15 Stair training }\end{array}$ & & & & \\
\hline & No data & $\begin{array}{l}\text { Functional } \\
\text { exercise training }\end{array}$ & $\begin{array}{l}\text { 1. Strength } \\
\text { 1.9 Heel raises - bilateral (calf raises) } \\
\text { 1.11 Hip abduction in sidelying } \\
\text { 1.31 Knee extension in sitting or supine (long arc } \\
\text { quad) } \\
\text { 1.32 Knee extension in sitting or supine (short arc } \\
\text { quad) } \\
\text { 1.37 Knee flexion in standing } \\
\text { 1.48 Sit-to-stand } \\
\text { 1.58 Straight leg raises } \\
\text { 1.61 Wall slides } \\
\text { 2. Aerobic } \\
\text { 2.2 Bike (Endurance) } \\
\text { 2.9 Walking } \\
\text { 3. Flexibility } \\
\text { 3.1 Ankle pumps } \\
\text { 3.6 Heel slides } \\
\text { 3.10 Knee extension AROM (unclear) } \\
\text { 3.11 Knee extension PROM in supine (unclear) } \\
\text { 3.12 Knee extension PROM in prone (unclear) } \\
\text { 3.13 Knee flexion AROM (unclear) } \\
\text { 3.14 Knee flexion PROM in sitting or supine } \\
\text { (unclear) } \\
\text { 5. Task specific training } \\
\text { 5.15 Stair training }\end{array}$ & $\mathrm{Y}(\mathrm{Y})$ & $\begin{array}{l}\text { Physical } \\
\text { therapist }\end{array}$ & $\begin{array}{l}\text { Self-guided } \\
\text { (unsupervised) }\end{array}$ & Home \\
\hline
\end{tabular}




\begin{tabular}{|c|c|c|c|c|c|c|c|}
\hline $\begin{array}{l}\text { Study, Year, } \\
\text { PMID, } \\
\text { Country }\end{array}$ & $\begin{array}{l}\text { Intended } \\
\text { Comparison }\end{array}$ & Arm & Components (Specific Exercises/Strategies) & $\begin{array}{l}\text { Progression } \\
\text { (Appropriate?) }\end{array}$ & Personnel & $\begin{array}{l}\text { Mode of } \\
\text { Delivery }\end{array}$ & Setting \\
\hline \multirow[t]{2}{*}{$\begin{array}{l}\text { Naylor, } \\
2017 \\
28899328 \\
\text { Australia }\end{array}$} & $\begin{array}{l}\text { Discharge to } \\
\text { inpatient } \\
\text { rehabilitation vs. } \\
\text { discharge to home } \\
\text { (observational) } \\
\end{array}$ & $\begin{array}{l}\text { Inpatient } \\
\text { rehabilitation }\end{array}$ & $\begin{array}{l}\text { [specific goals and exercises not defined; } \\
\text { comparison of setting] }\end{array}$ & NR & NR & NR & $\begin{array}{l}\text { Other inpatient } \\
\text { facility (not acute) }\end{array}$ \\
\hline & No data & $\begin{array}{l}\text { No inpatient } \\
\text { rehabilitation }\end{array}$ & $\begin{array}{l}\text { [specific goals and exercises not defined; } \\
\text { comparison of setting] }\end{array}$ & NR & NR & NR & $\begin{array}{l}\text { NR (other than not } \\
\text { inpatient) }\end{array}$ \\
\hline \multirow[t]{3}{*}{$\begin{array}{l}\text { Padgett, } \\
2018 \mathrm{a} \\
29352683 \\
\text { USA }\end{array}$} & $\begin{array}{l}\text { Discharge to home } \\
\text { vs. discharge to } \\
\text { inpatient } \\
\text { rehabilitation } \\
\text { Discharge to skilled } \\
\text { nursing facility vs. } \\
\text { discharge to } \\
\text { inpatient } \\
\text { rehabilitation }\end{array}$ & Home & $\begin{array}{l}\text { [specific goals and exercises not defined; } \\
\text { comparison of setting] }\end{array}$ & NR & NR & NR & Home \\
\hline & No data & $\begin{array}{l}\text { Long term care } \\
\text { facility }\end{array}$ & $\begin{array}{l}\text { [specific goals and exercises not defined; } \\
\text { comparison of setting] }\end{array}$ & NR & NR & NR & $\begin{array}{l}\text { Other inpatient } \\
\text { facility (long term } \\
\text { care facility) }\end{array}$ \\
\hline & No data & $\begin{array}{l}\text { Inpatient } \\
\text { rehabilitation }\end{array}$ & $\begin{array}{l}\text { [specific goals and exercises not defined; } \\
\text { comparison of setting] }\end{array}$ & NR & NR & NR & $\begin{array}{l}\text { Other inpatient } \\
\text { facility (inpatient } \\
\text { rehabilitation; not } \\
\text { acute) }\end{array}$ \\
\hline \multirow[t]{2}{*}{$\begin{array}{l}\text { Petersen, } \\
2018, \\
29294078 \\
\text { Netherlands }\end{array}$} & $\begin{array}{l}\text { Acupuncture and } \\
\text { exercise vs. } \\
\text { exercise alone }\end{array}$ & $\begin{array}{l}\text { Exercise \& } \\
\text { acupuncture }\end{array}$ & $\begin{array}{l}\text { 1. Strength } \\
\text { [specific exercises not defined] } \\
\text { 2. Aerobic } \\
\text { [specific exercises not defined] } \\
\text { 3. Flexibility } \\
\text { [specific exercises not defined] } \\
\text { 4. Balance-Motor Learning-Agility } \\
\text { [specific exercises not defined] } \\
\text { 5. Task specific training } \\
\text { [specific exercises not defined] } \\
\text { 7. Adjunctive modality } \\
\text { 7.16 Dry needling (acupuncture) }\end{array}$ & $\mathrm{Y}(\mathrm{Y})$ & $\begin{array}{l}\text { Physical } \\
\text { therapist }\end{array}$ & In-person & $\begin{array}{l}\text { Physical } \\
\text { therapy/rehabilitation } \\
\text { facility (outpatient) }\end{array}$ \\
\hline & No data & Exercise & $\begin{array}{l}\text { 1. Strength } \\
\text { [specific exercises not defined] } \\
\text { 2. Aerobic } \\
\text { [specific exercises not defined] } \\
\text { 3. Flexibility } \\
\text { [specific exercises not defined] } \\
\text { 4. Balance-Motor Learning-Agility } \\
\text { [specific exercises not defined] } \\
\text { 5. Task specific training } \\
\text { [specific exercises not defined] }\end{array}$ & $\mathrm{Y}(\mathrm{Y})$ & $\begin{array}{l}\text { Physical } \\
\text { therapist }\end{array}$ & In-person & $\begin{array}{l}\text { Physical } \\
\text { therapy/rehabilitation } \\
\text { facility (outpatient) }\end{array}$ \\
\hline $\begin{array}{l}\text { Petterson, } \\
2009 \\
19177542 \\
\text { USA }\end{array}$ & $\begin{array}{l}\text { Neuromuscular } \\
\text { electrical stimulation } \\
\text { plus progressive } \\
\text { volitional strength } \\
\end{array}$ & $\begin{array}{l}\text { Exercise \& } \\
\text { NMES }\end{array}$ & $\begin{array}{l}\text { 1. Strength } \\
1.11 \text { Hip abduction in sidelying } \\
1.31 \text { Knee extension in sitting or supine (long arc } \\
\text { quad) }\end{array}$ & $\mathrm{Y}(\mathrm{Y})$ & $\begin{array}{l}\text { Physical } \\
\text { therapist }\end{array}$ & In-person & $\begin{array}{l}\text { Physical } \\
\text { therapy/rehabilitation } \\
\text { facility (outpatient) }\end{array}$ \\
\hline
\end{tabular}




\begin{tabular}{|c|c|c|c|c|c|c|c|}
\hline $\begin{array}{l}\text { Study, Year, } \\
\text { PMID, } \\
\text { Country }\end{array}$ & $\begin{array}{l}\text { Intended } \\
\text { Comparison }\end{array}$ & Arm & Components (Specific Exercises/Strategies) & $\begin{array}{l}\text { Progression } \\
\text { (Appropriate?) }\end{array}$ & Personnel & $\begin{array}{l}\text { Mode of } \\
\text { Delivery }\end{array}$ & Setting \\
\hline & $\begin{array}{l}\text { training program vs. } \\
\text { progressive } \\
\text { volitional strength } \\
\text { training program } \\
\text { alone }\end{array}$ & & $\begin{array}{l}\text { 1.37 Knee flexion in standing } \\
\text { 1.41 Lunges } \\
\text { 1.43 Quad sets } \\
\text { 1.51 Standing terminal knee extension } \\
\text { 1.52 Step down } \\
\text { 1.55 Step up - forward } \\
\text { 1.58 Straight leg raises } \\
\text { 1.61 Wall slides } \\
\text { 3. Flexibility } \\
\text { 3.2 Bike (ROM) } \\
\text { 3.11 Knee extension PROM in supine } \\
\text { 3.12 Knee extension PROM in prone } \\
\text { 3.14 Knee flexion PROM in sitting or supine } \\
\text { 5. Task specific training } \\
\text { 5.8 Gait training } \\
\text { 5.15 Stair training } \\
\text { 7. Adjunctive modality } \\
\text { 7.5 E-stim for strength (NMES) } \\
\text { 7.11 Massage for scar mobility } \\
\text { 7.14 Mobilizations - Patellar }\end{array}$ & & & & \\
\hline & No data & Exercise & $\begin{array}{l}\text { 1. Strength } \\
\text { 1.11 Hip abduction in sidelying } \\
\text { 1.31 Knee extension in sitting or supine (long arc } \\
\text { quad) } \\
\text { 1.37 Knee flexion in standing } \\
\text { 1.41 Lunges } \\
\text { 1.43 Quad sets } \\
\text { 1.51 Standing terminal knee extension } \\
\text { 1.52 Step down } \\
\text { 1.55 Step up - forward } \\
\text { 1.58 Straight leg raises } \\
\text { 1.61 Wall slides } \\
\text { 3. Flexibility } \\
\text { 3.2 Bike (ROM) } \\
\text { 3.11 Knee extension PROM in supine } \\
\text { 3.12 Knee extension PROM in prone } \\
\text { 3.14 Knee flexion PROM in sitting or supine } \\
\text { 5. Task specific training } \\
\text { 5.8 Gait training } \\
\text { 5.15 Stair training } \\
\text { 7. Adjunctive modality } \\
\text { 7.11 Massage for scar mobility } \\
\text { 7.14 Mobilizations - Patellar }\end{array}$ & $\mathrm{Y}(\mathrm{Y})$ & $\begin{array}{l}\text { Physical } \\
\text { therapist }\end{array}$ & In-person & $\begin{array}{l}\text { Physical } \\
\text { therapy/rehabilitation } \\
\text { facility (outpatient) }\end{array}$ \\
\hline $\begin{array}{l}\text { Piqueras, } \\
2013, \\
23474735, \\
\text { Spain }\end{array}$ & $\begin{array}{l}\text { Interactive virtual } \\
\text { telerehabilitation } \\
\text { system vs. } \\
\text { conventional } \\
\text { outpatient physical } \\
\text { therapy }\end{array}$ & $\begin{array}{l}\text { Interactive virtual } \\
\text { telerehabilitation } \\
\text { system }\end{array}$ & $\begin{array}{l}\text { 1. Strength } \\
\text { [specific exercises not defined] } \\
\text { 3. Flexibility } \\
\text { [specific exercises not defined] } \\
\text { 5. Task specific training } \\
\text { [specific exercises not defined] } \\
\text { 5.8 Gait training }\end{array}$ & $\mathrm{Y}(\mathrm{N})$ & $\begin{array}{l}\text { Physical } \\
\text { therapist }\end{array}$ & $\begin{array}{l}\text { In-person; } \\
\text { Remote via } \\
\text { app or } \\
\text { telephone }\end{array}$ & $\begin{array}{l}\text { Acute inpatient } \\
\text { (postoperative); } \\
\text { Home }\end{array}$ \\
\hline
\end{tabular}




\begin{tabular}{|c|c|c|c|c|c|c|c|}
\hline \multirow[t]{3}{*}{$\begin{array}{l}\text { Study, Year, } \\
\text { PMID, } \\
\text { Country }\end{array}$} & $\begin{array}{l}\text { Intended } \\
\text { Comparison }\end{array}$ & Arm & Components (Specific Exercises/Strategies) & $\begin{array}{l}\text { Progression } \\
\text { (Appropriate?) }\end{array}$ & Personnel & $\begin{array}{l}\text { Mode of } \\
\text { Delivery }\end{array}$ & Setting \\
\hline & & & $\begin{array}{l}\text { 6. Patient education } \\
\text { 6.1 Activities of daily living }\end{array}$ & & & & \\
\hline & No data & $\begin{array}{l}\text { Conventional } \\
\text { outpatient } \\
\text { physical therapy }\end{array}$ & $\begin{array}{l}\text { 1. Strength } \\
\text { [specific exercises not defined] } \\
\text { 3. Flexibility } \\
\text { [specific exercises not defined] } \\
\text { 5. Task specific training } \\
\text { [specific exercises not defined] } \\
\text { 5.8 Gait training } \\
\text { 6. Patient education } \\
\text { 6.1 Activities of daily living }\end{array}$ & $\mathrm{N}(\mathrm{NA})$ & $\begin{array}{l}\text { Physical } \\
\text { therapist }\end{array}$ & In-person & $\begin{array}{l}\text { Acute inpatient } \\
\text { (postoperative); } \\
\text { Physical } \\
\text { therapy/rehabilitation } \\
\text { facility (outpatient) }\end{array}$ \\
\hline $\begin{array}{l}\text { Piva, } \\
2017, \\
28217891 \\
\text { USA }\end{array}$ & $\begin{array}{l}\text { Comprehensive } \\
\text { Behavioral } \\
\text { intervention (CBI) } \\
\text { that combines } \\
\text { intense exercises } \\
\text { with an education } \\
\text { program to promote } \\
\text { health and physical } \\
\text { activity vs. standard } \\
\text { of care exercise } \\
\text { program }\end{array}$ & $\begin{array}{l}\text { Comprehensive } \\
\text { behavioral } \\
\text { intervention }\end{array}$ & $\begin{array}{l}\text { 1. Strength } \\
\text { 1.11 Hip abduction in sidelying (position unclear) } \\
\text { 1.12 Hip abduction in standing (position unclear) } \\
\text { 1.13 Hip abduction in supine (position unclear) } \\
\text { 1.17 Hip extension in sidelying (position unclear) } \\
\text { 1.18 Hip extension in prone (position unclear) } \\
\text { 1.19 Hip extension in standing (position unclear) } \\
\text { 1.28 Knee extension machine (one-leg) (one or } \\
\text { two legs unclear) } \\
\text { 1.29 Knee extension machine (two-legs) (one or } \\
\text { two legs unclear) } \\
\text { 1.33 Knee flexion machine (Hamstring curl) one } \\
\text { knee (one or two legs unclear) } \\
\text { 1.34 Knee flexion machine (Hamstring curl) two } \\
\text { knees (one or two legs unclear) } \\
\text { 1.48 Sit-to-stand } \\
\text { 1.49 Squats } \\
\text { 1.50 Squats (one leg) } \\
\text { 2. Aerobic } \\
\text { 2.8 Treadmill walking } \\
\text { 3. Flexibility } \\
\text { 3.2 Bike (ROM) } \\
\text { 3.3 Calf stretch with knee bent (soleus) } \\
\text { 3.4 Calf stretch with knee straight (gastroc) } \\
\text { 3.5 Hamstring stretch in any position } \\
\text { 3.10 Knee extension AROM } \\
\text { 3.13 Knee flexion AROM } \\
\text { 3.16 Knee flexion PROM in prone (rectus femoris } \\
\text { stretch) } \\
\text { 4. Balance-Motor Learning-Agility } \\
\text { 4.3 Balance on unstable surface } \\
\text { 4.6 Marching } \\
\text { 4.10 Stepping multiple directions (grapevine) } \\
\text { 4.13 Step lateral (side step) } \\
\text { 4.17 Tandem walking } \\
\text { 5. Task specific training } \\
\text { 5.4 Gait backwards } \\
\text { 5.15 Stair training } \\
\text { 6. Patient education }\end{array}$ & $\mathrm{Y}(\mathrm{Y})$ & $\begin{array}{l}\text { Physical } \\
\text { therapist }\end{array}$ & In-person & $\begin{array}{l}\text { Physical } \\
\text { therapy/rehabilitation } \\
\text { facility (outpatient); } \\
\text { Home }\end{array}$ \\
\hline
\end{tabular}




\begin{tabular}{|c|c|c|c|c|c|c|c|}
\hline $\begin{array}{l}\text { Study, Year, } \\
\text { PMID, } \\
\text { Country }\end{array}$ & $\begin{array}{l}\text { Intended } \\
\text { Comparison }\end{array}$ & Arm & Components (Specific Exercises/Strategies) & $\begin{array}{l}\text { Progression } \\
\text { (Appropriate?) }\end{array}$ & Personnel & $\begin{array}{l}\text { Mode of } \\
\text { Delivery }\end{array}$ & Setting \\
\hline & & & $\begin{array}{l}\text { 6.2 Home exercise program } \\
6.3 \text { Life-style change }\end{array}$ & & & & \\
\hline & No data & $\begin{array}{l}\text { Standard care } \\
\text { exercise }\end{array}$ & $\begin{array}{l}\text { 1. Strength } \\
\text { 1.11 Hip abduction in sidelying (position unclear) } \\
1.12 \text { Hip abduction in standing (position unclear) } \\
\text { 1.13 Hip abduction in supine (position unclear) } \\
\text { 1.17 Hip extension in sidelying (position unclear) } \\
\text { 1.18 Hip extension in prone (position unclear) } \\
\text { 1.19 Hip extension in standing (position unclear) } \\
\text { 1.28 Knee extension machine (one-leg) (one or } \\
\text { two legs unclear) } \\
\text { 1.29 Knee extension machine (two-legs) (one or } \\
\text { two legs unclear) } \\
\text { 1.33 Knee flexion machine (Hamstring curl) one } \\
\text { knee (one or two legs unclear) } \\
\text { 1.34 Knee flexion machine (Hamstring curl) two } \\
\text { knees (one or two legs unclear) } \\
\text { 2. Aerobic } \\
\text { 2.8 Treadmill walking } \\
\text { 3. Flexibility } \\
\text { 3.2 Bike (ROM) } \\
\text { 3.3 Calf stretch with knee bent (soleus) } \\
\text { 3.4 Calf stretch with knee straight (gastroc) } \\
\text { 3.5 Hamstring stretch in any position } \\
\text { 3.10 Knee extension AROM } \\
\text { 3.13 Knee flexion AROM } \\
\text { 3.16 Knee flexion PROM in prone (rectus femoris } \\
\text { stretch) } \\
\text { 6. Patient education } \\
\text { 6.2 Home exercise program }\end{array}$ & $\mathrm{Y}(\mathrm{Y})$ & $\begin{array}{l}\text { Physical } \\
\text { therapist }\end{array}$ & In-person & $\begin{array}{l}\text { Physical } \\
\text { therapy/rehabilitation } \\
\text { facility (outpatient); } \\
\text { Home }\end{array}$ \\
\hline \multirow[t]{2}{*}{$\begin{array}{l}\text { Piva, } \\
2019, \\
30794296, \\
\text { USA }\end{array}$} & $\begin{array}{l}\text { Community-based } \\
\text { group exercise vs. } \\
\text { clinic-based } \\
\text { individual physical } \\
\text { therapy vs. usual } \\
\text { medical care }\end{array}$ & $\begin{array}{l}\text { Community- } \\
\text { based group } \\
\text { exercise }\end{array}$ & $\begin{array}{l}\text { 1. Strength } \\
\text { [specific exercises not defined] } \\
\text { 2. Aerobic } \\
\text { [specific exercises not defined] } \\
\text { 3. Flexibility } \\
\text { [specific exercises not defined] } \\
\text { 4. Balance-Motor Learning-Agility } \\
\text { [specific exercises not defined] } \\
\text { 5. Task specific training } \\
\text { [specific exercises not defined] }\end{array}$ & $\mathrm{N}(\mathrm{NA})$ & $\begin{array}{l}\text { Other (athletic } \\
\text { trainer) }\end{array}$ & In-person & $\begin{array}{l}\text { Gym or other } \\
\text { community center }\end{array}$ \\
\hline & No data & $\begin{array}{l}\text { Clinic-based } \\
\text { individual } \\
\text { physical therapy } \\
\text { exercise }\end{array}$ & $\begin{array}{l}\text { 1. Strength } \\
\text { 1.17 Hip extension in sidelying (position unclear) } \\
\text { 1.18 Hip extension in prone (position unclear) } \\
\text { 1.19 Hip extension in standing (position unclear) } \\
\text { 1.20 Hip flexion in sidelying (position unclear) } \\
\text { 1.21 Hip flexion in sitting (position unclear) } \\
\text { 1.22 Hip flexion in standing (position unclear) } \\
\text { 1.23 Hip flexion in supine (position unclear) } \\
\text { 1.30 Knee extension AAROM in sitting or supine } \\
\text { (short- or long arc quad) (position unclear) }\end{array}$ & $\mathrm{Y}(\mathrm{Y})$ & $\begin{array}{l}\text { Physical } \\
\text { therapist }\end{array}$ & In-person & $\begin{array}{l}\text { Physical } \\
\text { therapy/rehabilitation } \\
\text { facility (outpatient) }\end{array}$ \\
\hline
\end{tabular}




\begin{tabular}{|c|c|c|c|c|c|c|c|}
\hline $\begin{array}{l}\text { Study, Year, } \\
\text { PMID, } \\
\text { Country }\end{array}$ & $\begin{array}{l}\text { Intended } \\
\text { Comparison }\end{array}$ & Arm & Components (Specific Exercises/Strategies) & $\begin{array}{l}\text { Progression } \\
\text { (Appropriate?) }\end{array}$ & Personnel & $\begin{array}{l}\text { Mode of } \\
\text { Delivery }\end{array}$ & Setting \\
\hline & & & $\begin{array}{l}\text { 1.31 Knee extension in sitting or supine (long arc } \\
\text { quad) (position unclear) } \\
\text { 1.32 Knee extension in sitting or supine (short arc } \\
\text { quad) (position unclear) } \\
\text { 1.35 Knee flexion in prone (position unclear) } \\
\text { 1.36 Knee flexion in sitting or supine (position } \\
\text { unclear) } \\
\text { 1.37 Knee flexion in standing (position unclear) } \\
\text { 1.49 Squats } \\
\text { 2. Aerobic } \\
\text { 2.2 Bike (Endurance) } \\
\text { 2.8 Treadmill walking } \\
\text { 4. Balance-Motor Learning-Agility } \\
\text { [specific exercises not defined] } \\
\text { 5. Task specific training } \\
\text { 5.8 Gait training } \\
\text { 5.13 Sit-to-stand training } \\
\text { 5.15 Stair training } \\
\text { 6. Patient education } \\
\text { 6.2 Home exercise program }\end{array}$ & & & & \\
\hline & No data & Standard care & $\begin{array}{l}\text { NA } \\
\text { [Usual medical care with no interference from the } \\
\text { study; waitlist for intervention after data } \\
\text { collection] }\end{array}$ & $\mathrm{NA}$ & $\mathrm{NA}$ & $\mathrm{NA}$ & $\mathrm{NA}$ \\
\hline $\begin{array}{l}\text { Pua, } \\
2017, \\
27810379 \\
\text { Singapore }^{A}\end{array}$ & $\begin{array}{l}\text { Rehabilitation } \\
\text { attendance of } 2 \text { or } \\
\text { more sessions vs. } 1 \\
\text { session vs. } 0 \\
\text { sessions }\end{array}$ & $\begin{array}{l}\text { Rehabilitation } \\
\text { attendance ( } 2 \text { or } \\
\text { more sessions) }\end{array}$ & $\begin{array}{l}\text { 1. Strength } \\
\text { 1.28 Knee extension machine (one-leg) } \\
\text { 1.31 Knee extension in sitting or supine (long arc } \\
\text { quad) (long or short unclear) } \\
\text { 1.32 Knee extension in sitting or supine (short arc } \\
\text { quad) (long or short unclear) } \\
\text { 1.38 Leg Press (one leg) } \\
\text { 1.43 Quad sets } \\
\text { 1.48 Sit-to-stand } \\
\text { 1.52 Step down } \\
\text { 1.55 Step up - forward } \\
\text { 1.58 Straight leg raises } \\
\text { 1.62 Wall slides - Lateral (hip AB and ADductors) } \\
\text { 2. Aerobic } \\
\text { 2.7 Stepper (upright or sitting) } \\
\text { 3. Flexibility } \\
\text { 3.2 Bike (ROM) } \\
\text { 3.5 Hamstring stretch in any position } \\
\text { 3.11 Knee extension PROM in supine (seated) } \\
\text { 3.13 Knee flexion AROM (unclear) } \\
\text { 3.14 Knee flexion PROM in sitting or supine } \\
\text { (unclear) } \\
\text { 4. Balance-Motor Learning-Agility } \\
\text { 4.6 Marching } \\
\text { 4.8 Single leg stance } \\
\text { 4.13 Step lateral (side step) }\end{array}$ & $\mathrm{Y}(\mathrm{N})$ & $\begin{array}{l}\text { Physical } \\
\text { therapist }\end{array}$ & In-person & $\begin{array}{l}\text { Physical } \\
\text { therapy/rehabilitation } \\
\text { facility (outpatient) }\end{array}$ \\
\hline
\end{tabular}




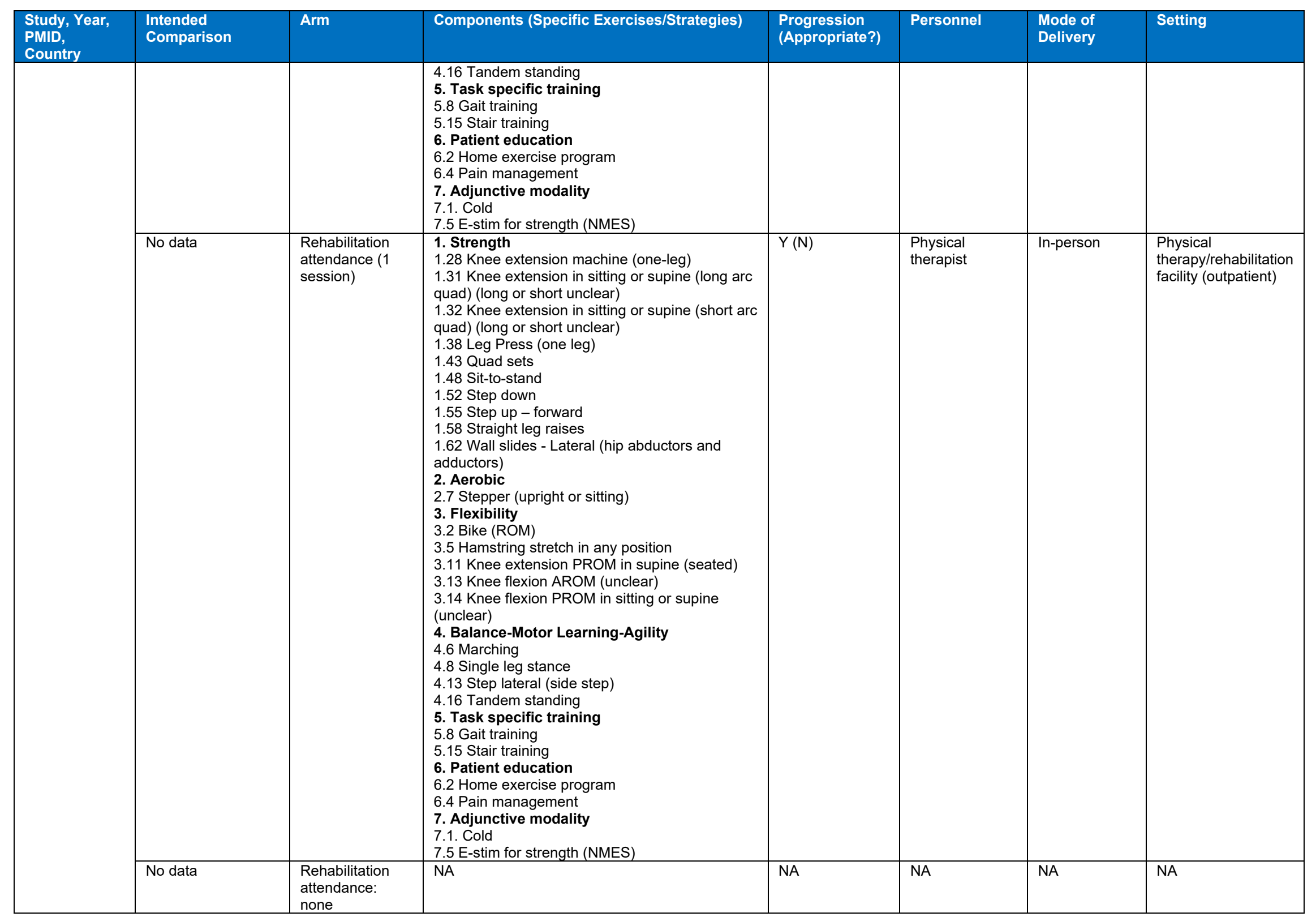




\begin{tabular}{|c|c|c|c|c|c|c|c|}
\hline $\begin{array}{l}\text { Study, Year, } \\
\text { PMID, } \\
\text { Country }\end{array}$ & $\begin{array}{l}\text { Intended } \\
\text { Comparison }\end{array}$ & Arm & Components (Specific Exercises/Strategies) & $\begin{array}{l}\text { Progression } \\
\text { (Appropriate?) }\end{array}$ & Personnel & $\begin{array}{l}\text { Mode of } \\
\text { Delivery }\end{array}$ & Setting \\
\hline \multirow[t]{2}{*}{$\begin{array}{l}\text { Rockstroh, } \\
2010, \\
20533147, \\
\text { Germany }\end{array}$} & $\begin{array}{l}\text { Microcurrent } \\
\text { therapy plus } \\
\text { conventional } \\
\text { postoperative } \\
\text { physiotherapy vs. } \\
\text { sham plus } \\
\text { conventional } \\
\text { postoperative } \\
\text { physiotherapy }\end{array}$ & $\begin{array}{l}\text { Physiotherapy \& } \\
\text { microcurrent }\end{array}$ & $\begin{array}{l}\text { 1. Strength } \\
\text { [specific exercises not defined] } \\
\text { 3. Flexibility } \\
\text { [specific exercises not defined] } \\
\text { 5. Task specific training } \\
\text { 5.8 Gait training } \\
\text { 7. Adjunctive modality } \\
\text { 7.4 E-stim for pain (TENS) }\end{array}$ & $N(N A)$ & Unclear & In-person & Acute Inpatient \\
\hline & No data & Physiotherapy & $\begin{array}{l}\text { 1. Strength } \\
\text { [specific exercises not defined] } \\
\text { 3. Flexibility } \\
\text { [specific exercises not defined] } \\
\text { 5. Task specific training } \\
\text { 5.8 Gait training }\end{array}$ & $\mathrm{N}(\mathrm{NA})$ & Unclear & In-person & Acute Inpatient \\
\hline \multirow[t]{2}{*}{$\begin{array}{l}\text { Sattler, } \\
2019, \\
30994586, \\
\text { Australia }\end{array}$} & $\begin{array}{l}\text { Pedaling-based } \\
\text { exercise protocol } \\
\text { vs. non-pedaling } \\
\text { (multi-exercise) } \\
\text { protocol }\end{array}$ & $\begin{array}{l}\text { Pedaling-based } \\
\text { protocol }\end{array}$ & $\begin{array}{l}\text { 3. Flexibility } \\
\text { 3.2 Bike (ROM) } \\
\text { 3.4 Calf stretch with knee straight (gastroc) } \\
\text { 3.5 Hamstring stretch in any position } \\
\text { 5. Task specific training } \\
\text { 5.8 Gait training }\end{array}$ & $\mathrm{N}(\mathrm{NA})$ & $\begin{array}{l}\text { Physical } \\
\text { therapist }\end{array}$ & In-person & Acute Inpatient \\
\hline & No data & $\begin{array}{l}\text { Non-pedaling } \\
\text { (multi-exercise] } \\
\text { protocol }\end{array}$ & $\begin{array}{l}\text { 1. Strength } \\
\text { 1.9 Heel raises - bilateral (calf raises) } \\
\text { 1.32 Knee extension in sitting or supine (short arc } \\
\text { quad) } \\
\text { 1.36 Knee flexion in sitting or supine } \\
\text { 1.43 Quad sets } \\
\text { 1.49 Squats } \\
\text { 1.58 Straight leg raises } \\
\text { 3. Flexibility } \\
\text { 3.1 Ankle pumps } \\
\text { 3.4 Calf stretch with knee straight (gastroc) } \\
\text { 3.6 Heel slides } \\
\text { 3.10 Knee extension AROM } \\
\text { 3.13 Knee flexion AROM } \\
\text { 5. Task specific training } \\
\text { 5.8 Gait training }\end{array}$ & $\mathrm{N}(\mathrm{NA})$ & $\begin{array}{l}\text { Physical } \\
\text { therapist }\end{array}$ & In-person & Acute Inpatient \\
\hline $\begin{array}{l}\text { Schache, } \\
2019, \\
31208916, \\
\text { Australia }\end{array}$ & $\begin{array}{l}\text { Standard } \\
\text { rehabilitation plus } \\
\text { hip abductor } \\
\text { strengthening vs. } \\
\text { standard } \\
\text { rehabilitation plus } \\
\text { general functional } \\
\text { exercise }\end{array}$ & $\begin{array}{l}\text { Standard } \\
\text { rehabilitation and } \\
\text { hip } \\
\text { strengthening } \\
\text { exercises }\end{array}$ & $\begin{array}{l}\text { 1. Strength } \\
\text { 1.9 Heel raises - bilateral (calf raises) } \\
\text { 1.11 Hip abduction in sidelying } \\
\text { 1.12 Hip abduction in standing } \\
\text { 1.18 Hip extension in prone } \\
\text { 1.24 Hip hikes in standing } \\
\text { 1.35 Knee flexion in prone } \\
\text { 1.37 Knee flexion in standing } \\
\text { 1.39 Leg Press (two legs) } \\
\text { 1.43 Quad sets } \\
\text { 1.49 Squats } \\
\text { 1.52 Step down }\end{array}$ & $\mathrm{Y}(\mathrm{Y})$ & $\begin{array}{l}\text { Physical } \\
\text { therapist }\end{array}$ & In-person & $\begin{array}{l}\text { Acute inpatient } \\
\text { (postoperative) (12 } \\
\text { days); Physical } \\
\text { therapy/rehabilitation } \\
\text { facility (outpatient) (6 } \\
\text { wks) }\end{array}$ \\
\hline
\end{tabular}




\begin{tabular}{|c|c|c|c|c|c|c|c|}
\hline $\begin{array}{l}\text { Study, Year, } \\
\text { PMID, } \\
\text { Country }\end{array}$ & $\begin{array}{l}\text { Intended } \\
\text { Comparison }\end{array}$ & Arm & Components (Specific Exercises/Strategies) & $\begin{array}{l}\text { Progression } \\
\text { (Appropriate?) }\end{array}$ & Personnel & $\begin{array}{l}\text { Mode of } \\
\text { Delivery }\end{array}$ & Setting \\
\hline & & & $\begin{array}{l}\text { 1.54 Step lateral } \\
\text { 1.55 Step up - forward } \\
\text { 2. Aerobic } \\
\text { 2.2 Bike (Endurance) } \\
\text { 3. Flexibility } \\
\text { 3.4 Calf stretch with knee straight (gastroc) } \\
\text { 3.7 Hip extensor stretch (knee to chest) } \\
\text { 3.11 Knee extension PROM in supine (sitting) } \\
\text { 3.12 Knee extension PROM in prone } \\
\text { 3.13 Knee flexion AROM (active or passive } \\
\text { unclear) } \\
\text { 3.14 Knee flexion PROM in sitting or supine } \\
\text { (active or passive unclear) } \\
\text { 4. Balance-Motor Learning-Agility } \\
\text { 4.6 Marching } \\
\text { 4.11 Step down } \\
\text { 4.14 Step up - forward } \\
\text { 5. Task specific training } \\
\text { 5.7 Gait sideways } \\
\text { 5.8 Gait training } \\
\text { 5.13 Sit-to-stand training } \\
\text { 6. Patient education } \\
\text { 6.2 Home exercise program } \\
\text { 7. Adjunctive modality } \\
\text { 7.10 Massage for edema control (goal unclear) } \\
\text { 7.11 Massage for scar mobility (goal unclear) } \\
\text { 7.12 Massage/myofascial techniques for soft } \\
\text { tissue (goal unclear) } \\
\text { 7.13 Mobilizations - Tibiofemoral (joint unclear) } \\
\text { 7.14 Mobilizations - Patellar (joint unclear) }\end{array}$ & & & & \\
\hline & No data & $\begin{array}{l}\text { Standard } \\
\text { rehabilitation } \\
\text { plus general } \\
\text { functional } \\
\text { exercise }\end{array}$ & $\begin{array}{l}\text { 1. Strength } \\
\text { 1.9 Heel raises - bilateral (calf raises) } \\
\text { 1.35 Knee flexion in prone } \\
\text { 1.37 Knee flexion in standing } \\
\text { 1.39 Leg Press (two legs) } \\
\text { 1.43 Quad sets } \\
\text { 1.49 Squats } \\
\text { 1.52 Step down } \\
\text { 1.55 Step up - forward } \\
\text { 2. Aerobic } \\
\text { 2.2 Bike (Endurance) } \\
\text { 3. Flexibility } \\
\text { 3.4 Calf stretch with knee straight (gastroc) } \\
\text { 3.7 Hip extensor stretch (knee to chest) } \\
\text { 3.11 Knee extension PROM in supine (sitting) } \\
\text { 3.12 Knee extension PROM in prone } \\
\text { 3.13 Knee flexion AROM (active or passive } \\
\text { unclear) } \\
\text { 3.14 Knee flexion PROM in sitting or supine } \\
\text { (active or passive unclear) } \\
\text { 4. Balance-Motor Learning-Agility }\end{array}$ & $\mathrm{Y}(\mathrm{Y})$ & $\begin{array}{l}\text { Physical } \\
\text { therapist }\end{array}$ & In-person & $\begin{array}{l}\text { Acute inpatient } \\
\text { (postoperative) (12 } \\
\text { days); Physical } \\
\text { therapy/rehabilitation } \\
\text { facility (outpatient) (6 } \\
\text { wks) }\end{array}$ \\
\hline
\end{tabular}




\begin{tabular}{|c|c|c|c|c|c|c|c|}
\hline $\begin{array}{l}\text { Study, Year, } \\
\text { PMID, } \\
\text { Country }\end{array}$ & $\begin{array}{l}\text { Intended } \\
\text { Comparison }\end{array}$ & Arm & Components (Specific Exercises/Strategies) & $\begin{array}{l}\text { Progression } \\
\text { (Appropriate?) }\end{array}$ & Personnel & $\begin{array}{l}\text { Mode of } \\
\text { Delivery }\end{array}$ & Setting \\
\hline & & & $\begin{array}{l}\text { 4.6 Marching } \\
\text { 4.11 Step down } \\
\text { 4.14 Step up - forward } \\
\text { 5. Task specific training } \\
\text { 5.8 Gait training } \\
\text { 5.13 Sit-to-stand training } \\
\text { 6. Patient education } \\
\text { 6.2 Home exercise program } \\
\text { 7. Adjunctive modality } \\
\text { 7.10 Massage for edema control (goal unclear) } \\
\text { 7.11 Massage for scar mobility (goal unclear) } \\
\text { 7.12 Massage/myofascial techniques for soft } \\
\text { tissue (goal unclear) } \\
\text { 7.13 Mobilizations - Tibiofemoral (joint unclear) } \\
\text { 7.14 Mobilizations - Patellar (joint unclear) }\end{array}$ & & & & \\
\hline \multirow[t]{2}{*}{$\begin{array}{l}\text { Shanb, } \\
\text { 2014, } \\
\text { CN-01041112, } \\
\text { Saudi Arabia }\end{array}$} & $\begin{array}{l}\text { Active exercise } \\
\text { training program } \\
\text { plus biofeedback } \\
\text { vs. active exercise } \\
\text { training program }\end{array}$ & $\begin{array}{l}\text { Active exercise } \\
\text { training program } \\
\& \text { biofeedback }\end{array}$ & $\begin{array}{l}\text { 1. Strength } \\
\text { 1.11 Hip abduction in sidelying } \\
\text { 1.30 Knee extension AAROM in sitting or supine } \\
\text { (short- or long arc quad) } \\
\text { 1.31 Knee extension in sitting or supine (long arc } \\
\text { quad) (long or short unclear) } \\
\text { 1.32 Knee extension in sitting or supine (short arc } \\
\text { quad) (long or short unclear) } \\
1.35 \text { Knee flexion in prone (position unclear) } \\
1.36 \text { Knee flexion in sitting or supine (position } \\
\text { unclear) } \\
1.37 \text { Knee flexion in standing (position unclear) } \\
1.51 \text { Standing terminal knee extension } \\
\text { 1.58 Straight leg raises } \\
\text { 1.63 Open chain ankle dorsiflexion/plantar } \\
\text { flexion/inversion/eversion } \\
\text { 7. Adjunctive modality } \\
\text { 7.14 Mobilizations - Patellar } \\
\text { 7.15 Biofeedback devices }\end{array}$ & $\mathrm{Y}(\mathrm{Y})$ & NR & In-person & $\begin{array}{l}\text { Physical } \\
\text { therapy/rehabilitation } \\
\text { facility (outpatient) }\end{array}$ \\
\hline & No data & $\begin{array}{l}\text { Active exercise } \\
\text { training program }\end{array}$ & $\begin{array}{l}\text { 1. Strength } \\
\text { 1.11 Hip abduction in sidelying } \\
\text { 1.30 Knee extension AAROM in sitting or supine } \\
\text { (short- or long arc quad) } \\
\text { 1.31 Knee extension in sitting or supine (long arc } \\
\text { quad) (long or short unclear) } \\
\text { 1.32 Knee extension in sitting or supine (short arc } \\
\text { quad) (long or short unclear) } \\
1.35 \text { Knee flexion in prone (position unclear) } \\
1.36 \text { Knee flexion in sitting or supine (position } \\
\text { unclear) } \\
1.37 \text { Knee flexion in standing (position unclear) } \\
\text { 1.51 Standing terminal knee extension } \\
\text { 1.58 Straight leg raises } \\
\text { 1.63 Open chain ankle dorsiflexion/plantar } \\
\text { flexion/inversion/eversion }\end{array}$ & $\mathrm{Y}(\mathrm{Y})$ & NR & In-person & $\begin{array}{l}\text { Physical } \\
\text { therapy/rehabilitation } \\
\text { facility (outpatient) }\end{array}$ \\
\hline
\end{tabular}




\begin{tabular}{|c|c|c|c|c|c|c|c|}
\hline $\begin{array}{l}\text { Study, Year, } \\
\text { PMID, } \\
\text { Country }\end{array}$ & $\begin{array}{l}\text { Intended } \\
\text { Comparison }\end{array}$ & Arm & Components (Specific Exercises/Strategies) & $\begin{array}{l}\text { Progression } \\
\text { (Appropriate?) }\end{array}$ & Personnel & $\begin{array}{l}\text { Mode of } \\
\text { Delivery }\end{array}$ & Setting \\
\hline & & & $\begin{array}{l}\text { 7. Adjunctive modality } \\
\text { 7.14 Mobilizations - Patellar }\end{array}$ & & & & \\
\hline $\begin{array}{l}\text { Stevens- } \\
\text { Lapsley, } \\
2012, \\
22095207, \\
\text { USA }\end{array}$ & $\begin{array}{l}\text { Early } \\
\text { neuromuscular } \\
\text { electrical stimulation } \\
\text { plus standard } \\
\text { rehabilitation vs. } \\
\text { standard } \\
\text { rehabilitation }\end{array}$ & $\begin{array}{l}\text { Standard } \\
\text { rehabilitation \& } \\
\text { NMES }\end{array}$ & $\begin{array}{l}\text { 1. Strength } \\
\text { 1.8 Gluteal Sets } \\
\text { 1.11 Hip abduction in sidelying } \\
\text { 1.13 Hip abduction in supine } \\
\text { 1.30 Knee extension AAROM in sitting or supine } \\
\text { (short- or long arc quad) } \\
\text { 1.31 Knee extension in sitting or supine (long arc } \\
\text { quad) } \\
\text { 1.32 Knee extension in sitting or supine (short arc } \\
\text { quad) } \\
\text { 1.37 Knee flexion in standing } \\
\text { 1.43 Quad sets } \\
\text { 1.49 Squats } \\
\text { 1.51 Standing terminal knee extension } \\
\text { 1.52 Step down } \\
\text { 1.55 Step up - forward } \\
\text { 1.58 Straight leg raises } \\
\text { 1.61 Wall slides } \\
\text { 2. Aerobic } \\
\text { 2.2 Bike (Endurance) } \\
\text { 3. Flexibility } \\
\text { 3.1 Ankle pumps } \\
\text { 3.2 Bike (ROM) } \\
\text { 3.6 Heel slides } \\
\text { 3.10 Knee extension AROM } \\
\text { 3.11 Knee extension PROM in supine (position } \\
\text { unclear) } \\
\text { 3.12 Knee extension PROM in prone (position } \\
\text { unclear) } \\
\text { 3.13 Knee flexion AROM } \\
\text { 3.14 Knee flexion PROM in sitting or supine } \\
\text { 3.17 Standing terminal knee extension } \\
\text { 4. Balance-Motor Learning-Agility } \\
\text { 4.8 Single leg stance } \\
\text { 4.11 Step down } \\
\text { 4.14 Step up - forward } \\
\text { 5. Task specific training } \\
\text { 5.1 Transfers } \\
\text { 5.8 Gait training } \\
\text { 5.13 Sit-to-stand training } \\
\text { 5.15 Stair training } \\
\text { 6. Patient education } \\
\text { 6.1 Activities of daily living } \\
\text { 6.2 Home exercise program } \\
\text { 7. Adjunctive modality } \\
\text { 7.1. Cold } \\
\text { 7.5 E-stim for strength (NMES) } \\
\text { 7.11 Massage for scar mobility } \\
\text { 7.13 Mobilizations - Tibiofemoral } \\
\text { (a) }\end{array}$ & $\mathrm{Y}(\mathrm{Y})$ & $\begin{array}{l}\text { Physical } \\
\text { therapist }\end{array}$ & $\begin{array}{l}\text { In-person; } \\
\text { Home }\end{array}$ & $\begin{array}{l}\text { Acute Inpatient; } \\
\text { Home; Physical } \\
\text { therapy/rehabilitation } \\
\text { facility (outpatient) }\end{array}$ \\
\hline
\end{tabular}




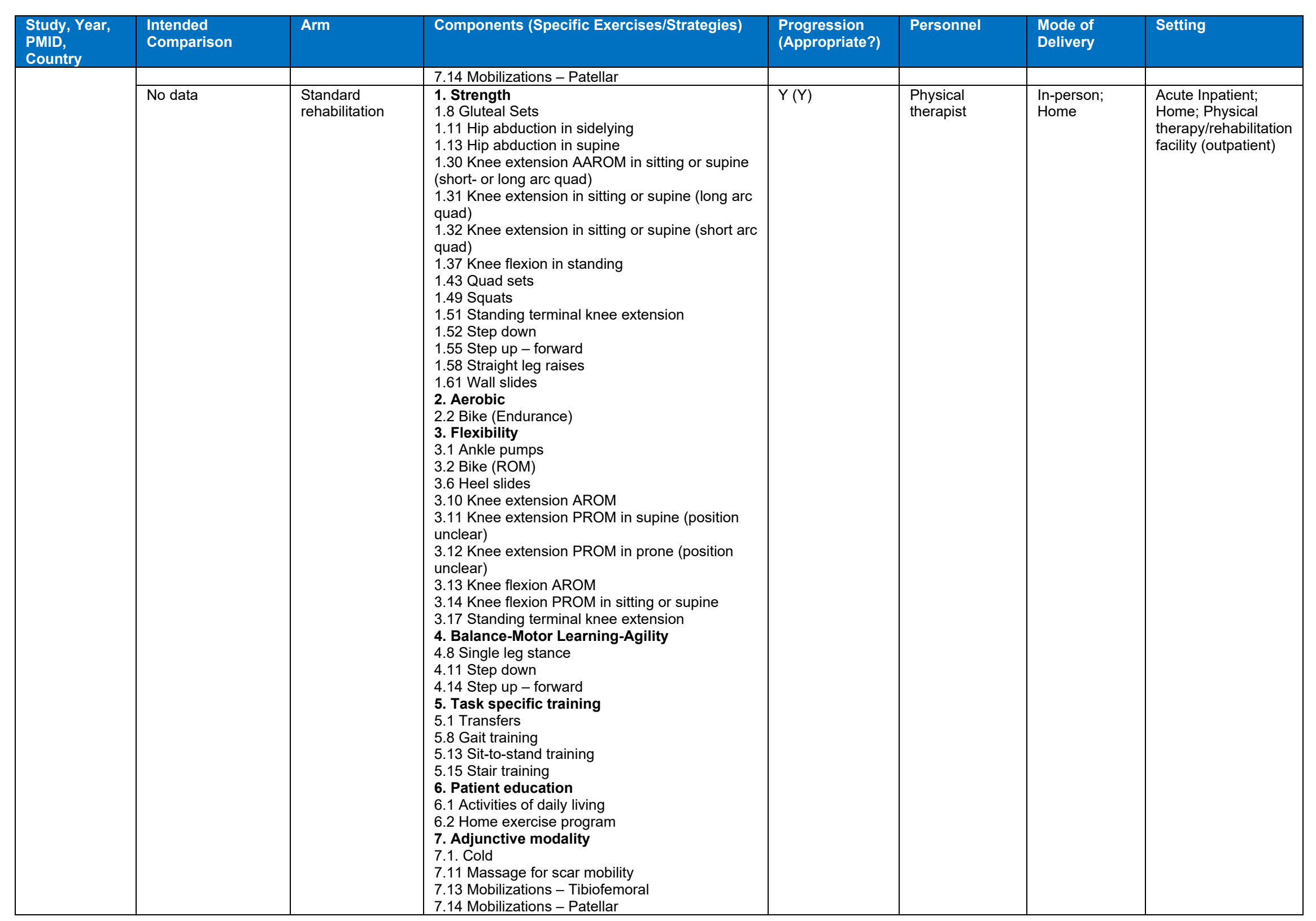




\begin{tabular}{|c|c|c|c|c|c|c|c|}
\hline $\begin{array}{l}\text { Study, Year, } \\
\text { PMID, } \\
\text { Country }\end{array}$ & $\begin{array}{l}\text { Intended } \\
\text { Comparison }\end{array}$ & Arm & Components (Specific Exercises/Strategies) & $\begin{array}{l}\text { Progression } \\
\text { (Appropriate?) }\end{array}$ & Personnel & $\begin{array}{l}\text { Mode of } \\
\text { Delivery }\end{array}$ & Setting \\
\hline \multirow[t]{2}{*}{$\begin{array}{l}\text { Tousignant, } \\
2011 \\
21398389 \\
\text { Canada }\end{array}$} & $\begin{array}{l}\text { Home } \\
\text { telerehabilitation vs. } \\
\text { conventional } \\
\text { rehabilitation }\end{array}$ & Telerehabilitation & $\begin{array}{l}\text { [specific goals and exercises not defined beyond } \\
\text { improving function and activities of daily living; } \\
\text { comparison of setting \& mode of delivery] } \\
\text { 6. Patient education } \\
\text { 6.1 Activities of daily living }\end{array}$ & $\mathrm{Y}(\mathrm{N})$ & $\begin{array}{l}\text { Physical } \\
\text { therapist }\end{array}$ & $\begin{array}{l}\text { Remote via } \\
\text { web }\end{array}$ & Home \\
\hline & No data & $\begin{array}{l}\text { Conventional } \\
\text { rehabilitation } \\
\text { (Home } \\
\text { care/outpatient } \\
\text { clinic) }\end{array}$ & $\begin{array}{l}\text { [specific goals and exercises not defined beyond } \\
\text { improving function and activities of daily living; } \\
\text { comparison of setting and mode of delivery] } \\
\text { 6. Patient education } \\
\text { 6.1 Activities of daily living }\end{array}$ & $\mathrm{Y}(\mathrm{N})$ & $\begin{array}{l}\text { Physical } \\
\text { therapist }\end{array}$ & In-person & $\begin{array}{l}\text { Physical } \\
\text { therapy/rehabilitation } \\
\text { facility (outpatient) }\end{array}$ \\
\hline $\begin{array}{l}\text { Tsukada, } \\
2020 \\
31723080 \\
\text { Japan }\end{array}$ & $\begin{array}{l}\text { Conventional } \\
\text { rehabilitation plus a } \\
\text { hybrid training } \\
\text { system vs. } \\
\text { conventional } \\
\text { rehabilitation alone }\end{array}$ & $\begin{array}{l}\text { Standard } \\
\text { rehabilitation \& } \\
\text { hybrid training } \\
\text { system }\end{array}$ & $\begin{array}{l}\text { 1. Strength } \\
\text { 1.8 Gluteal Sets } \\
\text { 1.13 Hip abduction in supine } \\
\text { 1.31 Knee extension in sitting or supine (long arc } \\
\text { quad) } \\
\text { 1.35 Knee flexion in prone (position unclear) } \\
\text { 1.36 Knee flexion in sitting or supine (position } \\
\text { unclear) } \\
\text { 1.37 Knee flexion in standing (position unclear) } \\
\text { 1.43 Quad sets } \\
\text { 1.58 Straight leg raises } \\
\text { 1.63 Open chain ankle dorsiflexion/plantar } \\
\text { flexion/inversion/eversion } \\
\text { 2. Aerobic } \\
\text { 2.2 Bike (Endurance) } \\
\text { 2.9 Walking } \\
\text { 3. Flexibility } \\
\text { 3.1 Ankle pumps } \\
\text { 3.2 Bike (ROM) } \\
\text { 3.6 Heel slides } \\
\text { 3.10 Knee extension AROM (active assisted) } \\
\text { 3.11 Knee extension PROM in supine (active } \\
\text { assisted) } \\
\text { 3.12 Knee extension PROM in prone (active } \\
\text { assisted) } \\
\text { 3.13 Knee flexion AROM (active assisted) } \\
\text { 3.14 Knee flexion PROM in sitting or supine } \\
\text { (active assisted) } \\
\text { 3.15 Knee flexion AROM in any position (rectus } \\
\text { femoris stretch) (active assisted) } \\
\text { 3.16 Knee flexion PROM in prone (rectus femoris } \\
\text { stretch) (active assisted) } \\
\text { 3.17 Standing terminal knee extension } \\
\text { 5. Task specific training } \\
\text { 5.1 Transfers } \\
\text { 5.8 Gait training } \\
\text { 5.15 Stair training } \\
\text { 6. Patient education } \\
\text { 6.1 Activities of daily living } \\
\text { 7. Adjunctive modality } \\
\end{array}$ & $\mathrm{Y}(\mathrm{N})$ & $\begin{array}{l}\text { Physical } \\
\text { therapist }\end{array}$ & In-person & Acute inpatient \\
\hline
\end{tabular}




\begin{tabular}{|c|c|c|c|c|c|c|c|}
\hline $\begin{array}{l}\text { Study, Year, } \\
\text { PMID, } \\
\text { Country }\end{array}$ & $\begin{array}{l}\text { Intended } \\
\text { Comparison }\end{array}$ & Arm & Components (Specific Exercises/Strategies) & $\begin{array}{l}\text { Progression } \\
\text { (Appropriate?) }\end{array}$ & Personnel & $\begin{array}{l}\text { Mode of } \\
\text { Delivery }\end{array}$ & Setting \\
\hline & & & $\begin{array}{l}\text { 7.1 Cold } \\
\text { 7.5 E-stim for strength (NMES) }\end{array}$ & & & & \\
\hline & No data & $\begin{array}{l}\text { Standard } \\
\text { rehabilitation }\end{array}$ & $\begin{array}{l}\text { 1. Strength } \\
\text { 1.8 Gluteal Sets } \\
\text { 1.13 Hip abduction in supine } \\
\text { 1.31 Knee extension in sitting or supine (long arc } \\
\text { quad) } \\
\text { 1.35 Knee flexion in prone (position unclear) } \\
\text { 1.36 Knee flexion in sitting or supine (position } \\
\text { unclear) } \\
\text { 1.37 Knee flexion in standing (position unclear) } \\
\text { 1.43 Quad sets } \\
\text { 1.58 Straight leg raises } \\
\text { 1.63 Open chain ankle dorsiflexion/plantar } \\
\text { flexion/inversion/eversion } \\
\text { 2. Aerobic } \\
\text { 2.2 Bike (Endurance) } \\
\text { 2.9 Walking } \\
\text { 3. Flexibility } \\
\text { 3.1 Ankle pumps } \\
\text { 3.2 Bike (ROM) } \\
\text { 3.6 Heel slides } \\
\text { 3.10 Knee extension AROM (active assisted) } \\
\text { 3.11 Knee extension PROM in supine (active } \\
\text { assisted) } \\
\text { 3.12 Knee extension PROM in prone (active } \\
\text { assisted) } \\
\text { 3.13 Knee flexion AROM (active assisted) } \\
\text { 3.14 Knee flexion PROM in sitting or supine } \\
\text { (active assisted) } \\
\text { 3.15 Knee flexion AROM in any position (rectus } \\
\text { femoris stretch) (active assisted) } \\
\text { 3.16 Knee flexion PROM in prone (rectus femoris } \\
\text { stretch) (active assisted) } \\
\text { 3.17 Standing terminal knee extension } \\
\text { 5. Task specific training } \\
\text { 5.1 Transfers } \\
\text { 5.8 Gait training } \\
\text { 5.15 Stair training } \\
\text { 6. Patient education } \\
\text { 6.1 Activities of daily living } \\
\text { 7. Adjunctive modality } \\
\text { 7.1 Cold }\end{array}$ & $\mathrm{Y}(\mathrm{N})$ & $\begin{array}{l}\text { Physical } \\
\text { therapist }\end{array}$ & In-person & Acute inpatient \\
\hline $\begin{array}{l}\text { Vuorenmaa, } \\
2014, \\
24241606, \\
\text { Finland }\end{array}$ & $\begin{array}{l}\text { Monitored home } \\
\text { exercise program } \\
\text { vs. normal care }\end{array}$ & Home exercise & $\begin{array}{l}\text { 1. Strength } \\
\text { 1.9 Heel raises - bilateral (calf raises) } \\
1.10 \text { Heel raises - unilateral } \\
1.31 \text { Knee extension in sitting or supine (long arc } \\
\text { quad) } \\
1.32 \text { Knee extension in sitting or supine (short arc } \\
\text { quad) }\end{array}$ & $\mathrm{Y}(\mathrm{N})$ & $\begin{array}{l}\text { Physical } \\
\text { therapist }\end{array}$ & $\begin{array}{l}\text { In-person; } \\
\text { Home }\end{array}$ & $\begin{array}{l}\text { Physical } \\
\text { therapy/rehabilitation } \\
\text { facility (outpatient); } \\
\text { Home }\end{array}$ \\
\hline
\end{tabular}




\begin{tabular}{|c|c|c|c|c|c|c|c|}
\hline $\begin{array}{l}\text { Study, Year, } \\
\text { PMID, } \\
\text { Country }\end{array}$ & $\begin{array}{l}\text { Intended } \\
\text { Comparison }\end{array}$ & Arm & Components (Specific Exercises/Strategies) & $\begin{array}{l}\text { Progression } \\
\text { (Appropriate?) }\end{array}$ & Personnel & $\begin{array}{l}\text { Mode of } \\
\text { Delivery }\end{array}$ & Setting \\
\hline & & & $\begin{array}{l}\text { 1.36 Knee flexion in sitting or supine } \\
\text { 1.48 Sit-to-stand } \\
\text { 1.49 Squats } \\
\text { 1.55 Step up - forward } \\
\text { 1.61 Wall slides } \\
\text { 3. Flexibility } \\
\text { 3.2 Bike (ROM) } \\
\text { 3.4 Calf stretch with knee straight (gastroc) } \\
\text { 3.5 Hamstring stretch in any position } \\
\text { 3.8 Hip flexor stretch (iliopsoas) } \\
\text { 3.16 Knee flexion PROM in prone (rectus femoris } \\
\text { stretch) } \\
\text { 6. Patient education } \\
\text { 6.2 Home exercise program }\end{array}$ & & & & \\
\hline & No data & Control & $\begin{array}{l}\text { [Normal care consisted of no additional guidance } \\
\text { after baseline measures] }\end{array}$ & $\mathrm{N}(\mathrm{NA})$ & None & NA & Home \\
\hline \multirow[t]{2}{*}{$\begin{array}{l}\text { Zapparoli, } \\
2020 \text {, } \\
32488010 \\
\text { Italy }\end{array}$} & $\begin{array}{l}\text { Motor imagery \& } \\
\text { rehabilitation vs. } \\
\text { rehabilitation }\end{array}$ & $\begin{array}{l}\text { Motor imagery \& } \\
\text { rehabilitation }\end{array}$ & $\begin{array}{l}\text { 1. Strength } \\
\text { 3. Flexibility } \\
\text { 3.10 Knee extension AROM } \\
\text { 3.11 Knee extension PROM in supine } \\
\text { 3.12 Knee extension PROM in prone } \\
\text { 3.13 Knee flexion AROM } \\
\text { 3.14 Knee flexion PROM in sitting or supine } \\
\text { 3.15 Knee flexion AROM in any position (rectus } \\
\text { femoris stretch) } \\
\text { 3.16 Knee flexion PROM in prone (rectus femoris } \\
\text { stretch) } \\
\text { 4. Balance-Motor Learning-Agility } \\
\text { 5. Task specific training } \\
\text { 5.8 Gait training } \\
\text { 7. Adjunctive modality } \\
\text { 7.17 Mindfulness, stress/anxiety-reduction } \\
\text { interventions }\end{array}$ & $\mathrm{N}(\mathrm{NA})$ & $\begin{array}{l}\text { Physical } \\
\text { therapist }\end{array}$ & In-person & Acute inpatient \\
\hline & No data & Rehabilitation & $\begin{array}{l}\text { 1. Strength } \\
\text { 3. Flexibility } \\
\text { 3.10 Knee extension AROM } \\
\text { 3.11 Knee extension PROM in supine } \\
\text { 3.12 Knee extension PROM in prone } \\
\text { 3.13 Knee flexion AROM } \\
\text { 3.14 Knee flexion PROM in sitting or supine } \\
\text { 3.15 Knee flexion AROM in any position (rectus } \\
\text { femoris stretch) } \\
\text { 3.16 Knee flexion PROM in prone (rectus femoris } \\
\text { stretch) } \\
\text { 4. Balance-Motor Learning-Agility } \\
\text { 5. Task specific training } \\
\text { 5.8 Gait training }\end{array}$ & $\mathrm{N}(\mathrm{NA})$ & $\begin{array}{l}\text { Physical } \\
\text { therapist }\end{array}$ & In-person & Acute inpatient \\
\hline
\end{tabular}

Non-randomized study 
Abbreviations: $\mathrm{AAROM}=$ assisted active range of motion, $\mathrm{ADL}=$ activities of daily living, $\mathrm{AROM}=$ active range of motion, $\mathrm{NA}=$ not applicable, $\mathrm{NMES}=$ neuromuscular electrical stimulation, $\mathrm{NR}=$ not reported, $\mathrm{PROM}=$ passive range of motion, $\mathrm{ROM}=$ range of motion, TENS $=$ transcutaneous electrical nerve stimulation, $\mathrm{TKA}=$ total knee arthroplasty. 
Table C-2.3. KQ 2. Risk of bias assessment for primary studies - randomized controlled trials (RCTs)

\begin{tabular}{|c|c|c|c|c|c|c|c|c|c|c|c|c|}
\hline Study, Year, PMID & Random & Allocation & $\begin{array}{l}\text { Blinding, } \\
\text { Participants }\end{array}$ & $\begin{array}{l}\text { Blinding, } \\
\text { Providers }\end{array}$ & $\begin{array}{l}\text { Blinding, Outcome, } \\
\text { Obj / Subj }\end{array}$ & Dropout & $\begin{array}{l}\text { Reporting } \\
\text { Bias }\end{array}$ & Other & Population & Intervention & Outcomes & Overall RoB \\
\hline $\begin{array}{l}\text { Andersen, 2018, No } \\
\text { PMID }\end{array}$ & Unsure & Unsure & High & High & Low & Unsure & Low & Low & No & No & No & High \\
\hline $\begin{array}{l}\text { Artz, 2017, } \\
27068368\end{array}$ & Low & Low & High & High & Unsure & High & Low & Low & No & No & No & High \\
\hline $\begin{array}{l}\text { Avramidis, 2011, } \\
21410130\end{array}$ & Low & Low & High & High & Low & Low & Unsure & Low & No & No & No & Moderate \\
\hline $\begin{array}{l}\text { Bade, 2017, } \\
27813347\end{array}$ & Low & Low & Low & High & Low & Low & Unsure & Low & No & No & No & Moderate \\
\hline $\begin{array}{l}\text { Bily, 2016, } \\
26763947\end{array}$ & Unsure & Low & High & High & High & Low & Low & Low & No & No & No & High \\
\hline $\begin{array}{l}\text { Bruun-Olsen, 2013, } \\
23614370\end{array}$ & Low & Low & High & Low & Low & Low & Low & Low & No & No & No & Moderate \\
\hline $\begin{array}{l}\text { Buhagiar, 2017, } \\
28291891\end{array}$ & Low & Low & High & High & Low & Low & Low & Low & No & No & No & Moderate \\
\hline Cai, 2018, 29239772 & Low & Low & High & Low & Low & Low & Low & Low & No & No & No & Moderate \\
\hline $\begin{array}{l}\text { DeJong, 2020, } \\
32360105\end{array}$ & Low & Unsure & High & High & Unsure & Low & Low & Low & No & No & No & High \\
\hline $\begin{array}{l}\text { Demircioglu, 2015, } \\
26355656\end{array}$ & High & Unsure & Unsure & Unsure & Unsure & Low & Low & Low & No & No & No & High \\
\hline $\begin{array}{l}\text { den, 2012, } \\
22643801 \\
\end{array}$ & Low & Low & High & High & Unsure & Low & Low & Low & No & No & No & Moderate \\
\hline $\begin{array}{l}\text { Eymir, 2020, } \\
32778907\end{array}$ & Low & High & High & High & Unsure & Low & Low & Low & No & No & No & High \\
\hline $\begin{array}{l}\text { Fransen, 2017, } \\
27868384\end{array}$ & Low & Low & High & Low & High & Low & Low & Low & No & No & No & Moderate \\
\hline $\begin{array}{l}\text { Hamilton, 2020, } \\
33051212\end{array}$ & Low & Low & High & High & High & Low & Low & Low & No & No & No & Moderate \\
\hline $\begin{array}{l}\text { Harmer, 2009, } \\
19177536\end{array}$ & Low & Low & High & High & Low & Low & Low & Low & No & No & No & Moderate \\
\hline $\begin{array}{l}\text { Heikkilä, 2017, } \\
28119232\end{array}$ & Low & Unsure & High & High & High & Low & Unsure & Low & No & No & Unsure & High \\
\hline $\begin{array}{l}\text { Iwakiri, 2020, } \\
32373475\end{array}$ & Low & Unsure & High & High & Low & Low & Low & Low & No & No & No & High \\
\hline $\begin{array}{l}\text { Jin, 2018, CN- } \\
01617489\end{array}$ & Low & Unsure & High & High & Unsure & Low & Low & Low & No & No & No & High \\
\hline $\begin{array}{l}\text { Kauppila, 2010, } \\
20354057\end{array}$ & Low & Low & High & High & High & Low & Low & Unsure & No & No & No & Moderate \\
\hline $\begin{array}{l}\text { Lenguerrand, 2020, } \\
31033232\end{array}$ & Low & Low & High & High & High & Low & Low & Low & No & No & No & Moderate \\
\hline $\begin{array}{l}\text { Lenssen, 2006, } \\
16942627\end{array}$ & Low & Low & High & Unsure & Low & Low & Low & Low & No & No & No & Moderate \\
\hline $\mathrm{Li}, 2014,23412304$ & Unsure & Unsure & High & High & Unsure & Low & Low & Low & No & No & No & High \\
\hline $\begin{array}{l}\text { Li, 2015, CN- } \\
01084888\end{array}$ & Unsure & Unsure & High & High & Unsure & Low & Low & Low & No & No & No & High \\
\hline
\end{tabular}




\begin{tabular}{|c|c|c|c|c|c|c|c|c|c|c|c|c|}
\hline Study, Year, PMID & Random & Allocation & $\begin{array}{l}\text { Blinding, } \\
\text { Participants }\end{array}$ & $\begin{array}{l}\text { Blinding, } \\
\text { Providers }\end{array}$ & $\begin{array}{l}\text { Blinding, Outcome, } \\
\text { Obj / Subj }\end{array}$ & Dropout & $\begin{array}{l}\text { Reporting } \\
\text { Bias }\end{array}$ & Other & Population & Intervention & Outcomes & Overall RoB \\
\hline $\mathrm{Li}, 2019,31003647$ & Low & Low & High & High & Unsure & Low & Low & Low & No & No & No & Moderate \\
\hline Li, 2017, No PMID & Low & Low & High & High & Low & Low & Low & Low & No & No & No & Moderate \\
\hline $\begin{array}{l}\text { Liao, } 2015 \\
25552523\end{array}$ & Low & Unsure & High & High & Unsure & Low & Low & Low & No & No & No & High \\
\hline $\begin{array}{l}\text { Liao, 2020, } \\
31687984\end{array}$ & Low & Low & High & High & Low & Low & Low & Low & No & No & No & Moderate \\
\hline $\begin{array}{l}\text { Liebs, 2010, } \\
20360503\end{array}$ & Low & Low & High & High & High & Low & Low & Low & No & No & No & Moderate \\
\hline $\begin{array}{l}\text { Liebs, 2012, } \\
22196125\end{array}$ & Low & Low & High & High & High & Low & Low & Low & No & No & No & Moderate \\
\hline $\begin{array}{l}\text { Madsen, 2013, } \\
23651717\end{array}$ & Unsure & Low & High & High & Unsure & Low & Low & Low & No & No & No & High \\
\hline $\begin{array}{l}\text { Minns Lowe, 2012, } \\
22180446\end{array}$ & Low & Low & High & High & Low & Low & Low & High & No & No & No & High \\
\hline $\begin{array}{l}\text { Mitchell, 2005, } \\
15869558\end{array}$ & Low & High & High & High & Unsure & Low & Low & Low & No & No & No & High \\
\hline $\begin{array}{l}\text { Moffet, 2015, } \\
26178888\end{array}$ & Low & Low & High & High & High & Low & Low & Low & No & No & No & Moderate \\
\hline $\begin{array}{l}\text { Monticone, 2013, } \\
23063624\end{array}$ & Low & Low & High & High & Low & Low & Low & Low & No & No & No & Moderate \\
\hline $\begin{array}{l}\text { Moutzouri, 2018, } \\
29473481\end{array}$ & Low & Low & High & High & Unsure & Low & Low & Low & No & No & No & Moderate \\
\hline $\begin{array}{l}\text { Petersen, 2018, } \\
29294078\end{array}$ & Low & Low & Unsure & Low & Low & Low & Low & Low & No & No & No & Moderate \\
\hline $\begin{array}{l}\text { Petterson, 2009, } \\
19177542\end{array}$ & Unsure & Unsure & High & High & Low & High & Low & Low & No & No & No & High \\
\hline $\begin{array}{l}\text { Piqueras, 2013, } \\
23474735\end{array}$ & Low & Low & High & High & Low & Low & Low & Low & No & No & No & Moderate \\
\hline $\begin{array}{l}\text { Piva, 2017, } \\
28217891\end{array}$ & Low & Low & Low & High & Low & Low & Low & Low & No & No & No & Moderate \\
\hline $\begin{array}{l}\text { Piva, 2019, } \\
30794296\end{array}$ & Low & Low & High & Low & Low & Low & Low & Low & No & No & No & Moderate \\
\hline $\begin{array}{l}\text { Rockstroh, 2010, } \\
20533147\end{array}$ & Low & Unsure & High & Unsure & High & Low & Unsure & Low & No & No & No & High \\
\hline $\begin{array}{l}\text { Sattler, 2019, } \\
30994586\end{array}$ & Low & Low & High & High & High & Low & Low & Low & No & No & No & Moderate \\
\hline $\begin{array}{l}\text { Schache, 2019, } \\
31208916\end{array}$ & Low & Low & High & Low & Low & Low & Low & Low & No & No & No & Moderate \\
\hline $\begin{array}{l}\text { Shanb, 2014, No } \\
\text { PMID }\end{array}$ & High & High & High & Low & High & Low & Low & Low & Yes & Yes & Yes & High \\
\hline $\begin{array}{l}\text { Stevens-Lapsley, } \\
2012,22095207\end{array}$ & Low & Low & High & High & High & Low & Low & Low & No & No & No & Moderate \\
\hline $\begin{array}{l}\text { Tousignant, 2011, } \\
21398389\end{array}$ & Low & Low & High & High & Unsure & Low & High & Low & Unsure & No & No & Moderate \\
\hline $\begin{array}{l}\text { Tsukada, 2020, } \\
31723080\end{array}$ & Low & Low & High & High & Unsure & Low & Low & Low & No & No & No & Moderate \\
\hline
\end{tabular}




\begin{tabular}{|c|c|c|c|c|c|c|c|c|c|c|c|c|}
\hline Study, Year, PMID & Random & Allocation & $\begin{array}{l}\text { Blinding, } \\
\text { Participants }\end{array}$ & $\begin{array}{l}\text { Blinding, } \\
\text { Providers }\end{array}$ & $\begin{array}{l}\text { Blinding, Outcome, } \\
\text { Obj / Subj }\end{array}$ & Dropout & $\begin{array}{l}\text { Reporting } \\
\text { Bias }\end{array}$ & Other & Population & Intervention & Outcomes & Overall RoB \\
\hline $\begin{array}{l}\text { Vuorenmaa, 2014, } \\
24241606\end{array}$ & Low & Low & High & High & Low & Low & Low & Low & No & No & No & Moderate \\
\hline $\begin{array}{l}\text { Zapparoli, 2020, } \\
32488010\end{array}$ & Low & Unsure & High & High & Low & Low & Low & Low & No & No & No & High \\
\hline
\end{tabular}

PMID = Obj = objective, PubMed Identifier, Subj = subjective

From the Cochrane Risk of Bias Tool (each item rated as Low, High, Unsure, or N/A). Ratings are color coded for emphasis only.

- Random: Random sequence generation (selection bias): Selection bias (biased allocation to interventions) due to inadequate generation of a randomized sequence;

- Allocation: Allocation concealment (selection bias): Selection bias (biased allocation to interventions) due to inadequate concealment of allocations prior to assignment;

- Blinding of participants (performance bias): Performance bias due to knowledge of the allocated interventions by participants during the study;

- Blinding of personnel/care providers (performance bias): Performance bias due to knowledge of the allocated interventions by personnel/care providers during the study;

- Blinding of outcome assessor (detection bias): Detection bias due to knowledge of the allocated interventions by outcome assessors;

- Dropout: Incomplete outcome data (attrition bias): Attrition bias due to amount, nature or handling of incomplete outcome data;

- Reporting Bias: Selective outcome reporting (outcome reporting bias): Bias arising from outcomes being selectively reported based on the direction and/or strength of the results;

- Other Bias: Bias due to problems not covered elsewhere in the table.

From the National Heart, Lung, and Blood Institute (NHLBI) Quality Assessment Tool (each item rated as No, Yes, or Unsure)

- Population: Eligibility criteria prespecified and clearly described: potentially related to selection bias;

- Intervention: Intervention clearly described and delivered consistently: potentially related to performance bias;

- Outcomes: Outcomes prespecified, clearly defined, valid, reliable, and assessed consistently: potentially related to detection bias.

Overall risk of bias assessed as HIGH, MODERATE, or LOW. 
Table C-2.4. KQ 2. Risk of bias assessment for primary studies - nonrandomized comparative studies (NRCSs) - assessment of confounding and selection bias

\begin{tabular}{|c|c|c|c|c|c|c|c|c|c|c|c|c|c|}
\hline Study, Year, PMID & 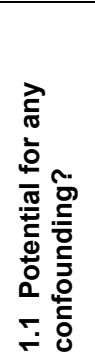 & 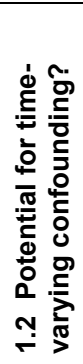 & 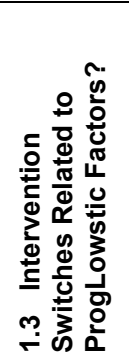 & 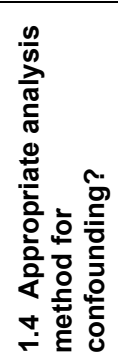 & 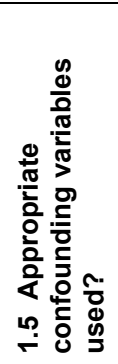 & 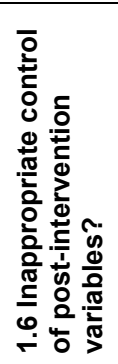 & 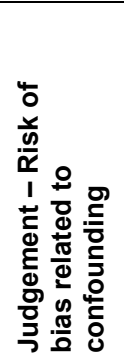 & 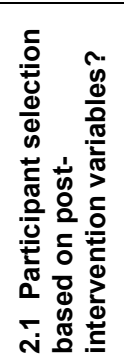 & 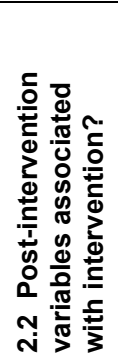 & 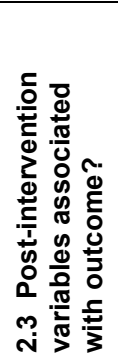 & 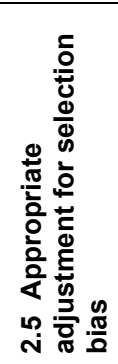 & 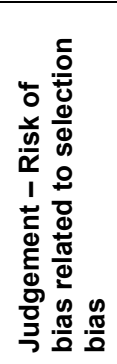 & $\begin{array}{l}\boldsymbol{m} \\
0 \\
\underline{\alpha} \\
\overline{\bar{\alpha}} \\
\overline{0} \\
\partial \\
0\end{array}$ \\
\hline $\begin{array}{l}\text { Chan, 2018, } \\
29372260\end{array}$ & Yes & No & No & Yes & Yes & No & Low & No & $\mathrm{N} / \mathrm{A}$ & $\mathrm{N} / \mathrm{A}$ & $\mathrm{N} / \mathrm{A}$ & Low & Moderate \\
\hline $\begin{array}{l}\text { Naylor, } 2017 \text {, } \\
28899328\end{array}$ & Yes & No & No & Yes & Yes & No & Low & No & $\mathrm{N} / \mathrm{A}$ & $\mathrm{N} / \mathrm{A}$ & $\mathrm{N} / \mathrm{A}$ & Low & Moderate \\
\hline $\begin{array}{l}\text { Padgett, 2018, } \\
29352683\end{array}$ & Yes & No & No & Yes & Yes & No & Low & No & $\mathrm{N} / \mathrm{A}$ & $\mathrm{N} / \mathrm{A}$ & $\mathrm{N} / \mathrm{A}$ & Low & Moderate \\
\hline Pua, 2017, 27810379 & Yes & No & No & Yes & Yes & No & Low & No & $\mathrm{N} / \mathrm{A}$ & $\mathrm{N} / \mathrm{A}$ & $\mathrm{N} / \mathrm{A}$ & Low & Moderate \\
\hline
\end{tabular}

- $\quad$ PMID = PubMed Identifier, Responses to Risk of Bias in Non-randomized Studies of Interventions (ROBINS-I) signaling questions 1.1 to 1.6 and 2.1 to 2.5 are in regular font. Each item rated as High, PY (probably High), NI (Low information), PN (probably Low), Low, or N/A (Not applicable).

- Judgments about confounding and selection bias are in bold font and each item is rated as Low, Moderate, Serious, or Critical. Ratings are color coded for emphasis only. 


\section{Key Question 3: Prehabilitation for Total Hip Arthroplasty}

Table C-3.1. KQ 3. Design details and arms

\begin{tabular}{|c|c|c|c|c|c|c|c|c|c|}
\hline $\begin{array}{l}\text { Study }{ }^{\mathrm{A}} \text {, Year, } \\
\text { PMID, } \\
\text { Country }\end{array}$ & $\begin{array}{l}\text { Funding } \\
\text { Source }\end{array}$ & $\begin{array}{l}\text { Overall } \\
\text { RoB }\end{array}$ & Eligibility Criteria & Intervention ${ }^{B}$ & $\begin{array}{l}\mathbf{N}, \\
\text { Enrolled }\end{array}$ & $\begin{array}{l}\text { Mean Age, } \\
\text { Years (SD) }\end{array}$ & $\begin{array}{l}\text { Female, } \\
\%\end{array}$ & $\begin{array}{l}\text { Mean } \\
\text { BMII }^{\mathrm{C}} \text { (SD) }\end{array}$ & $\begin{array}{l}\text { Prior } \\
\text { Contralateral } \\
\text { Arthroplasty }\end{array}$ \\
\hline \multirow[t]{2}{*}{$\begin{array}{l}\text { Bitterli, } \\
2011 \\
21630176 \\
\text { Switzerland }\end{array}$} & NR & Moderate & $\begin{array}{l}\text { INCLUSION: Unilateral arthrosis or femoral head } \\
\text { necrosis, first and unilateral THA. EXCLUSION: } \\
\text { Previous surgery on the affected joint, who had } \\
\text { been fitted with hip, knee or ankle joint prostheses, } \\
\text { for whom a double-sided TKA had been planned, } \\
\text { surgery }<3 \text { weeks, able to follow the training } \\
\text { program for }<15 \text { days, suffering from neurological } \\
\text { complaints. }\end{array}$ & $\begin{array}{l}\text { Pre-operative home exercise } \\
\text { sensorimotor training program } \\
\text { Comp: S-E } \\
\text { AdjMod: - } \\
\text { Set: H }\end{array}$ & 41 & $\begin{array}{l}65.4(10.8) \\
\text { Range (37, } \\
85)\end{array}$ & $46 \%$ & $\begin{array}{l}27.6(3.6) \\
\text { Range } \\
(20,40)\end{array}$ & $\mathrm{NR}$ \\
\hline & No data & No data & No data & $\begin{array}{l}\text { Control } \\
\text { Comp: - } \\
\text { AdjMod: - } \\
\text { Set: - }\end{array}$ & 39 & $\begin{array}{l}68.4(9.7) \\
\text { Range (40, } \\
86)\end{array}$ & $31 \%$ & $\begin{array}{l}27.1(3.6) \\
\text { Range } \\
(18,36)\end{array}$ & NR \\
\hline \multirow[t]{2}{*}{$\begin{array}{l}\text { Holsgaard- } \\
\text { Larsen, } \\
2020 \\
32376477 \\
\text { Denmark }\end{array}$} & NR & Moderate & $\begin{array}{l}\text { INCLUSION: } \geq 50 \text { yo, scheduled THA due to primary } \\
\text { OA. Minimum participation in } 80 \% \text { of training (no } \\
\text { more than } 2 \text { sequential skipped sessions. } \\
\text { EXCLUSION: Uremia, cancer, systemic treatment } \\
\text { with glucocorticoid }>3 \text { mo in the last } 5 \text { yrs with a daily } \\
\text { dose }>5 \mathrm{mg} \text {. Fracture of the hip (ipsi or contralateral). } \\
\text { Other fracture of the lower extremities within the last } \\
\text { year. Other condition with reduced function. }\end{array}$ & $\begin{array}{l}\text { Preoperative progressive } \\
\text { resistance training } \\
\text { Comp: S } \\
\text { AdjMod: - } \\
\text { Set: O }\end{array}$ & 40 & $70.0(7.7)$ & $27 \%$ & $28.2(5.3)$ & NR \\
\hline & No data & No data & No data & $\begin{array}{l}\text { Control } \\
\text { Comp: - } \\
\text { AdjMod: - } \\
\text { Set: - }\end{array}$ & 40 & $70.8(7.5)$ & $25 \%$ & $27.4(3.8)$ & NR \\
\hline \multirow[t]{2}{*}{$\begin{array}{l}\text { Pour, } \\
2007 \\
17768187 \\
\text { USA }\end{array}$} & Industry & High & $\begin{array}{l}\text { INCLUSION: } 18-75 \text { yo, diagnosis of OA. } \\
\text { EXCLUSION: BMI >30 kg/m², cognitive impairment } \\
\text { or severe psychiatric illness that would preclude } \\
\text { participation in the protocol-mandated procedures. }\end{array}$ & $\begin{array}{l}\text { Accelerated rehabilitation } \\
\text { Comp: T } \\
\text { AdjMod: - } \\
\text { Set: O } \\
\end{array}$ & 46 & $60.4(\mathrm{NR})^{*}$ & $48 \%$ & $25.5(\mathrm{NR})^{*}$ & NR \\
\hline & No data & No data & No data & $\begin{array}{l}\text { Standard rehabilitation } \\
\text { Comp: - } \\
\text { AdjMod: - } \\
\text { Set: - }\end{array}$ & 48 & $61.1(\mathrm{NR})^{\mathrm{D}}$ & $50 \%$ & $26.4(\mathrm{NR})^{\mathrm{d}}$ & NR \\
\hline $\begin{array}{l}\text { Rooks, } \\
2006 \\
17013852 \\
\text { USA }\end{array}$ & NR & Moderate & $\begin{array}{l}\text { INCLUSION: Unilateral, primary THA, advanced OA, } \\
\text { interval of 8-12 weeks between enrolment and } \\
\text { surgery. EXCLUSION: Inflammatory arthritis (i.e., } \\
\text { RA, systemic lupus erythematosus), Parkinsons } \\
\text { disease, or any medical condition in which a } \\
\text { moderate level of exercise was contraindicated (i.e., } \\
\text { uncontrolled diabetes or hypertension), bilateral joint } \\
\text { replacement or an extended out-of-town vacation } \\
\text { during the } 6 \text { wks prior to surgery. }\end{array}$ & $\begin{array}{l}\text { Preoperative exercise } \\
\text { Comp: S-A-F } \\
\text { AdjMod: - } \\
\text { Set: Gym or other community } \\
\text { center }\end{array}$ & 32 & $65(11)$ & $63 \%$ & $28.4(5.3)$ & NR \\
\hline
\end{tabular}




\begin{tabular}{|c|c|c|c|c|c|c|c|c|c|}
\hline $\begin{array}{l}\text { Study }{ }^{A}, \text { Year, } \\
\text { PMID, } \\
\text { Country }\end{array}$ & $\begin{array}{l}\text { Funding } \\
\text { Source }\end{array}$ & $\begin{array}{l}\text { Overall } \\
\text { RoB }\end{array}$ & Eligibility Criteria & Intervention ${ }^{B}$ & $\begin{array}{l}\mathbf{N}, \\
\text { Enrolled }\end{array}$ & $\begin{array}{l}\text { Mean Age, } \\
\text { Years (SD) }\end{array}$ & $\begin{array}{l}\text { Female, } \\
\%\end{array}$ & $\begin{array}{l}\text { Mean } \\
\text { BMIC (SD) }\end{array}$ & $\begin{array}{l}\text { Prior } \\
\text { Contralateral } \\
\text { Arthroplasty }\end{array}$ \\
\hline & No data & No data & No data & $\begin{array}{l}\text { Preoperative education } \\
\text { Comp: - } \\
\text { AdjMod: - } \\
\text { Set: - }\end{array}$ & 31 & $59(7)$ & $52 \%$ & $30.3(9.1)$ & NR \\
\hline \multirow[t]{2}{*}{$\begin{array}{l}\text { Soeters, } \\
2018 \\
29529614 \\
\text { USA }\end{array}$} & NR & Moderate & $\begin{array}{l}\text { INCLUSION: 18-85yo, scheduled unilateral TJA } \\
\text { (THA or TKA), able to independently ambulate a half } \\
\text { a block or more with or without an assistive device, } \\
\text { able to independently perform nonreciprocal stairs } \\
\text { with or without assistive devices, and planned to be } \\
\text { discharged home after surgery. EXCLUSION: } \\
\text { Patients who did not undergo scheduled surgery, } \\
\text { underwent a procedure other than primary TJA, or } \\
\text { were discharged to inpatient rehabilitation centers. }\end{array}$ & $\begin{array}{l}\text { Preoperative physical therapy } \\
\text { (PreopPTEd) } \\
\text { Comp: T-E } \\
\text { AdjMod: - } \\
\text { Set: NR }\end{array}$ & 31 & $\begin{array}{l}61(9) ; \\
\text { Range (37- } \\
98)^{\mathrm{E}}\end{array}$ & $44 \%$ & $\begin{array}{l}29(6) ; \\
\text { Range } \\
(19-46)\end{array}$ & NR \\
\hline & No data & No data & No data & $\begin{array}{l}\text { No preoperative physical } \\
\text { therapy education } \\
\text { Comp: - } \\
\text { AdjMod: - } \\
\text { Set: - }\end{array}$ & 32 & $\begin{array}{l}62(8) ; \\
\text { Range (45- } \\
85)\end{array}$ & $29 \%$ & $\begin{array}{l}29(6) ; \\
\text { Range } \\
(17-48)\end{array}$ & NR \\
\hline \multirow[t]{2}{*}{$\begin{array}{l}\text { Vukomanović, } \\
2008, \\
18499950, \\
\text { Serbia }\end{array}$} & NR & High & $\begin{array}{l}\text { INCLUSION: <70yo, primary and secondary OA, } \\
\text { ability to walk up and down stairs, no need for using } \\
\text { crutches while walking, no experience in walking } \\
\text { with crutches (because of opposite hip arthroplasty } \\
\text { or some other reasons), no coexisting morbidity } \\
\text { such as a history of severe cardiovascular, } \\
\text { respiratory, neuromuscular, rheumatic disease or } \\
\text { mental confusion. EXCLUSION: intraoperative } \\
\text { (femoral or acetabular fracture), postoperative } \\
\text { complications (postoperative disorientation, anemia, } \\
\text { circulatory collapse, orthostatic hypotension, chest } \\
\text { pain, sustained hypertension, deep venous } \\
\text { thrombosis, pulmonary embolism, hip dislocation) } \\
\text { which compromised or delayed the beginning of } \\
\text { physical therapy after the operation }\end{array}$ & $\begin{array}{l}\text { Short-term preoperative } \\
\text { physical therapy and education } \\
\text { Comp: T-E } \\
\text { AdjMod: - } \\
\text { Set: NR }\end{array}$ & 23 & $\begin{array}{l}60.1(11.0) \text {; } \\
\text { Range (30- } \\
70)\end{array}$ & $70 \%$ & NR & NR \\
\hline & No data & No data & No data & $\begin{array}{l}\text { Control } \\
\text { Comp: - } \\
\text { AdjMod: - } \\
\text { Set: - }\end{array}$ & 22 & $\begin{array}{l}56.2(18.5) \text {; } \\
\text { Range (19- } \\
70)\end{array}$ & $80 \%$ & NR & NR \\
\hline
\end{tabular}

Abbreviations: $\mathrm{BMI}=\mathrm{H}=$ home, $\mathrm{NA}=$ not applicable, $\mathrm{NMES}=$ neuromuscular electrical stimulation, $\mathrm{NR}=$ not reported, $\mathrm{O}=$ outpatient physiotherapy center, $\mathrm{OA}=$ osteoarthritis, $\mathrm{PMID}=$ PubMed identifier, RA = rheumatoid arthritis, $\mathrm{RoB}=$ risk of bias, $\mathrm{SD}=$ standard deviation, $\mathrm{SD}=$ standard deviation, TENS $=$ transcutaneous electrical nerve stimulation, THA = total hip arthroplasty, TJA = total joint arthroplasty, TKA $=$ total knee arthroplasty, yo = years old.

Components: $\mathrm{A}=$ aerobic exercise, $\mathrm{B}=$ Balance-motor/Learning-agility exercise, $\mathrm{E}=$ patient education, $\mathrm{F}=$ flexibility exercise, $\mathrm{S}=$ strengthening exercise, $\mathrm{T}=$ task-specific training.

A All randomized controlled trials, except as footnoted.

B Including Components (Comp)); Adjunctive modalities (AdjMod); and Setting (Set).

C $\mathrm{kg} / \mathrm{m}^{2}$ 
D Calculated

Reported age, gender, BMI data for total joint replacement population (TKA and THA) combined 
Table C-3.2. KQ 3. Prehabilitation component details

\begin{tabular}{|c|c|c|c|c|c|c|c|}
\hline $\begin{array}{l}\text { Study, Year, } \\
\text { PMID }\end{array}$ & $\begin{array}{l}\text { Intended } \\
\text { Comparison }\end{array}$ & Arm & Components (Specific Exercises/Strategies) & $\begin{array}{l}\text { Progression } \\
\text { (Appropriate?) }\end{array}$ & Personnel & $\begin{array}{l}\text { Mode of } \\
\text { Delivery }\end{array}$ & Setting \\
\hline \multirow[t]{2}{*}{$\begin{array}{l}\text { Bitterli, } \\
2011 \\
21630176 \\
\text { Switzerland }\end{array}$} & $\begin{array}{l}\text { Pre-operative } \\
\text { sensorimotor training } \\
\text { vs. no therapy }\end{array}$ & $\begin{array}{l}\text { Pre-operative } \\
\text { home exercise } \\
\text { sensorimotor } \\
\text { training program }\end{array}$ & $\begin{array}{l}\text { 1. Strength } \\
\text { 1.2 Bridges two-legged (supine hip extension) } \\
\text { 1.8 Gluteal Sets } \\
\text { 1.12 Hip abduction in standing } \\
\text { 1.13 Hip abduction in supine } \\
\text { 1.36 Knee flexion in sitting or supine } \\
\text { 1.43 Quad sets } \\
\text { 1.49 Squats } \\
\text { 6. Patient education } \\
\text { 6.2 Home exercise program }\end{array}$ & $N(N A)$ & $\begin{array}{l}\text { NR (who delivered the } \\
\text { instruction for the home } \\
\text { exercise program); None } \\
\text { (unsupervised) }\end{array}$ & $\begin{array}{l}\text { In-person; Self- } \\
\text { guided } \\
\text { (unsupervised) }\end{array}$ & Home \\
\hline & No data & Control & $\begin{array}{l}\text { NA } \\
\text { [No therapy] }\end{array}$ & NA & NA & NA & NA \\
\hline \multirow[t]{2}{*}{$\begin{array}{l}\text { Holsgaard- } \\
\text { Larsen, } \\
2020, \\
32376477 \\
\text { Denmark }\end{array}$} & $\begin{array}{l}\text { Preoperative } \\
\text { explosive type } \\
\text { progressive } \\
\text { resistance training vs. } \\
\text { care as usual }\end{array}$ & $\begin{array}{l}\text { Preoperative } \\
\text { progressive } \\
\text { resistance } \\
\text { training }\end{array}$ & $\begin{array}{l}\text { 1. Strength } \\
\text { 1.19 Hip extension in standing } \\
1.28 \text { Knee extension machine (one-leg) } \\
1.33 \text { Knee flexion machine (Hamstring curl) one } \\
\text { knee } \\
1.38 \text { Leg press (one leg) }\end{array}$ & $\mathrm{Y}(\mathrm{Y})$ & Physical therapist & In-person & $\begin{array}{l}\text { Physical } \\
\text { therapy/rehabilitation } \\
\text { facility (outpatient) }\end{array}$ \\
\hline & No data & Control & $\begin{array}{l}\text { NA } \\
\text { [Care as usual including no formal exercise] }\end{array}$ & NA & NA & NA & NA \\
\hline \multirow[t]{2}{*}{$\begin{array}{l}\text { Pour, } \\
2007 \\
17768187 \\
\text { USA }\end{array}$} & $\begin{array}{l}\text { Accelerated } \\
\text { preoperative protocol } \\
\text { vs. standard } \\
\text { preoperative protocol }\end{array}$ & $\begin{array}{l}\text { Accelerated } \\
\text { rehabilitation }\end{array}$ & $\begin{array}{l}\text { 5. Task specific training } \\
5.8 \text { Gait training }\end{array}$ & $N(N A)$ & Physical therapist & In-person & $\begin{array}{l}\text { Physical } \\
\text { therapy/rehabilitation } \\
\text { facility (outpatient) }\end{array}$ \\
\hline & No data & $\begin{array}{l}\text { Standard } \\
\text { rehabilitation }\end{array}$ & $\begin{array}{l}\text { NA } \\
\text { [No preoperative rehabilitation; standard pre- } \\
\text { operative appointment to discuss procedure and } \\
\text { post-operative rehabilitation expectations] }\end{array}$ & NA & NA & NA & NA \\
\hline
\end{tabular}




\begin{tabular}{|c|c|c|c|c|c|c|c|}
\hline $\begin{array}{l}\text { Study, Year, } \\
\text { PMID }\end{array}$ & $\begin{array}{l}\text { Intended } \\
\text { Comparison }\end{array}$ & Arm & Components (Specific Exercises/Strategies) & $\begin{array}{l}\text { Progression } \\
\text { (Appropriate?) }\end{array}$ & Personnel & $\begin{array}{l}\text { Mode of } \\
\text { Delivery }\end{array}$ & Setting \\
\hline \multirow[t]{2}{*}{$\begin{array}{l}\text { Rooks, } \\
2006 \\
17013852 \\
\text { USA }\end{array}$} & $\begin{array}{l}\text { Short preoperative } \\
\text { rehabilitation } \\
\text { intervention vs. } \\
\text { Education } \\
\text { intervention }\end{array}$ & $\begin{array}{l}\text { Preoperative } \\
\text { exercise }\end{array}$ & $\begin{array}{l}\text { 1. Strength } \\
\text { 1.6 Core strengthening } \\
\text { 1.38 Leg Press (one leg) } \\
\text { 1.39 Leg Press (two legs) } \\
\text { 1.60 Upper extremity strengthening } \\
\text { 2. Aerobic } \\
\text { 2.2 Bike (Endurance) } \\
\text { 2.3 Elliptical machine } \\
\text { 3. Flexibility } \\
\text { 3.1 Ankle pumps } \\
\text { 3.3 Calf stretch with knee bent (soleus) } \\
\text { 3.4 Calf stretch with knee straight (gastroc) } \\
\text { 3.7 Hip extensor stretch (knee to chest) } \\
\text { 3.8 Hip flexor stretch (iliopsoas) } \\
\text { 3.10 Knee extension AROM (unclear) } \\
\text { 3.11 Knee extension PROM in supine (unclear) } \\
\text { 3.12 Knee extension PROM in prone (unclear) } \\
\text { 3.13 Knee flexion AROM (unclear) } \\
\text { 3.14 Knee flexion PROM in sitting or supine } \\
\text { (unclear) } \\
\text { 3.15 Knee flexion AROM in any position (rectus } \\
\text { femoris stretch) (unclear) } \\
\text { 3.16 Knee flexion PROM in prone (rectus femoris } \\
\text { stretch) (unclear) }\end{array}$ & $\mathrm{Y}(\mathrm{N})$ & Physical therapist & In-person & $\begin{array}{l}\text { Gym or other } \\
\text { community center }\end{array}$ \\
\hline & No data & $\begin{array}{l}\text { Preoperative } \\
\text { education }\end{array}$ & $\begin{array}{l}\text { NA } \\
\text { [handout about home modifications and } \\
\text { preparation for surgery and hospital stay, no } \\
\text { advice on exercise; attention control] }\end{array}$ & NA & NA & NA & NA \\
\hline \multirow[t]{2}{*}{$\begin{array}{l}\text { Soeters, } \\
2018 \\
29529614 \\
\text { USA }\end{array}$} & $\begin{array}{l}\text { One-time } \\
\text { preoperative physical } \\
\text { therapy education } \\
\text { session with practice } \\
\text { plus microsite vs. no } \\
\text { preoperative physical } \\
\text { therapy education }\end{array}$ & $\begin{array}{l}\text { Preoperative } \\
\text { physical therapy } \\
\text { education } \\
\text { (PreopPTEd) }\end{array}$ & $\begin{array}{l}\text { 5. Task specific training } \\
\text { 5.1 Transfers } \\
\text { 5.13 Sit-to-stand training } \\
\text { 5.15 Stair training } \\
\text { 6. Patient education } \\
\text { 6.1 ADLs }\end{array}$ & NR & Physical therapist & In-person & $\begin{array}{l}\text { NR (Unclear if home } \\
\text { or outpatient) }\end{array}$ \\
\hline & No data & $\begin{array}{l}\text { No preoperative } \\
\text { physical therapy } \\
\text { education }\end{array}$ & $\begin{array}{l}\text { NA } \\
\text { [One group education class regarding surgery } \\
\text { specific information] }\end{array}$ & NA & NA & NA & NA \\
\hline \multirow[t]{2}{*}{$\begin{array}{l}\text { Vukomanović, } \\
2008, \\
18499950, \\
\text { Serbia }\end{array}$} & $\begin{array}{l}\text { Preoperative physical } \\
\text { therapy and } \\
\text { education vs. no } \\
\text { physical therapy or } \\
\text { education }\end{array}$ & $\begin{array}{l}\text { Short-term } \\
\text { preoperative } \\
\text { physical therapy } \\
\text { and education }\end{array}$ & $\begin{array}{l}\text { 5. Task specific training } \\
\text { 5.1 Transfers } \\
\text { 5.8 Gait training } \\
\text { 5.13 Sit-to-stand training } \\
\text { 5.15 Stair training } \\
\text { 6. Patient education } \\
\text { 6.1 ADLs } \\
\text { 6.2 Home exercise program } \\
\end{array}$ & $\mathrm{N}(\mathrm{NA})$ & Physical therapist & In-person & NR \\
\hline & No data & Control & [No additional care] & NA & NA & NA & NA \\
\hline
\end{tabular}

Abbreviations: $\mathrm{ADL}=$ activities of daily living, $\mathrm{AROM}=$ active range of motion, $\mathrm{NA}=$ not applicable, $\mathrm{NMES}=$ neuromuscular electrical stimulation, $\mathrm{NR}=$ not reported, $\mathrm{PROM}=$ passive range of motion, $\mathrm{ROM}=$ range of motion, TENS $=$ transcutaneous electrical nerve stimulation. 
Table C-3.3. KQ 3. Risk of bias assessment for primary studies - randomized controlled trials (RCTs)

\begin{tabular}{|c|c|c|c|c|c|c|c|c|c|c|c|c|}
\hline Study, Year, PMID & Random & Allocation & $\begin{array}{l}\text { Blinding, } \\
\text { Participants }\end{array}$ & $\begin{array}{l}\text { Blinding, } \\
\text { Providers }\end{array}$ & $\begin{array}{l}\text { Blinding, Outcome, } \\
\text { Obj / Subj }\end{array}$ & Dropout & $\begin{array}{l}\text { Reporting } \\
\text { Bias }\end{array}$ & Other & Population & Intervention & Outcomes & Overall \\
\hline $\begin{array}{l}\text { Bitterli, 2011, } \\
21630176\end{array}$ & Low & Low & High & Low & Low & Low & High & Low & No & No & No & Moderate \\
\hline $\begin{array}{l}\text { Holsgaard-Larsen, } \\
2020,32376477\end{array}$ & Low & Low & High & High & High & Low & Low & Low & No & No & No & Moderate \\
\hline $\begin{array}{l}\text { Pour, 2007, } \\
17768187\end{array}$ & Unsure & Unsure & High & High & Low & Low & Low & Low & No & No & No & High \\
\hline $\begin{array}{l}\text { Rooks, } 2006, \\
17013852 \\
\end{array}$ & Low & Unsure & High & Low & Unsure & Low & Low & Low & No & No & No & Moderate \\
\hline $\begin{array}{l}\text { Soeters, 2018, } \\
29529614\end{array}$ & Low & Low & High & Low & Low & Low & Low & Low & No & No & No & Moderate \\
\hline $\begin{array}{l}\text { Vukomanović, 2008, } \\
18499950\end{array}$ & Unsure & Unsure & High & High & Unsure & Low & Low & Low & No & No & No & High \\
\hline
\end{tabular}

PMID $=$ Obj $=$ objective, PubMed Identifier, Subj $=$ subjective

From the Cochrane Risk of Bias Tool (each item rated as Low, High, Unsure, or N/A). Ratings are color coded for emphasis only.

- Random: Random sequence generation (selection bias): Selection bias (biased allocation to interventions) due to inadequate generation of a randomized sequence;

- Allocation: Allocation concealment (selection bias): Selection bias (biased allocation to interventions) due to inadequate concealment of allocations prior to assignment;

- Blinding of participants (performance bias): Performance bias due to knowledge of the allocated interventions by participants during the study;

- Blinding of personnel/care providers (performance bias): Performance bias due to knowledge of the allocated interventions by personnel/care providers during the study;

- Blinding of outcome assessor (detection bias): Detection bias due to knowledge of the allocated interventions by outcome assessors;

- Dropout: Incomplete outcome data (attrition bias): Attrition bias due to amount, nature or handling of incomplete outcome data;

- Reporting Bias: Selective outcome reporting (outcome reporting bias): Bias arising from outcomes being selectively reported based on the direction and/or strength of the results;

- Other Bias: Bias due to problems not covered elsewhere in the table.

From the National Heart, Lung, and Blood Institute (NHLBI) Quality Assessment Tool (each item rated as No, Yes, or Unsure)

- Population: Eligibility criteria prespecified and clearly described: potentially related to selection bias;

- Intervention: Intervention clearly described and delivered consistently: potentially related to performance bias;

- Outcomes: Outcomes prespecified, clearly defined, valid, reliable, and assessed consistently: potentially related to detection bias.

Overall risk of bias assessed as HIGH, MODERATE, or LOW. 


\section{Key Question 4: Rehabilitation for Total Hip Arthroplasty}

Table C-4.1. KQ 4. Design details and arms

\begin{tabular}{|c|c|c|c|c|c|c|c|c|c|}
\hline $\begin{array}{l}\text { StudyA, } \\
\text { Year, } \\
\text { PMID, } \\
\text { Country }\end{array}$ & $\begin{array}{l}\text { Funding } \\
\text { Source }\end{array}$ & $\begin{array}{l}\text { Overall } \\
\text { RoB }\end{array}$ & Eligibility Criteria & Intervention ${ }^{\mathrm{B}}$ & $\begin{array}{l}\mathbf{N}, \\
\text { Enrolled }\end{array}$ & $\begin{array}{l}\text { Mean } \\
\text { Age, } \\
\text { Years } \\
\text { (SD) }\end{array}$ & $\begin{array}{l}\text { Female, } \\
\%\end{array}$ & $\begin{array}{l}\text { Mean } \\
\text { BMI }^{\mathrm{C}} \\
\text { (SD) }\end{array}$ & $\begin{array}{l}\text { Prior } \\
\text { Contralateral } \\
\text { Arthroplasty }\end{array}$ \\
\hline \multirow[t]{2}{*}{$\begin{array}{l}\text { Austin, } \\
2017 \\
28419032 \\
\text { USA }\end{array}$} & NR & Moderate & $\begin{array}{l}\text { INCLUSION: } 18-80 \text { yo, primary, THA for OA. } \\
\text { EXCLUSION: Those with inflammatory or } \\
\text { posttraumatic arthritis, those with a history of septic } \\
\text { arthritis of the involved hip, and those undergoing } \\
\text { revision THA or conversion THA with removal of } \\
\text { previously implanted components. Additionally, } \\
\text { patients requiring discharge to an acute } \\
\text { rehabilitation center, skilled nursing facility, } \\
\text { convalescent home, or long-term care facility }\end{array}$ & $\begin{array}{l}\text { Home exercise } \\
\text { Comp: E } \\
\text { AdjMod: - } \\
\text { Set: H }\end{array}$ & 60 & $\begin{array}{l}62.3^{\mathrm{d}} \\
(12.7)\end{array}$ & $26 \%$ & $\begin{array}{l}28.2 \\
(7.0)\end{array}$ & NR \\
\hline & No data & No data & No data & $\begin{array}{l}\text { Formal Physical Therapy } \\
\text { Comp: - } \\
\text { AdjMod: - } \\
\text { Set: H, O }\end{array}$ & 60 & $\begin{array}{l}61.2 \\
(8.4)\end{array}$ & $21 \%$ & $\begin{array}{l}30.4 \\
(5.2)\end{array}$ & NR \\
\hline $\begin{array}{l}\text { Beck, } \\
2019, \\
30782304, \\
\text { Germany }\end{array}$ & $\begin{array}{l}\text { Non- } \\
\text { industry }\end{array}$ & High & $\begin{array}{l}\text { INCLUSION: } \geq 18 y o \text {, general medical eligibility for } \\
\text { hip rehab sports therapy, a stable implant. } \\
\text { EXCLUSION: acute or chronic diseases and severe } \\
\text { pain in the affected hip joint. }\end{array}$ & $\begin{array}{l}\text { Sports therapy } \\
\text { Comp: S-A-F-B-T-E } \\
\text { AdjMod: Massage } \\
\text { Set: O }\end{array}$ & 80 & $\begin{array}{l}\text { Median } \\
(59.0) ; \\
\text { Range } \\
(51.1- \\
69.7)\end{array}$ & $53 \%$ & $\begin{array}{l}\text { Median } \\
(26.4) \\
\text { Range } \\
(23.8 \\
28.6) \\
\end{array}$ & NR \\
\hline \multirow[t]{2}{*}{$\begin{array}{l}\text { Coulter, } \\
2017 \\
28506775 \\
\text { Australia }\end{array}$} & NR & High & $\begin{array}{l}\text { INCLUSION: } \geq 18 \text { yo, having a primary elective THA. } \\
\text { EXCLUSION: Metastatic disease, pathological } \\
\text { fractures, infection, or acute trauma; revision THA; } \\
\text { University of California, Los Angeles (UCLA) activity } \\
\text { scale level <2 preoperatively; being able to bear } \\
\text { weight postoperatively; requiring inpatient } \\
\text { rehabilitation postoperatively }\end{array}$ & $\begin{array}{l}\text { Unsupervised home-based } \\
\text { exercises } \\
\text { Comp: S-F-E } \\
\text { AdjMod: - } \\
\text { Set: H }\end{array}$ & 42 & $\begin{array}{l}\text { Median } \\
(63) ; \text { IQR } \\
(53,87) ; \\
\text { Range } \\
(21,86)\end{array}$ & $50 \%$ & NR & NR \\
\hline & No data & No data & No data & $\begin{array}{l}\text { Supervised rehabilitation \& } \\
\text { unsupervised home-based } \\
\text { exercises } \\
\text { Comp: S-F-T-E } \\
\text { AdjMod: - } \\
\text { Set: O }\end{array}$ & 56 & $\begin{array}{l}\text { Median } \\
66 ; \text { IQR } \\
(57,88) ; \\
\text { Range } \\
(34,88)\end{array}$ & $64 \%$ & NR & NR \\
\hline
\end{tabular}




\begin{tabular}{|c|c|c|c|c|c|c|c|c|c|}
\hline $\begin{array}{l}\text { Study } \\
\text { Year, } \\
\text { PMID, } \\
\text { Country }\end{array}$ & $\begin{array}{l}\text { Funding } \\
\text { Source }\end{array}$ & $\begin{array}{l}\text { Overall } \\
\text { RoB }\end{array}$ & Eligibility Criteria & Intervention ${ }^{B}$ & $\begin{array}{l}\mathrm{N}, \\
\text { Enrolled }\end{array}$ & $\begin{array}{l}\text { Mean } \\
\text { Age, } \\
\text { Years } \\
\text { (SD) }\end{array}$ & $\begin{array}{l}\text { Female, } \\
\%\end{array}$ & $\begin{array}{l}\text { Mean } \\
\text { BMIc } \\
\text { (SD) }\end{array}$ & $\begin{array}{l}\text { Prior } \\
\text { Contralateral } \\
\text { Arthroplasty }\end{array}$ \\
\hline \multirow[t]{2}{*}{$\begin{array}{l}\text { Giaquinto } \\
2010 \\
19282040 \\
\text { Italy }\end{array}$} & NR & Low & $\begin{array}{l}\text { INCLUSION: (i) patients referred from district } \\
\text { hospitals; (ii) short interval from surgical intervention } \\
\text { (less than } 10 \text { days); (iii) good general conditions. } \\
\text { EXCLUSION: (i) presence of a physical or mental } \\
\text { acute illness; (ii) cognitive deterioration; (iii) inability } \\
\text { to complete questionnaires; (iv) no compliance }\end{array}$ & $\begin{array}{l}\text { Hydrotherapy } \\
\text { Comp: S-F } \\
\text { AdjMod: - } \\
\text { Set: Other inpatient facility }\end{array}$ & 31 & $\begin{array}{l}70.1 \\
(8.5)\end{array}$ & $68 \%$ & $\begin{array}{l}\text { Median } \\
(25.5)\end{array}$ & NR \\
\hline & No data & No data & No data & $\begin{array}{l}\text { No hydrotherapy } \\
\text { Comp: - } \\
\text { AdjMod: Massage } \\
\text { Set: Other inpatient facility }\end{array}$ & 33 & $\begin{array}{l}70.6 \\
(8.4)\end{array}$ & $67 \%$ & $\begin{array}{l}\text { Median } \\
(26.4)\end{array}$ & NR \\
\hline \multirow[t]{2}{*}{$\begin{array}{l}\text { Heiberg, } \\
2012 \\
22170790 \\
\text { Norway }\end{array}$} & $\begin{array}{l}\text { Non- } \\
\text { industry }\end{array}$ & Moderate & $\begin{array}{l}\text { INCLUSION: Diagnosis of OA of the hip joint. } \\
\text { EXCLUSION: Had OA in a knee or the contralateral } \\
\text { hip that restricted their walking, a neurologic } \\
\text { disease, dementia, heart disease, drug abuse }\end{array}$ & $\begin{array}{l}\text { Walking skill training program } \\
\text { Comp: S-A-B-T } \\
\text { AdjMod: - } \\
\text { Set: O }\end{array}$ & 35 & $\begin{array}{l}65 ; 95 \% \\
\mathrm{Cl}(63, \\
68)\end{array}$ & $60 \%$ & $\begin{array}{l}27(95 \% \\
\mathrm{Cl} 26, \\
29)\end{array}$ & NR \\
\hline & No data & No data & No data & $\begin{array}{l}\text { Standard care } \\
\text { Comp: - } \\
\text { AdjMod: - } \\
\text { Set: - }\end{array}$ & 33 & $\begin{array}{l}66 ; 95 \% \\
\mathrm{Cl}(63, \\
69)\end{array}$ & $42 \%$ & $\begin{array}{l}27(95 \% \\
\mathrm{Cl} 25, \\
28)\end{array}$ & NR \\
\hline $\begin{array}{l}\text { Liebs, } \\
2010, \\
20360503 \\
\text { Germany }\end{array}$ & No data & No data & No data & $\begin{array}{l}\text { Control } \\
\text { Comp: S-F-B-T } \\
\text { AdjMod: - } \\
\text { Set: O }\end{array}$ & 104 & $\begin{array}{l}67.2 \\
(8.5)\end{array}$ & $63 \%$ & $\begin{array}{l}27.4 \\
(4.5)\end{array}$ & NR \\
\hline \multirow[t]{2}{*}{$\begin{array}{l}\text { Liebs, } \\
2012, \\
22196125, \\
\text { Germany }\end{array}$} & $\begin{array}{l}\text { Non- } \\
\text { industry }\end{array}$ & Moderate & $\begin{array}{l}\text { INCLUSION: Unilateral hip or knee replacement } \\
\text { surgery at participating centers on an elective basis } \\
\text { after diagnosis of OA. EXCLUSION: History of } \\
\text { septic arthritis, hip or knee fracture, intraoperative } \\
\text { complications, revision arthroplasty, RA, } \\
\text { amputations, malignancy }\end{array}$ & $\begin{array}{l}\text { Early aquatic therapy } \\
\text { Comp: S-F-B-T } \\
\text { AdjMod: - } \\
\text { Set: O }\end{array}$ & 138 & $\begin{array}{l}66.7 \\
(10.3)\end{array}$ & $64 \%$ & $\begin{array}{l}27.6 \\
(4.4)\end{array}$ & NR \\
\hline & No data & No data & No data & $\begin{array}{l}\text { Late aquatic therapy (after } \\
\text { wound healing) } \\
\text { Comp: S-F-B-T } \\
\text { AdjMod: - } \\
\text { Set: O }\end{array}$ & 142 & $\begin{array}{l}69.1 \\
(9.8)\end{array}$ & $62 \%$ & $\begin{array}{l}26.8 \\
(4.6)\end{array}$ & NR \\
\hline $\begin{array}{l}\text { Łyp, } \\
2016, \\
27455419, \\
\text { Poland }\end{array}$ & NR & High & INCLUSION: Patients after THR. EXCLUSION: NR & $\begin{array}{l}\text { Kinesiotherapy, low-frequency } \\
\text { magnetic field and water } \\
\text { exercises } \\
\text { Comp: S-F } \\
\text { AdjMod: Complimentary and } \\
\text { alternative therapy } \\
\text { Set: O }\end{array}$ & 32 & $\begin{array}{l}59.84 \\
(6.00)\end{array}$ & NR & $\begin{array}{l}27.7 \\
(5.1)\end{array}$ & NR \\
\hline
\end{tabular}




\begin{tabular}{|c|c|c|c|c|c|c|c|c|c|}
\hline $\begin{array}{l}\text { Study } \\
\text { Year, } \\
\text { PMID, } \\
\text { Country }\end{array}$ & $\begin{array}{l}\text { Funding } \\
\text { Source }\end{array}$ & $\begin{array}{l}\text { Overall } \\
\text { RoB }\end{array}$ & Eligibility Criteria & Intervention ${ }^{B}$ & $\begin{array}{l}\mathbf{N}, \\
\text { Enrolled }\end{array}$ & $\begin{array}{l}\text { Mean } \\
\text { Age, } \\
\text { Years } \\
\text { (SD) }\end{array}$ & $\begin{array}{l}\text { Female, } \\
\%\end{array}$ & $\begin{array}{l}\text { Mean } \\
\text { BMIC } \\
\text { (SD) }\end{array}$ & $\begin{array}{l}\text { Prior } \\
\text { Contralateral } \\
\text { Arthroplasty }\end{array}$ \\
\hline & No data & No data & No data & $\begin{array}{l}\text { Kinesiotherapy, low-frequency } \\
\text { magnetic field, without water } \\
\text { exercises } \\
\text { Comp: S } \\
\text { AdjMod: Complimentary and } \\
\text { alternative therapy } \\
\text { Set: O }\end{array}$ & 32 & $\begin{array}{l}63.5 \\
(10.0)\end{array}$ & NR & $\begin{array}{l}27.1 \\
(4.6)\end{array}$ & NR \\
\hline \multirow[t]{2}{*}{$\begin{array}{l}\text { Mikkelsen, } \\
2014, \\
25305374, \\
\text { Denmark }\end{array}$} & $\begin{array}{l}\text { Non- } \\
\text { industry }\end{array}$ & Moderate & $\begin{array}{l}\text { INCLUSION: Primary unilateral THA for hip OA, } \\
\text { preoperative HOOS ADL } 67 \text {, age }>18 \text { years, willing } \\
\text { to participate in training twice a week for } 10 \text { weeks. } \\
\text { EXCLUSION: Resurfacing hip implant, body mass } \\
\text { index }(\mathrm{BMI})>35 \text {, pre-planned supervised } \\
\text { rehabilitation, pre-planned contralateral THA within } \\
6 \text { months, mental or physical conditions impeding } \\
\text { the intervention }\end{array}$ & $\begin{array}{l}\text { Early supervised progressive } \\
\text { resistance training } \\
\text { Comp: S-F-E } \\
\text { AdjMod: } \\
\text { Set: }\end{array}$ & 32 & $64.8(8)$ & $44 \%$ & $27.5(4)$ & $25 \%$ \\
\hline & No data & No data & No data & $\begin{array}{l}\text { Unsupervised home-based } \\
\text { exercise } \\
\text { Comp: S-F-E } \\
\text { AdjMod: } \\
\text { Set: }\end{array}$ & 30 & $65.1(10)$ & $40 \%$ & $25.4(4)$ & $23 \%$ \\
\hline $\begin{array}{l}\text { Monticone, } \\
2014, \\
24459172, \\
\text { Italy }\end{array}$ & NR & Moderate & $\begin{array}{l}\text { INCLUSION: Patients had to have undergone } \\
\text { primary traditional uncemented total hip } \\
\text { replacement because of osteoarthritis in the } \\
\text { dominant leg 4-7 days before admission to our } \\
\text { Rehabilitation Unit, be in the impossible-to-go- home } \\
\text { group after discharge from the Orthopaedic Unit } \\
\text { because of multiple comorbidities (e.g. cardiac, } \\
\text { respiratory, or endocrine diseases), still requiring } \\
\text { medical care and/or insufficient home support (e.g. } \\
\text { living alone, absence of familiar, and social helps, or } \\
\text { lack of transportation in order to access outpatient } \\
\text { services), be aged }>50 \text { years, and have a good } \\
\text { understanding of Italian. EXCLUSION: Cognitive } \\
\text { impairment and all other causes of hip pain, such as } \\
\text { previous lower limb surgery, infection, fracture, } \\
\text { osteonecrosis or malignancy, and systemic or } \\
\text { neuromuscular diseases; any subjects receiving } \\
\text { compensation for work-related disabilities }\end{array}$ & $\begin{array}{l}\text { Task-oriented exercises } \\
\text { Comp: S-F-B-T-E } \\
\text { AdjMod: - } \\
\text { Set: G; H }\end{array}$ & 50 & $\begin{array}{l}69.5 \\
(7.5)\end{array}$ & $64 \%$ & $\begin{array}{l}27.7 \\
(4.2)\end{array}$ & NR \\
\hline
\end{tabular}




\begin{tabular}{|c|c|c|c|c|c|c|c|c|c|}
\hline $\begin{array}{l}\text { Study } \\
\text { Year, } \\
\text { PMID, } \\
\text { Country }\end{array}$ & $\begin{array}{l}\text { Funding } \\
\text { Source }\end{array}$ & $\begin{array}{l}\text { Overall } \\
\text { RoB }\end{array}$ & Eligibility Criteria & Intervention ${ }^{B}$ & $\begin{array}{l}\mathbf{N}, \\
\text { Enrolled }\end{array}$ & $\begin{array}{l}\text { Mean } \\
\text { Age, } \\
\text { Years } \\
\text { (SD) }\end{array}$ & $\begin{array}{l}\text { Female, } \\
\%\end{array}$ & $\begin{array}{l}\text { Mean } \\
\text { BMIC }^{c} \\
\text { (SD) }\end{array}$ & $\begin{array}{l}\text { Prior } \\
\text { Contralateral } \\
\text { Arthroplasty }\end{array}$ \\
\hline \multirow[t]{2}{*}{$\begin{array}{l}\text { Naylor, } \\
2018 \\
30021552 \\
\text { Australia }^{\mathrm{E}}\end{array}$} & $\begin{array}{l}\text { Non- } \\
\text { industry }\end{array}$ & Moderate & $\begin{array}{l}\text { INCLUSION: Primary surgery, no further } \\
\text { arthroplasty planned within 3-months, and the ability } \\
\text { to understand the protocol and be followed-up by } \\
\text { telephone for } 1 \text { year, being privately insured. } \\
\text { EXCLUSION: Publicly insured patients, the privately } \\
\text { insured patients referred to inpatient rehabilitation } \\
\text { due to slow progress, inadequate social supports } \\
\text { (e.g living alone or without the help of an able } \\
\text { carer), and those with conditions that would alter } \\
\text { their rehabilitation pathway (e.g bilateral surgery or } \\
\text { significant complication (defined below) up to 90- } \\
\text { days post-surgery). }\end{array}$ & $\begin{array}{l}\text { Inpatient rehabilitation } \\
\text { Comp: - } \\
\text { AdjMod: - } \\
\text { Set: Other inpatient facility }\end{array}$ & 123 & $\begin{array}{l}67.8 \\
(10.0)\end{array}$ & $36 \%$ & $\begin{array}{l}27.8 \\
(4.8)\end{array}$ & NR \\
\hline & No data & No data & No data & $\begin{array}{l}\text { No inpatient rehabilitation } \\
\text { Comp: - } \\
\text { AdjMod: - } \\
\text { Set: Home }\end{array}$ & 123 & $\begin{array}{l}66.9 \\
(10.6)\end{array}$ & $32 \%$ & $\begin{array}{l}28.0 \\
(5.1)\end{array}$ & NR \\
\hline \multirow[t]{2}{*}{$\begin{array}{l}\text { Nelson, } \\
2020 \\
32026820 \\
\text { Australia }\end{array}$} & $\begin{array}{l}\text { Non- } \\
\text { industry }\end{array}$ & Moderate & $\begin{array}{l}\text { INCLUSION: Undergoing primary elective THR, } \\
\text { could attend five in-person appointments. } \\
\text { EXCLUSION: Comorbidities preventing participation } \\
\text { in rehabilitation, were undergoing revision THR, } \\
\text { experienced intraoperative complications that } \\
\text { prevented participation in the Queensland Health } \\
\text { inpatient THR clinical pathway, or were unable to } \\
\text { mobilize full weight-bearing. }\end{array}$ & $\begin{array}{l}\text { Telerehabilitation } \\
\text { Comp: S-T-E } \\
\text { AdjMod: - } \\
\text { Set: H }\end{array}$ & 35 & $62(9)$ & $66 \%$ & NR & NR \\
\hline & No data & No data & No data & $\begin{array}{l}\text { In-person usual care } \\
\text { Comp: S-T-E } \\
\text { AdjMod: - } \\
\text { Set: } \mathrm{H}, \mathrm{O}\end{array}$ & 35 & $67(11)$ & $60 \%$ & NR & NR \\
\hline \multirow[t]{2}{*}{$\begin{array}{l}\text { Rao, } \\
2021 \\
33863614 \\
\text { USA }\end{array}$} & Industry & High & $\begin{array}{l}\text { INCLUSION: older than } 18 \text { years of age undergoing } \\
\text { primary, unilateral THA for osteoarthritis. } \\
\text { EXCLUSION: inflammatory or post-traumatic } \\
\text { arthritis or underwent revision THA }\end{array}$ & $\begin{array}{l}\text { Home physical therapy } \\
\text { Comp: S-F } \\
\text { AdjMod: - } \\
\text { Set: H }\end{array}$ & 75 & $\begin{array}{l}56.0 \\
(10.7)\end{array}$ & $63 \%$ & $\begin{array}{l}28.0 \\
(5.0)\end{array}$ & NR \\
\hline & No data & No data & No data & $\begin{array}{l}\text { Formal physical therapy } \\
\text { Comp: S-F-E } \\
\text { AdjMod: - } \\
\text { Set: O; H }\end{array}$ & 72 & $\begin{array}{l}54.3 \\
(11.2)\end{array}$ & $60 \%$ & $\begin{array}{l}28.0 \\
(5.4)\end{array}$ & NR \\
\hline $\begin{array}{l}\text { Smith, } \\
2009, \\
19876883 \\
\text { UK }\end{array}$ & NR & Moderate & $\begin{array}{l}\text { INCLUSION: Patients undergoing unilateral primary } \\
\text { THR, aged } 18 \text { years-old or over of either gender. } \\
\text { EXCLUSION: Unable to undertake assessment and } \\
\text { treatment procedures, could not independently } \\
\text { mobilise with or without walking aids, required to be } \\
\text { non-weight- bearing post-operatively, complex } \\
\text { primary THRs requiring bone grafting and/or } \\
\text { acetabulum screw fixation }\end{array}$ & $\begin{array}{l}\text { Gait re-education program and } \\
\text { bed exercises } \\
\text { Comp: S-F } \\
\text { AdjMod: - } \\
\text { Set: Al }\end{array}$ & 30 & $\begin{array}{l}66.2 \\
(11.3)\end{array}$ & $70 \%$ & NR & NR \\
\hline
\end{tabular}




\begin{tabular}{|c|c|c|c|c|c|c|c|c|c|}
\hline $\begin{array}{l}\text { Study } \\
\text { Year, } \\
\text { PMID, } \\
\text { Country }\end{array}$ & $\begin{array}{l}\text { Funding } \\
\text { Source }\end{array}$ & $\begin{array}{l}\text { Overall } \\
\text { RoB }\end{array}$ & Eligibility Criteria & Intervention ${ }^{B}$ & $\begin{array}{l}\mathrm{N}, \\
\text { Enrolled }\end{array}$ & $\begin{array}{l}\text { Mean } \\
\text { Age, } \\
\text { Years } \\
\text { (SD) }\end{array}$ & $\begin{array}{l}\text { Female, } \\
\%\end{array}$ & $\begin{array}{l}\text { Mean } \\
\text { BMIC } \\
\text { (SD) }\end{array}$ & $\begin{array}{l}\text { Prior } \\
\text { Contralateral } \\
\text { Arthroplasty }\end{array}$ \\
\hline & No data & No data & No data & $\begin{array}{l}\text { Gait re-education program only } \\
\text { Comp: T } \\
\text { AdjMod: - } \\
\text { Set: Al }\end{array}$ & 30 & $\begin{array}{l}68.1 \\
(10.5)\end{array}$ & $63 \%$ & NR & NR \\
\hline \multirow[t]{2}{*}{$\begin{array}{l}\text { Winther, } \\
2020, \\
31977324, \\
\text { Norway }\end{array}$} & NR & Moderate & $\begin{array}{l}\text { INCLUSION: Scheduled for THA. Diagnosis of } \\
\text { primary osteoarthritis as the main cause for elective } \\
\text { THA. ASA score of I-III (stable).EXCLUSION: } \\
\text { Severe hip osteoarthritis of the contralateral hip, not } \\
\text { fully recovered from a previous THA, or any } \\
\text { illness/disease that could influence the ability to } \\
\text { accomplish training and/or physical testing } \\
\text { performance, such as muscular diseases, rest } \\
\text { symptoms from stroke, and paralysis of the } \\
\text { peroneus muscles. }\end{array}$ & $\begin{array}{l}\text { Strengthening exercise } \\
\text { Comp: S } \\
\text { AdjMod: - } \\
\text { Set: O }\end{array}$ & 27 & 60 (NR) & $52 \%$ & 28 & NR \\
\hline & No data & No data & No data & $\begin{array}{l}\text { Standard care } \\
\text { Comp: - } \\
\text { AdjMod: - } \\
\text { Set: - }\end{array}$ & 27 & 66 (NR) & $44 \%$ & 27 & NR \\
\hline
\end{tabular}

Blue coloring is only to visually separate different studies.

Abbreviations: $\mathrm{AI}=$ acute inpatient, $\mathrm{BMI}=$ body mass index, $\mathrm{G}=$ gym or other community center (outpatient) $\mathrm{H}=$ home, $\mathrm{NA}=$ not applicable, $\mathrm{NMES}=$ neuromuscular electrical stimulation, $\mathrm{NR}=$ not reported, $\mathrm{O}=$ outpatient physiotherapy center, $\mathrm{OA}=$ osteoarthritis, $\mathrm{PMID}=$ PubMed identifier, $\mathrm{RA}=$ rheumatoid arthritis, RoB $=$ risk of bias, $\mathrm{SD}=$ standard deviation, $\mathrm{SD}=$ standard deviation, TENS $=$ transcutaneous electrical nerve stimulation, THA = total hip arthroplasty, TJA = total joint arthroplasty, TKA $=$ total knee arthroplasty, yo $=$ years old.

A All randomized controlled trials, except as footnoted.

B Including Components (Comp)); Adjunctive modalities (AdjMod); and Setting (Set).

Components: $\mathrm{A}=$ aerobic exercise, $\mathrm{B}=$ Balance-motor/Learning-agility exercise, $\mathrm{E}=$ patient education, $\mathrm{F}=$ flexibility exercise, $\mathrm{S}=$ strengthening exercise, $\mathrm{T}=$ task-specific training.

C $\mathrm{kg} / \mathrm{m}^{2}$

D Reported age, gender, BMI data for patients included in final analysis only ( $\mathrm{n}=54$ for both home exercise and formal physical therapy groups)

E Non-randomized study 
Table C-4.2. KQ 4. Rehabilitation component details

\begin{tabular}{|c|c|c|c|c|c|c|c|}
\hline $\begin{array}{l}\text { Study, Year, } \\
\text { PMID }\end{array}$ & Intended Comparison & Arm & Components (Specific Exercises/Strategies) & $\begin{array}{l}\text { Progression } \\
\text { (Appropriate) }\end{array}$ & Personnel & Mode of Delivery & Setting \\
\hline \multirow[t]{2}{*}{$\begin{array}{l}\text { Austin, } \\
2017 \\
28419032 \\
\text { USA }\end{array}$} & $\begin{array}{l}\text { Self-directed home } \\
\text { exercise program vs. } \\
\text { standard home and } \\
\text { outpatient physical } \\
\text { therapy }\end{array}$ & Home exercise & $\begin{array}{l}\text { [specific goals and exercises not defined] } \\
\text { 6. Patient education } \\
6.2 \text { Home exercise program }\end{array}$ & $\mathrm{NR}$ & None & $\begin{array}{l}\text { Self-guided } \\
\text { (unsupervised) }\end{array}$ & Home \\
\hline & No data & $\begin{array}{l}\text { Formal Physical } \\
\text { Therapy }\end{array}$ & $\begin{array}{l}\text { [specific goals and exercises not defined] } \\
\text { [Conventional home and outpatient physical } \\
\text { therapy - specific interventions not described] }\end{array}$ & NR & $\begin{array}{l}\text { Physical } \\
\text { therapist }\end{array}$ & In-person & $\begin{array}{l}\text { Home (2wks); } \\
\text { Physical } \\
\text { therapy/rehabilitation } \\
\text { facility (outpatient) } \\
\text { (8wks) }\end{array}$ \\
\hline \multirow[t]{2}{*}{$\begin{array}{l}\text { Beck, } \\
2019, \\
30782304, \\
\text { Germany }\end{array}$} & $\begin{array}{l}\text { Intensive sport } \\
\text { rehabilitation vs. } \\
\text { control }\end{array}$ & Sports therapy & $\begin{array}{l}\text { 1. Strength } \\
\text { 1.49 Squats } \\
\text { 2. Aerobic } \\
\text { 2.2 Bike (Endurance) } \\
\text { 2.9 Walking } \\
\text { 3. Flexibility } \\
\text { 3.8 Hip flexor stretch (iliopsoas) } \\
\text { 4. Balance-Motor Learning-Agility } \\
\text { 4.3 Balance on unstable surface } \\
\text { 5. Task specific training } \\
\text { 5.8 Gait training } \\
\text { 6. Patient education } \\
\text { [specific education not defined] } \\
\text { 7. Adjunctive modality } \\
\text { 7.12 Massage/myofascial techniques for soft } \\
\text { tissue }\end{array}$ & $\mathrm{N}(\mathrm{NA})$ & $\begin{array}{l}\text { Other } \\
\text { ("rehabilitation } \\
\text { sports therapy } \\
\text { providers"; } \\
\text { unspecified) }\end{array}$ & In-person & $\begin{array}{l}\text { Physical } \\
\text { therapy/rehabilitation } \\
\text { facility (outpatient) }\end{array}$ \\
\hline & No data & Control & $\begin{array}{l}\text { NA } \\
\text { [No hip rehabilitation sports therapy] }\end{array}$ & $\mathrm{N}(\mathrm{NA})$ & NA & NA & $\mathrm{NA}$ \\
\hline $\begin{array}{l}\text { Coulter, } \\
2017, \\
28506775, \\
\text { Australia }\end{array}$ & $\begin{array}{l}\text { Unsupervised home- } \\
\text { based physiotherapy } \\
\text { vs. supervised } \\
\text { outpatient } \\
\text { physiotherapy }\end{array}$ & $\begin{array}{l}\text { Unsupervised home- } \\
\text { based exercises }\end{array}$ & $\begin{array}{l}\text { 1. Strength } \\
\text { [specific exercises not defined] } \\
\text { 3. Flexibility } \\
\text { [specific exercises not defined] } \\
\text { 6. Patient education } \\
6.2 \text { Home exercise program }\end{array}$ & $\mathrm{Y}(\mathrm{N})$ & $\begin{array}{l}\text { None } \\
\text { (unsupervised) }\end{array}$ & $\begin{array}{l}\text { Self-guided } \\
\text { (unsupervised) }\end{array}$ & Home \\
\hline
\end{tabular}




\begin{tabular}{|c|c|c|c|c|c|c|c|}
\hline $\begin{array}{l}\text { Study, Year, } \\
\text { PMID }\end{array}$ & Intended Comparison & Arm & Components (Specific Exercises/Strategies) & $\begin{array}{l}\text { Progression } \\
\text { (Appropriate) }\end{array}$ & Personnel & Mode of Delivery & Setting \\
\hline & & $\begin{array}{l}\text { Supervised } \\
\text { rehabilitation \& } \\
\text { unsupervised home- } \\
\text { based exercises }\end{array}$ & $\begin{array}{l}\text { 1. Strength } \\
\text { 1.2 Bridges Two-legged (supine hip extension) } \\
\text { 1.12 Hip abduction in standing } \\
\text { 1.13 Hip abduction in supine } \\
\text { 1.19 Hip extension in standing } \\
\text { 1.31 Knee extension in sitting or supine (long arc } \\
\text { quad) (unclear long or short) } \\
\text { 1.32 Knee extension in sitting or supine (short arc } \\
\text { quad) (unclear long or short) } \\
\text { 1.48 Sit-to-stand } \\
\text { 1.55 Step up - forward } \\
\text { 3. Flexibility } \\
\text { 3.2 Bike (ROM) } \\
\text { 5. Task specific training } \\
\text { 5.8 Gait training } \\
\text { 5.13 Sit-to-stand training } \\
\text { 5.15 Stair training } \\
\text { 6. Patient education } \\
\text { 6.2 Home exercise program }\end{array}$ & $\mathrm{Y}(\mathrm{Y})$ & $\begin{array}{l}\text { Physical } \\
\text { therapist }\end{array}$ & In-person & $\begin{array}{l}\text { Physical } \\
\text { therapy/rehabilitation } \\
\text { facility (outpatient) }\end{array}$ \\
\hline \multirow[t]{2}{*}{$\begin{array}{l}\text { Giaquinto, } \\
2010 \\
19282040 \\
\text { Italy }\end{array}$} & $\begin{array}{l}\text { Hydrotherapy vs. Land } \\
\text { therapy }\end{array}$ & Hydrotherapy & $\begin{array}{l}\text { 1. Strength } \\
\text { [specific exercises not defined] } \\
\text { 3. Flexibility } \\
\text { [specific exercises not defined] }\end{array}$ & $\mathrm{N}(\mathrm{NA})$ & Unclear & In-person & $\begin{array}{l}\text { Other inpatient } \\
\text { facility }\end{array}$ \\
\hline & No data & No hydrotherapy & $\begin{array}{l}\text { 7. Adjunctive modality } \\
7.11 \text { Massage for scar mobility } \\
\text { [Land therapy followed by scar mobility] }\end{array}$ & $\mathrm{N}(\mathrm{NA})$ & Unclear & In-person & $\begin{array}{l}\text { Other inpatient } \\
\text { facility }\end{array}$ \\
\hline
\end{tabular}




\begin{tabular}{|c|c|c|c|c|c|c|c|}
\hline $\begin{array}{l}\text { Study, Year, } \\
\text { PMID }\end{array}$ & Intended Comparison & Arm & Components (Specific Exercises/Strategies) & $\begin{array}{l}\text { Progression } \\
\text { (Appropriate) }\end{array}$ & Personnel & Mode of Delivery & Setting \\
\hline \multirow[t]{2}{*}{$\begin{array}{l}\text { Heiberg KE, } \\
2012 \\
22170790 \\
\text { Norway }\end{array}$} & $\begin{array}{l}\text { Walking skill training } \\
\text { program vs. control }\end{array}$ & $\begin{array}{l}\text { Walking skill training } \\
\text { program }\end{array}$ & $\begin{array}{l}\text { 1. Strength } \\
\text { 1.41 Lunges } \\
\text { 1.42 Lunges to side (lateral lunge) } \\
\text { 1.47 Single leg stance } \\
\text { 1.48 Sit-to-stand } \\
\text { 1.49 Squats } \\
\text { 1.52 Step down } \\
\text { 1.55 Step up - forward } \\
\text { 2. Aerobic } \\
\text { 2.9 Walking } \\
\text { 3.3 Calf stretch with knee bent (soleus) (position } \\
\text { unclear) } \\
\text { 3.4 Calf stretch with knee straight (gastroc) } \\
\text { (position unclear) } \\
\text { 3.5 Hamstring stretch in any position } \\
\text { 4. Balance-Motor Learning-Agility } \\
\text { 4.3 Balance on unstable surface } \\
\text { 4.4 Balance with perturbations } \\
\text { 4.8 Single leg stance } \\
\text { 4.11 Step down } \\
\text { 4.13 Step lateral (side step) } \\
\text { 4.14 Step up - forward } \\
\text { 5. Task specific training } \\
\text { 5.6 Gait on uneven surfaces } \\
\text { 5.10 Gait with perturbations } \\
\text { 5.12 Obstacle training } \\
\text { 5.15 Stair training }\end{array}$ & $\mathrm{Y}(\mathrm{Y})$ & $\begin{array}{l}\text { Physical } \\
\text { therapist }\end{array}$ & In-person & $\begin{array}{l}\text { Physical } \\
\text { therapy/rehabilitation } \\
\text { facility (outpatient) }\end{array}$ \\
\hline & No data & Standard care & $\begin{array}{l}\text { "The control group did not attend any supervised } \\
\text { physiotherapy programs during the same time } \\
\text { period, but were encouraged to continue with the } \\
\text { exercises" }\end{array}$ & $\mathrm{N}(\mathrm{NA})$ & NA & NA & NA \\
\hline $\begin{array}{l}\text { Liebs, } \\
2010, \\
20360503 \\
\text { Germany }\end{array}$ & $\begin{array}{l}\text { Ergometer cycling plus } \\
\text { standard physiotherapy } \\
\text { vs. standard } \\
\text { physiotherapy alone }\end{array}$ & Ergometer cycling & $\begin{array}{l}\text { 1. Strength } \\
\text { [specific exercises not defined] } \\
\text { 3. Flexibility } \\
\text { 3.2 Bike (ROM) } \\
\text { 4. Balance-Motor Learning-Agility } \\
\text { 4.3 Balance on unstable surface } \\
\text { 5. Task specific training } \\
\text { 5.1 Transfers } \\
\text { 5.6 Gait on uneven surfaces } \\
\text { 5.8 Gait training } \\
\text { 5.15 Stair training }\end{array}$ & $\mathrm{N}(\mathrm{NA})$ & $\begin{array}{l}\text { Physical } \\
\text { therapist }\end{array}$ & In-person & $\begin{array}{l}\text { Physical } \\
\text { therapy/rehabilitation } \\
\text { facility (outpatient) }\end{array}$ \\
\hline
\end{tabular}




\begin{tabular}{|c|c|c|c|c|c|c|c|}
\hline $\begin{array}{l}\text { Study, Year, } \\
\text { PMID }\end{array}$ & Intended Comparison & Arm & Components (Specific Exercises/Strategies) & $\begin{array}{l}\text { Progression } \\
\text { (Appropriate) }\end{array}$ & Personnel & Mode of Delivery & Setting \\
\hline & No data & Control & $\begin{array}{l}\text { 1. Strength } \\
\text { [specific exercises not defined] } \\
\text { 3. Flexibility } \\
\text { [specific exercises not defined] } \\
\text { 4. Balance-Motor Learning-Agility } \\
\text { 4.3 Balance on unstable surface } \\
\text { 5. Task specific training } \\
\text { 5.1 Transfers } \\
\text { 5.6 Gait on uneven surfaces } \\
\text { 5.8 Gait training } \\
\text { 5.15 Stair training }\end{array}$ & $N(N A)$ & $\begin{array}{l}\text { Physical } \\
\text { therapist }\end{array}$ & In-person & $\begin{array}{l}\text { Physical } \\
\text { therapy/rehabilitation } \\
\text { facility (outpatient) }\end{array}$ \\
\hline \multirow[t]{2}{*}{$\begin{array}{l}\text { Liebs, } \\
2012, \\
22196125, \\
\text { Germany }\end{array}$} & $\begin{array}{l}\text { Early aquatic therapy } \\
\text { vs. late aquatic therapy }\end{array}$ & Early aquatic therapy & $\begin{array}{l}\text { 1. Strength } \\
\text { [specific exercises not defined] } \\
\text { 3. Flexibility } \\
\text { [specific exercises not defined] } \\
\text { 4. Balance-Motor Learning-Agility } \\
\text { 4.3 Balance on unstable surface } \\
\text { 5. Task specific training } \\
\text { 5.1 Transfers } \\
\text { 5.6 Gait on uneven surfaces } \\
\text { 5.8 Gait training } \\
\text { 5.15 Stair training }\end{array}$ & $\mathrm{N}(\mathrm{NA})$ & $\begin{array}{l}\text { Physical } \\
\text { therapist }\end{array}$ & In-person & $\begin{array}{l}\text { Physical } \\
\text { therapy/rehabilitation } \\
\text { facility (outpatient) }\end{array}$ \\
\hline & No data & $\begin{array}{l}\text { Late Aquatic therapy } \\
\text { (after wound healing) }\end{array}$ & $\begin{array}{l}\text { 1. Strength } \\
\text { [specific exercises not defined] } \\
\text { 3. Flexibility } \\
\text { [specific exercises not defined] } \\
\text { 4. Balance-Motor Learning-Agility } \\
\text { 4.3 Balance on unstable surface } \\
\text { 5. Task specific training } \\
\text { 5.1 Transfers } \\
\text { 5.6 Gait on uneven surfaces } \\
\text { 5.8 Gait training } \\
\text { 5.15 Stair training }\end{array}$ & $\mathrm{N}(\mathrm{NA})$ & $\begin{array}{l}\text { Physical } \\
\text { therapist }\end{array}$ & In-person & $\begin{array}{l}\text { Physical } \\
\text { therapy/rehabilitation } \\
\text { facility (outpatient) }\end{array}$ \\
\hline \multirow[t]{3}{*}{$\begin{array}{l}\text { Łyp, } \\
2016, \\
27455419 \\
\text { Poland }\end{array}$} & $\begin{array}{l}\text { Kinesiotherapy, low- } \\
\text { frequency magnetic } \\
\text { field and water } \\
\text { exercises vs. } \\
\text { kinesiotherapy and } \\
\text { low-frequency } \\
\text { magnetic field vs. } \\
\text { control group }\end{array}$ & $\begin{array}{l}\text { Kinesiotherapy, low- } \\
\text { frequency magnetic } \\
\text { field and water } \\
\text { exercises }\end{array}$ & $\begin{array}{l}\text { 1. Strength } \\
\text { [specific exercises not defined] } \\
\text { 3. Flexibility } \\
\text { [specific exercises not defined] } \\
\text { 7. Adjunctive modality } \\
\text { 7.18 Complementary and alternative therapies }\end{array}$ & $\mathrm{N}(\mathrm{NA})$ & Unclear & In-person & $\begin{array}{l}\text { Physical } \\
\text { therapy/rehabilitation } \\
\text { facility (outpatient) }\end{array}$ \\
\hline & No data & $\begin{array}{l}\text { Kinesiotherapy, low- } \\
\text { frequency magnetic } \\
\text { field, without water } \\
\text { exercises }\end{array}$ & $\begin{array}{l}\text { 1. Strength } \\
\text { [specific exercises not defined] } \\
\text { 7. Adjunctive modality } \\
\text { 7.18 Complementary and alternative therapies }\end{array}$ & $\mathrm{N}(\mathrm{NA})$ & Unclear & In-person & $\begin{array}{l}\text { Physical } \\
\text { therapy/rehabilitation } \\
\text { facility (outpatient) }\end{array}$ \\
\hline & No data & Control & $\begin{array}{l}\text { [Awaiting rehabilitation and instructed not to do } \\
\text { activities that aggravated pain] }\end{array}$ & & & & \\
\hline
\end{tabular}




\begin{tabular}{|c|c|c|c|c|c|c|c|}
\hline $\begin{array}{l}\text { Study, Year, } \\
\text { PMID }\end{array}$ & Intended Comparison & Arm & Components (Specific Exercises/Strategies) & $\begin{array}{l}\text { Progression } \\
\text { (Appropriate) }\end{array}$ & Personnel & Mode of Delivery & Setting \\
\hline \multirow[t]{2}{*}{$\begin{array}{l}\text { Mikkelsen, } \\
2014, \\
25305374, \\
\text { Denmark }\end{array}$} & $\begin{array}{l}\text { Early supervised } \\
\text { progressive resistance } \\
\text { training vs. } \\
\text { unsupervised home- } \\
\text { based exercise }\end{array}$ & $\begin{array}{l}\text { Early supervised } \\
\text { progressive } \\
\text { resistance training }\end{array}$ & $\begin{array}{l}\text { 1. Strength } \\
\text { 1.11 Hip abduction in sidelying (position unclear) } \\
\text { 1.12 Hip abduction in standing (position unclear) } \\
\text { 1.13 Hip abduction in supine (position unclear) } \\
\text { 1.17 Hip extension in sidelying (position unclear) } \\
\text { 1.18 Hip extension in prone (position unclear) } \\
\text { 1.19 Hip extension in standing (position unclear) } \\
\text { 1.20 Hip flexion in sidelying (position unclear) } \\
\text { 1.21 Hip flexion in sitting (position unclear) } \\
\text { 1.22 Hip flexion in standing (position unclear) } \\
\text { 1.23 Hip flexion in supine (position unclear) } \\
\text { 1.28 Knee extension machine (one-leg) } \\
\text { 1.30 Knee extension AAROM in sitting or supine } \\
\text { (short- or long arc quad) (position unclear) } \\
\text { 1.31 Knee extension in sitting or supine (long arc } \\
\text { quad) (position unclear) } \\
\text { 1.32 Knee extension in sitting or supine (short arc } \\
\text { quad) (position unclear) } \\
\text { 1.35 Knee flexion in prone (position unclear) } \\
\text { 1.36 Knee flexion in sitting or supine (position } \\
\text { unclear) } \\
\text { 1.37 Knee flexion in standing (position unclear) } \\
\text { 1.38 Leg Press (one leg) } \\
\text { 3. Flexibility } \\
\text { 3.2 Bike (ROM) } \\
\text { 6. Patient education } \\
\text { 6.2 Home exercise program }\end{array}$ & $\mathrm{Y}(\mathrm{Y})$ & $\begin{array}{l}\text { Physical } \\
\text { therapist; } \\
\text { None } \\
\text { (unsupervised) }\end{array}$ & $\begin{array}{l}\text { In-person; Self- } \\
\text { guided } \\
\text { (unsupervised) }\end{array}$ & $\begin{array}{l}\text { Gym or other } \\
\text { community center } \\
\text { (outpatient); Home }\end{array}$ \\
\hline & No data & $\begin{array}{l}\text { Unsupervised home- } \\
\text { based exercise }\end{array}$ & $\begin{array}{l}\text { 1. Strength } \\
\text { 1.11 Hip abduction in sidelying (position unclear) } \\
\text { 1.12 Hip abduction in standing (position unclear) } \\
\text { 1.13 Hip abduction in supine (position unclear) } \\
\text { 1.17 Hip extension in sidelying (position unclear) } \\
\text { 1.18 Hip extension in prone (position unclear) } \\
\text { 1.19 Hip extension in standing (position unclear) } \\
\text { 1.20 Hip flexion in sidelying (position unclear) } \\
\text { 1.21 Hip flexion in sitting (position unclear) } \\
\text { 1.22 Hip flexion in standing (position unclear) } \\
\text { 1.23 Hip flexion in supine (position unclear) } \\
\text { 1.30 Knee extension AAROM in sitting or supine } \\
\text { (short- or long arc quad) (position unclear) } \\
\text { 1.31 Knee extension in sitting or supine (long arc } \\
\text { quad) (position unclear) } \\
\text { 1.32 Knee extension in sitting or supine (short arc } \\
\text { quad) (position unclear) } \\
\text { 1.35 Knee flexion in prone (position unclear) } \\
\text { 1.36 Knee flexion in sitting or supine (position } \\
\text { unclear) } \\
\text { 1.37 Knee flexion in standing (position unclear) } \\
\text { 3. Flexibility } \\
\text { 3.2 Bike (ROM) } \\
\text { 6. Patient education } \\
6.2 \text { Home exercise program }\end{array}$ & $\mathrm{Y}(\mathrm{N})$ & $\begin{array}{l}\text { None } \\
\text { (unsupervised) }\end{array}$ & $\begin{array}{l}\text { Self-guided } \\
\text { (unsupervised) }\end{array}$ & Home \\
\hline
\end{tabular}




\begin{tabular}{|c|c|c|c|c|c|c|c|}
\hline $\begin{array}{l}\text { Study, Year, } \\
\text { PMID }\end{array}$ & Intended Comparison & Arm & Components (Specific Exercises/Strategies) & $\begin{array}{l}\text { Progression } \\
\text { (Appropriate) }\end{array}$ & Personnel & Mode of Delivery & Setting \\
\hline \multirow[t]{2}{*}{$\begin{array}{l}\text { Monticone, } \\
2014 \\
24459172 \\
\text { Italy }\end{array}$} & $\begin{array}{l}\text { Task-oriented exercise } \\
\text { with early full weight } \\
\text { bearing vs. open chain } \\
\text { kinetic exercise with } \\
\text { partial weight bearing }\end{array}$ & $\begin{array}{l}\text { Task-oriented } \\
\text { exercises }\end{array}$ & $\begin{array}{l}\text { 1. Strength } \\
\text { 1.17 Hip extension in sidelying (sitting; option not } \\
\text { available) } \\
\text { 1.18 Hip extension in prone } \\
\text { 1.19 Hip extension in standing } \\
\text { 1.21 Hip flexion in sitting } \\
\text { 1.37 Knee flexion in standing } \\
\text { 3. Flexibility } \\
\text { 3.2 Bike (ROM) } \\
\text { 4. Balance-Motor Learning-Agility } \\
\text { 4.3 Balance on unstable surface } \\
\text { 4.10 Stepping multiple directions (grapevine) } \\
\text { 5. Task specific training } \\
\text { 5.8 Gait training } \\
\text { 5.10 Gait with perturbations } \\
\text { 5.12 Obstacle training } \\
\text { 5.13 Sit-to-stand training } \\
\text { 5.15 Stair training } \\
\text { 6. Patient education } \\
\text { 6.1 Activities of daily living } \\
\end{array}$ & $\mathrm{Y}(\mathrm{N})$ & $\begin{array}{l}\text { Physical } \\
\text { therapist }\end{array}$ & In-person & $\begin{array}{l}\text { Other inpatient } \\
\text { facility }\end{array}$ \\
\hline & No data & $\begin{array}{l}\text { Open chain kinetic } \\
\text { exercise }\end{array}$ & $\begin{array}{l}\text { 1. Strength } \\
\text { 1.13 Hip abduction in supine } \\
1.17 \mathrm{Hip} \text { extension in sidelying } \\
1.18 \mathrm{Hip} \text { extension in prone } \\
\text { 1.23 Hip flexion in supine } \\
\text { 1.26 Hip rotation external (lateral) } \\
\text { 1.31 Knee extension in sitting or supine (long arc } \\
\text { quad) } \\
1.32 \mathrm{Knee} \text { extension in sitting or supine (short arc } \\
\text { quad) } \\
\text { 1.36 Knee flexion in sitting or supine } \\
\text { 1.43 Quad sets } \\
\text { 5. Task specific training } \\
\text { 5.8 Gait training }\end{array}$ & $\mathrm{N}(\mathrm{NA})$ & $\begin{array}{l}\text { Physical } \\
\text { therapist }\end{array}$ & In-person & $\begin{array}{l}\text { Other inpatient } \\
\text { facility }\end{array}$ \\
\hline \multirow{2}{*}{$\begin{array}{l}\text { Naylor, } \\
2018, \\
30021552, \\
\text { Australia }\end{array}$} & $\begin{array}{l}\text { Discharge to inpatient } \\
\text { rehabilitation vs. } \\
\text { discharge to home }\end{array}$ & Inpatient rehabilitation & $\begin{array}{l}\text { [specific goals and exercises not defined; } \\
\text { comparison of setting] }\end{array}$ & NR & NR & In-person & $\begin{array}{l}\text { Other inpatient } \\
\text { facility }\end{array}$ \\
\hline & No data & $\begin{array}{l}\text { No inpatient } \\
\text { rehabilitation }\end{array}$ & $\begin{array}{l}\text { [specific goals and exercises not defined; } \\
\text { comparison of setting] }\end{array}$ & NR & NR & NR & Home \\
\hline
\end{tabular}




\begin{tabular}{|c|c|c|c|c|c|c|c|}
\hline $\begin{array}{l}\text { Study, Year, } \\
\text { PMID }\end{array}$ & Intended Comparison & Arm & Components (Specific Exercises/Strategies) & $\begin{array}{l}\text { Progression } \\
\text { (Appropriate) }\end{array}$ & Personnel & Mode of Delivery & Setting \\
\hline \multirow[t]{2}{*}{$\begin{array}{l}\text { Nelson, } \\
2020, \\
32026820, \\
\text { Australia }\end{array}$} & $\begin{array}{l}\text { Telerehabilitation and } \\
\text { technology-based } \\
\text { home exercise } \\
\text { program vs. in-person } \\
\text { physiotherapy care and } \\
\text { paper-based home } \\
\text { exercise program }\end{array}$ & Telerehabilitation & $\begin{array}{l}\text { 1. Strength } \\
\text { 1.11 Hip abduction in sidelying (position unclear) } \\
1.12 \text { Hip abduction in standing (position unclear) } \\
\text { 1.13 Hip abduction in supine (position unclear) } \\
\text { 1.17 Hip extension in sidelying (position unclear) } \\
\text { 1.18 Hip extension in prone (position unclear) } \\
\text { 1.19 Hip extension in standing (position unclear) } \\
\text { 1.20 Hip flexion in sidelying (position unclear) } \\
1.21 \text { Hip flexion in sitting (position unclear) } \\
1.22 \text { Hip flexion in standing (position unclear) } \\
\text { 1.23 Hip flexion in supine (position unclear) } \\
\text { 1.28 Knee extension machine (one-leg) (position } \\
\text { unclear) } \\
\text { 1.29 Knee extension machine (two-legs) (position } \\
\text { unclear) } \\
\text { 1.30 Knee extension AAROM in sitting or supine } \\
\text { (short- or long arc quad) (position unclear) } \\
\text { 1.31 Knee extension in sitting or supine (long arc } \\
\text { quad) (position unclear) } \\
\text { 1.32 Knee extension in sitting or supine (short arc } \\
\text { quad) (position unclear) } \\
\text { 5. Task specific training } \\
\text { 5.8 Gait training } \\
\text { 6. Patient education } \\
\text { 6.2 Home exercise program }\end{array}$ & $\mathrm{N}(\mathrm{NA})$ & $\begin{array}{l}\text { Physical } \\
\text { therapist }\end{array}$ & $\begin{array}{l}\text { Remote via } \\
\text { telephone }\end{array}$ & Home \\
\hline & No data & In-person usual care & $\begin{array}{l}\text { 1. Strength } \\
\text { 1.11 Hip abduction in sidelying (position unclear) } \\
\text { 1.12 Hip abduction in standing (position unclear) } \\
\text { 1.13 Hip abduction in supine (position unclear) } \\
1.17 \text { Hip extension in sidelying (position unclear) } \\
1.18 \text { Hip extension in prone (position unclear) } \\
\text { 1.19 Hip extension in standing (position unclear) } \\
\text { 1.20 Hip flexion in sidelying (position unclear) } \\
\text { 1.21 Hip flexion in sitting (position unclear) } \\
\text { 1.22 Hip flexion in standing (position unclear) } \\
\text { 1.23 Hip flexion in supine (position unclear) } \\
\text { 1.28 Knee extension machine (one-leg) (position } \\
\text { unclear) } \\
\text { 1.29 Knee extension machine (two-legs) (position } \\
\text { unclear) } \\
\text { 1.30 Knee extension AAROM in sitting or supine } \\
\text { (short- or long arc quad) (position unclear) } \\
\text { 1.31 Knee extension in sitting or supine (long arc } \\
\text { quad) (position unclear) } \\
\text { 1.32 Knee extension in sitting or supine (short arc } \\
\text { quad) (position unclear) } \\
\text { 5. Task specific training } \\
\text { 5.8 Gait training } \\
\text { 6. Patient education } \\
\text { 6.2 Home exercise program }\end{array}$ & $\mathrm{N}(\mathrm{NA})$ & $\begin{array}{l}\text { Physical } \\
\text { therapist }\end{array}$ & $\begin{array}{l}\text { Self-guided } \\
\text { (unsupervised); In- } \\
\text { person }\end{array}$ & $\begin{array}{l}\text { Home; Physical } \\
\text { therapy/rehabilitation } \\
\text { facility (outpatient) }\end{array}$ \\
\hline
\end{tabular}




\begin{tabular}{|c|c|c|c|c|c|c|c|}
\hline $\begin{array}{l}\text { Study, Year, } \\
\text { PMID }\end{array}$ & Intended Comparison & Arm & Components (Specific Exercises/Strategies) & $\begin{array}{l}\text { Progression } \\
\text { (Appropriate) }\end{array}$ & Personnel & Mode of Delivery & Setting \\
\hline \multirow[t]{2}{*}{$\begin{array}{l}\text { Rao, } \\
2021 \\
33863614 \\
\text { USA }\end{array}$} & $\begin{array}{l}\text { Home physical therapy } \\
\text { vs. formal physical } \\
\text { therapy }\end{array}$ & $\begin{array}{l}\text { Home physical } \\
\text { therapy }\end{array}$ & $\begin{array}{l}\text { 1. Strength } \\
\text { 1.2 Bridges Two-legged (supine hip extension) } \\
\text { 1.12 Hip abduction in standing } \\
\text { 3. Flexibility } \\
\text { 3.10 Knee extension AROM } \\
\text { 6. Patient education } \\
\text { 6.2 Home exercise program }\end{array}$ & $\mathrm{Y}(\mathrm{N})$ & $\begin{array}{l}\text { None } \\
\text { (unsupervised) }\end{array}$ & Self-guided & Home \\
\hline & No data & $\begin{array}{l}\text { Formal physical } \\
\text { therapy }\end{array}$ & $\begin{array}{l}\text { 1. Strength } \\
\text { 3. Flexibility } \\
\text { 6. Patient education } \\
6.2 \text { Home exercise program }\end{array}$ & $\mathrm{Y}(\mathrm{Y})$ & $\begin{array}{l}\text { Physical } \\
\text { therapist; } \\
\text { None } \\
\text { (unsupervised) }\end{array}$ & $\begin{array}{l}\text { In-person; self- } \\
\text { guided }\end{array}$ & $\begin{array}{l}\text { Physical } \\
\text { therapy/rehabilitation } \\
\text { facility (outpatient); } \\
\text { home }\end{array}$ \\
\hline \multirow[t]{2}{*}{$\begin{array}{l}\text { Smith, } \\
2009 \\
19876883 \\
\text { UK }\end{array}$} & $\begin{array}{l}\text { Standard gait re- } \\
\text { education program and } \\
\text { bed exercises vs. } \\
\text { standard gait re- } \\
\text { education program } \\
\text { alone }\end{array}$ & $\begin{array}{l}\text { Gait re-education } \\
\text { program \& bed } \\
\text { exercises }\end{array}$ & $\begin{array}{l}\text { 1. Strength } \\
\text { 1.8 Gluteal Sets } \\
\text { 1.23 Hip flexion in supine } \\
\text { 1.43 Quad sets } \\
\text { 3. Flexibility } \\
\text { 3.1 Ankle pumps } \\
\text { 3.6 Heel slides } \\
\text { 5. Task specific training } \\
\text { 5.8 Gait training } \\
\text { 5.15 Stair training }\end{array}$ & $\mathrm{Y}(\mathrm{Y})$ & $\begin{array}{l}\text { Physical } \\
\text { therapist }\end{array}$ & In-person & $\begin{array}{l}\text { Acute inpatient } \\
\text { (postoperative) }\end{array}$ \\
\hline & No data & $\begin{array}{l}\text { Gait re-education } \\
\text { program only }\end{array}$ & $\begin{array}{l}\text { 5. Task specific training } \\
\text { 5.8 Gait training } \\
\text { 5.15 Stair training }\end{array}$ & $\mathrm{Y}(\mathrm{Y})$ & $\begin{array}{l}\text { Physical } \\
\text { therapist }\end{array}$ & In-person & $\begin{array}{l}\text { Acute inpatient } \\
\text { (postoperative) }\end{array}$ \\
\hline \multirow[t]{2}{*}{$\begin{array}{l}\text { Winther, } \\
2020 \\
31977324 \\
\text { Norway }\end{array}$} & $\begin{array}{l}\text { Maximal strength } \\
\text { training vs. } \\
\text { conventional } \\
\text { rehabilitation }\end{array}$ & $\begin{array}{l}\text { Strengthening } \\
\text { exercise }\end{array}$ & $\begin{array}{l}\text { 1. Strength } \\
\text { 1.12 Hip abduction in standing } \\
\text { 1.38 Leg Press (one leg) }\end{array}$ & $\mathrm{Y}(\mathrm{Y})$ & $\begin{array}{l}\text { Physical } \\
\text { therapist }\end{array}$ & In-person & $\begin{array}{l}\text { Physical } \\
\text { therapy/rehabilitation } \\
\text { facility (outpatient) }\end{array}$ \\
\hline & No data & Standard care & $\begin{array}{l}\text { [Range of exercises suggested but not } \\
\text { standardized or described in sufficient detail to } \\
\text { code; includes strength exercises and warm up on } \\
\text { cycle, step, or treadmill] }\end{array}$ & $N(N A)$ & $\begin{array}{l}\text { Physical } \\
\text { therapist }\end{array}$ & In-person & $\begin{array}{l}\text { Physical } \\
\text { therapy/rehabilitation } \\
\text { facility (outpatient) }\end{array}$ \\
\hline
\end{tabular}

Abbreviations: $\mathrm{AAROM}=$ assisted active range of motion, $\mathrm{ADL}=$ activities of daily living, $\mathrm{AROM}=$ active range of motion, $\mathrm{NA}=$ not applicable, $\mathrm{NR}=$ not reported, $\mathrm{PROM}=$ passive range of motion, $\mathrm{ROM}=$ range of motion. 
Table C-4.3. KQ 4. Risk of bias assessment for primary studies - randomized controlled trials (RCTs)

\begin{tabular}{|c|c|c|c|c|c|c|c|c|c|c|c|c|}
\hline Study, Year, PMID & Random & Allocation & $\begin{array}{l}\text { Blinding, } \\
\text { Participants }\end{array}$ & $\begin{array}{l}\text { Blinding, } \\
\text { Providers }\end{array}$ & $\begin{array}{l}\text { Blinding, Outcome, } \\
\text { Obj / Subj }\end{array}$ & Dropout & $\begin{array}{l}\text { Reporting } \\
\text { Bias }\end{array}$ & Other & Population & Intervention & Outcomes & Overall \\
\hline $\begin{array}{l}\text { Austin, 2017, } \\
28419032\end{array}$ & Low & Low & High & High & High & Low & Low & Low & No & No & No & Moderate \\
\hline $\begin{array}{l}\text { Beck, 2019, } \\
30782304\end{array}$ & Low & High & High & High & Unsure & Low & Low & Low & No & No & No & High \\
\hline $\begin{array}{l}\text { Coulter, 2017, } \\
28506775\end{array}$ & Low & High & High & High & Low & Low & Low & Unsure & No & No & No & High \\
\hline $\begin{array}{l}\text { Giaquinto, NR, } \\
19282040\end{array}$ & Low & Low & Low & Low & Unsure & Low & Low & Low & No & No & No & Low \\
\hline $\begin{array}{l}\text { Heiberg, 2012, } \\
22170790\end{array}$ & Low & Low & High & High & Low & Low & Low & Low & No & No & No & Moderate \\
\hline $\begin{array}{l}\text { Liebs, 2010, } \\
20360503\end{array}$ & Low & Low & High & High & High & Low & Low & Low & No & No & No & Moderate \\
\hline $\begin{array}{l}\text { Liebs, 2012, } \\
22196125\end{array}$ & Low & Low & High & High & High & Low & Low & Low & No & No & No & Moderate \\
\hline Łyp, 2016, 27455419 & Low & Unsure & High & High & Unsure & Low & Low & Low & No & No & No & High \\
\hline $\begin{array}{l}\text { Mikkelsen, 2014, } \\
25305374\end{array}$ & Low & Low & High & High & Low & Low & Low & Low & No & No & No & Moderate \\
\hline $\begin{array}{l}\text { Monticone, 2014, } \\
24459172\end{array}$ & Unsure & Low & Low & High & Low & Low & Low & Low & No & No & No & Moderate \\
\hline $\begin{array}{l}\text { Nelson, } 2020 \text {, } \\
32026820\end{array}$ & Low & Low & High & High & Low & Low & Low & Low & No & No & No & Moderate \\
\hline $\begin{array}{l}\text { Rao, 2021, } \\
33863614\end{array}$ & Low & High & High & High & Unsure & Low & Low & Low & No & No & No & High \\
\hline $\begin{array}{l}\text { Smith, 2009, } \\
19876883\end{array}$ & Low & Low & High & Low & Low & Low & Low & Low & No & No & No & Moderate \\
\hline $\begin{array}{l}\text { Winther, 2020, } \\
31977324\end{array}$ & Low & Low & Low & High & Unsure & Low & Low & Low & No & No & No & Moderate \\
\hline
\end{tabular}

PMID $=$ Obj = objective, PubMed Identifier, Subj = subjective

From the Cochrane Risk of Bias Tool (each item rated as Low, High, Unsure, or N/A). Ratings are color coded for emphasis only.

- Random: Random sequence generation (selection bias): Selection bias (biased allocation to interventions) due to inadequate generation of a randomized sequence;

- Allocation: Allocation concealment (selection bias): Selection bias (biased allocation to interventions) due to inadequate concealment of allocations prior to assignment;

- Blinding of participants (performance bias): Performance bias due to knowledge of the allocated interventions by participants during the study;

- Blinding of personnel/care providers (performance bias): Performance bias due to knowledge of the allocated interventions by personnel/care providers during the study;

- Blinding of outcome assessor (detection bias): Detection bias due to knowledge of the allocated interventions by outcome assessors;

- Dropout: Incomplete outcome data (attrition bias): Attrition bias due to amount, nature or handling of incomplete outcome data;

- $\quad$ Reporting Bias: Selective outcome reporting (outcome reporting bias): Bias arising from outcomes being selectively reported based on the direction and/or strength of the results; - Other Bias: Bias due to problems not covered elsewhere in the table.

From the National Heart, Lung, and Blood Institute (NHLBI) Quality Assessment Tool (each item rated as No, Yes, or Unsure)

- Population: Eligibility criteria prespecified and clearly described: potentially related to selection bias;

- Intervention: Intervention clearly described and delivered consistently: potentially related to performance bias;

- Outcomes: Outcomes prespecified, clearly defined, valid, reliable, and assessed consistently: potentially related to detection bias.

Overall risk of bias assessed as HIGH, MODERATE, or LOW. 
Table C-4.4. KQ 4. Risk of bias assessment for primary studies - nonrandomized comparative studies (NRCSs) - assessment of confounding and selection bias

\begin{tabular}{|c|c|c|c|c|c|c|c|c|c|c|c|c|c|}
\hline Study, Year, PMID & 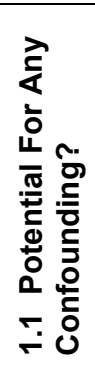 & 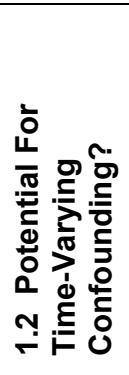 & 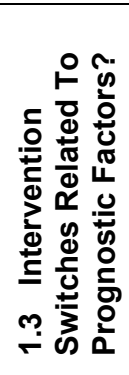 & 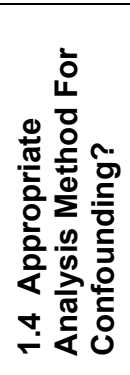 & 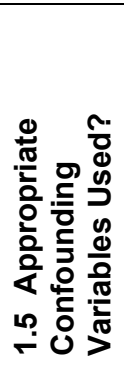 & 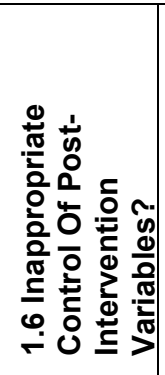 & 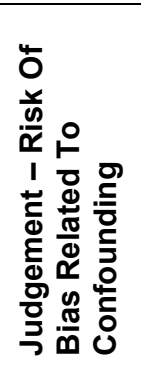 & 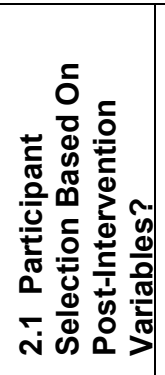 & 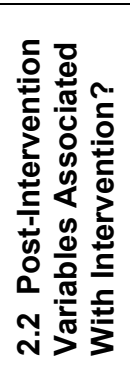 & 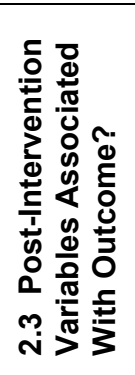 & 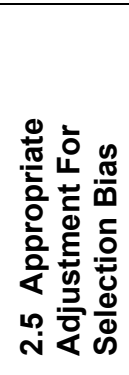 & 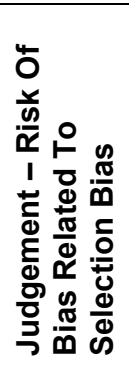 & 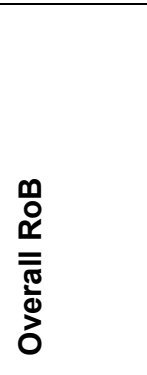 \\
\hline $\begin{array}{l}\text { Naylor, 2018, } \\
30021552\end{array}$ & No & No & No & No & $\mathrm{N} / \mathrm{A}$ & No & Low & No & $\mathrm{N} / \mathrm{A}$ & $\mathrm{N} / \mathrm{A}$ & $\mathrm{N} / \mathrm{A}$ & Low & Moderate \\
\hline
\end{tabular}

- $\quad$ PMID = PubMed Identifier, Responses to Risk of Bias in Nonrandomized Studies of Interventions (ROBINS-I) signaling questions 1.1 to 1.6 and 2.1 to 2.5 are in regular font. Each item rated as High, PY (probably High), NI (Low information), PN (probably Low), Low, or N/A (Not applicable).

- Judgments about confounding and selection bias are in bold font and each item is rated as Low, Moderate, Serious, or Critical. Ratings are color coded for emphasis only. 